\title{
AGED 539 Project
}

John Williams

Madera South High School

Winter 2014 


\section{COLLEGE OF AGRICULTURE, FOOD \& ENVIRONMENTAL SCIENCES}

CALTPSOLY

AGRICULTURE

fame: John S. Williams

FORMAL STUDY PLAN

Address: 175 Dwyer Street, Apt \#41

Date: $\quad 2 / 27 / 2014$

Telephone: $\underline{805.478 .0193}$

Madera, CA 93637

Student ID\#: 006442289

CURRENT ACADEMIC PROGRAM: Master of Agricultural Education

1. UNDERGRADUATE INFORMATION

Degree held: $\underline{B . S}$.

Institution: CSU-Fresno

Date granted: $\underline{\text { Fall } 2008}$

Major: Agricultural Education

Admission GPA: $\underline{3.68}$

2. GRADUATE ADMISSION $\square$ Classified $\square$ Conditional (list conditions below)

Qtr/Yr Admitted to Cal Poly Spring 2010

Qtr/Yr first work completed on plan Spring 2010

Qtr/Yr 7-yr limit for degree will expire Summer, 2017

3. STUDY PLAN: (List transfer or extension courses in Part $A$ of this section; Cal Poly courses in Part B.)

\begin{tabular}{|c|c|c|c|c|c|c|c|}
\hline A. Course & Units & Institution & Qtr/Yr & Grade & & & \\
\hline AGRI 280 & $\underline{4.5}$ & CSU-Fresno & Fall 2008 & $\underline{\underline{A}}$ & & & \\
\hline AGRI 281 & $\underline{4.5}$ & CSU-Fresno & Fall 2008 & $\underline{B}$ & & & \\
\hline EHD 155B & $\underline{3}$ & CSU-Fresno & Fall 2008 & CR & & & \\
\hline B. Course & $\underline{\text { Units }}$ & Grade & Qtr/Yr & Course & $\underline{\text { Units }}$ & $\underline{\text { Grade }}$ & Qtr/Yr \\
\hline AgEd S520 & 3 & A & SP 2012 & $\mathrm{AgEd} 500^{*}$ & 3 & IP & W 2014 \\
\hline AgEd S522 & 3 & A & SP 2011 & $\operatorname{AgEd} 500^{* *}$ & 3 & IP & W 2014 \\
\hline AgEd S525 & 3 & A & SP 2010 & AgEd 580 & 3 & IP & W 2014 \\
\hline AgEd S530 & 3 & A & SP 2010 & AgEd S581 & 3 & A & SP 2011 \\
\hline AgEd 539 & 6 & IP & W 2014 & BRAE S570 & 3 & B & SP 2012 \\
\hline
\end{tabular}

If courses above have variable title/content, asterisk them and give course information below: AgEd 500* Vineyard Ground Prep and Irrigation Design

$\underline{\text { AgEd } 580}$ Plant Vineyard and Train Vines

AgEd 500** Vineyard Trellis System

TOTAL UNITS IN PROGRAM 45

Check One: $\square$ Thesis $\square$ Project $\quad$ Internship = Units 6.0

(required in Ag. Education)

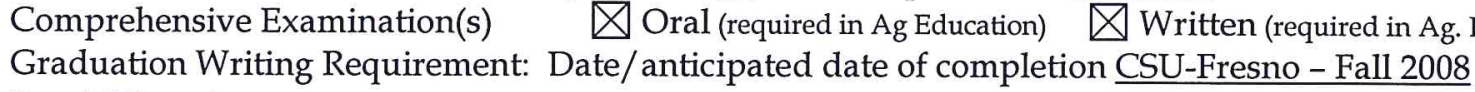

Special Requirements:

4. SIGNATURES:

Student

Date

Adviser

Date

Comm Member

Date

Comm Member

Date

Coord/Dean

Date

$R \& G P$

Date

NOTE: ANY CHANGES IN COURSES REQUIRE THE SUBMISSION OF AN "AMENDMENT TO FORMAL STUDY PLAN" FORM.

istribution: Evaluations; Adviser; Coordinator; Student; Research \& Graduate Programs

FSP form. Doc REV. 6-08 
Quality Criteria

One

Curriculum and Instruction

Madera South High School follows a career school model. In the School of Agriculture, we have full pathways and are the model for our school district. Students can choose a pathway of Plant Science, Animal Science, Power Mechanics, Floriculture, and Fabrication and Construction. Students are encouraged to pick a pathway as a freshmen and our counselors push to keep them in it throughout high school.

The plant science, animal science, and floral classes all start with the Agriscience 1 classes, the vet science then has animal care and vet aid; during students junior and senior year, the Ag. Science 3 and Veterinary Science classes alternate to complete the pathway. For the plant science pathway students take Horticulture 1, Horticulture 2, and then Viticulture and Crop Production. For the Floral Pathway Students take Floral Design, Advanced Floral Design and then Retail Floral.

The mechanics starts with Ag. Mechanics 1 and then the students choose to take the Power Mechanics emphasis or the Welding and Fabrication. As a sophomore in Power Mechanics, students take Diesel Engines, followed by ROP Small Engines or ROP Diesel Engines, these 2 classes are 2 period blocks and alternate every other year. For the welding and fabrication pathway students take Ag. Mechanics 2; followed by Ag Mech 3/ Ag Construction.

In addition to the classes in our pathways, we have a few other classes that get UC and CSU credit, our Ag. Biology course receives high school and college life science credit, our Earth Science counts for high school physical science and our Ag. Econ counts for High School and College history. In addition our floral class, which is a part of the floral pathway, received CSU/UC art credit and our Vet Science class is an A-G elective course. 
Incorporated in every class we teach at Madera South High School is an FFA, Careers and Recordkeeping unit. All of our student's record books are stored in file cabinets or in folders on the instructor's computer. Most of the teachers will even use guest speakers, during the career unit, to help and show students the opportunities they have in their career area.

Supporting documentation and evidence can be found in the following appendices:

Appendix a

Appendix b

Appendix c

Appendix d

Appendix e

Appendix g
Appendix s

Appendix $\mathrm{t}$

Appendix 11 


\section{Quality Criteria \\ Two \\ Leadership and Citizenship Development}

The Madera FFA chapter was the $17^{\text {th }}$ chapter chartered in the State of California. All students in the Madera FFA Chapter are required to participate in 3 FFA activities each semester, for a total of 6 a year. Each teacher checks this through the sign in sheets and it accounts for $10 \%$ of the semester grade. Madera FFA is a very active chapter and we participate in well above the 12 required activity and we have held the Sectional Participation Trophy as the most active chapter in the West Fresno/Madera Section for the last 6 years. Our students can participate in their choice of 3-4 fundraisers each semester, monthly FFA meetings, recruitment, Ag. Literacy Day, community service, as well as all the Sectional, Regional and State Activities.

The comprehensive program plan has been recently revised by Kristin Mckenna, it outlines the responsibilities of the teachers to participate and supervise student leadership activities. Students are graded for their participation in FFA activities and it is worth $10 \%$ of their overall grade in their Agriculture course. Many students participate in more than the required 3 activities for extra credit and they like most of the activities that our chapter officers plan for the year.

Included in all of our course curriculum is a careers unit. In this unit we talk about potential jobs students can get, as well as the organizations that help agriculture; such as water districts, Farm Bureau's, and USDA.

The Madera FFA has 7 fully credentialed agriculture teachers who hold both a single subject credential in agriculture and the ag. specialist degree from Fresno State. We all teach a full schedule of 5 classes each and the 2 FFA advisors have sold a prep to teach the zero period Ag. Leadership class. 
Our community service committee hosts the Ag. Literacy Day for 2,500 kindergarten and $1^{\text {st }}$ grade students, Coats for Kids, Canned Food Drive, and Bowling for Kids for Children's Hospital.

Appendix a

Appendix b

Appendix c

Appendix i

Appendix j
Appendix k

Appendix 1

Appendix $\mathrm{p}$

Appendix q

Appendix t 
Williams, John 2014

\section{Quality Criteria \\ Three \\ Practical Application of Occupational Skills}

I take pride in the fact that the program at Madera South emphasizes the need to continue growing our CTE courses to prepare student for industry needs. Our classes mimic real life job sites and often times our students are ready for entry level positions when they graduate from our program. In my diesel/small engine program, students are treated like employees, they must keep track of time they put in the shop and are paid (Grade) for the work they complete. They must also show up on time and if they are late, they lose points (money) just as if they were late for a job. The other mechanics classes do the same and the horticulture classes use time cards that students must fill out every time they work in the green house.

We also have a very strong SAE program that is very balanced and diverse. We have our regular show kids who show at both Madera and Chowchilla fairs. We also have kids who work on their family farms or in their family business and we have students who are farm laborers and work in the fields during the summers and weekends. These students took a lot of time to try and get them to show value in the work that society typically looks down upon, but not our students in this area are receiving their state and American degrees for their work. Teachers supervise these students based on their strengths and also if they have the student in their class. We share the responsibility to make sure we do project visits each semester. At every project visit we have forms that we fill out to keep a record of the student's project and student must work in their record books. 
We have 7 vehicles that belong to the agriculture department, we are all assigned to a vehicle to help keep them maintained properly and also to use as needed when we are out on project visits and other activities. Our fuel and maintenance is part of the district match for our Ag Incentive Grant.

Appendix a

Appendix b

Appendix c

Appendix d

Appendixe

Appendix $f$

Appendix g

Appendix h

Appendix i

Appendix I

Appendix m
Appendix $n$

Appendix o

Appendixp

Appendix $q$

Appendix s

Appendix u

Appendix v

Appendixy

Appendix z

Appendix kk

Appendix mm

Appendix oo 


\author{
Quality Criteria \\ Four \\ Qualified and Competent Personal
}

There are seven Ag. Teachers in the Madera South High School Agriculture Department; the years of service in our department ranges from two years all the way to thirty years. One interesting thing is that four of the seven teachers got their first job at Madera South and have been here since. All seven of us hold single subject agriculture credentials and agriculture specialist credentials from California State University, Fresno.

Many of the agriculture teachers at Madera South have gone through much training in effective teaching practices and we all follow our districts educational focus, of checking for understanding, re-teaching, and bell to bell instruction. All seven of us attend regional, sectional and state CATA meetings. All of our young teachers have attended new professionals each year, approximately five of us a year will attend roadshows or other professional development opportunities. The department head gets an additional prep period as well as a stipend in order to conduct the activities of the Madera South Agriculture Program.

In terms of our counselors and administrators, we are very fortunate since they all encourage students to continue in their selected pathways by choosing courses that are next in their pathway sequence. They also regularly attend our advisory meetings and help out when we need an extra hand at our banquets and will also judge if we need them to at section and regional contests. 
Williams, John 2014

Supporting documentation and evidence can be found in the appendices:

Appendix c

Appendix i

Appendix m

Appendix q

Appendix $\mathrm{t}$
Appendix v

Appendix w

Appendix z

Appendix aa

Appendix bb
Appendix ee

Appendix kk

Appendix 11

Appendix o 


\section{Quality Criteria}

Five

Facilities, Equipment, and Materials

The Madera South High School Agriculture Department was built 8 years ago; it is a twenty-acre, on campus school farm including 4 barns, 3 science classrooms, 4 shops and adjoining classrooms, a floral shop and laboratory, arena for horses, and 3 acres of pasture for large animals, as well as greenhouse facilities. Recently we planted a 2 acre vineyard that will be in full production by fall of 2015 . We have 4 varieties of wine grapes that will be student managed and it is the starting point of creating an enology program at Madera South High School. It is a very modern and state of the art facility and we frequently have other high schools and universities come out to look at it to change their own facilities. To get the facilities we have today, our advisory had started to negotiate and bargain for it 30 years ago and I am fortunate they did, because I am truly pleased with everything we have and am very fortunate to them.

In terms of the equipment being used in our facilities, these were not included when they built the farm, so we try and replace things, in rotation, in order to stay on top of industry trends and so that way our equipment matches that being used by industry in Madera. We purchase this through grants, or Agriculture Incentive, Perkins Funding, ROP and our Site Budget. All of our facilities are handicapped accessible so that they can learn and experience everything we do in our classes.

All of the classrooms on campus are equipped with a computer, and a projector tied into it. In the agriculture department we have further improved our technology through our own money to purchase Promethium boards, document cameras and 
additional computers in many of our classrooms. These additional technologies are nice to have for classroom instruction.

Some of the perks that I have from my facility are that I have a classroom and a shop. This way I can teach concepts in class and then the students are able to showcase what they learned in my shop. I would say that I do not have nearly enough storage as I would like, I have more engines than I know what to do with along with various pieces of equipment that needs to be stored. There are future plans set to build a storage building, but I do not know if this will happen before I retire.

I truly feel fortunate every day to have the facilities we have, however our facilities still are not complete. We have a very detailed plan to develop the farm in the future. Also within the next year we will be planting a variety block of citrus and fruit trees by the greenhouse. Both of these projects will increase students SAE projects. Down the road further is our dream of being able to build a vet science laboratory on the farm, where the animal science classes can perform routine procedures on small animals. The other thing down the road is an enclosed storage facility for our tractors, trailers and trucks.

Supporting documentation and evidence can be found in the appendices:

Appendix $n$

Appendix $p$

Appendix q

Appendix cc
Appendix gg

Appendix hh

Appendix kk 
Williams, John 2014

\section{Quality Criteria Six}

Community, Business, and Industry Involvement

Our agriculture advisory committee is made up of a minimum of 3 representatives from each career pathway, administrators from both our high school campus and district, parents, all 7 agriculture teachers and a school counselor. We meet as a large group twice a year and as subcommittees, as the need arises. We have a special committee in place for the implementation and design of our schools orchards and vines. At each advisory committee meeting, one pathway does a pathway presentation with current classes, projects and future plans and goals. From there the advisory committee gives advice and helps to shape the curriculum being taught from the current industry trends. Our advisory committee moves quickly on the things that we need, my job when I got hired was to plant permanent crops on our school farm. With the help of some of these members we now have a 2 acre vineyard that was at no cost to our department. Although they are not a boosters group, their influence in the community to get things donated has helped out tremendously. They truly have been a huge help with everything.

Our advisory committee reviews and updates our curriculum guides every 2 years in subcommittees, they also help with any issues that may come up with the school board. While looking at curriculum guides, they also look at textbooks and supplemental materials to make sure they are pertinent to the material being taught. The agendas and invitation to the meetings are sent out 3 weeks prior and minutes are sent to each member within one week. We always make sure we provide dinner for our members as well. 
Williams, John 2014

Supporting documentation and evidence can be found in the appendices:

Appendix q

Appendix ee

Appendix ff 
Williams, John 2014

\section{Quality Criteria}

Seven

Career Guidance

At the beginning of each school year every student enrolled in the agriculture classes fill out their course career planning guides. This helps out our 2 counselors designated to the school of agriculture tremendously, because when it comes time to register, students know what class they are supposed to take next in their respected pathway. These sheets are kept in their permanent files.

For the last 3 years we haven't been allowed to do any recruitment outside of our feeder schools. The extent of our recruitment, was being allowed to send a PowerPoint with our activities director and let her leadership students present it at $8^{\text {th }}$ grade enrollment. After approaching the school board and administration, we got approved to recruit at all schools in the district. Since most of the county schools are agriculture based, and our schools feed into Madera North and not us, we found it important to get more of these 4-H and agriculture families in our program. We went out to all $8^{\text {th }}$ grade classes in our district, and the private catholic school, and presented for an hour. The students gave a 10 minute presentation on each pathway and then the FFA. Students were given information to take home on the Career School Transfers and $8^{\text {th }}$ grade parent night that was held at the school farm. This didn't really help much as far as transfer coming in; we are now focusing more on the students on our campus that we have missed. Our program is now impacted to the point that if a student wants to take an ag class, they must switch careers schools to be in our classes.

Our capstone classes are full to the brim and we have approximately 150 program completers every year. We take pride in having 5 complete pathways for students to take part in 
so that they are ready for the workforce or college when they graduate. Our program also has a retention of 2.7 years which is one of the highest of all ag programs in the state.

Supporting documentation and evidence can be found in the appendices:

Appendix a

Appendix b

Appendix c
Appendix k

Appendix $n$

Appendix o
Appendix p

Appendix s

Appendix nn 


\section{Quality Criteria \\ Eight \\ Program Promotion}

Our main program promotion and recruitment activity is our annual Ag. Literacy Day; previously called Pumpkin Festival. It is a 2-day event in October, where more than 2,000 Kindergarten and $1^{\text {st }}$ grade students in the district, come out to the farm to participate in story time, plant a pumpkin, and apples journey, and a petting zoo activity; at the end each student gets a personal pumpkin. This event is one remembered by many of our students, and since it has been going on for 13 years, most of our high school students remember this activity from when they were in $1^{\text {st }}$ grade. We send out monthly updates with our happenings and placing's at contest to everyone in the district via email, as well as the Madera County Ag. Boosters and Ag. Advisory committees. Our Public Relation committee produces 2 newsletters a semester and sends them out to the same people and parents. Our Fall and Spring awards banquet always reaches occupancy in our cafeteria of 350 , and we make frequent trips in classes, and with teams to industry locations. During FFA week we have activities for all of campus and open our FFA meeting up to anyone during that week. The one thing we need to improve on is our visibility in our local newspaper the Madera Tribune. Although we have a few articles a year in their already, our goal is to start getting in a minimum of 1 article per month, which will widen our publicity in the whole community of Madera. This is increasingly important with the current budget cuts we are facing.

Our program brochure is our easiest source to pass out to interested students as well as our Program of Activities; we have extra copies of both on hand at all times. 
Supporting documentation and evidence can be found in the appendices:

Appendix I

Appendix J

Appendix M
Appendix P

Appendix Q

Appendix EE
Appendix KK

Appendix $\mathrm{OO}$ 


\section{Quality Criteria \\ Nine \\ Program Accountability and Planning}

In terms of program accountability we are pretty good about getting all of our reports filed in time. This past year we were due for an advisory committee review. So at our September advisory committee we broke up into groups and reviewed each of the quality criteria to see what we meet and what we needed to improve on. In terms of onsite reviews we haven't had one since 2011 , no the state staff are not allowed to travel far from their offices so we are not sure when we will have another review. Our comprehensive program plan has been updated this year and the department head tries to stay on top of it so that we do not get behind.

We send a follow up survey in August of every year to all of our program completers; typically only $50 \%$ of these are returned which leaves us to make phone calls to get the rest of our needed information. We get a wide variety of responses on these but the majority are attending a 2 or 4 year college and many of them are majoring in Agriculture Education. We also see a good majority working full time in welding or engines shops, which shows those programs are doing their job with training and keeping up partnerships in our community. Perhaps my favorite part of the survey is to look at what the students consider to be the part of the program that helped them the most and what parts need improvement.

Madera South gets many different forms of funding, we get a department budget each year from the school of approximately $\$ 8,000$ most of this is spent on gas for the welding and floral classes, we also have 2 classes that get an ROP budget for their supplies, in addition those of us who teach science classes do get some basic supplies 
from that department, however the majority of our funding comes from Ag. Incentive Grant and Perkins. The district actually over matches our funds, they pay for fuel for our vehicles, maintenance, and we get approximately $\$ 25,000$ in open PO's to local business, this money is used to maintain and improve our school farm. We have PO's to the irrigation company, 2 hardware stores, 2 feed stores, the local nursery, engine shop, welding shop, and a lumber yard. This money is super important to our classes for example I have $\$ 1,000$ to spend at the mower shop every year, it is primarily used on parts for the engines we work on in class.

Supporting documentation and evidence can be found in the appendices:

Appendix C

Appendix $\mathrm{p}$

Appendix q

Appendix s
Appendix $\mathrm{t}$

Appendix u

Appendix bb

Appendix gg
Appendix hh

Appendix ii

Appendix oo 
Quality Criteria

Ten

Student-Teacher Ration

By far the biggest weakness for the Madera South Agriculture Department is our class sizes. Our district doesn't follow Criteria 10 at all. All of our science and vet and animal elective classes are capped at 38 students and frequently we will carry that many students all year. They cap our shop classes at 32 but it frequently will go over 35. The biggest issue is that our counselors do not have anywhere to put their students, especially in elective courses because there are not enough electives on our campus. They figure the best place to put them is in the most dangerous classes on campus. We have fought on this topic, but our district does not see the importance of this and either does the site administration. I do not see this ever changing in the future because when you have 2,800 students on one campus, they have to put the students somewhere.

Supporting documentation and evidence can be found in the appendices: 


\section{Quality Criteria \\ Eleven \\ Full Year Employment}

All seven of the teachers receive an extended contract. Our extended contract amount is $20 \%$ of our salary. We are required to work 36 addition non working school days; these can be holidays, summer, or weekends, a day is considered 6 hours. Most of the teachers meet this prior to summer vacation but are still out here a minimum of 3 days a week during the summer, since we have animals on the farm. We are required to log these hours in a calendar which is turned in at the end of the year.

At Madera South high school the SAE periods were lost about 15 years ago, however both the Department Head still gets an SAE period. Also with the current budget situation I don't see these coming back in the near future. We also have a department head stipend of $\$ 2,000$, a FFA advisor stipend for the lead advisor of $\$ 1,400$ and an assistant FFA advisor stipend of $\$ 900$. These are all through our ASB on campus and added to the check monthly from August to June.

Supporting documentation and evidence can be found in the appendices:

Appendix e

Appendix $f$
Appendix $\mathrm{h}$

Appendix j
Appendix m

Appendix y 
A. Student Data Sheet 


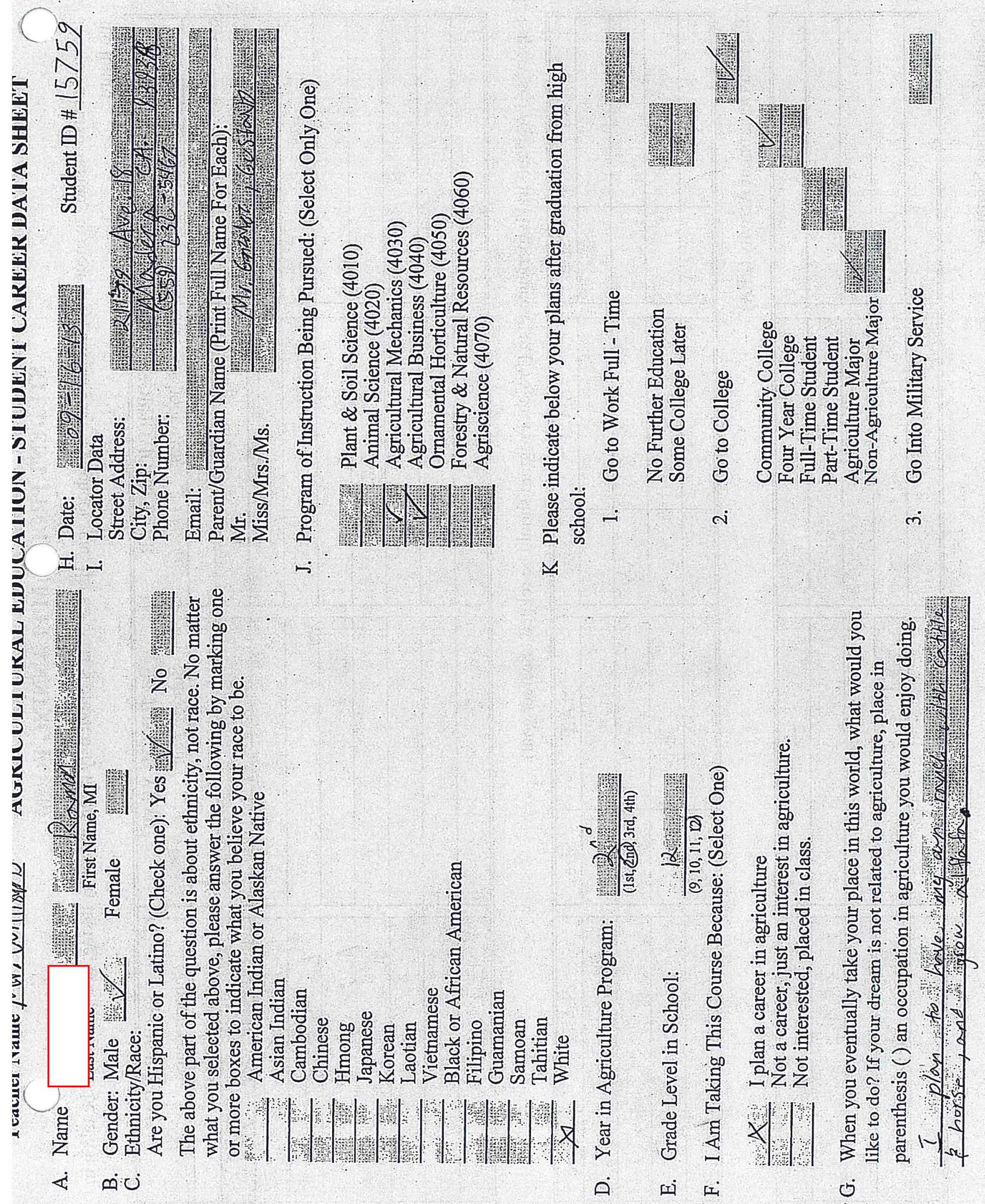




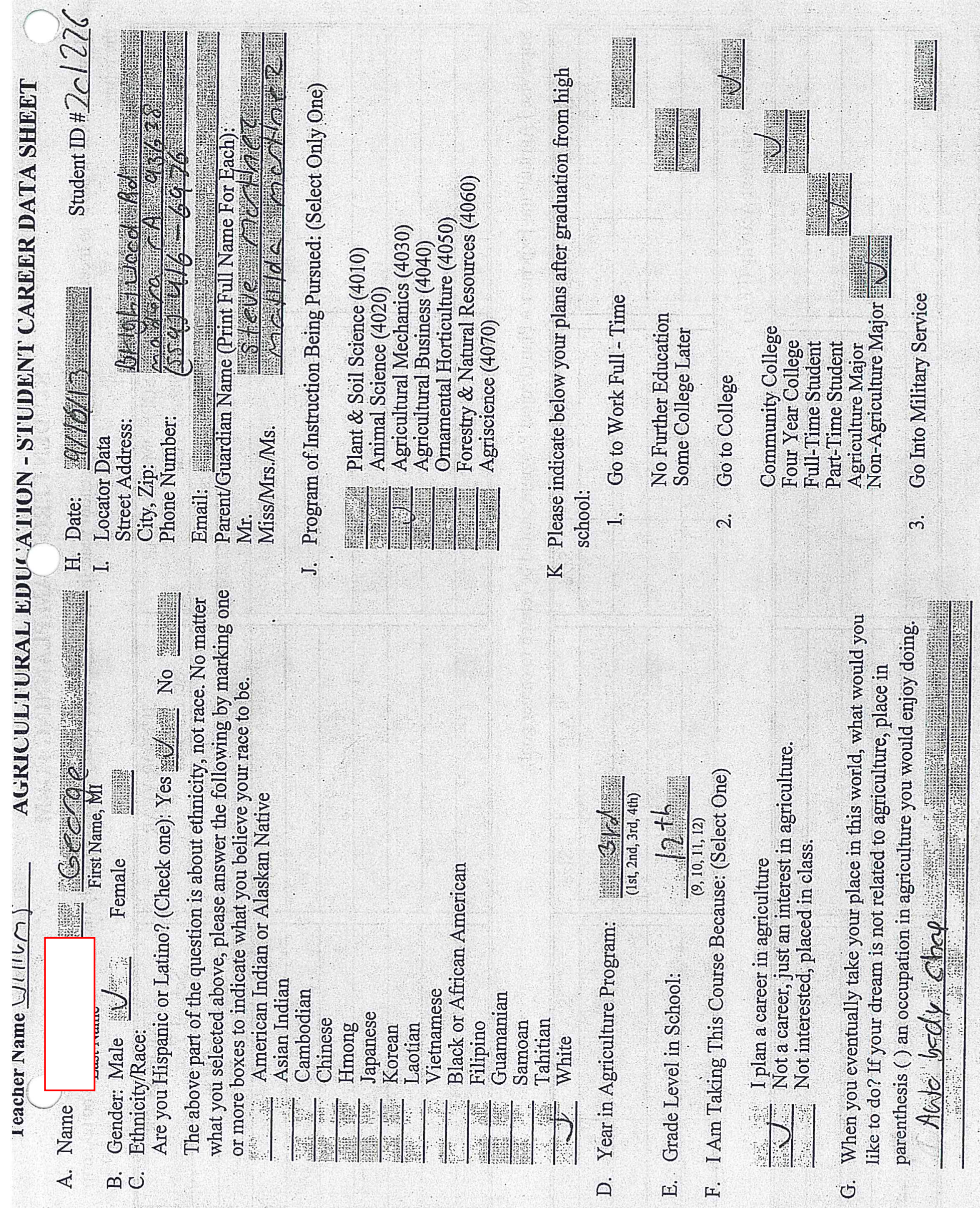




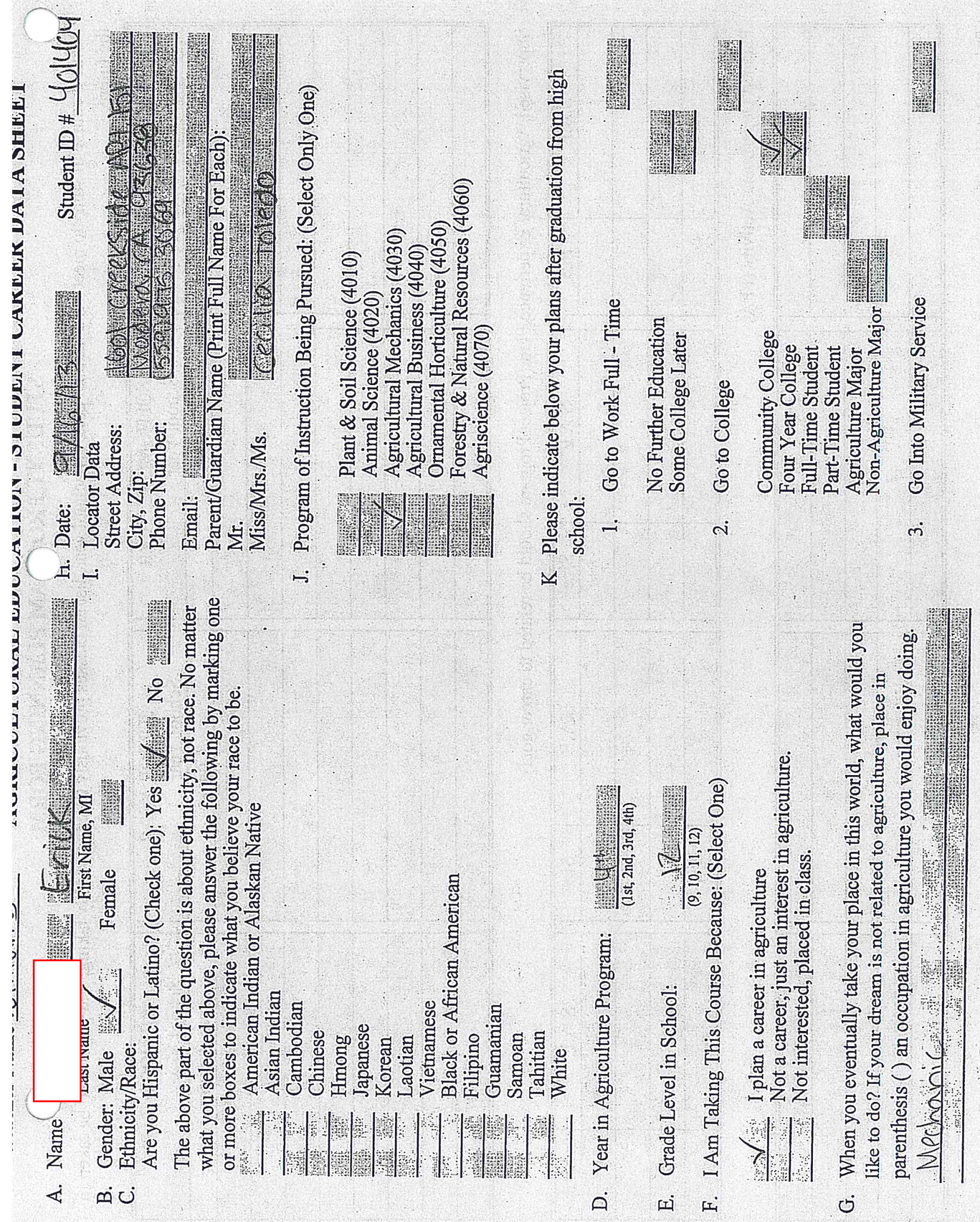



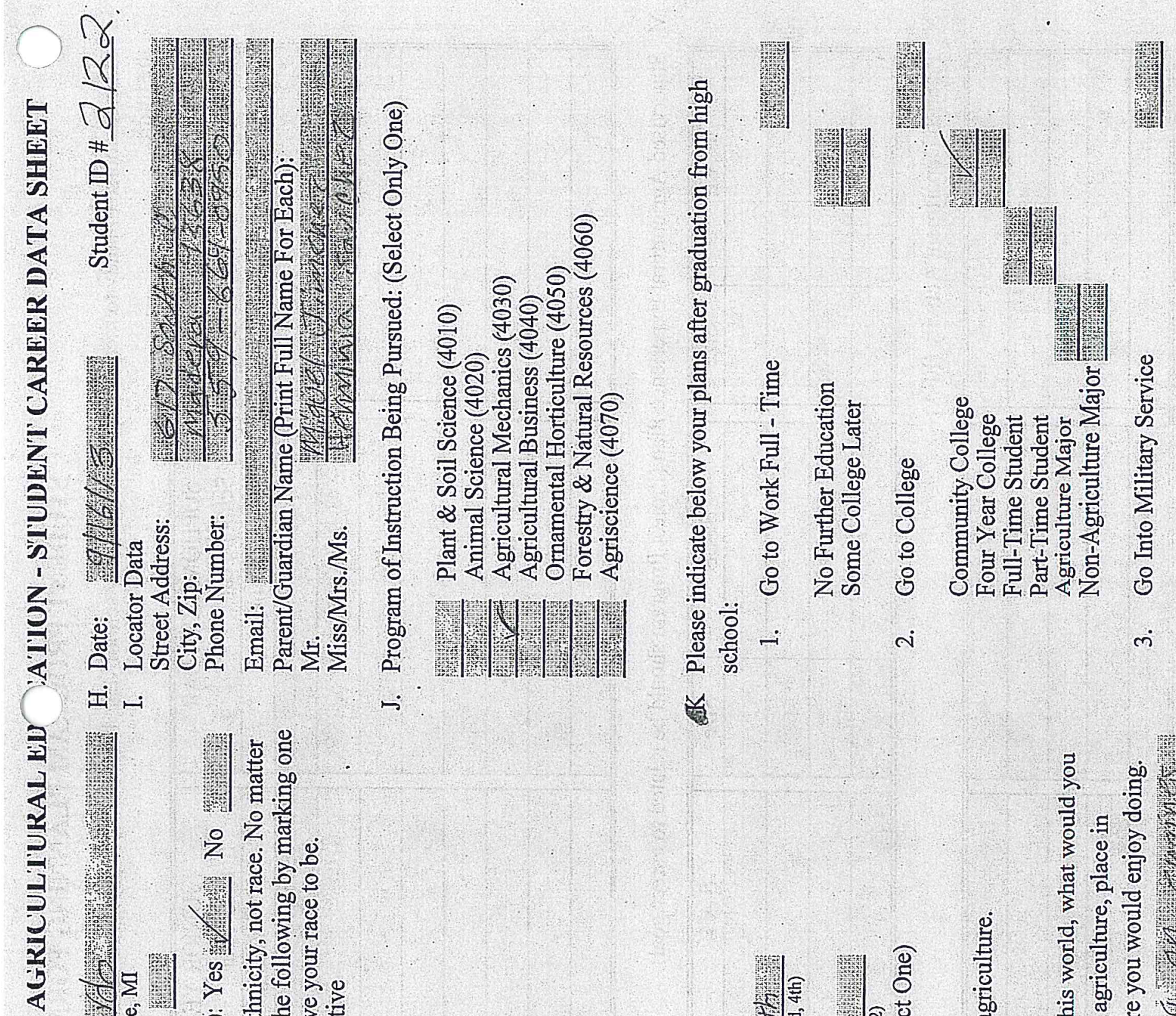

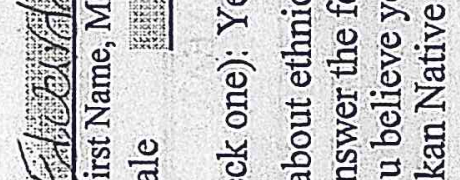

긴

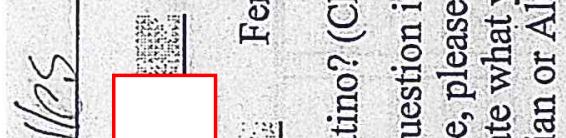

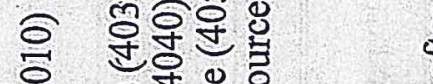

\%.ㄴ 2

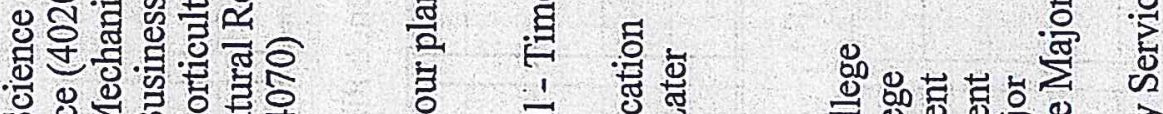

न.

\&

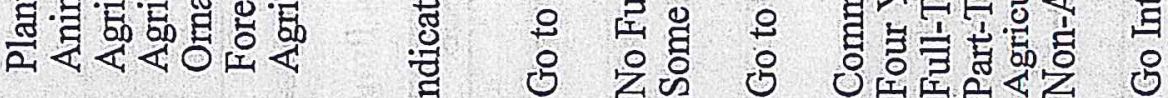

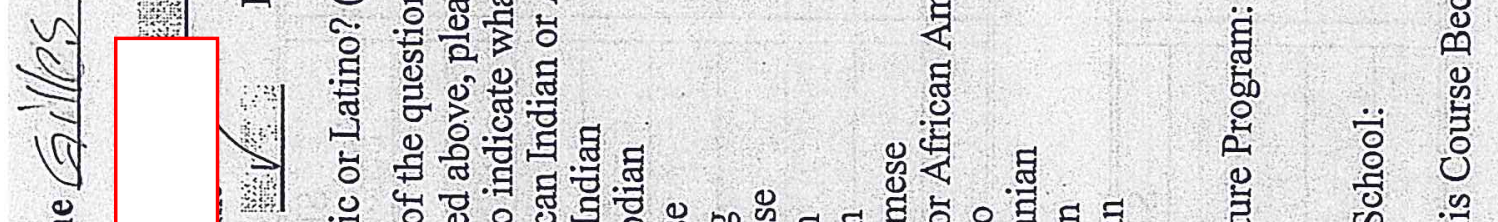

.

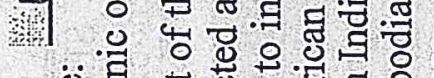

仓ं

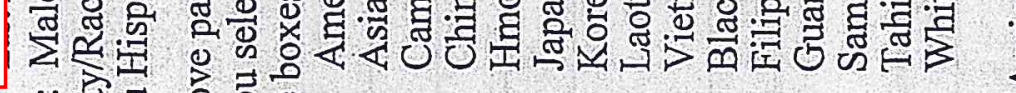

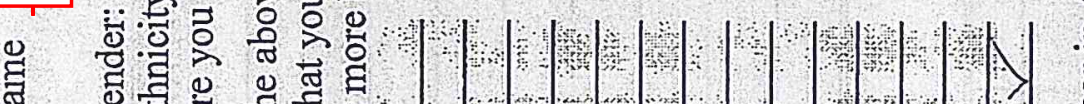

\& 


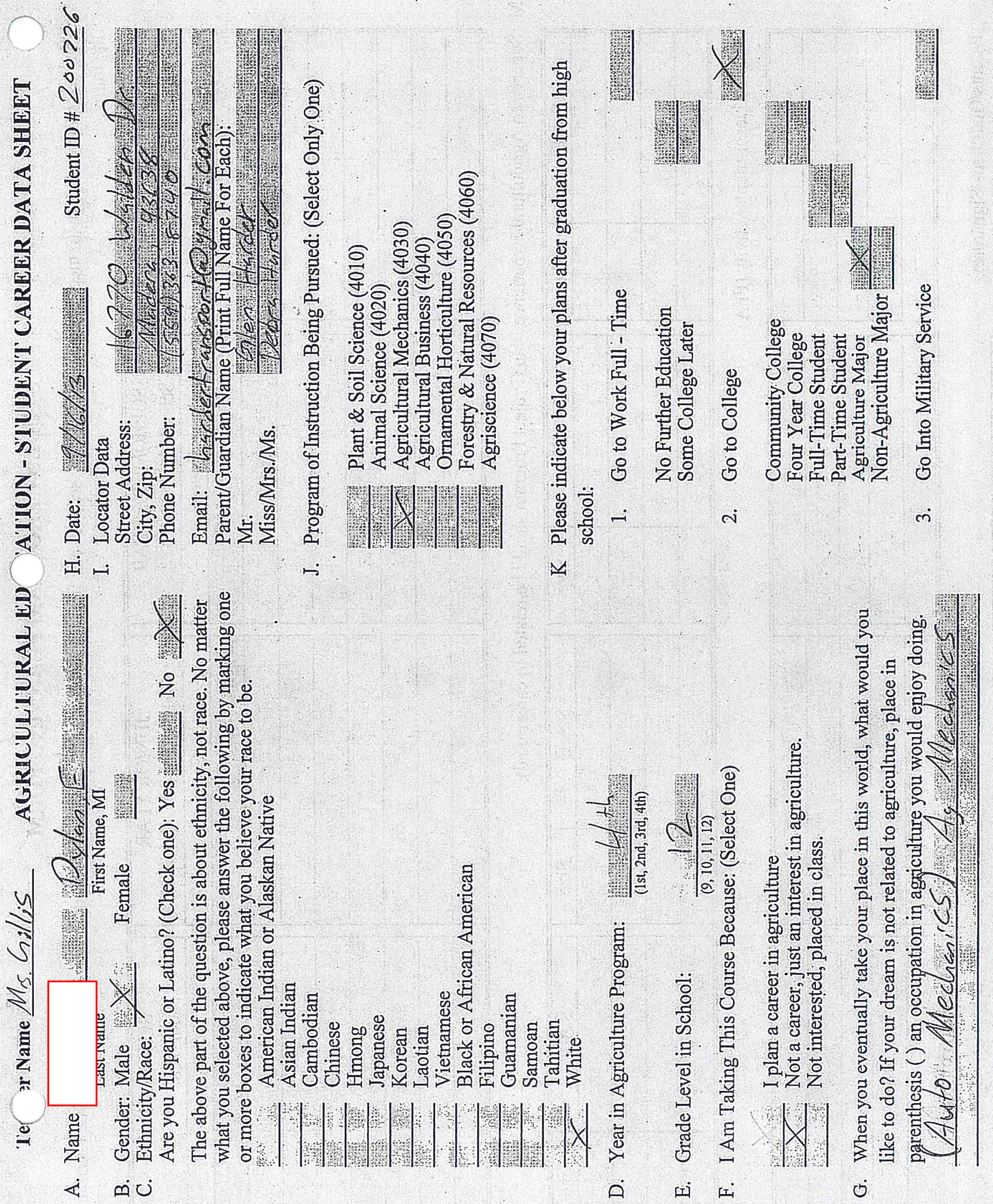




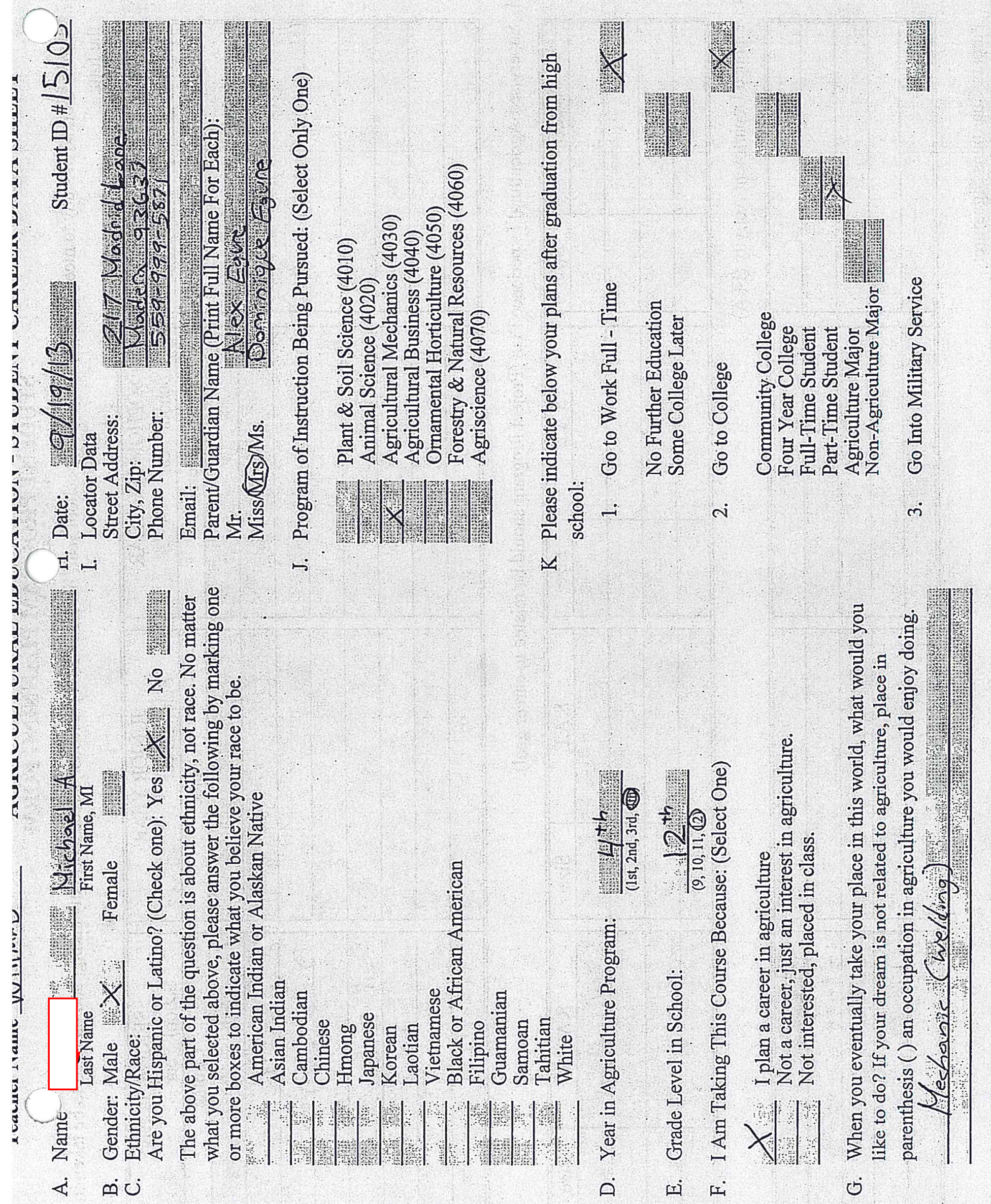



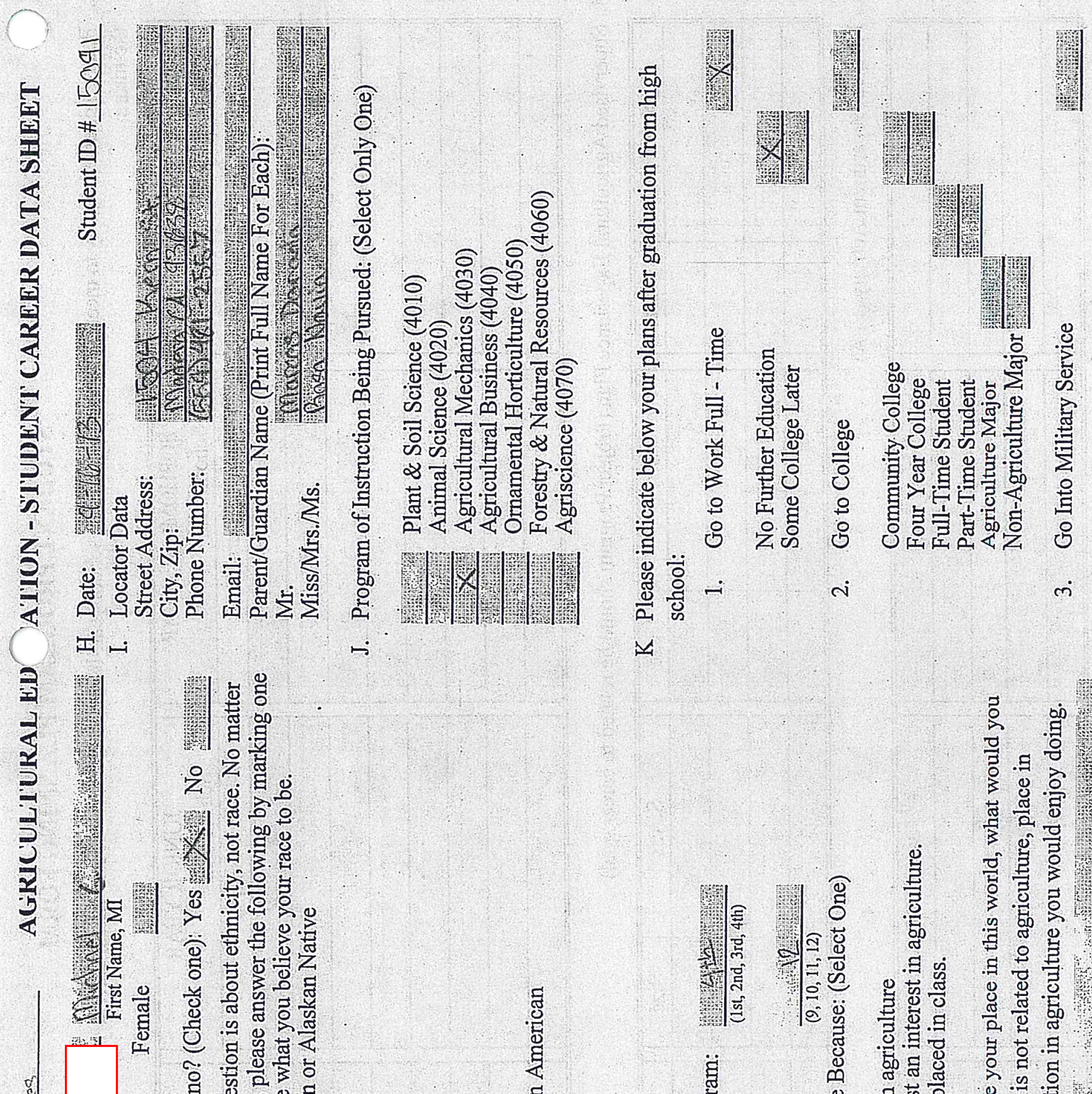

,

(5)

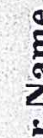

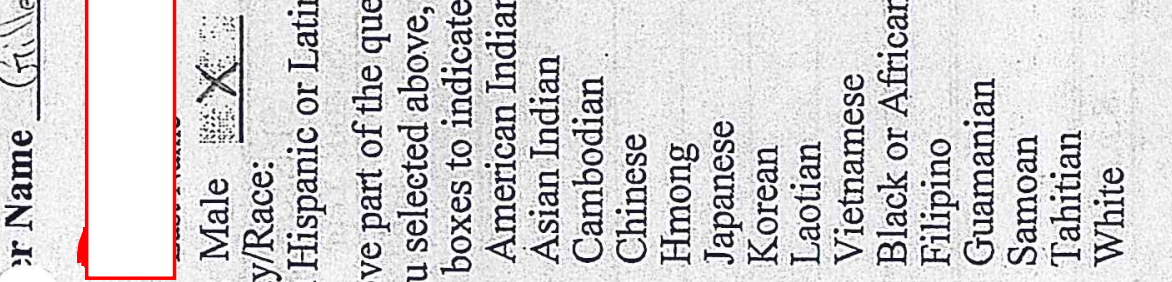

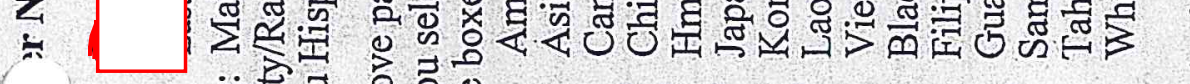

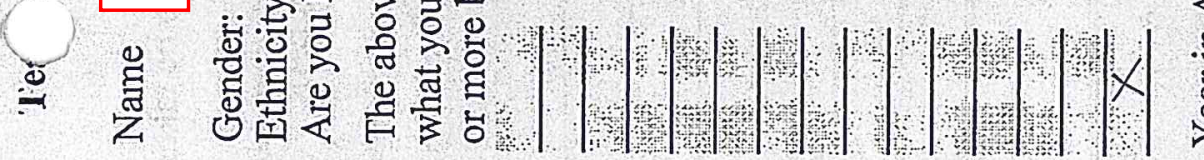
« $\oplus \dot{0}$

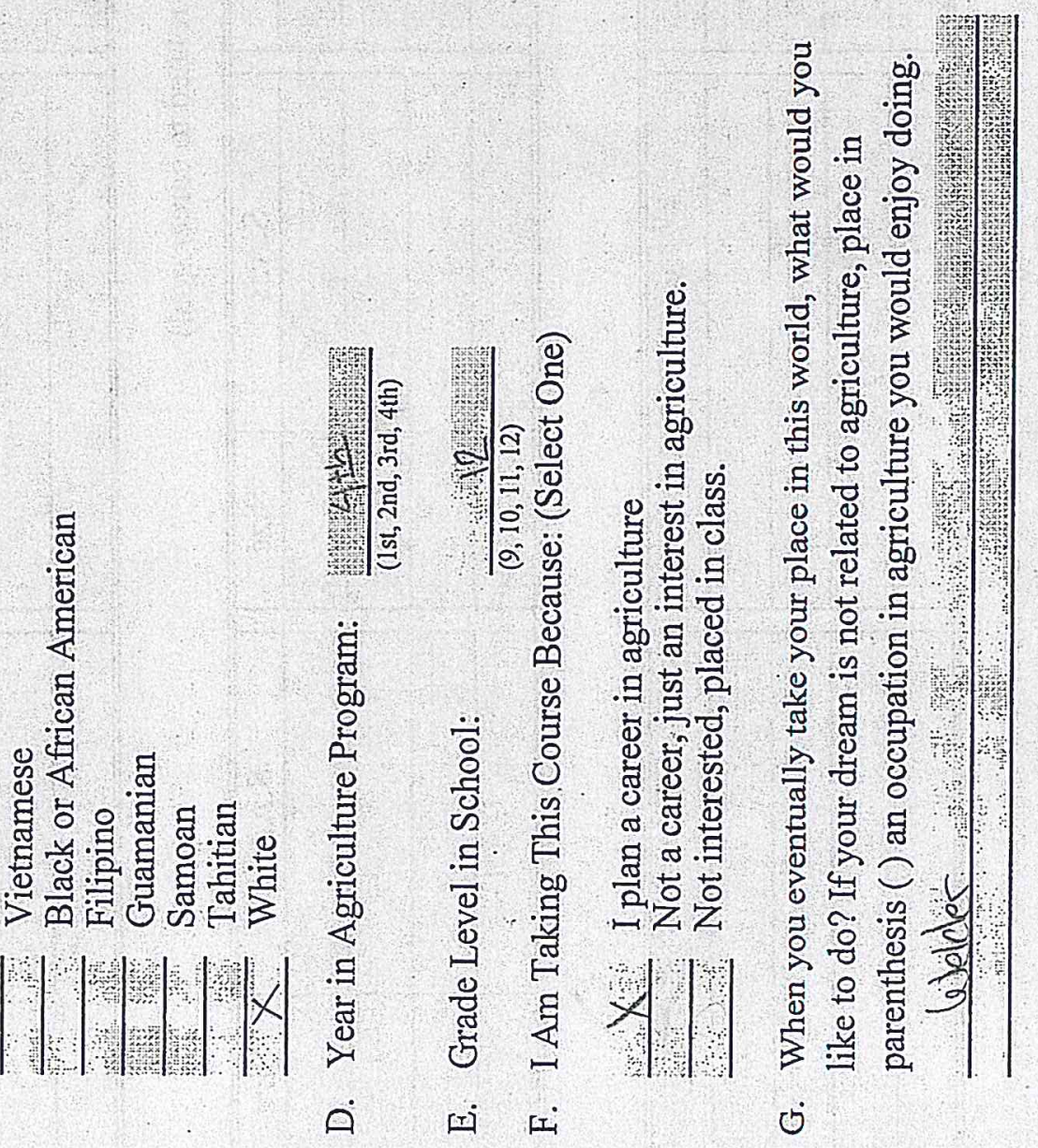




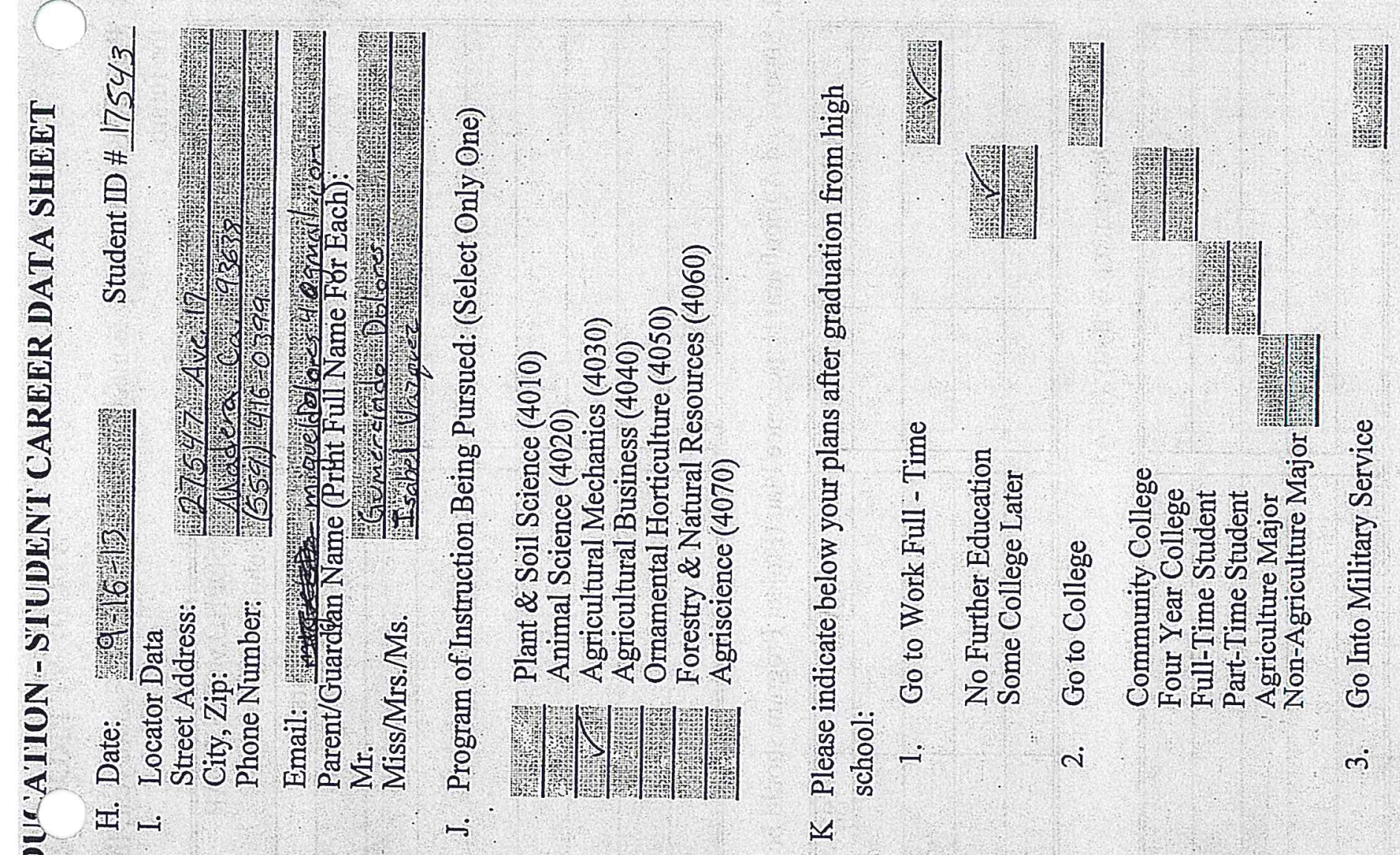




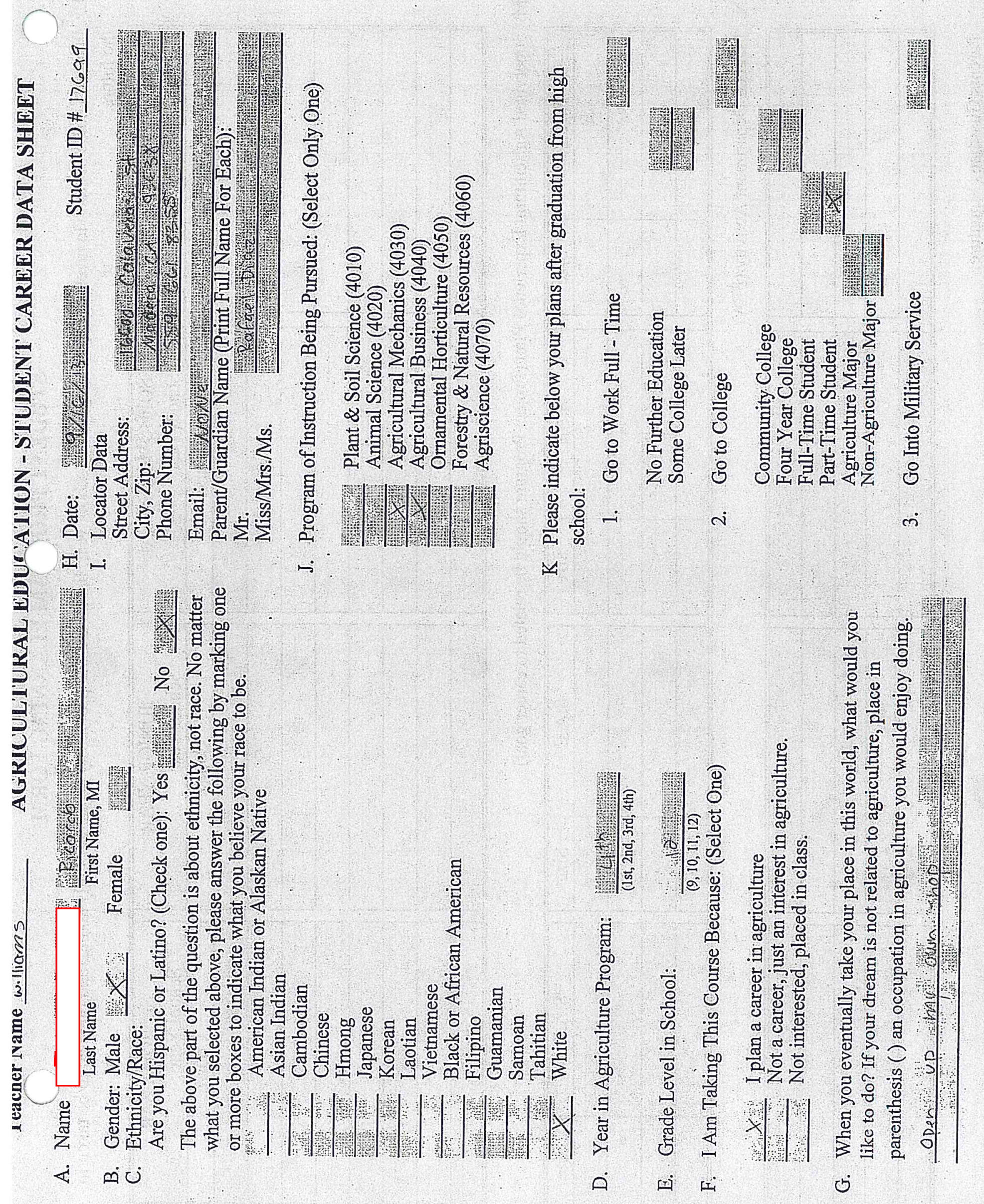


b. Permanent Vo-Ag Student Record 


\section{Permanent Vo-Ag Records}

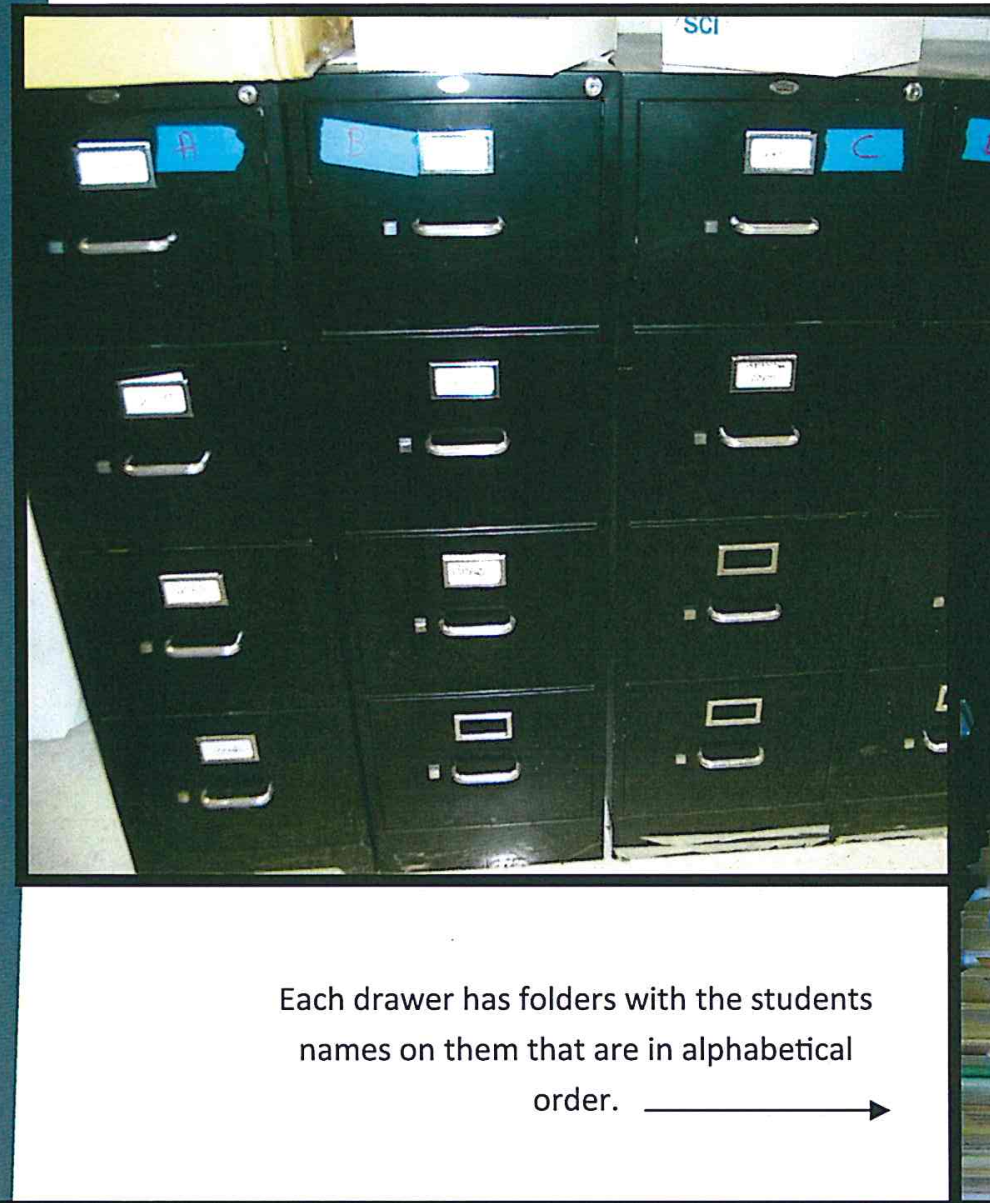

There are 4 file cabinets in the hallway between the science room. Each grade level has a file cabinet and seniors and graduates share there's. All old records are kept in these, current years books are stored in boxes in the teachers classroom. 


\section{Course Outlines}




\section{Diesel Engines/Small Engines \\ Expectations \& Rules}

Name:

ID\#

Period: Instructor:

1. Shop Safety: To ensure safety to all of our students, the following will be required for all students to wear during class and lab activities that require safety equipment.
A) Safety Glasses
B) Close-Toed Leather Shoes
C) Long Pants (Shorts are Not Allowed in the Shop)
D) Long Sleeve Shirts (When Applicable)
E) Proper Safety Equipment Needed for Specified Job.

\section{Failure to Wear Correct Safety Equipment Will Result in an Immediate Failure in this Class. Safety is Our Number 1 Priority!}

2. Do not wear loose fitting clothing, especially around moving equipment.

3. Do not misuse tools or equipment, if damage is done to tools as a result you are responsible for replacing the tool or equipment.

4. CELL PHONES are not allowed in class or lab. If cell phone is out during class, it will be taken away and given to career school.

5. Clean all spills once they happen, if unable to do so, stop and ask the instructor for help.

6. If in DOUBT at anytime, stop what you are doing and ask the instructor for help. It is important to be safe for ourselves and our equipment.

7. All students are required to take part in shop cleanup at the end of the period. Failure to do so will result in loss of daily points.

8. To be successful in this class and life, you are required to give $100 \%$ effort on all assignments and lab activities. This class is set up so that you are successful in your life and future.

9. A binder is required for this class to keep assignments, lab books, and other things needed to participate in class. You will also need to have a pencil or pen at every class meeting.

10. There will be no food or drink allowed in the shop at anytime!

I have read this with my parents and understand that I will be held accountable for all of the rules above and accept the consequences of not following them: DATE: 


\section{Diesels \\ Mr. Williams}

Prerequisites: Ag. Mech. 1

\section{Course description:}

Diesel Mechanics is designed to train students in the basics of diesel engines. This course emphasizes skills necessary to be competent in basic fundamentals of diesel engines, hydraulics, powertrains and farm machinery. This year we will focus on basic shop skills, the fundamentals and methodology of Diesel, Small Engines and Farm Machinery.

This course will also train students to real world specifications on all levels, to be included are: resume building, job application and job interview skills.

\section{Course Competencies:}

1. Demonstrate knowledge and understanding of classroom procedures

2. Demonstrate work ethic through classroom and lab activities

3. Be able to identify tools and parts that are used with diesel engines

4. Demonstrate safe work habits

5. Maintain a clean shop area

6. Operate equipment safely

7. Demonstrate ability to use precise measuring tools

8. Demonstrate knowledge and fundamentals of diesel engine mechanics

9. Demonstrate the ability to remove, inspect, recondition and install:
A) Cylinder block, end plates, covers, head, valves and liners
B) Crankshaft and bearings,
C) Flywheel, ring gear, clutch pilot bearing, flywheel housing, and gear train cover
D) Vibration damper
E) Pistons, connecting rods
F) Timing gears and camshaft

10. Demonstrate ability to know preventive maintenance

11. Demonstrate knowledge of fasteners, understanding grades of bolts, proper tightening techniques

12. Ability to demonstrate knowledge of torque wrenches in shop activities.

13. Demonstrate knowledge of horsepower and torque 


\section{Instructional Methods:}

The following instructional methods will be used in teaching the course:
a) Lecture
b) Audio visual
c) Laboratory activities
d) Tests and quizzes
e) Reading/writing assignments
f) Guest speakers
g) Classroom Discussion

\section{Books and Resources}

Diesel Technology, Norman, Corinchock, Scharf

We will also use internet resources and classroom discussion in daily activities.

\section{Grading:}

Grading will be as followed

45\% Tests/Quizzes

$45 \%$ Class Work/Projects

10\% Homework/FFA Activities 


\section{Intro to Diesel Mechanics}

Course Syllabus

\section{Instructor: Mr. Williams}

Madera South High School

\section{Course Description:}

This is an introductory level class in the theories of Diesel Technology. The main focus is to learn the theories and skills used in operation, breakdown, trouble shooting, maintenance, repair and safety. Our NUMBER ONE focus is shop safety at ALL TIMES to maintain a premier learning environment for all students. Since this class is based through agriculture education, all students are FFA members and a percentage of this class will be based on FFA activities. This class is designed to give students hands-on experience working on Diesel Engines.

\section{Student Expectations:}

1. Be prepared for every class

2. Bring a pencil or pen to every class

3. Bring class binder to every class

4. Turn assignments in on time.

5. Wear proper safety equipment at all times.

6. Treat everyone with respect

7. Come to class on time (1 free tardy)

8. Be honest

9. Always obey shop safety rules

10. No talking during class or instruction

11 . No cursing or rude comments

12. No food or drink in the shop

13. Take care of tools and equipment

14. Always clean up assigned area

\section{Goals}

1. Provide students with basic skills in diesel engines and there components.

2. Give students the opportunity to gain industry knowledge and skills in a premier learning environment. 
3. Show students the opportunities waiting for them once High School is completed.

4. Create an ideal work environment through safe practices and processes.

5. Develop problem solving skills.

6. Familiarize students with compact diesel engines to prepare them for more advance classes.

7. Strengthen students academic skills through classroom instruction and "hands on" practical training experiences within the field of agricultural mechanics.

\section{Course Objectives:}

1. Demonstrate ability to be a safe student through practical use of safety tools and methods.

2. Recognize and demonstrate the use of tools in their proper manner.

3. Breakdown and rebuild diesel engines through the supervision of the instructor.

4. Understand the methods and fundamentals of diesel engines.

5. Demonstrate the use of specialty measuring tools.

6. Understand the principles of diesel engines and the future of the industry.

Course Outline: 


\section{Madera South \\ AGRICULTURE DEPARTMENT \\ Course Outline}

\section{ROP Small Engines \\ Teacher - Mr. Williams}

\section{Small Engines}

1. Grade Level: $11-12$

2. Type of course: Elective

3. Prerequisite: Diesels

4. Length of course: 1 year, $1^{\text {st }} \& 2^{\text {nd }}$ Period

\section{Brief description:}

This course is designed to provide students with the basic skills and knowledge in the areas of small engine theory of operation, construction, and repair. Students will receive classroom instruction as well as "hands on" shop experiences. Each unit of instruction includes a required assignment that will allow the student to apply those skills learned.

Students will receive instruction in shop safety prior to being allowed to work in the agriculture power facility. They will be required to take a general safety test as well as other safety tests pertaining to each piece of equipment that will be used. Students must pass with no less than $90 \%$.

Students will have required troubleshooting lab assignments which will develop their skills in the various areas of engine theory and operation. These troubleshooting lab assignments will be reinforced with classroom instruction (lectures, demonstrations, worksheets, quizzes, tests, and videos) utilizing the textbook. When the student has completed all the required troubleshooting lab assignments he/she will be able to apply those skills on engines of their own.

Students will be required to have at least two projects. These projects must be engine or equipment related. Students will have to troubleshoot the engine problem(s) and then repair, rebuild, or tune up the specific engine and service it as well.

Students are required to wear protective clothing (coveralls, shop coats, or old clothes) to keep themselves from getting dirty. Students are also required to wear safety glasses at all times when they or other students are working in the shop. Students are also required to wear closed toe shoes. It is the student's responsibility to purchase these items. 


\section{Instructional materials:}

Text: Small Gas Engines

Author: Alfred C. Roth

Publisher: Goodheart Wilcox Co., Inc.

\section{Major goals and objectives:}

1. Students will develop an understanding of the four-stroke engine

2. Students will develop advanced technical skills and knowledge to perform routine maintenance on small engines.

3. Students will be able to properly troubleshoot and diagnose small engine problems.

4. Students will be able to properly read and understand a small engines part books and repair manuals.

5. Students will develop professional work habits and attitudes.

\section{Small Engine Basic Skills:}

During the course, the following skills will be mastered by the students.

- Understand the operation of the four stroke internal combustion engine.

- Perform routine maintenance on a small engine.

- Be able to work with engines and shop equipment safely.

- The proper terminology and use of tools.

- Be able to disassemble and re-assemble an engine to the specifications in the service manual.

- Engine troubleshooting and diagnosis.

- Engine overhaul (valves, valve guides, valve springs, pistons, piston rings)

- The different types of fuel and carburetion systems.

- The different types of ignition systems.

- The different types of governor systems.

\section{Course outline:}

Unit 1 - Safety

1. Classroom Instruction and Demonstration of Equipment

2. Safety Tests

\section{Unit 2 - Identification of tools, equipment, and parts}

1. Classroom Instruction of Different Tools, Equipment, and Engine Parts

2. Precision Measuring Instruments

\section{Unit 3 - Engine theory and operation}

1. 4 Stroke Engines

2. 2 Stroke Engines

3. Intake, Compression, Power, and Exhaust 


\section{Unit 4 - Carburetion systems}

1. Theory of Carburetion and Atomization

2. Flow Jet Carburetor

3. Vacu Jet Carburetor

4. Pulsa Jet Carburetor

5. Mixture Adjusting Screws

6. Venturi Action

7. Priming and Choking

8. Possible Problems

9. Troubleshooting Assignment

\section{Unit 5 - Ignition Systems}

1. Basic Theory

2. Types of Systems

3. Spark Plugs

4. Flywheel and Flywheel Key

\section{Unit 6-Compression System}

1. Pistons and Rings

2. Valves

3. Engine Displacement

\section{Unit 7 - Lubrication Systems}

1. Principles of Lubrication

2. Friction, Wear, and Sealing

3. SAE Viscosity Grade

4. API Engine Oil Service Classification

5. Splash Lubrication

6. Oil Pumps

7. Oil Filters

\section{Unit 8 - Cooling Systems}

1. Principles of Engine Cooling

2. How Air Cooling Works

3. How Exhaust Cooling Works

4. How Water Cooling Works

5. Water Pumps

6. Thermostats

7. Radiators 
Unit 9 - Fuel and Emissions

1. Engine Fuels

2. Tanks, Lines, and Fittings

3. Fuel Pumps

4. Exhaust Emissions

5. Environmental and Health Issues

Unit 10 - Preventive Maintenance and Troubleshooting

1. General Preventive Maintenance

2. Keeping Engines Clean

3. Servicing

4. Systematic Troubleshooting

Unit 11 - Engine Teardown and Re-assembly

1. Engine Inspection

2. Engine Disassembly

3. Organization

4. Fasteners, Sealers, and Gaskets

5. Engine Re-assembly

Unit 12 - Measuring Engine Performance

1. Basic Terminology

2. Engine Displacement

3. Compression Ratio

4. Torque and Horsepower

5. Volumetric Efficiency

6. Practical Efficiency

7. Mechanical Efficiency

8. Thermal Efficiency

Unit 13 - Employability

1. Resume

2. Application

3. Employee/Employer Expectations 


\section{Grading Policies and Procedures}

1. Grading Scale

$$
\begin{aligned}
& 90-100 \%=\mathrm{A} \\
& 80-89 \%=\mathrm{B} \\
& 70-79 \%=\mathrm{C} \\
& 60-69 \%=\mathrm{D} \\
& 0-59 \%=\mathrm{F}
\end{aligned}
$$

2. All assignments will be given a point value. Therefore, each student's grade will be determined by calculating the percentage of the points earned and the assigning the corresponding letter grade in accordance with the scale shown above. The main categories under which the students are given points are shown below.

1. Student Binders

2. Class Work

3. Daily Shop Points

4. Lab Exercises

5. Lab Projects

6. Tests and Quizzes

EACH STUDENT IS REQUIRED TO MAINTAIN A 3 RING BINDER SPECIFICALLY FOR THIS CLASS. Students will keep all notes, assignments, tests, handouts, and this syllabus in their binder. It will be checked weekly for neatness and completion. 


\section{Xerox Copy Of Daily grade Sheets From Each Class Taught}




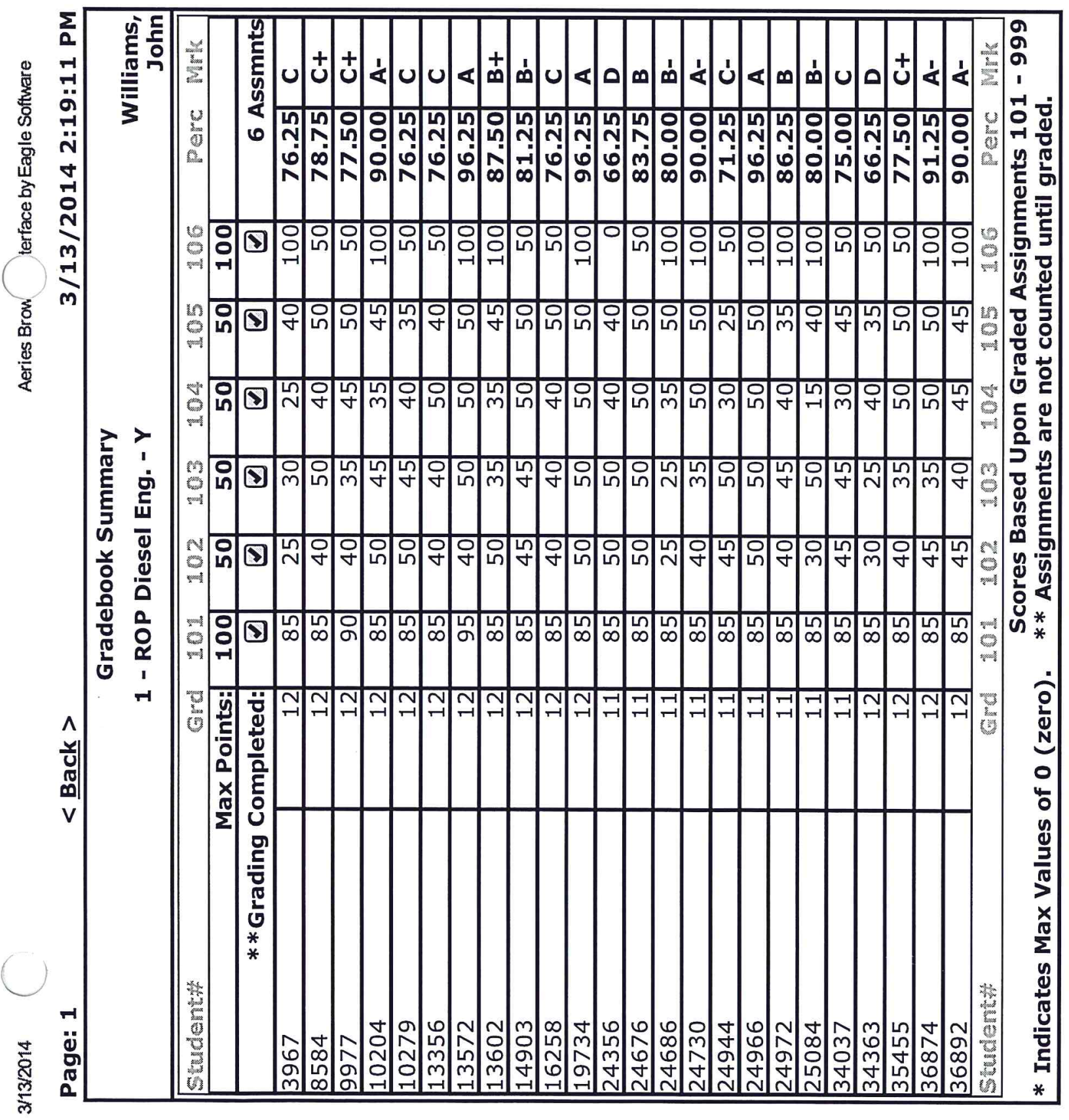




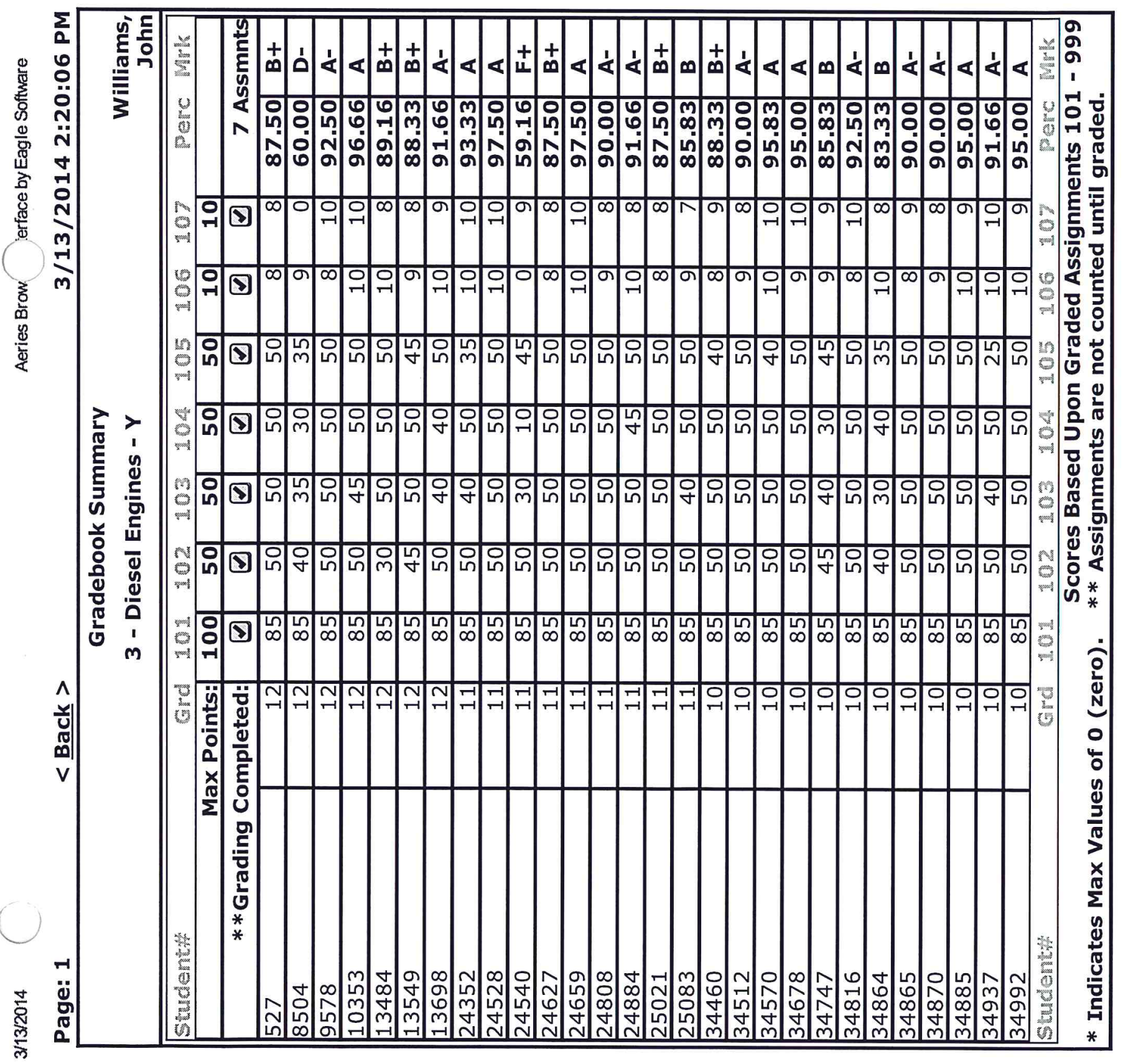




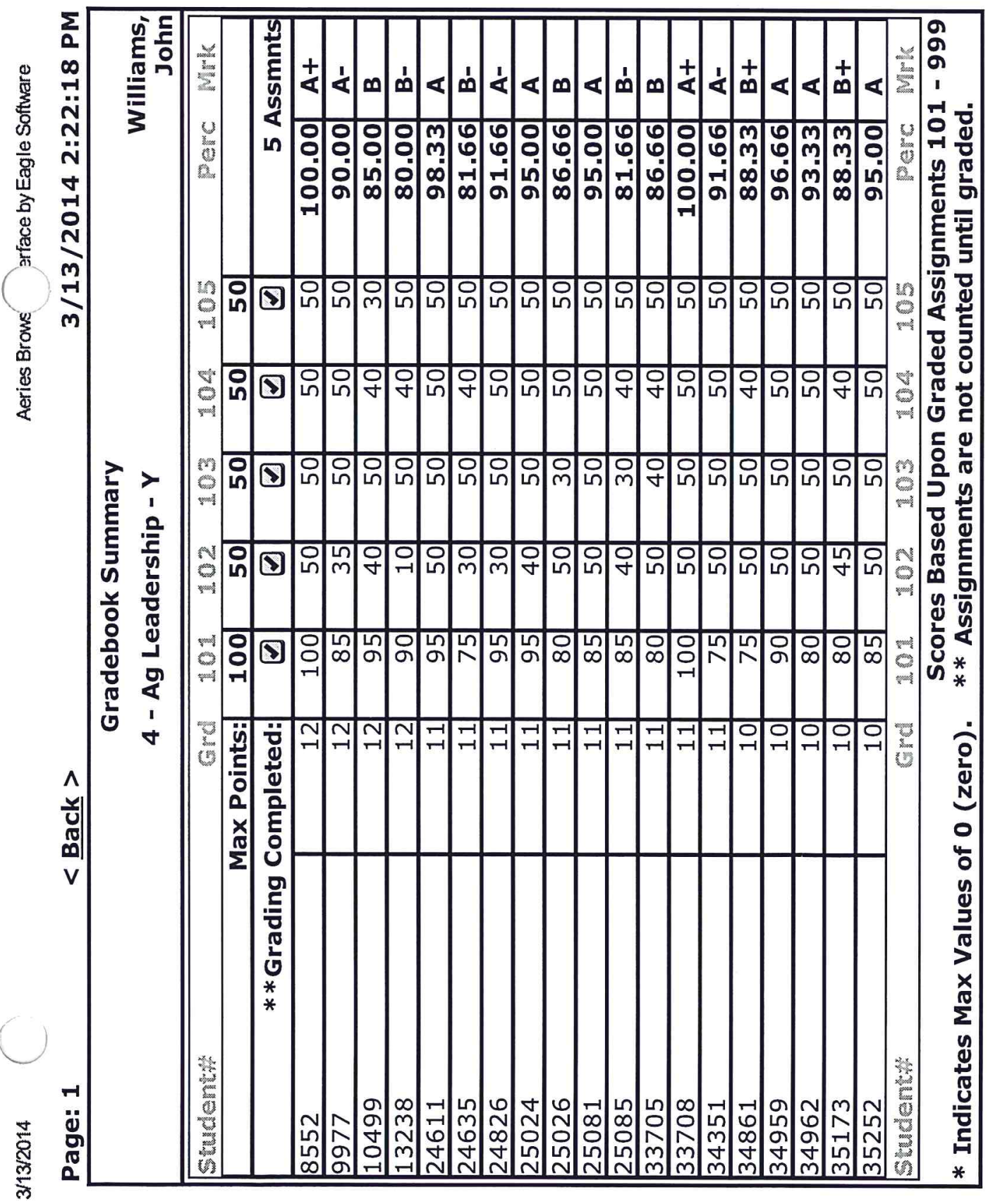




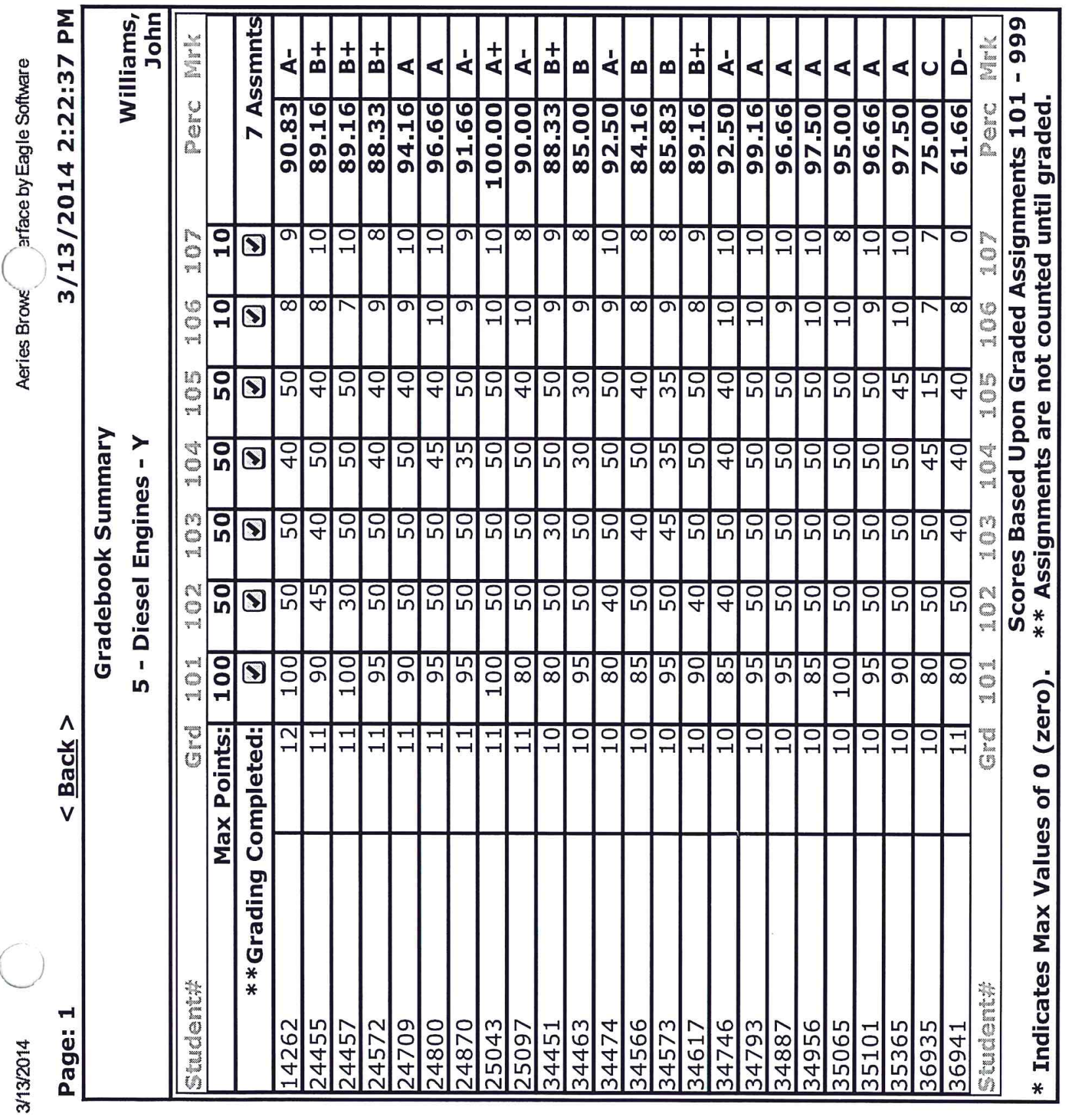




\section{E. SOE Supervision Forms Used on Project Visits}


Time:

Date: $7 / 10 / 13$

\section{Madera South High School Agriculture Department Project Supervision Form}

Name of Student: Jime t. (wevels

Project:Replacement dairy heifer

Scope: one

\begin{tabular}{l} 
Yes No \\
$X$ \\
\hline
\end{tabular}$-$ Clean Pen

$\frac{x}{x}-$ Feed

$\frac{x}{x}-$ Animal Clean

$\frac{x}{x} \quad$ Wormed $\geqslant / 10$ Date

$\underline{x}-$ Clean Water

$\frac{x}{x}-$ Healthy Animal

$\frac{x}{x}-$ Halter Broke

$\underline{X}-$ Student Present

Record Book Verification

\begin{tabular}{|l|l|l|l|}
\hline Weight & ADG & \\
\hline- Previous Weight & & $\mathrm{X}$ Days to Fair & \\
\hline $\begin{array}{l}\text { = Total Gain } \\
\text { I Days from last } \\
\text { weight = ADG }\end{array}$ & + Today's Weight & \\
\hline
\end{tabular}

Recommended feed ration: I flake of Alfalfa, 3 pounds of grain 1 flake of oats per feeding.

Recommended exercise program: $20 \quad$ minutes per day.

Recommended washing schedule: 1 times per week.

Current Project Size/Scope: I dary heiter

Overall Recommendations: Geod over all list needs to show whos boss

Future Project Plans: work her a little longer

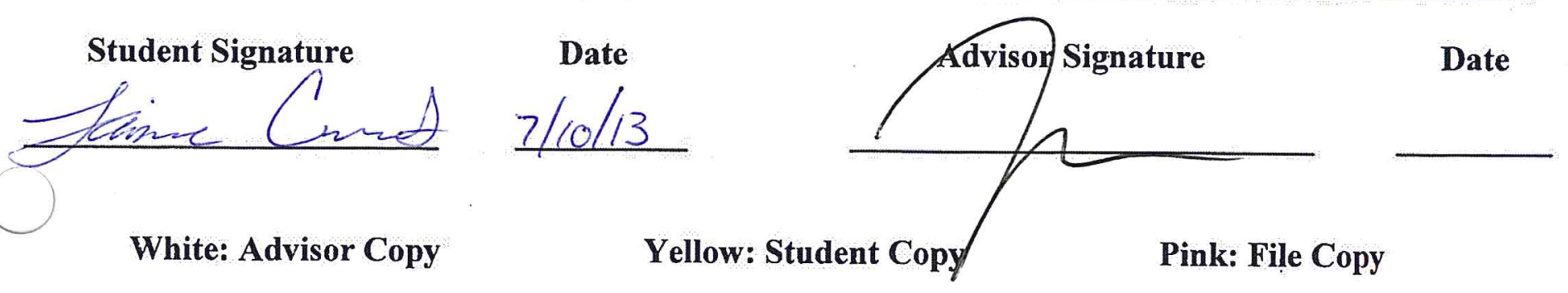


Time:

Date: $12 / 12 / 13$

\section{Madera South High School Agriculture Department Project Supervision Form}

Name of Student: Jdime $\epsilon_{i}$ luevas

Project:Replacement dairy heifer

Record Book Verification

Scope: one

\begin{tabular}{lll} 
Yes & No \\
$X$ & $-\quad$ Clean Pen \\
\hline
\end{tabular}

$\frac{x}{x}-$ Feed

$\frac{x}{x}-$ Animal Clean

$\frac{x}{x}-$ Wormed $12 / 12$ Date

$\begin{array}{ll}\frac{x}{x} & -\begin{array}{l}\text { Clean Water } \\ \text { Healthy Animal }\end{array}\end{array}$

$\frac{x}{x}-$ Halter Broke

$\frac{x}{x}$ - Student Present

\begin{tabular}{|l|l|l|l|}
\hline Weight & ADG & \\
\hline- Previous Weight & X_Days to Fair & \\
\hline $\begin{array}{l}\text { = Total Gain } \\
\text { /\# Days from last } \\
\text { weight = ADG }\end{array}$ & + Today's Weight & \\
\hline
\end{tabular}

Recommended feed ration: I flatse of Alfalfa, 3 peunds of

grain I flake of oats

per feeding.

Recommended exercise program: 20 minutes per day.

Recommended washing schedule:_ I_times per week.

Current Project Size/Scope: I dary heifer

Overall Recommendations: Improwement on Shereing whes boss, but needs to gain a little weight

Future Project Plans: feed half a pound more of grain

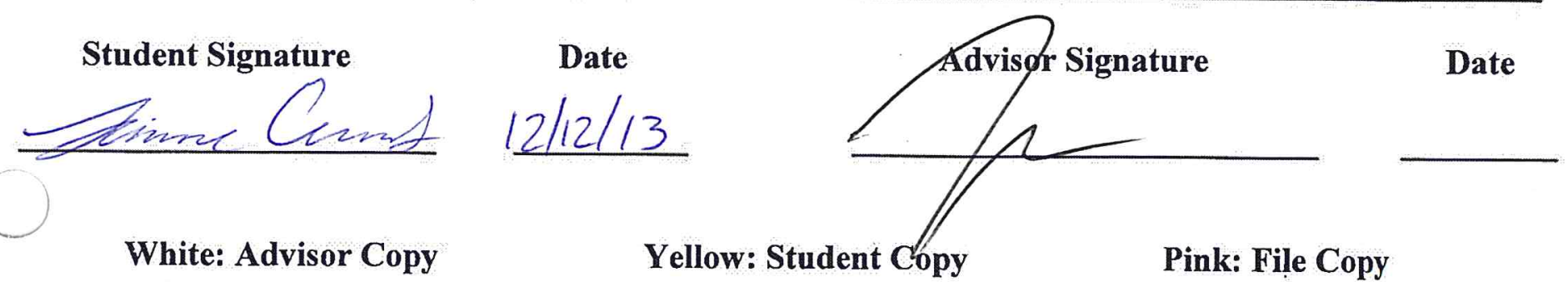

White: Advisor Copy

Yellow: Student Copy

Pink: File Copy 
Time: $12: 00$ PM

Date: $8 / 4 / 13$

Madeira South High School Agriculture Department Project Supervision Form

Name of Student: Clayton Sheehan

Project: Dairy Replacement Heifer

Record Book Verification

scope: I head

Yes No

$\underline{X} \quad$ Clean Pen

Feed

Animal Clean

Wormed $9 / 10 / 13$ Date

Clean Water

Healthy Animal

Halter Broke

Student Present

\begin{tabular}{|l|l|l|l|}
\hline Weight & & ALG & \\
\hline- Previous Weight & & $\mathrm{X}$ Days to Fair & \\
\hline$=$ Total Gain & & + Today's Weight & \\
\hline $\begin{array}{l}\text { /\# Days from last } \\
\text { weight = ADG }\end{array}$ & = Projected Fair Weight & \\
\hline
\end{tabular}

Recommended feed ration: One flake of alfalfa and

3 pounds of grain per feeding.

Recommended exercise program: 30 minutes per day.

Recommended washing schedule: __ I t t t t t

Current Project Size/Scope: I head

Overall Recommendations: You should work with the heifer

more.

Future Project Plans: Attain another heifer for the next

2 yrs.

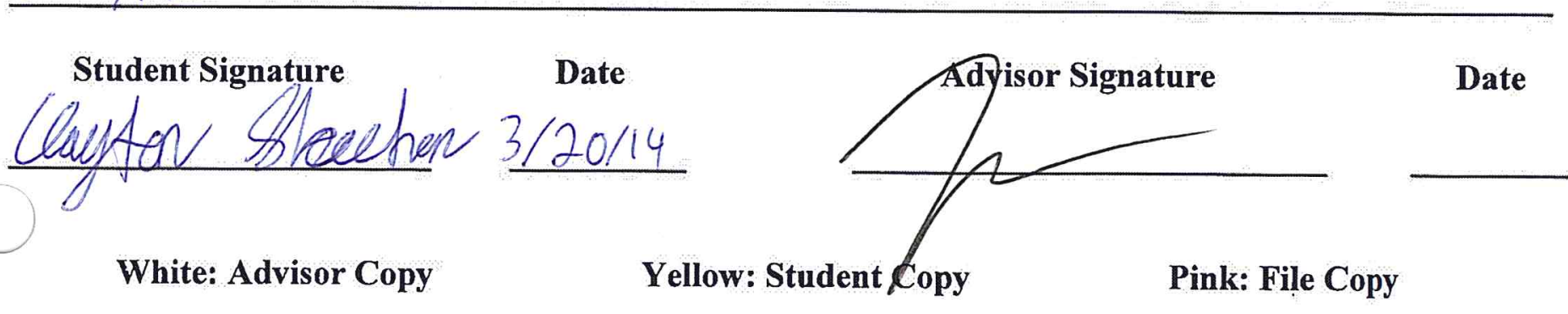


Time: $10: 00 \mathrm{AM}$

Date: $12 / 12 / 13$

\section{Madera South High School}

\section{Agriculture Department Project Supervision Form}

Name of Student: Clayton Sheehar

Project: Dairy replacement heifer

Record Book Verification

scope: I head

Yes No

$\frac{x}{x}-{ }_{\text {Feed }}^{\text {Clean Pen }}$

$\frac{\frac{x}{x}}{x}-$ Animal Clean

$\frac{x}{x}-$ Wormed $12 / 6 / 13$ Date

$\frac{x}{\frac{x}{x}}-\begin{aligned} & \text { Clean Water } \\ & \text { Healthy Animal }\end{aligned}$

$\frac{x}{x}-$ Halter Broke

$\underline{X}$ - Student Present

\begin{tabular}{|l|l|l|l|}
\hline Weight & ADG & \\
\hline- Previous Weight & & $\mathrm{X}$ _ Days to Fair & \\
\hline = Total Gain & & + Today's Weight & \\
\hline $\begin{array}{l}\text { /\# Days from last } \\
\text { weight = ADG }\end{array}$ & & $=$ Projected Fair Weight & \\
\hline
\end{tabular}

Recommended feed ration: One flake of alfalfa and 3 pounds of grain per feeding.

Recommended exercise program: 30 minutes per day.

Recommended washing schedule: ___ t times per week.

Current Project Size/Scope: I head

overall Recommendations: Should Spend more time with the animal

Future Project Plans: Breed the herfer to sell pregnant at the fair.

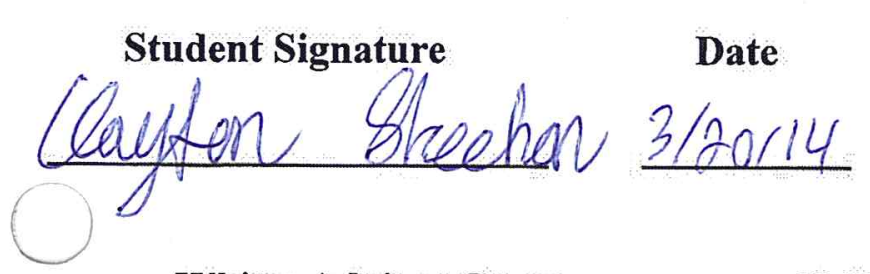

White: Advisor Copy
Yellow: Student Copy

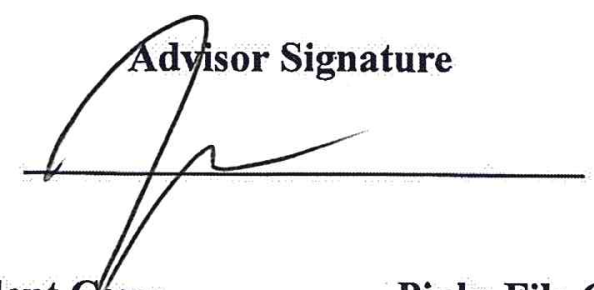

Pink: File Copy

Date 
Time: 3055 P.M.

Date: $11 / 26 / 13$

\section{Madera South High School Agriculture Department Project Supervision Form}

Name of Student: Virat Kang

Project:Dainy Replacement Heifer Record Book Verification

scope: I head

Yes No

$\frac{V}{V}-\quad \begin{aligned} & \text { Clean Pen } \\ & \text { Feed }\end{aligned}$

$\simeq \quad$ Animal Clean

$\checkmark-$ Wormed $1 / 17$ Date

$\underline{L}$ Clean Water

$\downarrow \quad$ Healthy Animal

$\sim \sim$ Halter Broke

Student Present

\begin{tabular}{|l|l|l|l|}
\hline Weight & ADG & \\
\hline- Previous Weight & X_ Days to Fair & \\
\hline$=$ Total Gain & & + Today's Weight & \\
\hline $\begin{array}{l}\text { I D Days from last } \\
\text { weight = ADG }\end{array}$ & = Projected Fair Weight & \\
\hline
\end{tabular}

Recommended feed ration: One flathe of alfalfa hay with 3 pounds of grain.

Recommended exercise program: 20 minutes per day.

Recommended washing schedule: 1 times per week.

Current Project Size/Scope: I head

Overall Recommendations: be calmer with animal and work with animal far loiyer secssions.

Future Project Plans: Mathe anrangementes in arder ter get treifer Ared.

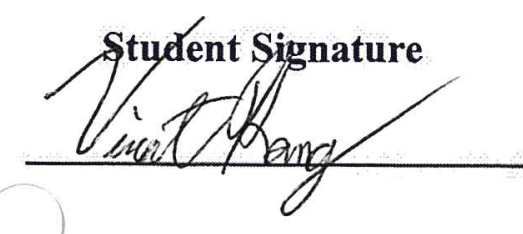

White: Advisor Copy

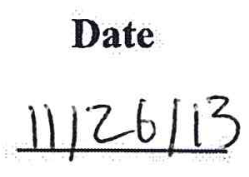

Yellow: Student Copy

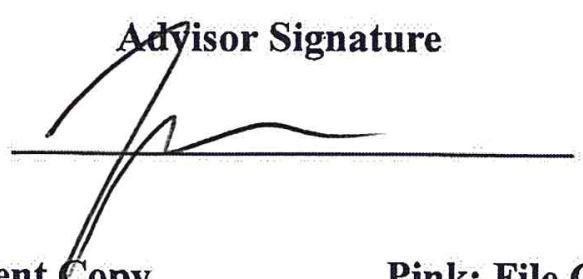

Pink: File Copy

Date 
Time: $4 \begin{array}{ll}0 \\ 0\end{array} \bar{n}$ D.m.

Date: $7 / 3 / 13$

\section{Madera South High School}

\section{Agriculture Department Project Supervision Form}

Name of Student: Virat Kang

Project: Dainy Replacement Hteifer Record Book Verification

scope: 1 head

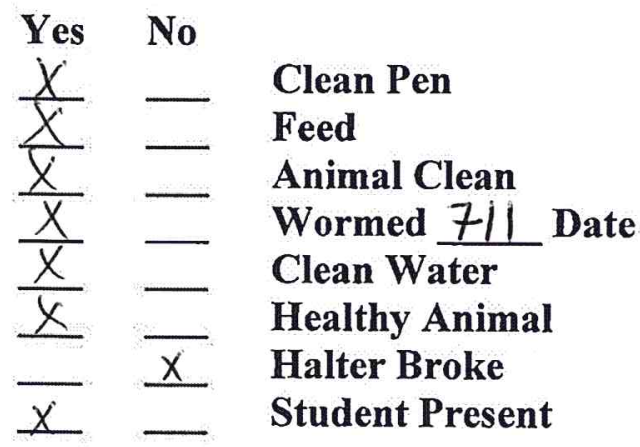

\begin{tabular}{|l|l|l|l|}
\hline Weight & ADG & \\
\hline - Previous Weight & X_ Days to Fair & \\
\hline $\begin{array}{l}=\text { Total Gain } \\
\begin{array}{l}\text { / Days from last } \\
\text { weight = ADG }\end{array}\end{array}$ & + Today's Weight & \\
\hline
\end{tabular}

Recommended feed ration: One flake of alfalfa hay with

3 pounds of $18 \%$ calf ration per feeding.

Recommended exercise program: 20 minutes per day.

Recommended washing schedule: 1 times per week.

Current Project Size/Scope: I head

Overall Recommendations: be more patient with neifer T warlin wim heifer for a longer perdad of lime

Future Project Plans: Consider purchasing another heiter

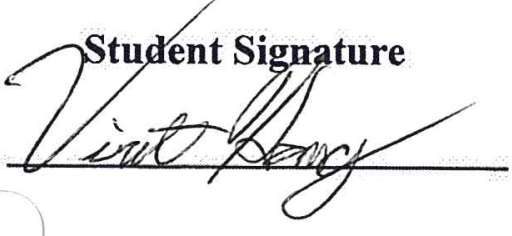

White: Advisor Copy

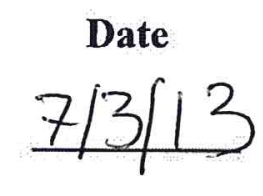

Yellow: Student Copy

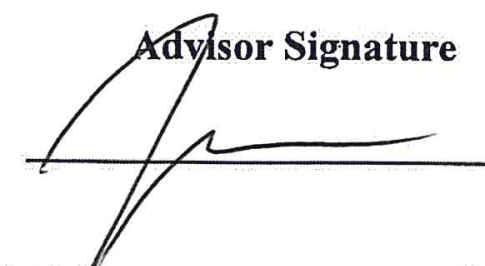

Date

Pink: File Copy 
Time: G:0OPM.

Date: $8 / 3 / B$

\section{Madera South High School}

\section{Agriculture Department Project Supervision Form}

Name of Student: Jenae Hansen

Project: Dairy Replacement Heifer

scope: 1 animal

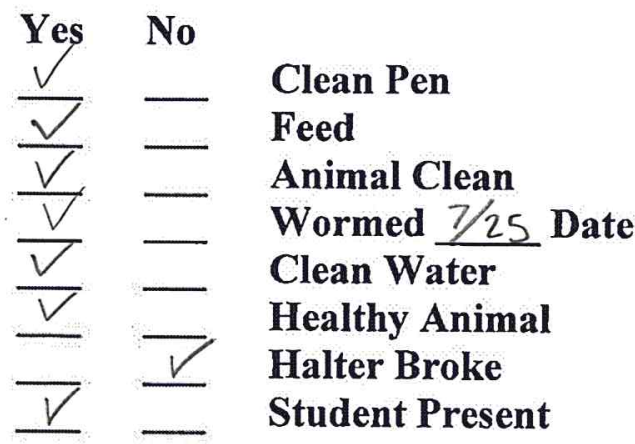

Record Book Verification

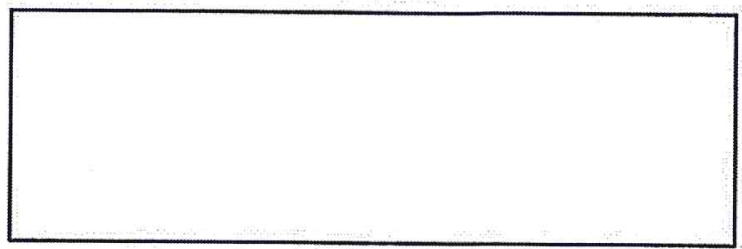

Recommended feed ration: one flake of alfal fa and $3 \mathrm{lbs}$

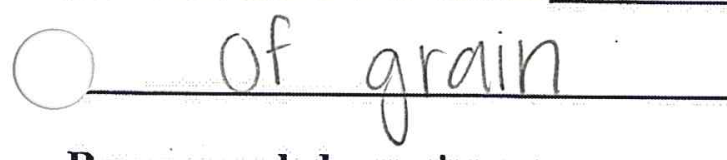

Recommended exercise program:

Recommended washing schedule:

Current Project Size/Scope: $\quad$ I ANIM AL

Overall Recommendations: NEEDS TO SPEND MORE TIMEV WFHH HELFER, THAT WILL MAKE ABETTER RELATIONSHIP BETWEEN THE TWO. ALSO WORK ON HALTER BREAKING.

Future Project Plans:

Student Signature

White: Advisor Copy

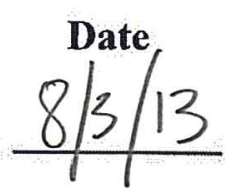

Yellow: Student Copy per feeding.

\begin{tabular}{|l|l|l|l|}
\hline Weight & ADG & \\
\hline- Previous Weight & & X_ Days to Fair & \\
\hline $\begin{array}{l}\text { = Total Gain } \\
\begin{array}{l}\text { I Days from last } \\
\text { weight = ADG }\end{array}\end{array}$ & + Today's Weight & \\
\hline
\end{tabular}
times per week.

20 minutes per day. 
Time: $6: 15$ P.M.

Date: $11 / 26 / 13$

\section{Madera South High School}

\section{Agriculture Department Project Supervision Form}

Name of Student: Jehae Hansen

Project:Dairy replacement Heifer Record Book Verification

scope: L animal

Yes No $\quad$ Clean Pen

$\frac{V}{V}-\begin{aligned} & \text { Clean Pen } \\ & \text { Feed } \\ & \text { Animal Cl }\end{aligned}$

$\frac{V}{V}-\quad$ Wormed $y_{25}$ Date

$V$ - Clean Water

$\checkmark$ - Healthy Animal

$\underline{V}$ - Halter Broke

$\underline{V}$ - Student Present

\begin{tabular}{|l|l|l|l|}
\hline Weight & ADG & \\
\hline- Previous Weight & & $\mathrm{X}$ _ Days to Fair & \\
\hline $\begin{array}{l}\text { = Total Gain } \\
\begin{array}{l}\text { /\# Days from last } \\
\text { weight = ADG }\end{array}\end{array}$ & + Today's Weight & \\
\hline
\end{tabular}

Recommended feed ration: 3 lbs of ofrain

per feeding.

Recommended exercise program: 20 minutes per day.

Recommended washing schedule: _ I t t times per week.

Current Project Size/Scope: I AN IMAL

Overall Recommendations: WOYK WIHH HEFFER FOR LOWGER PERFODS OF TIME.

Future Project Plans: $100 K$ INTO GETTING HEIFER BRED

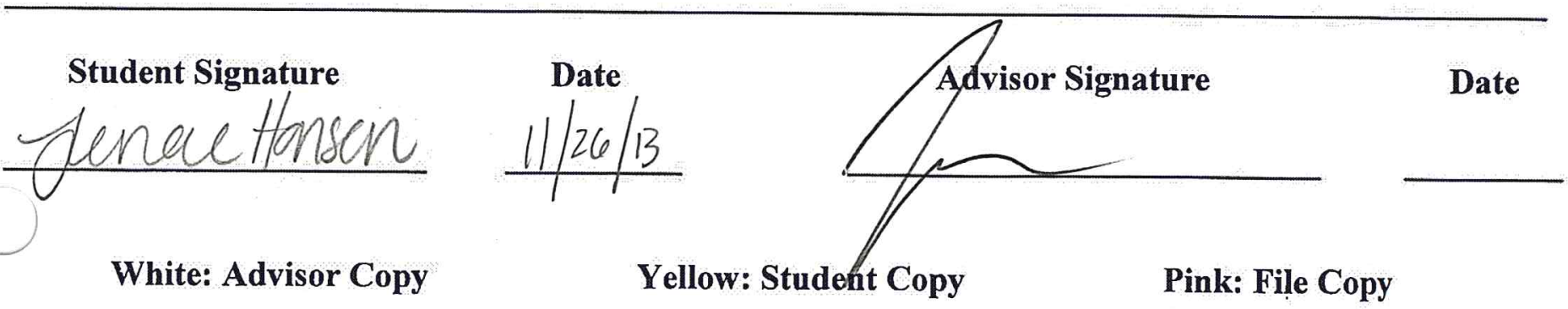


Time:
Date:
Alucust $5^{\text {th }}=2013$

\section{Madera South High School Agriculture Department Project Supervision Form}

Name of Student: Tams Bavers

Project: Dary thifer

Record Book Verification

Scope:

Yes No

$\frac{X}{x} \quad$ Clean Pen

$\underline{X}-$ Feed

$x$ - Animal Clean

$\underline{x}-$ Wormed

\& — Clean Water

$\div \quad$ Healthy Animal

$\triangle \quad X \quad$ Halter Broke

$\stackrel{X}{+}$ Student Present Date

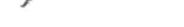

Recommended feed ration:

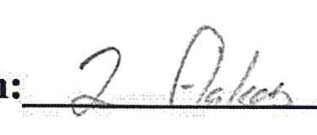

\begin{tabular}{|l|l|l|l|}
\hline Weight & ADG & \\
\hline - Previous Weight & & $\mathrm{X}$ Days to Fair & \\
\hline$=$ Total Gain & & + Today's Weight & \\
\hline $\begin{array}{l}\text { / \# Days from last } \\
\text { weight = ADG }\end{array}$ & = Projected Fair Weight & \\
\hline
\end{tabular}
gram: 25 minutes per day.

Recommended exercise program: 25 minutes per day.
Recommended washing schedule: 2 times per week.

Current Project Size/Scope: I Naify heitsen

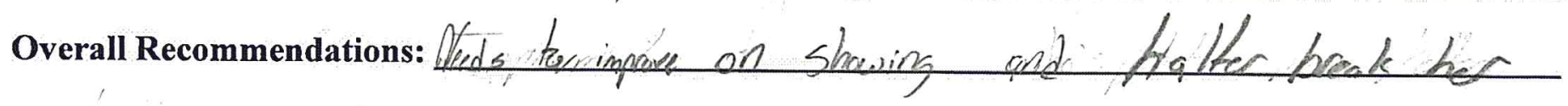
per feeding.

Future Project Plans: Have balter baken feoter tir.

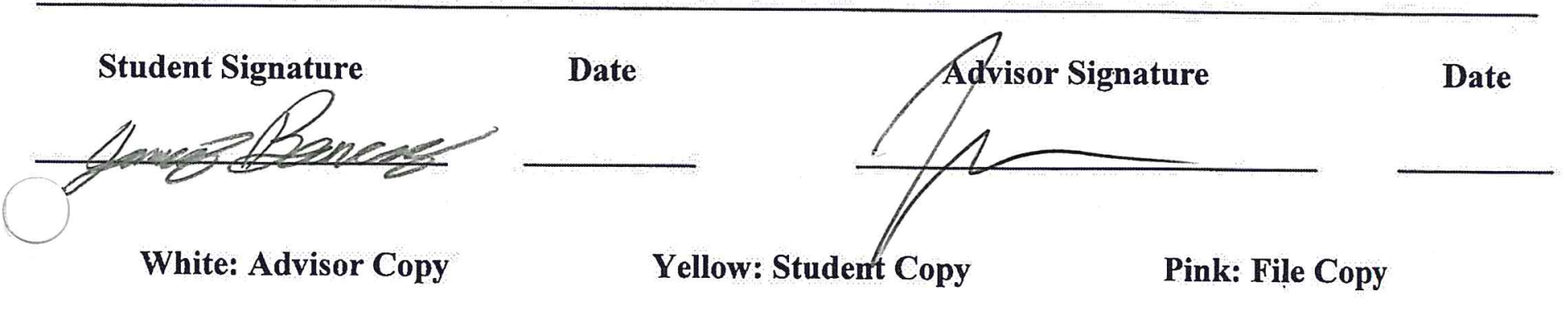


Time:

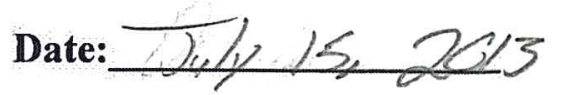

\section{Madera South High School Agriculture Department Project Supervision Form}

Name of Student: Jawes Seners

Project: Holecount Nare hetech

Record Book Verification

Scope:

Yes No

$\frac{x}{x} \quad-\quad$ Clean Pen

$\frac{x}{x}-$ Feed

$\frac{x}{x} \quad-\quad$ Animal Clean

$\frac{\frac{x}{y}}{\frac{y}{x}}-\begin{aligned} & \text { Wormed } \\ & \text { Clean Water }\end{aligned}$

$\frac{y}{x}-$ Healthy Animal

$\begin{array}{ll}\frac{X}{\chi} \quad & \quad \begin{array}{l}\text { Halter Broke } \\ \text { Student Present }\end{array}\end{array}$ Date

Recommended feed ration:

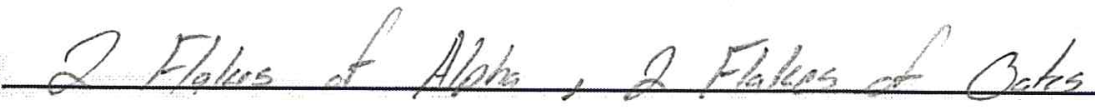

\begin{tabular}{|l|l|l|l|}
\hline Weight & ADG & \\
\hline - Previous Weight & X Days to Fair & \\
\hline = Total Gain & & + Today's Weight & \\
\hline $\begin{array}{l}\text { / Days from last } \\
\text { weight = ADG }\end{array}$ & = Projected Fair Weight & \\
\hline
\end{tabular}

Recommended exercise program: minutes per day.

Recommended washing schedule:

Current Project Size/Scope:

Overall Recommendations:

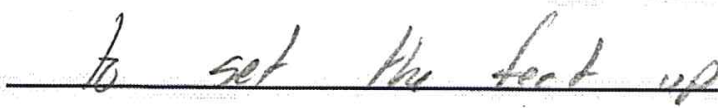

20

per feeding. times per week.
2
times per week. 
F. Wall Chart Of SOE Visits 


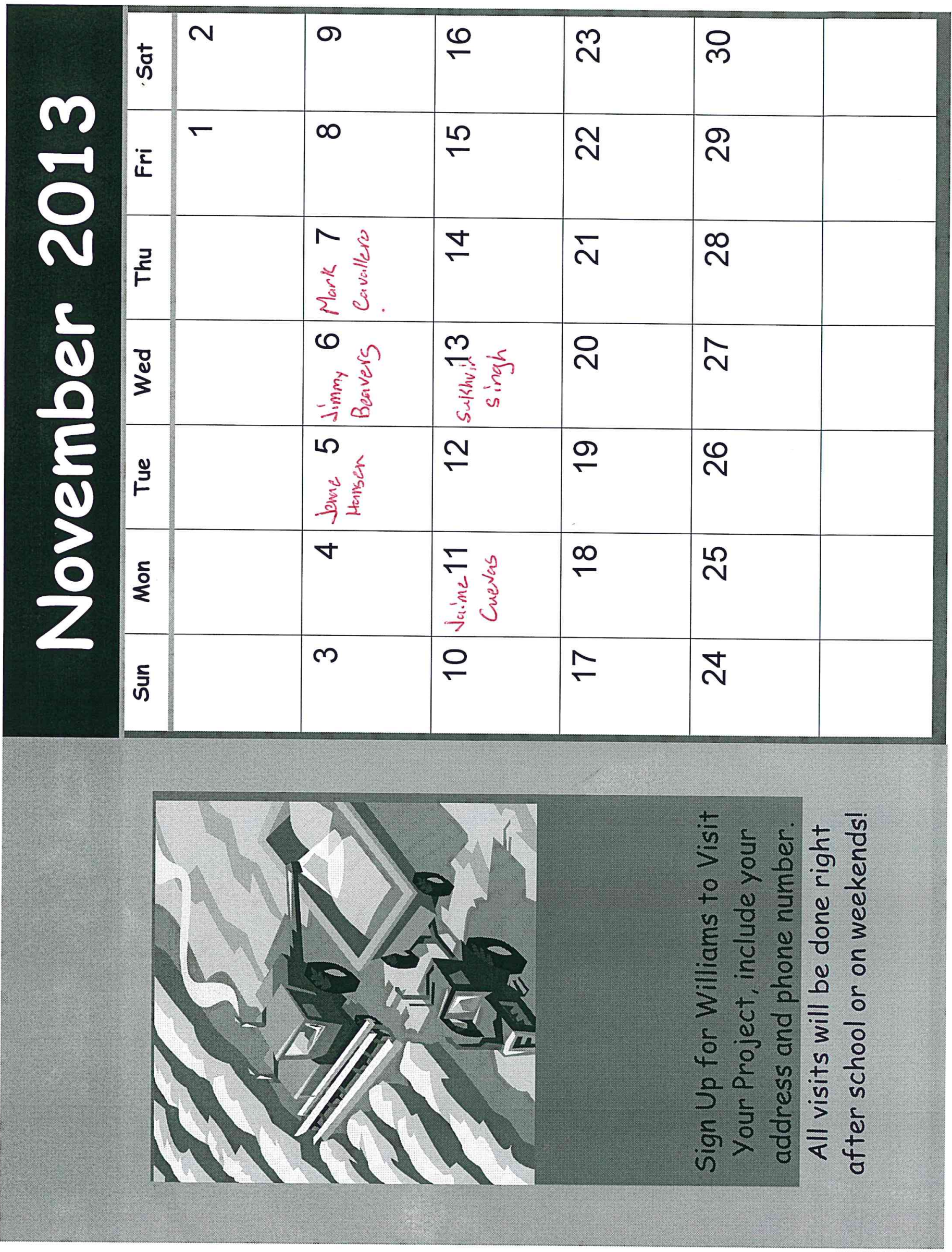


G. SOE summary By Individual Stusents 


\begin{tabular}{|c|c|c|c|c|}
\hline \multicolumn{5}{|c|}{ SAE Project List } \\
\hline Name & Grade & \begin{tabular}{|l|}
$\mathrm{Pd}$ \\
\end{tabular} & Current Project & Future Plans \\
\hline Jose Carrasqillo & 11 & 1 & Engine/Repair & Military \\
\hline Sergio Cortez & 12 & 1 & Engine/Repair & Military \\
\hline Jaime Cuevas & 12 & 1 & Chickens & College \\
\hline Ricardo Diaz & 12 & 1 & Engine/Repair & College \\
\hline Angel Fernandez & 11 & 1 & Landscape & College \\
\hline Ramon Gonzales & 12 & 1 & Beef Production & College \\
\hline Jose Guzman & 11 & 1 & Engine/Repair & Military \\
\hline Dylan Harder & 12 & 1 & Engine/Repair & College \\
\hline Erick Leyva & 12 & 1 & Engine/Repair & College \\
\hline Juan Manzano & 12 & 1 & Engine/Repair & Military \\
\hline George Martinez & 12 & 1 & Engine/Repair & College \\
\hline Matthew Mealy & 11 & 1 & Engine/Repair & Military \\
\hline Javier Moreno & 12 & 1 & Engine/Repair & College \\
\hline Tyler Morris & 11 & 1 & Engine/Repair & College \\
\hline Eric Ochoa & 11 & 1 & Engine/Repair & Work \\
\hline Juan Padilla Navarro & 12 & 1 & Engine/Repair & Work \\
\hline Bryan Pichardo & 12 & 1 & Engine/Repair & College \\
\hline Justin Reece & 12 & 1 & Engine/Repair & College \\
\hline Elwin Reyes & 11 & 1 & Engine/Repair & Military \\
\hline David Romero & 11 & 1 & Engine/Repair & Military \\
\hline Luis Salazar & 11 & 1 & Engine/Repair & Military \\
\hline Ignacio Segura & 12 & 1 & Engine/Repair & Military \\
\hline Sukvir Singh & 12 & 1 & Engine/Repair & College \\
\hline Juan Vargas & 12 & 1 & Engine/Repair & College \\
\hline Eduardo Castillo & 12 & 2 & Engine/Repair & College \\
\hline Anthony Cortazar & 11 & 2 & Engine/Repair & College \\
\hline Miguel Dolores & 12 & 2 & Engine/Repair & College \\
\hline Eduardo Jimenez & 12 & 2 & Engine/Repair & Military \\
\hline Angel Rodriguez & 11 & 2 & Engine/Repair & College \\
\hline Angel Aguierre & 10 & 3 & Engine/Repair & Military \\
\hline Edwin Alvarado & 12 & 3 & Engine/Repair & Military \\
\hline Cruz Becerra & 10 & 3 & Engine/Repair & College \\
\hline Steven Bennett & 12 & 3 & Ag Mech & College \\
\hline Andre Caballero & 12 & 3 & Engine/Repair & College \\
\hline Ivan Castillo & 10 & 3 & Engine/Repair & College \\
\hline Brian Cortez & 10 & 3 & Engine/Repair & College \\
\hline Micheal Egure & 12 & 3 & Engine/Repair & College \\
\hline Ernesto Escalante & 11 & 3 & Engine/Repair & College \\
\hline Charlie Hernandez & 11 & 3 & Engine/Repair & Military \\
\hline Gerardo Jayme & 10 & 3 & Engine/Repair & College \\
\hline CJ Leal & 11 & 3 & Engine/Repair & College \\
\hline Ricardo Lopez & 10 & 3 & Engine/Repair & College \\
\hline Danny Marquez & 12 & 3 & Engine/Repair & College \\
\hline Oscar Martines & 11 & 3 & Engine/Repair & College \\
\hline Cresce Martinez & 11 & 3 & Landscape & Military \\
\hline
\end{tabular}




\begin{tabular}{|c|c|c|c|c|}
\hline Gustavio Martinez & 11 & 3 & Engine/Repair & Work \\
\hline Eduardo Mendez & 10 & 3 & Engine/Repair & Work \\
\hline Arturo Molina & 11 & 3 & Engine/Repair & Work \\
\hline Luis Montano & 11 & 3 & Engine/Repair & Work \\
\hline Jevon Neely & 12 & 3 & Engine/Repair & Military \\
\hline Noel Ocegueda & 12 & 3 & Engine/Repair & Military \\
\hline Giovanni Perez & 10 & 3 & Engine/Repair & Military \\
\hline Christian Ramos & 10 & 3 & Engine/Repair & Military \\
\hline Troy Smith & 11 & 3 & Engine/Repair & Military \\
\hline Issac Trigos & 10 & 3 & Engine/Repair & Military \\
\hline Jonathan Vargas & 10 & 3 & Engine/Repair & College \\
\hline Victor Vasquez & 10 & 3 & Engine/Repair & College \\
\hline Mario Alvarez & 10 & 4 & Swine & Military \\
\hline James Beavers & 12 & 4 & Chickens & College \\
\hline Justin Bradford & 11 & 4. & Ag Mech & College \\
\hline Mark Cavallero & 11 & 4 & Pomology & College \\
\hline Jaime Cuevas & 12 & 4 & Chickens & College \\
\hline Vanessa Duarte & 12 & 4 & Swine & College \\
\hline Micheal Ewing & 11 & 4 & Swine & College \\
\hline Jenae Hansen & 11 & 4 & Viticulture & College \\
\hline Victoria Jones & 12 & 4 & sheep & College \\
\hline Virat Kang & 11 & 4 & Pomology & College \\
\hline Vanessa Maravilla & 11 & 4 & Beef Production & College \\
\hline Jorge Mendoza & 11 & 4 & sheep & College \\
\hline Chris Oharo & 11 & 4 & sheep & College \\
\hline Gabrielle Ortega & 10 & 4 & Swine & College \\
\hline Gissel Pedraza & 11 & 4 & sheep & College \\
\hline Sarah Reece & 10 & 4 & Swine & College \\
\hline Rodolfo Rodriguez & 10 & 4. & Swine & College \\
\hline Clayton Sheehan & 10 & $4:$ & Swine & College \\
\hline Quinn Shippey & 11 & 4 & Landscape & College \\
\hline Gilberto Antonio & 11 & 5 & Engine/Repair & Military \\
\hline Eduardo Daza & 10 & 5 & Engine/Repair & Military \\
\hline Evelyn Delarosa & 10 & 5 & Engine/Repair & Work \\
\hline Elias Delgado & 10 & 5 & Engine/Repair & Work \\
\hline Ismeal Guzman & 11 & 5 & Engine/Repair & Work \\
\hline Jacob hernandez & 11 & 5 & Engine/Repair & Work \\
\hline Marcos Jimenez & 11 & 5 & Engine/Repair & Military \\
\hline Daniel Lopez & 11 & 5 & Engine/Repair & College \\
\hline Jesus Lopez & 10 & 5 & Engine/Repair & Military \\
\hline David Martinez & 10 & 5 & Engine/Repair & College \\
\hline Alexis mendoza & 10 & 5 & Engine/Repair & Work \\
\hline Alexis Morales & 10 & 5 & Engine/Repair & Work \\
\hline Rodrigo Ortega & 11 & 5 & Engine/Repair & Work \\
\hline Fernando Perez & 12 & 5 & Engine/Repair & Military \\
\hline Gabriel Pimentel & 11 & 5 & Engine/Repair & Military \\
\hline Alfonso Rendon & 10 & 5 & Engine/Repair & College \\
\hline
\end{tabular}




\begin{tabular}{|l|r|r|l|l|}
\hline Juan Rendon & 11 & 5 & Engine/Repair & College \\
\hline Josue Sandoval & 11 & 5 & Engine/Repair & College \\
\hline Jesus Santos & 10 & 5 & Engine/Repair & College \\
\hline Raymond Serna & 10 & 5 & Engine/Repair & Military \\
\hline Michael Williams & 10 & 5 & Engine/Repair & Military \\
\hline Miguel Zaragoza & 10 & 5 & Engine/Repair & Military \\
\hline Yuvani Zarate & 10 & 5 & Engine/Repair & College \\
\hline Carlos Zarate & 10 & 5 & Engine/Repair & Work \\
\hline
\end{tabular}



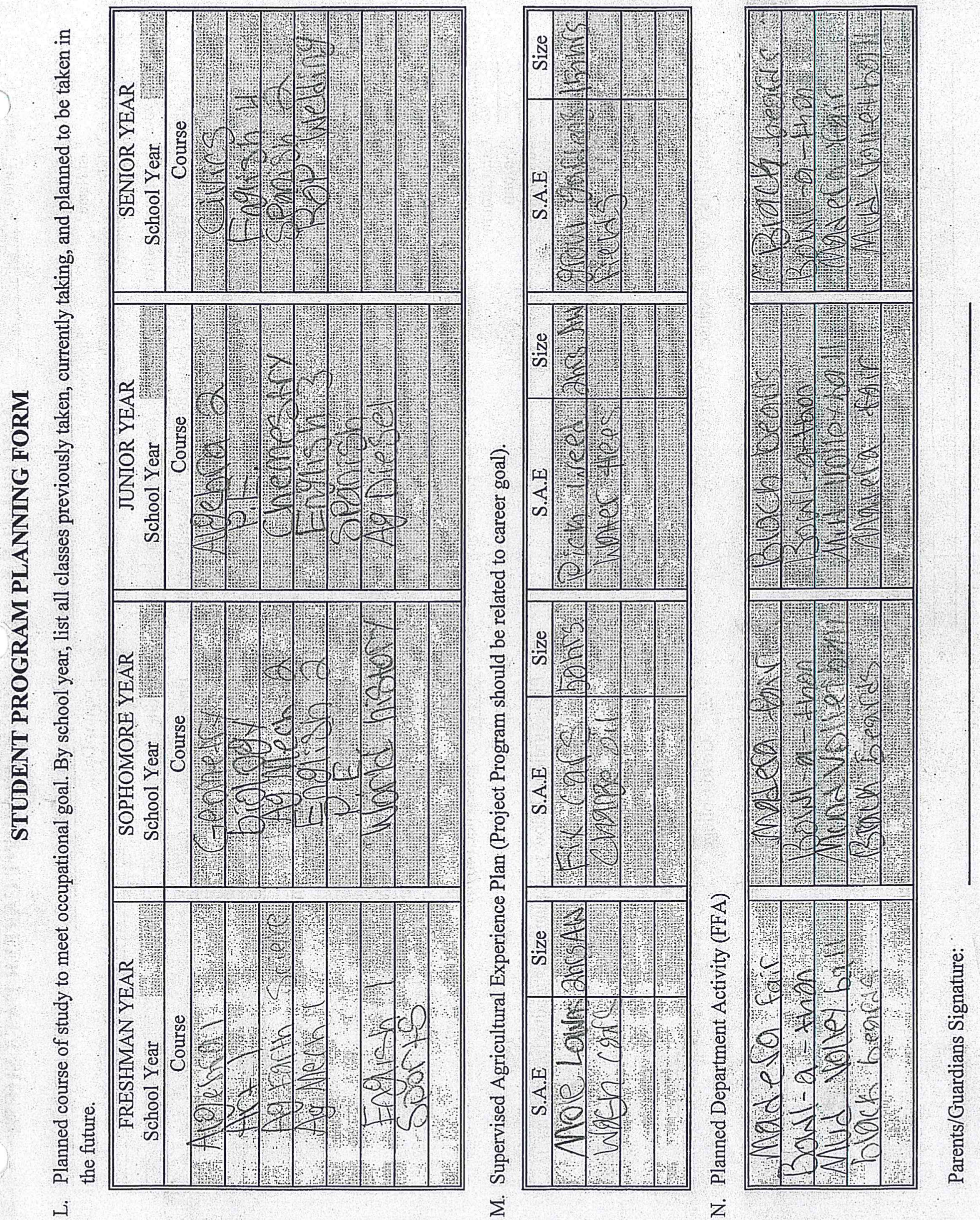


\section{H. Board Approve Department SOEP Policy, Procedures And Operation Statement}




\section{Madera FFA Exhibitors Contract}

As a member of the Madera FFA chapter, I realize that there are certain obligations on my part in order to assure a successful project. As part of my obligations, I agree that the following expectations will help me to complete my animal project. This contract shall begin on and terminate on

- All students shall be respectful to parents, advisors, staff and students.

- All students shall be in good standing, a current member of the FFA, and maintain a 2.0 GPA throughout the duration of the project.

- No students shall show/exhibit an animal project as a member of the Madera FFA without an Exhibitors Contract signed by 1) the exhibitor, 2) parents/guardians, 3) chapter advisor and 4) the vice principal.

- All school rules, district, and California State rules and the specific rules pertaining to the show in question shall be upheld by the exhibitor.

- Throughout the term of the project all exhibitors are to follow the directions and advice given to them by the designated advisor for that species.

- All exhibitors will be responsible for the care, feeding, exhibiting and marketing of their animals.

- All students will participate in showmanship and $80 \%$ of the scheduled showmanship practices prior to the fair.

- All students will show in the "Official FFA Show Uniform" 1) white pants, 2) white collared shirt, 3) FFA jacket and 4) FFA Tie/Scarf.

- No students shall be on the fairgrounds after 9:00 PM without the written permission from the parents/guardians and supervised Ag. Staff.

- Student may transport themselves and siblings with written approval from 1) Parents/Guardians, 2) School Administration (Permission in writing from the vice principal and parents/guardians and given to the advisor prior to the event.

- No student shall leave the fairgrounds at anytime without the supervision of his/her parent/guardian, designated district chaperone or chapter advisor.

- FFA members are required to obtain their homework from all of their teachers in advance of missing school for attending fairs.

- Each exhibitor must read and understand the rules and regulations in the fair's premium book.

- The student must sign-in and sign out every day during participation in the fair.

- Each exhibitor is required to serve barn duties as assigned and specified by the project advisor.

- All students will participate in the moving in and (Loading and setting-up) and removal (clean-up and removal of equipment from the show).

- ALL Students shall be responsible for completing the following prior to their premium or market check being issued: 1) A complete \& up-dated record book, 2) thank-you letters to buyers, award donors and add-on bids 3) all bills paid in full and 4) all hours worked at the farm.

Failure to comply with any of the above obligations due to extenuating circumstances must be approved by the advisor. Failure to do so is in violation of the contract and will result in the loss of
showing privileges effective immediately.

Student

Parent/Guardian

Advisor

School of Ag Vice Principal

\section{Date}

Date

Date

Date 


\section{Madera FFA Exhe ibitors Contract}

As a member of the Madera FFA chapter, I to complete my animal project. This contract shations, I agree that the following on my part in
terminate on

- All students shall be respectul to parents, advisors, help me

All students shall be in good standing a thro advisors, staff and students,

- No students shall stion of the project. Exhibitors Contract signed an animal project as a

vice principal.
All school rules, district, and

question shall be upheld by the exhibia State rules and the specific rules pertaining Throughout the term of the project altor.

- $\quad$ All by the designated advisoject all exhibitors are to follow

- All students will participate in showman care, feeding, exhibiting and and andice given to

-

All students will show in the "Orfar animals.

- No FFA jacket and 4) FFA Tie/Scarf.

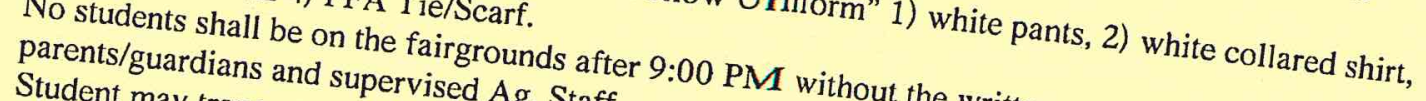

- Student may transport themselves and staff. 2) School Administration (Permission in wings with written approval from

- No student shallor prior to the event. parent/guardian, designated fairgrounds at anytime with

- FFA members are required district chaperone or chapter the supervision of his/her

missing school for attending fairs.
Each exhibitor mus homework from all of their teachers

- The student must sign-in and understand the rull

- Each exhibitor is required to serve barn duties as assigned participation in the fair.

- ALL Students equipment from the showing in and (Loading and setting-up) the project advisor. check being issuedl be responsible for completing the folting-up) and removal (clean-up donors and add iss: 1) A complete \& up-dated recollowing prior to their premiun follo 3) all bills paid in full and 4) all hook, 2) thank-you letters to buyers, award approved by the advish any of the above obligations due to

showing privileges effective immediately.
in violation of the contract and will result in the be Student

Parent/Guardian

Advisor

School of Ag Vice Principal

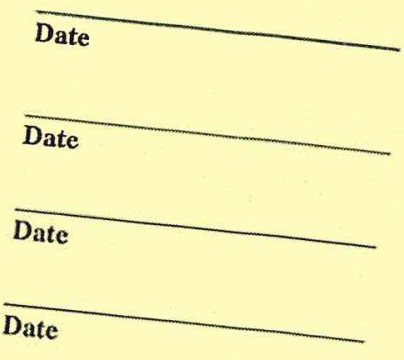




\section{Madera South High School Farm Laboratory Policy Regarding Use}

Project Owner:

Type of Project:

Advisor:

The sole purposes of the Madera High School Farm Laboratory are to provide a laboratory for hands-on instruction in a vocational agriculture class and to allow the students enrolled in vocational agriculture to conduct Supervised Agricultural Experience Projects (SAE).

1. Only a student currently enrolled in a vocational agriculture class or FFA program at Madera South High School shall have the privilege of using the School Farm Lab.

2. The School Farm Lab is part of the campus of Madera South High School; therefore, all Madera South High School and Madera Unified School District policies apply to the School Farm Lab.

3. The responsibility of the project owner (student) includes but is not limited to keeping all gates to the School Farm Lab locked, feeding, grooming, pen clean-up and maintenance, hauling feed/manure and other supplies, planting, irrigation, weeding, harvest, selling, and any care necessary to maintain the health and/or aesthetics of animals at the School Farm Lab.

4. The Madera Unified School District and its employees are NOT responsible for the loss, theft, disappearance, or death of any animal. The students are responsible for all personal equipment or materials of any kind.

5. No person shall drive or operate any vehicle on the School Farm Lab without prior permission or supervision from a Madera South High School vocational agriculture instructor.

6. School Farm Lab hours shall be 6:00 am to $8: 00 \mathrm{pm}$. No one shall conduct any activity on the school Farm Lab during closed hours without prior permission from the advisor. ONLY current FFA members are allowed on the school farm grounds.

7. In the event of an emergency, if services of a veterinarian are utilized the fees of the services are the responsibility of the project owner (student) and/or parent/guardian. This excludes breeding projects owned by the Madera South Ag Department.

8. To assure proper care of livestock animals, animals are expected to be fed and/or checked twice daily. Morning feeding hours must occur between $6-8$ am and evening feeding hours shall be from 5-8pm.

9. A student exhibiting a project at the fair must have a signed exhibitors contract to accompany this contract.

10. Any infraction of school or district policy, any infraction of this agreement, or any lack of responsibility on the part of the student may result in the indefinite removal of the project from the School Farm Lab and/or the indefinite revoking of School Farm Lab use privileges of the students.

11. Administration and interpretation of all policies regarding use of the Madera South High School Farm Laboratory shall be the responsibility of the project advisor.

12. Notification or infraction of any of the aforementioned policies shall inherent the following disciplinary actions:

$1^{\text {st }}$ offense: A written warning

$2^{\text {nd }}$ offense: A written warning, letter sent home and/or phone call to parents

$3^{\text {rd }}$ offense: Removal of student project from school farm

Upon receipt of third offense, the student will have 1 week to remove his/her animal from the farm. The project must be paid, in full, prior to removal. Projects not paid for in full will become school property. Projects will then be sold. Proceeds from the sale of the project will be credited to the student's financial obligations.

I have read and understand the policy of the Madera South High School Farm Laboratory and hereby comply in agreement with all aspects of this policy.

Parent/Guardian Signature

Student Signature

Advisor Signature

Vice Principal Signature

\section{Date}

Date

Date

Date 
I. Program Of work 


\section{Modera FFA \\ Prograce of Aativitios}

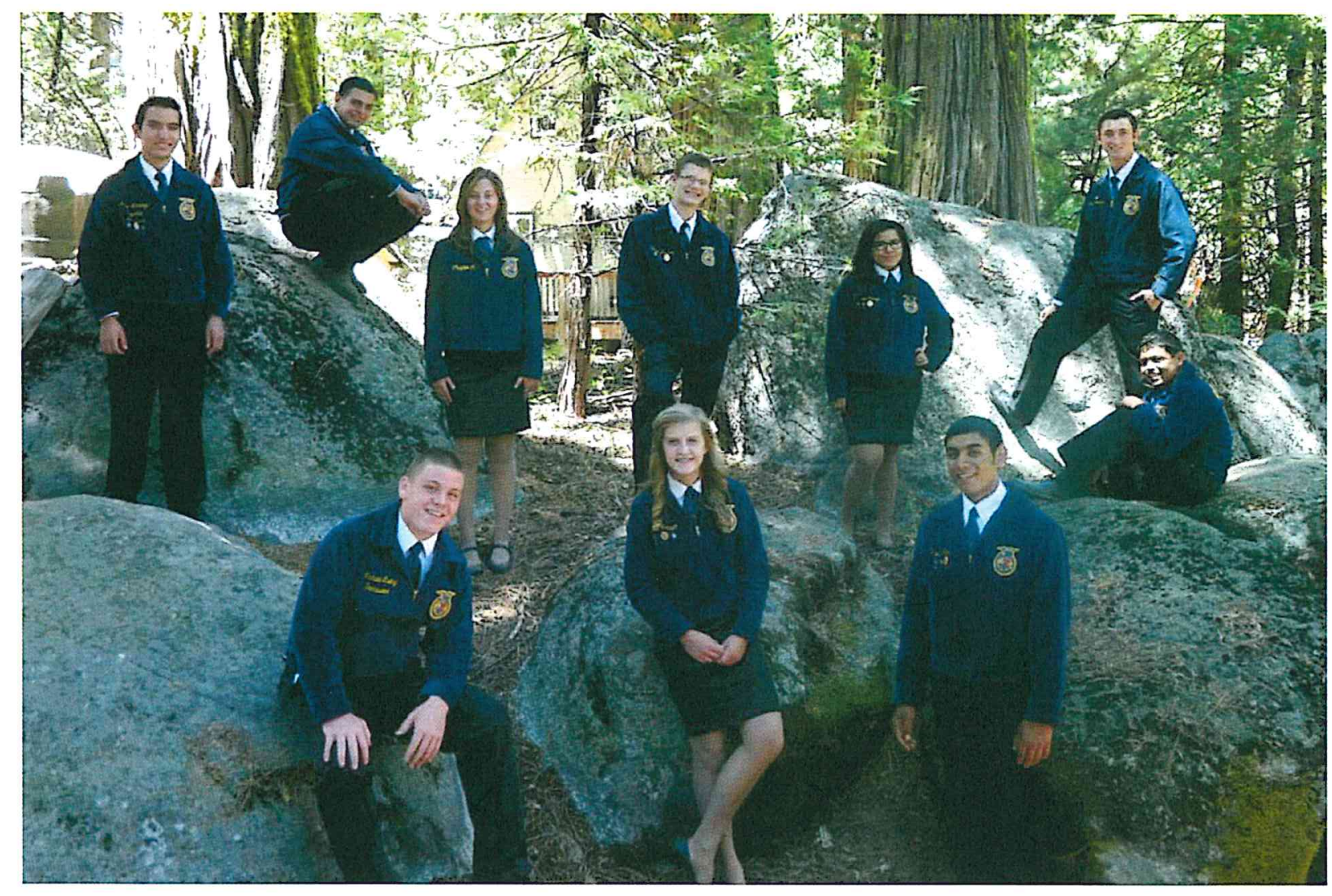

2

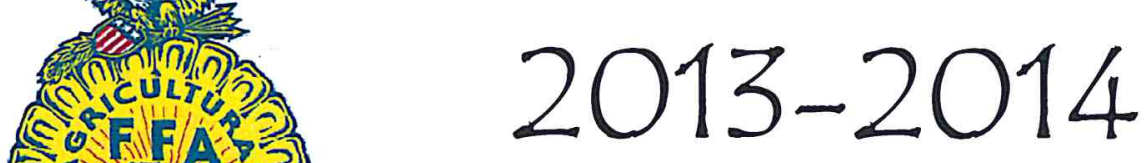

(c)

$[E-\infty]$

(C) OCATO

(1) (U) (U) (U) (1)

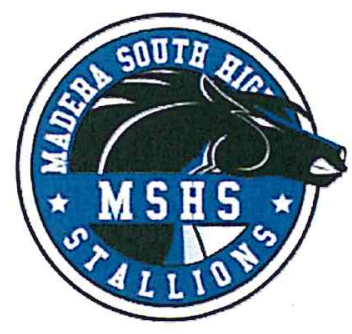




\section{Table of Contents}

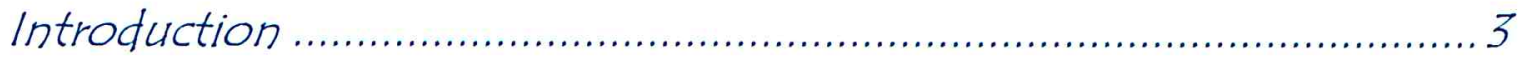

Advisors Greetings ..................................................................... 4

Officer's Message and Goals ........................................................ 5

FFA History .................................................................... $6-23$

Calendar of Events 2013-2014 .................................................. 24-36

FFA Activities Budget....................................................... 37-38

Community Service Activities............................................... 39

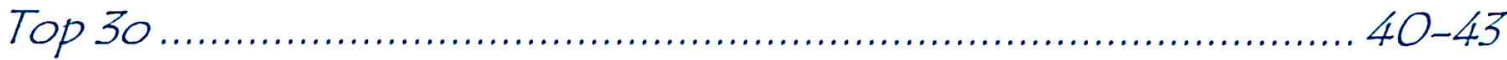

Madera FFA Constitution and Bylaws ................................... 44-49

FFA Awards and Recognition ............................................50-53

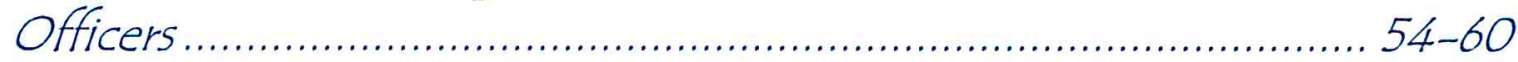

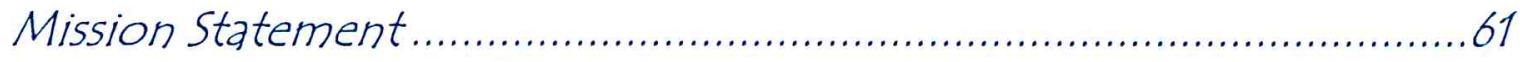

FFA Emblem and Uniform .....................................................62

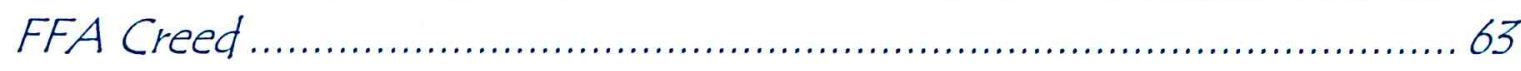

Career Development Events ..........................................6.64-66

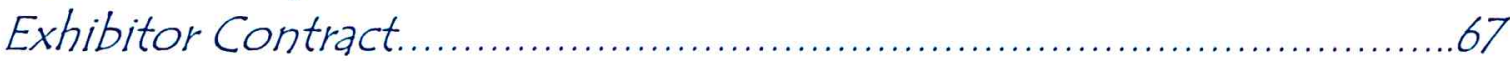

Project Facility Contract..............................................68

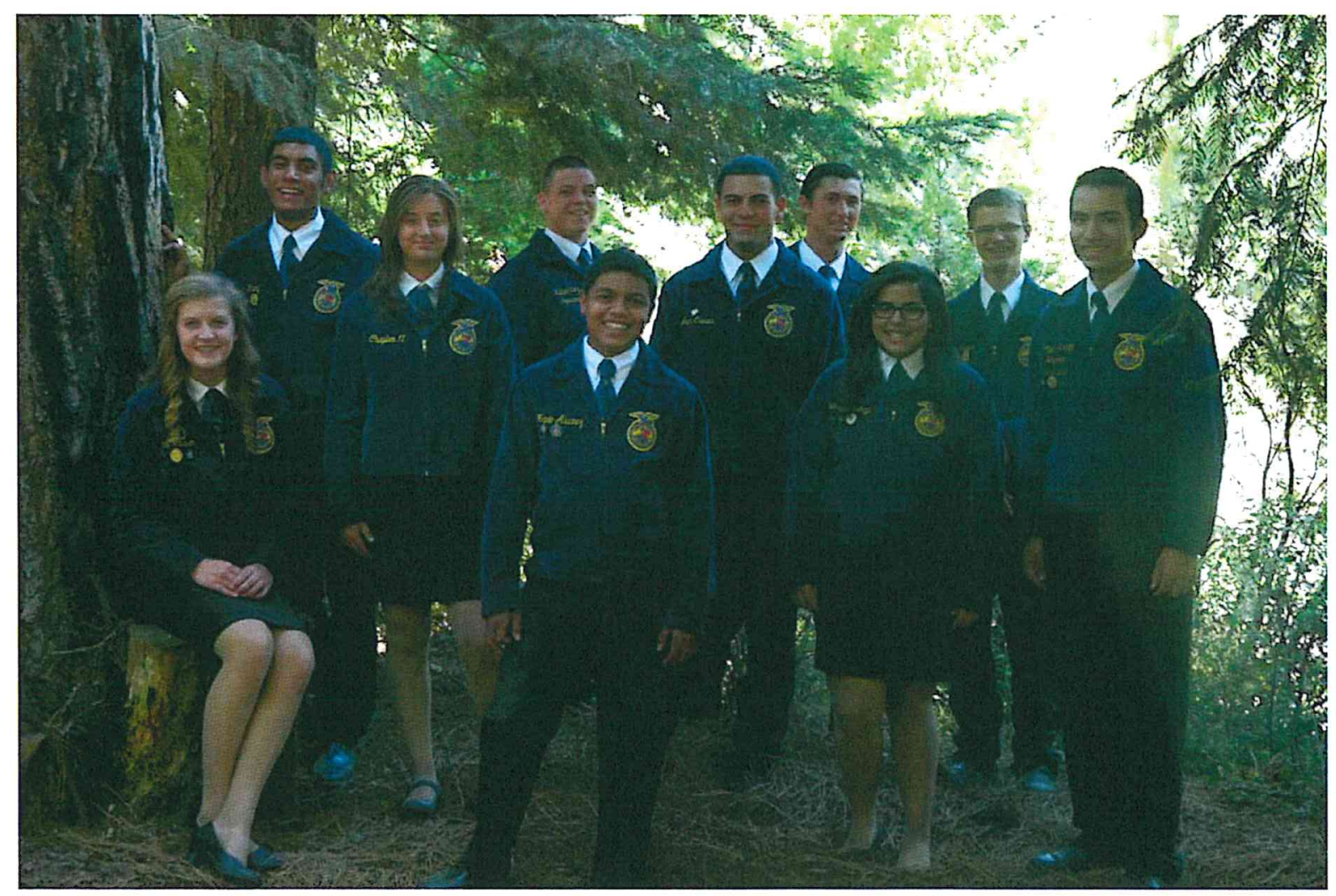




\section{InEIroduction to POA}

The Program of Activities can be used as a guide, outlining the variety of activities students can become involved with. Student involvement is the key to success for a powerful agriculture program. Without member involvement all the officer teams goals and missions along with advisor guidance wouldn't accomplish much. The success of your agriculture department is almost entirely dependent upon your involvement and your desire to do the very best you can do.

In this Program of Activities you will find the rules of the chapter through our constitution, a list of all activities you can become involved in within our calendar pages, money and awards available to you and how to get them, the History of the Madera FFA Chapter dating back to its beginnings, as well as History and information about the National FFA Organization.

Keep this copy of our Program of Activities as a reference as you go on through the year and as you take advantage of all the opportunities that Madera FFA can offer you.

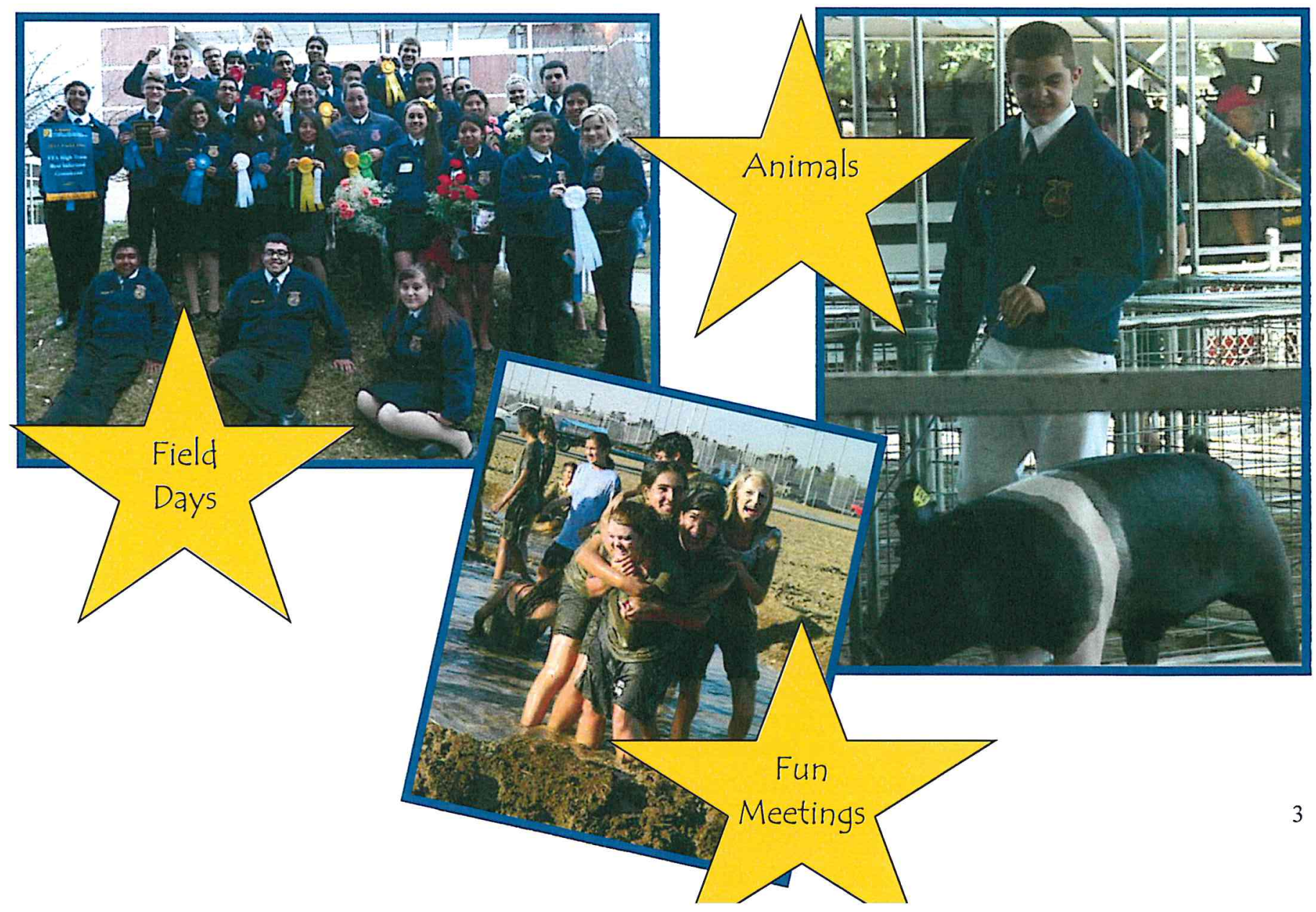




\section{Advisor's Greetings}

Welcome back to MSHS and the Madera FFA. Our advisors Mr. George, Mr. Deniz, Mr. Williams, Mrs. McKenna, Miss. Luera, Mrs. Sheehan, and Miss Gilles all agree that this is the best facility in the State of California. Our farm, classroom and laboratories are state of the art and as a student you have full access to them. It is our goal as a staff to provide our students with the greatest opportunities and learning experiences by fully utilizing the facilities we have been blessed with.

This year promises to be one of new growth and expansion. This year we will be taking a large group of students to both the Madera and Chowchilla Fairs where they will showcase their livestock, horticulture, and mechanics projects. The students and advisors have put in numerous hours of effort and hard work in expectations of an extremely successful year at both county fairs. During the year our farm facilities will be improved to include finalizing the school farm vineyard as well as general upkeep. For those of you with a stronger farming interest, there will be opportunities to plant row crops. The advisors will be looking for enthused hard working students to help develop our farm from barren land to a lush high producing operation.

As advisors we agree with the officers that individual student growth, both personally and professionally is the number one, most important element our department can offer each member. In order for this growth to occur we will provide many different opportunities for student involvement throughout the year. So once again, welcome back, and welcome to one of the most exciting years of your life. As advisors we look forward to working with each and every one of you and firmly believe that with your help we can ensure that Madera FFA remains a powerhouse agriculture program in the state of California!

Sincerely,

\section{The Madera FFA Advisors}

Mrs. McKenna Mrs. Sheehan

Miss Luera

Mr. Deniz

Mr. Williams

Mr. George

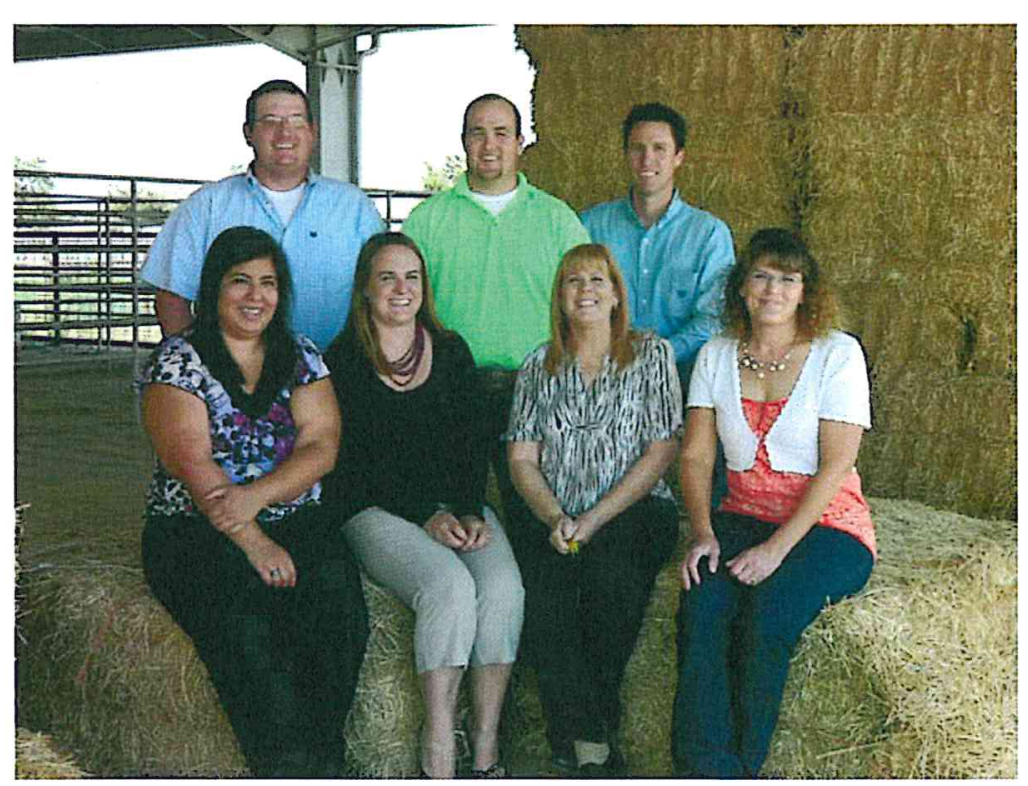




\section{Officers Message}

The Madera FFA Chapter officer team looks forward to the upcoming year in which we strive to serve our members by developing premier leadership, personal growth, and career success. through agricultural education.

\section{Mader FA Team Goals}

- Advertise meetings by fliers, posters, and slideshows done 2 weeks prior to the meeting

- Meet at least one new member a month and keep in touch with them.

- Have presentations for meetings done 1 week before FFA meeting.

- Officers must maintain a GPA of over 3.0 and have at least a $B$ in their AG class.

\section{Maderer FFA Chorpter Gool}

In the upcoming year we will strive to increase chapter participation by having at least one of our monthly meetings with $40 \%$ of our membership in attendance.

\section{Madera FA Theme}

Small acts impact beyond belief. 


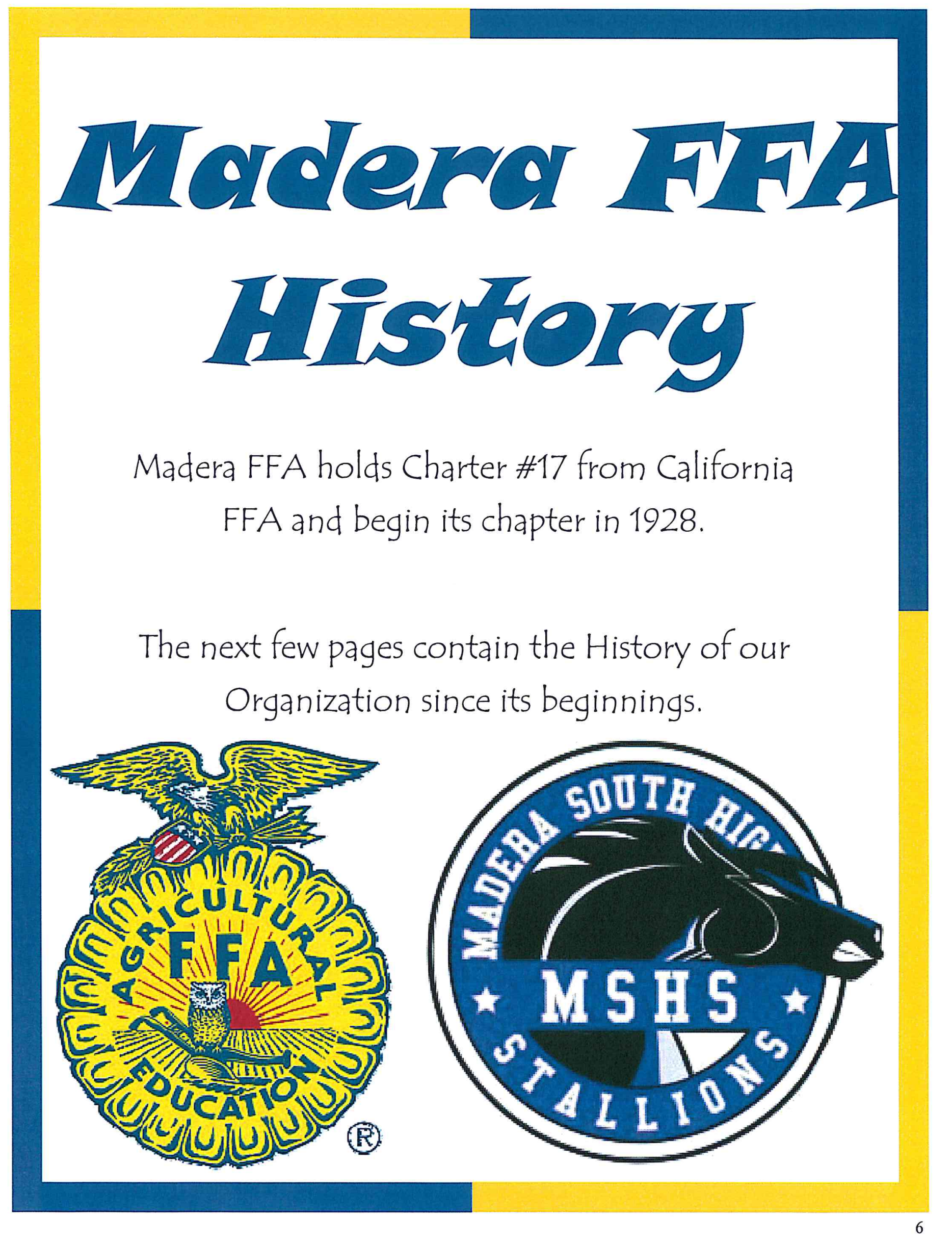




\section{Seationall Proficieney Winners}

\begin{tabular}{|c|c|c|}
\hline Matthew Chavira & Ag Mechanics Design/Fabrication Entrepreneur & $2005-06$ \\
\hline Enrique Hernandez & Agribusiness & $2005-06$ \\
\hline William Thornton & Diversified Crop Production & $2005-06$ \\
\hline Ectali Mendez & Floriculture Placement & $2006-07$ \\
\hline Michael Fincher & Beef Production Placement & $2006-07$ \\
\hline Cody Ogletree & Ag Mechanics Design/Fabrication Entrepreneur & $2006-07$ \\
\hline Enrique Hernandez & Agribusiness & $2008-09$ \\
\hline Enrique Hernandez & Turf Grass Management Entrepreneur & $2008-09$ \\
\hline Sierra Meyers & Beef Production & $2008-09$ \\
\hline Amy Evans & Beef Production & $2008-09$ \\
\hline Alonzo Hernandez & Diversified Livestock & $2008-09$ \\
\hline Jessica Sidney & Agriculture Education & $2008-09$ \\
\hline Ben Salazar & Agricultural Mechanics Design and Fabrication & $2009-10$ \\
\hline Amy Evans & Beef Production Entrepreneurship & $2009-10$ \\
\hline Sierra Meyers & Beef Production Placement & $2009-10$ \\
\hline Matthew Borges & Dairy Production Placement & $2009-10$ \\
\hline Gustavo Contreras & Diversified Crop Production Placement & $2009-10$ \\
\hline Caleb Hatfield & Equine Science Placement & $2009-10$ \\
\hline Magali Rodriguez & Floriculture & $2009-10$ \\
\hline Scott Thornton & Pomology Production Placement & $2009-10$ \\
\hline Lindsay Tasos & Swine Production (Entrepreneurship) & $2009-10$ \\
\hline Gabe Sanchez & Ag. Mechanics Design and Fabrication & $2010-2011$ \\
\hline Michael Valencia & Specialty Animal Production & $2010-2011$ \\
\hline Amy Evans & Beef Production Entrepreneurship & $2010-2011$ \\
\hline John McClure & Diversified Horticulture Placement & $2010-2011$ \\
\hline Lindsay Tasos & Swine Production (Entrepreneurship) & $2010-2011$ \\
\hline Magali Rodriguez & Floriculture & $2010-2011$ \\
\hline
\end{tabular}




\section{Seational Profiatenay hilnmers}

\begin{tabular}{|c|c|c|}
\hline David Nino & Viticulture Placement & $2010-2011$ \\
\hline Matthew Borges & Forage & $2010-2011$ \\
\hline Michael Valencia & Ag. Services & $2012-2013$ \\
\hline Jackie Vasquez & Ag. Sales Placement & $2012-13$ \\
\hline Chris Melikian & Ag. Services & $2012-13$ \\
\hline
\end{tabular}

\section{Regional Profiatenay Iinners}

\begin{tabular}{|l|l|l|}
\hline Enrique Hernandez & Turf Grass Management Entrepreneur & $2005-06$ \\
\hline Cinda Mattrocce & Diversified Livestock Production & $2005-06$ \\
\hline Warren Tucker & Grain Production & $2005-06$ \\
\hline Ectali Mendez & Floriculture Placement & $2006-07$ \\
\hline Enrique Hernandez & Turf grass Management Entrepreneur & $2008-09$ \\
\hline Matthew Chavira & Ag Sales & $2008-09$ \\
\hline Matthew Chavira & Ag Mechanics Fabrication/Design Entrepreneur & $2008-09$ \\
\hline Matthew Borges & Dairy Production Placement & $2009-10$ \\
\hline Gabriel Sanchez & Agriculture Mechanics Design/Fabrication & $2010-11$ \\
\hline Amy Evans & Beef Production Entrepreneurship & $2010-11$ \\
\hline Michael Valencia & Specialty Animal Production & $2010-11$ \\
\hline John McClure & Diversified Horticulture Placement & $2010-11$ \\
\hline Michael Valencia & Specialty Animal Production & $2011-12$ \\
\hline Michael Valencia & Ag. Services & $2012-2013$ \\
\hline
\end{tabular}

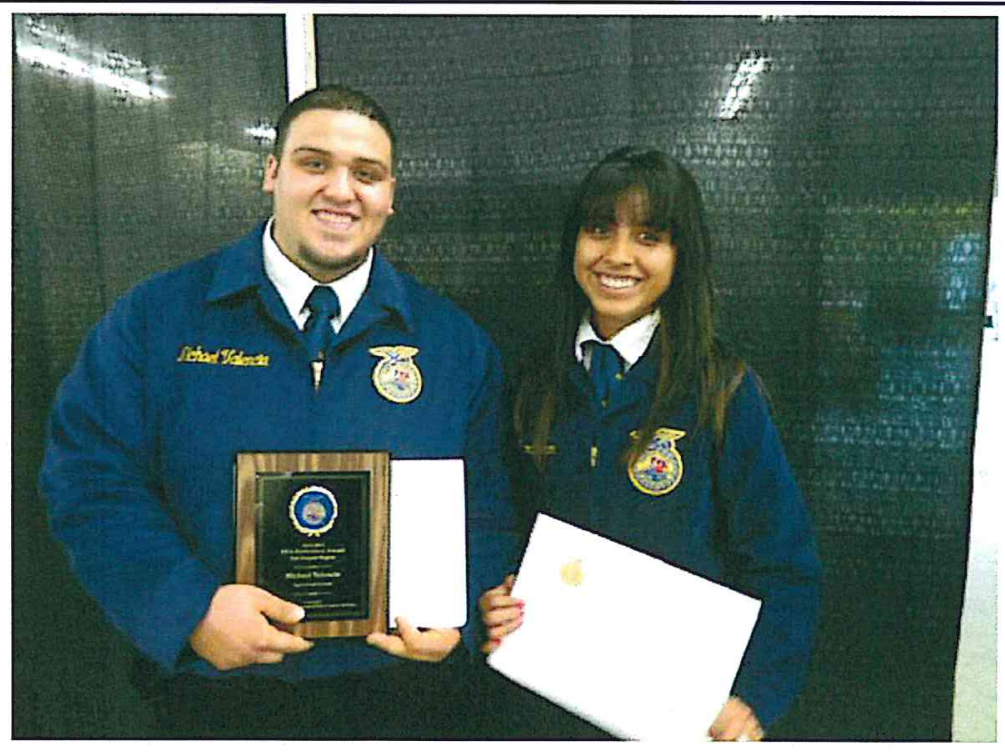




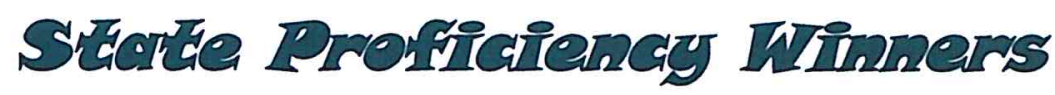

\begin{tabular}{|c|c|c|}
\hline & Farm Safety & 1954-1955 \\
\hline & Farm Safety & $1957-1958$ \\
\hline Daryl Schlumbohm & Farm Mechanics & $1959-1960$ \\
\hline Bruno Pelanconi & Crop Production & $1960-1961$ \\
\hline Steve Ficklin & Farm and Home Electrification & $1960-1961$ \\
\hline Tommy Sesock & Farm Mechanics & $1960-1961$ \\
\hline Carl Schroeder & Farm Mechanics & $1962-1963$ \\
\hline Terrel West & Farm Mechanics & $1963-1964$ \\
\hline Steve Grant & Natural Resources & $1968-1969$ \\
\hline Douglas Anderson & Ornamental Horticulture & $1969-1970$ \\
\hline Mark Carlson & Agricultural Electrification & $1970-1971$ \\
\hline Mark Foster & Fish and Wildlife Management & $1970-1971$ \\
\hline Sandy Konkol & Placement in Processing & $1970-1971$ \\
\hline Denis Prosperi & Soil, Water and Air Management & 1970-1971 \\
\hline Mark Lindsay & Agriculture Production & $1971-1972$ \\
\hline Conrad Bitter & Placement in Processing & $1971-1972$ \\
\hline Henry Oyler & Ag Sales and Services & $1973-1974$ \\
\hline Kevin Lee Peters & Home and Farmstead Improvement & $1976-1977$ \\
\hline Gary Agajanian & Soil, Water, and Air Management & $1976-1977$ \\
\hline Gary Agajanian & Ag Mechanics & $1977-1978$ \\
\hline John Koretoff & Soil and Water Management & $1977-1978$ \\
\hline Kurt Peters & Turf and Landscape Management & $1977-1978$ \\
\hline John Toschi & Nursery Operations & $1979-1980$ \\
\hline Bill Valorosi & Soil and Water Management & $1979-1980$ \\
\hline Bill Valorosi & Ag. Mechanics & $1980-1981$ \\
\hline Rob Hall & Floriculture & $1980-1981$ \\
\hline John Toschi & Nursery Operations & 1980-1981 \\
\hline John Toschi & Nursery Operations & $1981-1982$ \\
\hline Kenneth Tucker & Soil and Water Management & $1981-1982$ \\
\hline Michael S. McClaran & Soil and Water Management & $1982-1983$ \\
\hline Sam Weis & Agricultural Mechanics & $1983-1984$ \\
\hline Doug Reed & Agricultural Electrification & $1985-1986$ \\
\hline Todd Fischer & Soil and Water Management & 1986-1987 \\
\hline Kevin Bier & Agricultural Electrification & $1987-1988$ \\
\hline Barbara Turner & Horse Production & 1990-1991 \\
\hline Sahan Van Alen & Beef Production & $1992-1993$ \\
\hline
\end{tabular}




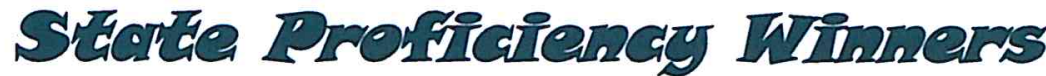

\begin{tabular}{|l|l|l|}
\hline Scott Bursey & Viticulture Production Entrepreneurship & $1997-1998$ \\
\hline Kyle Prosperi & Viticulture Production Entrepreneurship & $1999-2000$ \\
\hline Grant Tucker & Grain Production Entrepreneurship & $2003-2004$ \\
\hline Stephanie Bellew & Ag. Mechanics Energy System & $2003-2004$ \\
\hline Lauren Da Silva & Equine Science Entrepreneurship & $2005-2006$ \\
\hline Alexandria Wara & Vegetable Crop Entrepreneurship & $2005-2006$ \\
\hline Lauren DeSilva & Equine Entrepreneurship & $2005-2006$ \\
\hline Enrique Hernandez & Turf Grass Management & $2005-2006$ \\
\hline Warren Tucker & Grain Production Entrepreneurship & $2005-2006$ \\
\hline Cinda Mattrocce & Diversified Livestock Entrepreneurship & $2005-2006$ \\
\hline Enrique Hernandez & Turf Grass Management Entrepreneur & $2008-2009$ \\
\hline Matthew Borges & Dairy Production Placement & $2009-2010$ \\
\hline Michael Valencia & Specialty Animal Production & $2011-2012$ \\
\hline Michael Valencia & Ag. Services & $2012-2013$ \\
\hline
\end{tabular}

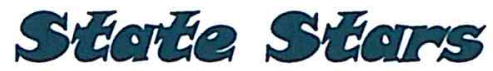

\begin{tabular}{|c|c|c|}
\hline Donald Cobb & State Star Farmer & $1940-1941$ \\
\hline Stephanie Bellew & State Star Reporter & $2002-2003$ \\
\hline Stephanie Bellew & State Star Reporter & $2003-2004$ \\
\hline Bret Theodozio & State Star Administrator & $2003-2004$ \\
\hline Enrique Hernandez Jr. & State Star Agribusiness & $2009-2010$ \\
\hline Michael Valencia & State Star Agribusiness & $2011-1012$ \\
\hline
\end{tabular}

\section{Nertionall Profiaienay Iilnners}

\begin{tabular}{|c|c|c|}
\hline John Sousa, Jr & Ag. Mechanics & 1983 \\
\hline Bruno Pelanconi, Jr. & Crop Production & $1961-1962$ \\
\hline Michael Valencia & Specialty Animal Production & 2012 \\
\hline
\end{tabular}




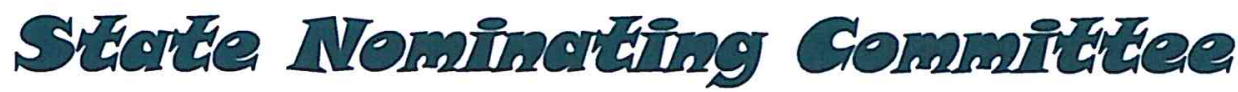

\begin{tabular}{|c|c|}
\hline Shirley Jones & $1935-1936$ \\
\hline Bill Spillane & $1938-1939$ \\
\hline Pete Laborde & $1939-1940$ \\
\hline Dino Petrucci & $1948-1949$ \\
\hline Dominic Bettini & $2009-2010$ \\
\hline Matthew Borges & $2010-2011$ \\
\hline
\end{tabular}

\section{Band, Ghorus Parritialpontes}

\begin{tabular}{|c|c|c|}
\hline Joseph Lilles, Jr. & National Chorus & $1956-1957$ \\
\hline Nicholas Lilles & State Chorus & $2000-2001$ \\
\hline Michael Croxen & State Band & $2002-2003$ \\
\hline Michael Croxen & National Band & $2003-2004$ \\
\hline Jorge Mendoza & State Band & $2011-2012$ \\
\hline Sukhvir Singh & State Band & $2011-2012$ \\
\hline
\end{tabular}

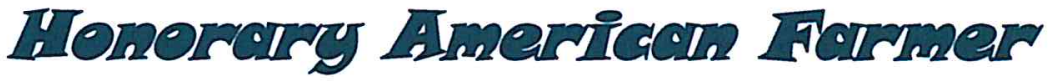

\begin{tabular}{|c|c|}
\hline Warren Smith & 1950 \\
\hline L.M. Dodd & 1951 \\
\hline Dino Petrucci & 1966 \\
\hline Jim Bomprezzi & 2007 \\
\hline
\end{tabular}




\section{Past Regional FIAI OAficias}

\begin{tabular}{|c|c|c|}
\hline Robert Crawford & President & $1934-1935$ \\
\hline Eugene Foust & Secretary & $1936-1937$ \\
\hline Ray Thomas & President & $1941-1942$ \\
\hline Dino Petrucci & President & $1947-1948$ \\
\hline Tom Westing & Reporter & $1953-1954$ \\
\hline Joe Stasulat & Secretary & $1960-1961$ \\
\hline Dan Chatman & Reporter & $1961-1962$ \\
\hline Wallace Emmert & President & $1963-1964$ \\
\hline Ralph Pistoresi & President & $1969-1970$ \\
\hline Roger Evans & Treasurer & $1971-1972$ \\
\hline Beth Boysen & Secretary & $1972-1973$ \\
\hline Beth Boysen & Vice President & $1973-1974$ \\
\hline John Koretoff & Sentinel & $1977-1978$ \\
\hline Shąna ValAlen & Vice President & $1992-1993$ \\
\hline Kyle Prosperi & Secretary & $2000-2001$ \\
\hline Megan Matteucci & Vice President & $2005-2006$ \\
\hline Zac Pruitt & Treasuer & $2006-2007$ \\
\hline Mika Petrucci & Reporter & $2007-2008$ \\
\hline Tiffany O'Haro & Vice President & $2008-2009$ \\
\hline Jessica Sidney & Treasurer & $2008-2009$ \\
\hline Dominic Bettini & Treasurer & $2009-2010$ \\
\hline Taylor Helton & Vice President & $2012-2013$ \\
\hline Virat Kang & Reporter & $2013-2014$ \\
\hline
\end{tabular}

\section{Pass Sterte FIFA Officens}

\begin{tabular}{|c|c|c|}
\hline Walter Ficklin & President & $1930-1931$ \\
\hline Calvin Jones & Vice President & $1931-1932$ \\
\hline Dino Petrucci & President & $1948-1949$ \\
\hline John Deniz & President & $1949-1950$ \\
\hline William Justice & Secretary & $1952-1953$ \\
\hline Tom Westing & Reporter & $1962-1963$ \\
\hline Dan Chatman & Secretary & $1963-1964$ \\
\hline Dan Chatman & Vice President & $1967-1968$ \\
\hline Larry Hirahara & President & $1967-1968$ \\
\hline David Loquaci & Secretary & 12 \\
\hline
\end{tabular}




\section{Past National Convention Dellegaries}

\begin{tabular}{|c|c|}
\hline Dino Petrucci & $1947-1948$ \\
\hline Dino Petrucci & 1948-1949 \\
\hline Dino Petrucci & $1949-1950$ \\
\hline Dan Chatman & $1962-1963$ \\
\hline Dan Chatman & $1963-1964$ \\
\hline Shana Van Alen & $1991-1992$ \\
\hline Michael Gomes & 1992-1993 \\
\hline Monica Williams & 1993-1994 \\
\hline Nicole Greci & 1993-1994 \\
\hline Tommy Greci & 1994-1995 \\
\hline Lenny Edlebacher & $1995-1996$ \\
\hline Tommy Greci & $1995-1996$ \\
\hline Shane Geist & $1995-1996$ \\
\hline Lisa McKinley & 1996-1997 \\
\hline Manuel Marin & $1997-1998$ \\
\hline Stacey Visscher & 1998-1999 \\
\hline Kyle Prosperi & 1998-1999 \\
\hline Chris Britton & 1998-1999 \\
\hline Kyle Prosperi & $1999-2000$ \\
\hline Megan Matteucci & $2005-2006$ \\
\hline Cinda Mattrocce & $2006-2007$ \\
\hline Mika Petrucci & $2007-2008$ \\
\hline Vincent Urena & $2007-2008$ \\
\hline Jessica Sidney & $2008-2009$ \\
\hline Matthew Borges & $2010-2011$ \\
\hline Michael Valencia & $2011-2012$ \\
\hline Taylor Helton & $2011-2012$ \\
\hline Taylor Helton & $2012-2013$ \\
\hline
\end{tabular}




\section{SEAE FA Degrees}

\begin{tabular}{|c|c|}
\hline Walter Ficklin & $1929-1930$ \\
\hline J.S. Davis & $1931-1932$ \\
\hline Robert Albonico & $1932-1933$ \\
\hline Williąm Gong & $1936-1937$ \\
\hline Donald Cobb & $1940-1941$ \\
\hline Avery Overgarrd & $1940-1941$ \\
\hline Raymond Thomas & $1941-1942$ \\
\hline Henry Janzen & $1945-1946$ \\
\hline John Mallory & $1945-1946$ \\
\hline Roy Mallory & $1945-1946$ \\
\hline Calvin Martin & $1945-1946$ \\
\hline Nat Morris & $1945-1946$ \\
\hline Dino Petrucci & $1945-1946$ \\
\hline Paul Toschi & $1945-1946$ \\
\hline Donald Bare & $1946-1947$ \\
\hline Bob Diebert & $1946-1947$ \\
\hline Raymond Dolio & $1946-1947$ \\
\hline William Eua & $1946-1947$ \\
\hline Richard Jensen & $1946-1947$ \\
\hline Dick Johnson & $1946-1947$ \\
\hline Gerald Montgomery & $1946-1947$ \\
\hline Travis Passmore & $1946-1947$ \\
\hline Don Tolladay & $1946-1947$ \\
\hline Aladino Unti & $1946-1947$ \\
\hline Charles Cox & $1947-1948$ \\
\hline Harvey Dane & $1947-1948$ \\
\hline Dale Evans & $1947-1948$ \\
\hline Pat Kennedy & $1947-1948$ \\
\hline Doyle Mascus & $1947-1948$ \\
\hline Earl Vanderburgh & $1947-1948$ \\
\hline Wayne Rogers & $1947-1948$ \\
\hline Travis Wisener & $1947-1948$ \\
\hline Douglas A. Wood & $1947-1948$ \\
\hline Mitsugyoshi Aoki & $1948-1949$ \\
\hline Johnny Deniz & $1948-1949$ \\
\hline Stephen Erickson & 1948-1949 \\
\hline Don Fortune & $1948-1949$ \\
\hline Joe Galliano & $1948-1949$ \\
\hline Johnny Martin & 1948-1949 \\
\hline Ray Whitacker & $1948-1949$ \\
\hline
\end{tabular}

\begin{tabular}{|c|c|}
\hline Raymond Dorn & $1949-1950$ \\
\hline Daniel Leach & $1949-1950$ \\
\hline Gene Lynch & $1949-1950$ \\
\hline Doyle Martin & $1949-1950$ \\
\hline Glenn Mays & $1949-1950$ \\
\hline Marvin Schmall & $1949-1950$ \\
\hline Dave Sesock & $1949-1950$ \\
\hline Frank Garner & $1950-1951$ \\
\hline Ronald Gruenwald & $1950-1951$ \\
\hline William Jantzen & $1950-1951$ \\
\hline Carl Simmons, Jr & $1950-1951$ \\
\hline Rocky Valorosi & $1950-1951$ \\
\hline Nello L. Bomprezzi & $1951-1952$ \\
\hline Duane L. Garner & $1951-1952$ \\
\hline Rudolph Gutierrez & $1951-1952$ \\
\hline William A. Justice & $1951-1952$ \\
\hline Michael S. Simmons & $1951-1952$ \\
\hline Leon LaMattina & $1952-1953$ \\
\hline Tommy Westing & $1952-1953$ \\
\hline Cliff Davis & $1953-1954$ \\
\hline Tommy Kenefick & $1953-1954$ \\
\hline Mike Ylarregui & $1953-1954$ \\
\hline Harold Ashton & $1955-1956$ \\
\hline Casey Campbell & $1955-1956$ \\
\hline William Dickey & $1955-1956$ \\
\hline Paul Mąrtines & $1955-1956$ \\
\hline Charles Mays & $1955-1956$ \\
\hline Jerry Siebert & $1955-1956$ \\
\hline Mike Allred & $1957-1958$ \\
\hline Melvin Aoki & $1957-1958$ \\
\hline Richard Dolio & $1957-1958$ \\
\hline Larry Jantzen & $1957-1958$ \\
\hline Ken Seibert & $1957-1958$ \\
\hline Ronald Smith & $1957-1958$ \\
\hline Robert Tate & $1957-1958$ \\
\hline Richard Williams & $1957-1958$ \\
\hline
\end{tabular}




\section{SEAte FFA Degrees Continved}

\begin{tabular}{|c|c|}
\hline Bob Prosperi & $1957-1958$ \\
\hline Wakao Aoki & $1958-1959$ \\
\hline James Chandler & $1958-1959$ \\
\hline Louis Contreras & $1958-1959$ \\
\hline Harold Giomi & $1958-1959$ \\
\hline Carl Janzen & $1958-1959$ \\
\hline Daryl Chlumbohm & 1958-1959 \\
\hline John Stasulat & $1958-1959$ \\
\hline Bruno Pelanconi Jr, & $1959-1960$ \\
\hline Robert Houlding & $1960-1961$ \\
\hline Robert Saulsbury & $1960-1961$ \\
\hline Harvey Aoki & $1961-1962$ \\
\hline Dan Chatman & $1961-1962$ \\
\hline Terry Cheek & $1961-1962$ \\
\hline Stan Hirahara & $1961-1962$ \\
\hline Dick McCollister & $1961-1962$ \\
\hline Robert McCollister & $1961-1962$ \\
\hline Carl Schroeder & $1961-1962$ \\
\hline Don Sellai & $1961-1962$ \\
\hline Richard E. Smith & $1961-1962$ \\
\hline Bobby Tate & $1961-1962$ \\
\hline Joe Camarillo & $1962-1963$ \\
\hline George Crafton & $1962-1963$ \\
\hline Wallace Emmert & $1962-1963$ \\
\hline Larry King & $1962-1963$ \\
\hline Frank Massetti & $1962-1963$ \\
\hline Marcell Monticello & $1962-1963$ \\
\hline Steve Sampaulesi & $1962-1963$ \\
\hline Doug Sordi & $1962-1963$ \\
\hline Steve Tomachoff & $1962-1963$ \\
\hline Terrel West & $1962-1963$ \\
\hline Phil Albonico & $1963-1964$ \\
\hline David Giomi & $1963-1964$ \\
\hline Steve Gist & $1963-1964$ \\
\hline Evert Plumb & $1963-1964$ \\
\hline Greg Desmond & $1964-1965$ \\
\hline Don Weins & $1964-1965$ \\
\hline Chester Andrew & $1965-1966$ \\
\hline Kenneth Aoki & $1965-1966$ \\
\hline
\end{tabular}

\begin{tabular}{|c|c|}
\hline Larry Hirahara & $1965-1966$ \\
\hline Roger Leach & $1965-1966$ \\
\hline David Loquaci & $1965-1966$ \\
\hline Victor Sahatdjian & $1965-1966$ \\
\hline Ray Seibert & $1965-1966$ \\
\hline Albert Lam & $1966-1967$ \\
\hline Norman Lincoln & $1966-1967$ \\
\hline Gary Bursery & $1967-1968$ \\
\hline Ronald Kelley & $1967-1968$ \\
\hline Walter Nelson & $1967-1968$ \\
\hline Ronald Pistoresi & $1967-1968$ \\
\hline Robert Rubottom & $1967-1968$ \\
\hline John Bese & $1968-1969$ \\
\hline Michael Camarillo & $1968-1969$ \\
\hline Lester Eddy & $1968-1969$ \\
\hline Mike Elliot & $1968-1969$ \\
\hline Paul Ely & $1968-1969$ \\
\hline Steve Emmert & $1968-1969$ \\
\hline Steve Grant & $1968-1969$ \\
\hline Dan Johnson & $1968-1969$ \\
\hline Gifford Johnson & $1968-1969$ \\
\hline Leslie Loquaci & $1968-1969$ \\
\hline Larry Moore & $1968-1969$ \\
\hline James Osterman & $1968-1969$ \\
\hline Frank Morgan & $1968-1969$ \\
\hline Ralph Pistoresi & $1968-1969$ \\
\hline Dan Prosperi & $1968-1969$ \\
\hline John Simpson & 1968-1969 \\
\hline Mark Carlson & $1969-1970$ \\
\hline Randall Chase & $1969-1970$ \\
\hline Jeff Coulthard & $1969-1970$ \\
\hline Pat Kirby & $1969-1970$ \\
\hline Eddie Martinazzi & $1969-1970$ \\
\hline Jerry Payne & $1969-1970$ \\
\hline Jim Pistoresi & $1969-1970$ \\
\hline Denis Prosperi & $1969-1970$ \\
\hline Douglas Row & $1969-1970$ \\
\hline Conrad Bitter & $1970-1971$ \\
\hline Roger Evans & $1970-1971$ \\
\hline
\end{tabular}




\section{State FFA Degrees Continued}

\begin{tabular}{|c|c|}
\hline Donald Parkey & $1970-1971$ \\
\hline Randy Belflower & $1971-1972$ \\
\hline Bob Creamer & $1971-1972$ \\
\hline Gene Ferretti & $1971-1972$ \\
\hline Sandy Konkol & $1971-1972$ \\
\hline Rick Osterman & $1971-1972$ \\
\hline Robert Simpson & $1971-1972$ \\
\hline Robery Bishel & $1972-1973$ \\
\hline Betsy Boysen & $1972-1973$ \\
\hline Mark Freeman & $1972-1973$ \\
\hline Randy Freeman & $1972-1973$ \\
\hline David Galleano & $1972-1973$ \\
\hline Rick Logoluso & $1972-1973$ \\
\hline LeRoy Marklund & $1972-1973$ \\
\hline Sandie McDonald & $1972-1973$ \\
\hline Linda Gąlleano & $1973-1974$ \\
\hline Jeff Joines & 1973-1974 \\
\hline Henry Oyler & $1973-1974$ \\
\hline Stephen Schafer & $1973-1974$ \\
\hline Marilyn Whiton & $1973-1974$ \\
\hline James Cavallero & $1975-1976$ \\
\hline Henry Contreras & $1975-1976$ \\
\hline Luanna James & $1975-1976$ \\
\hline Brad McDonald & $1975-1976$ \\
\hline Vince Petrucci & $1975-1976$ \\
\hline Mike Schafer & $1975-1976$ \\
\hline Greg Agajanian & $1976-1977$ \\
\hline John Koretoff & $1976-1977$ \\
\hline Kevin Peters & $1976-1977$ \\
\hline Kurt Peters & $1976-1977$ \\
\hline Cheryl Schąfer & $1976-1977$ \\
\hline Randall Armstrong & $1977-1978$ \\
\hline John Arnold & $1977-1978$ \\
\hline Darrel Bishel & $1977-1978$ \\
\hline Mark Doig & $1977-1978$ \\
\hline Rusty Jensen & $1977-1978$ \\
\hline Barbar Keller & $1977-1978$ \\
\hline Kevin Mercer & $1977-1978$ \\
\hline Kevin Richardson & $1977-1978$ \\
\hline Robyn Harper & $1978-1979$ \\
\hline
\end{tabular}

\begin{tabular}{|c|c|}
\hline Bill Anderson & $1979-1980$ \\
\hline Lorna Gunter & $1979-1980$ \\
\hline Lisa Peterson & $1979-1980$ \\
\hline Karla Stockli & $1979-1980$ \\
\hline Bob Hall & $1981-1982$ \\
\hline Sam Weis & $1982-1983$ \\
\hline Edward Correa & $1983-1984$ \\
\hline Kurt Fick & $1983-1984$ \\
\hline Phillip Montagna & $1983-1984$ \\
\hline Raymond Montagna & $1983-1984$ \\
\hline John Gray & $1984-1985$ \\
\hline Kevin Miles & $1984-1985$ \\
\hline Doug Reed & $1984-1985$ \\
\hline Jim Bell & $1985-1986$ \\
\hline Rhonda Long & $1985-1986$ \\
\hline Derek Sambueso & $1985-1986$ \\
\hline Brian Cox & $1986-1987$ \\
\hline Howard Eledge & $1986-1987$ \\
\hline Todd Fischer & $1986-1987$ \\
\hline Erin McCracken & $1986-1987$ \\
\hline Jeff Miles & $1986-1987$ \\
\hline Roy Morris & $1986-1987$ \\
\hline Heather Parks & $1986-1987$ \\
\hline Cathy Trautman & $1986-1987$ \\
\hline Kevin Bier & $1987-1988$ \\
\hline Mike Ervin & $1987-1988$ \\
\hline Miguel, A. Flores & $1987-1988$ \\
\hline Jeffrey Pacini & $1987-1988$ \\
\hline Suzanne Stretch & $1987-1988$ \\
\hline Terry Brand & $1988-1989$ \\
\hline Scott Jackson & $1988-1989$ \\
\hline Shanon Blackmore & 1990 \\
\hline Diane Hench & 1990 \\
\hline Shawn Johnson & 1990 \\
\hline Craig Waag & 1990 \\
\hline Kris Garzone & 1992 \\
\hline Angelo Gomes & 1992 \\
\hline Javier Guerra & 1992 \\
\hline Sherry Lee & 1992 \\
\hline
\end{tabular}


SErEe FA Degrees Continued

\begin{tabular}{|c|c|}
\hline Shawn Moore & 1992 \\
\hline Frank Reddell & 1992 \\
\hline Shana VanAlen & 1992 \\
\hline Kris Detjen & 1993 \\
\hline Alana Cervantes & 1993 \\
\hline Michael Gomes & 1993 \\
\hline LeAnn McPeters & 1993 \\
\hline Daniel Ogan & 1993 \\
\hline Steven Sesock & 1993 \\
\hline Timothy Carter & 1994 \\
\hline Jodi Hibdon & 1994 \\
\hline Carson Farino & 1994 \\
\hline Bryon Jones & 1994 \\
\hline Brian Fitzgerald & 1994 \\
\hline Stephanie Garzone & 1994 \\
\hline Ryan Logoluso & 1994 \\
\hline Travis Harris & 1994 \\
\hline Stacy Redding & 1994 \\
\hline Monica Williams & 1994 \\
\hline Alan Deniz & 1995 \\
\hline Mark Kazynski & 1995 \\
\hline Mitch Robinson & 1995 \\
\hline Daniel Sesock & 1995 \\
\hline Paulette Sesock & 1995 \\
\hline Deanna Ogan & 1995 \\
\hline Andrew Perreira & 1995 \\
\hline Bryan Perreira & 1995 \\
\hline Steven Clement & 1996 \\
\hline Shane Geist & 1996 \\
\hline Frank Lourenco & 1996 \\
\hline Jeff Perreira & 1996 \\
\hline Kathy Sesock & 1996 \\
\hline Tyler Berry & 1996 \\
\hline Jenny Edelbacher & 1996 \\
\hline Leon Prichard & 1996 \\
\hline
\end{tabular}

\begin{tabular}{|c|c|}
\hline Matt Redding & 1996 \\
\hline Melissa Redding & 1996 \\
\hline Julie Hallam & 1997 \\
\hline Colleen Miller & 1997 \\
\hline Heather Todisco & 1997 \\
\hline Lisa McKinley & 1997 \\
\hline Lisa McPeters & 1997 \\
\hline Matt Beechinor & 1998 \\
\hline Scott Bursey & 1998 \\
\hline Stacy Cook & 1998 \\
\hline Renee Crawford & 1998 \\
\hline Keri Jackson & 1998 \\
\hline Robbie Loquaci & 1998 \\
\hline Manuel Marin & 1998 \\
\hline Jeffrey Riddle & 1998 \\
\hline Susan Riddle & 1998 \\
\hline Steven Rodriguez & 1998 \\
\hline Alyson Seibert & 1998 \\
\hline Sarah Stutler & 1998 \\
\hline Shawn Liles & 1999 \\
\hline Steve Calderon & 1999 \\
\hline Bryan Rodriguez & 1999 \\
\hline Shanna Rodriguez & 1999 \\
\hline Rene Gonzalez & 1999 \\
\hline Jennifer Tune & 1999 \\
\hline Stacey Visscher & 1999 \\
\hline Jason Erickson & 2000 \\
\hline Audrey Estabrook & 2000 \\
\hline Antionette Francher & 2000 \\
\hline Andrea Hench & 2000 \\
\hline Laura Del Bianco & 2001 \\
\hline Erick Buckley & 2001 \\
\hline Jesse Croxen & 2001 \\
\hline Robert Fahey & 2001 \\
\hline Cassie McKienly & 2001 \\
\hline
\end{tabular}




\section{SEate FA Degrees Continued}

\begin{tabular}{|c|c|}
\hline Jessica Miller & 2001 \\
\hline Colin Rock & 2001 \\
\hline Loriann Sesock & 2001 \\
\hline Jason Wara & 2001 \\
\hline Alyson Padgett & 2002 \\
\hline Trevor Meyers & 2002 \\
\hline Monica Medina & 2002 \\
\hline Jennifer Mansell & 2002 \\
\hline Brian Schafer & 2002 \\
\hline Brandon Visscher & 2002 \\
\hline Kevin Willet & 2002 \\
\hline Stephanie Bellew & 2003 \\
\hline Donald Doyle & 2003 \\
\hline Lee Erickson & 2003 \\
\hline Resse Fahey & 2003 \\
\hline Amanda Hallam & 2003 \\
\hline Garret Mattrocce & 2003 \\
\hline Giana Toschi & 2003 \\
\hline Cody Waltz & 2003 \\
\hline Garth Wara & 2003 \\
\hline Michael Croxen & 2004 \\
\hline Adam Pistoresi & 2004 \\
\hline Melissa Alley & 2005 \\
\hline Jessica Alcorn & 2005 \\
\hline Howard Beach & 2005 \\
\hline Amy Bonander & 2005 \\
\hline Gabriel Garcia & 2005 \\
\hline Landon Gill & 2005 \\
\hline Cory Padgett & 2005 \\
\hline Megan Mettucci & 2005 \\
\hline Brett Martinazzi & 2005 \\
\hline Christopher Rippee & 2005 \\
\hline Andrea Bartley & 2006 \\
\hline Margarito Cervantes & 2006 \\
\hline Thomas Lovelady & 2006 \\
\hline
\end{tabular}

\begin{tabular}{|c|c|}
\hline Cinda Mattrocce & 2006 \\
\hline Jeffrey Moosios & 2006 \\
\hline Cody Ogletree & 2006 \\
\hline Steve Pistoresi & 2006 \\
\hline William Thornton & 2006 \\
\hline Warren Tucker & 2006 \\
\hline Alexandria Wara & 2006 \\
\hline Kendra Willet & 2006 \\
\hline Jose Farias & 2007 \\
\hline Michael Fincher & 2007 \\
\hline Glen Gil & 2007 \\
\hline Kaysy Hopson & 2007 \\
\hline Jacob Maggiore & 2007 \\
\hline Mika Petrucci & 2007 \\
\hline Bobby Sholler & 2007 \\
\hline Anthony Tates & 2007 \\
\hline Vincent Urena & 2007 \\
\hline Victoria Barros & 2008 \\
\hline Matthew Chavira & 2008 \\
\hline Alonzo hernandez & 2008 \\
\hline Tori Isaac & 2008 \\
\hline Hector lopez & 2008 \\
\hline Ritchie Lopez & 2008 \\
\hline Tiffany O'haro & 2008 \\
\hline Rodolfo Pineda & 2008 \\
\hline Ramiro Sanchez & 2008 \\
\hline Jessica Sidney & 2008 \\
\hline Nicole Sidney & 2008 \\
\hline Dominic Bettini & 2009 \\
\hline Megan Christiansen & 2009 \\
\hline Caleb Hatfield & 2009 \\
\hline Enrique Hernandez & 2009 \\
\hline Melissa Hubbard & 2009 \\
\hline Riley Lovelady & 2009 \\
\hline Sierra Meyers & 2009 \\
\hline
\end{tabular}




\section{Stiterte Degrees Conthilnued}

\begin{tabular}{|c|c|}
\hline Wesley Ogletree & 2009 \\
\hline Gabriel Sanchez & 2009 \\
\hline Scott Thornton & 2009 \\
\hline Henry Bales & 2010 \\
\hline Taylor Bese & 2010 \\
\hline Matthew Borges & 2010 \\
\hline Veronica Cervantes & 2010 \\
\hline Gustavo Contreras & 2010 \\
\hline Jerry Cook & 2010 \\
\hline Jonathan Costa & 2010 \\
\hline Santiago DeLaCruz & 2010 \\
\hline Spencer Deniz & 2010 \\
\hline Amy Evąns & 2010 \\
\hline Whitney Laymon & 2010 \\
\hline Jordan Lyons & 2010 \\
\hline Alejandro Madrigal & 2010 \\
\hline John McClure & 2010 \\
\hline Benjamin Salazar & 2010 \\
\hline Shannon Sumpter & 2010 \\
\hline Adam Taylor & 2010 \\
\hline Cody Ward & 2010 \\
\hline Shelby Caraway & 2011 \\
\hline Matthew Cavallero & 2011 \\
\hline Matthew Cavaletto & 2011 \\
\hline Leo Cervantes & 2011 \\
\hline Amy Dierberger & 2011 \\
\hline Jorge Garcia & 2011 \\
\hline Luis Mancillas & 2011 \\
\hline Shelby Moit & 2011 \\
\hline David Nino & 2011 \\
\hline Norrin Pecarrovich & 2011 \\
\hline
\end{tabular}

\begin{tabular}{|c|c|}
\hline Magali Rodriguez & 2011 \\
\hline Santiago Santos & 2011 \\
\hline Lindsay Tasos & 2011 \\
\hline Alex Teran & 2011 \\
\hline Jessica Trembley & 2011 \\
\hline Michael Valencia & 2011 \\
\hline Alexis Aguilar & 2012 \\
\hline Rochelle Brewer & 2012 \\
\hline Scott Bullis & 2012 \\
\hline Jessica Davila & 2012 \\
\hline Emily Fernandez & 2012 \\
\hline Dalice Garcia & 2012 \\
\hline Molly Gilbert & 2012 \\
\hline Kasey Griffin & 2012 \\
\hline Taylor Helton & 2012 \\
\hline Carla Johnson & 2012 \\
\hline Luz Lopez & 2012 \\
\hline Chris Melikian & 2012 \\
\hline Jevan Grewal & 2012 \\
\hline Crystal Bazante & 2013 \\
\hline Jimmy Beavers & 2013 \\
\hline Jaime Cuevąs & 2013 \\
\hline Vannessa Duarte & 2013 \\
\hline Brianna Gagliardi & 2013 \\
\hline Rostia Galindo & 2013 \\
\hline Marcelina Gonzalez & 2013 \\
\hline Allison Helton & 2013 \\
\hline Alexus Hernandez & 2013 \\
\hline Cody Knott & 2013 \\
\hline Kayla Melikian & 2013 \\
\hline Jacqueline Morales & 2013 \\
\hline
\end{tabular}




\section{Strite Degrees Continued}

\begin{tabular}{|c|c|}
\hline Dominique Ortega & 2013 \\
\hline Jamie Oyler & 2013 \\
\hline Eduardo Rodriguez & 2013 \\
\hline Joalex Sanchez & 2013 \\
\hline Sukhvir Singh & 2013 \\
\hline Spenser Smith & 2013 \\
\hline Whitney Swengel & 2013 \\
\hline Hailey Wilberg & 2013 \\
\hline
\end{tabular}
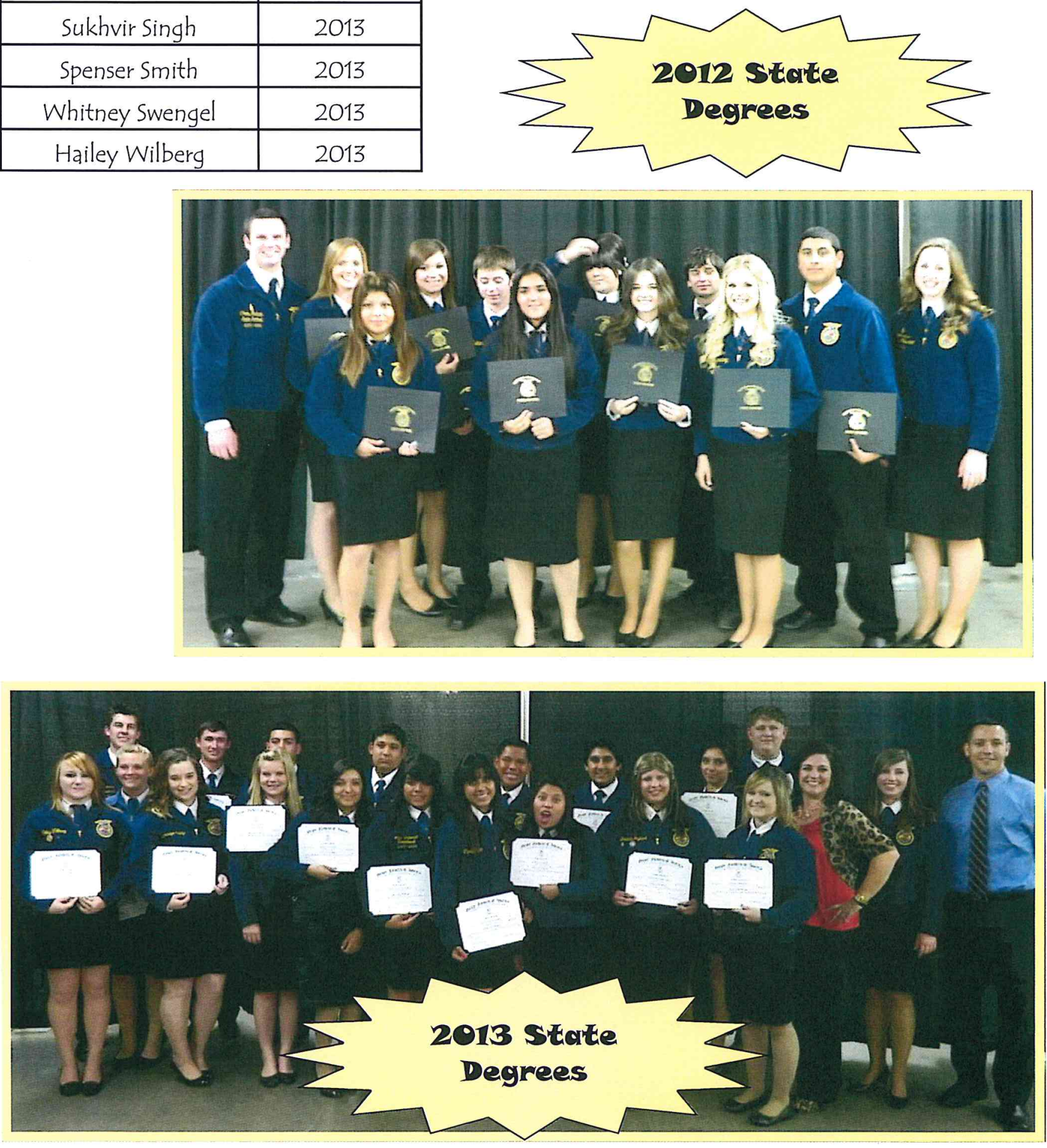


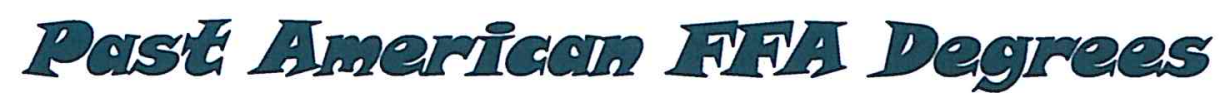

\begin{tabular}{|c|c|}
\hline Raymond Thomas & $1945-1946$ \\
\hline Dino Petrucci & $1948-1949$ \\
\hline Johnny Deniz & $1951-1952$ \\
\hline Robert Prosperi & $1960-1961$ \\
\hline Joe Stasulat & $1962-1963$ \\
\hline Larry Hirahara & $1968-1969$ \\
\hline Roger Leach & $1968-1969$ \\
\hline Gary Lee Agajanian & $1978-1979$ \\
\hline John Koretoff & $1978-1979$ \\
\hline Karla J. Stockli & $1984-1985$ \\
\hline Samuel K. Weis & $1984-1985$ \\
\hline Angela Gomes & 1993 \\
\hline Carson Farino & 1995 \\
\hline Brian Fitzgerald & 1995 \\
\hline Michael Gomes & 1995 \\
\hline Ryan Logoluso & 1995 \\
\hline LeAnn Iva McPeters & 1995 \\
\hline Steven Sesock & 1995 \\
\hline Shana Van Alen & 1995 \\
\hline Nicole Greci & 1996 \\
\hline Monica Denise Williams & 1996 \\
\hline Shane Geist & 1999 \\
\hline Tomas Greci & 1999 \\
\hline Brandon Rodriquez & 1999 \\
\hline Kathy Sesock & 1999 \\
\hline Paulette Sesock & 1999 \\
\hline Matt Beechinor & 2000 \\
\hline Matt Redding & 2000 \\
\hline Lisa McPeters & 2000 \\
\hline Steve Rodriquez & 2000 \\
\hline Julie Hallum & 2001 \\
\hline
\end{tabular}

\begin{tabular}{|c|c|}
\hline Robbie Loquaci & 2001 \\
\hline Manuel Marin & 2001 \\
\hline Lisa McKinley & 2001 \\
\hline Alyson Seibert & 2001 \\
\hline Jason Erickson & 2002 \\
\hline Kyle Prosperi & 2002 \\
\hline Brian Davis & 2003 \\
\hline Nick Davis & 2003 \\
\hline Laura McGee & 2003 \\
\hline Loriann Sesock & 2003 \\
\hline Jason Wara & 2003 \\
\hline Robert Fahey & 2004 \\
\hline Casandra McKinley & 2004 \\
\hline Jessica Miller & 2004 \\
\hline Michael Croxen & 2005 \\
\hline Lee Erickson & 2005 \\
\hline Amanda Hallum & 2005 \\
\hline Garrett Mattrocce & 2005 \\
\hline Brian Schafer & 2005 \\
\hline Cody Waltz & 2005 \\
\hline Garth Wara & 2005 \\
\hline Megan Matteucci & 2007 \\
\hline Melissa Alley & 2007 \\
\hline Cory Padgett & 2007 \\
\hline Jessica Alcorn & 2007 \\
\hline Steve Pistoresi & 2007 \\
\hline Brett Martinazzi & 2007 \\
\hline Ross Dellaualle & 2007 \\
\hline Thomas Lovelady & 2008 \\
\hline Christopher Rippee & 2008 \\
\hline Kendra Willet & 2008 \\
\hline Alex Wara & 2008 \\
\hline
\end{tabular}




\section{Pars Imeriach FITI Degrees}

\section{Congunded}

\begin{tabular}{|c|c|}
\hline Cinda Mattrocce & 2008 \\
\hline Glen Gil & 2009 \\
\hline William Thornton & 2009 \\
\hline Michael Fincher & 2009 \\
\hline Mika Petrucci & 2009 \\
\hline Matthew Chavira & 2009 \\
\hline Margarito Cervantes & 2009 \\
\hline Anthony Tates & 2009 \\
\hline Enrique Hernandez & 2010 \\
\hline Alonzo Espinoza & 2010 \\
\hline Brittany Cavaletto & 2011 \\
\hline Gabriel Sanchez & 2011 \\
\hline Dominic Bettini & 2011 \\
\hline Sierra Meyers & 2011 \\
\hline Matthew Borges & 2012 \\
\hline John McClure & 2012 \\
\hline David Nino & 2012 \\
\hline Scott Thornton & 2012 \\
\hline Shannon Sumpter & 2012 \\
\hline
\end{tabular}
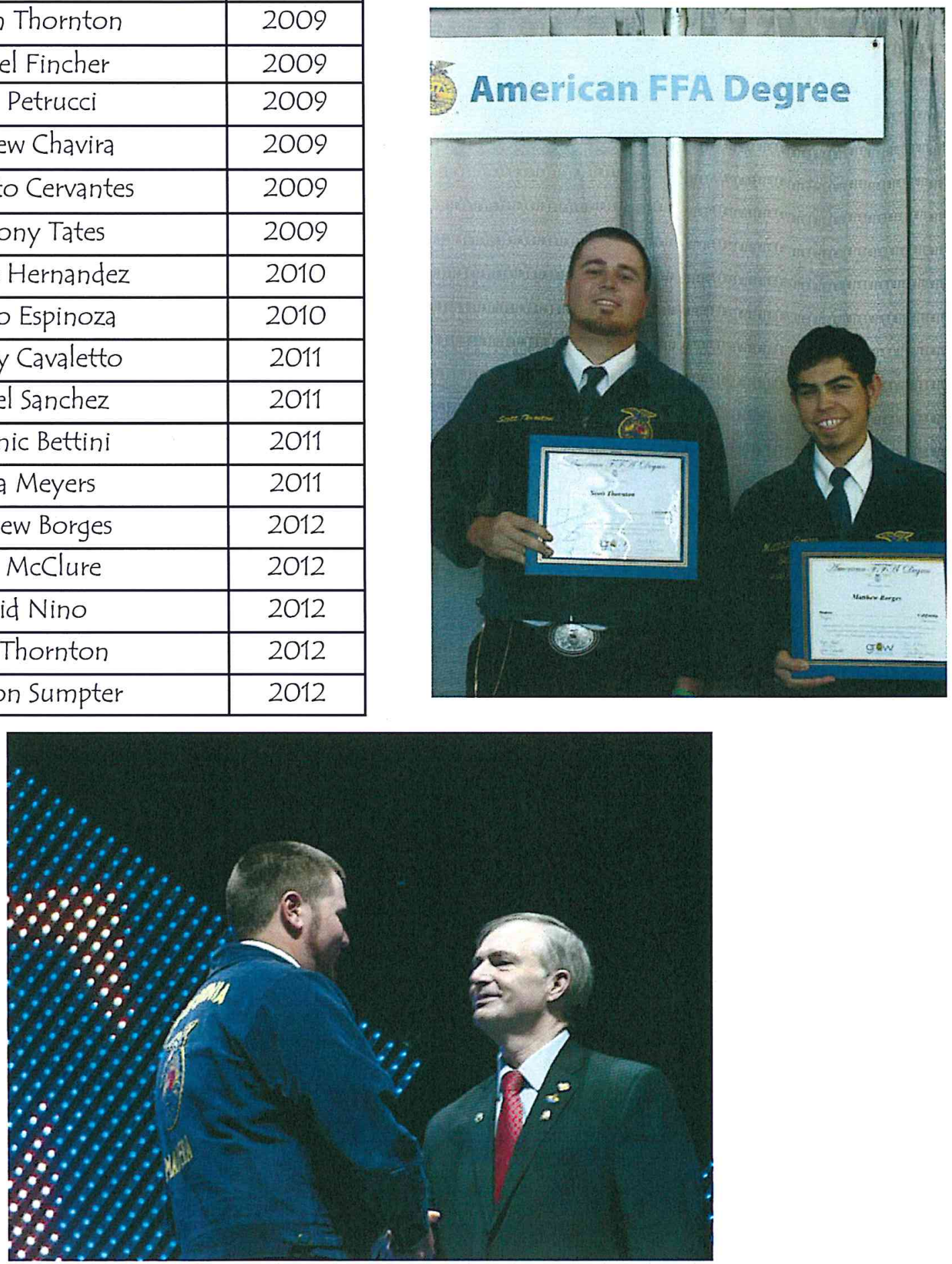


\section{StEotE Wunning Tearms}

Trees, 1933-1934

Trees, 1936-1937

Dino Petrucci, Public Speaking, 1946-47

${ }^{* *}$ Livestock, 1948

Milk 1950-1951

Ag. Marketing, 1955-1956

Ag. Mechanics, 1959-1960

Ag Mechanics, 1960-1961

Agronomy, 1960-1961

Truck Crops, 1960-1961

Land, 1961-1962

Truck Crops, 1961-1962

Grapevine Judging, 1961-1962

Public Speaking- Dan Chatman, 1962-1963

Agronomy, 1962-1963

Truck Crops, 1962-1963

Cotton, 1963-1964

Grapevine Pruning, 1964-1965

Farm Power, 1965-1966

Truck Crops, 1965-1966

Grapevine Judging, 1965-1966

Livestock, 1966-1967

Public Speaking-Dave Loquaci, 1966-67

Truck Crops, 1967-1968

Grapevine Pruning, 1967-1968

Agronomy, 1968-1969

Ag. Pest Control, 1968-1969

Grapevine Judging, 1968-1969

Grapevine Pruning, 1968-1969

Tree Pruning, 1969-1970

Cotton, 1974-1975

Citrus, 1975-1976

Citrus, 1977-1978

Livestock, 1980-1981

Small Engines, 1980-1981

Small Engines, 1981-1982
Fruit Tree Pruning, 1982-1983

Grapevine Pruning, 1982-1983

Land Judging, 1984-1985

Light Horse Judging, 1984-1985

Small Engines, 1986-1987

Small Engines, 1987-1988

Small Engines, 1988-1989

Fruit Tree Pruning-1989-90

Small Engines-1990-91

Grapevine Pruning- 1992-93

Fruit Tree Pruning- 1992-93

Grapevine Pruning-1993-94

Fruit Tree Pruning-1993-94

Grapevine Judging - 1993-1994

Farm Power and Machine, 1995-1996

Grapevine Pruning-1995-96

Fruit Tree Pruning-1996-1997

Fruit Tree Pruning-1997-1998

Small Engines- 1999-2000

Floriculture- 2001-2002

Small Engines, 2002-2003

Best Informed Greenhand- 2004-2005

Best Informed Greenhand-2006-2007

Best Informed Greenhand-2007-2008

Small Engines- 2007-2008

Meats- 2007-2008

Vine Pruning -2009- 2010

Vine Pruning-2010-2011

Best Informed Greenhand-2010-2011

Floriculture-2010-2011

Best Informed Greenhand-2011-2012

Vine Pruning - 2012-2013

Meats Judging-2012-2013

Best Informed Greenhand -2012-2013 


\section{Madera ALA}
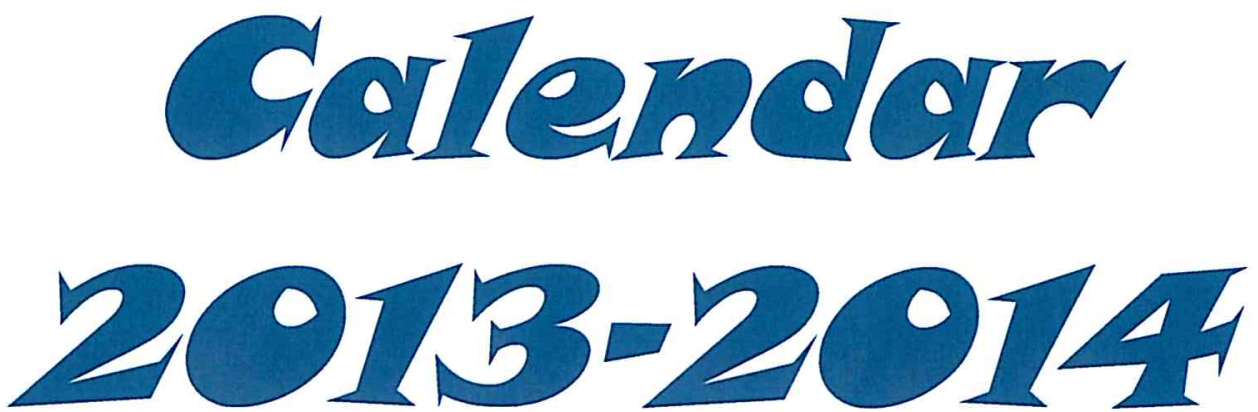

Use the following pages to see the opportunities available to you as an FFA member or community member who would like to get more involved.
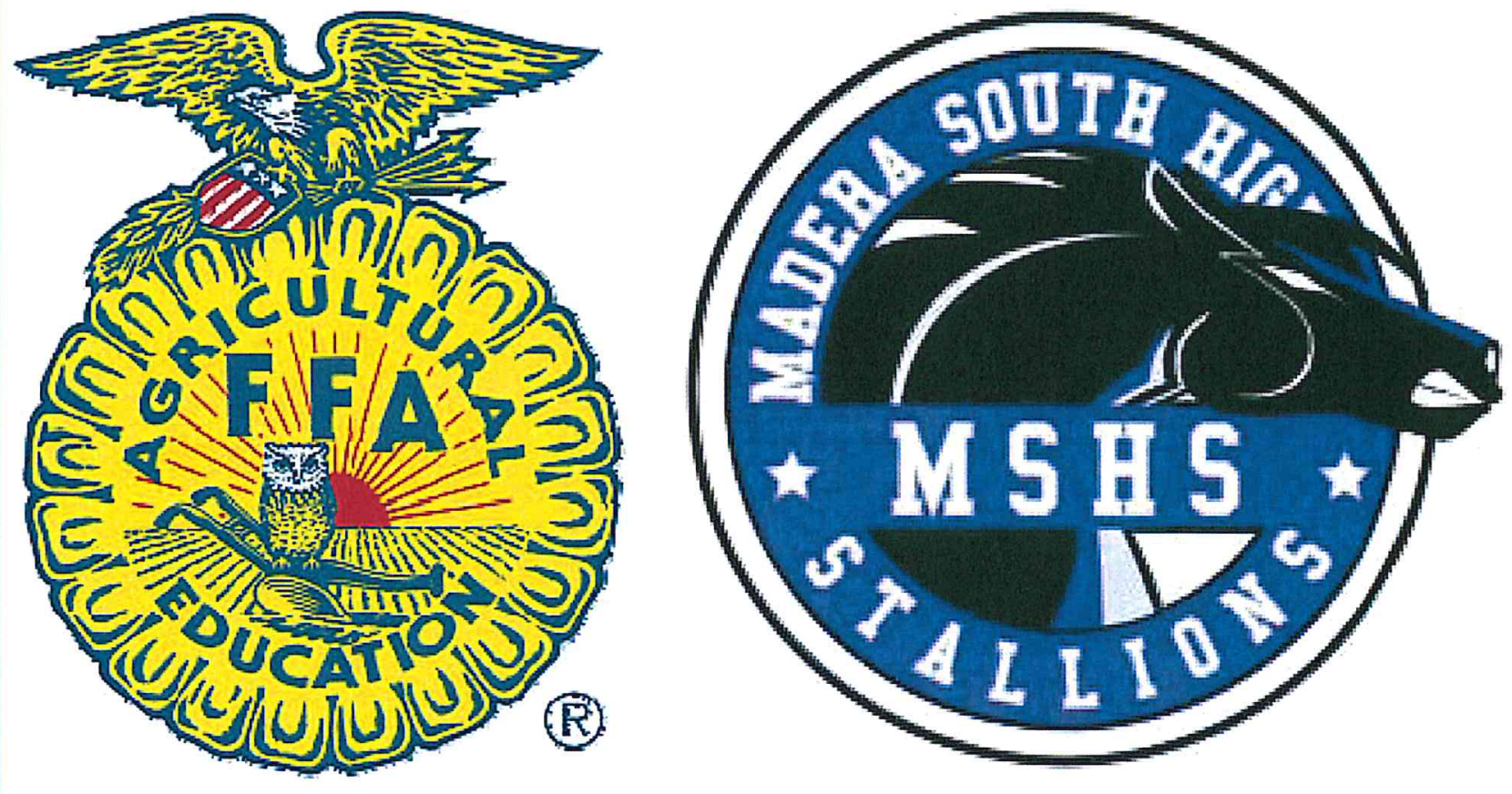


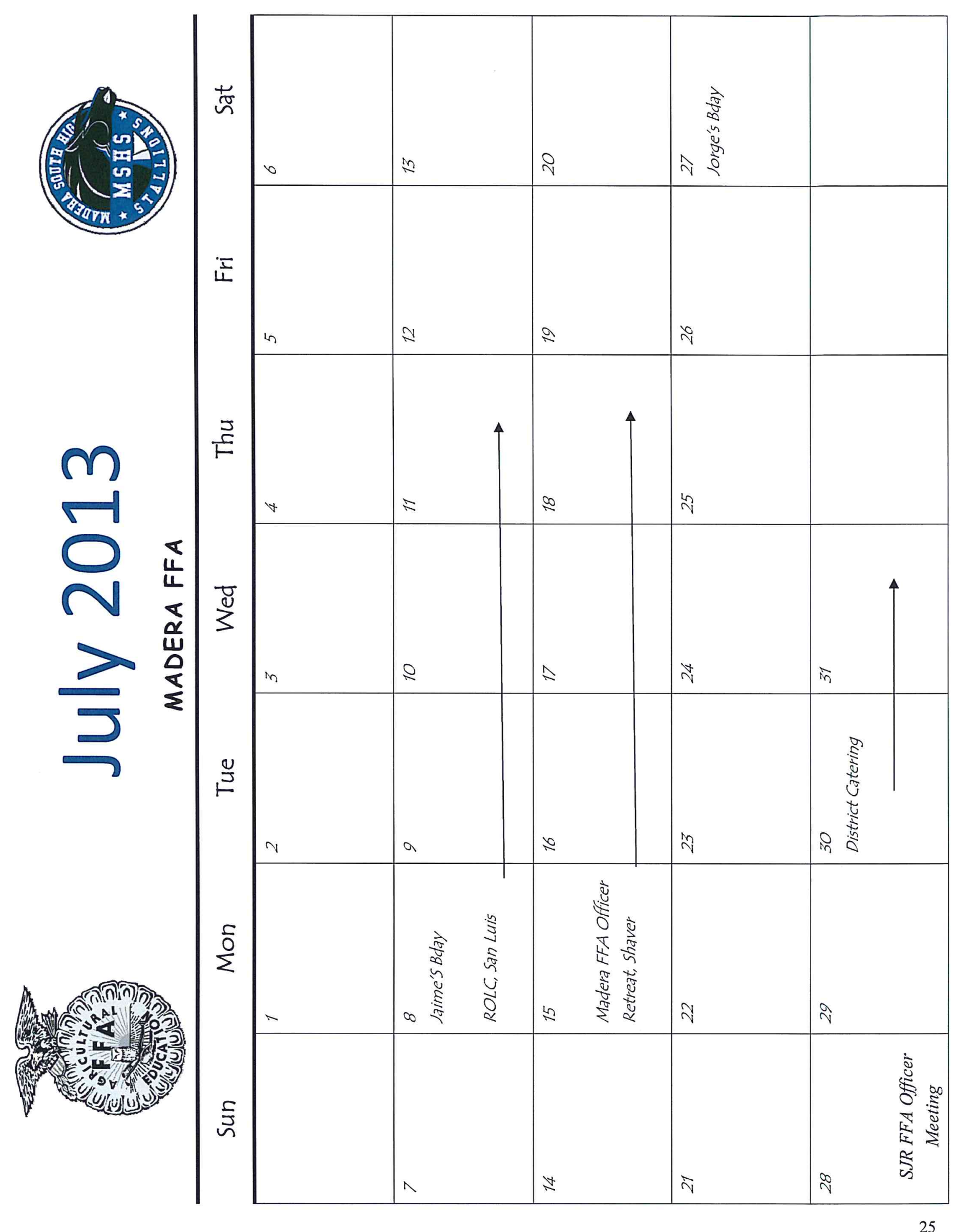




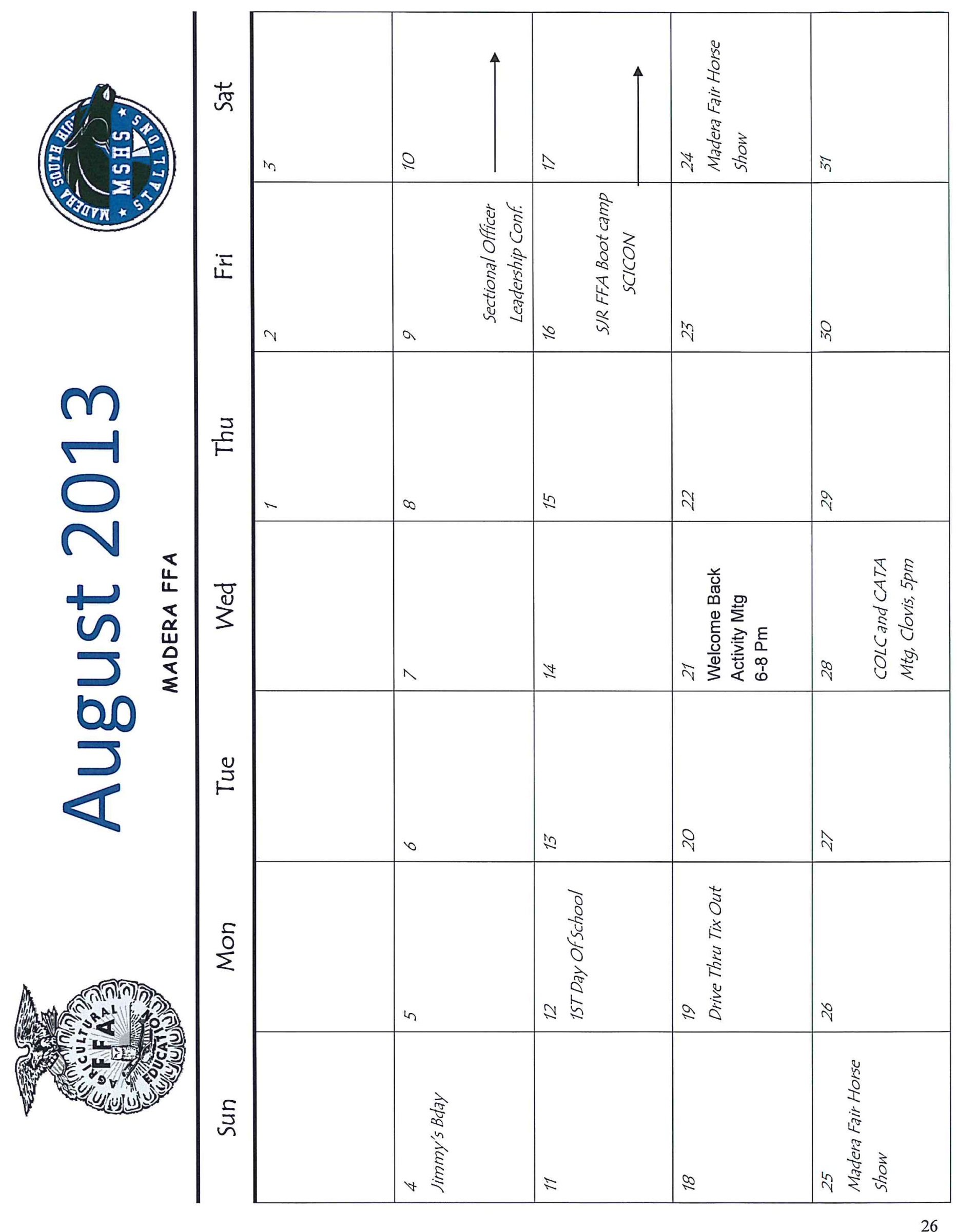




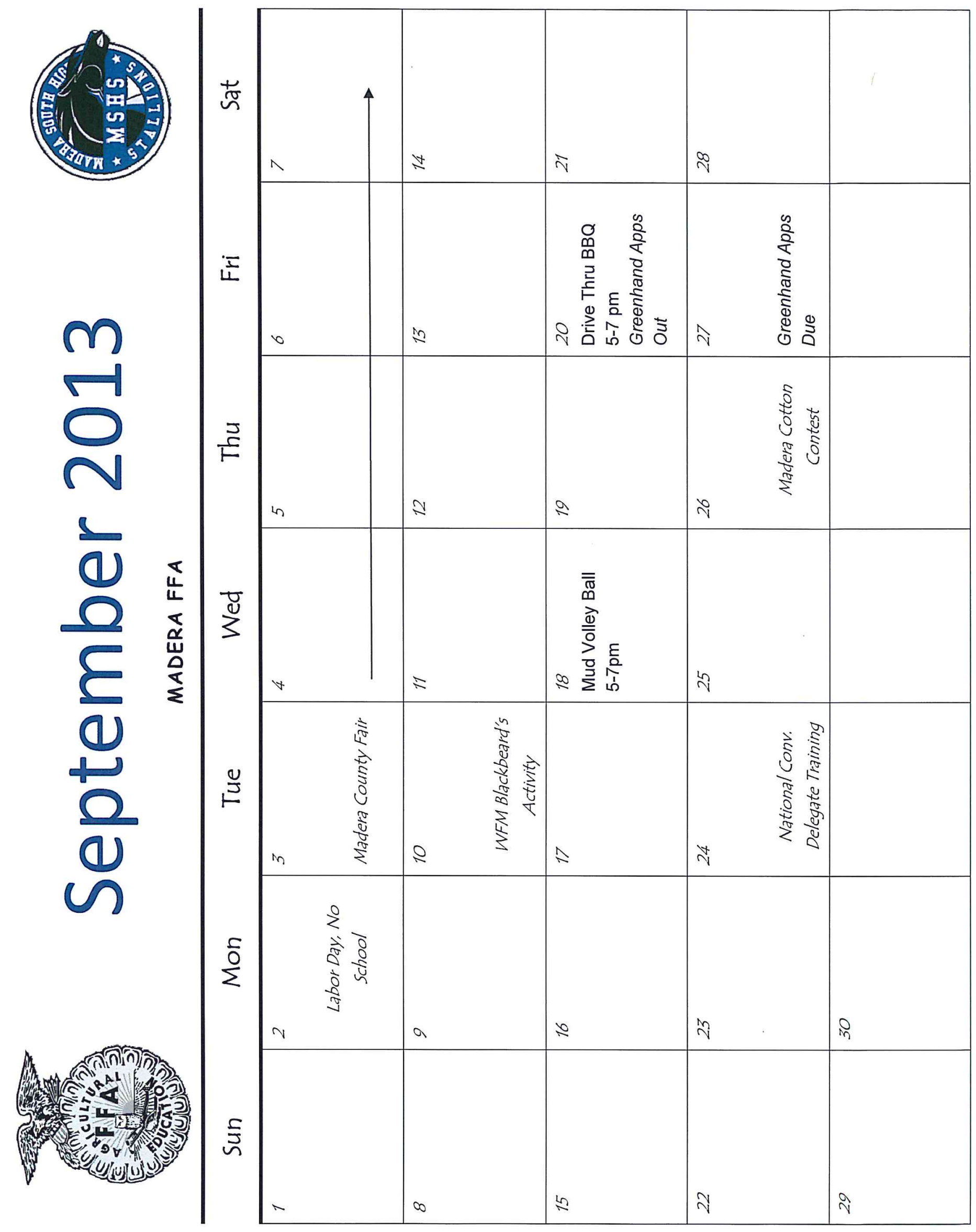




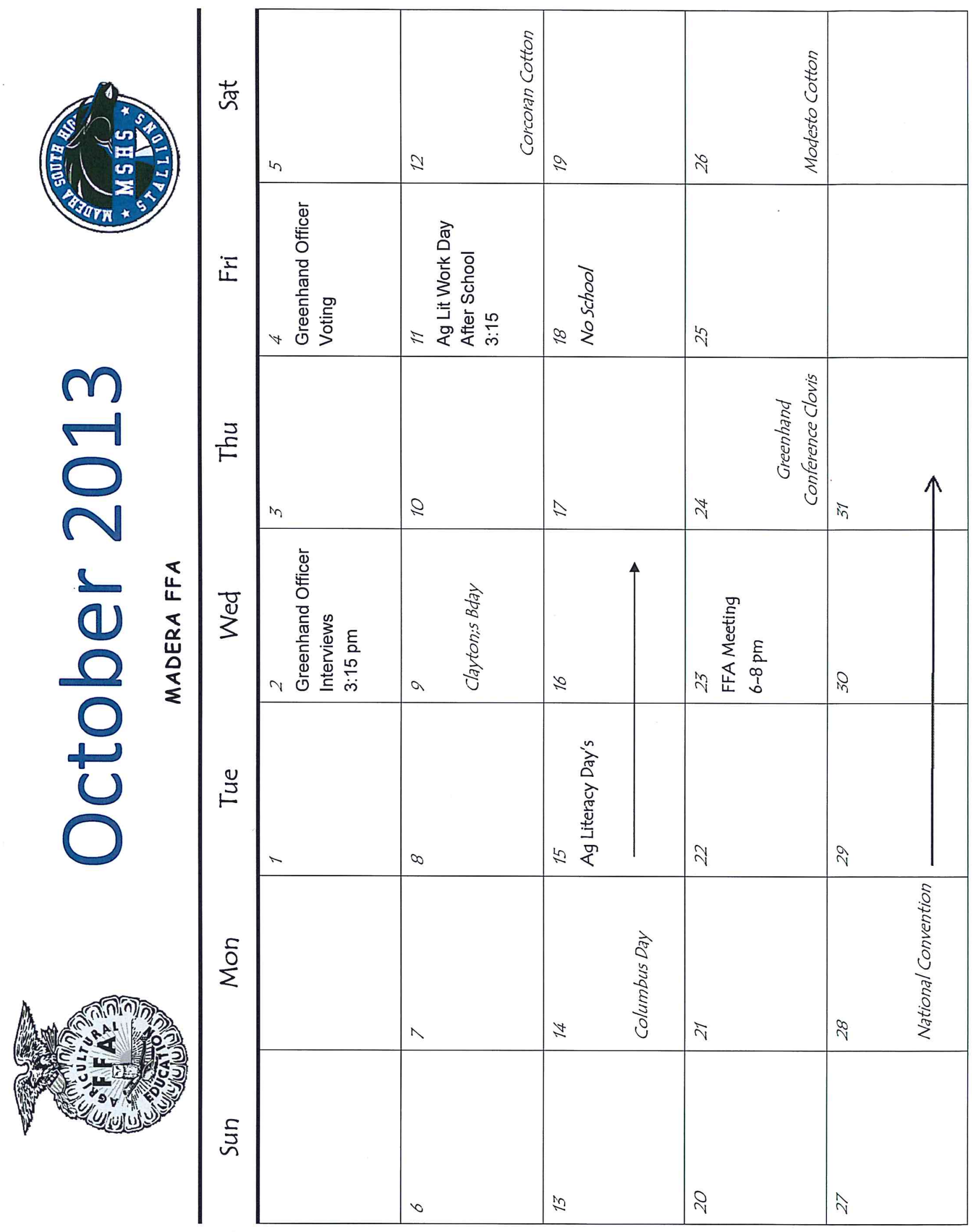




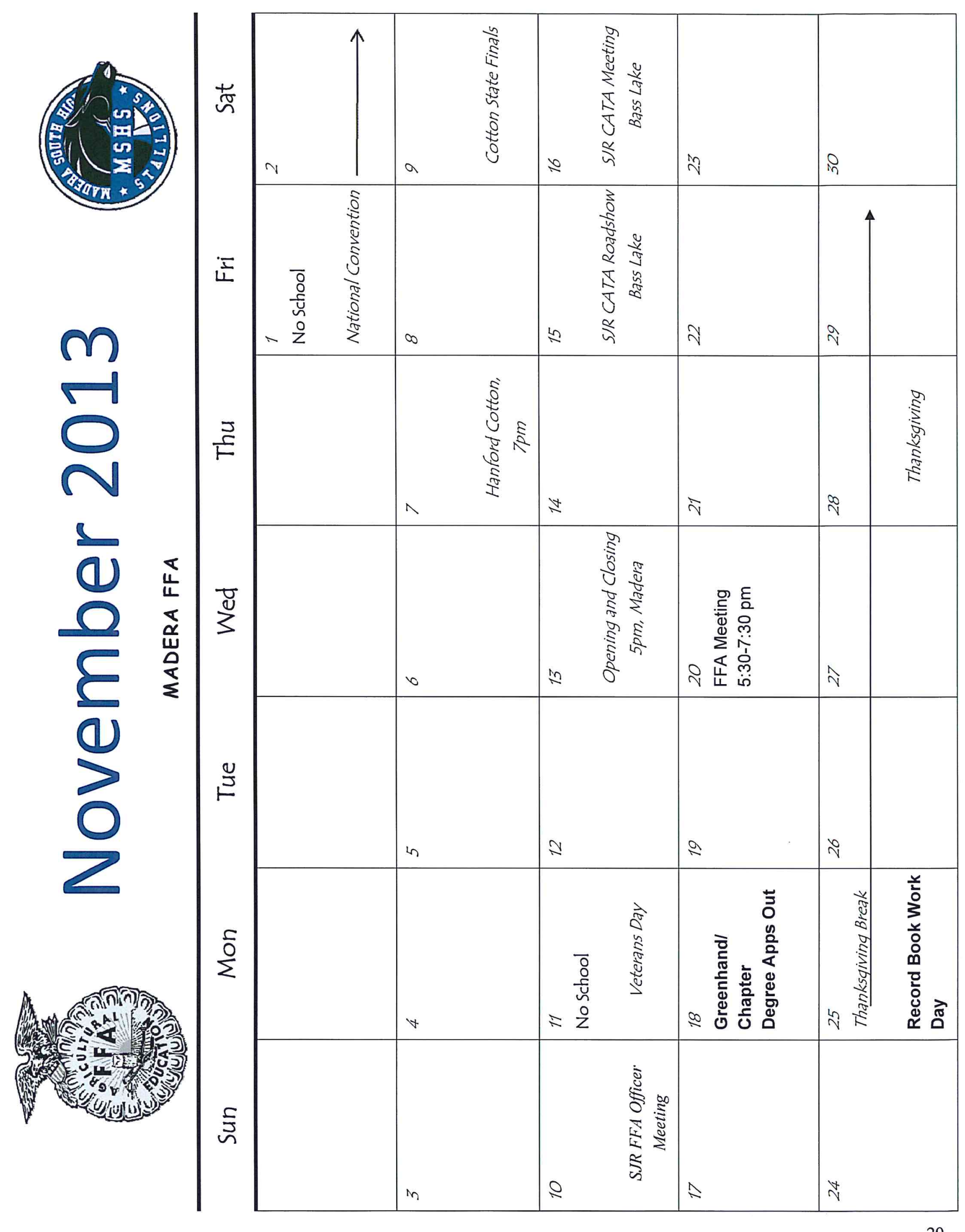




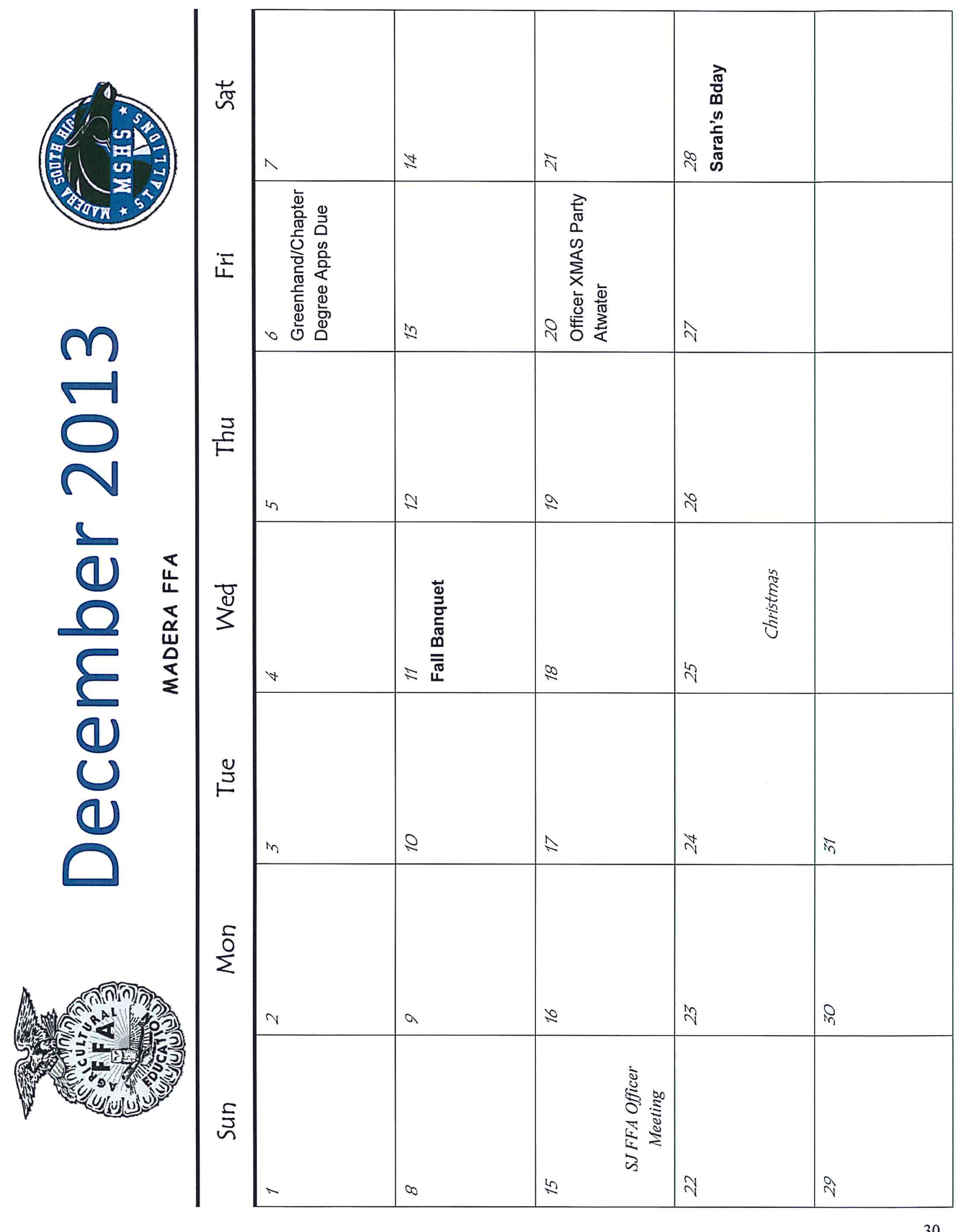




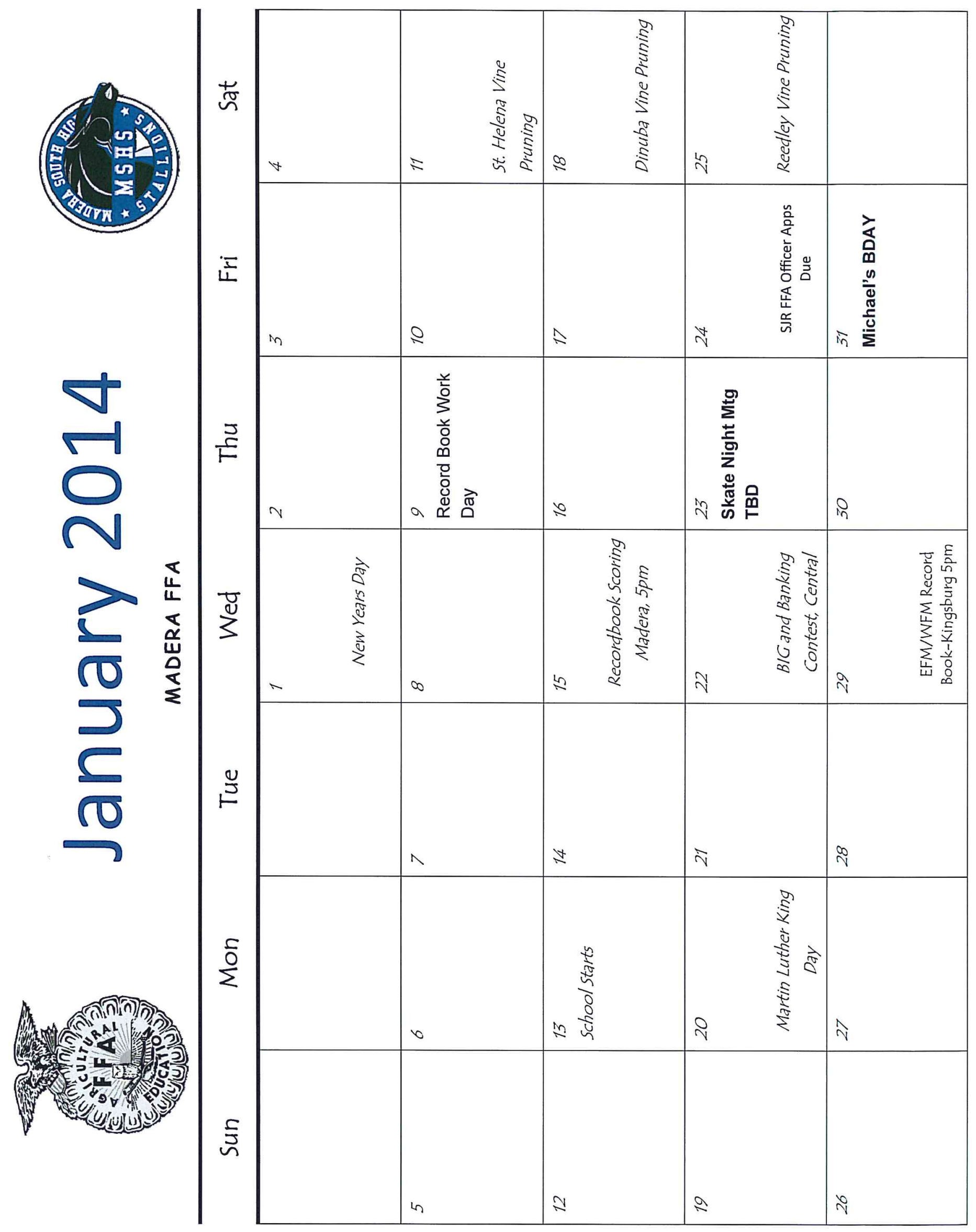




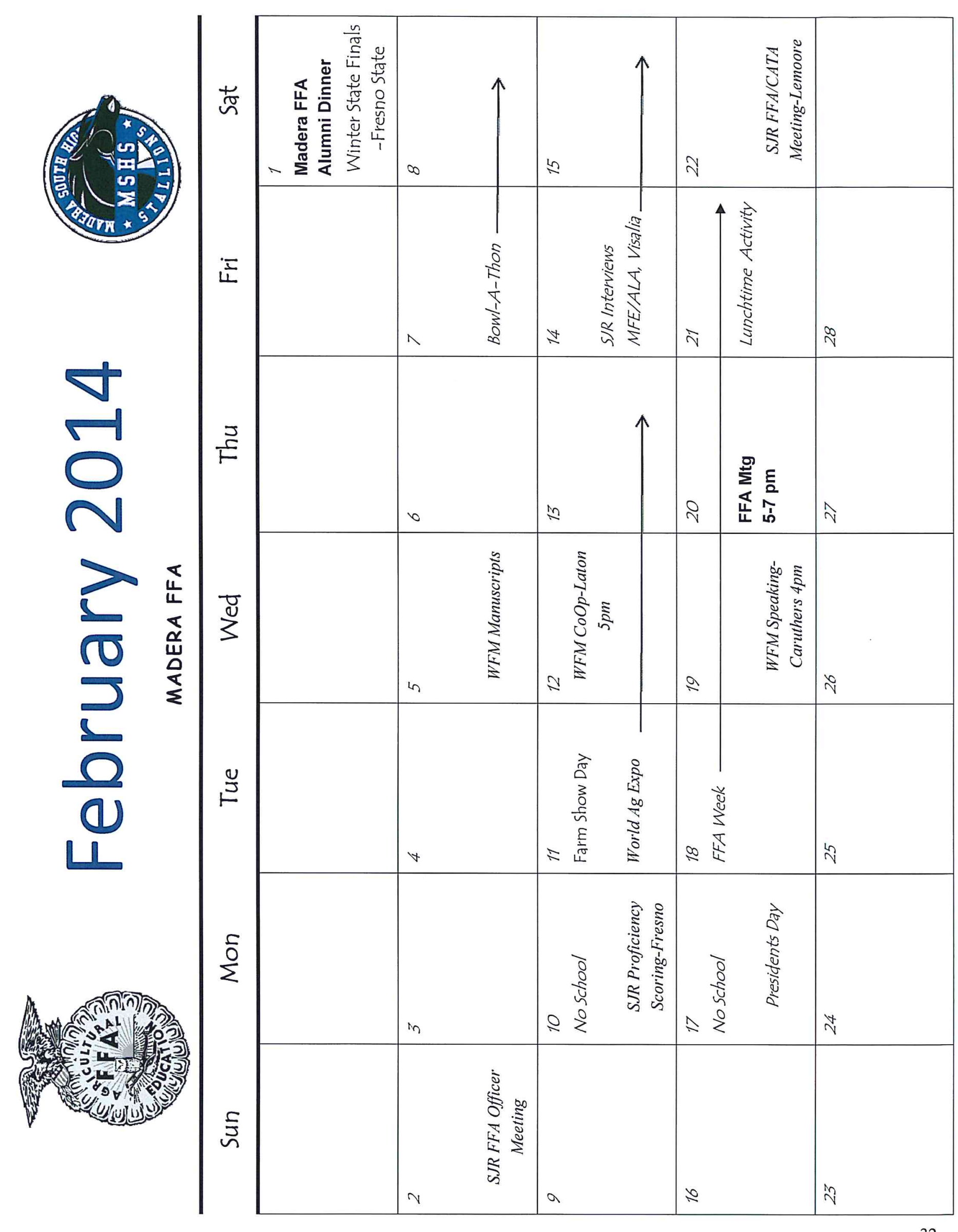




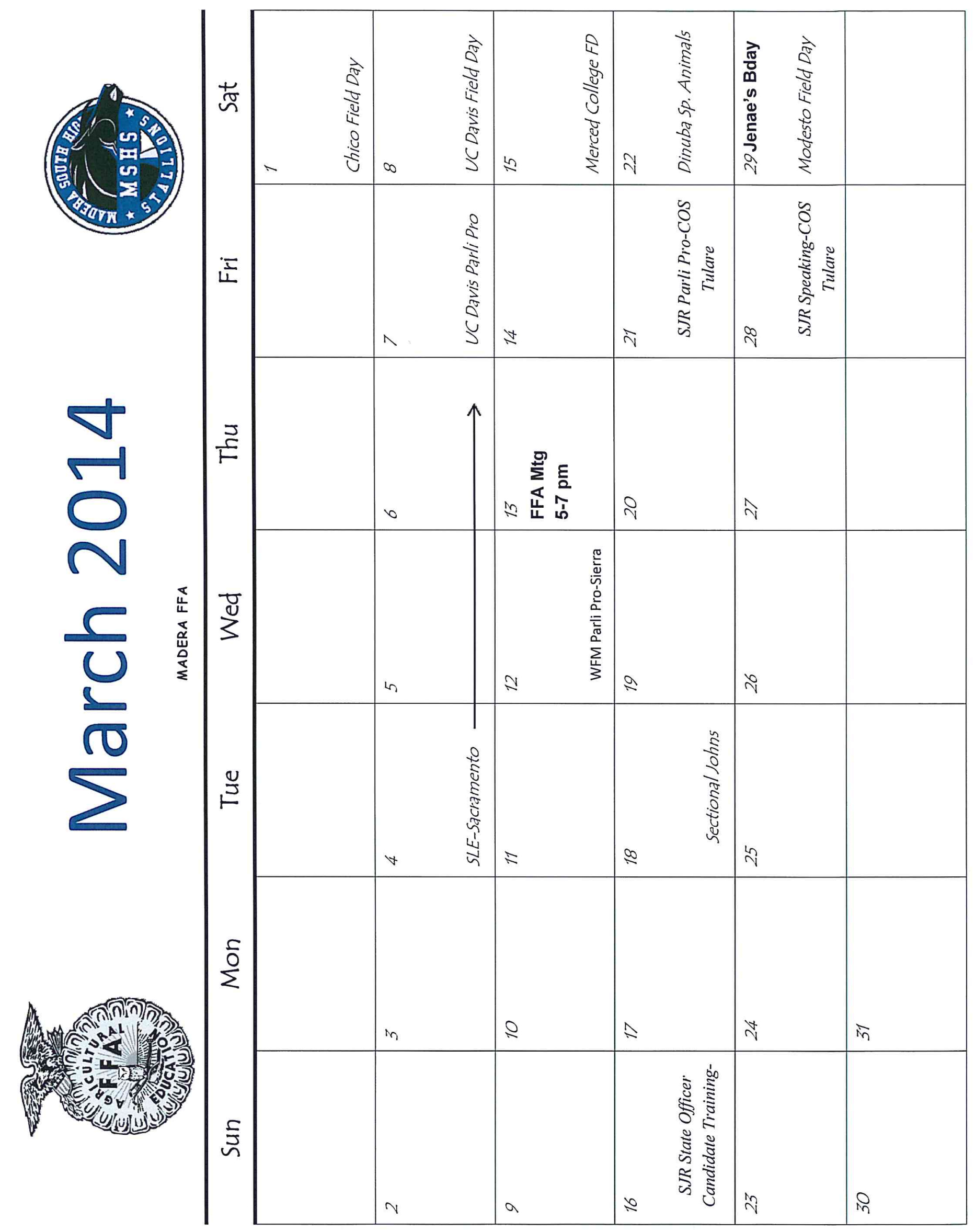




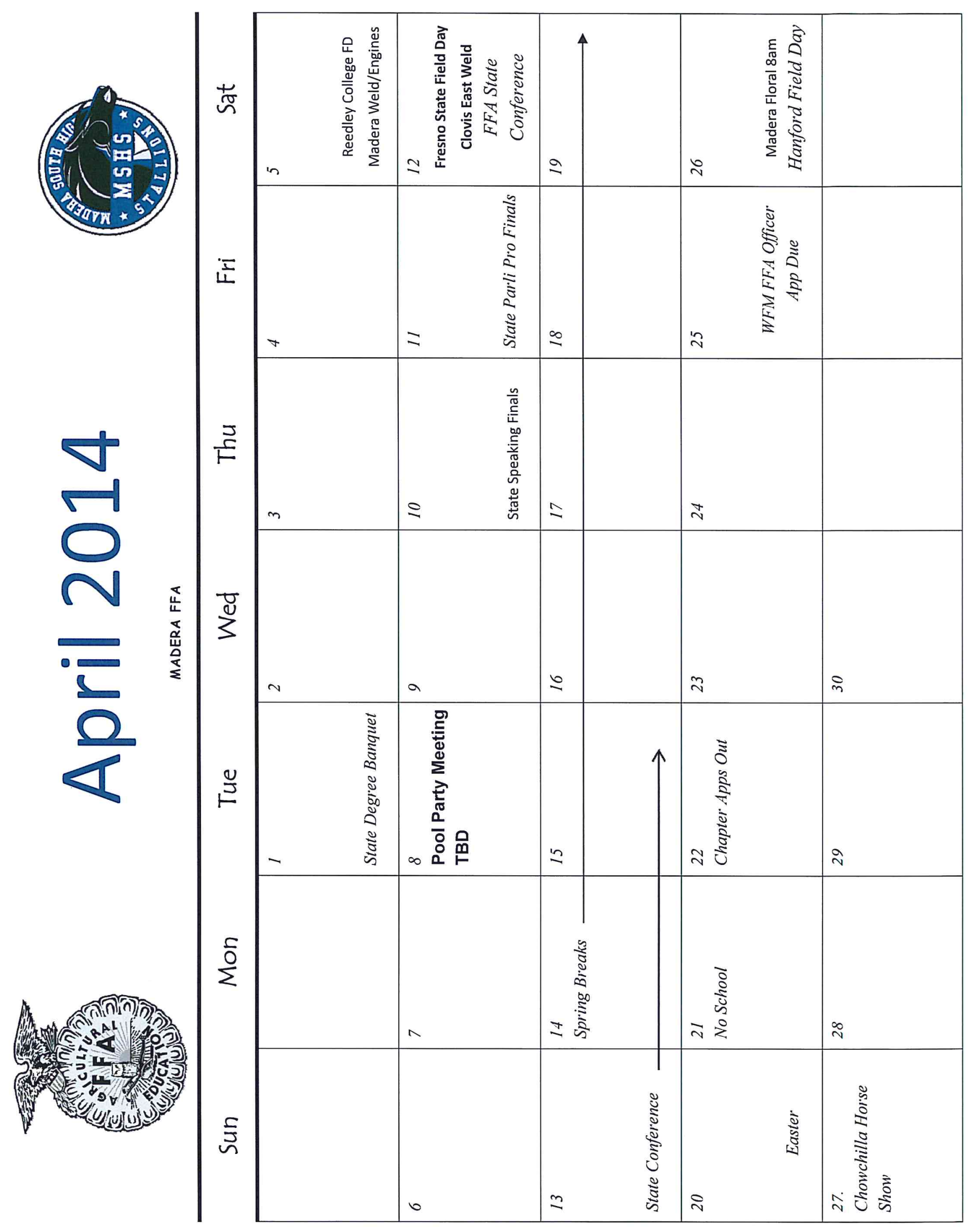




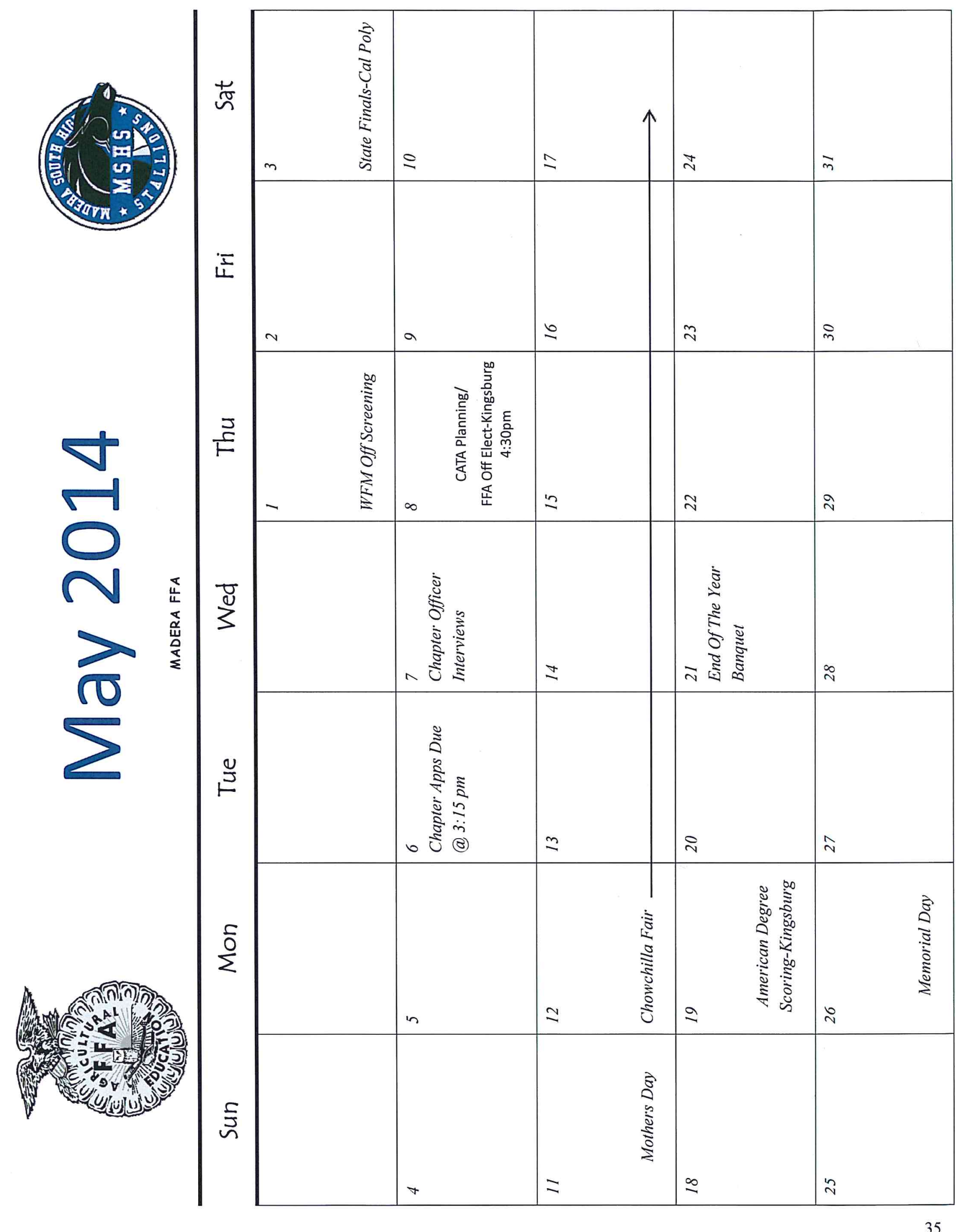




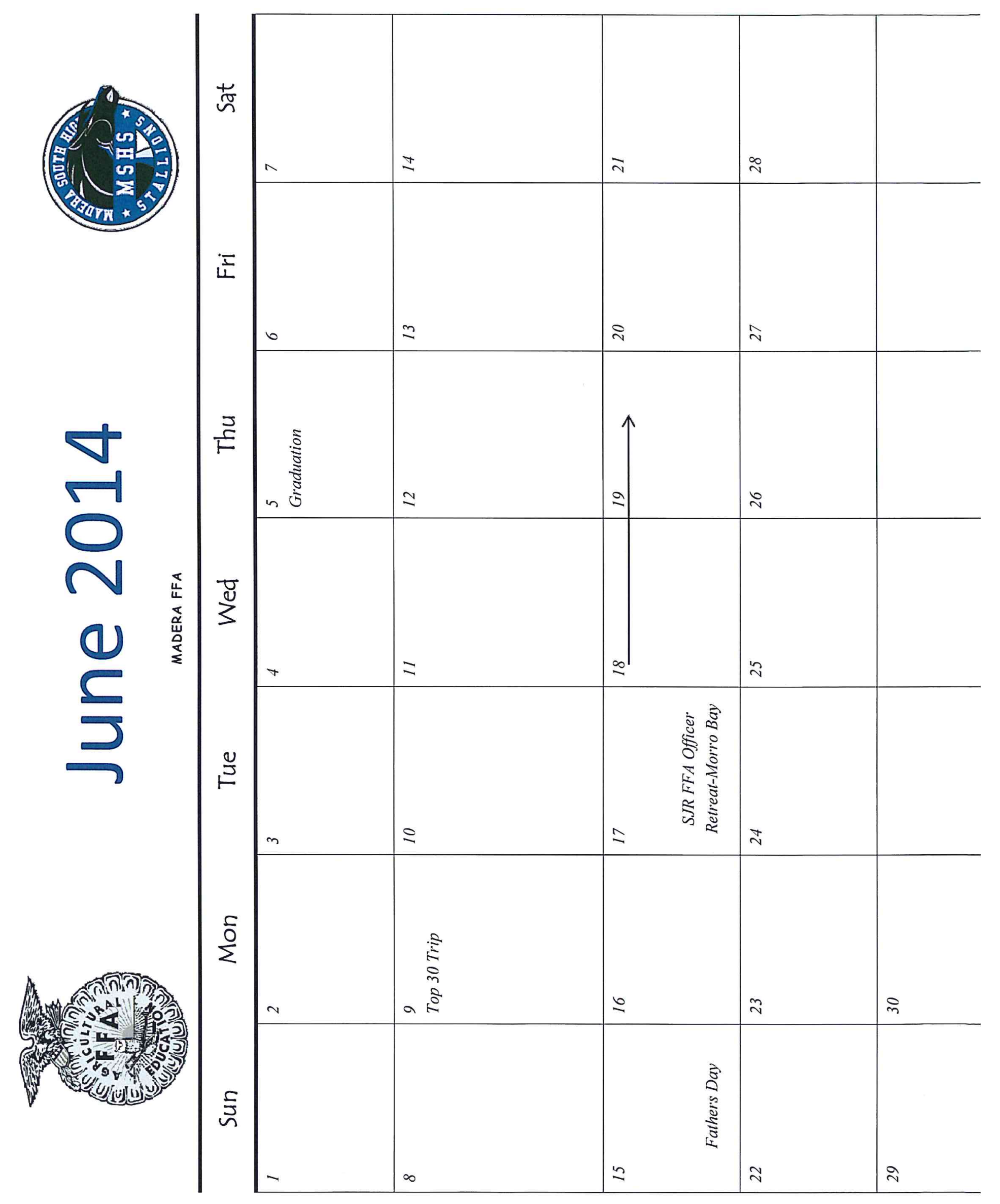




\section{Madera FIA}
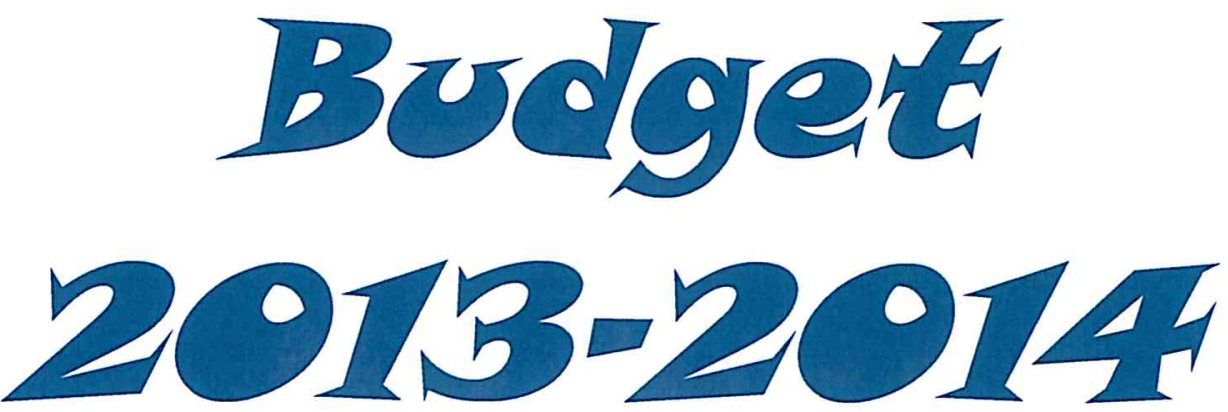

Attached is the FFA Activity Budget which was developed by the officers at retreat and approved by the ASB Director and Bookkeeper on

$$
\text { August 30, } 2013 .
$$
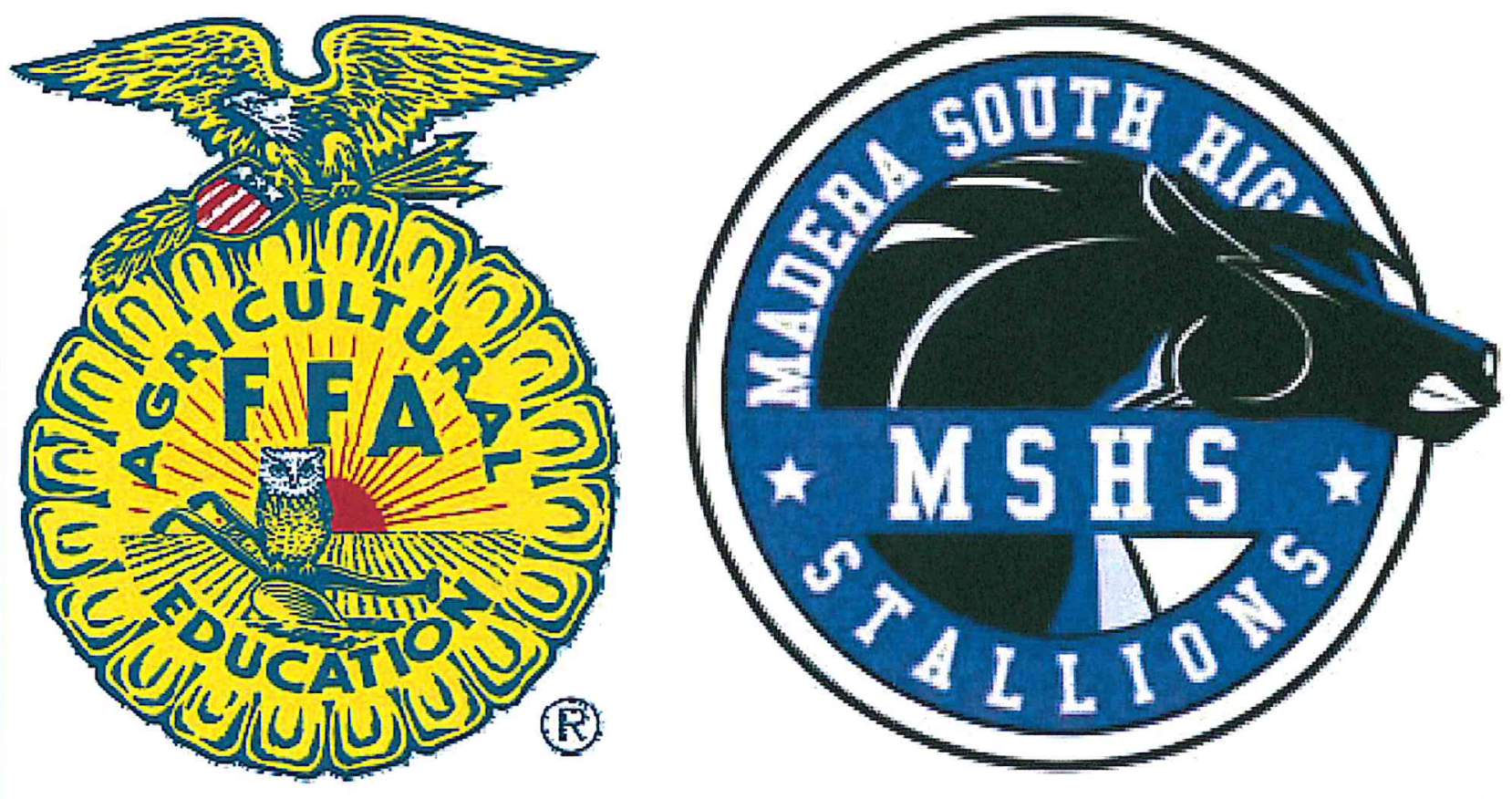


\section{Madera FA Budget \\ 2013-2014}

\begin{tabular}{|c|c|}
\hline \multicolumn{2}{|c|}{ Income } \\
\hline District Lunches & $\$ \quad 5,000.00$ \\
\hline Opening and Closing & $\$ 1,000.00$ \\
\hline Ag. Literacy Day & $\$ 2,500.00$ \\
\hline SHARES & $\$ \quad 600.00$ \\
\hline Snack Sales & $\$ 12,000.00$ \\
\hline Alumni Dinner & $\$ 5,000.00$ \\
\hline Drive Thru BBO & $\$ \quad 4,000.00$ \\
\hline TOTAL INCOME & $\$ 30,100.00$ \\
\hline
\end{tabular}

\begin{tabular}{|c|c|}
\hline \multicolumn{2}{|c|}{ Expenses } \\
\hline Banquet Awards and Décor & $\$ 2,000.00$ \\
\hline Banquet Food & $\$ 700.00$ \\
\hline Bowl A Thon Bus & 350.00 \\
\hline Casino Night & 200.00 \\
\hline Chapter Meetings & $\$ 1,000.00$ \\
\hline Hotels for Field Days & $\$ \quad 1800.00$ \\
\hline COLC & $\$ \quad 120.00$ \\
\hline District Lunches & $\$ 2,000.00$ \\
\hline Field Day Entries & $\$ 2,000.00$ \\
\hline Fall Banquet & $\$ 500.00$ \\
\hline Fall and Spring Regional FFA Meeting & 250.00 \\
\hline Greenhand Conference & 450.00 \\
\hline Grub Down & 200.00 \\
\hline Homecoming Float & 200.00 \\
\hline Officer Polos and Retreat & 800.00 \\
\hline Opening and Closing Food & 675.00 \\
\hline POA Printing & 150.00 \\
\hline Pumpkin Festival Supplies & $\$ 1,900.00$ \\
\hline Scrapbook Stuff & $\$ \quad 200.00$ \\
\hline Skating Meeting & $\$ \quad 450.00$ \\
\hline Snack Sale & $\$ \quad 5,000.00$ \\
\hline Top 30 Tickets & $\$ 1,200.00$ \\
\hline Tri Tip BBO & $\$ 4,000.00$ \\
\hline TOTAL EXPENSES & $\$ 26,145.00$ \\
\hline
\end{tabular}




\section{Gommunity Service}

At Madera FFA we feel community service is not a service at all. As community members we feel it is our duty to step in and help when it is needed; whether it is making sure community members have food at Thanksgiving or making sure children have a present to open on Christmas morning. Madera FFA is there and willing to help when the community needs it.

Ag. Literacy Day- We host a two day activity in which we recognize the importance of Agriculture to the youth within our community.

Bowling-4-Kids-Sectional Bowl-A-Thon is an event awarded to those individuals who help raise money for Children's Hospital.

Can Food Drive- We will host a food drive during the Christmas season, working with local community shelters to distribute the food to the less fortunate in our community.

Old Timer's Parade- FFA members clean up the parade route.

Farm Bureau Scholarship Event- FFA members serve as event staff parking cars, serving, busing tables, and cleaning up.

Relay for Life- FFA members help in the fight against cancer at the Madera Relay for Life where they participate in painting luminary bags and setting up the relay course.

Toys for Tots- FFA members partner with the Toys for Tots organization to provide children with a gift during the holiday season. 


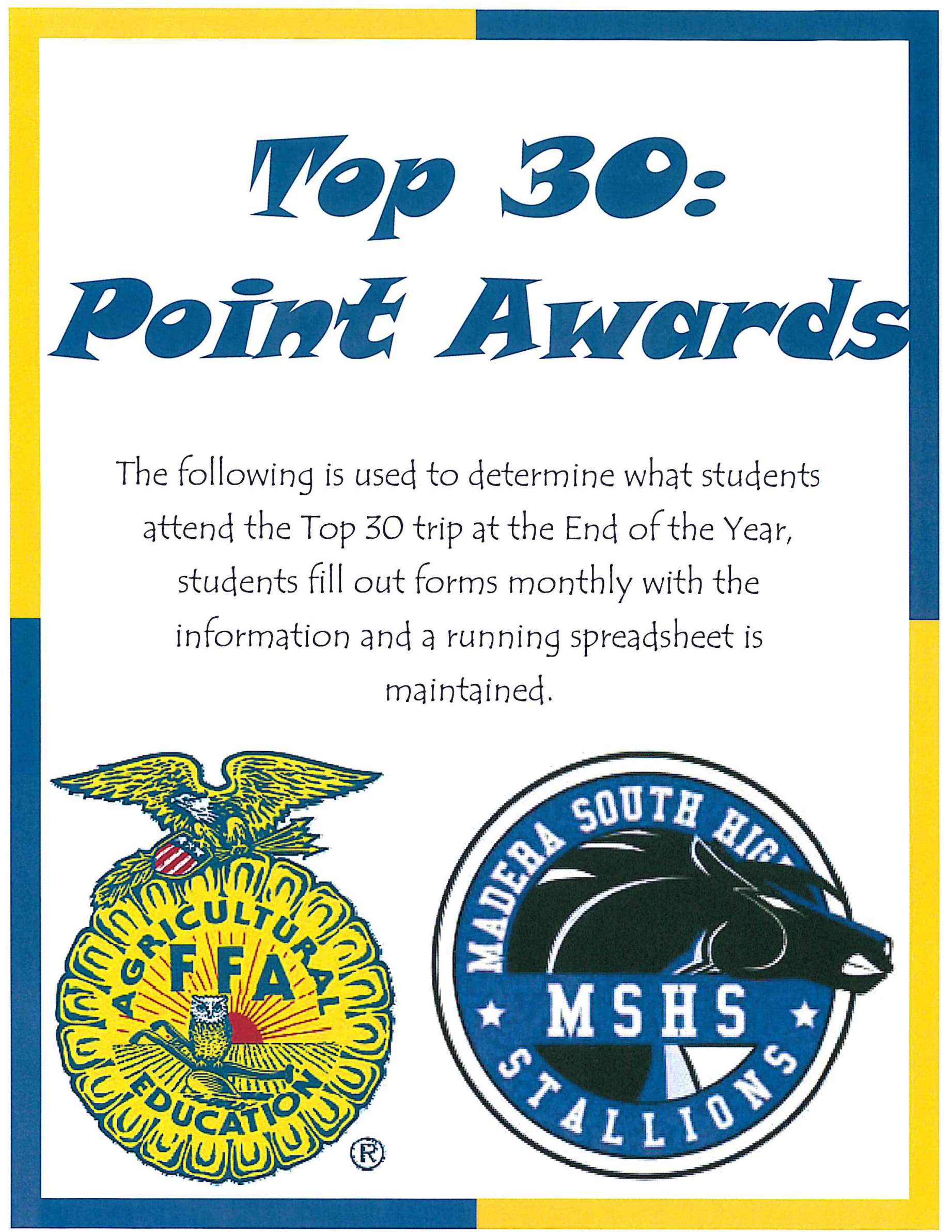




\section{Top Thirety Triip}

Top Seller Trip

Throughout the year, Madera FFA members are given the opportunity to help raise money for our chapter. The money raised goes towards numerous events and activities that take place in our FFA chapter. For example, Madera FFA plans a minimum of one activity per month during our school year that is free of charge to our students. These activities include skating, bowling, and movie night just to name a few. In order to fund these trips, we put on different fundraising events throughout the year. This year we carry on the traditional fundraisers such as the Drive thru BBQ, Snack Sales in the Fall and Spring, and our Alumni Dinner Dance. If a member has sold a total of 40 tickets or items at the end of the last fundraiser, he or she will be invited to the Top Seller Trip at the end of the year. The Top Seller Trip is designed to give every student in our department the opportunity to receive a free trip to a designated theme park.

\section{Top Thirty Trip}

As mentioned above, Madera FFA members are given numerous opportunities to participate in FFA activities. Throughout the year we give students points for attending and participating in FFA activities, events, and contests. For example, the top thirty students are announced at the annual end of the year banquet and then those students are taken on an achievement trip to Magic Mountain. The students fill out point award sheets at the end of each month and a running total is kept until the end of the year. The winner of the Top Thirty Trip is announced at the chapter banquet.

I. Leadership

A. Greenhand Degree (December of each year awarded) 50

B. Chapter FFA Degree (December of year awarded) $\quad 75$

C. State FFA Degree

100

D. Star State Farmer (In addition to degree) 125

11. Offices (Points awarded in May)

A. Chapter Officer 100

B. Committee members 75

C. Sectional Officer 100

D. Regional Officer 100

III. Meetings/Conferences

A. Attend Chapter FFA Meetings/Activities/Banquets 50

B. Attend Sectional/Regional Meeting or Activity 75

C. Attend State Convention 100

D. Attend One Day State Convention 25

E. Attend National Convention 150

F. Delegate for conferences 50

G. Chapter, Section, Region Banquets 50

H. Greenhand, MFE, ALA, SLE, or WLC 75 
IV. Community/Service
A. Ag. Literacy Day (each day)
25
B. $8^{\text {th }}$ Grade Visitation
50
C. Canned Food Drive
50
D. Bowl-a-Thon
50
E. Love Madera
50
F. Other Activities as Developed
50

Fundraisers
A. Tri Tip and Alumni Dinner Ticket Sales
1 pt per $\$$
B. Snack Bags
C. Other Activities as Developed
1 pt per\$
1 pt per $\$$

VI. Other FFA Activities
A. Purchase FFA T-Shirt/Sweatshirt
20
B. Purchase FFA Jacket
50

VII. Supervised Agriculture Experience

A. Approved Enterprise: (Each project)

Large Mechanics, Market Beef, Dairy, Sheep, 50

Swine, and Goats

2. Small Mechanics, Market Poultry, and Rabbits 30

3. Breeding Projects 75

B. Exhibit at Shows and Fairs: Mechanics, Landscape, Floral, and Animal Projects

1. First Award 25

2. Second Award 20

3. Third Award 15

4. Fourth Award - tenth award 10

5. Round Robin Participant

1st

50

2nd $\quad 40$

3 rd 30

4th $\quad 25$

5th 20

6th 15

7th 10

6. Best of Show (Ag Mech., Land, Floral) 50

7. Outstanding Exhibitor (any species) 50 


\section{Top Thintey Trip}

C. Animal Projects: Market or Breeding

1. Grand Champion 50

2. Reserve Grand Champion 35

3. FFA Champion 25

4. FFA Reserve Champion 20

5. Breed Champion 15

6. Reserve Breed Champion 10

D. S.A.E. Hours

1. 1 point per hour worked ( must be proved by record book)

VIII. Proficiency Awards

$\begin{array}{lll}\text { A. } & \text { Applicant } & 20 \\ \text { B. Sectional Winner } & 25 \\ \text { C. } & \text { Regional Winner } & 50 \\ \text { D. State Winner } & 75 \\ \text { E. National Winner } & 100\end{array}$

IX. Contests

A. Participation 15

B. Placement

1. Individual and Team Placing

a. First High Overall 25

b. Second High Overall 20

c. Third High Overall 15

d. Fourth High Overall 10

e. Fifth High Overall 5

The above points for a State Finals Contest are doubled!

The above points for a National Finals Contest are tripled!

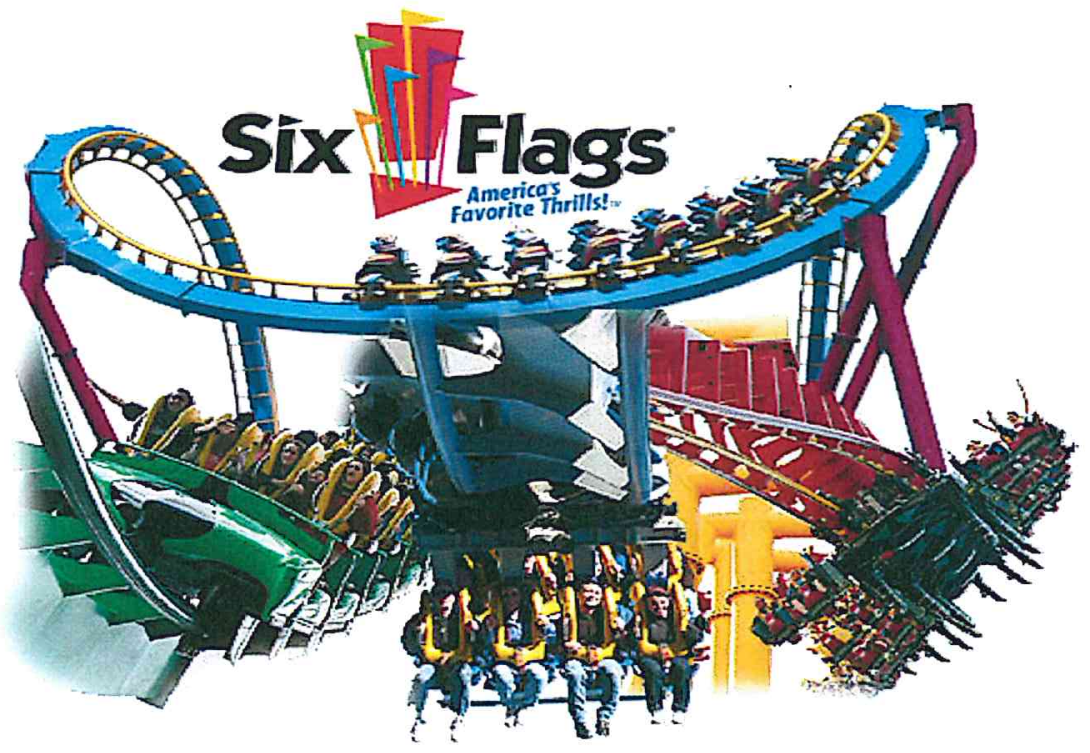




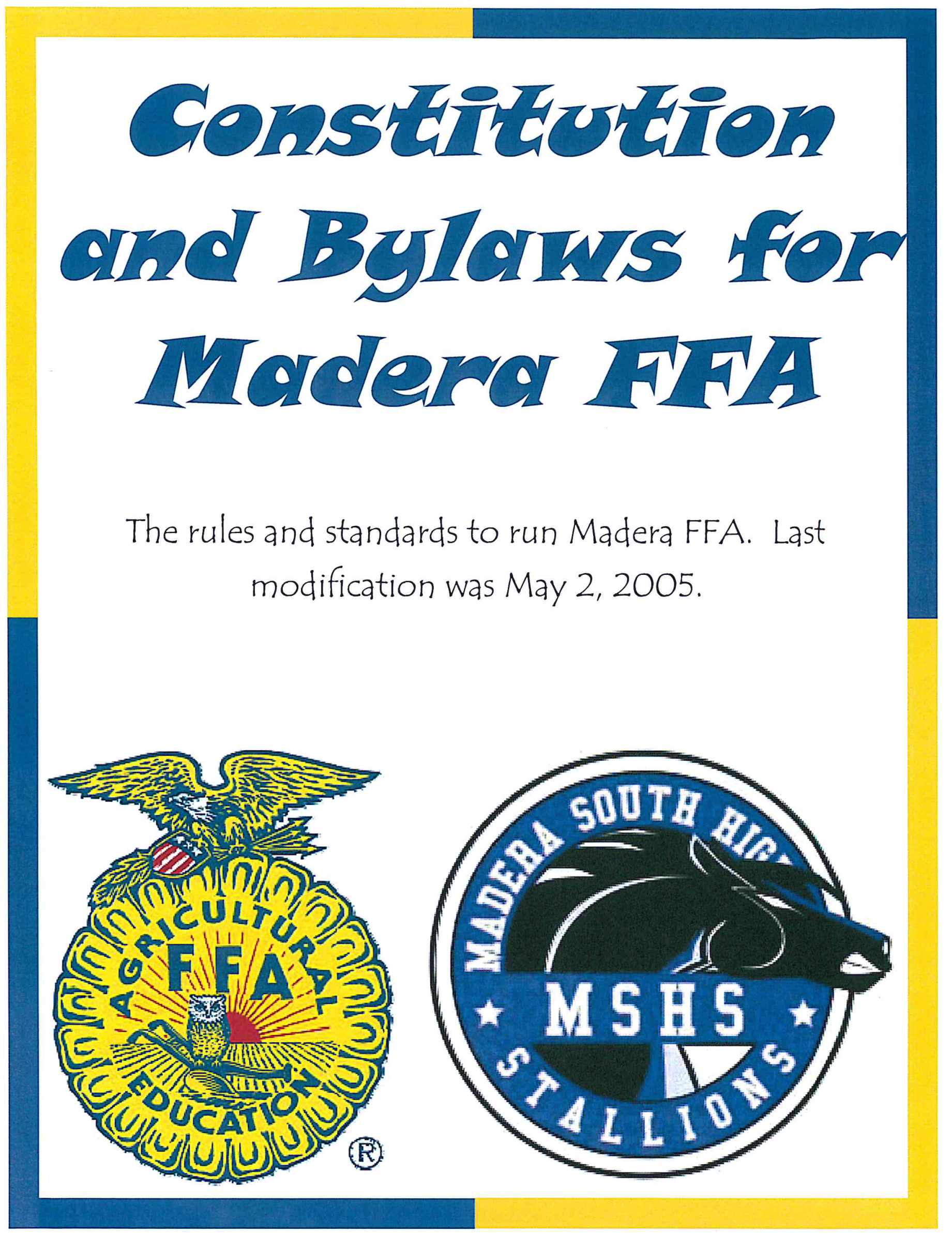




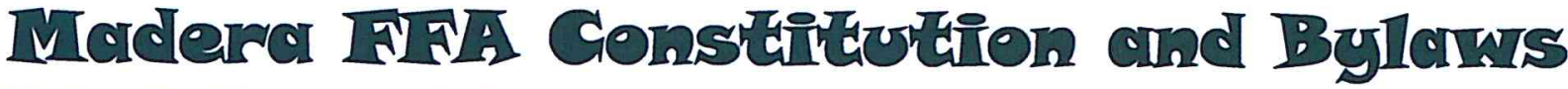 Ariticile I. Name and Purposes}

Section A. The name of this organization shall be the Madera FFA Chapter. The letters "FFA" will be used to designate the chapter, its activities, and its members.

Section B. The purpose for which this chapter is formed by is as follows:

1. To develop agricultural leadership skills among all members.

2. To develop a global awareness of agriculture.

3. To bestow confidence among agricultural students and the work.

4. To promote agriculture career opportunities through hands-on training.

5. To develop competencies in communication, human relations, and social abilities.

6. To build cooperative attitudes among agricultural students.

7. To encourage improvement in scholastics.

8. To provide organized recreational activities for agriculture students.

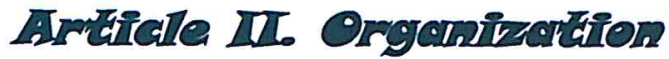

Section A. The Madera FFA Chapter is a chartered local entity of the West Fresno - Madera Section of the California Association, made up of local members.

Section B. This chapter accepts in full, the provision in the constitution and bylaws of the California Association of the Future Farmers of America as well as those of the National FFA Organization.

\section{Articile III. Membership}

Section A. Membership is limited to students enrolled in Agriculture Education at Madera SouthHigh School.

Section B. Membership of graduates is limited to students that were active members in high school.

Section C. The Madera FFA is a $100 \%$ affiliated chapter with every student becoming a member of the FFA when they enroll in an agriculture class.

Section D. No student may participate in any FFA activities unless they are members in good standing with the FFA. In order to be in good standing with the FFA a student must owe no money to the FFA, and their name must not appear on the ineligible list.

Section E. The FFA advisors at their own discretion have the right to dismiss any members from the FFA organization at anytime with approval of the administration.

Section F. Membership in this chapter shall be of three kinds: 


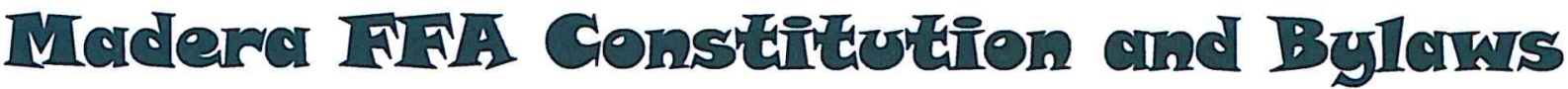

1. Active - Any student enrolled in an agriculture education program.

2. Alumni - Any person who has formerly been enrolled in an agriculture education program or in other ways interested in supporting the FFA.

3. Honorary - Any person who has helped to advance agriculture education and the FFA and who have rendered outstanding service may be elected to honorary membership.

Section G. The regular work of this chapter shall be carried on by the active membership.

Section $\mathrm{H}$. Honorary membership in the chapter shall be limited to the Honorary FFA Degree.

Section I. There shall be four levels of active degree attainment in the Madera FFA Chapter.

The Greenhand FFA Degree

1. All Greenhand Degree recipients are entitled to wear the regulation bronze emblem charm.

2. The Chapter FFA Degree

All members holding the Chapter FFA Degree are entitled to wear the silver emblem pin.

3. The State FFA Degree

All members holding the State FFA Degree are entitled to wear the regulation gold emblem charm.

4. The American FFA Degree

All members holding the American FFA Degree are entitled to wear the regulation gold emblem key.

Section ). Greenhand FFA Degree. Minimum qualifications for election:

1. Be enrolled in agricultural education and have satisfactory plans for a Supervised Agricultural Experience Program.

2. Learn and explain the FFA Creed, Motto, and Salute.

3. Describe and explain the meaning of the FFA emblem and colors.

4. Demonstrate a knowledge of the FFA Code of Ethics and the proper use of the FFA jacket.

5. Demonstrate knowledge of history of the organization, chapter constitution and bylaws and the chapter Program of Activities.

6. Have access to the Official FFA Manual and the FFA Student Handbook.

7. Submit a written application for the Greenhand FFA Degree. 
Section K. Chapter FFA Degree. Minimum qualifications for election:

1. Must have received the Greenhand FFA Degree.

2. Must be enrolled in their second year of agricultural education and have an approved Supervised Agricultural Experience Program.

3. Participate in planning and conducting of at least three official chapter functions.

4. Have earned at least $\$ 150.00$ or worked at least 45 hours and have developed plans for the growth of their SOEP.

5. Have effectively led a group discussion for 15 minutes.

6. Have demonstrated five procedures of Procedure Law.

7. Show progress towards individual achievement in the FFA awards' programs.

8. Have a satisfactory scholastic record.

9. Submit a written application for the Chapter FFA Degree.

Section L. State FFA Degree. Minimum qualifications for election:

1. Qualifications for the State FFA Degree are those set forth in the Constitution of the National FFA Organization.

Section M. American FFA Degree. Minimum qualifications for election:

1. Qualifications for the American FFA Degree are those set forth in the Constitution of the National FFA Organization.

Section N. Special committees shall review the qualifications of members and make recommendations to the chapter concerning degree advancement.

\section{Firiale II. OAficens}

Section A. The possible FFA offices for the Madera FFA Chapter shall be as follows:
1.) President
6.) Reporter
11.) Sweetheart
2.) Vice President
7.) Sentinel
3.) Second Vice President
8.) Historian
4.) Secretary
9.) Chaplain
5.) Treasurer
10.) Parliamentarian

Section B. The Officers shall be elected or confirmed by a majority vote of the active members.

* The advisors and current chapter officers have the right to operate outside of the constitution for special circumstances not addressed.

Section C. If more than one student desires the office of president, the candidates ranking 1, 2, \& 3 by vote for office of chapter president shall be elected as president, vice president, and 2 nd vice president, respectively. 
Section D. The nominating committee shall be composed of the 12th grade chapter officers and the FFA advisors, others to be appointed if necessary. Upon reviewing officer applications for chapter office, students will be slated as candidates on the ballot.

Section E. All officer vacancies, during the term of office, shall be filled by a majority vote of the chapter officers with the exception of the president. The 1st Vice-President shall fill vacancy. The president shall nominate candidates for the committee's consideration.

Section F. Officers Eligibility. Minimum qualifications to run for chapter office:

1. Must be academically eligible to run for a FFA office.

2. For offices of President and Vice President, the applicant must have already completed at least three years of Agriculture classes, and/or hold the Chapter FFA Degree.

3. Other offices require that they have completed a year of an Ag class, and hold the Greenhand Degree.

4. Must enroll in the agriculture leadership class.

Section G. Officer Probation due to academic ineligibility

Any officer who becomes academically ineligible during their term of office will be put on a one-time probationary six-week suspension. At the end of six weeks a grade check will be due to the advisors. While officers are on probation they will not participate in any FFA affiliated activities. If the student meets grade requirements they will be immediately reinstated. If the officer remains academically ineligible they will be immediately removed from office.

* Grades will be based on quarter report cards and grade checks

* Officer participation during the probationary period will be at the advisors discretion.

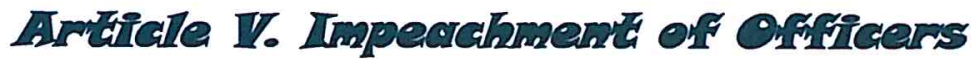

Section A. Immediate Impeachment.

The FFA advisors may at any time at their own discretion remove an officer who has repeatedly disregarded his/her duties by not fulfilling them to his/her best ability.

Section B. Steps of Impeachment.

1. Any FFA chapter officer not fulfilling the duties of the office as described by this constitution will be required to meet with fellow officers and advisors to discuss a plan for improvement.

2. A written plan for improvement will be drawn up by the advisor based on the conversation of the meeting in Step 1 , and will be confirmed and signed by the FFA President, Vice President, and the officer in question.

3. If the officer in question still does not fulfill his/her duties, then a $2 / 3$ vote of the executive committee will remove that officer from office. 


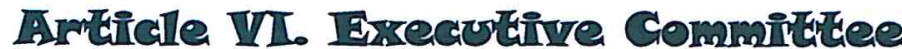

Section A. Executive Meetings shall be held as needed.

Section B. $\quad$ Standard meeting paraphernalia shall be used at each meeting. All special meetings shall open and close with the official ceremony. Parliamentary Procedure shall be used in transacting all business at each meeting.

Section C. Hats shall not be worn in the meeting room.

Section D. Poor conduct will result in that member being dismissed from the meeting room.

Section E. Delegates go through an application process headed by the Ag staff to be able to represent the chapter at the State Convention. The delegates may be named as necessary in order to have proper representation at various other FFA meetings within the state.

\section{Firiale ILI. Dues}

Section A. As long as Incentive Grant funds are available dues shall be paid for all members through that source.

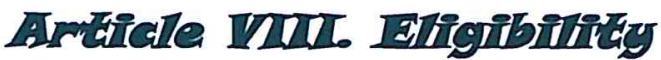

Section A. Eligibility of members exhibiting at fairs and shows will be based on the advisors discretion.

Section B. Members must be academically eligible to participate above the chapter level.

Section C. $\quad$ See rules of article IV section G of the Madera FFA Chapter

\section{Fintiale LX. Amendmentis}

Section A. To amend the constitution, a majority vote of the Executive Committee is required.

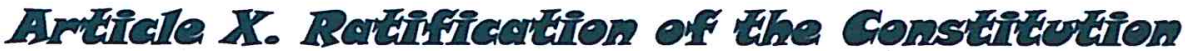

Section A. This constitution shall become effective when passed by the executive committee and advisors. 


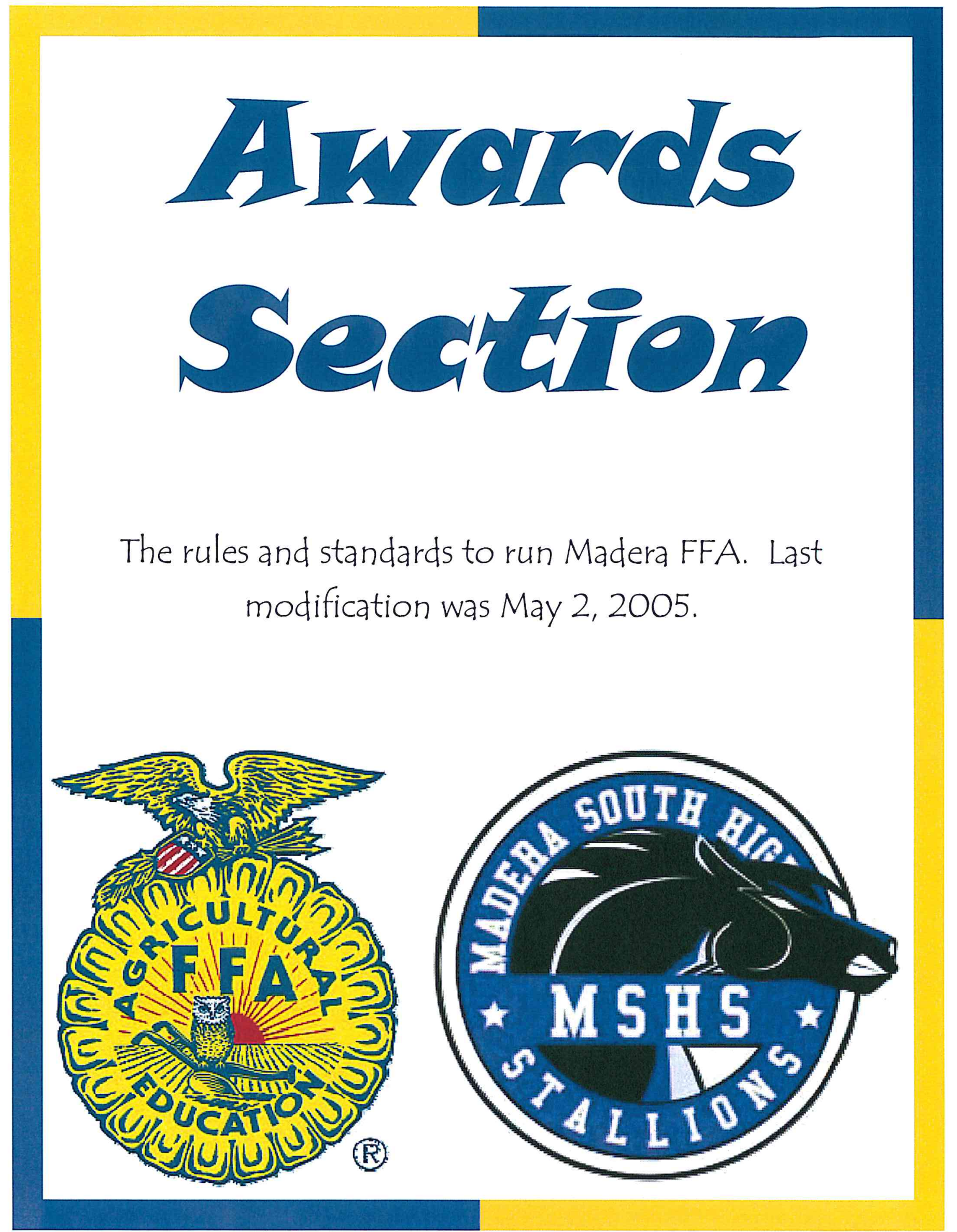


Madera FFA holds a fall and spring banquet where degrees and the following awards are given out.

\section{Degrees}

- Greenhand Degree: For $1^{\text {st }}$ year Ag students.

- Star Greenhand: Most Outstanding $1^{\text {st }}$ year Ag student.

- Chapter FFA Degree: For $2^{\text {nd }}$ year Ag students.

- Chapter Star Farmer: Most Outstanding $2^{\text {nd }}$ year Ag student

- Chapter Star in Agribusiness: Most Outstanding 3rd or 4th year Ag students.

- State FFA Degree: For $3^{\text {rd }}$ and $4^{\text {th }}$ year Ag students.

- State Star Farmer: For $3^{\text {rd }}$ or $4^{\text {th }}$ year Ag students.

- State Star in Agribusiness: For $3^{\text {rd }}$ or 4th year Ag students.

- American FFA Degree: For $5^{\text {th }}$ year Ag student.

- American Star Farmer: For $5^{\text {th }}$ year Ag students.

- American Star in Agribusiness: For $5^{\text {th }}$ year Ag students.

\section{Judging Team Awards - See Career Development Event Pages}

Students who competed in any Career Development Events offered by Madera FFA will receive a certificate and pin. State Winning teams will be awarded with special recognition.

\section{Outstanding Ag Student Awards}

This award is given to the outstanding student in each grade level based on their academic success as well as involvement in the FFA.

\section{Outstanding Herdsmen Award}

This award is presented to a student in each specie category. The student must show great dedication and commitment to their animal project to even be considered for this award. This time spent with their specie includes at the farm as well as at livestock fairs and shows.

\section{Top 30 Award}

FFA points are awarded for students' involvement in FFA activities throughout the entire school year. The top 30 students earn an all expenses paid trip at the end of the year.

\section{Top Seller Award}

Any student who participates in fundraising activities and sells 20 tickets or items receives a free trip at the end of the school year. 


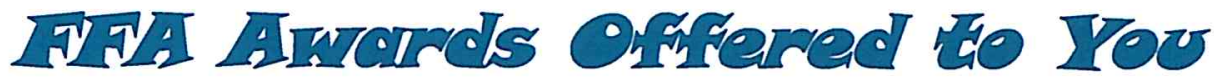

\section{Proficiency Awards}

These awards are given to students based on their SAE projects, and they are given at the Sectional, Regional, State, and National levels.

Outstanding Ornamental Horticulture Student

This award goes to a student who has a strong SAE in this project area. The student must also be enrolled in Ornamental Horticulture classes.

Outstanding Small Engines Student

For any student who shows their dedication to the Small Engines class, career development event, or SAE project.

\section{Outstanding Ag Mechanics Student}

This award is given to a student representing each grade level. The students must be enrolled in some kind of Agriculture Mechanics class, have a relating SAE, or be involved in the Ag Mechanics career development event.

\section{Outstanding Floral Design Student}

For a student who is taking any level of Floral Design classes. The recipient must have a relating SAE or career development event.

\section{Outstanding Animal Science Student}

This award goes to a student who has a strong SAE in this project area. The student must also be enrolled in Animal Science classes.

\section{Outstanding Overall SAE Project}

Presented to the student with the chapter's best SAE project. It can be from any project area.

\section{Petrucci Participation Award}

This award is given to the student who shows dedication to all areas of the Madera FFA Chapter; including classes, SAE's, career development events, fundraisers, and activities.

\section{Teco Award}

Given to a senior in the Ag Mechanics class with an outstanding SAE project.

\section{Stockli-Weiss Award}

This award is presented to a hard working individual who is devoted to the success of the chapter by involving themselves in activities outside their own projects on the school farm. The recipient of this award must be very dedicated person who is always willing to help others. 


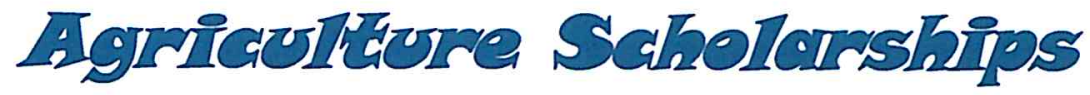

\section{Scholarship applications are available through the high school coun- seling office. It is your responsibility to get an application and return it by the deadline - GOOD LUCK!}

\author{
Matthew Roussel Memorial \\ $\$ 250$
}

This is a memorial scholarship to honor an alumni FFA member who has passed away. It is awarded to a student who has been active in our FFA Organization. The application for this scholarship is the general MHS scholarship application form. By meeting the application requirements and deadline, the school scholarship committee will select a recipient.

\section{Madera Agriculture Youth Association (M.A.Y.A.) \\ $\$ 500$}

The MAYA association is pleased to offer a scholarship program for those high school students pursuing a career in an agriculture related field.

Eligibility requirements:

1. A 2.5 cumulative grade point average.

2. Application must be planning to enroll as a full time student (MINIMUM 12 UNITS)

3. Must be a resident of Madera County and an active FFA member of Chowchilla, Madera, Firebaugh or Yosemite High Schools or a Madera County 4-H Club.

Friends of Madera FFA

This is a scholarship is awarded to a student who has been active in the Madera FFA Organization. The application for this scholarship is the general MSHS scholarship application form. By meeting the application requirements and deadline, the school scholarship committee will select a recipient.

\section{Outside Organizations}

Other Agricultural Community Organizations also have scholarships available to students pursuing degrees in Agriculture, check with the following organizations for specific qualifications and deadlines.

- Calcot Seitz Foundations

- California Women for Agriculture

- World Ag. Expo

- National FFA (Online)

- California FFA

- Madera County Farm Bureau - California State Fair

- California Farm Bureau Fed- - California Table Grape eration
Commission
- Cattleman's

- CANERS Foundation

- Chowchilla Western Stampede

- Also check with the campus' you are applying to for other scholarships. 


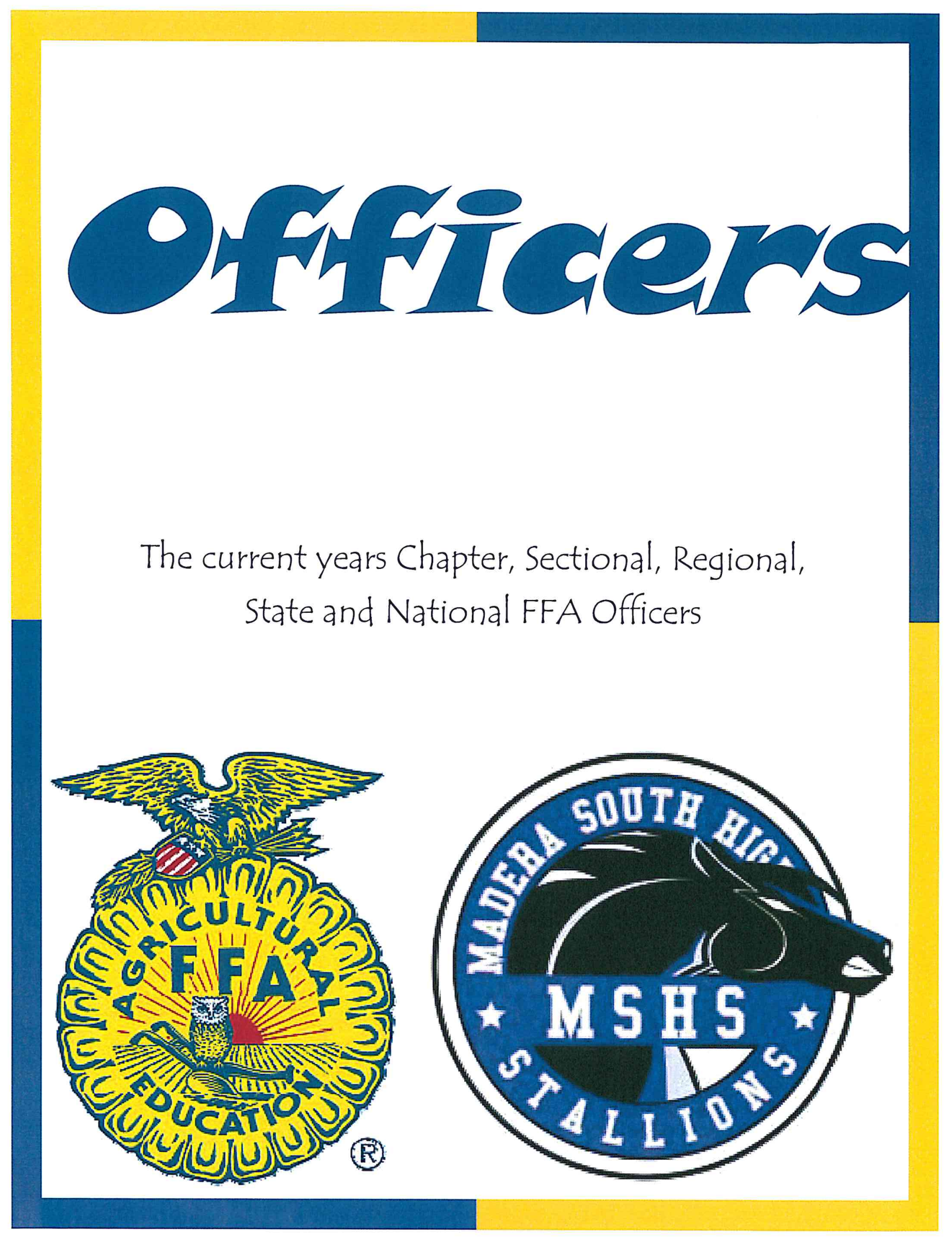




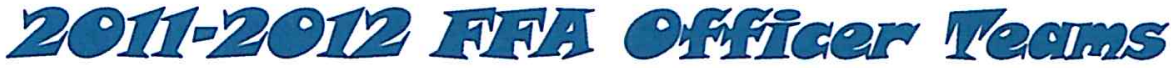

\section{Madera FFA Chapter Officers}

President

Vice President

2nd Vice President

Secretary

Treasurer

Reporter

Sentinel

Historian

Chaplain

Parliamentarian
Virat Kang

Jimmy Beavers

Jaime Cuevas

Jenae Hansen

Michael Ewing

Jorge Mendoza

Gabrielle Ortega

Mario Alvarez

Sarah Reece

Clayton Sheehan
President

Vice-President

$2^{\text {nd }}$ Vice President

Secretary

Treasurer

Reporter

Sentinel

Historian

Chaplin

Parliamentarian
West Fresno Madera Sectional FFA Officers

Amber LaSalle, Firebaugh

Brianne LeBeau, Fresno Central

Kellen Habib, Caruthers

Mackenzie Meek, Central West

Sean Pimentel, Central West

Kiana Peter, Chowchilla

Jimmy Beavers, Madera

Brandon Miller, Central West

Llimy Garcia, Mendota

Jacob Quinteros, Firebaugh

\section{San Joaquin Regional FFA Officers}

President

East Fresno/Madera VP

Tulare Kings VP

Sequoia VP

South Valley VP

Kern VP

WF/M VP

Secretary

Treasurer

Reporter

Sentinel

Advisor
Dipak Kumar, Tulare

Emilie Gambril, Clovis East

Hattie Jameson, Golden Valley

Bailey Minday, Tulare

Madion Zittle, Frontier

Joseph Agular

Amber LaSalle, Firebaugh

Evie Starich, Hanford

Brant Hall, O'neals Minarets

Virat Kang, Madera

Kristyin Fletcher, Sierra

Charles Parker 


\section{California State FFA Officers}

President

Vice President

Secretary

Treasurer

Reporter

Sentinel

Advisor
Riley Nelson

Valerie Canas

Gabrielle Franke

Gage Willey

Sheldon Overton

Hunter Berry

Bob Hueval

\section{National FFA Officers}

President

Secretary

Eastern Vice-President Central Vice-President

Southern Vice-President Western Vice -President
Clay Sapp, Florida

Katie Hall, Georgia

Joenelle Futrell, Kentucky

Brennan Costello, Nebraska

Wiley Bailey, Alabama

Lindsey Anderson, California

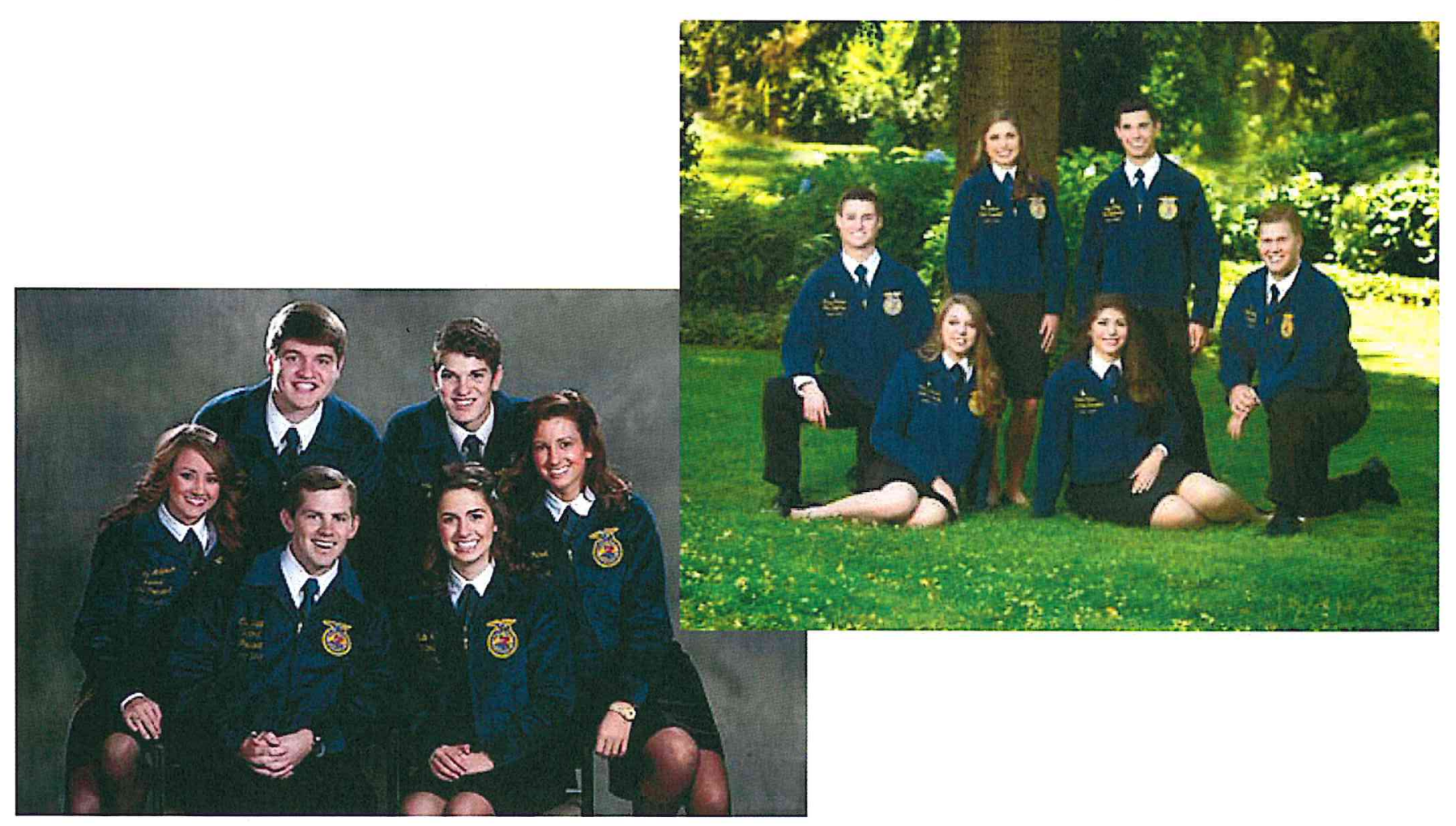




\section{3-2014 Madera FIA Officer Info}

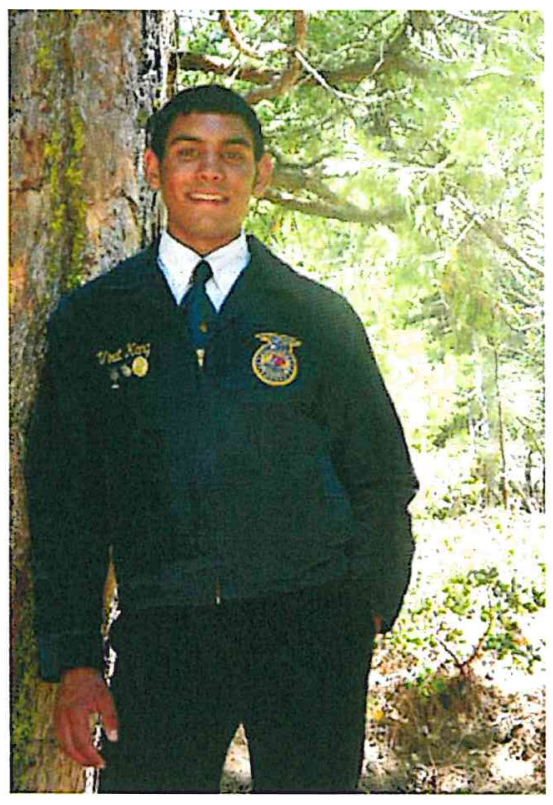

Vice President, James Beavers

Grade: Senior

SAE: Dairy Replacement Heifer, Market Hogs

CDE: Vine Pruning, Extemporaneous Public Speaking

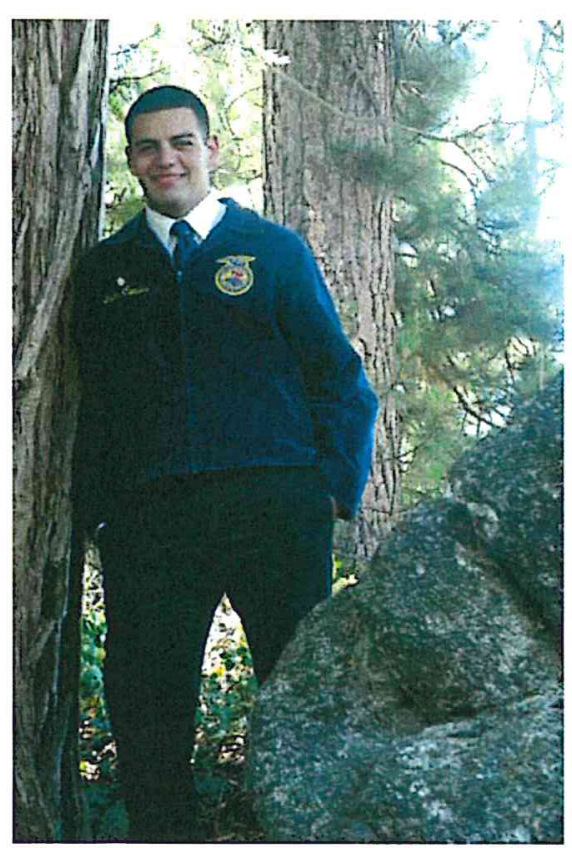

\section{President, Virat Kang}

Grade: Junior

SAE: Ag Sales, Forage Production, Dairy Replacement Heifer CDE: Small Engines, Extemporaneous Public Speaking, Tree Pruning

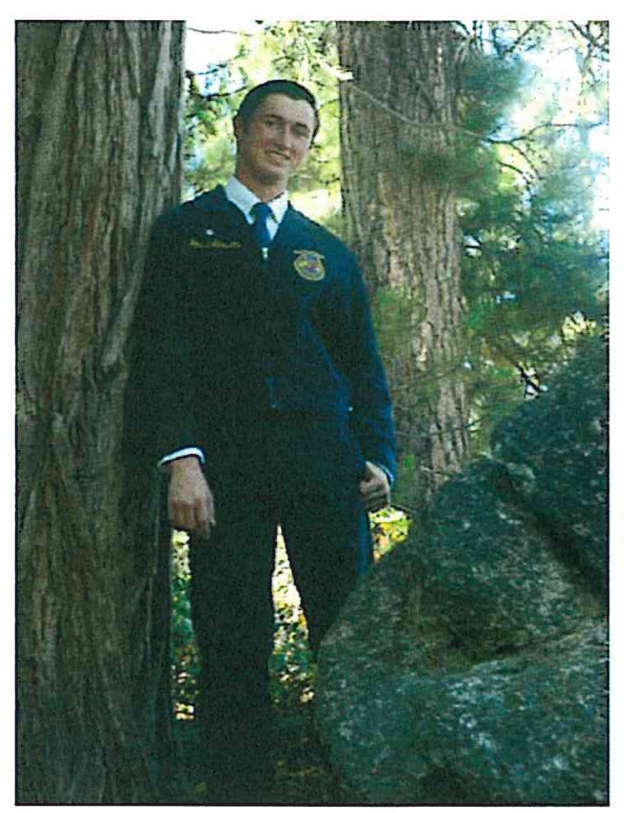

\section{Vice President, Jaime Cuevas}

Grade: Senior

SAE: Dairy Replacement Heifer

CDE: Small Engines, Extemporaneous Public Speaking 


\section{3-2014 Madera FAA OAficer Info}

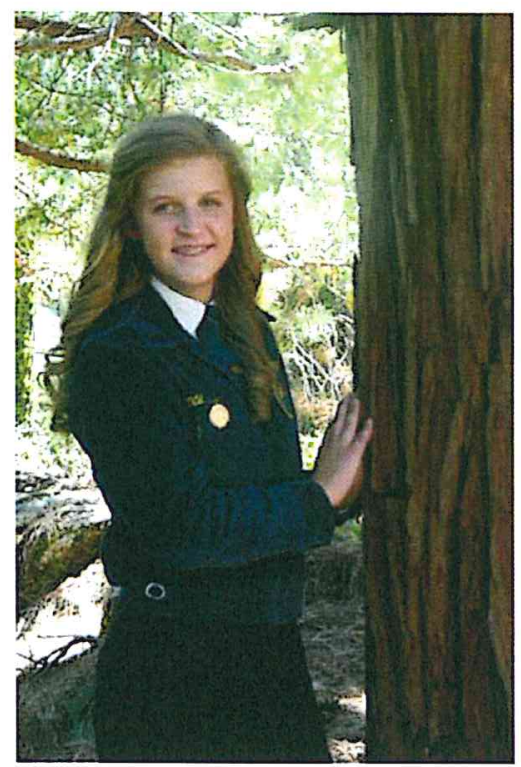

\section{Secretary, Jence Hansen}

Grade: Junior

SAE: Dairy Replacement Heifer, Market Hogs, Viticulture CDE: Prepared Public Speaking, Tree Pruning, Cotton Judging, Vine Judging

Treasurer, Michael Ewing

Grade: Junior

SAE: Market swine

CDE: Job interview, Vine Judging
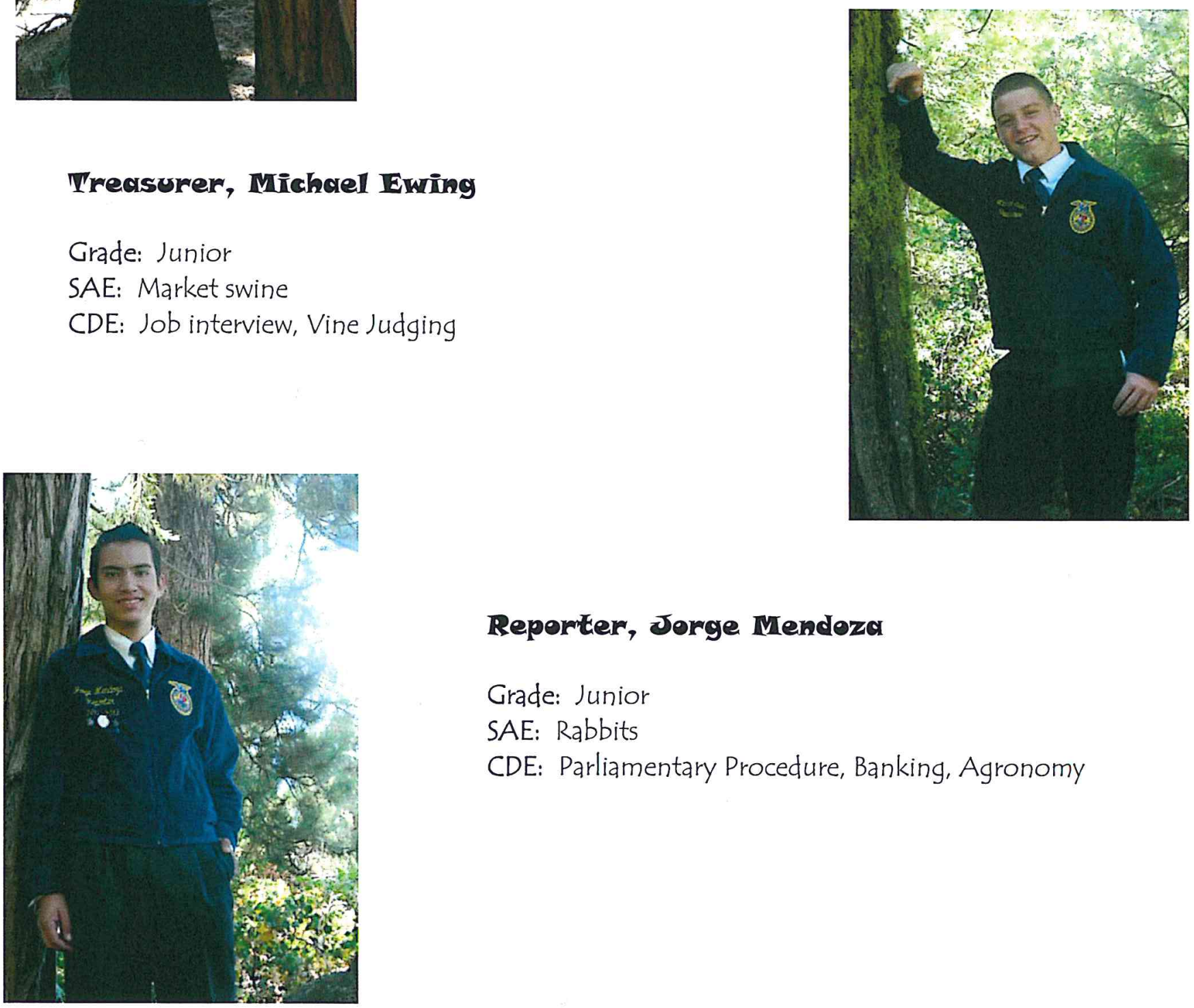

Reporter, Jorge Mendozo

Grade: Junior

SAE: Rabbits

CDE: Parliamentary Procedure, Banking, Agronomy 


\section{3-2014 Madera FNA OAficer Info}

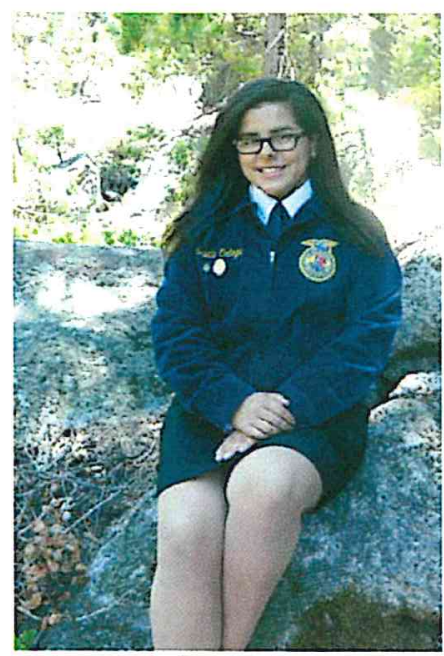

Mistorian, IMrio Alvarez

Grade: Sophomore

SAE: Market sheep

CDE: Banking, Impromptu Public Speaking

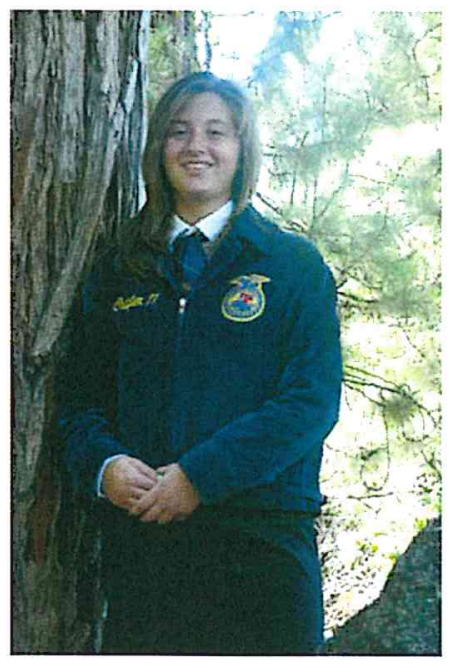

Parlimmentarian, Glayton

Sheehmin

Grade: Sophomore

SAE: Diary Replacement Heifer, Swine Breeding, Market Swine

CDE: Vine Pruning, Agronomy, Advance
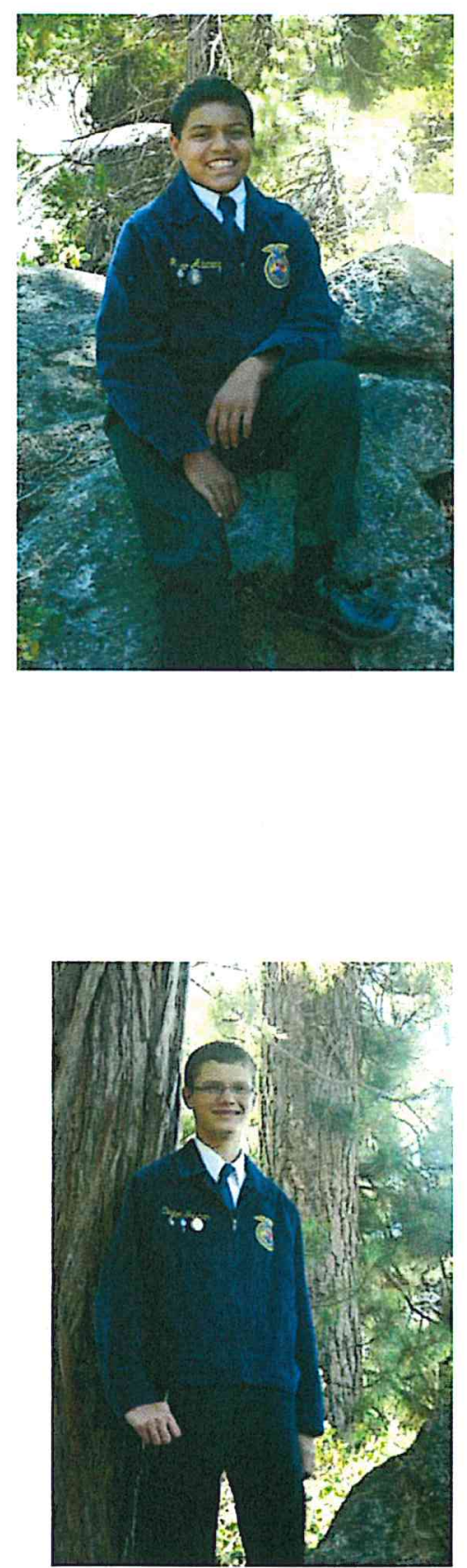


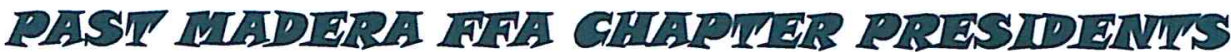

2001-02

2002-03

2003-04

2004-05

2005-06

2006-07

2007-08

2008-09

2009-10

2010-11

2011-12

2012-13

2013-14

* Took over after resignation
Nick Davis

Brandon Visscher

Giana Toschi

Megan Matteucci *

Megan Matteucci

Cody Ogletree

Mika Petrucci

Jessica Sydney

Dominic Bettini

Amy Evans

Michael Valencia

Taylor Helton

Virat Kang

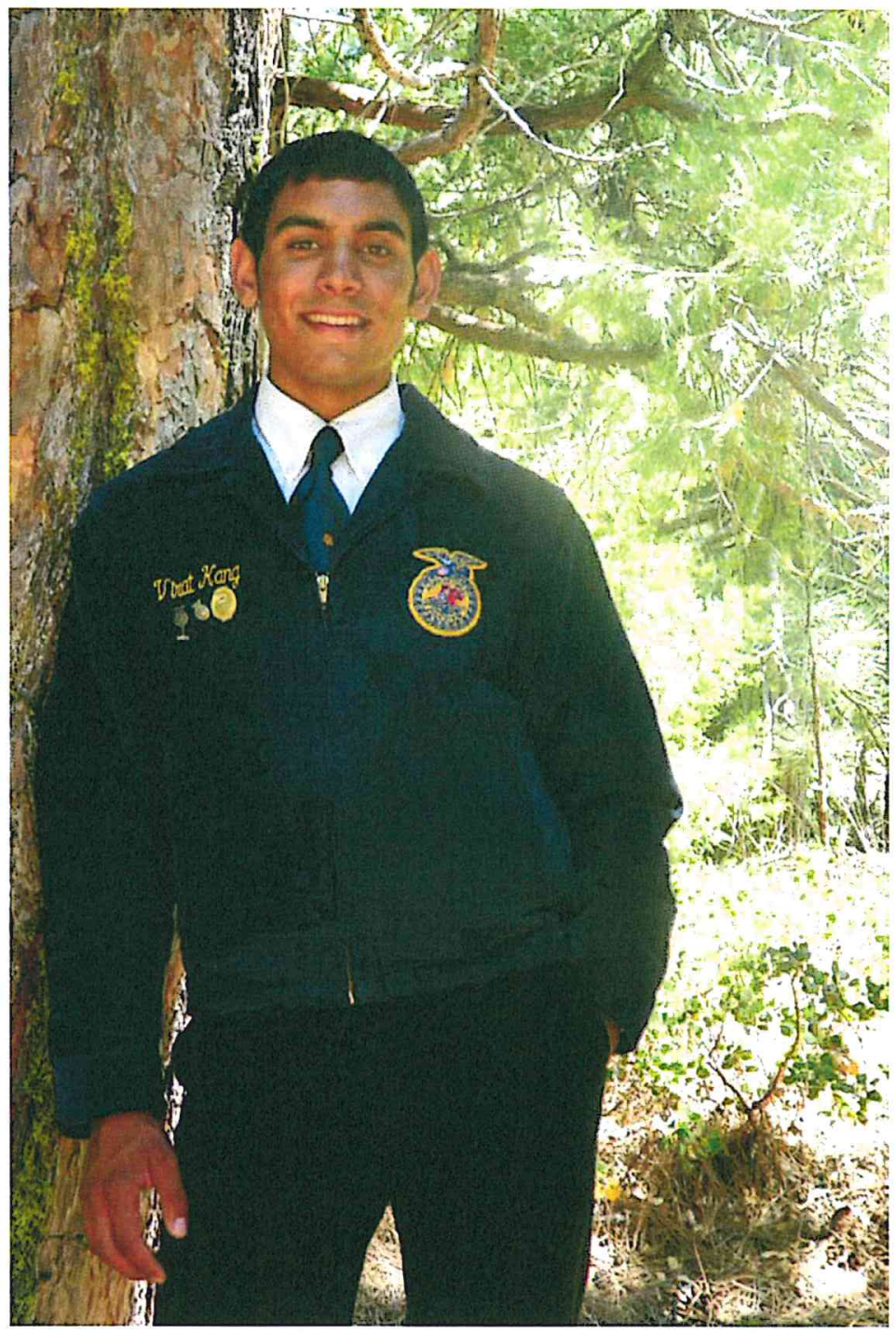




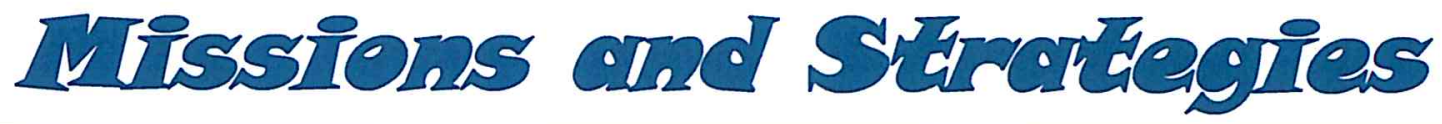

"FFA makes a positive difference in the lives of others by developing their potential for premier leadership, personal growth and career success through agricultural education."

To accomplish this mission, we the FFA will:

1. Develop a competent and assertive agricultural leadership program.

2. Increase awareness of the global and technological importance of Agriculture and its contribution to our well-being.

3. Strengthen the confidence of agriculture students in themselves and their work.

4. Promote the intelligent choice and establishment of an agricultural career.

5. Encourage achievement in supervised occupational experience programs.

6. Encourage wise management of the economic, environmental, and human resources of the community.

7. Develop interpersonal skills in teamwork, communication, and human relations.

8. Build character; promote citizenship, volunteerism, and patriotism in our students and community.

9. Promote cooperation and cooperative attitudes among all people. 10. Promote healthy lifestyles.

11. Encourage excellence in scholarship.

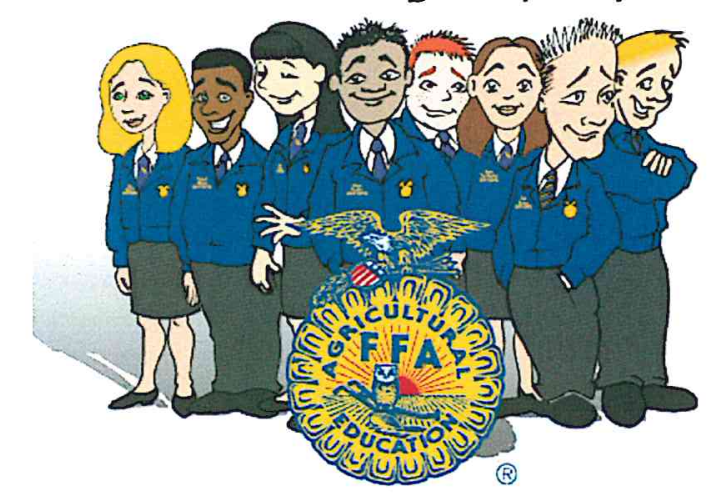




\section{FFA Emblem, OAficiol Colors, \& FA MotEo}

The National FFA emblem consists of five symbols and represents the history, goals and future of the organization. As a whole, the emblem covers the broad spectrum of the FFA and agriculture. Each element within the emblem has unique significance. THE CROSS SECTION OF THE EAR OF CORN provides the foundation of American agriculture. It is also a symbol of unity, because corn is grown in every state of our nation. THE RISING SUN signifies progress and holds a promise that tomorrow will bring a new day glowing with opportunity. THE PLOW signifies labor and tillage of the soil, the backbone of agriculture and the historic foundation of our country's strength. THE EAGLE is a national symbol, which serves as a reminder of our freedom and ability to explore new horizons for the future of agriculture. THE OWL, long recognized for its wisdom, symbolizes the knowledge required to be successful in the agriculture industry. The words "AGRICULTURE EDUCATION" and "FFA" are emblazoned in the center to signify the combination of learning and leadership necessary for progressive agriculture.

\section{Colors}

As the blue field of our nation's flag and the golden fields of ripened corn unify our country, the FFA colors of "NATIONAL BLUE and CORN GOLD" give unity to the organization. All FFA functions and paraphernalia should proudly display the colors.

\section{Motto}

The FFA motto gives members twelve short words to live by as they experience the opportunities in the organization.

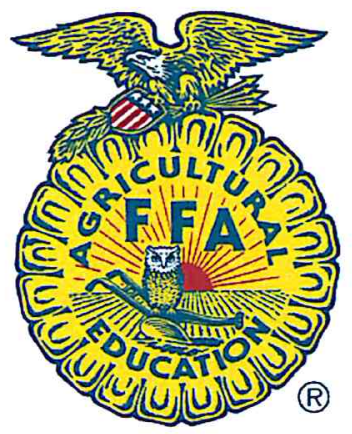

Learning to Do,

Doing to Learn,

Earning to Live,

Living to Serve.

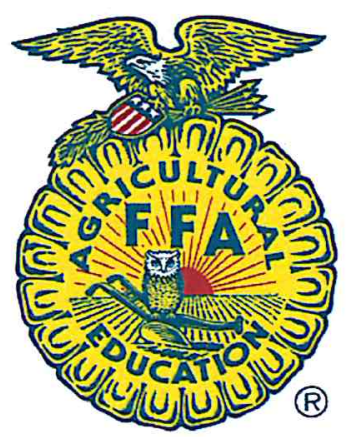




\section{The Fin Greed}

The creed was written by E.M. Tiffany, and adopted at the 3rd National Convention of the FFA. It was revised at the 38th Convention and the 63rd Convention.

I believe in the future of agriculture, with a faith born not of words but of deeds-achievements won by the present and past generations of agriculturists; in the promise of better days through better ways, even as the better things we now enjoy have come to us from the struggles of former years.

I believe that to live and work on a good farm, or to be engaged in other agricultural pursuits, is pleasant as well as challenging; for I know the joys and discomforts of agricultural life and hold an inborn fondness for those associations which, even in hours of discouragement, I cannot deny.

I believe in leadership from ourselves and respect from others. I believe in my own ability to work efficiently and think clearly, with such knowledge and skill as I can secure and in the ability of progressive agriculturists to serve our own and the public interest in producing and marketing the product of our toil.

I believe in less dependence on begging and more power in bargaining; in the life abundant and enough honest wealth to help make it so - for others as well as myself; in less need for charity and more of it when needed; in being happy myself and playing square with those whose happiness depends upon me.

I believe that American agriculture can and will hold true to the best traditions of our national life and that I can exert an influence in my home and community which will stand solid for my part in that inspiring task. 


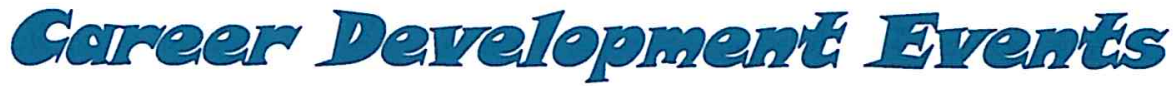

The following list are CDE teams and competitions you can get more involved in check with the Ag. Teacher for more Information.

\section{Agrricuriture Tlelling}

To evaluate the contestant's manipulative skills, general knowledge and professional presentation as these correlate to his/her preparation for employment in the broad field of welding (agricultural, industrial, or other). Coach: Mr. Deniz

\section{Bernising}

Members complete a written test made up of financial standings, such as checks and bank statements. You will be expected to know the different rates of credit that may be given to you. Coach: Mrs. McKenna

\section{Besis Intormed Greenlherned}

This contest is for freshmen FFA students only. Members on this team complete a written test on their knowledge of the FFA. Coach: Mrs. Sheehan

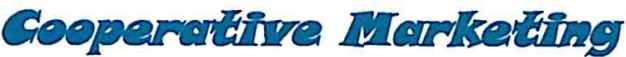

This contest is designed to create an awareness and understanding of the basic elements of farm product marketing and farmer cooperation in marketing, purchasing, bargaining, and service. Coach: Mr. Williams

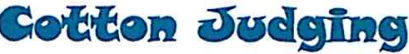

This contest is made up of a three-member team. Members are required to take a written exam of their knowledge of cotton. Members will also be required to judge and score different types of cotton.

\section{Coach: Ms. Gilles}

\section{Greed Spectingeg}

This contest is for freshmen FFA students only. Students memorize and recite the FFA Creed written by E.M. Tiffany and answer three oral questions from a panel of judges. Coach: Mr. George

\section{Deiliry Proderéts}

In this contest students learn about the Milk Industry through a test. In addition students verify whether dairy products are real vs. artificial, know different cheese varieties and are able to identify off flavors in milk. Coach: Ms. Luera

\section{Eximemporaneous Public Speaking}

Members deliver a speech on one of three agricultural topics after they are given thirty minutes to pre-

pare a four-to-six minute speech. At the conclusion of the speech, the judges may question the speaker for five-minutes. Decisions are based on factors similar to the prepared event. Coach: Miss. Luera

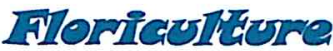

Members demonstrate proficiency in plant identification, judgment of floral and foliage arrangements, problem solving, and skills that include flower arranging and corsage construction. Coach: Ms. Gilles 


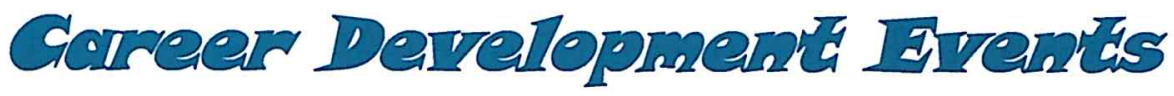

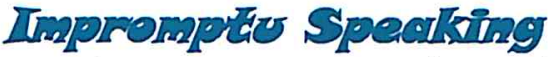

Members are required to learn about many agriculture topics, and will be given 1 minute to think about a topic they draw and then deliver a 1-2 minute speech from memory. There is a question and quote round. Coach: Mrs. McKenna

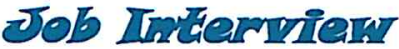

Members are required to create a cover letter and resume prior to participating in a job interview. You are evaluated and placed according to your resume, cover letter, and interview scores. Coach: Mr. Deniz

\section{Mears}

Members complete a written test; evaluate beef carcasses in terms of quality and yield grades; and judge and place beef, lamb and pork classes. Members also complete a meat formation problem and cooperatively fulfill a beef carcass order. Coach: Mr. George

\section{Nurserv/_andsempe}

The Nursery/Landscape contest prepares students for careers in the nursery and landscaping. Topics include plant identification, plant physiology, soil science, plant reproduction, and nursery production, as well as landscaping design, installation, and maintenance. Coach: Mrs. McKenna

\section{Opening/Glosing}

This contest is made up of a six-person team. Each member of the team is responsible for memorizing one officer part of the opening and closing ceremonies and reciting it at the sectional contest. Coach: All Ag. Teachers

\section{Preperred Publia Spearings}

The member that chooses this speaking contest is to write and memorize a six-eight-minute speech on a major agriculture issue. The individual will be scored on his or her ability to speak and also on how well they can answer questions on the topic they choose. Coach: Mr. Williams

\section{Perilienenentirny Procedure}

The team consists of six members. One member of the six person team serves as the chair and another as the secretary. Participants enter a room and have one minute to study a card that has five motions on it. The card also has a main motion on the card that the members must debate. In order to score top points from the judges the team must perform the five motions on the card and five more motions that are not listed on the card. Each team member will also debate the main motion four times each. A round in Parliamentary Procedure is timed for ten minutes and thirty seconds. At the end of the round judges ask the team questions relating to Parliamentary Procedure. There is also a twenty five question written test. Coach: Novice, Ms. Luera Advanced: Mrs. Sheehan

\section{serrerpbook}

The Nursery/Landscape contest prepares students for careers in the nursery and landscaping. Topics include plant identification, plant physiology, soil science, plant reproduction, and nursery production, as well as landscaping design, installation, and maintenance. Coach: Mrs. McKenna 


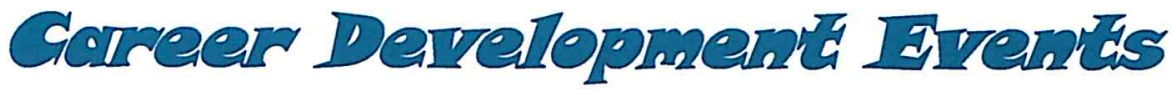

\section{Smalli Engines}

A team is made up of three members. Members are tested on identification, theory, problem solving and troubleshooting related to small engine repair. They are required to fix an engine and take a written test. Coach: Mr. Williams

\section{Vine Judging}

The teams consist of three of four members. There are four classes of pruned vines that will be judged by each member of the team for twenty minutes apiece. The member will then give oral reasons for each class of vines. Coach: Mr. Williams and Mrs. McKenna

\section{Vine Prorning}

Members prune three classes of grapevines ranging from table grapes to wine grapes. The contest consists of a timed pruning and a written exam. The contest may include questions from the judges after a participant is done pruning it. Coach: Mr. Williams and Mrs. McKenna
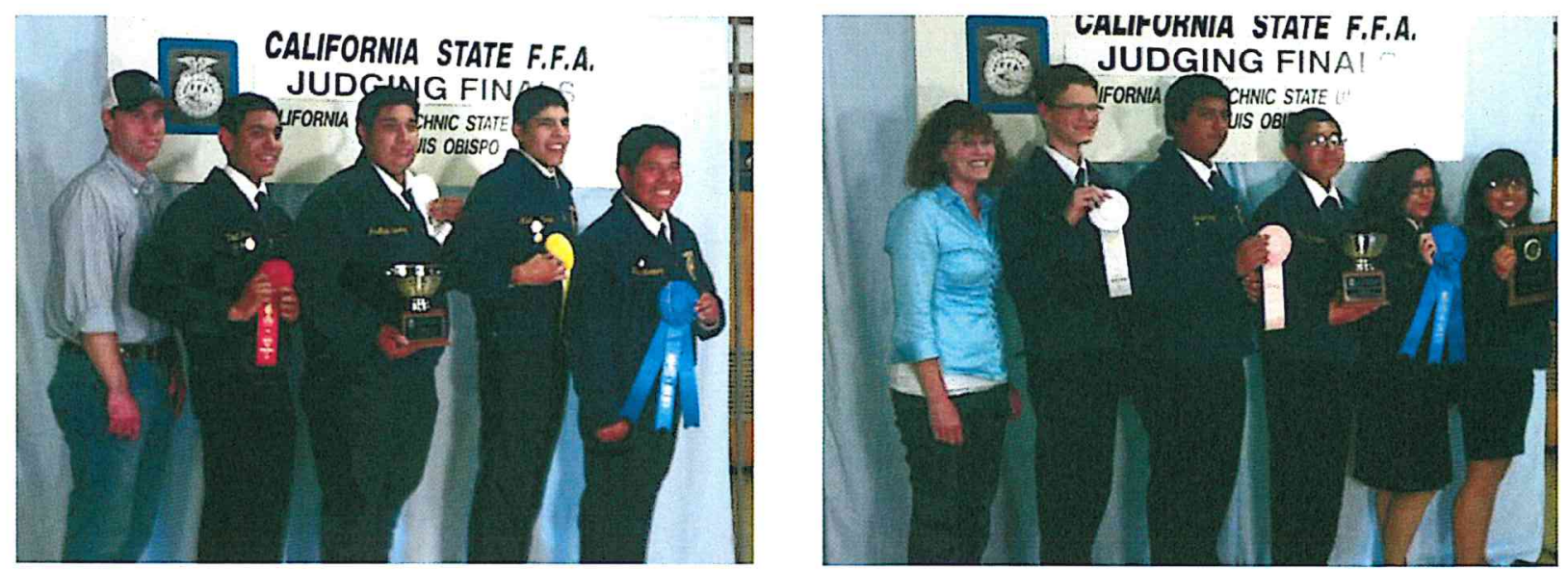

Last years State Winning Vine Pruning, Best Informed Greenhand and Meats Evaluation

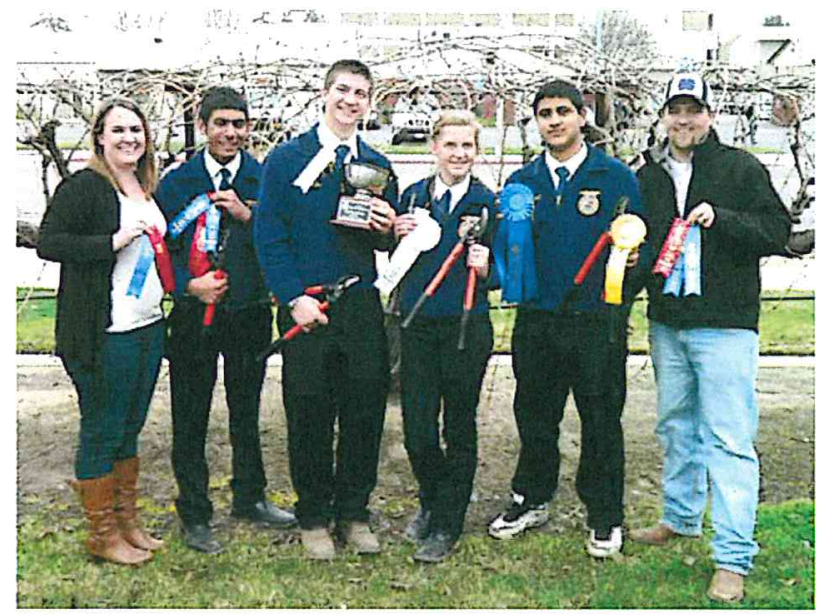




\section{Here is arn exrmple of the Exhibitors Contract Signed by mem- bers a carbon copy sheet must be obtained by a project advisor.}

\section{Madera FFA Exhibitors Contract}

As a member of the Madera FFA chapter, I realize that there are certain obligations on my part in order to assure a successful project. As part of my obligations, I agree that the following expectations will help me to complete my animal project. This contract shall begin on and terminate on

All students shall be respectful to parents, advisors, staff and students.

All students shall be in good standing, a current member of the FFA, and maintain a 2.0 GPA throughout the duration of the project.

No students shall show/exhibit an animal project as a member of the Madera FFA without an Exhibitors Contract signed by 1) the exhibitor, 2) parents/guardians, 3) chapter advisor and 4) the vice principal.

All school rules, district, and California State rules and the specific rules pertaining to the show in question shall be upheld by the exhibitor.

Throughout the term of the project all exhibitors are to follow the directions and advice given to them by the designated advisor for that species.

All exhibitors will be responsible for the care, feeding, exhibiting and marketing of their animals.

$\downarrow$ All students will participate in showmanship and $80 \%$ of the scheduled showmanship practices prior to the fair.

^ All students will show in the "Official FFA Show Uniform" 1) white pants, 2) white collared shirt, 3) FFA jacket and 4) FFA Tie/Scarf.

$\uparrow$ No students shall be on the fairgrounds after 9:00 PM without the written permission from the parents/guardians or supervised Ag. Staff.

$\uparrow$ Student may transport themselves and siblings with written approval from 1) Parents/Guardians, 2) School Administration (Permission in writing from the vice principal and parents/guardians and given to the advisor prior to the event.

$\star$ No student shall leave the fairgrounds at anytime without the supervision of his/her parent/guardian, designated district chaperone or chapter advisor.

$\downarrow$ FFA members are required to obtain their homework from all of their teachers in advance of missing school for attending fairs.

$\downarrow$ Each exhibitor must read and understand the rules and regulations in the fair's premium book.

+ Each exhibitor is required to serve barn duties as assigned and specified by the project advisor.

All students will participate in the moving in and (Loading and setting-up) and removal (clean-up and removal of equipment from the show).

ALL Students shall be responsible for completing the following prior to their premium or market check being issued: 1) A complete \& up-dated record book, 2) thank-you letters to buyers, award donors and add-on bids 3) all bills paid in full and 4) all hours worked at the farm.

Failure to comply with any of the above obligations due to extenuating circumstances must be approved by the advisor. Failure to do so is in violation of the contract and will result in the loss of showing privileges effective immediately.

Student

Parent/Guardian

Advisor

School of Ag Vice Principal

\begin{tabular}{l}
$\overline{\text { Date }}$ \\
$\overline{\text { Date }}$ \\
$\overline{\text { Date }}$ \\
\hline Date
\end{tabular}




\title{
Here is aln exrmple of the Form Policy signed by members or corrbon copy sheet must be obtoined by a project advisor.
}

\author{
Madera South High School Farm Laboratory Policy Regarding Use
}

Project Owner:

Type of Project:

Advisor:

The sole purposes of the Madera High School Farm Laboratory are to provide a laboratory for hands-on instruction in a vocational agriculture class and to allow the students enrolled in vocational agriculture to conduct Supervised Agricultural Experience Projects (SAE).

1. Only a student currently enrolled in a vocational agriculture class or FFA program at Madera South High School shall have the privilege of using the School Farm Lab.

2.The School Farm Lab is part of the campus of Madera South High School; therefore, all Madera South High School and Madera Unified School District policies apply to the School Farm Lab.

3.The responsibility of the project owner (student) includes but is not limited to keeping all gates to the School Farm Lab locked, feeding, grooming, pen clean-up and maintenance, hauling feed/manure and other supplies, planting, irrigation, weeding, harvest, selling, and any care necessary to maintain the health and/or aesthetics of animals at the School Farm Lab.

4.The Madera Unified School District and its employees are NOT responsible for the loss, theft, disappearance, or death of any animal. The students are responsible for all personal equipment or materials of any kind.

5.No person shall drive or operate any vehicle on the School Farm Lab without prior permission or supervision from a Madera South High School vocational agriculture instructor.

6.School Farm Lab hours shall be 7:00 am to 9:00 pm. No one shall conduct any activity on the school Farm Lab during closed hours without prior permission from the advisor. ONLY current FFA members are allowed on the school farm grounds.

7.In the event of an emergency, if services of a veterinarian are utilized the fees of the services are the responsibility of the project owner (student) and/or parent/guardian. This excludes breeding projects owned by the Madera South Ag Department.

8.To assure proper care of livestock animals, animals are expected to be fed and/or checked twice daily. Morning feeding hours must occur between 7-9 am and evening feeding hours shall be from 5-8pm.

9.A student exhibiting a project at the fair must have a signed exhibitors contract to accompany this contract.

10.Any infraction of school or district policy, any infraction of this agreement, or any lack of responsibility on the part of the student may result in the indefinite removal of the project from the School Farm Lab and/or the indefinite revoking of School Farm Lab use privileges of the students.

11.Administration and interpretation of all policies regarding use of the Madera South High School Farm Laboratory shall be the responsibility of the project advisor.

Notification or infraction of any of the aforementioned policies shall inherent the following disciplinary actions:

$1^{\text {st }}$ offense: A written warning

$2^{\text {nd }}$ offense: $\quad$ A written warning, letter sent home and phone call to parents

$3^{\text {rd }}$ offense: $\quad$ Removal of student project from school farm

Upon receipt of second offense, the student will have 1 week to remove his/her animal from the farm. The project must be paid, in full, prior to removal. Projects are not paid for in full will become school property. Projects will then be sold. Proceeds from the sale of the project will be credited to the student's financial obligations.

I have read and understand the policy of the Madera South High School Farm Laboratory and hereby comply in agreement with all aspects of this policy.

Parent/Guardian Signature

Date

Student Signature

Date

Advisor Signature

Date 
J. Copy Of Board Approved Policy Statement Pertaining To FFA As An Integral Part Of The Ag Program 


\section{AGENDA ITEM \\ MADERA UNIFIED SCHOOL DISTRICT}

Date:

October 10, 2013

Subject: $\quad$ Request approval of Madera FFA Program of Activities

Responsible Staff: Dr. Ed Gonzalez, Superintendent

Agenda Placement: Information/Reports

Background/ rationale:

- FFA Program of Activities

- Annually the FFA Program of Activities is presented to MUSD Board of Trustees. The purpose of the Program of Activities (POA) is to inform all stakeholders of the program plan for Madera FFA. The POA is updated annually by the Madera FFA Officer team and submitted to the Regional FFA Supervisor for approval, it also must be presented to the Board of Trustees for review.

- Madera FFA Chapter Officers will make a 5-10 minute presentation

Financial impact:

- "none."

\section{Superintendent's recommendation:}

Supporting documents attached:

- 16 copies of the Madera FFA Program of Activities 

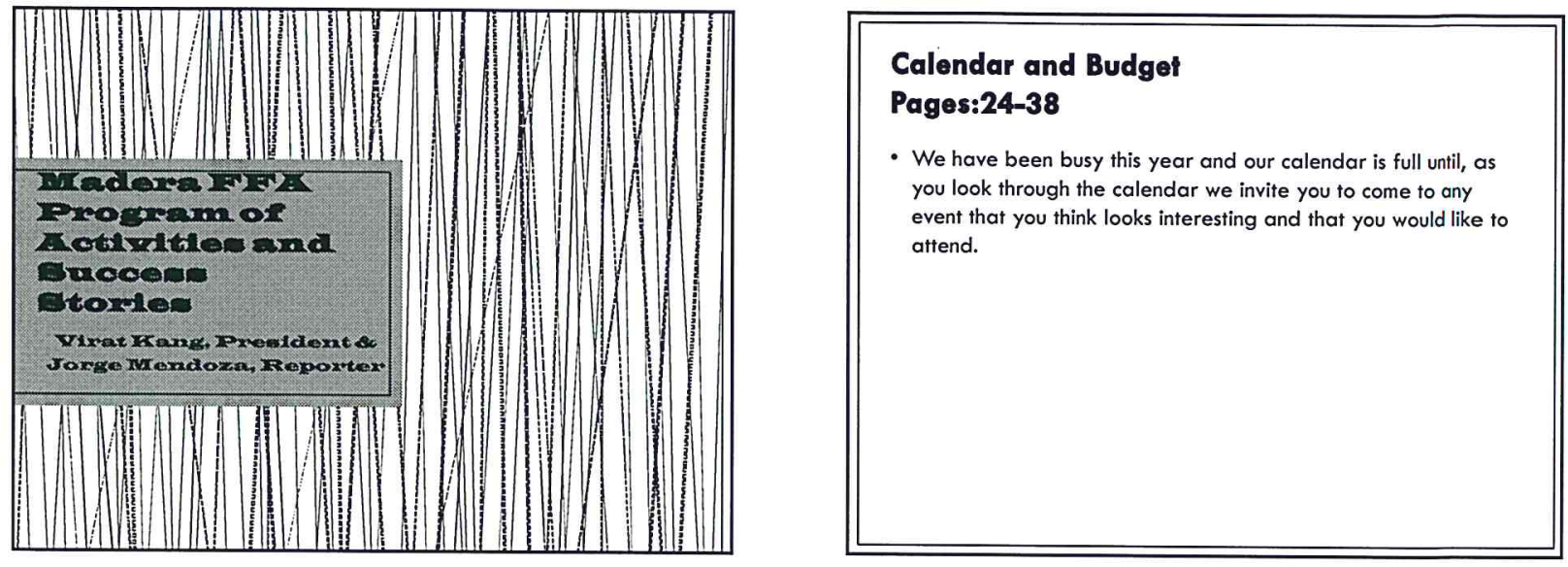

Madera FFA Goals
Page 5
- Madera FFA team goals:
- Advertise meetings by fliers, posters, and slideshows done 2
weeks prior to the meeting.
- Meet at least one new member a month and keep in touch with
them.
- Have presentations for meetings done 1 week before FFA meeting.
- Officers must maintain a GPA of over 3.0 and have at least a B in
their AG class.
- Madera FFA chapter goal: In the upcoming year we will strive to
increase chapter participation by having at least one of our
monthly meetings with $40 \%$ of our membership in attendance.
- Madera FFA theme: Small acts impact beyond belief.

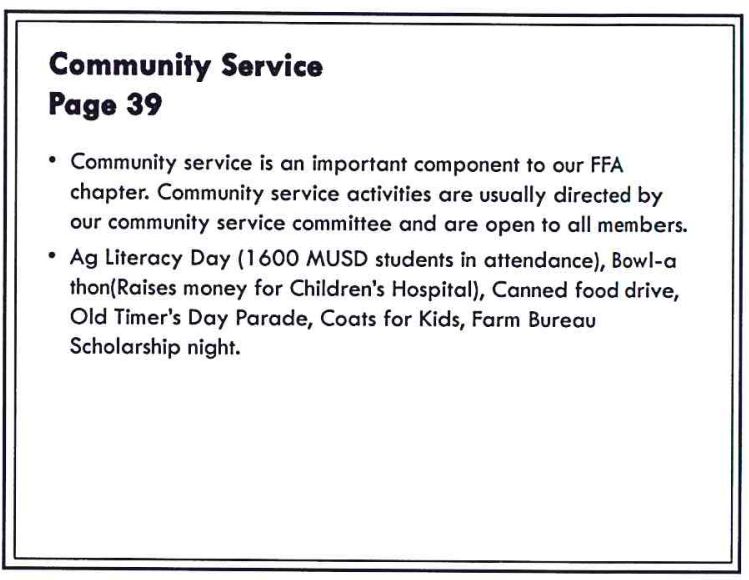

Madera FFA Hisfory
Pages 7-23
- Madera FFA was one of the first 20 Chapters started in 1928.
- This section covers Proficiency Award winners, State Champion
Teams and Degree Recipients.
- Proficiencies are awards received by students who are
proficient in their S.A.E. projects.
- These are available at the sectional, regional, state, and
national levels.

\section{Incentives for Participation Pages 40-43}

- The Madera FFA Chapter encourages active participation among its members.

- To offer incentive for participation, we have developed a point awards and top seller system.

- We give points for meetings, fairs, contests and fundraisers which are updated monthly

- At the end of the year, the top thirty most active members and top salespersons are awarded with an expense paid trip to Six Flags Magic Mountain 


\section{Madera FFA Constitution and Bylaws Pages 44-49}

- Was drafted to establish the principles and rules of the Madera FFA chapter. Rules that we follow.

- Article I. Name and Purposes

- Article II. Organization

- Article III. Membership

- Article IV \& V. Officers and their impeachment.

- Article VI. Executive Committee

- Article VII \& VIII. Dues and Eligibility

- Article IX \&X. Amendments and Ratification of Constitution

- (Official calendar attached pgs. 24-36)

\section{Chapter Officers \\ Pages 57-60}

- Student leaders who are responsible for planning, organizing and running meetings and chapter events.

- Required to have:

- Supervised Agriculture Experience

- Career Development Event

- Public Speaking Event

- Slated by advisors and past senior chapter officers, elected by student vote.

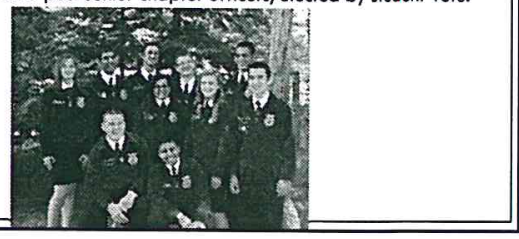

\section{FFA Awards offered to you}

Pages 51,52

- Multiple degrees are available for different levels of students in the organization.

- Awards are offered for various successes at and above the chapter level.

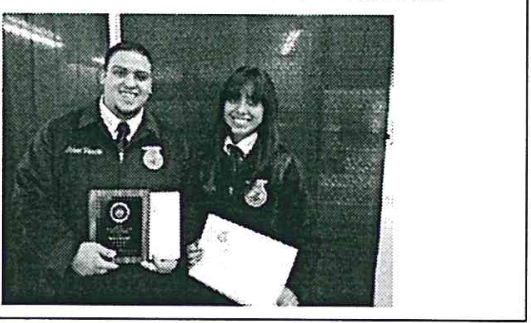

\section{Career Development Events Pages 64-66}

- Career Development Events are used to give FFA members experience in a field of their interest.

- There are approximately 15 field days/CDE competitions per year which the Madera FFA attends

- Some of these locations include UC Davis, Fresno State, Cal Poly SLO and Reedley

- CDEs can focus on public speaking, hands-on/technical skills, Ag business, and plant or animal science.

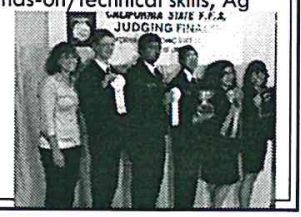

\section{Agriculture Scholarships Page 53}

- Our FFA chapter and its partners and former members offer a variety of scholarships to senior members. Here are just a few

- Matthew Roussel Memorial: $\$ 250$

- California Women for Agriculture: $\$ 250$

- Madera Agriculture Youth Association: $\$ 500$

- CALCOT: $\$ 3000$

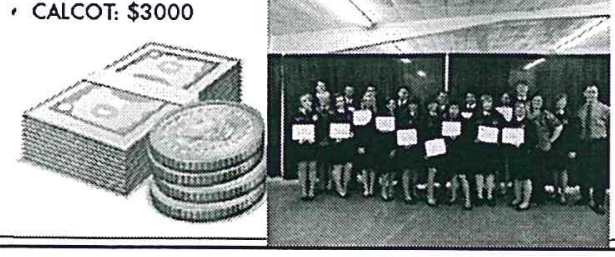

\section{Exhibitor Coniracts} page $67 \& 68$

- Before entering into an S.A.E. project, members must sign a contract amongst themselves, their parents, and their agricultural advisors

- These contracts specify the rules and expectations of the particular S.A.E. project

- They are meant to maintain a sense of professionalism and responsibility for members

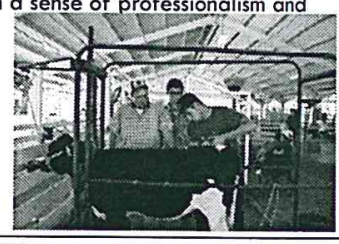



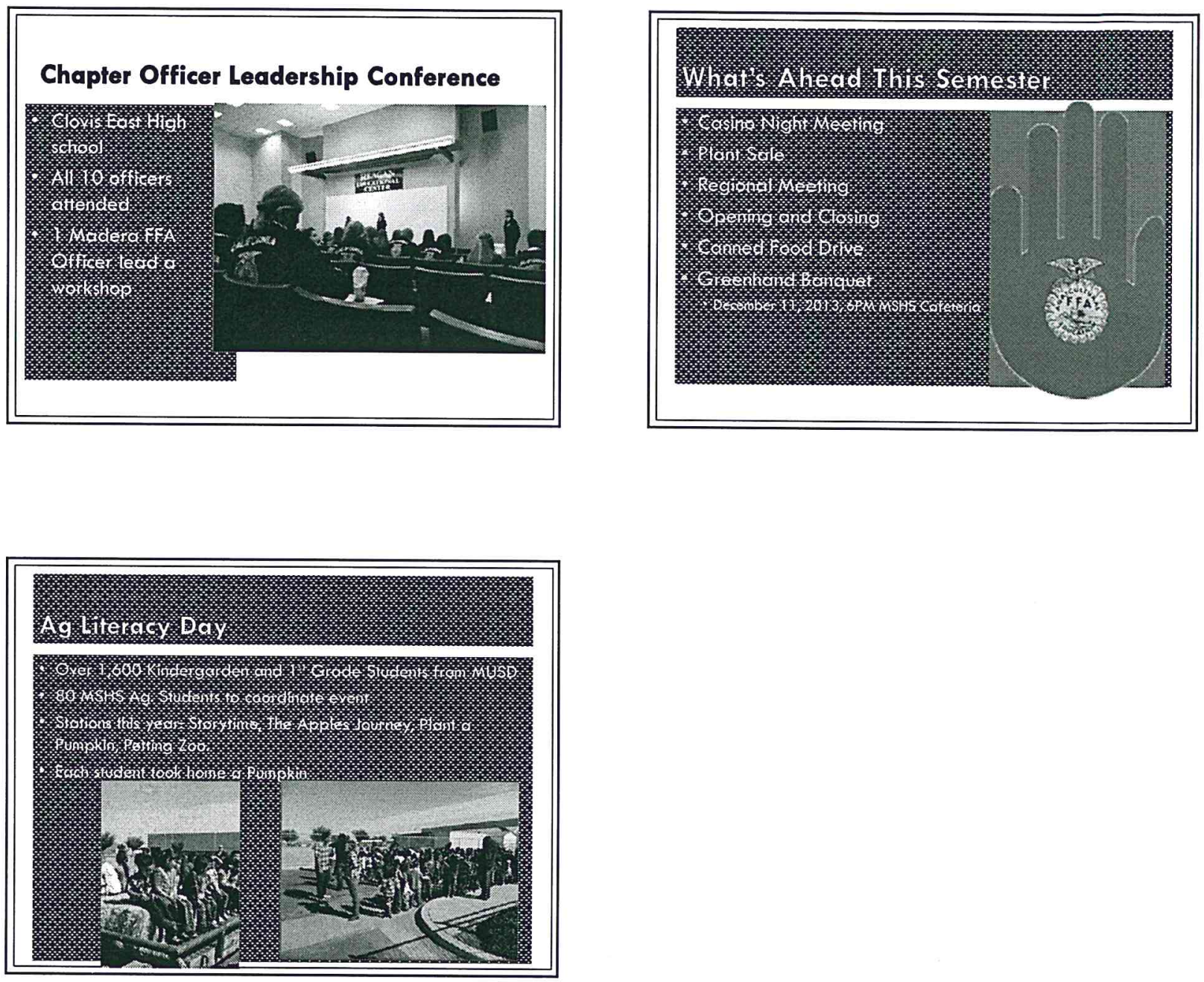

\section{National Convention 2013}

- This year Madera FFA had a very successful National convention.

- Michael Valencia was the National champion in the Ag services Proficiency. 2 years in a row.

- The Best Informed Greenhand team Placed $2^{\text {nd. }}$

- The Meats Evaluation \& Technology team placed $6^{\text {th }}$.

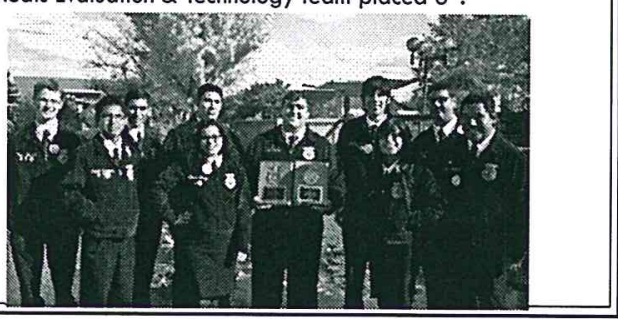


K. Recruitment Program 


\section{Madera FA Recruitment}

Madera FFA goes to our feeder middle schools, on the day they get to register for their classes in the spring each year. Two-three officers, go in uniform, with the advisor to present the attached PowerPoint and talk about the opportunities Madera FFA offers. We have a table set up with the scrapbook and other student projects, for the students, along with a brochure that students can take home with them. There are also 2 country schools that are supposed to go to Madera North, but we go and do the same presentation to them with the hope of them doing a Career School transfer and come to Madera South High School for the Ag. program.

Each year we also have a pumpkin festival where 2,000 Kindergarten and 1st grade students from all schools in Madera Unified, come to the school farm to learn about agriculture. I am always amazed by how many of our incoming Freshmen remember this activity and how much of an impact it made on them. So although it is not intended as a recruitment activity, it does help us in recruitment.
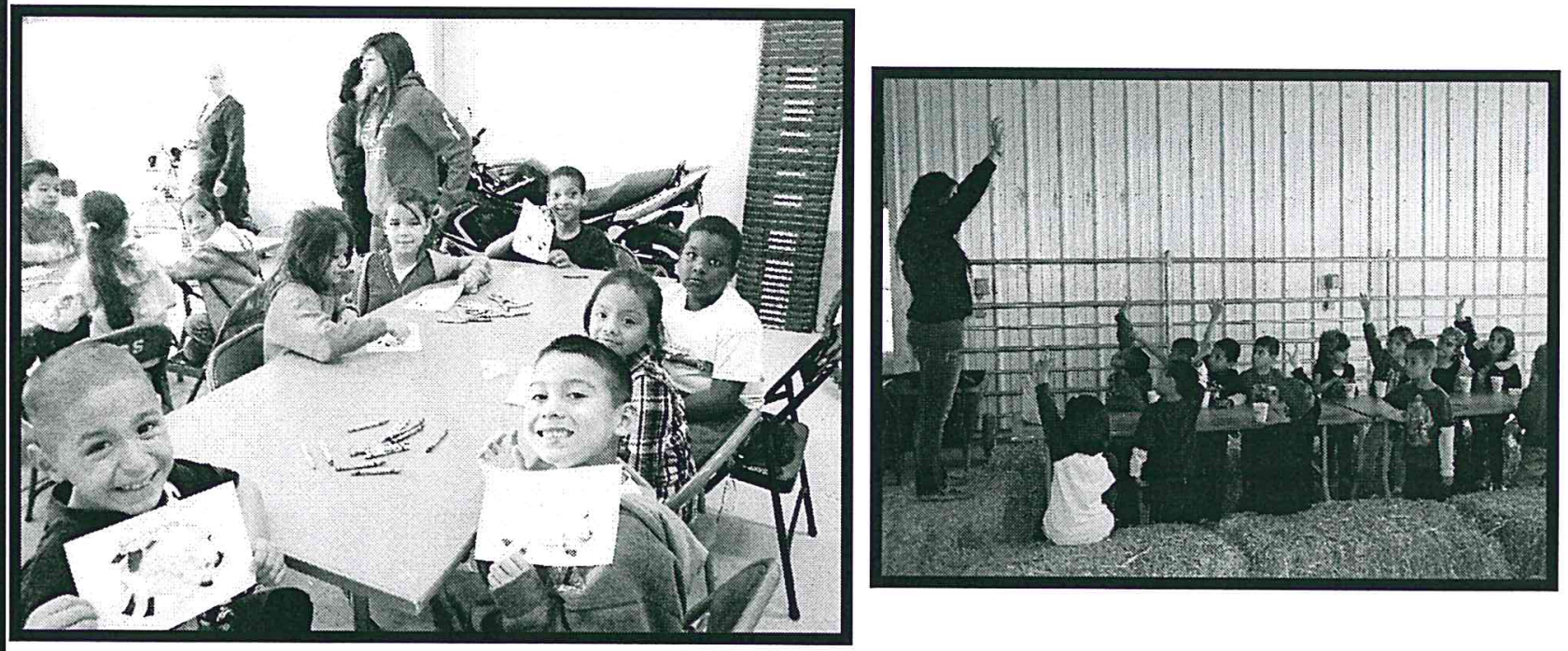


\section{Chapter Scrapbook}




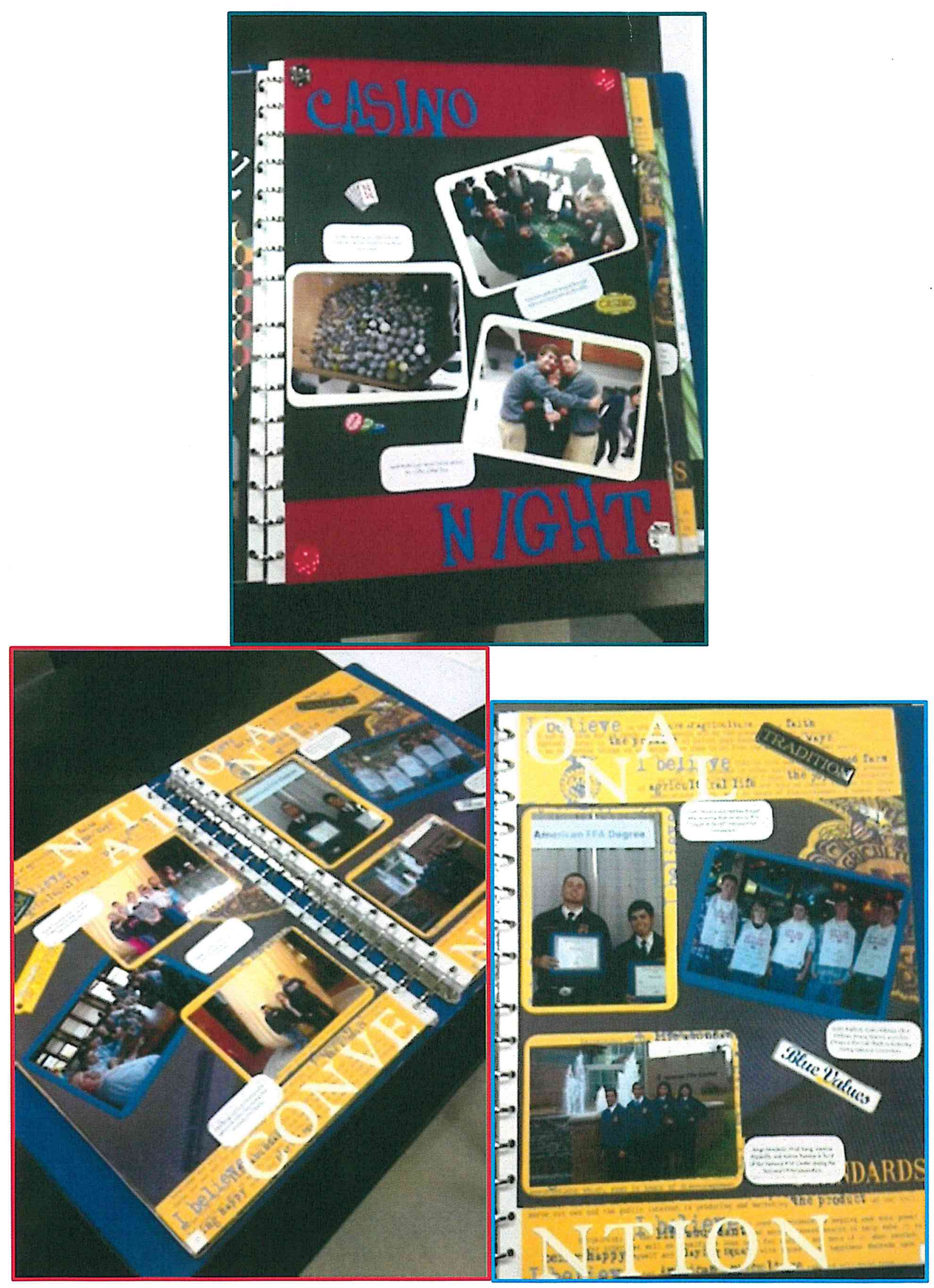


M. Summer Activities Schedule 


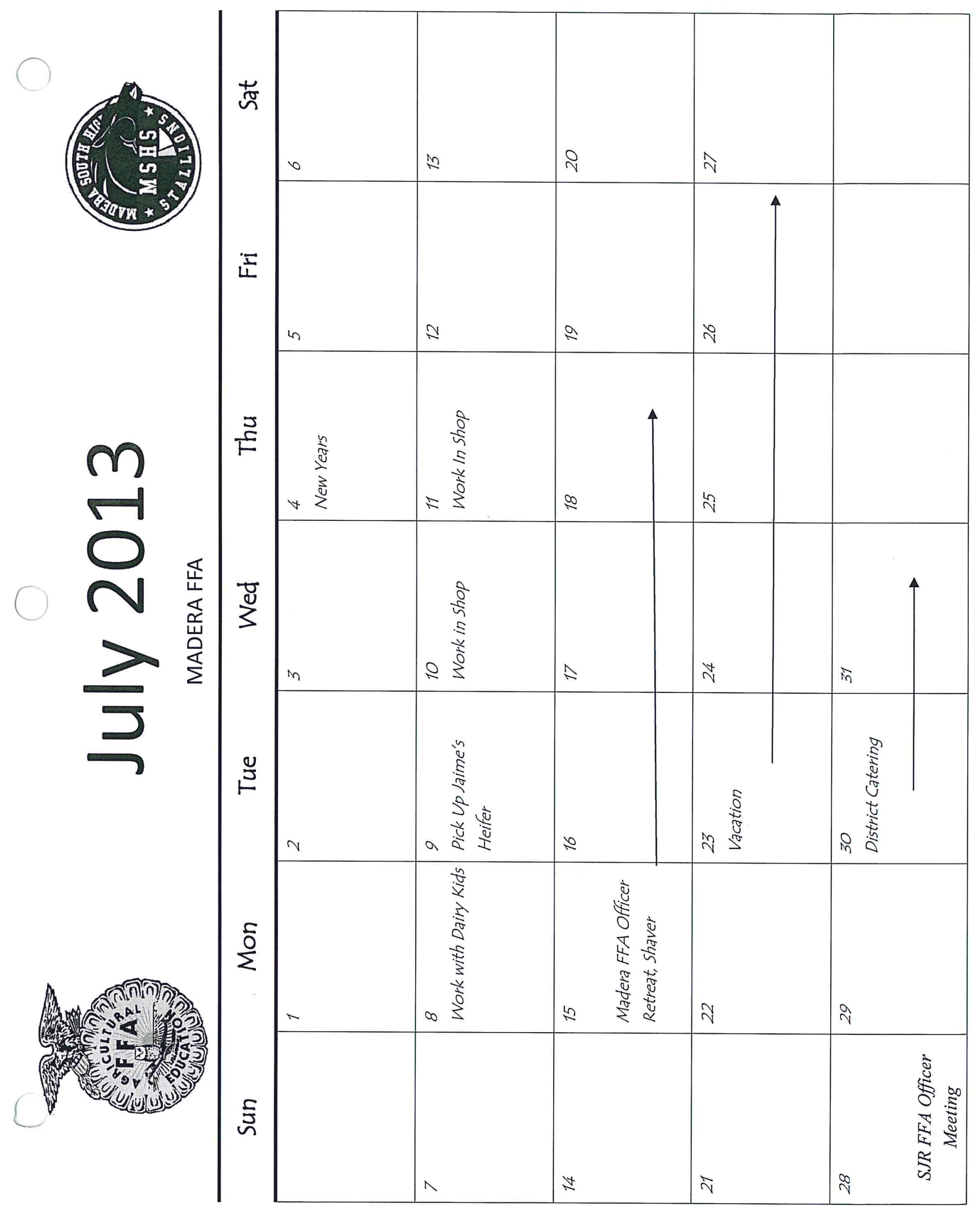




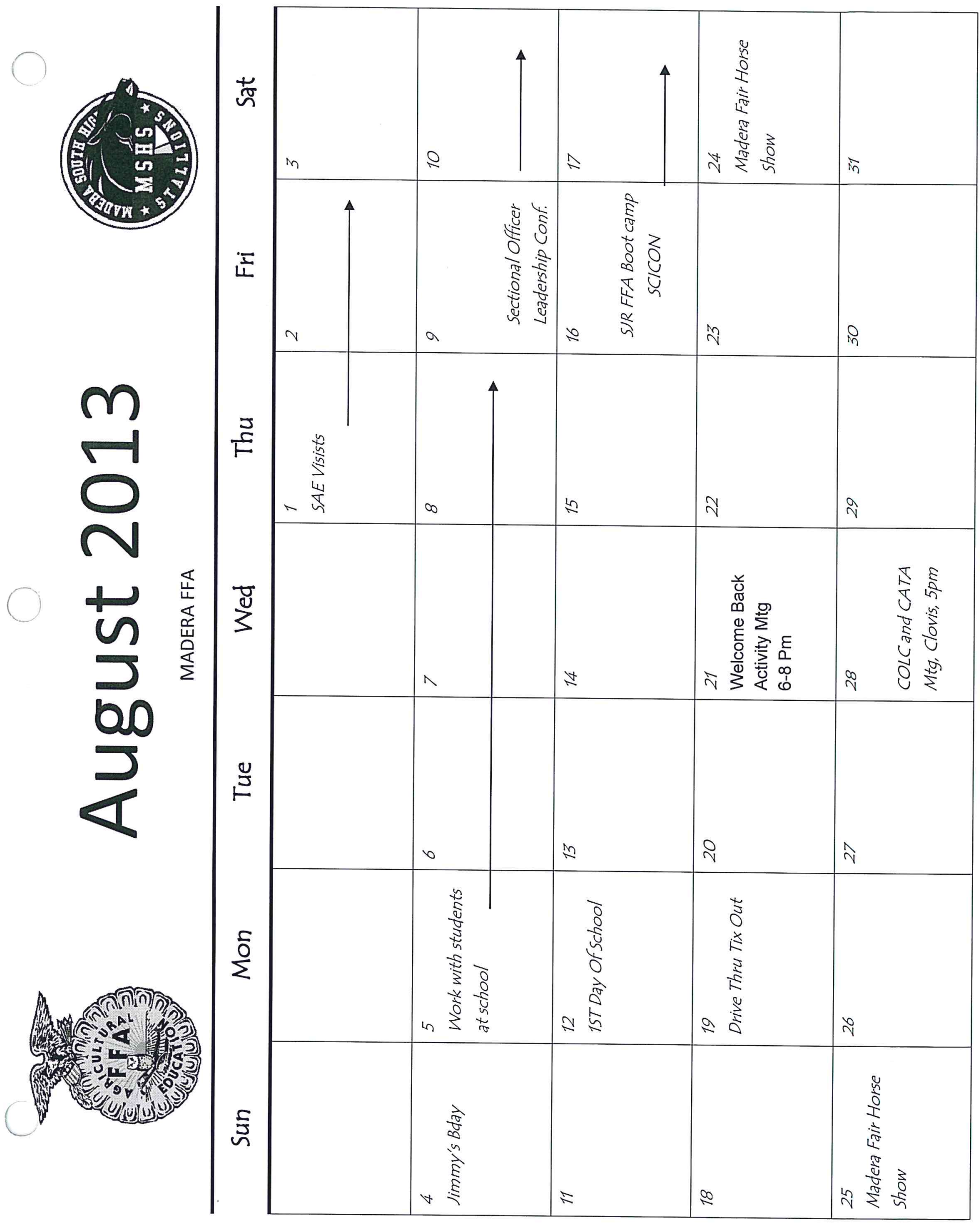


N. Sample Of Vo-Ag Follow -Up Survey 

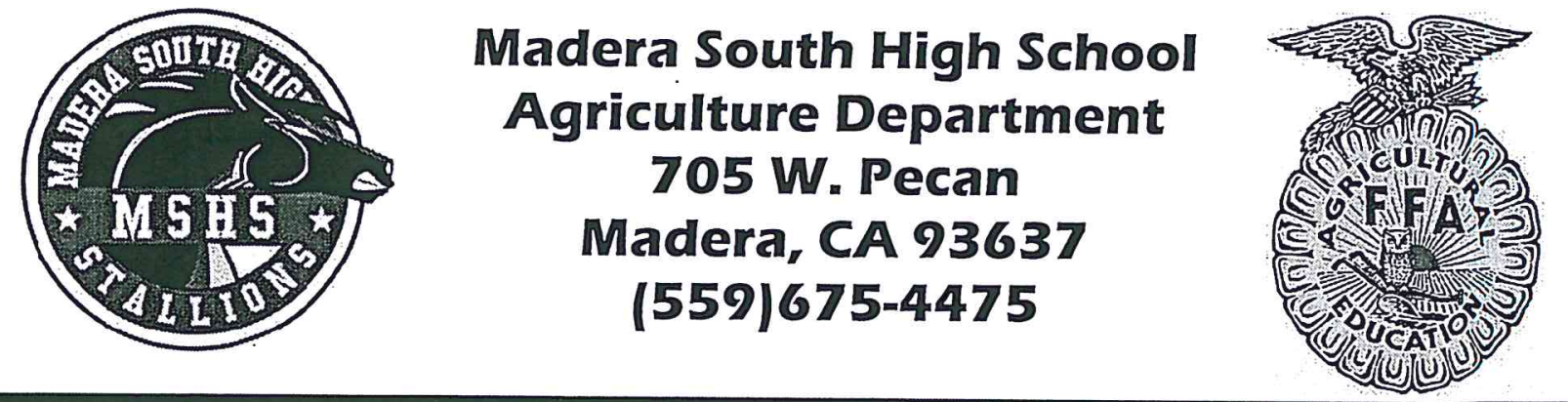

\section{Madera South Agriculture Graduate Follow Up System}

Madera South High School calls all graduates in August and September following graduation to find out they key information for the R-2. We keep their contact information and if we cannot get a hold of the student, we call the parent. The questions asked include:

- Name

- Address

- Phone Number

- What are you doing now?

* Attending School?

$\downarrow$ Full Time or Part Time

४What School

$\downarrow$ Major

*Working?

$\downarrow$ Full Time or Part Time

- What type of business or industry are you involved in?

- Job Title or Description

-What skills from MSHS Ag. are you using in your job?

*Military or Other

- What experiences in Ag. and FFA were most valuable for you?

- Any other comments?!? 


\section{O. Up-To-Date file On Status Of Graduates}




\section{Graduate Follow-up Report}

Filing Year $=2013$

\# CA0141 Madera-Madera South

Madera South HS

705 West Pecan

Madera, CA 93637

Printed: 3/13/2014 10:53:56 AM

\begin{tabular}{|l|l|}
\hline Total Seniors (Year=2012 ): & 135 \\
\hline $\begin{array}{l}\text { Total Seniors having completed } \\
\text { 3 or more years of Ag Instruction: }\end{array}$ & 71 \\
\hline \multicolumn{2}{|l|}{ Program Completer Status } \\
\hline Two Year College Ag Major & 14 \\
\hline Two Year College Non-Ag Major & 23 \\
\hline Four Year College Ag Major & 5 \\
\hline Four Year College Non-Ag Major & 6 \\
\hline Employed - Parttime Ag Job & 4 \\
\hline Employed - Parttime Non-Ag Job & 2 \\
\hline Employed - Fulltime Ag Job & 8 \\
\hline Employed - Fulltime Non-Ag Job & 4 \\
\hline Military & 3 \\
\hline Location or Position Unknown & 2 \\
\hline
\end{tabular}

Site developed and maintained by the Californt: PAA Association. 


\section{Graduate Follow-up}

\# CA0141 Madera - Madera South

Madera South HS

705 West Pecan

Madera, CA 93637

Graduates for Spring: 2013

\begin{tabular}{|c|c|c|}
\hline & First Name & Graduate Status \\
\hline & Marco A & Military- \\
\hline & Jose $\mathrm{G}$ & Two Year College-Non-Ag Majo \\
\hline & Juan D & Employed - Fulltime-Ag Job \\
\hline & Rosita & Four Year College-Non-Ag Major \\
\hline & Dominique $\mathrm{R}$ & Two Year College-Non-Ag Major \\
\hline & Taylor B & Four Year College-Ag Major \\
\hline & Marcos G & Two Year College-Non-Ag Majol \\
\hline & Cristian & Two Year College-Ag Major \\
\hline & Blake & Two Year College-Non-Ag Majo \\
\hline & Brooklynn A & Four Year College-Ag Major \\
\hline & Crystal & Four Year College-Ag Major \\
\hline & Stanley & Two Year College-Ag Major \\
\hline & Jessica & Four Year College-Ag Major \\
\hline & Miguel A & Two Year College-Ag Major \\
\hline & Juan E & Two Year College-Non-Ag Major \\
\hline & Matthew C & Two Year College-Ag Major \\
\hline & Arthur R & Two Year College-Non-Ag Major \\
\hline & Valentin & Two Year College-Ag Major \\
\hline & Jose A & Two Year College-Non-Ag Major \\
\hline & Renee A & Employed - Fulltime-Ag Job \\
\hline & Hailey L & Military- \\
\hline & Amado S & Employed - Parttime-Ag Job \\
\hline & Whitney A & Four Ycar College-Non-Ag Major \\
\hline & Alexander & Two Year College-Non-Ag Major \\
\hline & Cody C & Location or Position Unknown- \\
\hline & Pedro A & Two Ycur College-Ag Major \\
\hline & Dalice J & Two Yinn college-Ag Major \\
\hline & Alexus R & Four Ycur cillege-Ag Major \\
\hline & Marcelina & Two Y ir ( wlege-Non- Ag Major \\
\hline & Luis & Emplad fullume-Non-Ag.Job \\
\hline & Anna $\mathrm{O}$ & Fou \\
\hline & Brandon & Empl ! ! iltime-Non-Ag Job \\
\hline & Matthew T & I:mpi : Iltime-Ag Lob \\
\hline
\end{tabular}




\begin{tabular}{|c|c|c|}
\hline & Maximilion & Four Year College-Non-Ag Major \\
\hline & Rochelle & Two Year College-Non-Ag Major \\
\hline & Spenser M & Two Year College-Non-Ag Major \\
\hline & Mikayla S & Two Year College-Non-Ag Major \\
\hline & JoAlex & Two Year College-Ag Major \\
\hline & Christopher M & Two Year College-Non-Ag Major \\
\hline & Ricardo E & Two Year College-Non-Ag Major \\
\hline & Christopher M & Two Year College-Ag Major \\
\hline & Erik & Two Year College-Non-Ag Major \\
\hline & Ivan & Two Year College-Non-Ag Major \\
\hline & Leonel & Employed - Fulltime-Ag Job \\
\hline & Garon & Two Year College-Ag Major \\
\hline & Esperanza & Employed - Fulltime-Non-Ag Job \\
\hline & Eduardo & Employed - Fulltime-Ag Job \\
\hline & Federico & Employed - Fulltime-Ag Job \\
\hline & Jacqueline & Two Year College-Non-Ag Major \\
\hline & Erika & Two Year College-Non-Ag Major \\
\hline & Jose & Employed - Fulltime-Ag Job \\
\hline & Ana & Two Year College-Non-Ag Major \\
\hline & Timothy & Two Year College-Non-Ag Major \\
\hline & Adrian & Employed - Fulltime-Non-Ag Job \\
\hline & Luis & Employed - Parttime-Ag Job \\
\hline & Enrique & Employed - Parttime-Ag Job \\
\hline & German & Two Year College-Ag Major \\
\hline & Rebekah F & Two Year College-Ag Major \\
\hline & Elmer & Employed - Parttime-Non-Ag Job \\
\hline & Isac $\mathrm{A}$ & Two Year College-Non-Ag Major \\
\hline & Ivette & Employed - Parttime-Non-Ag Job \\
\hline & Ezequiel & Military- \\
\hline & David O & Four Year College-Non-Ag Major \\
\hline & Antoinette V & Two Year College-Non-Ag Major \\
\hline & Luis $\mathrm{F}$ & Two Year College-Ag Major \\
\hline & Martin & Two Year College-Non-Ag Major \\
\hline & Tyra & Two Year College-Ag Major \\
\hline & Sebastian & Employed - Fulltime-Ag Job \\
\hline & Sergio & Employed - Parttime-Ag Job \\
\hline & Adrian & Four Year College-Non-Ag Major \\
\hline & Scott & Location or Position Unknown- \\
\hline
\end{tabular}

Printed ; : 3/2014 10:53:49 AM

Count -

$\therefore$ 


\section{P. Comprehensive Program}



A. Job Market Descriptions
B. Targeted Occupations
C. Total Program Goals and Objectives
D. Program (s) Description of Included Courses, SAE, and Leadership Development

E. Program (s) and/or Course Subject Matter Content Outlines

F. Program Completion Standards

G. Description of Major Facilities and Equipment

H. Five (5) Year Facility and Equipment Acquisition Schedule

I. Staff Assignments

J. FFA Program of Activities

K. School and/or Department Policies Pertaining to:

- $\quad$ Student Eligibility to Participate in Out-of-Class Activities

- Leadership Development Integration into Program

- SOE Integration into Program and other Policies

L. Proficiency Standards for Program Completers

M. Teacher Data Sheets for each Teacher

N. Roster of Agriculture Advisory Committee

O. Advisory Committee Minutes

P. Current Year Budget

Q. Signed Articulation Agreement and/or Evidence of Articulation

R. Graduate Follow-up System

S. List of Active Placement Sites

T. Recruitment Activities and Materials

U. Staff In-service Record

V. Staff Minutes

W. Department Inventory

X. List of Courses that Qualify for Alternative Credit

Y. 


\section{Top Paying Jobs in Madera County}

Occupation Title

Chief Executives

Lawyers

Dentists, General

Pharmacists

Legal Occupations

Engineering Managers

Construction Managers

Medical and Health Services Managers

General and Operations Managers

Industrial Engineers

Managers, All Other

Computer and Information Systems Managers

Computer Software Engineers, Systems Software

Clinical, Counseling, and School Psychologists

Industrial Production Managers

Physician Assistants

Civil Engineers

Financial Managers

Management Occupations

Occupational Therapists

Dental Hygienists

Social and Community Service Managers

Healthcare Practitioners and Technical Workers, All

Other

Registered Nurses

Computer Programmers

Physical Therapists

Market Research Analysts

Diagnostic Medical Sonographers
2009 Median Wage

Hourly

$\begin{array}{ll}\$ 78.00 & \$ 162,234 \\ \$ 74.75 & \$ 155,463 \\ \$ 64.24 & \$ 133,624 \\ \$ 60.80 & \$ 126,464 \\ \$ 60.68 & \$ 126,228\end{array}$

$\$ 56.92$

$\$ 118,406$

$\$ 48.91$

$\$ 101,725$

$\$ 48.14$

$\$ 100,116$

$\$ 47.18$

$\$ 98,120$

$\$ 46.03$

$\$ 95,735$

$\$ 45.71$

$\$ 95,085$

$\$ 44.61$

$\$ 92,773$

$\$ 44.14$

$\$ 91,814$

$\$ 43.27$

$\$ 90,006$

$\$ 42.55$

$\$ 88,510$

$\$ 42.31$

$\$ 87,995$

$\$ 39.88$

$\$ 82,950$

$\$ 38.41$

$\$ 79,905$

$\$ 37.88$

$\$ 78,780$

$\$ 37.72$

$\$ 78,462$

$\$ 37.44$

$\$ 77,894$

$\$ 36.31$

$\$ 75,511$

$\$ 35.85$

$\$ 74,578$

$\$ 35.81$

$\$ 74,495$

$\$ 35.72$

$\$ 74,306$

$\$ 35.31$

$\$ 73,449$

$\$ 34.75$

$\$ 72,294$

$\$ 34.35$ 
Management Analysts

$\$ 33.86$

$\$ 70,422$

Protective Service Occupations

$\$ 33.24$

$\$ 69,139$

Sales Representatives, Services, All Other

$\$ 33.18$

$\$ 69,002$

Healthcare Practitioners and Technical Occupations

Teachers and Instructors, All Other

Paralegals and Legal Assistants

Medical and Public Health Social Workers

$\$ 32.90$

$\$ 68,440$

See Footnote

$\$ 67,949$

$\$ 32.44$

$\$ 67,486$

$\$ 32.36$

$\$ 67,317$

Speech-Language Pathologists

$\$ 32.26$

$\$ 67,097$

First-Line Supervisors/Managers of Non-Retail Sales

$\$ 32.13$

$\$ 66,843$

Workers

Sales Managers

$\$ 31.75$

$\$ 66,044$

Librarians

$\$ 31.70$

$\$ 65,927$

Transportation, Storage, and Distribution Managers

$\$ 31.52$

$\$ 65,562$

Network and Computer Systems Administrators

Postmasters and Mail Superintendents

$\$ 30.78$

$\$ 64,028$

Network Systems and Data Communications Analysts

$\$ 30.71$

$\$ 63,881$

Environmental Scientists and Specialists, Including

Health

$\$ 30.32$

$\$ 63,060$

$\$ 30.29$

$\$ 63,005$

Dietitians and Nutritionists

$\$ 30.16$

$\$ 62,726$

$\underline{\text { Radiologic Technologists and Technicians }}$

$\$ 29.83$

$\$ 62,052$

Accountants and Auditors

$\$ 29.56$

$\$ 61,492$

Administrative Services Managers

$\$ 29.11$

$\$ 60,555$

Architecture and Engineering Occupations

$\$ 29.09$

$\$ 60,497$

Rehabilitation Counselors

$\$ 28.99$

$\$ 60,281$

Computer Systems Analysts

$\$ 28.88$

$\$ 60,073$

Farm, Ranch, and Other Agricultural Managers

$\$ 28.88$

$\$ 60,071$

Sales Representatives, Wholesale and

Manufacturing, Except Technical and Scientific

$\$ 28.82$

$\$ 59,954$

Products

Computer and Mathematical Occupations

$\$ 28.77$

$\$ 59,852$

Employment, Recruitment, and Placement Specialists

$\$ 28.36$

$\$ 58,992$

$\$ 28.18$

$\$ 58,607$

First-Line Supervisors/Managers of Construction 
Trades and Extraction Workers

Legal Secretaries

Police and Sheriff Patrol Officers

Postal Service Mail Sorters, Processors, and

Processing Machine Operators

Urban and Regional Planners

First-Line Supervisors/Managers of Helpers, Laborers, and Material Movers, Hand

Human Resources, Training, and Labor Relations

Specialists, All Other

Postal Service Clerks

Elementary School Teachers, Except Special

Education

Cost Estimators

Water and Liquid Waste Treatment Plant and System Operators

Purchasing Agents, Except Wholesale, Retail, and

Farm Products

First-Line Supervisors/Managers of Mechanics,

Installers, and Repairers

Business and Financial Operations Occupations

Electricians

Structural Iron and Steel Workers

Architectural and Civil Drafters

Clergy

Loan Officers

Business Operations Specialists, All Other

Life, Physical, and Social Science Occupations

First-Line Supervisors/Managers of Transportation and Material-Moving Machine and Vehicle Operators

Postal Service Mail Carriers

Compensation, Benefits, and Job Analysis Specialists

Emergency Medical Technicians and Paramedics
$\$ 28.14$

$\$ 58,544$

$\$ 28.05$

$\$ 58,338$

$\$ 27.62$

$\$ 57,459$

$\$ 27.38$

$\$ 56,940$

$\$ 27.31$

$\$ 56,790$

$\$ 27.03$

$\$ 56,218$

$\$ 26.80$

$\$ 55,749$

See Footnote

$\$ 54,819$

$\$ 26.32$

$\$ 54,746$

$\$ 26.04$

$\$ 54,170$

$\$ 25.84$

$\$ 53,766$

$\$ 25.84$

$\$ 53,763$

$\$ 25.70$

$\$ 53,456$

$\$ 25.67$

$\$ 53,395$

$\$ 25.57$

$\$ 53,182$

$\$ 25.51$

$\$ 25.36$

$\$ 25.25$

$\$ 24.87$

$\$ 53,061$

$\$ 52,740$

$\$ 52,506$

$\$ 51,730$

$\$ 24.81$

$\$ 51,591$

$\$ 24.69$

$\$ 51,360$

$\$ 24.67$

$\$ 51,312$

$\$ 23.91$

$\$ 49,738$

$\$ 23.67$

$\$ 49,232$

$\$ 23.64$

$\$ 49,172$ 
First-Line Supervisors/Managers of Production and

Operating Workers

First-Line Supervisors/Managers of Office and

$\$ 23.21$

$\$ 48,277$

Administrative Support Workers

Roofers

$\$ 23.14$

$\$ 48,125$

Operating Engineers and Other Construction

$\$ 22.99$

$\$ 47,815$

Equipment Operators

Stonemasons

Heating, Air Conditioning, and Refrigeration

Mechanics and Installers

Education, Training, and Library Occupations

Compliance Officers, Except Agriculture.

Construction, Health and Safety, and Transportation

Tile and Marble Setters

$\$ 22.95$

$\$ 47,732$

$\$ 22.94$

$\$ 47,714$

$\$ 22.68$

$\$ 47,176$

$\$ 22.47$

$\$ 46,751$

$\$ 22.26$

$\$ 46,300$

Computer Support Specialists

$\$ 22.23$

$\$ 46,239$

Production. Planning, and Expediting Clerks

First-Line Supervisors/Managers of Landscaping.

$\$ 22.16$

$\$ 46,085$

Lawn Service, and Groundskeeping Workers

Maintenance Workers, Machinery

$\$ 22.08$

$\$ 45,922$

Education Administrators, Preschool and Child Care

Center/Program

$\$ 21.98$

$\$ 45,712$

$\$ 21.69$

$\$ 45,110$

Lodging Managers

$\$ 21.65$

$\$ 45,028$

Police, Fire, and Ambulance Dispatchers

$\$ 21.62$

$\$ 44,972$

Licensed Practical and Licensed Vocational Nurses

$\$ 21.61$

$\$ 44,933$

Community and Social Services Occupations

$\$ 21.59$

$\$ 44,896$

Construction and Building Inspectors

$\$ 21.52$

$\$ 44,768$

(1) For some occupations, workers may not work full-time all year-round. For these occupations it is not feasible to calculate an hourly wage.

Search by Topic

Search by Keyword 


\section{Top 100 Fastest Growing Occupations in Madera County, 2006-2016}

Occupation Title

Control and Valve Installers and Repairers, Except

Mechanical Door

Personal and Home Care Aides

Amusement and Recreation Attendants

Hotel, Motel, and Resort Desk Clerks

Network Systems and Data Communications Analysts

Pharmacy Technicians

Correctional Officers and Jailers

First-Line Supervisors/Managers of Landscaping, Lawn

Service, and Groundskeeping Workers

Health Educators

Retail Salespersons

First-Line Supervisors/Managers of Correctional

Officers

Medical Assistants

Home Health Aides

Medical Records and Health Information Technicians

First-Line Supervisors/Managers of Police and

Detectives

Gaming and Sports Book Writers and Runners

Bill and Account Collectors

Customer Service Representatives

Network and Computer Systems Administrators

Vocational Education Teachers, Postsecondary

Vocational Education Teachers, Secondary School

Environmental Scientists and Specialists, Including

Health

Cost Estimators

Appraisers and Assessors of Real Estate

Automotive Body and Related Repairers
Employment

2006

2016 Change

Percent

$\begin{array}{rrr}30 & 50 & 66.7 \% \\ 590 & 890 & 50.8 \% \\ 80 & 120 & 50.0 \% \\ 80 & 120 & 50.0 \% \\ 40 & 60 & 50.0 \% \\ & & \\ 170 & 240 & 41.2 \% \\ 880 & 1,240 & 40.9 \% \\ 50 & 70 & 40.0 \% \\ 50 & 70 & 40.0 \% \\ 650 & 900 & 38.5 \%\end{array}$

$\begin{array}{rrr}190 & 260 & 36.8 \% \\ 280 & 380 & 35.7 \% \\ 170 & 230 & 35.3 \% \\ 30 & 40 & 33.3 \% \\ & & \\ 30 & 40 & 33.3 \% \\ & & \\ 30 & 40 & 33.3 \% \\ 90 & 120 & 33.3 \% \\ 270 & 360 & 33.3 \% \\ 30 & 40 & 33.3 \% \\ 30 & 40 & 33.3 \%\end{array}$

30

40

$33.3 \%$

60

80

$33.3 \%$

60

80

$33.3 \%$

30

40

$33.3 \%$

30

$33.3 \%$ 
Slaughterers and Meat Packers

Paving, Surfacing, and Tamping Equipment Operators

Helpers--Carpenters

Construction and Building Inspectors

Taxi Drivers and Chauffeurs

Service Station Attendants

Maids and Housekeeping Cleaners

Gaming Dealers

Special Education Teachers, Preschool, Kindergarten, and Elementary School

$\underline{\text { Pharmacists }}$

Landscaping and Groundskeeping Workers

First-Line Supervisors/Managers of Housekeeping and Janitorial Workers

Kindergarten Teachers, Except Special Education

Computer Support Specialists

Management Analysts

Tire Repairers and Changers

First-Line Supervisors/Managers of Retail Sales

Workers

Receptionists and Information Clerks

First-Line Supervisors/Managers of Personal Service

Workers

Medical Transcriptionists

Emergency Medical Technicians and Paramedics

Detectives and Criminal Investigators

Dental Assistants

Computer Software Engineers, Applications

Industrial Production Managers

Lodging Managers

Probation Officers and Correctional Treatment

Specialists

$\begin{array}{lll}60 & 80 & 33.3 \% \\ 30 & 40 & 33.3 \% \\ 30 & 40 & 33.3 \% \\ 30 & 40 & 33.3 \% \\ 30 & 40 & 33.3 \%\end{array}$

$60 \quad 80 \quad 33.3 \%$

$310 \quad 410 \quad 32.3 \%$

$130 \quad 170 \quad 30.8 \%$

$100 \quad 130 \quad 30.0 \%$

$100 \quad 130 \quad 30.0 \%$

$370 \quad 480 \quad 29.7 \%$

$70 \quad 90 \quad 28.6 \%$

$140 \quad 180 \quad 28.6 \%$

$70 \quad 90 \quad 28.6 \%$

$70 \quad 90 \quad 28.6 \%$

$70 \quad 90 \quad 28.6 \%$

$340 \quad 430 \quad 26.5 \%$

$190 \quad 240 \quad 26.3 \%$

$40 \quad 50 \quad 25.0 \%$

$40 \quad 50 \quad 25.0 \%$

$80 \quad 100 \quad 25.0 \%$

$40 \quad 50 \quad 25.0 \%$

$120 \quad 150 \quad 25.0 \%$

$80 \quad 100 \quad 25.0 \%$

$40 \quad 50 \quad 25.0 \%$

$40 \quad 50 \quad 25.0 \%$

$160 \quad 200 \quad 25.0 \%$ 
Coaches and Scouts

Food Batchmakers

Industrial Machinery Mechanics

Painters, Transportation Equipment

Elementary School Teachers, Except Special Education

Automotive Service Technicians and Mechanics

Police and Sheriff Patrol Officers

Physicians and Surgeons, All Other

First-Line Supervisors/Managers of Mechanics,

Installers, and Repairers

Human Resources Assistants, Except Payroll and

Timekeeping

First-Line Supervisors/Managers of Production and

Operating Workers

First-Line Supervisors/Managers of Transportation and

Material-Moving Machine and Vehicle Operators

Bakers

Extruding, Forming, Pressing, and Compacting Machine Setters, Operators, and Tenders

Dental Hygienists

Interpreters and Translators

Middle School Teachers, Except Special and Vocational Education

Mental Health and Substance Abuse Social Workers

Social and Community Service Managers

Pharmacy Aides

Slot Key Persons

Gaming Service Workers, All Other

Bartenders

Security Guards

Maintenance and Repair Workers, General

Packaging and Filling Machine Operators and Tenders

Lawyers

Farm, Ranch, and Other Agricultural Managers

$\begin{array}{rrr}40 & 50 & 25.0 \% \\ 40 & 50 & 25.0 \% \\ 40 & 50 & 25.0 \% \\ & & \\ 40 & 50 & 25.0 \% \\ 1,030 & 1,280 & 24.3 \% \\ 170 & 210 & 23.5 \% \\ 130 & 160 & 23.1 \% \\ 90 & 110 & 22.2 \%\end{array}$

$\begin{array}{rrr}90 & 110 & 22.2 \% \\ 50 & 60 & 20.0 \% \\ 250 & 300 & 20.0 \% \\ & & \\ 50 & 60 & 20.0 \% \\ 50 & 60 & 20.0 \%\end{array}$

$\begin{array}{rrr}50 & 60 & 20.0 \% \\ 50 & 60 & 20.0 \% \\ 50 & 60 & 20.0 \% \\ & & \\ 150 & 180 & 20.0 \% \\ 50 & 60 & 20.0 \%\end{array}$

$\begin{array}{rrr}50 & 60 & 20.0 \% \\ 50 & 60 & 20.0 \% \\ 150 & 180 & 20.0 \% \\ 50 & 60 & 20.0 \% \\ 100 & 120 & 20.0 \%\end{array}$

$310 \quad 370 \quad 19.4 \%$

$360 \quad 430 \quad 19.4 \%$

$160 \quad 190 \quad 18.8 \%$

$110 \quad 130 \quad 18.2 \%$

$840 \quad 990 \quad 17.9 \%$ 
Education Administrators, Elementary and Secondary School

Child, Family, and School Social Workers

Merchandise Displayers and Window Trimmers

Dentists, General

Social and Human Service Assistants

First-Line Supervisors/Managers of Food Preparation and Serving Workers

Gaming Cage Workers

Recreation Workers

Counter and Rental Clerks

First-Line Supervisors/Managers of Helpers, Laborers, and Material Movers, Hand

Bus Drivers, School

Helpers--Installation, Maintenance, and Repair Workers

Food Preparation Workers

Child Care Workers

Registered Nurses

Gaming Change Persons and Booth Cashiers

Executive Secretaries and Administrative Assistants

Combined Food Preparation and Serving Workers, Including Fast Food

Secondary School Teachers, Except Special and

Vocational Education

Welders, Cutters, Solderers, and Brazers

$\begin{array}{rrr}240 & 280 & 16.7 \% \\ 60 & 70 & 16.7 \% \\ 60 & 70 & 16.7 \% \\ 60 & 70 & 16.7 \% \\ 120 & 140 & 16.7 \%\end{array}$

$180 \quad 210 \quad 16.7 \%$

$\begin{array}{lll}60 & 70 \quad 16.7 \%\end{array}$

$\begin{array}{lll}60 & 70 \quad 16.7 \%\end{array}$

$180 \quad 210 \quad 16.7 \%$

$60 \quad 70 \quad 16.7 \%$

$180 \quad 210 \quad 16.7 \%$

$\begin{array}{lll}60 & 70 \quad 16.7 \%\end{array}$

$250 \quad 290 \quad 16.0 \%$

$450 \quad 520 \quad 15.6 \%$

$1,280 \quad 1,480 \quad 15.6 \%$

$130 \quad 150 \quad 15.4 \%$

$390 \quad 450 \quad 15.4 \%$

$530 \quad 610 \quad 15.1 \%$

$600 \quad 690 \quad 15.0 \%$

$200 \quad 230 \quad 15.0 \%$

Search by Topic

Search by Keyword 


\section{$\underline{A} \underline{B} \underline{C} \underline{D} \underline{E} \underline{F} \underline{G} \underline{H} \underline{I} \underline{J} \underline{K} \underline{L} \underline{M} \underline{N} \underline{0} \underline{P} \underline{Q} \underline{R} \underline{S} \underline{T} \underline{U} \underline{V} \underline{W} \underline{X} \underline{Y} \underline{Z}$ \\ Occupations with the title starting with $A$ \\ Occupation

\author{
Occupation
}

Abdominal Sonographers

Account Executives

Account Managers

Account Representatives

Account Services Representatives

Accountants and Auditors

Accounting Assistants

Accounting Associates

Accounting Clerks

Accounting Managers

Accounting Representatives

Accounting Technicians, and Audit Clerks.

Accounts Payables Clerks

Accounts Receivable Clerks

Acoustical Carpenters

Acoustical Installers

Activities of Daily Living Specialists

Activity Coordinators

Activity Directors

Actors

Actuaries

Addictions Counselor Assistants

Addictions Nurses

Administrative Aides

Administrative Assistants

Administrative Associates

$\underline{\text { Administrative Clerks }}$

Administrative Coordinators

Administrative Law Judges and Hearing
Amusement and Recreation Attendants

Anesthesiologists

Animal Breeders

Animal Control Workers

Animal Trainers

Announcers

Anthropologists and Archeologists

Anthropology and Archeology Teachers, Postsecondary

Anthropology/Archeology Teachers. Postsecondary

Apartment Managers

Application Developers

Application Integration Engineers

Applications Developers

Applications Programmers and Systems Programmers.

Appointment Clerks

Appraisers

Appraisers and Assessors of Real Estate

Arbitrators, Mediators, and Conciliators

Arc Cutters

Arc Welders

Architects, Except Landscape and Naval

Architectural Coatings Finishers

Architectural Inspectors

Architectural and Civil Drafters

Architecture Teachers, Postsecondary

Archivists, Curators, and Museum

Technicians

Area and Ethnic Studies Teachers, Postsecondary

Area, Ethnic, and Cultural Studies Teachers. Postsecondary

Armored Car Guards, and Store Detectives. 
$\underline{\text { Officers }}$

Administrative Law Judges, Adjudicators, and Hearing Officers

Administrative Secretaries

Administrative Services Assistants.

$\underline{\text { Administrative Services Managers }}$

Administrative Support Assistants

Administrative Technicians

Adobe Layers

Adoption Specialists

Adult Literacy and GED Teachers

Adult Literacy, Remedial Education, and GED Teachers and Instructors

Advertising Layout Workers

Advertising Sales Agents

Advertising and Promotions Managers

Advocates

Aerospace Engineering and Operations

Technicians

Aerospace Engineers

Agents and Business Managers of Artists, Performers, and Athletes

Agricultural Engineers

Agricultural Equipment Operators

Agricultural Inspectors

Agricultural Microbiologists

Agricultural Sciences Teachers.

Postsecondary

Agricultural and Food Science Technicians

Agricultural and Food Scientists

Air Traffic Control Specialists

Air Traffic Controllers

Air Traffic Controllers (Enroute Option)

Air Traffic Controllers (Tower Option)

Aircraft Assemblers

$\underline{\text { Art Directors }}$

Art Teachers

Art, Drama, and Music Teachers, Postsecondary

Artists. Performers, and Athletes

Agents/Managers

Assembly Line Workers

Assembly Operators

Assembly Technicians

Assistant Designers

Assisted Living and Senior Housing Managers

Associate Attorneys

Associates

Assurance Managers

Assurance Seniors

Astronomers

Astronomers and Physicists

Athletes and Sports Competitors

Athletic Trainers

Atmospheric and Space Scientists

Atmospheric, Earth, Marine, and Space

Sciences Teachers, Postsecondary

Atmospheric/Space Sciences Teachers, Postsecondary

Attorney Assistants.

Attorneys

Attorneys at Law

Audio and Video Equipment Technicians

Audio-Visual Collections Specialists

Audiologists

Auditors-in-Charge

Auditors.

Auto Brake Mechanics 
Aircraft Cargo Handling Supervisors Auto Technicians

Aircraft Mechanics and Service Technicians Auto Transmission Specialists.

Aircraft Structure, Surfaces, Rigging, and

Systems Assemblers

Airfield Operations Specialists

Automotive Air-Conditioning Repairers

Airline Pilots, Copilots, and Flight Engineers

Airport Tower or Terminal Controllers

Ambulance Drivers and Attendants

Ambulance Drivers and Attendants, Except

Emergency Medical Technicians

Automotive Body and Related Repairers

Automotive Glass Installers and Repairers

Automotive Service Technicians and

Mechanics

Avionics Technicians

Real Estate Appraisers and Assessors 


\section{Standard Occupational Classification (SOC) Code Groups}

Click on a job family below to see detailed occupations:

11-0000 Management Occupations

13-0000 Business and Financial Operations Occupations

15-0000 Computer and Mathematical Occupations

17-0000 Architecture and Engineering Occupations

19-0000 Life, Physical, and Social Science Occupations

21-0000 Community and Social Services Occupations

$\underline{23-0000}$ Legal Occupations

25-0000 Education, Training, and Library Occupations

27-0000 Arts, Design, Entertainment, Sports, and Media

29-0000 Healthcare Practitioners and Technical Occupations

31-0000 Healthcare Support Occupations

33-0000 Protective Service Occupations

35-0000 Food Preparation and Serving Related Occupations

$\underline{37-0000}$ Building, Grounds, and Maintenance Occupations

39-0000 Personal Care and Service Occupations

41-0000 Sales and Related Occupations

43-0000 Office and Administrative Support Occupations

45-0000 Farming, Fishing, and Forestry Occupations

47-0000 Construction and Extraction Occupations

49-0000 Installation, Maintenance, and Repair Occupations

51-0000 Production Occupations

53-0000 Transportation and Material-Moving Occupations

55-0000 Military Specific Occupations 


\section{$\underline{A} \underline{B} \underline{\mathbf{C}} \underline{\mathrm{E}} \underline{\mathrm{E}} \underline{\mathrm{G}} \underline{\mathrm{G}} \underline{\mathrm{H}} \underline{\mathrm{J}} \underline{\mathrm{K}} \underline{\mathrm{L}} \underline{\mathrm{M}} \underline{\mathrm{N}} \underline{\mathrm{O}} \underline{\mathrm{P}} \underline{\mathrm{Q}} \underline{\mathrm{R}} \underline{\mathrm{S}} \underline{\mathrm{T}} \underline{\mathrm{U}} \underline{\mathrm{V}} \underline{\mathrm{W}} \underline{\mathrm{X}} \underline{\mathrm{Y}} \underline{\mathrm{Z}}$ \\ Occupations with the title starting with $B$ \\ Occupation \\ Occupation}

Backhoe Operators

Bacteriologists

Baggage Porters and Bellhops

Baggers

Bailiffs

Bakers

Bakery Delivery Persons

Bank Tellers

Banquet Cooks, and Dinner Cooks.

Banquet Directors

Barbers

Bartenders

Beauticians

Beauty Operators, and Stylists.

Bench Stamping Die Makers

Bench Tool Makers

Bicycle Repairers

Bill and Account Collectors

Billing and Posting Clerks and Machine

Operators

Billing and Posting Workers

Bindery Workers

Biochemists and Biophysicists

Biological Science Teachers, Postsecondary

Biological Scientists

Biological Scientists, All Other

Biological Technicians

Biology Teachers

Biomedical Engineers

Blocklayers
Bodyguards

Boilermakers

Bookbinders

Bookkeepers

Bookkeeping, Accounting, and Auditing

Clerks

Bouncers

Boxers

Brake Repairers

Bread Distributors

Breast Sonographers

Bricklayers

Brickmasons and Blockmasons

Bridge Painters

Bridge and Lock Tenders

Broadcast Technicians

Broiler Cooks

Brokerage Clerks

Budget Analysts

Building Maintenance Repairer

Bus Drivers, School

Bus Drivers, Transit and Intercity

Bus and Truck Mechanics

Bus and Truck Mechanics and Diesel Engine

Specialists

Business Analysts

Business Consultants

Business Management Analysts

Business Systems Analysts

Business Teachers, Postsecondary

Butchers and Meat Cutters 


\section{$\underline{A} \underline{B} \underline{C} \underline{D} \underline{E} \underline{F} \underline{G} \underline{H} \underline{I} \underline{\mathrm{K}} \underline{\mathrm{L}} \underline{\mathrm{M}} \underline{\mathrm{N}} \underline{\mathrm{O}} \underline{\mathrm{P}} \underline{\mathrm{Q}} \underline{\mathrm{R}} \underline{\mathrm{S}} \underline{\mathrm{T}} \underline{\mathrm{U}} \underline{\mathrm{V}} \underline{\mathrm{W}} \underline{\mathrm{X}} \underline{\mathrm{Y}} \underline{\mathrm{Z}}$ \\ Occupations with the title starting with $C$ \\ Occupation

\author{
Occupation
}

$\underline{\text { CAT Scan Operators }}$

Cabinet Assemblers

Cabinet Builders

Cabinet Installers

Cabinet Makers

Cabinetmakers and Bench Carpenters

Cafeteria Managers

Cafeteria Supervisors

Calibration Laboratory Technicians

California Highway Patrol (CHP) Officers

Call Center Representatives

Camera Operators, Television, Video, and

Motion Picture

Camera Operators, Television, and Motion

Picture

Camera and Photographic Equipment

Repairers

Campus Security Officers

Cancer Registrars

Captains, Mates, and Pilots of Water

Vessels

$\underline{\text { Cardiac Sonographers }}$

$\underline{\text { Cardiac Technicians }}$

Cardiac and Vascular Nurses

Cardiographers

Cardiology Technologists

Cardiopulmonary Technologists

Cardiovascular Technologists and

Technicians

Cargivers

$\underline{\text { Cargo and Freight Agents }}$
Combined Food Preparation and Serving Workers

Combined Food Preparation and Serving

Workers, Including Fast Food

Commercial Carpenters

Commercial Divers

Commercial Interior Designers

Commercial Pilots

Commercial Trailer Truck Drivers.

Commercial and Industrial Designers

Communications Teachers, Postsecondary

Community Dietitians

Community Liaisons

Community Managers

Community Services Officers

Companions

Compensation and Benefits Managers

Compensation and Job Analysis Specialists

Compensation, Benefits, and Job Analysis

Specialists

Compliance Officers, Except Agriculture

Compliance Officers, Except Agriculture. Construction, Health and Safety, and

Transportation

Computer Control Programmers

Computer Hardware Engineers

Computer Operators

Computer Programmers

Computer Science Teachers, Postsecondary

Computer Security Specialists

Computer Software Engineers, Applications

Computer Software Engineers, Systems 
Carpenters

Carpet Installers

Cartographers and Photogrammetrists

Case Aides

Case Managers

Casework Specialists

Caseworkers

Cashiers

$\underline{\text { Catalog Illustrators }}$

$\underline{\text { Caterers }}$

Catering Managers.

Ceiling Installers

$\underline{\text { Cell Biologists }}$

Cement Masons and Concrete Finishers

Cementing and Gluing Machine Operators and Tenders

Cementing and Gluing Machine Workers

Ceramic Tile Installers

Certified Kitchen Designers

Certified Medical Assistants (CMA)

Certified Nurse Assistants

Certified Nurse Midwives

Certified Ophthalmic Technicians

Certified Personal Chefs.

Certified Pharmacy Technicians

Certified Professional Controllers

Certified Public Accountants (CPA)

Certified Public Health Microbiologists

Certified Registered Dental Assistants

Certified Registered Nurse Anesthetists

Certified Therapeutic Recreation Specialists
Software

Computer Support Specialists

Computer Systems Analysts

Computer Technicians

Computer Tomography (CT) Technologists.

Computer and Information Scientists,

Research

Computer and Information Systems

Managers

Computer and Office Machine Repairers

Computer, Automated Teller, and Office

Machine Repairers

Computer-Aided Design (CAD)

Programmers

Computer-Aided Manufacturing (CAM)

Programmers

Computer-Controlled Machine Tool

Operators

Computer-Controlled Machine Tool

Operators, Metal and Plastic

Concierges

Concrete Finishers

Concrete Floor Installers

Condominium Managers

Conservation Scientists

Consolidators

Construction Foremen

Construction Laborers

Construction Managers

Construction Supervisors

Construction Trades Supervisors

Construction Workers

Construction and Building Inspectors

Consultant Dietitians

Continuous Mining Machine Operators

Contract Specialists

Control and Valve Installers and Repairers. Except Mechanical Door 
Charge Nurse Specialists

Charge Nurses

Chefs and Head Cooks

Chemical Engineers

Chemical Equipment Operators and Tenders

Chemical Equipment Workers

Chemical Plant and System Operators

Chemical Technicians

Chemistry Teachers, Postsecondary

Chemists

Chief Electrician.

Chief Executives

Chief Operating Officers (COO)

Chief Operations Officers.

Child Care Workers

Child Protective Services Specialists

Child Welfare Workers

Child, Family, and School Social Workers

Childhood Development Teachers

Chiropractor Assistants

Chiropractors

Choreographers

City Police Officers

City Route Drivers.

Civil Attorneys

Civil Engineering Technicians

Civil Engineers

Claims Adjusters, Examiners, and

Investigators

Claims Agents

Claims Analysts

Claims Representatives

Claims Specialists

Classroom Teachers
Control and Valve Workers, Except

Mechanical Door

Conveyor Operators and Tenders

Cooks, Fast Food

Cooks, Institution and Cafeteria

Cooks, Private Household

Cooks, Restaurant

Cooks, Short Order

Cooling and Freezing Equipment Operators

and Tenders

Cooling and Freezing Equipment Workers

Corporate Lawyers

Correctional Officer Supervisors

Correctional Officers and Jailers

Correspondence Clerks

Cost Accountants

Cost Estimators

Costume Attendants

Costume Designers

Counselors, All Other

Counter Attendants, Cafeteria, Food

Concession, and Coffee Shop

Counter Attendants, Concession, and Coffee

Shop

Counter and Rental Clerks

Couriers and Messengers

Court Clerks

Court Reporters

Court, Municipal, and License Clerks

Courtesy Clerks

Crane and Tower Operators

Credit Analysts

Credit Authorizers, Checkers, and Clerks

Credit Reporting Clerks.

Criminal Justice Teachers, Postsecondary

Criminal Justice and Law Enforcement

Teachers, Postsecondary

Criminal Lawyers 
Cleaners of Vehicles and Equipment

Cleaning. Washing, and Metal Pickling

Equipment Operators and Tenders

Cleaning, Washing, and Pickling Equipment

Workers

Clergy

Clerk Typists

Client Services Representatives

Clinical Assistants

Clinical Data Specialists

Clinical Dietetic Technicians

Clinical Dietitians

Clinical Directors

Clinical Microbiologists

Clinical Nurse Specialists

Clinical Pharmacists

Clinical, Counseling, and School

Psychologists

Coaches and Scouts

Coating, Painting, and Spraying Machine

Setters, Operators, and Tenders

Coating, Painting, and Spraying Machine Workers

$\underline{\text { Coil Winders, Tapers, and Finishers }}$

Coin, Vending, and Amusement Machine Servicers and Repairers

Coin, Vending, and Amusement Machine Workers

$\underline{\text { Collection Clerks }}$

Colorists

Combination Welders
Critical Care Nurses

Crossing Guards

Crushing, Grinding, and Polishing Machine

Setters, Operators, and Tenders

Crushing, Grinding, and Polishing Machine

Workers

Curators

Curb Builders

Custody Assistants.

Custom Furriers

Customer Account Technicians

Customer Care Specialists

Customer Service Associates (CSA)

Customer Service Representatives

Customer Support Analysts

Cutters and Trimmers

Cutters and Trimmers, Hand

Cutting Torch Operators

Cutting and Slicing Machine Setters, Operators, and Tenders

Cutting and Slicing Machine Workers

Cutting, Punching, and Press Machine Setters, Operators, and Tenders, Metal and Plastic

Cutting. Punching, and Press Machine

Workers

$\underline{\text { Cytologists }}$

First-Line Supervisors/Managers of Construction Trades and Extraction Workers First-Line Supervisors/Managers of Correctional Officers 


\section{$\underline{A} \underline{B} \underline{C} \underline{D} \underline{E} \underline{F} \underline{G} \underline{H} \underline{I} \underline{\mathrm{J}} \underline{\mathrm{L}} \underline{\mathrm{M}} \underline{\mathrm{N}} \underline{\mathrm{O}} \underline{\mathrm{P}} \underline{\mathrm{Q}} \underline{\mathrm{R}} \underline{\mathrm{S}} \underline{\mathrm{T}} \underline{\mathrm{U}} \underline{\mathrm{V}} \underline{\mathrm{W}} \underline{\mathrm{X}} \underline{\mathrm{Y}} \underline{\mathrm{Z}}$ \\ Occupations with the title starting with $D$ \\ Occupation}

Dancers

Data Entry Keyers

Data Processing Managers

Database Administrators

Delivery Persons

Demonstrators and Product Promoters

Dental Assistants

Dental Hygienists

Dental Laboratory Technicians

Dentists

Dentists, General

Department Secretaries

Deputy Jailers

Deputy Sheriffs

Derrick Operators, Oil and Gas

Designers

Designers, All Other

Desktop Publishers

Desktop Support Technicians

Detectives and Criminal Investigators

Detention Deputies

Detention Officers

Diagnostic Medical Sonographers

Diagnostic Radiologic Technologists

Die Casting and Plastic Molding Mold Makers

Die Finishers

Die Sinkers

Diesel Mechanics

Diet Consultants

Diet Technicians Registered (DTR)

Dietary Aides
Dietary Assistants

Dietary Managers

Dietary Supervisors

Dietetic Technicians

Dietitians and Nutritionists

Digital Artists

Dining Room and Cafeteria Attendants and

Bartender Helpers

Dining Room, Cafeteria, and Bartender Helpers

Directors of Application Development

Directors of Data Operations

Directors of Health Services

Directors of Nursing

Directors of Operations

Directors, Religious Activities and Education

Dishwashers

Dispatchers, Except Police, Fire, and

Ambulance

Dispensary Attendants

Doctors of Pharmacy

Door-To-Door Sales Workers, News and Street Vendors, and Related Workers

Door-to-Door Sales Workers

Drafters, All Other

Dredge Operators

Drilling and Boring Machine Tool Setters, Operators, and Tenders, Metal and Plastic

Drilling and Boring Machine Workers

Driver/Sales Workers

Drug Clerks

Drywall Finishers

Drywall Hangers

Drywall and Ceiling Tile Installers

Drywall-Lathers 


\section{$\underline{A} \underline{B} \underline{C} \underline{D} \underline{E} \underline{F} \underline{G} \underline{H} \underline{I} \underline{\mathrm{K}} \underline{\mathrm{L}} \underline{\mathrm{M}} \underline{\mathrm{N}} \underline{\mathrm{O}} \underline{\mathrm{P}} \underline{\mathrm{Q}} \underline{\mathrm{R}} \underline{\mathrm{S}} \underline{\mathrm{T}} \underline{\mathrm{U}} \underline{\mathrm{V}} \underline{\mathrm{W}} \underline{\mathrm{X}} \underline{\mathrm{Y}} \underline{\mathrm{Z}}$ \\ Occupations with the title starting with $E$ \\ Occupation \\ Occupation}

EKG Technicians

EMTs

Early Childhood Teachers

Earth Drillers, Except Oil and Gas

Echocardiographers

Economics Teachers, Postsecondary

Economists

Editors

Education Administrators

Education Administrators, All Other

Education Administrators, Elementary and Secondary

Education Administrators, Elementary and Secondary School

Education Administrators, Postsecondary

Education Administrators, Preschool and Child Care

Education Administrators, Preschool and Child Care Center/Program

Education Teachers, Postsecondary

Educational, Vocational, and School

Counselors

Educators

Electric Motor, Power Tool, and Related

Repairers

Electrical Engineers

Electrical Inspectors

Electrical Mechanical Technicians

Electrical Power-Line Installers and

$\underline{\text { Repairers }}$

Electrical Sign Servicer

Electrical Technicians
Elevator Installers and Repairers

Eligibility Interviewers, Government

Programs

Embalmers

Emergency Management Specialists

Emergency Medical Technicians and

Paramedics

Emergency or Trauma Nurses

Employment Programs Analysts

Employment, Recruitment, and Placement

Specialists

En Route Controllers

Engine Performance Technicians.

Engine and Other Machine Assemblers

Engineering Managers

Engineering Teachers, Postsecondary

Engineering Technicians, All Other

Engineering Technicians, Except Drafters, All Other

English Language Teachers, Postsecondary

English Language and Literature Teachers,

Postsecondary

English Teachers

Entertainment Lawyers

Environmental Engineering Technicians

Environmental Engineers

Environmental Lawyers

Environmental Microbiologists

Environmental Science Teachers, Postsecondary

Environmental Science and Protection Technicians, Including Health 
Electrical and Electronic Engineering Technicians

Electrical and Electronic Equipment Assemblers

Electrical and Electronics Drafters

Electrical and Electronics Installers and Repairers, Transportation Equipment

Electrical and Electronics Repairers, Commercial

Electrical and Electronics Repairers, Commercial and Industrial Equipment

Electrical and Electronics Repairers, Powerhouse

Electrical and Electronics Repairers, Powerhouse, Substation, and Relay

Electrical and Electronics Workers.

Transportation

Electricians

Electro-Mechanical Technicians

Electrocardiograph Technicians

Electromechanical Equipment Assemblers

Electronic Data Processing (EDP) Auditors

Electronic Data Processing (EDP) Systems Engineers.

Electronic Equipment Installers and

Repairers, Motor Vehicles

Electronic Equipment Workers, Motor

Vehicles

Electronic Home Entertainment Equipment Installers and Repairers

Electronic Home Entertainment Equipment Workers

Electronics Engineers, Except Computer

Electronics Technicians

Elementary Education Teachers

Elementary School Teachers, Except Special Educ

Elementary School Teachers, Except Special Education
Environmental Scientists and Specialists, Including Health

Environmental Scientists, Including Health

Environmental Technicians, Including Health

Epidemiologists

Estate Lawyers

Etchers and Engravers

Examination Proctors

Excavating and Loading Machine Operators

Excavating and Loading Machine and

Dragline Operators

Excavator Operators

Executive Administrative Assistants

Executive Assistants

Executive Chefs

Executive Secretaries

Executive Secretaries and Administrative

Assistants

Expediters

Explosives Workers

Explosives Workers, Ordnance Handling Experts, and Blasters

Exterminator Helpers

Exterminators

Extruding and Drawing Machine Setters, Operators, and Tenders, Metal and Plastic

Extruding and Drawing Machine Workers

Extruding and Forming Machine Setters, Operators, and Tenders, Synthetic and Glass Fibers

Extruding and Forming Machine Workers

Extruding, Forming, Pressing, and 


\section{Elementary Teachers}

Elevator Inspectors
Compacting Machine Setters, Operators, and Tenders

Extruding, Forming, and Pressing Machine Workers 


\section{$\underline{A} \underline{B} \underline{\mathrm{C}} \underline{\mathrm{D}} \underline{\mathrm{F}} \underline{\mathrm{G}} \underline{\mathrm{H}} \underline{\underline{J}} \underline{\mathrm{J}} \underline{\mathrm{L}} \underline{\mathrm{M}} \underline{\mathrm{N}} \underline{\mathrm{N}} \underline{\mathrm{O}} \underline{\mathrm{P}} \underline{\mathrm{Q}} \underline{\mathrm{R}} \underline{\mathrm{S}} \mathrm{I} \underline{\mathrm{U}} \underline{\mathrm{V}} \underline{\mathrm{W}} \underline{\mathrm{X}} \underline{\mathrm{Y}} \underline{\mathrm{Z}}$ \\ Occupations with the title starting with $F$ \\ Occupation \\ Occupation}

Construction Trades Supervisors

Correctional Officer Supervisors

Fabric Menders, Except Garment

Fabric and Apparel Patternmakers

Fabricators

Facilities Painters

Fallers

Family Development Specialists

Family Lawyers

Family Practice Physician Assistants

Family Preservation Caseworkers

Family Self-Sufficiency Specialists

Family Service Caseworkers

Family and General Practitioners

Farm Equipment Engine Mechanics

Farm Equipment Mechanics

Farm Labor Contractors

Farm and Home Management Advisors

Farm, Ranch, and Other Agricultural

Managers

Farmers and Ranchers

Farming, Fishing, and Forestry Supervisors

Farming, Fishing, and Forestry Supervisors

Farmworkers and Laborers, Crop and

Nursery

Farmworkers and Laborers, Crop, Nursery, and Greenhouse

Farmworkers, Farm and Ranch Animals

Fashion Advisors

Fashion Designers
First-Line Supervisors/Managers of

Transportation and Material-Moving Machine and Vehicle Operators

Fiscal Technicians

Fish and Game Wardens

Fishers and Related Fishing Workers

Fitness Coordinators

Fitness Directors

Fitness Instructors

Fitness Technicians

Fitness Trainers and Aerobics Instructors

Flatbed Truck Drivers

Fleet Mechanics

Flight Attendants

Flight Paramedics

Flight Service Specialists

Floor Layers, Except Carpet, Wood, and

Hard Tiles

Floor Sanders and Finishers

Floral Designers

Fluoroscopy Radiologic Technologists

Food Batchmakers

Food Cooking Machine Operators and Tenders

Food Preparation Workers

Food Preparation and Serving Worker

Supervisors

Food Scientists and Technologists

Food Servers, Nonrestaurant

Food Service Managers

Food Service Supervisors

Food and Tobacco Roasting, Baking, and Drying Machine Operators and Tenders 
Fast Food Service Managers

Fence Erectors

Fiberglass Laminators and Fabricators

Field Adjusters

Field Installers

Field Technicians.

File Clerks

Film and Video Editors

Financial Analysts

Financial Auditors

Financial Examiners

Financial Managers

Financial Services Sales Agents
Food and Tobacco Workers

Foreign Language Teachers, Postsecondary

Foreign Language and Literature Teachers.

Postsecondary

Foremen

Forensic Science Technicians

Forest Fire Fighters

Forest Fire Inspectors

Forest Fire Inspectors and Prevention Specialists

Forest Ranger Technicians, and Foresters.

Forest and Conservation Technicians

Forest and Conservation Workers

Foresters

Forestry and Conservation Science

Teachers, Postsecondary

Fine Artists, Including Painters and Sculptors

Fine Artists, Including Painters, Sculptors, and Illustrators

Forestry and Conservation Teachers,

Postsecondary

Forging Machine Setters, Operators, and

Tenders, Metal and Plastic

Forging Machine Workers

Foster Care Social Workers

Foundry Mold and Coremakers

Frame Builders

Front Desk Clerks

Front Desk Receptionists

Fuel Injection Servicers

Fumigators.

Funeral Attendants

Funeral Directors

Furnace Converters

Furnace Liners

Furnace, Kiln, Oven, Drier, and Kettle

Construction Trades and Extraction Workers

First-Line Supervisors/Managers of

Correctional Officers

\section{Operators and Tenders}

Furnace, Kiln, Oven, Drier, and Kettle Workers

First-Line Supervisors/Managers of Farming. Fishing, and Forestry Workers

Furniture Finishers 
First-Line Supervisors/Managers of Fire Fighting and Prevention Workers

First-Line Supervisors/Managers of Food Preparation and Serving Workers

First-Line Supervisors/Managers of Helpers, Landscaping and Groundskeeping Laborers, and Material Movers, Hand

First-Line Supervisors/Managers of Housekeeping and Janitorial Workers

First-Line Supervisors/Managers of Landscaping, Lawn Service, and Groundskeeping Workers

First-Line Supervisors/Managers of Mechanics, Installers, and Repairers

First-Line Supervisors/Managers of NonRetail Sales Workers First-Line Supervisors/Managers of Office and Administrative Support Workers First-Line Supervisors/Managers of Personal Service Workers

First-Line Supervisors/Managers of Police and Detectives

First-Line Supervisors/Managers of Production and Operating Workers

First-Line Supervisors/Managers of Retail Sales Workers
Helpers, Laborers, and Material-Moving Supervisors

Housekeeping and Janitorial Supervisors Supervisors

Mechanics, Installers, and Repair Supervisors

Non-Retail Sales Supervisors

Office Support Supervisors

Personal Service Worker Supervisors

Police and Detective Supervisors

Production and Operating Supervisors

$\underline{\text { Retail Sales Supervisors }}$

Securities, Commodities, and Financial Services Sales Agents

Transportation and Material-Moving Supervisors 


\section{$\underline{A} \underline{B} \underline{C} \underline{D} \underline{E} \underline{F} \underline{G} \underline{H} \underline{I} \underline{J} \underline{K} \underline{L} \underline{M} \underline{N} \underline{0} \underline{P} \underline{Q} \underline{R} \underline{S} I \underline{U} \underline{v} \underline{W} \underline{X} \underline{Y} \underline{Z}$ \\ Occupations with the title starting with $G$ \\ Occupation \\ Occupation}

Gaming Cage Workers

Gaming Change Persons and Booth

Cashiers

Gaming Dealers

Gaming Managers

Gaming Service Workers, All Other

Gaming Supervisors

Gaming Surveillance Officers and Gaming $\underline{\text { Investigators }}$

Gaming Surveillance Officers and

Investigators

Gaming and Sports Book Writers and

Runners

Gas Compressor and Gas Pumping Station

Operators

Gas Furnace Installers

Gas Line Installers

Gas Plant Operators

Gas Welders

General Accountants

$\underline{\text { General Managers }}$

General and Operations Managers

Geographers

Geography Teachers, Postsecondary

Geological and Petroleum Technicians

Geoscientists, Except Hydrologists and

Geographers

Geriatric Aides
Geriatric Social Workers

Gerontological Nutrition Dietitians

Gift Wrappers

Glass Glaziers

Glazier Apprentices

Glaziers

Grader Operators

Graders and Sorters, Agricultural Products

Graduate Teaching Assistants

Granite Setters

Graphic Artists

Graphic Designers

Greeters

Grinding and Polishing Workers

Grinding and Polishing Workers, Hand

Grinding, Lapping, Polishing, and Buffing

Machine Tool Setters, Operators, and

Tenders, Metal and Plastic

Grinding, Polishing, and Buffing Machine

Workers

Grounds Maintenance Workers, All Other

Group Exercise Instructors

Group Fitness Instructors

Gynecologic Sonographers

Gynecology Nurses 


\section{$\underline{A} \underline{B} \underline{C} \underline{D} \underline{E} \underline{F} \underline{G} \underline{H} \underline{J} \underline{K} \underline{L} \underline{M} \underline{N} \underline{0} \underline{P} \underline{Q} \underline{R} \underline{S} \underline{T} \underline{U} \underline{W} \underline{X} \underline{Y} \underline{Z}$ \\ Occupations with the title starting with $H$ \\ Occupation \\ Occupation}

First-Line Supervisors/Managers of Helpers Laborers, and Material Movers, Hand

First-Line Supervisors/Managers of Housekeeping and Janitorial Workers

HVAC Technicians

HVACR Technicians

Hairdressers, Hairstylists, and

Cosmetologists

Hardwood Floorlayers

Hazardous Materials Removal Workers

Head Designers

Head Start Teachers

Head Teachers.

Health Care Administrators

Health Educators

Health Information Administrators

Health Information Analysts

Health Information Coders

Health Information Management Specialists

Health Information Management Technicians

Health Information Specialists

Health Information Systems Technicians

Health Insurance Adjusters

Health Specialties Teachers. Postsecondary

Health and Safety Engineers, Except Mining

Safety

Health and Safety Engineers, Except Mining

Safety Engineers and Inspectors

Heat Treating Equipment Setters, Operators, and Tenders, Metal and Plastic

Heat Treating Equipment Workers

Heating and Air-Conditioning Technicians

and Installers

Heating, Air Conditioning, and Refrigeration

Mechanics and Installers
Helpers--Installation. Maintenance, and

Repair Workers

Helpers--Painters, Paperhangers, Plasterers, and Stucco Masons

Helpers--Pipelayers, Plumbers, Pipefitters, and Steamfitters

Helpers--Production Workers

Helpers--Roofers

High School Teachers

Highway Maintainers

Highway Maintenance Crew Workers

Highway Maintenance Workers

Highway Painters

Highway Workers

Histocompatibility Laboratory Directors

Historians

History Teachers, Postsecondary

Hoist and Winch Operators

Holistic Nurses.

Home Appliance Repairers

Home Attendants.

Home Care Workers

Home Economics Teachers, Postsecondary

Home Health Aides

Home Health Care Nurses

Home Health Care Providers.

Home Health Directors

Home Health Nurses

Home Inspectors

Home Visitors 
Heating, Air-Conditioning, and Refrigeration Technicians and Installers

Heating, Ventilating, and Air Conditioning Sheet Metal Installers

Heating, Ventilating, and Air Conditioning Technicians

Heating/Air Conditioning and Refrigeration

Workers

Heavy Equipment Operators

Heavy Truck Drivers

Help Desk Analysts or Technicians

Helpers, Brickmasons and Tile Setters

Helpers, Carpenters

Helpers, Construction Trades, All Other

Helpers, Electricians

Helpers, Extraction Workers

Helpers. Installation and Repair Workers

Helpers, Laborers, and Material-Moving

Supervisors

Helpers, Painters, Paperhangers, and

Plasterers

Helpers, Pipelayers, Plumbers, and

Pipefitters

Helpers, Production Workers

Helpers, Roofers

Helpers--Brickmasons, Blockmasons, Stonemasons, and Tile and Marble Setters

Helpers--Carpenters

Helpers--Electricians

Helpers--Extraction Workers $\underline{\text { Homemaker Health Aides }}$

$\underline{\text { Hospital Attendants }}$

Hospital Directors

Hospital Nurses

Hospital Pharmacists

Hosts and Hostesses, Restaurant, Lounge. and Coffee Shop

Hosts, Hostesses, Restaurant, and Coffee Shop

Hot Water Heater Installers

Hotel, Motel, and Resort Desk Clerks

House Painters

House Wirer

Housekeeping and Janitorial Supervisors

Human Resources Assistants

Human Resources Assistants, Except

Payroll and Timekeeping

Human Resources Managers

Human Resources Managers, All Other

Human Resources and Labor Specialists. All

Other

Human Resources, Training, and Labor

Relations Specialists, All Other

Human Services Program Specialists

Human Services Workers.

Hydrologists

Truck Drivers, Heavy and Tractor-Trailer 


\section{$\underline{A} \underline{B} \underline{C} \underline{D} \underline{E} \underline{F} \underline{G} \underline{H} \underline{I} \underline{\mathrm{K}} \underline{\mathrm{L}} \underline{\mathrm{M}} \underline{\mathrm{N}} \underline{\mathrm{O}} \underline{\mathrm{P}} \underline{\mathrm{Q}} \underline{\mathrm{R}} \underline{\mathrm{S}} \underline{\mathrm{T}} \underline{\mathrm{U}} \underline{\mathrm{V}} \underline{\mathrm{W}} \underline{\mathrm{X}} \underline{\mathrm{Y}} \underline{\mathrm{Z}}$ \\ Occupations with the title starting with I \\ Occupation \\ Occupation}

$\underline{\text { Immunologists }}$

$\underline{\text { Independent Living Specialists }}$

Industrial Analysts

Industrial Engineering Technicians

$\underline{\text { Industrial Engineers }}$

Industrial Machinery Mechanics

$\underline{\text { Industrial Microbiologists }}$

Industrial Painters

Industrial Production Managers

Industrial Therapists

Industrial Truck and Tractor Operators

Industrial-Organizational Psychologists

Infection Control Specialists

Information Systems Consultants

Information Systems Directors

Information Technology (IT) Specialists

Information Technology (IT) Specialists,

Managers, or Directors

Information Technology Directors

Information Technology Managers

Information Technology Systems Directors

Inside Sales Persons

Inspectors, Testers, Sorters, Samplers, and

Weighers

Inspectors. Testers, Sorters, and Weighers

Installment Agents.

$\underline{\text { Instructional Aides }}$
Instructional Assistants. Instructional Coordinators

Instrumentation Technicians.

Insulation Workers

Insulation Workers, Floor, Ceiling, and Wall

Insulation Workers, Mechanical

Insulators

Insurance Appraisers

Insurance Appraisers, Auto Damage

Insurance Attorneys

Insurance Auditors

Insurance Claims and Policy Processing

$\underline{\text { Clerks }}$

Insurance Sales Agents

Insurance Underwriters

Intellectual Property Lawyers

Interior Decorators

Interior Design Consultants

Interior Design Coordinators.

Interior Designers

Internal Auditors

Internists, General

Interpreters and Translators

Interviewers, Except Eligibility and Loan

Iron Cutters 


\section{$\underline{A} \underline{B} \underline{C} \underline{D} \underline{E} \underline{\mathbf{G}} \underline{\mathrm{H}} \underline{\underline{J}} \underline{\mathrm{K}} \underline{\mathrm{L}} \underline{\mathrm{M}} \underline{\mathrm{N}} \underline{\mathrm{O}} \underline{\mathrm{P}} \underline{\mathrm{Q}} \underline{\mathrm{R}} \underline{\mathrm{S}} \underline{\mathrm{T}} \underline{\mathrm{U}} \underline{\mathrm{W}} \underline{\mathrm{X}} \underline{\mathrm{Y}} \underline{\mathrm{Z}}$ Occupations with the title starting with $J$ Occupation Occupation}

Janitors and Cleaners, Except Maids and Housekeeping Cleaners Janitors and Cleaners, Except

Maids/Housekeeping

Judges, Magistrate Judges, and Magistrates

$\underline{\text { Judicial Assistants }}$

Jewelers and Precious Stone and Metal

Workers 


\section{$\underline{A} \underline{B} \underline{\mathrm{C}} \underline{\mathrm{D}} \underline{\mathrm{E}} \underline{\mathrm{G}} \underline{\mathrm{H}} \underline{\underline{J}} \underline{\mathrm{J}} \underline{\mathrm{K}} \underline{\mathrm{L}} \underline{\mathrm{M}} \underline{\mathrm{N}} \underline{\mathrm{O}} \mathrm{P} \underline{\mathrm{Q}} \underline{\mathrm{R}} \underline{\mathrm{S}} \mathrm{I} \underline{\mathrm{U}} \underline{\mathrm{V}} \underline{\mathrm{W}} \underline{\mathrm{X}} \underline{\mathrm{Y}} \underline{\mathrm{Z}}$ Occupations with the title starting with $K$ Occupation Occupation}

Kindergarten Teachers, Except Special Education 


\section{$\underline{A} \underline{B} \underline{C} \underline{D} \underline{E} \underline{F} \underline{G} \underline{H} \underline{I} \underline{\mathrm{K}} \underline{\mathrm{L}} \underline{\mathrm{M}} \underline{\mathrm{N}} \underline{\mathrm{O}} \mathrm{P} \underline{\mathrm{Q}} \underline{\mathrm{R}} \underline{\mathrm{S}} \underline{\mathrm{T}} \underline{\mathrm{U}} \underline{\mathrm{V}} \underline{\mathrm{W}} \underline{\mathrm{X}} \underline{\mathrm{Y}} \underline{\mathrm{Z}}$ \\ Occupations with the title starting with $L$ \\ Occupation \\ Occupation}

First-Line Supervisors/Managers of

Landscaping, Lawn Service, and

Groundskeeping Workers

Laborers

Laborers and Freight, Stock, and Material

Movers, Hand

Laborers and Material Movers

Landlords

Landscape Architects

Landscaping and Groundskeeping

Supervisors

Landscaping and Groundskeeping Workers

Lathe and Turning Machine Tool Setters,

Operators, and Tenders, Metal and Plastic

Lathe and Turning Machine Workers

Lathers

Laundry and Dry-Cleaning Workers

Law Clerks

Law Enforcement Officers

Law Secretaries

Law Teachers, Postsecondary

Lawyers

Lay-Out Workers, Metal and Plastic

Layout Artists

Lead Teachers

Leasing Managers

Legal Administrative Assistants

Legal Counselors.

Legal Document Assistants

Legal Investigators

Legal Secretaries

Licensed Physical Therapy Assistants

Licensed Practical Nurses

Licensed Practical and Licensed Vocational

Nurses

Licensed Vocational Nurses

Life Coaches

Lifeguards, Ski Patrol, and Other

Recreational Protective Service Workers

Lifeguards, Ski Patrol, and Other Service

Workers

Light Truck Drivers

Line Haul Drivers

Litigation Examiners

Loader Operators

Loading Machine Operators, Underground

Mining

Loan Counselors

Loan Interviewers and Clerks

Loan Officers

Local Area Network (LAN) or Wide Area

Network (WAN) Administrators.

Local Truck Drivers

Locker Room, Coatroom, and Dressing

Room Attendants

Locker, Coat, and Dressing Room

Attendants

Locksmiths and Safe Repairers

Locomotive Engineers

Locomotive Engineers and Operators

Locomotive Firers

Lodging Managers

Log Graders and Scalers

Logging Equipment Operators 
Legislators

Librarians

Library Assistants, Clerical

Library Science Teachers, Postsecondary

Library Technicians

Licensed Attendants

Licensed Clinical Social Workers
Logisticians

Logistics Analysts

Logistics Engineers

Logistics Planners

Long Haul Truck Drivers

Long-Term Care Nurse Managers

Truck Drivers, Light or Delivery Services 


\section{$\underline{A} \underline{B} \underline{\mathrm{C}} \underline{\mathrm{D}} \underline{\mathrm{F}} \underline{\mathrm{G}} \underline{\mathrm{H}} \underline{\mathrm{I}} \underline{\mathrm{J}} \underline{\mathrm{K}} \underline{\mathrm{L}} \underline{\mathrm{M}} \underline{\mathrm{N}} \underline{\mathrm{O}} \underline{\mathrm{P}} \underline{\mathrm{Q}} \underline{\mathrm{R}} \underline{\mathrm{S}} \mathrm{I} \underline{\mathrm{U}} \underline{\mathrm{V}} \underline{\mathrm{W}} \underline{\mathrm{X}} \underline{\mathrm{Y}} \underline{\mathrm{Z}}$ \\ Occupations with the title starting with $M$ \\ Occupation \\ Occupation}

First-Line Supervisors/Managers of

Mechanics, Installers, and Repairers

Machine Feeders and Offbearers

Machinists

Machinists Tool and Die

Magnetic Resonance Imaging (MRI)

Technologists

Maids and Housekeeping Cleaners

Mail Clerks and Mail Machine Operators,

Except Postal Service

Mail Workers, Except Postal Service

Mainframe Programmers

Maintenance Machinists and Experimental

Machinists.

Maintenance Mechanic.

Maintenance Painters

Maintenance Welders

Maintenance Workers, Machinery

Maintenance and Repair Workers, General

Makeup Artists, Theatrical and Performance

Mammographic Radiologic Technologists

Management Analysts

Management Consultants

Management Dietitians

Management Information Systems Directors

Management Information Systems

Managers.

Manicurists and Pedicurists

Manufactured Building and Mobile Home Installers

Marble Finishers

Marine Engineers and Naval Architects
Medical Stenographers

Medical Transcribers

Medical Transcriptionists

Medical and Clinical Laboratory Technicians

Medical and Clinical Laboratory

Technologists

Medical and Health Services Managers

Medical and Public Health Social Workers

Medium and Heavy Truck Mechanics

Meeting and Convention Planners

Member Service Representatives

Member Services Representatives

Member Services Representatives.

Mental Health Counselors

Mental Health Technicians

Mental Health and Substance Abuse Social

Workers

Merchandise Displayers and Window

Trimmers

Metal Framers

Metal Welders

Metal-Refining Furnace Operators and

$\underline{\text { Tenders }}$

Meter Readers, Utilities

Microbiologists

Middle School Teachers, Except Special

Education

Middle School Teachers, Except Special and

Vocational Education

Mildly Disabled Student Special Education

Teachers

Military Lawyers

Milk Route Deliverers 
Market Research Analysts

Marketing Associates.

Marketing Managers

Marriage and Family Therapists

Masons

Massage Therapists

Material Planners

Material Planning Clerks

Materials Engineers

Materials Scientists

Math Teachers

Mathematical Science Teachers,

Postsecondary

Mathematical Scientists, Miscellaneous

Mathematical Technicians

Mathematicians

Meat and Dairy Driver/Sales Workers

Meat, Poultry, and Fish Cutters and

Trimmers

Mechanical Door Repairers

Mechanical Drafters

Mechanical Engineering Technicians

Mechanical Engineers

Mechanical Inspectors

Mechanics, Installers, and Repair

Supervisors

Medical Appliance Technicians

Medical Assistants

Medical Directors

Medical Equipment Preparers

Medical Equipment Repairers
Milling and Planing Machine Setters, Operators, and Tenders, Metal and Plastic

Milling and Planing Machine Workers

Millwrights

Mine Cutting and Channeling Machine Operators

Mini Shifters

Mining Machine Operators, All Other

Mining and Geological Engineers, Including Mining

Mining and Geological Engineers, Including Mining Safety Engineers

Miscellaneous Mathematical Scientists

Mixing and Blending Machine Setters, Operators, and Tenders

Mixing and Blending Machine Workers

Mobile Heavy Equipment Mechanics, Except Engines

Mobile Home Park Managers

Model Makers and Patternmakers, Wood

Model Makers, Metal and Plastic

Model Makers, Wood

Models

Molders, Shapers, and Casters

Molders, Shapers, and Casters, Except

Metal and Plastic

Molding, Coremaking, and Casting Machine Setters, Operators, and Tenders, Metal and Plastic

Molding, Coremaking, and Casting Machine Workers

Motion Picture Projectionists

Motor Grader Operators

Motorboat Mechanics

Motorboat Operators

Motorcycle Mechanics

Multi-Media Artists and Animators

Multimedia Specialists. 
Medical Microbiologists

Medical Office Assistants

Medical Record Technicians

Medical Records and Health Information

Technicians

Medical Scientists

Medical Scientists, Except Epidemiologists

Medical Secretaries
Multiple Machine Tool Setters, Operators, and Tenders, Metal and Plastic

Mültiple Machine Tool Workers

Museum Technicians and Conservators

Music Directors and Composers

Musical Instrument Repairers and Tuners

Musicians and Singers

Mycologists 


\section{$\underline{A} \underline{B} \underline{C} \underline{D} \underline{E} \underline{F} \underline{G} \underline{H} \underline{I} \underline{\mathrm{J}} \underline{\mathrm{L}} \underline{\mathrm{M}} \underline{\mathrm{N}} \underline{\mathrm{O}} \underline{\mathrm{P}} \underline{\mathrm{Q}} \underline{\mathrm{R}} \underline{\mathrm{S}} \underline{\mathrm{T}} \underline{\mathrm{U}} \underline{\mathrm{V}} \underline{\mathrm{W}} \underline{\mathrm{X}} \underline{\mathrm{Z}}$ \\ Occupations with the title starting with $\mathbf{N}$ \\ Occupation \\ Occupation}

First-Line Supervisors/Managers of NonRetail Sales Workers

Natural Sciences Managers

Network Consultants, Engineers,

Technicians, or Managers

Network Control Operators

Network Engineers, Administrators,

Specialists, or Managers

Network Security Administrators

Network Systems and Data Communications

Analysts

Network and Computer Systems

Administrators

Networking Systems and Distributed

Systems Engineers.

Neurosonographers

New Accounts Clerks

News Analysts, Reporters and

Correspondents

Newspaper Delivery Drivers

Non-Retail Sales Supervisors

Nonfarm Animal Caretakers

Nuclear Engineers

$\underline{\text { Nuclear Medicine Technologists }}$ $\underline{\text { Nuclear Power Reactor Operators }}$

Nuclear Technicians

Numerical Control Programmers

Numerical Tool Programmers

Numerical Tool and Process Control

Programmers

Nurse Administrators

Nurse Aides

Nurse Assistants

Nurse Educators

Nurse Practitioners

Nurse Supervisors

Nursery School Teachers

Nursing Aides, Orderlies, and Attendants

Nursing Instructors and Teachers,

Postsecondary

Nursing Technicians

Nutrition Technicians 


\section{A $\underline{B} \underline{\mathrm{C}} \underline{\mathrm{D}} \underline{\mathrm{E}} \underline{\mathrm{F}} \underline{\mathrm{G}} \underline{\mathrm{H}} \underline{\mathrm{J}} \underline{\mathrm{K}} \underline{\mathrm{L}} \underline{\mathrm{M}} \underline{\mathrm{N}} \underline{\mathrm{O}} \underline{\mathrm{P}} \underline{\mathrm{Q}} \underline{\mathrm{R}} \underline{\mathrm{S}} \underline{\mathrm{T}} \underline{\mathrm{U}} \underline{\mathrm{V}} \underline{\mathrm{W}} \underline{\mathrm{X}} \underline{\mathrm{Y}} \underline{\mathrm{Z}}$ \\ Occupations with the title starting with $O$ \\ Occupation}

First-Line Supervisors/Managers of Office and Administrative Support Workers

Obstetric Sonographers

Obstetricians and Gynecologists

Occupational Health and Safety Specialists

Occupational Health and Safety Specialists and Technicians

Occupational Health/Safety

Specialists/Technicians

Occupational Social Workers.

Occupational Therapist Aides

Occupational Therapist Assistants

Occupational Therapists

Office Administrators

Office Assistants

Office Clerks, General

Office Coordinators

Office Machine Operators, Except Computer

Office Managers

Office Managers.

Office Nurses

Office Support Supervisors

Office Systems Coordinators

On-Site Managers.
Operating Engineers and Other Construction

Equipment Operators

Operating Room Nurses

Operations Managers

Operations Research Analysts

Ophthalmic Assistants.

Ophthalmic Laboratory Technicians

Ophthalmic Sonographers

Opticians, Dispensing

Optometric Assistants

Optometrists

Order Clerks

Order Fillers

Orthodontic Assistants.

Orthopedic Physician Assistants

Orthotists and Prosthetists

Outdoor Power Equipment Mechanics

Outdoor Power Equipment and Other Small

Engine Mechanics

Outside Sales Representatives

Over the Road Drivers

Owner Operators 


\section{$\underline{A} \underline{B} \underline{C} \underline{D} \underline{E} \underline{F} \underline{G} \underline{H} \underline{I} \underline{\mathrm{K}} \underline{\mathrm{L}} \underline{\mathrm{M}} \underline{\mathrm{N}} \underline{\mathrm{O}} \underline{\mathrm{P}} \underline{\mathrm{Q}} \underline{\mathrm{R}} \underline{\mathrm{S}} \mathrm{I} \underline{\mathrm{U}} \underline{\mathrm{v}} \underline{\mathrm{W}} \underline{\mathrm{X}} \underline{\mathrm{Y}} \underline{\mathrm{Z}}$ \\ Occupations with the title starting with $\boldsymbol{P}$ \\ Occupation}

First-Line Supervisors/Managers of Personal Service Workers

First-Line Supervisors/Managers of Police and Detectives

First-Line Supervisors/Managers of

Production and Operating Workers

Package Makers

Packaging and Filling Machine Operators and Tenders

Packaging and Filling Machine Workers

Packers and Packagers

Packers and Packagers, Hand

Painters and Decorators

Painters, Construction and Maintenance

Painters, Transportation Equipment

Painting, Coating, and Decorating Workers

Paper Goods Machine Setters, Operators. and Tenders

Paper Goods Machine Workers

Paperhangers

Paraeducators

Paralegal Secretaries

Paralegals and Legal Assistants

Paraprofessionals

Parking Enforcement Workers

Parking Lot Attendants

Partners

Parts Salespersons

Pastry Chefs, and Sous Chefs.

Patient Care Orderlies

Patient Care Technicians

Patient Escorts.

Patternmakers, Metal and Plastic
Pile-Driver Operators

Pipelayers

Pizza Delivery Drivers

Plasterers and Stucco Masons

Plastic Fixture Builders

Plastic Tool Makers

Plating and Coating Machine Setters, Operators, and Tenders, Metal and Plastic

Plating and Coating Machine Workers

Plumbers, Pipefitters, and Steamfitters

Plumbing Inspectors

Podiatrists

Police and Detective Supervisors

Police and Sheriff Patrol Officers

Police, Fire, and Ambulance Dispatchers

Political Science Teachers, Postsecondary

Political Scientists

Postal Service Clerks

Postal Service Mail Carriers

Postal Service Mail Processors

Postal Service Mail Sorters, Processors, and

Processing Machine Operators

Postmasters and Mail Superintendents

Pourers and Casters, Metal

Power Distributors and Dispatchers

Power Plant Operators

Pre-Kindergarten Teachers

Precision Instrument Repairers, All Other

Precision Instrument and Equipment

Repairers, All Other

Precision Machine Operators 
Patternmakers, Wood

Pavement Stripers

Paving. Surfacing, and Tamping Equipment Operators

Payment Collectors

Payroll and Timekeeping Clerks

Peace Officers

Pediatric Clinical Dietitians

Pediatric Nurses

Pediatric Physical Therapists

Pediatric Physician Assistants

Pediatricians, General

Perfusionists

Personal Attendants.

Personal Banking Representatives

Personal Chefs

Personal Financial Advisors

Personal Injury Lawyers

Personal Secretaries.

Personal Service Worker Supervisors

Personal Trainers

Personal and Home Care Aides

Pest Control Technicians

Pest Control Workers

Pesticide Handlers, Sprayers, and

Applicators, Vegetation

Pesticide Sprayers and Applicators.

Vegetation

Petroleum Engineers

Petroleum Pump System Operators,

Refinery Operators, and Gaugers

Petroleum Pump System Workers

Pharmaceutical Care Associates

Pharmacist Assistants

Pharmacist Assistants.

Pharmacist Technicians

Pharmacists
Prepress Technicians and Workers

Preschool Special Education Teachers

Preschool Teachers, Except Special

Education

Prescription Clerks

Prescriptionists

Pressers, Textile, Garment, and Related

Materials

Prevention Specialists.

Primary Teachers

Printing Machine Operators

Prison Guards

Private Chefs

Private Detectives and Investigators

Private Trainers.

Probate Lawyers

Probate Paralegals

Probation Officers and Correctional

Treatment Specialists

Probation Officers and Treatment Specialists

Procurement Clerks

Procurement Specialists

Producers and Directors

Product Engineering Technicians

Production Assistants

Production Control Clerks

Production Dispatchers

Production Line Workers

Production Machinists

Production Planners

Production Scheduling Clerks

Production and Operating Supervisors

Production, Planning, and Expediting Clerks

Program Management Analysts

Programmer Analysts

Programmers 
Pharmacists in Charge.

Pharmacy Aides

Pharmacy Clerks

Pharmacy Consultants

Pharmacy Laboratory Technicians

Pharmacy Technicians

Pharmacy Technologists

Philosophy and Religion Teachers,

Postsecondary

Photographers

Photographic Process Workers

Photographic Processing Machine Operators

Physical Therapist Aides

Physical Therapist Assistants

Physical Therapists

Physician Assistant Certified Assistants

Physician Assistants

Physician's Assistants

Physicians and Surgeons

Physicians and Surgeons. All Other

Physicists

Physics Teachers, Postsecondary

Physiotherapists.

Pick Up Drivers

Pick Up Truck Drivers
Programmers.

Proofreaders and Copy Markers

Property and Community Association

Managers

Property, Real Estate, and Community

Association Managers

Prototype Machinists

Psychiatric Aides

Psychiatric Nurses

Psychiatric Technicians

Psychiatrists

Psychologists, All Other

Psychology Teachers, Postsecondary

Public Address System and Other

Announcers

Public Health Administrators

Public Health Nurses

Public Relations Managers

Public Relations Specialists

Public Works Inspectors

Publishing Systems Analysts

Pulmonary Physical Therapists

Pump Operators, Except Wellhead Pumpers

Purchasing Agents and Buyers, Farm

Products

Purchasing Agents, Except Wholesale, Retail, and Farm Products

Purchasing Agents, Except Wholesale, Retail/Farm

Purchasing Managers 


\section{$\underline{A} \underline{B} \underline{C} \underline{D} \underline{E} \underline{G} \underline{G} \underline{H} \underline{I} \underline{\mathrm{K}} \underline{\mathrm{L}} \underline{\mathrm{M}} \underline{\mathrm{N}} \underline{\mathrm{O}} \underline{\mathrm{P}} \underline{\mathrm{Q}} \underline{\mathrm{R}} \underline{\mathrm{S}} \underline{\mathrm{T}} \underline{\mathrm{U}} \underline{\mathrm{V}} \underline{\mathrm{W}} \underline{\mathrm{X}} \underline{\mathrm{Y}} \underline{\mathrm{Z}}$ Occupations with the title starting with $Q$ Occupation \\ Occupation}

Quality Assurance Analysts

Quality Assurance Inspectors

Quality Assurance Specialists

Quality Control Analysts

Quality Control Inspectors
Quality Control Technicians

Quality Control Testers

Quality Inspectors

Quality Technicians 


\section{A $\underline{B} \underline{\mathrm{C}} \underline{\mathrm{D}} \underline{\mathrm{E}} \underline{\mathrm{G}} \underline{\mathrm{H}} \underline{\mathrm{I}} \underline{\mathrm{J}} \underline{\mathrm{K}} \underline{\mathrm{L}} \underline{\mathrm{M}} \underline{\mathrm{N}} \underline{\mathrm{O}} \underline{\mathrm{P}} \underline{\mathrm{Q}} \underline{\mathrm{R}} \underline{\mathrm{S}} \underline{\mathrm{T}} \underline{\mathrm{U}} \underline{\mathrm{W}} \underline{\mathrm{X}} \underline{\mathrm{Y}} \underline{\mathrm{Z}}$ \\ Occupations with the title starting with $R$ \\ Occupation

\author{
Occupation
}

Appraisers and Assessors of Real Estate

First-Line Supervisors/Managers of Retail Sales Workers

Radiation Therapists

Radio Mechanics

Radio Operators

Radio and Television Announcers

Radiographers

Radiologic Technologists and Technicians

Rail Car Repairers

Rail Yard Engineers

Rail Yard Engineers, Dinkey Operators, and Hostlers

Rail-Track Laying and Maintenance

Equipment Operators

Rail-Track Laying and Maintenance Workers

Railroad Brake, Signal, and Switch

Operators

Railroad Conductors and Yardmasters

Reading Recovery Teachers

Real Estate Appraisers and Assessors

Real Estate Brokers

Real Estate Clerks.

Real Estate Lawyers

Real Estate Paralegals

Real Estate Sales Agents

Receiving Clerks

Receiving Managers

Receptionists and Information Clerks

Recreation Therapists

Recreation Workers

Recreation and Fitness Studies Teachers, Postsecondary
Registered Diagnostic Sonographers

$\underline{\text { Registered Dietitians }}$

Registered Health Information Technicians (RHIT)

Registered Medical Assistants

Registered Nurses

Registered Pharmacists

Rehabilitation Counselors

Rehabilitation Engineers

Rehabilitation Therapists

Reinforcing Iron and Rebar Workers

Reporters and Correspondents

Research Chefs and Supermarket Chefs.

Research Electricians

Reservation and Transportation Ticket

Agents

Reservation and Transportation Ticket

Agents and Travel Clerks

Resident Assessment Specialists

Residential Advisors

Residential Carpenters

Resource Program Teachers

Respiratory Therapists

Respiratory Therapy Technicians

Restaurant Managers

Retail Managers

Retail Sales Supervisors

Retail Salespersons

Retail Store Managers

Retail Supervisors and Managers

Revenue Tax Specialists. 
Recreation and Fitness Teachers,

Postsecondary

Recreational Therapists

$\underline{\text { Recreational Vehicle Service Technicians }}$

Referral and Information Aides

Refractory Bricklayers

Refractory Masons

Refractory Materials Repairers, Except

Brickmasons

Refuse and Recyclable Material Collectors

Regional Sales Managers.

Registered Dental Assistants (RDA)

Registered Dental Hygienists (RDH) and Hygienists. $\underline{\text { Riggers }}$

Rock Splitters, Quarry

Rolling Machine Setters, Operators, and

Tenders, Metal and Plastic

Rolling Machine Workers

Roofers

Rotary Drill Operators, Oil and Gas

Roustabouts, Oil and Gas

$\underline{\text { Route Deliverers }}$

Route Drivers

Route Sales Drivers

Roving Tellers 


\section{$\underline{A} \underline{B} \underline{C} \underline{D} \underline{E} \underline{F} \underline{G} \underline{H} \underline{I} \underline{\mathrm{K}} \underline{\mathrm{L}} \underline{\mathrm{M}} \underline{\mathrm{N}} \underline{\mathrm{O}} \underline{\mathrm{P}} \underline{\mathrm{Q}} \underline{\mathrm{R}} \underline{\mathrm{S}} \underline{\mathrm{U}} \underline{\mathrm{U}} \underline{\mathrm{W}} \underline{\mathrm{X}} \underline{\mathrm{Y}} \underline{\mathrm{Z}}$ \\ Occupations with the title starting with $S$}

Occupation

Financial Services Sales Agents

Sailors and Marine Oilers

Sales Consultants

Sales Directors

Sales Engineers

Sales Executives

Sales Managers

Sales Representatives

Sales Representatives, Except Technical

Sales Representatives, Technical and

$\underline{\text { Scientific }}$

Sales Representatives, Wholesale and

Manufacturing. Except Technical and

Scientific Products

Sales Representatives, Wholesale and

Manufacturing. Technical and Scientific

Products

Salesmen

Saw Makers

Sawing Machine Setters, Operators, and

Tenders, Wood

Sawing Machine Workers

Scaffold Erectors.

$\underline{\text { Schedule Clerks }}$

Schedulers

Science Teachers

Secondary School Teachers, Except Special Educ

Secondary School Teachers, Except Special and Vocational Education

Secondary Teachers

Secretaries

Secretaries, Except Legal, Medical, and
Social Work Teachers, Postsecondary

Social Workers, All Other

Social and Community Service Managers

Social and Human Service Assistants

Sociologists

Sociology Teachers, Postsecondary

Software Analysts, Architects, Developers,

Designers, or Engineers

Software Developers or Engineers

Software Development Engineers

Software Engineers or Developers

$\underline{\text { Soil and Plant Scientists }}$

Solar Panel Installers and Technicians

Sonographers

Sound Engineering Technicians

Spanish Teachers

Special Education Aides

Special Education Teachers, Elementary School

Special Education Teachers, Middle School

Special Education Teachers, Preschool. Kindergarten, and Elementary School

Special Education Teachers, Secondary School

Specialty Carpenters.

Specialty Cooks

Specialty Designers

Specification Inspectors 
Executive

Secretaries.

Securities, Commodities, and Financial

Services Sales Agents

Security Guards

Security Officers

Security and Fire Alarm Systems Installers

Segmental Pavers

Self-Enrichment Education Teachers

Semiconductor Development Technicians

Semiconductor Processors

Separating and Filtering Machine Workers

Separating, Filtering, Clarifying.

Precipitating, and Still Machine Setters,

Operators, and Tenders

Septic Tank Servicers and Sewer Pipe

Cleaners

Service Assistants (Maintenance)

Service Station Attendants

Service Technicians.

Service Unit Operators, Oil, Gas, and Mining

Set and Exhibit Designers

Severe Emotional Disorders Teachers

Sewers, Hand

Sewing Machine Operators

Shampooers

Sheet Metal Installers

Sheet Metal Layout Mechanics

Sheet Metal Mechanics

Sheet Metal Workers

Ship Engineers

Ship and Boat Captains and Operators

Shippers

Shipping Clerks.

Shipping, Receiving, and Traffic Clerks

Shipyard Painters

Shoe Machine Operators and Tenders
Speech-Language Pathologists

Sports Lawyers

Sports Physical Therapists

Sprinkling System Installers.

Staff Accountants

Staff Development Specialists

Staff Nurses

Staff Pharmacists

Staff Therapists

Stained Glass Glaziers

Stamping Die Makers

Stationary Engineers and Boiler Operators

$\underline{\text { Statistical Assistants }}$

Statisticians

Steel Welders.

Stock Clerks and Order Fillers

Stone Derrickmen and Riggers

Stone Setters

Stonemasons

Store Managers

Store Managers.

Structural Iron and Steel Workers

Structural Metal Fabricators and Fitters

Stylists.

Substance Abuse and Behavioral Disorder Counselors

Subway and Streetcar Operators

Supervisors of Food and Nutrition Services. Supply Chain Directors

Surgeons

Surgical Dental Assistants

Surgical Physician Assistants

Surgical Technologists

Survey Researchers 
Shoe Machine Workers

Shoe and Leather Workers and Repairers

Shuttle Car Operators

Signal and Track Switch Repairers

Skin Care Specialists

Slaughterers and Meat Packers

Slot Key Persons

Social Science Research Assistants

Social Sciences Teachers, Postsecondary, All Other

Social Studies Teachers
Surveying and Mapping Technicians

Surveyors

Switchboard Operators

Switchboard Operators, Including Answering Service

Systems Administrators, Engineers, or Programmers

Systems Architects

Systems Developers.

Systems Engineers or Administrators

Systems Engineers or Programmers

Systems Integrators or Specialists 


\section{A $\underline{B} \underline{\mathrm{C}} \underline{\mathrm{D}} \underline{\mathrm{F}} \underline{\mathrm{G}} \underline{\mathrm{H}} \underline{\mathrm{I}} \underline{\mathrm{J}} \underline{\mathrm{K}} \underline{\mathrm{L}} \underline{\mathrm{M}} \underline{\mathrm{N}} \underline{\mathrm{O}} \underline{\mathrm{P}} \underline{\mathrm{Q}} \underline{\mathrm{R}} \underline{\mathrm{S}} \mathrm{I} \underline{\mathrm{U}} \underline{\mathrm{V}} \underline{\mathrm{W}} \underline{\mathrm{X}} \underline{\mathrm{Y}} \underline{\mathrm{Z}}$ \\ Occupations with the title starting with $T$ \\ Occupation \\ Occupation}

First-Line Supervisors/Managers of

Transportation and Material-Moving Machine Therapeutic Radiologic Technologists and Vehicle Operators

Heavy Truck Drivers

Light Truck Drivers

Tailors, Dressmakers, and Custom Sewers

Tank Car, Truck, and Ship Loaders

Tap and Die Maker Technicians

Tapers

Tax Attorneys

Tax Examiners, Collectors, and Revenue Agents

Tax Preparers

Taxi Drivers and Chauffeurs

Teacher Aides

Teacher Assistants

Teachers

Teachers of Developmentally Delayed

Teachers of Emotionally Disturbed

Teachers of Hearing Impaired Students

Teachers of Students with Learning and

Behavior Disabilities

Teachers of Visually Impaired

Teaching Assistants

Team Assemblers

Technical Services Managers

Technical Writers

Telecommunications Equipment Installers and Repairers, Except Line Installers

Telecommunications Equipment Workers, Except Line

Telecommunications Line Installers and Repairers

Telecommunications Managers

Telemarketers
Therapeutic Recreation Specialists

Therapists, All Other

Tile Finishers

Tile Mechanics

Tile and Marble Installers

Tile and Marble Setters

Timing Device Assemblers, Adjusters, and

Calibrators

Timing Device Workers

Tire Builders

Tire Repairers and Changers

Title Attorneys.

Title Examiners, Abstractors, and Searchers

Title One Reading Teachers

Tool Grinders, Filers, and Sharpeners

Tool Makers

Tool and Die Machinists

Tool and Die Makers

Tour Guides and Escorts

Tour and Travel Guides

Track Hoe Operators.

Tractor Trailer Mechanics

Traffic Managers

Traffic Technicians

$\underline{\text { Trainers }}$

Training and Development Managers

Training and Development Specialists

Transit and Railroad Police 
Telephone Operators

Teller Coordinators.

Tellers

Termite Control Technicians

Terrazzo Workers and Finishers

Testing and Regulating Technicians

Textile Bleaching and Dyeing Machine Operators and Tenders

Textile Bleaching and Dyeing Machine Workers

Textile Cutting Machine Setters, Operators, and Tenders

Textile Cutting Machine Workers

Textile Knitting and Weaving Machine Setters, Operators, and Tenders

Textile Knitting and Weaving Machine Workers

Textile Winding and Twisting Machine Workers

Textile Winding. Twisting, and Drawing Out Machine Setters, Operators, and Tenders Theatrical Costume Designers.
Transmission Technicians

Transportation Attendants

Transportation Attendants, Except Flight

Attendants and Baggage Porters

Transportation Inspectors

Transportation and Material-Moving

Supervisors

Transportation, Storage, and Distribution Managers

Travel Agents

Travel Guides

Tree Trimmers and Pruners

$\underline{\text { Trim Die Makers }}$

Truck Drivers, Heavy and Tractor-Trailer

Truck Drivers, Light or Delivery Services

$\underline{\text { Tunnel Laborers }}$

$\underline{\text { Tunnel Miners }}$ 


\section{$\underline{A} \underline{B} \underline{C} \underline{D} \underline{E} \underline{F} \underline{G} \underline{H} \underline{I} \underline{\mathrm{K}} \underline{\mathrm{L}} \underline{\mathrm{M}} \underline{\mathrm{N}} \underline{\mathrm{O}} \underline{\mathrm{P}} \underline{\mathrm{Q}} \underline{\mathrm{R}} \underline{\mathrm{S}} \underline{\mathrm{U}} \underline{\mathrm{U}} \underline{\mathrm{W}} \underline{\mathrm{X}} \underline{\mathrm{Y}} \underline{\mathrm{Z}}$ Occupations with the title starting with $U$ Occupation Occupation}

University of California (UC) and California

U.S. Border Patrol and Customs Agents. State University (CSU) Campus Police Officers

Ultrasound Technicians

Upholsterers

Ultrasound Technologists

Urban and Regional Planners

Umpires, Referees, and Other Sports

Ushers, Lobby Attendants, and Ticket

$\underline{\text { Officials }}$

Takers

Undercar Technicians

Utility Baggers

Underwater Welders.

Utility Operators. 


\section{$\underline{A} \underline{B} \underline{C} \underline{D} \underline{E} \underline{F} \underline{G} \underline{H} \underline{I} \underline{\mathrm{J}} \underline{\mathrm{L}} \underline{\mathrm{M}} \underline{\mathrm{N}} \underline{\mathrm{O}} \underline{\mathrm{P}} \underline{\mathrm{Q}} \underline{\mathrm{R}} \underline{\mathrm{S}} \underline{\mathrm{T}} \underline{\mathrm{U}} \underline{\mathrm{V}} \underline{\mathrm{W}} \underline{\mathrm{X}} \underline{\mathrm{Z}} \underline{\mathrm{Z}}$ Occupations with the title starting with $V$ \\ Occupation \\ Occupation}

Vascular Technologists

Veterinarians

Veterinary Assistants and Laboratory Animal Caretakers

Veterinary Assistants/Laboratory Animal

Caretakers

Veterinary Microbiologists
Vocational Education Teachers, Middle School

Vocational Education Teachers, Postsecondary

Vocational Education Teachers, Secondary School

Vocational Rehabilitation Specialists 


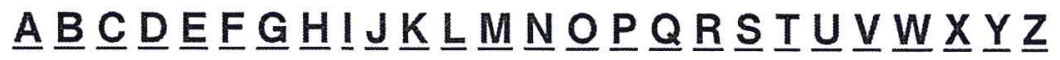 \\ Occupations with the title starting with $W$ \\ Occupation \\ Occupation}

Waiters and Waitresses

Warehouse Workers

Watch Repairers

Water and Liquid Waste Treatment Plant Workers

Water and Liquid Waste Treatment Plant and System Operators

Web Application Developers.

Web Programmers

Weighers, Measurers, Checkers, and Samplers

Weighers, Measurers, Checkers, and Samplers, Recordkeeping

Welders, Cutters, Solderers, and Brazers

Welding, Soldering, and Brazing Machine Setters, Operators, and Tenders
Wholesale and Retail Buyers, Except Farm Products

Window Glaziers

Wire Drawing Die Makers

Wood Machinists.

Woodworking Machine Setters, Operators, and Tenders, Except Sawing

Woodworking Machine Workers, Except

Sawing

Word Processors and Typists

Work Station Support Specialists.

Workers' Compensation Examiners

Writers and Authors 
$\underline{A} \underline{B} \underline{C} \underline{D} \underline{E} \underline{F} \underline{G} \underline{H} \underline{I} \underline{\mathrm{K}} \underline{L} \underline{\mathrm{M}} \underline{\mathrm{N}} \underline{\mathrm{O}} \underline{\mathrm{P}} \underline{\mathrm{Q}} \underline{\mathrm{R}} \underline{\mathrm{S}} \underline{\mathrm{T}} \underline{\mathrm{U}} \underline{\mathrm{W}} \underline{\mathrm{X}} \underline{\mathrm{Y}} \underline{\mathrm{Z}}$ Occupations with the title starting with $X$ Occupation Occupation

$\underline{\text { X-ray Technicians. }}$ 
$\underline{A} \underline{B} \underline{C} \underline{D} \underline{E} \underline{F} \underline{G} \underline{H} \underline{I} \underline{J} \underline{K} \underline{L} \underline{M} \underline{N} \underline{O} \underline{P} \underline{Q} \underline{R} \underline{S} \underline{T} \underline{U} \underline{V} \underline{W} \underline{X} \underline{Y} \underline{Z}$ Occupations with the title starting with $Y$ Occupation

Youth Correctional Counselors Occupation

Youth Correctional Officers 
$\underline{A} \underline{B} \underline{C} \underline{D} \underline{E} F \underline{G} \underline{H} \underline{I} \underline{\mathrm{J}} \underline{\mathrm{L}} \underline{\mathrm{M}} \underline{\mathrm{N}} \underline{\mathrm{O}} \underline{\mathrm{P}} \underline{\mathrm{Q}} \underline{\mathrm{R}} \underline{\mathrm{S}} \underline{\mathrm{T}} \underline{\mathrm{U}} \underline{\mathrm{V}} \underline{\mathrm{W}} \underline{\mathrm{X}} \underline{\mathrm{Z}} \underline{\mathrm{Z}}$ Occupations with the title starting with $Z$ Occupation Occupation

$\underline{\text { Zoologists and Wildlife Biologists }}$ 


\section{Some of the careers this school prepares students for include:}

Veterinarian

Geneticist

Livestock Rancher

Dairy Nutrition

Specialist

Landscape Architect

Soil Scientist

Farm Appraiser

Ag Loan Officer

Wildlife Manager

Ag Inspector

Ag Construction

Engineer

Diesel Mechanic

Fruit \& Vine Grower

Water Engineer

Produce Buyer

Welder

Ag Lawyer

Ag Teacher

\section{FFA teams that can} assist in the pursuit of career opportunities:

Banking

Horse Evaluation

Small Engines

Vine Pruning

Public Speaking

Parliamentary

Procedure

Cotton Judging

Floriculture

Livestock Evaluation

....and many more

\section{SCHOOL OF AGRICULTURE, SCIENCE AND ENGINEERING}

The school of Agriculture, Science and Engineering prepares students for modern careers in the agriculture, environmental science and $A G$ engineering industries. Both new and traditional methods in these fields are fully explored which provides students with a strong foundation for future college coursework or entry into a career field.

Only a small percentage of jobs in the agriculture industry are directly involved in traditional agriculture. This school provides academic training and hands-on coursework, preparing students to enter

engineering, agribusiness, horticulture, communications, science and research, processing and distribution, marketing and sales, food processing and dozens of other occupations which serve the agricultural industry.

Instructions in the School of Agriculture, Science \& Engineering provide a personal approach and work together with counselors to assist students in making academic choices to support their professional career interests and future educational goals.

\section{SPECIAL FEATURES}

\section{FFA}

The Madera FFA is one of California's largest FFA chapters. Active participation in FFA builds leadership skills for life, reinforces instruction, recognizes excellence and gives students opportunities to make a positive difference in their school and community. Participation in FFA helps students make connections between school, their lives and future careers.

\section{ENGINEERING, FABRICATION AND POWER EQUIPMENT}

A fully equipped, state of the art computer aided drafting lab allows School of Agriculture, Science and Engineering students to learn skills in drafting currently used in industry. The electronic classes prepare students with the foundation of electrical systems used in many different professions. The School of Agriculture, Science and Engineering combine all of this with a metal fabrication and power equipment program that is among the best in the state with new facilities that are second to none!

\section{NEW SCHOOL FARM LABORATORY}

The newly built 20-acre school farm laboratory gives students from every background the opportunity for hands-on-learning. A new greenhouse, swine barn, sheep/goat barn, beef/dairy barn and horse facility allows students the opportunity to apply what they learned in the classroom into real life applications. Students will continue to develop the school farm through special class projects.

\section{LAB-ORIENTED INSTRUCTION AND SPECIALTY CLASSES}

Students can immediately apply what they learned in the classroom through intensive labs and practical hands-on experience in nearly every career area. Technical training is provided through an $\mathrm{Ag}$ Mechanics program which is a four-year course of study emphasizing welding, construction and small engines work. Landscape/Horticulture offers hands-on application of the theories of landscaping. This provides students with a variety of skills to carry to the world of work. Animal Care and Veterinary Aide is designed to prepare students to work in animal care facilities at all levels of expertise. 


\section{Cal Grant GPA Information Release Form}

Students, please complete this form and return it to the high school counselor so that the school will release your GPA information to the California Student Aid Commission for use in the Cal Grant awards.

Do not send this form to the Commission.

Please print your full name as it appears on your social security card:

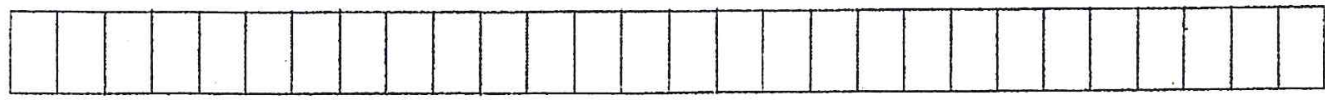

Student's last name

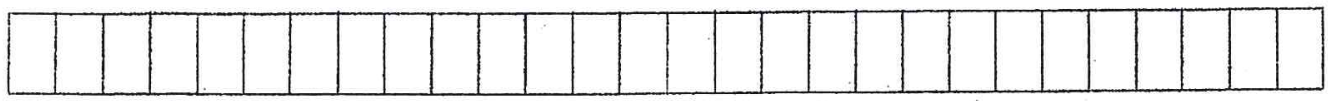

Student's first name

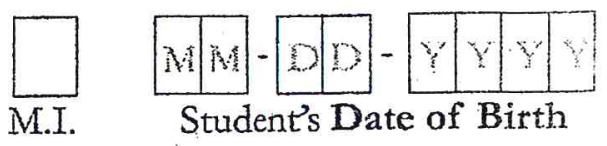

Permanent Mailing Address

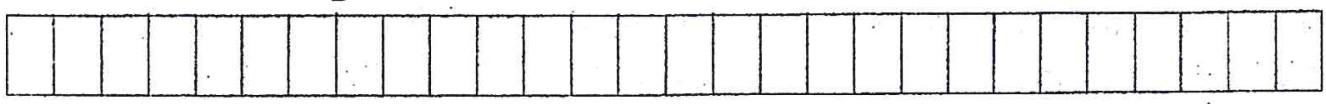

Number and Street

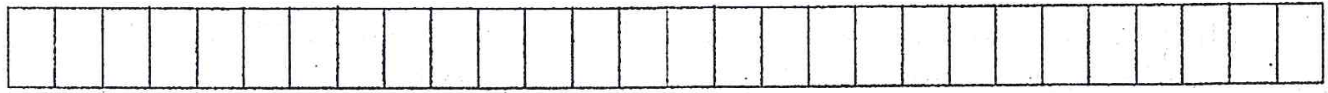

City

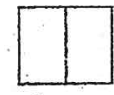

State

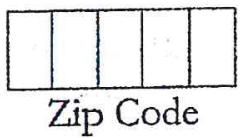

Student's Social Security Number

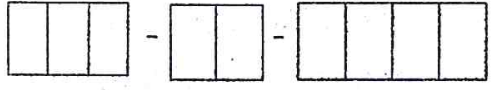

Tonth and year of high school graduation

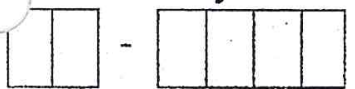

Spring School Code - if you are enrolled for Spring

\begin{tabular}{|l|l|l|l|l|l|}
\hline 0 & 5 & 1 & 8 & 7 & 4 \\
\hline
\end{tabular} classes, enter school code.

(Example: 06-2008)

By signing this release form, I authorize the release of my high school Cal Grant GPA information and social security number to the California Student Aid Commission, for use in the Cal Grant application process.

\section{Student Signature}

Date

I am the parent or legal guardian of the above named minor, and I authorize the release of this minor's high school GPA information and social security number to the California Student Aid Commission for use in the Ca! Grant application process.

Parent/Legal Guardian Signature (required if student is under 18)

Date

School rse Only

SSN

H.S. GPA

Sehoolcode

IS GPA

Ir. S. Orad

Spring Schoe:

Flag

Dite

Code

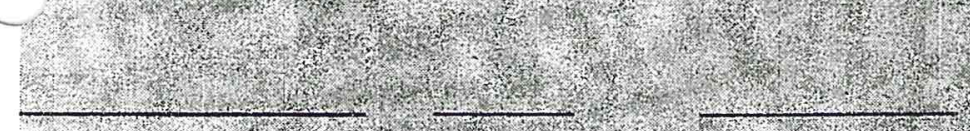




\section{CTC - Continued}

\section{Opening Doors To Employment}

The Career \& Technology Center offers support for individuals committed to improving themselves. The unique, hands-on programs offer:

Open entry

Low Cost

Financial aid

Year-round registration

Industry specific training

Classes at all levels of academic achievement

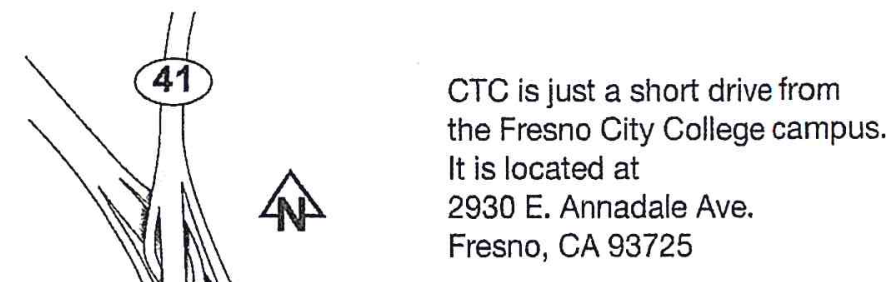

वृ 486-0173

\section{CTC Programs}

\section{Auto Collision Repair}

30-week program. Sheet metal repair, power tools, plastic body fillers, fiberglass, painting and refinishing.

\section{Engthe Performance/} Electricallheating \& $A C$

30-week program. Computer controlled vehicles, driveability, electrical, fuel injection and sensor diagnosis. Troubleshooting and repairing, plus working with portable test equipment, engine oscilloscopes and computerized emis- . sion control engine analyzer. Diagnose and repair $A C$ systems.

\section{Engine Repaili}

20-week program. Practical and theoretical training in engine diagnosis, heads, valves, engine block, lubrication and cooling.

\section{Fire Academy}

20- or 31-week program. Extensive hands-on training. Strong emphasis on physical training and testing. HazMat FRO, Confined Space, I-100 and I-200, Auto Extrication, Fire Control 3, Low Angle Rope Rescue, and CDF Wildland certification.

\section{Fire Technology}

Associate degree program in Fire Technology. Emergency Medical Technician 1 training. California State Fire Marshall Fire Officer certification classes, specialized courses preparing students for advancement in the Fire Service.

\section{Maintenance Mechanic}

30-week program. Theory, application, troubleshooting and repair in welding, hydraulics and pneumatics, machining and electrical fundamentals.

Warehouse \& Distribution Careers 12-week program. Forklift training, inventory control, blueprint reading, computer and math literacy. 


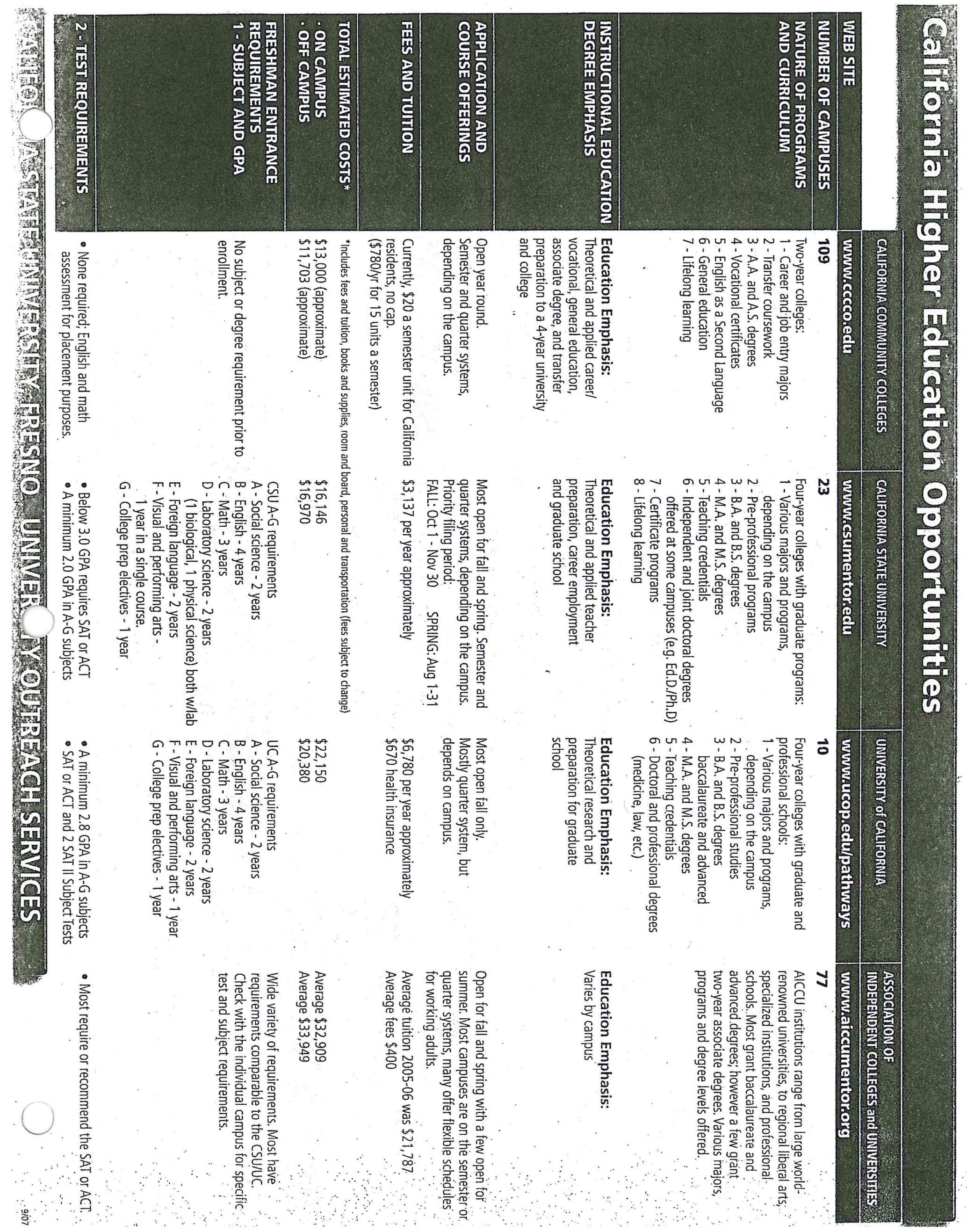




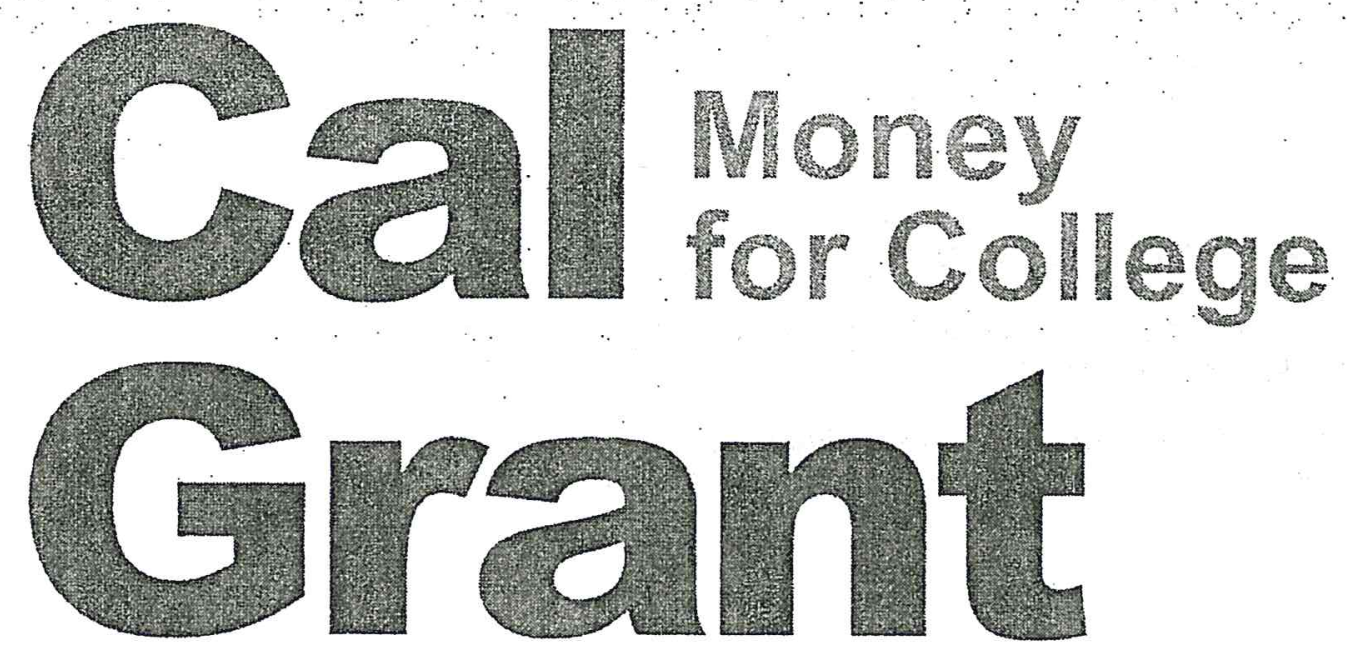

\section{Cal Grant Awards guaranteed for students who qualify}

College is now more affordable than ever for students with good grades and financial need. The best part is that Cal Grants cost nothing to apply for and do not have to be repaid!

Students who meet the following criteria are guaranteed grants:

\section{Cal Grant A Entitlement Awards}

Requirements include financial and basic eligibility (see box lower right), and a minimum 3.0 grade point average (GPA). Students must apply by March 2 nd either the year they graduate from high school or the following year. This Entitlement Award provides for fees at the California State University and the University of California, as well as tuition support at private California colleges and universities.*

\section{Cal Grant B Entitlement Awards}

Requirements include financial and basic eligibility and a minimum 2.0 GPA. Students must apply by March 2nd either the year they graduate from high school or the following year. This Entitlement Award provides up to $\$ 1,551$ for books and living expenses for students in their first year of college.
For the second and subsequent years, the award also helps pay for tuition and fees at the California State University and the University of California, as well as tuition support at private California colleges and universities."

\section{California Community College Transfer Entitlement Awards} Students who meet financial and basic eligibility requirements, have a minimum 2.4 GPA from a California Community College, were California residents when they graduated from high school, and graduate from high school July 1, 2000, or later are eligible for this award when transferring from a community college to a fouryear institution. This Entitlement Award is offered to California Community College students who were not awarded a Cal Grant within a year of graduating from high school, but meet certain eligibility requirements at the time of transfer from a California Community College to most four-year colleges or universities in California.

\section{Basic Cal Grant eligibility requirements}

All Cal Grant applicants must.

- Be California residents

- Be US. citizens or eligible non-citizens

- Meet U.S. Selective Service requirements

- Attend a qualifying California posisecondary institution

- Be enrolled at least half-time

- Maintain satisfactory academic progress as defined at the school of attendance

- Have family income and assets below the established ceilings

- Not be in default on any student loan

- Not owe any federal or state grant refund

*Students attending a California Community College may have their fees waived through the Board of Governor's Fee Waiver Program (BOGFW). Contact your local comminity college financial aid office for more information. 

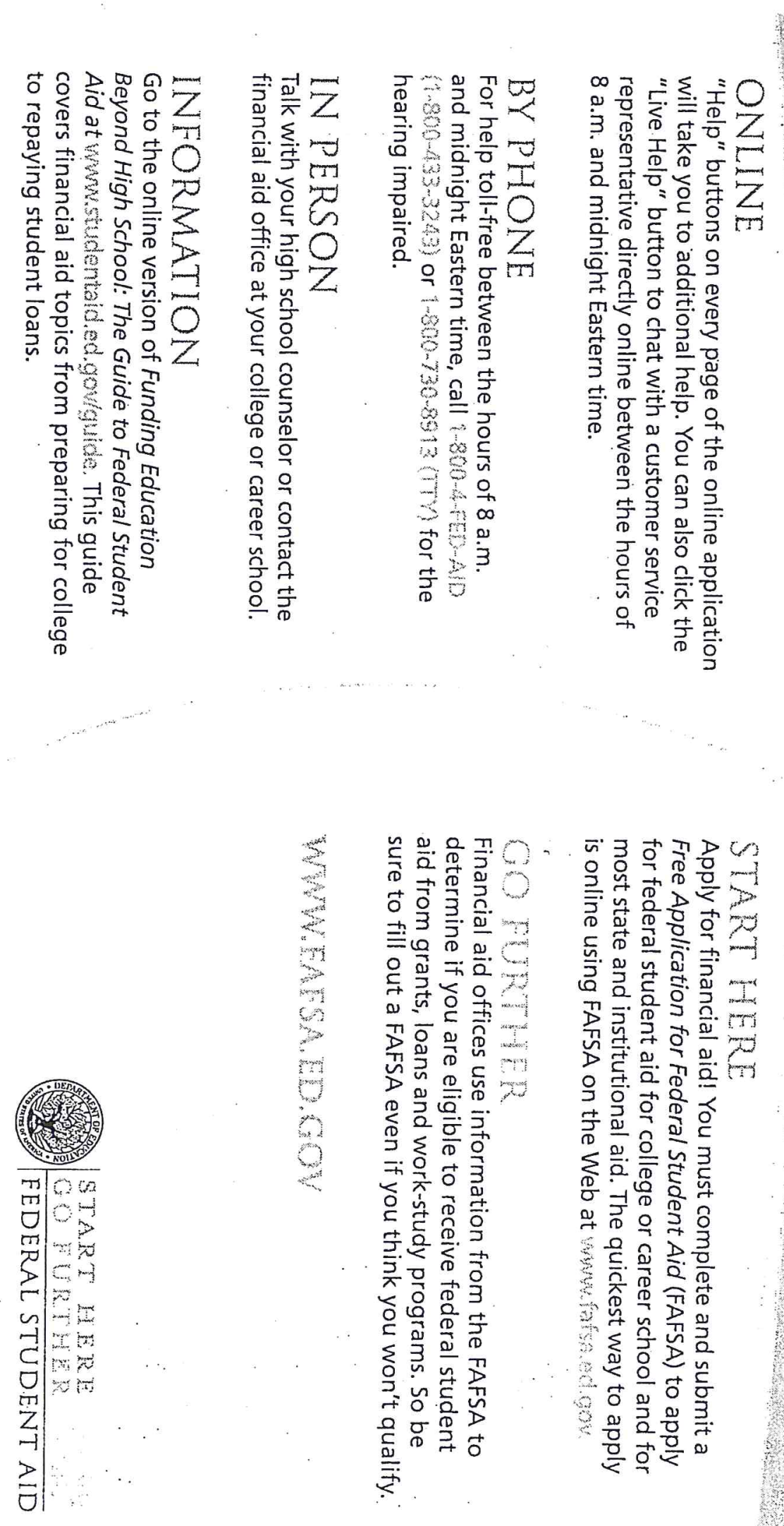


\section{I have to wark}

You aren't alone. Many high school students work parttime and still manage to get good grades. In college, that won't change. You'll find that many college students work and carry a full load of classes. Tip: Look for a school that offers flexibility, such as online classes, night classes, weekend classes and part-time study.

\section{My friends aren't planning to go to college}

That's too bad! Maybe they will change their minds. In the meantime, don't let your friends discourage you. Getting an education is the right thing for you. Follow your own dreams - not someone else's.

\section{I have a baby}

You won't be the only one. Many college students juggle school and family obligations, but they keep going because they want to make a better life for themselves and their children. Look for a college that provides day care and other assistance-many do.

\section{Lodk at YquR Options}

Going to college doesn't have to mean living in a dorm at a state university. You have many options for higher education-including options for where you live.

\section{Community colleges}

Community colleges offer two-year degree and certificate courses. They're usually flexible in admissions policies, so if you haven't done well in high school, they can offer you a fresh start. Some states are introducing new programs that offer automatic acceptance into a university if you've succeeded at a community college.

Another plus: Community colleges are cheaper. You can complete your first two years of study at a community college, and then transfer your credits to a four-year college or university to obtain your bachelor's degree.

\section{Four-year colleges or universities}

A four-year college or university will offer you more advanced classes and may have a more challenging environment. If you want to pursue a graduate degree, you may be able to go straight from your bachelor's courses to master's level courses at the same university.

\section{Live at home or on campus}

You'll also have to look ahead and decide if you want (or need) to live at home during college. Often, you'll find that colleges near home offer some great programs. Just make sure that your local college has the classes you need. One other thing to consider: While it probably will be less expensive to live at home during college, you may miss out on some of the social life and experiences that come with living on campus.

\section{ANOTHEROPION:

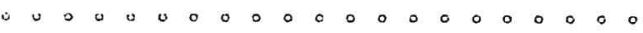

\section{Career or Technical Schools}

\author{
If your dream job requires techni- \\ cal training, you may want to go to \\ a career or technical school. As with \\ any callege, it's important to check \\ out a technical school's curricu- \\ lum, credentials and job-place- \\ ment success to ensure it \\ meets your goals.
}




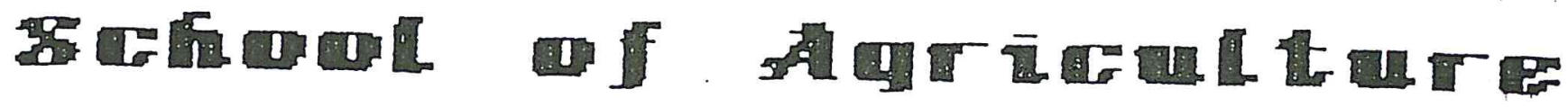
con al

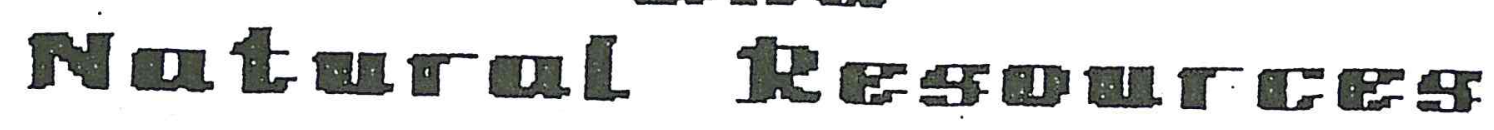

\section{Categt Wfitert}

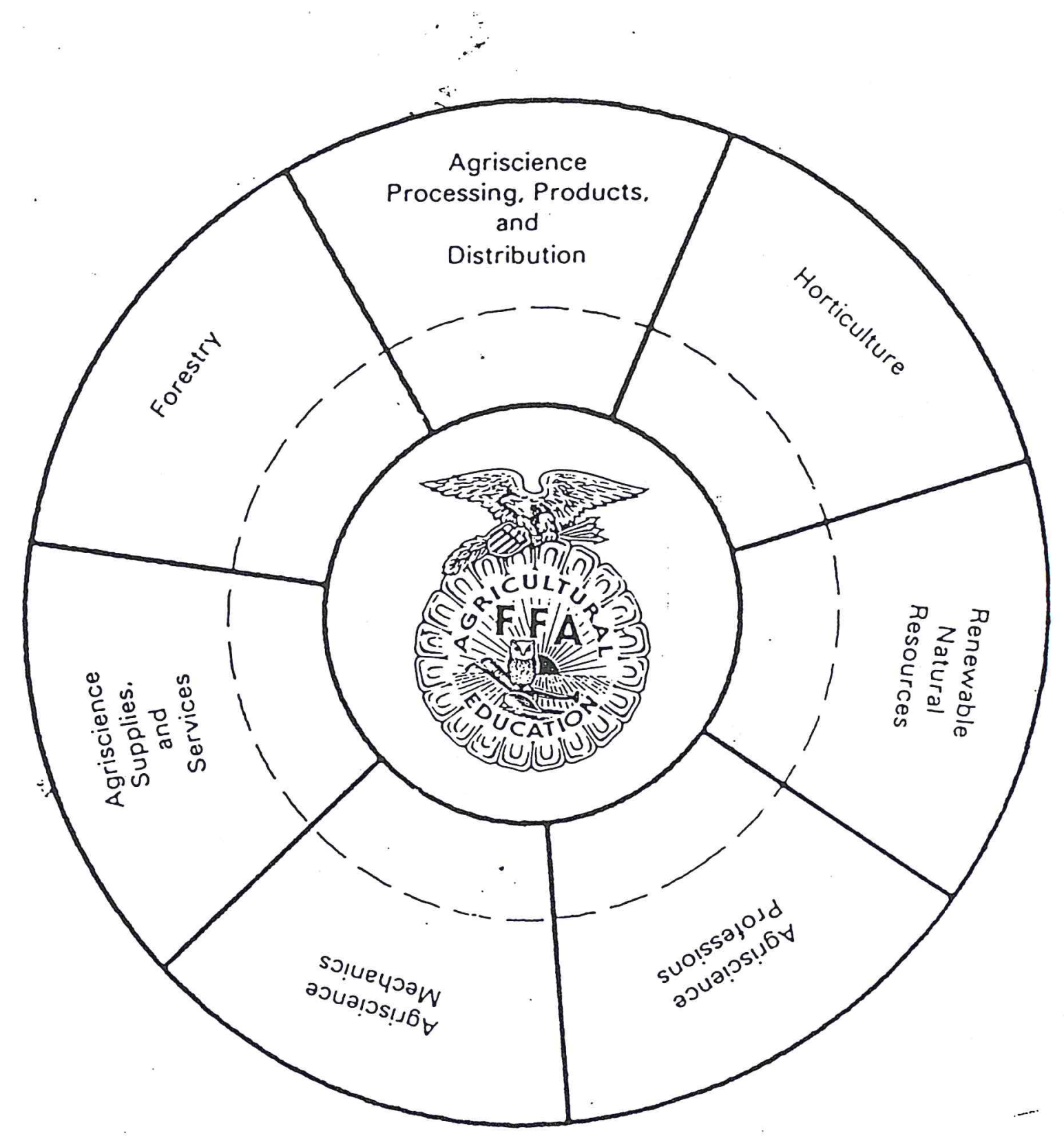




\section{Supplies, and Services}

Aerial Crop Duster

Ag Aviator

Ag Chemical Dealer

Ag Equipment Dealer

Animal Groomer

Animal Health Products Distributor

Animal Inspector

Animal Keeper

Animal Trainer

Artificial Breeding Distributor

Artificial Breeding Technician

Artificial Inseminator

Biostatician

Chemical Applicator

Chemical Distributor

Computer Analyst

Computer Operator

Computer Programmer

Computer Salesperson

Custom Operator

Dairy Management Specialist

Dog Groomer

Farm Appraiser

Farm Auctioneer

Farrier

Feed Mill Operator
Feed Ration'Developer \& Analyst

Fertilizer Plant Supervisor

Fiber Technologist

Field Inspector

Field Sales Representative, Agricultural Equipment Field Sales Representative, Animal Health Products

Field Sales Representative, Crop Chemicals, Machinery Harness Maker

Harvest Contractor

Horse Trainer

Insect \& Disease Inspector

Kennel Operator

Lab Technician

Meteorological Analyst

Pest Control Technician

Pet Shop Operator

Poultry Field Service Technician

Poultry Hatchery Manager

Poultry Inseminator

Sales Manager

Salesperson

Service Technician

Sheep Shearer 


\section{Renewable \\ Natural \\ Resources}

Animal Behaviorist

Animal Ecologist

Animal Taxonomist

Environmental Conservation Officer

Environmentalist

Fire Warden

Forest Fire Fighter/Warden

Forest Ranger

Game Farm Supervisor
Game Warden

Ground Water Geologist

Park Ranger

Range Conservationist

Resource Manager

Soil Conservationist

Trapper

Water Resources Manager

Wildlife Manager

\section{Mechanics}

Ag Construction Engineer

Ag Electrician

Ag Equipment Designer

Ag Plumber

Ag Safety Engineer

Diesel Mechanic

Equipment Operator

Hydraulic Engineer
Irrigation Engineer

Land Surveyor

Machinist

Parts Manager

Research Engineer

Safety Inspector

Soil Engineer

Welder

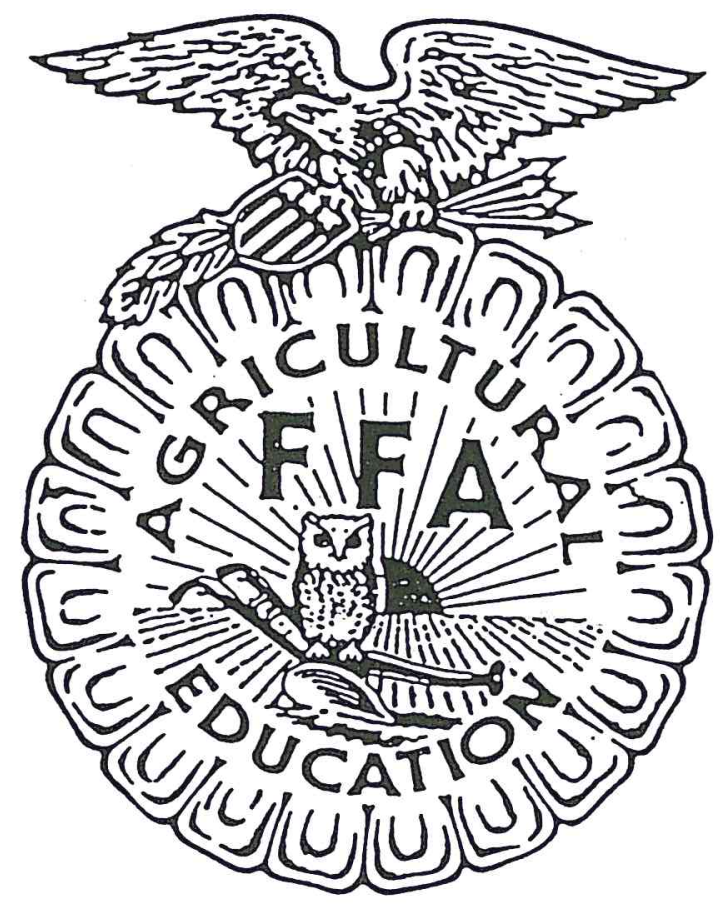



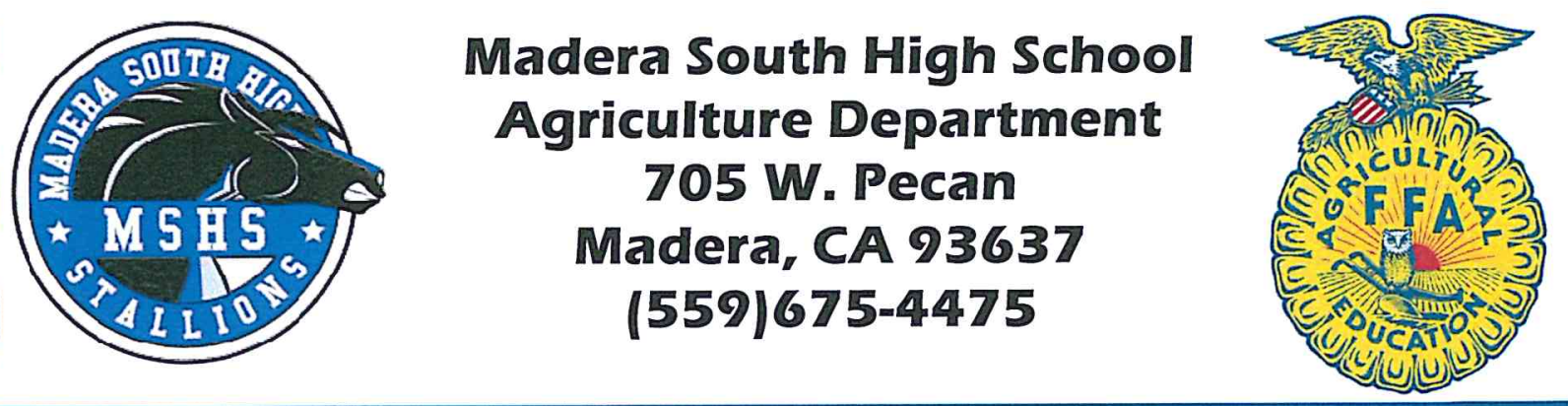

\section{Madera South Program Goals and Objectives}

Goal: $\quad$ The goal of the MSHS Agriculture Department is to increase participation in all SAE's and develop a work experience program for students to participate in.

Objective(s):

- Incorporate innovative SAE opportunities for members, by utilizing all 7 kinds of SAE.

- Develop a career day with industry representatives to come and talk to the FFA members.

- Take the officers to local businesses during the summer to see what is happening in Madera County. 


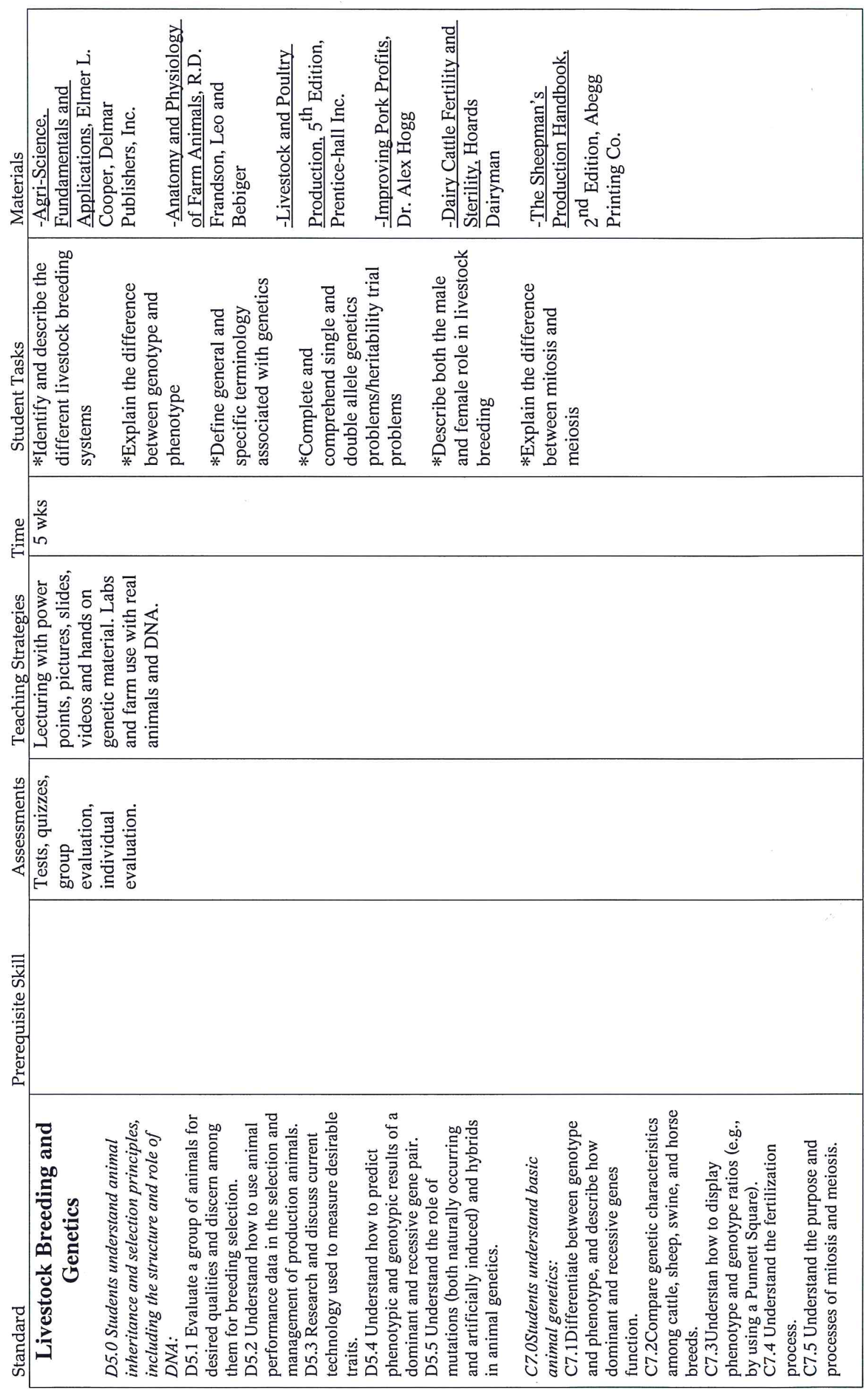



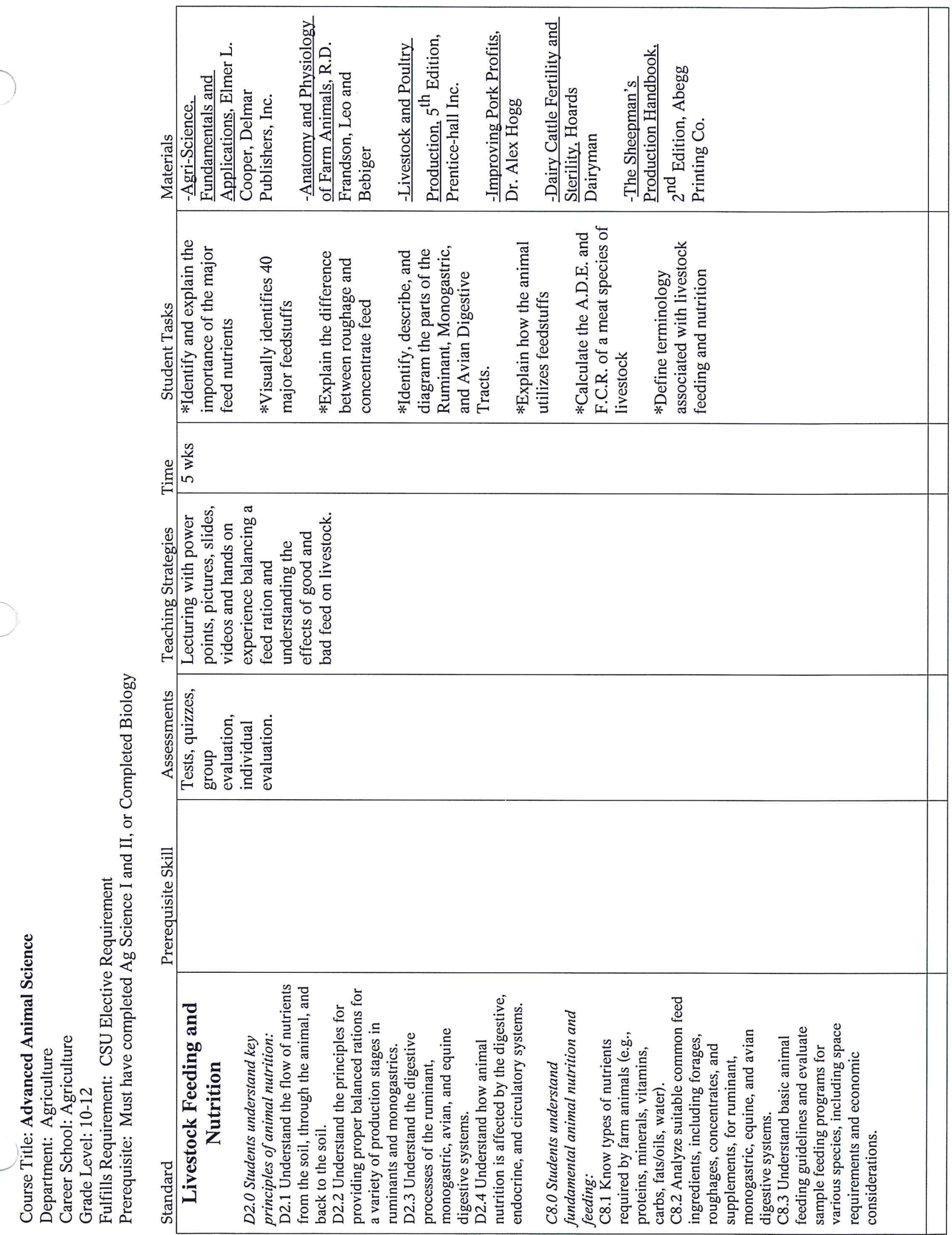

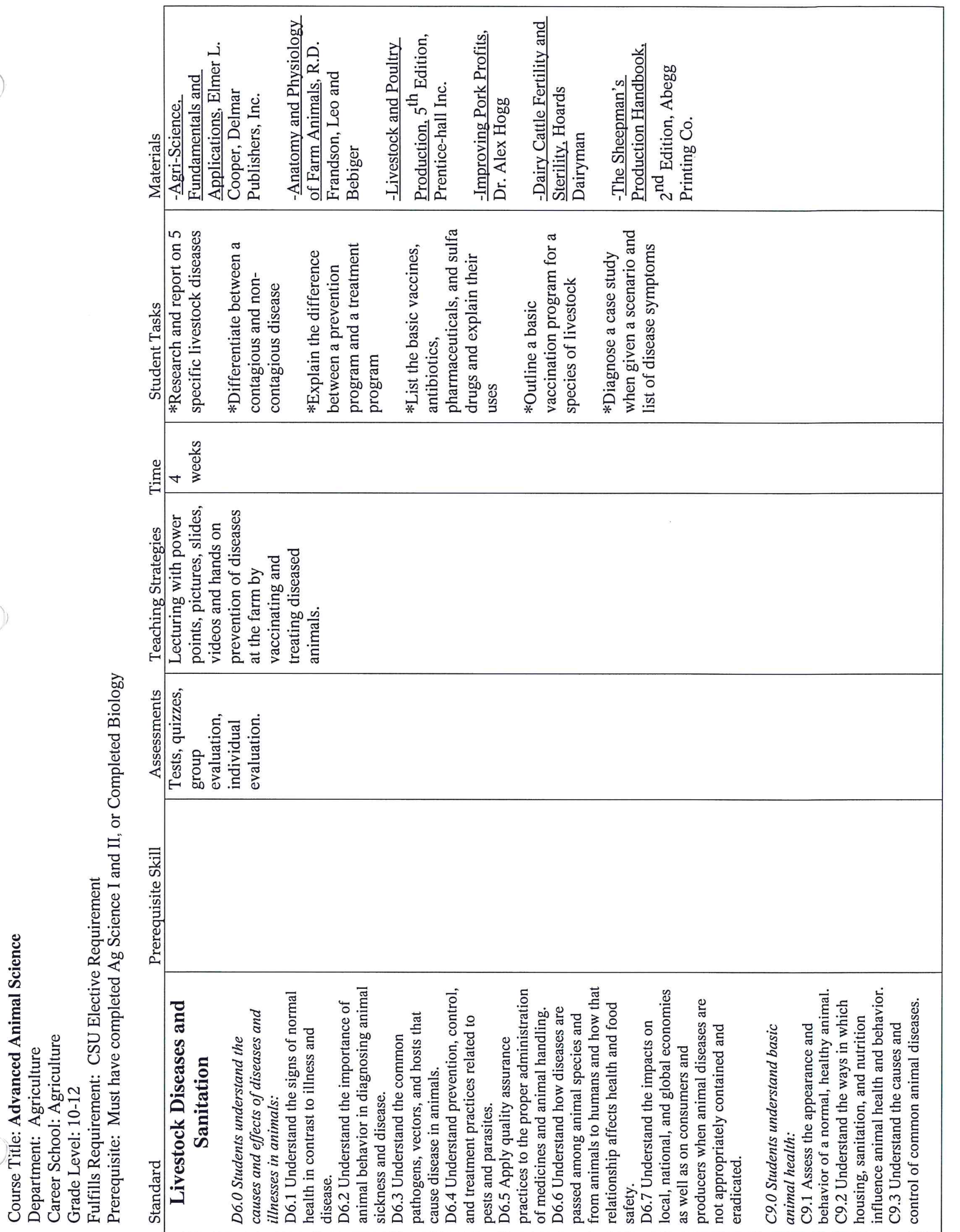

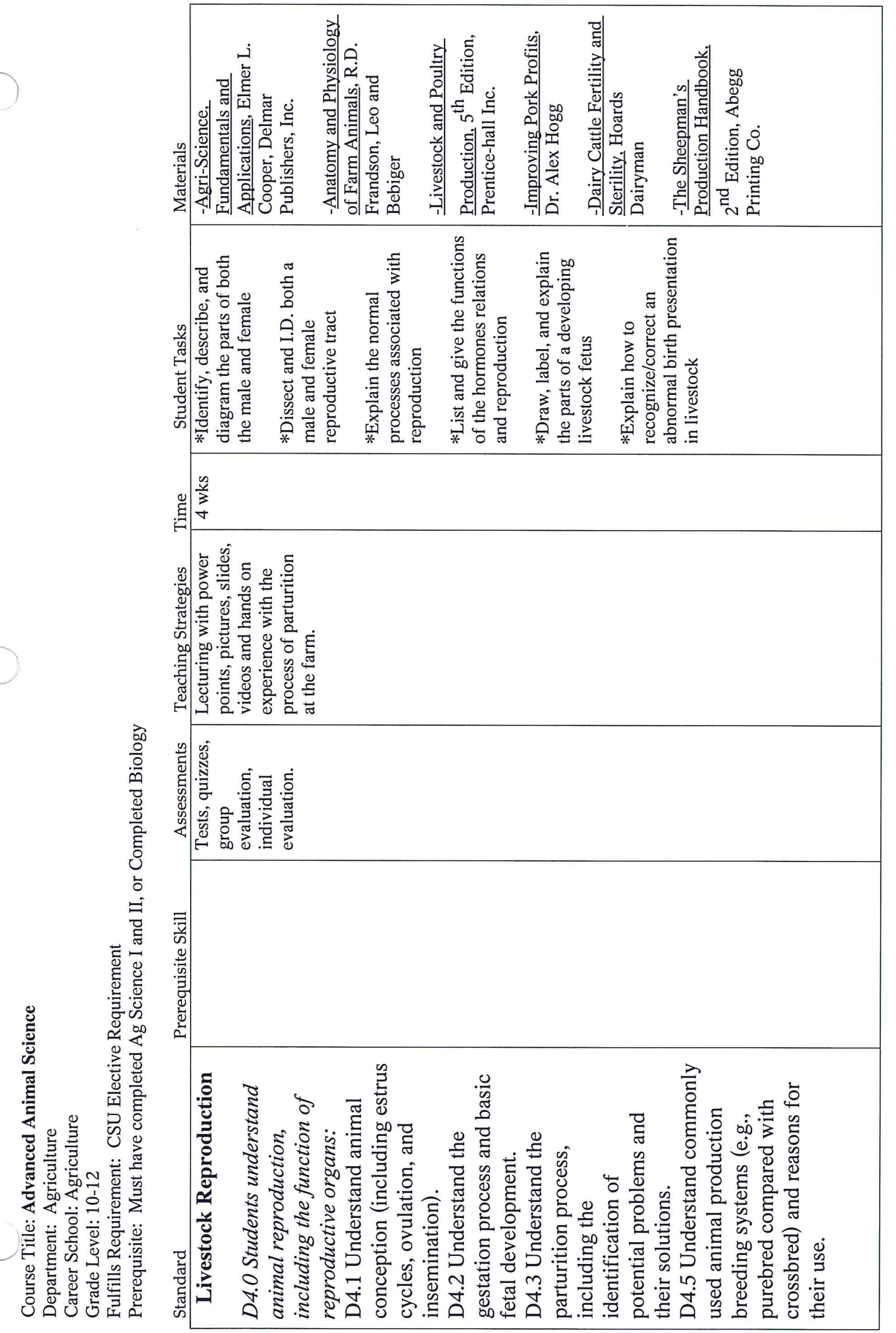


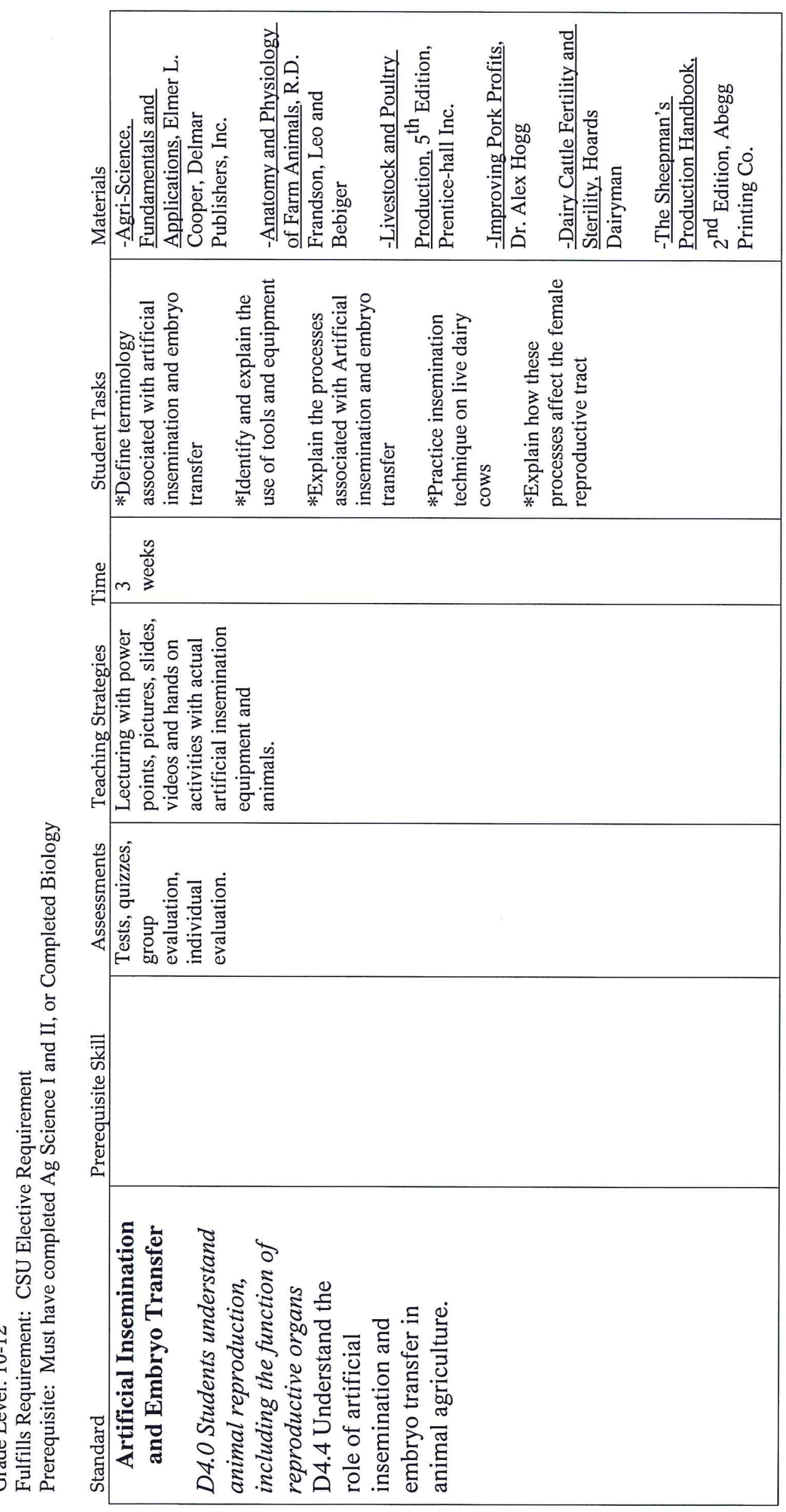




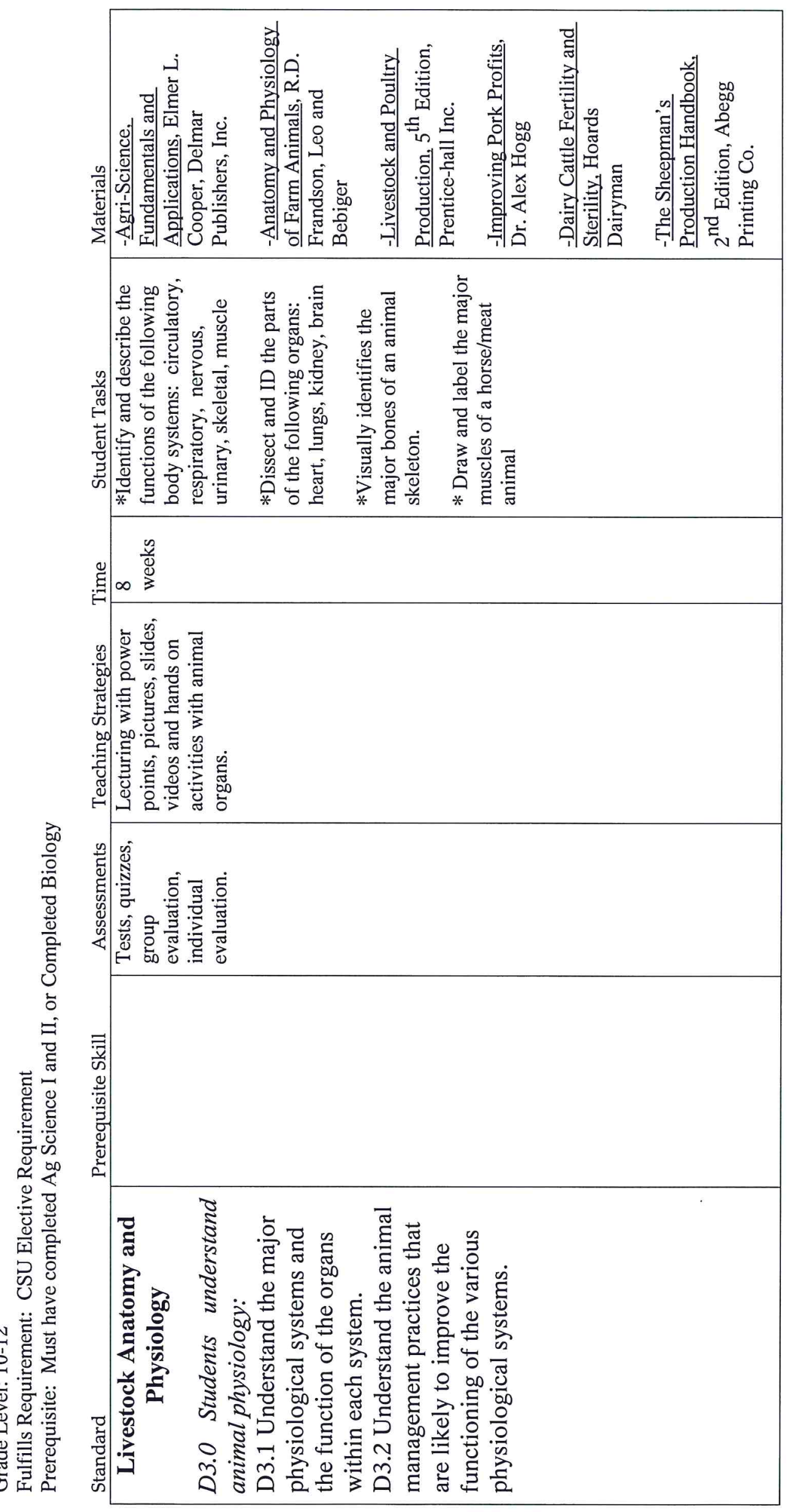

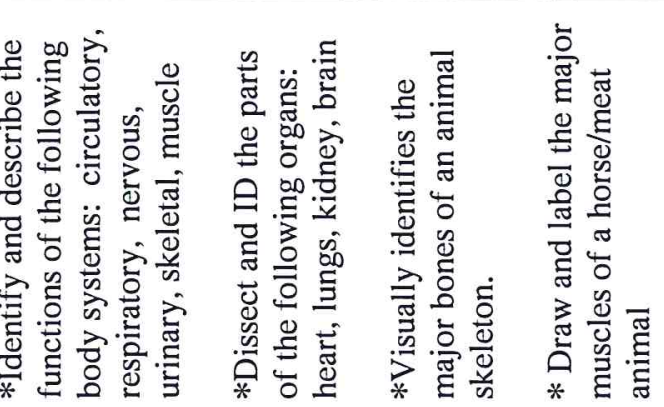



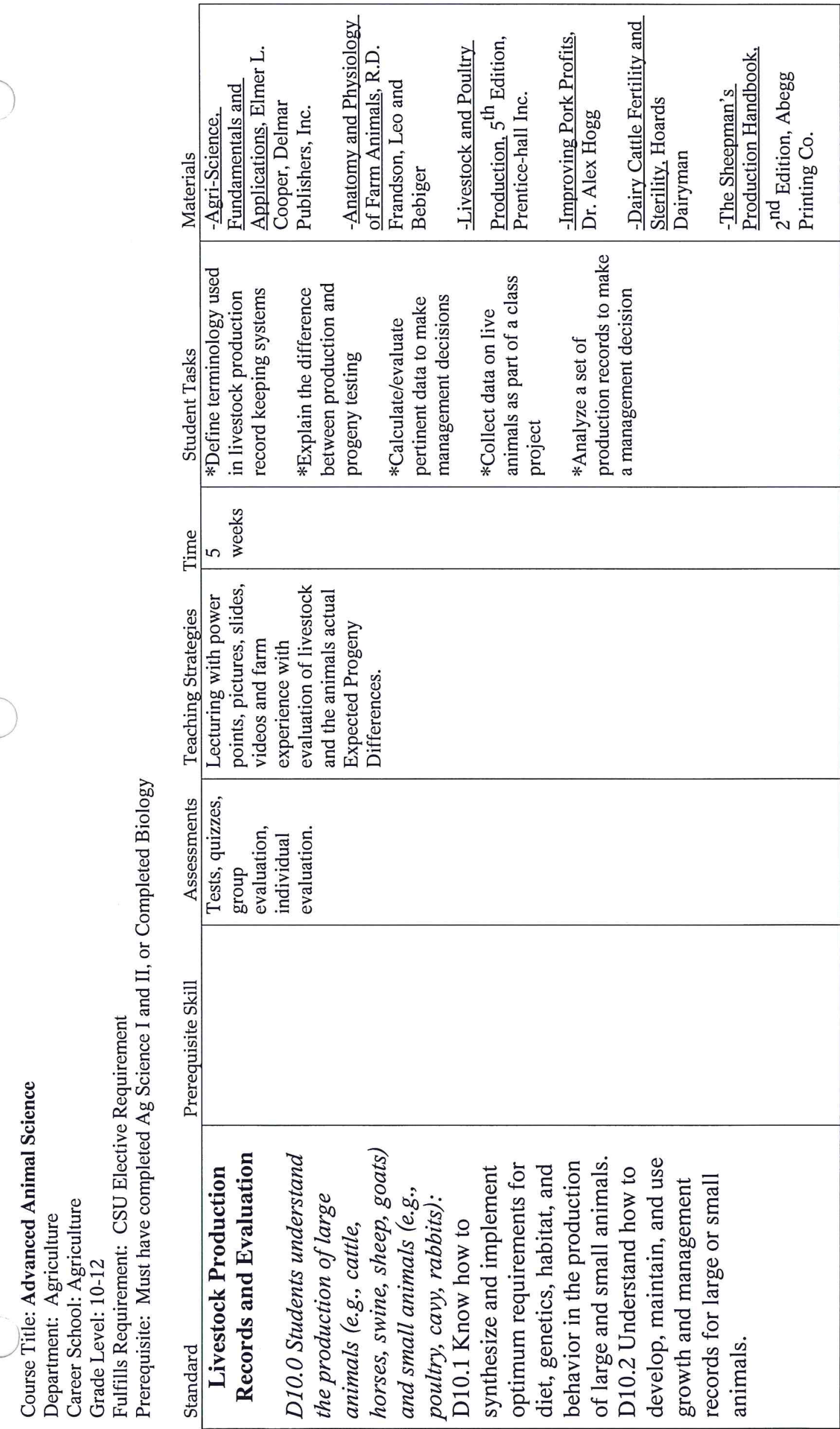

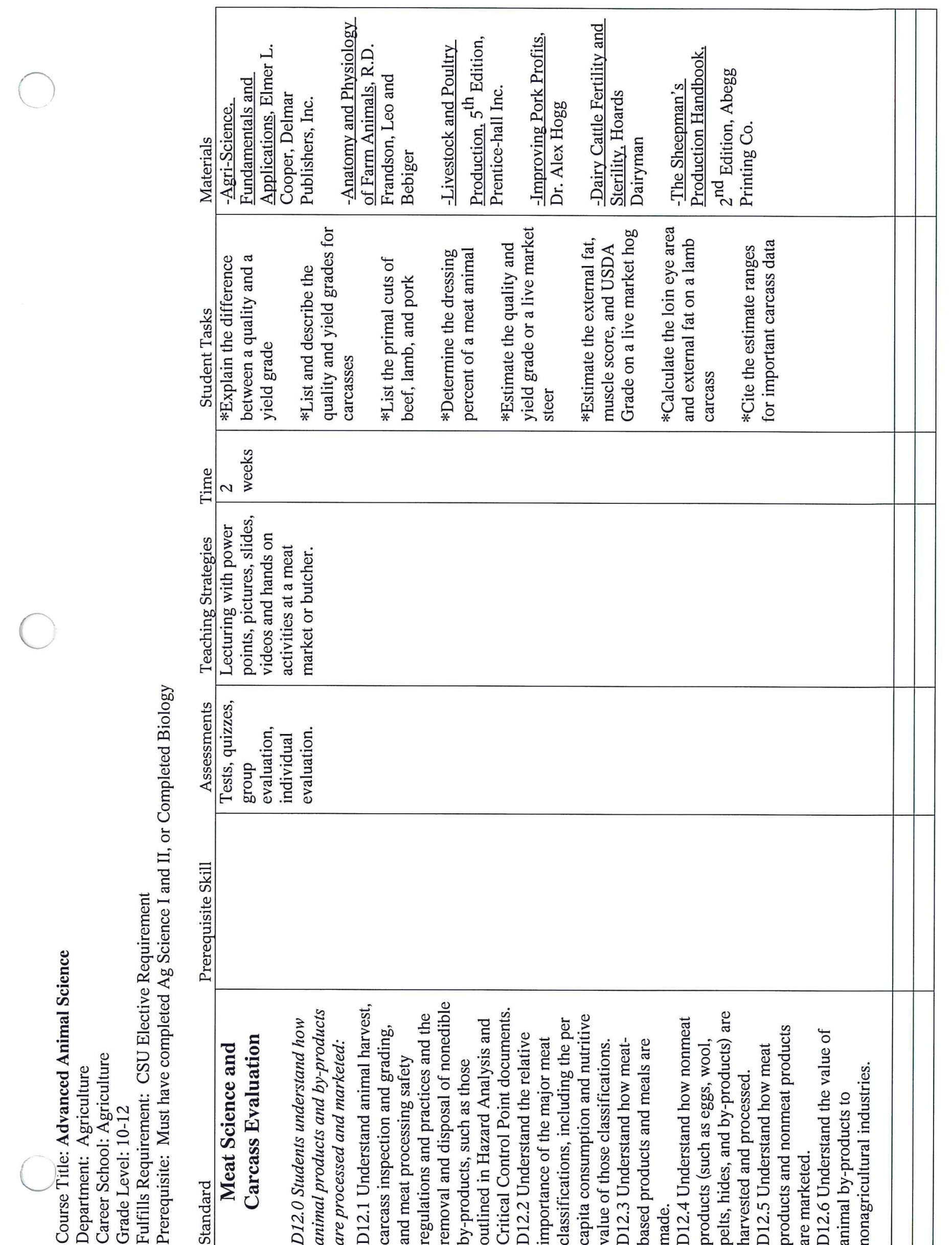

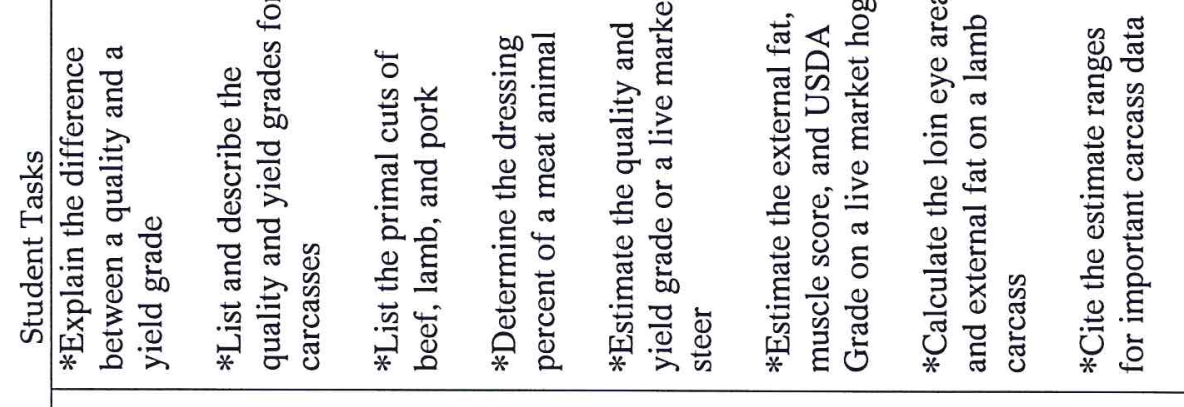

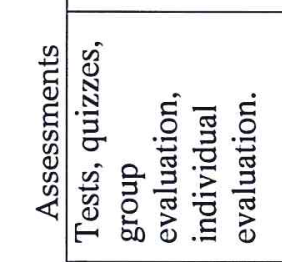

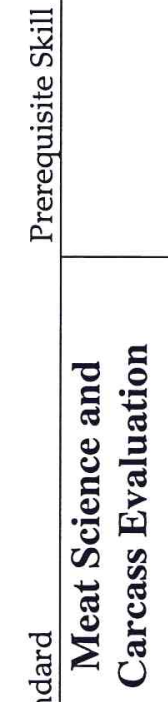

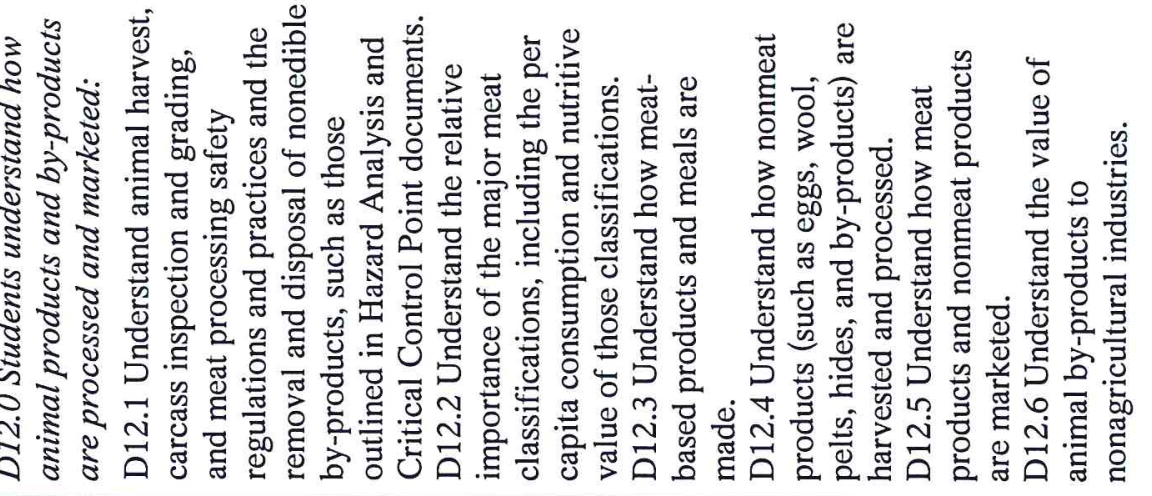




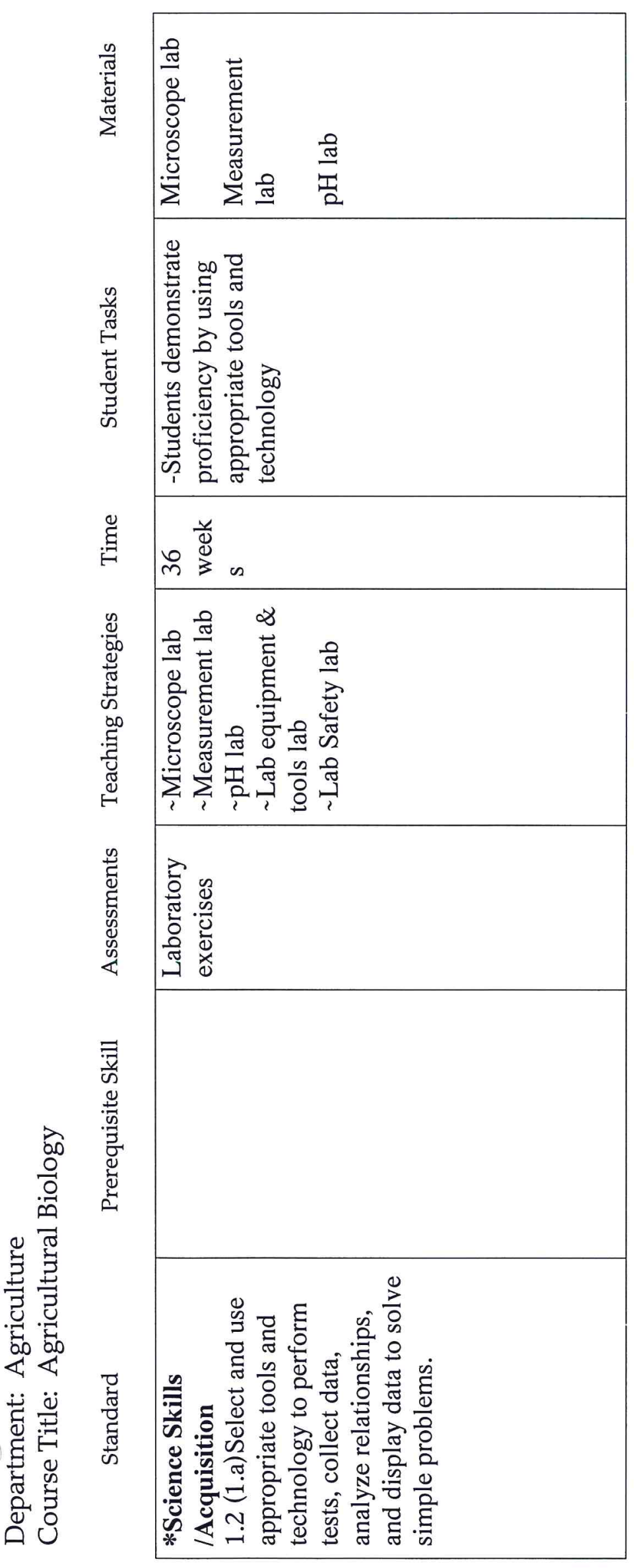




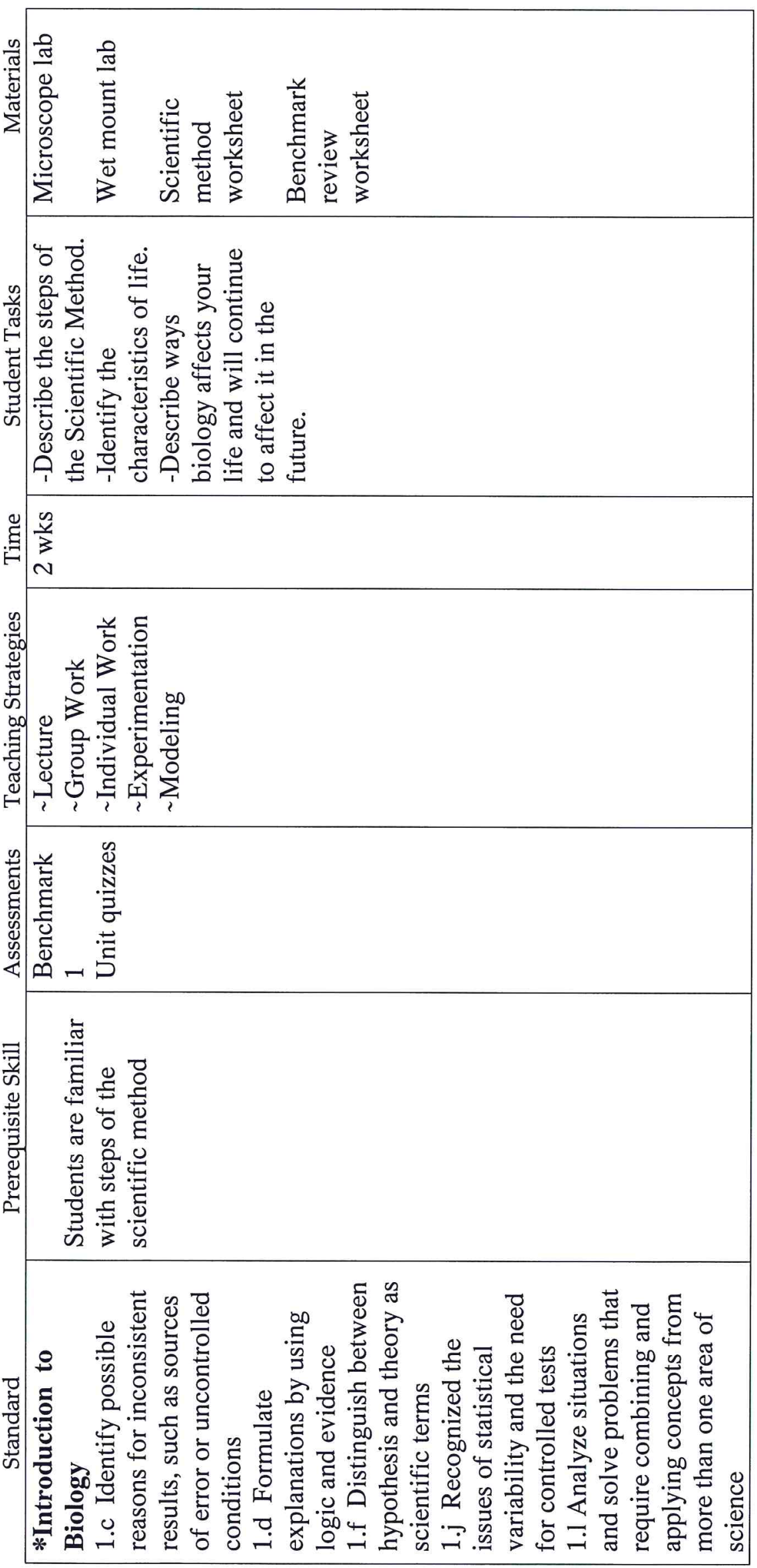




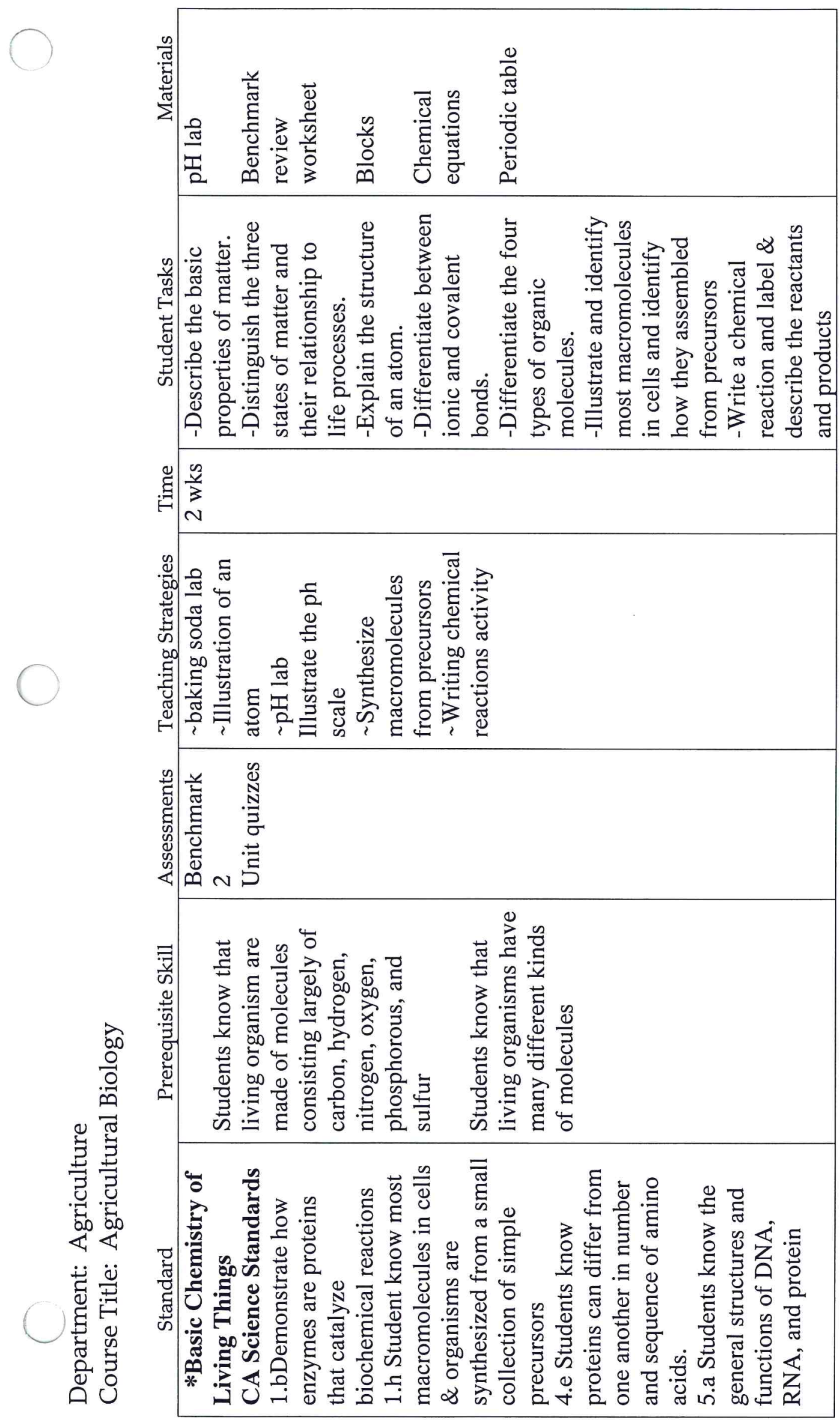



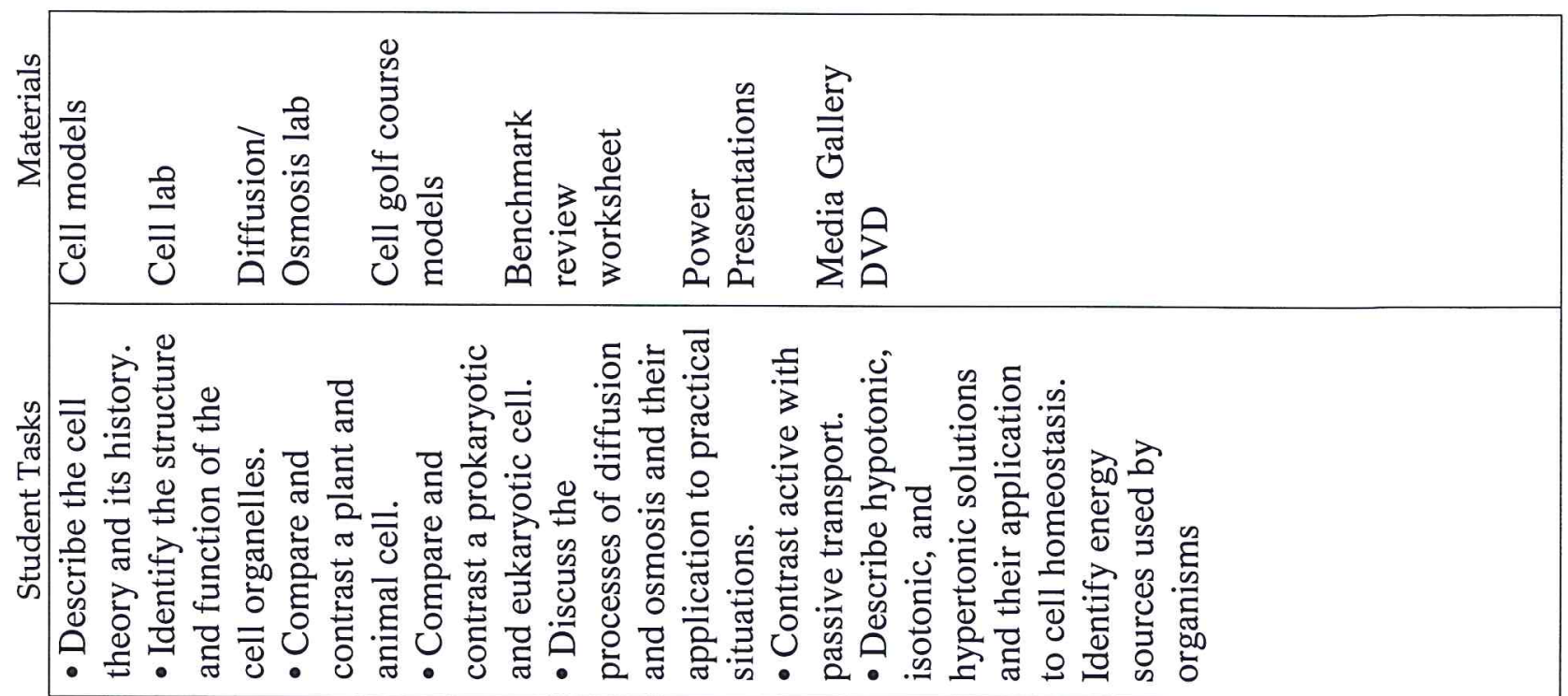

:

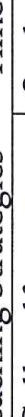

离

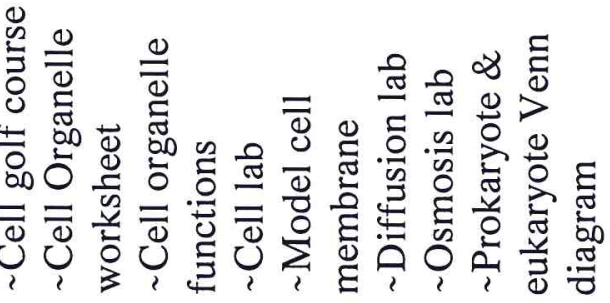

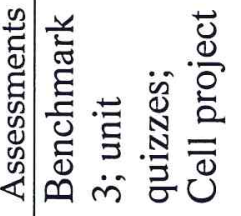

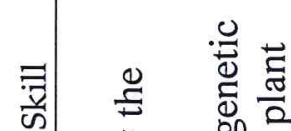

:

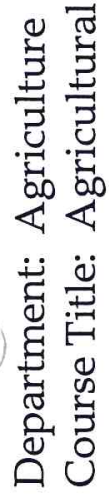

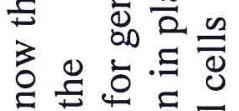

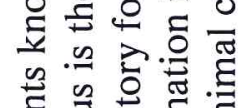

$$
\begin{aligned}
& \text { 氜 }
\end{aligned}
$$

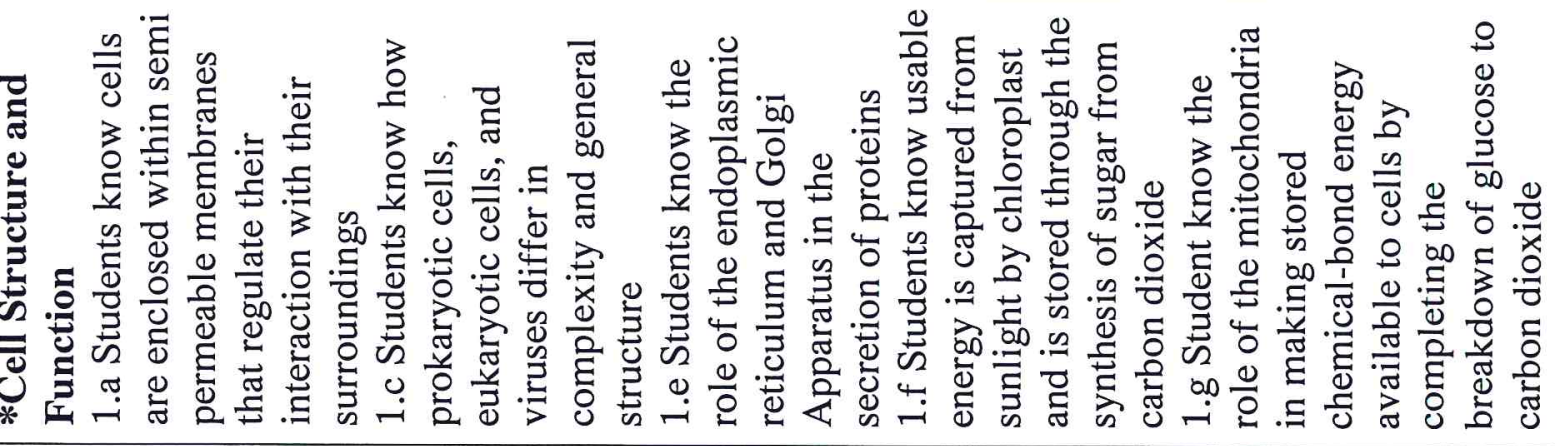




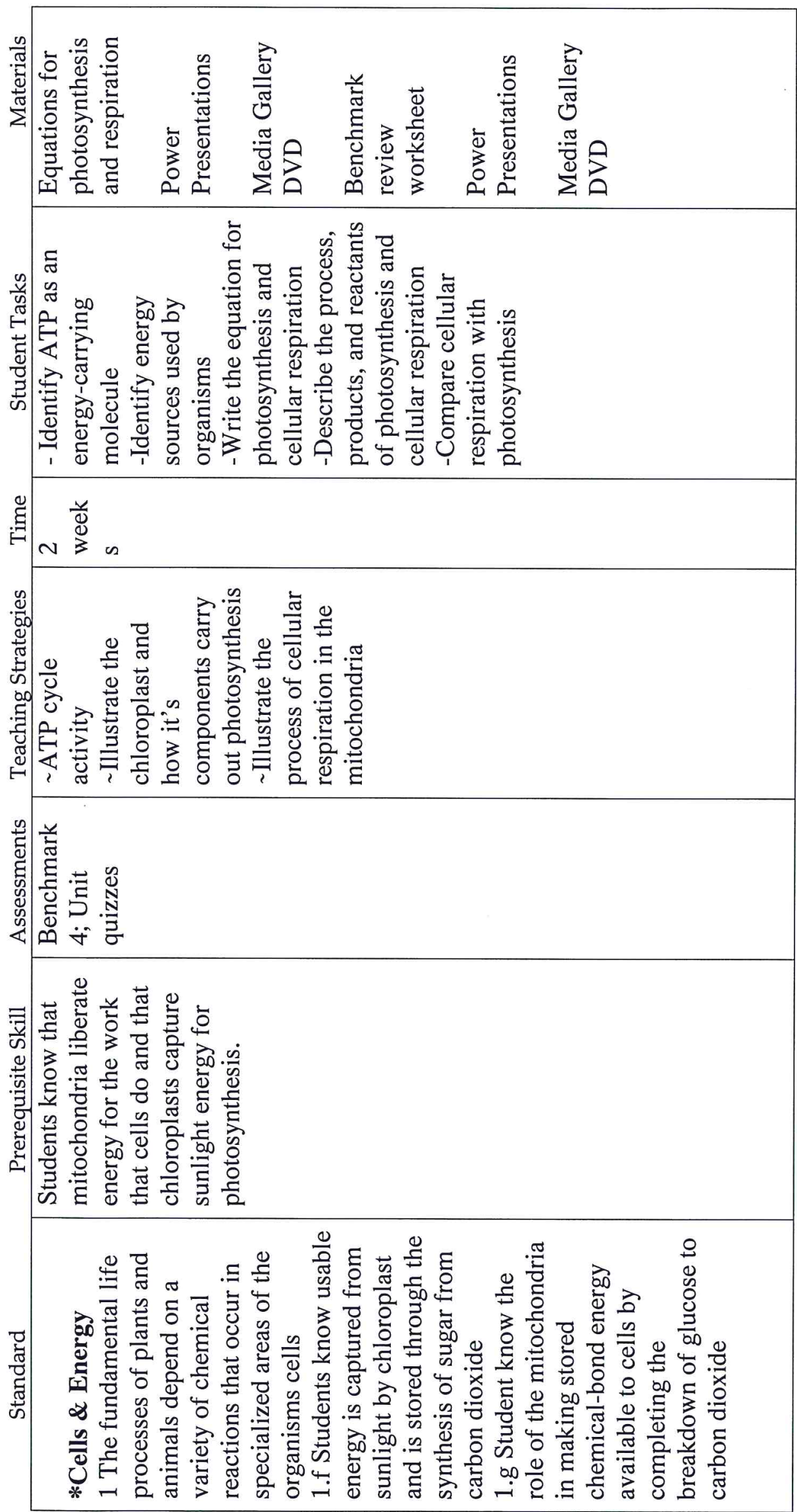




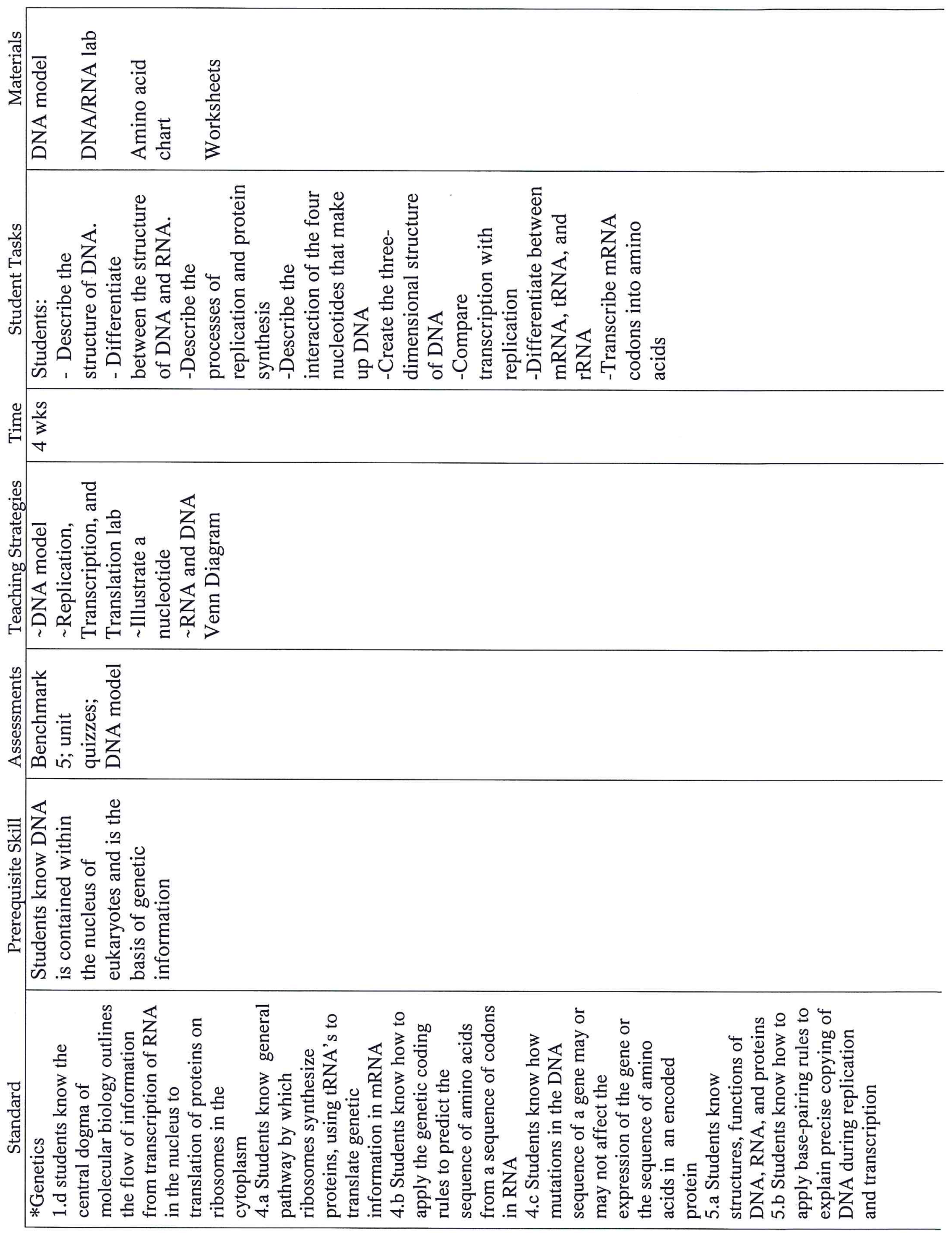




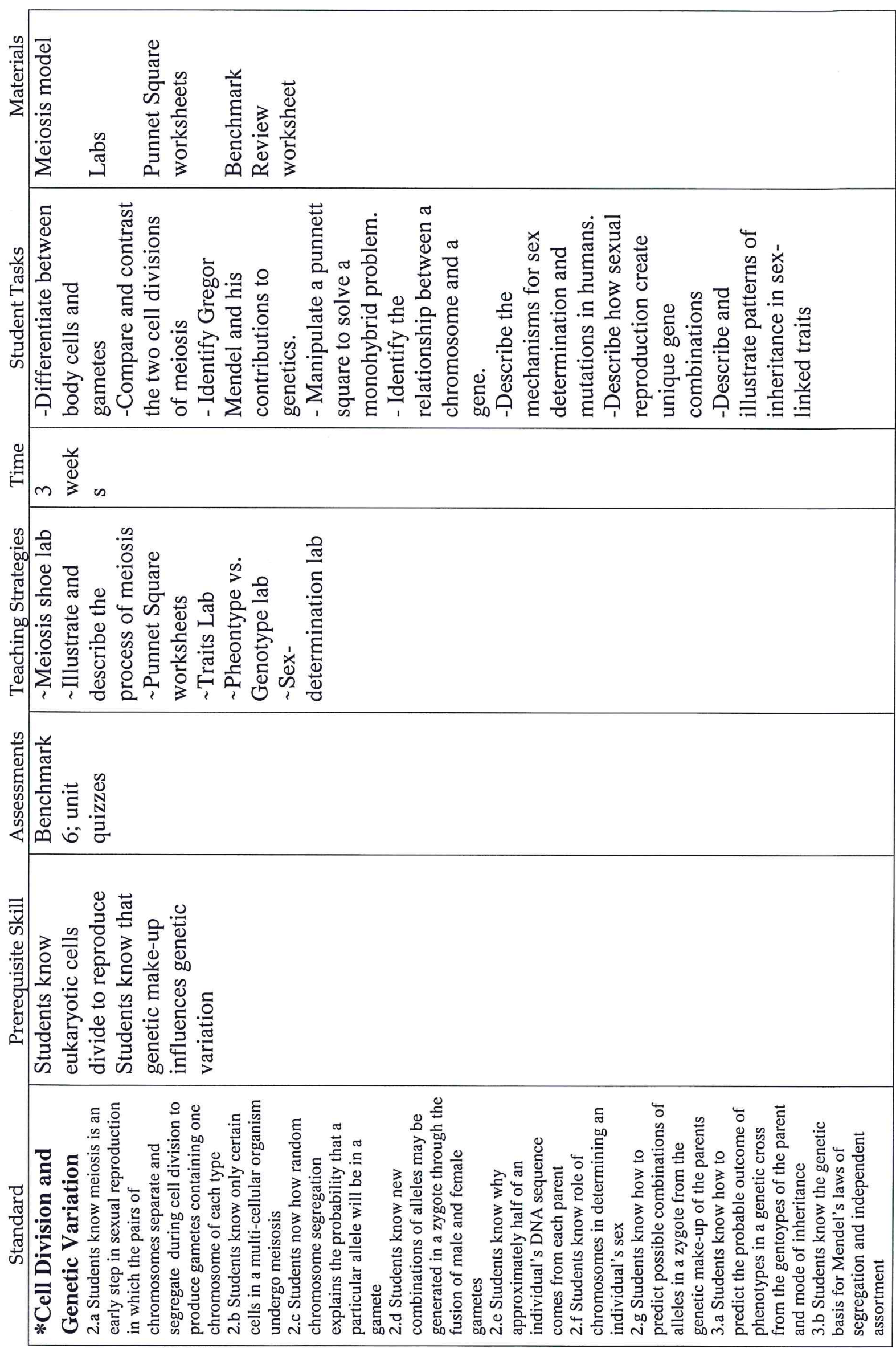




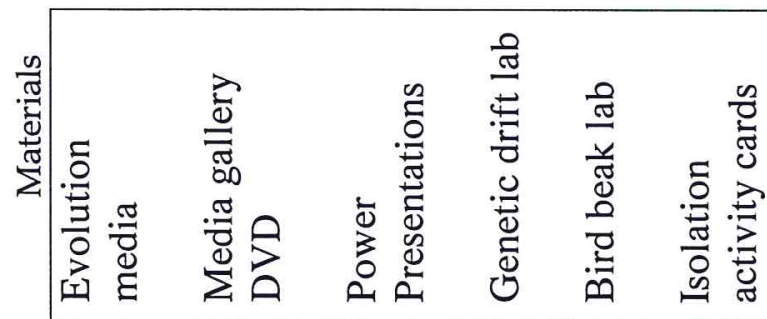
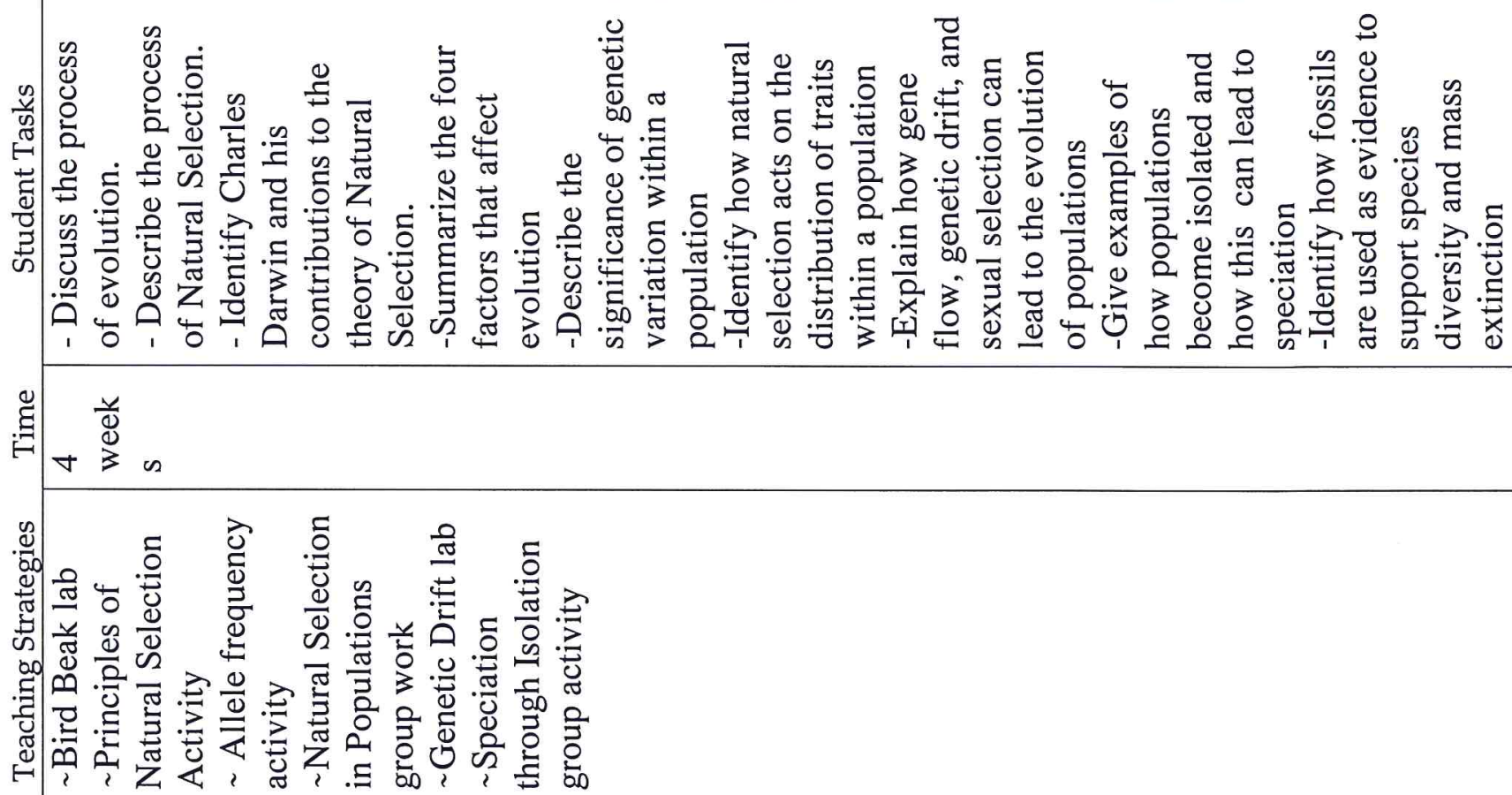

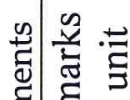

空

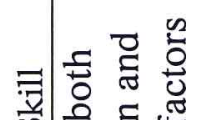

क

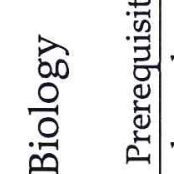

苟

窇营

苟 0

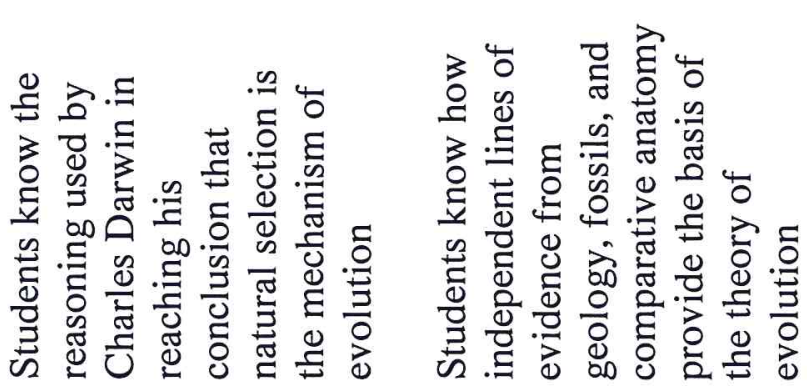

कू

$\varangle<$

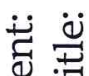

记

द्व के

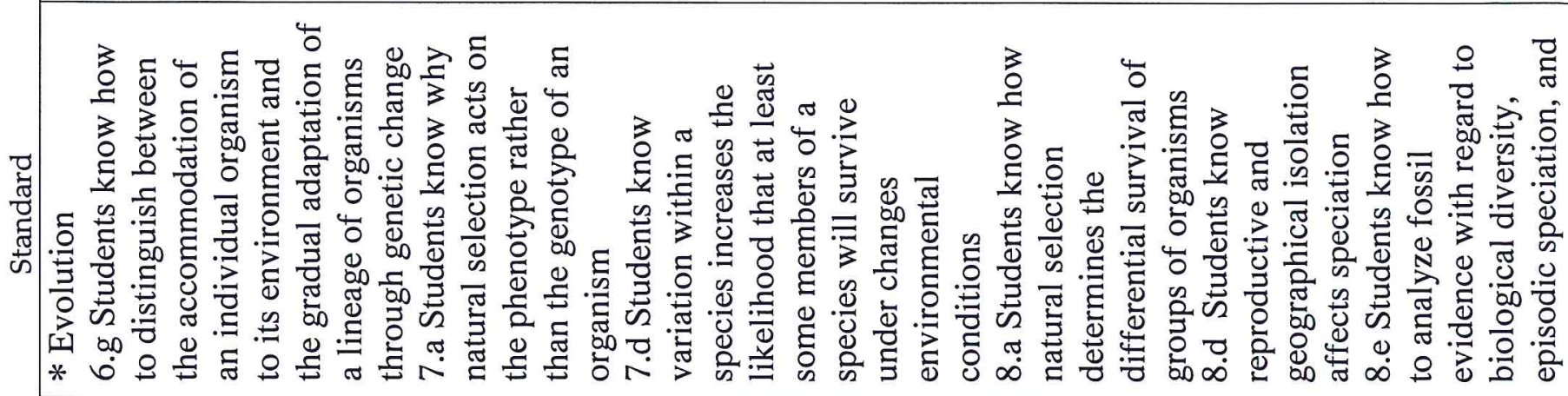




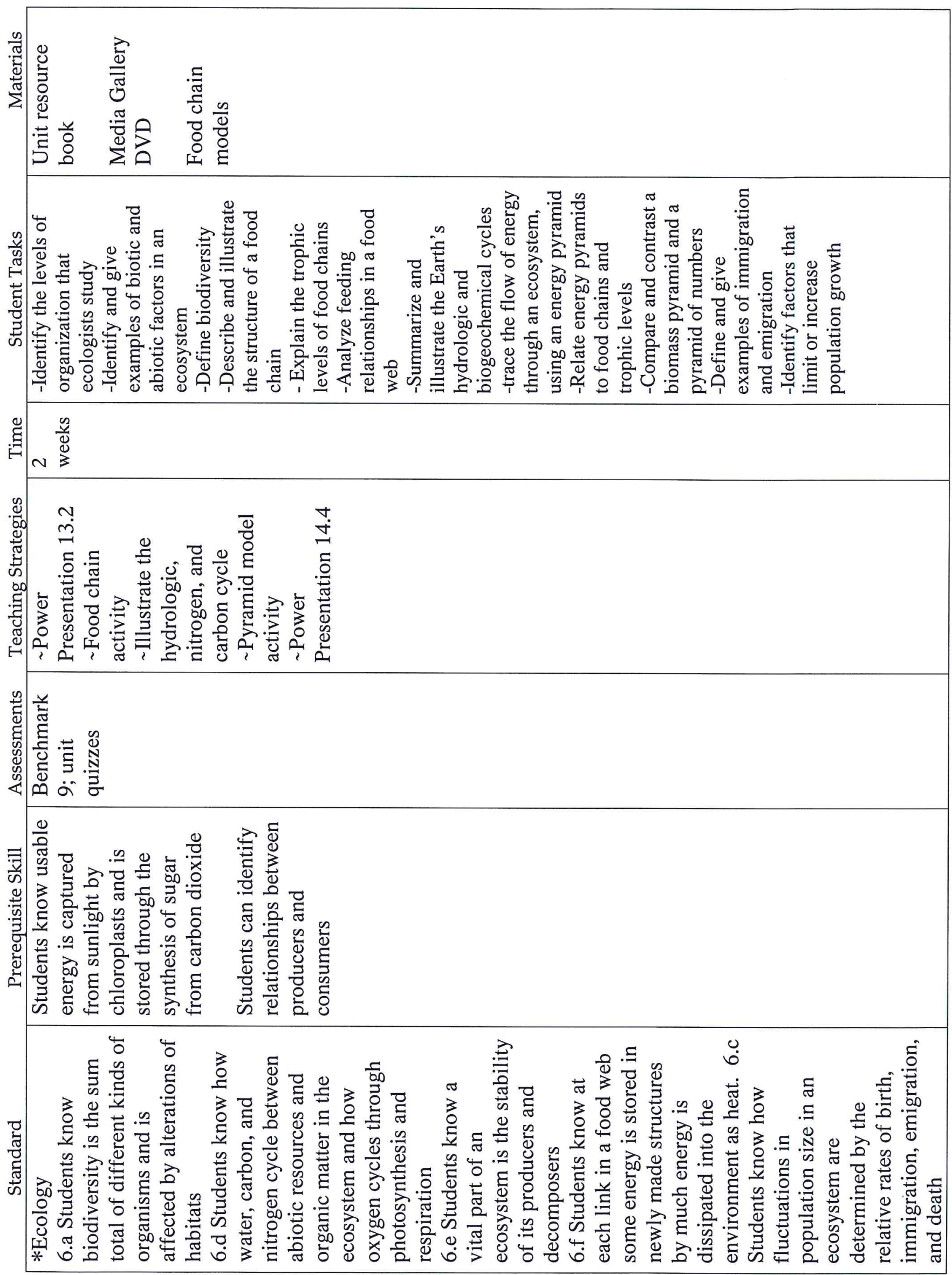




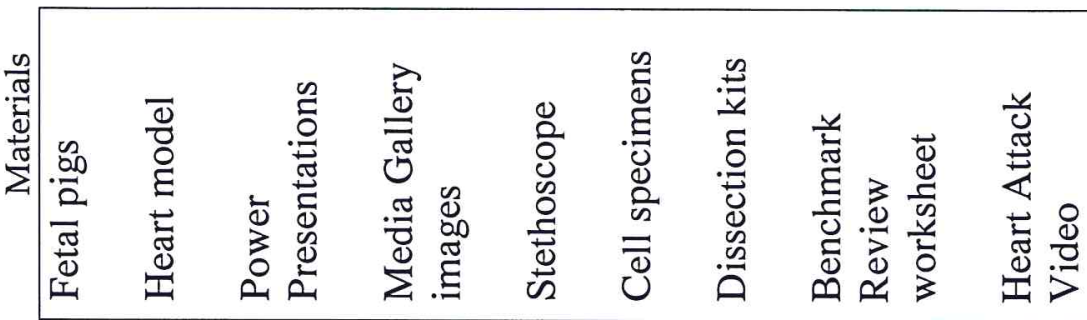

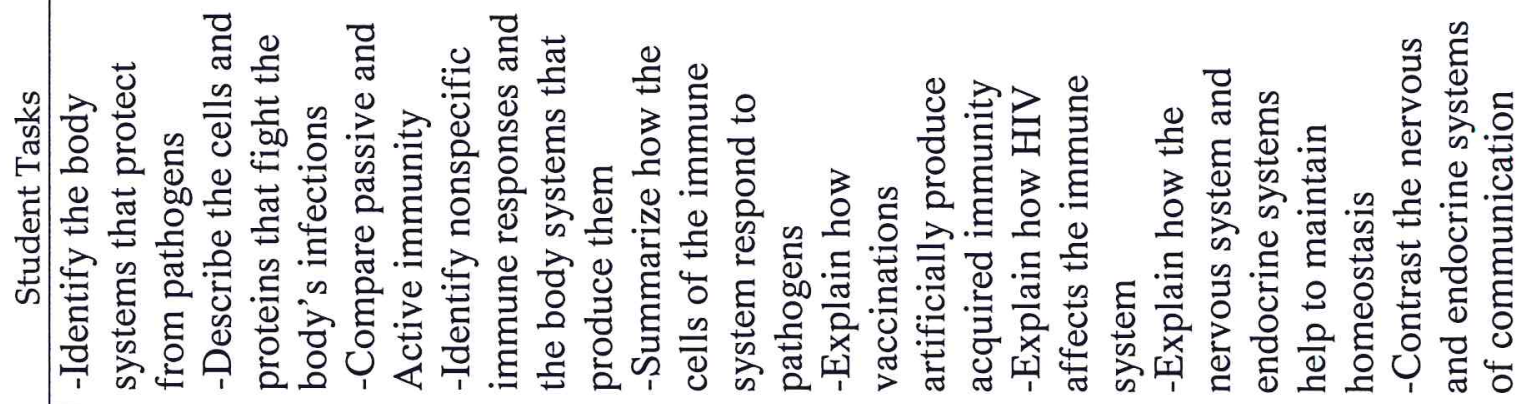

:

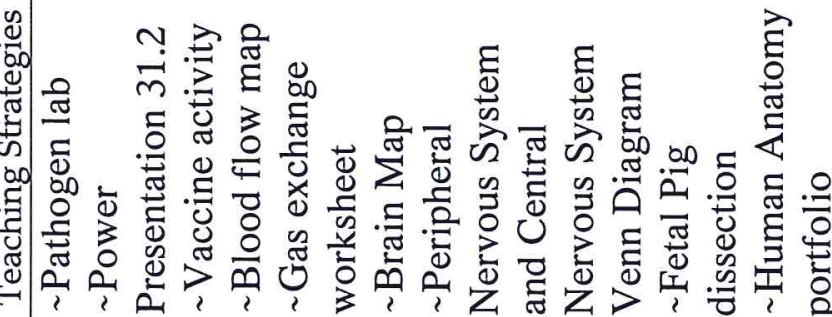

䒕

E.

ص⿺辶一

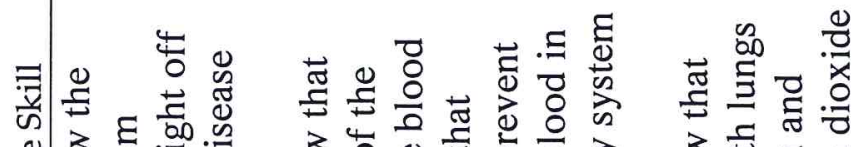

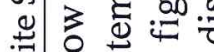

응

望壱

常

$\varangle$

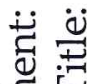

ह

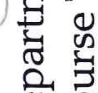

峛

㲾品

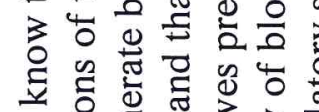

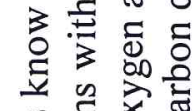

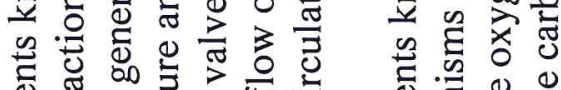

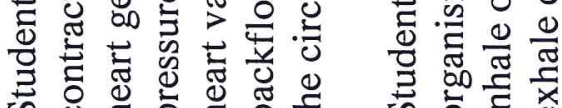

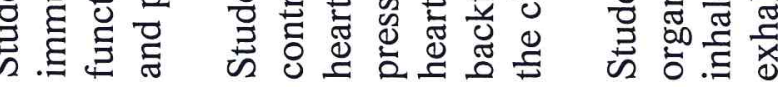

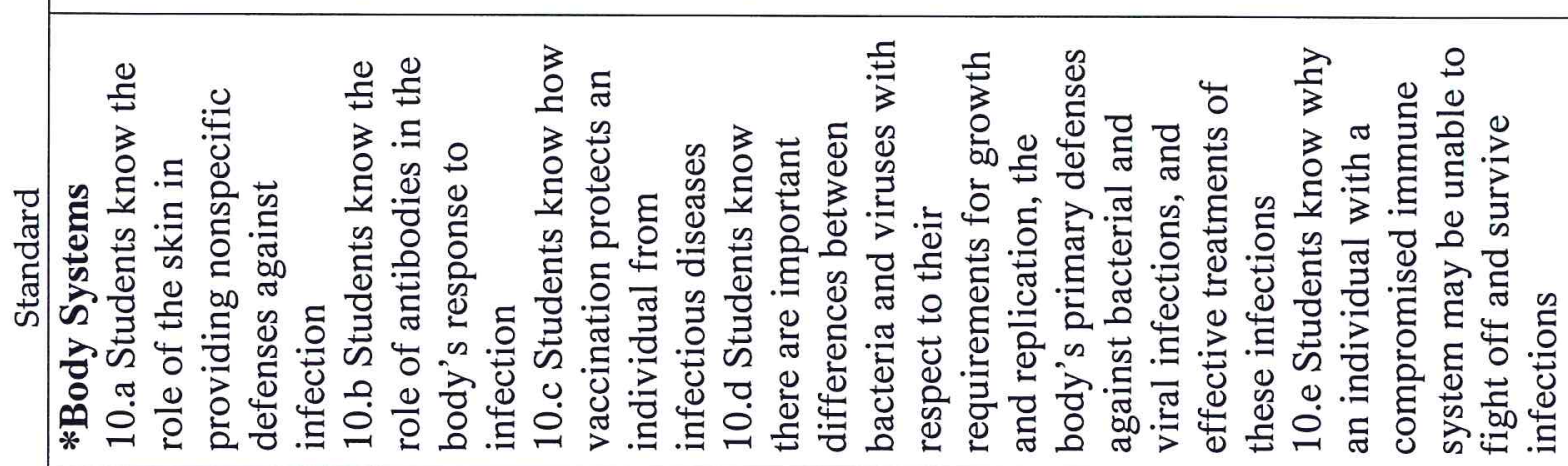




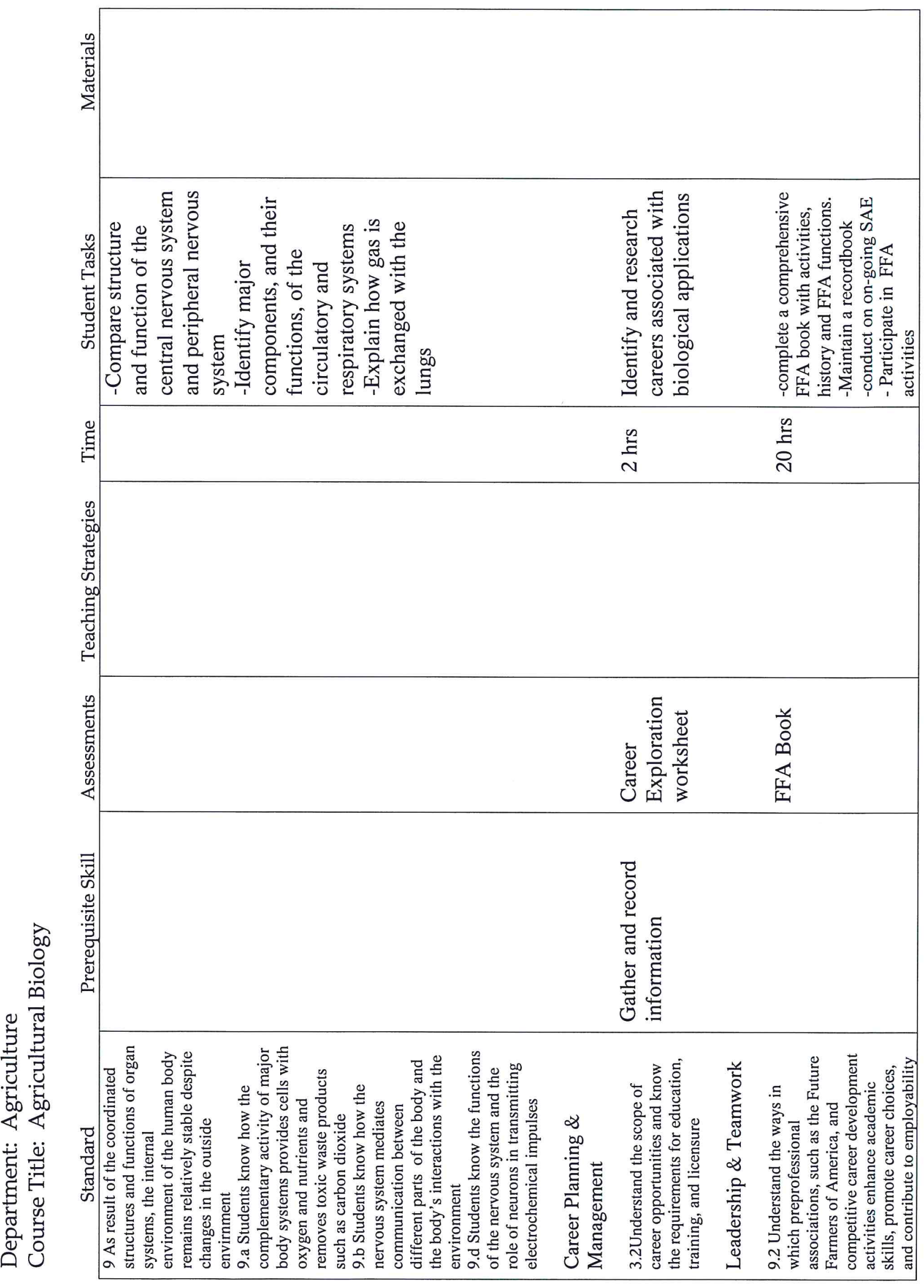




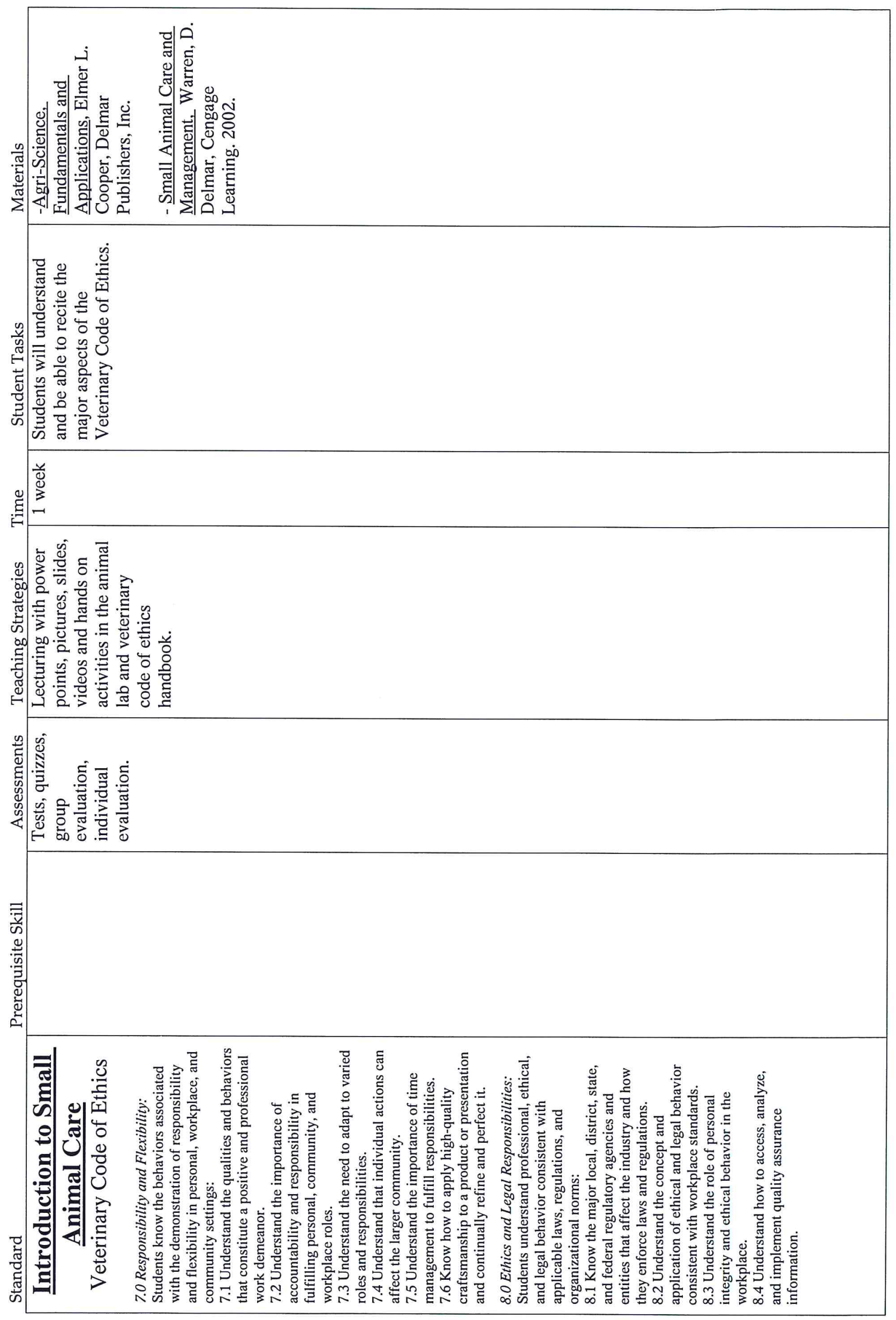




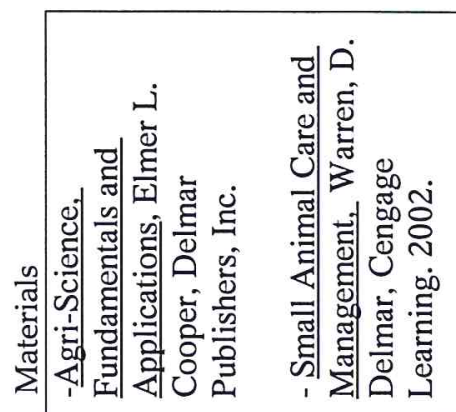

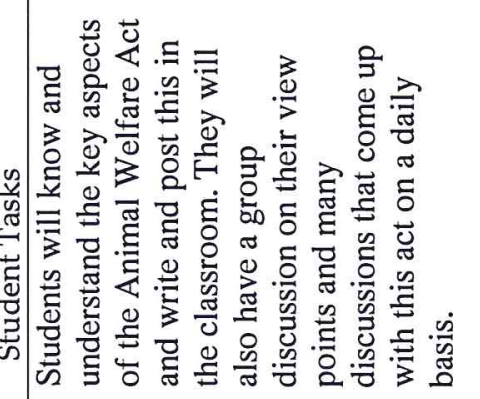

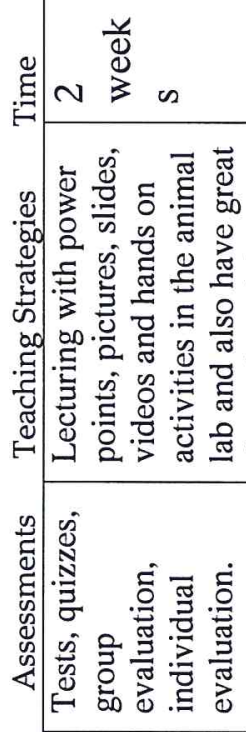
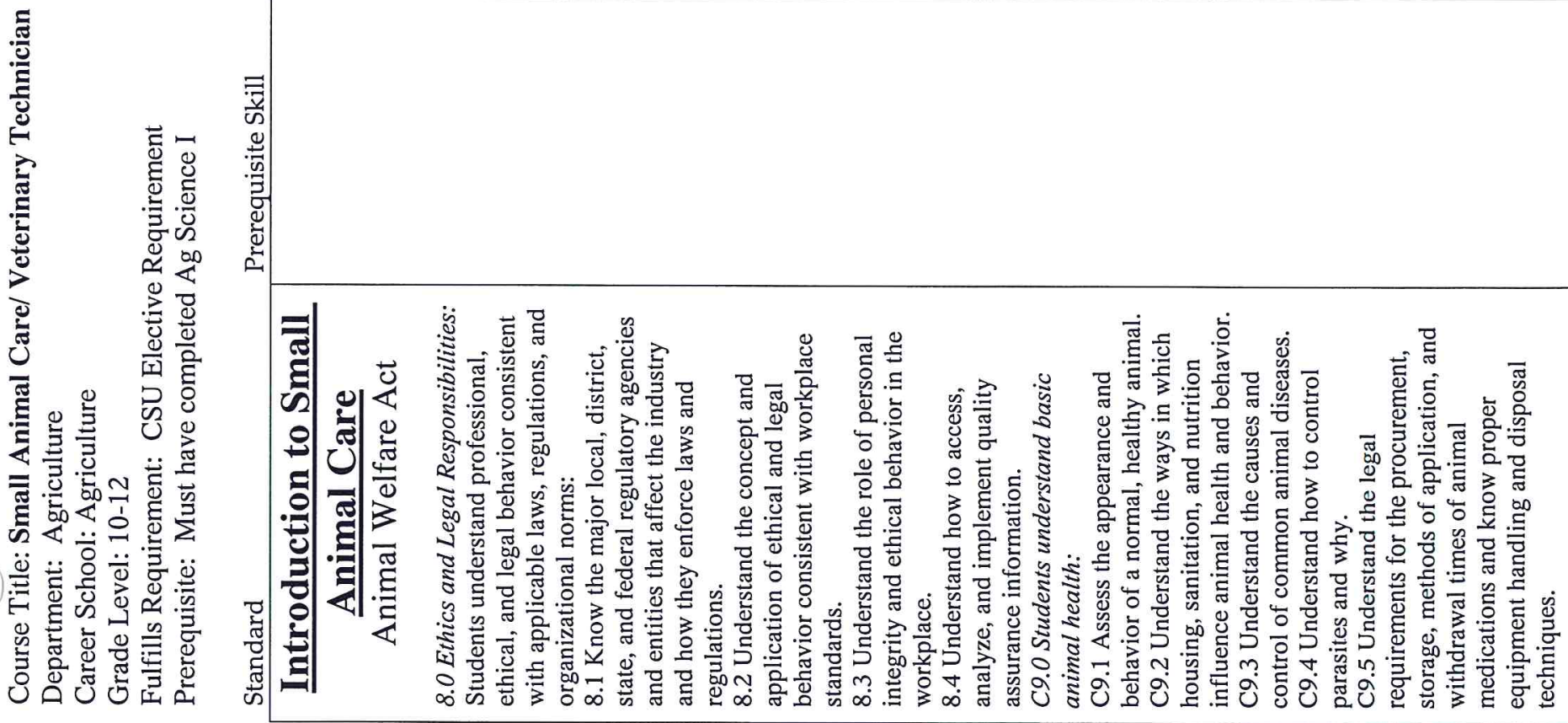

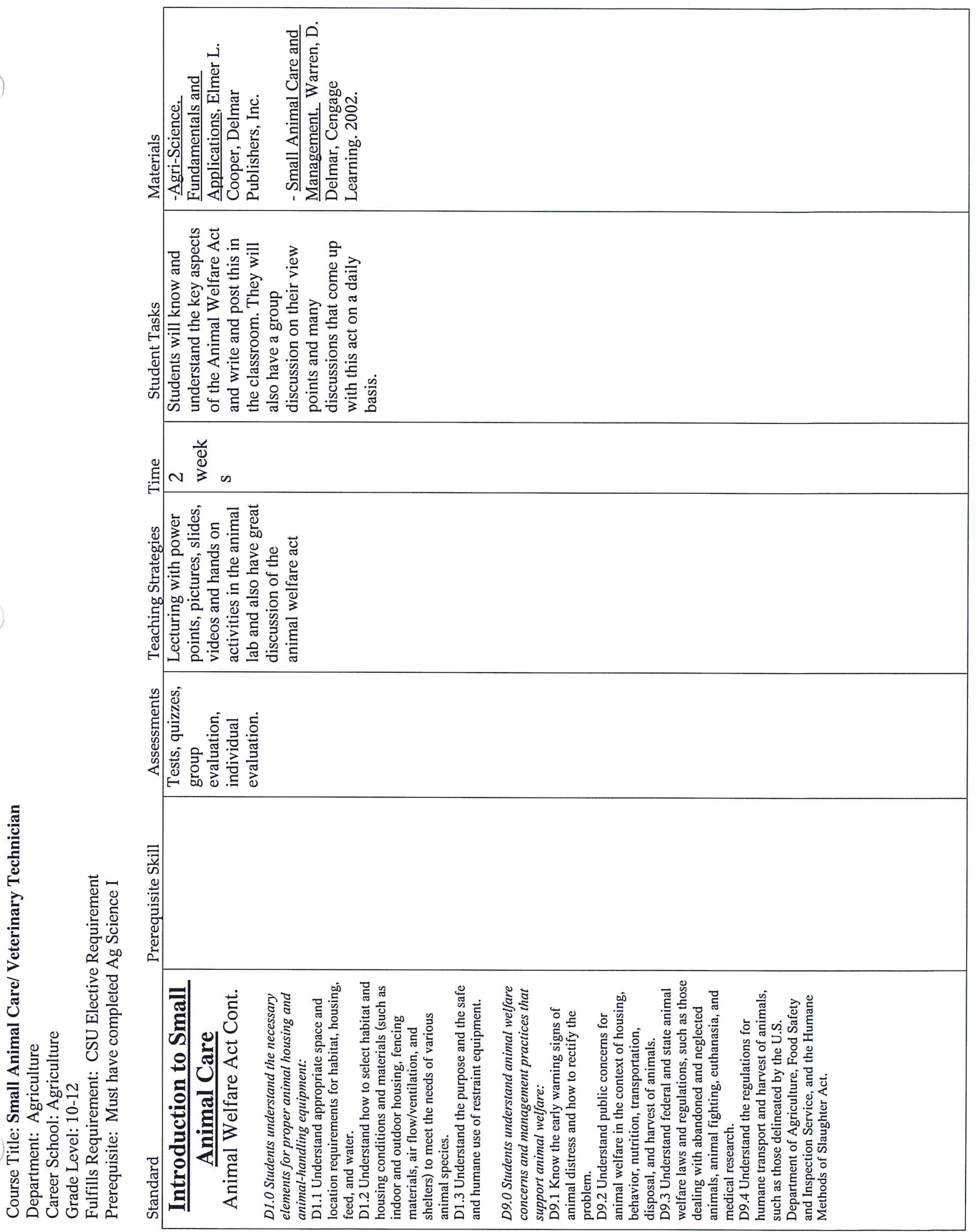


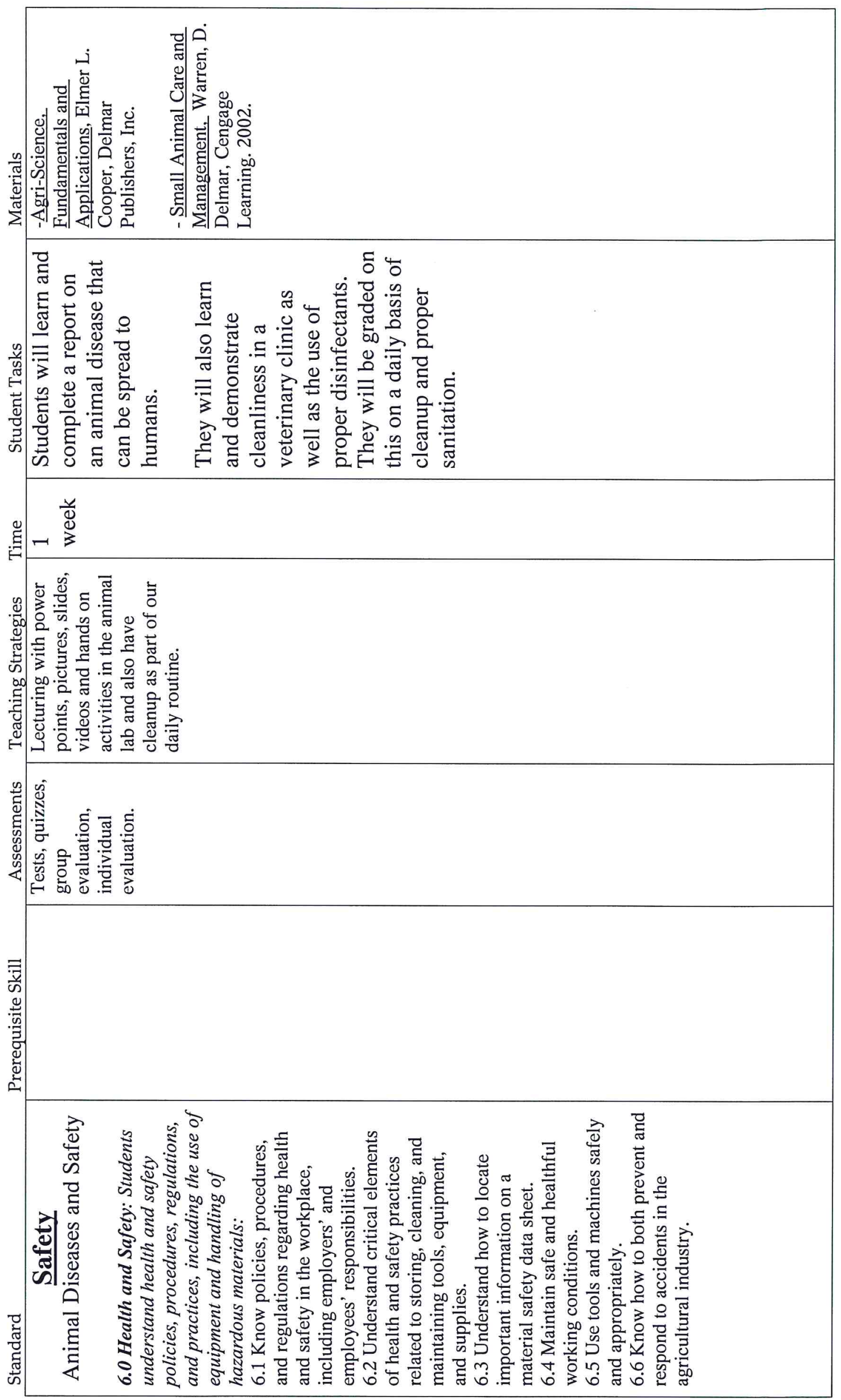



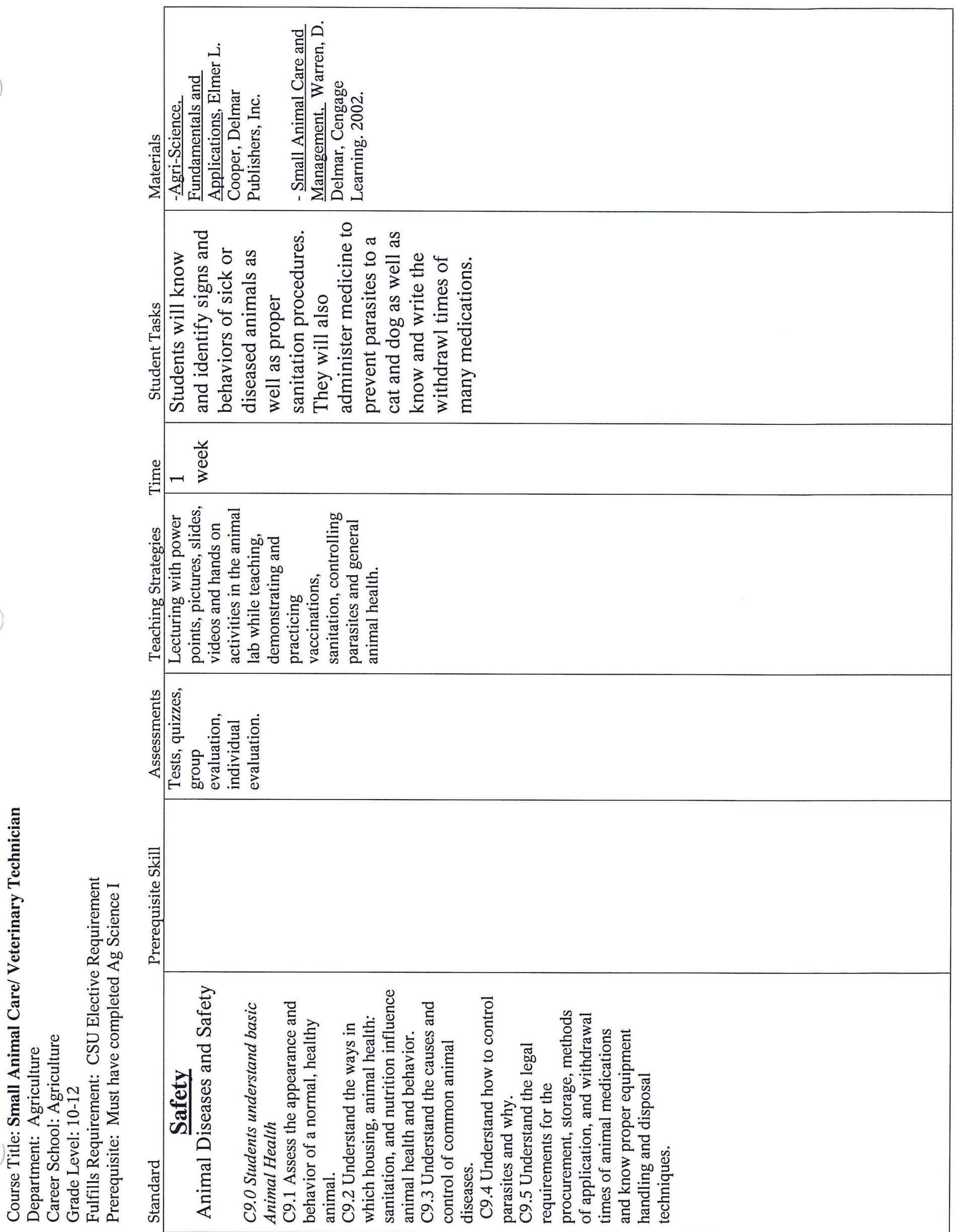

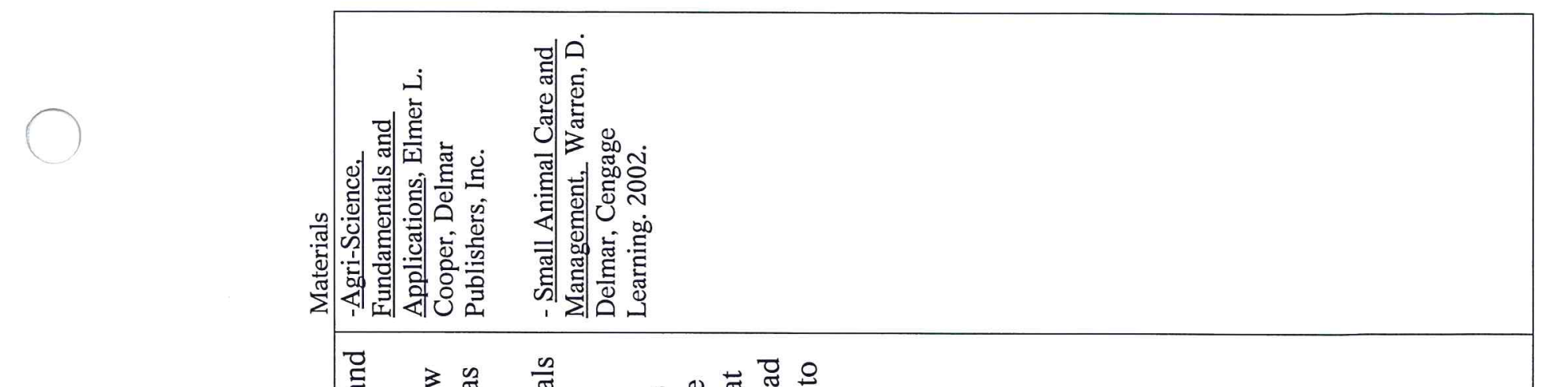


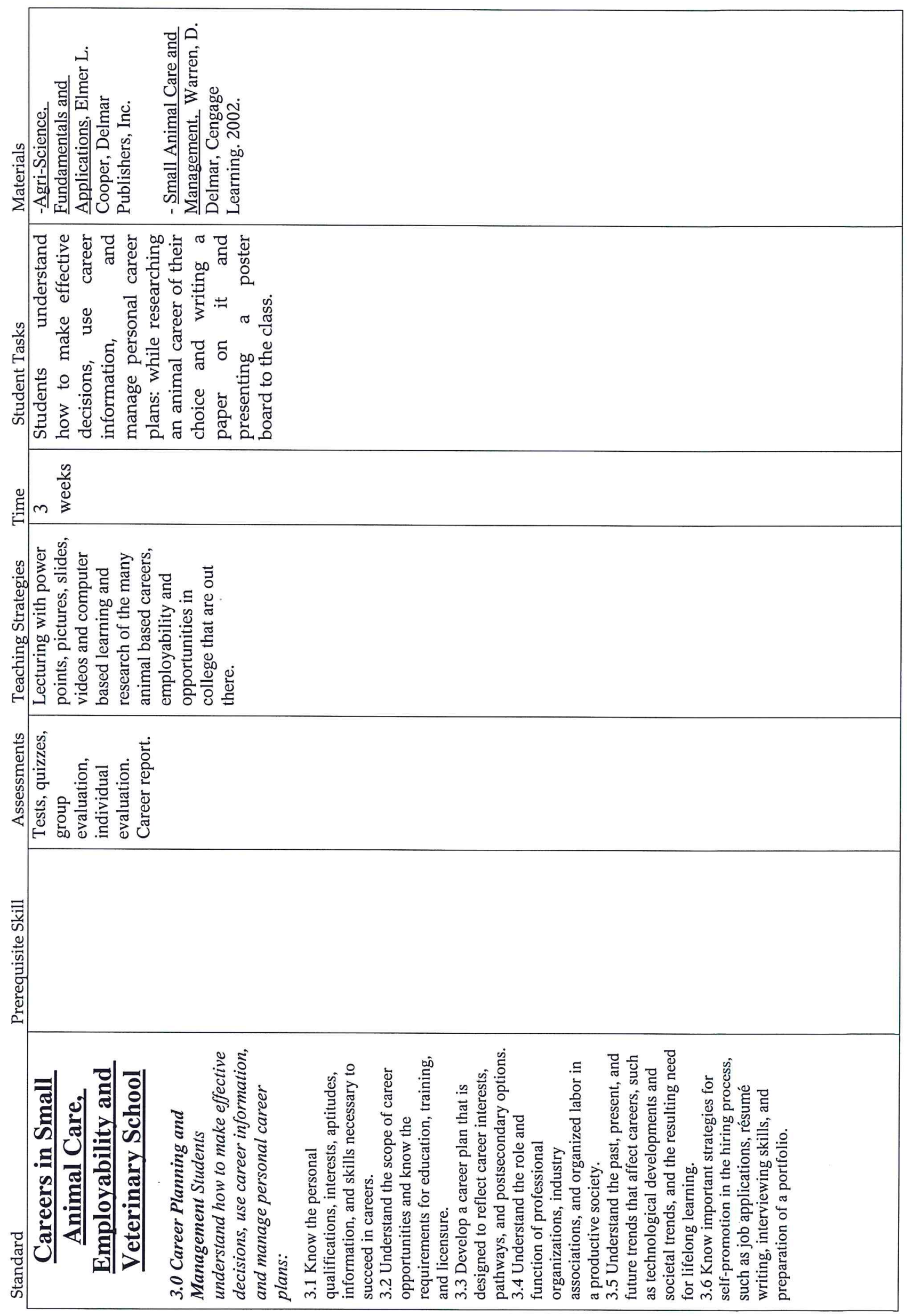




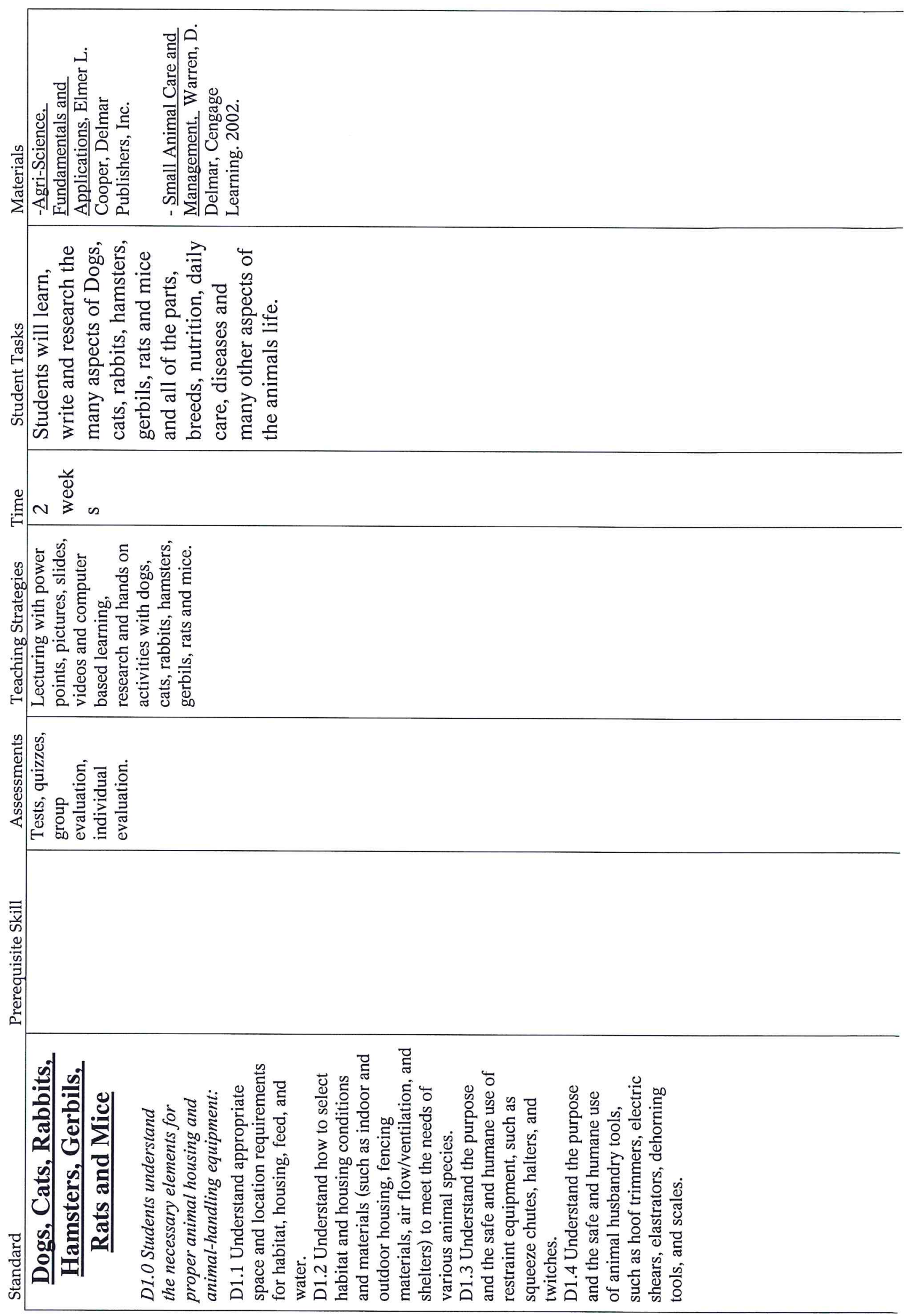



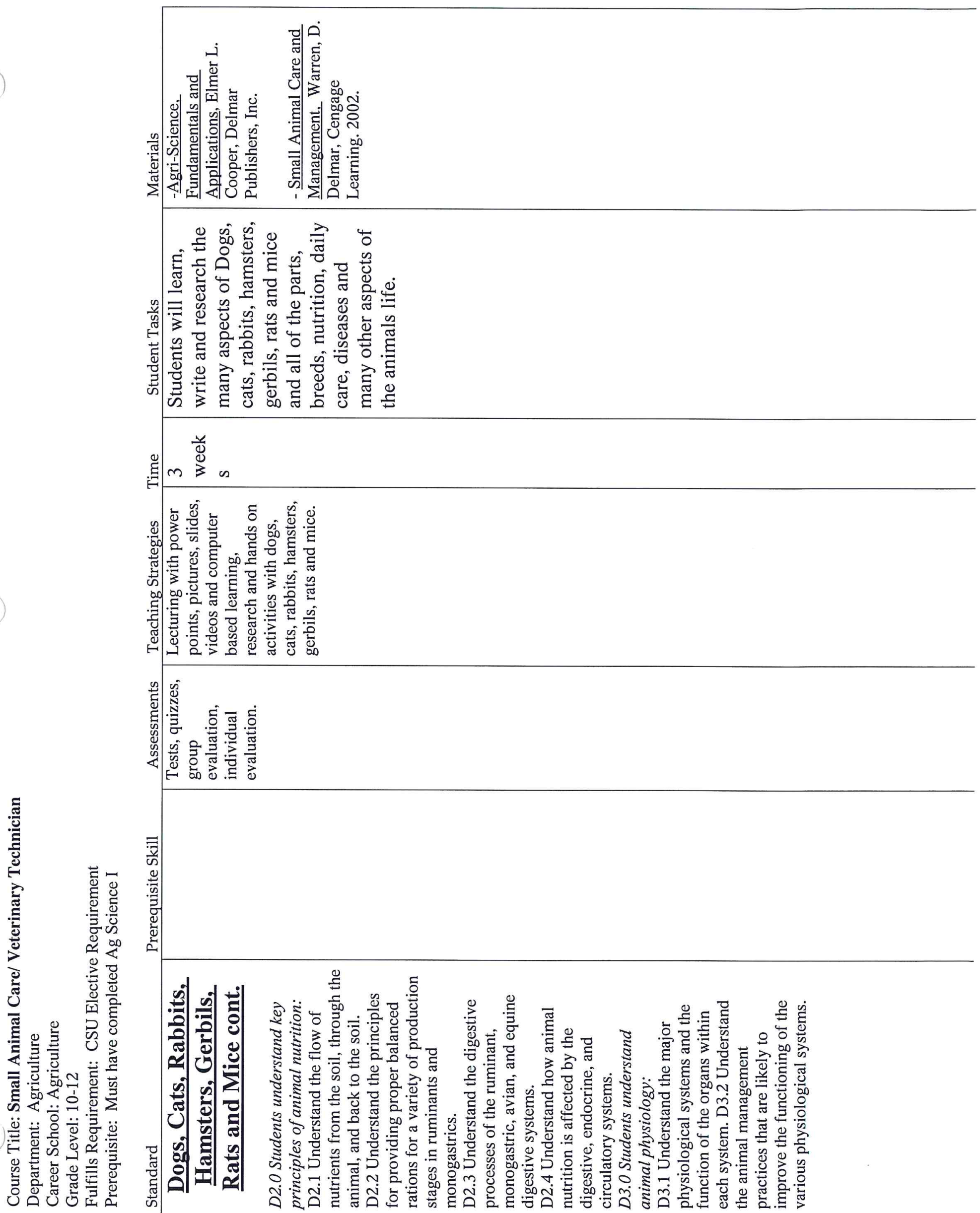

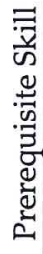



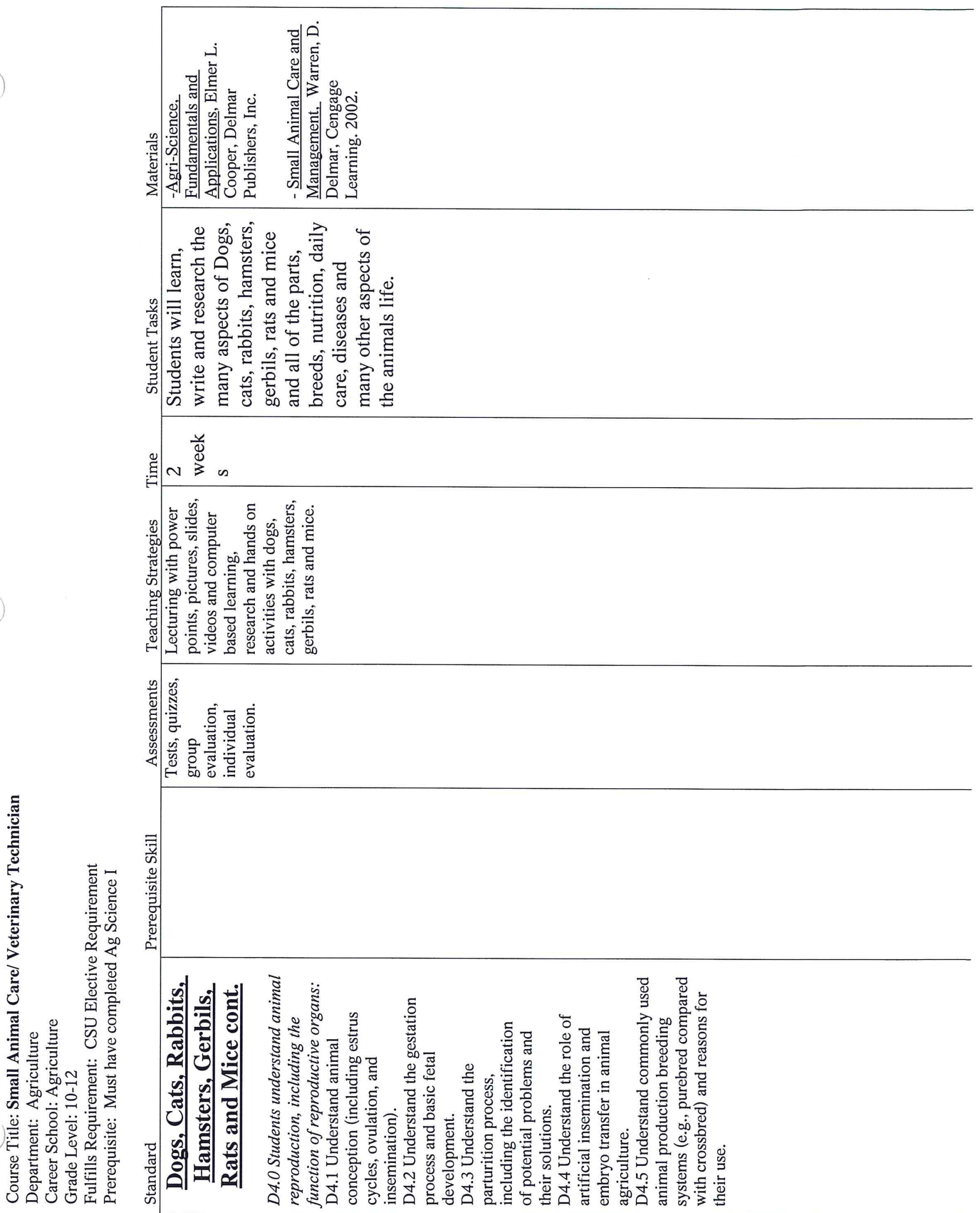

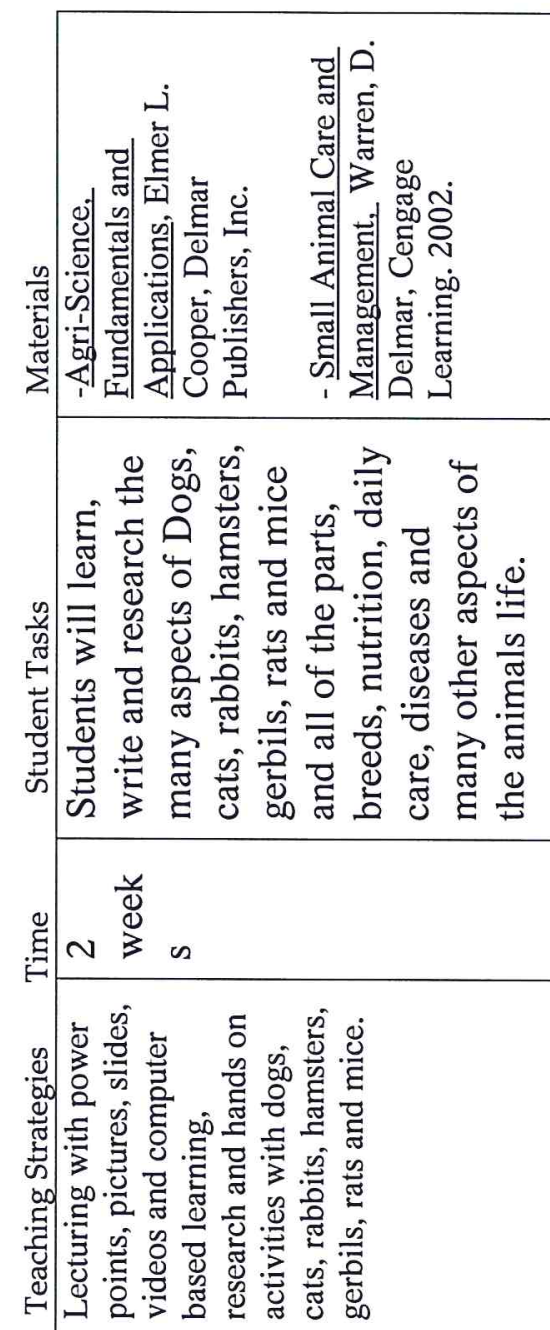


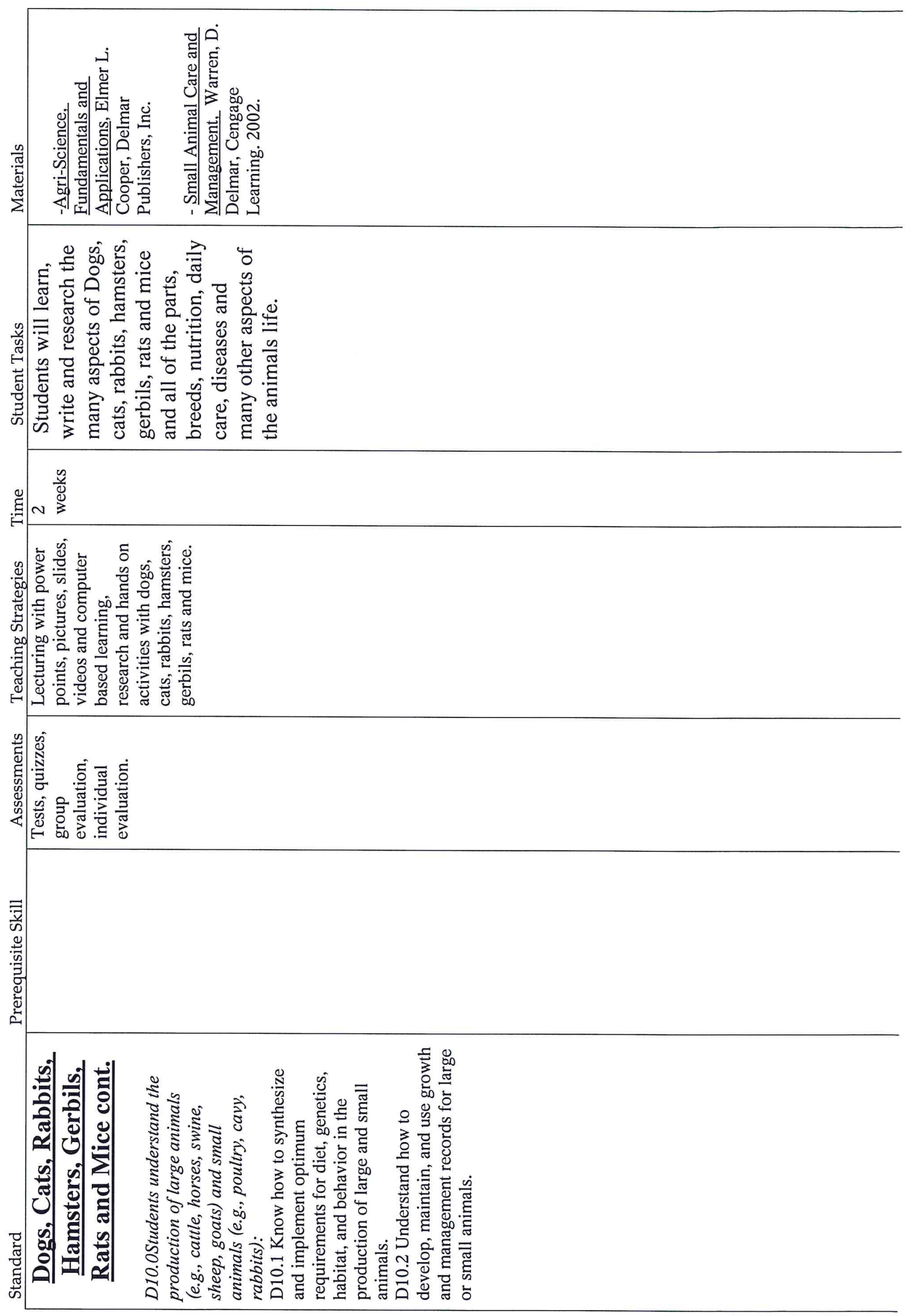




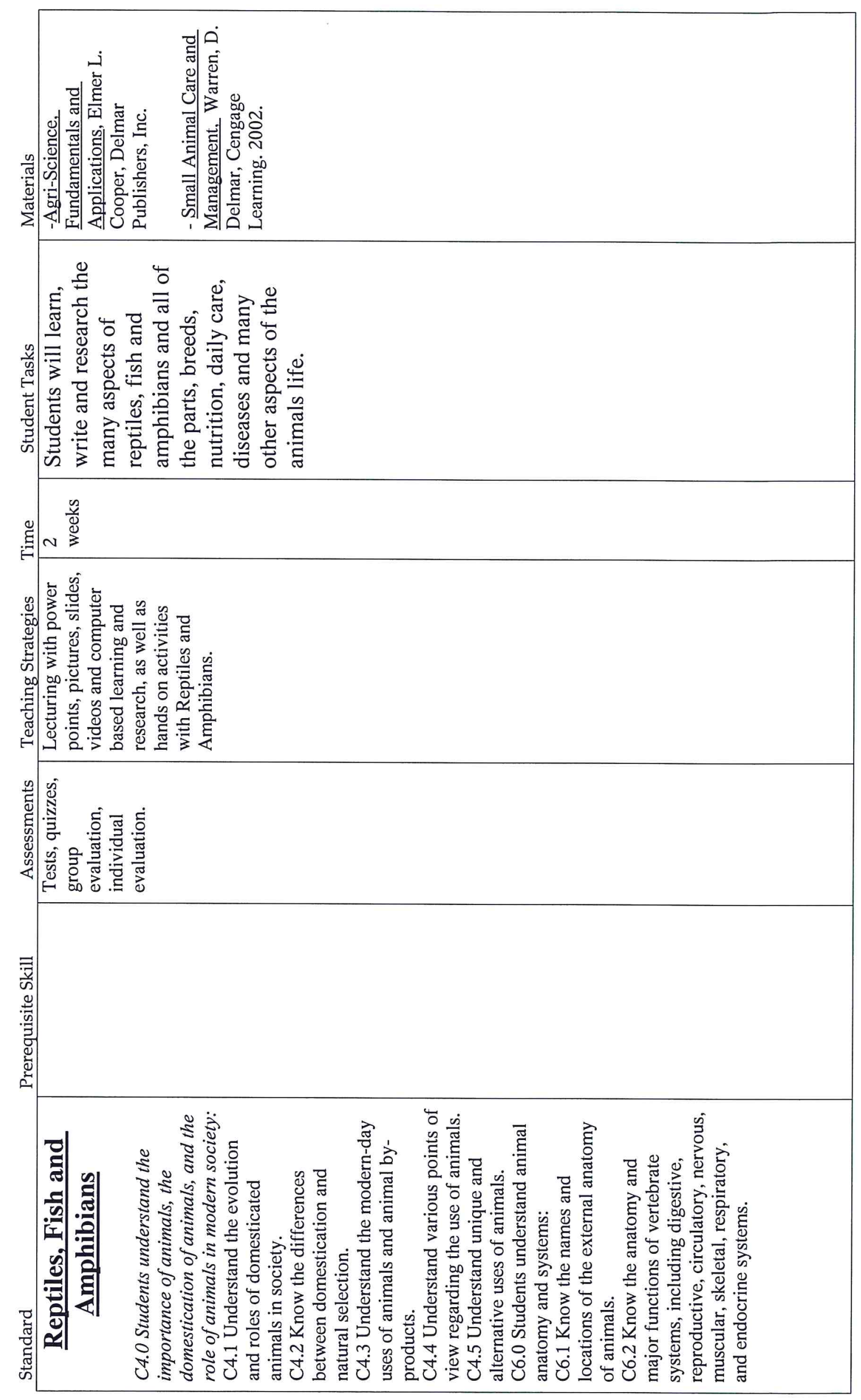



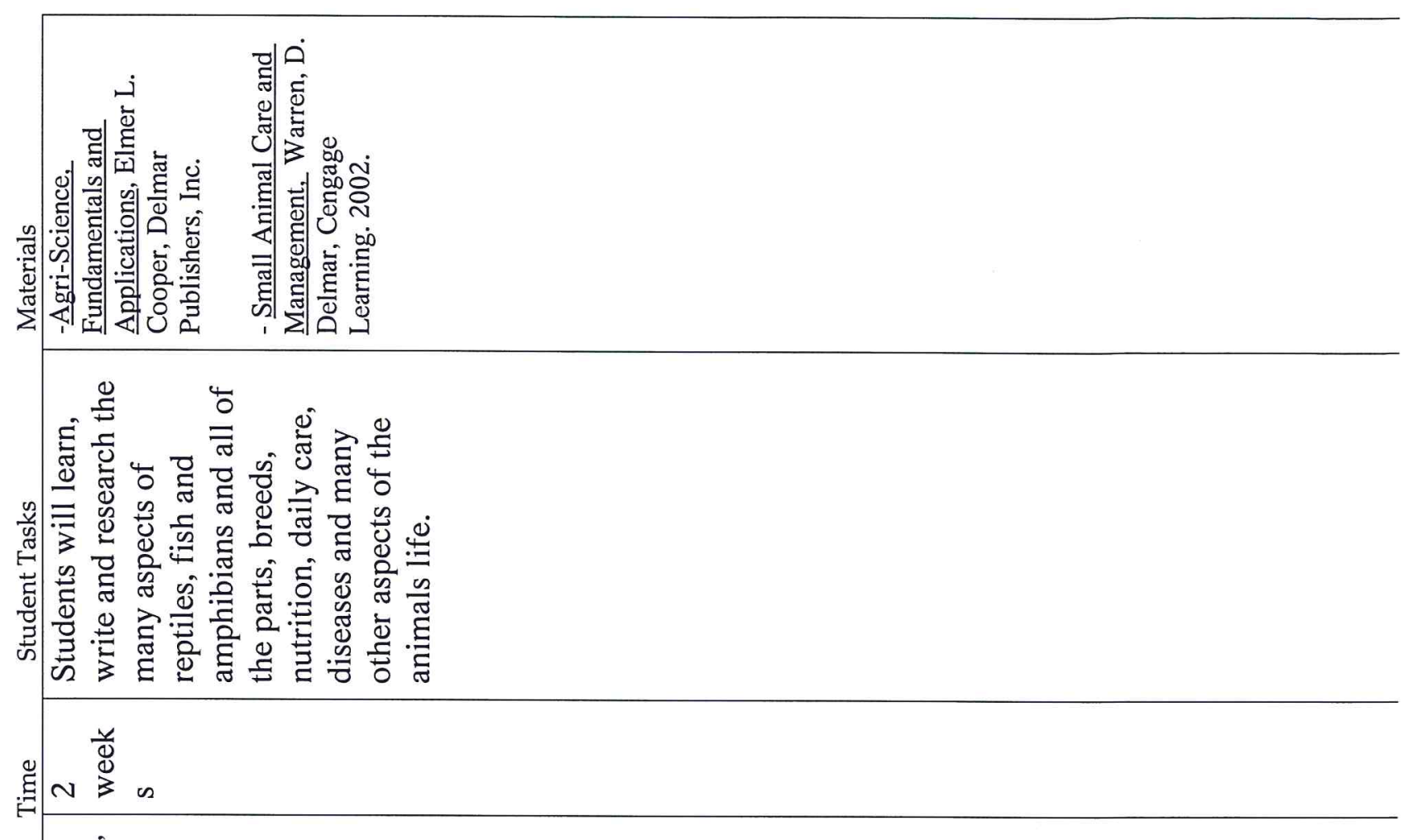

$\overline{0} \frac{\tilde{\theta}}{0}$

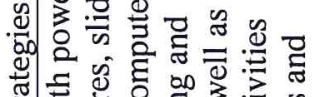

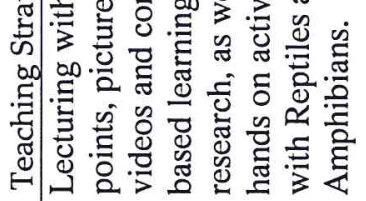

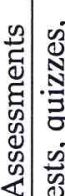

.

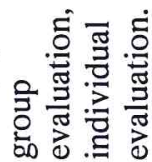

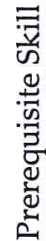

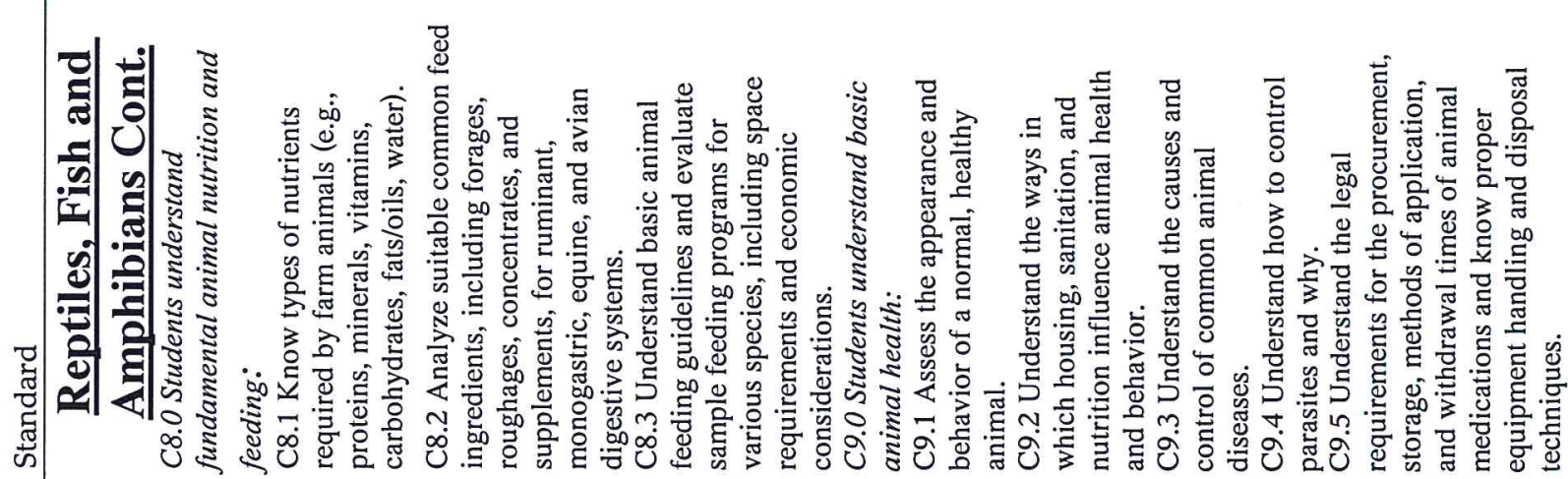




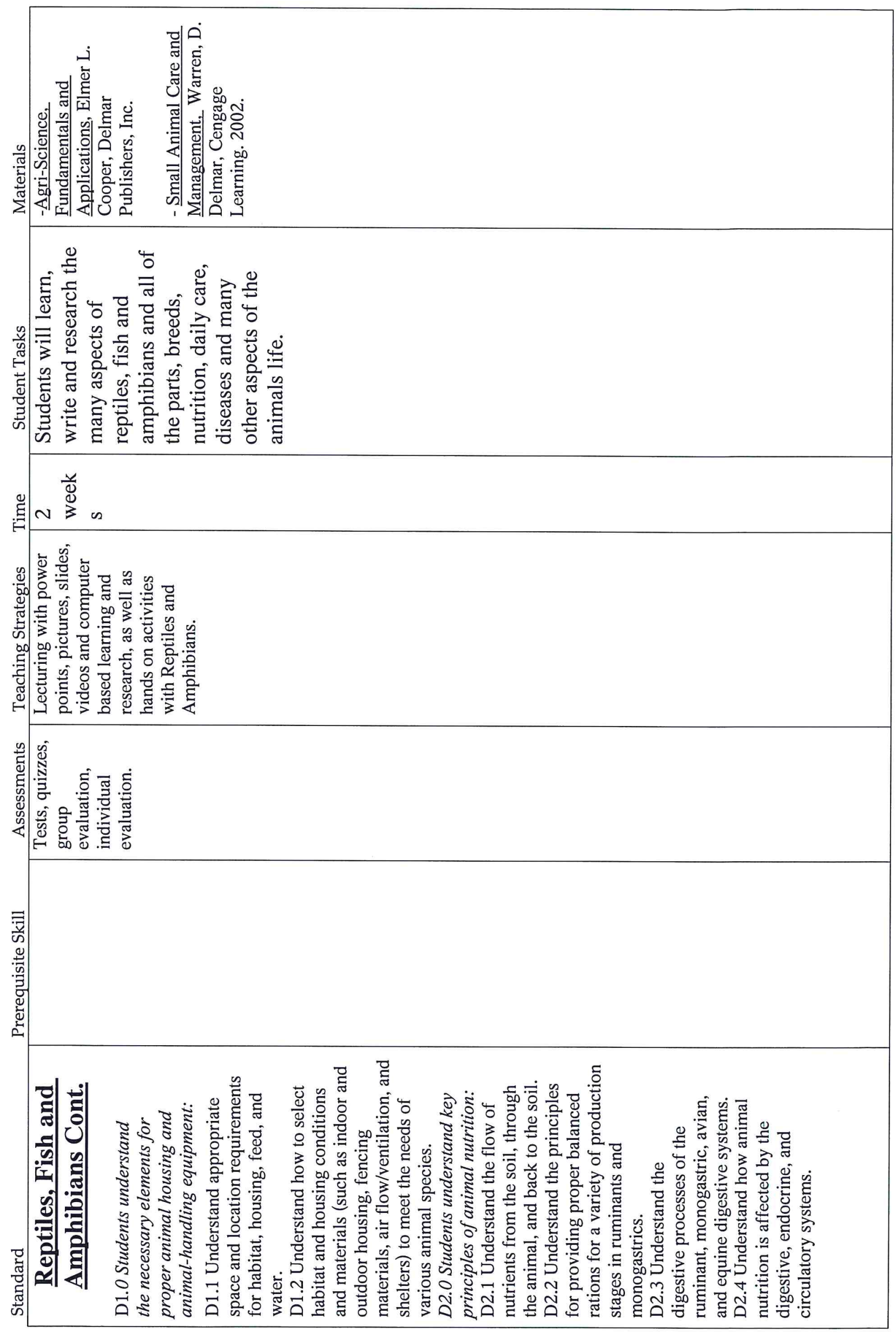



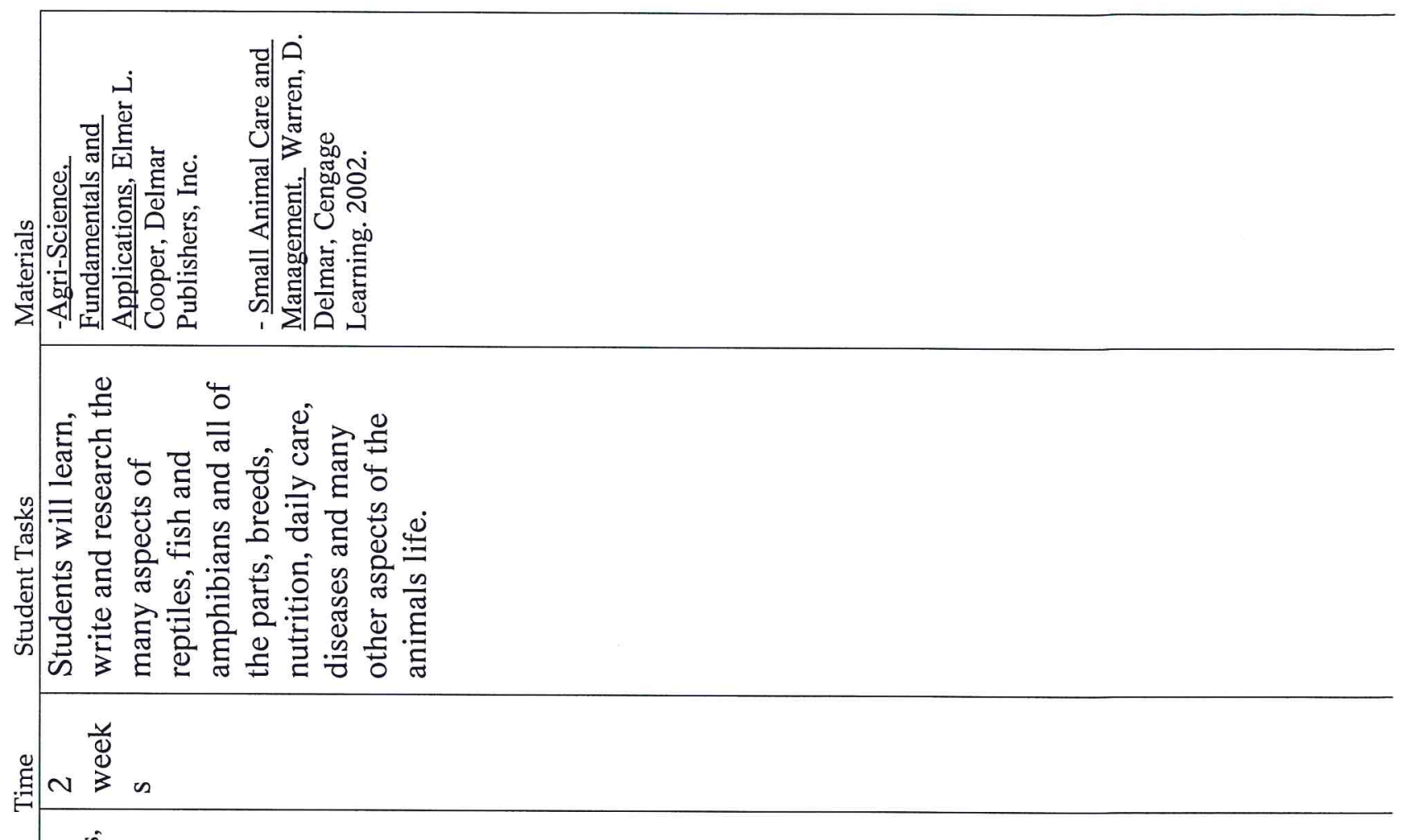

ఫิ
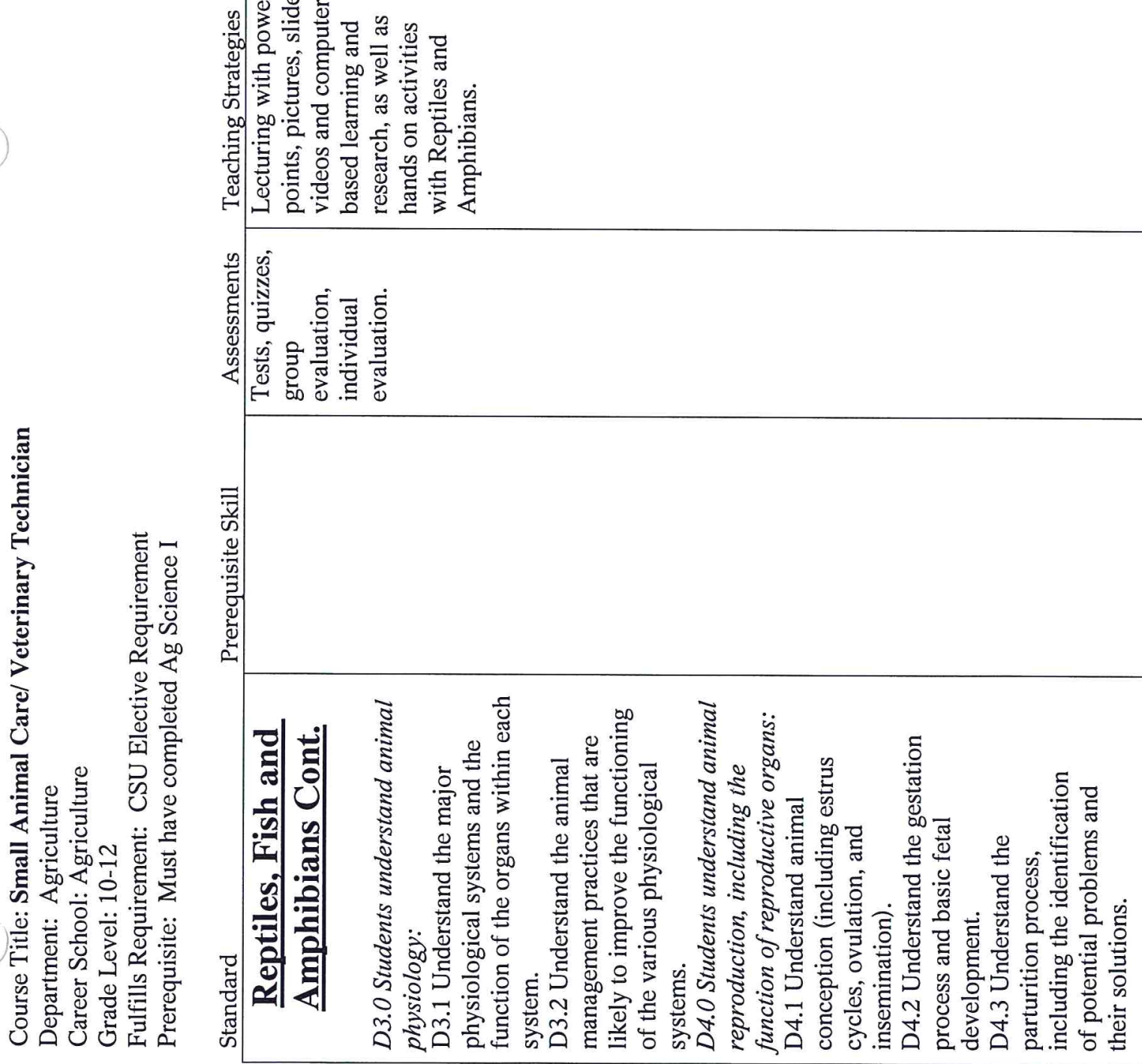


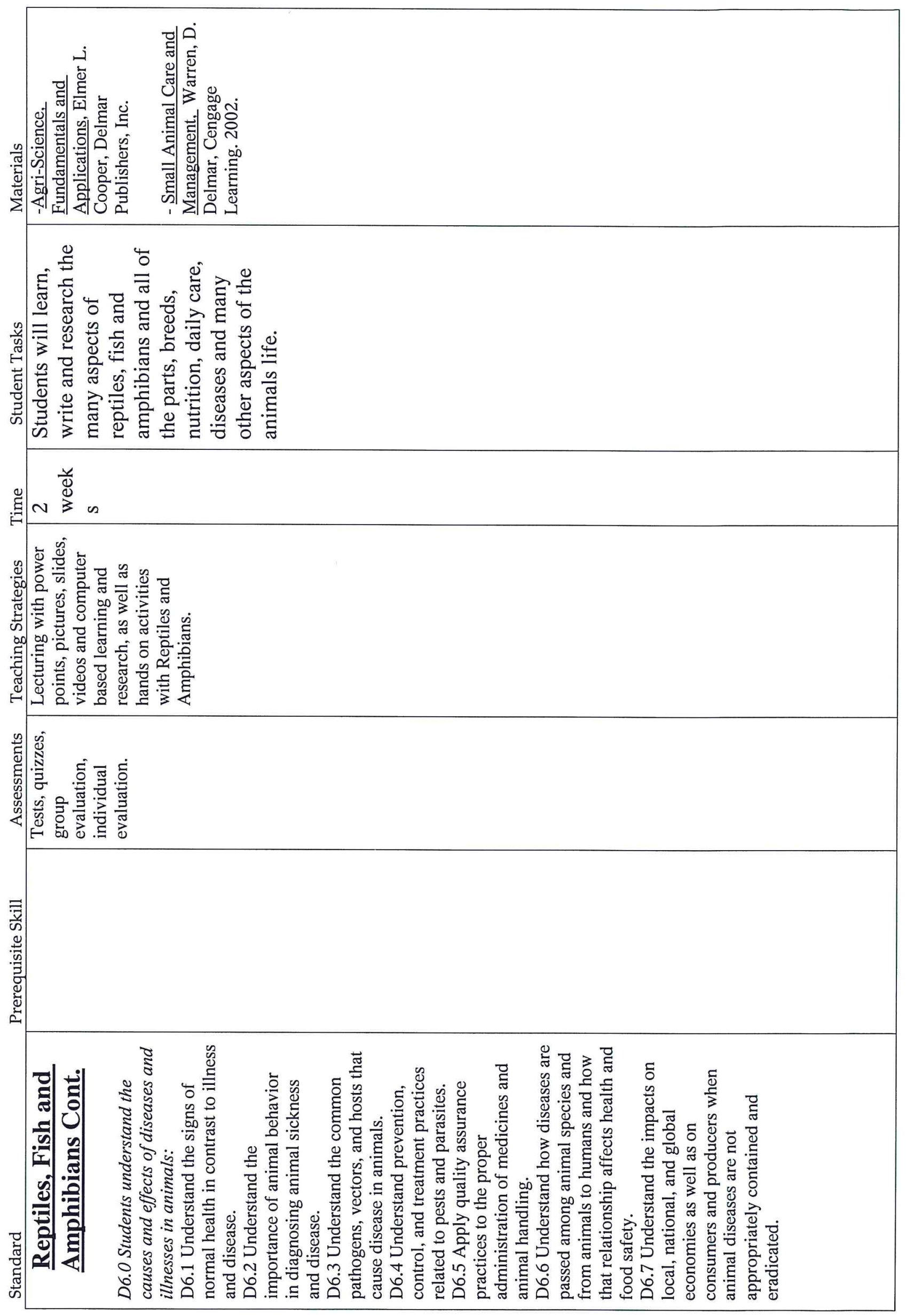



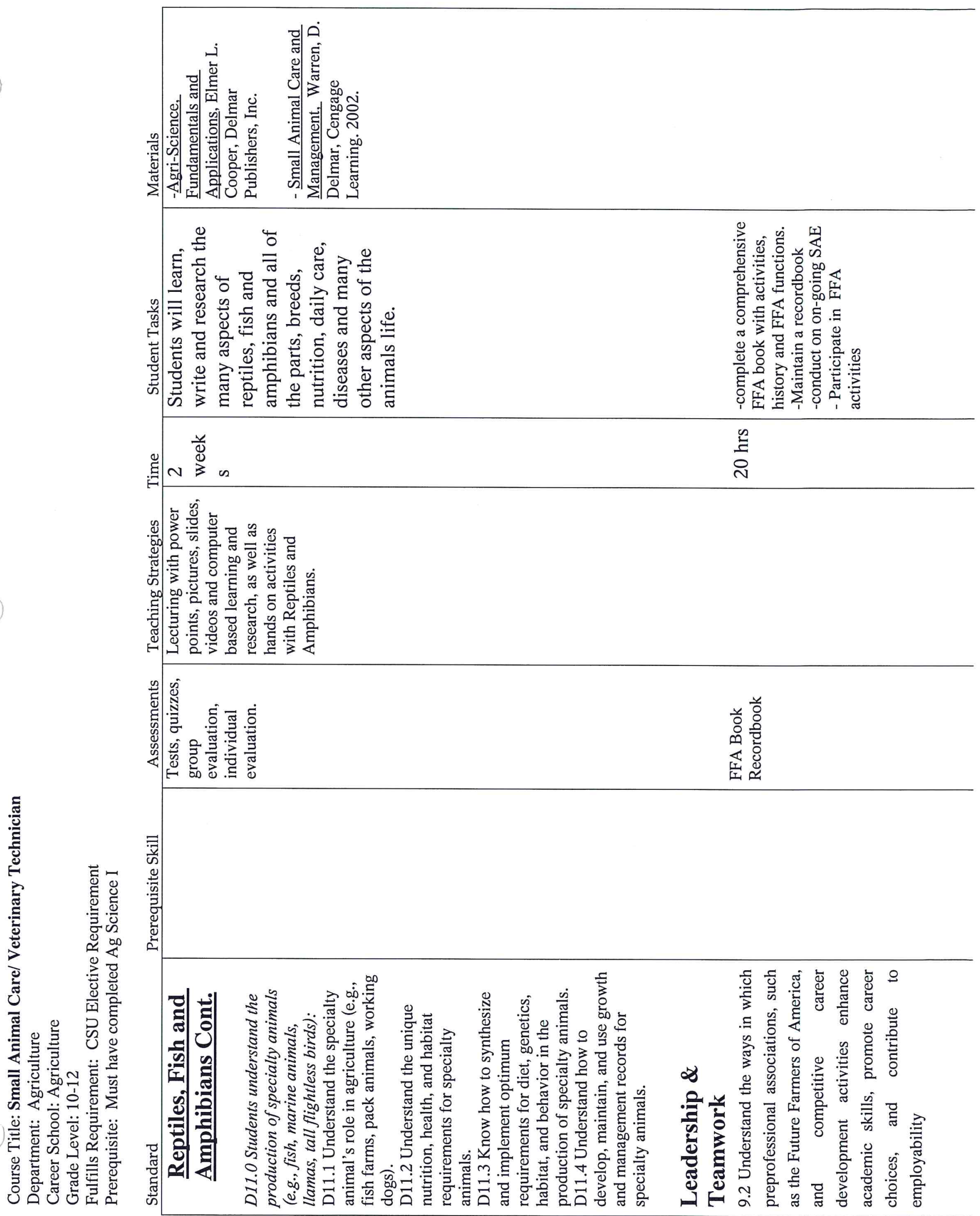


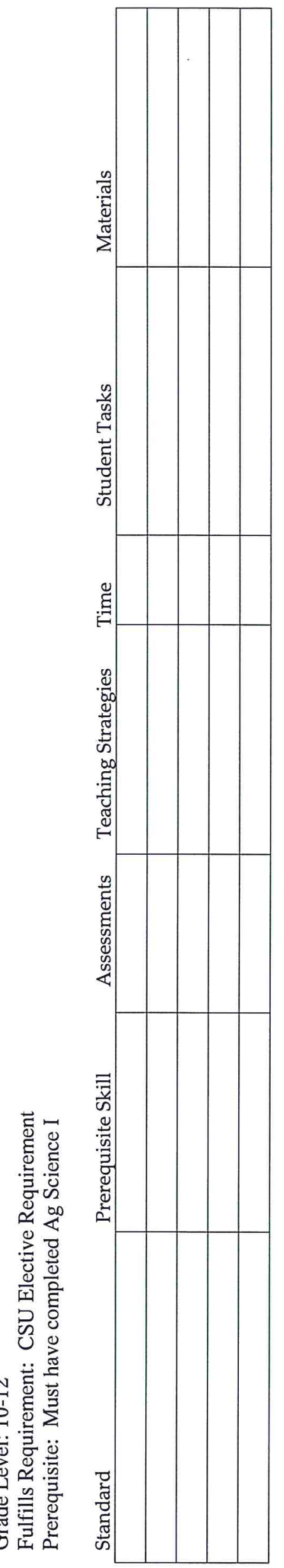

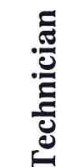




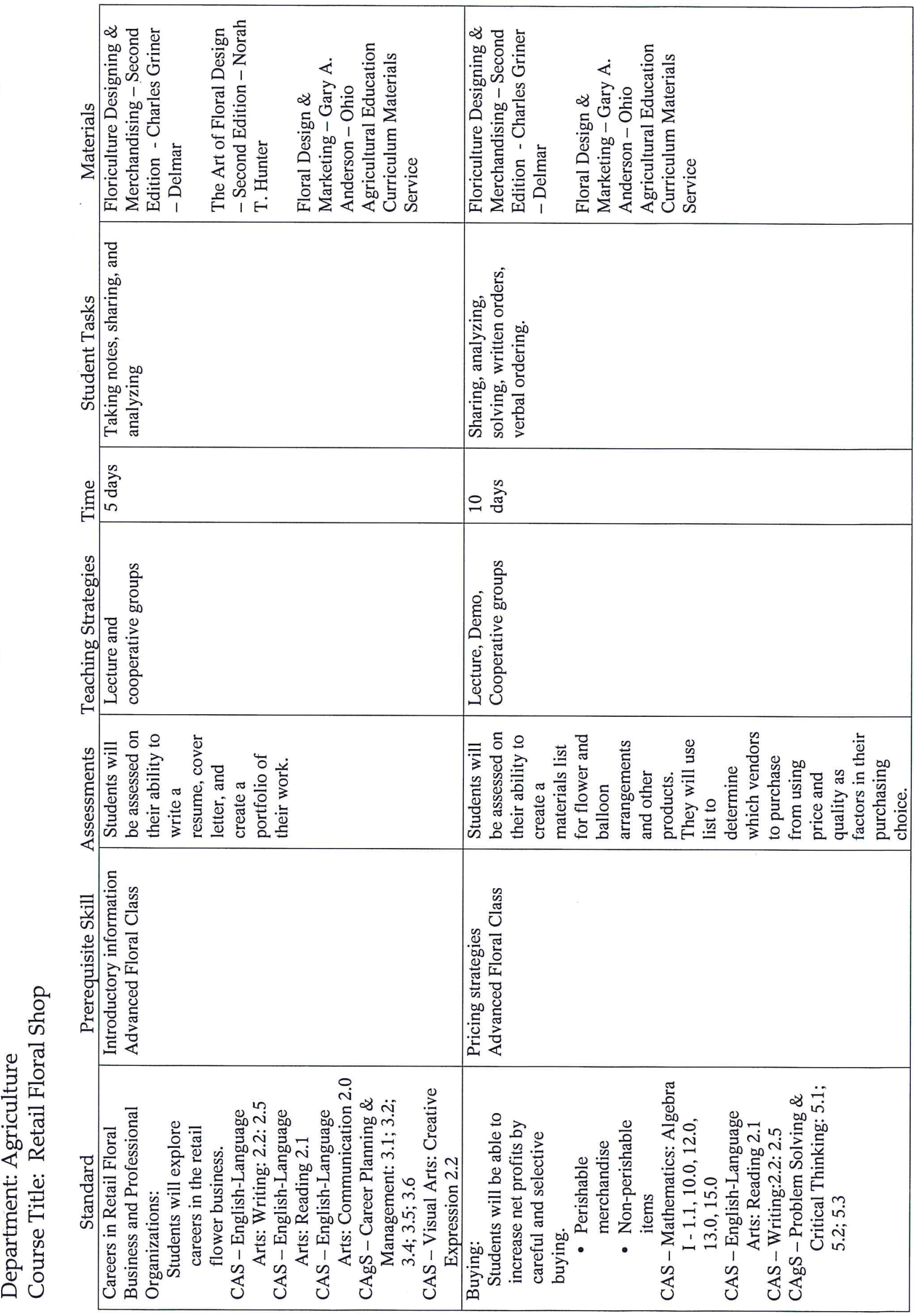



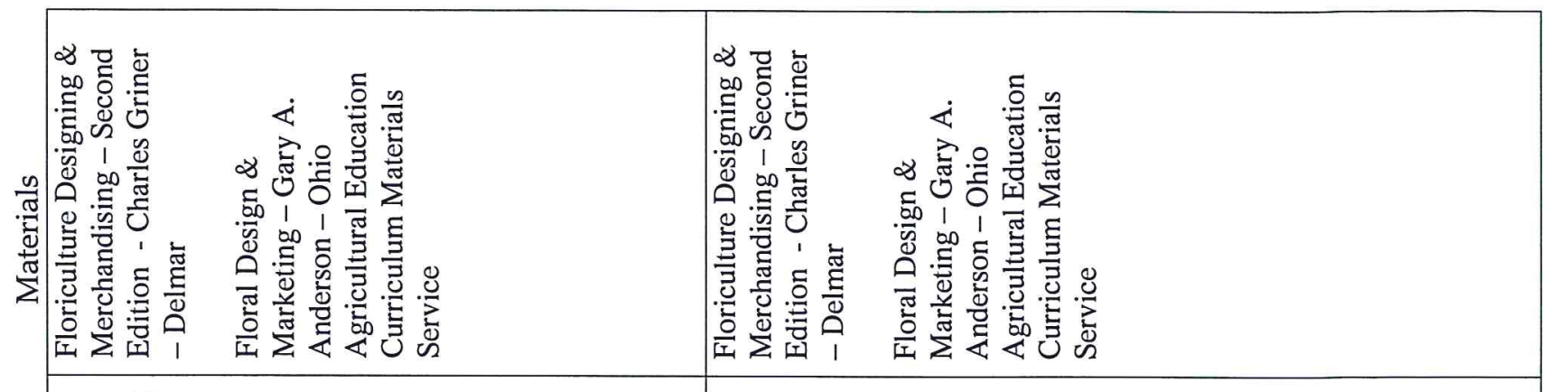

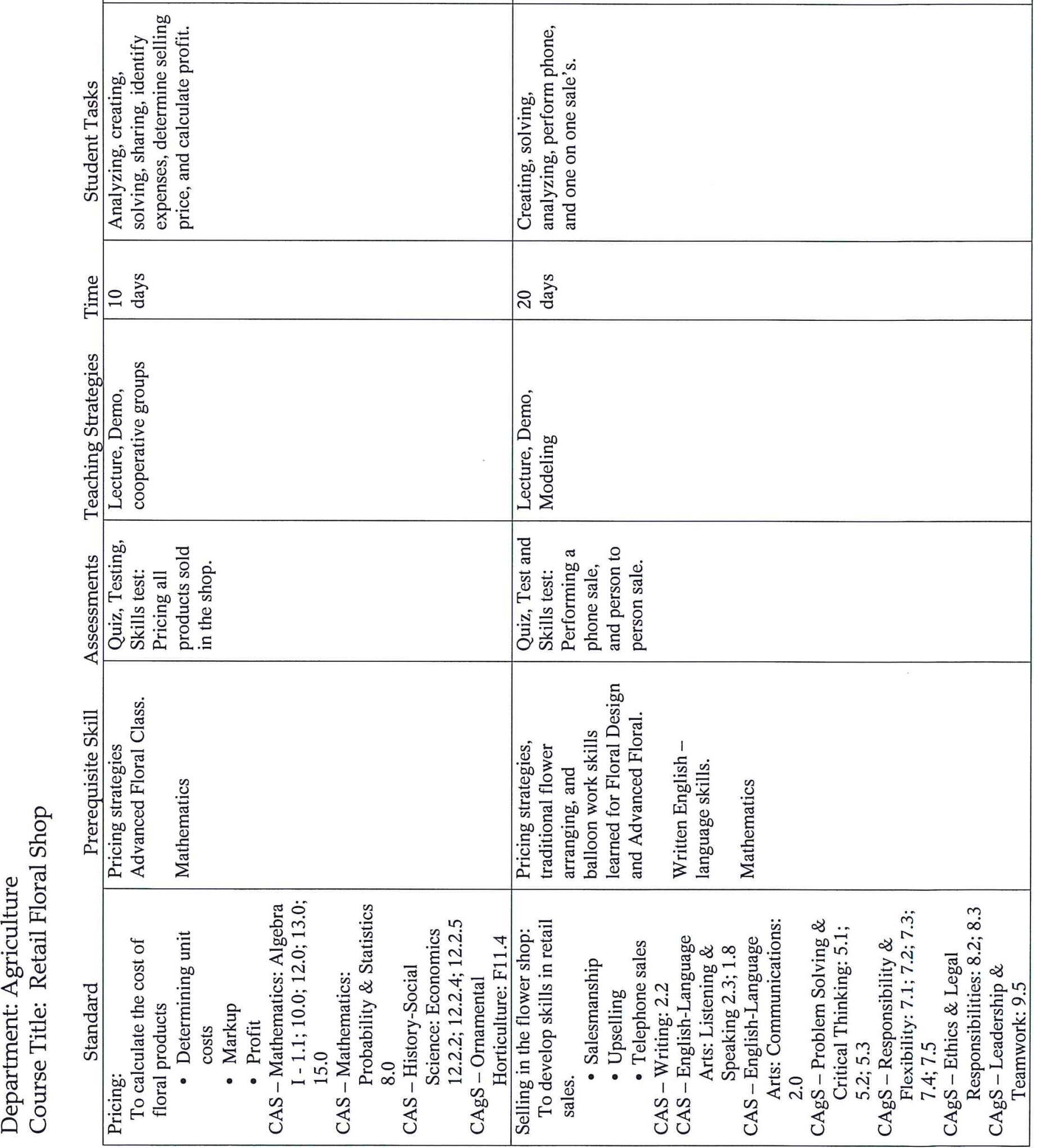




\begin{tabular}{|c|c|}
\hline 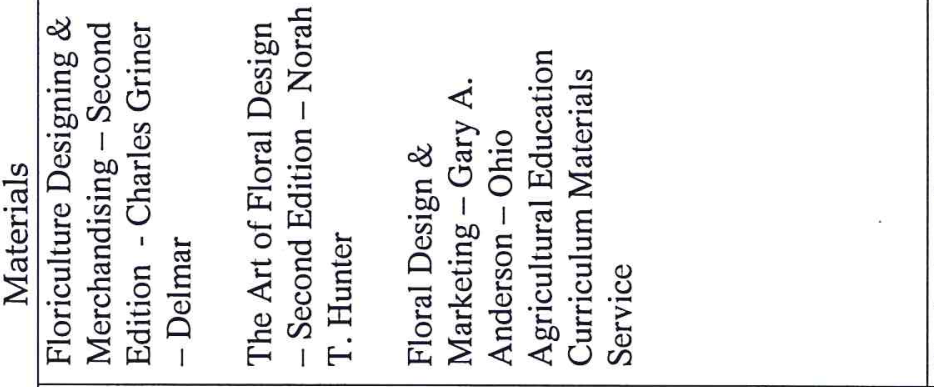 & 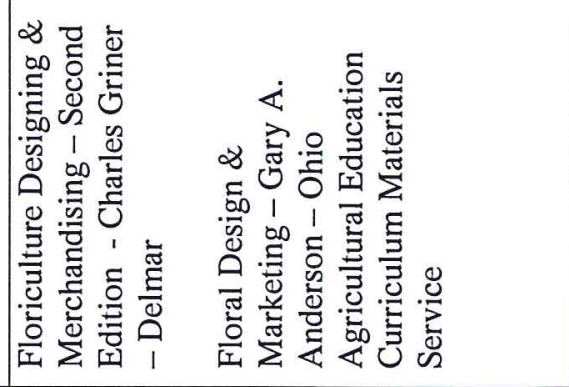 \\
\hline 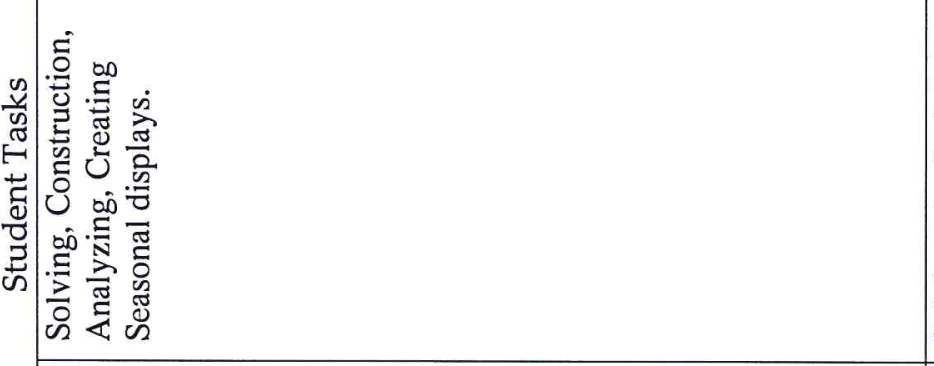 & 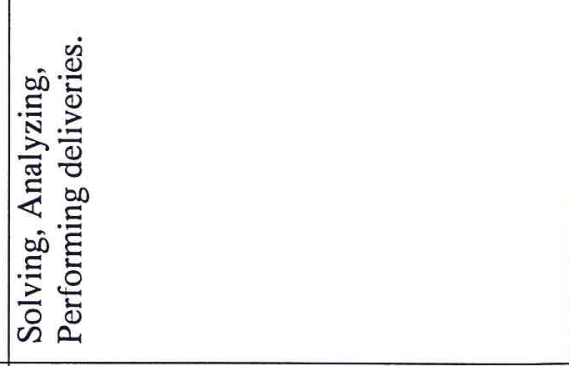 \\
\hline 兄 & O气 \\
\hline 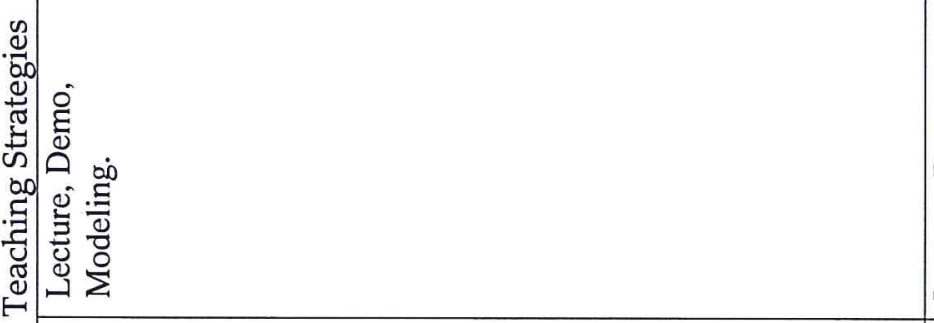 & 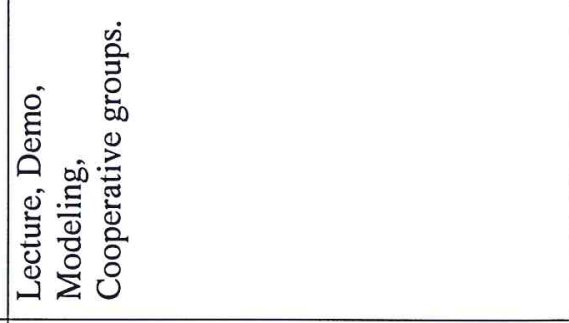 \\
\hline 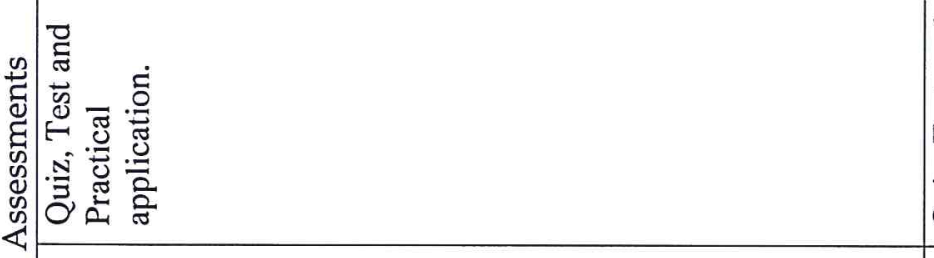 & 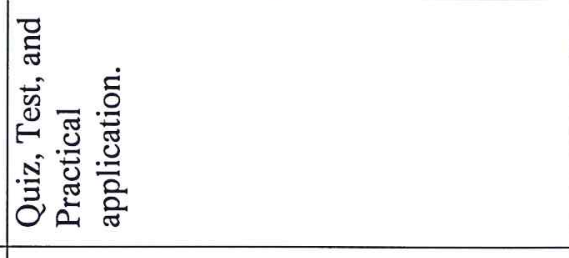 \\
\hline 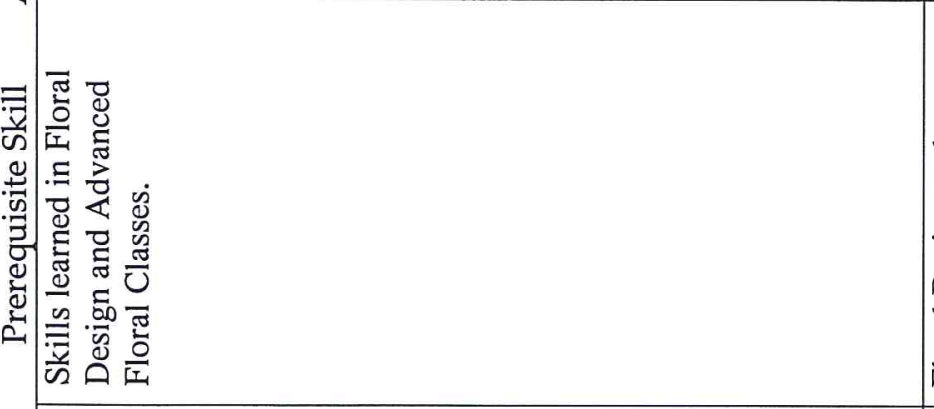 & 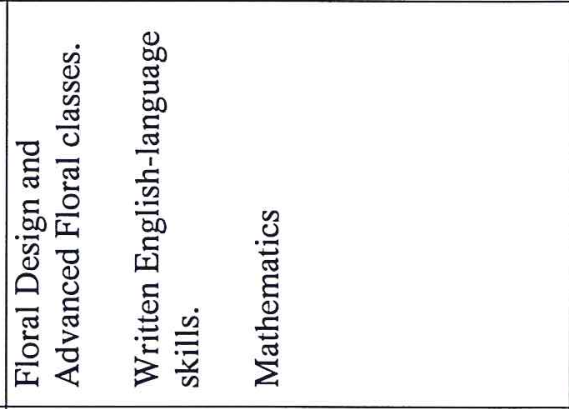 \\
\hline 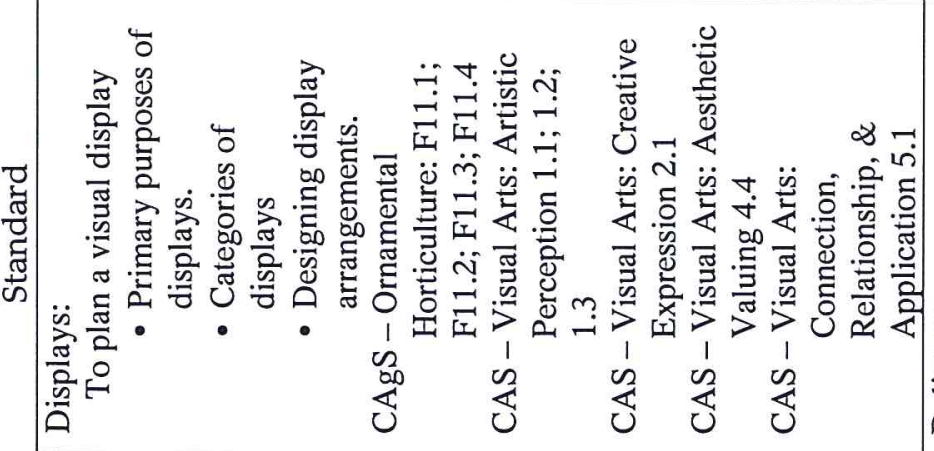 & 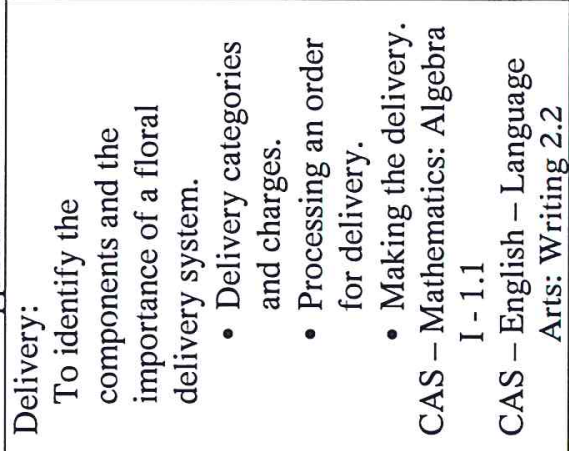 \\
\hline
\end{tabular}




\begin{tabular}{|c|c|}
\hline 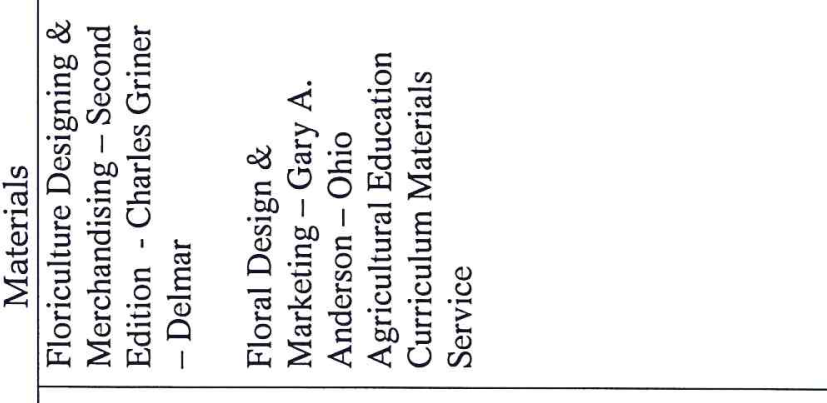 & 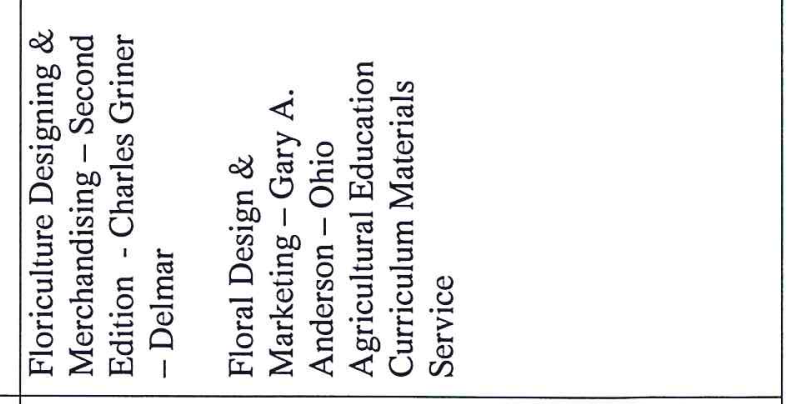 \\
\hline 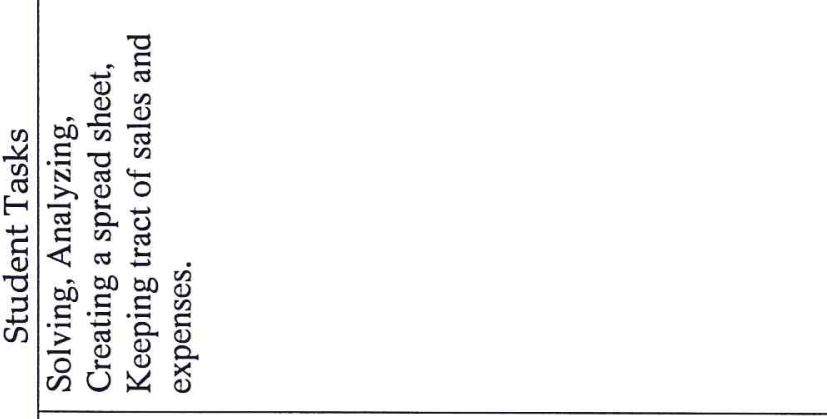 & 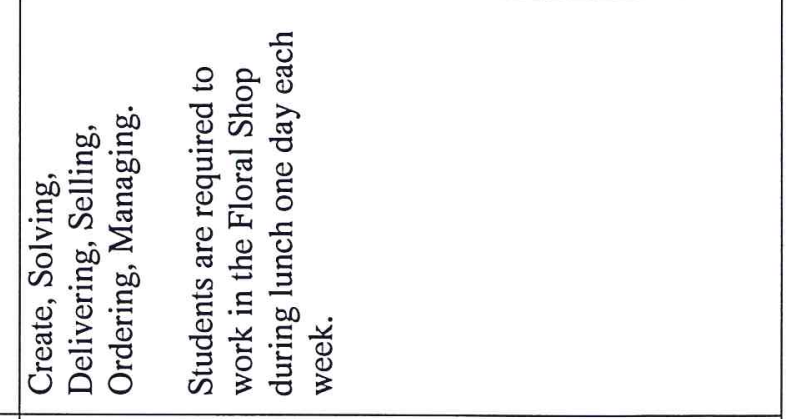 \\
\hline 方 & R. \\
\hline 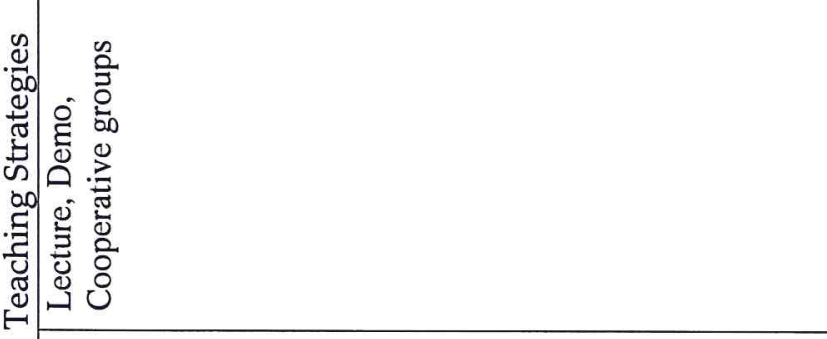 & 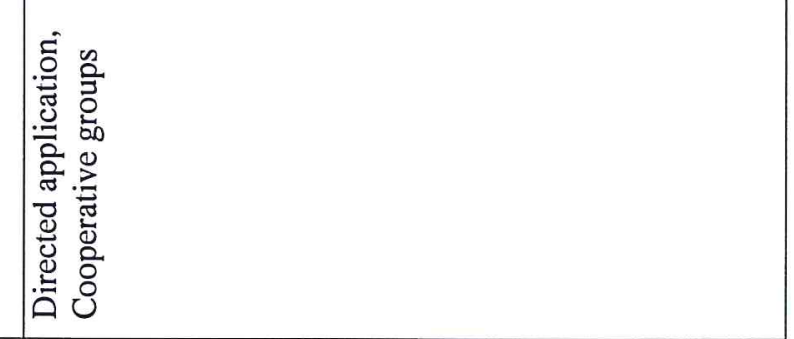 \\
\hline 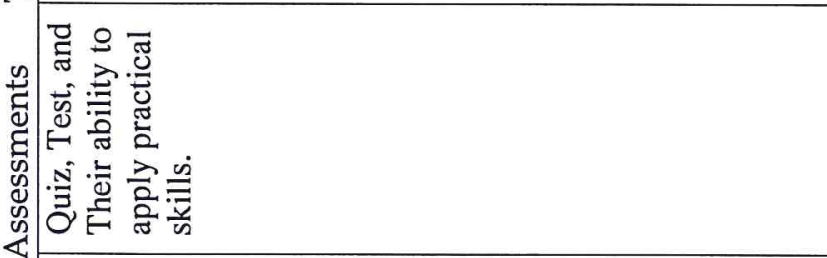 & 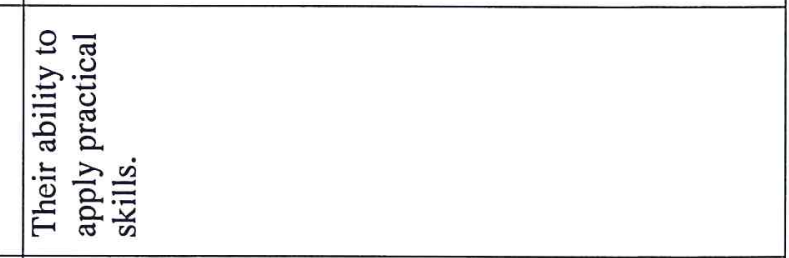 \\
\hline 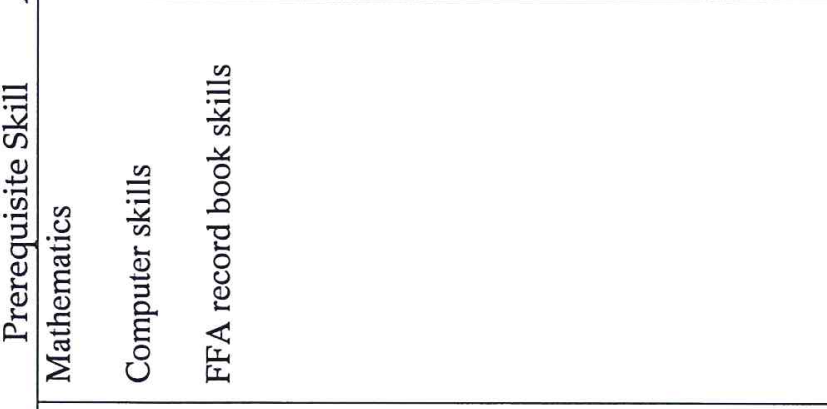 & 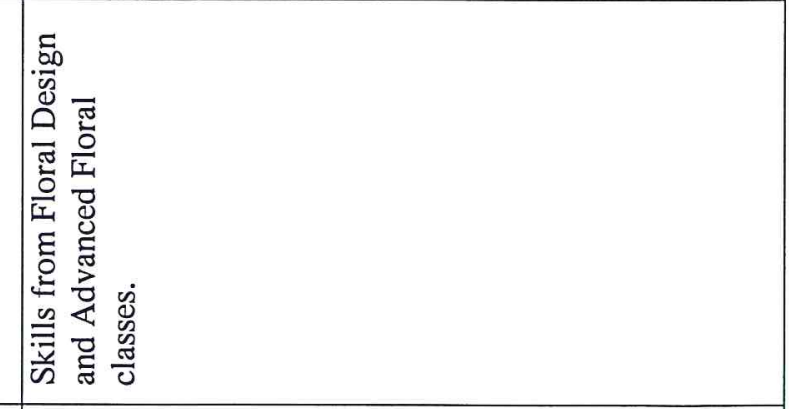 \\
\hline 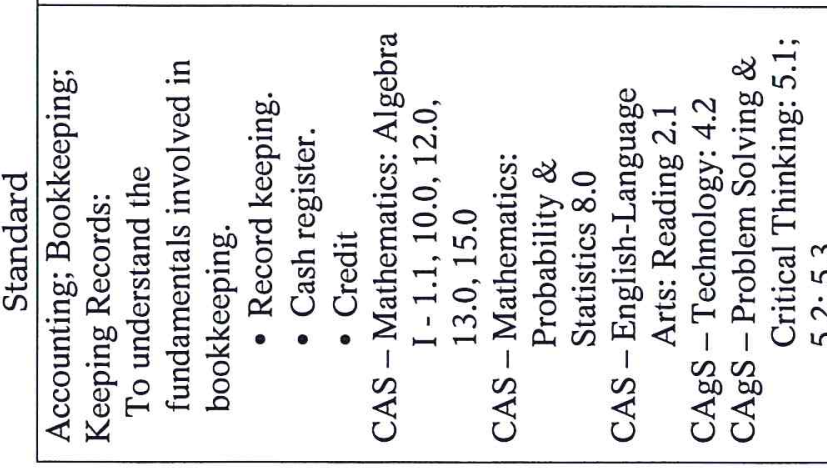 & 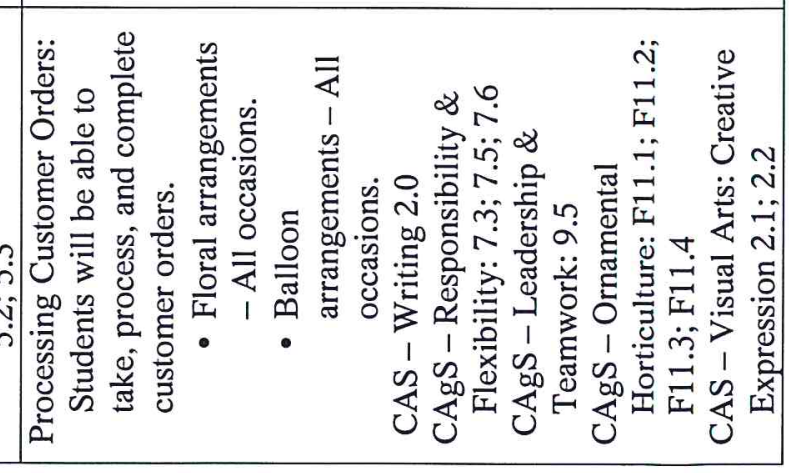 \\
\hline
\end{tabular}




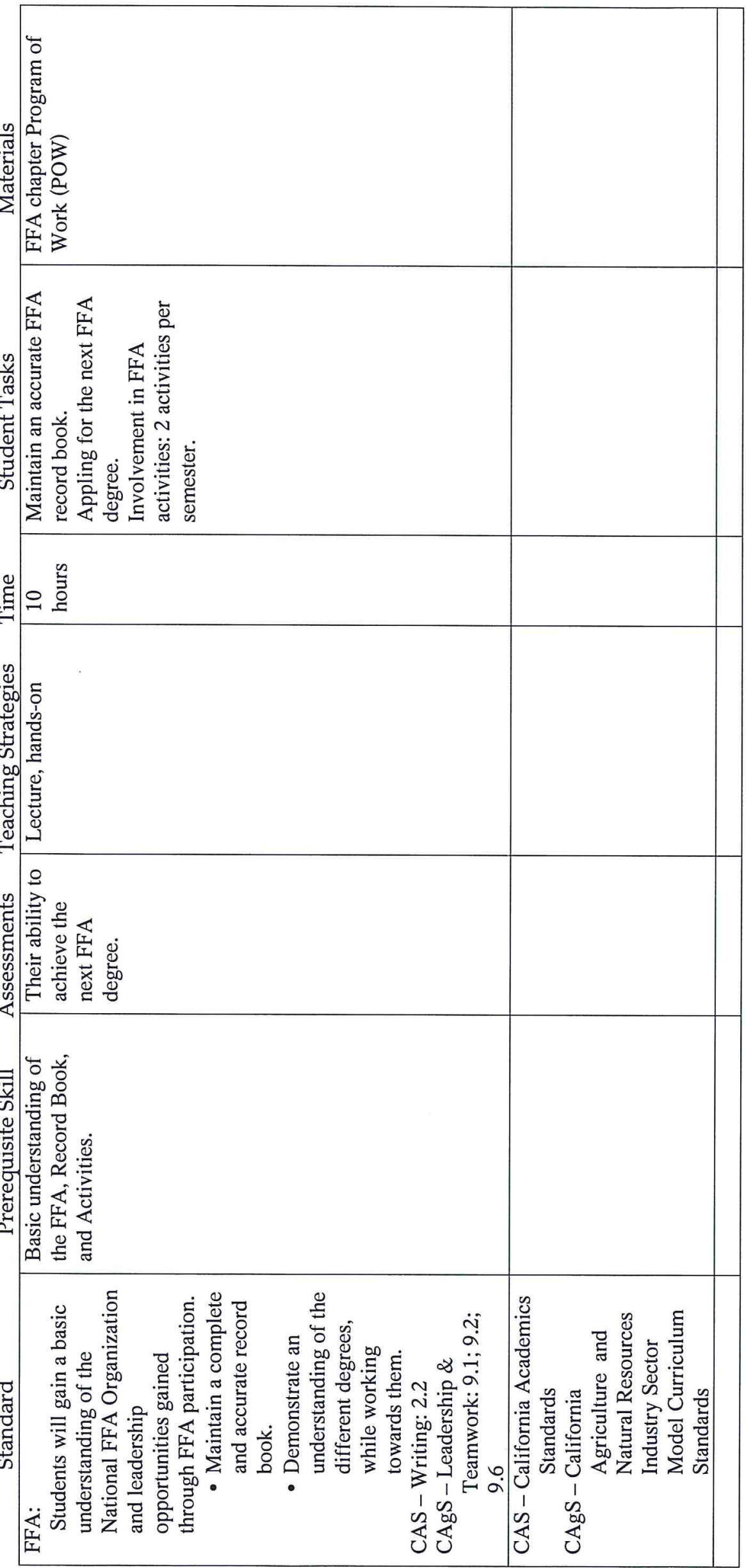




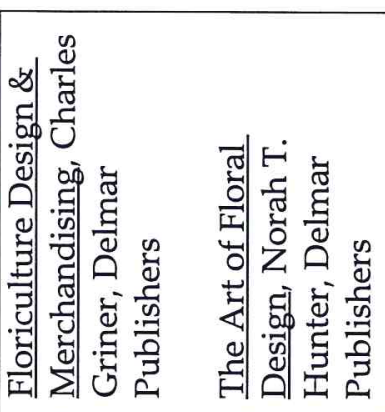

菊

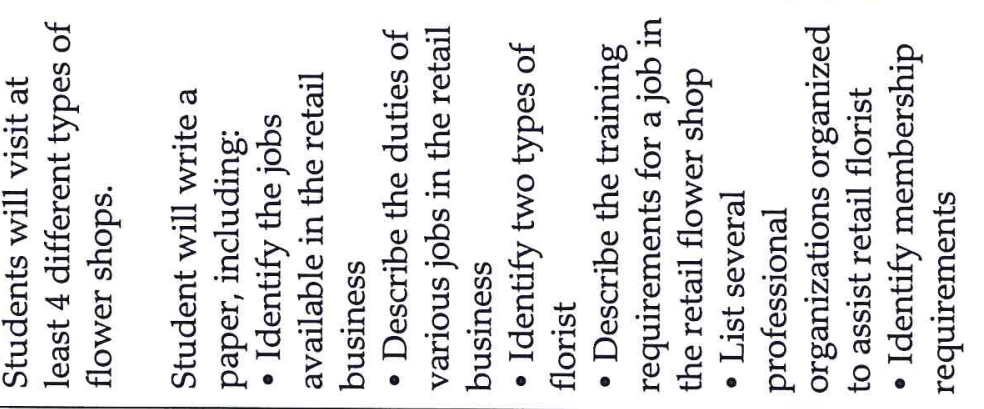

늠 


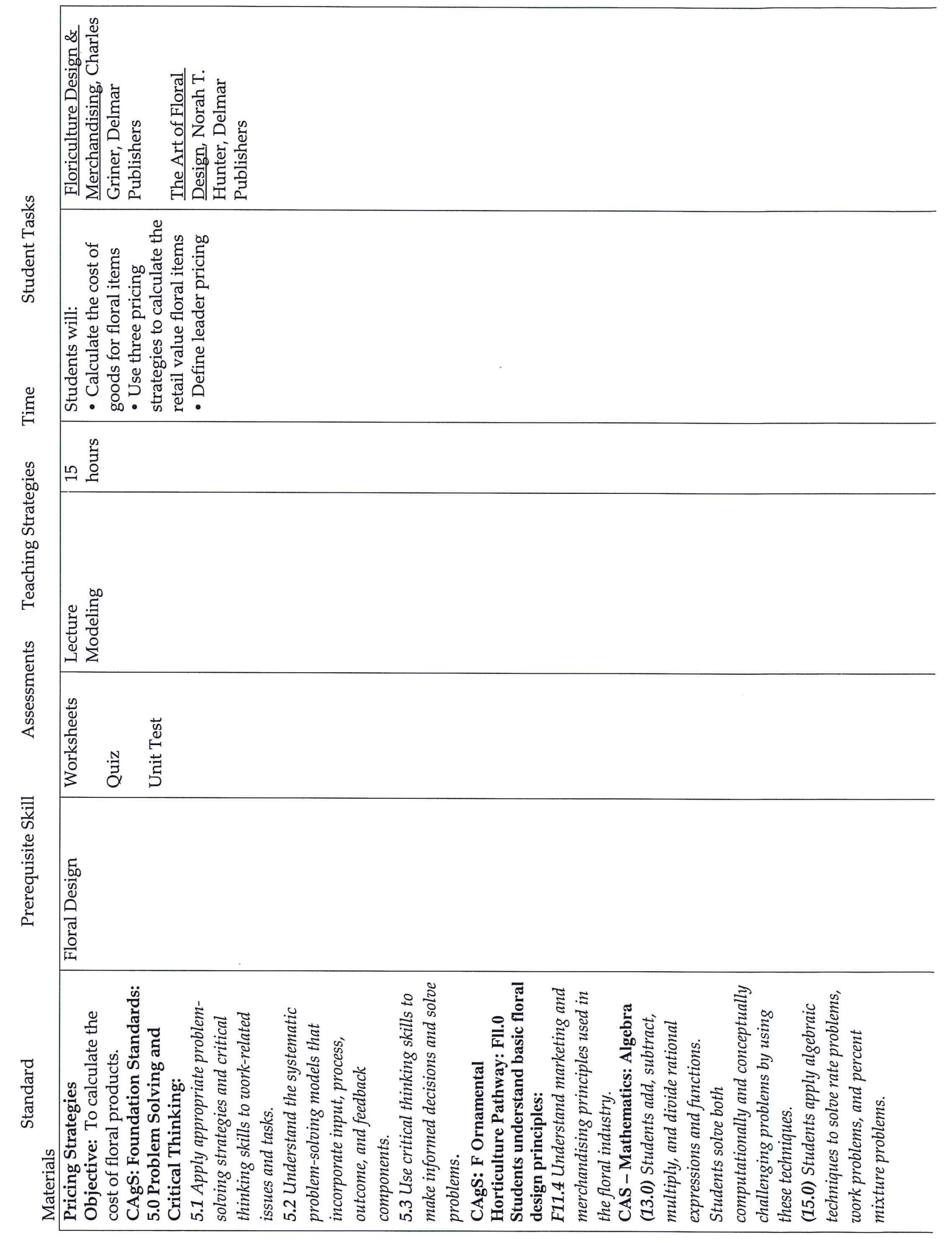




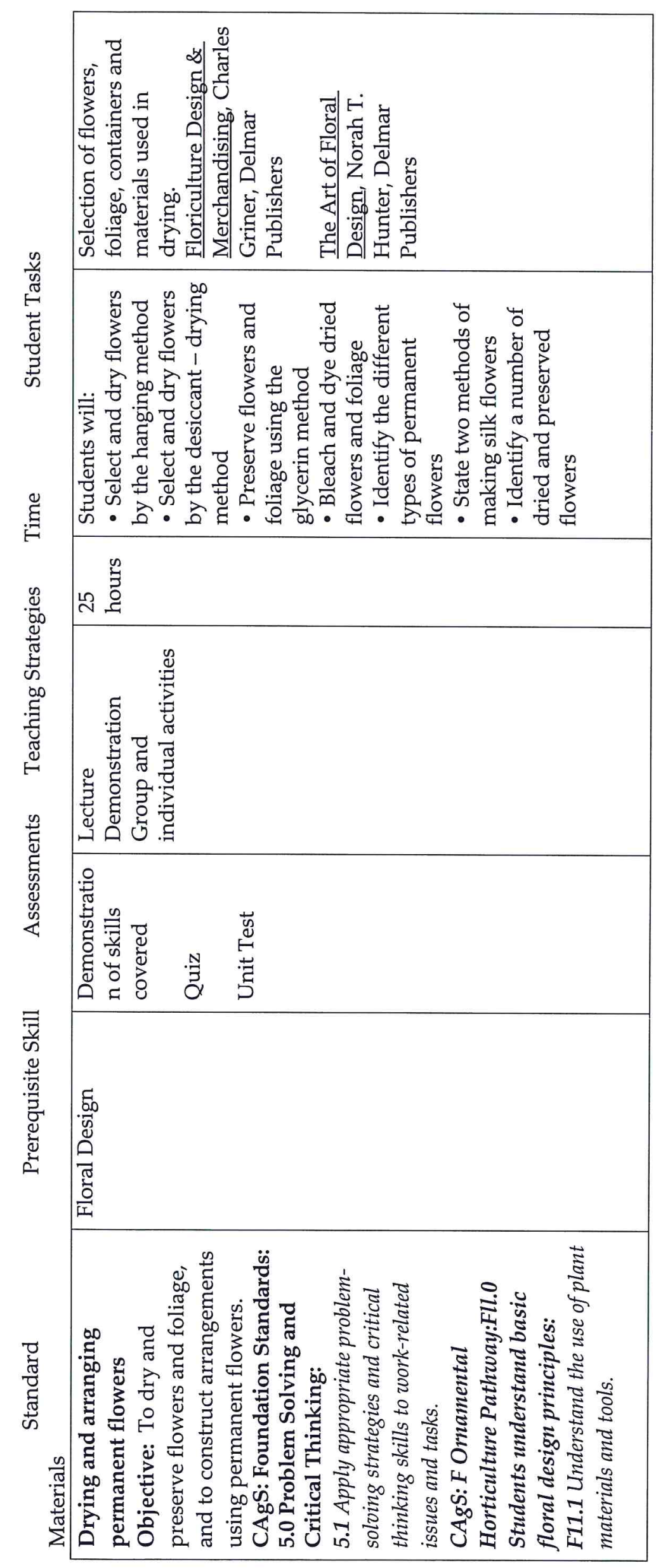




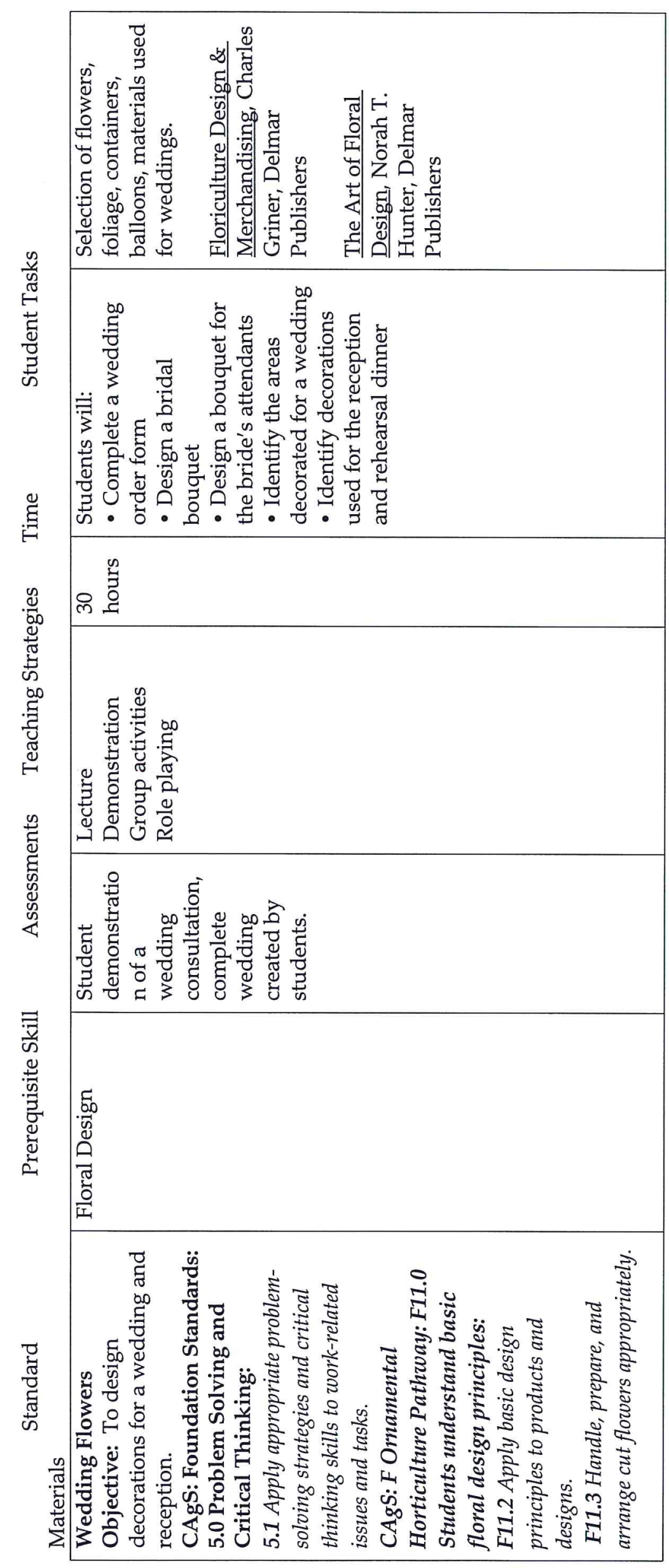




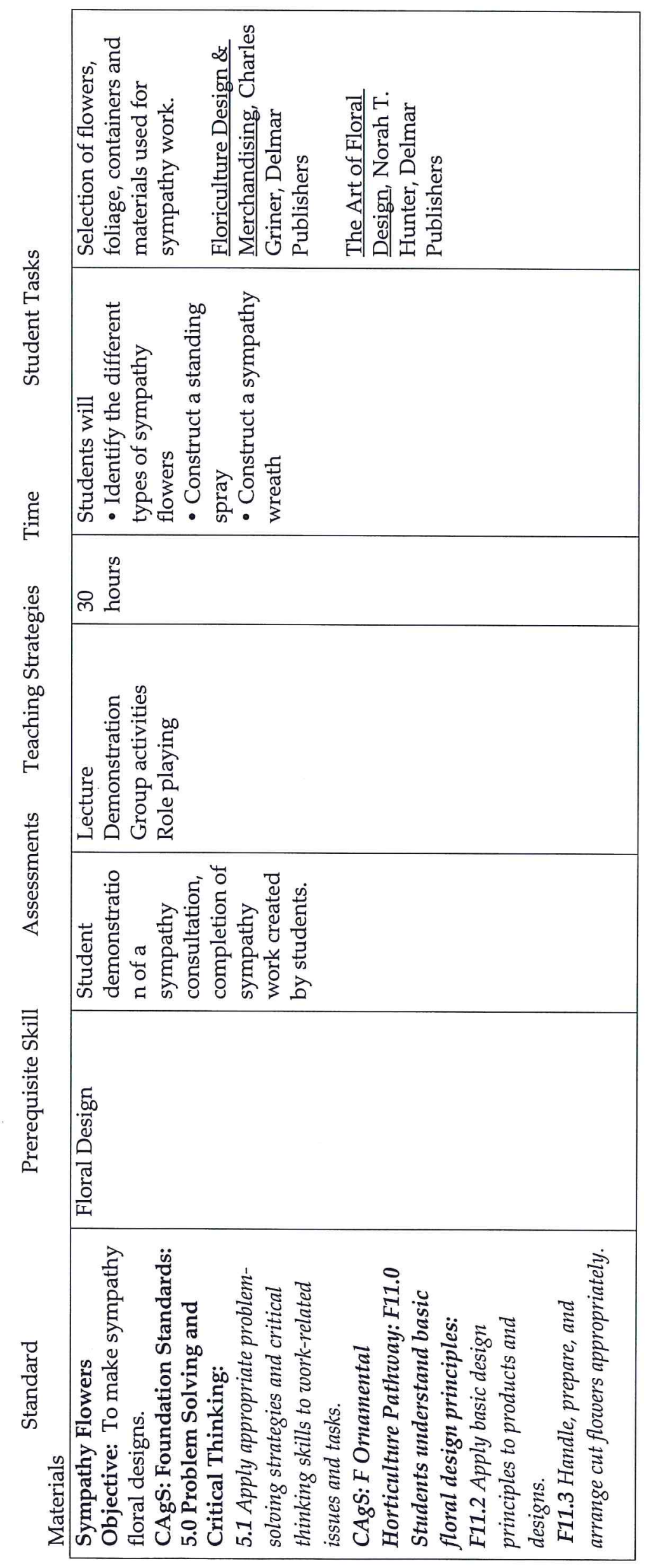




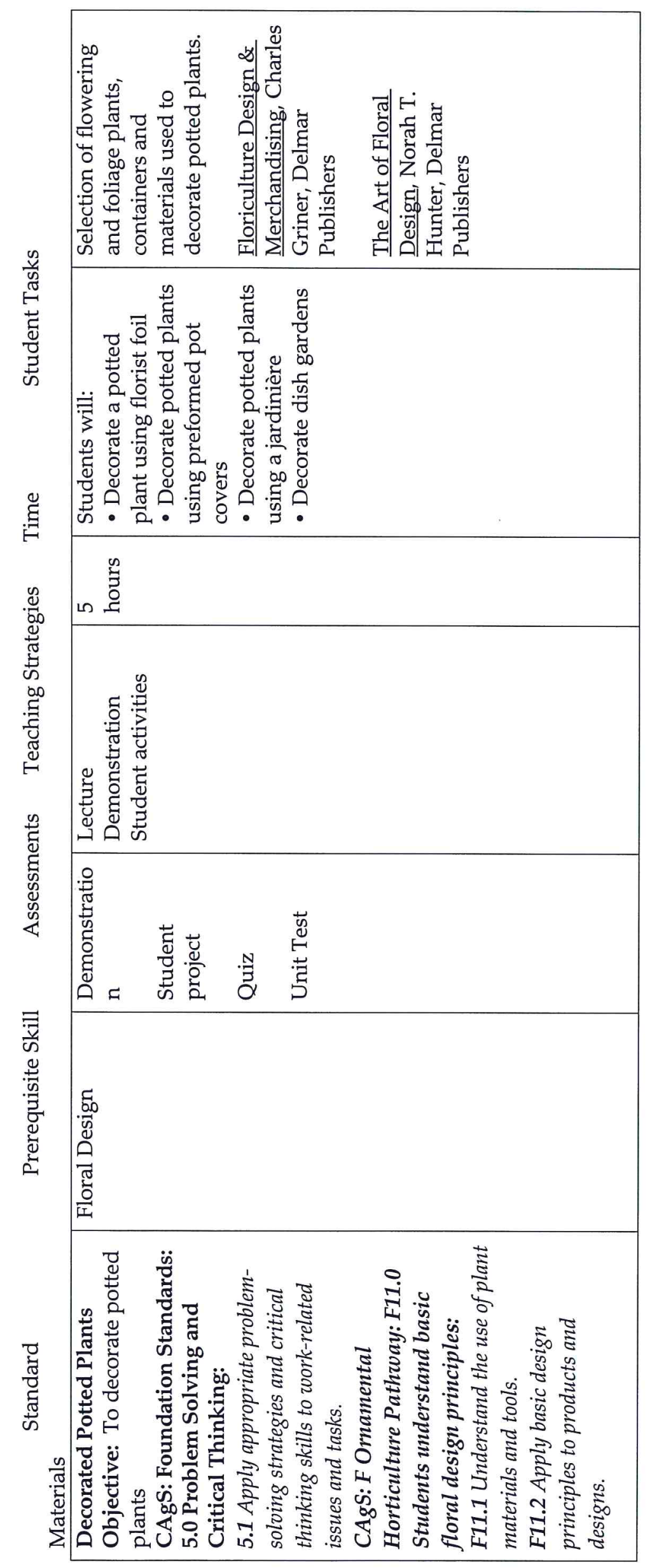




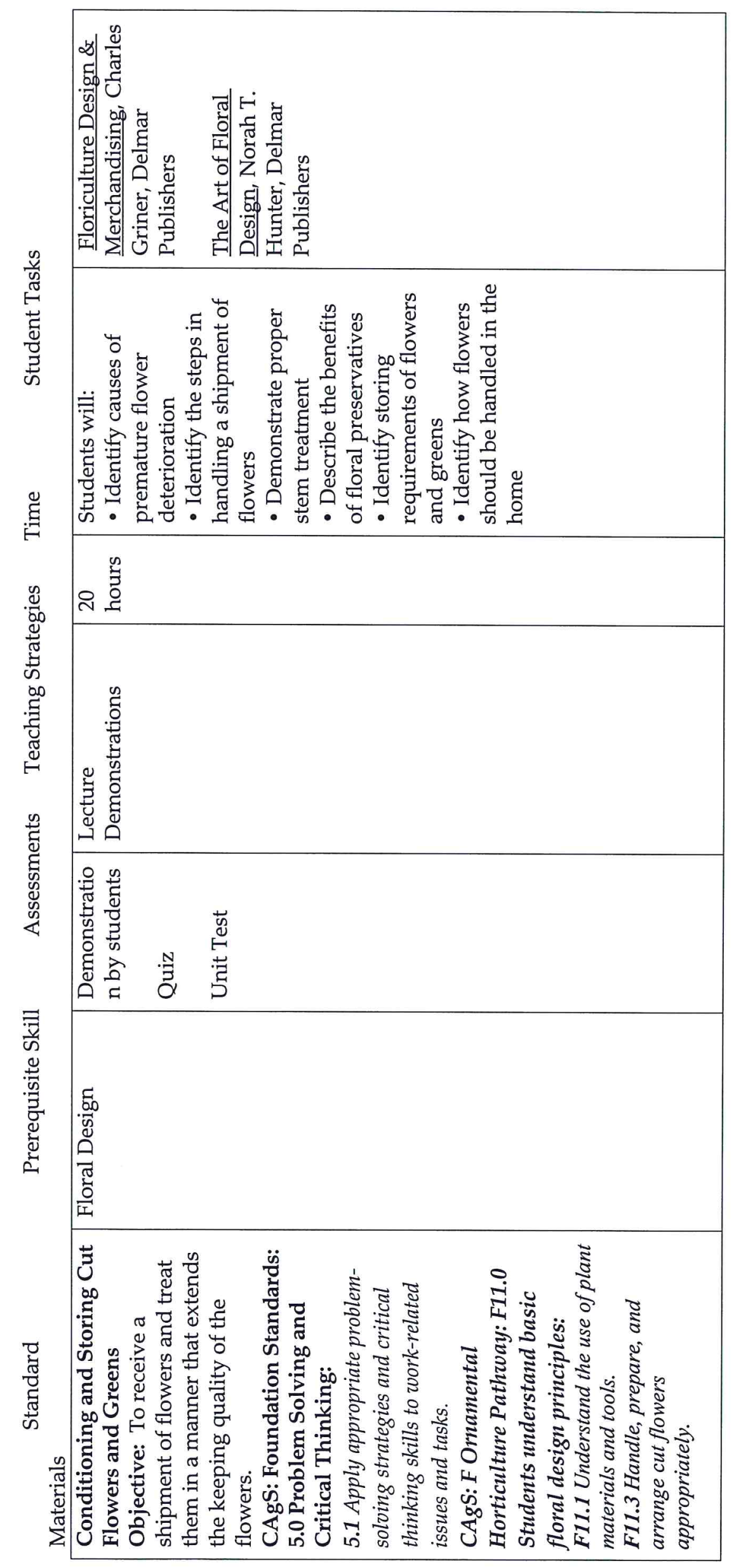




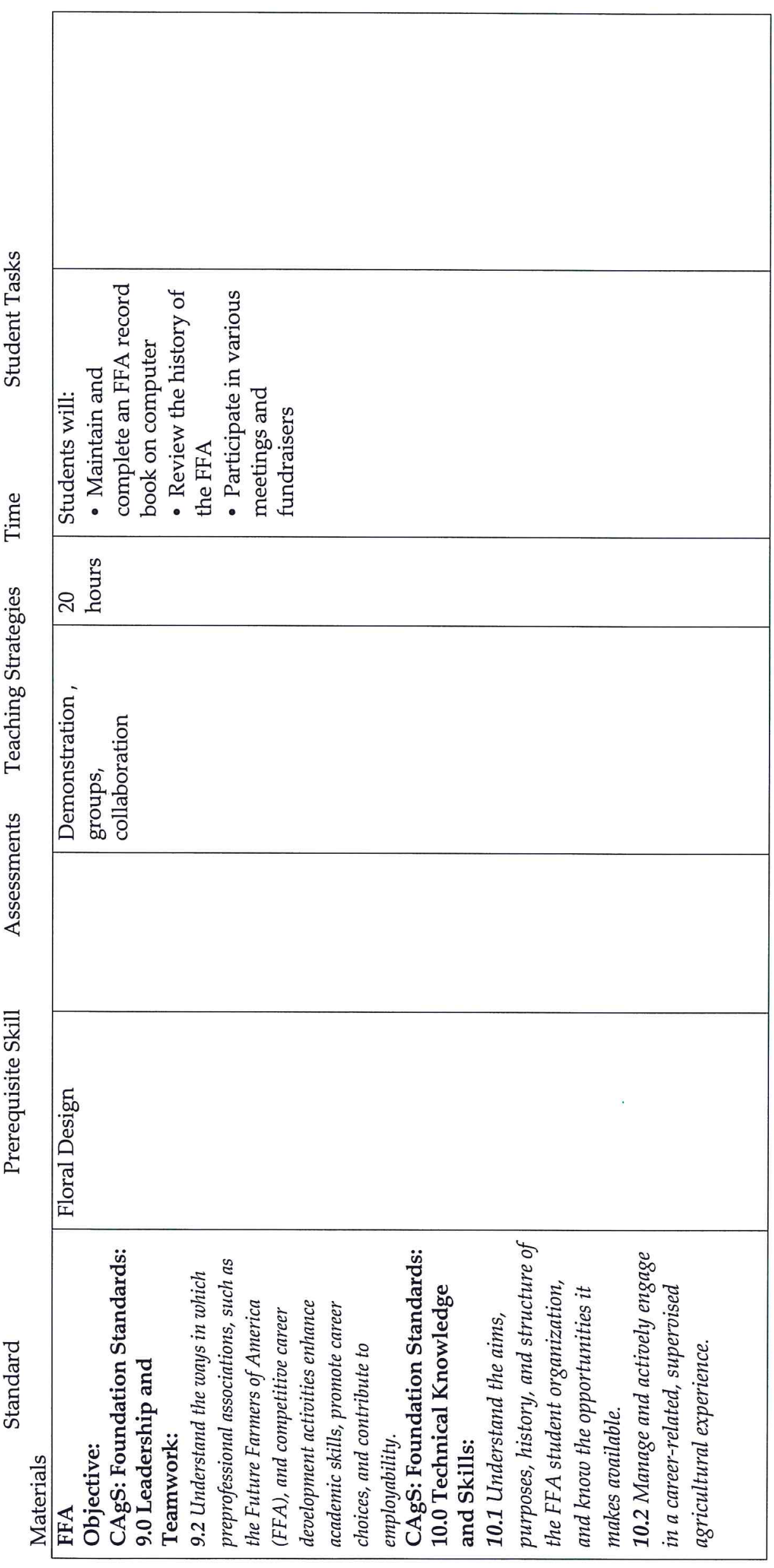




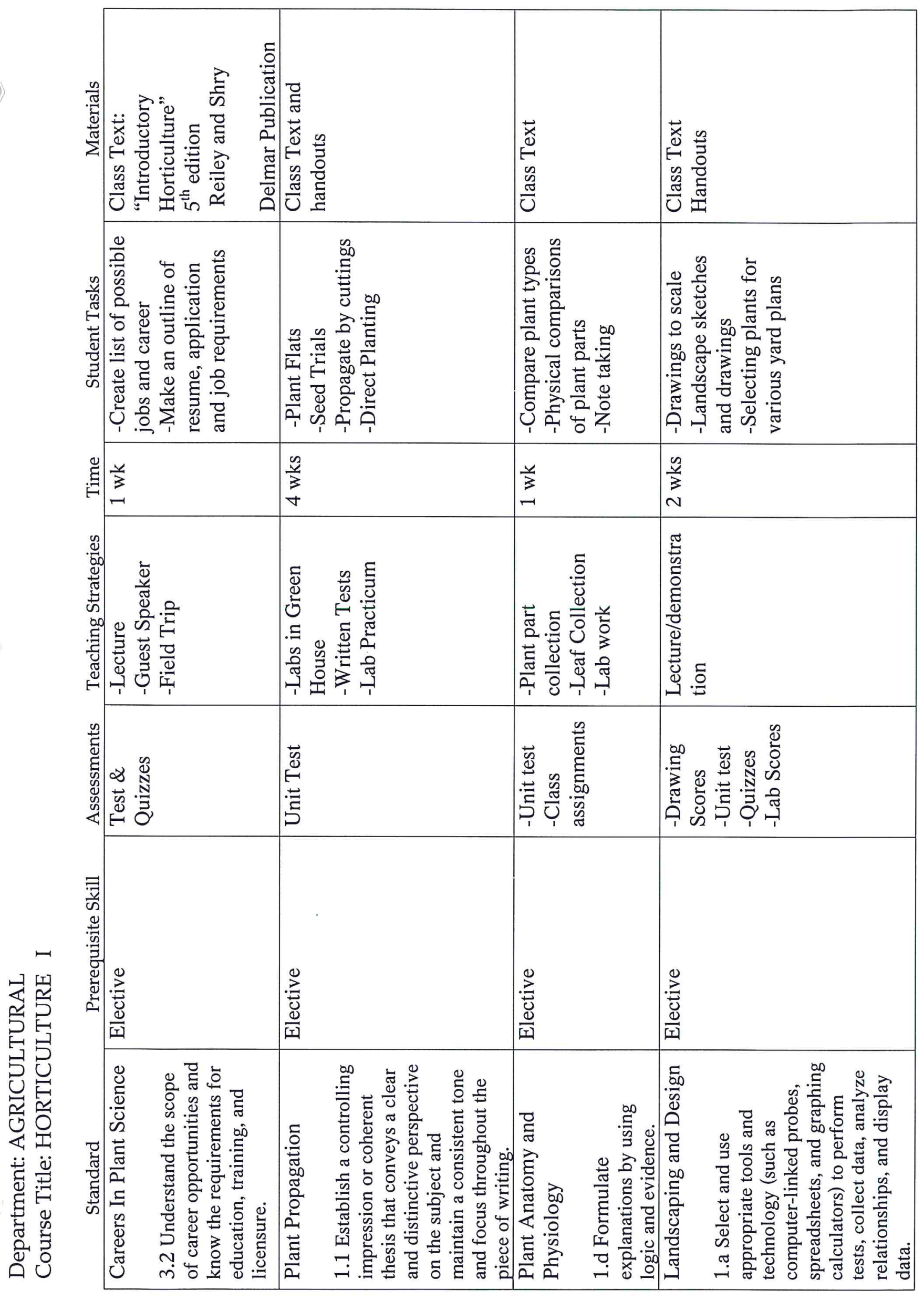




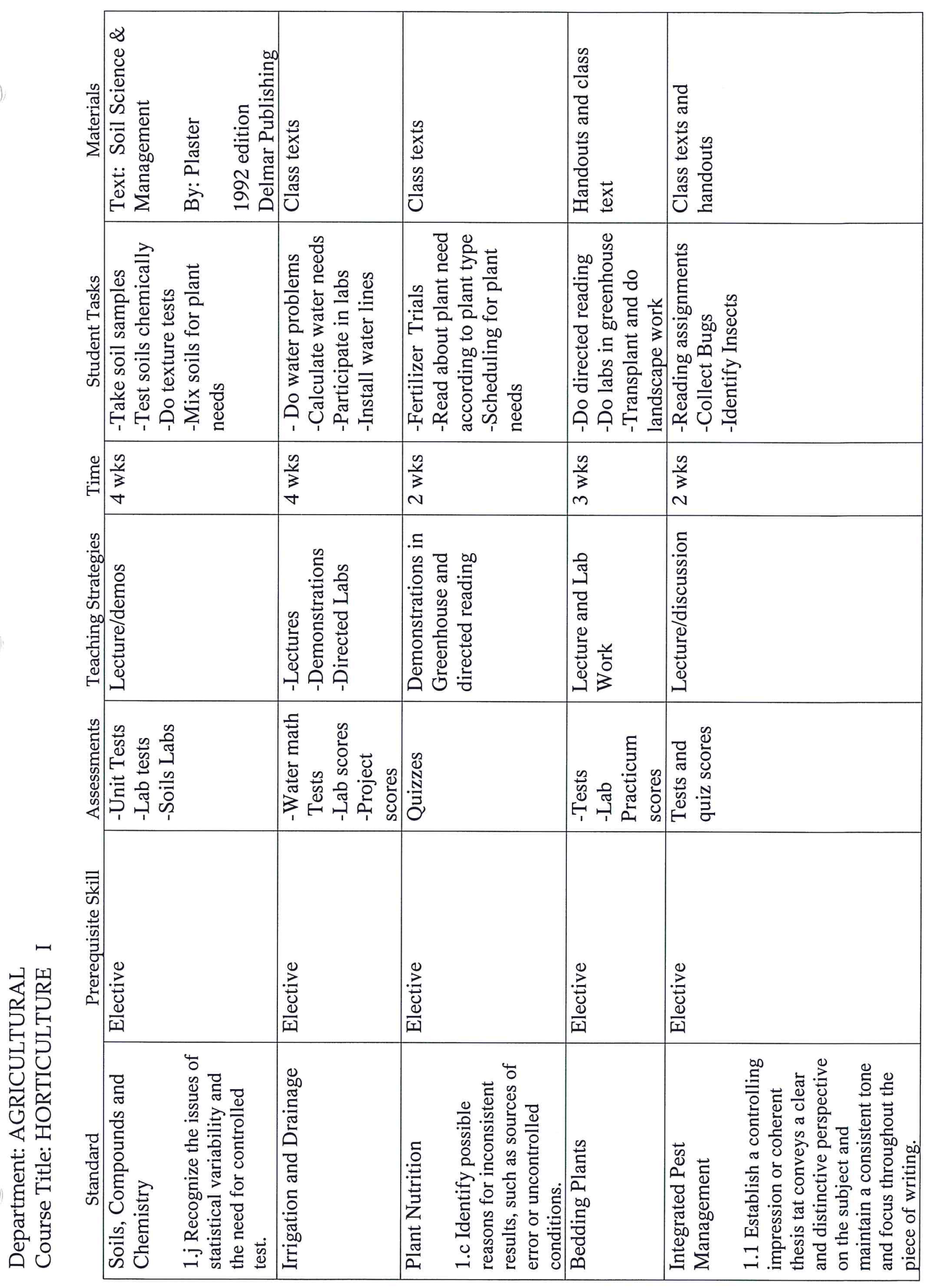




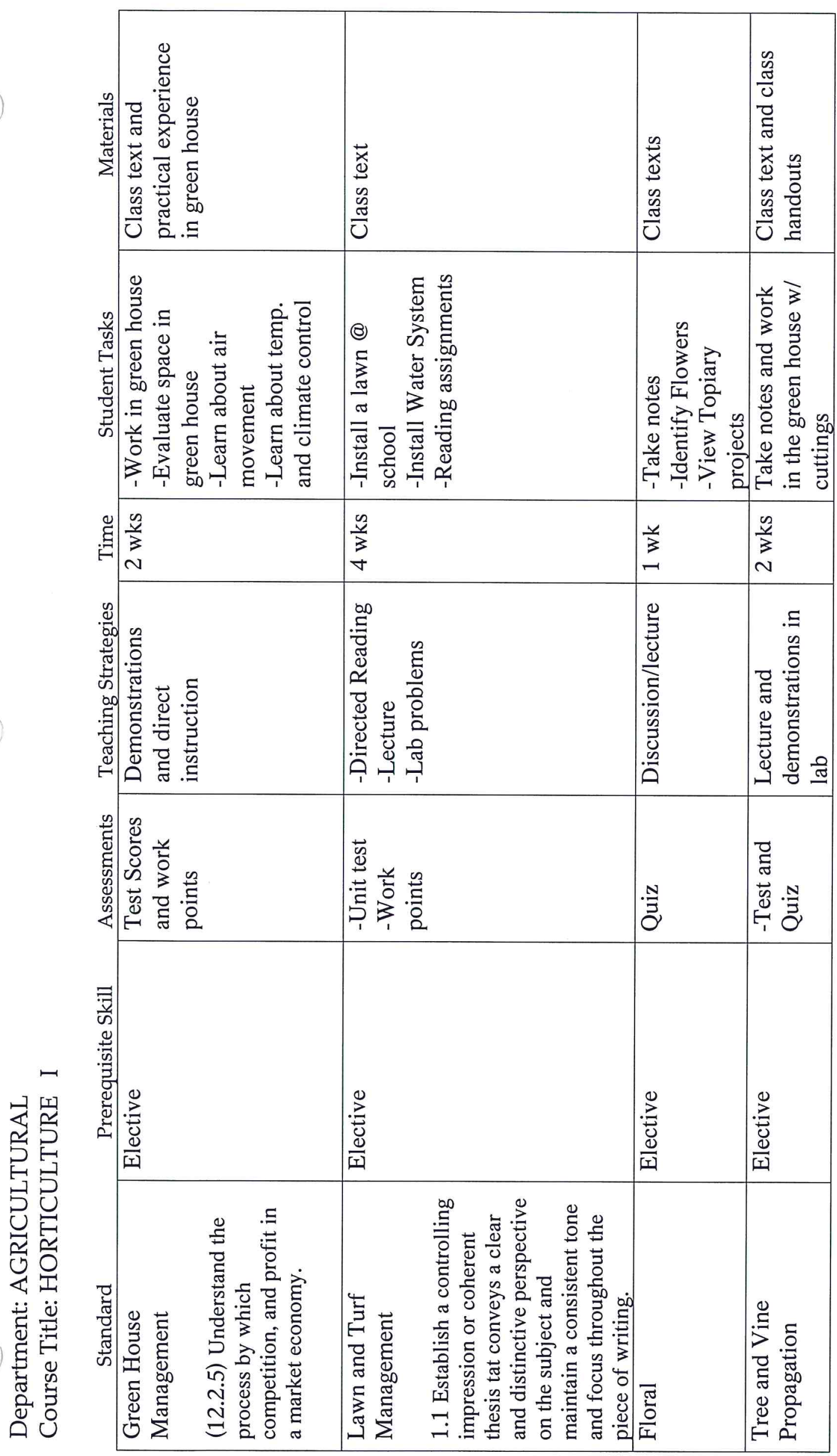




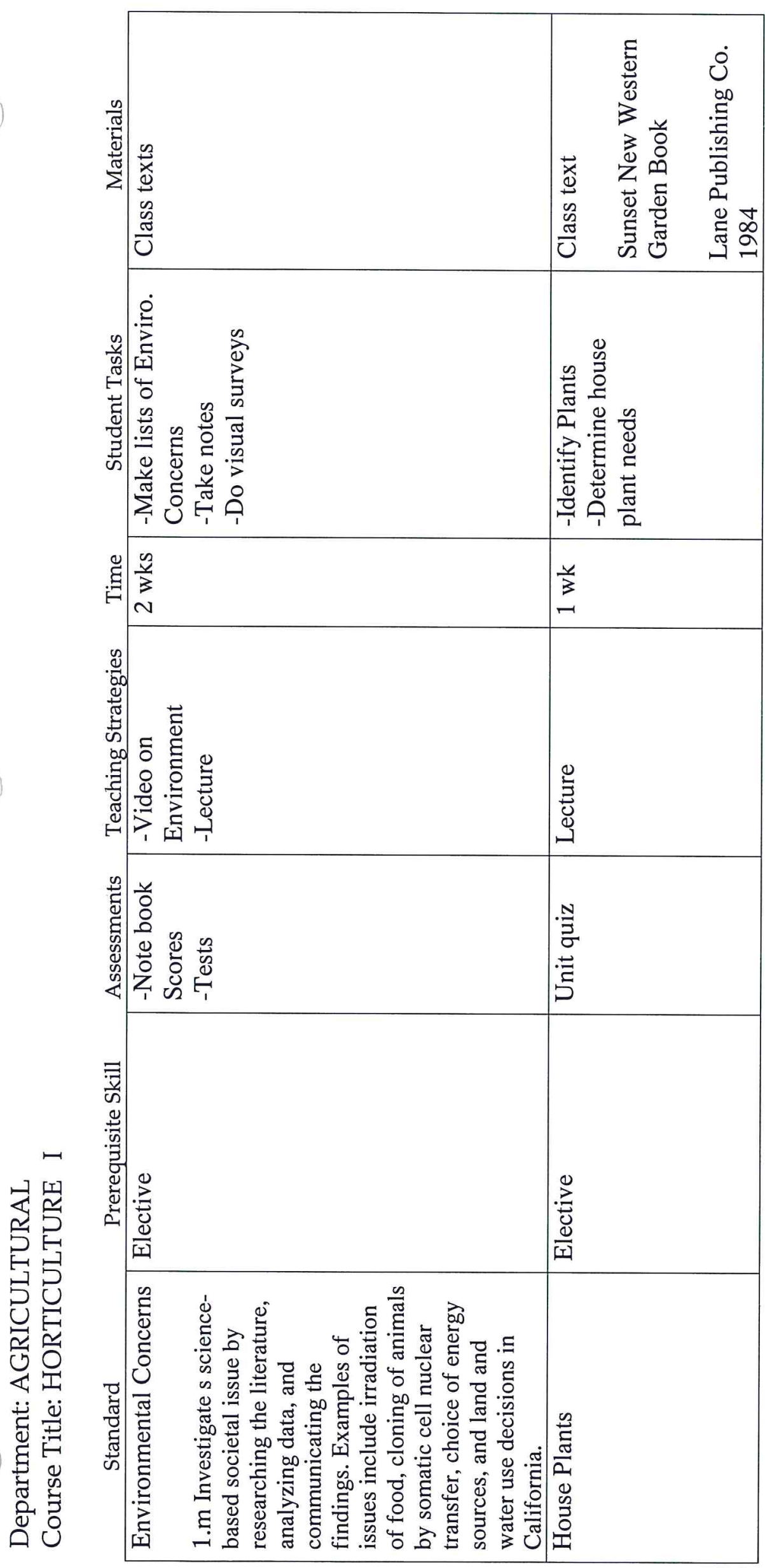




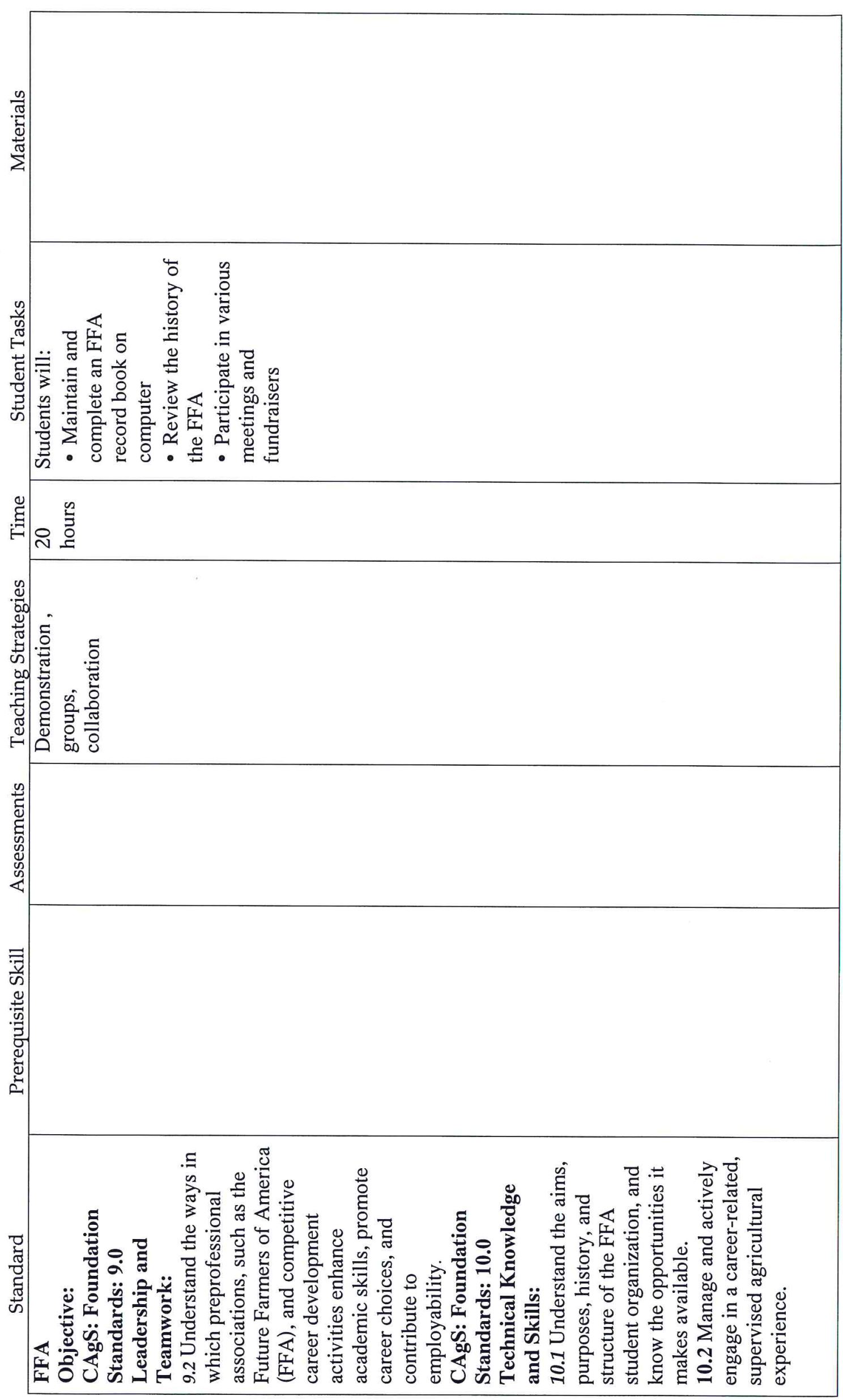




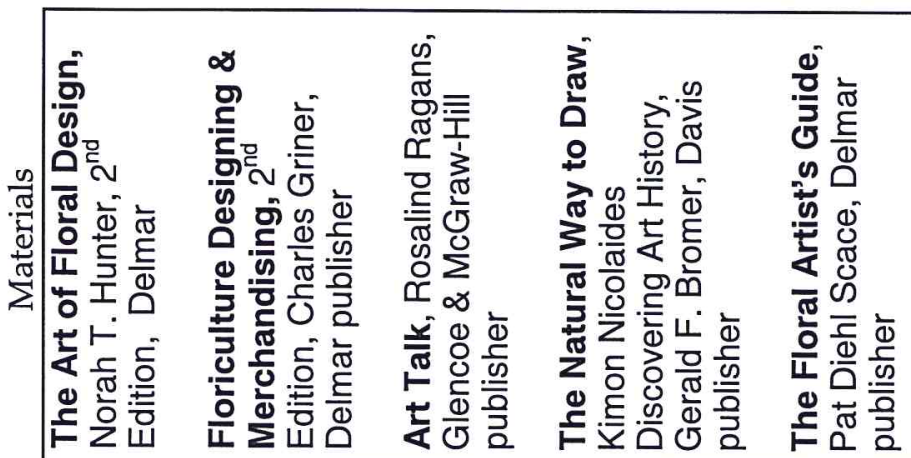

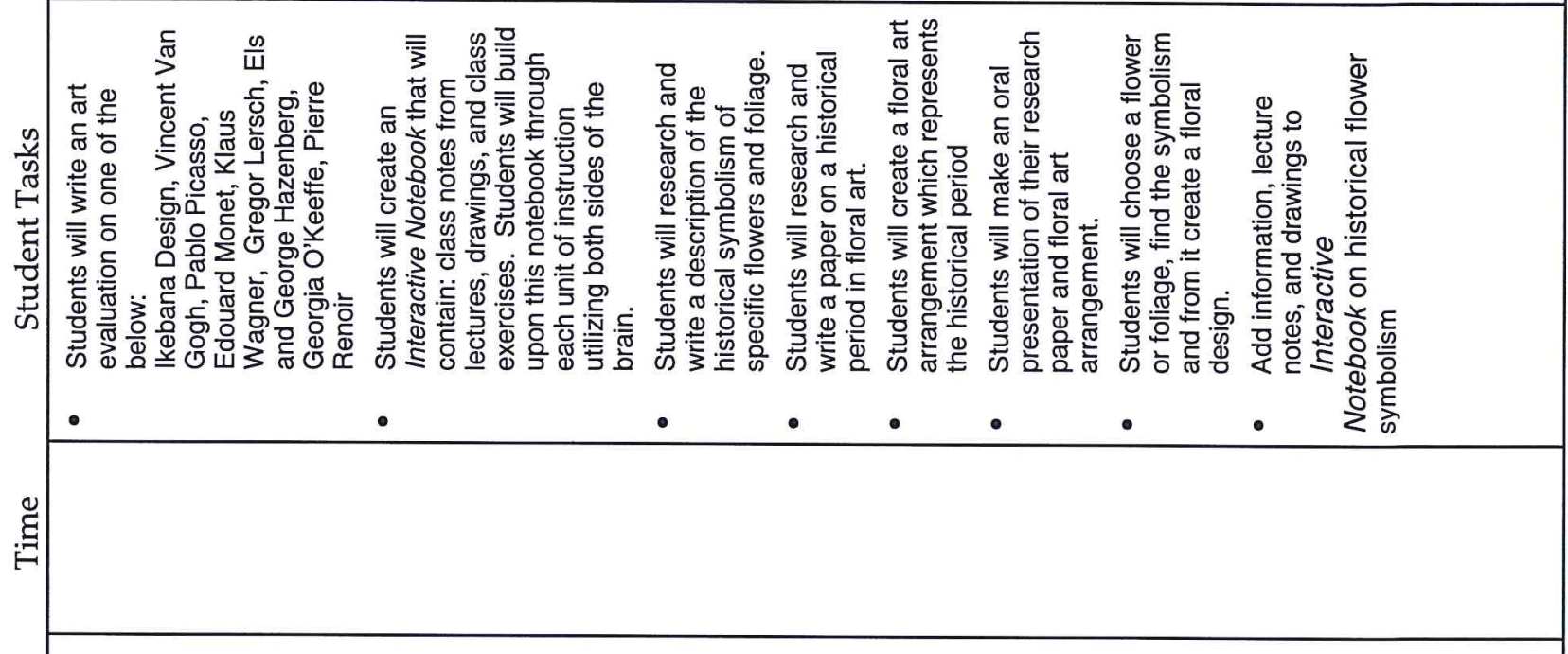

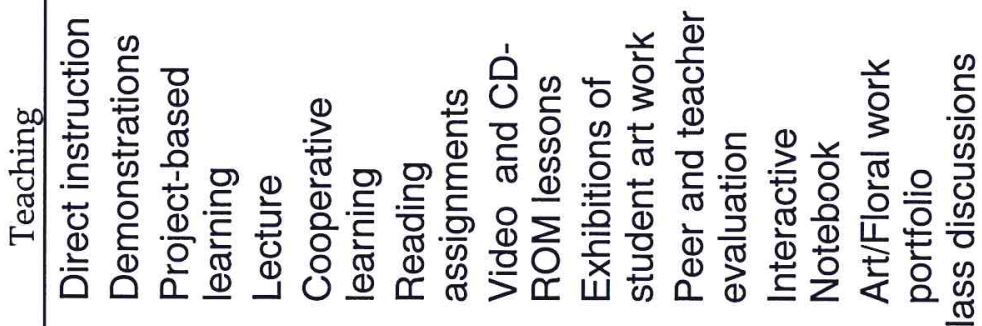
- $\quad$ m

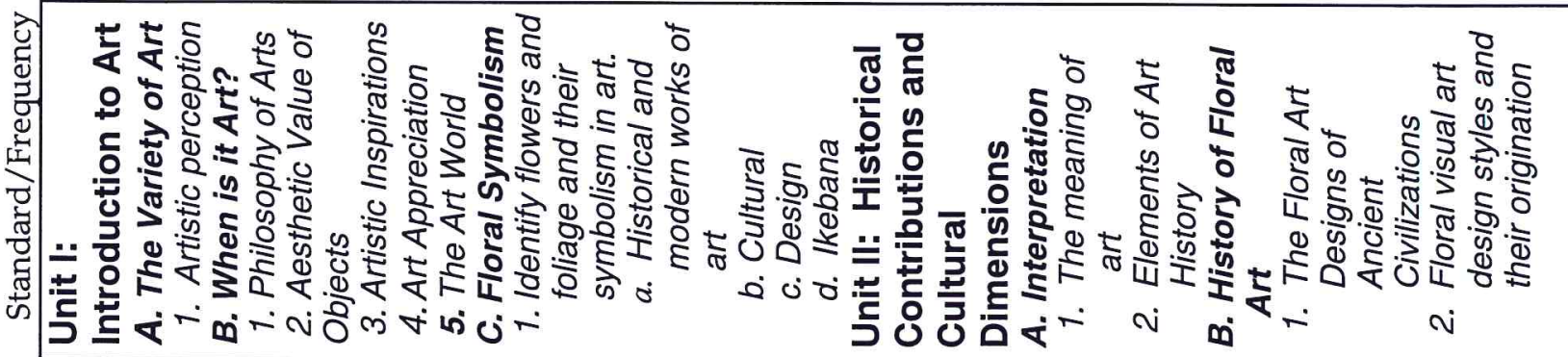




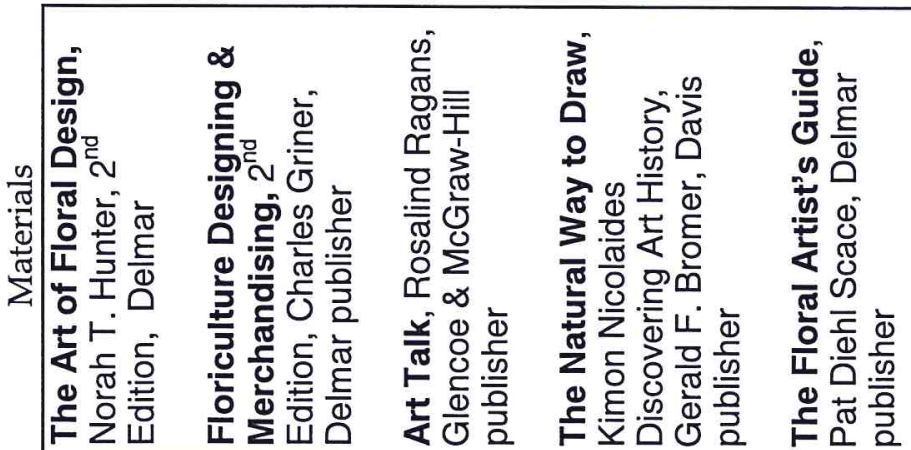

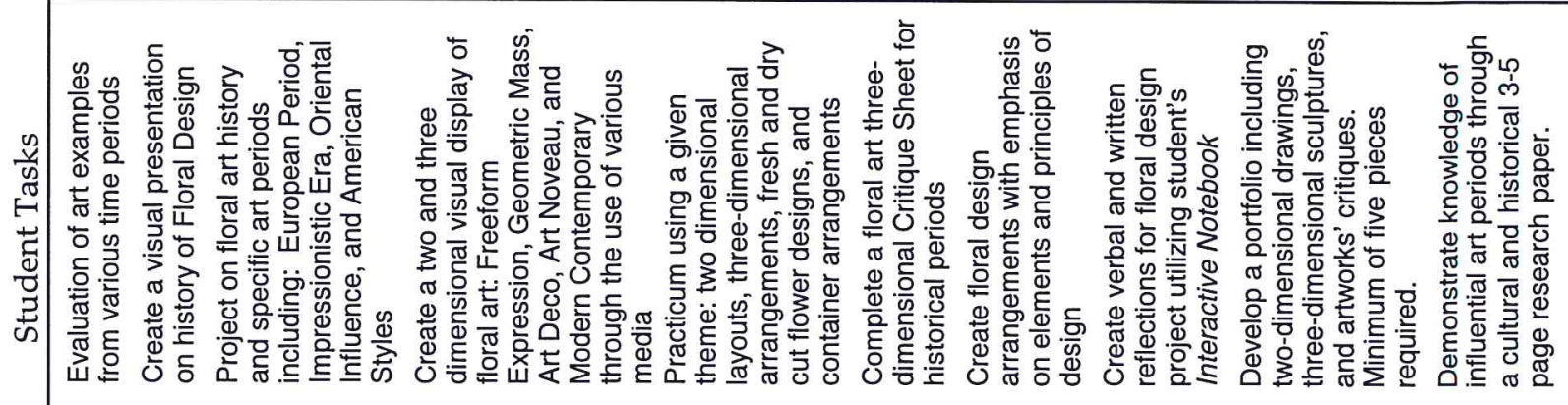

๕్

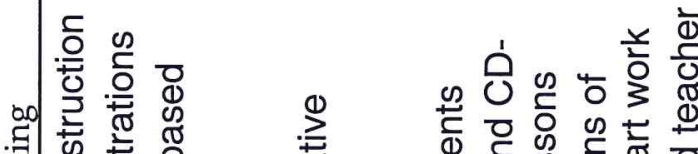

:

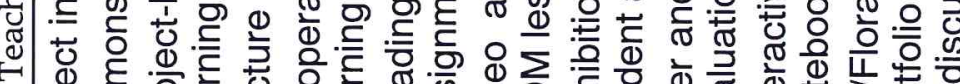
ब

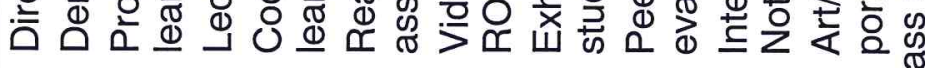

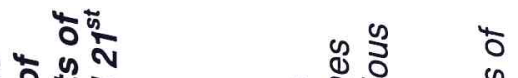

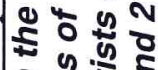

0

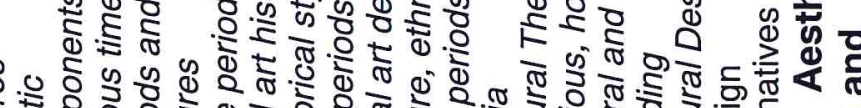

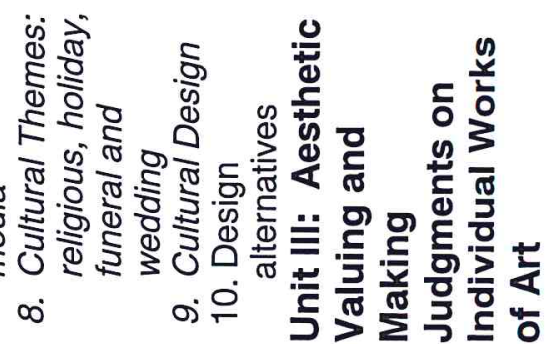



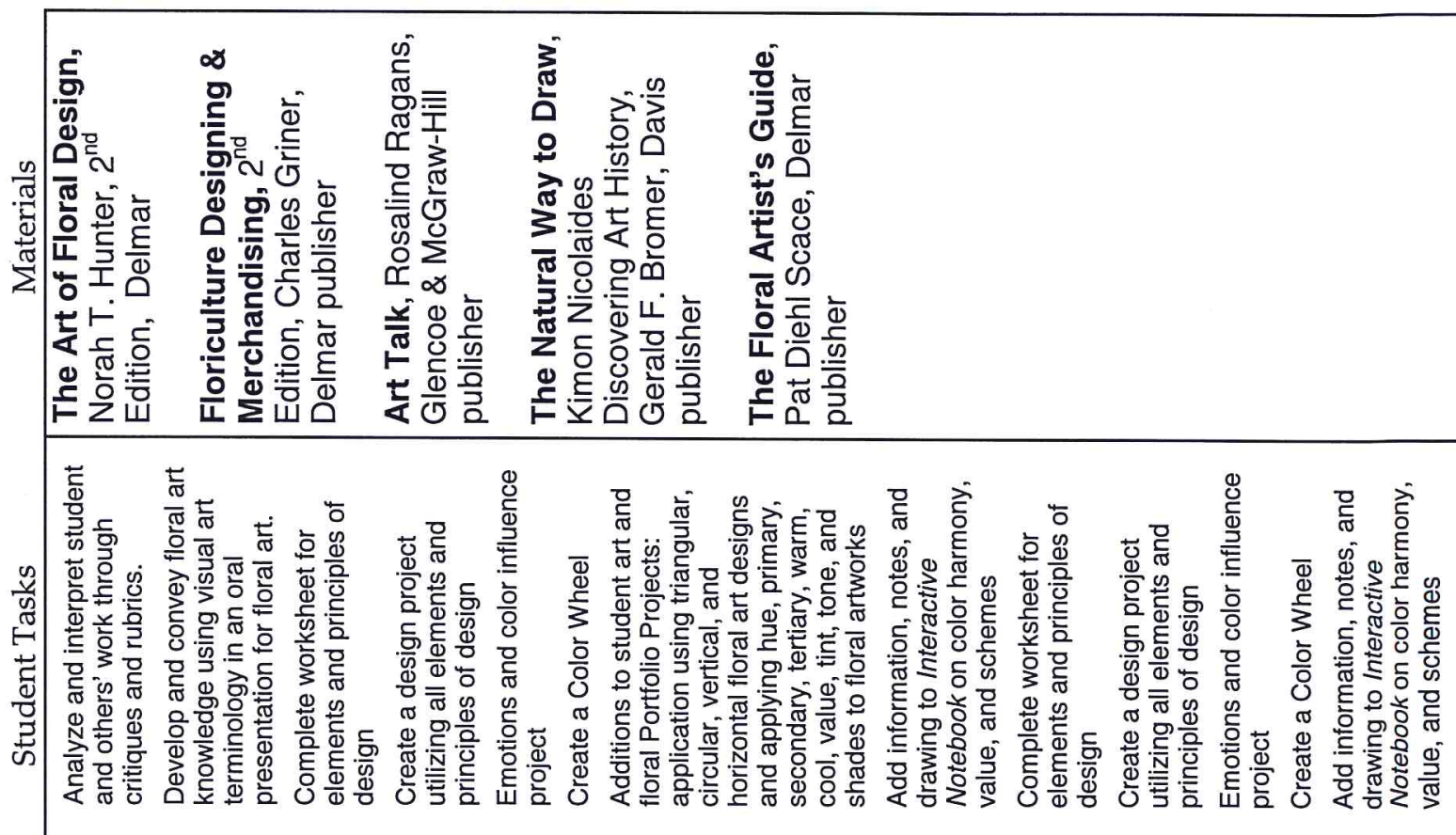

$\stackrel{\mathscr{Q}}{\mathrm{E}}$

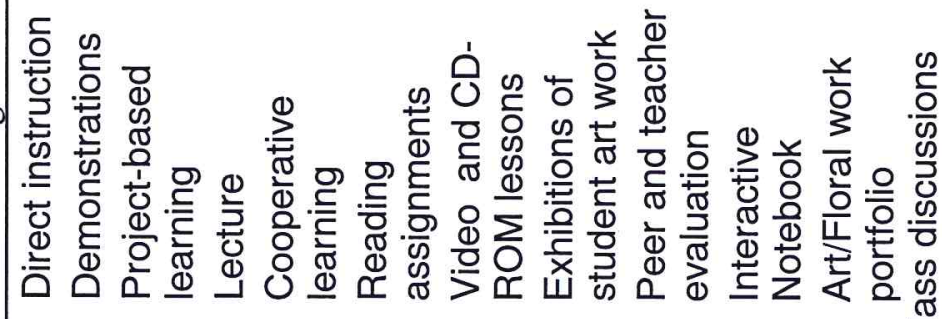

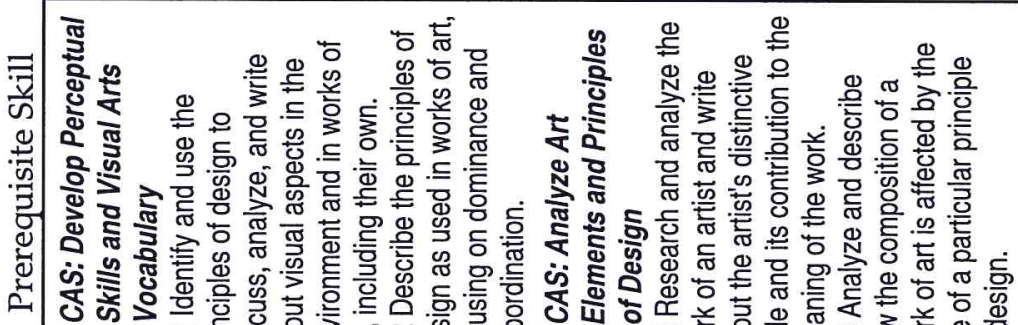

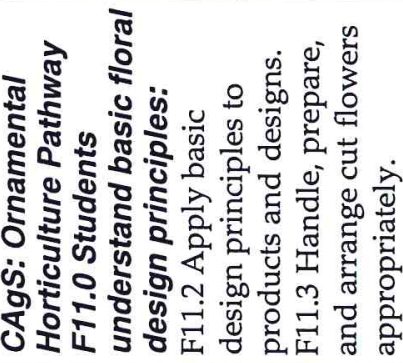

弐

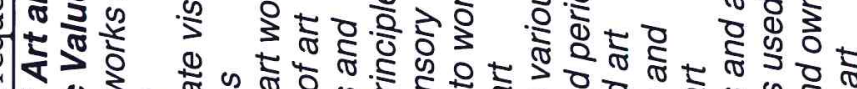



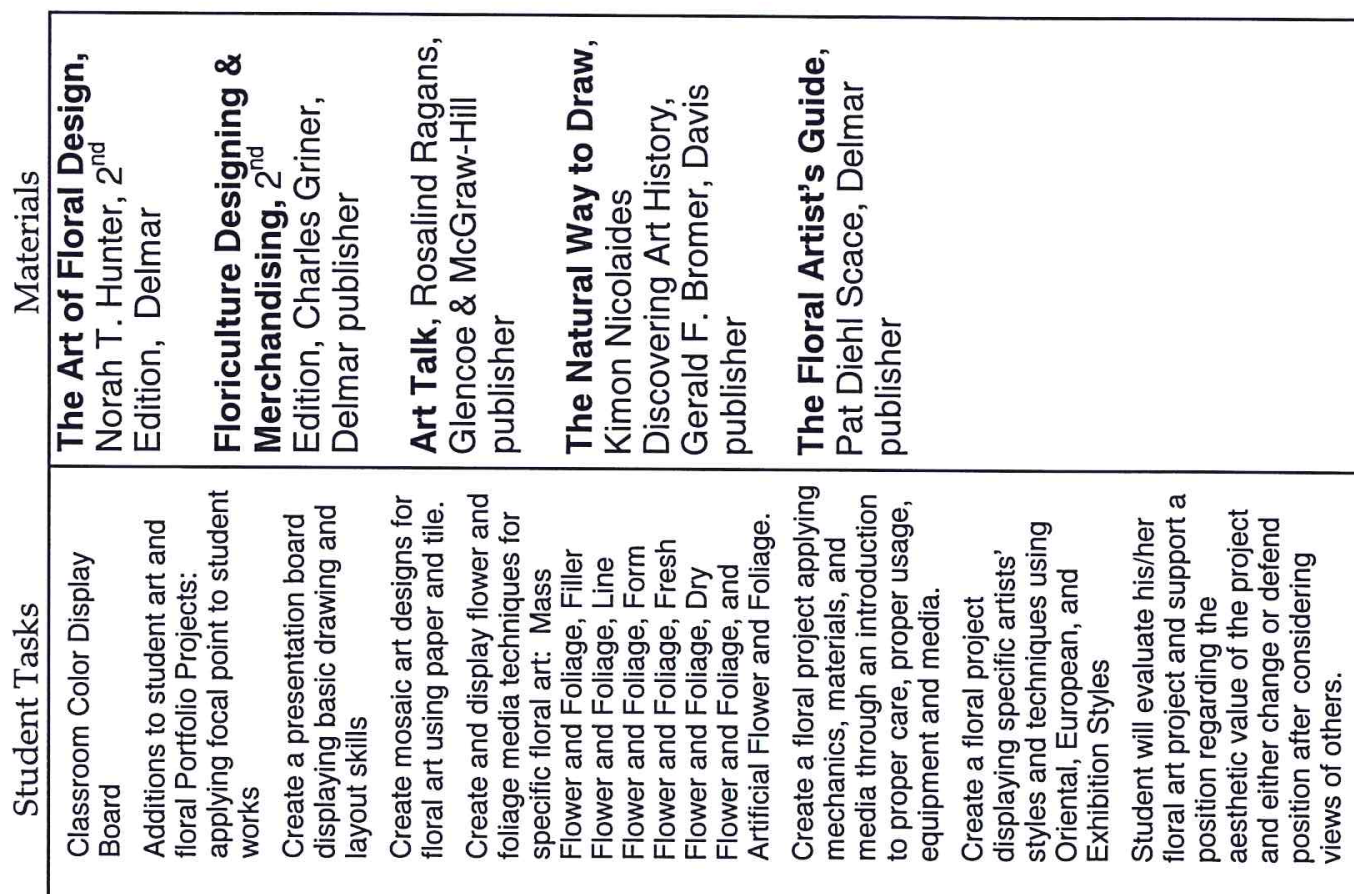

छี

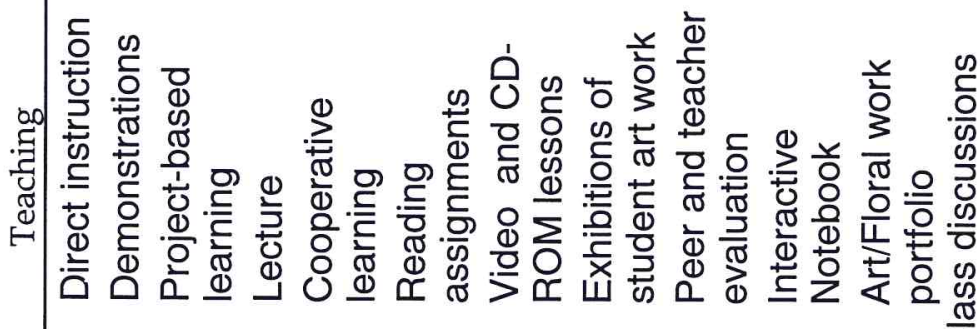

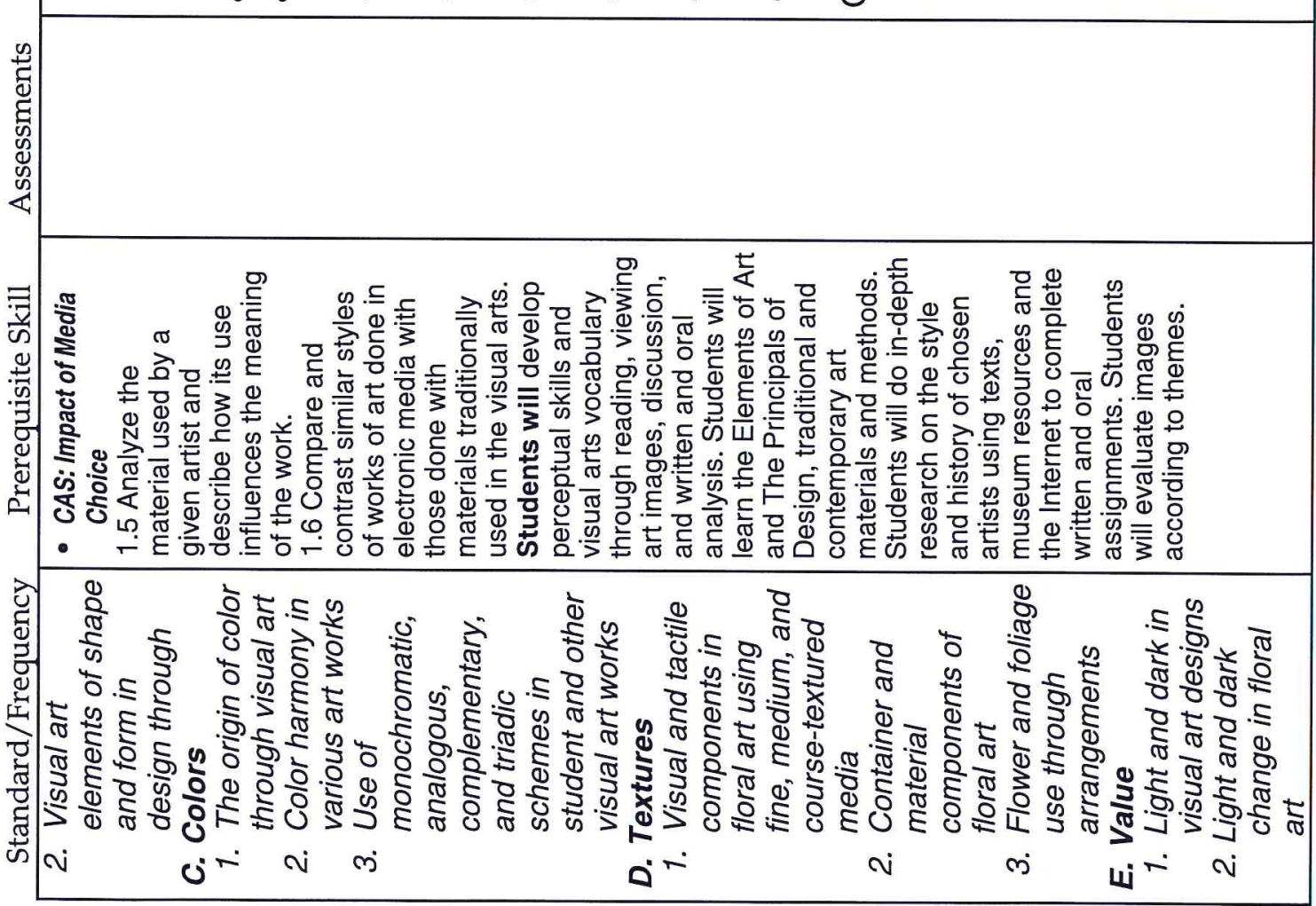



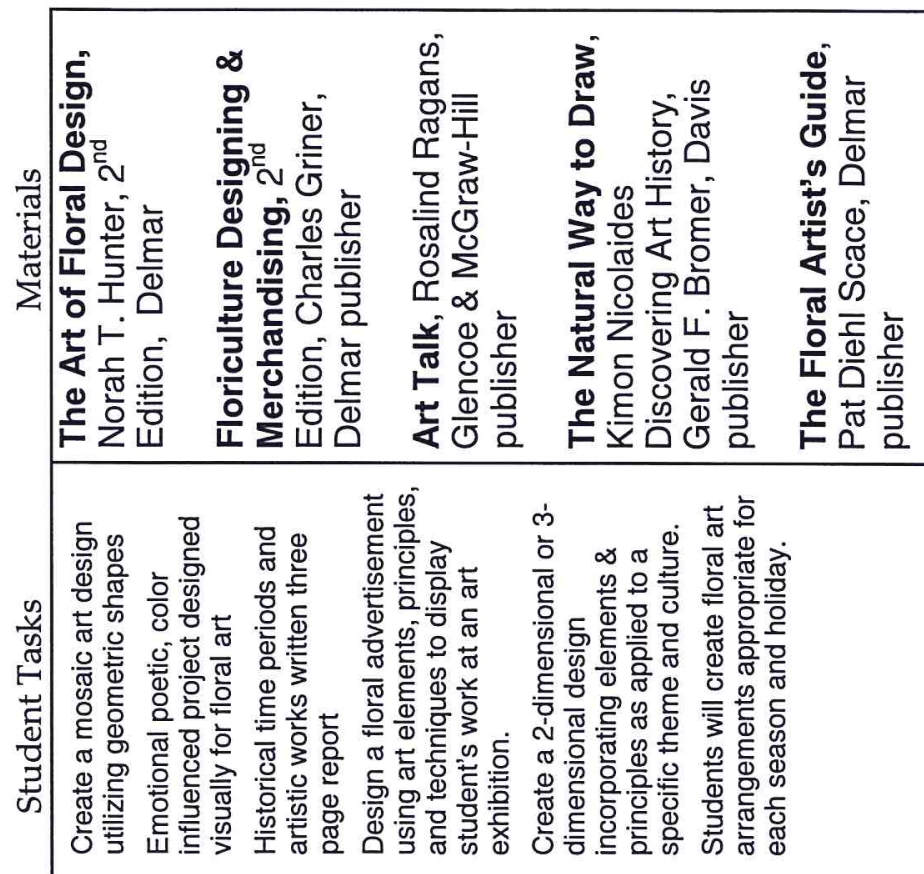

్ㅗㅂ

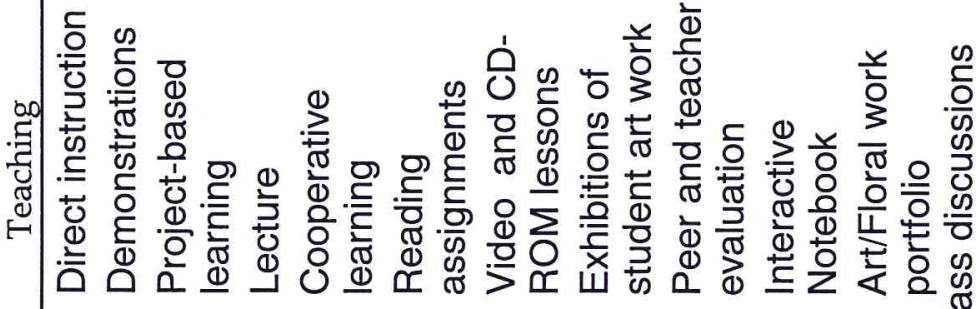

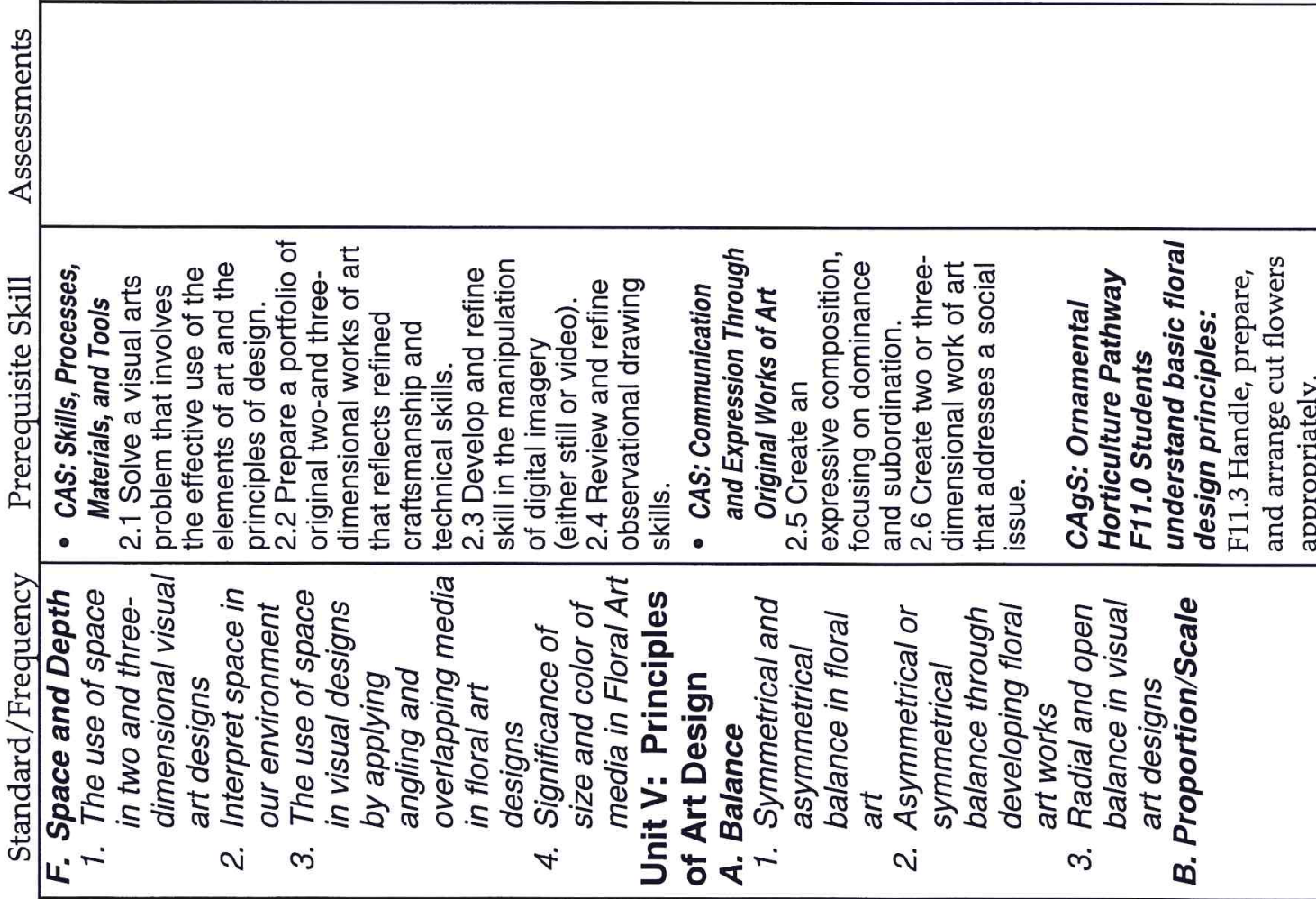




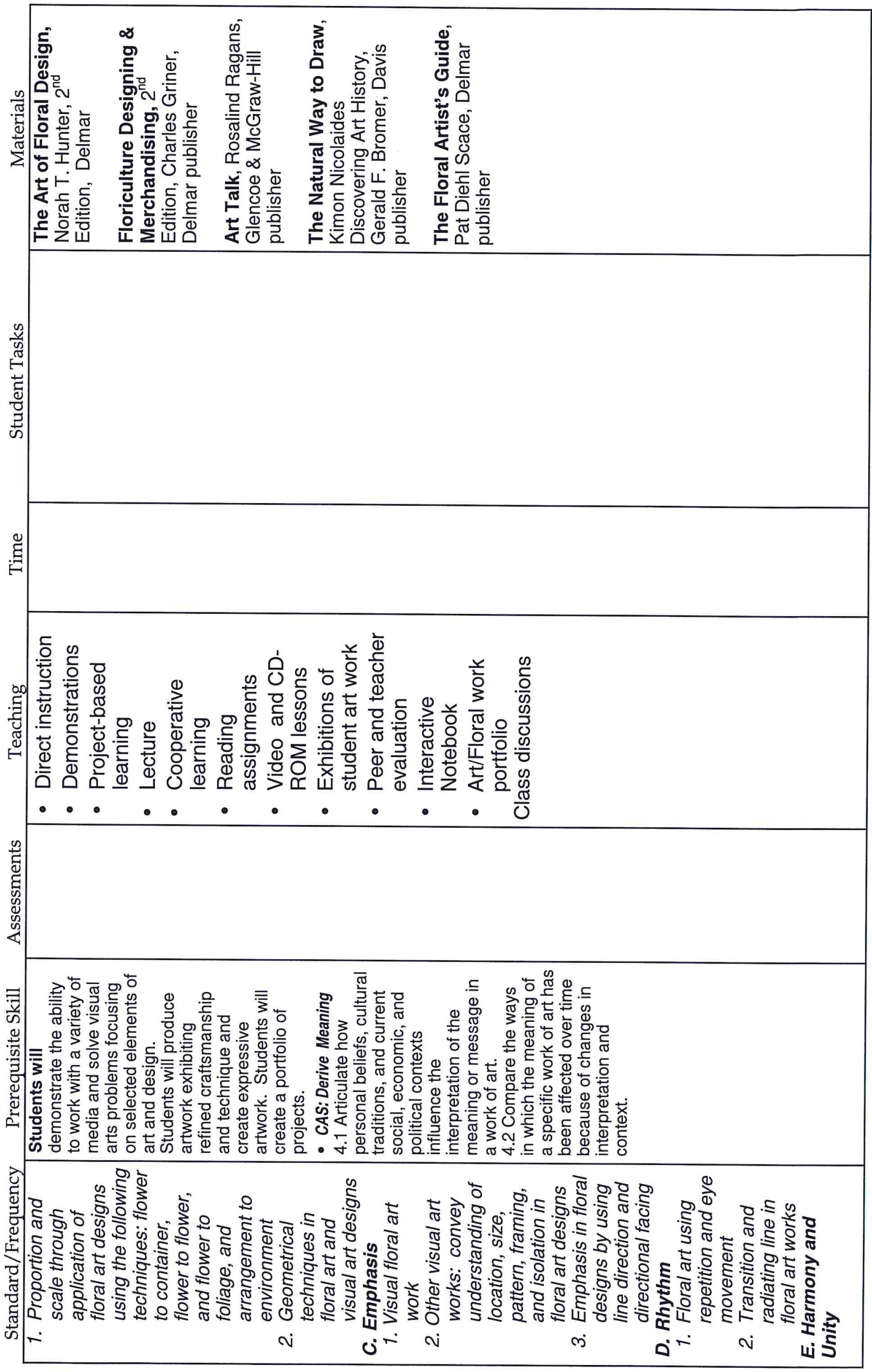




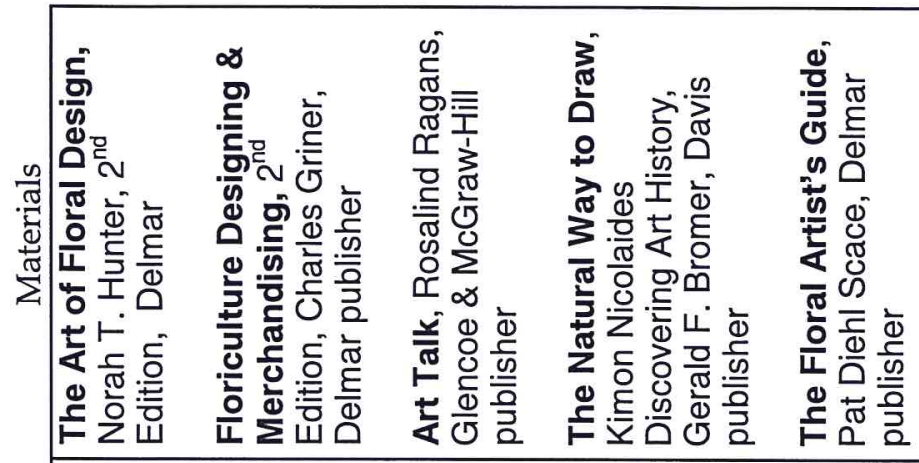

क्ष

๕્g

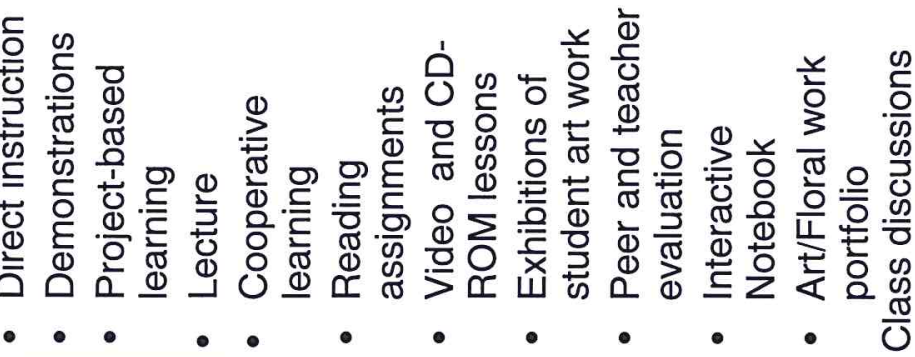

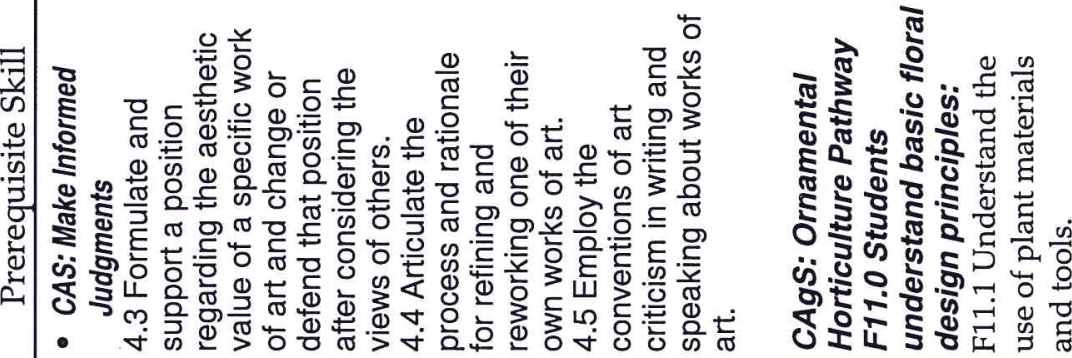




\begin{tabular}{|c|c|c|c|c|}
\hline 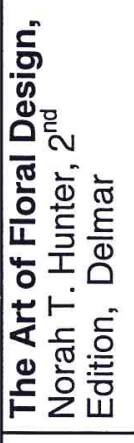 & 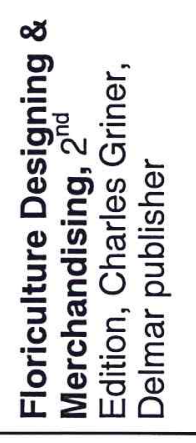 & 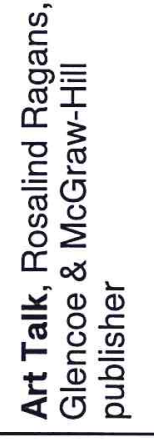 & 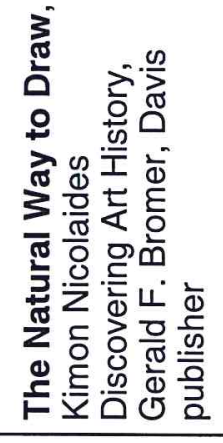 & 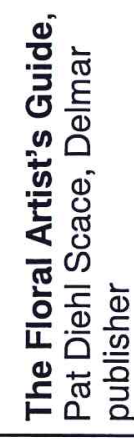 \\
\hline
\end{tabular}

$\frac{\text { कै }}{\text { कै }}$

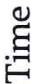

:

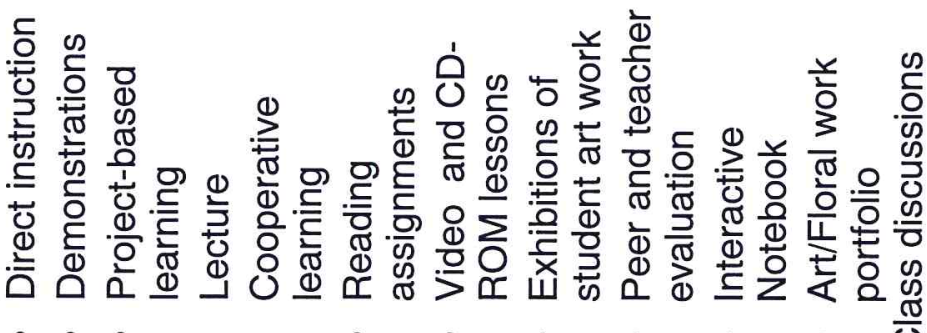

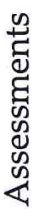

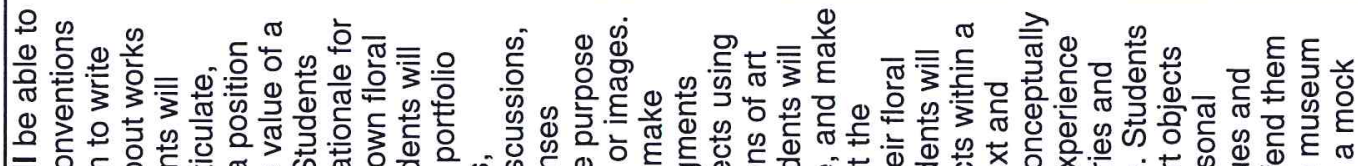

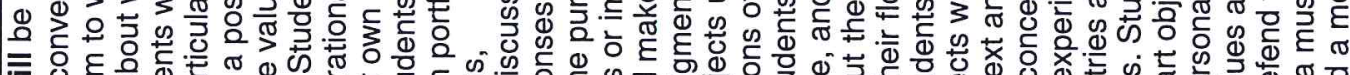

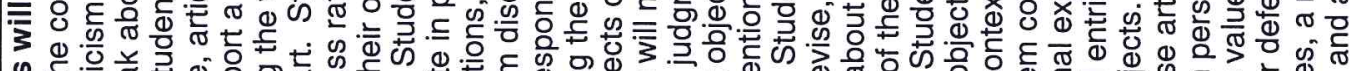

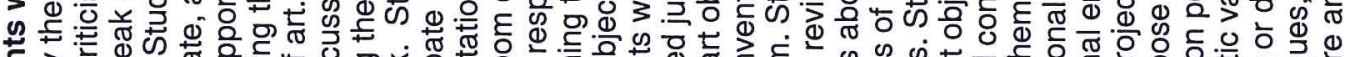

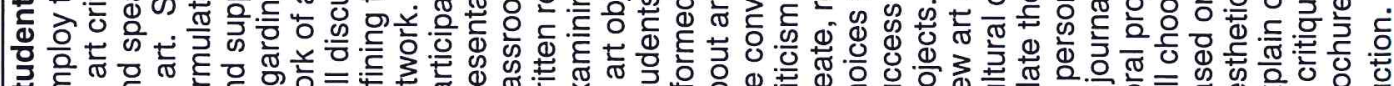

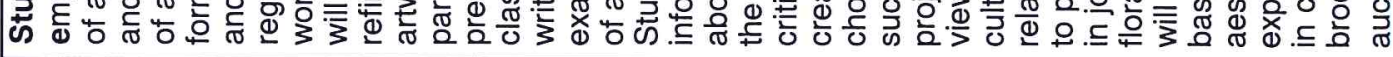

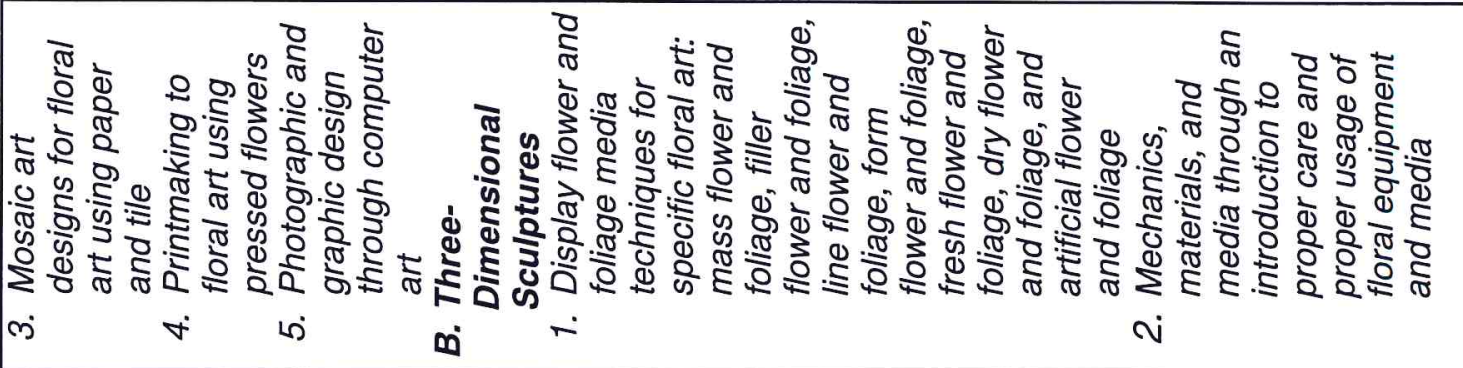




\begin{tabular}{|c|c|c|c|c|}
\hline 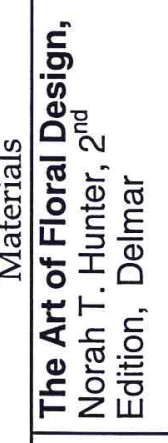 & 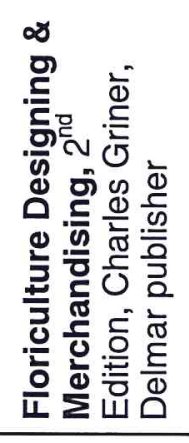 & 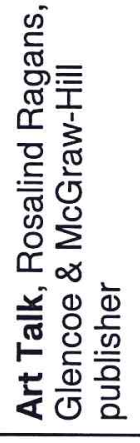 & 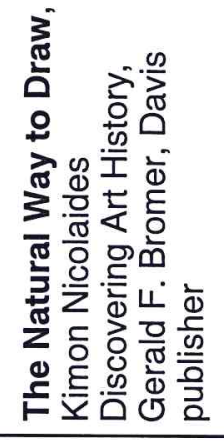 & 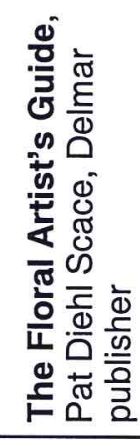 \\
\hline
\end{tabular}

छ్

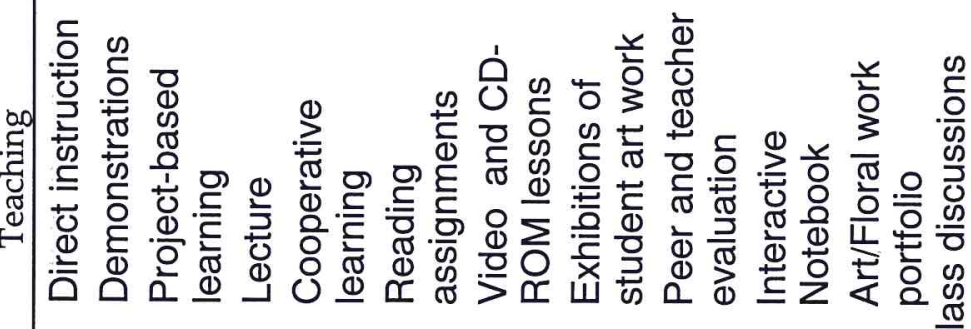

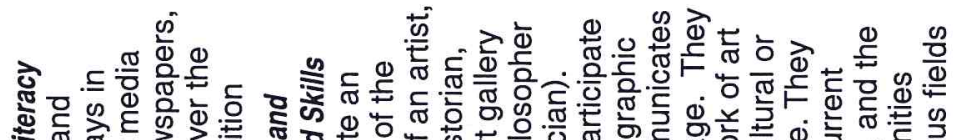

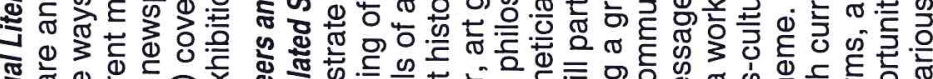

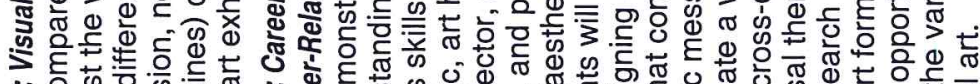

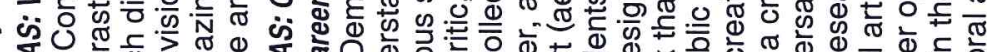

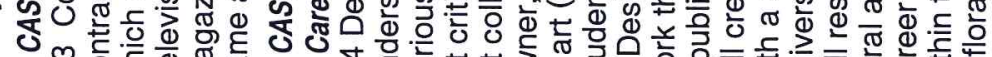

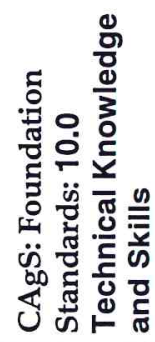

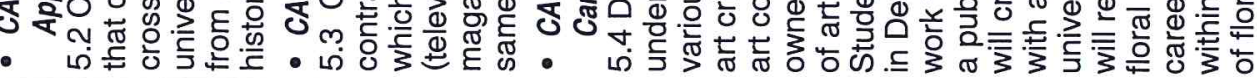

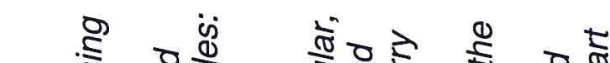

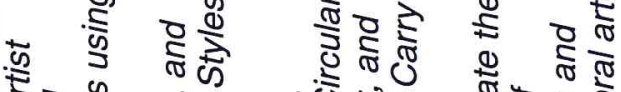
कृ

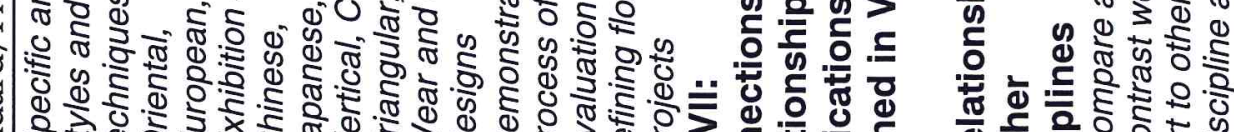

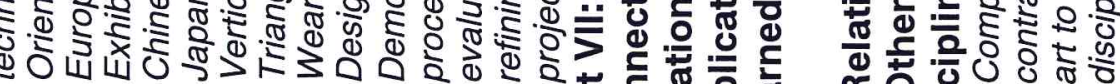
लं

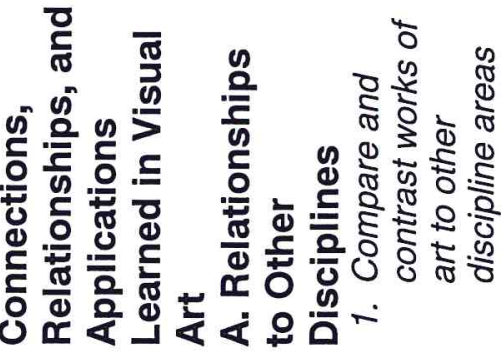




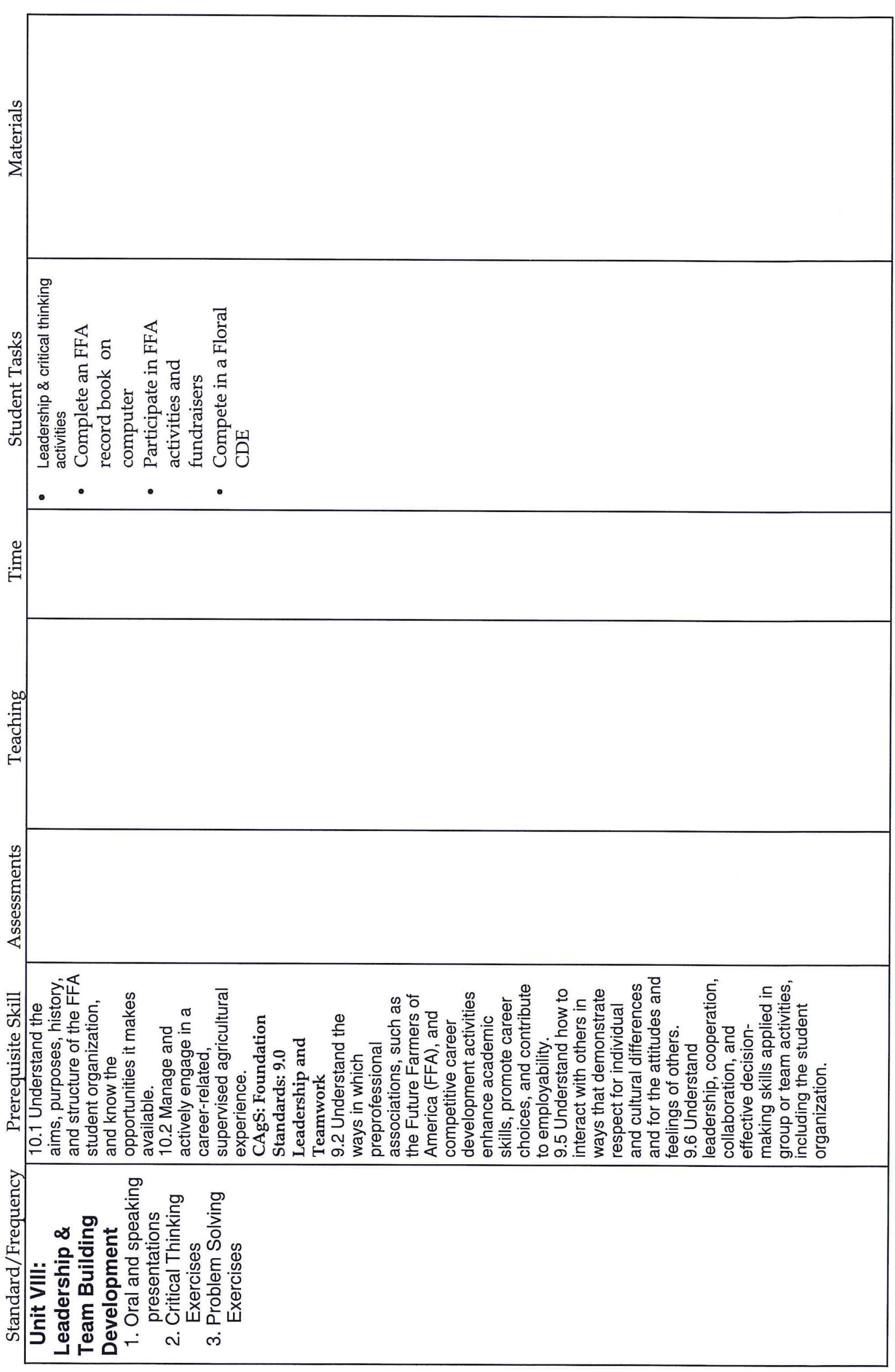


$\frac{0}{\sqrt[3]{2}}$

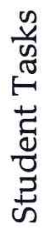

$\underset{\Xi}{\Xi}$

.ే

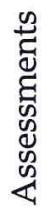

जี

苟

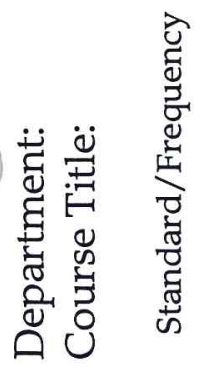




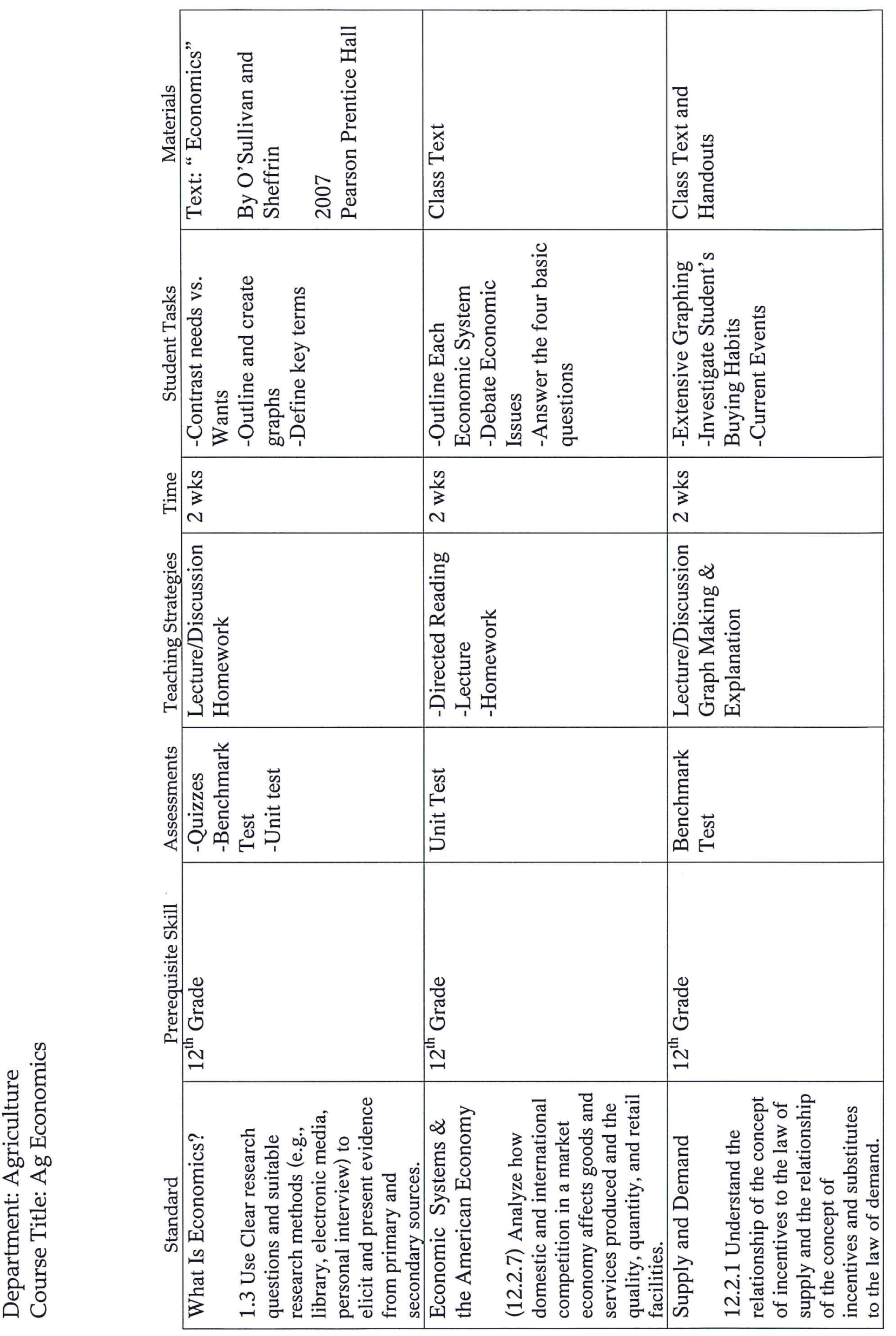




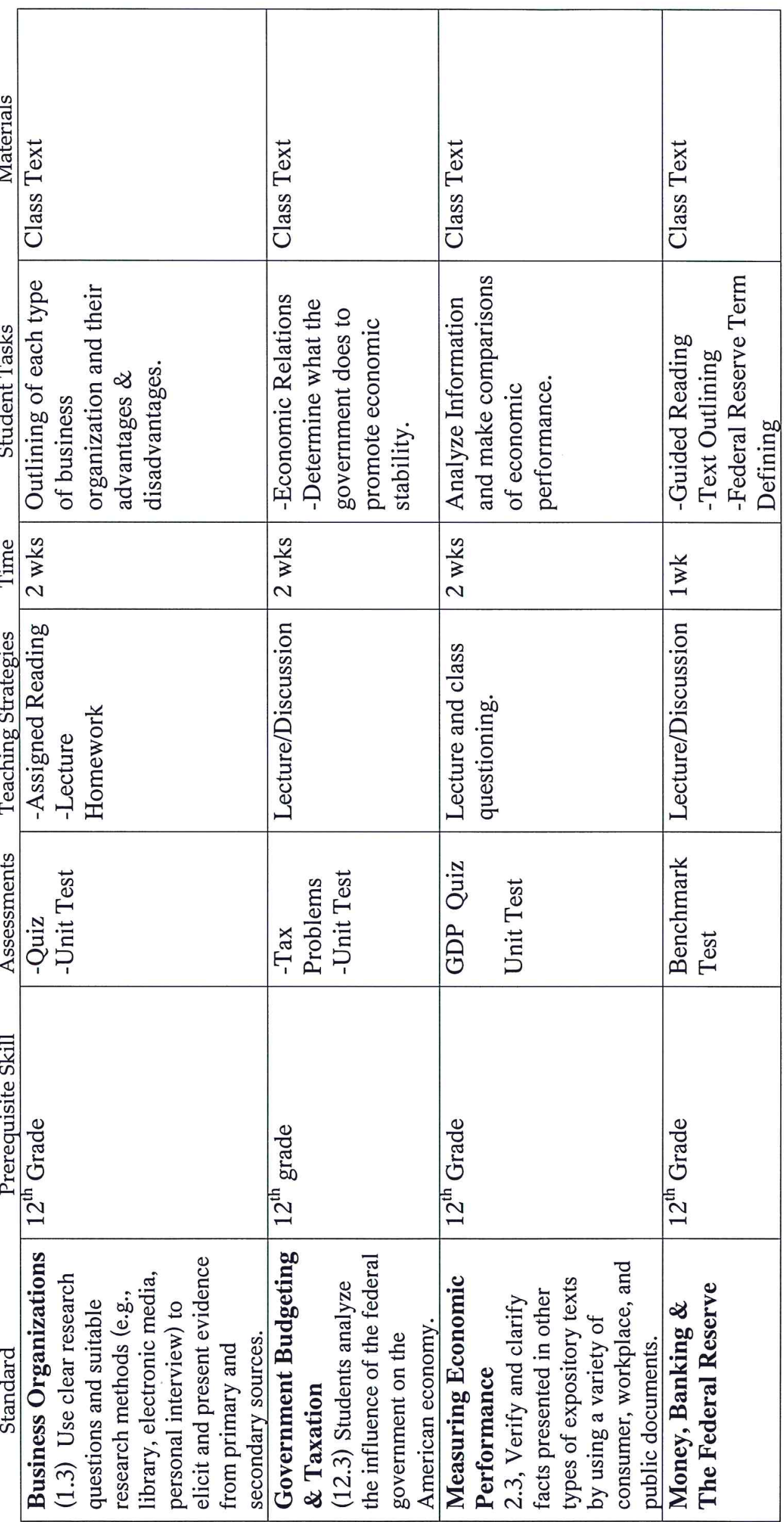




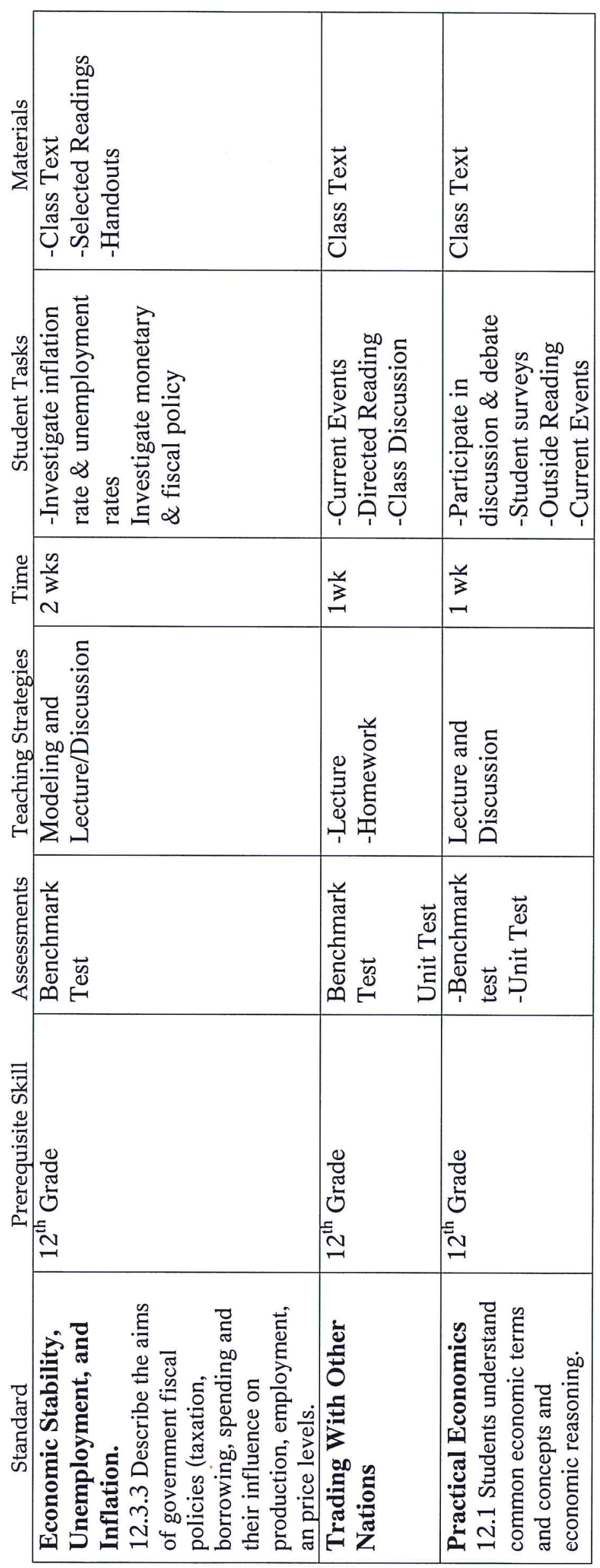




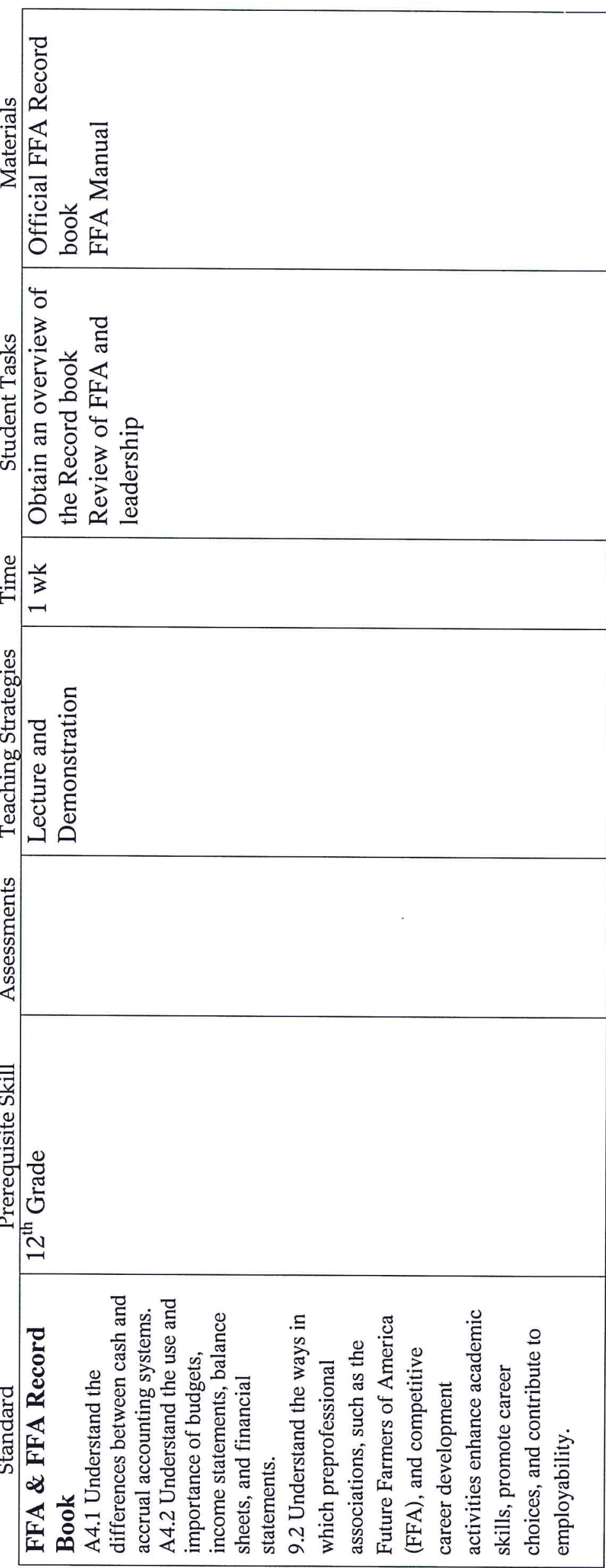




\begin{tabular}{|c|c|c|c|c|}
\hline 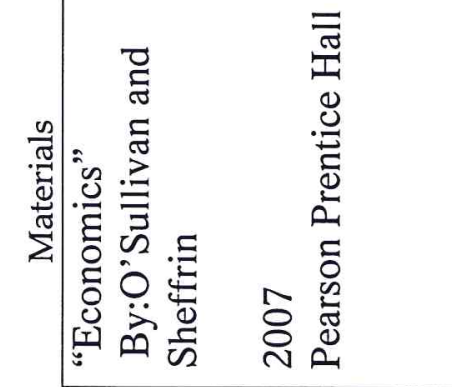 & 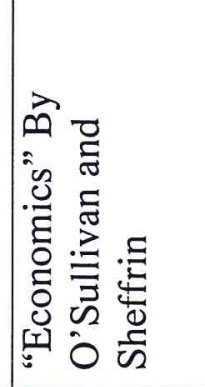 & 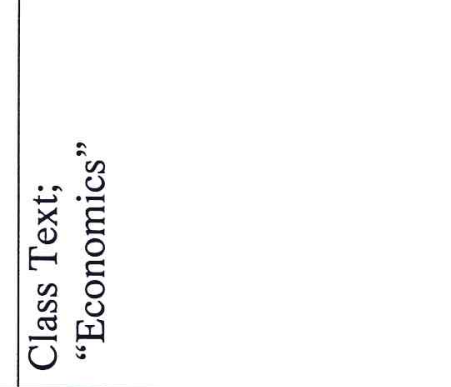 & 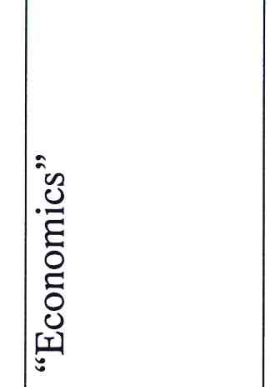 & 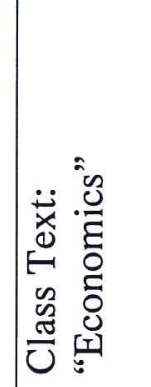 \\
\hline 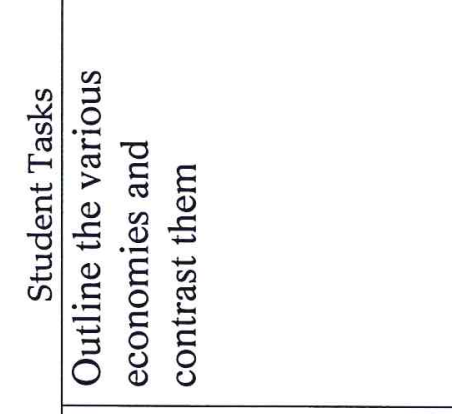 & 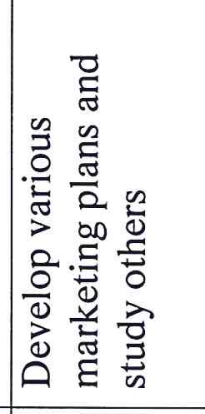 & 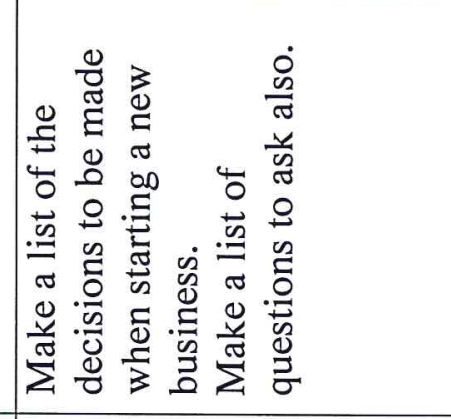 & 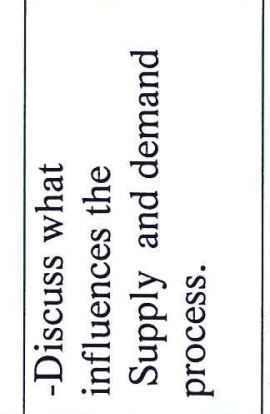 & 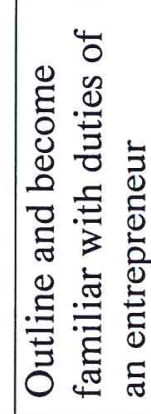 \\
\hline 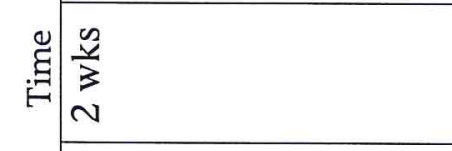 & $\begin{array}{l}\frac{0}{3} \\
\frac{1}{3} \\
v\end{array}$ & \begin{tabular}{|l|l|} 
\\
\\
$m$ \\
$m$
\end{tabular} & 管 & \\
\hline 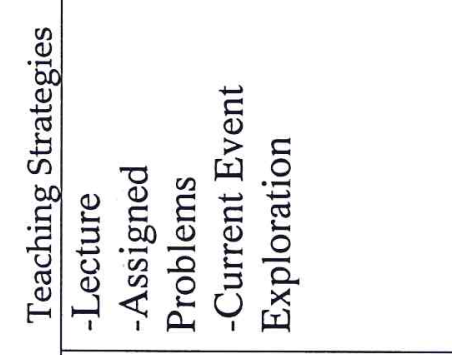 & 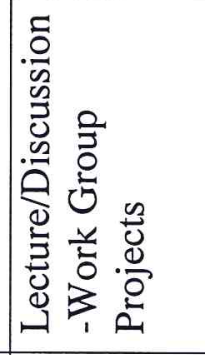 & 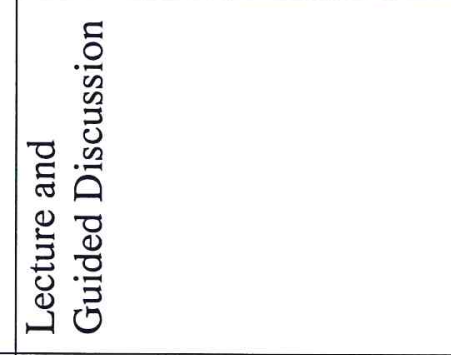 & 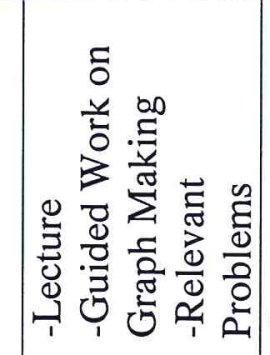 & 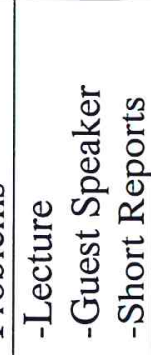 \\
\hline 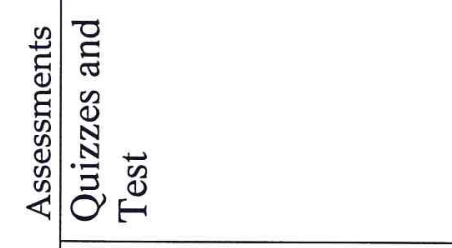 & 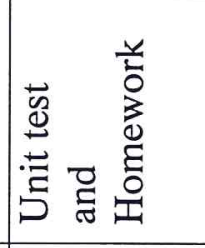 & 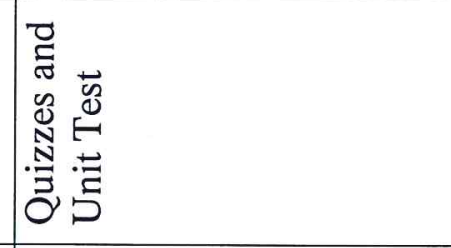 & 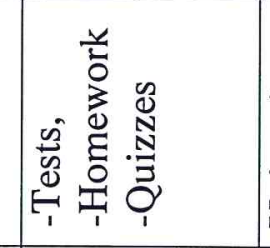 & 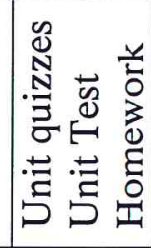 \\
\hline 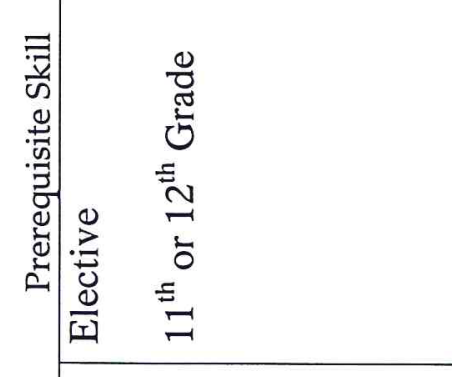 & 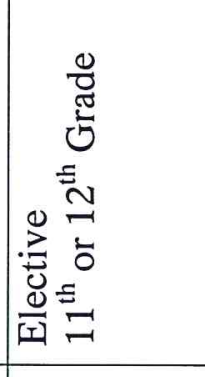 & 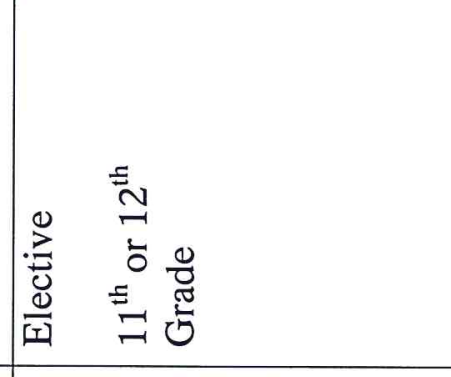 & 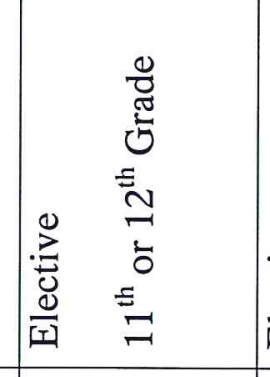 & 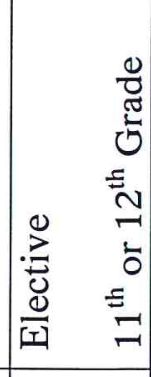 \\
\hline 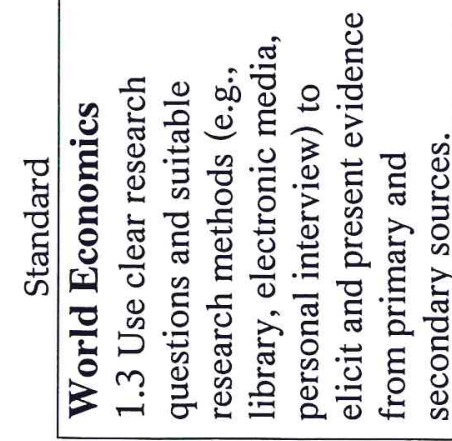 & 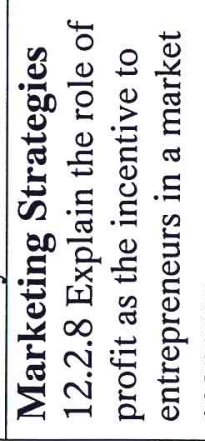 & 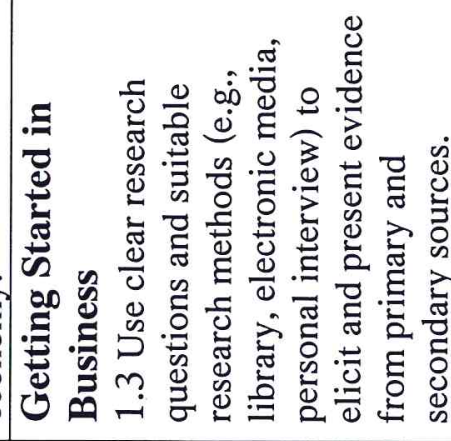 & 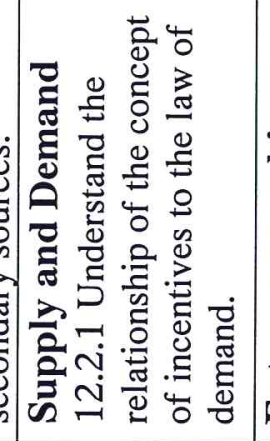 & 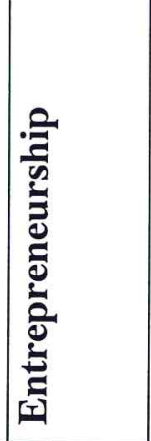 \\
\hline
\end{tabular}




\begin{tabular}{|c|c|c|c|c|}
\hline 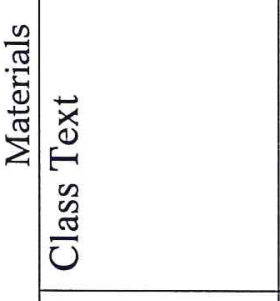 & 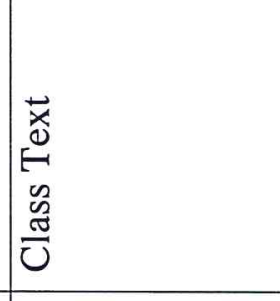 & 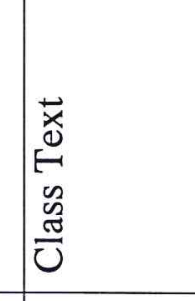 & 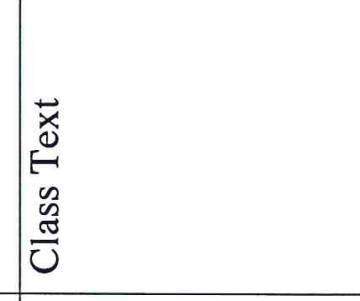 & 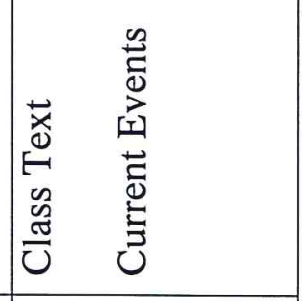 \\
\hline 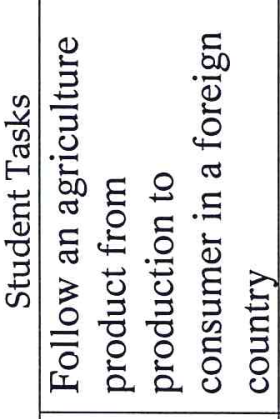 & 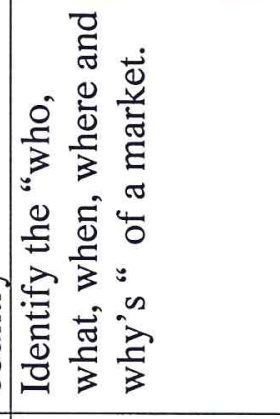 & 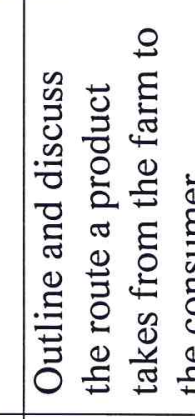 & 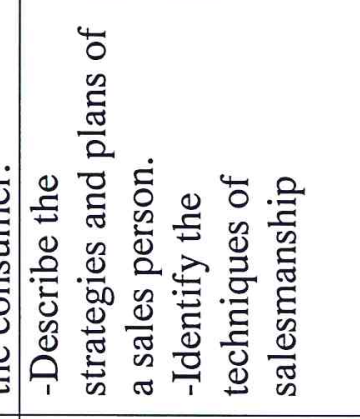 & 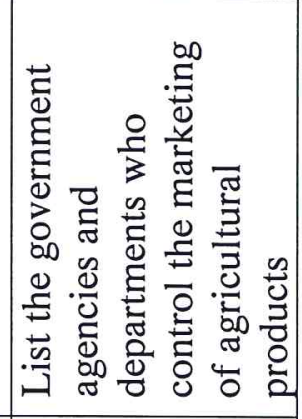 \\
\hline $\begin{array}{l}\frac{y}{3} \\
m \\
m\end{array}$ & & $\begin{array}{l}\frac{\infty}{3} \\
\frac{1}{3} \\
m\end{array}$ & 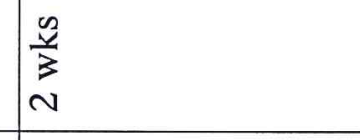 & \begin{tabular}{|l}
$\frac{w}{y}$ \\
$\frac{y}{m}$ \\
$m$
\end{tabular} \\
\hline 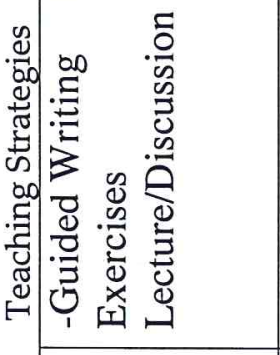 & 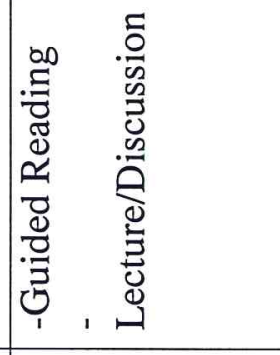 & 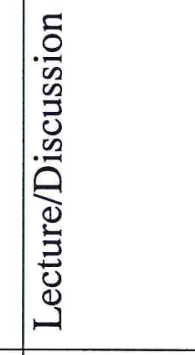 & 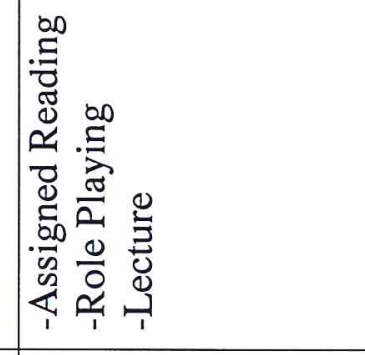 & 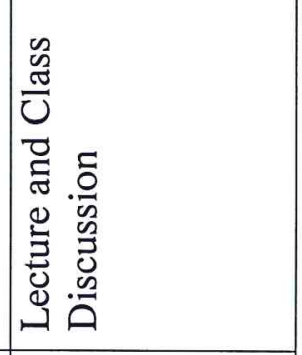 \\
\hline 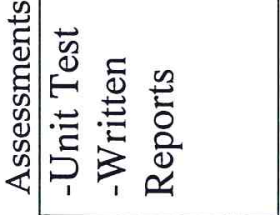 & 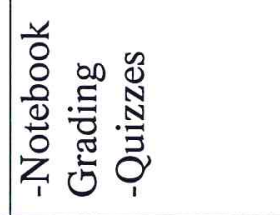 & 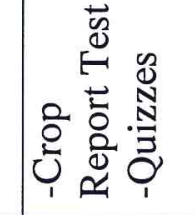 & إن & 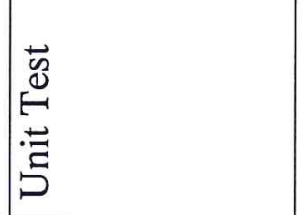 \\
\hline 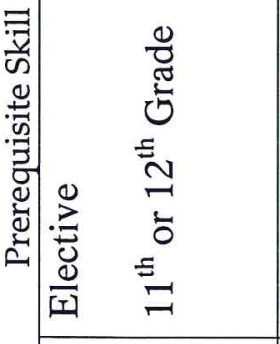 & 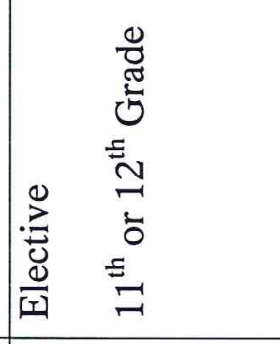 & 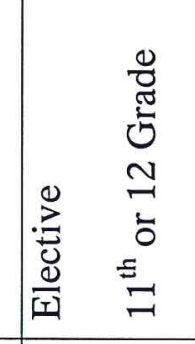 & 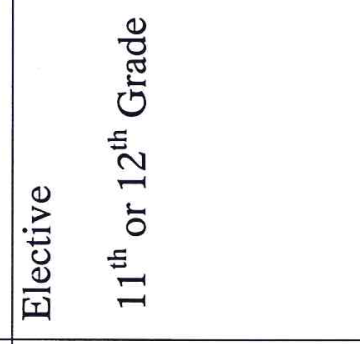 & 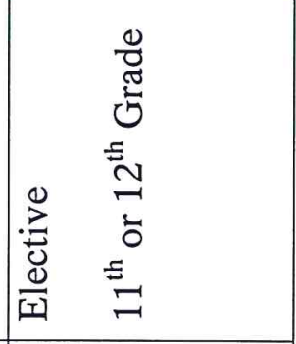 \\
\hline 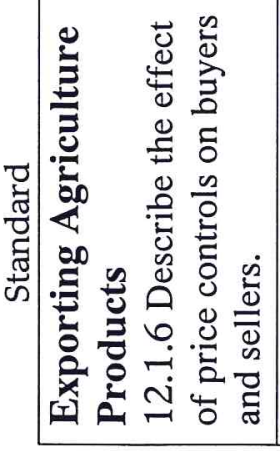 & 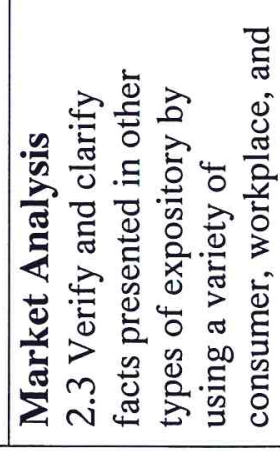 & 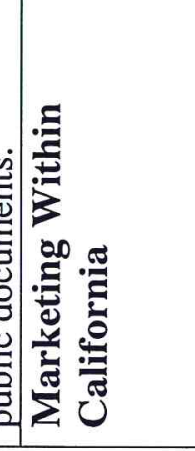 & 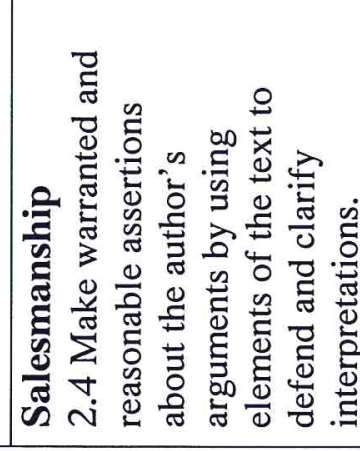 & 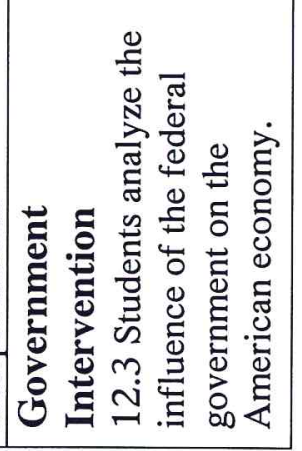 \\
\hline
\end{tabular}




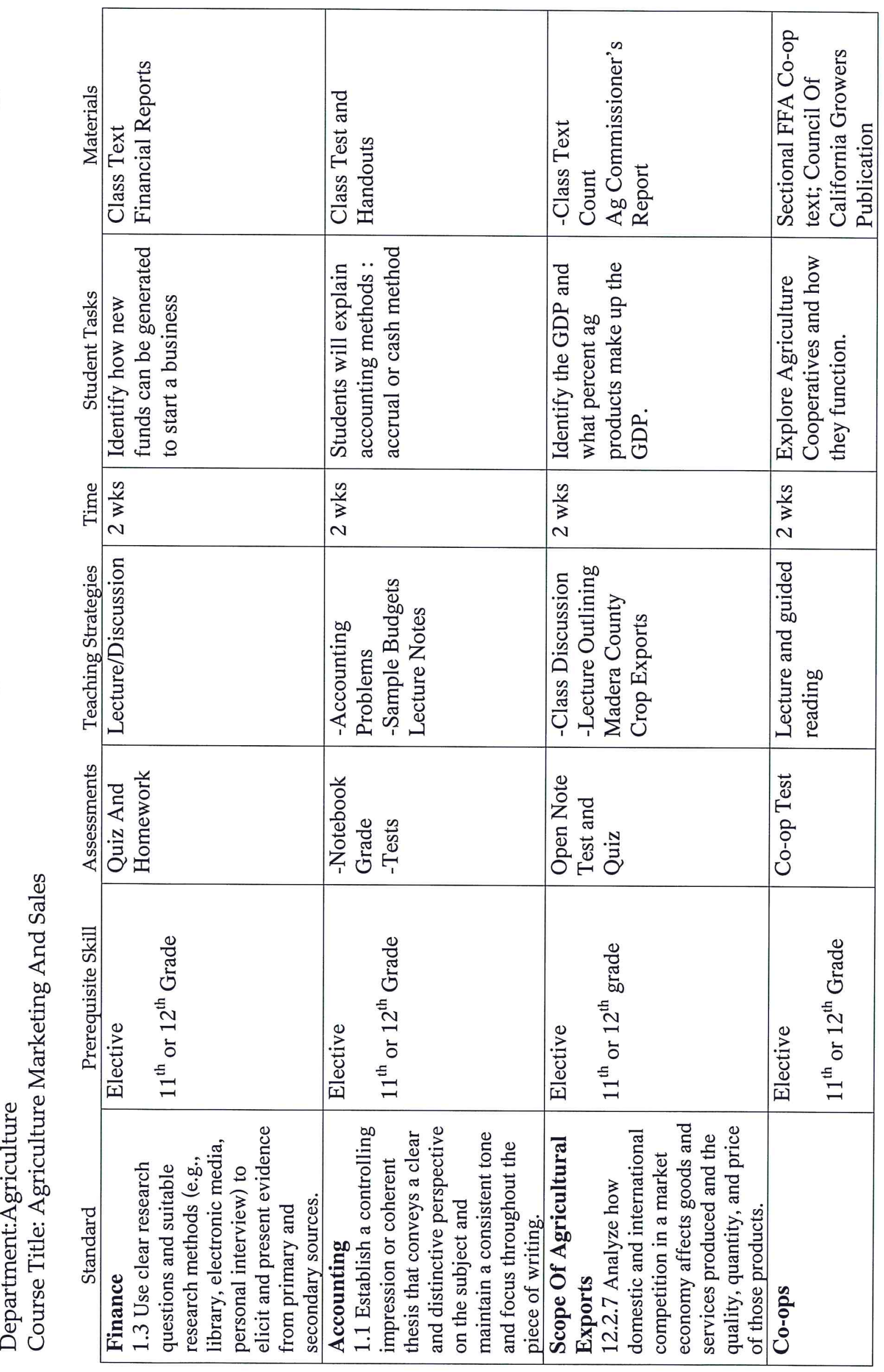




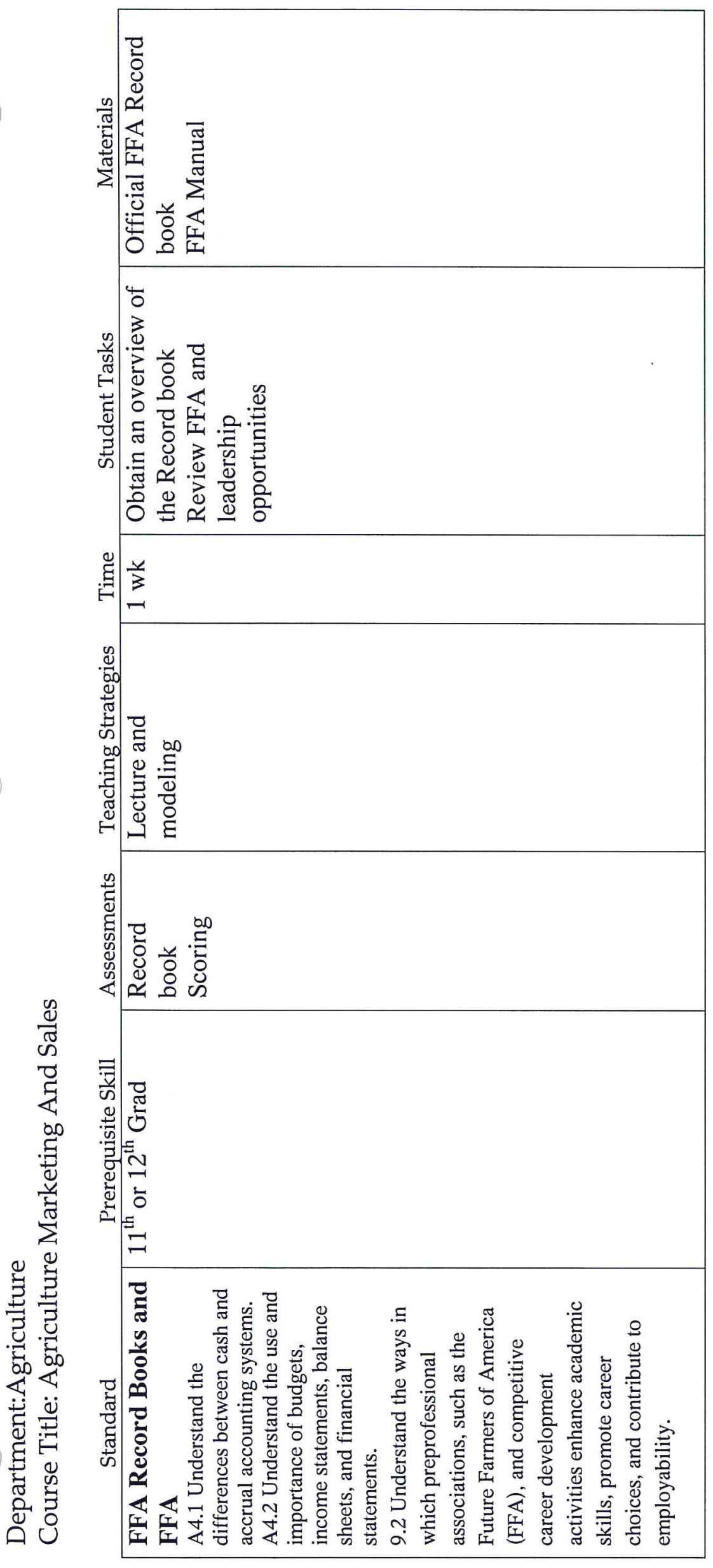




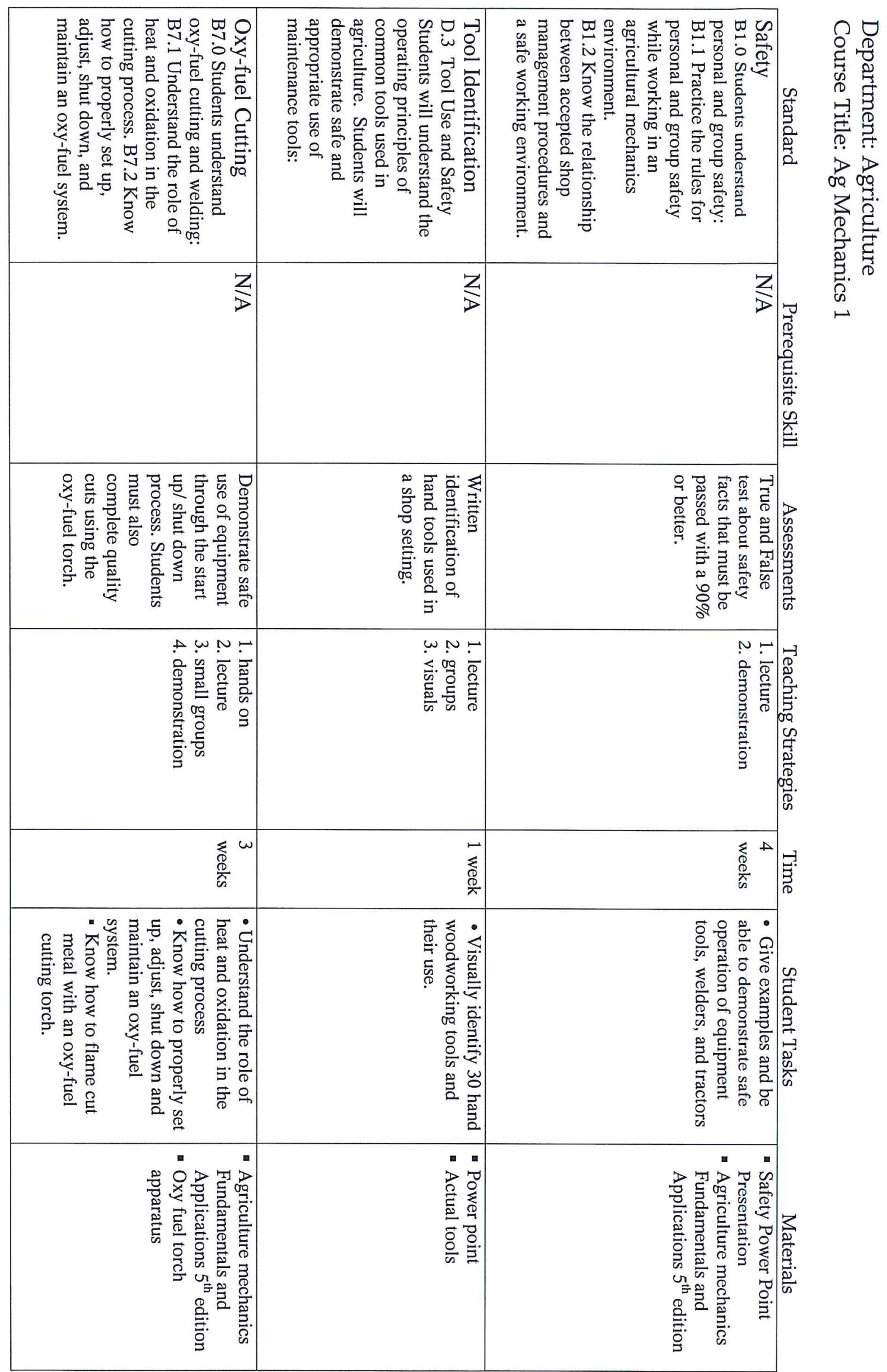




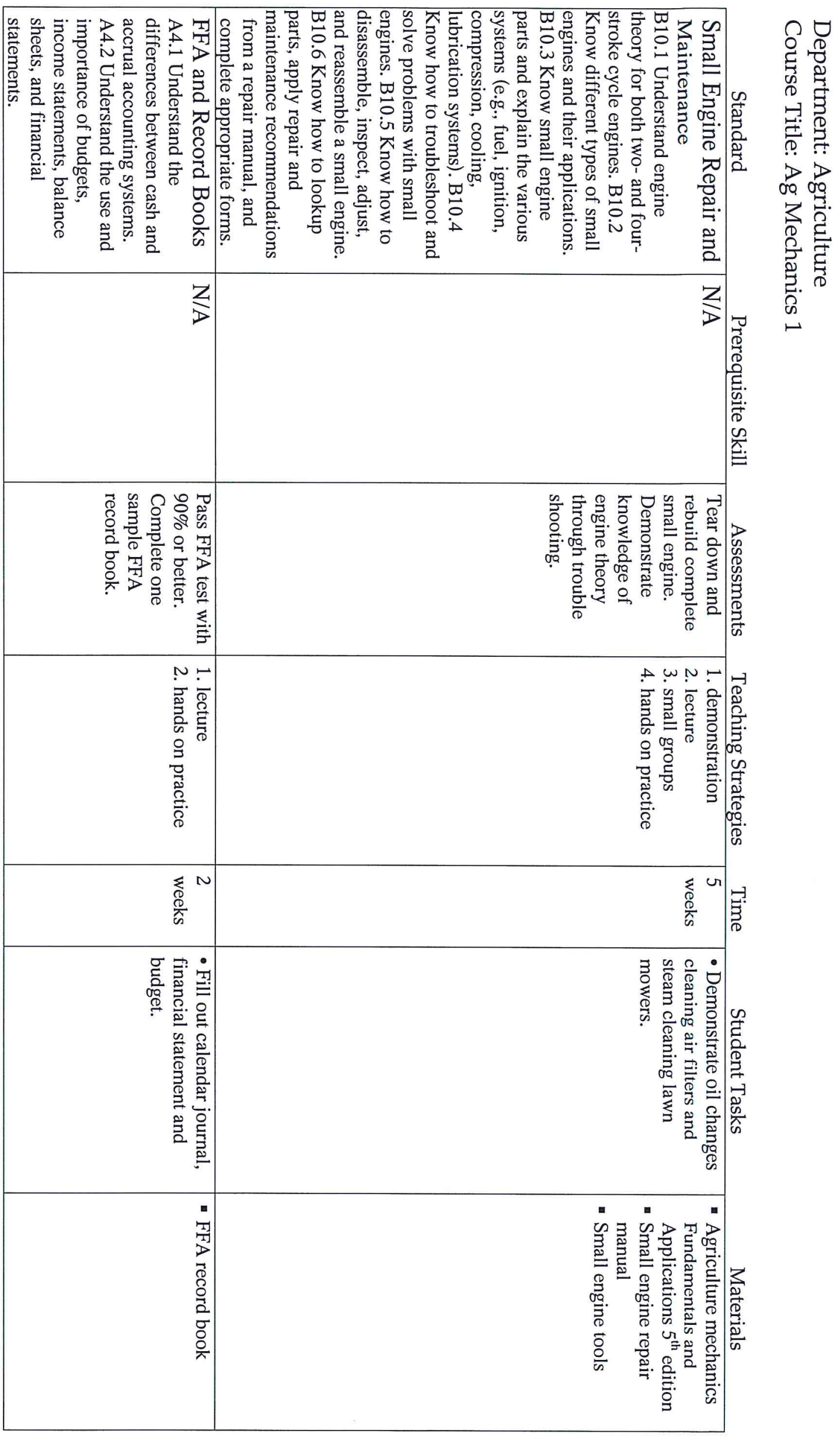




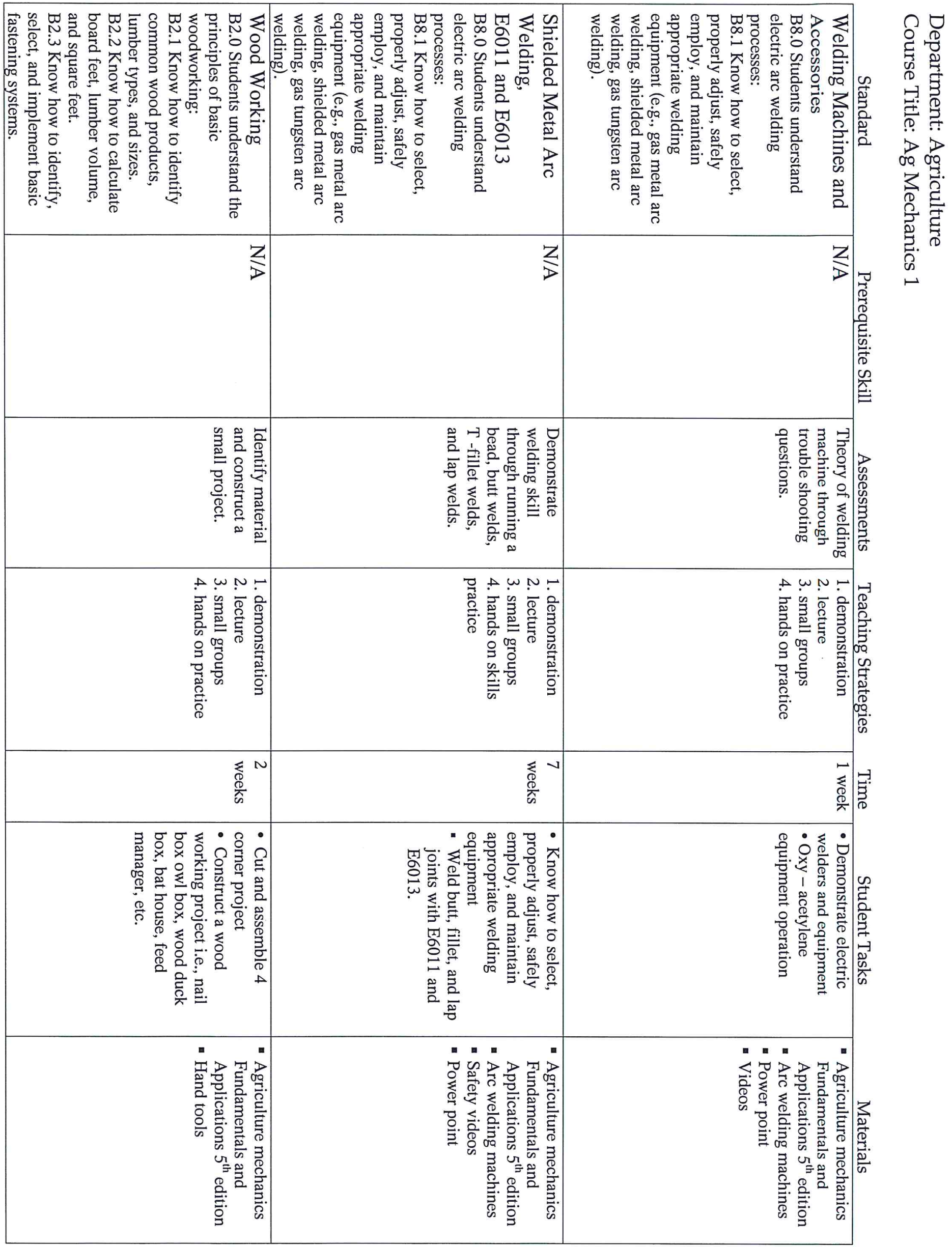




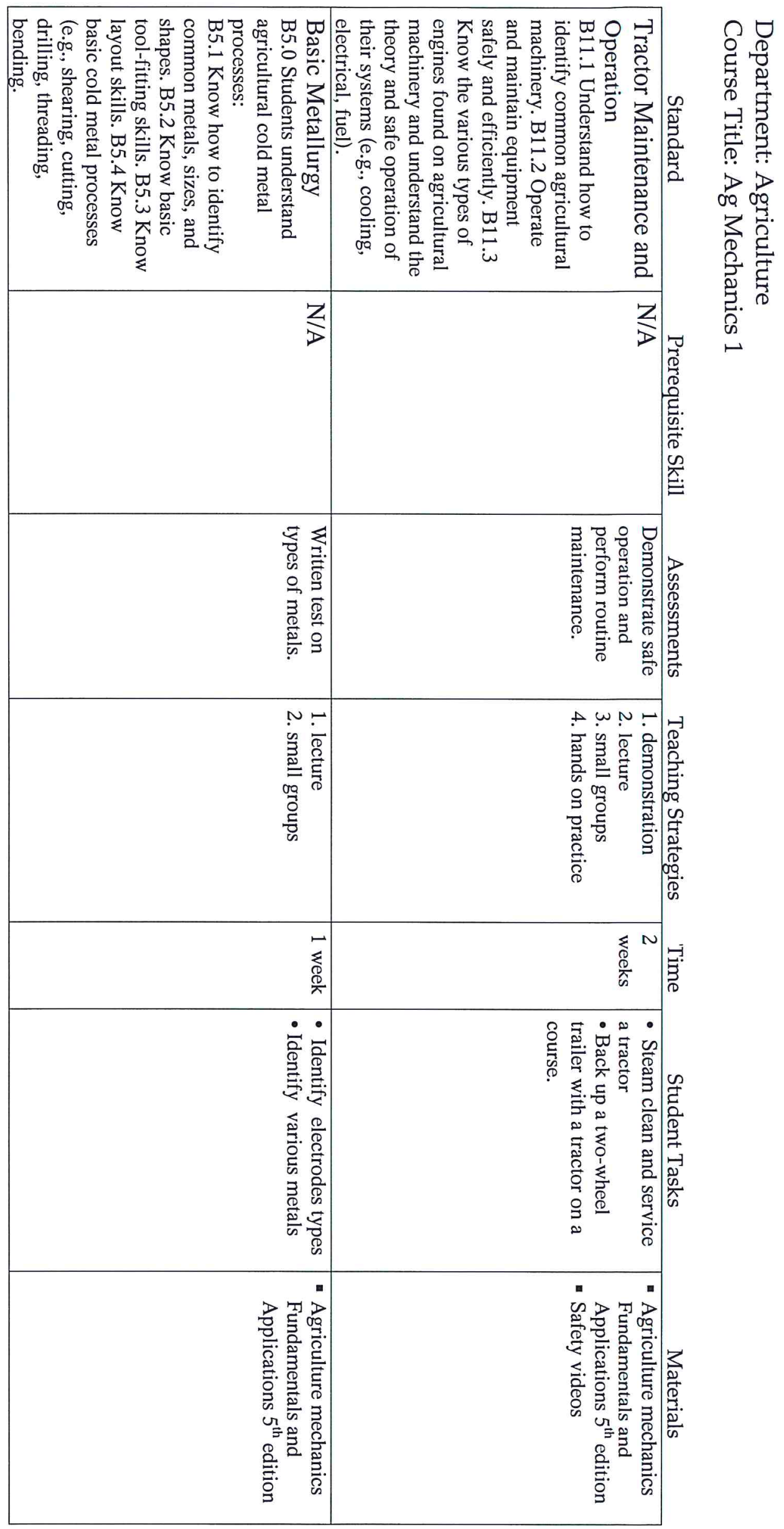




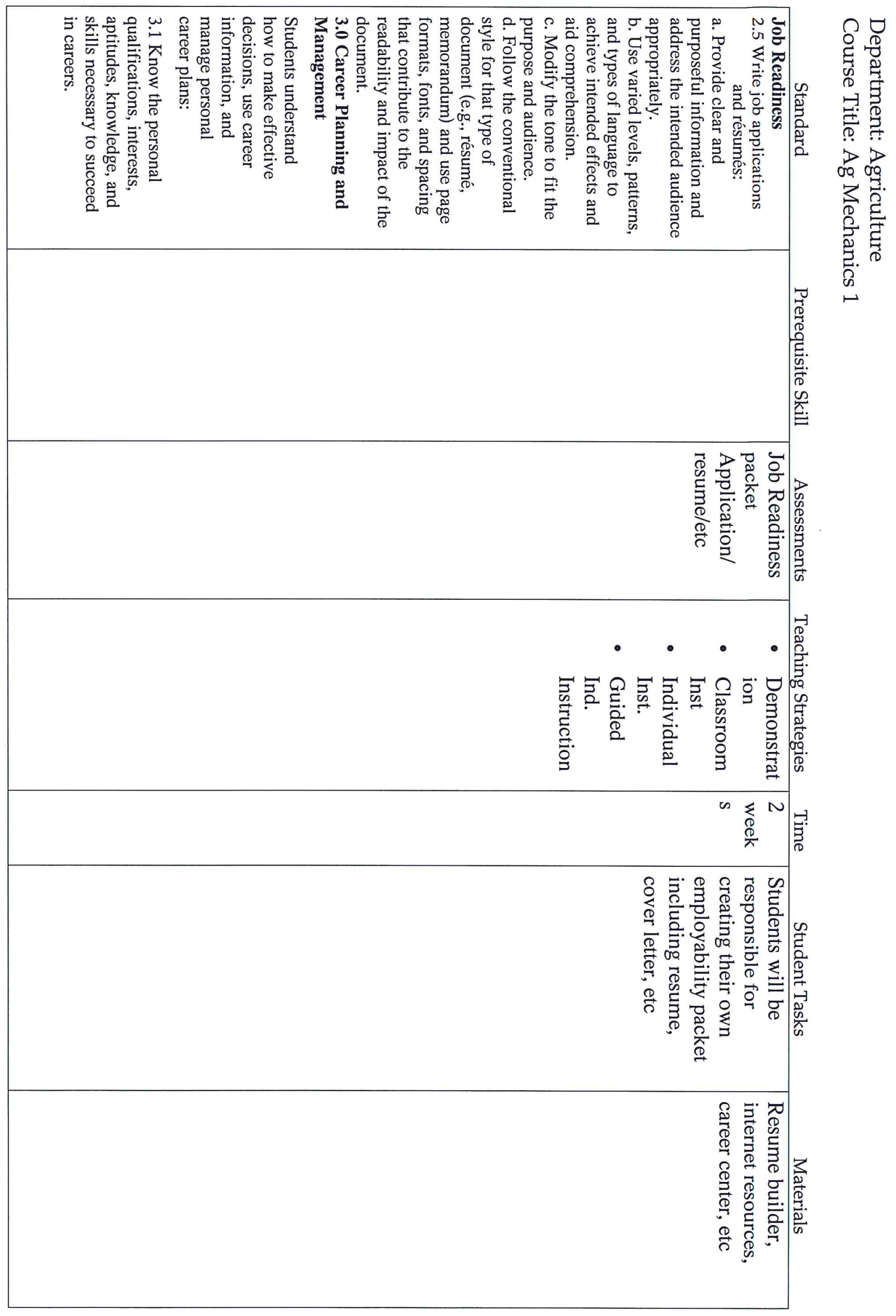




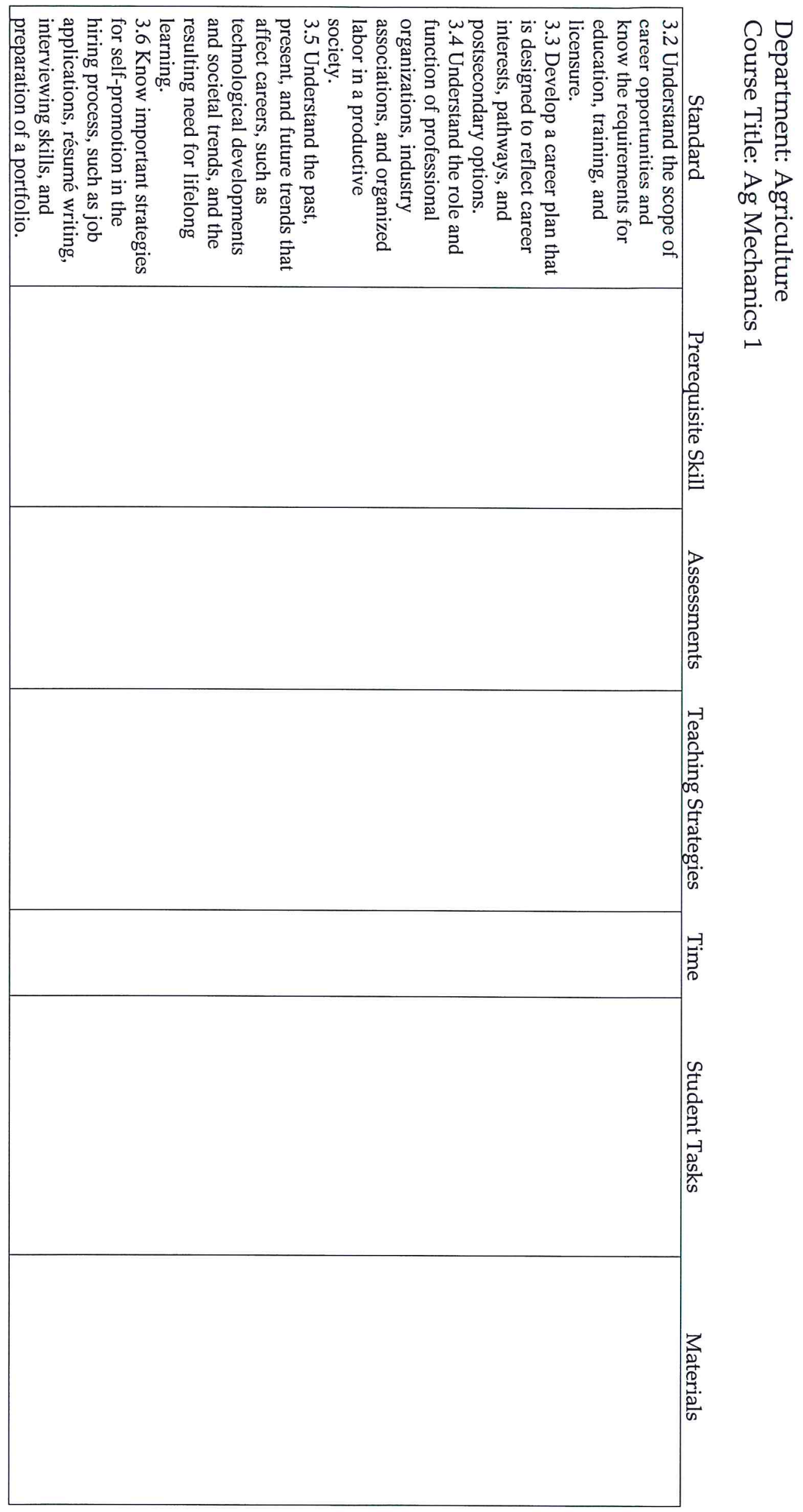




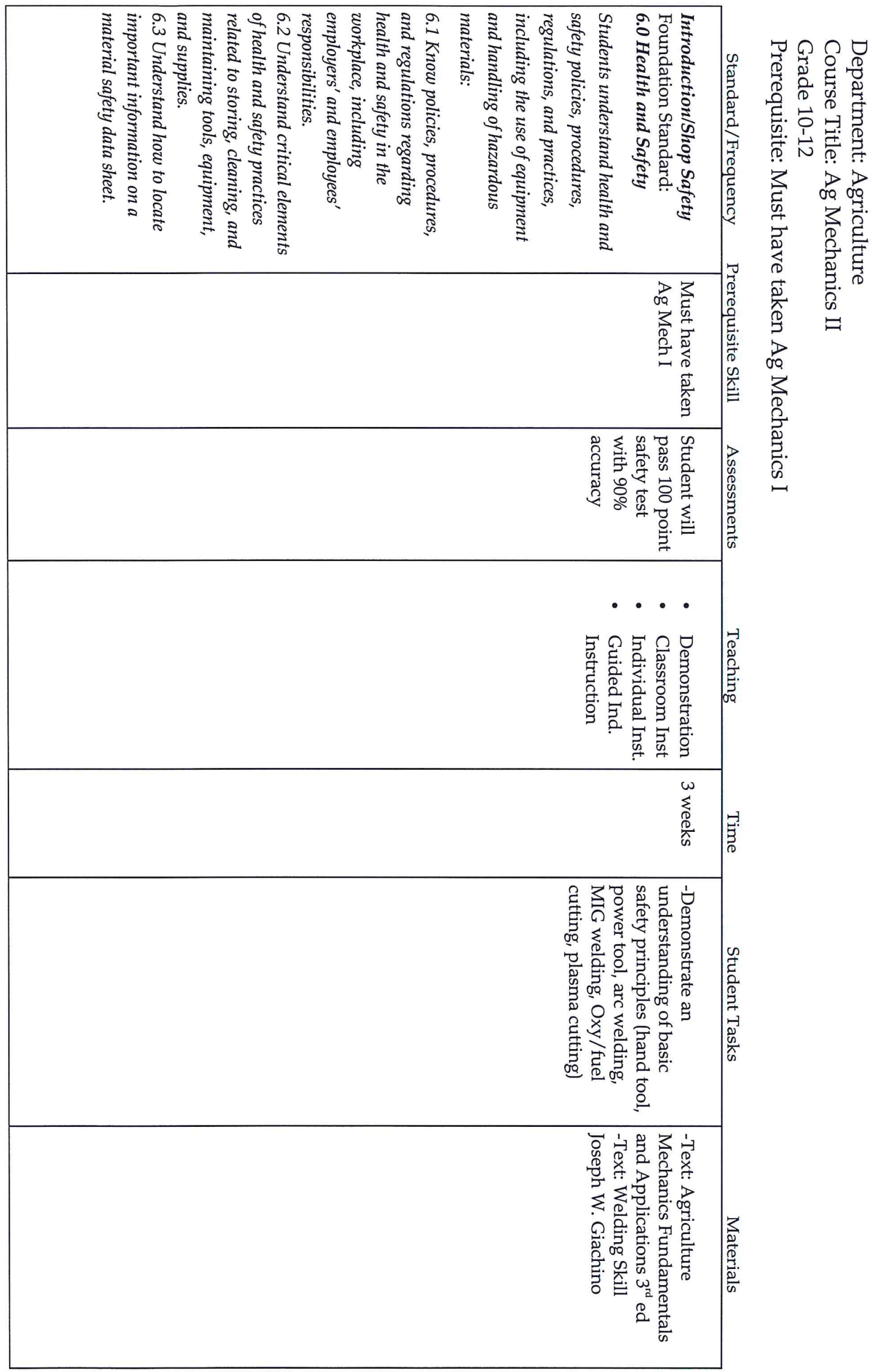




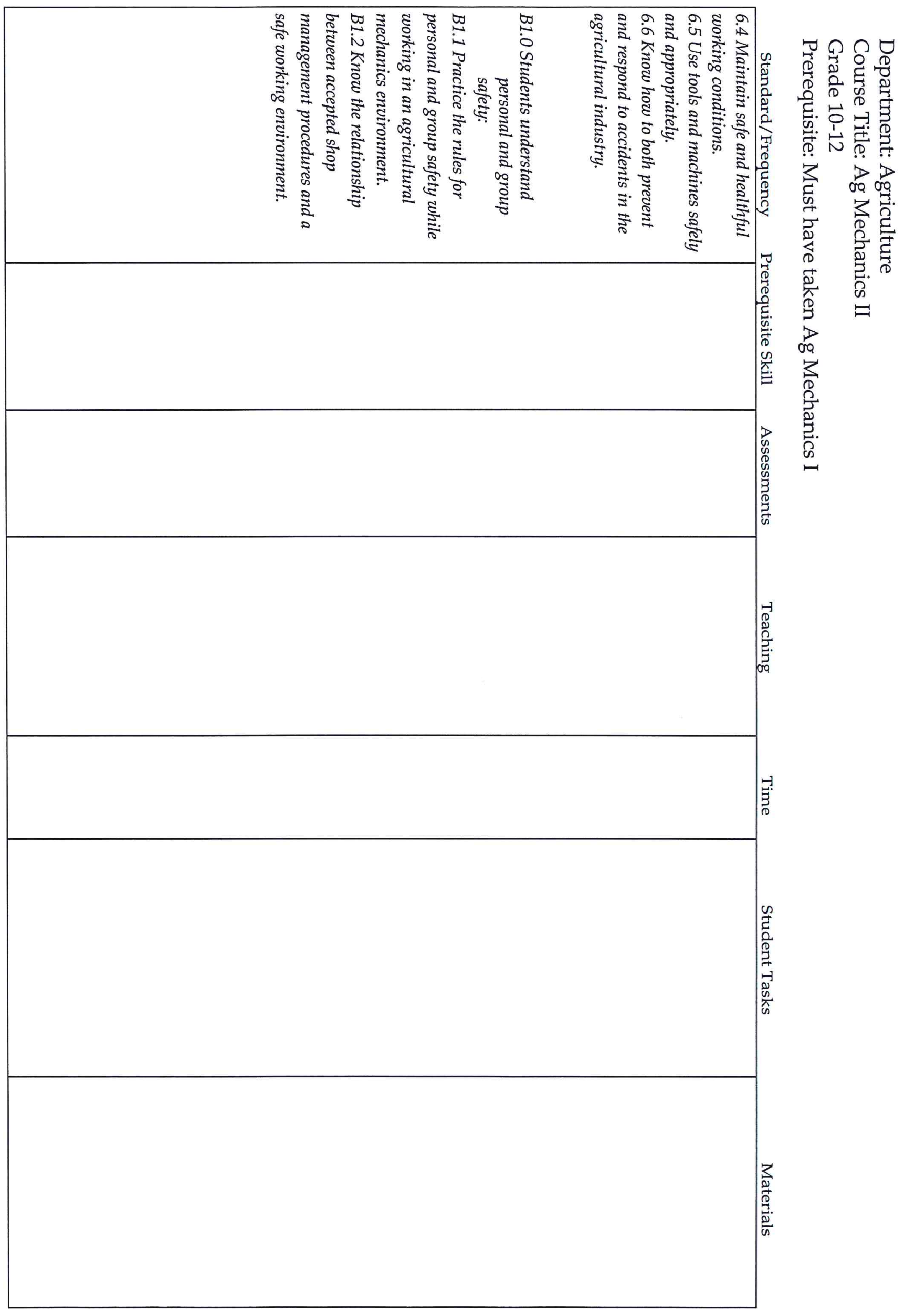




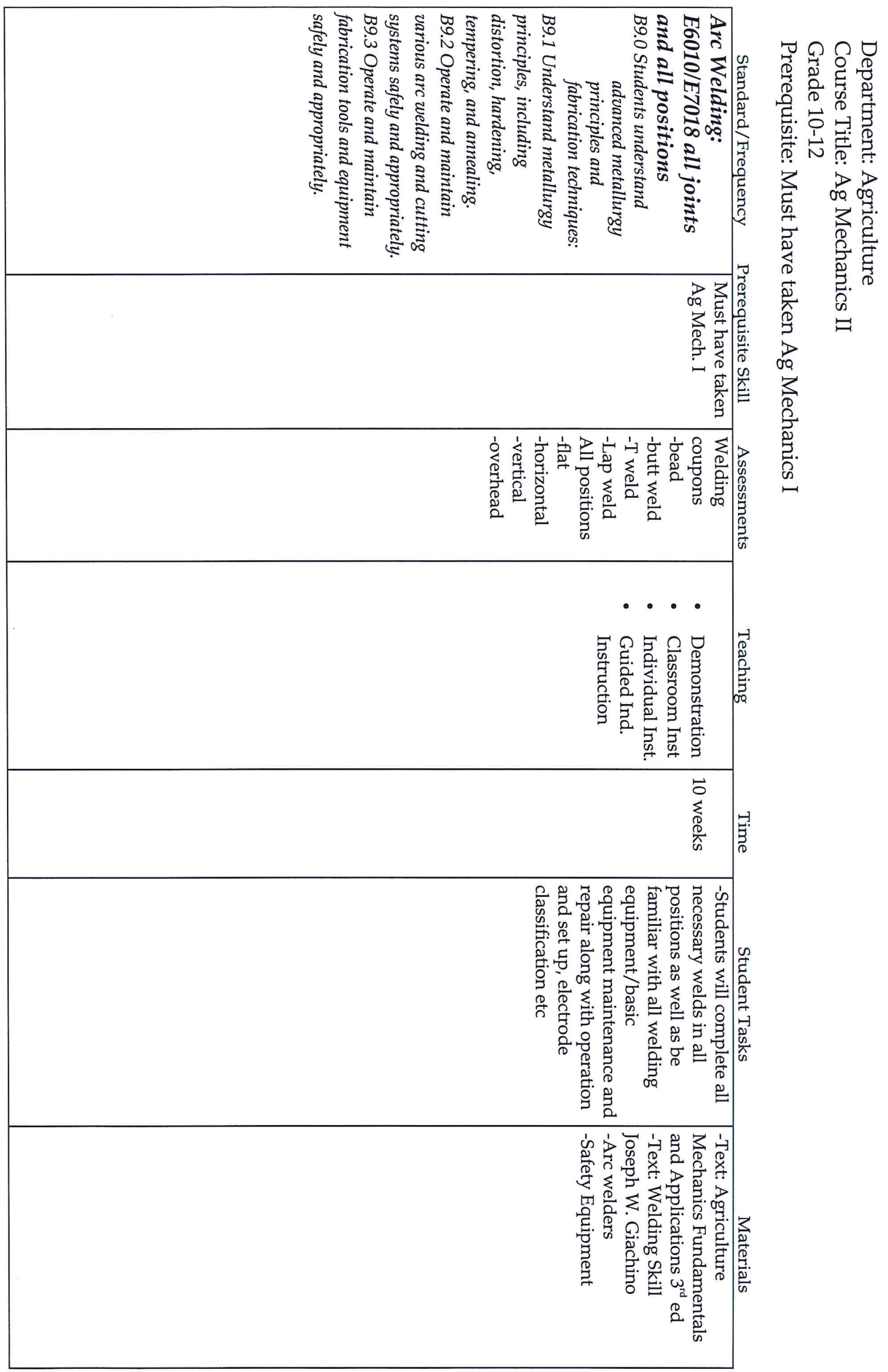




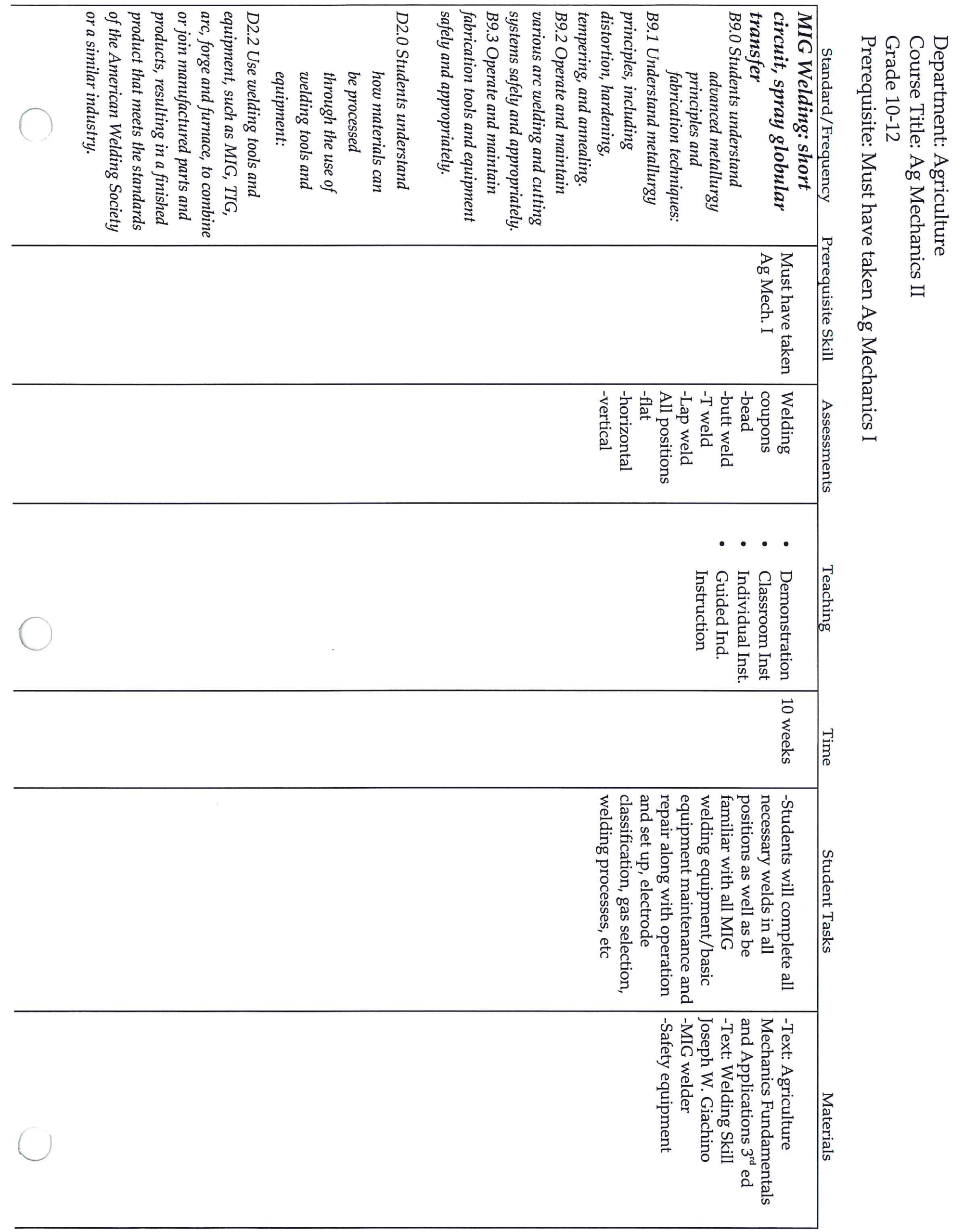




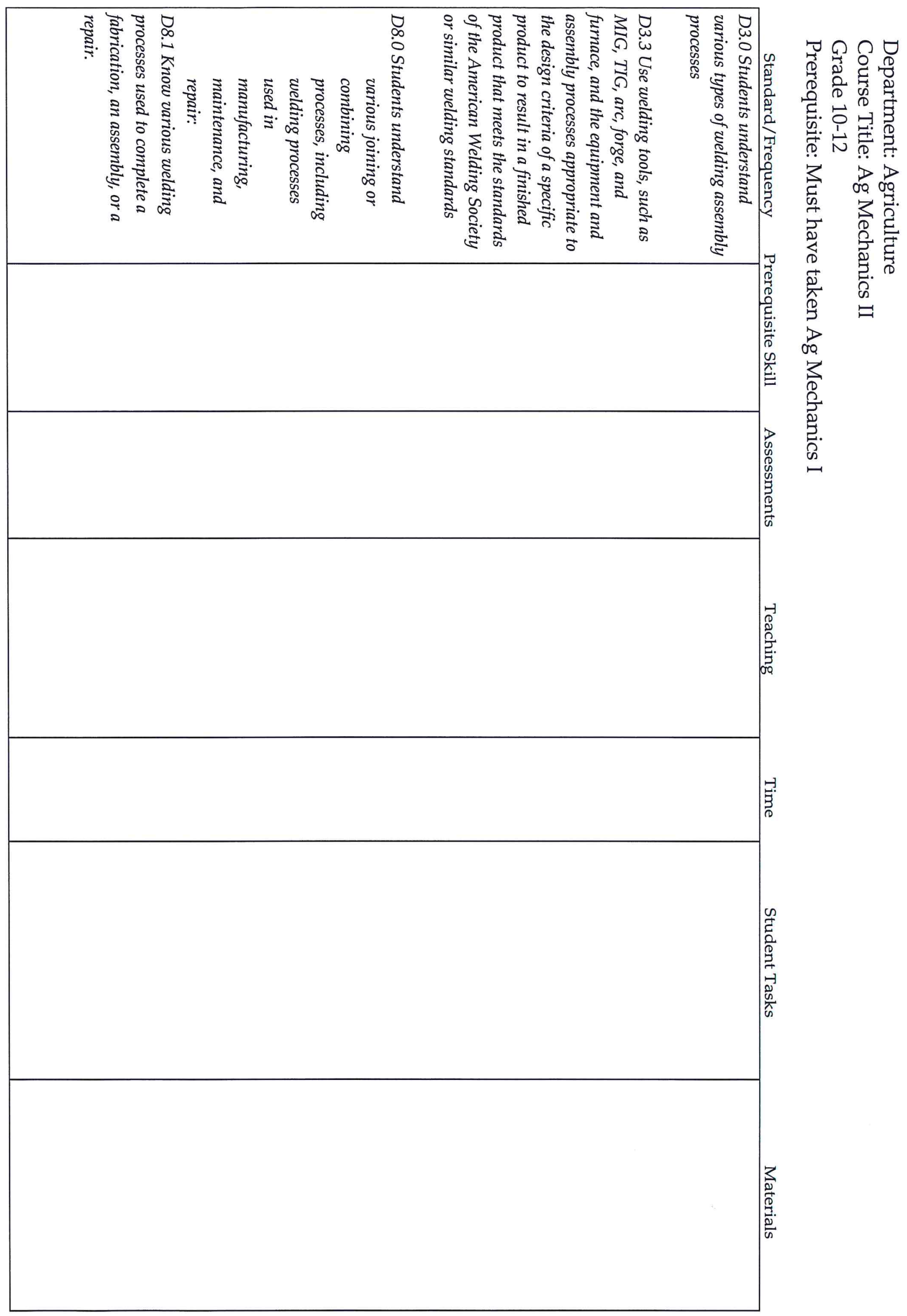




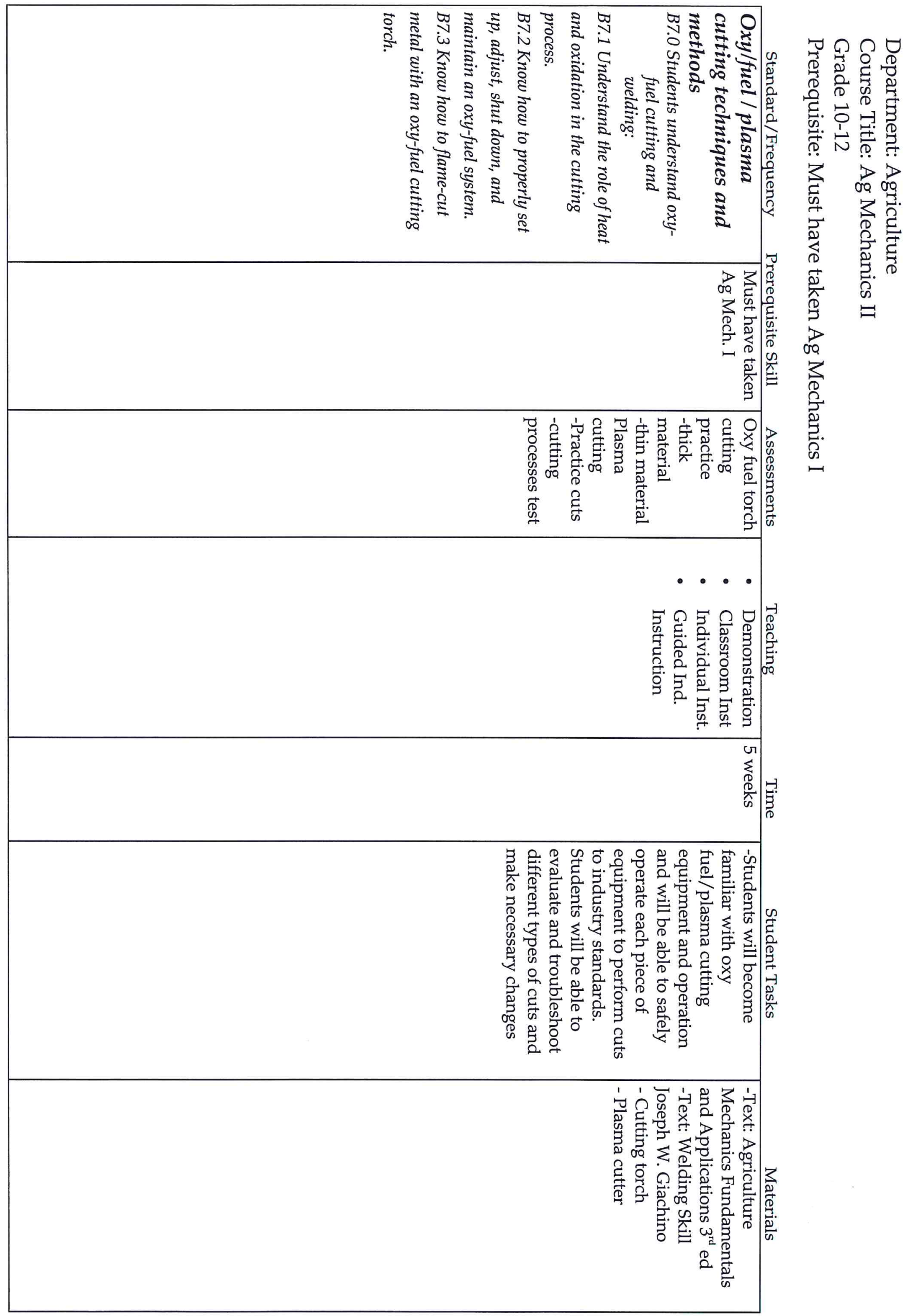




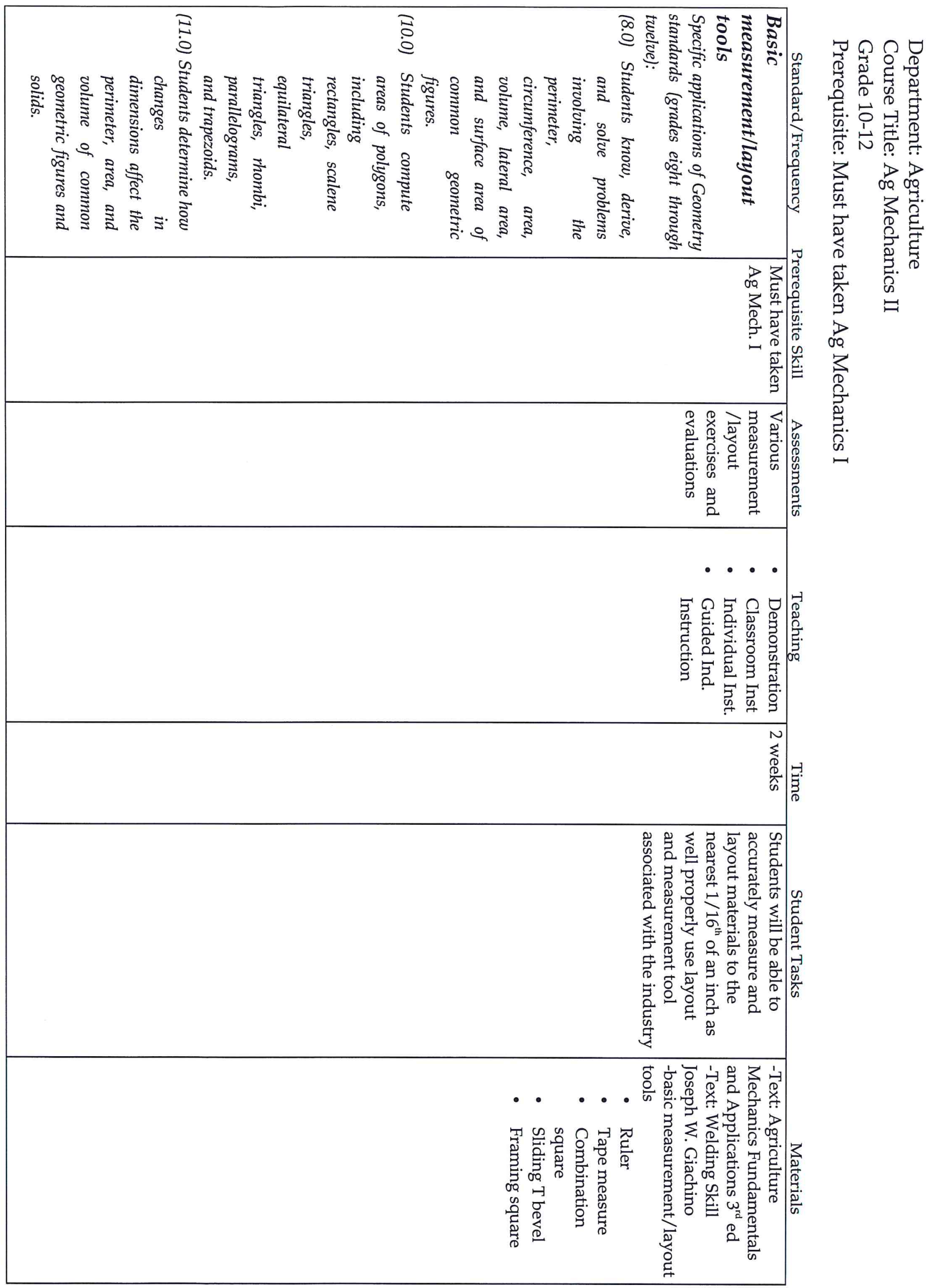




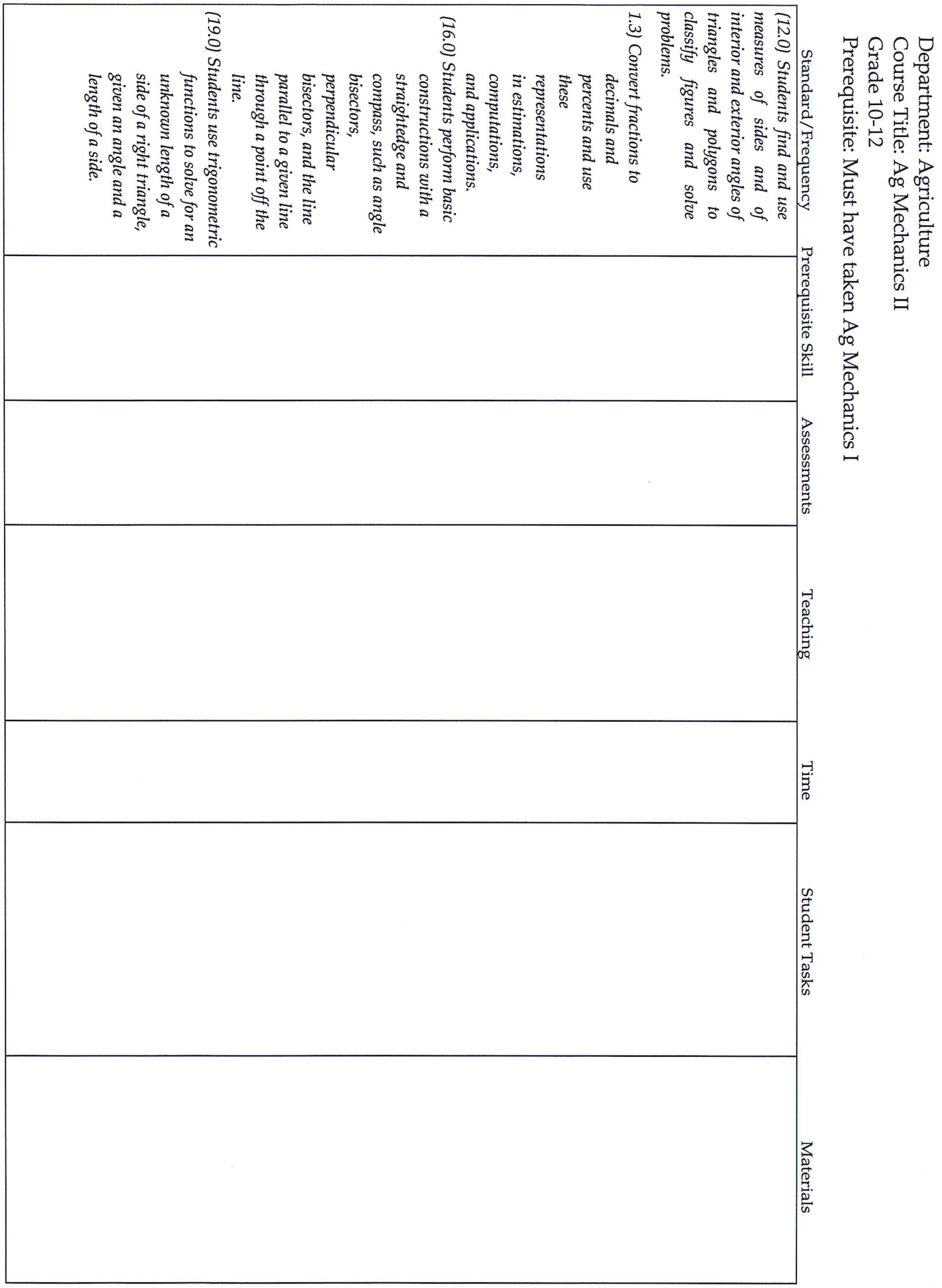




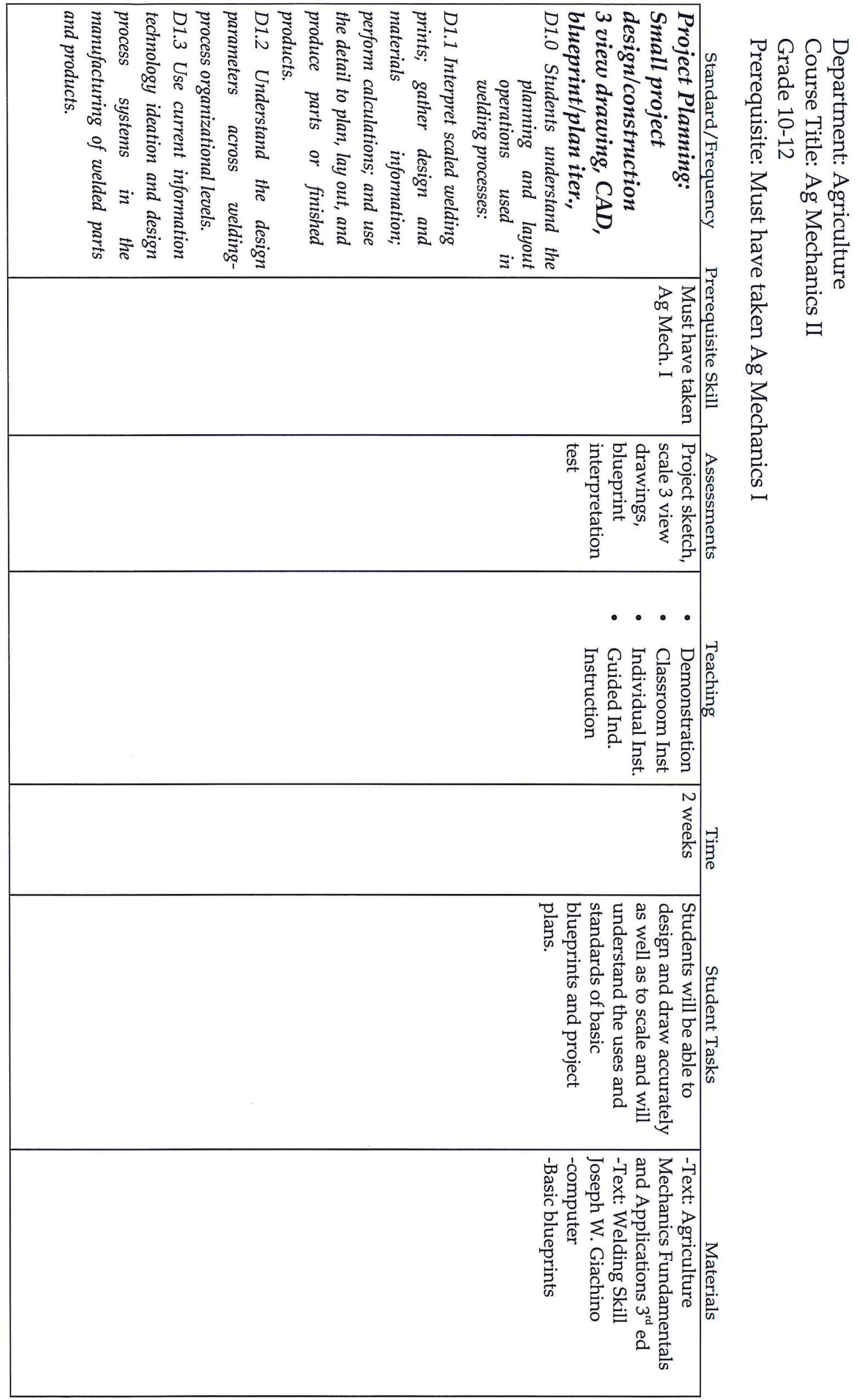




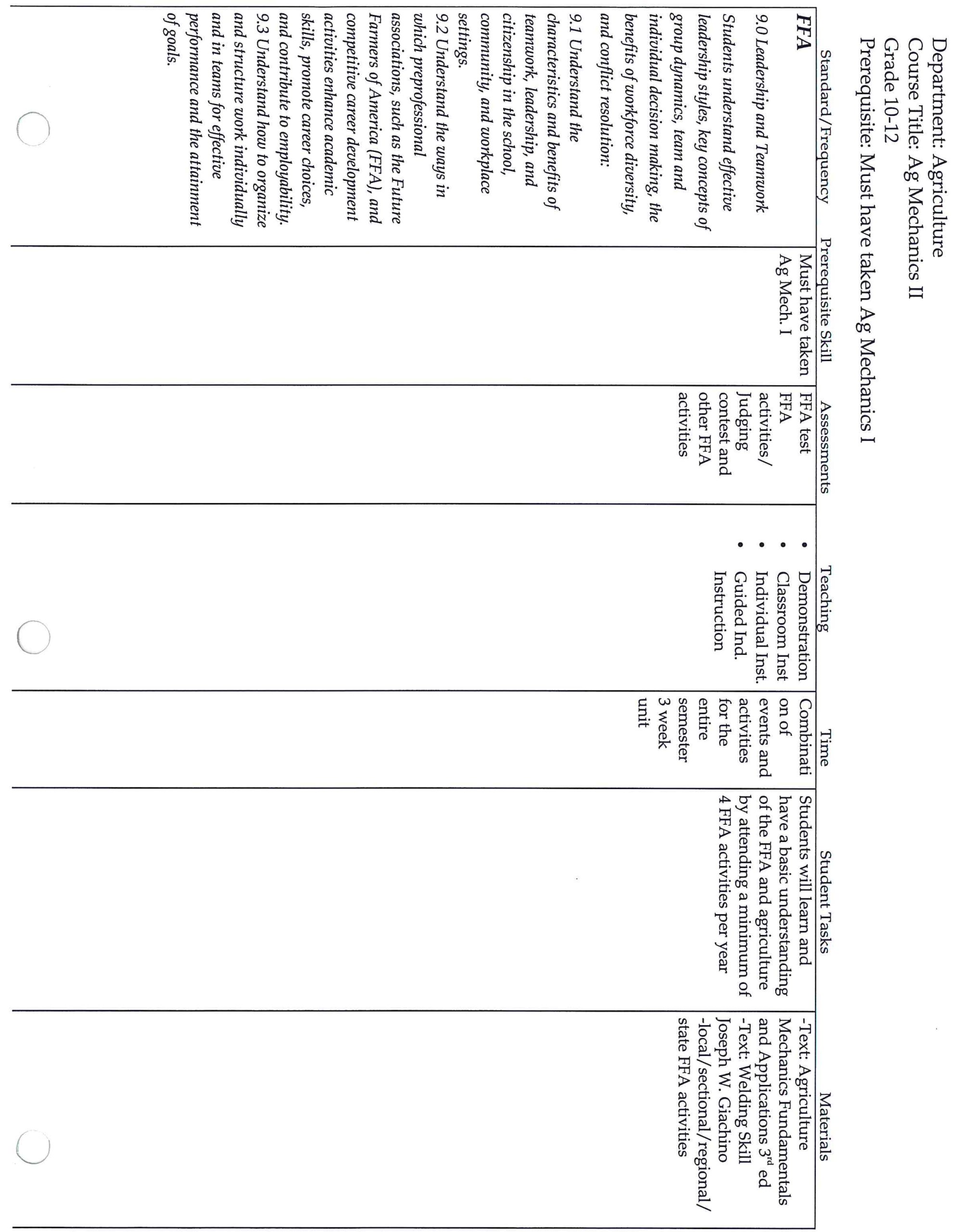




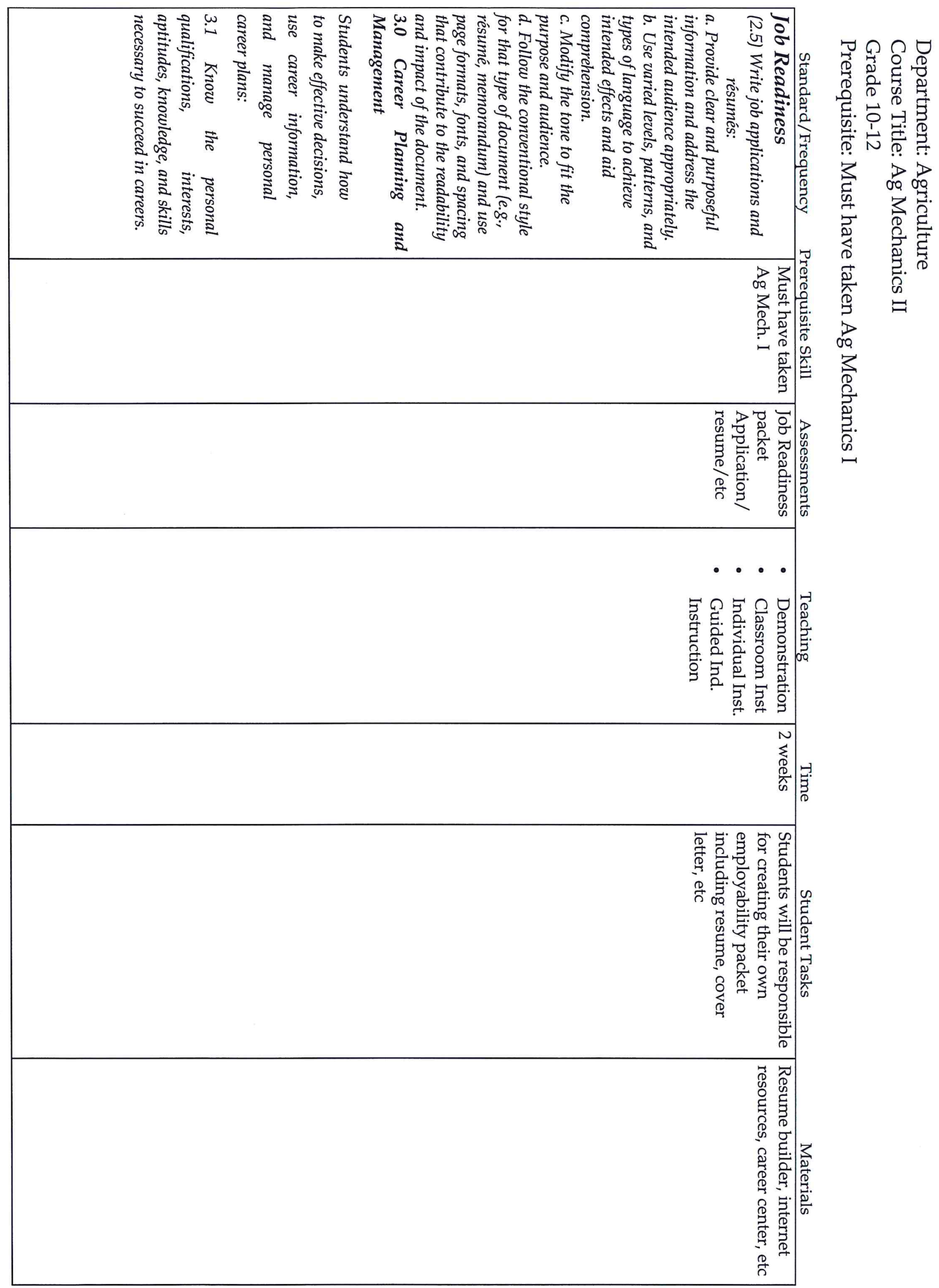




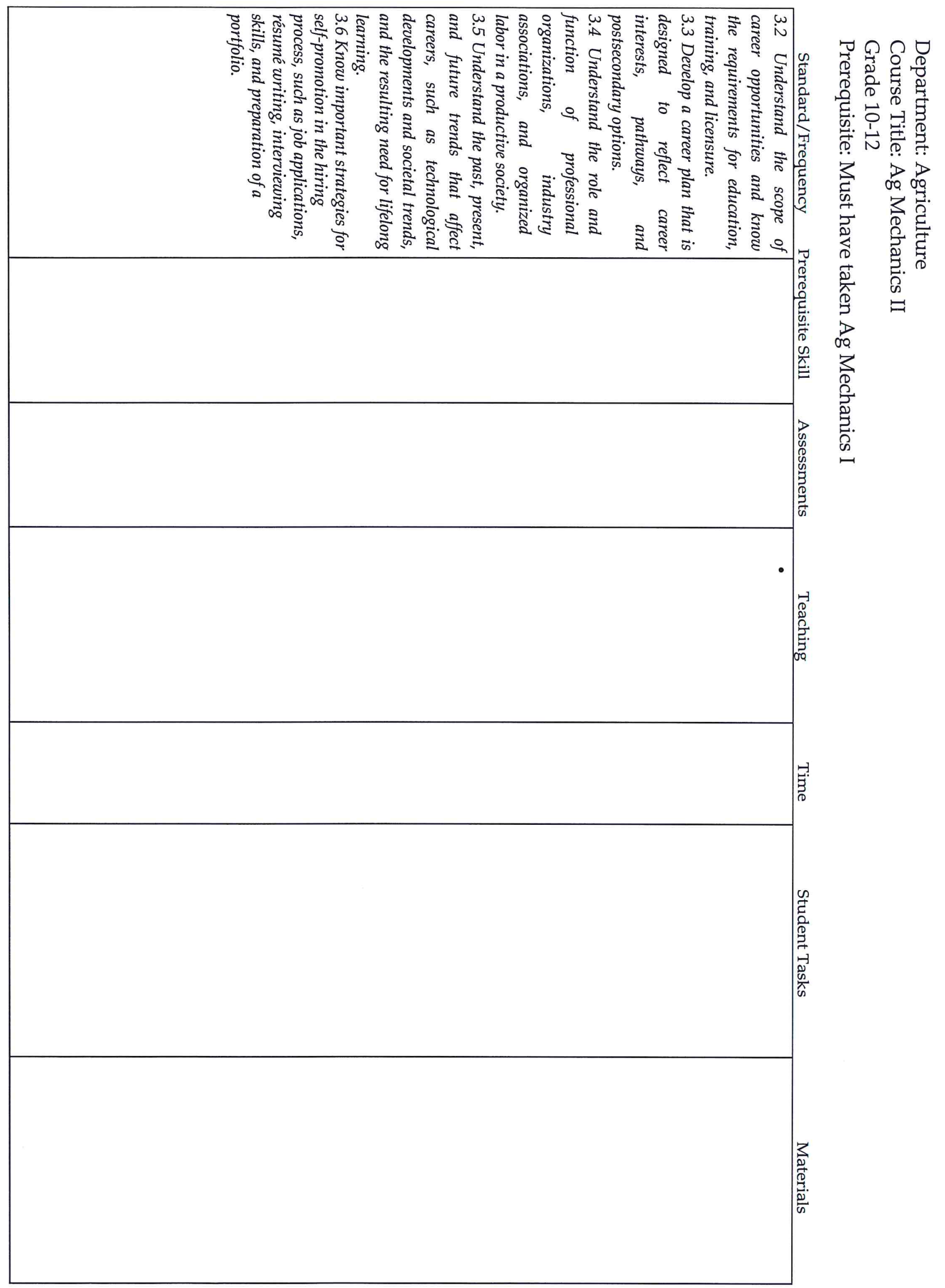




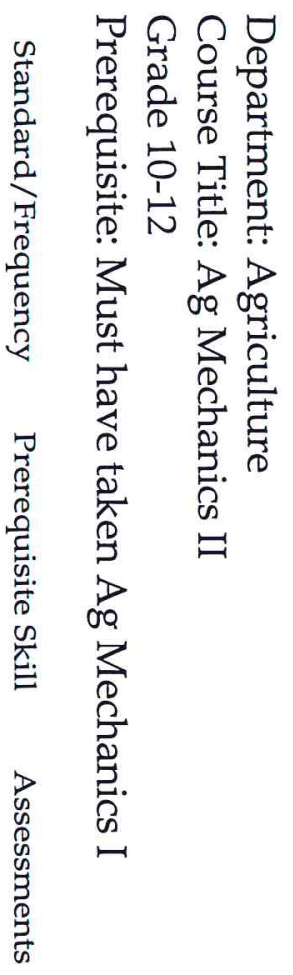

范

붕

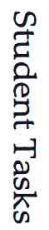

交. 


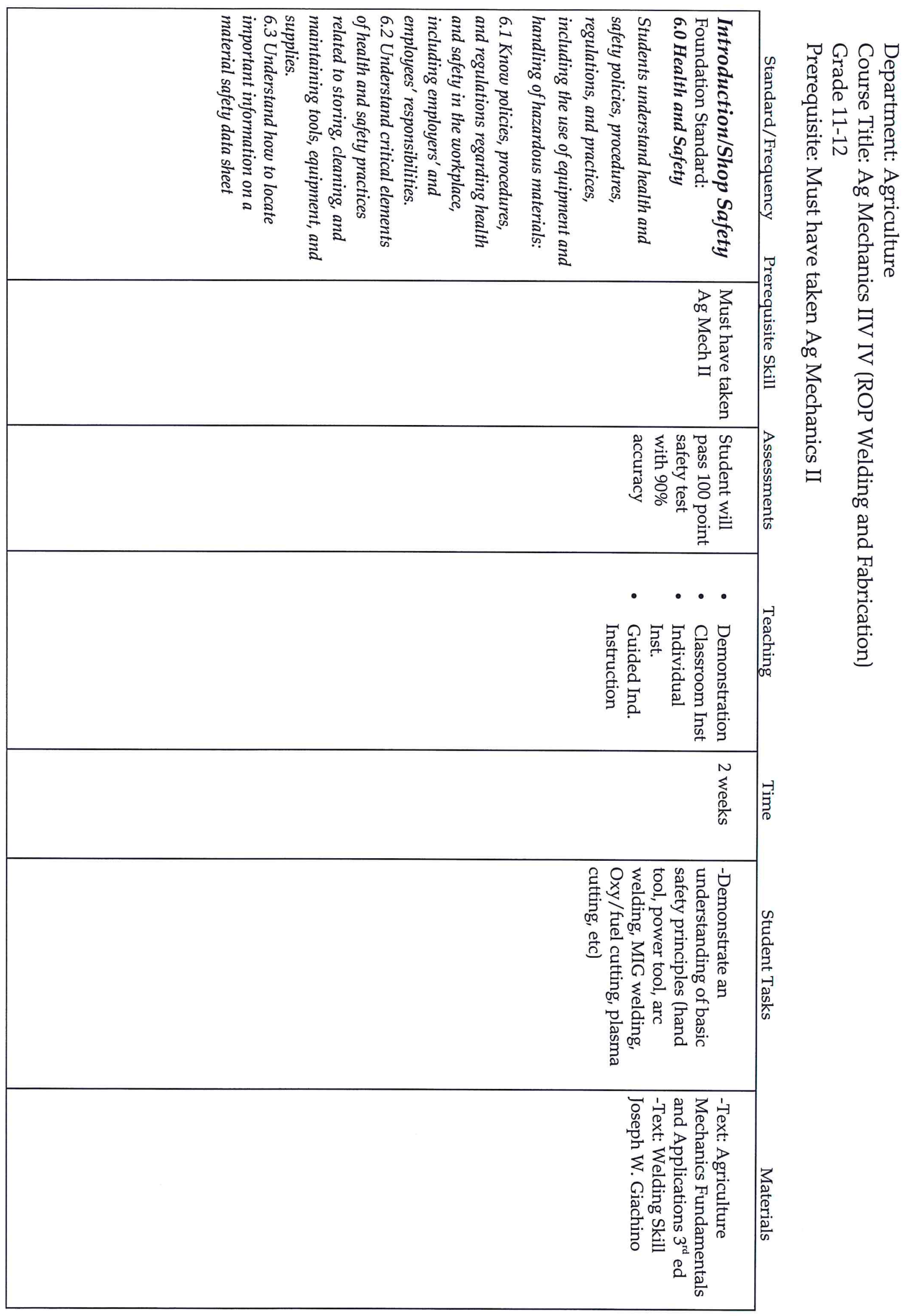




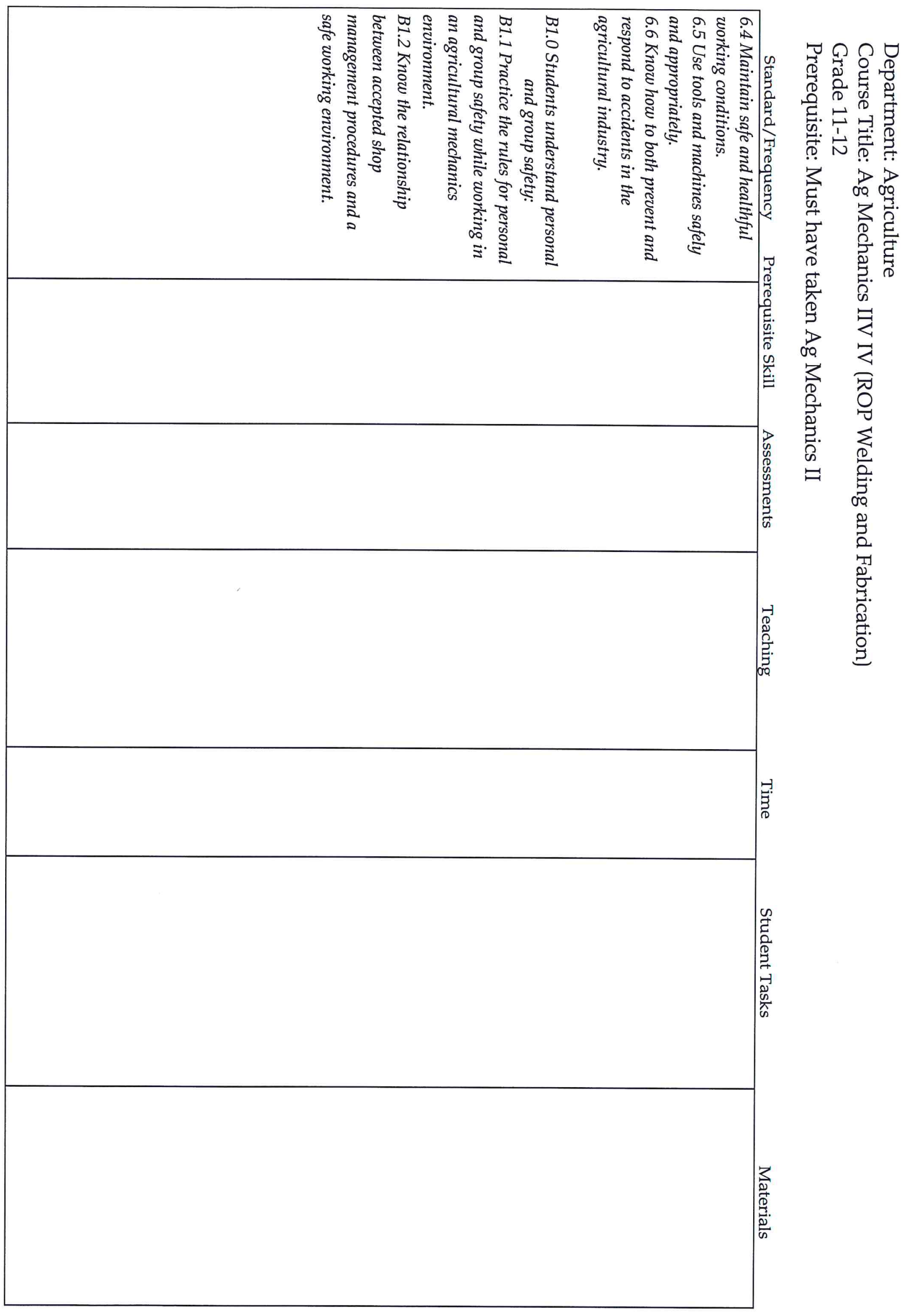




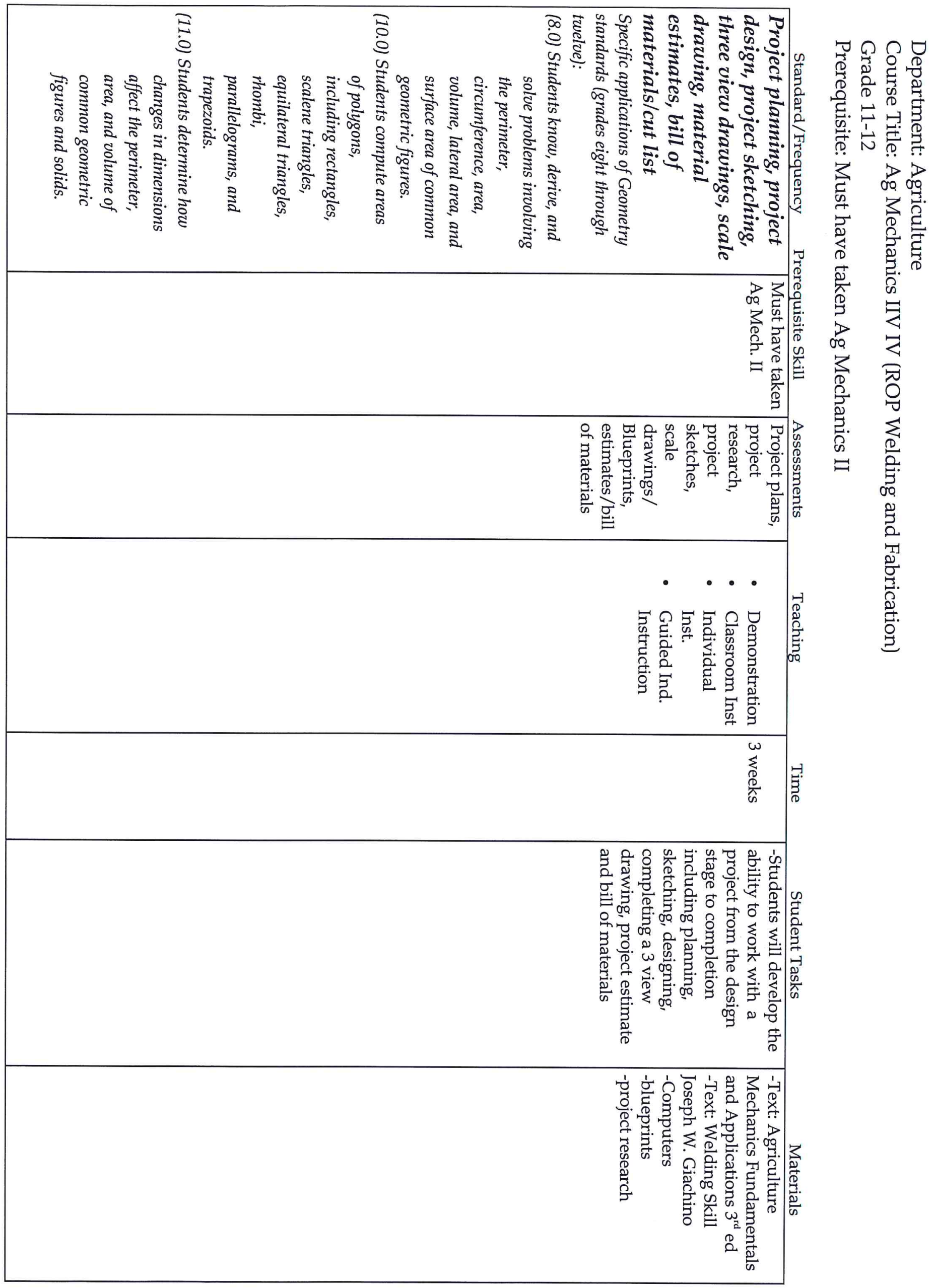




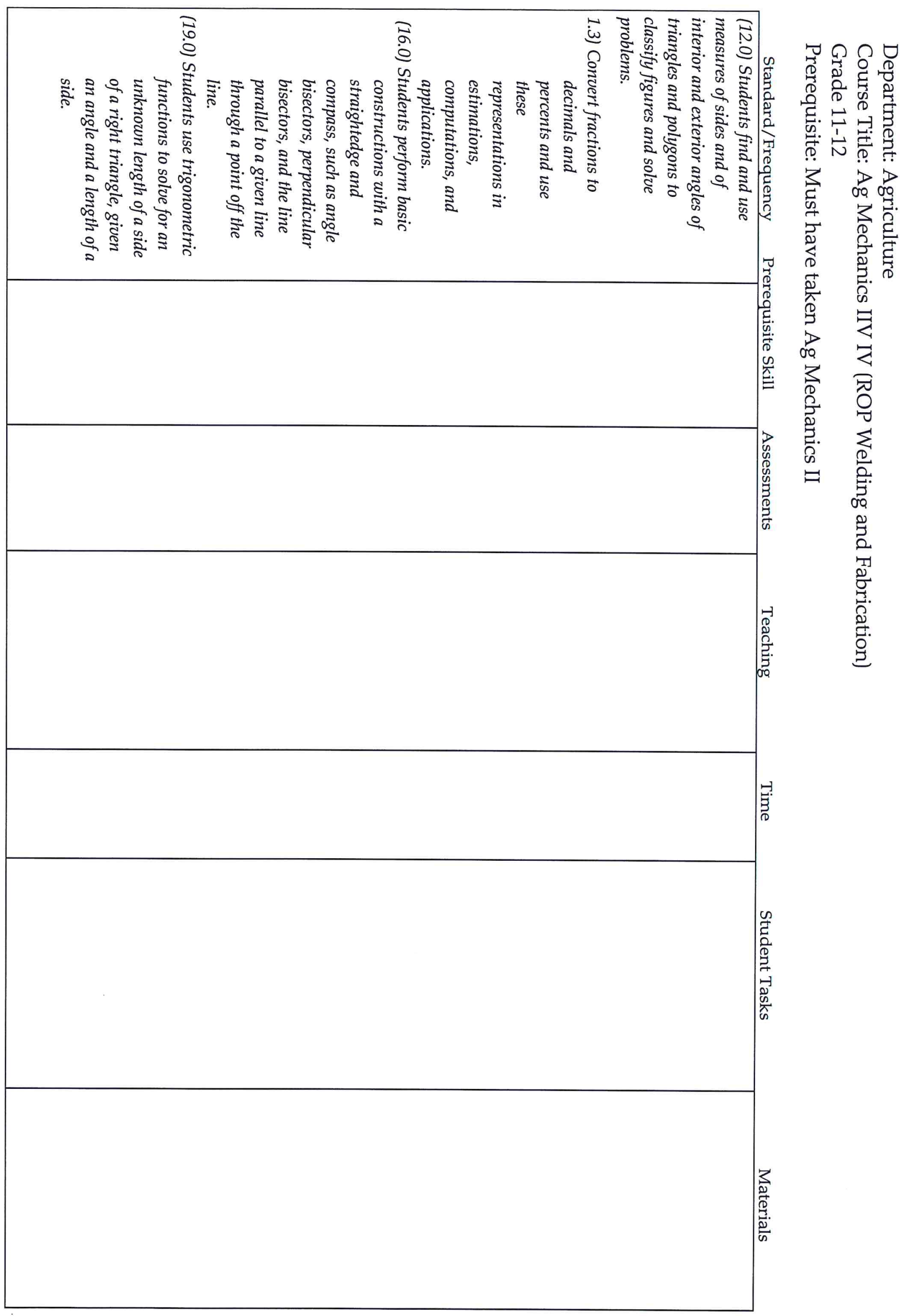




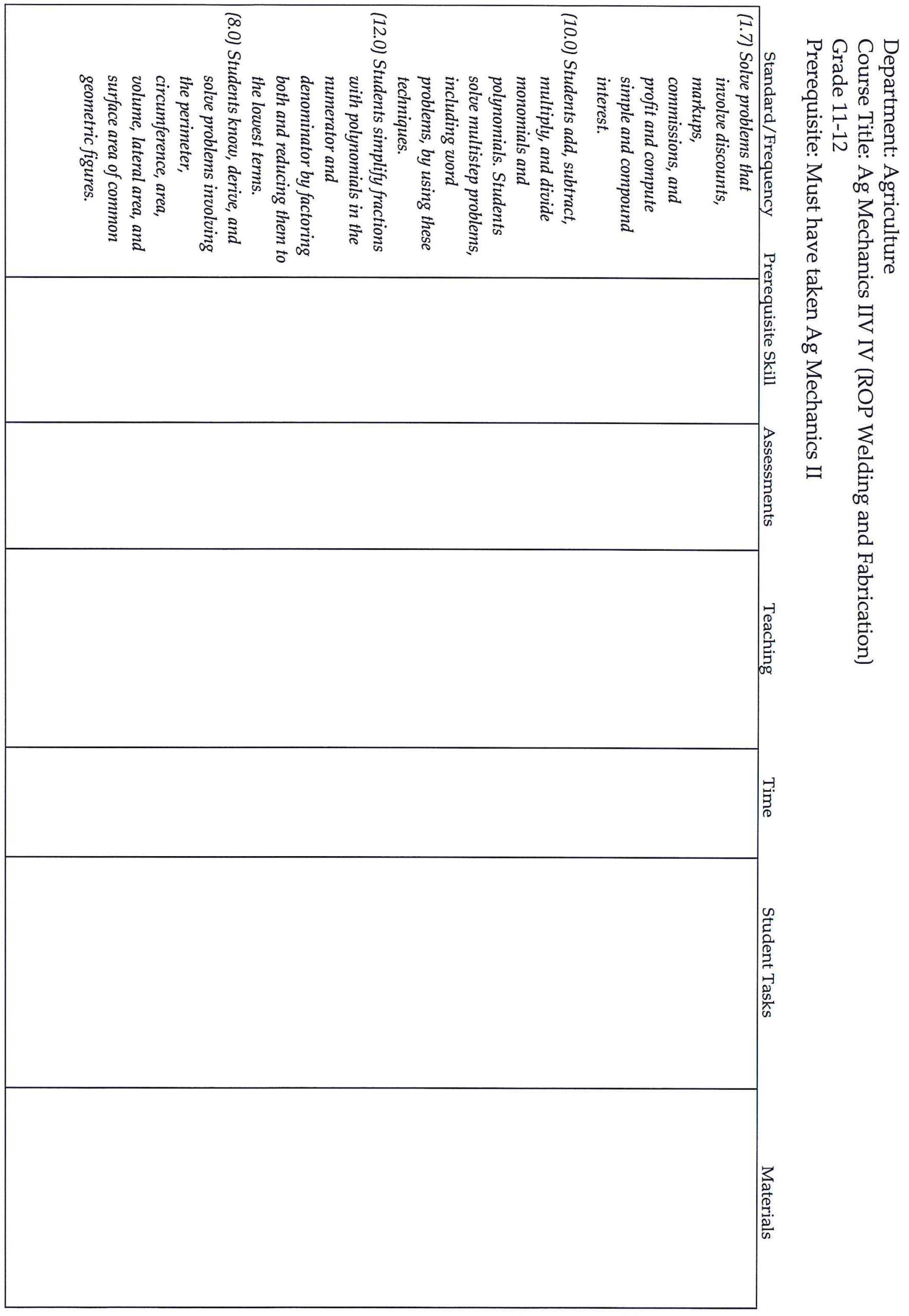




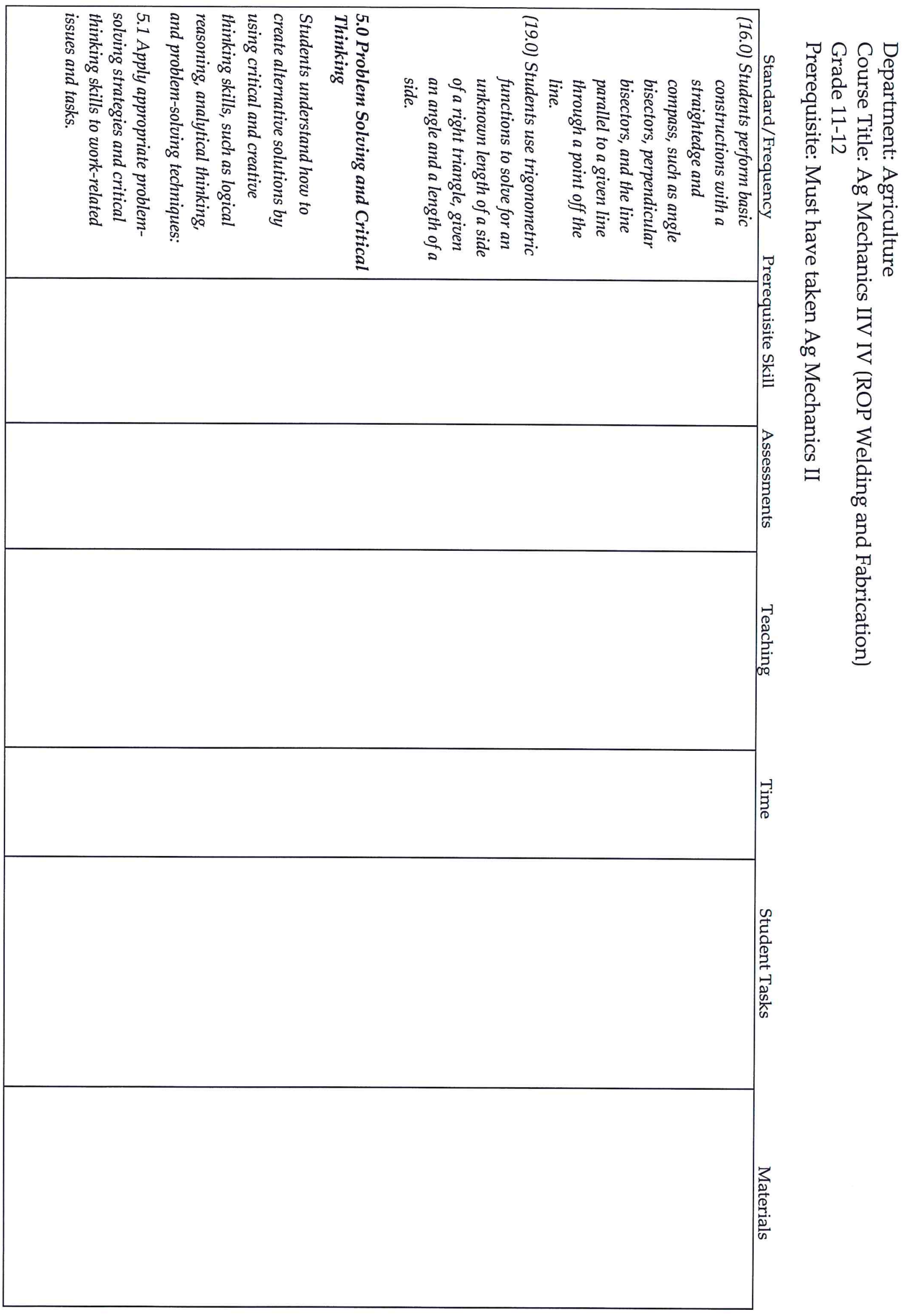




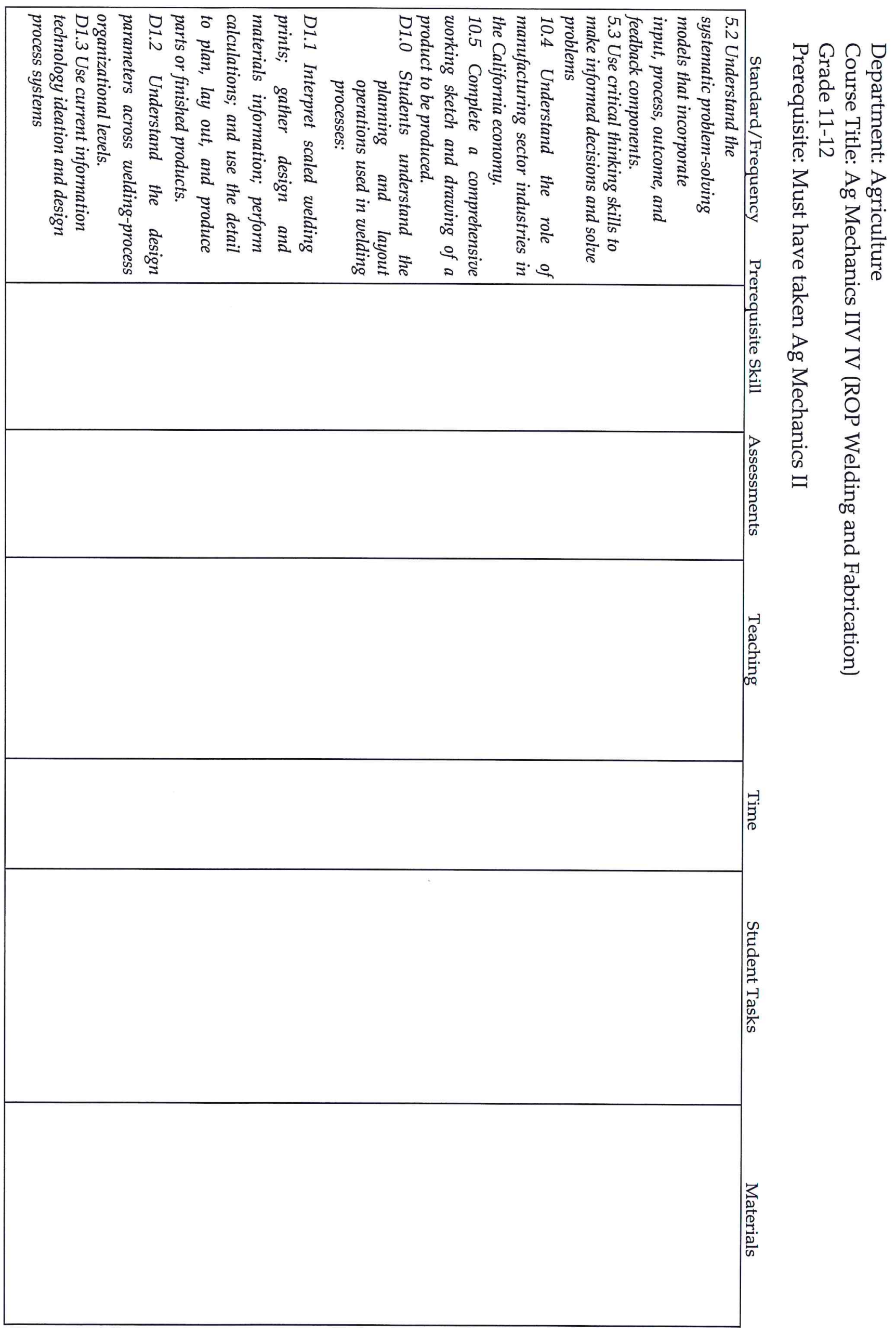




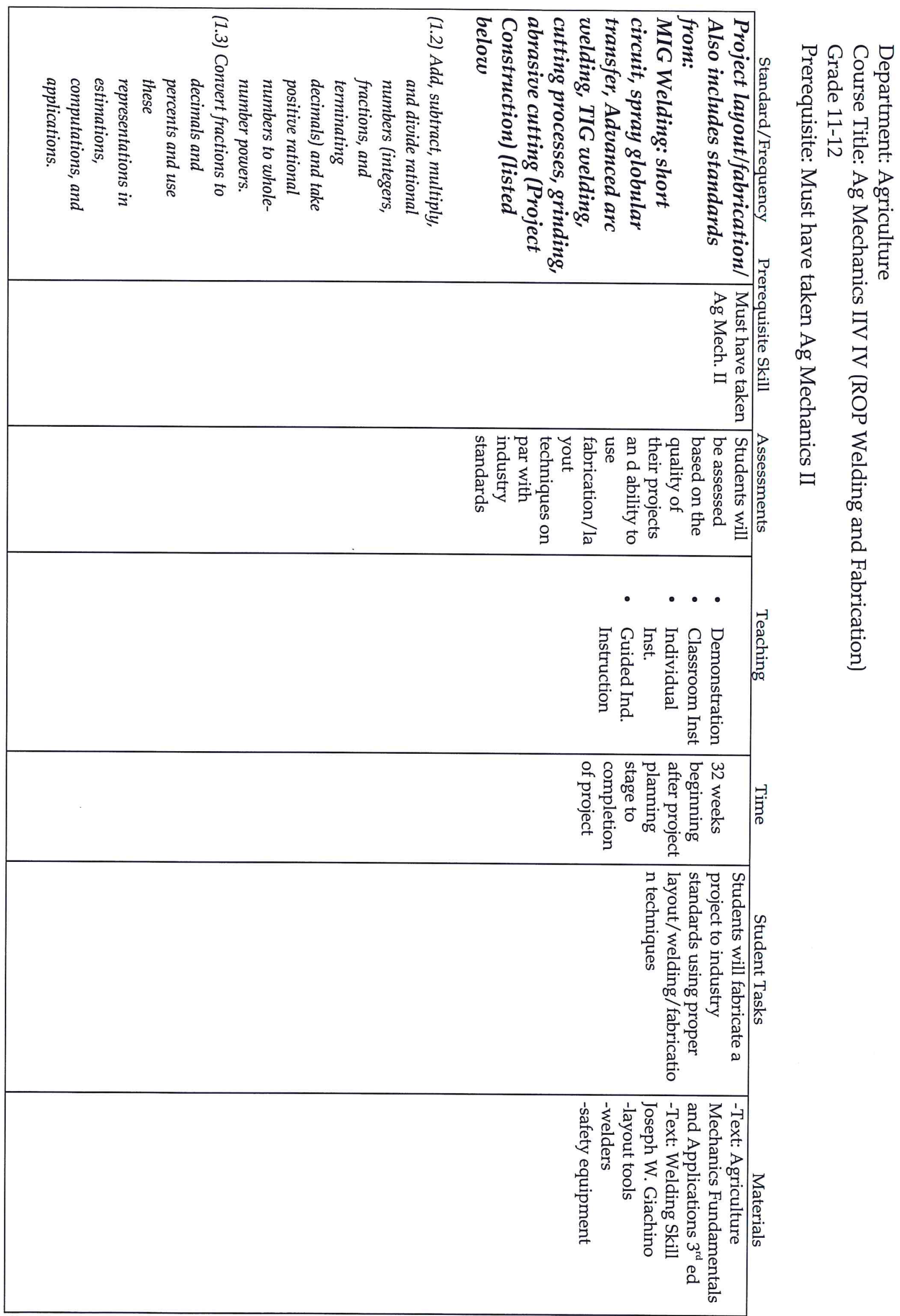




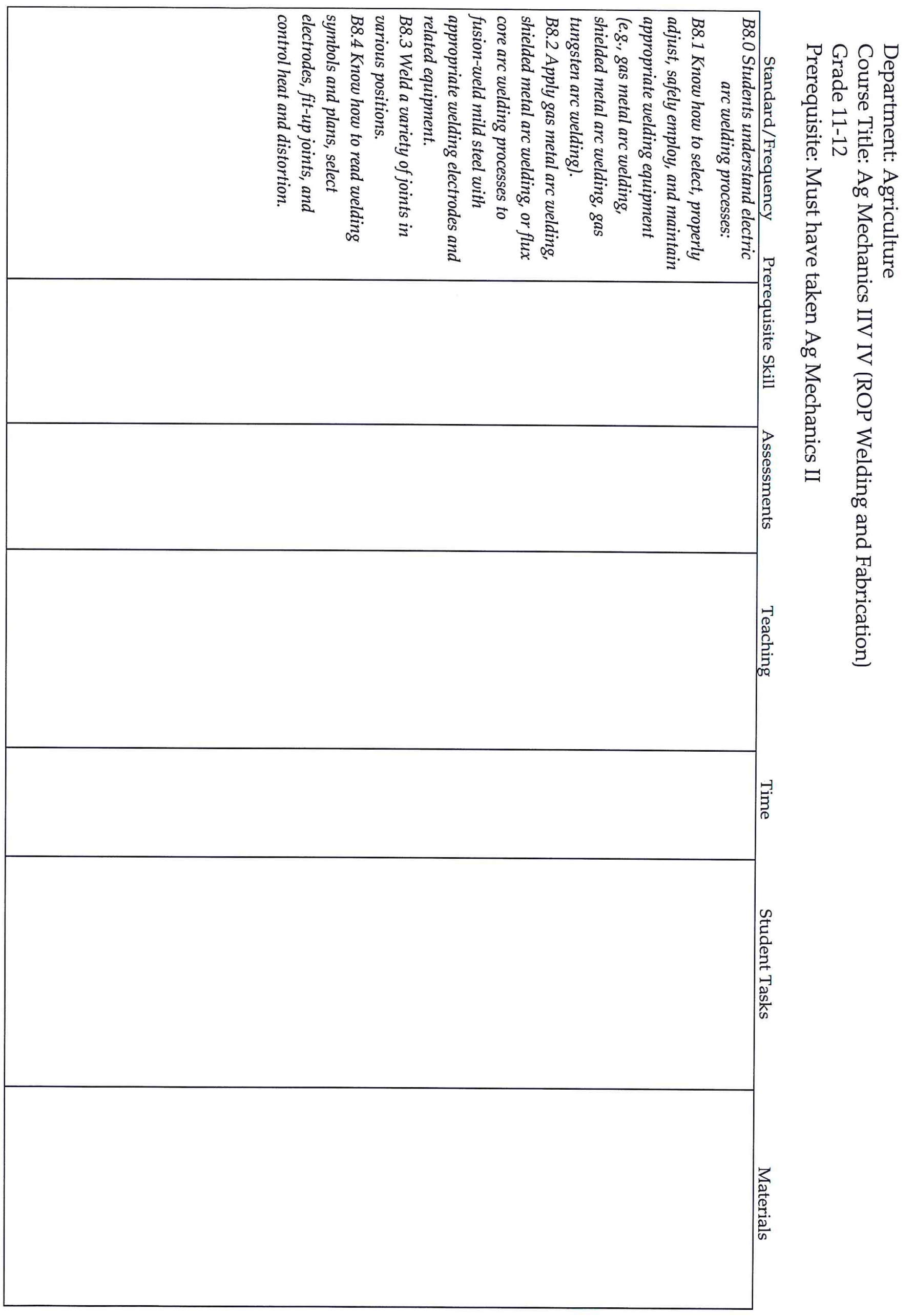




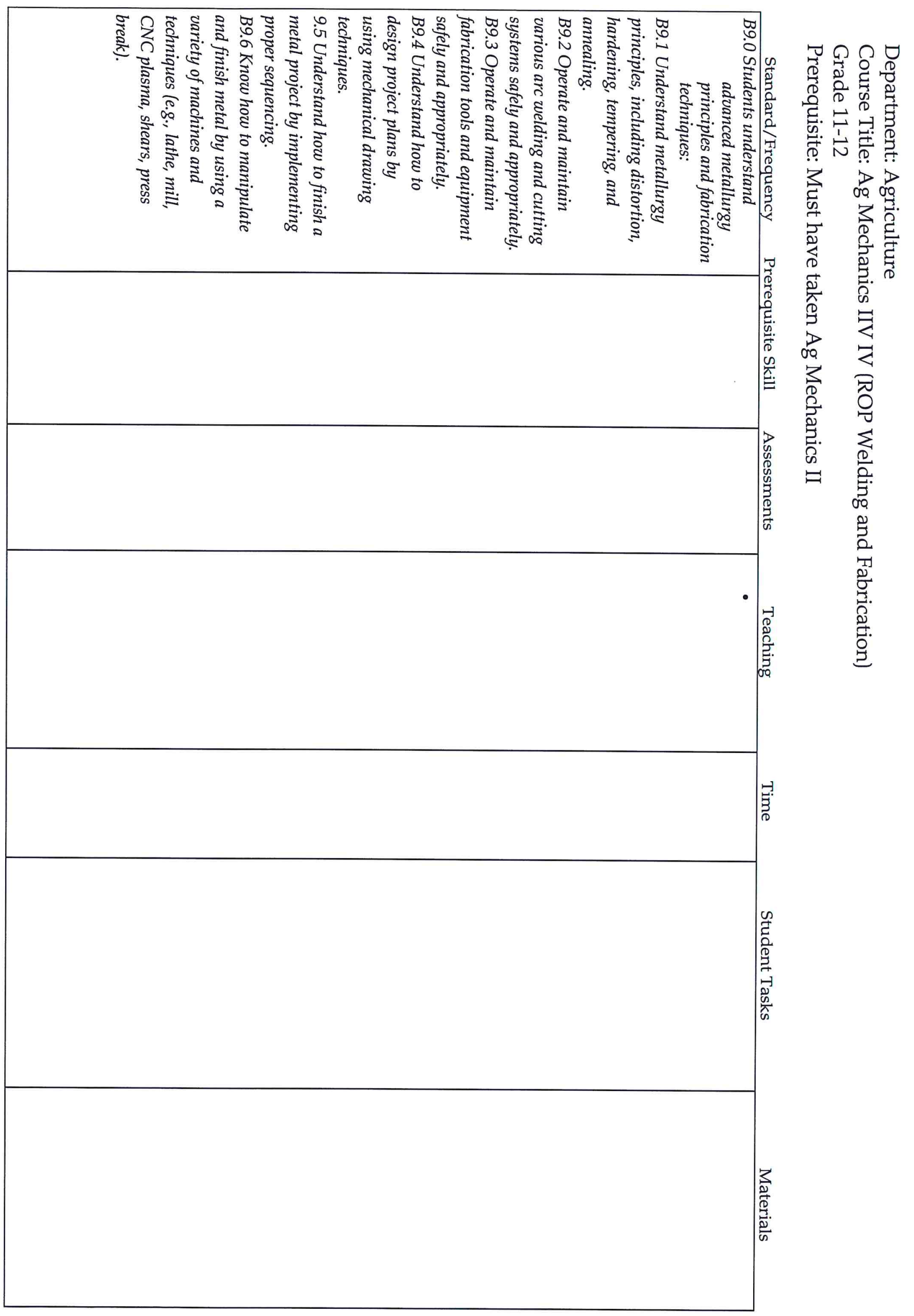




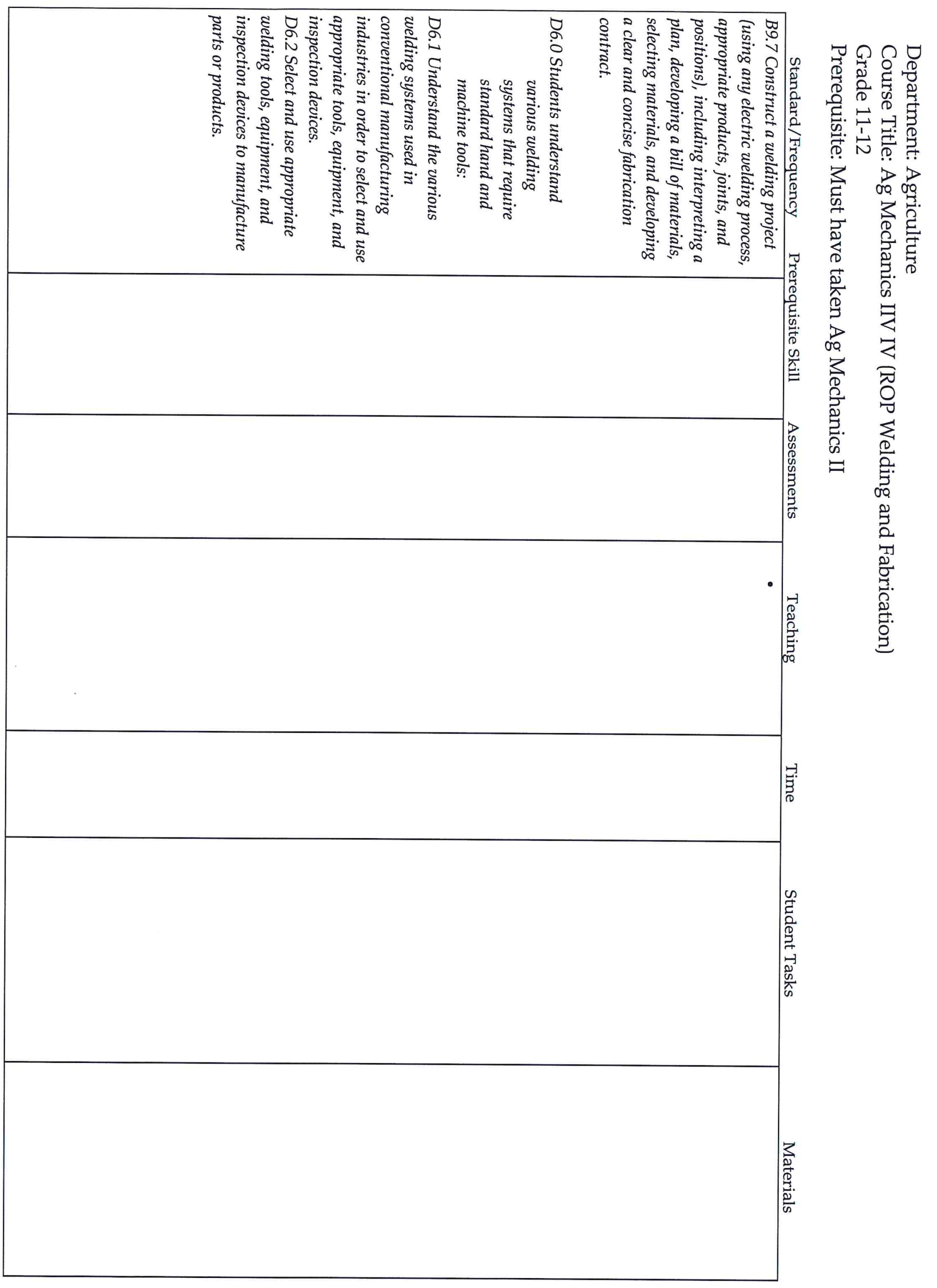




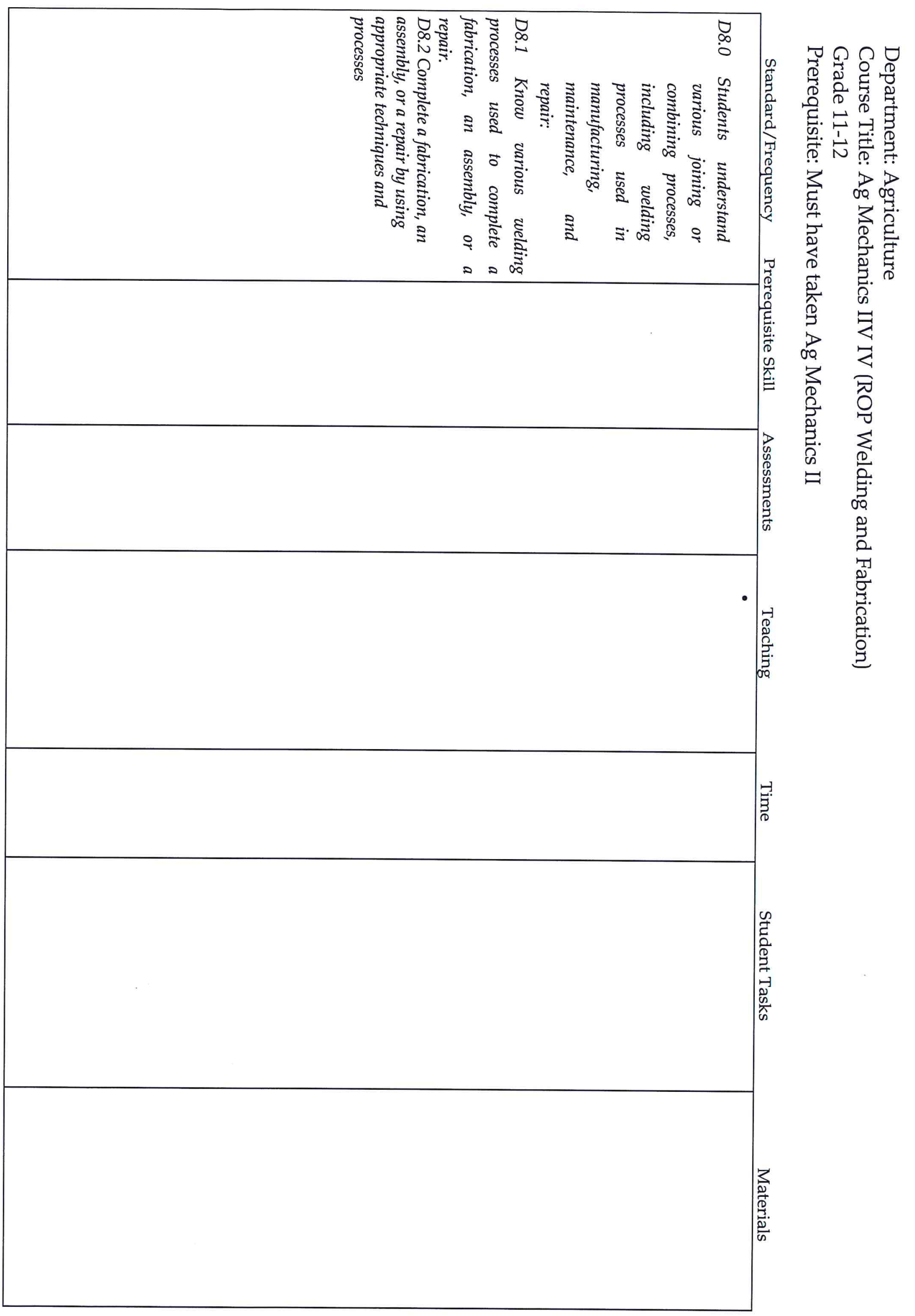




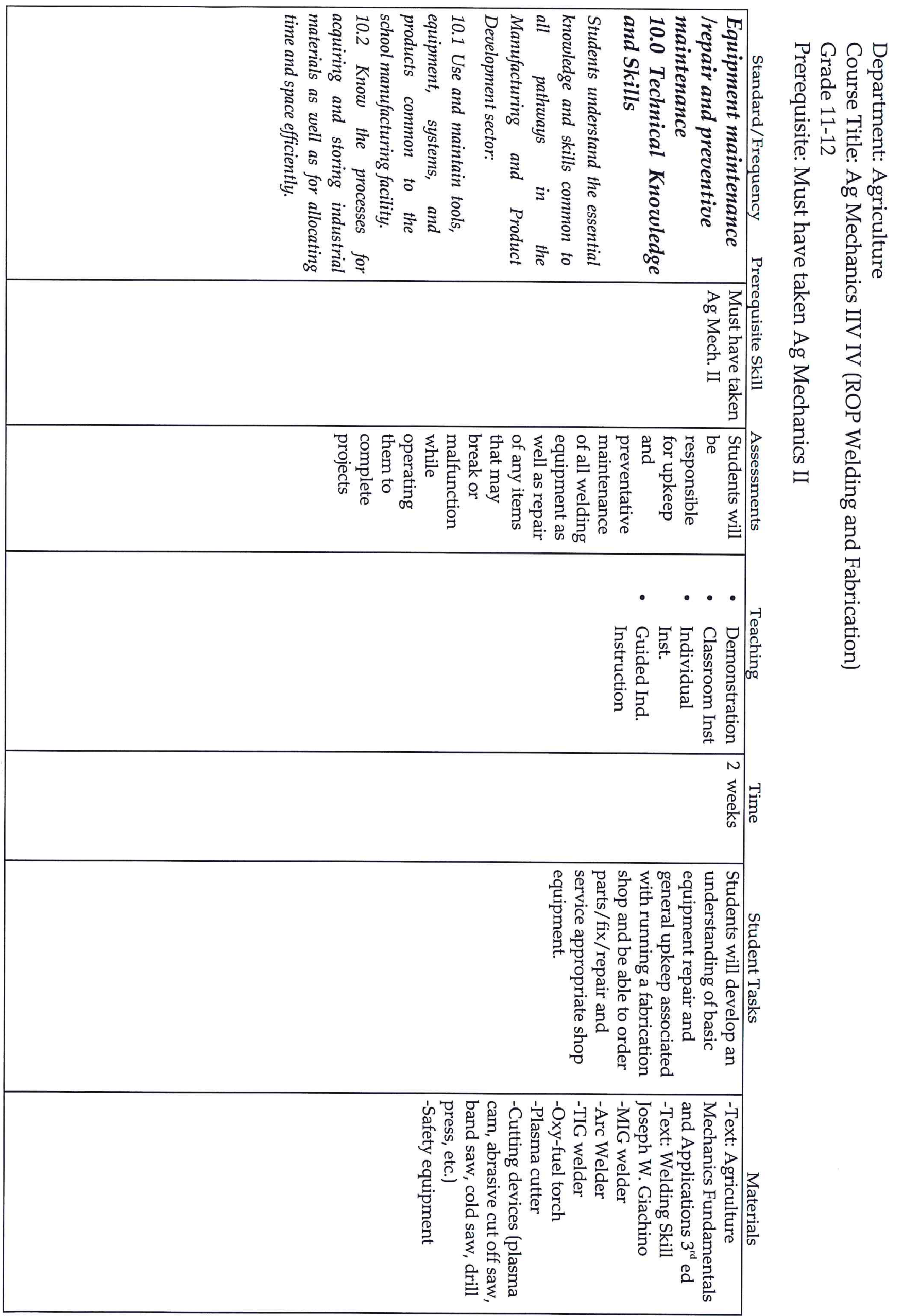




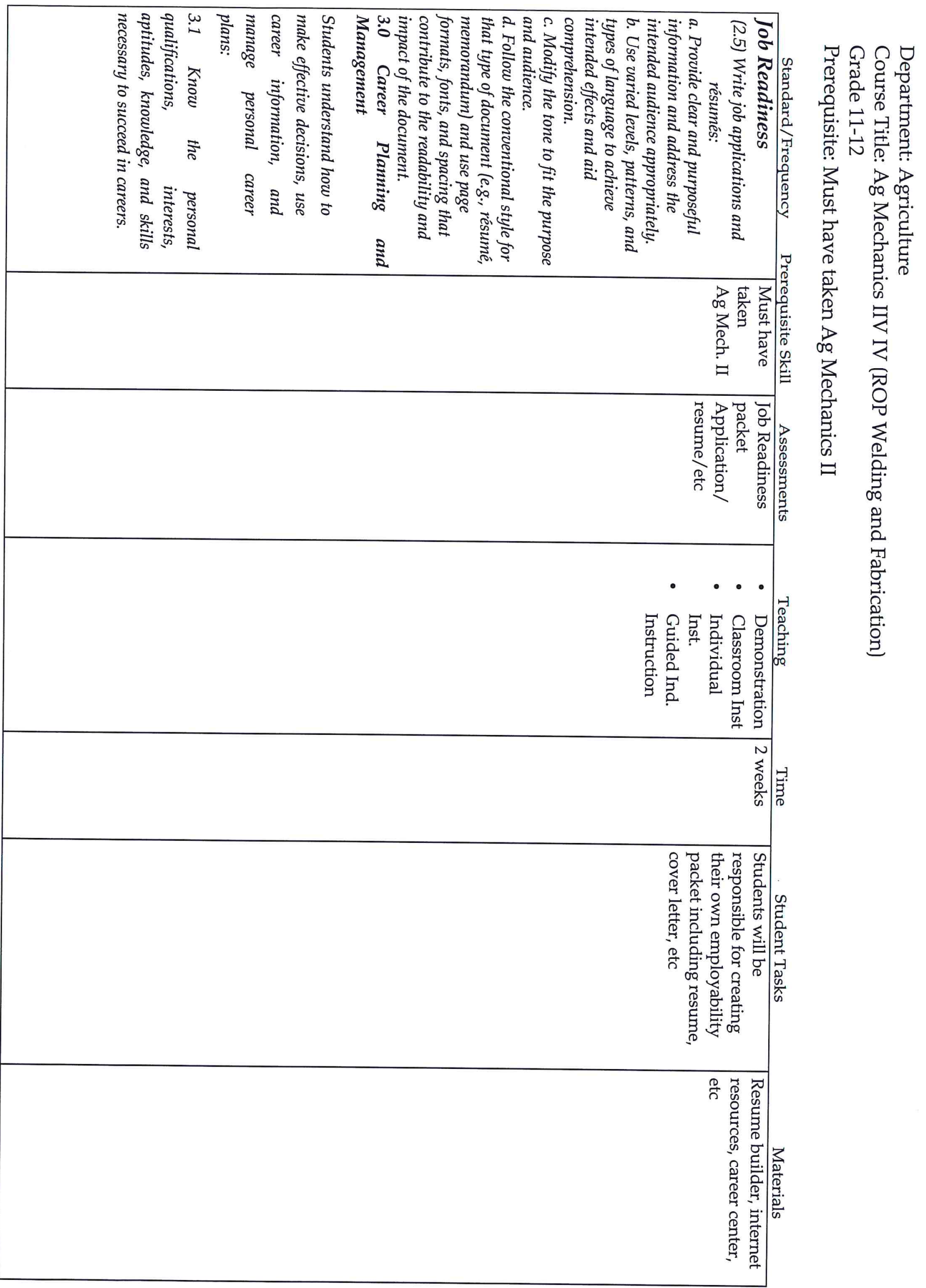




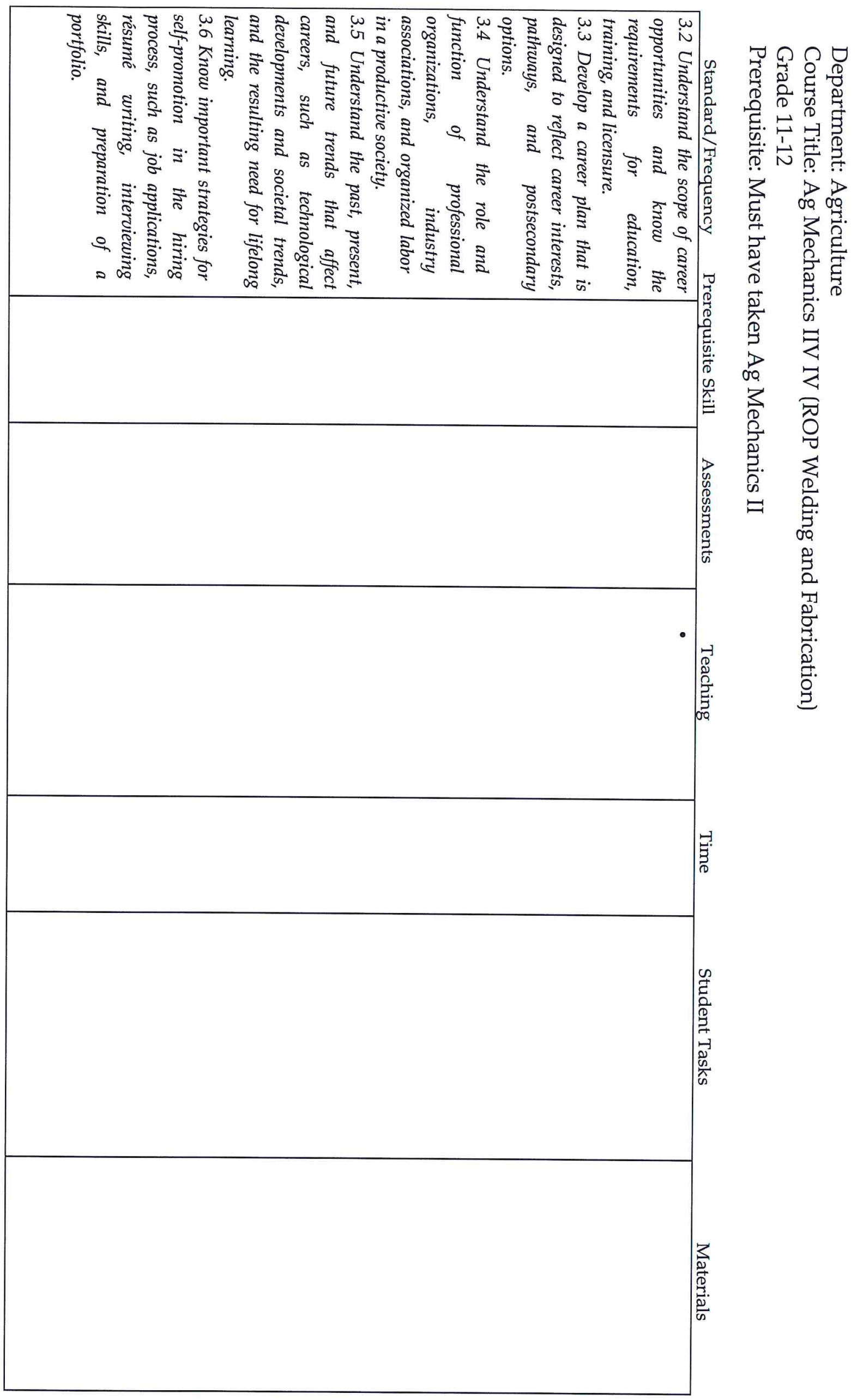



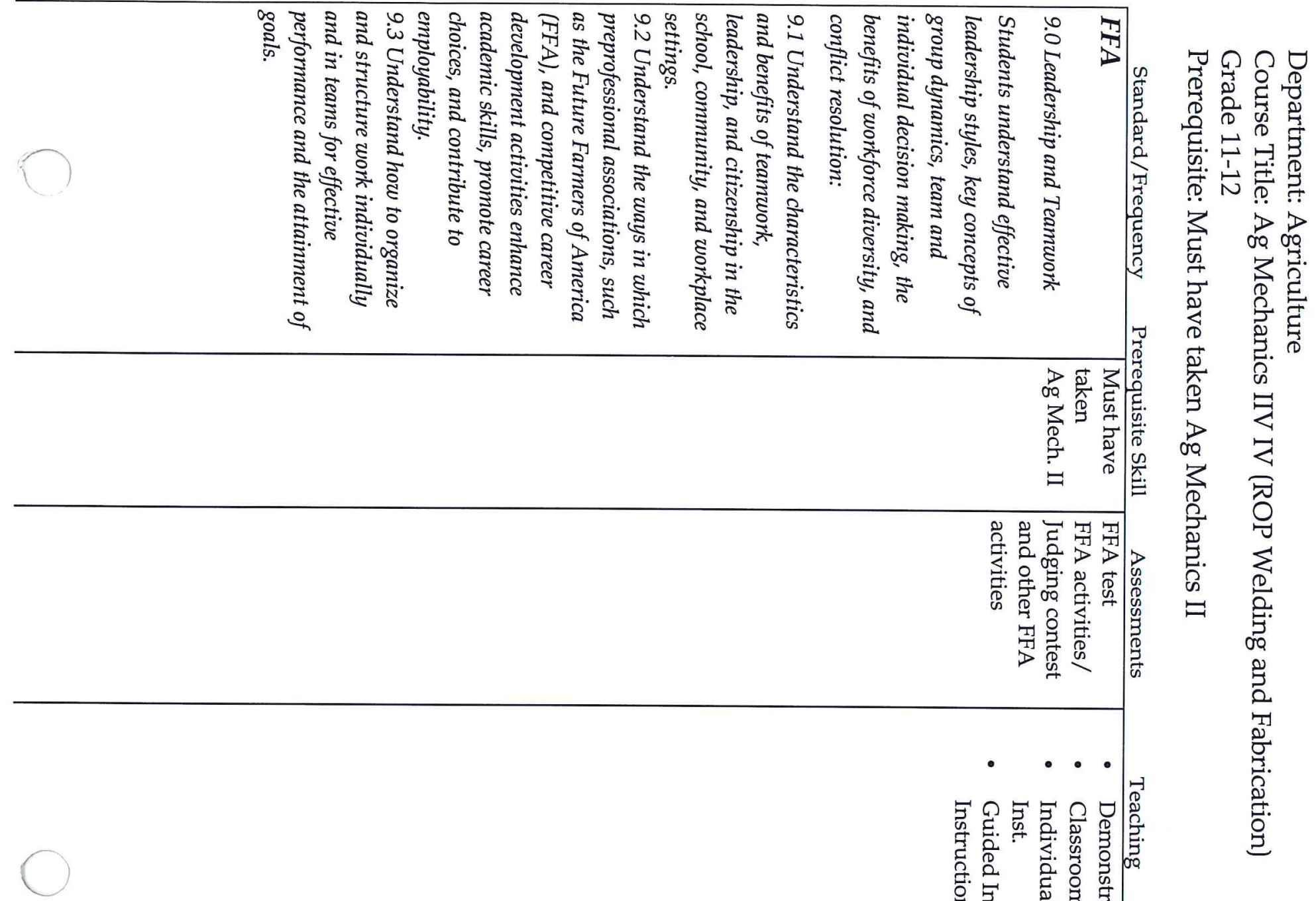

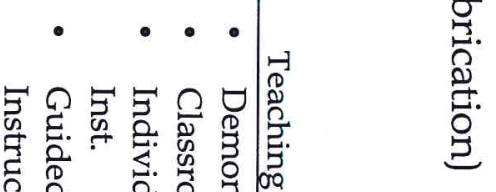



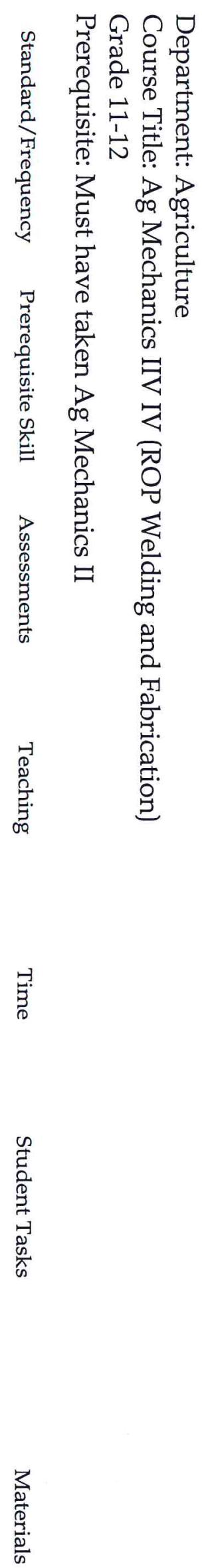

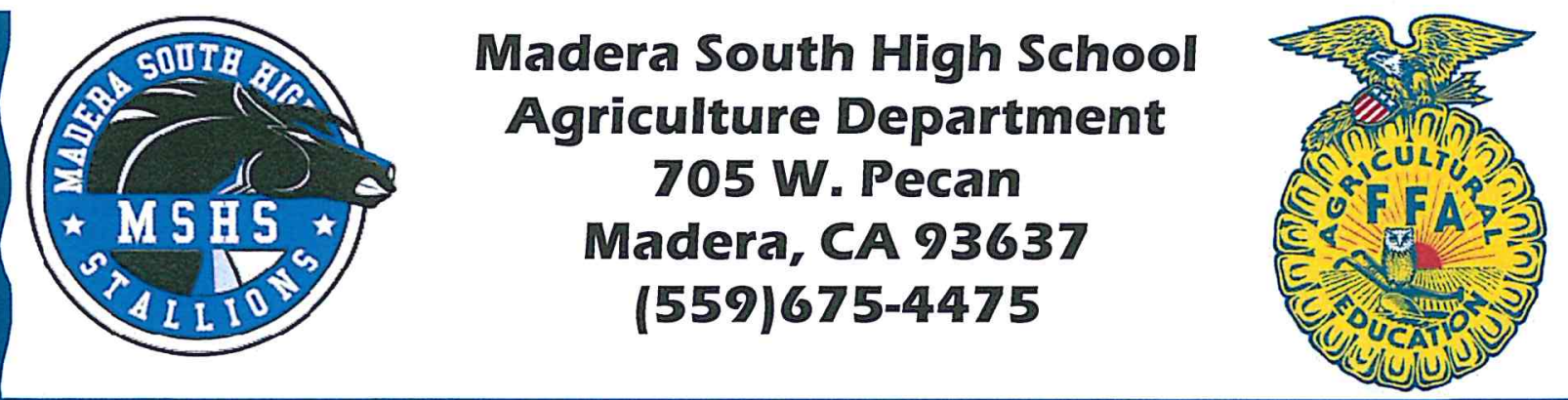

\section{Guidelines to Become a Program Completer}

In order to be recognized by the Madera South High School Agriculture Department as a program completer a student must meet all of the following guidelines.

- Must have been enrolled in an agricultural class for 3 of their 4 years at MSHS.

- Must have an ongoing Supervised Agriculture Experience Project

- Attend and participate in FFA activities and functions.

If the above guidelines are meet the student will receive their program completer status at the Spring Awards Banquet during their senior year. 


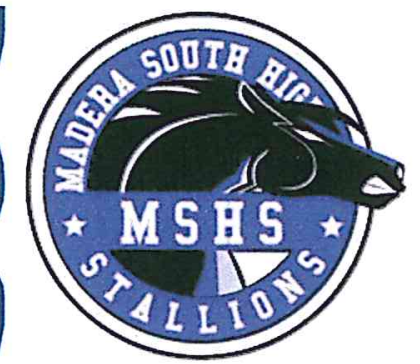

\section{Madera South High School Agriculture Department 705 W. Pecan \\ Madera, CA 23637 (552)1675-4475}

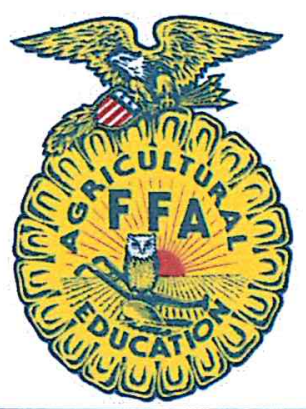

\section{Madera South Agriculture Facility and Equipment List}

Madera South High School Facilities

- 20 acre school laboratory

- 4 livestock barns, show rings, and storage facilities (Beef/Dairy, Sheep/Goat, Pig, and Horse).

- Greenhouse Unit and Storage Shed

- 3 State of the art shops and classrooms

- 3 State of the art Ag. Science classrooms and laboratories

- 1 Floral Shop and Classroom

Madera South Major Equipment

- 2 school vans

- 4 school trucks

- Tractors

- Gooseneck Livestock Trailer

- Bumper Pull Trailer

- Flat Bed Trailer

- See Inventory List under W for a complete list. 


\section{Madera South High School \\ Agriculture Department \\ 5 year Plan}

\section{2/13}

Planting of Vines

Irrigation for Vines

Horticulture Area: 2nd greenhouse

Poultry Unit

Develop SAE Grant Program

\section{3/14}

Replacement of Vehicles

Develop a Work Experience program

Student Field Crop Program

Horse Arena - Bleachers

\section{4/15}

Storage Buildings for: Farm Manager; Ag Mechanics; Floral

Develop Ag Business pathway/computers

Floral Delivery Cart

Horse Arena - Lighting

\section{5/16}

Veterinarian Science Laboratory

Crop Science Pathway

Tree Crops

2016/17

Replacement of Vehicles 

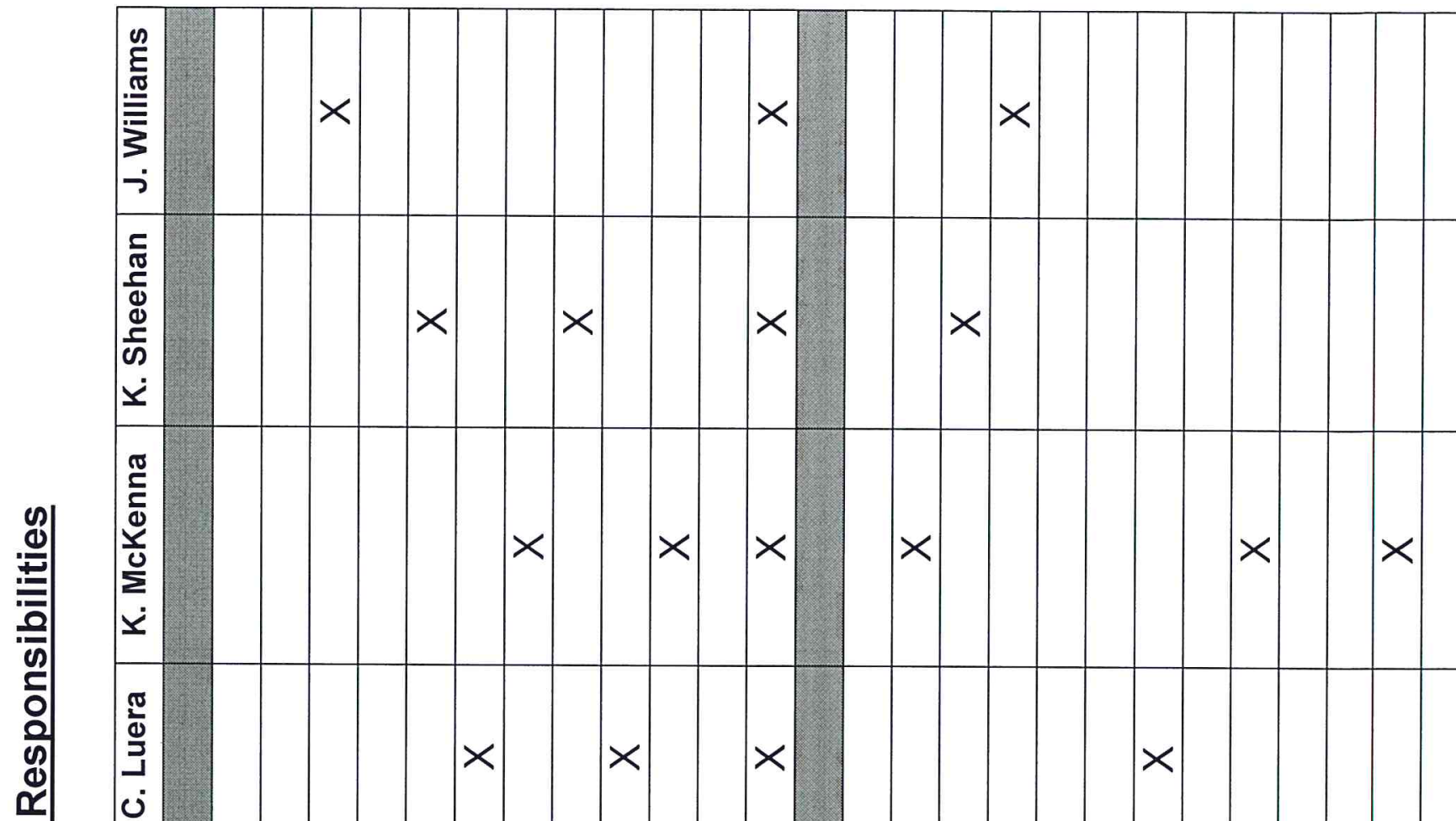

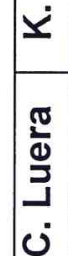

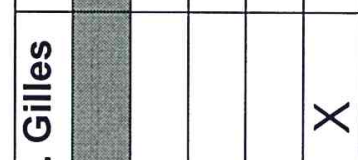

$x \quad x \quad x$

$\times$

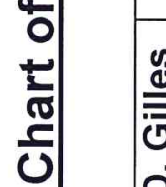

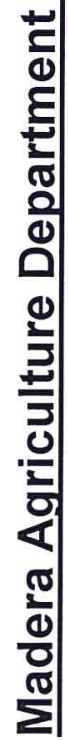

ơ

๓

赵

$\times \quad x$

$\times$

$\vdash$

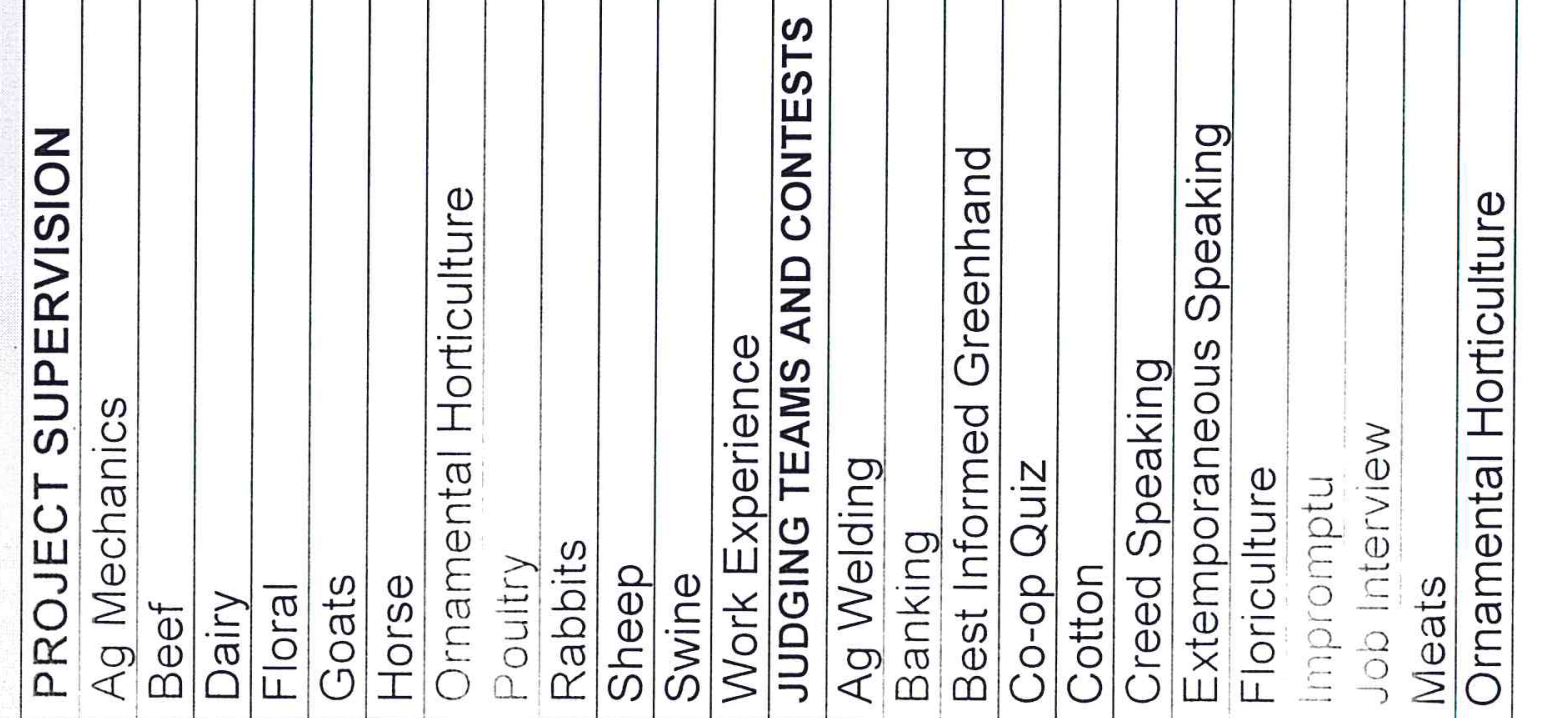



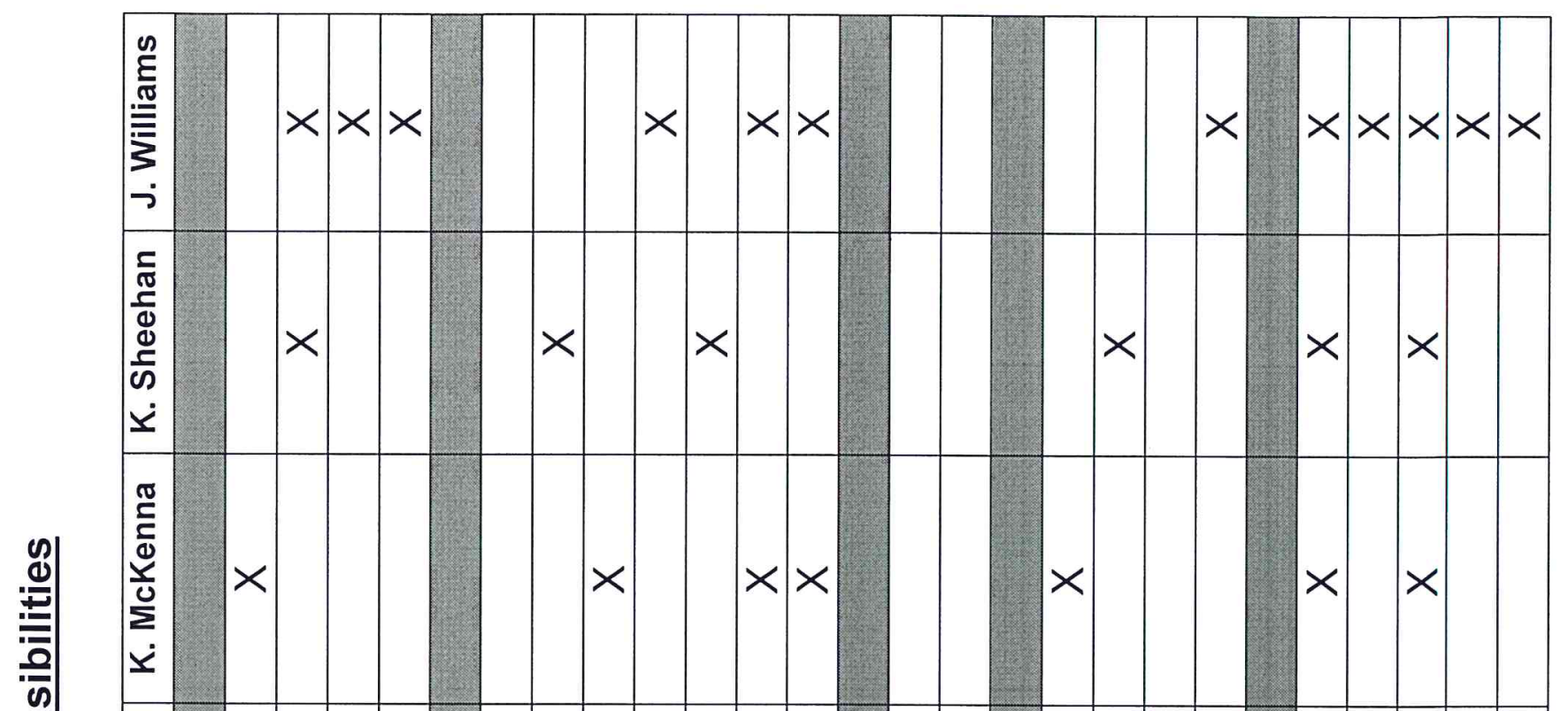

! $\times$

I $x$

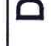

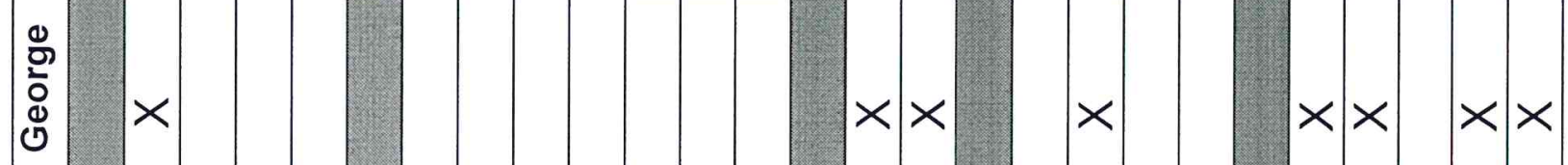
๓

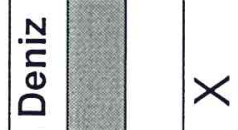
$\vdash$

$x \times$

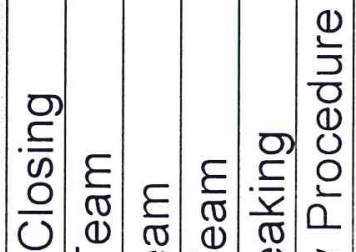

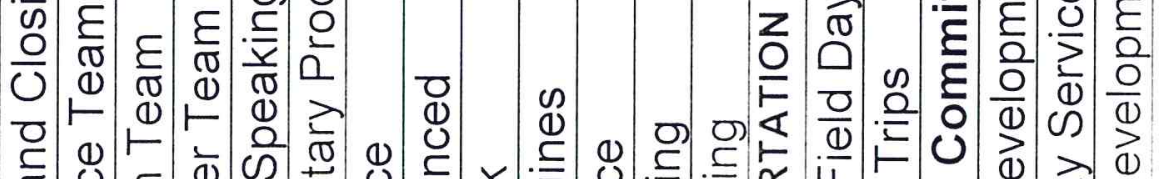

占

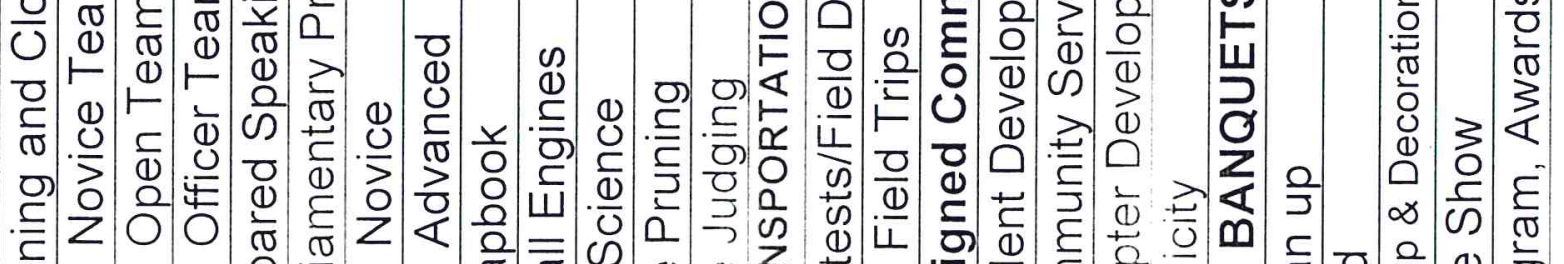

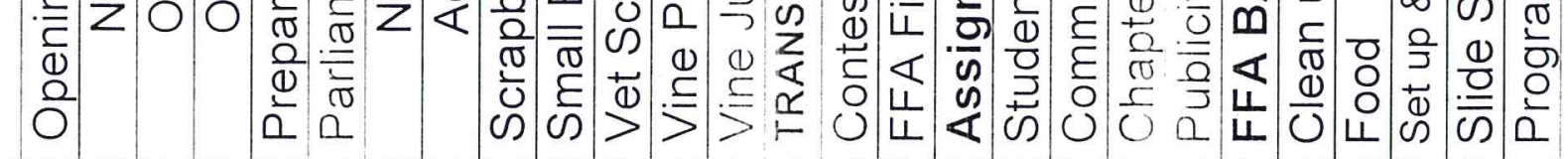




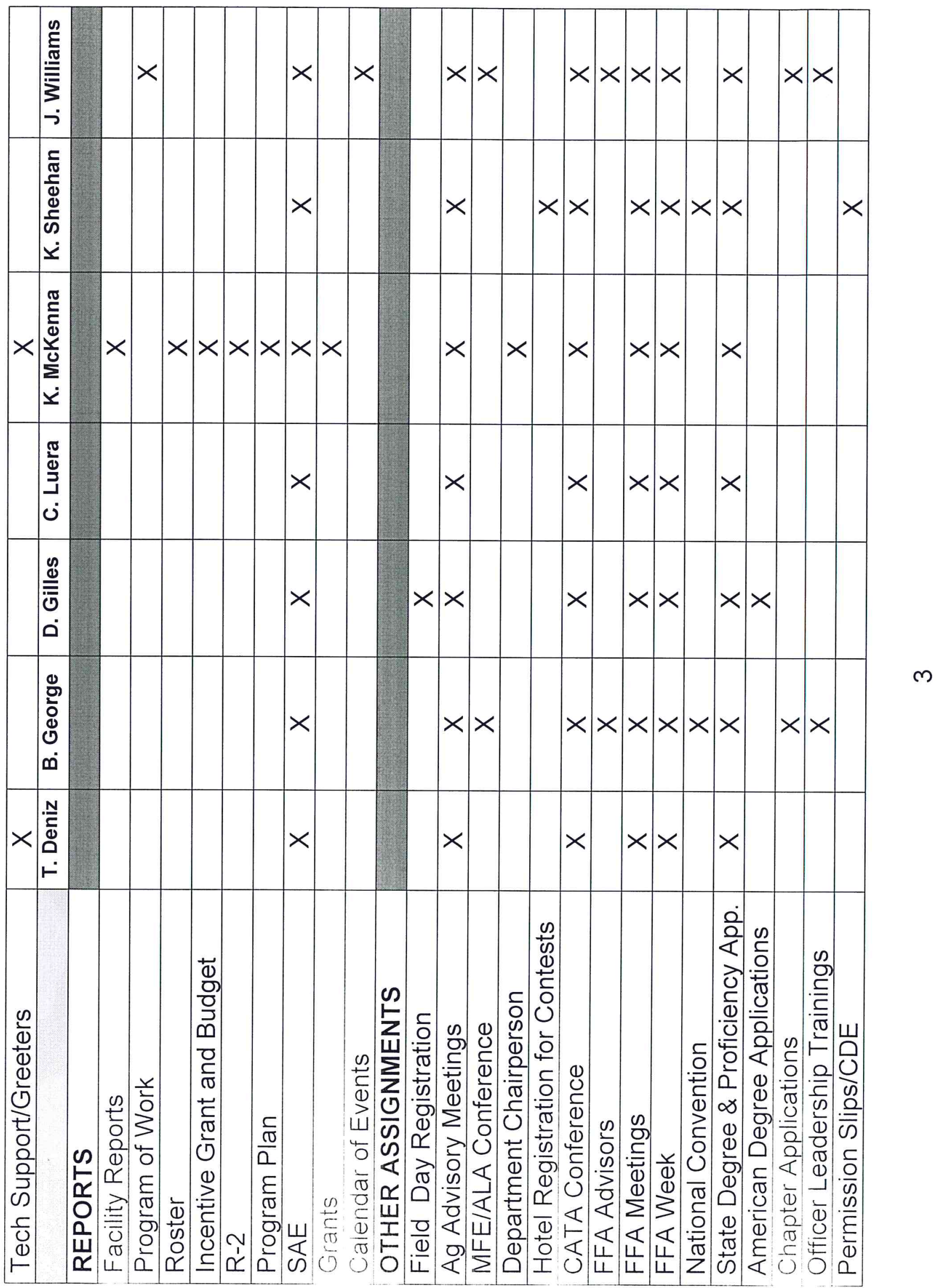




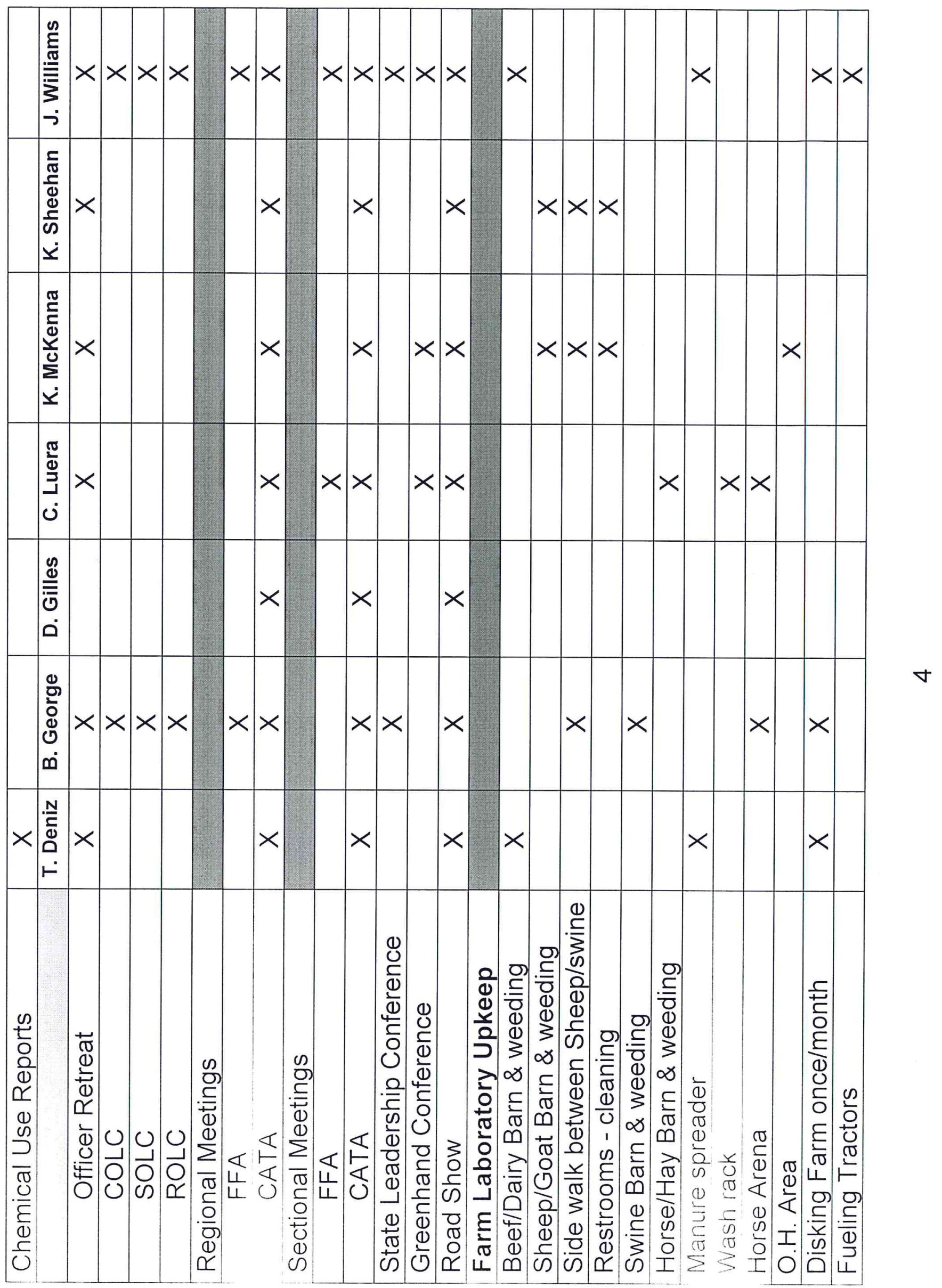



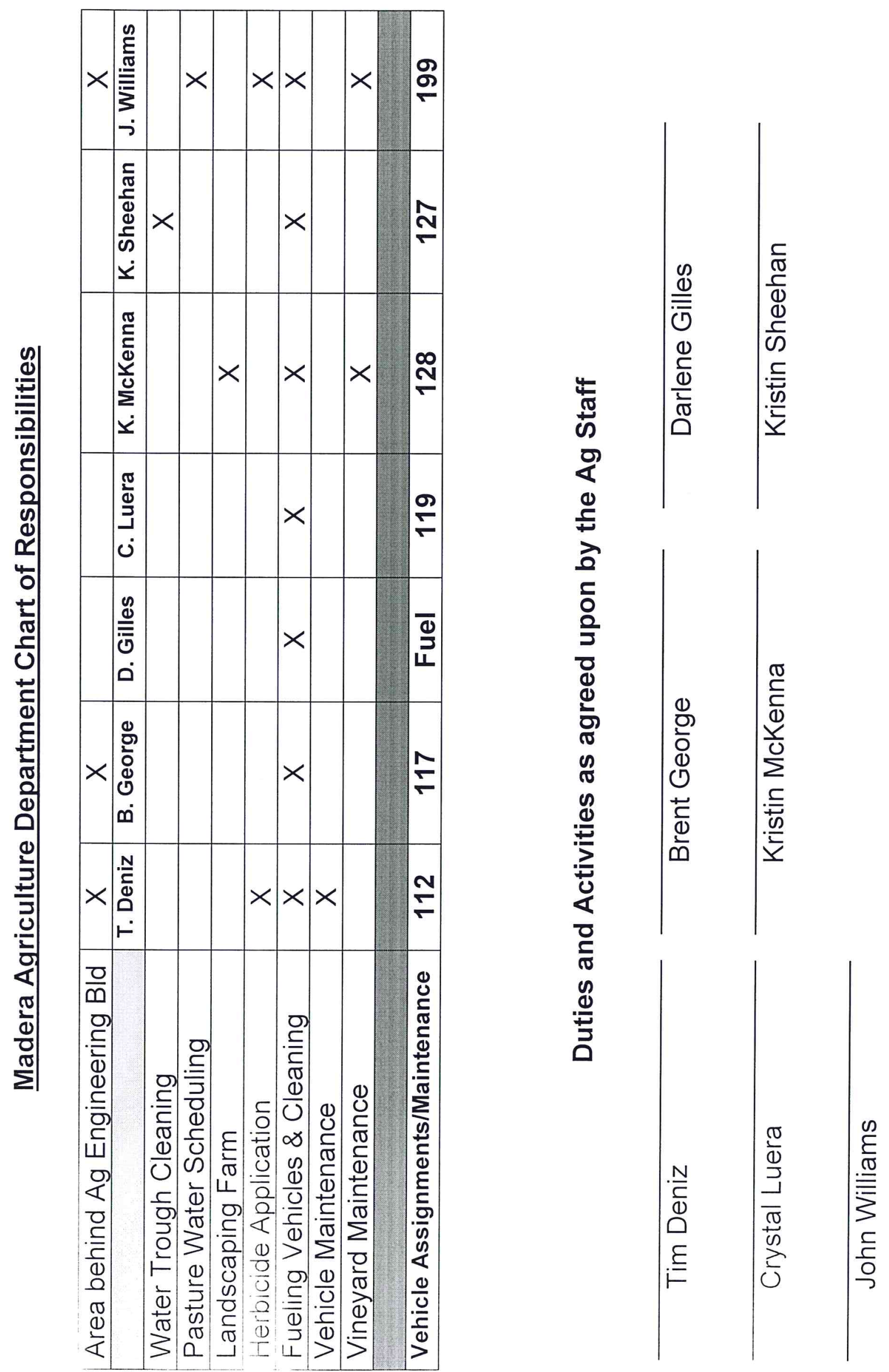

10 


\section{Maderd FFA \\ Progracin of Iativitios}

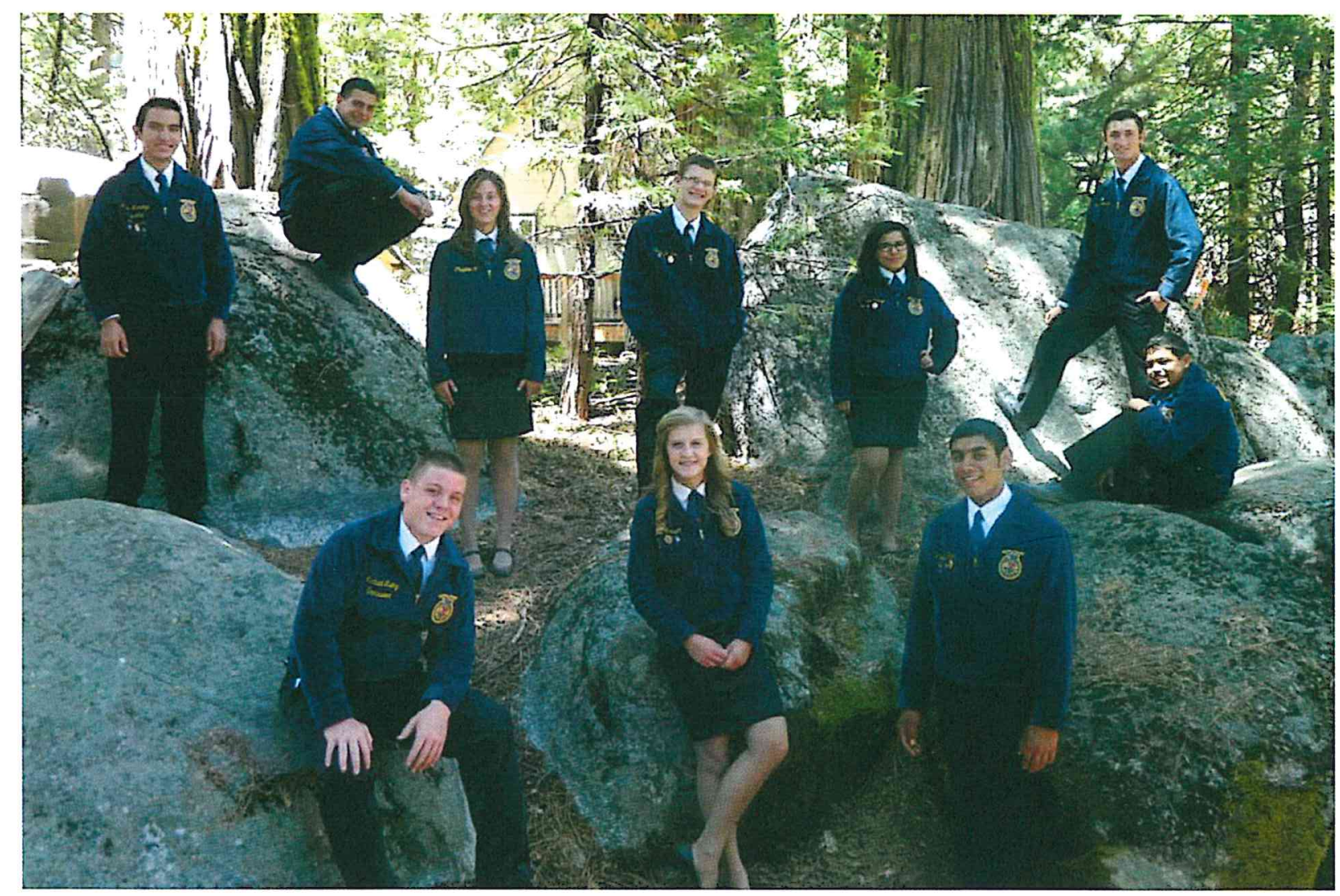

$\longrightarrow \sqrt{7}$

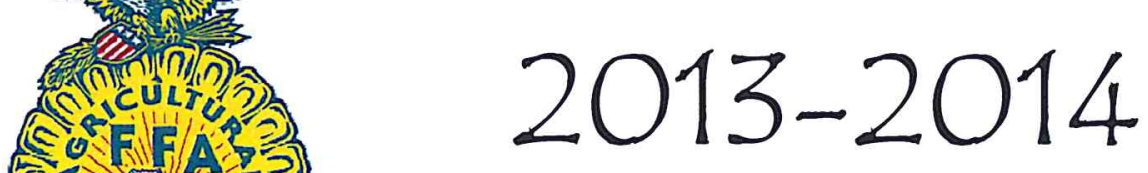

\& o n $\quad A$ ?

E - $)$

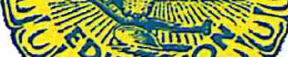

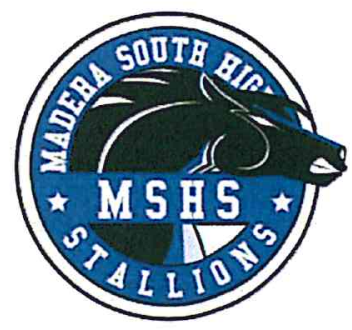




\section{Table of Contents}

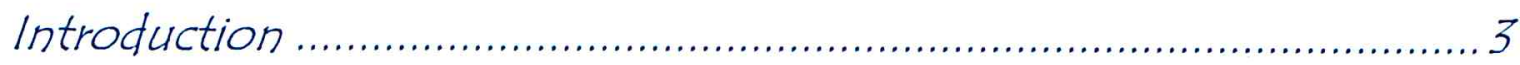

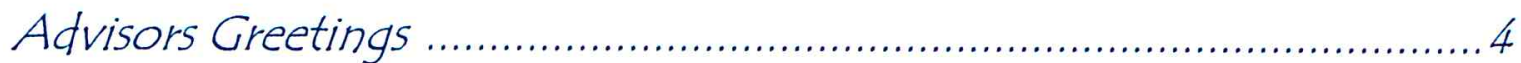

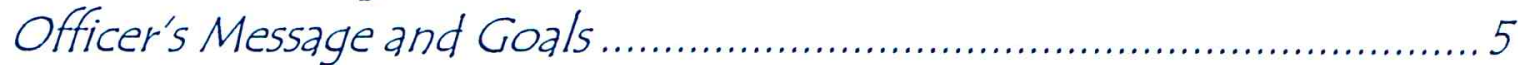

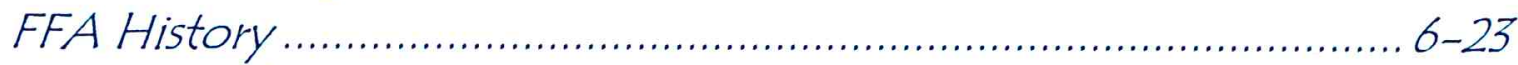

Calendar of Events 2013-2014 ..................................................24-36

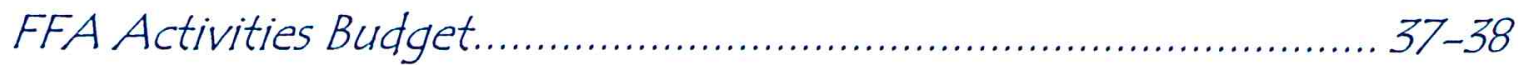

Community Service Activities..................................................... 39

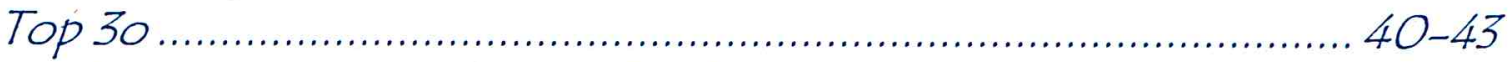

Madera FFA Constitution and Bylaws ..................................... 44-49

FFA Awards and Recognition .................................................50-53

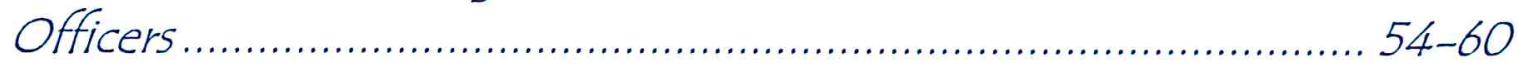

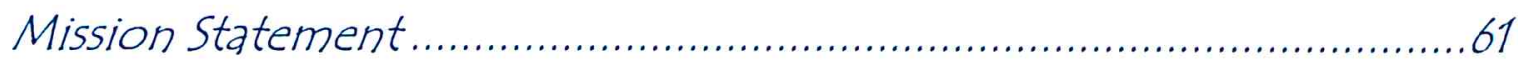

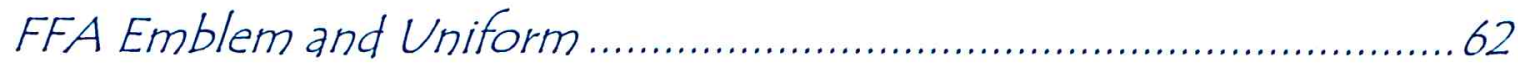

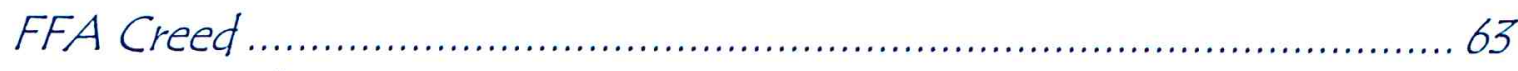

Career Development Events ..........................................................64-66

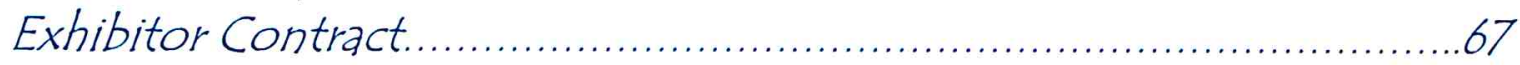

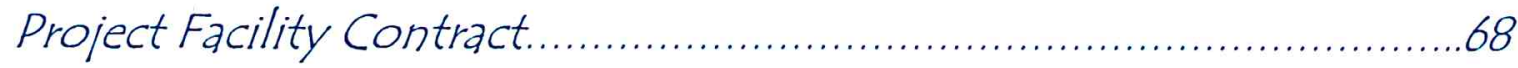

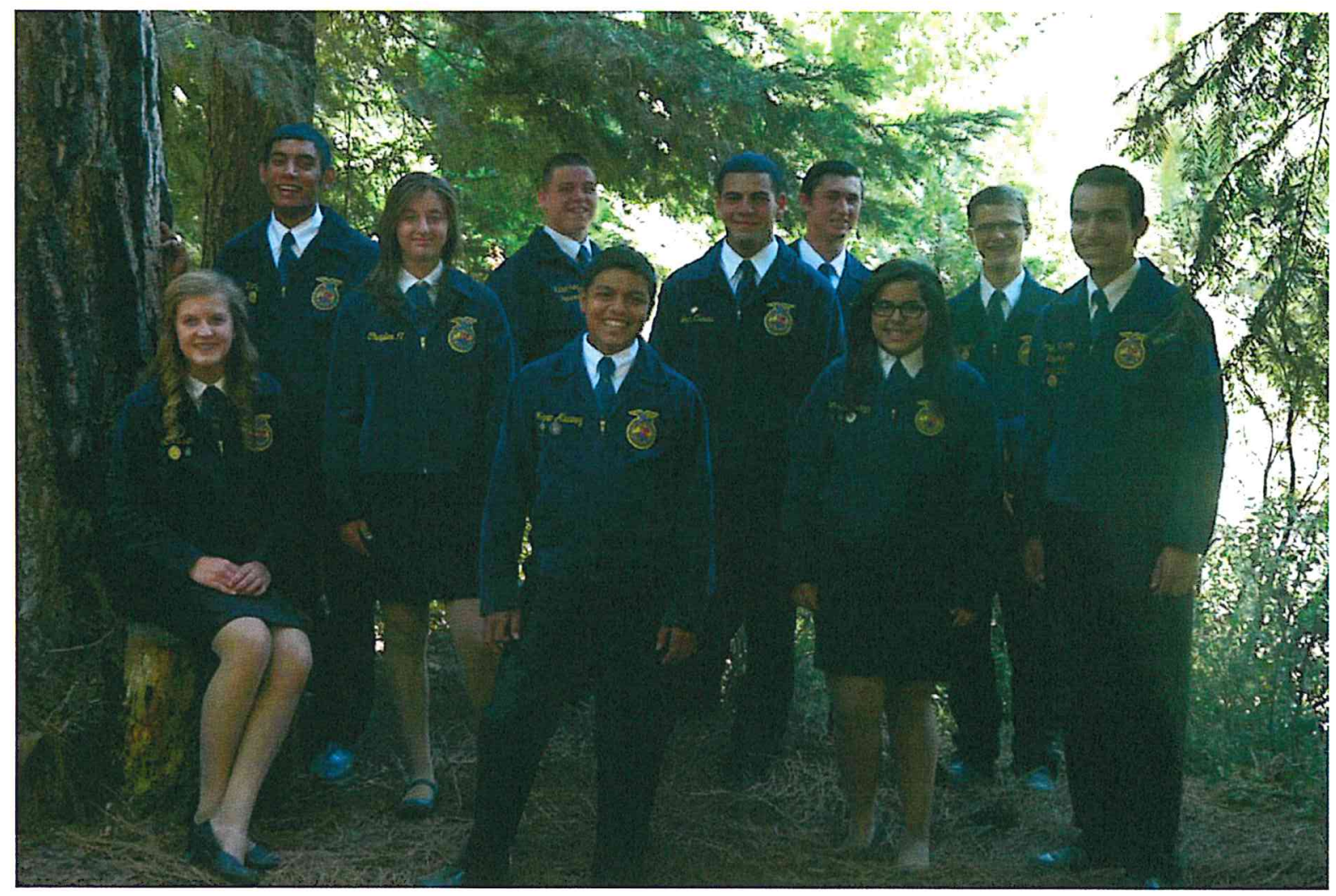




\section{Introduation EO POA}

The Program of Activities can be used as a guide, outlining the variety of activities students can become involved with. Student involvement is the key to success for a powerful agriculture program. Without member involvement all the officer teams goals and missions along with advisor guidance wouldn't accomplish much. The success of your agriculture department is almost entirely dependent upon your involvement and your desire to do the very best you can do.

In this Program of Activities you will find the rules of the chapter through our constitution, a list of all activities you can become involved in within our calendar pages, money and awards available to you and how to get them, the History of the Madera FFA Chapter dating back to its beginnings, as well as History and information about the National FFA Organization.

Keep this copy of our Program of Activities as a reference as you go on through the year and as you take advantage of all the opportunities that Madera FFA can offer you.

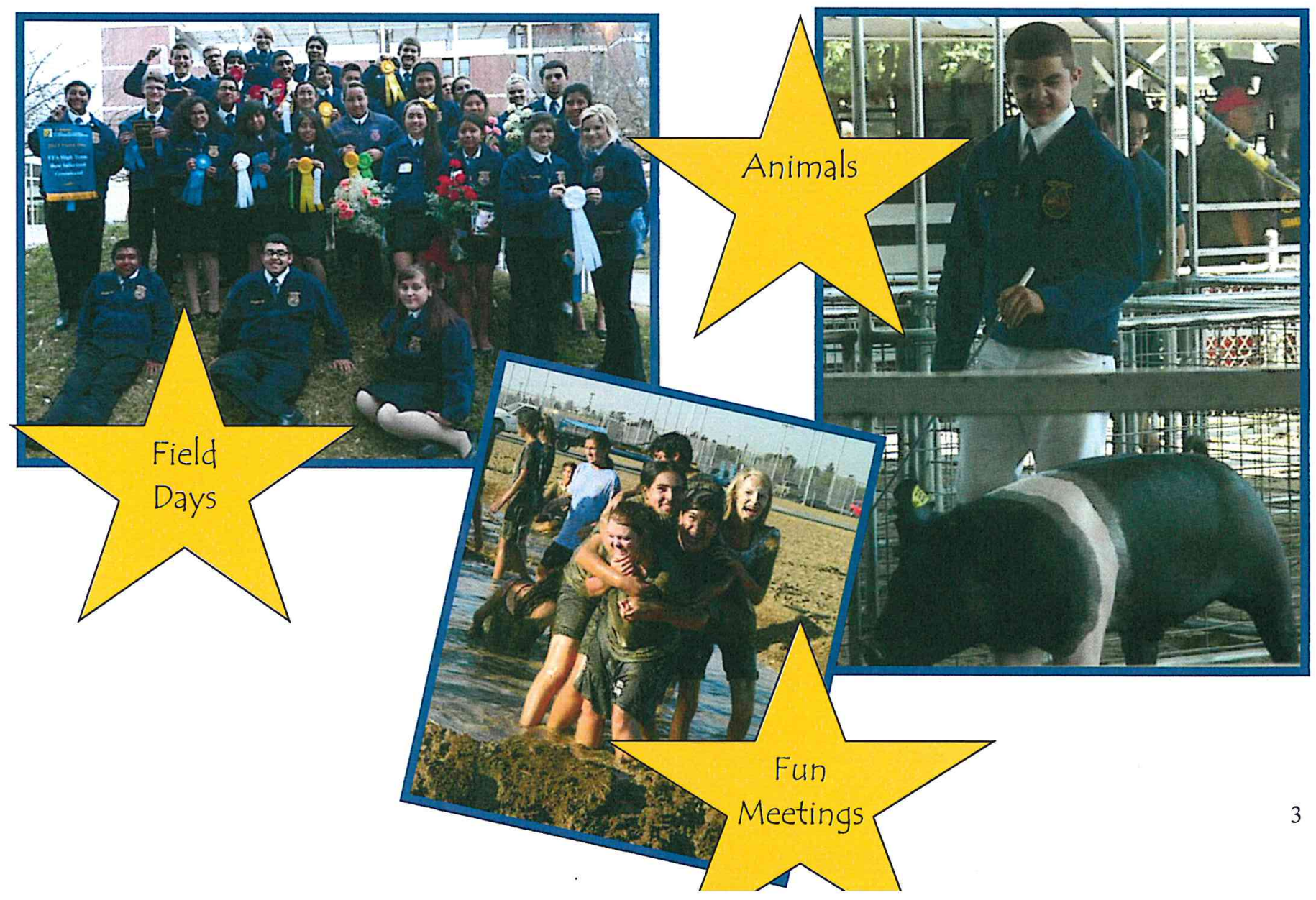




\section{Advisor's Greetings}

Welcome back to MSHS and the Madera FFA. Our advisors Mr. George, Mr. Deniz, Mr. Williams, Mrs. McKenna, Miss. Luera, Mrs. Sheehan, and Miss Gilles all agree that this is the best facility in the State of California. Our farm, classroom and laboratories are state of the art and as a student you have full access to them. It is our goal as a staff to provide our students with the greatest opportunities and learning experiences by fully utilizing the facilities we have been blessed with.

This year promises to be one of new growth and expansion. This year we will be taking a large group of students to both the Madera and Chowchilla Fairs where they will showcase their livestock, horticulture, and mechanics projects. The students and advisors have put in numerous hours of effort and hard work in expectations of an extremely successful year at both county fairs. During the year our farm facilities will be improved to include finalizing the school farm vineyard as well as general upkeep. For those of you with a stronger farming interest, there will be opportunities to plant row crops. The advisors will be looking for enthused hard working students to help develop our farm from barren land to a lush high producing operation.

As advisors we agree with the officers that individual student growth, both personally and professionally is the number one, most important element our department can offer each member. In order for this growth to occur we will provide many different opportunities for student involvement throughout the year. So once again, welcome back, and welcome to one of the most exciting years of your life. As advisors we look forward to working with each and every one of you and firmly believe that with your help we can ensure that Madera FFA remains a powerhouse agriculture program in the state of California!

Sincerely,

\section{The Madera FFA Advisors}

Mrs. McKenna

Miss Luera

Mr. Deniz

Mr. George

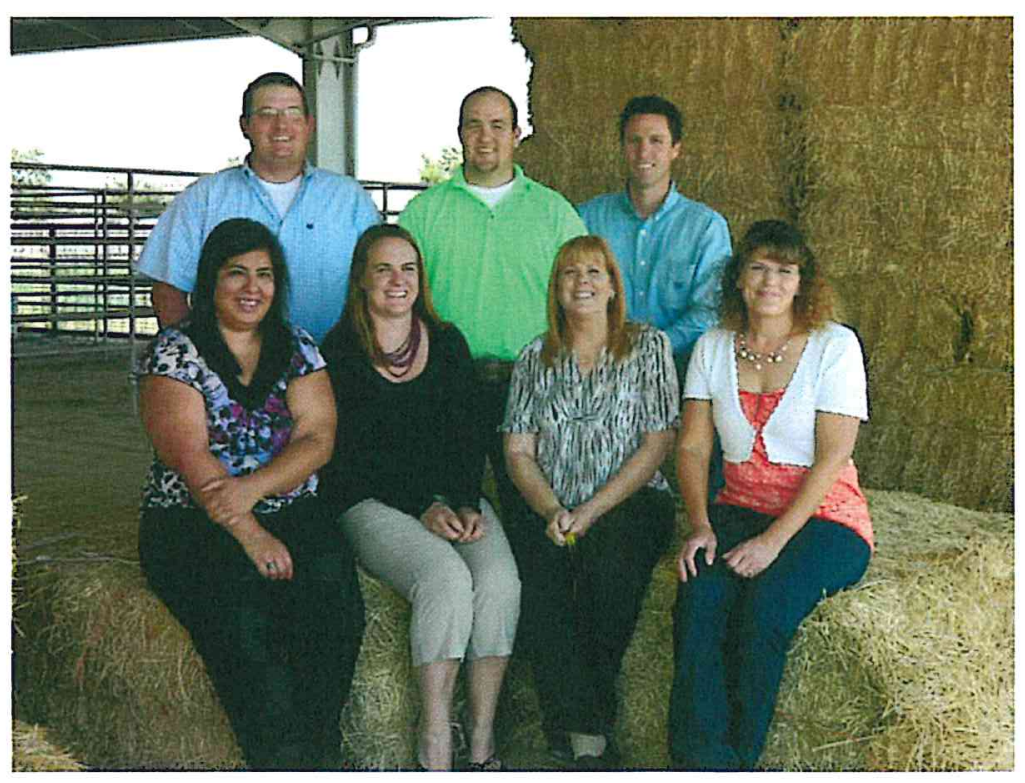




\section{Officers Message}

The Madera FFA Chapter officer team looks forward to the upcoming year in which we strive to serve our members by developing premier leadership, personal growth, and career success. through agricultural education.

\section{Madera Fa Team Goals}

- Advertise meetings by fliers, posters, and slideshows done 2 weeks prior to the meeting

- Meet at least one new member a month and keep in touch with them.

- Have presentations for meetings done 1 week before FFA meeting.

- Officers must maintain a GPA of over 3.0 and have at least a $B$ in their AG class.

\section{Madera FA Gherpter GodI}

In the upcoming year we will strive to increase chapter participation by having at least one of our monthly meetings with $40 \%$ of our membership in attendance.

\section{Madera FFA Theme}

Small acts impact beyond belief. 


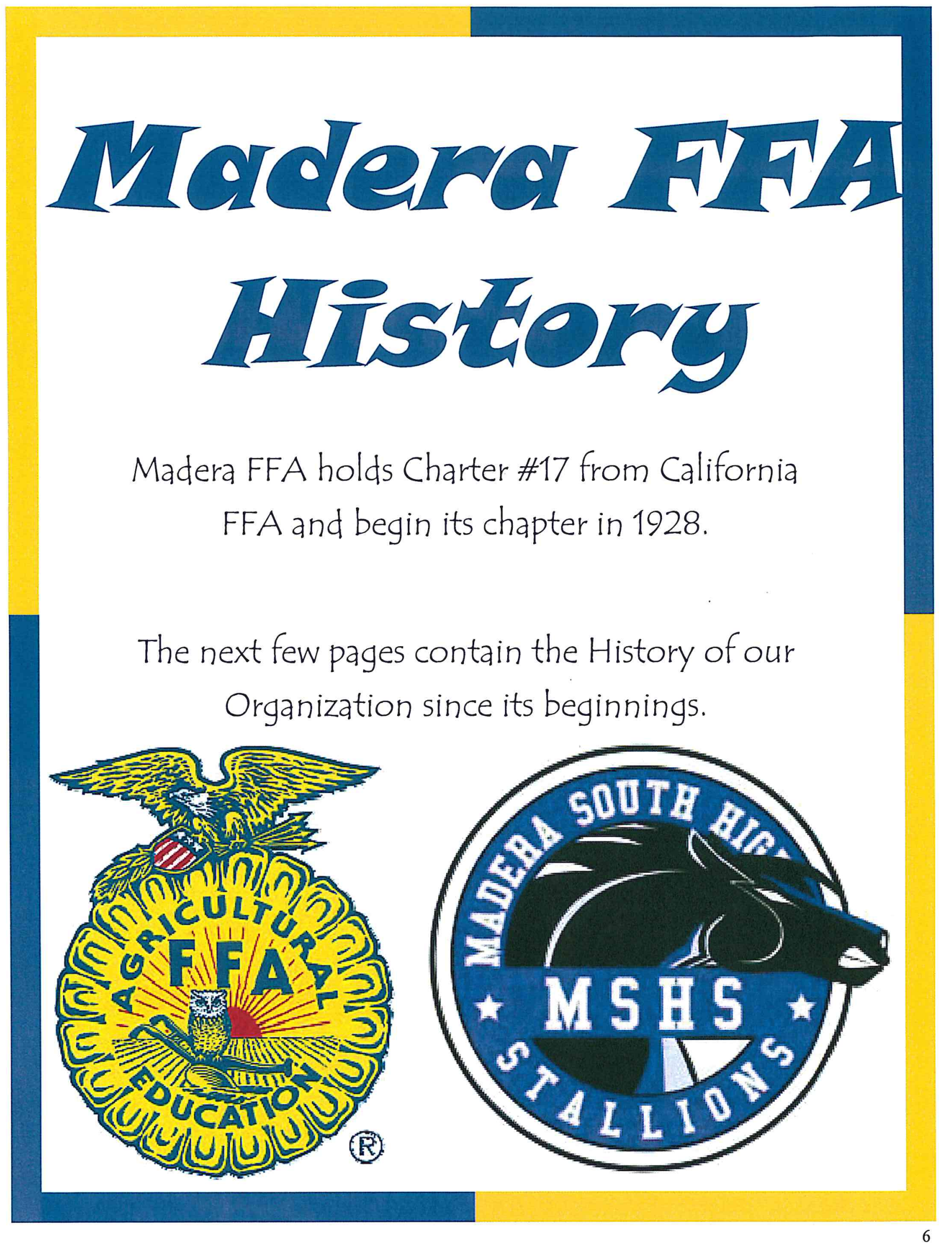




\section{Sectionerl Proficiency Winners}

\begin{tabular}{|c|c|c|}
\hline Matthew Chavira & Ag Mechanics Design/Fabrication Entrepreneur & $2005-06$ \\
\hline Enrique Hernandez & Agribusiness & $2005-06$ \\
\hline William Thornton & Diversified Crop Production & $2005-06$ \\
\hline Ectali Mendez & Floriculture Placement & $2006-07$ \\
\hline Michael Fincher & Beef Production Placement & $2006-07$ \\
\hline Cody Ogletree & Ag Mechanics Design/Fabrication Entrepreneur & $2006-07$ \\
\hline Enrique Hernandez & Agribusiness & $2008-09$ \\
\hline Enrique Hernandez & Turf Grass Management Entrepreneur & $2008-09$ \\
\hline Sierra Meyers & Beef Production & $2008-09$ \\
\hline Amy Evans & Beef Production & $2008-09$ \\
\hline Alonzo Hernandez & Diversified Livestock & $2008-09$ \\
\hline Jessica Sidney & Agriculture Education & $2008-09$ \\
\hline Ben Salazar & Agricultural Mechanics Design and Fabrication & $2009-10$ \\
\hline Amy Evans & Beef Production Entrepreneurship & $2009-10$ \\
\hline Sierra Meyers & Beef Production Placement & $2009-10$ \\
\hline Matthew Borges & Dairy Production Placement & $2009-10$ \\
\hline Gustavo Contreras & Diversified Crop Production Placement & $2009-10$ \\
\hline Caleb Hatfield & Equine Science Placement & $2009-10$ \\
\hline Magali Rodriguez & Floriculture & $2009-10$ \\
\hline Scott Thornton & Pomology Production Placement & $2009-10$ \\
\hline Lindsay Tasos & Swine Production (Entrepreneurship) & $2009-10$ \\
\hline Gabe Sanchez & Ag. Mechanics Design and Fabrication & $2010-2011$ \\
\hline Michael Valencia & Specialty Animal Production & $2010-2011$ \\
\hline Amy Evans & Beef Production Entrepreneurship & $2010-2011$ \\
\hline John McClure & Diversified Horticulture Placement & $2010-2011$ \\
\hline Lindsay Tasos & Swine Production (Entrepreneurship) & $2010-2011$ \\
\hline Magali Rodriguez & Floriculture & $2010-2011$ \\
\hline
\end{tabular}




\section{Seational Proficienay Iilnners}

\begin{tabular}{|c|c|c|}
\hline David Nino & Viticulture Placement & $2010-2011$ \\
\hline Matthew Borges & Forage & $2010-2011$ \\
\hline Michael Valencia & Ag. Services & $2012-2013$ \\
\hline Jackie Vasquez & Ag. Sales Placement & $2012-13$ \\
\hline Chris Melikian & Ag. Services & $2012-13$ \\
\hline
\end{tabular}

\section{Regional Profialenay ITinners}

\begin{tabular}{|l|l|l|}
\hline Enrique Hernandez & Turf Grass Management Entrepreneur & $2005-06$ \\
\hline Cinda Mattrocce & Diversified Livestock Production & $2005-06$ \\
\hline Warren Tucker & Grain Production & $2005-06$ \\
\hline Ectali Mendez & Floriculture Placement & $2006-07$ \\
\hline Enrique Hernandez & Turf grass Management Entrepreneur & $2008-09$ \\
\hline Matthew Chavira & Ag Sales & $2008-09$ \\
\hline Matthew Chavira & Ag Mechanics Fabrication/Design Entrepreneur & $2008-09$ \\
\hline Matthew Borges & Dairy Production Placement & $2009-10$ \\
\hline Gabriel Sanchez & Agriculture Mechanics Design/Fabrication & $2010-11$ \\
\hline Amy Evans & Beef Production Entrepreneurship & $2010-11$ \\
\hline Michael Valencia & Specialty Animal Production & $2010-11$ \\
\hline John McClure & Diversified Horticulture Placement & $2010-11$ \\
\hline Michael Valencia & Specialty Animal Production & $2011-12$ \\
\hline Michael Valencia & Ag. Services & $2012-2013$ \\
\hline
\end{tabular}

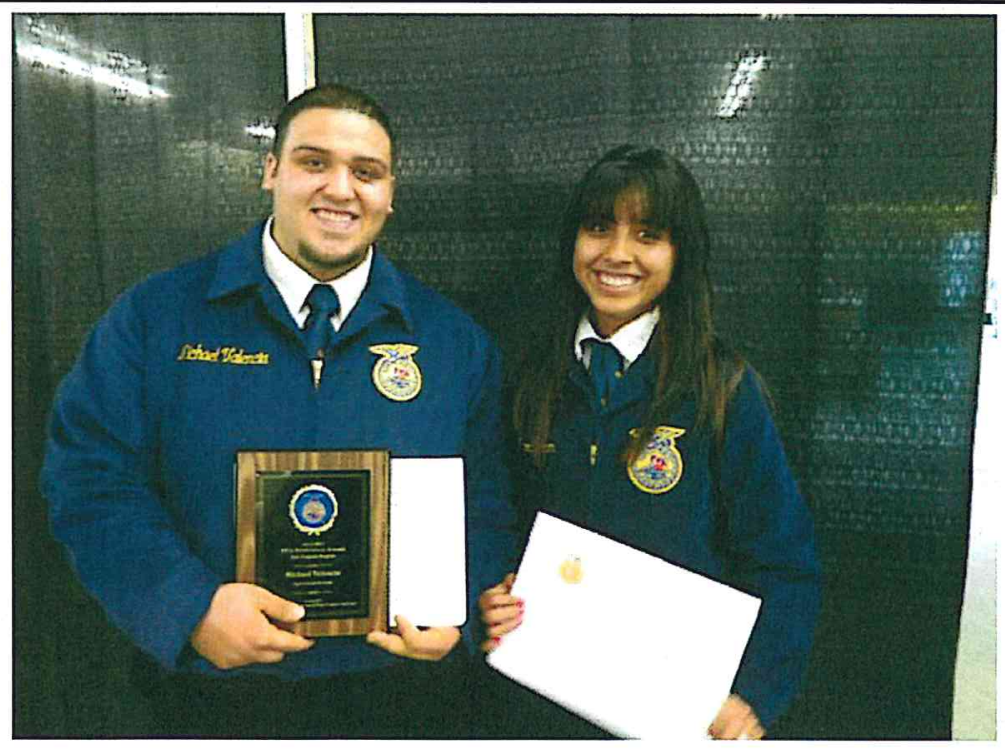




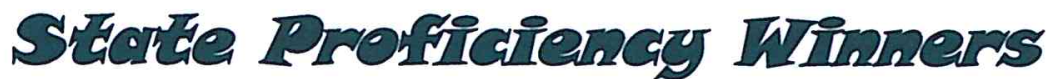

\begin{tabular}{|l|l|l|}
\hline & Farm Safety & $1954-1955$ \\
\hline & Farm Safety & $1957-1958$ \\
\hline Daryl Schlumbohm & Farm Mechanics & $1959-1960$ \\
\hline Bruno Pelanconi & Crop Production & $1960-1961$ \\
\hline Steve Ficklin & Farm and Home Electrification & $1960-1961$ \\
\hline Tommy Sesock & Farm Mechanics & $1960-1961$ \\
\hline Carl Schroeder & Farm Mechanics & $1962-1963$ \\
\hline Terrel West & Farm Mechanics & $1963-1964$ \\
\hline Steve Grant & Natural Resources & $1968-1969$ \\
\hline Douglas Anderson & Ornamental Horticulture & $1969-1970$ \\
\hline Mark Carlson & Agricultural Electrification & $1970-1971$ \\
\hline Mark Foster & Fish and Wildlife Management & $1970-1971$ \\
\hline Sandy Konkol & Placement in Processing & $1970-1971$ \\
\hline Denis Prosperi & Soil, Water and Air Management & $1970-1971$ \\
\hline Mark Lindsay & Agriculture Production & $1971-1972$ \\
\hline Conrad Bitter & Placement in Processing & $1971-1972$ \\
\hline Henry Oyler & Ag Sales and Services & $1973-1974$ \\
\hline Kevin Lee Peters & Home and Farmstead Improvement & $1976-1977$ \\
\hline Gary Agajanian & Soil, Water, and Air Management & $1976-1977$ \\
\hline Gary Agajanian & Ag Mechanics & $1977-1978$ \\
\hline John Koretoff & Soil and Water Management & $1977-1978$ \\
\hline Kurt Peters & Turfand Landscape Management & $1977-1978$ \\
\hline John Toschi & Nursery Operations & $1979-1980$ \\
\hline Bill Valorosi & Soil and Water Management & $1979-1980$ \\
\hline Bill Valorosi & Ag. Mechanics & $1980-1981$ \\
\hline Rob Hall & Floriculture & $1980-1981$ \\
\hline John Toschi & Nursery Operations & $1980-1981$ \\
\hline John Toschi & Nursery Operations & $1981-1982$ \\
\hline Kenneth Tucker & Soil and Water Management & $1981-1982$ \\
\hline Michael S. McClaran & Soil and Water Management & $1982-1983$ \\
\hline Sam Weis & Agricultural Mechanics & $1983-1984$ \\
\hline Doug Reed & Agricultural Electrification & $1985-1986$ \\
\hline Todd Fischer & Soil and Water Management & $1986-1987$ \\
\hline Kevin Bier & Agricultural Electrification & $1987-1988$ \\
\hline Barbara Turner & Horse Production & $1990-1991$ \\
\hline Sahan Van Alen & Beef Production & \\
\hline & & \\
\hline
\end{tabular}




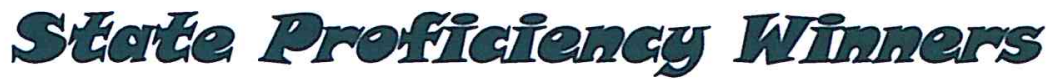

\begin{tabular}{|l|l|l|}
\hline Scott Bursey & Viticulture Production Entrepreneurship & $1997-1998$ \\
\hline Kyle Prosperi & Viticulture Production Entrepreneurship & $1999-2000$ \\
\hline Grant Tucker & Grain Production Entrepreneurship & $2003-2004$ \\
\hline Stephanie Bellew & Ag. Mechanics Energy System & $2003-2004$ \\
\hline Lauren Da Silva & Equine Science Entrepreneurship & $2005-2006$ \\
\hline Alexandria Wara & Vegetable Crop Entrepreneurship & $2005-2006$ \\
\hline Lauren DeSilva & Equine Entrepreneurship & $2005-2006$ \\
\hline Enrique Hernandez & Turf Grass Management & $2005-2006$ \\
\hline Warren Tucker & Grain Production Entrepreneurship & $2005-2006$ \\
\hline Cinda Mattrocce & Diversified Livestock Entrepreneurship & $2005-2006$ \\
\hline Enrique Hernandez & Turf Grass Management Entrepreneur & $2008-2009$ \\
\hline Matthew Borges & Dairy Production Placement & $2009-2010$ \\
\hline Michael Valencia & Specialty Animal Production & $2011-2012$ \\
\hline Michael Valencia & Ag. Services & $2012-2013$ \\
\hline
\end{tabular}

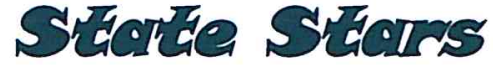

\begin{tabular}{|c|c|c|}
\hline Donald Cobb & State Star Farmer & 1940-1941 \\
\hline Stephanie Bellew & State Star Reporter & $2002-2003$ \\
\hline Stephanie Bellew & State Star Reporter & $2003-2004$ \\
\hline Bret Theodozio & State Star Administrator & $2003-2004$ \\
\hline Enrique Hernandez Jr. & State Star Agribusiness & $2009-2010$ \\
\hline Michael Valencia & State Star Agribusiness & $2011-1012$ \\
\hline
\end{tabular}

\section{Naritionall Profiaienay Itinners}

\begin{tabular}{|c|c|c|}
\hline John Sousa, Jr & Ag. Mechanics & 1983 \\
\hline Bruno Pelanconi, Jr. & Crop Production & $1961-1962$ \\
\hline Michael Valencia & Specialty Animal Production & 2012 \\
\hline
\end{tabular}




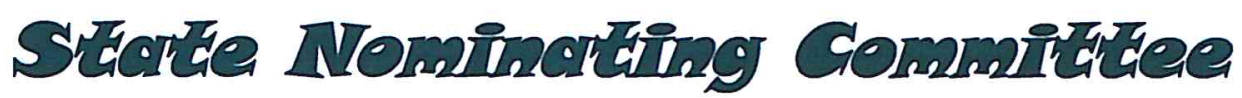

\begin{tabular}{|c|c|}
\hline Shirley Jones & $1935-1936$ \\
\hline Bill Spillane & $1938-1939$ \\
\hline Pete Laborde & $1939-1940$ \\
\hline Dino Petrucci & $1948-1949$ \\
\hline Dominic Bettini & $2009-2010$ \\
\hline Matthew Borges & $2010-2011$ \\
\hline
\end{tabular}

\section{Band, Ghoress Parritiaipantess}

\begin{tabular}{|c|c|c|}
\hline Joseph Lilles, Jr. & National Chorus & 1956-1957 \\
\hline Nicholas Lilles & State Chorus & $2000-2001$ \\
\hline Michael Croxen & State Band & $2002-2003$ \\
\hline Michael Croxen & National Band & $2011-2012$ \\
\hline Jorge Mendoza & State Band & $2011-2012$ \\
\hline Sukhvir Singh & State Band & \\
\hline
\end{tabular}

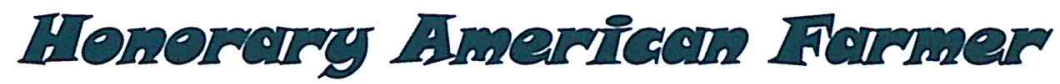

\begin{tabular}{|c|c|}
\hline Warren Smith & 1950 \\
\hline L.M. Dodd & 1951 \\
\hline Dino Petrucci & 1966 \\
\hline Jim Bomprezzi & 2007 \\
\hline
\end{tabular}




\section{Past Regional FIFA OAficiars}

\begin{tabular}{|c|c|c|}
\hline Robert Crawford & President & $1934-1935$ \\
\hline Eugene Foust & Secretary & $1936-1937$ \\
\hline Ray Thomas & President & $1941-1942$ \\
\hline Dino Petrucci & President & $1947-1948$ \\
\hline Tom Westing & Reporter & $1953-1954$ \\
\hline Joe Stasulat & Secretary & $1960-1961$ \\
\hline Dan Chatman & Reporter & $1961-1962$ \\
\hline Wallace Emmert & President & $1963-1964$ \\
\hline Ralph Pistoresi & President & $1969-1970$ \\
\hline Roger Evans & Treasurer & $1971-1972$ \\
\hline Beth Boysen & Secretary & $1972-1973$ \\
\hline Beth Boysen & Vice President & $1973-1974$ \\
\hline John Koretoff & Sentinel & $1977-1978$ \\
\hline Shana ValAlen & Vice President & $1992-1993$ \\
\hline Kyle Prosperi & Secretary & $2000-2001$ \\
\hline Megan Matteucci & Vice President & $2005-2006$ \\
\hline Zac Pruitt & Treasuer & $2006-2007$ \\
\hline Mika Petrucci & Reporter & $2007-2008$ \\
\hline Tiffany O'Haro & Vice President & $2008-2009$ \\
\hline Jessica Sidney & Treasurer & $2008-2009$ \\
\hline Dominic Bettini & Treasurer & $2009-2010$ \\
\hline Taylor Helton & Vice President & $2012-2013$ \\
\hline Virat Kang & Reporter & $2013-2014$ \\
\hline & & \\
\hline & & \\
\hline
\end{tabular}

\section{Persi ST}

\begin{tabular}{|c|c|c|}
\hline Walter Ficklin & President & $1930-1931$ \\
\hline Calvin Jones & Vice President & $1931-1932$ \\
\hline Dino Petrucci & President & $1948-1949$ \\
\hline John Deniz & President & $1949-1950$ \\
\hline William Justice & Secretary & $1952-1953$ \\
\hline Tom Westing & Reporter & $1954-1955$ \\
\hline Dan Chatman & Secretary & $1962-1963$ \\
\hline Dan Chatman & Vice President & $1963-1964$ \\
\hline Larry Hirahara & President & $1967-1968$ \\
\hline David Loquaci & Secretary & $1967-1968$ \\
\hline
\end{tabular}




\section{Past National Contrention Dellegrites}

\begin{tabular}{|c|c|}
\hline Dino Petrucci & $1947-1948$ \\
\hline Dino Petrucci & 1948-1949 \\
\hline Dino Petrucci & $1949-1950$ \\
\hline Dan Chatman & $1962-1963$ \\
\hline Dan Chatman & $1963-1964$ \\
\hline Shana Van Alen & 1991-1992 \\
\hline Michael Gomes & 1992-1993 \\
\hline Monica Williams & $1993-1994$ \\
\hline Nicole Greci & 1993-1994 \\
\hline Tommy Greci & 1994-1995 \\
\hline Lenny Edlebacher & $1995-1996$ \\
\hline Tommy Greci & $1995-1996$ \\
\hline Shane Geist & $1995-1996$ \\
\hline Lisa McKinley & 1996-1997 \\
\hline Manuel Marin & $1997-1998$ \\
\hline Stacey Visscher & $1998-1999$ \\
\hline Kyle Prosperi & 1998-1999 \\
\hline Chris Britton & $1998-1999$ \\
\hline Kyle Prosperi & $1999-2000$ \\
\hline Megan Matteucci & $2005-2006$ \\
\hline Cinda Mattrocce & $2006-2007$ \\
\hline Mika Petrucci & $2007-2008$ \\
\hline Vincent Urena & $2007-2008$ \\
\hline Jessica Sidney & $2008-2009$ \\
\hline Matthew Borges & $2010-2011$ \\
\hline Michael Valencia & $2011-2012$ \\
\hline Taylor Helton & $2011-2012$ \\
\hline Taylor Helton & $2012-2013$ \\
\hline
\end{tabular}




\section{SERE FA Degrees}

\begin{tabular}{|c|c|}
\hline Walter Ficklin & $1929-1930$ \\
\hline J.S. Davis & $1931-1932$ \\
\hline Robert Albonico & $1932-1933$ \\
\hline William Gong & $1936-1937$ \\
\hline Donald Cobb & 1940-1941 \\
\hline Avery Overgarrd & 1940-1941 \\
\hline Raymond Thomas & $1941-1942$ \\
\hline Henry Janzen & $1945-1946$ \\
\hline John Mallory & $1945-1946$ \\
\hline Roy Mallory & $1945-1946$ \\
\hline Calvin Martin & $1945-1946$ \\
\hline Nat Morris & $1945-1946$ \\
\hline Dino Petrucci & $1945-1946$ \\
\hline Paul Toschi & $1945-1946$ \\
\hline Donald Bare & 1946-1947 \\
\hline Bob Diebert & 1946-1947 \\
\hline Raymond Dolio & $1946-1947$ \\
\hline William Eua & 1946-1947 \\
\hline Richard Jensen & $1946-1947$ \\
\hline Dick Johnson & $1946-1947$ \\
\hline Gerald Montgomery & $1946-1947$ \\
\hline Travis Passmore & $1946-1947$ \\
\hline Don Tolladay & $1946-1947$ \\
\hline Aladino Unti & $1946-1947$ \\
\hline Charles Cox & $1947-1948$ \\
\hline Harvey Dane & $1947-1948$ \\
\hline Dale Evans & $1947-1948$ \\
\hline Pat Kennedy & $1947-1948$ \\
\hline Doyle Mascus & $1947-1948$ \\
\hline Earl Vanderburgh & $1947-1948$ \\
\hline Wayne Rogers & $1947-1948$ \\
\hline Travis Wisener & $1947-1948$ \\
\hline Douglas A. Wood & $1947-1948$ \\
\hline Mitsugyoshi Aoki & $1948-1949$ \\
\hline Johnny Deniz & $1948-1949$ \\
\hline Stephen Erickson & $1948-1949$ \\
\hline Don Fortune & $1948-1949$ \\
\hline Joe Galliano & 1948-1949 \\
\hline Johnny Martin & $1948-1949$ \\
\hline Ray Whitacker & $1948-1949$ \\
\hline
\end{tabular}

\begin{tabular}{|c|c|}
\hline Raymond Dorn & $1949-1950$ \\
\hline Dąniel Leach & $1949-1950$ \\
\hline Gene Lynch & $1949-1950$ \\
\hline Doyle Martin & $1949-1950$ \\
\hline Glenn Mays & $1949-1950$ \\
\hline Marvin Schmall & $1949-1950$ \\
\hline Dave Sesock & $1949-1950$ \\
\hline Frank Garner & $1950-1951$ \\
\hline Ronald Gruenwald & $1950-1951$ \\
\hline William Jantzen & $1950-1951$ \\
\hline Carl Simmons, Jr & $1950-1951$ \\
\hline Rocky Valorosi & $1950-1951$ \\
\hline Nello L. Bomprezzi & $1951-1952$ \\
\hline Duane L. Garner & $1951-1952$ \\
\hline Rudolph Gutierrez & $1951-1952$ \\
\hline William A. Justice & $1951-1952$ \\
\hline Michael S. Simmons & $1951-1952$ \\
\hline Leon LaMattina & $1952-1953$ \\
\hline Tommy Westing & $1952-1953$ \\
\hline Cliff Davis & $1953-1954$ \\
\hline Tommy Kenefick & $1953-1954$ \\
\hline Mike Ylarregui & $1953-1954$ \\
\hline Harold Ashton & $1955-1956$ \\
\hline Casey Campbell & $1955-1956$ \\
\hline William Dickey & $1955-1956$ \\
\hline Paul Mąrtines & $1955-1956$ \\
\hline Charles Mays & $1955-1956$ \\
\hline Jerry Siebert & $1955-1956$ \\
\hline Mike Allred & $1957-1958$ \\
\hline Melvin Aoki & $1957-1958$ \\
\hline Richard Dolio & $1957-1958$ \\
\hline Larry Jantzen & $1957-1958$ \\
\hline Ken Seibert & $1957-1958$ \\
\hline Ronald Smith & $1957-1958$ \\
\hline Robert Tate & $1957-1958$ \\
\hline Richard Williąms & $1957-1958$ \\
\hline
\end{tabular}


SErEe FA Degrees Contilnued

\begin{tabular}{|c|c|}
\hline Bob Prosperi & $1957-1958$ \\
\hline Wakao Aoki & $1958-1959$ \\
\hline James Chandler & $1958-1959$ \\
\hline Louis Contreras & $1958-1959$ \\
\hline Harold Giomi & $1958-1959$ \\
\hline Carl Janzen & $1958-1959$ \\
\hline Daryl Chlumbohm & $1958-1959$ \\
\hline John Stasulat & $1958-1959$ \\
\hline Bruno Pelanconi Ir, & $1959-1960$ \\
\hline Robert Houlding & $1960-1961$ \\
\hline Robert Saulsbury & $1960-1961$ \\
\hline Harvey Aoki & $1961-1962$ \\
\hline Dan Chatman & $1961-1962$ \\
\hline Terry Cheek & $1961-1962$ \\
\hline Stan Hirahara & $1961-1962$ \\
\hline Dick McCollister & $1961-1962$ \\
\hline Robert McCollister & $1961-1962$ \\
\hline Carl Schroeder & $1961-1962$ \\
\hline Don Sellai & $1961-1962$ \\
\hline Richard E. Smith & $1961-1962$ \\
\hline Bobby Tate & $1961-1962$ \\
\hline Joe Camarillo & $1962-1963$ \\
\hline George Crafton & $1962-1963$ \\
\hline Wallace Emmert & $1962-1963$ \\
\hline Larry King & $1962-1963$ \\
\hline Frank Massetti & $1962-1963$ \\
\hline Marcell Monticello & $1962-1963$ \\
\hline Steve Sampaulesi & $1962-1963$ \\
\hline Doug Sordi & $1962-1963$ \\
\hline Steve Tomachoff & $1962-1963$ \\
\hline Terrel West & $1962-1963$ \\
\hline Phil Albonico & $1963-1964$ \\
\hline David Giomi & $1963-1964$ \\
\hline Steve Gist & $1963-1964$ \\
\hline Evert Plumb & $1963-1964$ \\
\hline Greg Desmond & $1964-1965$ \\
\hline Don Weins & $1964-1965$ \\
\hline Chester Andrew & $1965-1966$ \\
\hline Kenneth Aoki & $1965-1966$ \\
\hline
\end{tabular}

\begin{tabular}{|c|c|}
\hline Larry Hirahara & \multicolumn{1}{|c}{$1965-1966$} \\
\hline Roger Leach & $1965-1966$ \\
\hline David Loquaci & $1965-1966$ \\
\hline Victor Sahatdiian & $1965-1966$ \\
\hline Ray Seibert & $1965-1966$ \\
\hline Albert Lam & $1966-1967$ \\
\hline Norman Lincoln & $1966-1967$ \\
\hline Gary Bursery & $1967-1968$ \\
\hline Ronald Kelley & $1967-1968$ \\
\hline Walter Nelson & $1967-1968$ \\
\hline Ronald Pistoresi & $1967-1968$ \\
\hline Robert Rubottom & $1967-1968$ \\
\hline John Bese & $1968-1969$ \\
\hline Michael Camarillo & $1968-1969$ \\
\hline Lester Eddy & $1968-1969$ \\
\hline Mike Elliot & $1968-1969$ \\
\hline Paul Ely & $1968-1969$ \\
\hline Steve Emmert & $1968-1969$ \\
\hline Steve Grant & $1968-1969$ \\
\hline Dan Johnson & $1968-1969$ \\
\hline Gifford Johnson & $1968-1969$ \\
\hline Leslie Loquaci & $1968-1969$ \\
\hline Larry Moore & $1968-1969$ \\
\hline James Osterman & $1968-1969$ \\
\hline Frank Morgan & $1968-1969$ \\
\hline Ralph Pistoresi & $1968-1969$ \\
\hline Dan Prosperi & $1968-1969$ \\
\hline John Simpson & $1968-1969$ \\
\hline Mark Carlson & $1969-1970$ \\
\hline Randall Chase & $1969-1970$ \\
\hline Jeff Coulthard & $1969-1970$ \\
\hline Pat Kirby & $1969-1970$ \\
\hline Eddie Martinazzi & $1969-1970$ \\
\hline Jerry Payne & $1969-1970$ \\
\hline Jim Pistoresi & $1969-1970$ \\
\hline Denis Prosperi & $1969-1970$ \\
\hline Douglas Row & $1969-1970$ \\
\hline Conrad Bitter & $1970-1971$ \\
\hline Roger Evans & $1970-1971$ \\
\hline & \\
\hline &
\end{tabular}


State FFA Degrees Continued

\begin{tabular}{|c|c|}
\hline Donald Parkey & 1970-1971 \\
\hline Randy Belflower & $1971-1972$ \\
\hline Bob Creamer & $1971-1972$ \\
\hline Gene Ferretti & $1971-1972$ \\
\hline Sandy Konkol & $1971-1972$ \\
\hline Rick Osterman & $1971-1972$ \\
\hline Robert Simpson & $1971-1972$ \\
\hline Robery Bishel & $1972-1973$ \\
\hline Betsy Boysen & $1972-1973$ \\
\hline Mark Freeman & $1972-1973$ \\
\hline Randy Freeman & $1972-1973$ \\
\hline David Galleano & $1972-1973$ \\
\hline Rick Logoluso & $1972-1973$ \\
\hline LeRoy Marklund & $1972-1973$ \\
\hline Sandie McDonald & $1972-1973$ \\
\hline Linda Galleano & $1973-1974$ \\
\hline Jeff Joines & $1973-1974$ \\
\hline Henry Oyler & $1973-1974$ \\
\hline Stephen Schafer & $1973-1974$ \\
\hline Marilyn Whiton & $1973-1974$ \\
\hline James Cavallero & $1975-1976$ \\
\hline Henry Contreras & $1975-1976$ \\
\hline Luanna James & $1975-1976$ \\
\hline Brad McDonald & $1975-1976$ \\
\hline Vince Petrucci & $1975-1976$ \\
\hline Mike Schafer & $1975-1976$ \\
\hline Greg Agajanian & $1976-1977$ \\
\hline John Koretoff & $1976-1977$ \\
\hline Kevin Peters & $1976-1977$ \\
\hline Kurt Peters & $1976-1977$ \\
\hline Cheryl Schafer & $1976-1977$ \\
\hline Randall Armstrong & $1977-1978$ \\
\hline John Arnold & $1977-1978$ \\
\hline Darrel Bishel & 1977-1978 \\
\hline Mark Doig & $1977-1978$ \\
\hline Rusty Jensen & $1977-1978$ \\
\hline Barbar Keller & $1977-1978$ \\
\hline Kevin Mercer & $1977-1978$ \\
\hline Kevin Richardson & $1977-1978$ \\
\hline Robyn Harper & 1978-1979 \\
\hline
\end{tabular}

\begin{tabular}{|c|c|}
\hline Bill Anderson & $1979-1980$ \\
\hline Lorna Gunter & $1979-1980$ \\
\hline Lisa Peterson & $1979-1980$ \\
\hline Karla Stockli & $1979-1980$ \\
\hline Bob Hall & $1981-1982$ \\
\hline Sam Weis & $1982-1983$ \\
\hline Edward Correa & $1983-1984$ \\
\hline Kurt Fick & $1983-1984$ \\
\hline Phillip Montagna & $1983-1984$ \\
\hline Raymond Montagna & $1983-1984$ \\
\hline John Gray & $1984-1985$ \\
\hline Kevin Miles & $1984-1985$ \\
\hline Doug Reed & $1984-1985$ \\
\hline Jim Bell & $1985-1986$ \\
\hline Rhonda Long & $1985-1986$ \\
\hline Derek Sambueso & $1985-1986$ \\
\hline Brian Cox & $1986-1987$ \\
\hline Howard Eledge & $1986-1987$ \\
\hline Todd Fischer & $1986-1987$ \\
\hline Erin McCracken & $1986-1987$ \\
\hline Jeff Miles & $1986-1987$ \\
\hline Roy Morris & $1986-1987$ \\
\hline Heather Parks & 1986-1987 \\
\hline Cathy Trautman & $1986-1987$ \\
\hline Kevin Bier & $1987-1988$ \\
\hline Mike Ervin & $1987-1988$ \\
\hline Miguel, A. Flores & $1987-1988$ \\
\hline Jeffrey Pacini & $1987-1988$ \\
\hline Suzanne Stretch & $1987-1988$ \\
\hline Terry Brand & $1988-1989$ \\
\hline Scott Jackson & $1988-1989$ \\
\hline Shanon Blackmore & 1990 \\
\hline Diane Hench & 1990 \\
\hline Shawn Johnson & 1990 \\
\hline Craig Waag & 1990 \\
\hline Kris Garzone & 1992 \\
\hline Angelo Gomes & 1992 \\
\hline Javier Guerra & 1992 \\
\hline Sherry Lee & 1992 \\
\hline
\end{tabular}




\section{SErEe FA Degrees Continued}

\begin{tabular}{|c|c|}
\hline Shawn Moore & 1992 \\
\hline Frank Reddell & 1992 \\
\hline Shana VanAlen & 1992 \\
\hline Kris Detjen & 1993 \\
\hline Alana Cervantes & 1993 \\
\hline Michael Gomes & 1993 \\
\hline LeAnn McPeters & 1993 \\
\hline Daniel Ogan & 1993 \\
\hline Steven Sesock & 1993 \\
\hline Timothy Carter & 1994 \\
\hline Jodi Hibdon & 1994 \\
\hline Carson Farino & 1994 \\
\hline Bryon Jones & 1994 \\
\hline Brian Fitzgerald & 1994 \\
\hline Stephanie Garzone & 1994 \\
\hline Ryan Logoluso & 1994 \\
\hline Travis Harris & 1994 \\
\hline Stacy Redding & 1994 \\
\hline Monica Williams & 1994 \\
\hline Alan Deniz & 1995 \\
\hline Mark Kazynski & 1995 \\
\hline Mitch Robinson & 1995 \\
\hline Daniel Sesock & 1995 \\
\hline Paulette Sesock & 1995 \\
\hline Deanna Ogan & 1995 \\
\hline Andrew Perreira & 1995 \\
\hline Bryan Perreira & 1995 \\
\hline Steven Clement & 1996 \\
\hline Shane Geist & 1996 \\
\hline Frank Lourenco & 1996 \\
\hline Jeff Perreira & 1996 \\
\hline Kathy Sesock & 1996 \\
\hline Tyler Berry & 1996 \\
\hline Jenny Edelbacher & 1996 \\
\hline Leon Prichard & 1996 \\
\hline
\end{tabular}

\begin{tabular}{|c|c|}
\hline Matt Redding & 1996 \\
\hline Melissa Redding & 1996 \\
\hline Julie Hallam & 1997 \\
\hline Colleen Miller & 1997 \\
\hline Heather Todisco & 1997 \\
\hline Lisa McKinley & 1997 \\
\hline Lisa McPeters & 1997 \\
\hline Mątt Beechinor & 1998 \\
\hline Scott Bursey & 1998 \\
\hline Stacy Cook & 1998 \\
\hline Renee Crawford & 1998 \\
\hline Keri Jackson & 1998 \\
\hline Robbie Loquaci & 1998 \\
\hline Manuel Marin & 1998 \\
\hline Jeffrey Riddle & 1998 \\
\hline Susan Riddle & 1998 \\
\hline Steven Rodríguez & 1998 \\
\hline Alyson Seibert & 1998 \\
\hline Sarah Stutler & 1998 \\
\hline Shawn Liles & 1999 \\
\hline Steve Calderon & 1999 \\
\hline Bryan Rodriguez & 1999 \\
\hline Shanna Rodriguez & 1999 \\
\hline Rene Gonzalez & 1999 \\
\hline Jennifer Tune & 1999 \\
\hline Stacey Visscher & 1999 \\
\hline Jason Erickson & 2000 \\
\hline Audrey Estabrook & 2000 \\
\hline Antionette Francher & 2000 \\
\hline Andrea Hench & 2000 \\
\hline Laura Del Bianco & 2001 \\
\hline Erick Buckley & 2001 \\
\hline Jesse Croxen & 2001 \\
\hline Robert Fahey & 2001 \\
\hline Cassie Mckienly & 2001 \\
\hline
\end{tabular}




\section{SEate FA Degrees Continued}

\begin{tabular}{|c|c|}
\hline Jessica Miller & 2001 \\
\hline Colin Rock & 2001 \\
\hline Loriann Sesock & 2001 \\
\hline Jason Wara & 2001 \\
\hline Alyson Padgett & 2002 \\
\hline Trevor Meyers & 2002 \\
\hline Monica Medina & 2002 \\
\hline Jennifer Mansell & 2002 \\
\hline Brian Schafer & 2002 \\
\hline Brandon Visscher & 2002 \\
\hline Kevin Willet & 2002 \\
\hline Stephanie Bellew & 2003 \\
\hline Donald Doyle & 2003 \\
\hline Lee Erickson & 2003 \\
\hline Resse Fahey & 2003 \\
\hline Amanda Hallam & 2003 \\
\hline Garret Mattrocce & 2003 \\
\hline Giąna Toschi & 2003 \\
\hline Cody Waltz & 2003 \\
\hline Garth Wara & 2003 \\
\hline Michael Croxen & 2004 \\
\hline Adam Pistoresi & 2004 \\
\hline Melissa Alley & 2005 \\
\hline Jessica Alcorn & 2005 \\
\hline Howard Beach & 2005 \\
\hline Amy Bonander & 2005 \\
\hline Gabriel Garcia & 2005 \\
\hline Landon Gill & 2005 \\
\hline Cory Padgett & 2005 \\
\hline Megan Mettucci & 2005 \\
\hline Brett Martinazzi & 2005 \\
\hline Christopher Rippee & 2005 \\
\hline Andrea Bartley & 2006 \\
\hline Margarito Cervantes & 2006 \\
\hline Thomas Lovelady & 2006 \\
\hline
\end{tabular}

\begin{tabular}{|c|c|}
\hline Cinda Mattrocce & 2006 \\
\hline Jeffrey Moosios & 2006 \\
\hline Cody Ogletree & 2006 \\
\hline Steve Pistoresi & 2006 \\
\hline William Thornton & 2006 \\
\hline Warren Tucker & 2006 \\
\hline Alexandria Wara & 2006 \\
\hline Kendra Willet & 2006 \\
\hline Jose Farias & 2007 \\
\hline Michael Fincher & 2007 \\
\hline Glen Gil & 2007 \\
\hline Kaysy Hopson & 2007 \\
\hline Jacob Maggiore & 2007 \\
\hline Mika Petrucci & 2007 \\
\hline Bobby Sholler & 2007 \\
\hline Anthony Tates & 2007 \\
\hline Vincent Urena & 2007 \\
\hline Victoria Barros & 2008 \\
\hline Matthew Chavira & 2008 \\
\hline Alonzo hernandez & 2008 \\
\hline Tori Isaac & 2008 \\
\hline Hector lopez & 2008 \\
\hline Ritchie Lopez & 2008 \\
\hline Tiffany O'haro & 2008 \\
\hline Rodolfo Pineda & 2008 \\
\hline Rąmiro Sanchez & 2008 \\
\hline Jessica Sidney & 2008 \\
\hline Nicole Sidney & 2008 \\
\hline Dominic Bettini & 2009 \\
\hline Megan Christiansen & 2009 \\
\hline Caleb Hatfield & 2009 \\
\hline Enrique Hernandez & 2009 \\
\hline Melissa Hubbard & 2009 \\
\hline Riley Lovelady & 2009 \\
\hline Sierra Meyers & 2009 \\
\hline
\end{tabular}




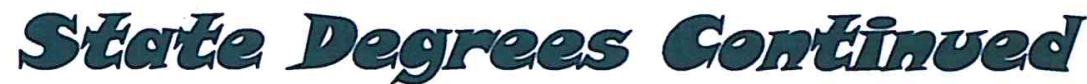

\begin{tabular}{|c|c|}
\hline Wesley Ogletree & 2009 \\
\hline Gabriel Sanchez & 2009 \\
\hline Scott Thornton & 2009 \\
\hline Henry Bales & 2010 \\
\hline Taylor Bese & 2010 \\
\hline Matthew Borges & 2010 \\
\hline Veronica Cervantes & 2010 \\
\hline Gustavo Contreras & 2010 \\
\hline Jerry Cook & 2010 \\
\hline Jonathan Costa & 2010 \\
\hline Santiago DeLaCruz & 2010 \\
\hline Spencer Deniz & 2010 \\
\hline Amy Evans & 2010 \\
\hline Whitney Laymon & 2010 \\
\hline Jordan Lyons & 2010 \\
\hline Alejandro Madrigal & 2010 \\
\hline John McClure & 2010 \\
\hline Benjamin Salazar & 2010 \\
\hline Shannon Sumpter & 2010 \\
\hline Adam Taylor & 2010 \\
\hline Cody Ward & 2010 \\
\hline Shelby Caraway & 2011 \\
\hline Matthew Cavallero & 2011 \\
\hline Matthew Cavaletto & 2011 \\
\hline Leo Cervantes & 2011 \\
\hline Amy Dierberger & 2011 \\
\hline Jorge Garcia & 2011 \\
\hline Luis Mancillas & 2011 \\
\hline Shelby Moit & 2011 \\
\hline David Nino & 2011 \\
\hline Norrin Pecarrovich & 2011 \\
\hline
\end{tabular}

\begin{tabular}{|c|c|}
\hline Magali Rodriguez & 2011 \\
\hline Santiago Santos & 2011 \\
\hline Lindsay Tasos & 2011 \\
\hline Alex Teran & 2011 \\
\hline Jessica Trembley & 2011 \\
\hline Michael Valencia & 2011 \\
\hline Alexis Aguilar & 2012 \\
\hline Rochelle Brewer & 2012 \\
\hline Scott Bullis & 2012 \\
\hline Jessica Davila & 2012 \\
\hline Emily Fernandez & 2012 \\
\hline Dalice Garcia & 2012 \\
\hline Molly Gilbert & 2012 \\
\hline Kasey Griffin & 2012 \\
\hline Taylor Helton & 2012 \\
\hline Carla Johnson & 2012 \\
\hline Luz Lopez & 2012 \\
\hline Chris Melikian & 2012 \\
\hline Jevan Grewal & 2012 \\
\hline Crystal Bazante & 2013 \\
\hline Jimmy Beavers & 2013 \\
\hline Jaime Cuevas & 2013 \\
\hline Vanessa Duarte & 2013 \\
\hline Brianna Gagliardi & 2013 \\
\hline Rostia Galindo & 2013 \\
\hline Marcelina Gonzalez & 2013 \\
\hline Allison Helton & 2013 \\
\hline Alexus Hernandez & 2013 \\
\hline Cody Knott & 2013 \\
\hline Kayla Melikian & 2013 \\
\hline Jacqueline Morales & 2013 \\
\hline
\end{tabular}




\section{Stiterte Degrees Continued}

\begin{tabular}{|c|c|}
\hline Dominique Ortega & 2013 \\
\hline Jamie Oyler & 2013 \\
\hline Eduardo Rodriguez & 2013 \\
\hline Joalex Sanchez & 2013 \\
\hline Sukhvir Singh & 2013 \\
\hline Spenser Smith & 2013 \\
\hline Whitney Swengel & 2013 \\
\hline Hailey Wilberg & 2013 \\
\hline
\end{tabular}
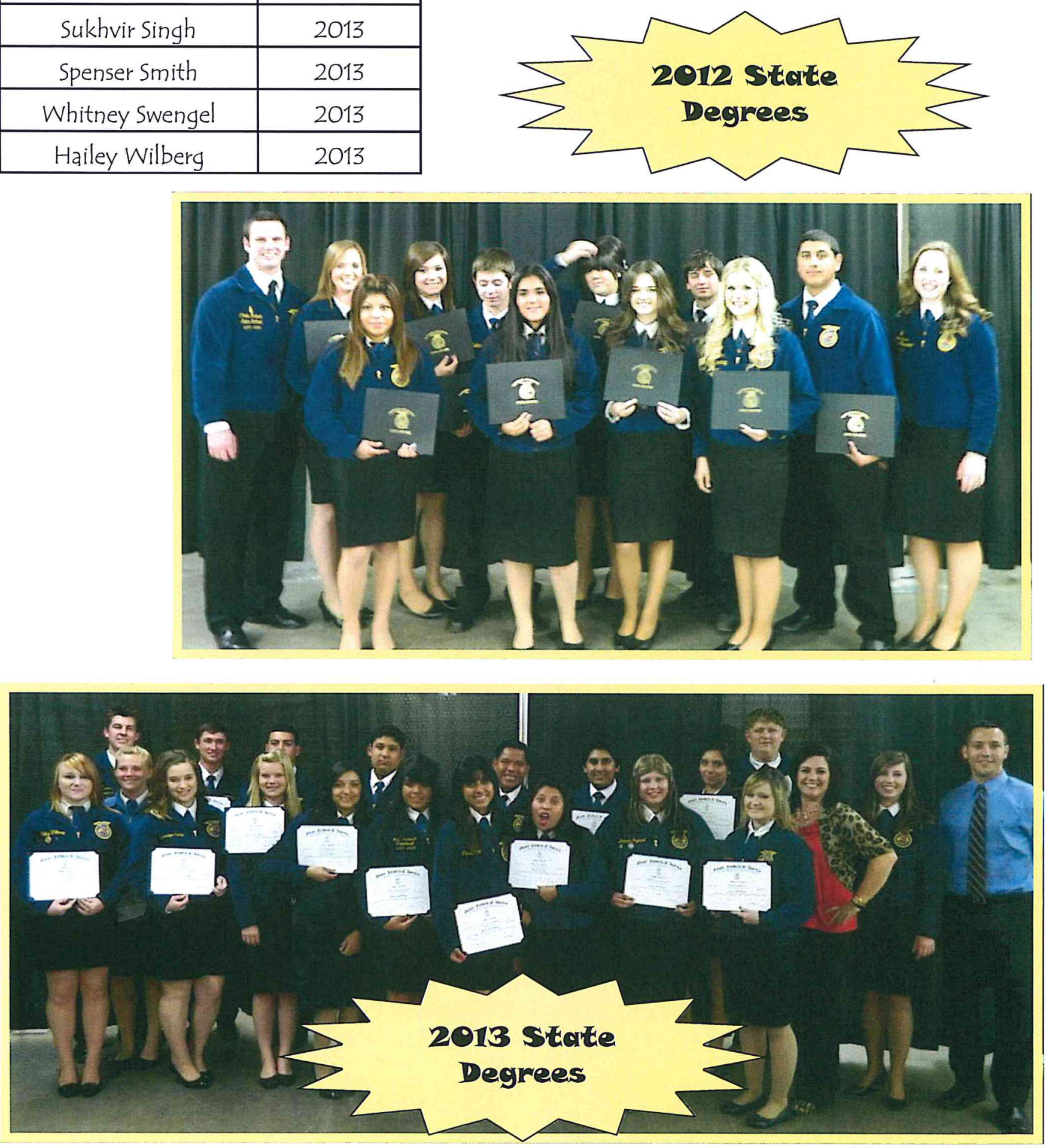


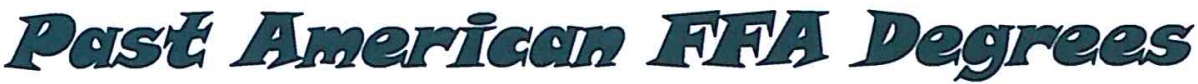

\begin{tabular}{|c|c|}
\hline Raymond Thomas & $1945-1946$ \\
\hline Dino Petrucci & $1948-1949$ \\
\hline Johnny Deniz & $1951-1952$ \\
\hline Robert Prosperi & $1960-1961$ \\
\hline Joe Stasulat & $1962-1963$ \\
\hline Larry Hirahara & $1968-1969$ \\
\hline Roger Leach & $1968-1969$ \\
\hline Gary Lee Agajanian & $1978-1979$ \\
\hline John Koretoff & $1978-1979$ \\
\hline Karla J. Stockli & 1984-1985 \\
\hline Samuel K. Weis & $1984-1985$ \\
\hline Angela Gomes & 1993 \\
\hline Carson Farino & 1995 \\
\hline Brian Fitzgerald & 1995 \\
\hline Michael Gomes & 1995 \\
\hline Ryan Logoluso & 1995 \\
\hline LeAnn Iva McPeters & 1995 \\
\hline Steven Sesock & 1995 \\
\hline Shana Van Alen & 1995 \\
\hline Nicole Greci & 1996 \\
\hline Monica Denise Williams & 1996 \\
\hline Shane Geist & 1999 \\
\hline Tomas Greci & 1999 \\
\hline Brandon Rodriquez & 1999 \\
\hline Kathy Sesock & 1999 \\
\hline Paulette Sesock & 1999 \\
\hline Matt Beechinor & 2000 \\
\hline Matt Redding & 2000 \\
\hline Lisa McPeters & 2000 \\
\hline Steve Rodriquez & 2000 \\
\hline Julie Hallum & 2001 \\
\hline
\end{tabular}

\begin{tabular}{|c|c|}
\hline Robbie Loquaci & 2001 \\
\hline Manuel Marin & 2001 \\
\hline Lisa McKinley & 2001 \\
\hline Alyson Seibert & 2001 \\
\hline Jason Erickson & 2002 \\
\hline Kyle Prosperi & 2002 \\
\hline Brian Davis & 2003 \\
\hline Nick Davis & 2003 \\
\hline Laura McGee & 2003 \\
\hline Loriann Sesock & 2003 \\
\hline Jason Wara & 2003 \\
\hline Robert Fahey & 2004 \\
\hline Casandra McKinley & 2004 \\
\hline Jessica Miller & 2004 \\
\hline Michael Croxen & 2005 \\
\hline Lee Erickson & 2005 \\
\hline Amanda Hallum & 2005 \\
\hline Garrett Mattrocce & 2005 \\
\hline Brian Schafer & 2005 \\
\hline Cody Waltz & 2005 \\
\hline Garth Wara & 2005 \\
\hline Megan Matteucci & 2007 \\
\hline Melissa Alley & 2007 \\
\hline Cory Padgett & 2007 \\
\hline Jessica Alcorn & 2007 \\
\hline Steve Pistoresi & 2007 \\
\hline Brett Martinazzi & 2007 \\
\hline Ross Dellaualle & 2007 \\
\hline Thomas Lovelady & 2008 \\
\hline Christopher Rippee & 2008 \\
\hline Kendra Willet & 2008 \\
\hline Alex Wara & 2008 \\
\hline
\end{tabular}




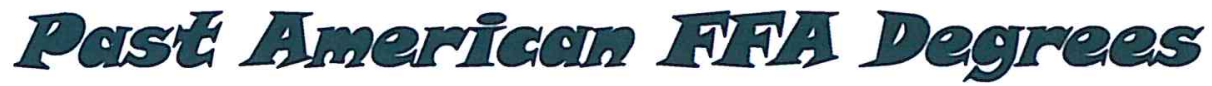

\section{Corratined}

\begin{tabular}{|c|c|}
\hline Cinda Mattrocce & 2008 \\
\hline Glen Gil & 2009 \\
\hline William Thornton & 2009 \\
\hline Michael Fincher & 2009 \\
\hline Mika Petrucci & 2009 \\
\hline Matthew Chavira & 2009 \\
\hline Margarito Cervantes & 2009 \\
\hline Anthony Tates & 2009 \\
\hline Enrique Hernandez & 2010 \\
\hline Alonzo Espinoza & 2010 \\
\hline Brittany Cavaletto & 2011 \\
\hline Gabriel Sanchez & 2011 \\
\hline Dominic Bettini & 2011 \\
\hline Sierra Meyers & 2011 \\
\hline Matthew Borges & 2012 \\
\hline John McClure & 2012 \\
\hline David Nino & 2012 \\
\hline Scott Thornton & 2012 \\
\hline Shannon Sumpter & 2012 \\
\hline
\end{tabular}
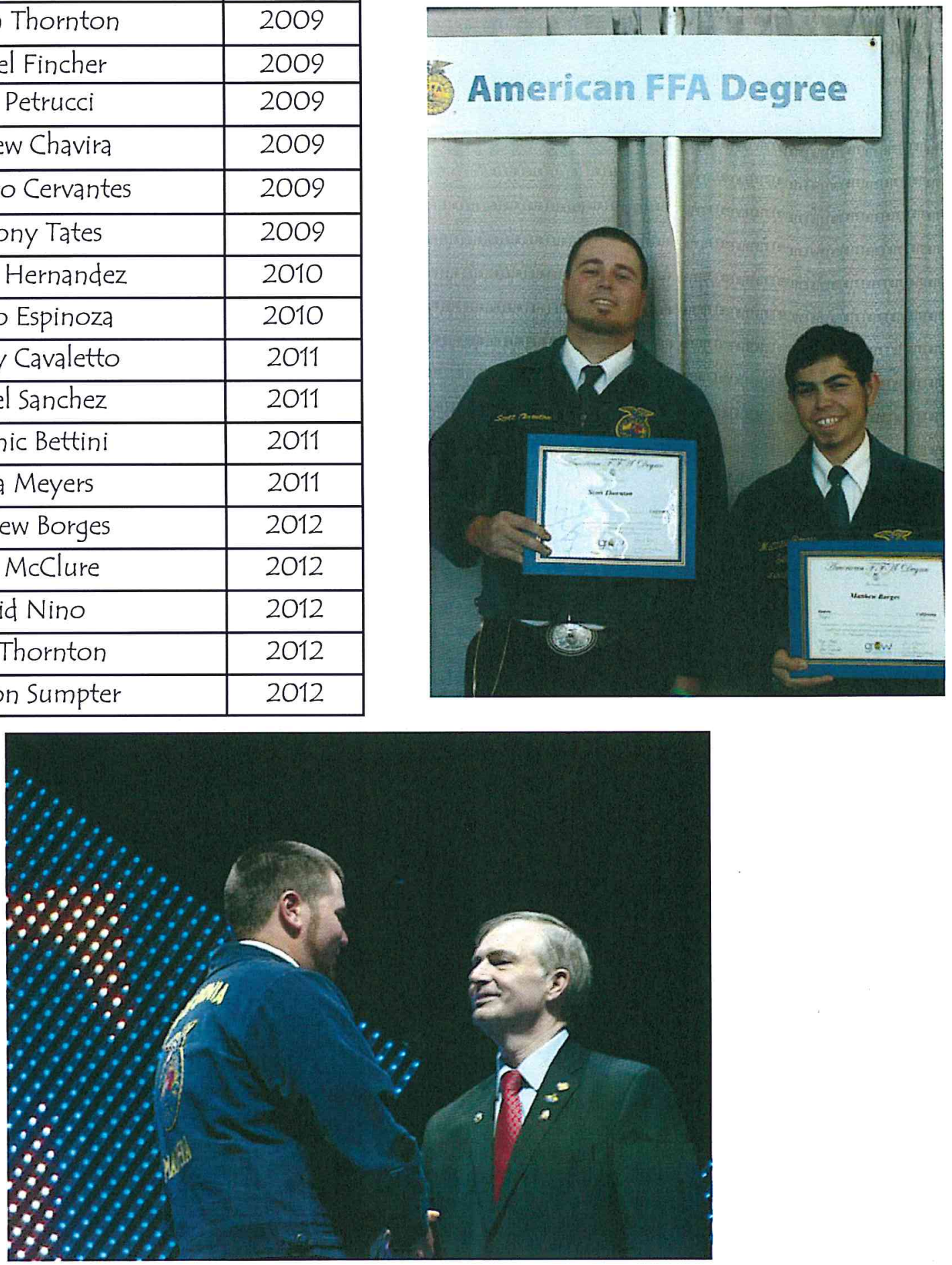


\section{StEerte Winning Teoms}

Trees, 1933-1934

Trees, 1936-1937

Dino Petrucci, Public Speaking, 1946-47

${ }^{* *}$ Livestock, 1948

Milk 1950-1951

Ag. Marketing, 1955-1956

Ag. Mechanics, 1959-1960

Ag Mechanics, 1960-1961

Agronomy, 1960-1961

Truck Crops, 1960-1961

Land, 1961-1962

Truck Crops, 1961-1962

Grapevine Judging, 1961-1962

Public Speaking- Dan Chatman, 1962-1963

Agronomy, 1962-1963

Truck Crops, 1962-1963

Cotton, 1963-1964

Grapevine Pruning, 1964-1965

Farm Power, 1965-1966

Truck Crops, 1965-1966

Grapevine Judging, 1965-1966

Livestock, 1966-1967

Public Speaking-Dave Loquaci, 1966-67

Truck Crops, 1967-1968

Grapevine Pruning, 1967-1968

Agronomy, 1968-1969

Ag. Pest Control, 1968-1969

Grapevine Judging, 1968-1969

Grapevine Pruning, 1968-1969

Tree Pruning, 1969-1970

Cotton, 1974-1975

Citrus, 1975-1976

Citrus, 1977-1978

Livestock, 1980-1981

Small Engines, 1980-1981

Small Engines, 1981-1982
Fruit Tree Pruning, 1982-1983

Grapevine Pruning, 1982-1983 Land Judging, 1984-1985

Light Horse Judging, 1984-1985

Small Engines, 1986-1987

Small Engines, 1987-1988

Small Engines, 1988-1989

Fruit Tree Pruning-1989-90

Small Engines-1990-91

Grapevine Pruning- 1992-93

Fruit Tree Pruning- 1992-93

Grapevine Pruning-1993-94

Fruit Tree Pruning-1993-94

Grapevine Judging - 1993-1994

Farm Power and Machine, 1995-1996

Grapevine Pruning-1995-96

Fruit Tree Pruning- 1996-1997

Fruit Tree Pruning-1997-1998

Small Engines-1999-2000

Floriculture- 2001-2002

Small Engines, 2002-2003

Best Informed Greenhand-2004-2005

Best Informed Greenhand-2006-2007

Best Informed Greenhand-2007-2008

Small Engines- 2007-2008

Meats- 2007-2008

Vine Pruning -2009- 2010

Vine Pruning-2010-2011

Best Informed Greenhand-2010-2011

Floriculture-2010-2011

Best Informed Greenhand-2011-2012

Vine Pruning-2012-2013

Meats Judging - 2012-2013

Best Informed Greenhand -2012-2013 


\section{Madera FIA}
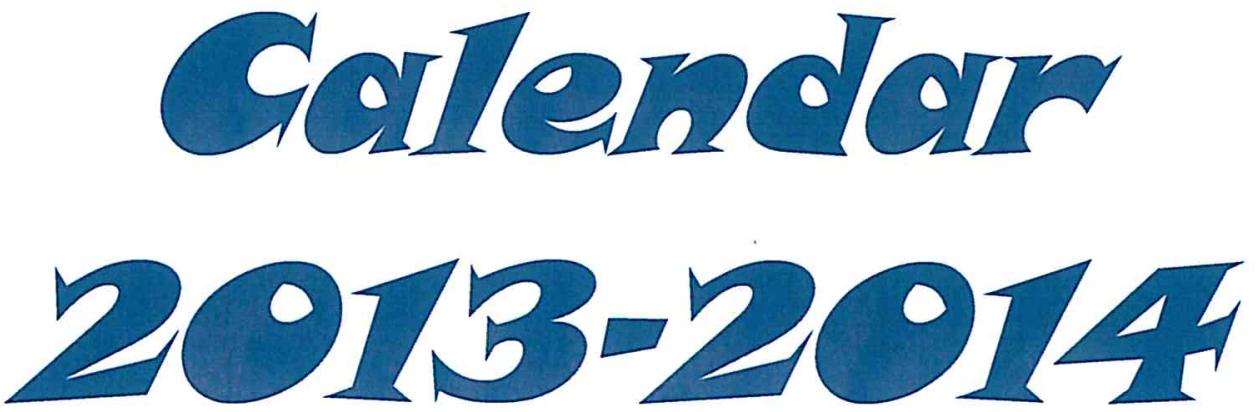

Use the following pages to see the opportunities available to you as an FFA member or community member who would like to get more involved.
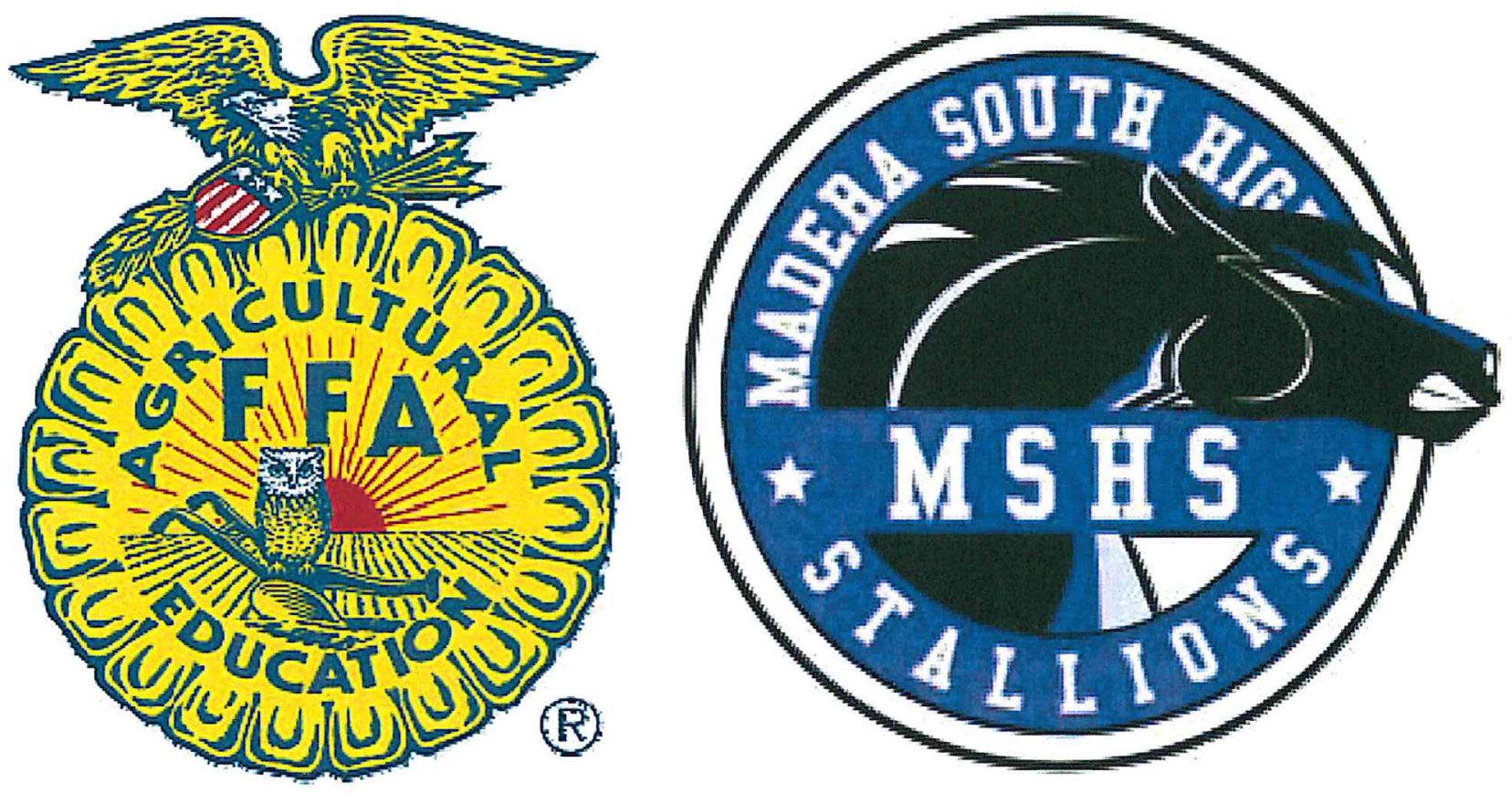


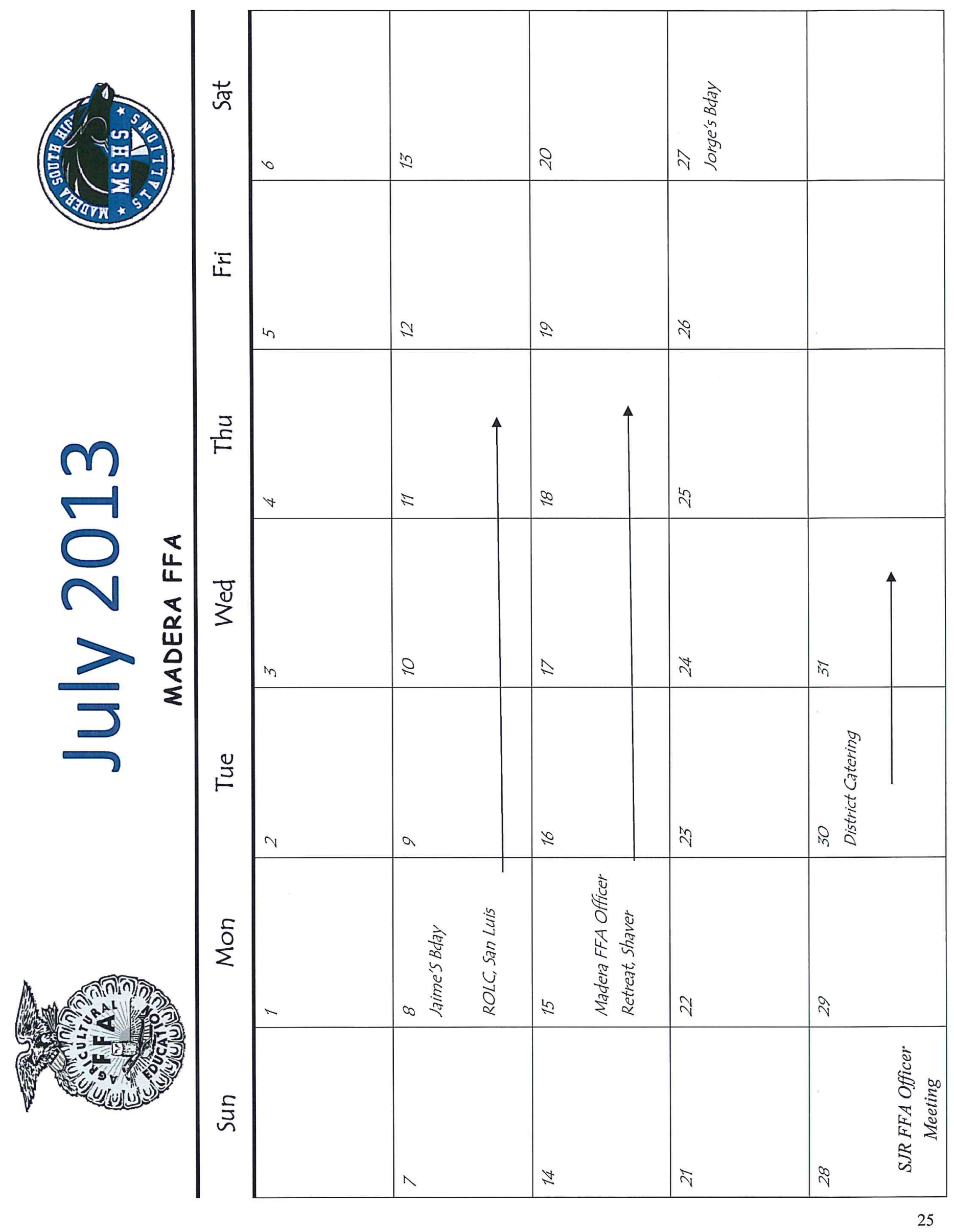




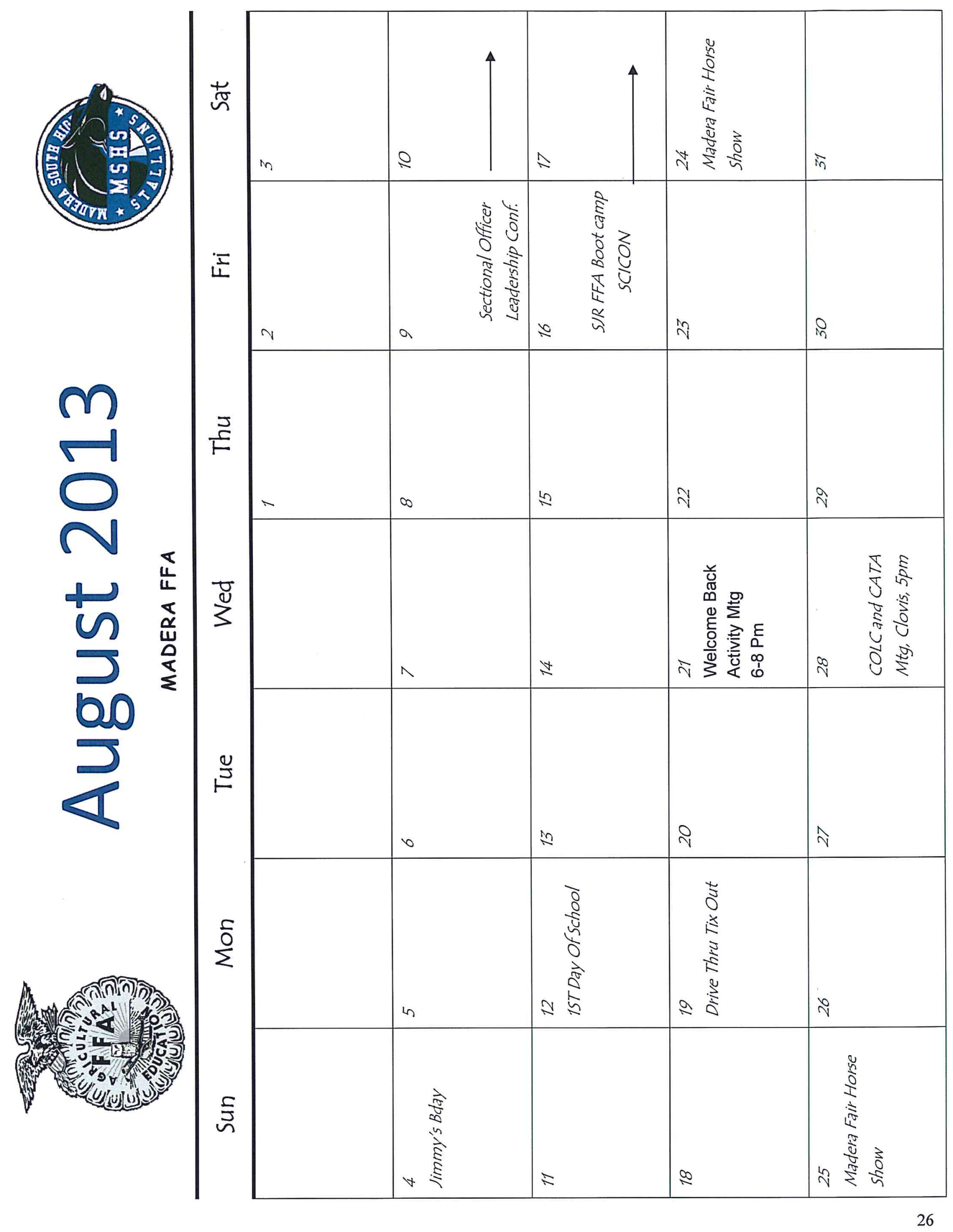




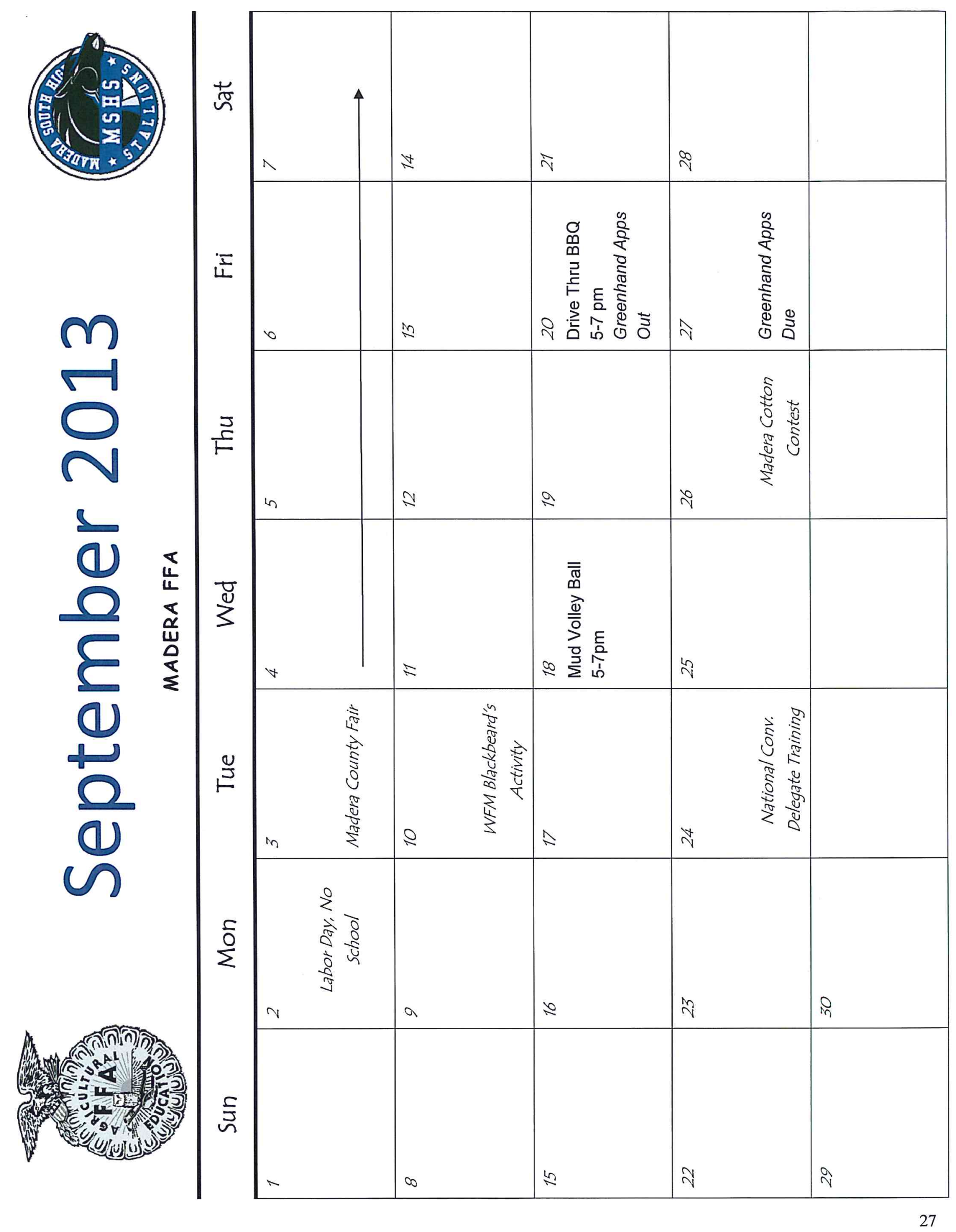




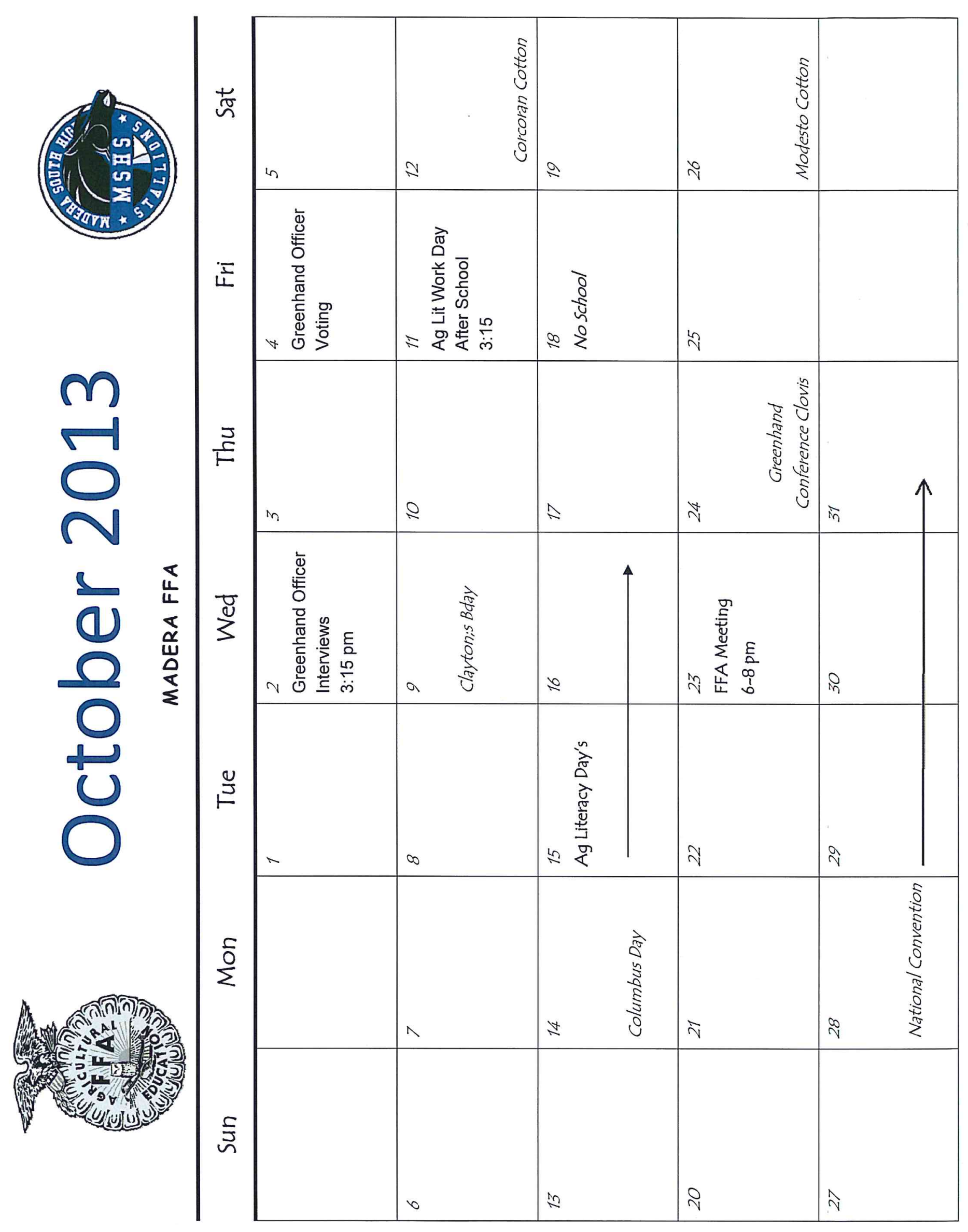




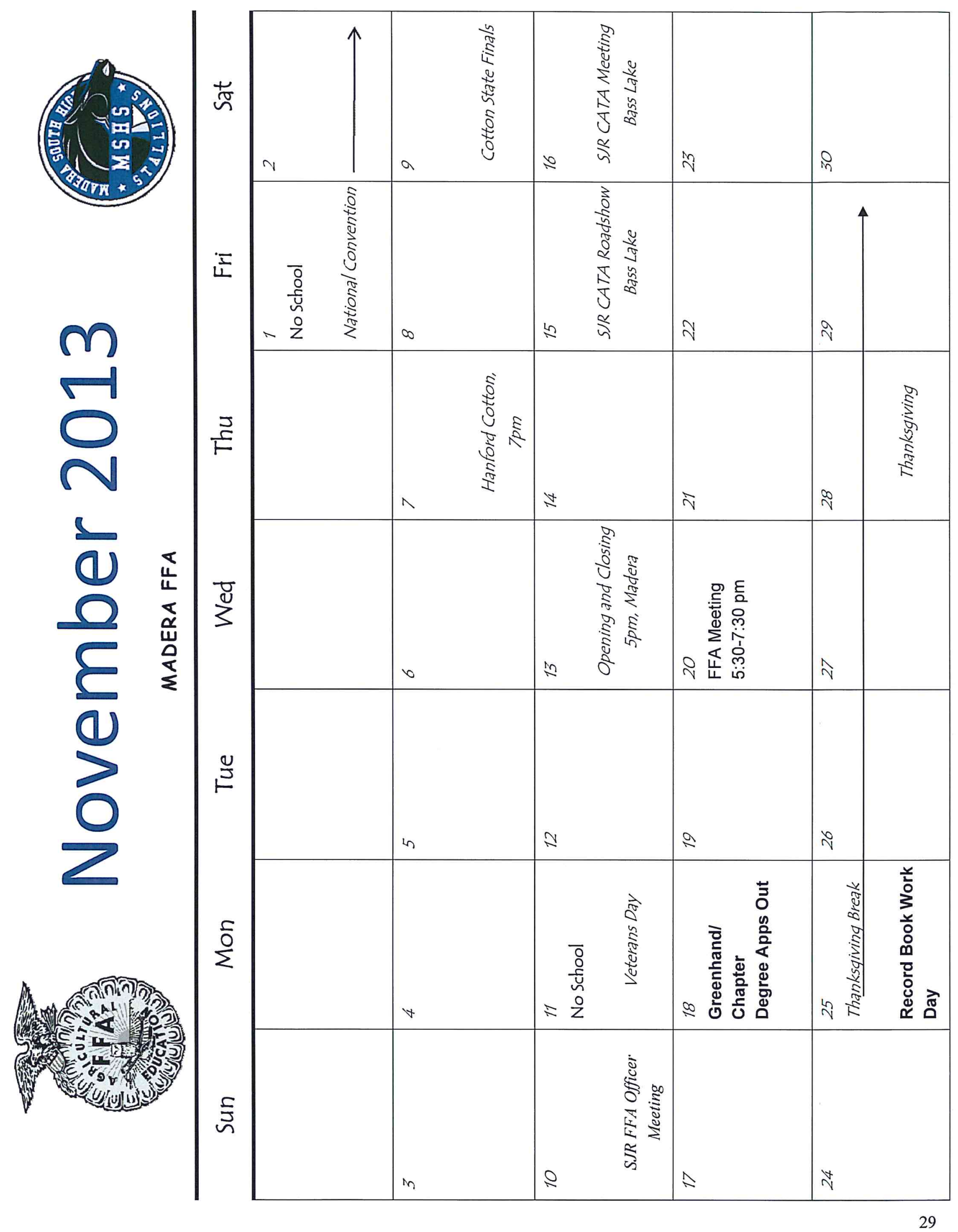




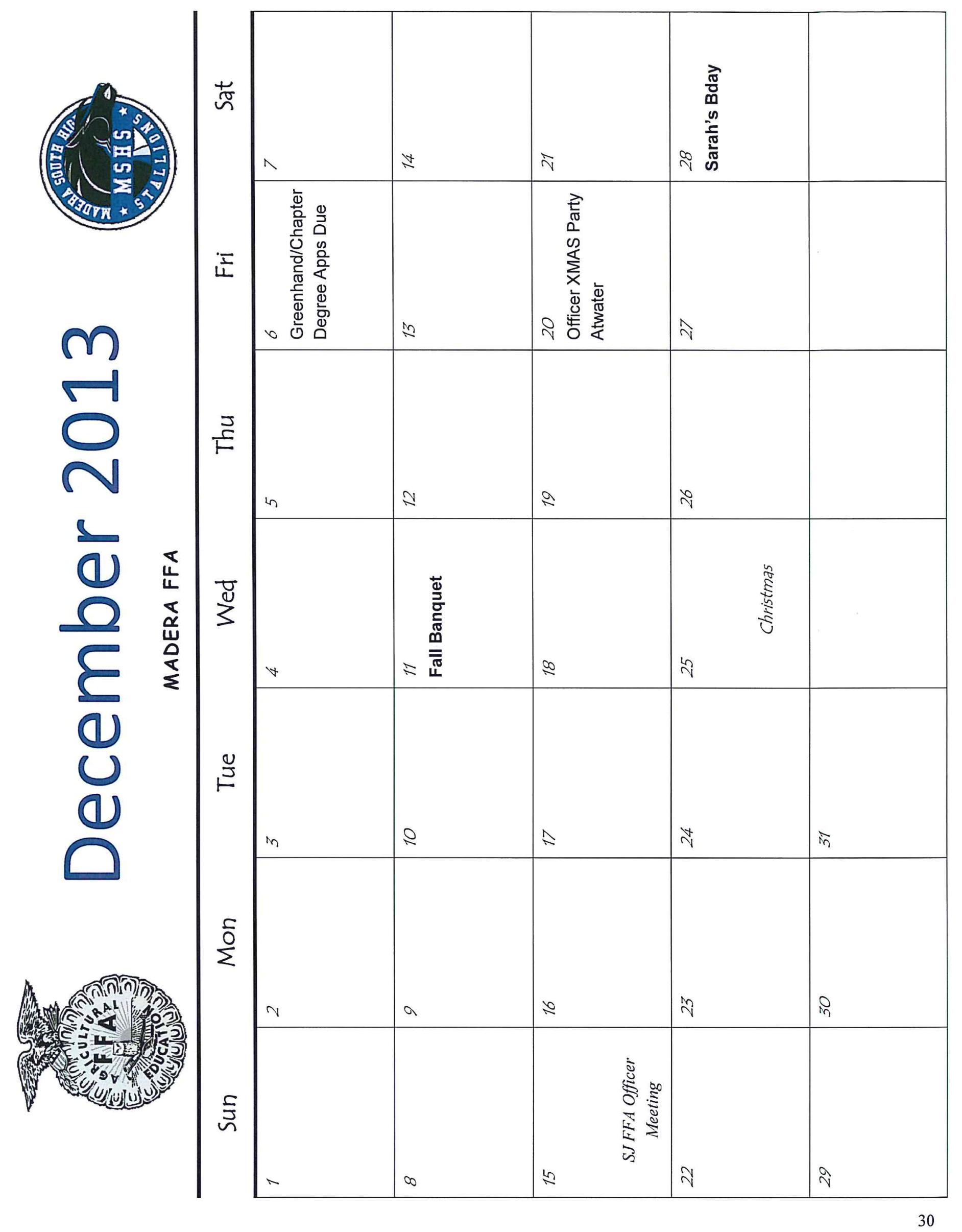




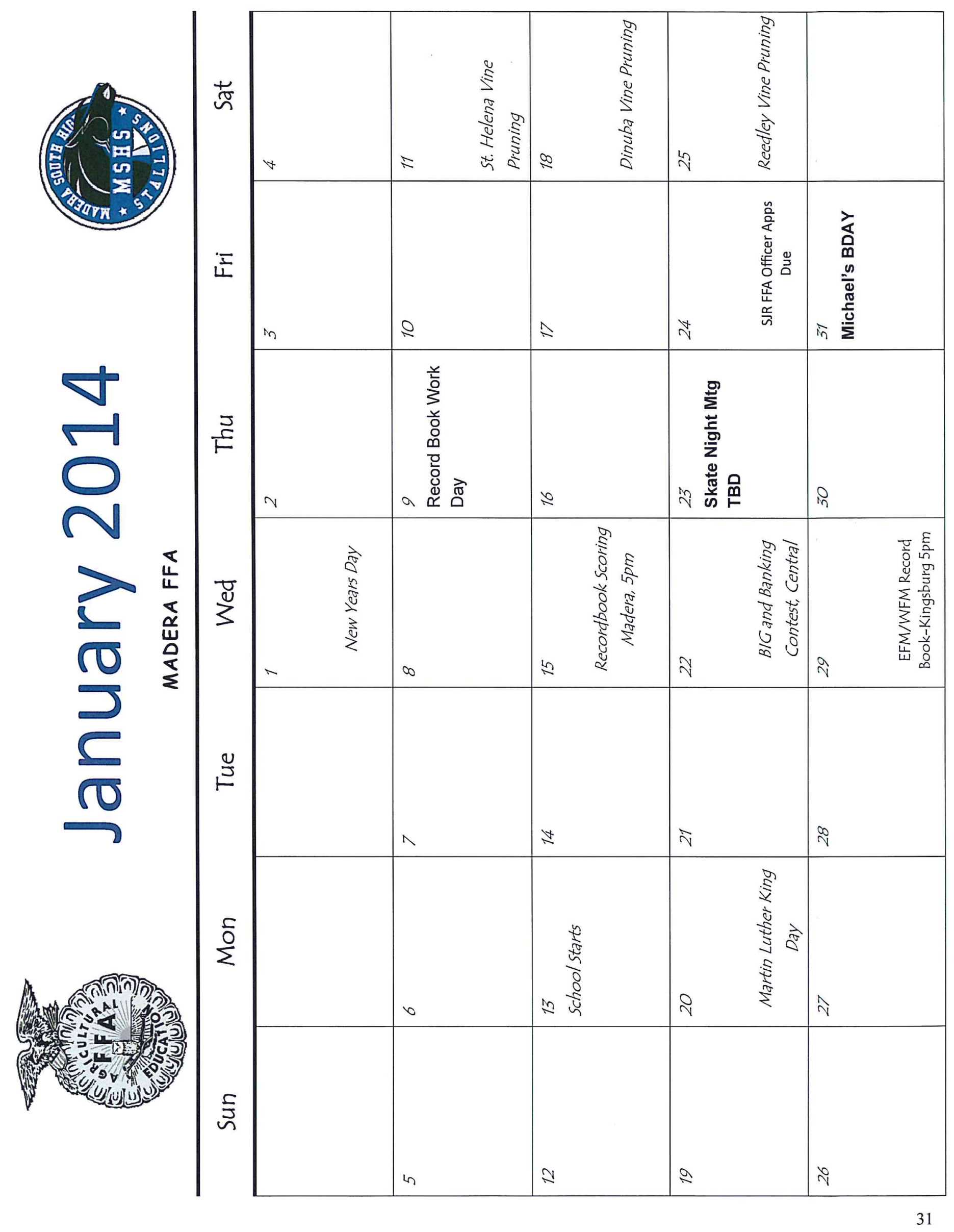




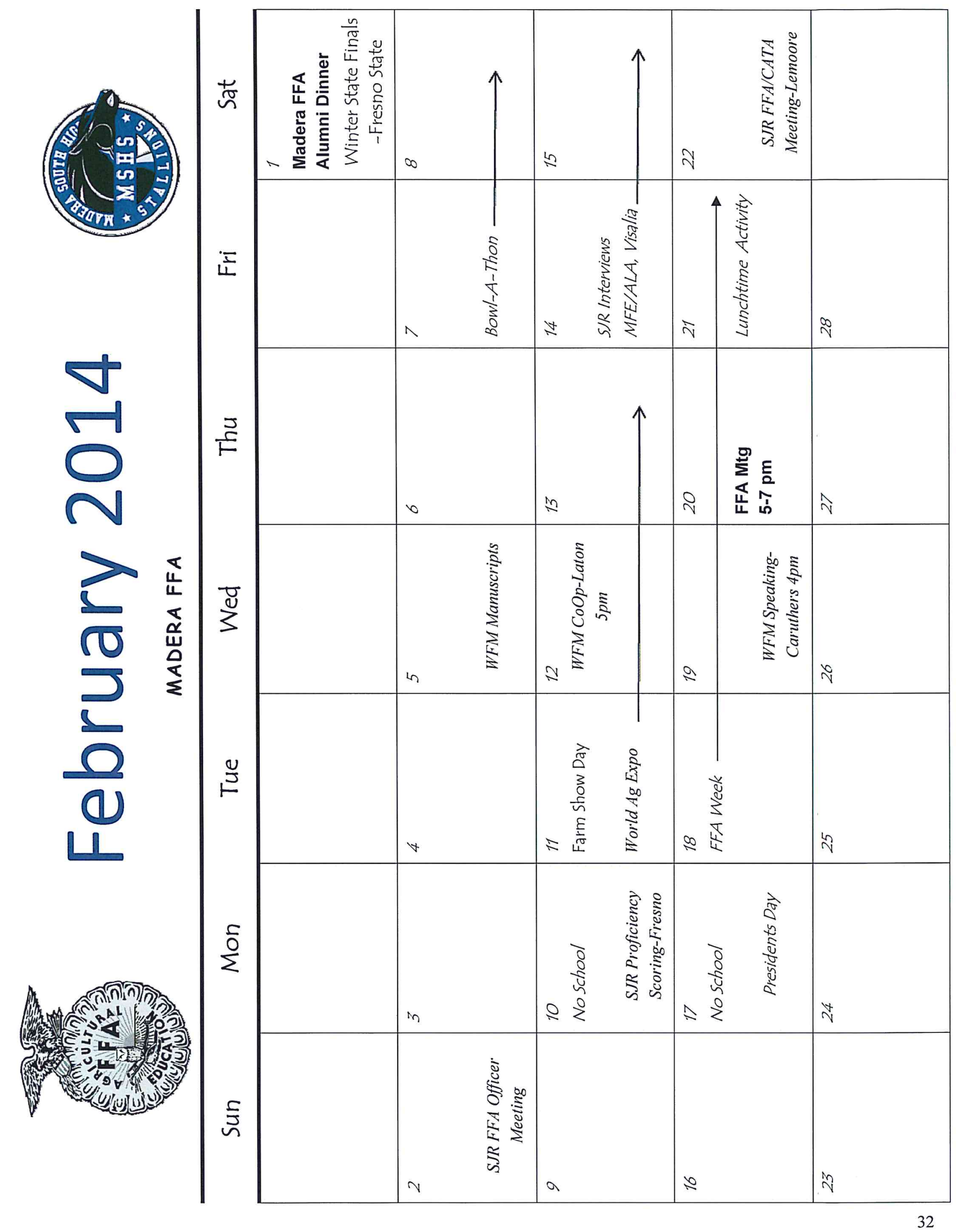




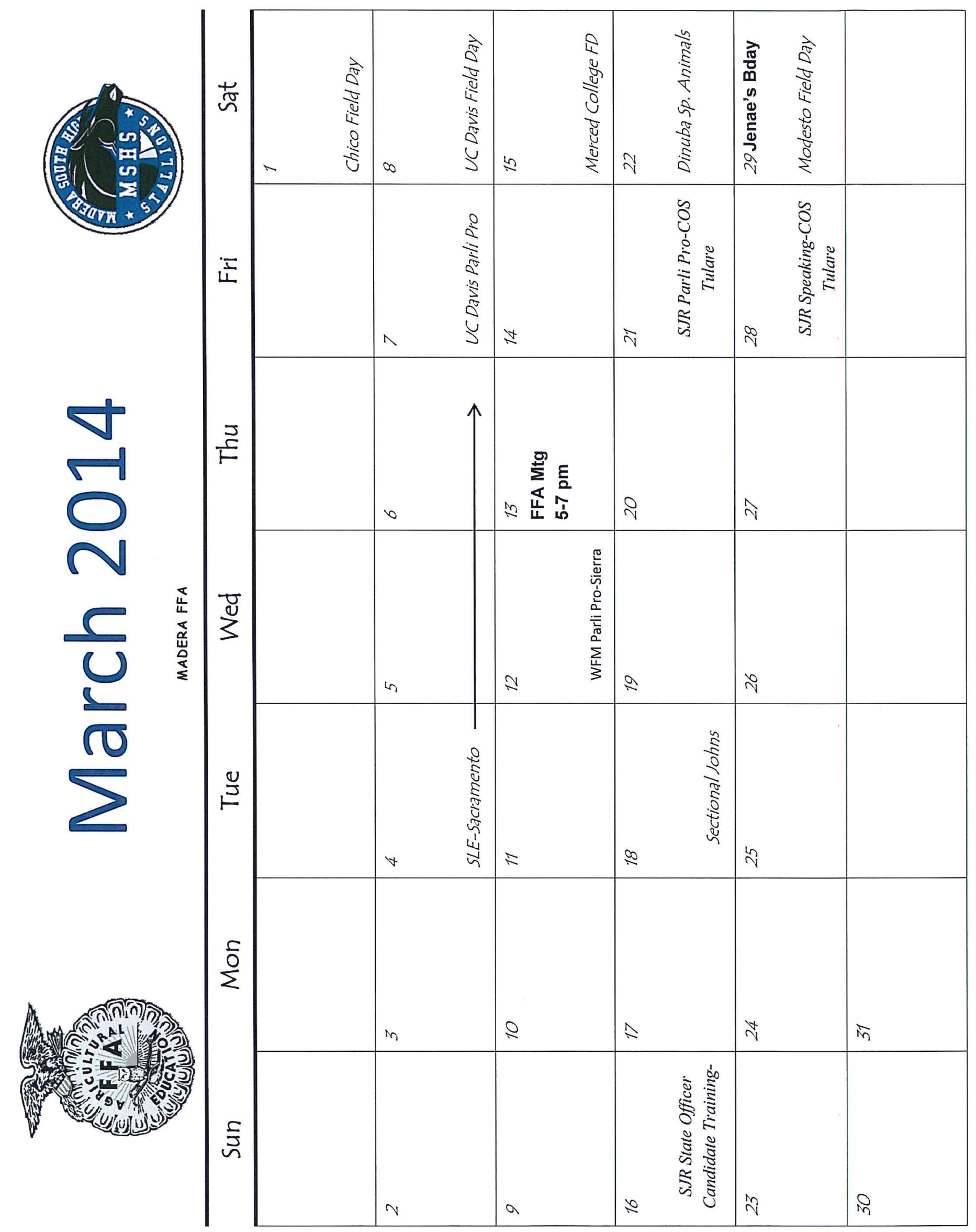




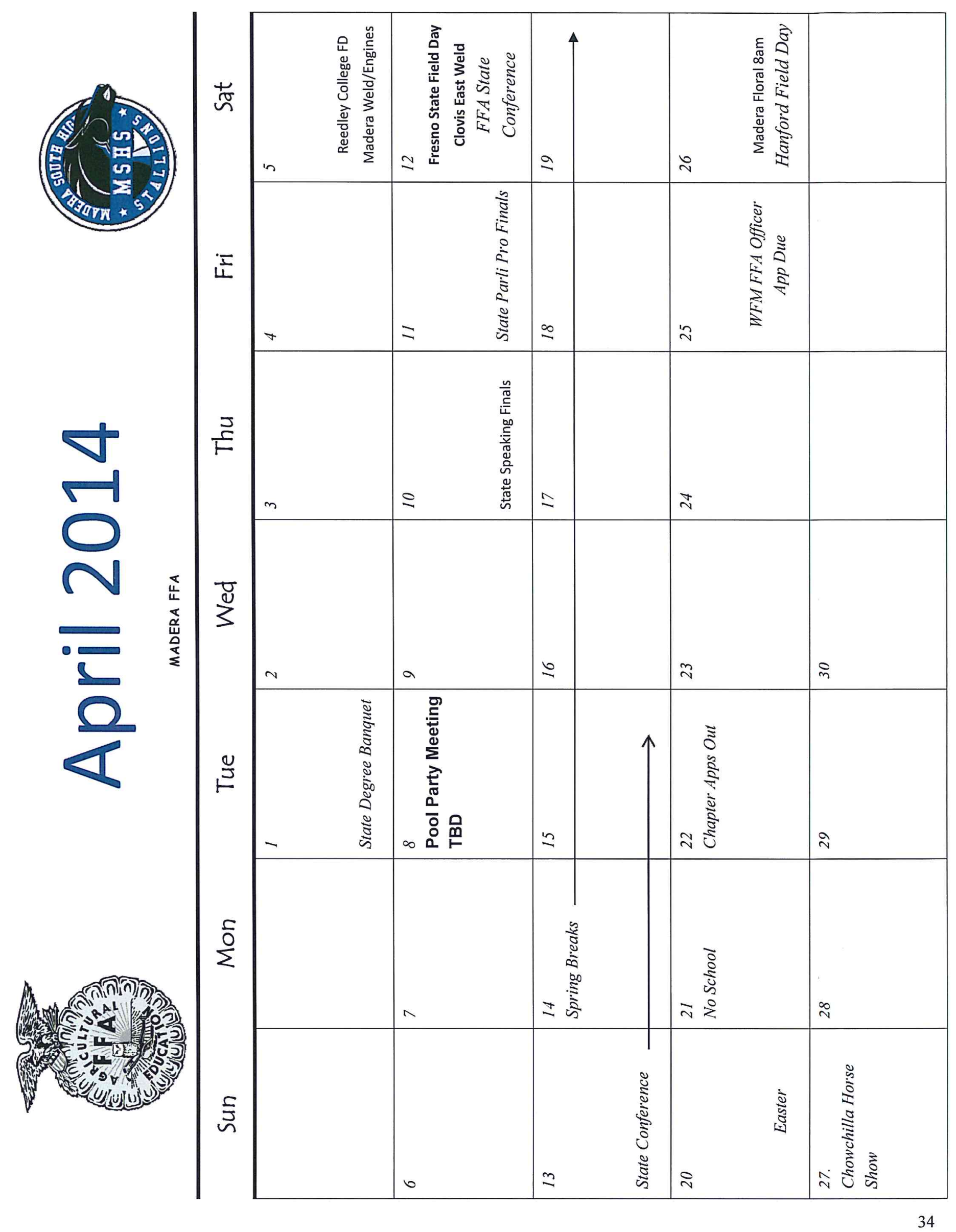




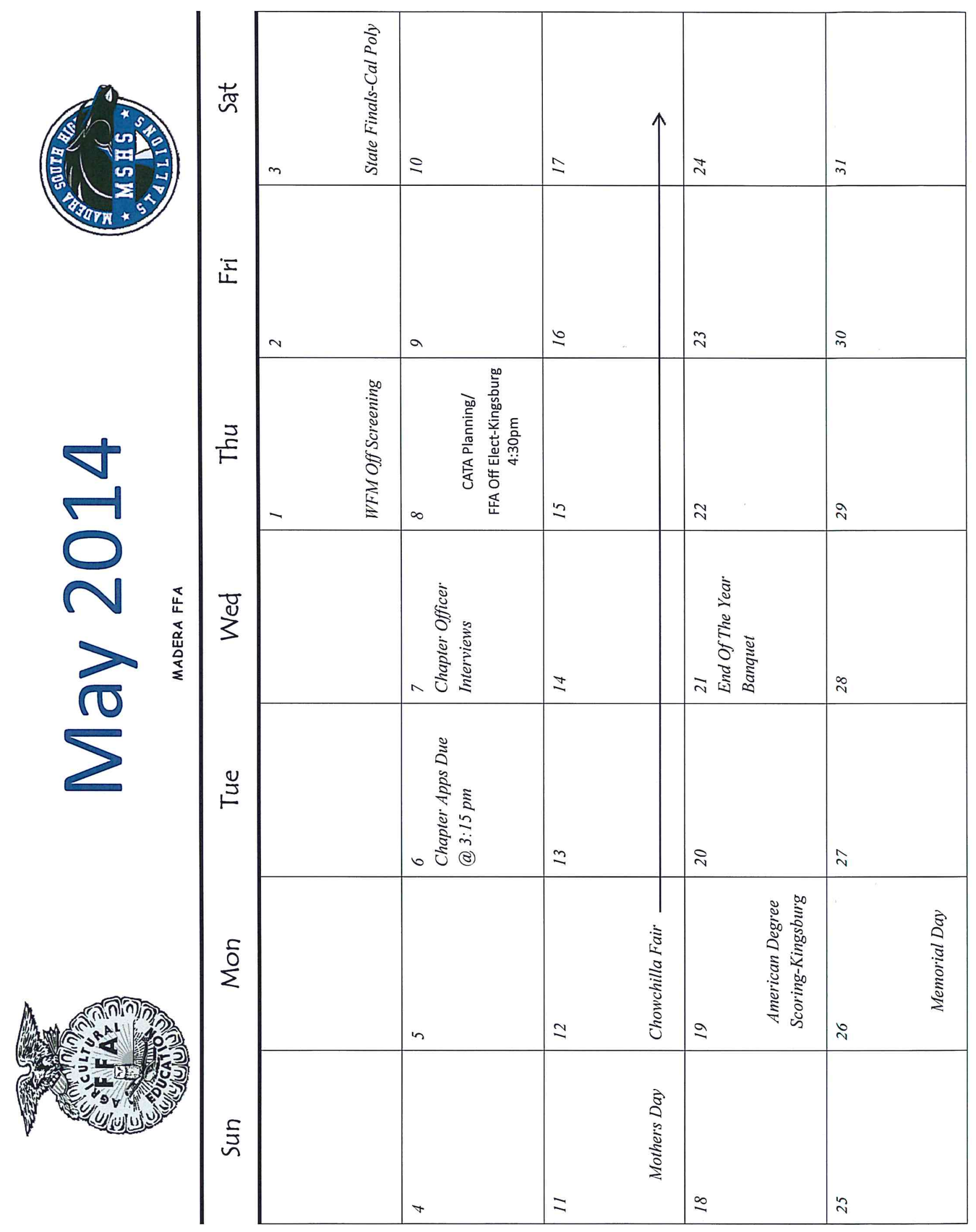




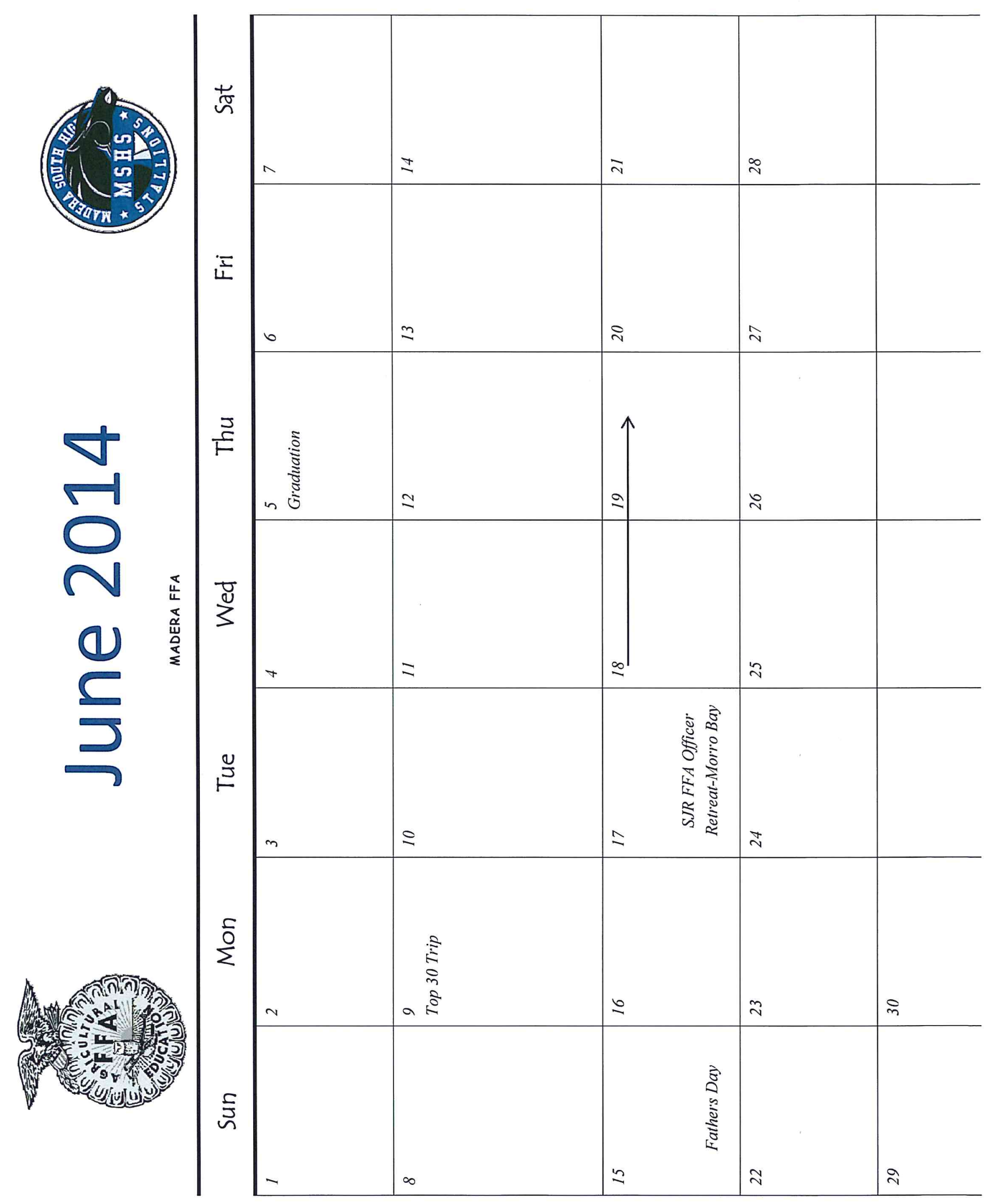


Maderd FIA
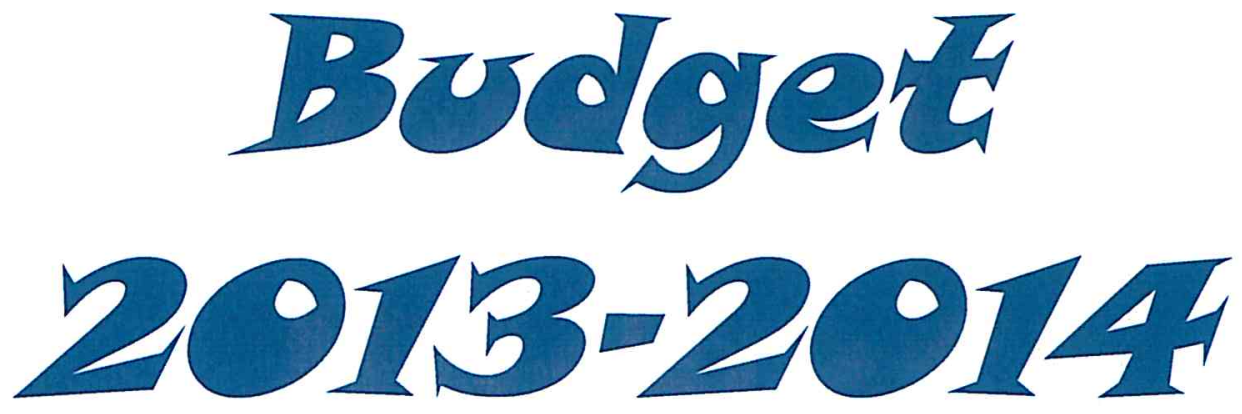

Attached is the FFA Activity Budget which was developed by the officers at retreat and approved by the ASB Director and Bookkeeper on August 30, 2013.
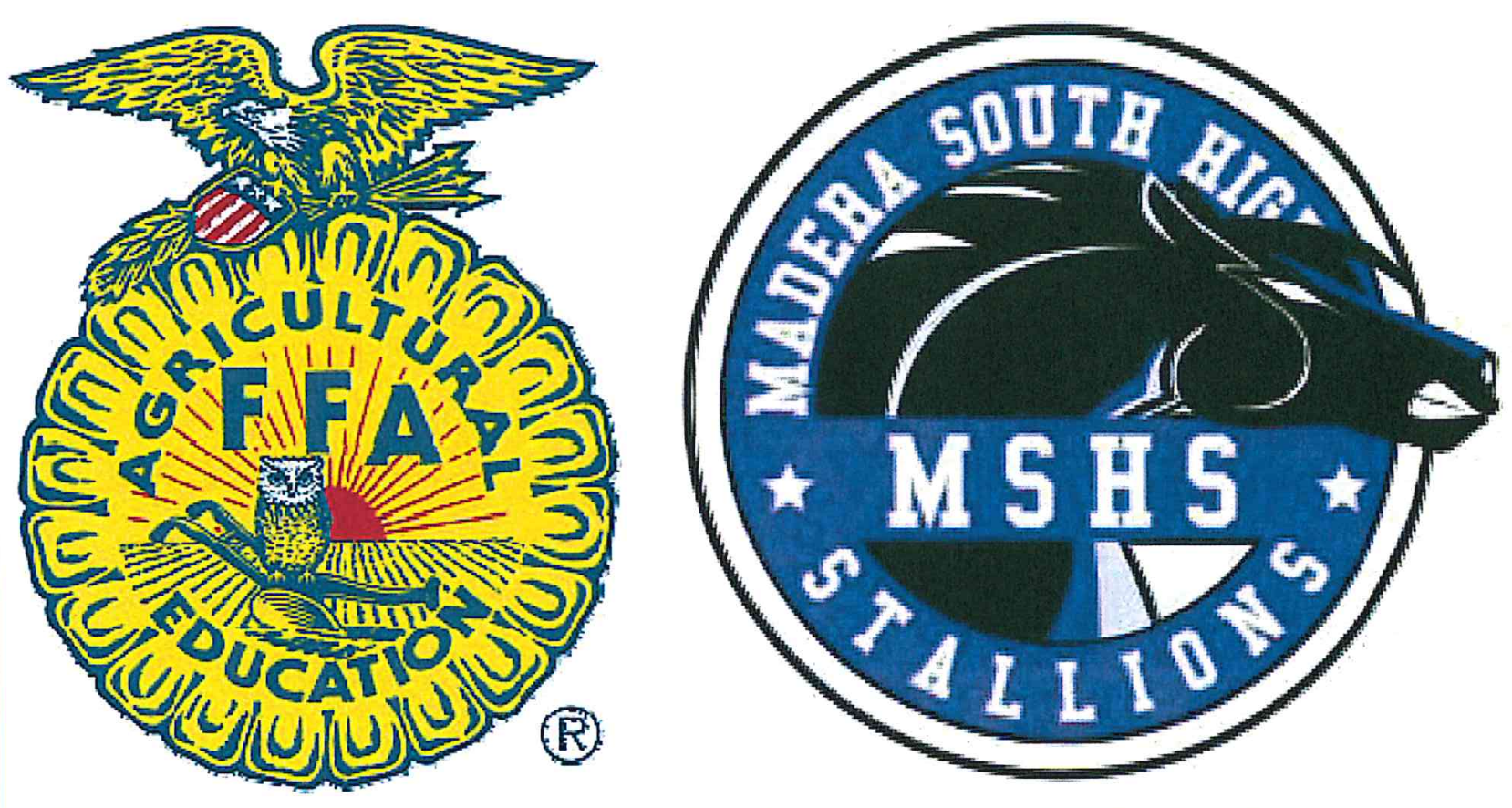


\section{Mader FFA Budget 2013-2014}

\begin{tabular}{|l|c|}
\multicolumn{2}{|c|}{ Income } \\
\hline District Lunches & $\$ 5,000.00$ \\
\hline Opening and Closing & $\$ 1,000.00$ \\
\hline Ag. Literacy Day & $\$ 2,500.00$ \\
\hline SHARES & $\$ 600.00$ \\
\hline Snack Sales & $\$ 12,000.00$ \\
\hline Alumni Dinner & $\$ 5,000.00$ \\
\hline Drive Thru BBO & $\$ 4,000.00$ \\
\hline TOTAL INCOME & $\$ 30,100.00$ \\
\hline
\end{tabular}

\begin{tabular}{|c|c|}
\hline \multicolumn{2}{|c|}{ Expenses } \\
\hline Banquet Awards and Décor & $2,000.00$ \\
\hline Banquet Food & 700.00 \\
\hline Bowl A Thon Bus & 350.00 \\
\hline Casino Night & 200.00 \\
\hline Chapter Meetings & $\$ 1,000.00$ \\
\hline Hotels for Field Days & 1800.00 \\
\hline COLC & 120.00 \\
\hline District Lunches & $\$ 2,000.00$ \\
\hline Field Day Entries & $\$ 2,000.00$ \\
\hline Fall Banquet & $\$ 500.00$ \\
\hline Fall and Spring Regional FFA Meeting & 250.00 \\
\hline Greenhand Conference & 450.00 \\
\hline Grub Down & 200.00 \\
\hline Homecoming Float & 200.00 \\
\hline Officer Polos and Retreat & 800.00 \\
\hline Opening and Closing Food & 675.00 \\
\hline POA Printing & 150.00 \\
\hline Pumpkin Festival Supplies & $1,900.00$ \\
\hline Scrapbook Stuff & 200.00 \\
\hline Skating Meeting & 450.00 \\
\hline Snack Sale & $\$ \quad 5,000.00$ \\
\hline Top 30 Tickets & $\$ 1,200.00$ \\
\hline Tri Tip BBO & $\$ \quad 4,000.00$ \\
\hline TOTAL EXPENSES & $\$ 26,145.00$ \\
\hline
\end{tabular}




\section{Community Service}

At Madera FFA we feel community service is not a service at all. As community members we feel it is our duty to step in and help when it is needed; whether it is making sure community members have food at Thanksgiving or making sure children have a present to open on Christmas morning. Madera FFA is there and willing to help when the community needs it.

Ag. Literacy Day- We host a two day activity in which we recognize the importance of Agriculture to the youth within our community.

Bowling-4-Kids-Sectional Bowl-A-Thon is an event awarded to those individuals who help raise money for Children's Hospital.

Can Food Drive- We will host a food drive during the Christmas season, working with local community shelters to distribute the food to the less fortunate in our community.

Old Timer's Parade- FFA members clean up the parade route.

Farm Bureau Scholarship Event- FFA members serve as event staff parking cars, serving, busing tables, and cleaning up.

Relay for Life- FFA members help in the fight against cancer at the Madera Relay for Life where they participate in painting luminary bags and setting up the relay course.

Toys for Tots- FFA members partner with the Toys for Tots organization to provide children with a gift during the holiday season. 


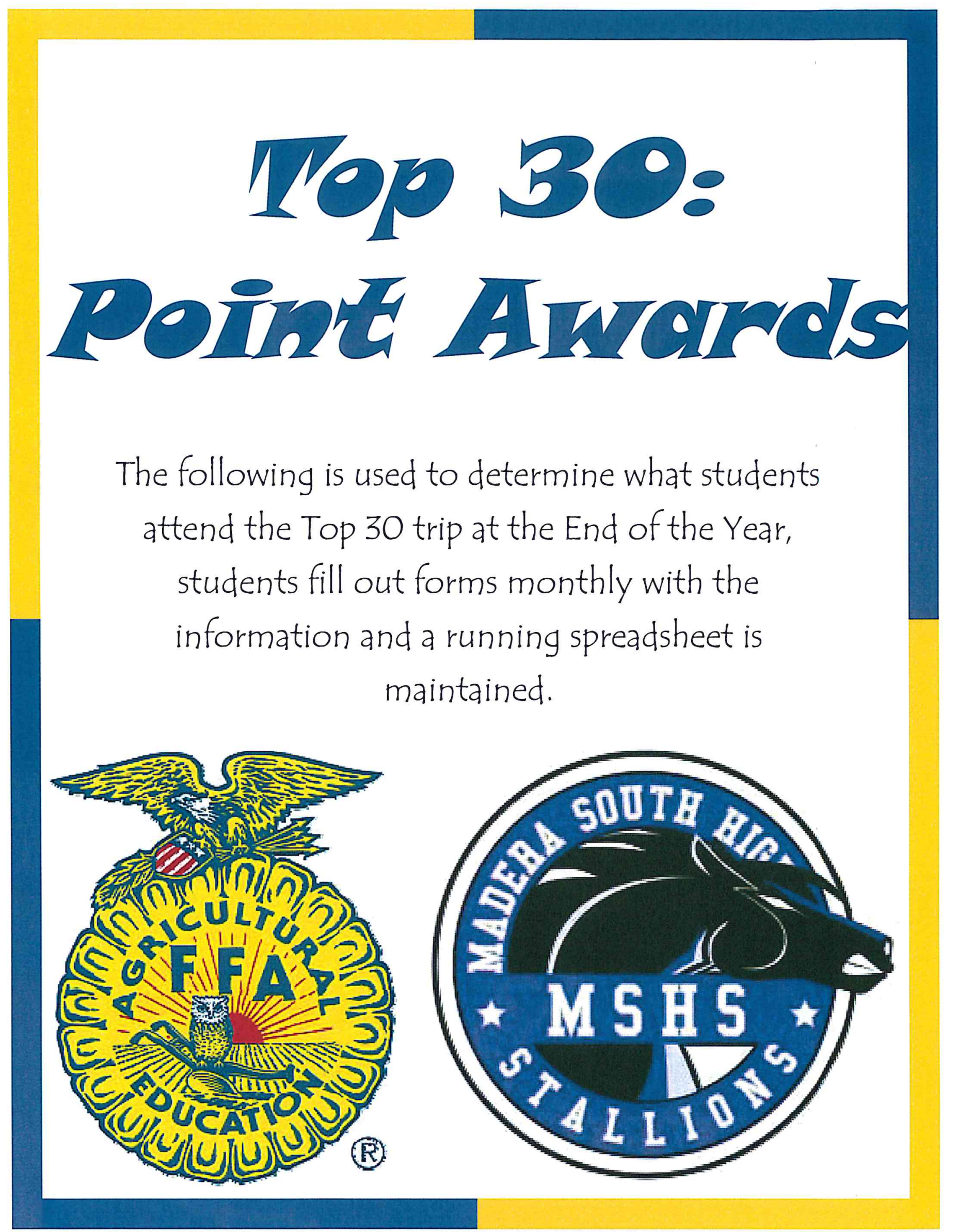




\section{Top Thirrty Trip}

Top Seller Trip

Throughout the year, Madera FFA members are given the opportunity to help raise money for our chapter. The money raised goes towards numerous events and activities that take place in our FFA chapter. For example, Madera FFA plans a minimum of one activity per month during our school year that is free of charge to our students. These activities include skating, bowling, and movie night just to name a few. In order to fund these trips, we put on different fundraising events throughout the year. This year we carry on the traditional fundraisers such as the Drive thru BBQ, Snack Sales in the Fall and Spring, and our Alumni Dinner Dance. If a member has sold a total of 40 tickets or items at the end of the last fundraiser, he or she will be invited to the Top Seller Trip at the end of the year. The Top Seller Trip is designed to give every student in our department the opportunity to receive a free trip to a designated theme park.

\section{Top Thirty Trip}

As mentioned above, Madera FFA members are given numerous opportunities to participate in FFA activities. Throughout the year we give students points for attending and participating in FFA activities, events, and contests. For example, the top thirty students are announced at the annual end of the year banquet and then those students are taken on an achievement trip to Magic Mountain. The students fill out point award sheets at the end of each month and a running total is kept until the end of the year. The winner of the Top Thirty Trip is announced at the chapter banquet.

I. Leadership

A. Greenhand Degree (December of each year awarded) 50

B. Chapter FFA Degree (December of year awarded) $\quad 75$

C. State FFA Degree 100

D. Star State Farmer (In addition to degree) 125

II. Offices (Points awarded in May)
A. Chapter Officer
100
B. Committee members
75
C. Sectional Officer
100
D. Regional Officer
100

III. Meetings/Conferences
A. Attend Chapter FFA Meetings/Activities/Banquets
50
B. Attend Sectional/Regional Meeting or Activity
75
C. Attend State Convention
100
D. Attend One Day State Convention
25
E. Attend National Convention
150
F. Delegate for conferences
50
G. Chapter, Section, Region Banquets
50
H. Greenhand, MFE, ALA, SLE, or WLC
75 


\section{Top Thinty Trip}

IV. Community/Service
A. Ag. Literacy Day (each day)
25
B. $\quad 8^{\text {th }}$ Grade Visitation
50
C. Canned Food Drive
50
D. Bowl-a-Thon
50
E. Love Madera
50
F. Other Activities as Developed
50

Fundraisers
A. Tri Tip and Alumni Dinner Ticket Sales
1 pt per $\$$
B. Snack Bags
1 pt per\$
C. Other Activities as Developed
1pt per \$

VI. Other FFA Activities
A. Purchase FFA T-Shirt/Sweatshirt
20
B. Purchase FFA Jacket
50

VII. Supervised Agriculture Experience

A. Approved Enterprise: (Each project)

Large Mechanics, Market Beef, Dairy, Sheep, $\quad 50$

Swine, and Goats

2. Small Mechanics, Market Poultry, and Rabbits 30

3. Breeding Projects 75

B. Exhibit at Shows and Fairs: Mechanics, Landscape, Floral, and Animal Projects

1. First Award 25

2. Second Award 20

3. Third Award 15

4. Fourth Award - tenth award 10

5. Round Robin Participant

1st

50

2nd $\quad 40$

3rd 30

4th 25

5th 20

6th 15

7 th 10

6. Best of Show (Ag Mech., Land, Floral) 50

7. Outstanding Exhibitor (any species) 50 


\section{Top Thirety Trip}

C. Animal Projects: Market or Breeding

1. Grand Champion 50

2. Reserve Grand Champion 35

3. FFA Champion 25

4. FFA Reserve Champion 20

5. Breed Champion 15

6. Reserve Breed Champion 10

D. S.A.E. Hours

1. 1 point per hour worked ( must be proved by record book)

VIII. Proficiency Awards

$\begin{array}{lll}\text { A. } & \text { Applicant } & 20 \\ \text { B. } & \text { Sectional Winner } & 25 \\ \text { C. } & \text { Regional Winner } & 50 \\ \text { D. State Winner } & 75 \\ \text { E. National Winner } & 100\end{array}$

IX. Contests

A. Participation 15

B. Placement

1. Individual and Team Placing

a. First High Overall 25

b. Second High Overall 20

c. Third High Overall 15

d. Fourth High Overall 10

e. Fifth High Overall 5

The above points for a State Finals Contest are doubled!

The above points for a National Finals Contest are tripled!

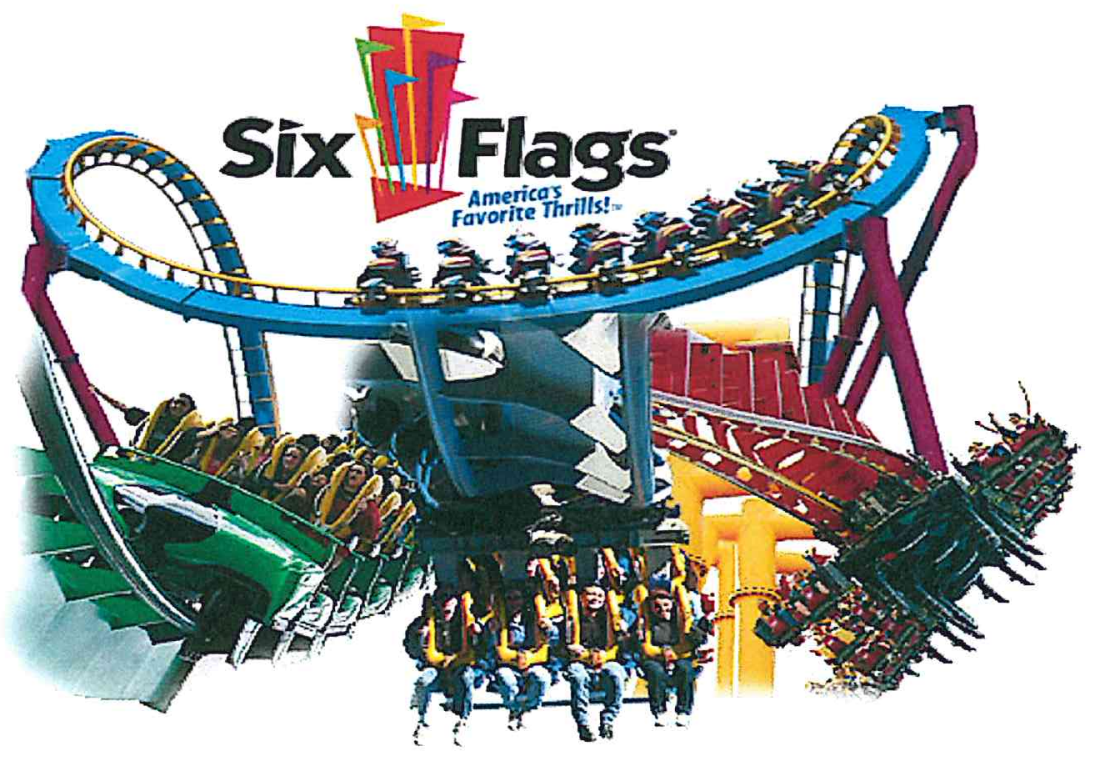


Gonstitution and Bylaris for Maderd FIA

The rules and standards to run Madera FFA. Last modification was May 2, 2005.
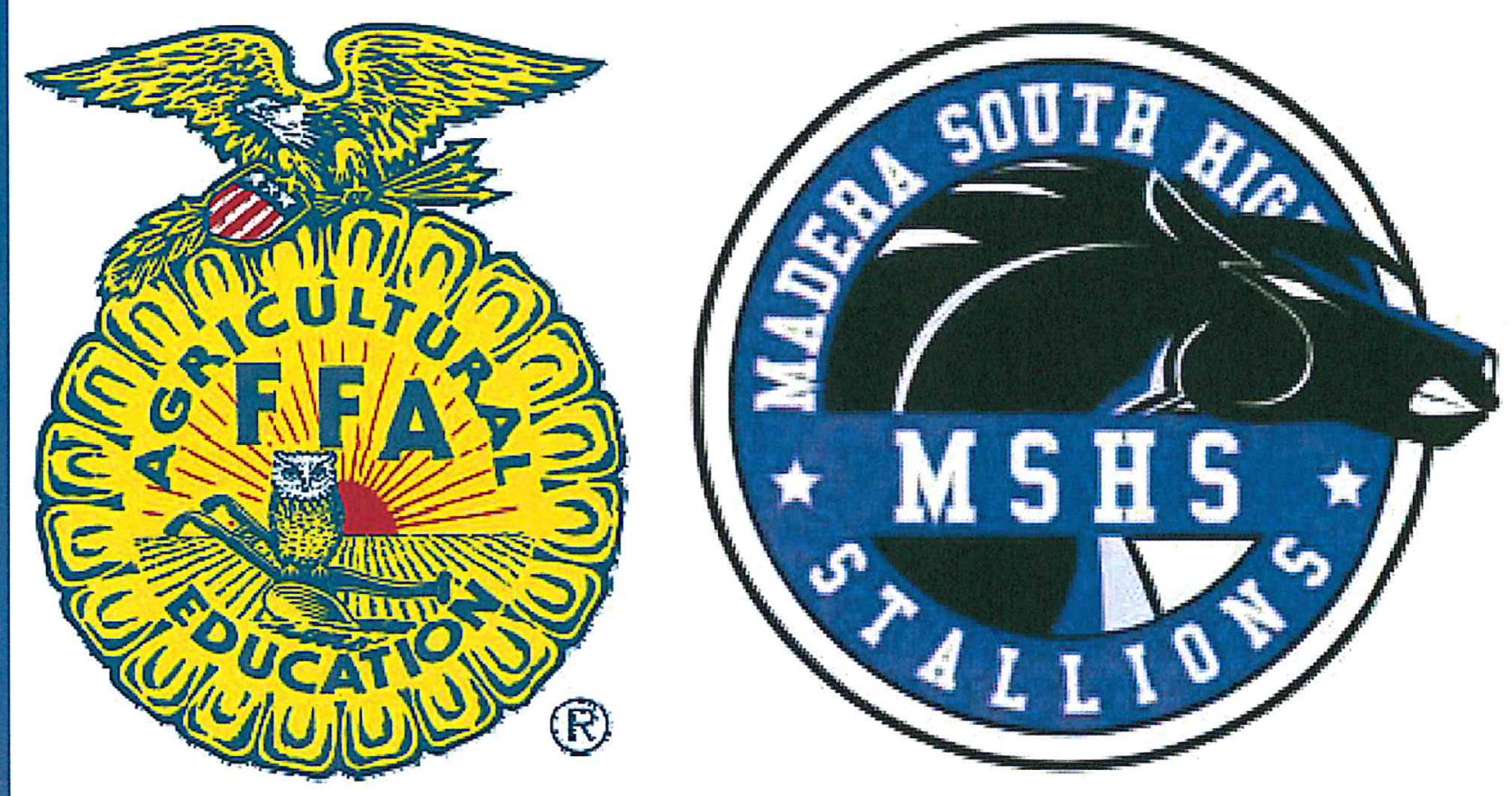


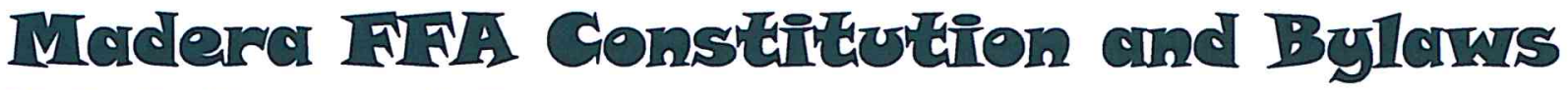 Aritille I. Nerme and Purposes}

Section A. The name of this organization shall be the Madera FFA Chapter. The letters "FFA" will be used to designate the chapter, its activities, and its members.

Section B. The purpose for which this chapter is formed by is as follows:

1. To develop agricultural leadership skills among all members.

2. To develop a global awareness of agriculture.

3. To bestow confidence among agricultural students and the work.

4. To promote agriculture career opportunities through hands-on training.

5. To develop competencies in communication, human relations, and social abilities.

6. To build cooperative attitudes among agricultural students.

7. To encourage improvement in scholastics.

8. To provide organized recreational activities for agriculture students.

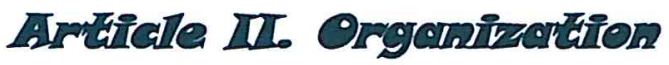

Section A. The Madera FFA Chapter is a chartered local entity of the West Fresno - Madera Section of the California Association, made up of local members.

Section B. This chapter accepts in full, the provision in the constitution and bylaws of the California Association of the Future Farmers of America as well as those of the National FFA Organization.

\section{Articile III. Membership}

Section A. Membership is limited to students enrolled in Agriculture Education at Madera SouthHigh School.

Section B. Membership of graduates is limited to students that were active members in high school.

Section C. The Madera FFA is a $100 \%$ affiliated chapter with every student becoming a member of the FFA when they enroll in an agriculture class.

Section D. No student may participate in any FFA activities unless they are members in good standing with the FFA. In order to be in good standing with the FFA a student must owe no money to the FFA, and their name must not appear on the ineligible list.

Section $\mathrm{E}$. The FFA advisors at their own discretion have the right to dismiss any members from the FFA organization at anytime with approval of the administration.

Section F. Membership in this chapter shall be of three kinds: 


\section{IMadera Fin Gonstitumen and Bylans}

1. Active - Any student enrolled in an agriculture education program.

2. Alumni - Any person who has formerly been enrolled in an agriculture education program or in other ways interested in supporting the FFA.

3. Honorary - Any person who has helped to advance agriculture education and the FFA and who have rendered outstanding service may be elected to honorary membership.

Section G. The regular work of this chapter shall be carried on by the active membership.

Section $\mathrm{H}$. Honorary membership in the chapter shall be limited to the Honorary FFA Degree.

Section I. There shall be four levels of active degree attainment in the Madera FFA Chapter.

The Greenhand FFA Degree

1. All Greenhand Degree recipients are entitled to wear the regulation bronze emblem charm.

2. The Chapter FFA Degree

All members holding the Chapter FFA Degree are entitled to wear the silver emblem pin.

3. The State FFA Degree

All members holding the State FFA Degree are entitled to wear the regulation gold emblem charm.

4. The American FFA Degree

All members holding the American FFA Degree are entitled to wear the regulation gold emblem key.

Section J. Greenhand FFA Degree. Minimum qualifications for election:

1. Be enrolled in agricultural education and have satisfactory plans for a Supervised Agricultural Experience Program.

2. Learn and explain the FFA Creed, Motto, and Salute.

3. Describe and explain the meaning of the FFA emblem and colors.

4. Demonstrate a knowledge of the FFA Code of Ethics and the proper use of the FFA jacket.

5. Demonstrate knowledge of history of the organization, chapter constitution and bylaws and the chapter Program of Activities.

6. Have access to the Official FFA Manual and the FFA Student Handbook.

7. Submit a written application for the Greenhand FFA Degree. 
Section K. Chapter FFA Degree. Minimum qualifications for election:

1. Must have received the Greenhand FFA Degree.

2. Must be enrolled in their second year of agricultural education and have an approved Supervised Agricultural Experience Program.

3. Participate in planning and conducting of at least three official chapter functions.

4. Have earned at least $\$ 150.00$ or worked at least 45 hours and have developed plans for the growth of their SOEP.

5. Have effectively led a group discussion for 15 minutes.

6. Have demonstrated five procedures of Procedure Law.

7. Show progress towards individual achievement in the FFA awards' programs.

8. Have a satisfactory scholastic record.

9. Submit a written application for the Chapter FFA Degree.

Section L. State FFA Degree. Minimum qualifications for election:

1. Qualifications for the State FFA Degree are those set forth in the Constitution of the National FFA Organization.

Section M. American FFA Degree. Minimum qualifications for election:

1. Qualifications for the American FFA Degree are those set forth in the Constitution of the National FFA Organization.

Section N. Special committees shall review the qualifications of members and make recommendations to the chapter concerning degree advancement.

\section{Firiciale II. Orficars}

Section A. The possible FFA offices for the Madera FFA Chapter shall be as follows:
1.) President
6.) Reporter
11.) Sweetheart
2.) Vice President
7.) Sentinel
3.) Second Vice President
8.) Historian
4.) Secretary
9.) Chaplain
5.) Treasurer
10.) Parliamentarian

Section B. The Officers shall be elected or confirmed by a majority vote of the active members.

* The advisors and current chapter officers have the right to operate outside of the constitution for special circumstances not addressed.

Section C. If more than one student desires the office of president, the candidates ranking $1,2, \& 3$ by vote for office of chapter president shall be elected as president, vice president, and 2 nd vice president, respectively. 
Section D. The nominating committee shall be composed of the 12th grade chapter officers and the FFA advisors, others to be appointed if necessary. Upon reviewing officer applications for chapter office, students will be slated as candidates on the ballot.

Section E. All officer vacancies, during the term of office, shall be filled by a majority vote of the chapter officers with the exception of the president. The 1st Vice-President shall fill vacancy. The president shall nominate candidates for the committee's consideration.

Section F. Officers Eligibility. Minimum qualifications to run for chapter office:

1. Must be academically eligible to run for a FFA office.

2. For offices of President and Vice President, the applicant must have already completed at least three years of Agriculture classes, and/or hold the Chapter FFA Degree.

3. Other offices require that they have completed a year of an Ag class, and hold the Greenhand Degree.

4. Must enroll in the agriculture leadership class.

Section G. Officer Probation due to academic ineligibility

Any officer who becomes academically ineligible during their term of office will be put on a one-time probationary six-week suspension. At the end of six weeks a grade check will be due to the advisors. While officers are on probation they will not participate in any FFA affiliated activities. If the student meets grade requirements they will be immediately reinstated. If the officer remains academically ineligible they will be immediately removed from office.

* Grades will be based on quarter report cards and grade checks

* Officer participation during the probationary period will be at the advisors discretion.

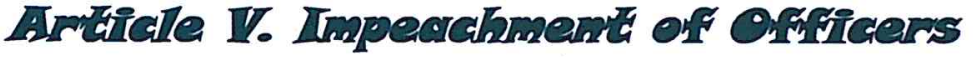

Section A. Immediate Impeachment.

The FFA advisors may at any time at their own discretion remove an officer who has repeatedly disregarded his/her duties by not fulfilling them to his/her best ability.

Section B. Steps of Impeachment.

1. Any FFA chapter officer not fulfilling the duties of the office as described by this constitution will be required to meet with fellow officers and advisors to discuss a plan for improvement.

2. A written plan for improvement will be drawn up by the advisor based on the conversation of the meeting in Step 1 , and will be confirmed and signed by the FFA President, Vice President, and the officer in question.

3. If the officer in question still does not fulfill his/her duties, then a $2 / 3$ vote of the executive committee will remove that officer from office. 


\section{Aricile VI. Executive Committee}

Section A. Executive Meetings shall be held as needed.

Section B. Standard meeting paraphernalia shall be used at each meeting. All special meetings shall open and close with the official ceremony. Parliamentary Procedure shall be used in transacting all business at each meeting.

Section C. Hats shall not be worn in the meeting room.

Section D. Poor conduct will result in that member being dismissed from the meeting room.

Section E. Delegates go through an application process headed by the Ag staff to be able to represent the chapter at the State Convention. The delegates may be named as necessary in order to have proper representation at various other FFA meetings within the state.

\section{Frifiale IIIT Dues}

Section A. As long as Incentive Grant funds are available dues shall be paid for all members through that source.

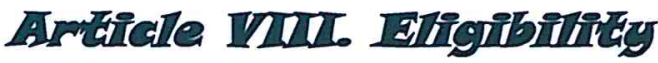

Section A. Eligibility of members exhibiting at fairs and shows will be based on the advisors discretion.

Section B. Members must be academically eligible to participate above the chapter level.

Section C. See rules of article IV section G of the Madera FFA Chapter

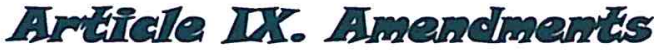

Section A. To amend the constitution, a majority vote of the Executive Committee is required.

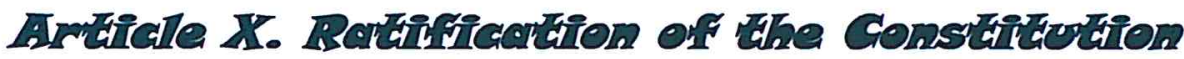

Section A. This constitution shall become effective when passed by the executive committee and advisors. 


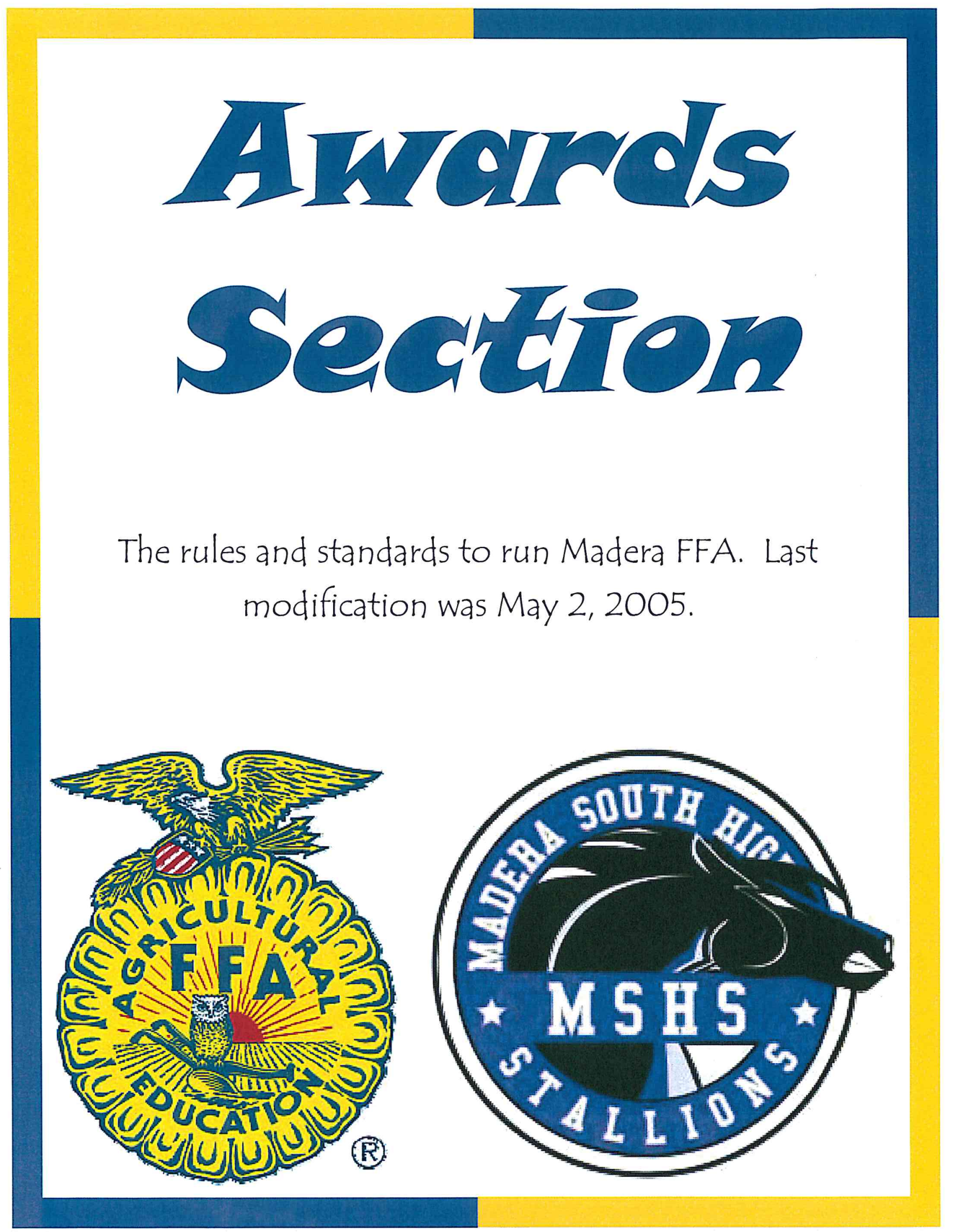




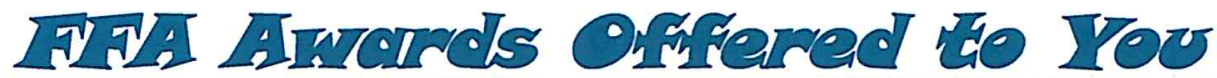

Madera FFA holds a fall and spring banquet where degrees and the following awards are given out.

\section{Degrees}

- Greenhand Degree: For $1^{\text {st }}$ year Ag students.

- Star Greenhand: Most Outstanding $1^{\text {1st }}$ year Ag student.

- Chapter FFA Degree: For $2^{\text {nd }}$ year Ag students.

- Chapter Star Farmer: Most Outstanding $2^{\text {nd }}$ year Ag student

- Chapter Star in Agribusiness: Most Outstanding 3rd or 4th year Ag students.

- State FFA Degree: For $3^{\text {rd }}$ and $4^{\text {th }}$ year Ag students.

- State Star Farmer: For $3^{\text {rd }}$ or $4^{\text {th }}$ year Ag students.

- State Star in Agribusiness: For $3^{\text {rd }}$ or 4th year Ag students.

- American FFA Degree: For $5^{\text {th }}$ year Ag student.

- American Star Farmer: For $5^{\text {th }}$ year Ag students.

- American Star in Agribusiness: For $5^{\text {th }}$ year Ag students.

\section{uudging Team Awards - See Career Development Event Pages}

Students who competed in any Career Development Events offered by Madera FFA will receive a certificate and pin. State Winning teams will be awarded with special recognition.

\section{Outstanding Ag Student Awards}

This award is given to the outstanding student in each grade level based on their academic success as well as involvement in the FFA.

\section{Outstanding Herdsmen Award}

This award is presented to a student in each specie category. The student must show great dedication and commitment to their animal project to even be considered for this award. This time spent with their specie includes at the farm as well as at livestock fairs and shows.

\section{Top 30 Award}

FFA points are awarded for students' involvement in FFA activities throughout the entire school year. The top 30 students earn an all expenses paid trip at the end of the year.

\section{Top Seller Award}

Any student who participates in fundraising activities and sells 20 tickets or items receives a free trip at the end of the school year. 


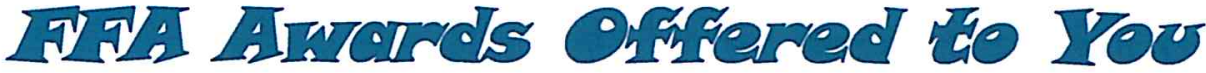

\section{Proficiency Awards}

These awards are given to students based on their SAE projects, and they are given at the Sectional, Regional, State, and National levels.

\section{Outstanding Ornamental Horticulture Student}

This award goes to a student who has a strong SAE in this project area. The student must also be enrolled in Ornamental Horticulture classes.

\section{Outstanding Small Engines Student}

For any student who shows their dedication to the Small Engines class, career development event, or SAE project.

\section{Outstanding Ag Mechanics Student}

This award is given to a student representing each grade level. The students must be enrolled in some kind of Agriculture Mechanics class, have a relating SAE, or be involved in the Ag Mechanics career development event.

\section{Outstanding Floral Design Student}

For a student who is taking any level of Floral Design classes. The recipient must have a relating SAE or career development event.

\section{Outstanding Animal Science Student}

This award goes to a student who has a strong SAE in this project area. The student must also be enrolled in Animal Science classes.

\section{Outstanding Overall SAE Project}

Presented to the student with the chapter's best SAE project. It can be from any project area.

\section{Petrucci Participation Award}

This award is given to the student who shows dedication to all areas of the Madera FFA Chapter; including classes, SAE's, career development events, fundraisers, and activities.

\section{Teco Award}

Given to a senior in the Ag Mechanics class with an outstanding SAE project.

\section{Stockli-Weiss Award}

This award is presented to a hard working individual who is devoted to the success of the chapter by involving themselves in activities outside their own projects on the school farm. The recipient of this award must be very dedicated person who is always willing to help others. 


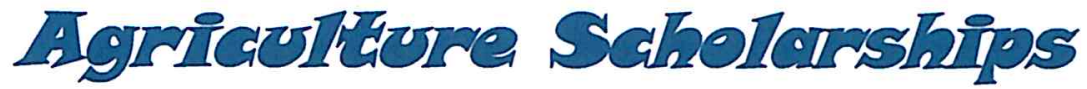

\section{Scholarship applications are available through the high school coun- seling office. It is your responsibility to get an application and return it by the deadline - GOOD LUCK!}

\author{
Matthew Roussel Memorial \\ $\$ 250$
}

This is a memorial scholarship to honor an alumni FFA member who has passed away. It is awarded to a student who has been active in our FFA Organization. The application for this scholarship is the general MHS scholarship application form. By meeting the application requirements and deadline, the school scholarship committee will select a recipient.

\section{Madera Agriculture Youth Association (M.A.Y.A.) $\$ 500$}

The MAYA association is pleased to offer a scholarship program for those high school students pursuing a career in an agriculture related field.

Eligibility requirements:

1. A 2.5 cumulative grade point average.

2. Application must be planning to enroll as a full time student (MINIMUM 12 UNITS)

3. Must be a resident of Madera County and an active FFA member of Chowchilla, Madera, Firebaugh or Yosemite High Schools or a Madera County 4-H Club.

\section{Friends of Madera FFA}

This is a scholarship is awarded to a student who has been active in the Madera FFA Organization. The application for this scholarship is the general MSHS scholarship application form. By meeting the application requirements and deadline, the school scholarship committee will select a recipient.

\section{Outside Organizations}

Other Agricultural Community Organizations also have scholarships available to students pursuing degrees in Agriculture, check with the following organizations for specific qualifications and deadlines.

- Calcot Seitz Foundations

- California Women for Agriculture

- Madera County Farm Bureau

- California Farm Bureau Fed- . eration
- World Ag. Expo

- National FFA (Online)

- California FFA

- California State Fair

- California Table Grape Commission
- Cattleman's

- CANERS Foundation

- Chowchilla Western Stampede

- Also check with the campus' you are applying to for other scholarships. 


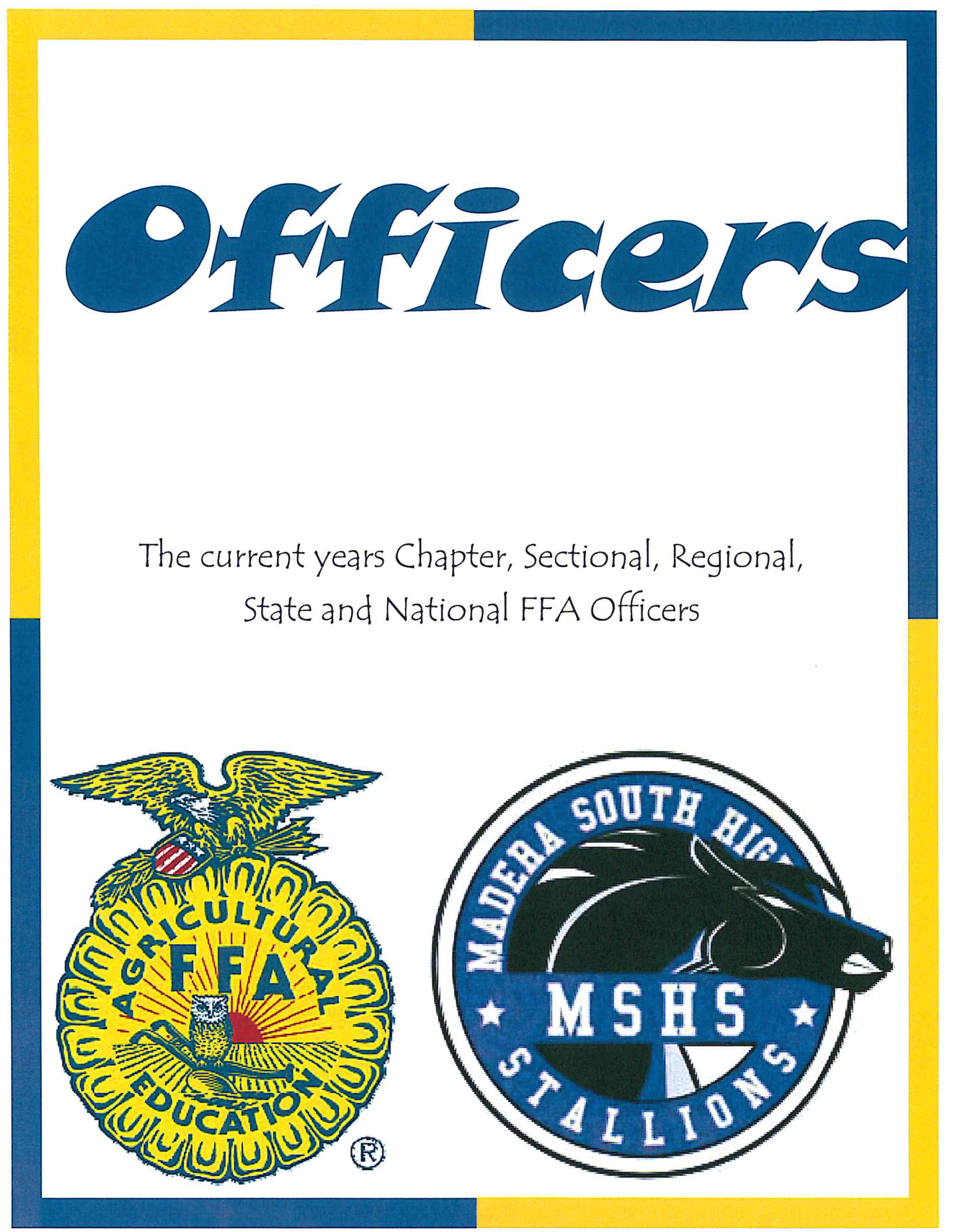


Madera FFA Chapter Officers

President

Vice President

2nd Vice President

Secretary

Treasurer

Reporter

Sentinel

Historian

Chaplain

Parliamentarian
Virat Kang

Jimmy Beavers

Jaime Cuevas

Jenae Hansen

Michael Ewing

Jorge Mendoza

Gabrielle Ortega

Mario Alvarez

Sarah Reece

Clayton Sheehan

\section{West Fresno Madera Sectional FFA Officers}

President

Vice-President

$2^{\text {nd }}$ Vice President

Secretary

Treasurer

Reporter

Sentinel

Historian

Chaplin

Parliamentarian
Amber LaSalle, Firebaugh

Brianne LeBeau, Fresno Central

Kellen Habib, Caruthers

Mackenzie Meek, Central West

Sean Pimentel, Central West

Kiana Peter, Chowchilla

Jimmy Beavers, Madera

Brandon Miller, Central West

Llimy Garcia, Mendota

Jacob Quinteros, Firebaugh
President

East Fresno/Madera VP

Tulare Kings VP

Sequoia VP

South Valley VP

Kern VP

$W F / M V P$

Secretary

Treasurer

Reporter

Sentinel

Advisor

\section{San Joaquin Regional FFA Officers}

Dipak Kumar, Tulare

Emilie Gambril, Clovis East

Hattie Jameson, Golden Valley

Bailey Minday, Tulare

Madion Zittle, Frontier

Joseph Agular

Amber LaSalle, Firebaugh

Evie Starich, Hanford

Brant Hall, O'neals Minarets

Virat Kang, Madera

Kristyin Fletcher, Sierra

Charles Parker 


\section{California State FFA Officers}

President

Vice President

Secretary

Treasurer

Reporter

Sentinel

Advisor
Riley Nelson

Valerie Canas

Gabrielle Franke

Gage Willey

Sheldon Overton

Hunter Berry

Bob Hueval

\section{National FFA Officers}

President

Secretary

Eastern Vice-President

Central Vice-President

Southern Vice-President

Western Vice - President
Clay Sapp, Florida

Katie Hall, Georgia

Joenelle Futrell, Kentucky

Brennan Costello, Nebraska

Wiley Bailey, Alabama

Lindsey Anderson, California

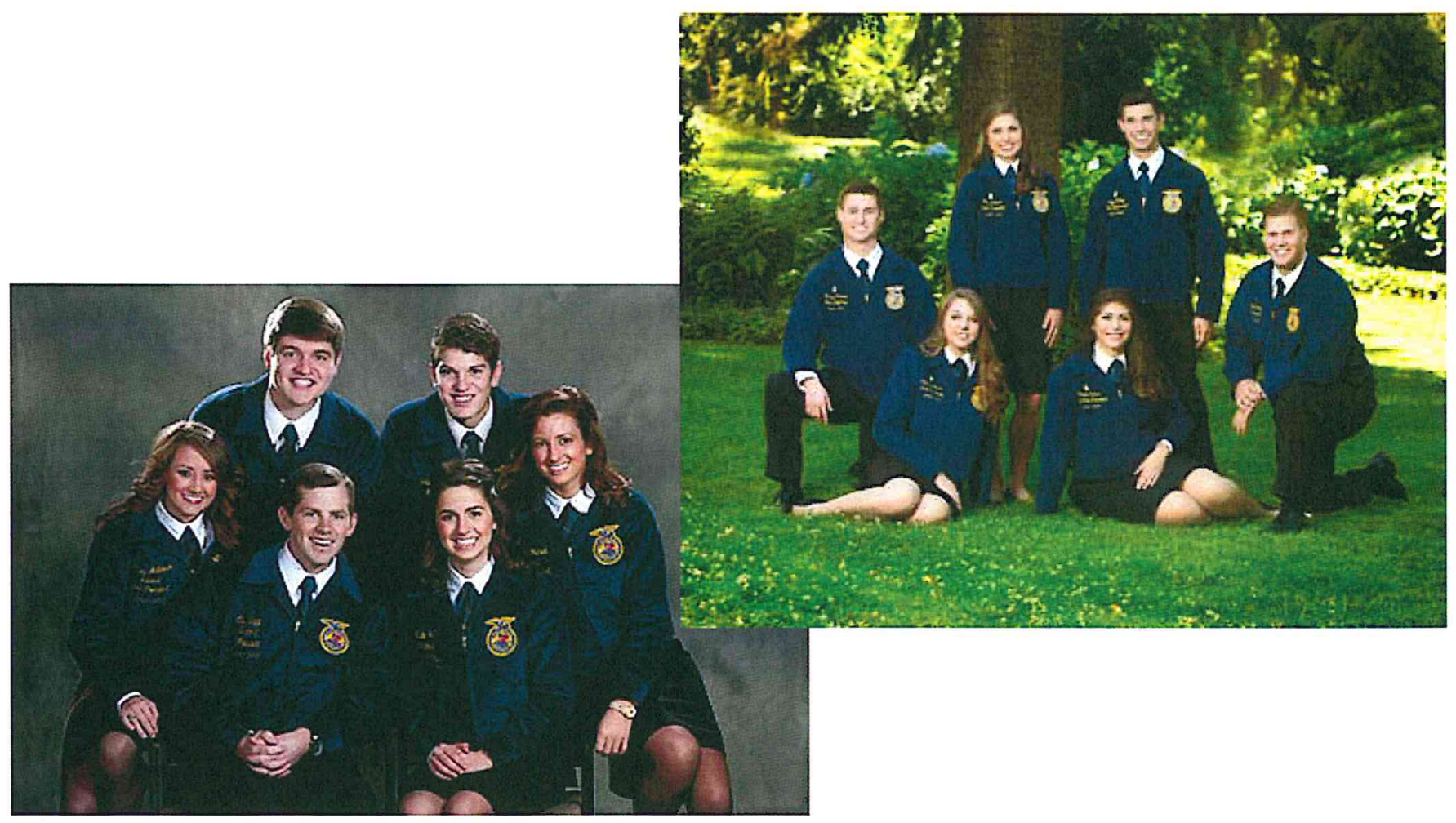




\section{3-2014 Madera FIA Officer Info}

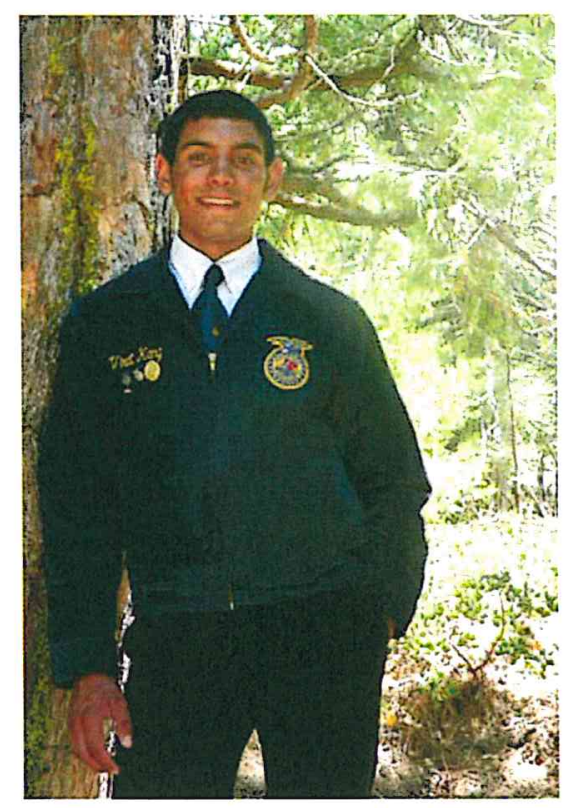

\section{President, Vurert Kang}

Grade: Junior

SAE: Ag Sales, Forage Production, Dairy Replacement Heifer CDE: Small Engines, Extemporaneous Public Speaking, Tree Pruning

Vice President, James Beavers

Grade: Senior

SAE: Dairy Replacement Heifer, Market Hogs

CDE: Vine Pruning, Extemporaneous Public Speaking
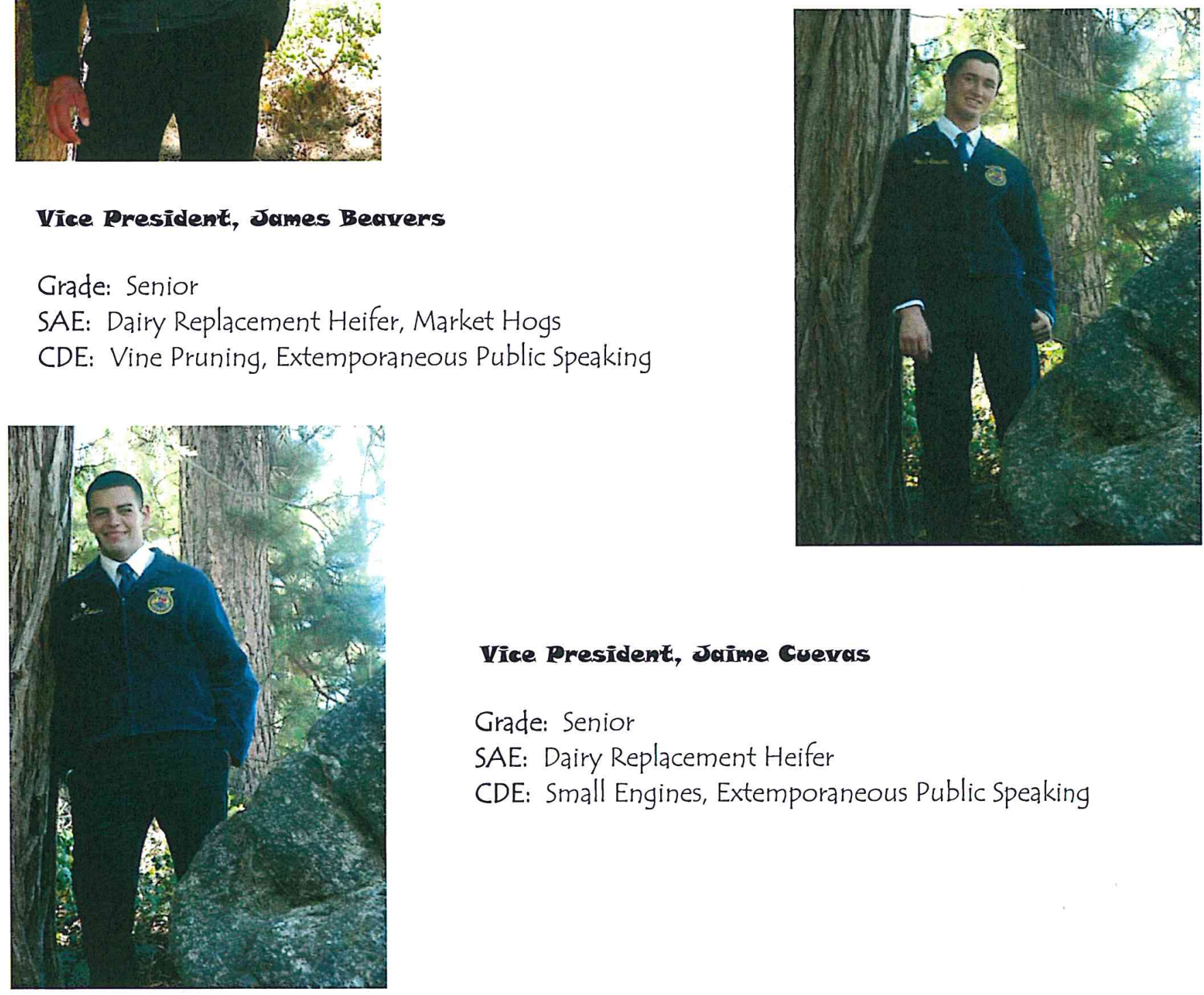

\section{Vice President, Jaime Cuevas}

Grade: Senior

SAE: Dairy Replacement Heifer

CDE: Small Engines, Extemporaneous Public Speaking 


\section{B-2014 Madera FAR Onficer Info}

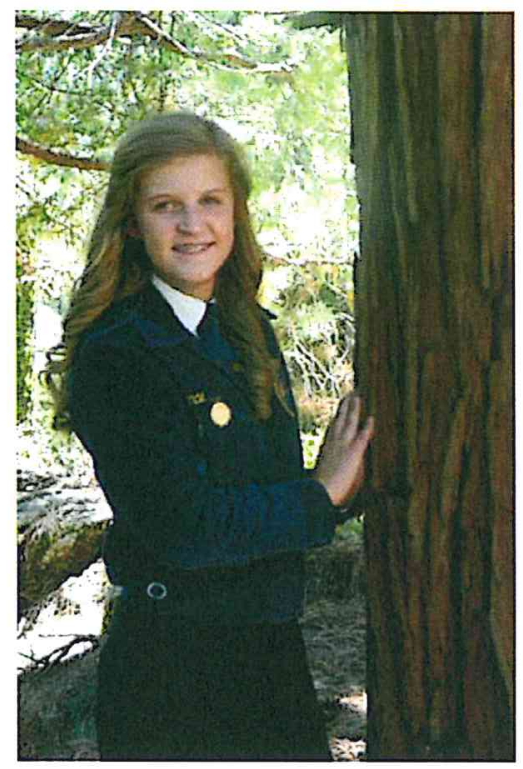

\section{Secretary, Jenae Hansen}

Grade: Junior

SAE: Dairy Replacement Heifer, Market Hogs, Viticulture CDE: Prepared Public Speaking, Tree Pruning, Cotton Judging, Vine Judging

Treasurer, IMichael Ewing

Grade: Junior

SAE: Market swine

CDE: Job interview, Vine Judging
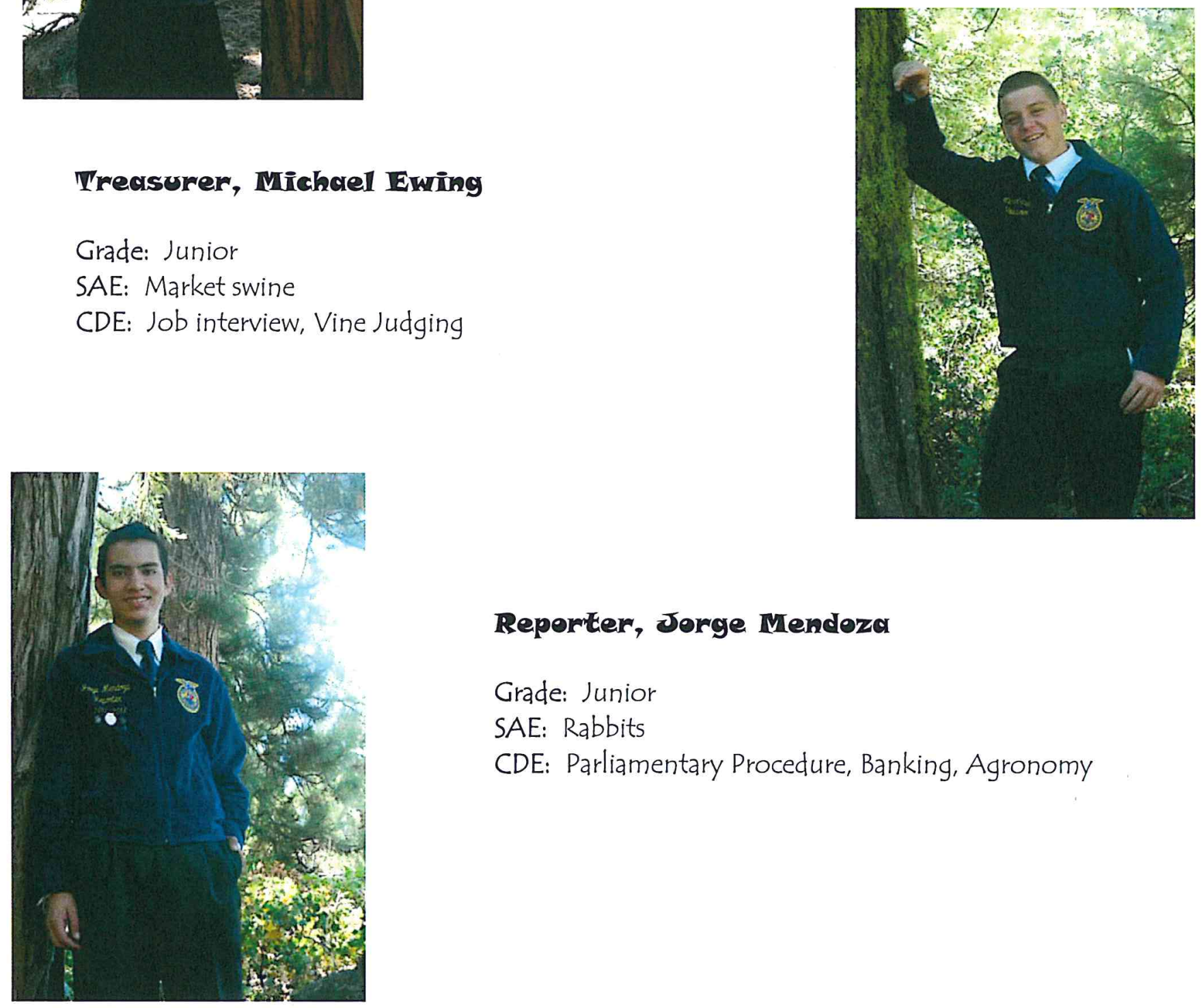

\section{Reporter, Jorge Mendozd}

Grade: Junior

SAE: Rabbits

CDE: Parliamentary Procedure, Banking, Agronomy 


\section{3-2014 Madera FAA Onficer Info}

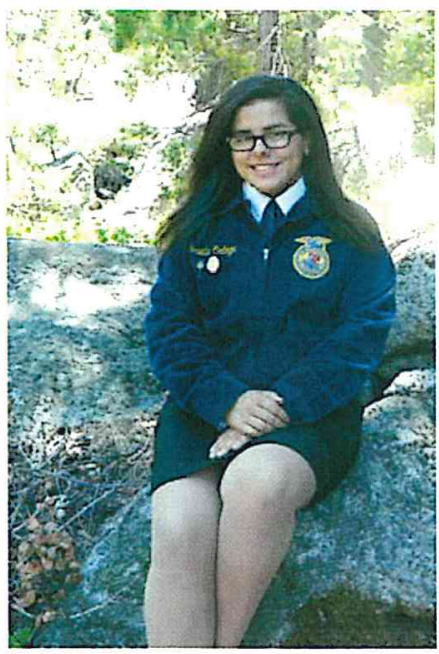

Mistorien, IMario Alverez

Grade: Sophomore

SAE: Market sheep

CDE: Banking, Impromptu Public Speaking

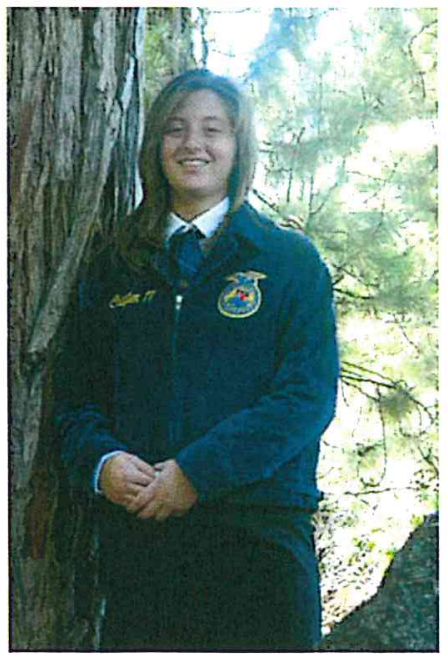

Poriliamentarion, Glayton

Sheehan

Grade: Sophomore

SAE: Diary Replacement Heifer, Swine Breeding, Market Swine

$C D E$ : Vine Pruning, Agronomy, Advance

\section{Choplain, Sarrah Reece}

Grade: Sophomore

SAE: Market Hogs

CDE: Vine Pruning, Impromptu Public Speaking
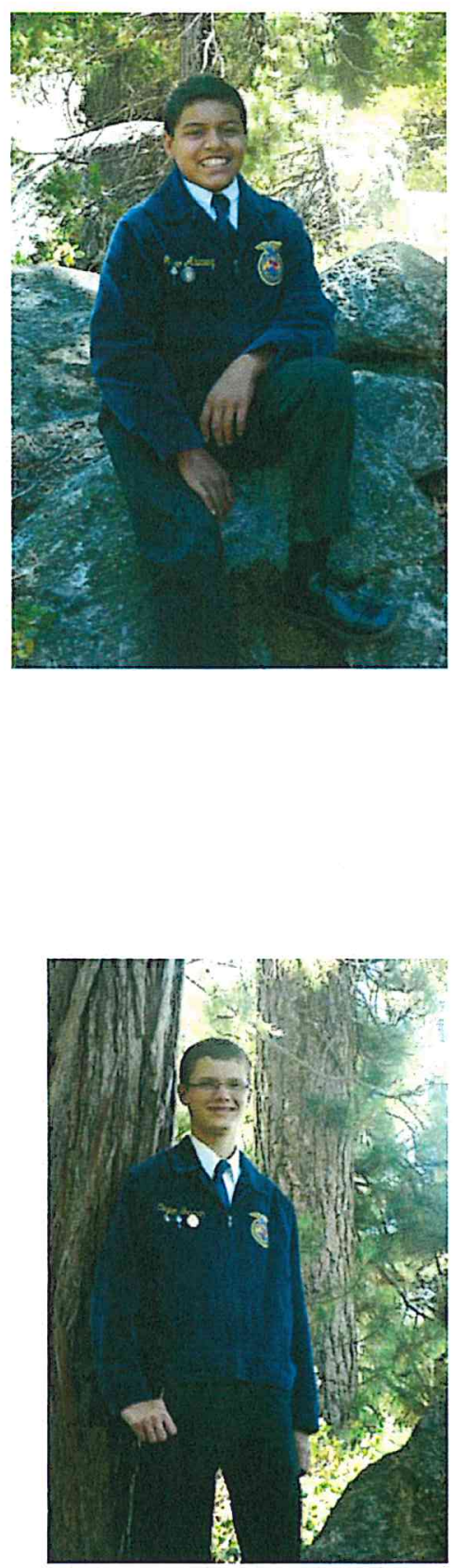


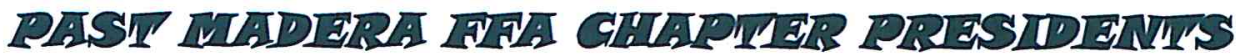

2001-02

2002-03

2003-04

2004-05

2005-06

2006-07

2007-08

2008-09

2009-10

2010-11

$2011-12$

2012-13

2013-14

* Took over after resignation
Nick Davis

Brandon Visscher

Giana Toschi Megan Matteucci *

Megan Matteucci

Cody Ogletree

Mika Petrucci

Jessica Sydney

Dominic Bettini

Amy Evans

Michael Valencia

Taylor Helton

Virat Kang

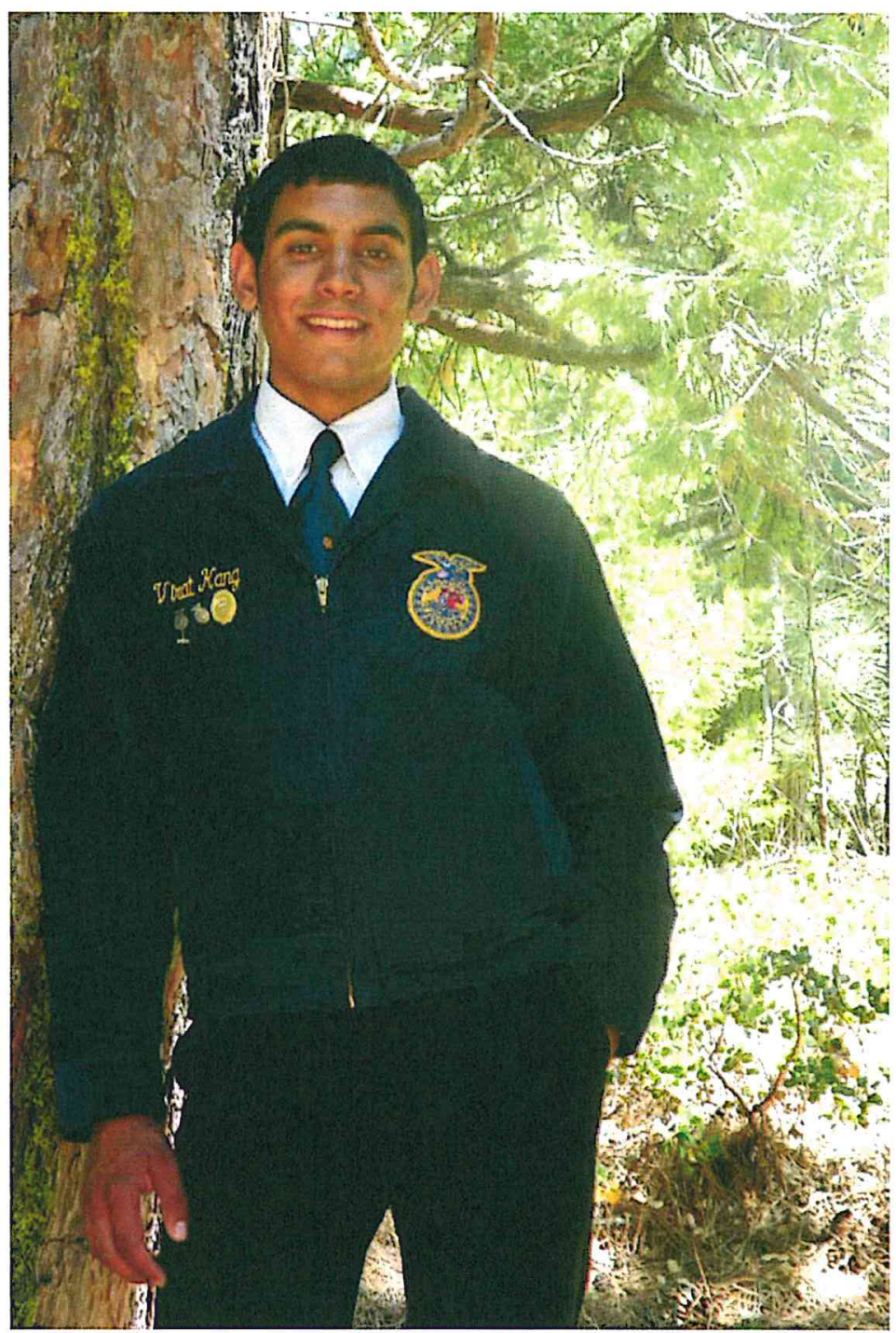




\section{ITissions and Striträties}

"FFA makes a positive difference in the lives of others by developing their potential for premier leadership, personal growth and career success through agricultural education."

To accomplish this mission, we the FFA will:

1. Develop a competent and assertive agricultural leadership program.

2. Increase awareness of the global and technological importance of Agriculture and its contribution to our well-being.

3. Strengthen the confidence of agriculture students in themselves and their work.

4. Promote the intelligent choice and establishment of an agricultural career.

5. Encourage achievement in supervised occupational experience programs.

6. Encourage wise management of the economic, environmental, and human resources of the community.

7. Develop interpersonal skills in teamwork, communication, and human relations.

8. Build character; promote citizenship, volunteerism, and patriotism in our students and community.

9. Promote cooperation and cooperative attitudes among all people.

10. Promote healthy lifestyles.

11. Encourage excellence in scholarship.

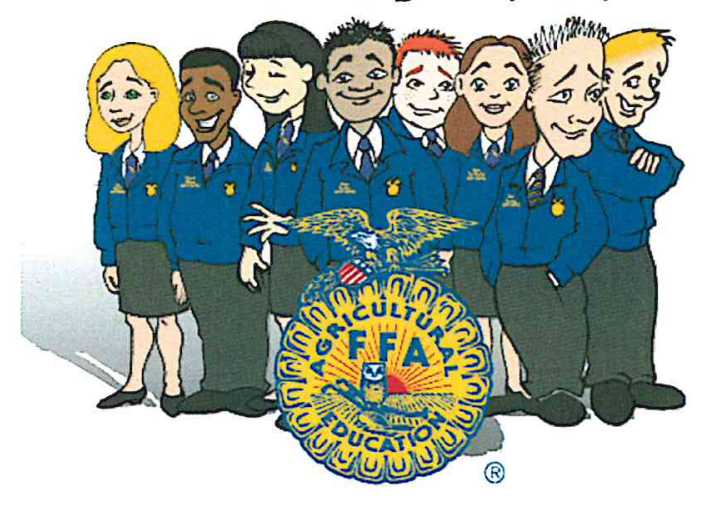




\section{FFA Emblem, Officior Colors, E FA Motto}

The National FFA emblem consists of five symbols and represents the history, goals and future of the organization. As a whole, the emblem covers the broad spectrum of the FFA and agriculture. Each element within the emblem has unique significance. THE CROSS SECTION OF THE EAR OF CORN provides the foundation of American agriculture. It is also a symbol of unity, because corn is grown in every state of our nation. THE RISING SUN signifies progress and holds a promise that tomorrow will bring a new day glowing with opportunity. THE PLOW signifies labor and tillage of the soil, the backbone of agriculture and the historic foundation of our country's strength. THE EAGLE is a national symbol, which serves as a reminder of our freedom and ability to explore new horizons for the future of agriculture. THE OWL, long recognized for its wisdom, symbolizes the knowledge required to be successful in the agriculture industry. The words "AGRICULTURE EDUCATION" and "FFA" are emblazoned in the center to signify the combination of learning and leadership necessary for progressive agriculture.

\section{Colors}

As the blue field of our nation's flag and the golden fields of ripened corn unify our country, the FFA colors of "NATIONAL BLUE and CORN GOLD" give unity to the organization. All FFA functions and paraphernalia should proudly display the colors.

\section{Motto}

The FFA motto gives members twelve short words to live by as they experience the opportunities in the organization.

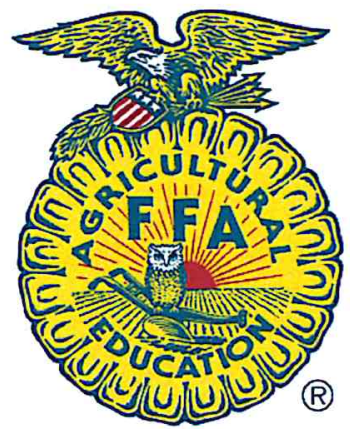

Learning to Do,

Doing to Learn,

Earning to Live,

Living to Serve.

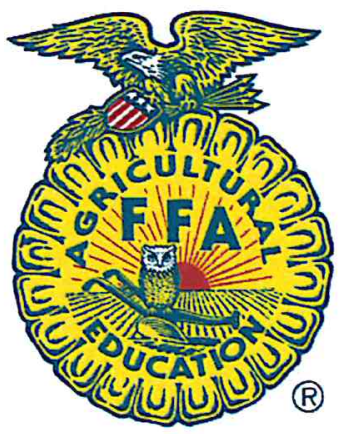




\section{The Fir Greed}

The creed was written by E.M. Tiffany, and adopted at the 3rd National Convention of the FFA. It was revised at the 38th Convention and the 63rd Convention.

I believe in the future of agriculture, with a faith born not of words but of deeds-achievements won by the present and past generations of agriculturists; in the promise of better days through better ways, even as the better things we now enjoy have come to us from the struggles of former years.

I believe that to live and work on a good farm, or to be engaged in other agricultural pursuits, is pleasant as well as challenging; for I know the joys and discomforts of agricultural life and hold an inborn fondness for those associations which, even in hours of discouragement, I cannot deny.

I believe in leadership from ourselves and respect from others. I believe in my own ability to work efficiently and think clearly, with such knowledge and skill as I can secure and in the ability of progressive agriculturists to serve our own and the public interest in producing and marketing the product of our toil.

1 believe in less dependence on begging and more power in bargaining; in the life abundant and enough honest wealth to help make it so - for others as well as myself; in less need for charity and more of it when needed; in being happy myself and playing square with those whose happiness depends upon me.

I believe that American agriculture can and will hold true to the best traditions of our national life and that I can exert an influence in my home and community which will stand solid for my part in that inspiring task. 


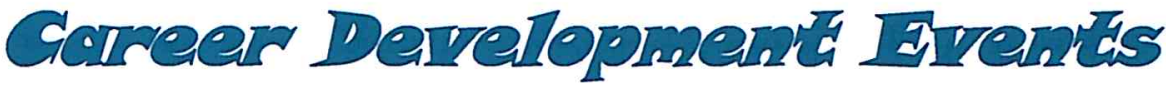

The following list are CDE teams and competitions you can get more involved in check with the Ag. Teacher for more Information.

\section{Agrriculiture. Telliling}

To evaluate the contestant's manipulative skills, general knowledge and professional presentation as these correlate to his/her preparation for employment in the broad field of welding (agricultural, industrial, or other). Coach: Mr. Deniz

\section{Bernking}

Members complete a written test made up of financial standings, such as checks and bank statements. You will be expected to know the different rates of credit that may be given to you. Coach: Mrs. McKenna

\section{Bestis Intormed Greenthernd}

This contest is for freshmen FFA students only. Members on this team complete a written test on their knowledge of the FFA. Coach: Mrs. Sheehan

\section{Cooperaritive IITrikering}

This contest is designed to create an awareness and understanding of the basic elements of farm product marketing and farmer cooperation in marketing, purchasing, bargaining, and service. Coach: Mr. Williams

\section{Cotion Judging}

This contest is made up of a three-member team. Members are required to take a written exam of their knowledge of cotton. Members will also be required to judge and score different types of cotton.

Coach: Ms. Gilles

\section{Greed Specting}

This contest is for freshmen FFA students only. Students memorize and recite the FFA Creed written by E.M. Tiffany and answer three oral questions from a panel of judges. Coach: Mr. George

\section{Dexirg Proderéts}

In this contest students learn about the Milk Industry through a test. In addition students verify whether dairy products are real vs. artificial, know different cheese varieties and are able to identify off flavors in milk. Coach: Ms. Luera

\section{Extemporaneous Publia Specking}

Members deliver a speech on one of three agricultural topics after they are given thirty minutes to prepare a four-to-six minute speech. At the conclusion of the speech, the judges may question the speaker for five-minutes. Decisions are based on factors similar to the prepared event. Coach: Miss. Luera

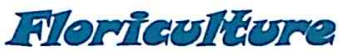

Members demonstrate proficiency in plant identification, judgment of floral and foliage arrangements, problem solving, and skills that include flower arranging and corsage construction. Coach: Ms. Gilles 


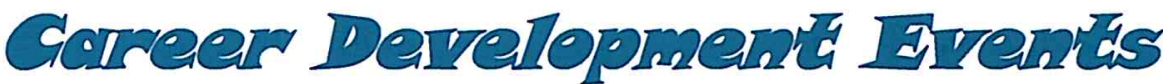

\section{Impromptü Spearking}

Members are required to learn about many agriculture topics, and will be given 1 minute to think about a topic they draw and then deliver a 1-2 minute speech from memory. There is a question and quote round. Coach: Mrs. McKenna

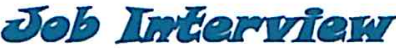

Members are required to create a cover letter and resume prior to participating in a job interview. You are evaluated and placed according to your resume, cover letter, and interview scores. Coach: Mr. Deniz

\section{Mears}

Members complete a written test; evaluate beef carcasses in terms of quality and yield grades; and judge and place beef, lamb and pork classes. Members also complete a meat formation problem and cooperatively fulfill a beef carcass order. Coach: Mr. George

\section{Norserey/Landsempe}

The Nursery/Landscape contest prepares students for careers in the nursery and landscaping. Topics include plant identification, plant physiology, soil science, plant reproduction, and nursery production, as well as landscaping design, installation, and maintenance. Coach: Mrs. McKenna

\section{Opening/Giosing}

This contest is made up of a six-person team. Each member of the team is responsible for memorizing one officer part of the opening and closing ceremonies and reciting it at the sectional contest. Coach: All Ag. Teachers

\section{Preparied Publia Speaking}

The member that chooses this speaking contest is to write and memorize a six-eight-minute speech on a major agriculture issue. The individual will be scored on his or her ability to speak and also on how well they can answer questions on the topic they choose. Coach: Mr. Williams

\section{Penriliementisnry Procedure}

The team consists of six members. One member of the six person team serves as the chair and another as the secretary. Participants enter a room and have one minute to study a card that has five motions on it. The card also has a main motion on the card that the members must debate. In order to score top points from the judges the team must perform the five motions on the card and five more motions that are not listed on the card. Each team member will also debate the main motion four times each. A round in Parliamentary Procedure is timed for ten minutes and thirty seconds. At the end of the round judges ask the team questions relating to Parliamentary Procedure. There is also a twenty five question written test. Coach: Novice, Ms. Luera Advanced: Mrs. Sheehan

\section{Sarerpbook}

The Nursery/Landscape contest prepares students for careers in the nursery and landscaping. Topics include plant identification, plant physiology, soil science, plant reproduction, and nursery production, as well as landscaping design, installation, and maintenance. Coach: Mrs. McKenna 


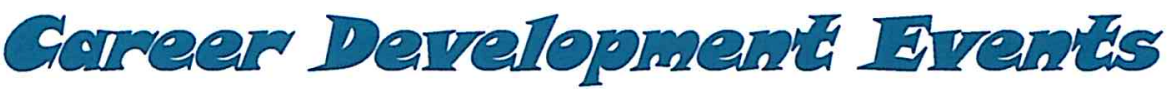

\section{Simerill Engines}

A team is made up of three members. Members are tested on identification, theory, problem solving and troubleshooting related to small engine repair. They are required to fix an engine and take a written test. Coach: Mr. Williams

\section{Vine Judging}

The teams consist of three of four members. There are four classes of pruned vines that will be judged by each member of the team for twenty minutes apiece. The member will then give oral reasons for each class of vines. Coach: Mr. Williams and Mrs. McKenna

\section{Vine Proing}

Members prune three classes of grapevines ranging from table grapes to wine grapes. The contest consists of a timed pruning and a written exam. The contest may include questions from the judges after a participant is done pruning it. Coach: Mr. Williams and Mrs. McKenna
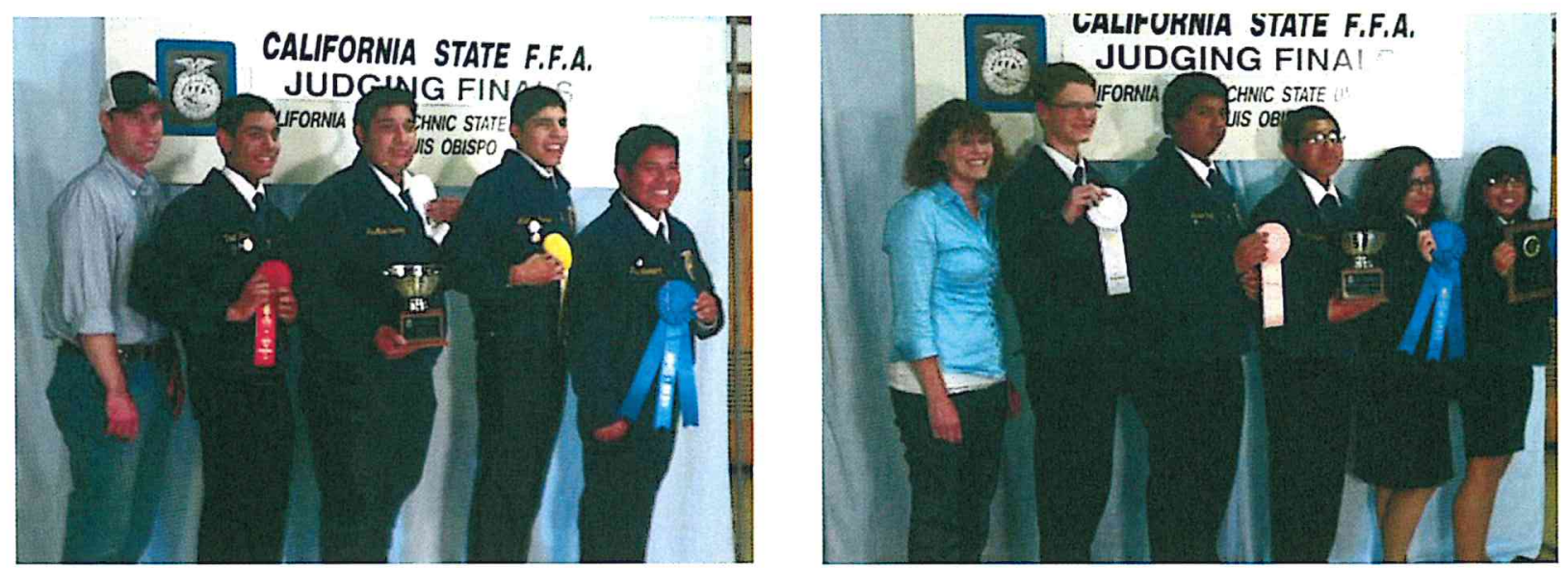

Last years State Winning Vine Pruning, Best Informed Greenhand and Meats Evaluation

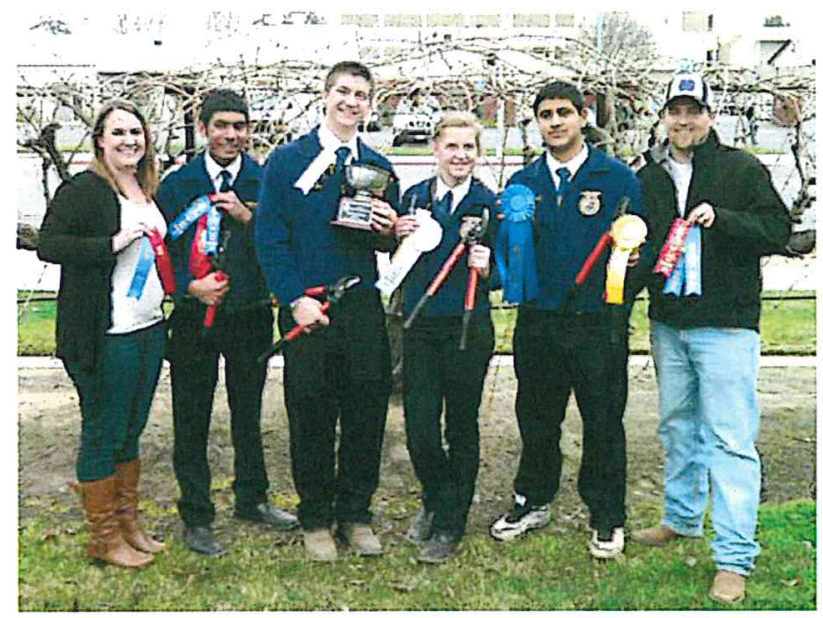




\section{Here is an excmple of the Exhibitors Controat Signed by mem= bers a carbon copy sheet must be obtained by a projeat ddivisor.}

\section{Madera FFA Exhibitors Contract}

As a member of the Madera FFA chapter, I realize that there are certain obligations on my part in order to assure a successful project. As part of my obligations, I agree that the following expectations will help me to complete my animal project. This contract shall begin on and terminate on

All students shall be respectful to parents, advisors, staff and students.

All students shall be in good standing, a current member of the FFA, and maintain a 2.0 GPA throughout the duration of the project. No students shall show/exhibit an animal project as a member of the Madera FFA without an Exhibitors Contract signed by 1) the exhibitor, 2) parents/guardians, 3) chapter advisor and 4) the vice principal.

All school rules, district, and California State rules and the specific rules pertaining to the show in question shall be upheld by the exhibitor.

Throughout the term of the project all exhibitors are to follow the directions and advice given to them by the designated advisor for that species.

All exhibitors will be responsible for the care, feeding, exhibiting and marketing of their animals.

$\downarrow$ All students will participate in showmanship and $80 \%$ of the scheduled showmanship practices prior to the fair.

$\downarrow$ All students will show in the "Official FFA Show Uniform" 1) white pants, 2) white collared shirt, 3) FFA jacket and 4) FFA Tie/Scarf.

$\downarrow$ No students shall be on the fairgrounds after 9:00 PM without the written permission from the parents/guardians or supervised Ag. Staff.

$\downarrow$ Student may transport themselves and siblings with written approval from 1) Parents/Guardians, 2) School Administration (Permission in writing from the vice principal and parents/guardians and given to the advisor prior to the event.

$\downarrow$ No student shall leave the fairgrounds at anytime without the supervision of his/her parent/guardian, designated district chaperone or chapter advisor.

$\downarrow$ FFA members are required to obtain their homework from all of their teachers in advance of missing school for attending fairs.

+ Each exhibitor must read and understand the rules and regulations in the fair's premium book.

+ Each exhibitor is required to serve barn duties as assigned and specified by the project advisor.

All students will participate in the moving in and (Loading and setting-up) and removal (clean-up and removal of equipment from the show).

ALL Students shall be responsible for completing the following prior to their premium or market check being issued: 1) A complete \& up-dated record book, 2) thank-you letters to buyers, award donors and add-on bids 3) all bills paid in full and 4) all hours worked at the farm.

Failure to comply with any of the above obligations due to extenuating circumstances must be approved by the advisor. Failure to do so is in violation of the contract and will result in the loss of showing privileges effective immediately.

Student

Parent/Guardian

Advisor

School of Ag Vice Principal

\begin{tabular}{l}
$\overline{\text { Date }}$ \\
$\overline{\text { Date }}$ \\
$\overline{\text { Date }}$ \\
\hline Date
\end{tabular}




\title{
Here is on exrmple of the Farm Pollicy signed by members a carbon copy sheet must be obtorined by a project adivisor.
}

\author{
Madera South High School Farm Laboratory Policy Regarding Use
}

Project Owner:

Type of Project:

Advisor:

The sole purposes of the Madera High School Farm Laboratory are to provide a laboratory for hands-on instruction in a vocational agriculture class and to allow the students enrolled in vocational agriculture to conduct Supervised Agricultural Experience Projects (SAE).

1.Only a student currently enrolled in a vocational agriculture class or FFA program at Madera South High School shall have the privilege of using the School Farm Lab.

2.The School Farm Lab is part of the campus of Madera South High School; therefore, all Madera South High School and Madera Unified School District policies apply to the School Farm Lab.

3.The responsibility of the project owner (student) includes but is not limited to keeping all gates to the School Farm Lab locked, feeding, grooming, pen clean-up and maintenance, hauling feed/manure and other supplies, planting, irrigation, weeding, harvest, selling, and any care necessary to maintain the health and/or aesthetics of animals at the School Farm Lab.

4.The Madera Unified School District and its employees are NOT responsible for the loss, theft, disappearance, or death of any animal. The students are responsible for all personal equipment or materials of any kind.

5.No person shall drive or operate any vehicle on the School Farm Lab without prior permission or supervision from a Madera South High School vocational agriculture instructor.

6.School Farm Lab hours shall be 7:00 am to 9:00 pm. No one shall conduct any activity on the school Farm Lab during closed hours without prior permission from the advisor. ONLY current FFA members are allowed on the school farm grounds.

7.In the event of an emergency, if services of a veterinarian are utilized the fees of the services are the responsibility of the project owner (student) and/or parent/guardian. This excludes breeding projects owned by the Madera South Ag Department.

8.To assure proper care of livestock animals, animals are expected to be fed and/or checked twice daily. Morning feeding hours must occur between 7- 9 am and evening feeding hours shall be from 5-8pm.

9.A student exhibiting a project at the fair must have a signed exhibitors contract to accompany this contract.

10. Any infraction of school or district policy, any infraction of this agreement, or any lack of responsibility on the part of the student may result in the indefinite removal of the project from the School Farm Lab and/or the indefinite revoking of School Farm Lab use privileges of the students.

11.Administration and interpretation of all policies regarding use of the Madera South High School Farm Laboratory shall be the responsibility of the project advisor.

Notification or infraction of any of the aforementioned policies shall inherent the following disciplinary actions:

$1^{\text {st }}$ offense: A written warning

$2^{\text {nd }}$ offense: $\quad$ A written warning, letter sent home and phone call to parents

$3^{\text {rd }}$ offense: $\quad$ Removal of student project from school farm

Upon receipt of second offense, the student will have 1 week to remove his/her animal from the farm. The project must be paid, in full, prior to removal. Projects are not paid for in full will become school property. Projects will then be sold. Proceeds from the sale of the project will be credited to the student's financial obligations.

I have read and understand the policy of the Madera South High School Farm Laboratory and hereby comply in agreement with all aspects of this policy.

Parent/Guardian Signature

Date

Student Signature

Date

Advisor Signature

Date 


\section{MADERA SOUTH HIGH SCHOOL AGRICLTURE DEPARTMENT \\ EXHIBITOR CONTRACT}

FFA members have the opportunity to raise animal projects to exhibit at the fairs. This is a great learning experience but also a commitment. In order for all students to learn and work together, the following requirements are expected of each exhibitor regardless of where the animal is housed.

1. Attend all scheduled exhibitor meetings and work days.

2. Purchase the show supplies necessary for project.

3. Secure a buyer prior to the fair, by sending out buyers letters to community supporters.

4. Have a complete FFA show uniform.

5. Prior to receiving fair checks, students must update record books, clean facilities, write Thank You letters with an envelope and stamp (all letters need to be approved by advisor)

Animals housed off campus will be the sole responsibility of the students. Students who keep their animal at home are required to make arrangements with the advisor for project visits as well as contact the advisor when they need assistance.

\section{Student}

Parent

\section{Advisor}




\section{MADERA SOUTH HIGH SCHOOL AGRICULTURE DEPARTMENT PROJECT FACILITY CONTRACT}

If you choose to keep your animal at the MSHS farm you are required to maintain the following for the duration of your project.

1. Grades and Eligibility

* You must have a 2.0 GPA currently

* You must have a satisfactory attendance and behavior record

2. Project and Facility requirements - Feeding and Cleaning

* Feed animals according to the feed schedule

* Contact advisor when feed is low (10 bags)

* Clean your pen and surrounding areas every scheduled day you are assigned

* Attend all required workdays

* Feed and Clean on your assigned time and day

$\circ$ Morning feeding - 6:00 A.M. - 8:00 A.M.

- Evening feeding - 6:00 A.M. - 8:00 P.M.

- Sweep and wash alley

- Sweep feed room

- Take garbage to dumpster

- Shut all gates

- Turn lights on and off

- Turn fans and misters on and off

FAILURE TO FOLLOW THE ABOVE STANDARDS WILL RESULT IN THE "3 STRIKE METHOD". THE THIRD STRIKE WILL RESULT IN THE ANIMAL BEING REMOVED FROM THE BARN WITHIN 1 WEEK. IF YOU FAIL TO REMOVE THE ANIMAL IN THE ALLOTED TIME IT WILL BE REMOVED FOR YOU.

THIS CONTRACT IS DUE TO THE ADVISOR PRIOR TO KEEPING THE ANIMAL AT THE FARM

STUDENT

PARENT

ADVISOR 


\section{Madera Agriculture Department Steer Project Contract}

We are privileged to have a steer project facility for students to raise animals. You are not required to keep your steer at our facility. However, you are required to maintain the following standards for the duration of your project.

1. If the project is student owned (no loan with Madera FFA as co-signer) then the project may be kept at home. If the project was purchased by bank loan (with Madera FFA as co-signer) then the steer will be kept at the school farm facility.

2. Student must maintain a 2.0 grade point average, must not be on the non-privilege list, must not be on the "debt" list, and in good standing with the FFA chapter.

3. Daily observation and maintenance of you animal must be as follows:

- Feed and water at the appropriate times which will be arranged by project advisor

- Check your animals health (any signs of sickness notify your advisor or other Ag teacher)

- Clean pens \& surrounding areas (on-campus projects)

- Check feed - contact advisor when feed is down to 3 days, if purchased by school

- Attend all workdays if project is on campus, if animal is at home get in contact with advisor to go over any questions or concerns you may have about your project or vice-versa.

- All necessary feed for the project (if kept at school farm) will be provided by the school and billed to the student at the completion or sale of the project

4. Daily general facility responsibilities for projects kept on campus

- Sweep and clean alley way

- Sweep feed room

- Return equipment to proper place daily

- Take garbage to dumpster

- Shut all gates

5. Project Visits

- On campus projects will be supervised a minimum of 1 time a week.

- Off campus projects will be supervised periodically as arranged by advisor and student. A project supervision form will be filled following each visit and will be signed by the student, parent, and advisor.

6. The advisor will be the final say on whether the animal is being taken care of properly. If this is deemed to be a problem, see consequences below

7. After sale of the project at the fair, the student will pay off any and all loans and will be presented with a bill to include all feed, show supplies, etc. The bill is to be paid as soon as all fair checks are received or will be turned over to the district bookkeeper for collections. 


\section{Failure to follow the above standards will result in the following actions:}

\section{Student Project (no bank loan)}

1. Animal must be removed from farm within 1 week of notice if animal is being kept at school farm facility.

2. Student will not be allowed to show at this fair or the next fair (any animal or any project). This pertains to student's projects that are kept on campus and off campus.

3. If the animal is not removed within the 1-week time period the MSHS Ag department will remove the animal and take it to the auction where it will be sold for auction price. The student will still be responsible for paying the remainder of the cost of the project (feed, housing, medication, hoof trimming, etc.) if the auction price is not enough to cover the entire project cost.

\section{Student Project (bank loan)}

1. MSHS Ag department will remove and sell the animal at auction price.

2. Student is still responsible for covering the remainder of the cost of project (feed, medication, hoof trimming, etc.)

3. Student will not show at the fair and will not be eligible to show at the next fair (any animal project or other project)

Parents and students are encouraged to read this carefully. This contract will be followed strictly in order to ensure the proper use of the facility and care of the animal.

This contract is due to the advisor prior to purchasing the animal.

$\begin{array}{llll}\overline{\text { Student }} & \overline{\text { Date }} & \overline{\text { Principal }} & \overline{\text { Date }} \\ \overline{\text { Parent }} & \overline{\text { Date }} & \overline{\text { Advisor }} & \overline{\text { Date }}\end{array}$




\section{BEEF PROJECT PURCHASING CONTRACT}

Student must meet the following criteria in order to purchase a market hog:

1. This contract must be turned in to the project advisor by the assigned date or an animal will not be purchased for the student.

2. Once the animal has been purchased for the student, the student becomes liable for the entire cost of the project even if the student backs out of the project $\underline{O R}$ loses the project due to ineligibility (less than $2.0 \mathrm{GPA}$ ) or breaking contract rules.

3. Student must pay the cost of the steer ( $\$ 1000)$ but have the option of getting a loan through Madera Farm Credit for the above amount

4. The remaining balance of the project must be paid by June $1^{\text {st }}$ for Chowchilla Fair and September 30th for Madera Fair. However, no project is taken to the fair unless the project has been paid in full before the fair.

5. The project will become property of the Agriculture Department if proper payments are not received by the given due dates.

I understand and agree to the above terms.

Student

Date

Parent

Date

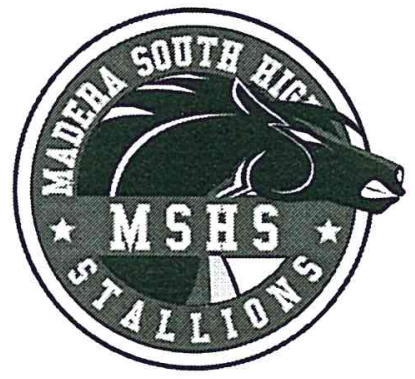




\section{Madera South High School Farm Laboratory
Policy Regarding Use - Goats}

Project owner:

Advisor:

The sole purposes of the Madera South High School Farm Laboratory are to provide a laboratory for hands-on instruction in a vocational agriculture class and to allow the Projects (SAE). at Madera South High School shall a vocational agriculture class or FFA program Lab.

2. The School Farm Lab is part of the campus of Madera South High School; therefore, all Madera South High School and Madera Unified School District

3. The responsibility of the project owner (student) includes but is not limited to supplies, and any care necessary to maintain the health and/or aesthetics of
animals at the School Farm Lab.

4. The Madera Unified School District and its employees are NOT responsible for the loss, theft, disappearance, or death of any animal. The students are responsible for all personal equipment or materials of any kind.

5. No person shall drive or operate any vehicle on the School Farm Lab without prior permission or supervision from a Madera South High School vocational

6. School Farm Lab hours shall be 7:00 am to 9:00 pm. No one shall conduct any from thive school Farm Lab during closed hours without prior permission grounds. This includes individuals feeding ans are allowed on the school farm

7. In the event of an emergency, if services of a veterinarian are utilized the fees of the services are the responsibility of the project owner (student) and/or parent/guardian. This excludes breeding projects owned by the MSHS farm.

8. Students must maintain a 2.0 GPA to maintain project animals with the Madera South High School Agriculture Department. The student shall provide the specie advisor with proof of academic eligibility. 


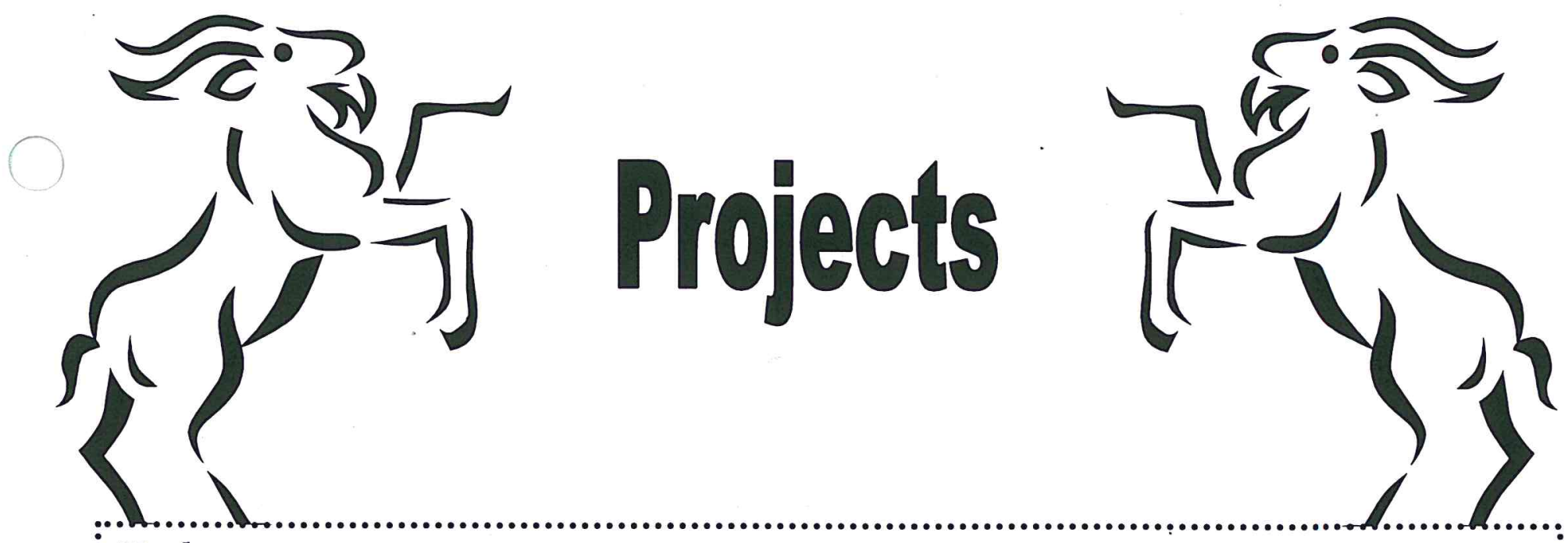

Herdsman:

Designed for students interested in a long -term project caring for MSHS goat herd

Responsibilities: Maintain herd through feeding, maintenance, and health care. General duties include, but are not limited to feeding, hoof trimming, vaccination, kid processing, veterinary care, pen/barn maintenance, kid management, and showing.

Time: 6 months; June to December or January to June

Requirements: Must have previously shown goats for the Madera FFA

*Assuming student has met appropriate requirements, the herdsman will receive 1 kid in return for adequate care of herd. Kid will be chosen with approval from the goat advisor.

Breeding Project:

Designed for students to achieve an introduction to goat management and goat showmanship

Responsibilities: Maintain MSHS doe(s) and/or kids through feeding, pen maintenance, and general health care

Time: 1 yr

Requirements: see contract

Market Project:

Designed for students interested in a short term market project for profit

Responsibilities: General care and maintenance of the students' goat, feed all market goats according to the market goat feed schedule, general care and maintenance of school facilities

Time: From time of purchase to time of sale (usually 60-90 days)

Requirements: Show project at the Fair 


\section{BREEDING ANIMAL CONTRACT}

The purpose of this project is to introduce the student to the responsibility of raising an animal. It is also designed to help the student learn responsibility while gaining animal science experience. To complete the project the student needs to abide by the following rules:

1. The student understands that the project animal is property of the Madera South High School Agriculture Department and cannot be sold by the student.

2. The student will be responsible for care and maintenance of the project animal. This includes, but is not limited to, feeding, watering, exercising, and vaccinating. The student will also agree to maintain facilities on the farm through weeding, repairs, raking, construction of new facilities, and/or removal of manure.

3. The student agrees to attend and assist with the birth and processing of offspring.

4. Madera South High School Agriculture Department will pay for all expenses incurred for the project. This includes feed, veterinary services, breeding fees, medicinal costs, and supplies.

5. Care of the project animal must extend a minimum of 60 days prior to the fair in which the animal will be shown.

6. If the student has done an acceptable job, the student may re-apply to care for a breeding project for another term.

7. If the student breeches this contract or the farm policy the project will no longer be the responsibility of the student upon notice from the advisor.

I have read the above breeding animal contract and agree to all items within: 

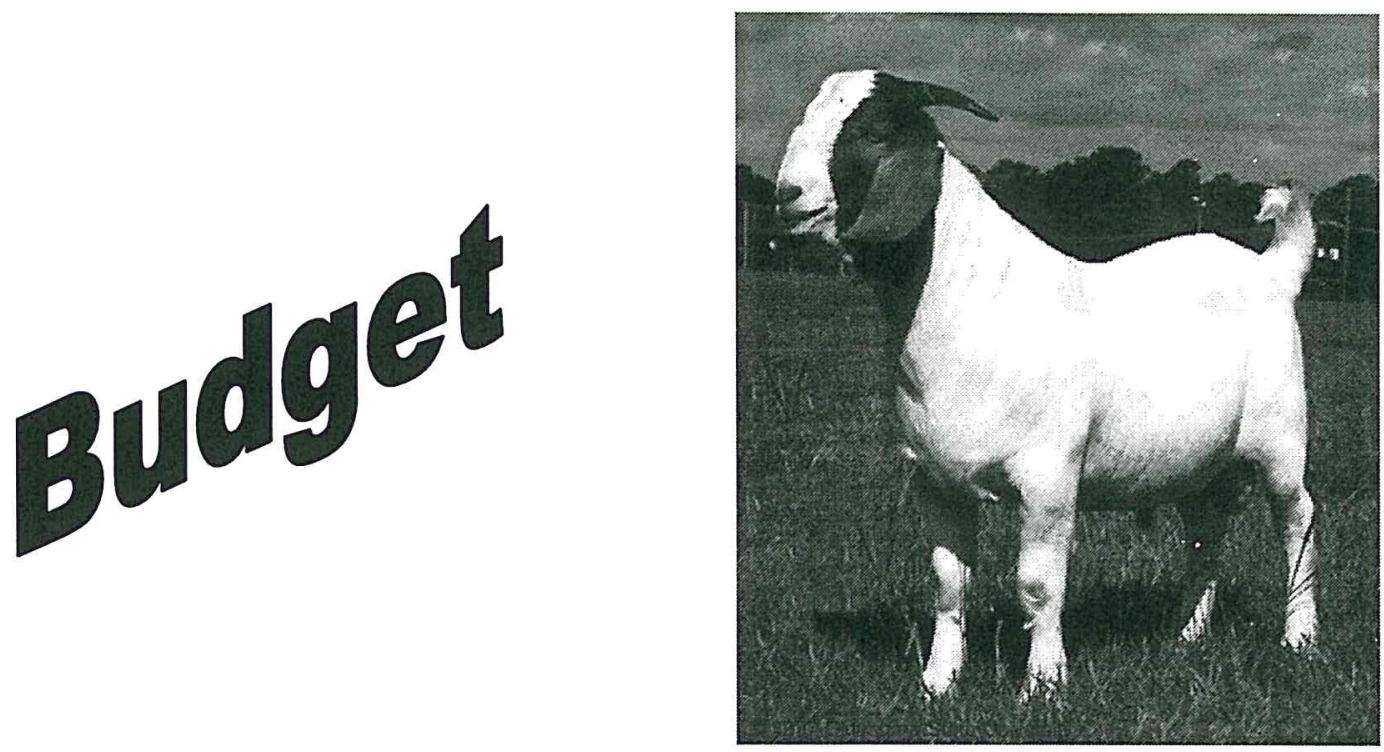

\section{Expenses}

\begin{tabular}{|c|c|}
\hline Cost & $\$ 200$ \\
\hline Feed & $\$ 100$ \\
\hline Mise & $\$ 40$ \\
\hline
\end{tabular}

Income

$\begin{array}{ll}\text { I00 Ib.x \$3.50/1b } & \$ 350 \\ \text { Bump bids } & \$ 100 \\ & \$ 450\end{array}$


Sheep Project

Information

Student Name

Advisor

Today's Date

Fair Destination

Payment Method

Date Check Needed

\section{Project Budget (Estimated Costs)}

Expense

Sheep

Feed (4 months)

Entry Fee

Insurance

Show/Vet Supply
$\$ 250.00$

$\$ 130.00$

$\$ 12.00$

$\$ 12.00$

$\$ 10.00$

Total Estimated Cost $\overline{\$ 414.00}$

Receipt

Sale Sheep @ Fair

This is a low estimate

130 lbs. @ \$3.25/lb.

$\$ 422.50$

Total Net Income

$\$ 8.50$ 


\section{SHEEP PROJECT PURCHASING CONTRACT}

Student must meet the following criteria in order to purchase a market lamb:

1. This contract must be turned in to the project advisor by the assigned date or an animal will not be purchased for the student.

2. Once the animal has been purchased for the student, the student becomes liable for the entire cost of the project even if the student backs out of the project $\underline{O R}$ loses the project due to ineligibility (less than 2.0 GPA or any F's) or breaking contract rules.

3. Student must pay the cost of the Sheep ( $\$ 250$ ) by January 9th for Chowchilla Fair and May $28^{\text {th }}$ for the Madera Fair.

4. The remaining balance of the project must be paid by June $1^{\text {st }}$ for Chowchilla Fair and September 30th for Madera Fair. However, no project is taken to the fair unless the project has been paid in full before the fair.

5. The project will become property of the Agriculture Department if proper payments are not received by the given due dates.

I understand and agree to the above terms.

Student

Date

Parent

Date

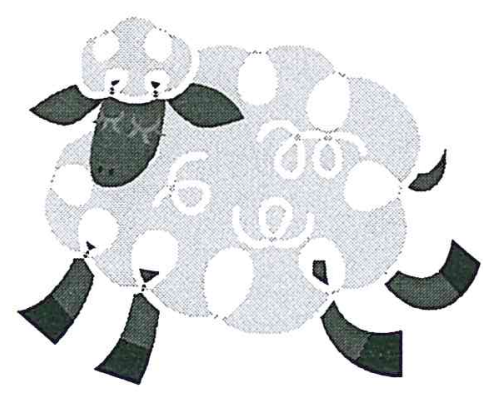




\section{Madera South High School \\ Greenhouse Use Contract}

Student (print)

Date

Year

The Madera South High School Agriculture Department will provide a greenhouse facility at 705 W. Pecan Ave. Madera, CA 93637 under the following terms:
A. The student agrees to pay all expenses incurred to Madera FFA.
B. Make sure that the plants are properly cared for on a daily basis during the school week based on the project advisor's instructions.
C. Keep the greenhouse, outdoor areas, container storage, and tool room clean at all times including proper supply storage and labeling.
D. Students must maintain communication with the Ag. Instructor with any problems they encounter.
E. Participate in scheduled school farm clean-up days.
F. Students must be present at the scheduled plant sales (Saturday) in order to make a profit off of their projects.
G. Students may leave both class projects and their own plants (with permission of the instructor) in the greenhouse.

Your signature below verifies that you have read, discussed, understand, and agree to abide by these rules. Please sign and return this form to the project advisor. If you have any questions at any time, please feel free to contact the advisor at the Madera Ag Department (675-4475).

Parent Signature:

Student Signature: 


\section{MADERA FUTURE FARMERS OF AMERICA AG DEPARTMENT-CONDUCT RULES}

The Madera Chapter of the Future Farmers of America (FFA) has established rules and regulations to increase the learning activity at FFA activities.

Exhibitors will be required to comply with the following rules and regulations without exception. Violations of any of the provisions listed in this contract will result in suspension from Madera FFA for a period of one year and loss of FFA office if he/she is an officer.

A student may appeal for probation status at the end of six months. It may or may not be granted depending on the rule or rules violated.

\section{IF AN EXHIBITOR'S CONDUCT IS SUCH AS TO REFLECT DISCREDIT ON HIS/HER CHAPTER OR CLUB, IT MAY BE NECESSARY TO SEND THE EXHIBITOR HOME.}

\section{HOTEL - JUDGING TEAMS-OVERNIGHT TRIPS}

a. Lights shall be turned off at 10:30 pm or on special occasions at the discretion of adult supervisor

b. Quiet is expected after 10:30 pm

c. Bed check is 11:00

d. As an FFA member you are expected to keep your room area clean of refuse and respect the property of others.

\section{EXHIBITS:}

a. You, your animal, and your organization are on exhibit during fairs, you will be expected to keep your exhibit area and adjacent aisles clean 
b. Stalls must be cleaned, with old bedding put into designated areas. Keep the aisles clean at all times- this is a safety and health factor as well as a feature of your exhibit.

c. Destruction of property, not cooperating with the employees of the show or cooperating groups all add up to a bad image; thus you will be expected to cooperate at all times.

d. Exhibitor will be required to show in chapter group if selected by supervisor.

e. An exhibitor must be in good standing with the Madera FFA Chapter and be approved by the appropriate supervisor before purchasing a show animal to be exhibited under Madera FFA at any fair or livestock show.

f. Each FFA exhibitor will be required to show in showmanship.

9. Exhibitor understands that he/she is under the supervision of the agriculture supervisors and is required to follow all instructions given by an advisor at any time.

h. Exhibitor will be subjected to all rules submitted by the FFA and Madera South High School.

i. All animals will be washed, and fitted properly before showing

\section{GENERAL CONDUCT RULES}

\section{GENERAL:}

Due to fire hazards and the welfare of exhibits and others, the following rules must be accepted by you as a condition of exhibiting.

a. No smoking will be permitted by exhibitors

b. The use of, or possession of firecrackers will be grounds for immediate suspension from the show.

c. Lariats or other pieces of equipment subjecting anyone to injury, unless used specifically while controlling animals, are taboo.

d. No exhibitor is to leave the grounds without permission of the adult he or she is responsible to. No cars are to be used at any time without the approval of the adult in charge.

e. Respectable conduct is expected at all times. Obscene language and roughhousing will not be tolerated at any time. 
f. Appropriate dress will be required at activities participated in by exhibitors, wearing their uniform when applicable.

9. Any display of overly affectionate attention between boys and girls shall be discouraged. Persistent abuse of this rule shall be cause for suspension.

Individuals who violate any rules or who does anything that may bring discredit to their organization will be subject to one-year ineligibility in FFA activities at the show, and all or any part of his or her premium money may be withheld. An FFA officer will lose his/her office. The animal could be scratched from show and/or sale.

Exhibitor will not be allowed to bring a dog or pets.

\section{ALL MADERA SOUTH HIGH SCHOOL RULES AND REGULATIONS ARE IN EFFECT}

The undersigned have read and understand the above rules and regulations. I also understand that in the event of my misconduct my parents may be notified and informed of the action to be taken. This action may include my parents being responsible for picking me up immediately from the fair.

Signed:

Signed:

Exhibitor

Parent or Guardian

Signed:

AG Advisor

Date:

THIS FORM MUST BE ON FILE BEFORE THE STUDENT WILL BE ALLOWED TO LEAVE

Please return this form. We will put it on file for your student's high school career. 


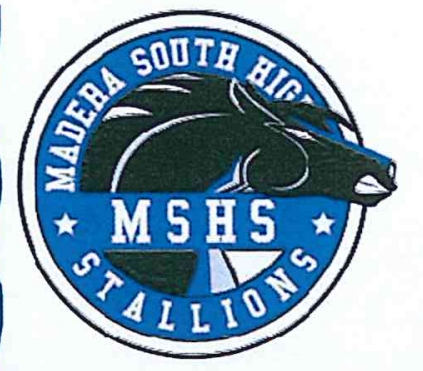

Madera South High School

Agriculture Department

$705 \mathrm{~W}$. Pecan

Madera, CA 93637

(552)675-4475

\section{Proficiency Standards for Program Completers}

Students who meet the guidelines to be classified as a program completer should be able to show the following level of proficiency.

- Students should be able to show or have completed $75 \%$ of the state standards in their area of emphasis. 


\section{Agriculture and Natural Resources Industry Sector}

Career Pathways

- Agricultural Business

- Agricultural Mechanics

- Agriscience

- Animal Science

- Forestry and Natural Resources

- Ornamental Horticulture

- Plant and Soil Science 


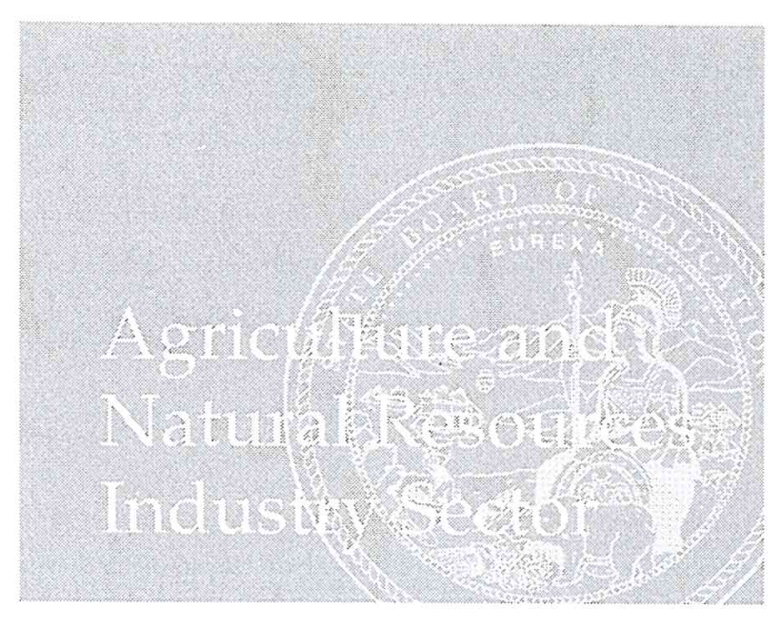

The Agriculture and Natural Resources sector is designed to provide a foundation in agriculture for all agriculture students in California. Students engage in an instructional program that integrates academic and technical preparation and focuses on career awareness, career exploration, and skill preparation in seven pathways. The pathways emphasize real-world, occupationally relevant experiences of significant scope and depth in Agricultural Business, Agricultural Mechanics, Agriscience, Animal Science, Forestry and Natural Resources, Ornamental Horticulture, and Plant and Soil Science. Integral components of classroom and laboratory instruction, supervised agricultural experience projects, and leadership and interpersonal skills development prepare students for continued training, advanced educational opportunities, or entry to a career.

\section{FOUNDATION STANDARDS}

\subsection{Academics}

Students understand the academic content required for entry into postsecondary education and employment in the Agriculture and Natural Resources sector.

(The standards listed below retain in parentheses the numbering as specified in the mathematics, science, and history-social science content standards adopted by the State Board of Education.)

\subsection{Mathematics}

Specific applications of Algebra I standards (grades eight through twelve):

(10.0) Students add, subtract, multiply, and divide monomials and polynomials. Students solve multistep problems, including word problems, by using these techniques.

(12.0) Students simplify fractions with polynomials in the numerator and denominator by factoring both and reducing them to the lowest terms. 
(13.0) Students add, subtract, multiply, and divide rational expressions and functions. Students solve both computationally and conceptually challenging problems by using these techniques.

(15.0) Students apply algebraic techniques to solve rate problems, work problems, and percent mixture problems.

Specific applications of Geometry standards (grades eight through twelve):

(8.0) Students know, derive, and solve problems involving the perimeter, circumference, area, volume, lateral area, and surface area of common geometric figures.

(10.0) Students compute areas of polygons, including rectangles, scalene triangles, equilateral triangles, rhombi, parallelograms, and trapezoids.

(11.0) Students determine how changes in dimensions affect the perimeter, area, and volume of common geometric figures and solids.

(12.0) Students find and use measures of sides and of interior and exterior angles of triangles and polygons to classify figures and solve problems.

Specific applications of Probability and Statistics standards (grades eight through twelve):

(8.0) Students organize and describe distributions of data by using a number of different methods, including frequency tables, histograms, standard line and bar graphs, stem-and-leaf displays, scatterplots, and box-and-whisker plots.

1.2 Science

Specific applications of Investigation and Experimentation standards (grades nine through twelve):

(1.a) Select and use appropriate tools and technology (such as computer-linked probes, spreadsheets, and graphing calculators) to perform tests, collect data, analyze relationships, and display data.

(1.c) Identify possible reasons for inconsistent results, such as sources of error or uncontrolled conditions.

(1.d) Formulate explanations by using logic and evidence.

(1.f) Distinguish between hypothesis and theory as scientific terms.

(1.j) Recognize the issues of statistical variability and the need for controlled tests.

(1.1) Analyze situations and solve problems that require combining and applying concepts from more than one area of science.

(1.m) Investigate a science-based societal issue by researching the literature, analyzing data, and communicating the findings. Examples of issues include irradiation of food, cloning of animals by somatic cell nuclear transfer, choice of energy sources, and land and water use decisions in California.

\subsection{History-Social Science \\ Specific applications of Principles of Economics standards (grade twelve): \\ (12.2) Students analyze the elements of America's market economy in a global setting.}


(12.2.2) Discuss the effects of changes in supply and/or demand on the relative scarcity, price, and quantity of particular products.

(12.2.3) Explain the roles of property rights, competition, and profit in a market economy.

(12.2.5) Understand the process by which competition among buyers and sellers determines a market price.

(12.2.6) Describe the effect of price controls on buyers and sellers.

(12.2.7) Analyze how domestic and international competition in a market economy affects goods and services produced and the quality, quantity, and price of those products.

(12.2.10) Discuss the economic principles that guide the location of agricultural production and industry and the spatial distribution of transportation and retail facilities.

(12.4) Students analyze the elements of the U.S. labor market in a global setting.

(12.4.3) Discuss wage differences among jobs and professions, using the laws of demand and supply and the concept of productivity.

\subsection{Communications}

Students understand the principles of effective oral, written, and multimedia communication in a variety of formats and contexts.

(The standards listed below retain in parentheses the numbering as specified in the Englishlanguage arts content standards adopted by the State Board of Education.)

\subsection{Reading}

Specific applications of Reading Comprehension standards (grades nine and ten):

Analyze the structure and format of functional workplace documents, including the graphics and headers, and explain how authors use the features to achieve their purposes.

(2.2) Prepare a bibliography of reference materials for a report using a variety of consumer, workplace, and public documents.

(2.3) Generate relevant questions about readings on issues that can be researched.

(2.6) Demonstrate use of sophisticated learning tools by following technical directions (e.g., those found with graphic calculators and specialized software programs and in access guides to World Wide Web sites on the Internet).

(2.7) Critique the logic of functional documents by examining the sequence of information and procedures in anticipation of possible reader misunderstandings.

(2.8) Evaluate the credibility of an author's argument or defense of a claim by critiquing the relationship between generalizations and evidence, the comprehensiveness of evidence, and the way in which the author's intent affects the structure and tone of the text (e.g., in professional journals, editorials, political speeches, primary source material). 
Specific applications of Reading Comprehension standards (grades eleven and twelve):

(2.1) Analyze both the features and the rhetorical devices of different types of public documents (e.g., policy statements, speeches, debates, platforms) and the way in which authors use those features and devices.

(2.3) Verify and clarify facts presented in other types of expository texts by using a variety of consumer, workplace, and public documents.

(2.4) Make warranted and reasonable assertions about the author's arguments by using elements of the text to defend and clarify interpretations.

\subsection{Writing}

Specific applications of Writing Strategies and Applications standards (grades nine and ten):

(1.1) Establish a controlling impression or coherent thesis that conveys a clear and distinctive perspective on the subject and maintain a consistent tone and focus throughout the piece of writing.

(1.2) Use precise language, action verbs, sensory details, appropriate modifiers, and the active rather than the passive voice.

(1.3) Use clear research questions and suitable research methods (e.g., library, electronic media, personal interview) to elicit and present evidence from primary and secondary sources.

(1.5) Synthesize information from multiple sources and identify complexities and discrepancies in the information and the different perspectives found in each medium (e.g., almanacs, microfiche, news sources, in-depth field studies, speeches, journals, technical documents).

(2.3) Write expository compositions, including analytical essays and research reports:

a. Marshal evidence in support of a thesis and related claims, including information on all relevant perspectives.

b. Convey information and ideas from primary and secondary sources accurately and coherently.

c. Make distinctions between the relative value and significance of specific data, facts, and ideas.

d. Include visual aids by employing appropriate technology to organize and record information on charts, maps, and graphs.

e. Anticipate and address readers' potential misunderstandings, biases, and expectations.

f. Use technical terms and notations accurately.

(2.5) Write business letters:

a. Provide clear and purposeful information and address the intended audience appropriately.

b. Use appropriate vocabulary, tone, and style to take into account the nature of the relationship with, and the knowledge and interests of, the recipients.

c. Highlight central ideas or images. 
d. Follow a conventional style with page formats, fonts, and spacing that contribute to the documents' readability and impact.

(2.6) Write technical documents (e.g., a manual on rules of behavior for conflict resolution, procedures for conducting a meeting, minutes of a meeting):

a. Report information and convey ideas logically and correctly.

b. Offer detailed and accurate specifications.

c. Include scenarios, definitions, and examples to aid comprehension (e.g., troubleshooting guide).

d. Anticipate readers' problems, mistakes, and misunderstandings.

Specific applications of Writing Strategies and Applications standards (grades eleven and twelve):

(1.3) Structure ideas and arguments in a sustained, persuasive, and sophisticated way and support them with precise and relevant examples.

(1.6) Develop presentations by using clear research questions and creative and critical research strategies (e.g., field studies, oral histories, interviews, experiments, electronic sources).

(1.7) Use systematic strategies to organize and record information (e.g., anecdotal scripting, annotated bibliographies).

(1.8) Integrate databases, graphics, and spreadsheets into word-processed documents.

(2.5) Write job applications and résumés:

a. Provide clear and purposeful information and address the intended audience appropriately.

b. Use varied levels, patterns, and types of language to achieve intended effects and aid comprehension.

c. Modify the tone to fit the purpose and audience.

d. Follow the conventional style for that type of document (e.g., résumé, memorandum) and use page formats, fonts, and spacing that contribute to the readability and impact of the document.

(2.6) Deliver multimedia presentations:

a. Combine text, images, and sound and draw information from many sources (e.g., television broadcasts, videos, films, newspapers, magazines, CD-ROMs, the Internet, electronic media-generated images).

b. Select an appropriate medium for each element of the presentation.

c. Use the selected media skillfully, editing appropriately and monitoring for quality.

d. Test the audience's response and revise the presentation accordingly.

\subsection{Written and Oral English Language Conventions}

Specific applications of English Language Conventions standards (grades eleven and twelve):

(1.1) Demonstrate control of grammar, diction, and paragraph and sentence structure and an understanding of English usage. 
(1.2) Produce legible work that shows accurate spelling and correct punctuation and capitalization.

(1.3) Reflect appropriate manuscript requirements in writing.

\subsection{Listening and Speaking}

Specific applications of Listening and Speaking Strategies and Applications standards (grades nine and ten):

(1.1) Formulate judgments about the ideas under discussion and support those judgments with convincing evidence.

(1.7) Use props, visual aids, graphs, and electronic media to enhance the appeal and accuracy of presentations.

(2.2) Deliver expository presentations:

a. Marshal evidence in support of a thesis and related claims, including information on all relevant perspectives.

b. Convey information and ideas from primary and secondary sources accurately and coherently.

c. Make distinctions between the relative value and significance of specific data, facts, and ideas.

d. Include visual aids by employing appropriate technology to organize and display information on charts, maps, and graphs.

e. Anticipate and address the listener's potential misunderstandings, biases, and expectations.

f. Use technical terms and notations accurately.

(2.3) Apply appropriate interviewing techniques:

a. Prepare and ask relevant questions.

b. Make notes of responses.

c. Use language that conveys maturity, sensitivity, and respect.

d. Respond correctly and effectively to questions.

e. Demonstrate knowledge of the subject or organization.

f. Compile and report responses.

g. Evaluate the effectiveness of the interview.

Specific applications of Listening and Speaking Strategies and Applications standards (grades eleven and twelve):

(1.8) Use effective and interesting language, including:

a. Informal expressions for effect

b. Standard American English for clarity

c. Technical language for specificity

(1.14) Analyze the techniques used in media messages for a particular audience and evaluate their effectiveness (e.g., Orson Welles' radio broadcast "War of the Worlds"). 
(2.4) Deliver multimedia presentations:

a. Combine text, images, and sound by incorporating information from a wide range of media, including films, newspapers, magazines, CD-ROMs, online information, television, videos, and electronic media-generated images.

b. Select an appropriate medium for each element of the presentation.

c. Use the selected media skillfully, editing appropriately and monitoring for quality.

d. Test the audience's response and revise the presentation accordingly

\subsection{Career Planning and Management}

Students understand how to make effective decisions, use career information, and manage personal career plans:

3.1 Know the personal qualifications, interests, aptitudes, information, and skills necessary to succeed in careers.

3.2 Understand the scope of career opportunities and know the requirements for education, training, and licensure.

3.3 Develop a career plan that is designed to reflect career interests, pathways, and postsecondary options.

3.4 Understand the role and function of professional organizations, industry associations, and organized labor in a productive society.

3.5 Understand the past, present, and future trends that affect careers, such as technological developments and societal trends, and the resulting need for lifelong learning.

3.6 Know important strategies for self-promotion in the hiring process, such as job applications, résumé writing, interviewing skills, and preparation of a portfolio.

\subsection{Technology}

Students know how to use contemporary and emerging technological resources in diverse and changing personal, community, and workplace environments:

4.1 Understand past, present, and future technological advances as they relate to a chosen pathway.

4.2 Understand the use of technological resources to gain access to, manipulate, and produce information, products, and services.

4.3 Understand the influence of current and emerging technology on selected segments of the economy.

4.4 Understand geographic information systems (G.I.S.).

4.5 Determine the validity of the content and evaluate the authenticity, reliability, and bias of electronic and other resources.

4.6 Differentiate among, select, and apply appropriate tools and technology. 


\subsection{Problem Solving and Critical Thinking}

Students understand how to create alternative solutions by using critical and creative thinking skills, such as logical reasoning, analytical thinking, and problem-solving techniques:

5.1 Apply appropriate problem-solving strategies and critical thinking skills to work-related issues and tasks.

5.2 Understand the systematic problem-solving models that incorporate input, process, outcome, and feedback components.

5.3 Use critical thinking skills to make informed decisions and solve problems.

\subsection{Health and Safety}

Students understand health and safety policies, procedures, regulations, and practices, including the use of equipment and handling of hazardous materials:

6.1 Know policies, procedures, and regulations regarding health and safety in the workplace, including employers' and employees' responsibilities.

6.2 Understand critical elements of health and safety practices related to storing, cleaning, and maintaining tools, equipment, and supplies.

6.3 Understand how to locate important information on a material safety data sheet.

6.4 Maintain safe and healthful working conditions.

6.5 Use tools and machines safely and appropriately.

6.6 Know how to both prevent and respond to accidents in the agricultural industry.

\subsection{Responsibility and Flexibility}

Students know the behaviors associated with the demonstration of responsibility and flexibility in personal, workplace, and community settings:

7.1 Understand the qualities and behaviors that constitute a positive and professional work demeanor.

7.2 Understand the importance of accountability and responsibility in fulfilling personal, community, and workplace roles.

7.3 Understand the need to adapt to varied roles and responsibilities.

7.4 Understand that individual actions can affect the larger community.

7.5 Understand the importance of time management to fulfill responsibilities.

7.6 Know how to apply high-quality craftsmanship to a product or presentation and continually refine and perfect it. 


\subsection{Ethics and Legal Responsibilities}

Students understand professional, ethical, and legal behavior consistent with applicable laws, regulations, and organizational norms:

8.1 Know the major local, district, state, and federal regulatory agencies and entities that affect the industry and how they enforce laws and regulations.

8.2 Understand the concept and application of ethical and legal behavior consistent with workplace standards.

8.3 Understand the role of personal integrity and ethical behavior in the workplace.

8.4 Understand how to access, analyze, and implement quality assurance information.

\subsection{Leadership and Teamwork}

Students understand effective leadership styles, key concepts of group dynamics, team and individual decision making, the benefits of workforce diversity, and conflict resolution:

9.1 Understand the characteristics and benefits of teamwork, leadership, and citizenship in the school, community, and workplace settings.

9.2 Understand the ways in which preprofessional associations, such as the Future Farmers of America (FFA), and competitive career development activities enhance academic skills, promote career choices, and contribute to employability.

9.3 Understand how to organize and structure work individually and in teams for effective performance and the attainment of goals.

9.4 Know multiple approaches to conflict resolution and their appropriateness for a variety of situations in the workplace.

9.5 Understand how to interact with others in ways that demonstrate respect for individual and cultural differences and for the attitudes and feelings of others.

9.6 Understand leadership, cooperation, collaboration, and effective decision-making skills applied in group or team activities, including the student organization.

\subsection{Technical Knowledge and Skills}

Students understand the essential knowledge and skills common to all pathways in the Agriculture and Natural Resources sector:

10.1 Understand the aims, purposes, history, and structure of the FFA student organization, and know the opportunities it makes available.

10.2 Manage and actively engage in a career-related, supervised agricultural experience. 
10.3 Understand the importance of maintaining and completing the California Agricultural Record Book.

10.4 Maintain and troubleshoot equipment used in the agricultural industry.

\subsection{Demonstration and Application}

Students demonstrate and apply the concepts contained in the foundation and pathway standards. 


\section{Pathway Standards}

\section{A. Agricultural Business Pathway}

In the Agricultural Business Pathway, students learn about agricultural business operation and management. Topics include accounting, finance, economics, business organization, marketing, and sales.

A1.0 Students understand decision-making processes within the American free enterprise system:

A1.1 Differentiate among the components of the American free enterprise system and other forms of economic systems.

A1.2 Distinguish among the main characteristics of individual proprietorships, partnerships, corporations, and cooperatives.

A1.3 Understand the advantages and disadvantages of the four types of business ownership.

A1.4 Analyze appropriate decision-making tools and financial records to make key management decisions.

A1.5 Analyze physical production relationships to determine optimum use levels.

A1.6 Understand how to calculate the fixed and variable costs associated with the production of agricultural products and determine the output level that will yield maximum profit.

A2.0 Students understand the fundamental economic principles of agribusiness and agricultural production:

A2.1 Understand how basic economic factors affect agricultural production and agribusiness management decisions.

A2.2 Know basic agricultural economic terminology.

A2.3 Understand the law of supply and demand as it effects price determination.

A2.4 Analyze how agriculture uses scarce resources to meet the needs and demands of its consumers.

A2.5 Differentiate between elastic and inelastic supply and demand.

A2.6 Understand the law of diminishing returns and its impact on agricultural production.

A3.0 Students understand the role of credit in agribusiness and agricultural production:

A3.1 Analyze the factors that determine the cost of credit in order to select optimum credit sources (e.g., the advantages and disadvantages of borrowing from the various types of credit providers and sources for short-, intermediate-, and longterm credit).

A3.2 Know the criteria lenders use to evaluate repayment capacity.

A3.3 Analyze balance sheets and cash-flow statements to determine the ability to repay loans. 
A4.0 Students understand proper accounting principles and procedures used in business management and tax planning:

A4.1 Understand the differences between cash and accrual accounting systems.

A4.2 Understand the use and importance of budgets, income statements, balance sheets, and financial statements.

A4.3 Understand the basis of taxation within the tax system and its impact on the economy, including the role of taxes in agribusiness.

A4.4 Analyze the role of depreciation and purchasing in tax planning and liability.

A4.5 Understand how to determine property values and how to complete a depreciation schedule.

A4.6 Understand how to determine the tax obligations for an agribusiness.

A5.0 Students understand basic risk management principles and their impact on economic viability:

A5.1 Understand environmental responsibility and its impact on agribusiness.

A5.2 Understand the concept of liability and the economic impact of being held liable.

A5.3 Understand the concept and process of risk management, including the use of risk management tools such as insurance.

A5.4 Understand how recordkeeping, farm plans, and an analysis of best practices affect risk management decisions.

A5.5 Understand the role of contingency plans in risk management.

A6.0 Students understand the role and value of agricultural organizations:

A6.1 Understand the benefits of private, public, and governmental organizations, including the value and impact of cooperatives.

A6.2 Understand how participation within organizations would be beneficial in supporting various agricultural operations.

A6.3 Understand how to identify and electronically access public and private agricultural organizations.

A7.0 Students understand agricultural marketing systems:

A7.1 Understand how marketing functions in a free market society.

A7.2 Understand the advantages and disadvantages of the various marketing options for agricultural products and services.

A7.3 Understand how the law of comparative advantage affects agricultural production.

A7.4 Understand the impact of advertising and promotion on the marketing of agricultural products and services.

A7.5 Understand how promotion trends for agricultural products influence individuals.

A7.6 Understand how to develop a marketing plan for an agricultural product or service. 
A8.0 Students understand the sales of agricultural products and services:

A8.1 Determine the most effective methods for assessing customer needs and wants.

A8.2 Understand the stages in making a successful sale and the various techniques used to approach potential customers and overcome their objections.

A8.3 Examine the physiological and psychological factors that influence motivation to purchase, including the fundamental steps in making a purchase.

A9.0 Students understand local, national, and international agricultural markets and how trade affects the economy:

A9.1 Understand how the importance of agricultural imports and exports affects state and national economies.

A9.2 Know how governmental, economic, and cultural factors affect international trade.

A9.3 Compare and contrast United States trade policies with those of other important trading partners.

A9.4 Understand how biotechnology affects trade and global economies.

A9.5 Understand how different cultural values affect agricultural production and marketing.

A9.6 Understand how negotiations and bargaining agreements affect trade agreements.

A9.7 Analyze agricultural marketing strategies in other parts of the world. 


\section{B. Agricultural Mechanics Pathway}

The Agricultural Mechanics Pathway prepares students for careers related to the construction, operation, and maintenance of equipment used by the agriculture industry. Basic agricultural mechanics skills and safety, standards B1.0 through B8.0, cover woodworking, electrical systems, plumbing, cold metal work, concrete, and welding technology. Advanced topics, standards B9.0 through B12.0, deal with metal fabrication, small engines, agriculture power and technology, and agriculture construction.

\section{B1.0 Students understand personal and group safety:}

B1.1 Practice the rules for personal and group safety while working in an agricultural mechanics environment.

B1.2 Know the relationship between accepted shop management procedures and a safe working environment.

B1.3 Know how to safely secure loads on a variety of vehicles.

\section{B2.0 Students understand the principles of basic woodworking:}

B2.1 Know how to identify common wood products, lumber types, and sizes.

B2.2 Know how to calculate board feet, lumber volume, and square feet.

B2.3 Know how to identify, select, and implement basic fastening systems.

B2.4 Complete a woodworking project, including interpreting a plan, developing a bill of materials and cutting list, selecting materials, shaping, joining, and finishing.

B3.0 Students understand the basic electricity principles and wiring practices commonly used in agriculture:

B3.1 Understand the relationship between voltage, amperage, resistance, and power in single-phase alternating current $(\mathrm{AC})$ circuits.

B3.2 Know how to use proper electrical test equipment for $\mathrm{AC}$ and direct current (DC).

B3.3 Analyze and correct basic circuit problems (e.g., open circuits, short circuits, incorrect grounding).

B3.4 Understand proper basic electrical circuit and wiring techniques with nonmetallic cable and conduit as defined by the National Electric Code.

B3.5 Interpret basic agricultural electrical plans.

B4.0 Students understand plumbing system practices commonly used in agriculture:

B4.1 Know basic plumbing fitting skills with a variety of materials, such as copper, PVC (polyvinyl chloride), steel, polyethylene, and ABS (acrylonitrile butadiene styrene).

B4.2 Understand the environmental influences on plumbing system choices (e.g., filter systems, water disposal). 
B4.3 Know how various plumbing and irrigation systems are used in agriculture.

B4.4 Complete a plumbing project, including interpreting a plan, developing a bill of materials and cutting list, selecting materials, joining, and testing.

B5.0 Students understand agricultural cold metal processes:

B5.1 Know how to identify common metals, sizes, and shapes.

B5.2 Know basic tool-fitting skills.

B5.3 Know layout skills.

B5.4 Know basic cold metal processes (e.g., shearing, cutting, drilling, threading, bending.).

B5.5 Complete a cold metal project, including interpreting a plan, developing a bill of materials, selecting materials, shaping, fastening, and finishing.

B6.0 Students understand concrete and masonry practices commonly used in agriculture:

B6.1 Understand how to accurately calculate volume, materials needed, and project costs for a concrete or masonry project.

B6.2 Know proper bed preparation, concrete forms layout, and construction.

B6.3 Complete a concrete or masonry project, including developing a bill of materials, assembling, mixing, placing, and finishing.

B7.0 Students understand oxy-fuel cutting and welding:

B7.1 Understand the role of heat and oxidation in the cutting process.

B7.2 Know how to properly set up, adjust, shut down, and maintain an oxy-fuel system.

B7.3 Know how to flame-cut metal with an oxy-fuel cutting torch.

B7.4 Know how to fusion-weld mild steel with and without filler rod by using oxyfuel equipment.

B7.5 Know basic repair skills using a variety of techniques, such as brazing or hard surfacing.

B8.0 Students understand electric arc welding processes:

B8.1 Know how to select, properly adjust, safely employ, and maintain appropriate welding equipment (e.g., gas metal arc welding, shielded metal arc welding, gas tungsten arc welding).

B8.2 Apply gas metal arc welding, shielded metal arc welding, or flux core arc welding processes to fusion-weld mild steel with appropriate welding electrodes and related equipment.

B8.3 Weld a variety of joints in various positions.

B8.4 Know how to read welding symbols and plans, select electrodes, fit-up joints, and control heat and distortion. 
Agricultural Mechanics Pathway

B9.0 Students understand advanced metallurgy principles and fabrication techniques:

B9.1 Understand metallurgy principles, including distortion, hardening, tempering, and annealing.

B9.2 Operate and maintain various arc welding and cutting systems safely and appropriately.

B9.3 Operate and maintain fabrication tools and equipment safely and appropriately.

B9.4 Understand how to design project plans by using mechanical drawing techniques.

B9.5 Understand how to finish a metal project by implementing proper sequencing.

B9.6 Know how to manipulate and finish metal by using a variety of machines and techniques (e.g., lathe, mill, CNC plasma, shears, press break).

B9.7 Construct a welding project (using any electric welding process, appropriate products, joints, and positions), including interpreting a plan, developing a bill of materials, selecting materials, and developing a clear and concise fabrication contract.

\section{B10.0 Students understand small and compact engines:}

B10.1 Understand engine theory for both two- and four-stroke cycle engines.

B10.2 Know different types of small engines and their applications.

B10.3 Know small engine parts and explain the various systems (e.g., fuel, ignition, compression, cooling, lubrication systems).

B10.4 Know how to troubleshoot and solve problems with small engines.

B10.5 Know how to disassemble, inspect, adjust, and reassemble a small engine.

B10.6 Know how to look up parts, apply repair and maintenance recommendations from a repair manual, and complete appropriate forms, including work orders.

B11.0 Students understand the principles and applications of various engines and machinery used in agriculture:

B11.1 Understand how to identify common agricultural machinery.

B11.2 Operate and maintain equipment safely and efficiently.

B11.3 Know the various types of engines found on agricultural machinery and understand the theory and safe operation of their systems (e.g., cooling, electrical, fuel).

B11.4 Know the theory and operation of mobile hydraulic systems and power take-off systems.

B11.5 Troubleshoot common problems with engines and agricultural equipment.

B11.6 Understand the theory and operation of 12-volt DC electronic and electrical systems (e.g., circuit design, starting, charging, and safety circuits). 
B12.0 Students understand land measurement and construction techniques commonly used in agriculture:

B12.1 Understand common surveying techniques used in agriculture (e.g., leveling, land measurement, building layout).

B12.2 Know how to draw and interpret architectural plans.

B12.3 Know how to install single- and three-phase wiring and control systems found in agricultural structures, pumps, and irrigation systems.

B12.4 Install plumbing in agricultural structures (e.g., potable water, sewer, irrigation).

B12.5 Form, place, and finish concrete or masonry (e.g., concrete block).

B12.6 Understand how to construct agricultural structures by using wood framing and steel framing systems (e.g., barns, shops, greenhouses, animal structures).

B12.7 Develop clear and concise agricultural construction contracts. 


\section{Agriscience Pathway}

The Agriscience Pathway helps students acquire a broad understanding of a variety of agricultural areas, develop an awareness of the many career opportunities in agriculture, participate in occupationally relevant experiences, and work cooperatively with a group to develop and expand leadership abilities. Students study California agriculture, agricultural business, agricultural technologies, natural resources, and animal, plant, and soil sciences.

\section{C1.0 Students understand the role of agriculture in the California economy:}

C1.1 Understand the history of the agricultural industry in California.

C1.2 Understand how California agriculture affects the quality of life.

C1.3 Understand the interrelationship of California agriculture and society at the local, state, national, and international levels.

C1.4 Understand the economic impact of leading California agricultural commodities.

C1.5 Understand the economic impact of major natural resources in California.

C1.6 Know the economic importance of major agricultural exports and imports.

C2.0 Students understand the interrelationship between agriculture and the environment:

C2.1 Understand important agricultural environmental impacts on soil, water, and air.

C2.2 Understand current agricultural environmental challenges.

C2.3 Understand how natural resources are used in agriculture.

C2.4 Compare and contrast practices for conserving renewable and nonrenewable resources.

C2.5 Understand how new energy sources are developed from agricultural products (e.g., gas-cogeneration and ethanol).

C3.0 Students understand the effects of technology on agriculture:

C3.1 Understand how an agricultural commodity moves from producer to consumer.

C3.2 Understand how technology influences factors such as labor, efficiency, diversity, availability, mechanization, communication, and so forth.

C3.3 Understand public concern for technological advancements in agriculture, such as genetically modified organisms.

C3.4 Understand the laws and regulations concerning biotechnology.

C4.0 Students understand the importance of animals, the domestication of animals, and the role of animals in modern society:

C4.1 Understand the evolution and roles of domesticated animals in society.

C4.2 Know the differences between domestication and natural selection.

C4.3 Understand the modern-day uses of animals and animal by-products. 
C4.4 Understand various points of view regarding the use of animals.

C4.5 Understand unique and alternative uses of animals (e.g., Handi-Riders and companion animals).

C5.0 Students understand the cell structure and function of plants and animals:

C5.1 Understand the purpose and anatomy of cells.

C5.2 Know how cell parts function.

C5.3 Understand various cell actions, such as osmosis and cell division.

C5.4 Understand how plant and animal cells are alike and different.

C6.0 Students understand animal anatomy and systems:

C6.1 Know the names and locations of the external anatomy of animals.

C6.2 Know the anatomy and major functions of vertebrate systems, including digestive, reproductive, circulatory, nervous, muscular, skeletal, respiratory, and endocrine systems.

C7.0 Students understand basic animal genetics:

C7.1 Differentiate between genotype and phenotype, and describe how dominant and recessive genes function.

C7.2 Compare genetic characteristics among cattle, sheep, swine, and horse breeds.

C7.3 Understand how to display phenotype and genotype ratios (e.g., by using a Punnett Square).

C7.4 Understand the fertilization process.

C7.5 Understand the purpose and processes of mitosis and meiosis.

C8.0 Students understand fundamental animal nutrition and feeding:

C8.1 Know types of nutrients required by farm animals (e.g., proteins, minerals, vitamins, carbohydrates, fats/oils, water).

C8.2 Analyze suitable common feed ingredients, including forages, roughages, concentrates, and supplements, for ruminant, monogastric, equine, and avian digestive systems.

C8.3 Understand basic animal feeding guidelines and evaluate sample feeding programs for various species, including space requirements and economic considerations.

C9.0 Students understand basic animal health:

C9.1 Assess the appearance and behavior of a normal, healthy animal.

C9.2 Understand the ways in which housing, sanitation, and nutrition influence animal health and behavior.

C9.3 Understand the causes and control of common animal diseases. 
C9.4 Understand how to control parasites and why.

C9.5 Understand the legal requirements for the procurement, storage, methods of application, and withdrawal times of animal medications and know proper equipment handling and disposal techniques.

C10.0 Students understand soil science principles:

C10.1 Recognize the major soil components and types.

C10.2 Understand how soil texture, structure, $\mathrm{pH}$, and salinity affect plant growth.

C10.3 Understand water delivery and irrigation system options.

C10.4 Understand the types, uses, and applications of amendments and fertilizers.

C11.0 Students understand plant growth and development:

C11.1 Understand the anatomy and functions of plant systems and structures.

C11.2 Understand plant growth requirements.

C11.3 Know annual, biennial, and perennial life cycles.

C11.4 Examine plant sexual and asexual reproduction.

C11.5 Understand the photosynthesis process and the roles of the sun, chlorophyll, sugar, oxygen, carbon dioxide, and water in the process.

C11.6 Understand the respiration process in the breakdown of food and organic matter.

C12.0 Students understand fundamental pest management:

C12.1 Understand the major classifications of pests (e.g., insects, weeds, disease, vertebrate pests).

C12.2 Understand chemical, mechanical, cultural, and biological methods of plant pest control.

C12.3 Understand the major principles, advantages, and disadvantages of integrated pest management.

C13.0 Students understand the scientific method:

C13.1 Understand the steps of the scientific method.

C13.2 Analyze an animal or plant problem and devise a solution based on the scientific method.

C13.3 Use the scientific method to conduct agricultural experiments. 


\section{Animal Science Pathway}

In the Animal Science Pathway, students study large, small, and specialty animals. Students explore the necessary elements-such as diet, genetics, habitat, and behaviorto create humane, ecologically and economically sustainable animal production systems. The pathway includes the study of animal anatomy and physiology, nutrition, reproduction, genetics, health and welfare, animal production, technology, and the management and processing of animal products and by-products.

\section{D1.0 Students understand the necessary elements for proper animal housing and animal-} handling equipment:

D1.1 Understand appropriate space and location requirements for habitat, housing, feed, and water.

D1.2 Understand how to select habitat and housing conditions and materials (such as indoor and outdoor housing, fencing materials, air flow/ventilation, and shelters) to meet the needs of various animal species.

D1.3 Understand the purpose and the safe and humane use of restraint equipment, such as squeeze chutes, halters, and twitches.

D1.4 Understand the purpose and the safe and humane use of animal husbandry tools, such as hoof trimmers, electric shears, elastrators, dehorning tools, and scales.

D2.0 Students understand key principles of animal nutrition:

D2.1 Understand the flow of nutrients from the soil, through the animal, and back to the soil.

D2.2 Understand the principles for providing proper balanced rations for a variety of production stages in ruminants and monogastrics.

D2.3 Understand the digestive processes of the ruminant, monogastric, avian, and equine digestive systems.

D2.4 Understand how animal nutrition is affected by the digestive, endocrine, and circulatory systems.

D3.0 Students understand animal physiology:

D3.1 Understand the major physiological systems and the function of the organs within each system.

D3.2 Understand the animal management practices that are likely to improve the functioning of the various physiological systems. 
D4.0 Students understand animal reproduction, including the function of reproductive organs:

D4.1 Understand animal conception (including estrus cycles, ovulation, and insemination).

D4.2 Understand the gestation process and basic fetal development.

D4.3 Understand the parturition process, including the identification of potential problems and their solutions.

D4.4 Understand the role of artificial insemination and embryo transfer in animal agriculture.

D4.5 Understand commonly used animal production breeding systems (e.g., purebred compared with crossbred) and reasons for their use.

D5.0 Students understand animal inheritance and selection principles, including the structure and role of DNA:

D5.1 Evaluate a group of animals for desired qualities and discern among them for breeding selection.

D5.2 Understand how to use animal performance data in the selection and management of production animals.

D5.3 Research and discuss current technology used to measure desirable traits.

D5.4 Understand how to predict phenotypic and genotypic results of a dominant and recessive gene pair.

D5.5 Understand the role of mutations (both naturally occurring and artificially induced) and hybrids in animal genetics.

D6.0 Students understand the causes and effects of diseases and illnesses in animals:

D6.1 Understand the signs of normal health in contrast to illness and disease.

D6.2 Understand the importance of animal behavior in diagnosing animal sickness and disease.

D6.3 Understand the common pathogens, vectors, and hosts that cause disease in animals.

D6.4 Understand prevention, control, and treatment practices related to pests and parasites.

D6.5 Apply quality assurance practices to the proper administration of medicines and animal handling.

D6.6 Understand how diseases are passed among animal species and from animals to humans and how that relationship affects health and food safety.

D6.7 Understand the impacts on local, national, and global economies as well as on consumers and producers when animal diseases are not appropriately contained and eradicated. 
D7.0 Students understand common rangeland management practices and their impact on a balanced ecosystem:

D7.1 Understand the role of rangeland use in an effective animal production program.

D7.2 Know how rangeland management practices affect pasture production, erosion control, and the general balance of the ecosystem.

D7.3 Understand how to manage rangelands (including how to calculate carrying capacity) for a variety of animal species and locations.

D7.4 Understand how to balance rangeland use for animal grazing and for wildlife habitat.

D8.0 Students understand the challenges associated with animal waste management:

D8.1 Understand animal waste treatment and disposal management systems.

D8.2 Understand various methods for using animal waste and their environmental impacts.

D8.3 Understand the health and safety regulations that are an integral part of properly managed animal waste systems.

D9.0 Students understand animal welfare concerns and management practices that support animal welfare:

D9.1 Know the early warning signs of animal distress and how to rectify the problem.

D9.2 Understand public concerns for animal welfare in the context of housing, behavior, nutrition, transportation, disposal, and harvest of animals.

D9.3 Understand federal and state animal welfare laws and regulations, such as those dealing with abandoned and neglected animals, animal fighting, euthanasia, and medical research.

D9.4 Understand the regulations for humane transport and harvest of animals, such as those delineated by the U.S. Department of Agriculture, Food Safety and Inspection Service, and the Humane Methods of Slaughter Act.

D10.0 Students understand the production of large animals (e.g., cattle, horses, swine, sheep, goats) and small animals (e.g., poultry, cavy, rabbits):

D10.1 Know how to synthesize and implement optimum requirements for diet, genetics, habitat, and behavior in the production of large and small animals.

D10.2 Understand how to develop, maintain, and use growth and management records for large or small animals. 
D11.0 Students understand the production of specialty animals (e.g., fish, marine animals, llamas, tall flightless birds):

D11.1 Understand the specialty animal's role in agriculture (e.g., fish farms, pack animals, working dogs).

D11.2 Understand the unique nutrition, health, and habitat requirements for specialty animals.

D11.3 Know how to synthesize and implement optimum requirements for diet, genetics, habitat, and behavior in the production of specialty animals.

D11.4 Understand how to develop, maintain, and use growth and management records for specialty animals.

D12.0 Students understand how animal products and by-products are processed and marketed:

D12.1 Understand animal harvest, carcass inspection and grading, and meat processing safety regulations and practices and the removal and disposal of nonedible byproducts, such as those outlined in Hazard Analysis and Critical Control Point documents.

D12.2 Understand the relative importance of the major meat classifications, including the per capita consumption and nutritive value of those classifications.

D12.3 Understand how meat-based products and meals are made.

D12.4 Understand how nonmeat products (such as eggs, wool, pelts, hides, and byproducts) are harvested and processed.

D12.5 Understand how meat products and nonmeat products are marketed.

D12.6 Understand the value of animal by-products to nonagricultural industries. 


\section{E. Forestry and Natural Resources Pathway}

The Forestry and Natural Resources Pathway helps students understand the relationships between California's natural resources and the environment. Topics include energy and nutrient cycles, water resources and management, soil conservation, wildlife preservation and management, forest and fire management, and lumber production. In addition, students study the outdoor recreation industry and multiple-use management.

\section{E1.0 Students understand the importance of energy and energy cycles:}

E1.1 Understand the oxygen, carbon, nitrogen, and water cycles.

E1.2 Understand the difference between renewable and nonrenewable energy sources.

E1.3 Understand the difference between natural resource management conservation strategies and preservation strategies.

E1.4 Compare the effects on air and water quality of using different forms of energy.

E1.5 Analyze the way in which human activities influence energy cycles and natural resource management.

E2.0 Students understand air and water use, management practices, and conservation strategies:

E2.1 Understand the government's role in regulating air, soil, and water use management practices and conservation strategies.

E2.2 Understand air and water conservation issues.

E2.3 Understand appropriate water conservation measures.

E2.4 Understand the component of a plan that monitors water quality.

E2.5 Understand the component of a plan that monitors air quality.

E2.6 Analyze the way in which water management affects the environment and human needs.

\section{E3.0 Students understand soil composition and soil management:}

E3.1 Understand the systems used to classify soils.

E3.2 Understand the reasons for and importance of soil conservation.

E3.3 Understand how to analyze soils found in the different natural resource management areas.

E3.4 Understand how to develop and implement a soil management plan for a natural resource management area.

E3.5 Understand how to analyze existing soil surveys to develop effective management plans. 
E4.0 Students understand rangeland management:

E4.1 Know the locations of major U.S. and California rangeland areas.

E4.2 Understand the interrelationship of rangeland management, the environment, wildlife management, and the livestock industry.

E4.3 Understand practices used to improve rangeland quality.

E4.4 Analyze the carrying capacity in various rangelands for both wildlife species and domestic livestock.

E4.5 Distinguish among different browse and forage species in California rangelands.

E4.6 Understand the components of a rangeland monitoring plan.

E4.7 Understand the requirements and rights accompanying public land grazing permits and the government agencies involved (e.g., Bureau of Land Management and U.S. Forest Service).

\section{E5.0 Students understand wildlife management and habitat:}

E5.1 Understand the relationship between habitat and wildlife population.

E5.2 Understand habitat requirements for different species and identify factors that influence population dynamics.

E5.3 Understand the methods for determining existing wildlife species populations.

E5.4 Understand mammalian and avian reproductive processes and explain how nutrition and habitat affect reproduction and population.

E5.5 Understand a variety of management practices used to manage wildlife populations for hunting and other recreational purposes.

E5.6 Analyze the economic and environmental significance of sport hunting and fishing industries.

E5.7 Understand the purpose, history, terminology, and challenges of the Endangered Species Act and current activities related to the Act.

E6.0 Students understand aquatic resource use and management:

E6.1 Understand the different types of aquatic resources.

E6.2 Know the major body parts, digestive systems, and reproductive organs of aquatic species.

E6.3 Understand a variety of methods to determine the populations of existing aquatic species.

E6.4 Analyze the relationship between water quality and aquatic species habitat.

E6.5 Understand a variety of management practices for managing aquatic species for sport fishing and other purposes.

E6.6 Understand how to make financial and production decisions and maintain growth and management records for a selected aquatic species. 
E7.0 Students understand the outdoor recreation industry:

E7.1 Understand the potential environmental impacts of recreational activities and how to manage the resources affected.

E7.2 Understand basic survival skills and first-aid procedures.

E7.3 Understand appropriate trail construction and maintenance techniques.

E7.4 Understand how to select appropriate recreational gear for trips of varying types and durations and how to use it safely and appropriately (for minimum environmental impact).

E7.5 Know how to set up a campsite for minimum environmental impact.

E8.0 Students understand basic plant physiology, anatomy, and taxonomy:

E8.1 Understand the scientific method of animal classification, including order, family, genus, and species.

E8.2 Know how to use a dichotomous key to identify plants and animals.

E8.3 Know how to identify local trees, shrubs, grasses, forbs, and wildlife species by common name.

E8.4 Recognize the factors that influence plant growth, such as respiration, temperature, nutrients, and photosynthesis.

E9.0 Students understand the role of fire in natural resource management:

E9.1 Understand the role of fire in forest and rangeland ecosystems.

E9.2 Understand the significance of each of the components of the "fire triangle."

E9.3 Know appropriate wildland fire-suppression practices.

E9.4 Understand the components of a fire-control plan.

E9.5 Know how to use fire-control tools safely.

E9.6 Know the training requirements for fire-suppression certification.

E10.0 Students understand forest management practices:

E10.1 Understand how social, political, and economic factors can affect the use of forests.

E10.2 Understand the California Forest Practice Act and the requirements for Timber Harvest and Habitat Conservation Plans.

E10.3 Analyze forest management systems (e.g., sustained yield, watershed management, ecosystem management, multiple-use management).

E10.4 Analyze harvest and renewability (e.g., re-seeding and thinning) systems and identify the impact of each on the land.

E10.5 Understand Silvicultural systems and skills, including appropriate tool use.

E10.6 Understand how to identify and diagnose damage from destructive insects, diseases, and weather, and know methods for their management. 
E11.0 Students understand the basic concepts of measurement, surveying, and mapping:

E11.1 Understand the Public Land Survey System.

E11.2 Use surveying equipment, including global positioning satellites, maps, and a compass to determine area, boundaries, and elevation differences.

E11.3 Know how to apply timber-cruising and log-scaling skills to determine timber and log volume for management and marketing.

E11.4 Understand how to create a management plan map that includes layer information and data points from global information systems.

E12.0 Students understand the use, processing, and marketing of products from natural resource industries:

E12.1 Know the marketing processes and manufacturing standards for a variety of natural resource products, including mining, quarrying, and drilling.

E12.2 Know how to manufacture a product (to manufacturing standards) from a natural resource.

E12.3 Analyze the production of specialty and seasonal products from natural resources.

E12.4 Know different wood types and their uses.

E12.5 Know lumber manufacturing processes.

E13.0 Students understand public and private land issues:

E13.1 Understand the differences between publicly and privately held lands.

E13.2 Understand the differences between public land designations (e.g., State Park, National Forest, wilderness areas, wild and scenic areas).

E13.3 Understand the role of public and private property rights and how they affect agriculture.

E13.4 Understand the role of government in managing public and private property rights. 


\section{F. Ornamental Horticulture Pathway}

The Ornamental Horticulture Pathway prepares students for careers in the nursery, landscaping, and floral industries. Topics include plant identification, plant physiology, soil science, plant reproduction, nursery production, and floriculture as well as landscaping design, installation, and maintenance.

\section{F1.0 Students understand plant classification and use principles:}

F1.1 Understand how to classify and identify plants by order, family, genus, and species.

F1.2 Understand how to identify plants by using a dichotomous key.

F1.3 Understand how common plant parts are used to classify the plants.

F1.4 Understand how to classify and identify plants by using botanical growth habits, landscape uses, and cultural requirements.

F1.5 Understand plant selection and identification for local landscape applications.

F2.0 Students understand plant physiology and growth principles:

F2.1 Understand plant systems, nutrient transportation, structure, and energy storage.

F2.2 Understand the seed's essential parts and functions.

F2.3 Understand how primary, secondary, and trace elements are used in plant growth.

F2.4 Understand the factors that influence plant growth, including water, nutrients, light, soil, air, and climate.

F2.5 Understand the tissues seen in a cross section of woody and herbaceous plants.

F2.6 Understand the factors that affect plant growth.

F3.0 Students understand sexual and asexual plant reproduction:

F3.1 Understand the different forms of sexual and asexual plant reproduction.

F3.2 Understand the various techniques for successful plant propagation (e.g., budding, grafting, cuttings, seeds).

F3.3 Understand how to monitor plant reproduction for the development of a saleable product.

F4.0 Students understand basic integrated pest management principles:

F4.1 Read and interpret pesticide labels and understand safe pesticide management practices.

F4.2 Understand how pesticide regulations and government agencies affect agriculture.

F4.3 Understand common horticultural pests and diseases and methods of controlling them.

F4.4 Understand the systematic approach to solving plant problems. 
F5.0 Students understand water and soil (media) management practices:

F5.1 Understand how basic soil science and water principles affect plant growth.

F5.2 Know basic irrigation design and installation methods.

F5.3 Prepare and amend soils, implement soil conservation methods, and compare results.

F5.4 Understand major issues related to water sources and water quality.

F5.5 Know the components of soilless media and the use of those media in various types of containers.

F6.0 Students understand ornamental plant nutrition practices:

F6.1 Analyze how primary and secondary nutrients and trace elements affect ornamental plants.

F6.2 Understand basic nutrient testing procedures on soil and plant tissue.

F6.3 Analyze organic and inorganic fertilizers to understand their appropriate uses.

F6.4 Understand how to read and interpret labels to properly apply fertilizers.

F7.0 Students understand the selection, installation, and maintenance of turf:

F7.1 Understand the selection and management of landscape and sports field turf.

F7.2 Understand how to select, install, and maintain a designated turfgrass area.

F7.3 Understand how the use of turf benefits the environment.

F8.0 Students understand nursery production principles:

F8.1 Understand how to properly use production facilities and common nursery equipment.

F8.2 Understand common nursery production practices.

F8.3 Understand how to propagate and maintain a horticultural crop to the point of sale.

F8.4 Understand marketing and merchandising principles used in nursery production.

F9.0 Students understand the use of containers and horticultural tools, equipment, and facilities:

F9.1 Understand the use of different types of containers and demonstrate how to maintain growing containers in controlled environments.

F9.2 Operate and maintain selected hand and power equipment safely and appropriately.

F9.3 Select proper tools for specific horticultural jobs.

F9.4 Understand how to install landscape components and electrical land and water features. 
Agriculture and Natural Resources Industry Sector

F10.0 Students understand basic landscape planning, design, construction, and maintenance:

F10.1 Know the terms associated with landscape and design and their appropriate use.

F10.2 Understand the principles of residential design, including how to render design to scale.

F10.3 Understand proper landscape planting and maintenance practices.

F10.4 Prune ornamental shrubs, trees, and fruit trees.

F10.5 Develop clear and concise landscape business contracts.

F11.0 Students understand basic floral design principles:

F11.1 Understand the use of plant materials and tools.

F11.2 Apply basic design principles to products and designs.

F11.3 Handle, prepare, and arrange cut flowers appropriately.

F11.4 Understand marketing and merchandising principles used in the floral industry. 


\section{G. Plant and Soil Science Pathway}

The Plant and Soil Science Pathway covers topics such as plant classification, physiology, reproduction, plant breeding, biotechnology, and pathology. In addition, students learn about soil management, water, pests, and equipment as well as cultural and harvest practices.

\section{G1.0 Students understand plant classification principles:}

G1.1 Understand how to classify and identify plants by order, family, genus, and species.

G1.2 Understand how to identify plants by using a dichotomous key.

G1.3 Understand how common plant parts are used to classify the plants.

G1.4 Understand the differences between and uses of native and nonnative plants.

G1.5 Understand the differences between monocots and dicots.

G1.6 Understand the differences between plants under production and weeds.

\section{G2.0 Students understand cell biology:}

G2.1 Understand the differences between prokaryotic cells and plant and animal eukaryotic cells and how viruses differ from them in complexity and general structure.

G2.2 Understand plant cellular function reactions when plants are grown under different conditions.

G2.3 Understand what functions organelles play in the health of the cell.

G2.4 Understand the part of the cell that is responsible for the genetic information that controls plant growth and development.

G2.5 Understand plant inheritance principles, including the structure and role of DNA.

G2.6 Understand which organelles in plant cells carry out photosynthesis.

G3.0 Students understand plant physiology and growth principles:

G3.1 Understand plant systems, nutrient transportation, structure, and energy storage.

G3.2 Understand the seed's essential parts and functions.

G3.3 Understand how primary, secondary, and trace elements are used in plant growth.

G3.4 Understand the factors that influence plant growth, including water, nutrients, light, soil, air, and climate.

G3.5 Understand the tissues seen in a cross section of woody and herbaceous plants.

G3.6 Understand the factors that affect plant growth and predict plant response. 
G4.0 Students understand sexual and asexual reproduction of plants:

G4.1 Understand the different forms of sexual and asexual plant reproduction.

G4.2 Understand the various techniques for successful plant propagation (e.g., budding, grafting, cuttings, and seeds).

G4.3 Understand the proper sterile technique used in tissue culture.

G5.0 Students understand pest problems and management:

G5.1 Understand how to categorize insects as pests, beneficial, or neutral and their roles.

G5.2 Understand the role of other pests, such as nematodes, molds, mildews, and weeds.

G5.3 Know conventional, sustainable, and organic management methods to prevent or treat plant disease symptoms.

G5.4 Understand integrated pest management to prevent, treat, and control plant disease symptoms (including conventional, sustainable, and organic management methods).

G5.5 Understand how biotechnology can be used to manage pests.

\section{G6.0 Students understand soils and plant production:}

G6.1 Understand soil types, soil texture, structure, and bulk density and explain the U.S. Department of Agriculture (USDA) soil-quality rating procedure.

G6.2 Understand soil properties necessary for successful plant production, including $\mathrm{pH}, \mathrm{EC}$, and essential nutrients.

G6.3 Understand soil biology and diagram the soil food chain.

G6.4 Understand how soil biology affects the environment and natural resources.

G7.0 Students understand effective tillage and soil conservation management practices:

G7.1 Understand how to effectively manage and conserve soil through conventional, minimum, conservation, and no-tillage irrigation and through drainage and tillage practices.

G7.2 Understand how global positioning systems, surveying, laser leveling, and other tillage practices conserve soil.

G7.3 Use tools such as the USDA and the local Resource Conservation District soil survey maps to determine appropriate soil management practices.

\section{G8.0 Students understand effective water management practices:}

G8.1 Understand California water history, current issues, water rights, water law, and water transfer through different distribution projects throughout the state.

G8.2 Understand the local, state, and federal agencies that regulate water quality and availability in California. 
G8.3 Understand the definition of a watershed and how it is used to measure water quality.

G8.4 Understand effective water management and conservation practices, including the use of tailwater ponds.

G8.5 Know water-testing standards and perform bioassay and macro-invertebrate protocols to assess water quality.

G9.0 Students understand the concept of an "agrosystem" approach to production:

G9.1 Understand how to identify and classify the plants and animals in an agricultural system (as producers, consumers, or decomposers).

G9.2 Understand the elements of conventional, sustainable, and organic production systems.

G9.3 Understand the components of "whole-system management."

G10.0 Students understand local crop management and production practices:

G10.1 Understand local cultural techniques, including monitoring, pruning, fertilization, planting, irrigation, harvest treatments, processing, and packaging practices for various tree, grain, hay, and vegetable classes.

G10.2 Understand common marketing and shipping characteristics of local commodities.

G10.3 Understand general maturity and harvest-time guidelines for specific local plant products.

\section{G11.0 Students understand plant biotechnology:}

G11.1 Understand how changing technology - such as micropropagation, biological pest controls, and genetic engineering (including DNA extraction and gel electrophoresis) - affects plant production, yields, and management.

G11.2 Understand the various technology advancements that affect plant and soil science (such as global positioning systems, global information systems, variable rate technology, and remote sensing).

G11.3 Know how herbicide-resistant plant genes can affect the environment.

G11.4 Understand how genetic engineering techniques have been used to improve crop yields.

G11.5 Understand the effects of agricultural biotechnology, including genetically modified organisms, on the agriculture industry and the larger society and the pros and cons of such use. 


\section{R2 Teacher Information Madera South HS,Madera Year: 2013}

\begin{tabular}{|c|c|c|c|c|c|c|c|c|c|c|c|}
\hline $\begin{array}{l}\text { Last } \\
\text { Name }\end{array}$ & $\begin{array}{l}\text { First } \\
\text { Name }\end{array}$ & MI & Gender & Ethnicity & $\begin{array}{c}\text { Total } \\
\text { Years } \\
\text { Teaching } \\
\text { Ag. }\end{array}$ & Credential Type & $\begin{array}{l}\text { 9-Month } \\
\text { Salary }\end{array}$ & $\begin{array}{l}\text { Extended } \\
\text { Contract } \\
\text { Stipend }\end{array}$ & $\begin{array}{c}\text { FFA } \\
\text { Stipend }\end{array}$ & $\begin{array}{c}\text { Department } \\
\text { Head } \\
\text { Stipend }\end{array}$ & $\begin{array}{c}\text { SOE } \\
\text { Period }\end{array}$ \\
\hline George & Brent & & Male & White & 9 & $\begin{array}{l}\text { Agriculture } \\
\text { Specialist }\end{array}$ & & & 1500 & 0 & $\mathrm{~N}$ \\
\hline Mckenna & Kristin & A & Female & White & 5 & $\begin{array}{l}\text { Agriculture } \\
\text { Specialist }\end{array}$ & & & 0 & and & $\mathrm{Y}$ \\
\hline Williams & John & $S$ & Male & White & 5 & $\begin{array}{l}\text { Agriculture } \\
\text { Specialist }\end{array}$ & & & Dod & 0 & $\mathrm{~N}$ \\
\hline Deniz & Tim & W & Male & White & 11 & $\begin{array}{l}\text { Agriculture } \\
\text { Specialist }\end{array}$ & & & 0 & 0 & $\mathrm{Y}$ \\
\hline Gilles & Darlene & E & Female & White & 30 & $\begin{array}{l}\text { Agriculture } \\
\text { Specialist }\end{array}$ & $\longdiv { 7 4 3 8 }$ & & 0 & 0 & $\mathrm{~N}$ \\
\hline Luera & Crystal & & Female & Hispanic & 2 & $\begin{array}{l}\text { Agriculture } \\
\text { Specialist }\end{array}$ & & & 0 & 0 & $\mathrm{~N}$ \\
\hline
\end{tabular}

\begin{tabular}{|l|l|l|l|l|l|}
\hline Deniz, Tim & \multicolumn{5}{|l|}{$\mid$} \\
\hline Schedule & Period & Beginning Time & Course Title & Enrollment & Type \\
\hline 1 & 0 & $6: 40$ & ROP Welding & 22 & Ag Mechanics \\
\hline 1 & 1 & $7: 40$ & ROP Welding & 22 & Ag Mechanics \\
\hline 1 & 2 & $8: 54$ & Ag. Mech II & 16 & Ag Mechanics \\
\hline 1 & 3 & $10: 01$ & Ag. Mech II & 22 & Ag Mechanics \\
\hline 1 & 4 & $11: 15$ & SAE Period & 0 & SAE \\
\hline 1 & 5 & $12: 56$ & Prep & 0 & Prep \\
\hline
\end{tabular}

\begin{tabular}{|l|l|l|l|l|l|}
\hline \multicolumn{6}{|l|}{ George, Brent } \\
\hline Schedule & Period & Beginning Time & Course Title & Enrollment & Type \\
\hline 1 & 1 & $7: 40$ & Ag. Mech I & 31 & Ag Mechanics \\
\hline 1 & 2 & $8: 54$ & Prep & 0 & Prep \\
\hline 1 & 3 & $111: 01$ & Ag. Mech 1 & 32 & Ag Mechanics \\
\hline 1 & 4 & $11: 15$ & Ag. Mech 1 & 35 & Ag Mechanics \\
\hline 1 & 5 & $12: 56$ & Ag. Mech 1 & 33 & Ag Mechanics \\
\hline 1 & 6 & $2 \cdot 10$ & Ag. Mech 1 & 32 & Ag Mechanics \\
\hline
\end{tabular}

\begin{tabular}{|c|c|c|c|c|c|}
\hline \multicolumn{6}{|c|}{ Gilles, Darlene } \\
\hline Schedule & Period & $\mathrm{Bc}-$ :nu: Time & Course linle & Enrollment & $\mathrm{T}: \mathrm{C}$ \\
\hline 1 & 1 & 7:4: & Prep & 0 & Prep? \\
\hline 1 & 2 & $8:-: 4$ & Floral & 36 & () $1 ! 1$-ko: \\
\hline 1 & 3 & 10 & Ag latth Sch & 38 & 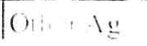 \\
\hline
\end{tabular}




\begin{tabular}{|l|l|l|l|l|l|}
\hline 1 & 4 & $11: 15$ & Advanced/Retail Floral & 14 & O.H./Floral \\
\hline 1 & 5 & $12: 56$ & Ag. Economics & 32 & Ag Bus Mgt \\
\hline 1 & 6 & $2: 10$ & Floral & 32 & O.H./Floral \\
\hline
\end{tabular}

\begin{tabular}{|c|c|c|c|c|c|}
\hline \multicolumn{6}{|c|}{ Luera, Crystal } \\
\hline Schedule & Period & Beginning Time & Course Title & Enrollment & Type \\
\hline 1 & 1 & $7: 40$ & Ag. Earth Science & 38 & Other Ag \\
\hline 1 & 2 & 8:54 & Ag. Earth Science & 38 & Other Ag \\
\hline 1 & 3 & 10:01 & Ag. Biology & 36 & Ag Biology \\
\hline 1 & 4 & 11:15 & Prep & 0 & Prep \\
\hline 1 & 5 & $12: 56$ & Ag. Biology & 36 & Ag Biology \\
\hline \multirow[t]{2}{*}{1} & 6 & $2: 10$ & Ag. Science 1 - & 29 न & Agrišcience I \\
\hline & & & $\because \cdots$ & 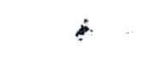 & \\
\hline \multicolumn{3}{|c|}{ Mckenna, Kristin } & . & \multirow{2}{*}{ Enrollment } & " \\
\hline Schedule & Period & Beginning Time & Course Title & & Type \\
\hline 1 & 1 & $7: 40$ & SAE Period & $0 \% "$ & SAE \\
\hline 1 & 2 & $8: 54$ & Ag. Science 1 & 27 & Agriscience I \\
\hline 1 & 3 & $9: 44$ & Horticulture 1 & 28 & O.H./Floral \\
\hline 1 & 4 & $11: 15$ & Horticulture 1 and 2 & 38 & O.H./Floral \\
\hline 1 & 5 & $12: 56$ & Ag. Science 1 & 35 & Agriscience I \\
\hline 1 & 6 & $2: 10$ & $* \quad$ & 0 & Prep \\
\hline
\end{tabular}

\begin{tabular}{|l|l|l|l|l|l|}
\hline \multicolumn{6}{|l|}{ Sheehan, Kristin } \\
\hline Schedule & Period & Beginning Time & Course Title & Enrollment & Type \\
\hline 1 & 1 & $7: 40$ & Veterinary Science & 38 & Animal Science \\
\hline 1 & 2 & $8: 54$ & Small Animal Care & 30 & Animal Science \\
\hline 1 & 3 & $10: 01$ & Prep & 0 & Prep \\
\hline 1 & 4 & $11: 15$ & Ag. Biology & 38 & Ag Biology \\
\hline 1 & 5 & $12: 56$ & Ag. Biology & 33 & Ag Biology \\
\hline 1 & 6 & $2: 10$ & Ag. Biology & 34 & Ag Biology \\
\hline
\end{tabular}

\begin{tabular}{|l|l|l|l|l|l|}
\hline \multicolumn{2}{|l|}{ Willians, John } & \multicolumn{2}{l|}{$\mid$} \\
\hline Schedule & Period & Beginning Time & Course Title & Enrollment & Type \\
\hline 1 & 1 & $7: 40$ & ROP Diesel & 24 & Ag Mechanics \\
\hline 1 & 2 & $8: 54$ & ROP Diesel & 24 & Ag Mcchanics \\
\hline 1 & 3 & $10: 01$ & Diesels & 31 & Ag Mechanics \\
\hline 1 & 4 & $11: 15$ & Ag. Leadership & 20 & Othu 1g \\
\hline 1 & 5 & $12: 56$ & Diesels & 29 & Ag Vuchanics \\
\hline 1 & 6 & $2: 10$ & Prep & 0 & Prep \\
\hline
\end{tabular}


Matt Angell

24220 Ave $131 / 2$

Madera, CA 93638

James Cavallero

10759 Road 24

Madera, CA 93637

Craig Farmer

8981 Ave. $231 / 2$

Chowchilla, CA 93610

Richard Haupt

2622 N. Vagedes Ave.

Fresno, CA 93705

Todd Houlding

119 East Loop

Madera, CA 93637

Richard Kuckenbecker

800 Madera Ave

Madera, CA 93637

Tim Leach

32288 Ave.12

Madera, CA 93638

Eddie Martinazzi

14390 Silk Oak Lane

Madera, CA 93637

Jeff McKinney

22457 Ave 131/2

Madera, CA 93637

Don Nelson

1901 W.Cleveland

Madera, CA 93637
Rik Wilson

28777 Ave 15 1/2

Madera, CA 93637

Robert Chavez 1902 Howard Road Madera, CA 93637

Don Farnesi

35155 Mustang Lane

Madera, CA 93637

Shelly Head

23296 Road 24

Madera, CA 93610

Phillip Janzen

19930 Del Mar Road

Madera, CA 93638

Joe Lilles

P.O. Box 506

Madera, CA 93639

Ed LeTourneau DVM

P.O. Box 990

Madera, CA 93639

Brad McDonald

1200 Maple

Madera, CA 93637

Bret Mercer

12872 Road 36 1/2

Madera, CA 93636

Bruce Norton

3055 Fairmont Ct

Madera, CA 93637
Jim Bomprezzi

2105 National Ave

Madera, CA 93637

Jim Erickson

10797 Road 29 1/2

Madera, CA 93637

Gary Geist

17663 Island Drive

Madera, CA 93636

Bob Houlding

15782 Road 21

Madera, CA 93637

John Koretoff

2300 Riverview Drive \#101

Madera, CA 93637

Bob Labrucherie

9671 Road $281 / 2$

Madera, CA 93637

David Loquaci

131 East Loop

Madera, CA 93637

Neil McDougal

332 Madera Ave

Madera, CA 93637

Bob Naden

39880 Ave. 71/2

Madera, CA 93638

Kevin Peters

1135 South Granada Drive Madera, CA 93637 
Dennis Prosperi

11409 Road $261 / 2$

Madera, CA 93637

Michael Salvador

25742 El Vado

Madera, CA 93638

Mike Schafer

4614 Road $241 / 2$

Madera, CA 93637

John Stafford

1902 Howard Road

Madera, CA 93637

Maddera FFA

705 W. Pecan

Madera, CA 93637

Madera FFA

705 W. Pecan

Madera, CA 93637

Madera FFA

705 W. Pecan

Madera, CA 93637

Madera FFA

705 W. Pecan

Madera, CA 93637

Madera FFA

705 W. Pecan

Madera, CA 93637

Madera FFA

705 W. Pecan

Madera, CA 93637
Kelly Porterfield

1902 Howard Road

Madera, CA 93637

Stan Samuelson

800 S. Madera Ave

Madera, CA 93637

Carl Schroeder

20769 Road 30

Madera, CA 93638

Stan Williams

400 Fairview Ave.

Madera, CA 93637
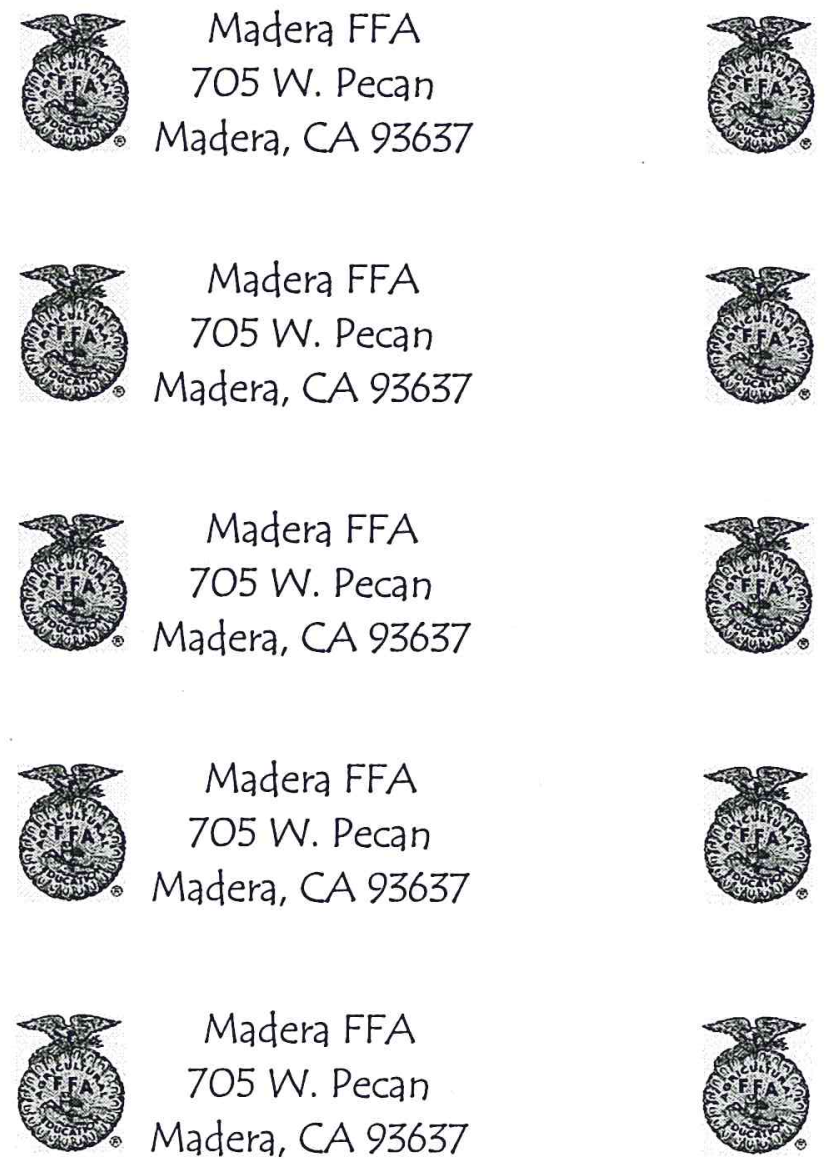

Madera FFA 705 W. Pecan Madera, CA 93637

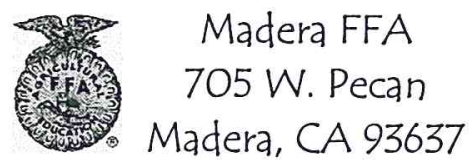

Madera FFA

705 W. Pecan

Madera, CA 93637

Madera FFA 705 W. Pecan

Madera, CA 93637

Madera FFA

705 W. Pecan

Madera, CA 93637 332 Madera Ave Madera, CA 93637

Steve Schafer 14484 Road 21

Madera, CA 93637

Ray Seibert 15252 Road 23

Madera, CA 93637

Madera FFA

705 W. Pecan

Madera, CA 93637
Robert Roland 


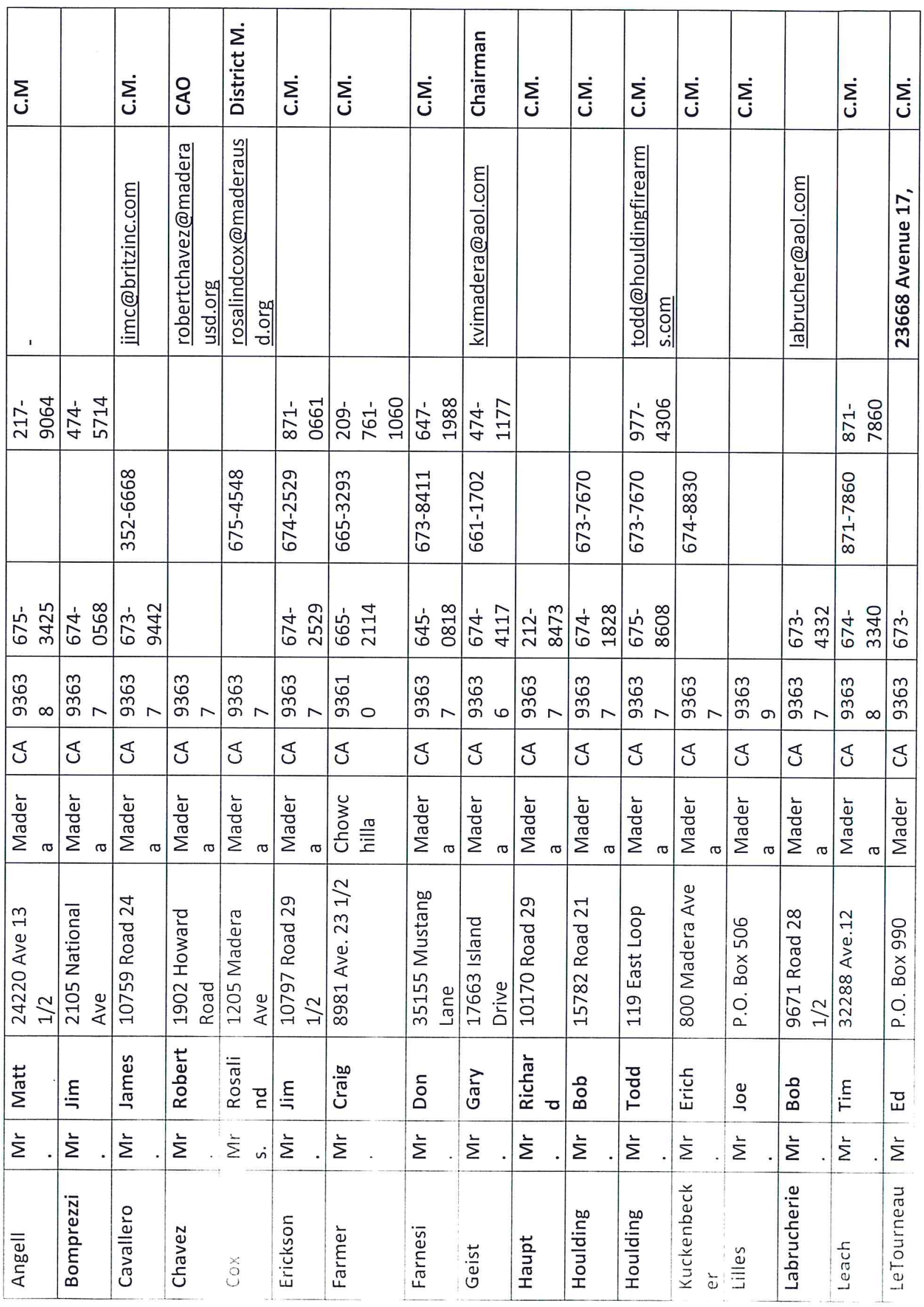




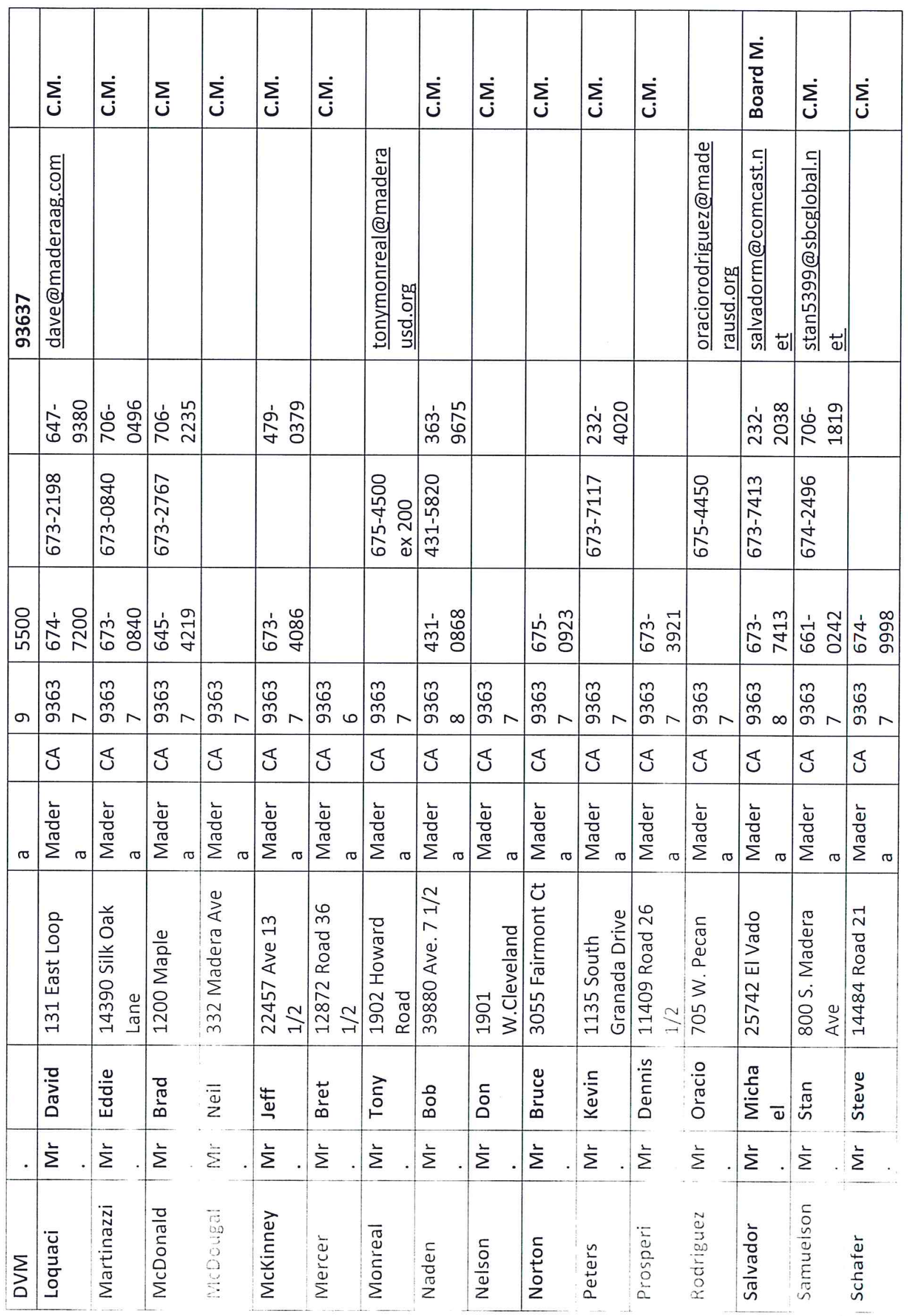




\begin{tabular}{|c|c|c|c|c|c|c|c|}
\hline غ & $\sum_{j}^{\infty}$ & 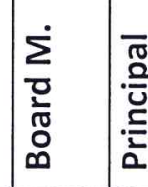 & 产产 & 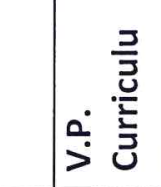 & $\varepsilon \sum_{\dot{\mathcal{j}}}$ & 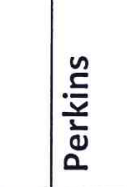 & غن \\
\hline . & & 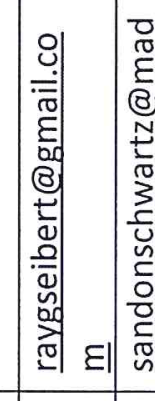 & 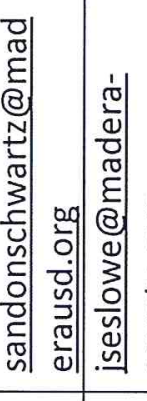 & | & 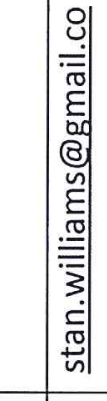 & 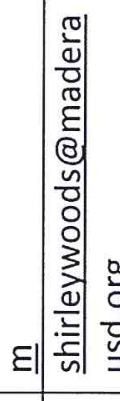 & 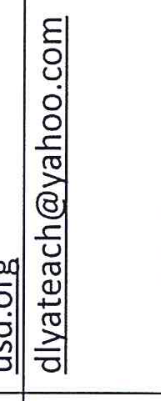 \\
\hline 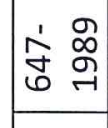 & & 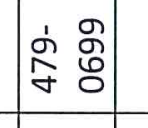 & & & & & 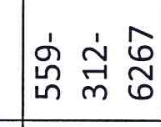 \\
\hline & & 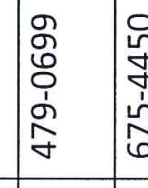 & & & & 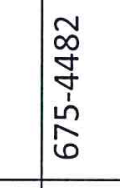 & \\
\hline & & $\begin{array}{l}1 \\
\vdots \\
0\end{array}$ & & & 童 & & 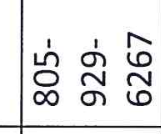 \\
\hline 惫, & & 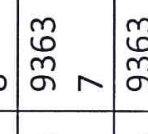 & 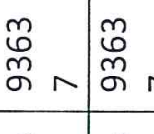 & 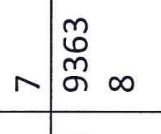 & 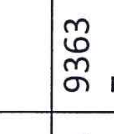 & 、罢 & 敕 \\
\hline ভ & $\mathbb{s}$ & 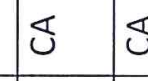 & $\begin{array}{lll} & \mathbb{8}\end{array}$ & $\varangle$ & $\mathbb{J}$ & S & $\mathbb{8}$ \\
\hline 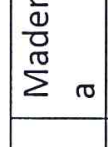 & & 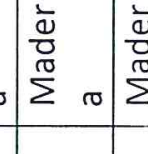 & 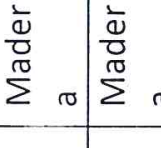 & ${ }_{\sigma} \sum_{\sigma}^{\frac{\bar{\nu}}{\omega}} \sum_{\sigma}$ & $\mid \begin{array}{l}\frac{\mathrm{u}}{\mathrm{u}} \\
\frac{\mathrm{a}}{\mathrm{a}}\end{array}$ & 。 & 。 \\
\hline 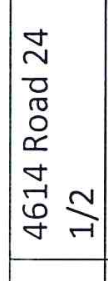 & 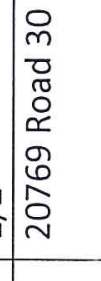 & 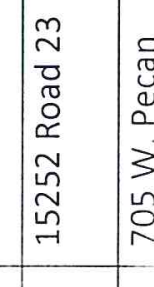 & 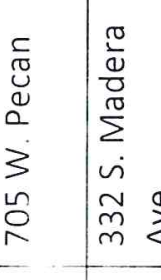 & 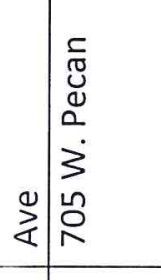 & 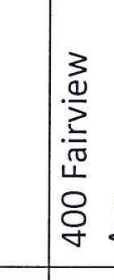 & 雷 & $\mid$ \\
\hline 送 & 焉 & 俤 & 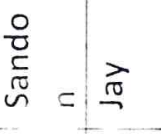 & 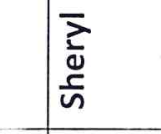 & 点 & 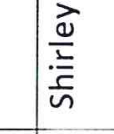 & \begin{tabular}{|l} 
\\
$\bar{a}$ \\
0
\end{tabular} \\
\hline$\Sigma$ & $\Sigma$ & $\Sigma$ ¿. & $\Sigma$ ¿ & $\Sigma \dot{~} \dot{ }$ & $\Sigma$ & $\bar{\Sigma}$ & $\Sigma$ \\
\hline & & 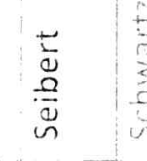 & & $\overline{\overline{\bar{n}}}$ & 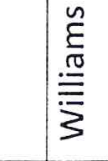 & $\begin{array}{l}\frac{n}{0} \\
\vdots \\
3\end{array}$ & 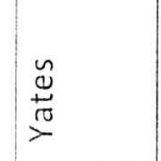 \\
\hline
\end{tabular}


Agriculture Advisory Meeting

Madera South High School

October 10, 3013

In attendance: See attachment \#1

$\begin{array}{lll}\text { Oracio Rodriguez } & \text { Michael Salvador } & \text { Sheryl Sisil } \\ \text { Dick Haupt } & \text { Jim Bomprezzi } & \text { Bob Naden } \\ \text { Carl Schroeder } & \text { Don Farnesi } & \text { Bob Labrucherie } \\ \text { Dave Loquaci } & \text { Jim Cavallero } & \text { Shirley Woods } \\ \text { Prince Marshall } & \text { Sandon Schwartz } & \text { Ed LeTourneau } \\ \text { Jim Erickson } & \text { Kristin McKenna } & \text { John Williams } \\ \text { Darlene Gilles } & \text { Tim Deniz } & \text { Brent George } \\ \text { Kristin Sheehan } & \text { Crystal Luera } & \text { Johnny Lopes }\end{array}$

The meeting was called to order by the department chairperson Kristin McKenna in Gary Geist's absence at 6:05 p.m. After a brief introduction the members were dismissed for dinner.

The meeting resumed at 6:15 p.m. and introductions were made of all present. Our new teacher Crystal Luera and student teacher Johnny Lopes were highlighted. Darlene Gilles was presented a plaque for 7 years of outstanding service as the department head.

A motion to approve the minutes from the March 2013 advisory meeting was made by Jim Erickson and seconded by Jim Cavallero, minutes approved, voice vote.

The agenda started with John Williams presenting a power point listing the accomplishments and many activities of the Madera FFA members from May to the present.

Kristin McKenna led a review of the Agriculture Incentive Grant checklist that needed to be certified by the advisory committee. The findings were we don't meet criteria's $10 \mathrm{~A}, 11 \mathrm{~B}$ and 12A. (Attachment 2) The goal of the department is to work towards meeting 12A, however with the current budget issues, criteria's $10 \mathrm{~A}$ and $11 \mathrm{~B}$ are out of reach at this time. The checklist was approved with a motion by Jim Cavallero and $2^{\text {nd }}$ by Dave Loquaci, checklist approved, voice vote.

John Williams reported on the status of the vineyard. The work started shortly after our last Ag. Advisory committee meeting; with trenching and manifolds being put in, stakes and wiring being hung, drip lines installed, and the vines being planted. All of the work done on the vineyard was completed by students. list of donors can be found in (attachment 3).

Tim Deniz and Brent George pr...ented the Ag. Mechanics Welding and Fabrication Pathway. Thev reviewed with everyon: the courses that make up the pathway, topics that are covered and career opportunities for dents that pursue the pathway. Tim also inform-d the 
committee that the classes are now articulated with Butte College and articulations are in the works for Fresno City and Merced. (Attachment 4)

Dave Loquaci asked a question about what effects common core will have on the Ag. Department and the time frame until full implementation. Sheryl Sisil stated that step one is rewriting core courses and then step 2 will be re-working elective classes. It is her opinion that common core will help our courses since the focus is in real life applications rather than memorization. Some of our courses may change in content taught in them but we will still be providing real life skills.

Meeting adjourned 7:07 p.m.

Respectfully submitted

Kristin McKenna

MSHS Ag Dept. 


\section{Madera South High School Agriculture Department Budget}

Perkins 2013-2014

42,000

Books/Supplies

3,200

Services/Operating

Expenditures:

Transportation

11,800

Instruction

5,000

Professional Development

8,000

New Professionals

National FFA convention

San Joaquin region road show

Regional Meetings

CATA Summer Conference

Substitute's

8,000

FFA Leadership Packets

6,000

Instructional Supplies

16,000

Travel

5,000

Equipment Replacement

5,000 


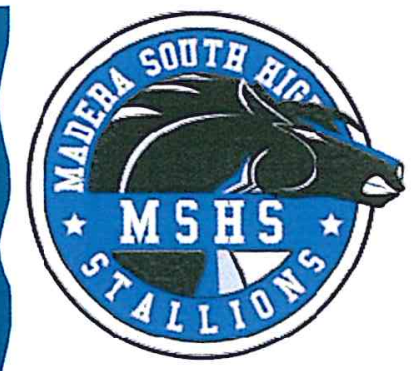

Madera South High School

Agriculture Department

$705 \mathrm{~W}$. Pecan

Madera, CA 23637

(552)675-4475

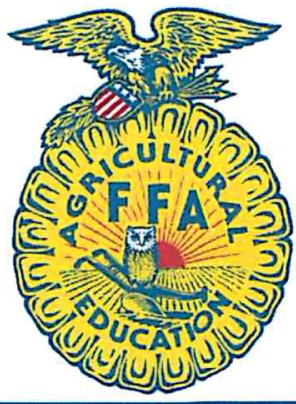

\section{Madera South Agriculture Graduate Follow Up System}

Madera South High School calls all graduates in August and September following graduation to find out they key information for the R-2. We keep their contact information and if we cannot get a hold of the student, we call the parent. The questions asked include:

- Name

- Address

- Phone Number

- What are you doing now?

* Attending School?

- Full Time or Part Time

هhat School

$\bullet$ Major

*Working?

- Full Time or Part Time

- What type of business or industry are you involved in?

- Job Title or Description

-What skills from MSHS Ag. are you using in your job? *Military or Other

- What experiences in Ag. and FFA were most valuable for you?

- Any other comments?!? 


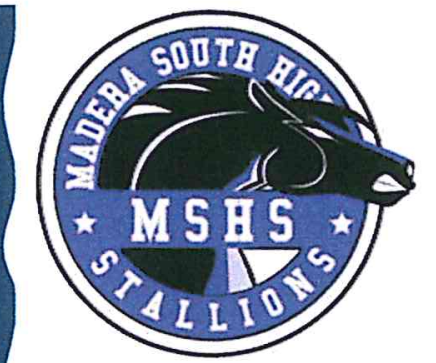

Madera South High School Agriculture Department 705 W. Pecan Madera, CA 23637 (552)675-4475

\section{Madera South Agriculture Placement Sites}

The following is a list of places students are currently interning or working at, or are places we could potentially send students.

- Midland Tractor

- Kuckenbecker Tractor

- Madera Tractor Company

- Mower Plus

- Farm Machinery Corporation

- Baltimore Air Coil

- Madera Welding and Manufacturing

- Blue Arc Welding

- U.S. Rental

- Madera Pump Company

- Jim Erickson Farms

- Talley Concrete

- Producers Livestock

- Production Credit Association

- Gus' Food Locker

- Madera Thoroughbreds

- TECO Feed and Hardware

- Valley Feed

- Evans Feed and Livestock Supply

- Floral Fantasy

- Purl Sheet Metal

- Schafer Metal Stake

- Madera Ag. Services

- Madera District Fairgrounds

- Schaffer Ranches

- S\&J Lumber
- H\&H Welding and Fabrication

- Warnock Food Products

- Valley Grain Products

- Valley Wide Irrigation

- Haupt Custom Almond Harvesting

- Evapco West Inc.

- Madera Animal Hospital

- Ag-Right Enterprises

- Tolmachoff \& Son Custom Harvesting

- Valley Pistachio Inc.

- Western Farm Service

- Advanced Drainage

- Ambroso Veterinary Hospital

- Bass \& Sons Septic Tank

- Bolderoff Custom Harvesting

- Britz Fertilizers Inc.

- Chavira Trucking

- General Builder Supply Company

- Steve's Wrought Iron

- Peters Brothers Nursery .

- Kings Valley Industry

- Landmark Irrigation

- American Grape Harvester

- Cherokee

- Dave Loqacci Famrs 


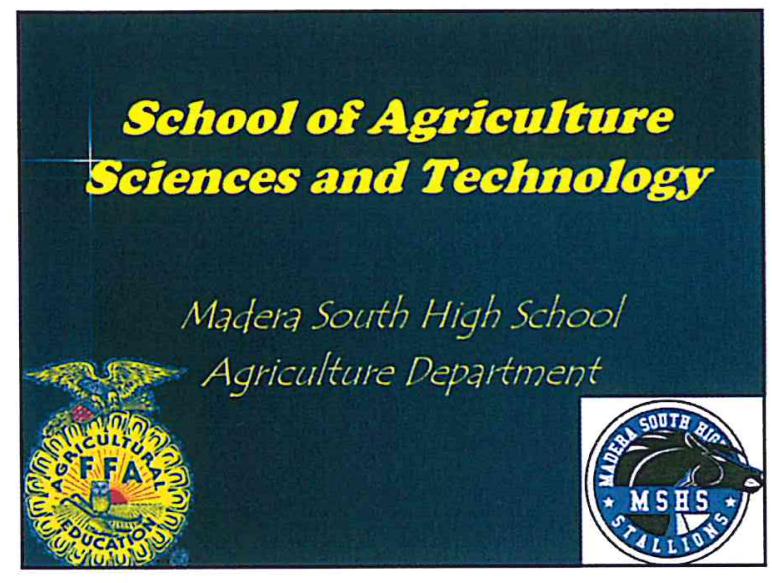

\section{CAREDR PATHWATS}

- Environmental Science

- Environmental Sciences I - HS Physical

Science Requirement

- Environmental Science II - HS Life Science Requirement

- Floriculture

- Floral Design I - HS Fine Art Requirement

- Advanced Floral Design

- Retail Floral shop

\section{CAREDR PATEIWATS}

- Animal Science

- Ag science I

- Ag science II - HS Life Science Graduation

Requirement

- Ay Biology - CSU/VC Lab science Requirement

- Veterinary Science -

CSU/UC Elective Requirement

- Veterinary Care \&s Handling

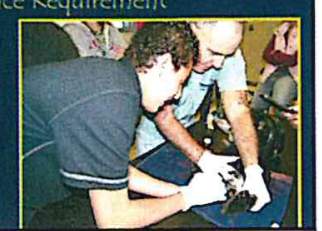

\section{CAREER PATHWATS}

- Agriculture Mechanics \& Technology

- Welding/Fabrication

- Ayriculture Mechanios

- Agriculture Mechanics II

- Ayriculture Mechanics III

- Ayriculture Mechanies IV

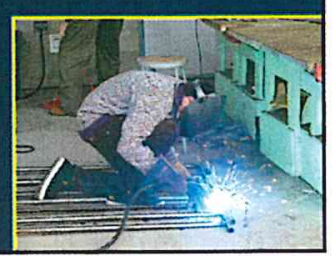

\section{FACILITIDS}

\section{CAREDR PATHWATS}

- Agriculture Mechanics \& Technology

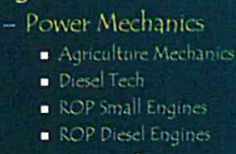

- Ornamental Horticulture

- Horticulture - HS Life Science

- Horticulture II - HS Physical Science Requirement

- Nursery Management - 2 hour course
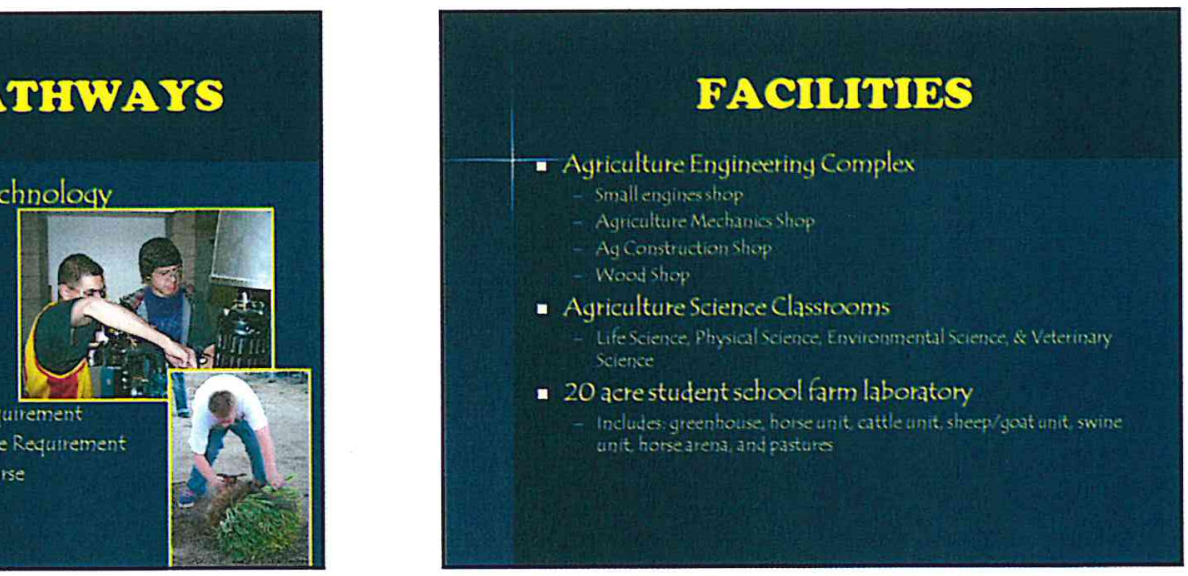

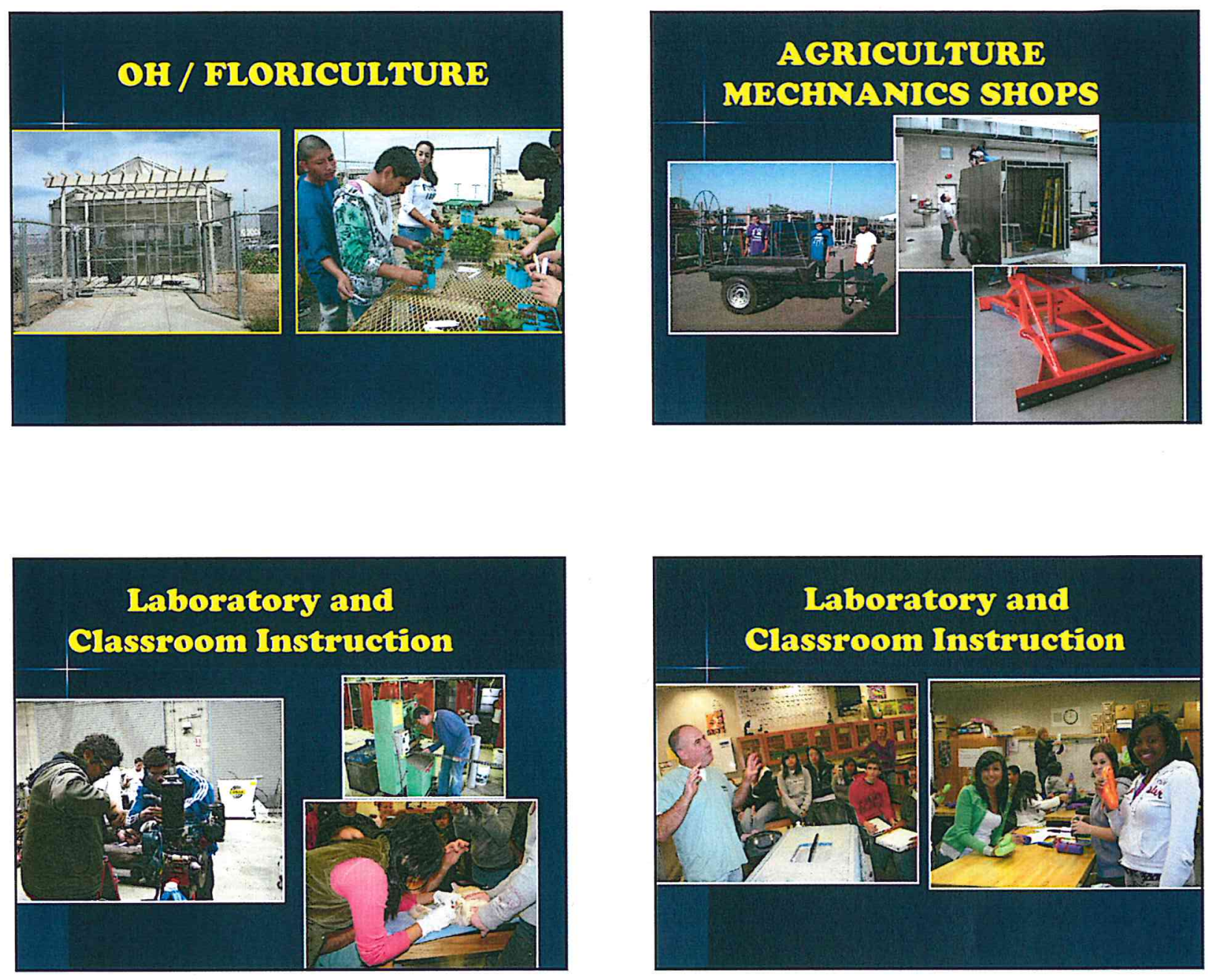

\section{STUDENT IEADERSAIP}

- National FFA Association

- Part of Ag Education Classes

- Personal Growth, Premier Leadership, \& Career Success

- Madera FFA is represented at the local, sectional, regional, \& state levels

- Madera FFA has 670 members

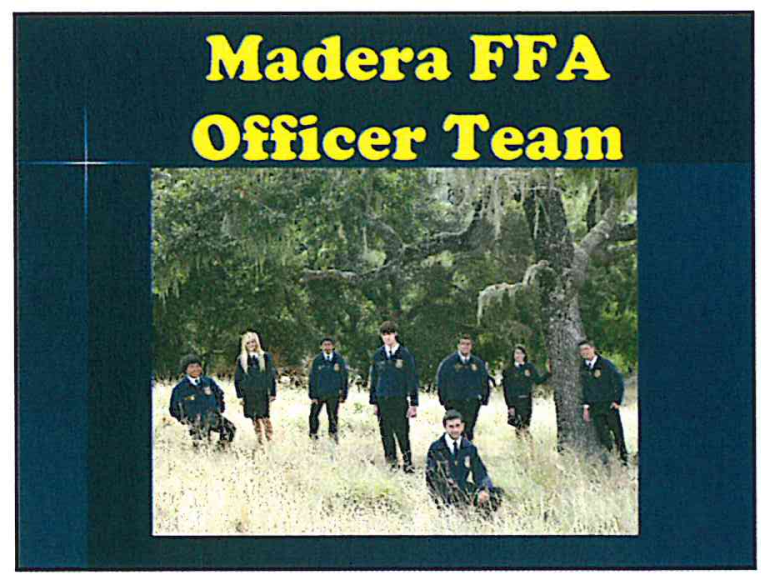



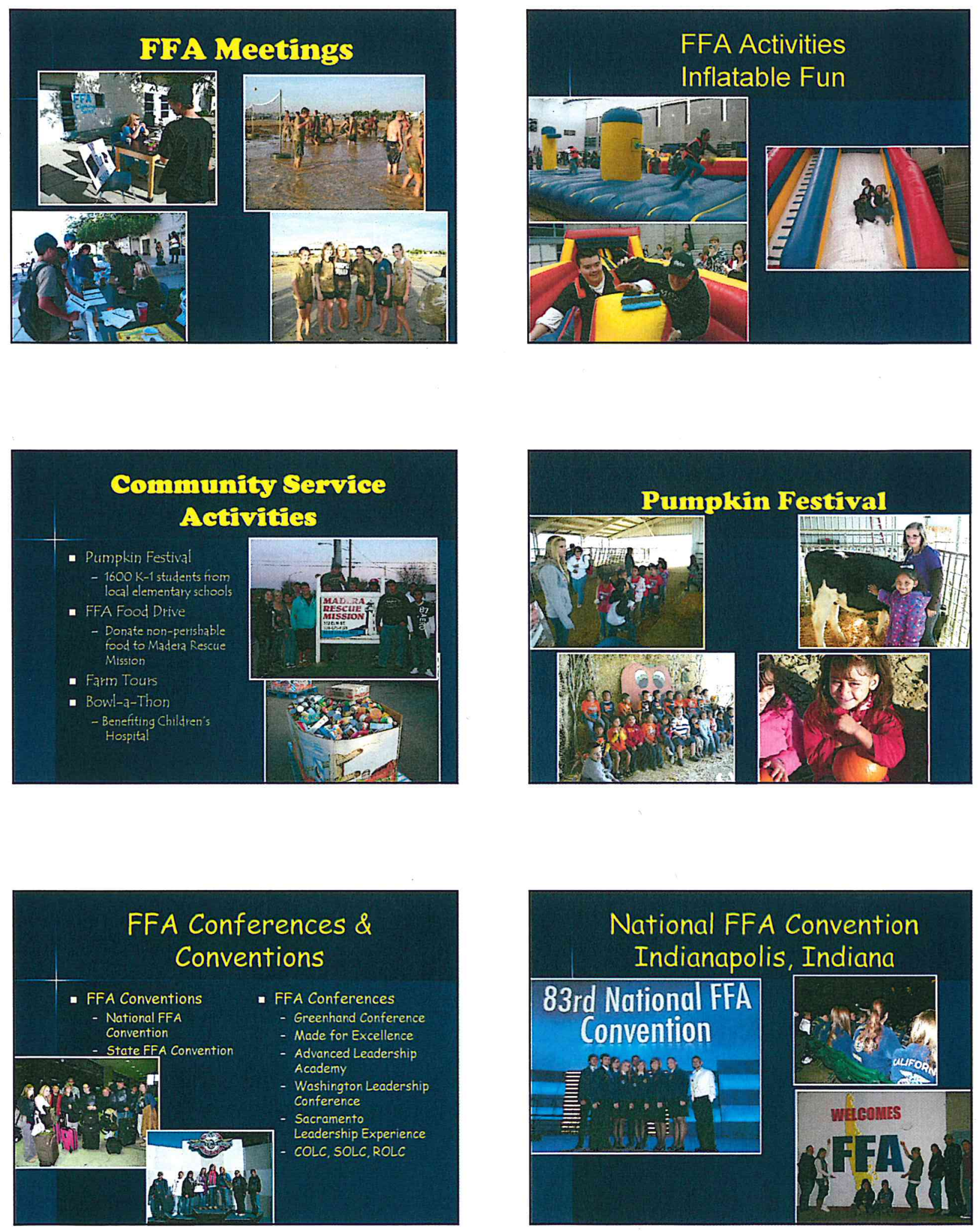


\section{STUDENT PROJECTS}

- Students will be required to participate in a supervised agriculture experience project.

- You gain real life experience and in most cases earn MONEY.

- An extremely diverse range of acceptable projects.

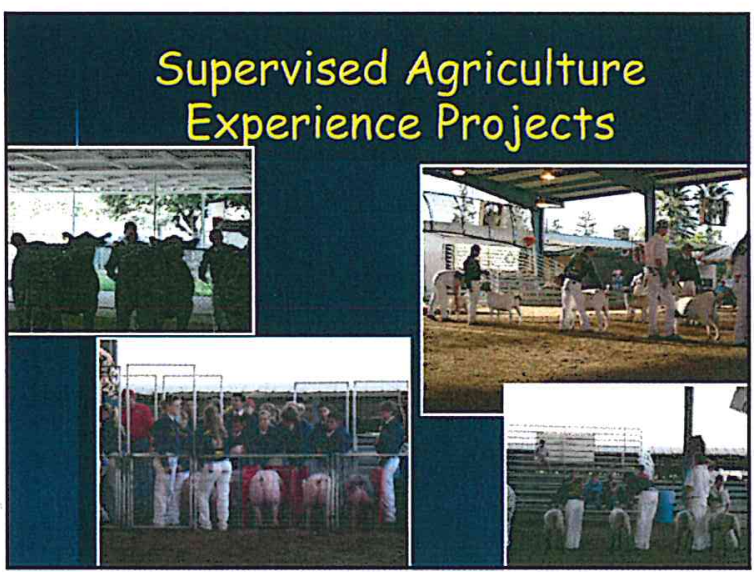

Career Development Events \& FFA Competitions

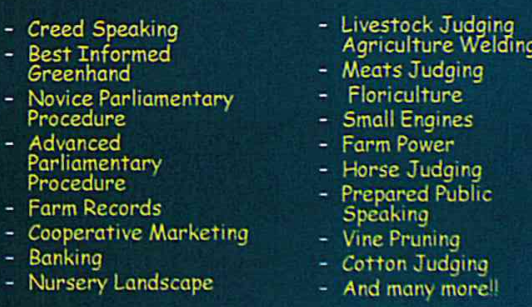

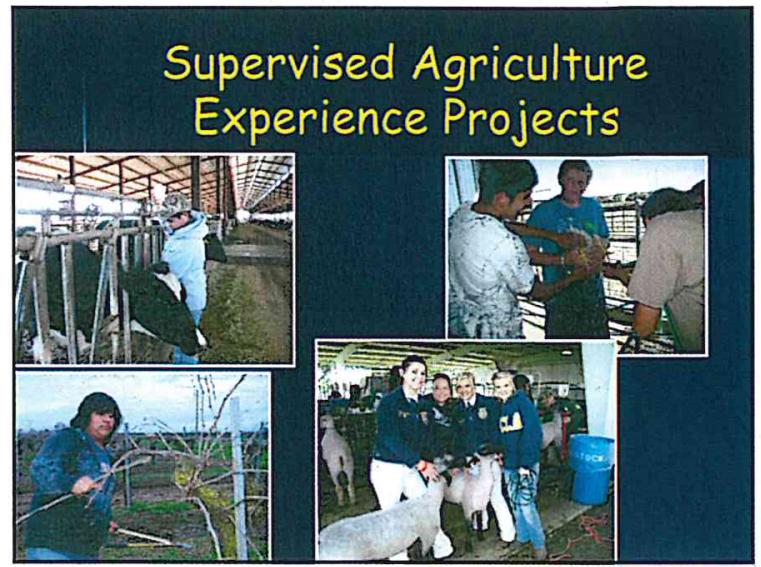
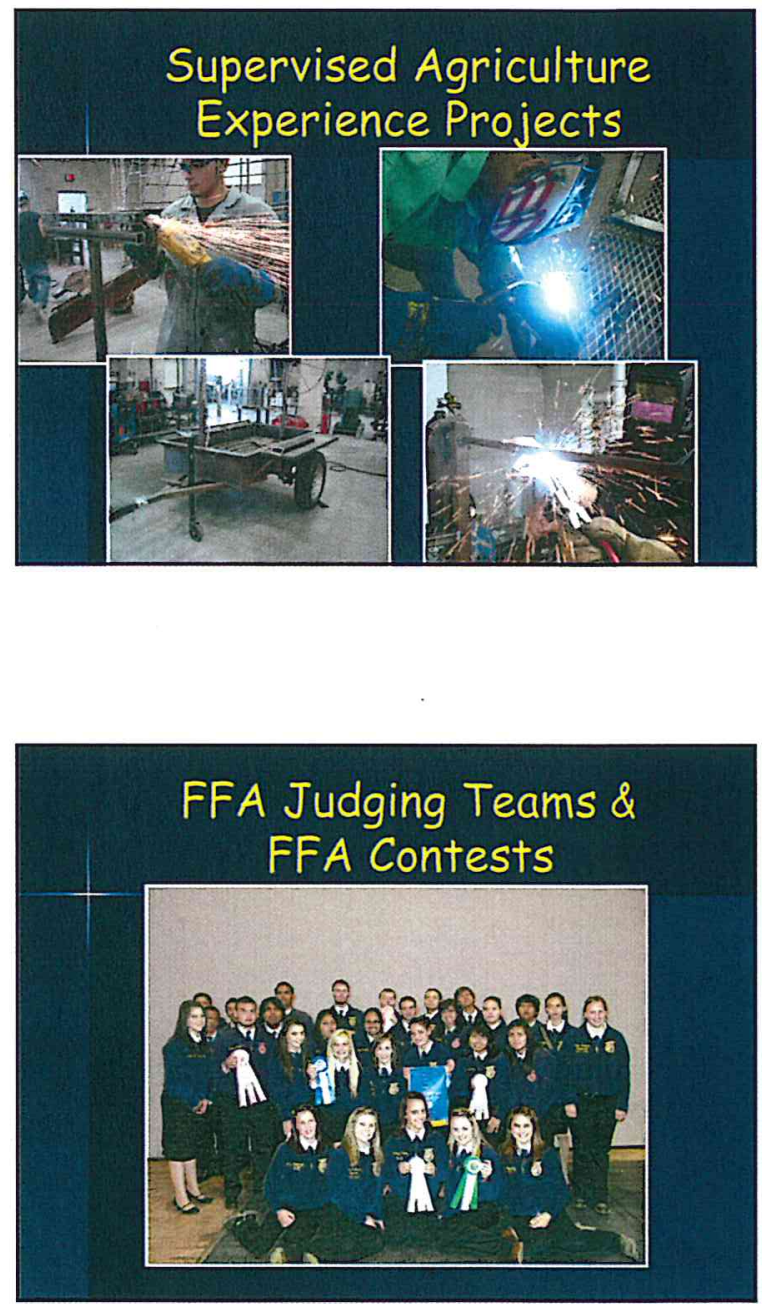


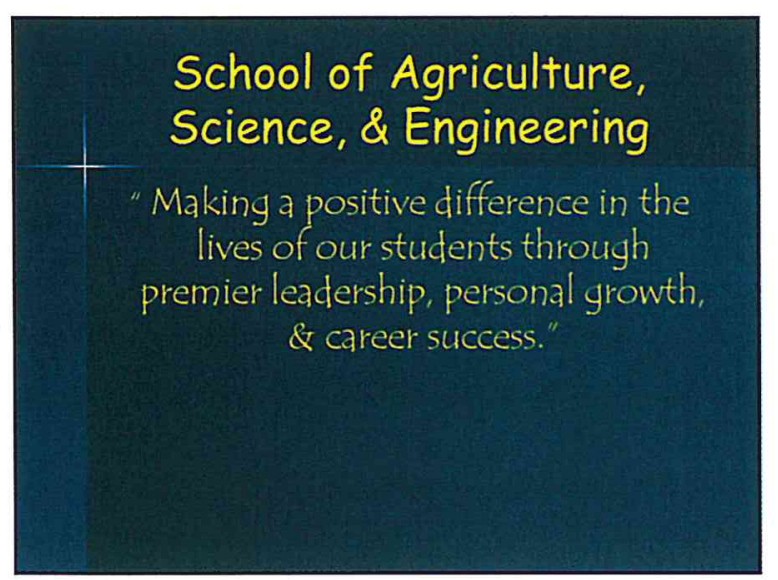



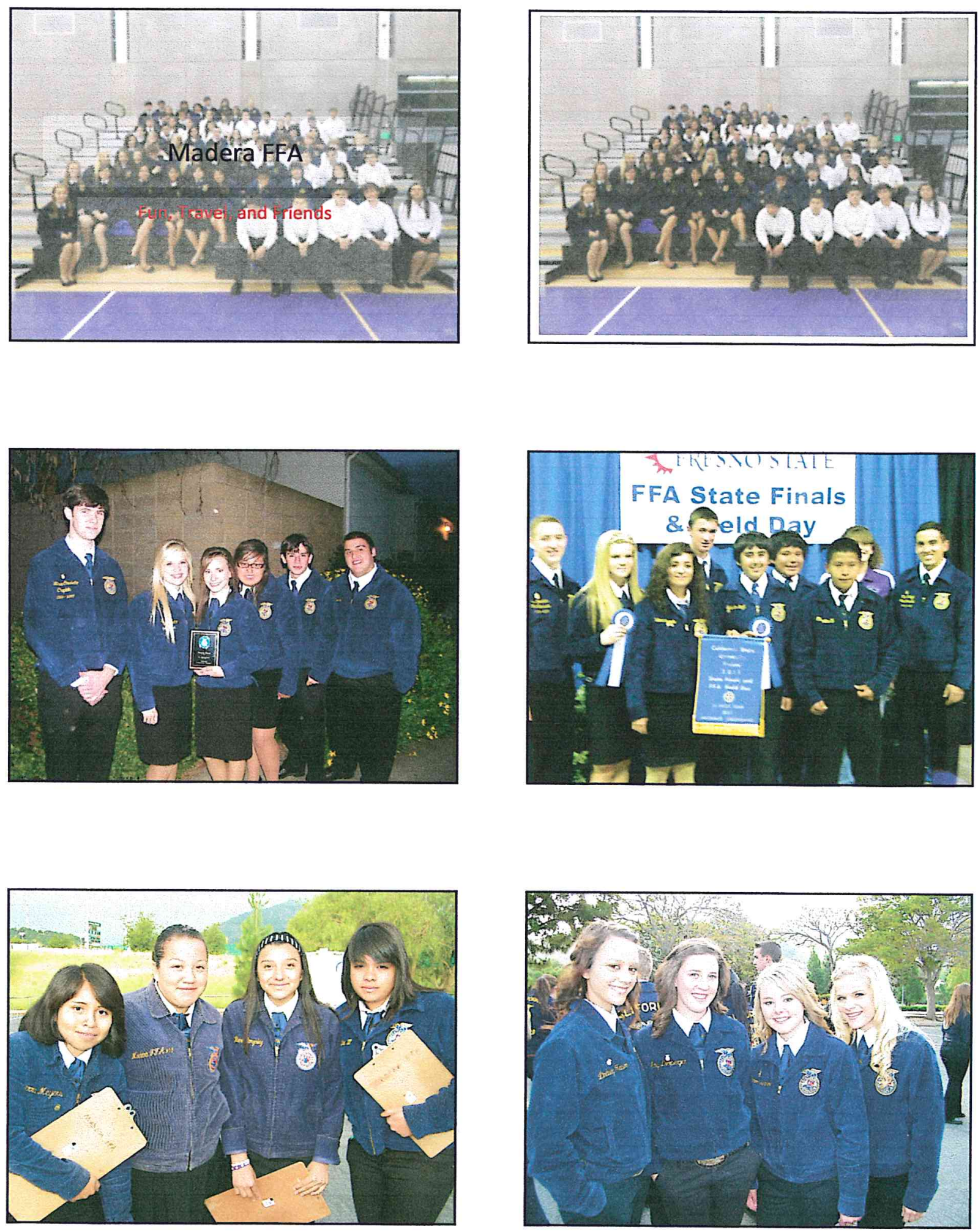

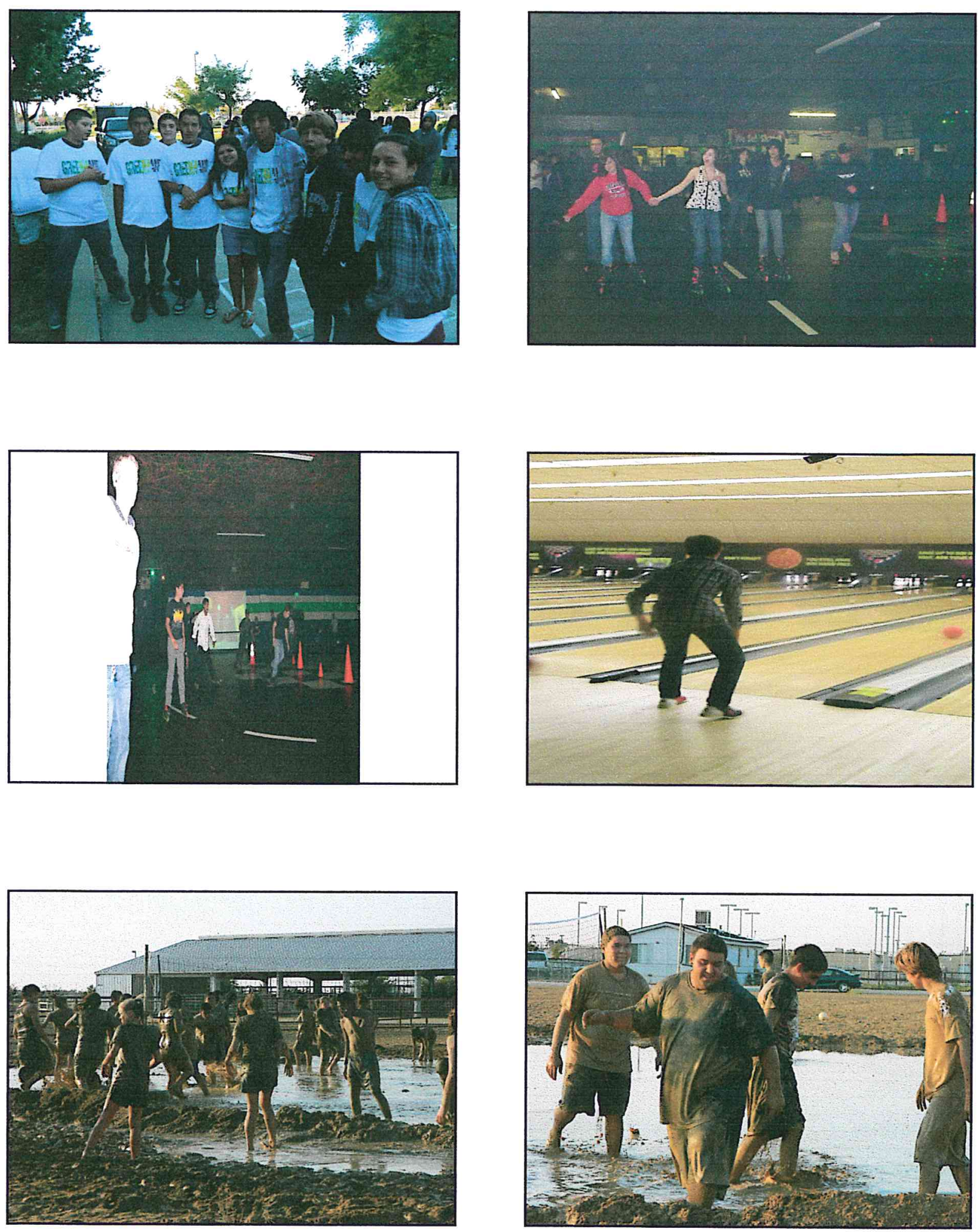

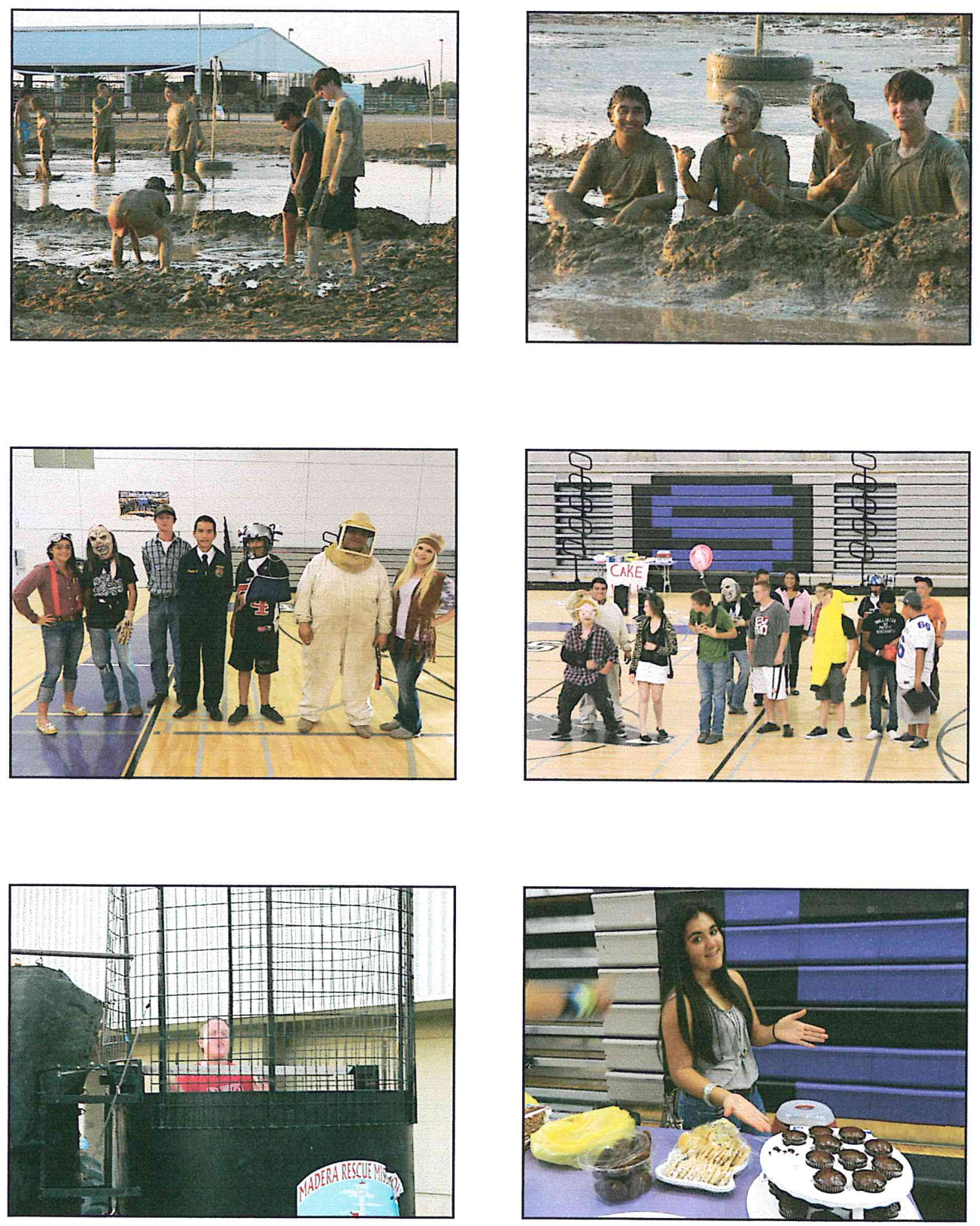

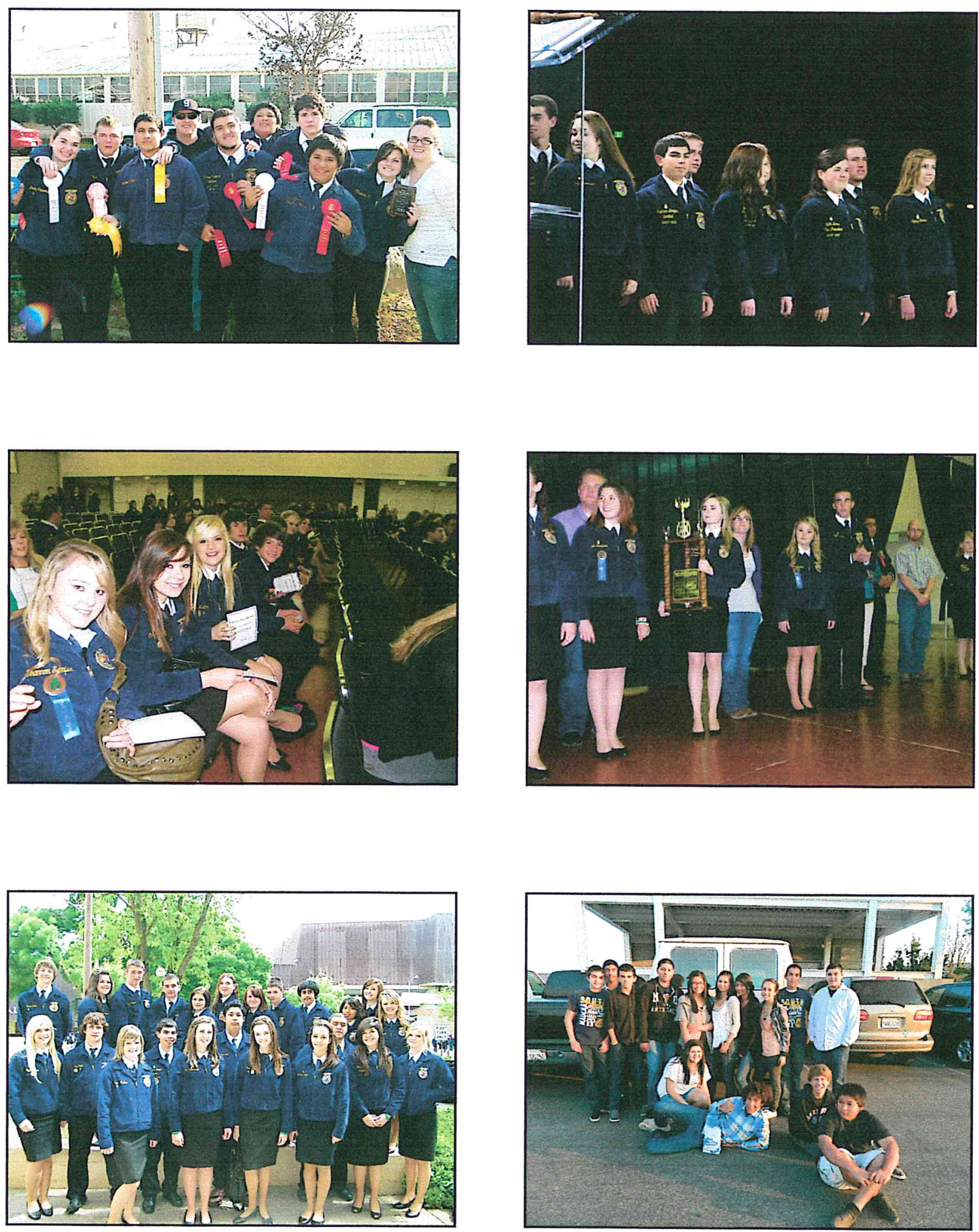

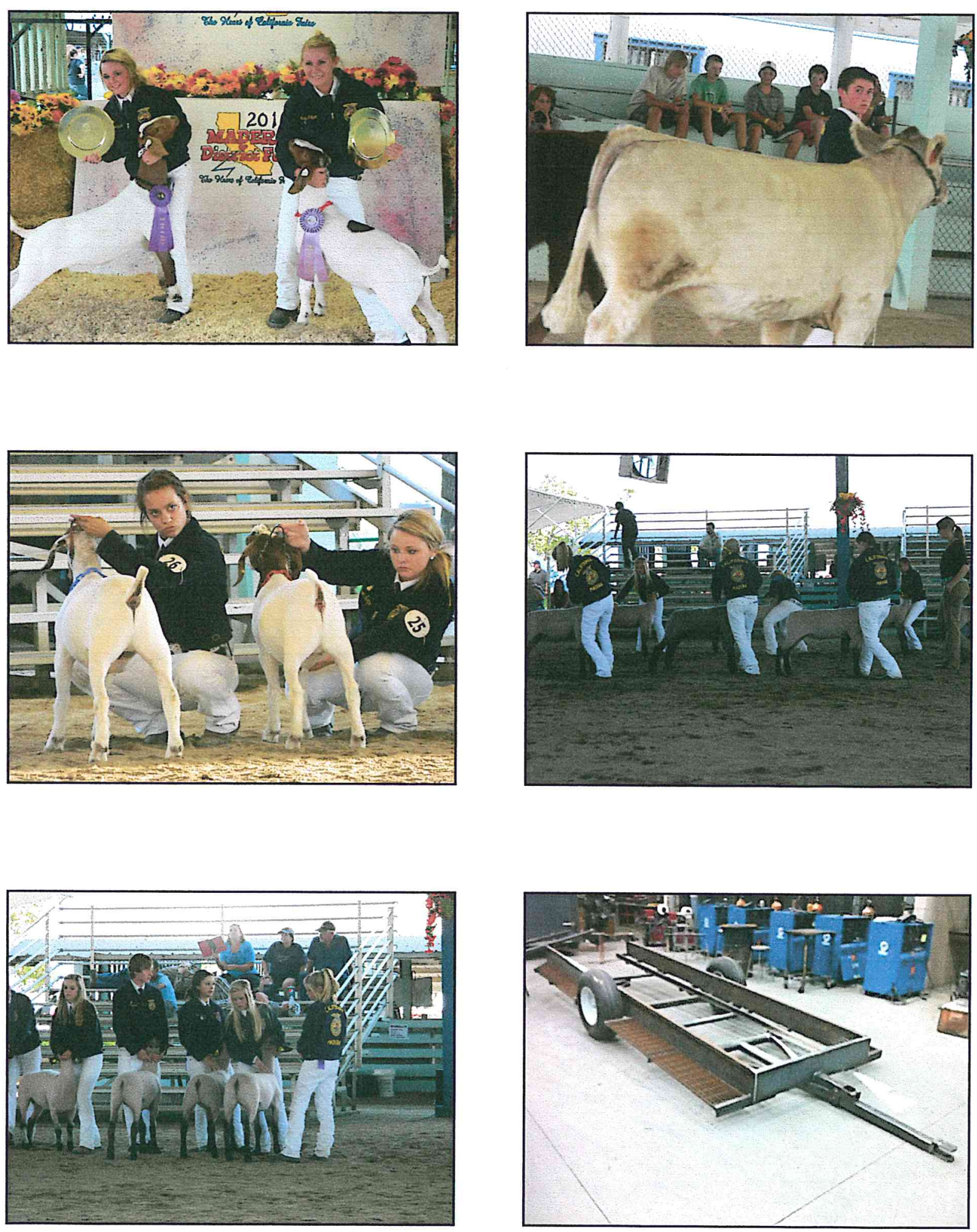

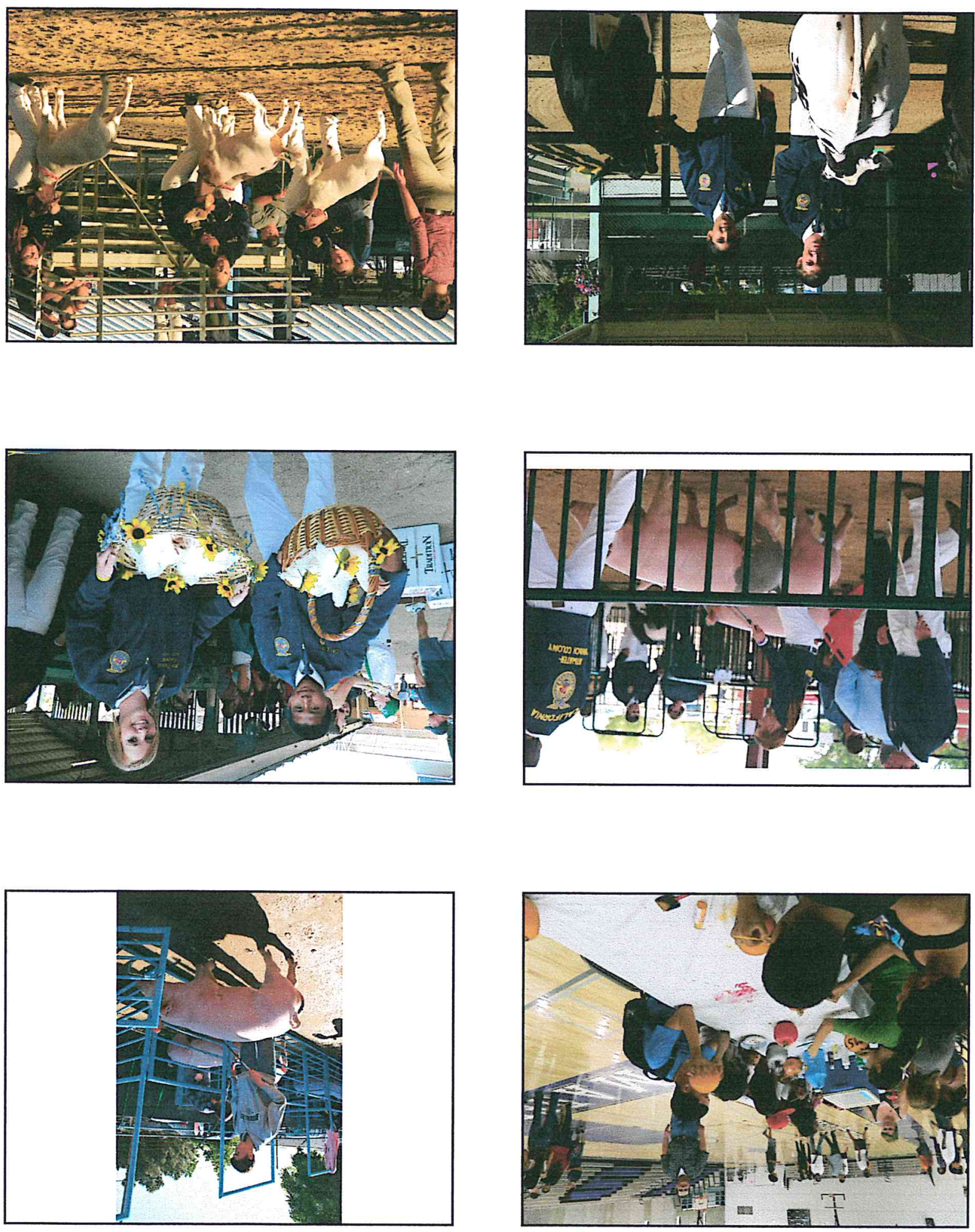


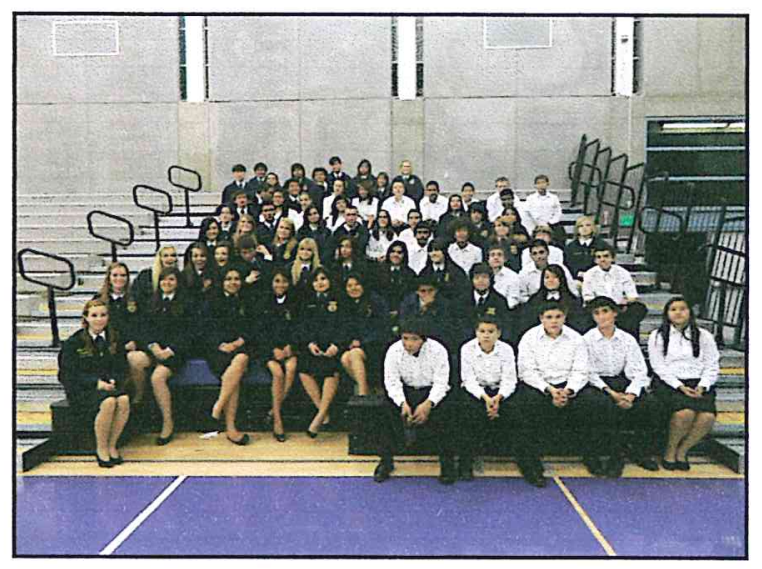



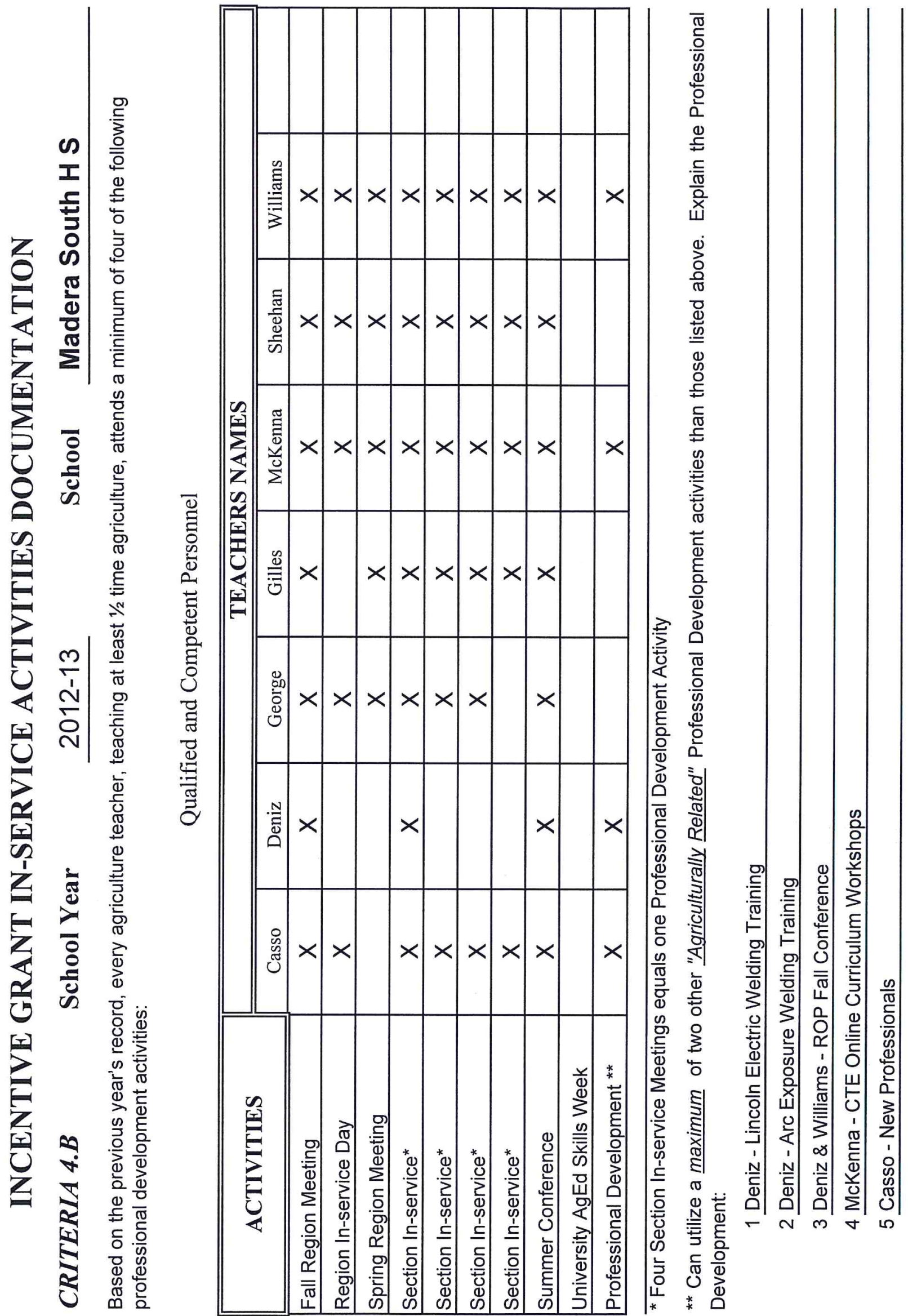


\section{Ag Dept Meeting Friday, August $9^{\text {th }}, 2013$}

1. Welcome to Student Teacher Johnny Lopes

CELL PHONE NUMBER 209-564-0902

Period 1 -ROP Diesels -Williams, Unit towards end of semester

Period 2 - Ag Mech II - Deniz - Start September 9

Period 3 - Ag. Earth Science - Gilles - Start 8-12-13

Period 4 - Ag. Mech I - George - Start 8-26-13

Period 5 -Diesels - Williams, Start September 16

Period 6 - Prep

2. Welcome Crystal to our Staff, Cell 661-444-1968

3. Ag. Incentive Money - Leftover must be spent September 30 and items received. PO for $\$ 1800$ Poppper has been submitted.

4. Roadshow/Regional Meeting - Tenaya Lodge, November 15-16

a. Who is going for both- All Teachers

b. Must make own Hotel Reservations through Regional Block, will be reimbursed for $1 / 2$, so find a roommate.

c. Chuckchansi Bus - Head Count

5. Fair Entries- Due August $13^{\text {th }}$

6. Chart of Responsibilities: Signed and returned to department head.

7. FFA - None at this time

Fall Activities - August and September

SOLC- Fri/Sat - 8-9 and 8-10

Boot Camp, SCICON - August 16-17

Welcome Back BBQ - August 21

Madera Fair Horse Show - August $24^{\text {th }}$

COLC/CATA Meetings - August $28^{\text {th }}$

Madera Fair - September 3-8
Blackbeard's - September 10

Mud Volleyball - September 18

Drive Thru BBQ - September 20

Madera Cotton Contest - September 26

Greenhand Applications Due - September 27 


\section{Ag Dept Meeting \\ Monday, August 19, 2013}

1. Ag. Incentive Money - PO's in by Thursday August $22^{\text {nd }}$

2. Roadshow/Regional Meeting - Tenaya Lodge, November 15-16

a. Don't forget to make hotel reservation. Online use group code 30U01G.

3. Fair Paperwork/Subs - Get subs called in, make sure you put the pay code under Professional Leave-AG

4. Dates for R-2 Data Sheets - We chose the week after fair to do R-2

5. Superintendent Visit-September 11 $1^{\text {th }} @ 3: 30 \mathrm{pm}$

6. Student Teacher Project - Build Engine stands for the diesel classes.

7. FFA -

- Bootcamp Recap - All officers went it was a great bonding activity.

- Welcome Back BBQ - All teachers need to help setup after school, Deniz and George are BBQing, Sheehan and Luera are doing Sign In table, all other teachers will help with food prep.

- ASB Accounts - Budgets and Minutes need to be turned into me by next Friday.

- Blackbeards - Williams, Lopes, Luera and George are riding the busses with the students.

- Drive Thru BBQ - This Friday in williams' classroom, all teachers are needed to help after school.

Fall Activities - August and September

Welcome Back BBQ - August 22

Madera Fair Horse Show - August $24^{\text {th }}$
COLC/CATA Meetings - August $28^{\text {th }}$

Poultry Show - August $31^{\text {st }}$

Madera Fair - September 3-8 


\section{Ag Dept Meeting \\ Monday, August 26, 2013}

1. Horse Show Results - Miranda was $2^{\text {nd }}$ in Showmanship

2. Ag. Incentive Money - Remaining $\$ 300$ will open for Farm Tools

3. Fair Paperwork/Subs - Make sure you put in for your subs.

4. Superintendent Visit-September 11 ${ }^{\text {th }} @ 3: 30$ pm

5. Advisory Meeting Dates - We will select the dates that work best for our advisory members.

6. Advisory Member Update - List was looked over in meeting to check and make sure advisors still want to be a part of the committee

7. CATA Meeting Wednesday, 5pm Clovis East

8. FFA -

- Welcome Back BBQ Recap - Over 300 students and family members attended.

- Blackbeards - FULL, all students will be responsible for money for dinner.

- Tee Shirt Sales - Please sell t-shirts in your classes, money will be due next week.

- Committee Meetings - All committee meetings will need to be done this week.

Fall Activities - August to October 15

COLC/CATA Meetings - August $28^{\text {th }}$

Poultry Show - August $31^{\text {st }}$

Madera Fair - September 3-8

Blackbeard's - September 10

Mud Volleyball - September 18

Drive Thru BBQ - September 20
Madera Cotton Contest - September 26

Greenhand Applications Due - Sept 27

Greenhand Interviews - October 2

Greenhand Voting -October 4

Ag. Literacy Clean Up - October $11^{\text {th }}$

Corcoran Cotton Contest - October $12^{\text {th }}$

Ag. Literacy Day - October 15-16 


\section{Vehicle Use and Service Needs}

\begin{tabular}{|c|c|c|c|c|c|c|}
\hline & Truck 127 & Truck 128 & Truck 119 & Truck 112 & Van 117 & Van 199 \\
\hline \multicolumn{7}{|l|}{ Mon 8-26 } \\
\hline Tues 8-27 & & & & & & \\
\hline Weds 8-28 & & & & & & \\
\hline Thurs 8-29 & & & & & & \\
\hline Fri 8-30 & & & & & & \\
\hline Sat 8-31 & & & & & & \\
\hline Sun 9-1 & & & & & & \\
\hline $\begin{array}{c}\text { Vehicle } \\
\text { Service } \\
\text { Needs }\end{array}$ & & & & & & \\
\hline $\begin{array}{c}\text { FARM } \\
\text { NEEDS }\end{array}$ & & & & & & \\
\hline Date & Truck 127 & Truck 128 & Truck 119 & Truck 112 & Van 117 & Van 199 \\
\hline Mon 9-2 & & & & & & \\
\hline Tues 9-3 & & & & & & \\
\hline Weds 9-4 & & & & & & \\
\hline Thurs 9-5 & & & & & & \\
\hline Fri 9-6 & & & & & & \\
\hline Sat 9-7 & & & & & & \\
\hline Sun 9-8 & & & & & & \\
\hline
\end{tabular}




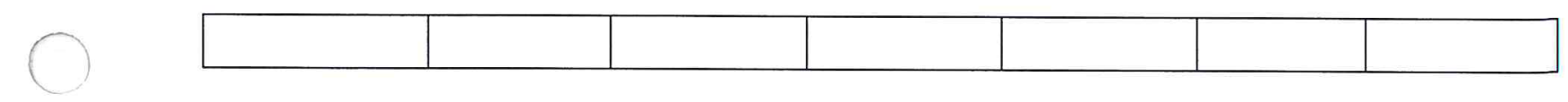




\section{Ag Dept Meeting \\ Monday, September 9, 2013}

1. Fair Results, Email -

2. Superintendent Visit - September $16^{\text {th }} @ 3: 30 \mathrm{pm}$

3. Advisory Meeting Dates - October 8 or 10 ?

4. Advisory Agenda - Incentive Advisory Review, Pathway, Etc??

5. FFA -

- Blackbeards -

- Drive Thru BBQ -

- Tee Shirt Sales -

- Committee Meetings -

- Mud Volleyball -

- Homecoming-

Fall Activities - September - October

Blackbeard's - September 10

Mud Volleyball - September 18

Drive Thru BBQ - September 20

Madera Cotton Contest - September 26

Greenhand Applications Due - Sept 27

Greenhand Interviews - October 2

Greenhand Voting -October 4
Ag. Literacy Clean Up - October $11^{\text {th }}$ Corcoran Cotton Contest - October $12^{\text {th }}$ Ag. Literacy Day - October 15-16 FFA Meeting - October 23rd Greenhand Conference - October $24^{\text {th }}$ Modesto Cotton - October 26th

Nation Convention - October 27-Nov. 2 


\section{Ag Dept. Meeting \\ Monday, September 16, 2013}

1. R-2 Reminders - In by Friday, Double check addresses, separate by year in Ag.

2. Fair Results, Email -

3. Superintendent Visit-September 16 ${ }^{\text {th }} @ 3: 30$ pm

4. Advisory Meeting Dates - October 10

5. Teacher Schedules-

6. FFA -

- Drive Thru BBQ -

- Tee Shirt Sales -

- Committee Meetings -

- Mud Volleyball -

- Homecoming-

- National Money

- Aggie of the Month

- Greenhand Office

Fall Activities - September - October

Mud Volleyball - September 18

Drive Thru BBQ - September 20

Madera Cotton Contest - September 26

Greenhand Applications Due - Sept 27

Greenhand Interviews - October 2

Greenhand Voting -October 4
Ag. Literacy Clean Up - October $11^{\text {th }}$

Corcoran Cotton Contest - October $12^{\text {th }}$

Ag. Literacy Day - October 15-16

FFA Meeting - October 23rd

Greenhand Conference - October $24^{\text {th }}$

Modesto Cotton - October 26th

Nation Convention - October 27-Nov. 2 


\section{Ag Department Meeting Monday, September 23, 2013}

1. Turn in Student Data Sheets -

2. Alumni Dinner -

3. Cotton Contest- Rooms, Help?

4. Five Year Facility and Acquisition Schedule

5. FFA -

- Homecoming-

○ Spirit Walk

- Aggie of the Month Lunch

- Greenhand Office -

Fall Activities - September - November

Madera Cotton Contest - September 26

Greenhand Applications Due - Sept 27

Greenhand Interviews - October 2

Greenhand Voting -October 4

Ag. Literacy Clean Up - October $11^{\text {th }}$

Corcoran Cotton Contest - October $12^{\text {th }}$

Ag. Literacy Day - October 15-16

FFA Meeting - October 23rd

Greenhand Conference - October $24^{\text {th }}$

Modesto Cotton - October 26th
Nation Convention - October 27-Nov. 2 Hanford Cotton-November 7

State Cotton Finals-November 9

Region Officer Meeting-November 10 Veterans Day (NO SCHOOL)-Nov 11 Opening and Closing - Nov 13

SJ Roadshow and Meeting-Nov 15-16

FFA Meeting - Nov. 20

Recordbook Work Day- Nov 25

Thanksgiving Break -Nov 25-29 


\section{Ag Department Meeting Monday, September 30, 2013}

1. R2 - 1,003 Contacts, Unduplicated 597, additional \$2k for Tim's classes.

2. Tenaya Lodge - Online Code 30U01G

3. Advisory Agenda- Any Additions?

4. Incentive Carryover - $\$ 160$ at Lowe's

5. FFA -

- Greenhand Office -

- MFE/ALA -

- Ag. Literacy Day -

- Snack Sale -

- Community Service Meeting - Tuesday @ Lunch

Fall Activities - October - November

Greenhand Interviews - October 2

Greenhand Voting-October 4

Ag. Literacy Clean Up - October $11^{\text {th }}$

Corcoran Cotton Contest - October $12^{\text {th }}$

Ag. Literacy Day - October 15-16

FFA Meeting - October 23rd

Greenhand Conference - October $24^{\text {th }}$

Modesto Cotton - October 26th

Nation Convention - October 27-Nov. 2
Hanford Cotton-November 7

State Cotton Finals-November 9

Region Officer Meeting-November 10

Veterans Day (NO SCHOOL)-Nov 11

Opening and Closing - Nov 13

SJ Roadshow and Meeting-Nov 15-16

FFA Meeting - Nov. 20

Recordbook Work Day- Nov 25

Thanksgiving Break -Nov 25-29 


\section{Ag Department Meeting Monday, October 7, 2013}

1. Tenaya Lodge - Online Code 30U01G

2. Advisory Meeting - Thursday, Reminder Calls, Dinner

3. Incentive Carryover -4 New Brooms, Spent All

4. Alegria Guild - Thanks Darlene for Handling it!!!

5. Corcoran Cotton Contest - October $12^{\text {th }}$

6. FFA -

- Greenhand Officers -

- Ag. Literacy Day -

- Snack Sale -

Fall Activities - October - November

Ag. Literacy Clean Up - October $11^{\text {th }}$ Corcoran Cotton Contest - October $12^{\text {th }}$

Ag. Literacy Day - October 15-16

FFA Meeting - October 23rd

Greenhand Conference - October $24^{\text {th }}$

Modesto Cotton - October 26th

Nation Convention - October 27-Nov. 2

Hanford Cotton -November 7
State Cotton Finals-November 9

Region Officer Meeting-November 10

Veterans Day (NO SCHOOL)-Nov 11

Opening and Closing - Nov 13

SJ Roadshow and Meeting-Nov 15-16

FFA Meeting - Nov. 20

Recordbook Work Day- Nov 25

Thanksgiving Break -Nov 25-29 


\section{Ag Department Meeting Monday, October 14, 2013}

1. Cotton Contest Results -

2. Grades - Due by Midnight -

3. Tenaya Lodge - Chuckchansi Bus??

4. District Advisory Meeting - Wednesday October $16 @ 6 \mathrm{pm}, \mathrm{DO}$

5. Curriculum Meeting - Wednesday October 23rd 3:30-5:30pm Cafeteria

6. FFA -

- Ag. Literacy Day -

- Snack Sale -

- FFA Meeting, Haunted Farm -

Fall Activities - October - November

Ag. Literacy Day - October 15-16

NO SCHOOL - October 18

FFA Meeting - October 23rd

Greenhand Conference - October $24^{\text {th }}$

Modesto Cotton - October 26th

Nation Convention - October 27-Nov. 2

Hanford Cotton-November 7
State Cotton Finals-November 9

Region Officer Meeting-November 10

Veterans Day (NO SCHOOL)-Nov 11

Opening and Closing - Nov 13

SJ Roadshow and Meeting-Nov 15-16

FFA Meeting - Nov. 20

Recordbook Work Day- Nov 25

Thanksgiving Break -Nov 25-29 


\section{Ag Department Meeting Monday, October 21, 2013}

1. Tenaya Lodge -Deniz, McKenna, W

2. Curriculum Meeting - Wednesday October 23rd 3:30-5:30pm Cafeteria

3. Student Recruitment - Dates and Career School Switches

4. Ag. Incentive Support Letters -

5. FFA -

- Snack Sale -

- FFA Meeting, Haunted Farm -

- Greenhand Conference -

Fall Activities - October - December

FFA Meeting - October 23rd

Greenhand Conference - October $24^{\text {th }}$

Modesto Cotton - October 26th

Nation Convention - October 27-Nov. 2

Hanford Cotton -November 7

State Cotton Finals-November 9

Region Officer Meeting-November 10

Veterans Day (NO SCHOOL)-Nov 11
Opening and Closing - Nov 13

SJ Roadshow and Meeting-Nov 15-16

FFA Meeting - Nov. 20

Recordbook Work Day- Nov 25

Thanksgiving Break -Nov 25-29

Greenhand/Chapter App Due -Dec 6

Greenhand Banquet - December $11^{\text {th }}$

Officer Christmas Party- December $20^{\text {th }}$ 


\section{Ag Department Meeting Monday, November 4, 2013}

1. National Convention- Great Job, BIG $\left(2^{\text {nd }}\right)$, Meats $\left(6^{\text {th }}\right)$, National Winner

2. New Attendance Policies -

3. Cotton Contests - Thursday and Saturday

4.

5. FFA -

- Opening and Closing -

- $\mathrm{CLC}-$

- Friday Tailgate -

- Casino Night Meeting -

Fall Activities - October - December Hanford Cotton-November 7

State Cotton Finals-November 9

Region Officer Meeting-November 10

Veterans Day (NO SCHOOL)-Nov 11

Opening and Closing - Nov 13

SJ Roadshow and Meeting-Nov 15-16

FFA Meeting - Nov. 20
Recordbook Work Day- Nov 25

Thanksgiving Break -Nov 25-29

Greenhand/Chapter App Due -Dec 6

Greenhand Banquet - December $11^{\text {th }}$

Officer Christmas Party- December $20^{\text {th }}$

Christmas Vacation - December 20-Jan 13

Recordbook Work Day - January $9^{\text {th }}$ 


\section{Ag Department Meeting Monday, November 12, 2013}

1. Cotton Contests $-4^{\text {th }}$ High Team, Jenae $2^{\text {nd }}$ High Individual

2. Board Meeting - Tonight @7pm

3. Judging Team Contracts - Brainstorm

4. Subs for Friday - SB-Ag

5. Cal Poly Visit- Thursday

6. FFA -

- Opening and Closing -

- Casino Night Meeting - Date Change, November $21^{\text {st }}$

- Assemblymen Visit -

Fall Activities - October - December

Opening and Closing - Nov 13

Cal Poly Visit - Nov 14

SJ Roadshow and Meeting-Nov 15-16

FFA Meeting - Nov. 21

Recordbook Work Day- Nov 25
Thanksgiving Break -Nov 25-29

Greenhand/Chapter App Due -Dec 6

Greenhand Banquet - December $11^{\text {th }}$

Officer Christmas Party- December $20^{\text {th }}$

Christmas Vacation - December 20-Jan 13

Recordbook Work Day - January $9^{\text {th }}$ 


\section{Ag Department Meeting Monday, November 18, 2013}

1. Judging Team Contracts - Changes?

2. Reimbursement Forms - Turn in

3. Cal Poly Visit- Thursday

4. Record book Work Days?

5. PG\&E Requests -

6. FFA -

- Casino Night Meeting -

- Banquet -

- Assemblymen Visit -

- Other needs for John -

Fall Activities - October - December FFA Meeting - Nov. 21

Recordbook Work Day- Nov 25

Thanksgiving Break -Nov 25-29

Greenhand/Chapter App Due -Dec 6
Greenhand Banquet - December $11^{\text {th }}$ Officer Christmas Party- December $20^{\text {th }}$ Christmas Vacation - December 20-Jan 13 Recordbook Work Day - January $9^{\text {th }}$ 


\section{Ag Department Meeting Monday, December 2, 2013}

1. Record book Work Day Reminders -

2. PG\&E Requests -

3. Course Sequencing-

4. Bigelow/Cannella Visit- December $19^{\text {th }}$

5. FFA -

- Banquet -

- Applications and RSVP Due Friday $-\pi$

Fall Activities - October - January

Greenhand/Chapter App Due -Dec 6

Greenhand Banquet - December $11^{\text {th }}$

Officer Christmas Party- December $20^{\text {th }}$

Christmas Vacation - December 20-Jan 13

Recordbook Work Day - January $8^{\text {th }}-9^{\text {th }}$

St. Helena Vine Pruning - January 10-11

Start of $2^{\text {nd }}$ Semester - January $13^{\text {th }}$
Recordbook Scoring-Madera - January $15^{\text {th }}$ Dinuba Vine Pruning - January $18^{\text {th }}$

No School-MLK Day - January $20^{\text {th }}$

BIG and Banking - January $22^{\text {nd }}$

Skate Night Meeting - January $23^{\text {rd }}$

Regional Officer Applications Due - Jan. 24

Reedley Vine Pruning - January 25th

Recordbooks Scoring, Kingsburg - Jan. 29 


\section{Ag Department Meeting Monday, December 9, 2013}

1. Bigelow/Cannella/Berryhill Visit- December $19^{\text {th }}-$ Students Names

2. Chico and Davis Registration -

3. FFA -

- Canned Food Drive

- Banquet -

Fall Activities - October - January Greenhand Banquet - December $11^{\text {th }}$ Farm Clean Up - December $18^{\text {th }} 1 \mathrm{pm}$ AIG Vist - December $19^{\text {th }}$

Officer Christmas Party- December $20^{\text {th }}$

Christmas Vacation - December 20-Jan 13 Recordbook Work Day - January $8^{\text {th }}-9^{\text {th }}$ St. Helena Vine Pruning - January 10-11 Start of $2^{\text {nd }}$ Semester - January $13^{\text {th }}$
Recordbook Scoring-Madera - January $15^{\text {th }}$ Dinuba Vine Pruning - January $18^{\text {th }}$

No School-MLK Day - January $20^{\text {th }}$ BIG and Banking - January $22^{\text {nd }}$ Skate Night Meeting - January $23^{\text {rd }}$ Regional Officer Applications Due - Jan. 24 Reedley Vine Pruning - January 25th

Recordbooks Scoring, Kingsburg - Jan. 29 


\section{Ag Department Meeting Monday, December 16, 2013}

1. Bigelow/Cannella/Berryhill Visit- December $19^{\text {th }}-$ Students Names, Video
a. Farm Clean Up/Classroom Clean Up
b. Vehicle Cleaning

2. Chico and Davis Registration -Anything you need?

3. Thursday Lunch -

4. Student Teacher Schedule -

$$
\begin{aligned}
& 1^{\text {st }} \\
& 2^{\text {nd }} \\
& 3^{\text {rd }} \\
& 4^{\text {th }} \text { - George, Ag. Mech } 1 \\
& 5^{\text {th }} \\
& 6 \text { th }
\end{aligned}
$$

5. FFA -

- Canned Food Drive Reflection

- Banquet Reflection

○ Solving Greenhand/Chapter Degree Issue

Fall Activities - October - January Farm Clean Up - December $18^{\text {th }} 1 \mathrm{pm}$ AIG Vist - December $19^{\text {th }}$ Officer Christmas Party- December $20^{\text {th }}$ Christmas Vacation - December 20-Jan 13 Recordbook Work Day - January $8^{\text {th }}-9^{\text {th }}$ St. Helena Vine Pruning - January 10-11 Start of $2^{\text {nd }}$ Semester - January $13^{\text {th }}$
Recordbook Scoring-Madera - January $15^{\text {th }}$ Dinuba Vine Pruning - January $18^{\text {th }}$ No School-MLK Day - January $20^{\text {th }}$ BIG and Banking - January $22^{\text {nd }}$ Skate Night Meeting - January $23^{\text {rd }}$ Regional Officer Applications Due - Jan. 24 Reedley Vine Pruning - January 25th Recordbooks Scoring, Kingsburg - Jan. 29 


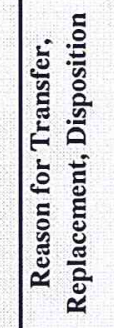

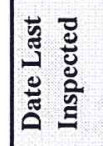

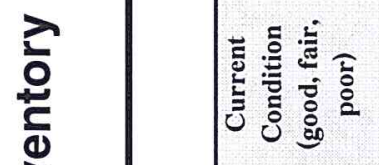

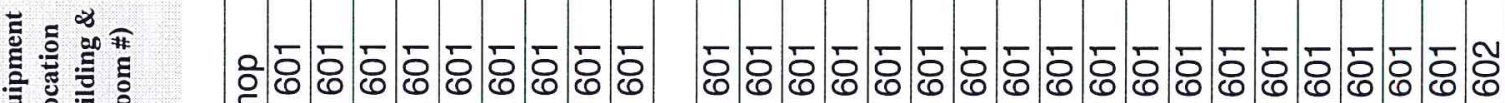

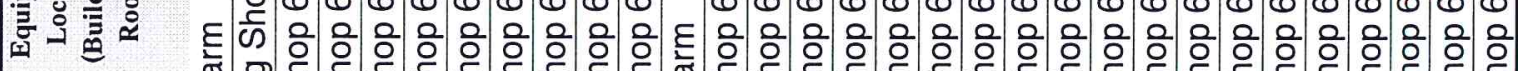

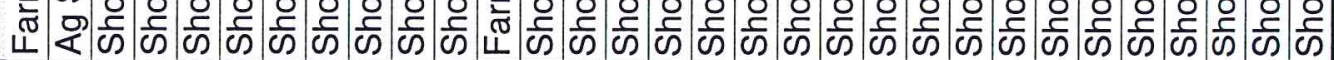
:

恶部 톤

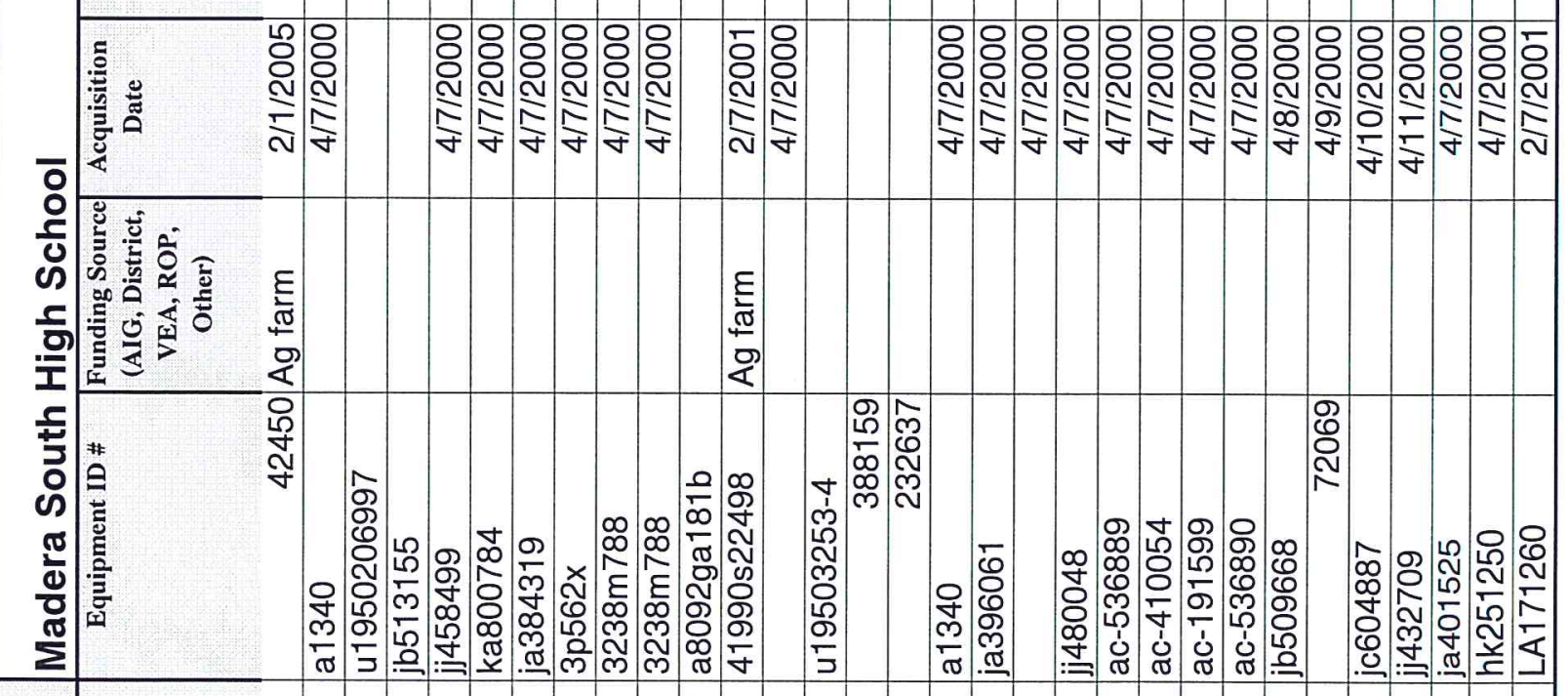




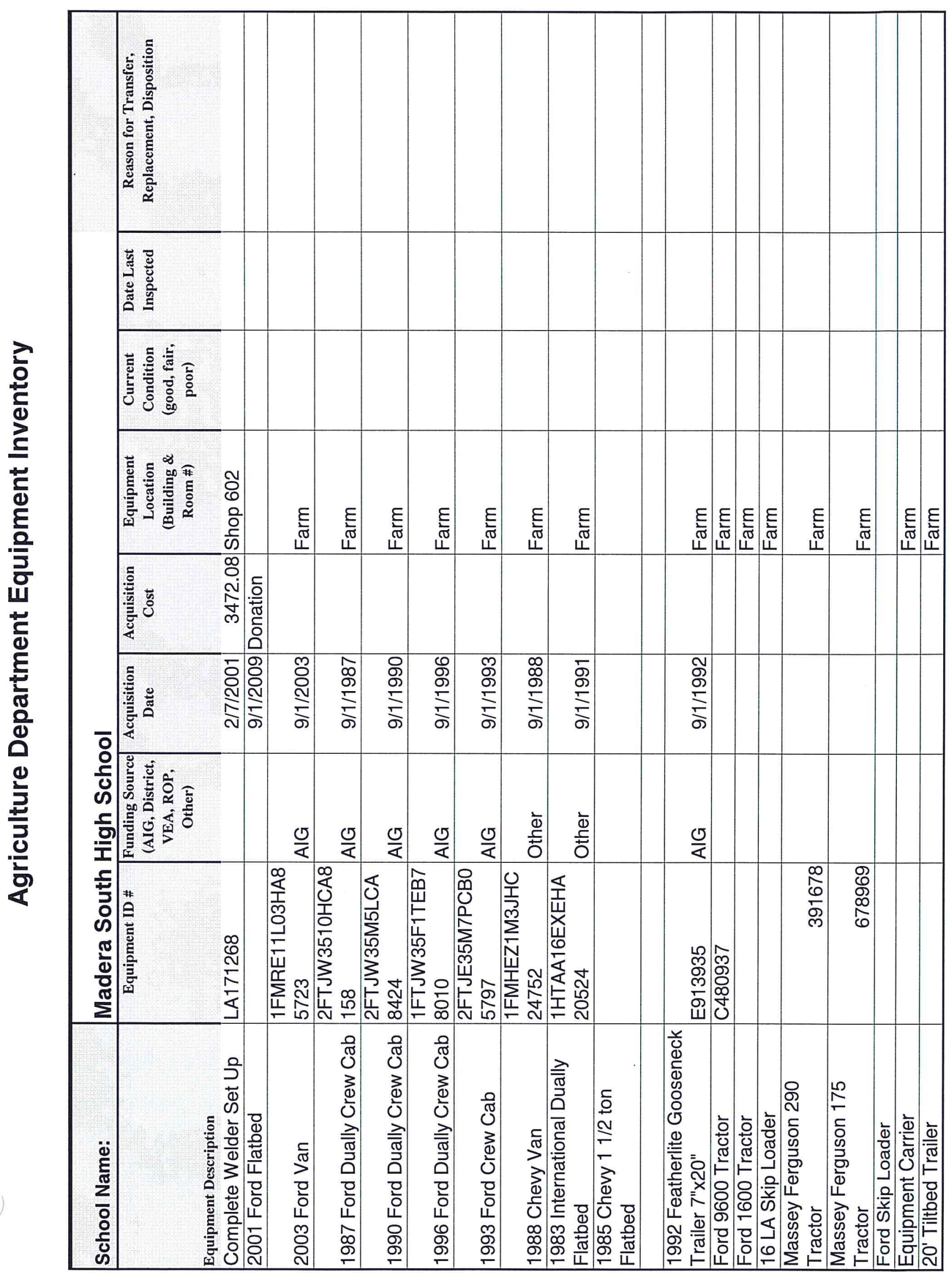




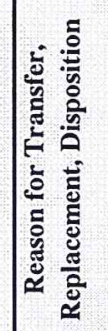

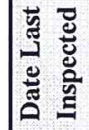

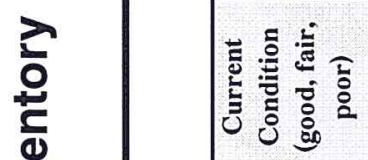

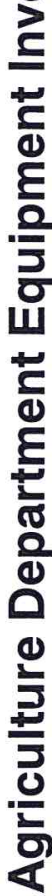

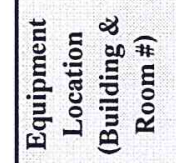

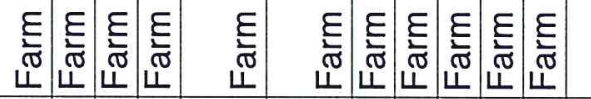

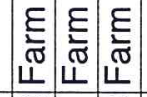

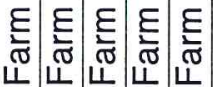

ธேீீ

5.

施

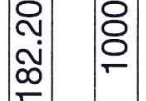

$\overline{0}$

产

ज̄

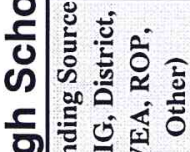

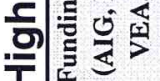

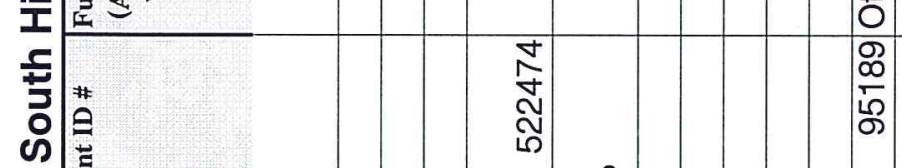

苋

옹

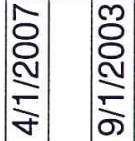

这

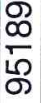

客 


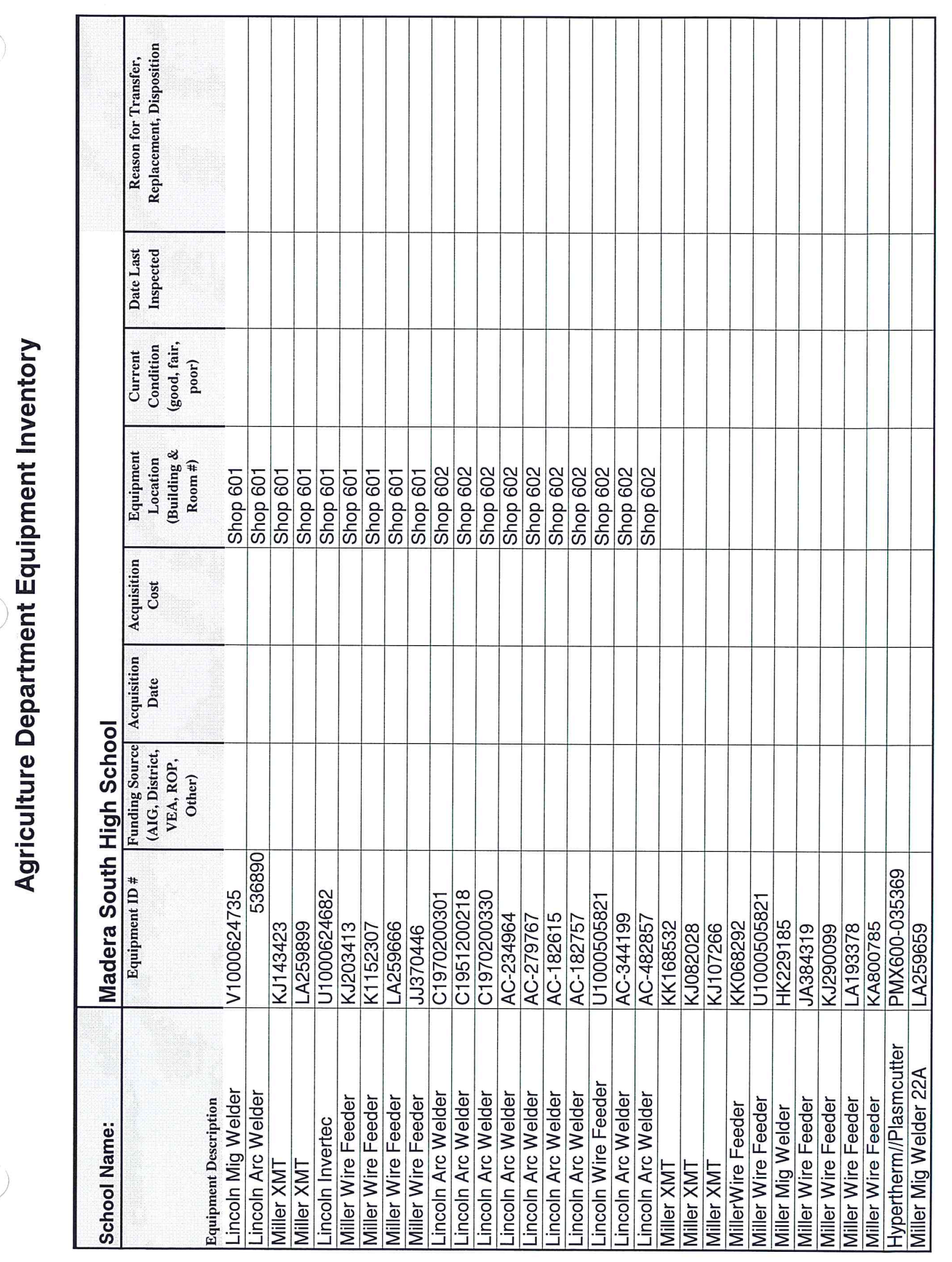




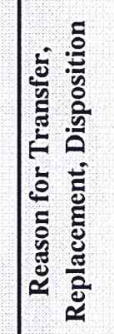

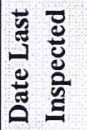

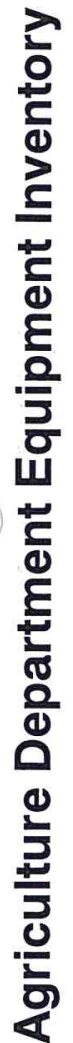

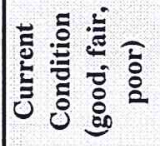

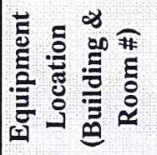

.

ज़े

这

옹

8 :

帘

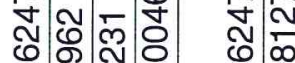

ญั

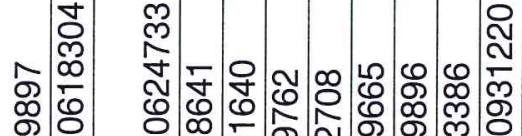

นㅇ ठ

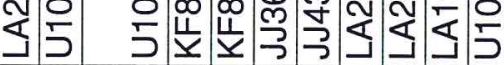

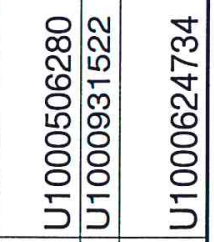

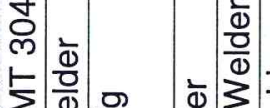

$\sum^{\infty} \frac{0}{3}$

은 $3 \frac{0}{2}$ 일

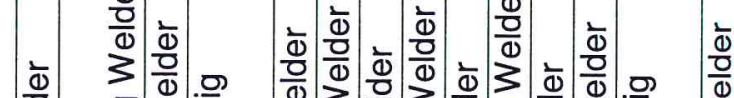

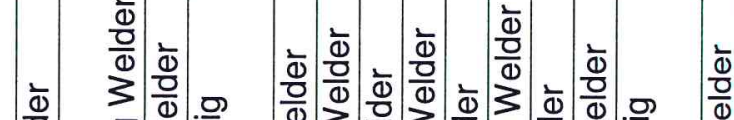

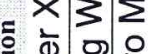

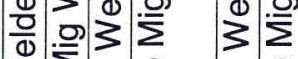

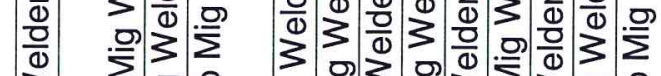

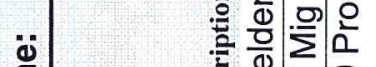

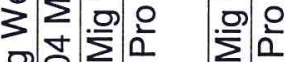

$\sum_{4}$

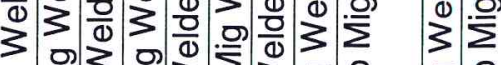

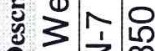

이잉

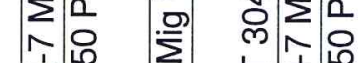

. 일 $\sum \sum 0$

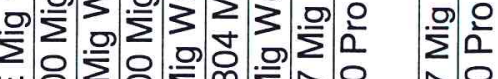




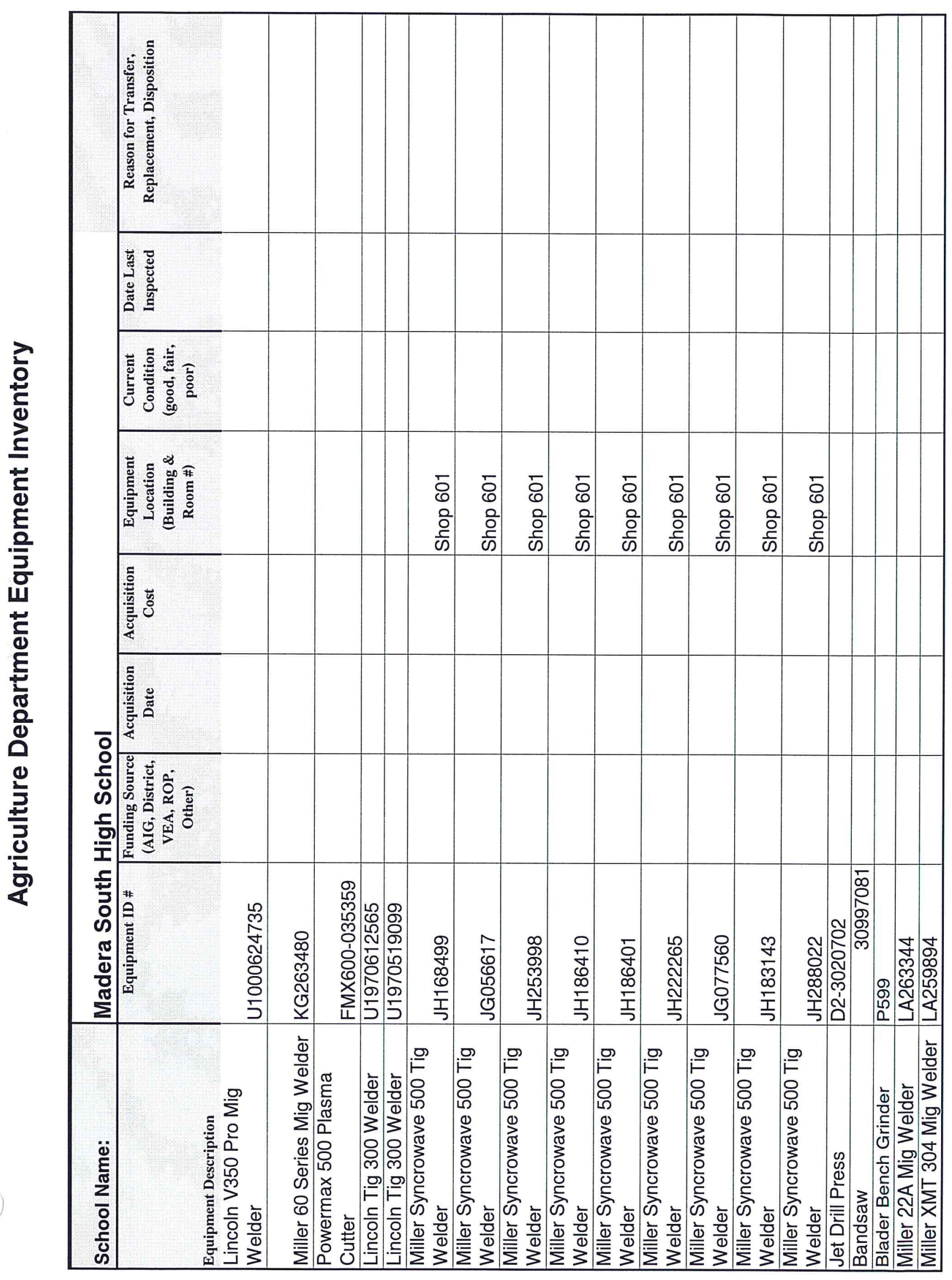




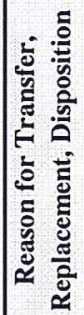

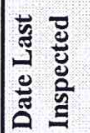

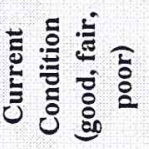

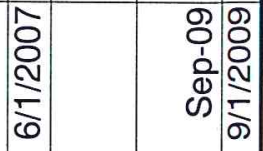

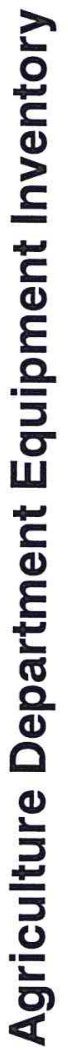

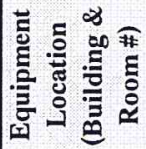

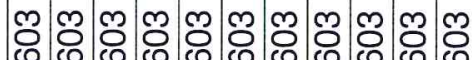

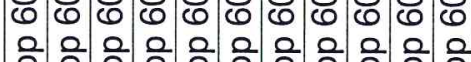

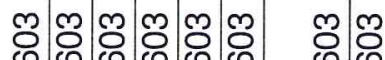

\begin{tabular}{|c|}
\hline 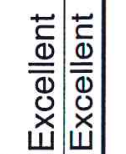 \\
\hline
\end{tabular}

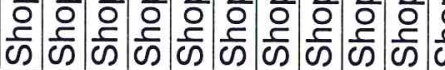

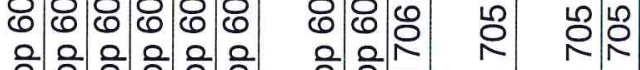

.อิ : हुㅇ

范

苋

遏

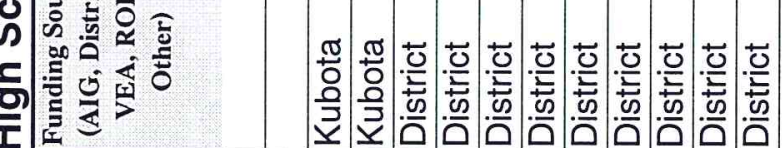

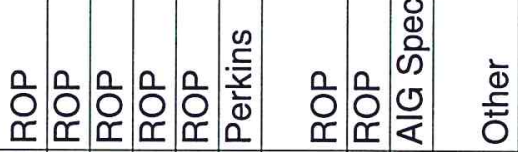

ஜ পுల $\checkmark$ ᄂ

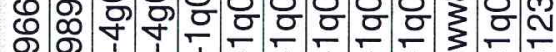

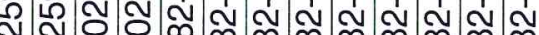

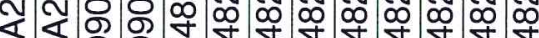

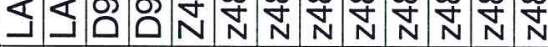
స్心

\section{$\frac{\Phi}{2}$}

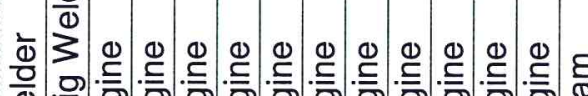

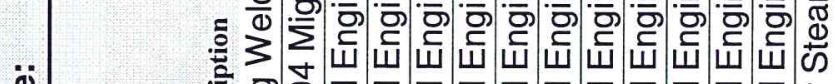



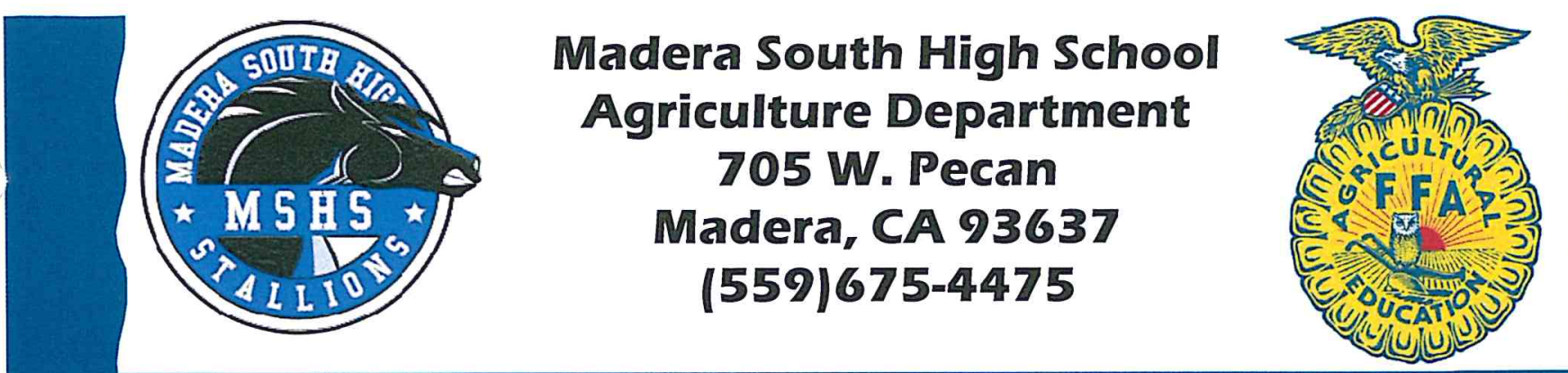

\section{Madera South Courses for Alternative Credit}

The following courses count for alternative credit.

- Agriculture Biology-A-G Biological Science

- Floral Design-A-G Fine Art

- Environmental Science I and II-High School Physical Science

- Ag. Econ and Government-A-G Social Science

- Vet Science-A-G Elective Credit

- Ag. Science I and II-High School Life Science

- Natural Resources I-High School Physical Science

- Ag. Mech IV (2 periods) - High School Fine Art

- Horticulture II-High School Fine Art 


\section{A student graduating from a Madera Unified high school must meet the following requirements:}

1) Shall have earned or successfully completed 230 credits.

2) Shall have passed the California High School Exit Examination CAHSEE) in both English language arts and mathematics.

3) Shall have completed:

a) 30 credits in English:

To include: one semester of composition and one semester of grammar.

b) $\mathbf{3 0}$ credits in social science:

To include: 10 credits in world history (freshman or sophomore), 10 credits in U.S. history (junior), 5 credits in civics (senior), 5 credits in economics (senior).

c) 20 credits in math:

(Including passing Algebra I)

d) 20 credits in science:

10 credits in biology or 20 credits in agricultural science I \& agricultural science II

10 credits in physical science, chemistry, physics or natural resources I

e) $\mathbf{3 0}$ credits in physical education:

(Ten credits may be waived upon application and authorization).

f) 10 credits in fine arts:

To be selected from foreign language, music, art, or one of these specific classes: horticulture II, floriculture; drafting technology, drama or agricultural mechanics IV (2 periods).

4) Shall have earned a 2.0 or above grade point average.

5) Shall have achieved proficiency level (score of 4 or higher) on the graduation requirement for occupational education (G2).

A student shall be eligible for graduation, and the receipt of a diploma, if they have completed all the requirements listed in 1,2,3,4 and 5.

UPDATE MAY 13, 2008: Students who fulfill all graduation requirements above EXCEPT for No. 2 ("shall have passed the California High School Exit Examination in both English language arts and mathematics") may participate in graduation ceremonies but WILL NOT receive a diploma. Instead, they will receive a certificate of completion. 


\section{Q. One Copy of Each Advisory Committee Minutes for Current Year}




\section{Agriculture Advisory Meeting \\ Madera South High School}

October 10, 3013

In attendance: See attachment \#1

Oracio Rodriguez
Dick Haupt
Carl Schroeder
Dave Loquaci
Prince Marshall
Jim Erickson
Darlene Gilles
Kristin Sheehan

Michael Salvador
Jim Bomprezzi
Don Farnesi
Jim Cavallero
Sandon Schwartz
Kristin McKenna
Tim Deniz
Crystal Luera

Sheryl Sisil

Bob Naden

Bob Labrucherie

Shirley Woods

Ed LeTourneau

John Williams

Brent George

Johnny Lopes

The meeting was called to order by the department chairperson Kristin McKenna in Gary Geist's absence at 6:05 p.m. After a brief introduction the members were dismissed for dinner.

The meeting resumed at 6:15 p.m. and introductions were made of all present. Our new teacher Crystal Luera and student teacher Johnny Lopes were highlighted. Darlene Gilles was presented a plaque for 7 years of outstanding service as the department head.

A motion to approve the minutes from the March 2013 advisory meeting was made by Jim Erickson and seconded by Jim Cavallero, minutes approved, voice vote.

The agenda started with John Williams presenting a power point listing the accomplishments and many activities of the Madera FFA members from May to the present.

Kristin McKenna led a review of the Agriculture Incentive Grant checklist that needed to be certified by the advisory committee. The findings were we don't meet criteria's 10A, 11B and 12A. (Attachment 2) The goal of the department is to work towards meeting 12A, however with the current budget issues, criteria's $10 \mathrm{~A}$ and $11 \mathrm{~B}$ are out of reach at this time. The checklist was approved with a motion by Jim Cavallero and $2^{\text {nd }}$ by Dave Loquaci, checklist approved, voice vote.

John Williams reported on the status of the vineyard. The work started shortly after our last Ag. Advisory committee meeting; with trenching and manifolds being put in, stakes and wiring being hung, drip lines installed, and the vines being planted. All of the work done on the vineyard was completed by students. A list of donors can be found in (attachment 3 ).

Tim Deniz and Brent George presented the Ag. Mechanics Welding and Fabrication Pathway. They reviewed with everyone the courses that make up the pathway, topics that are covered and career opportunities for students that pursue the pathway. Tim also informed the 
committee that the classes are now articulated with Butte College and articulations are in the works for Fresno City and Merced. (Attachment 4)

Dave Loquaci asked a question about what effects common core will have on the Ag. Department and the time frame until full implementation. Sheryl Sisil stated that step one is rewriting core courses and then step 2 will be re-working elective classes. It is her opinion that common core will help our courses since the focus is in real life applications rather than memorization. Some of our courses may change in content taught in them but we will still be providing real life skills.

Meeting adjourned 7:07 p.m.

Respectfully submitted

Kristin McKenna

MSHS Ag Dept. 


\section{Agriculture Advisory Meeting \\ Madera South High School}

March 18, 2013

In attendance: See attachment \#1

$\begin{array}{lll}\text { Ray Seibert } & \text { Shirley Woods } & \text { Oracio Rodriguez } \\ \text { Stan Williams } & \text { Bob Labrucherie } & \text { Robert Chavez } \\ \text { Sheryl Sisil } & \text { Jim Cavallero } & \text { Bob Naden } \\ \text { Mike Schafer } & \text { Sandon Schwartz } & \text { Jim Erickson } \\ \text { Todd Houlding } & \text { Gary Geist } & \text { Brent George } \\ \text { John Williams } & \text { Tim Deniz } & \text { Kristin Mckenna } \\ \text { Kristin Sheehan } & \text { Celia Casso } & \text { Darlene Gilles }\end{array}$

The meeting was called to order by the chairman Gary Geist at 6:20 p.m. After a brief introduction the members were dismissed for dinner.

The meeting resumed at 6:40 p.m. and introductions where made of all present.

The agenda started with Kristin McKenna presenting a power point listing the accomplishments and many activities of the Madera FFA members from October to the present. Following her remarks freshman Clayton Sheehan recited the FFA Creed. Clayton is preparing for the Regional finals in Creed Speaking.

The Ag Departments purposed Perkins budget was presented by Darlene Gilles for the committee's approval. See attachment \#2. Discussion followed as the purposed expenditures were explained. Questions were asked and answered about:

How much Ag Incentive Grants does the department receive?

How much does the District contribute to the department?

How much Perkins does the department receive on average?

Sheryl Sisil and Shirley Woods explained the criteria used to determine the amount of Perkins a program will receive.

Jim Cavallero moved that the committee except the Perkins budget as presented. Stan Williams seconded the motion and the motion passed by voice vote.

John Williams gave the committee an update on the vineyard. Western Ag trenched and put in the pipe, etc for the irrigation. The rows will be set up and done on Saturday. There will be 12 rows of wine grapes planted in 4 different varieties. The department used Perkins money this year to purchase a vineyard disc and a sulfur sprayer.

During the Fall Advisory Meeting there was discussion by the members on the need for a viticulture class because of the vineyard going in. Celia Casso shared with the committee the draft of a new class "Viticulture and Crops. She explained the different units for class would 
cover. See attachment \#3. Members were asked for feedback on the purposed class; any other areas that needed to be included?

Questions: Could students get their personal applicators license while in taking the class? No not till they turn 18 years of age. Will this class receive UC Credit? UC Credit will be applied for. Will there be a possibility to job shadowing with a PCA? That can be added to the course. What about adding a business component: cost of raising a crop/doing business/can money be made?

Committee members asked about the possibility of a Ag Math class.

Bob Naden asked about a refrigeration class. John explained he does a brief unit in his ROP Diesel's class as a trouble shooting skill. We do not have the equipment or money for equipment to get deeper into the subject. John will contact Midland Tractor and Caterpillar for additional information and help.

Dr. Ed LeTourneau presented information on the Chowchilla Western Stampede Scholarship. He needs a committee of Ag Advisory members from each High School that the scholarship is available to, to chose the winning recipient. The following members volunteered to be on the committee:

Jim Cavallero

Bob Labrucherie

Mike Schafer

Dr. Ed LeTourneau

The scholarship committee will meet on Wednesday April $3^{\text {rd }}$ at $5 \mathrm{pm}$ on campus in room 706. Darlene Gilles will call to remind them of the meeting.

Tim Deniz shared that Gary Geist and Kuckenbecker Tractor are the recipients of the ROP Noley Award which will be presented on April 23 at Clovis North High School.

Jim Cavallero asked how the department was doing with transportation; are we able to get additional vehicles if and when we need them? The department has been able to manage with easily with the addition of the new van and truck. To date we have not needed to request any additional vehicles for events the department needs to attend.

The meeting was adjourned at 8:10 p.m.

Respectfully submitted

Darlene Gilles

MSHS Ag Dept. 


\section{R. One Copy Of A Completed Student Program Plan}




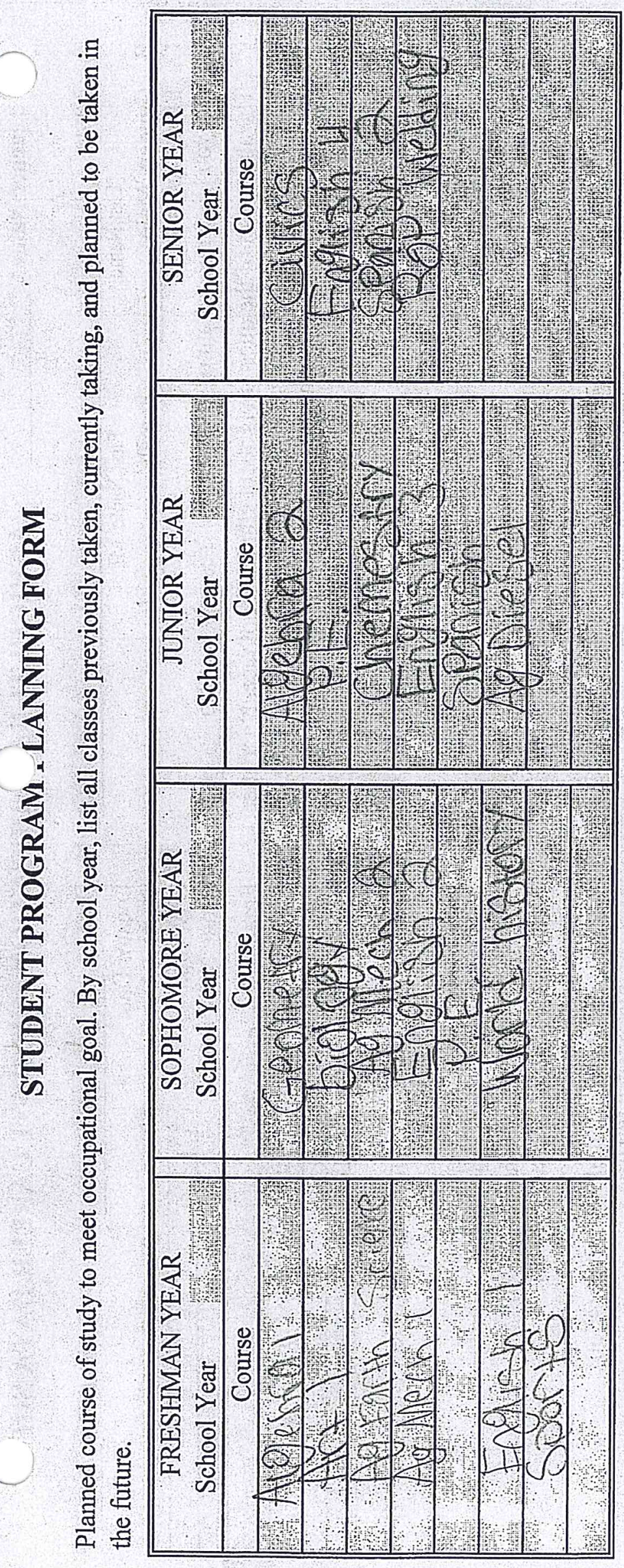

मi

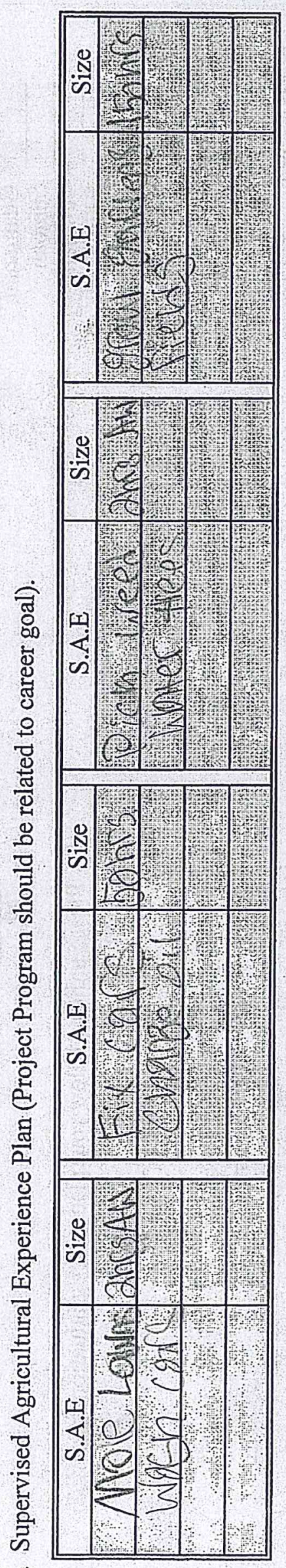

$\Sigma$

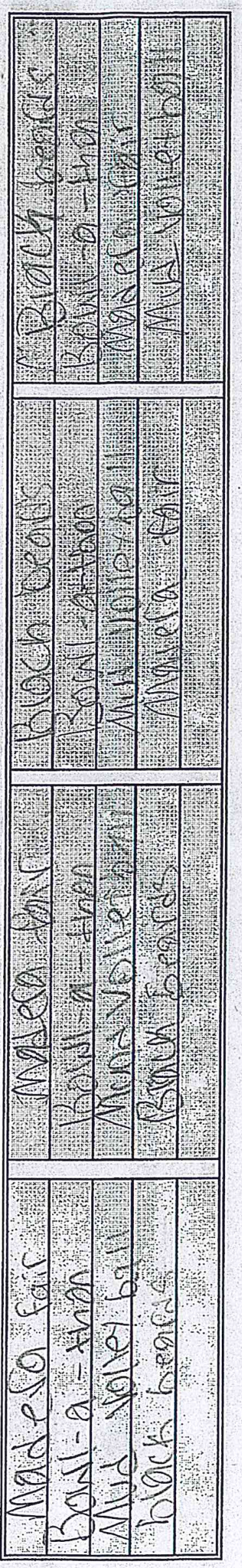

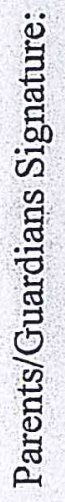


S. Copy Of Proficiency Standards Or Work In Progress On Proficiency

Standards 


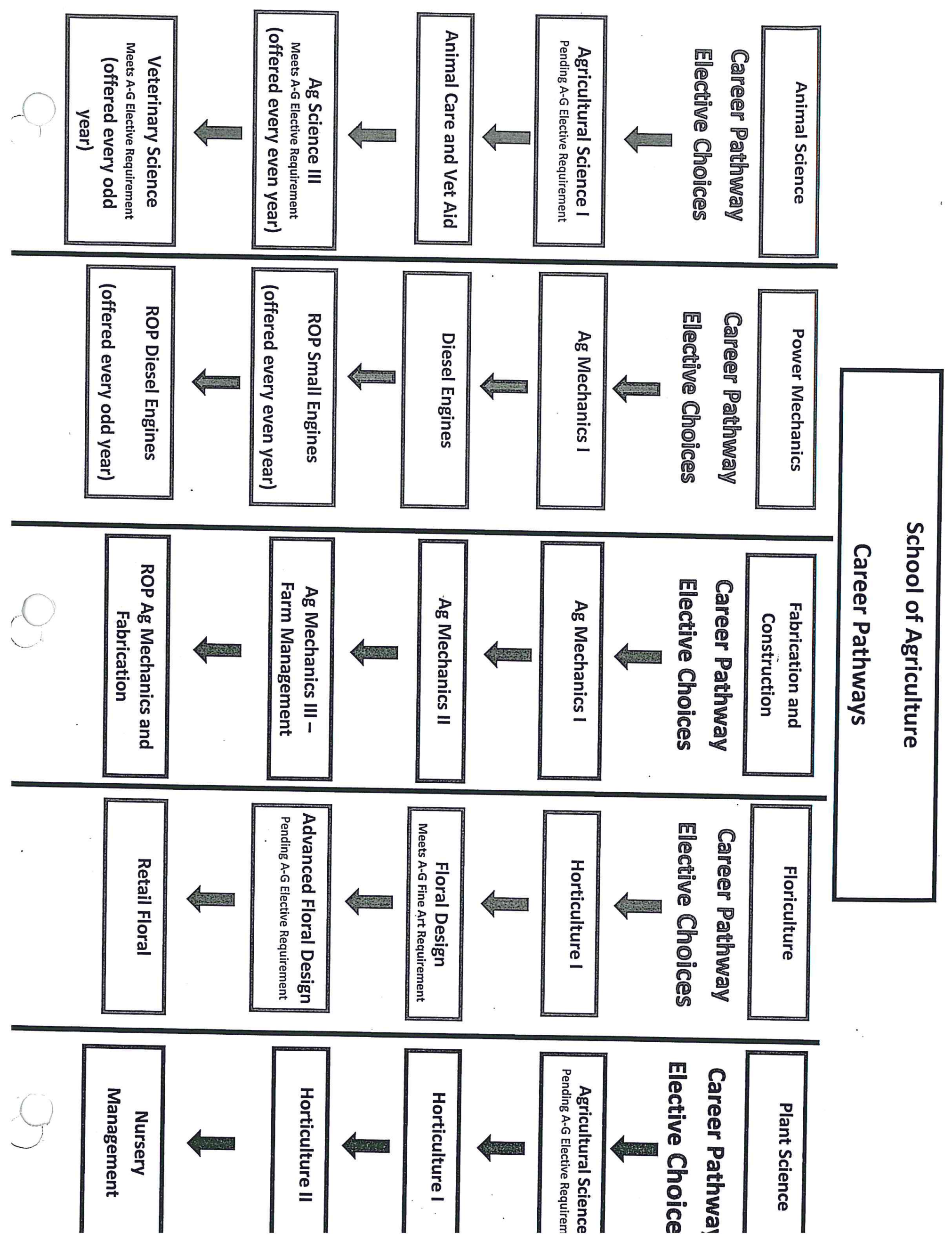




\section{Madera South High School Agriculture Department Proficiency Standard}

Upon completion of the following pathway, students should be proficient in the following:

\section{Agriculture Mechanies:}

1. Students show competency in measurement

2. Students understand personal and group safety in the shop

3. Students understand the basic electricity principles and wiring practices commonly used in agriculture

4. Students understand basic plumbing system practices commonly used in agriculture

5. Students understand cold metal processes

6. Students understand concrete and masonry practices commonly used in agriculture

7. Students understand oxy-fuel cutting and welding

8. Students understand electric arc welding processes

9. Students understand the principles of basic woodworking

\section{Agriculture Science:}

1. Students can identify and understand the importance of production agriculture

2. Students understand the importance of the role of agriculture in the California economy

3. Students understand the interrelationship between agriculture and the environment

4. Identify lab equipment and materials used in Ag Science lab settings

5. Students understand the effects of technology on agriculture

6. Students understand the importance of animals of animals, the domestication of animals, and the role of animals in modern society

7. Students understand basic animal health

8. Students understand plant growth and development

9. Students understand soils and plant production

10. Students understand the scientific method 


\section{Madera South High School Agriculture Department Proficiency Standard}

\section{O.I.}

1. To identify and understand the importance of horticulture production

2. Students understand plant classification and use principles

3. Students understand sexual and asexual plant reproduction

4. Students understand ornamental and floral plant nutrition practices and needs

5. Students understand the use of containers and horticulture tools, equipment, and facilities

7. Students understand agribusiness as it applies to the horticulture industry

\section{Floral Design}

1. Students know principles of all basic arrangements

2. Students are able to identify common tools, plants and materials

3. Students know the eras and art for each period of design

Students understand agribusiness as it applies to the floral industry 


\section{T. A Copy Of Credential Or Authorization Letter}




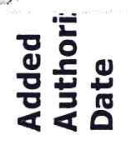

훙ำ

$\frac{2}{2}$

$\frac{\bar{T}}{\Sigma}$

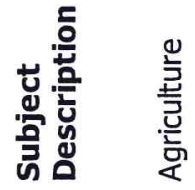

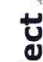

营蹗 总

岩

त్

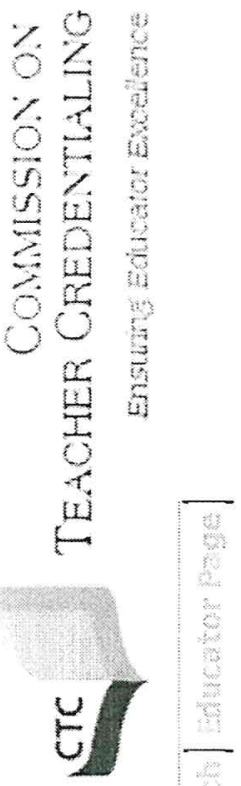

믐

荧 है

究荡

한

突苞

록

음

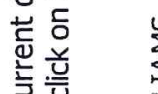

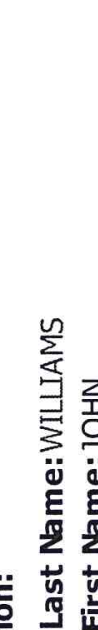

气

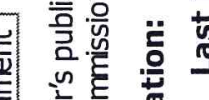

z-

矛 总高
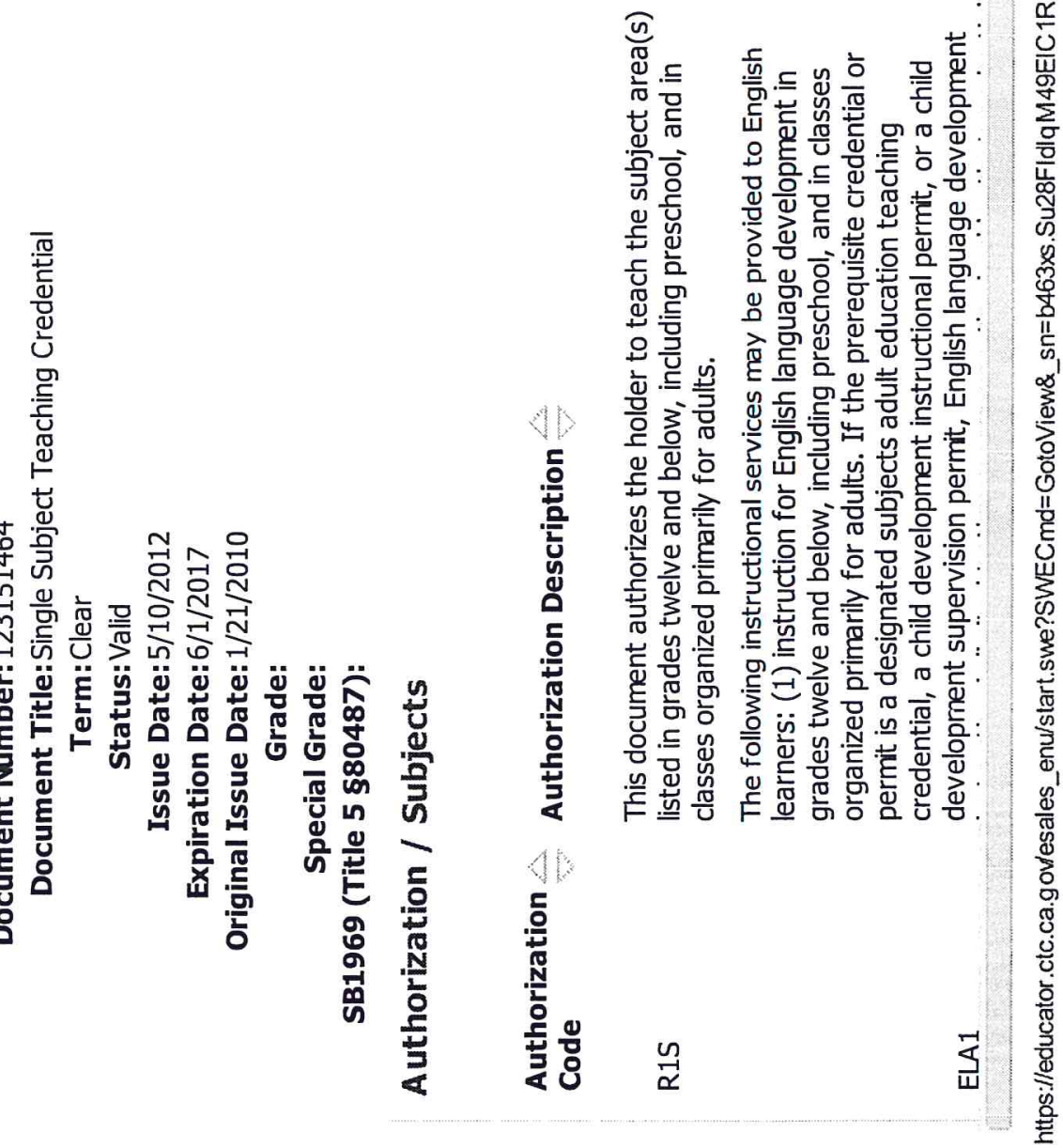

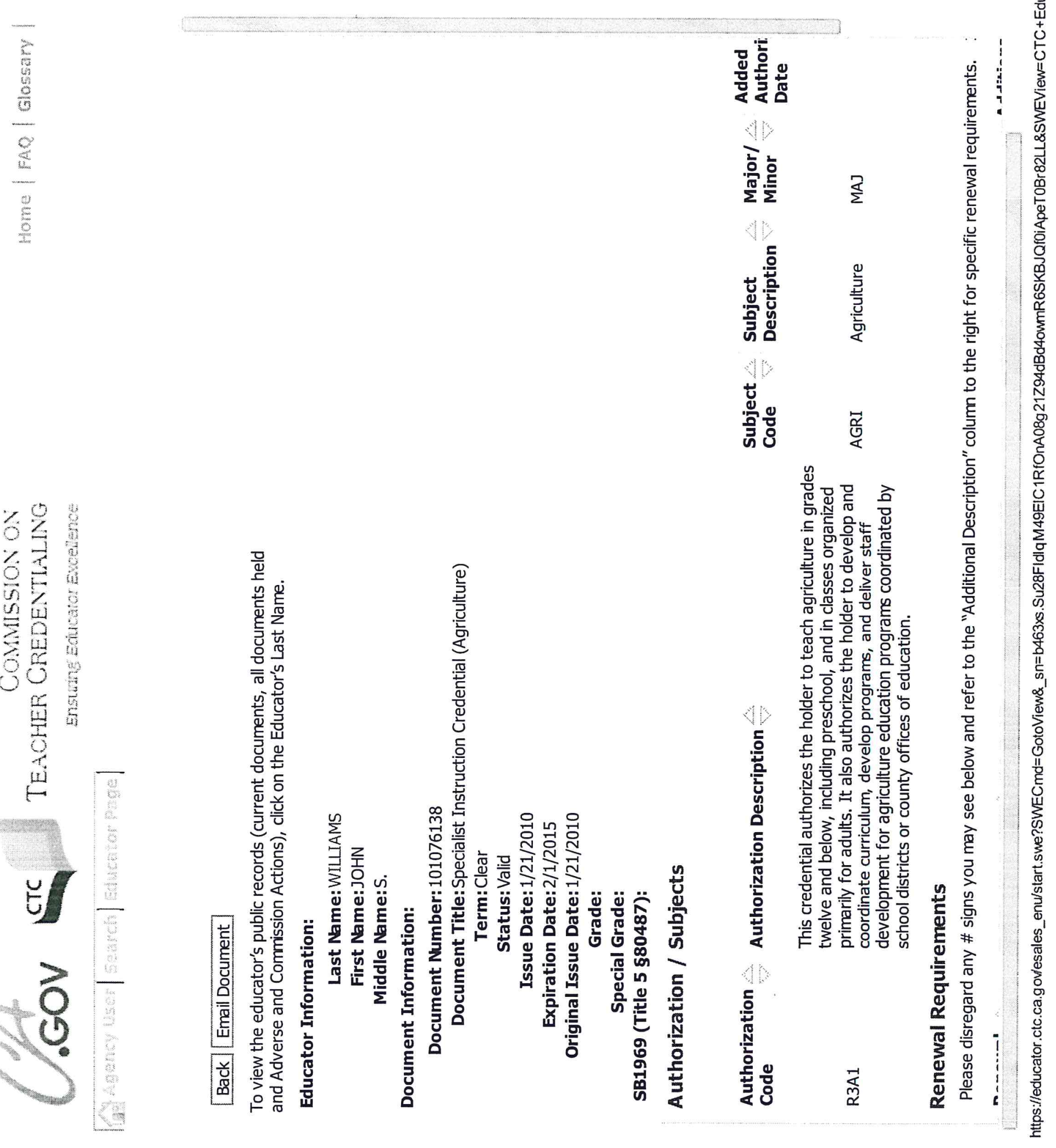
U. Calendar Of

Your/Department/Chapter Activities 


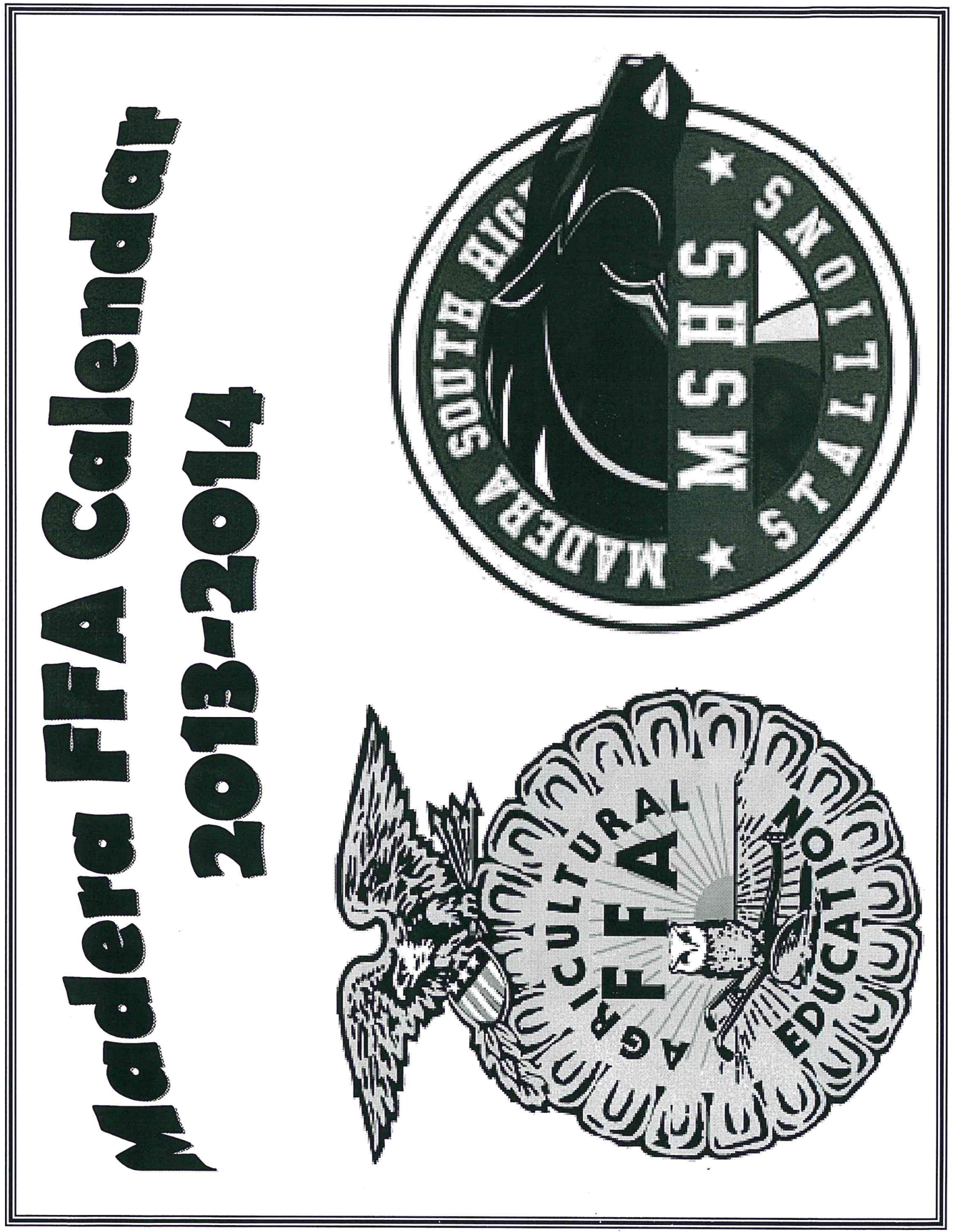




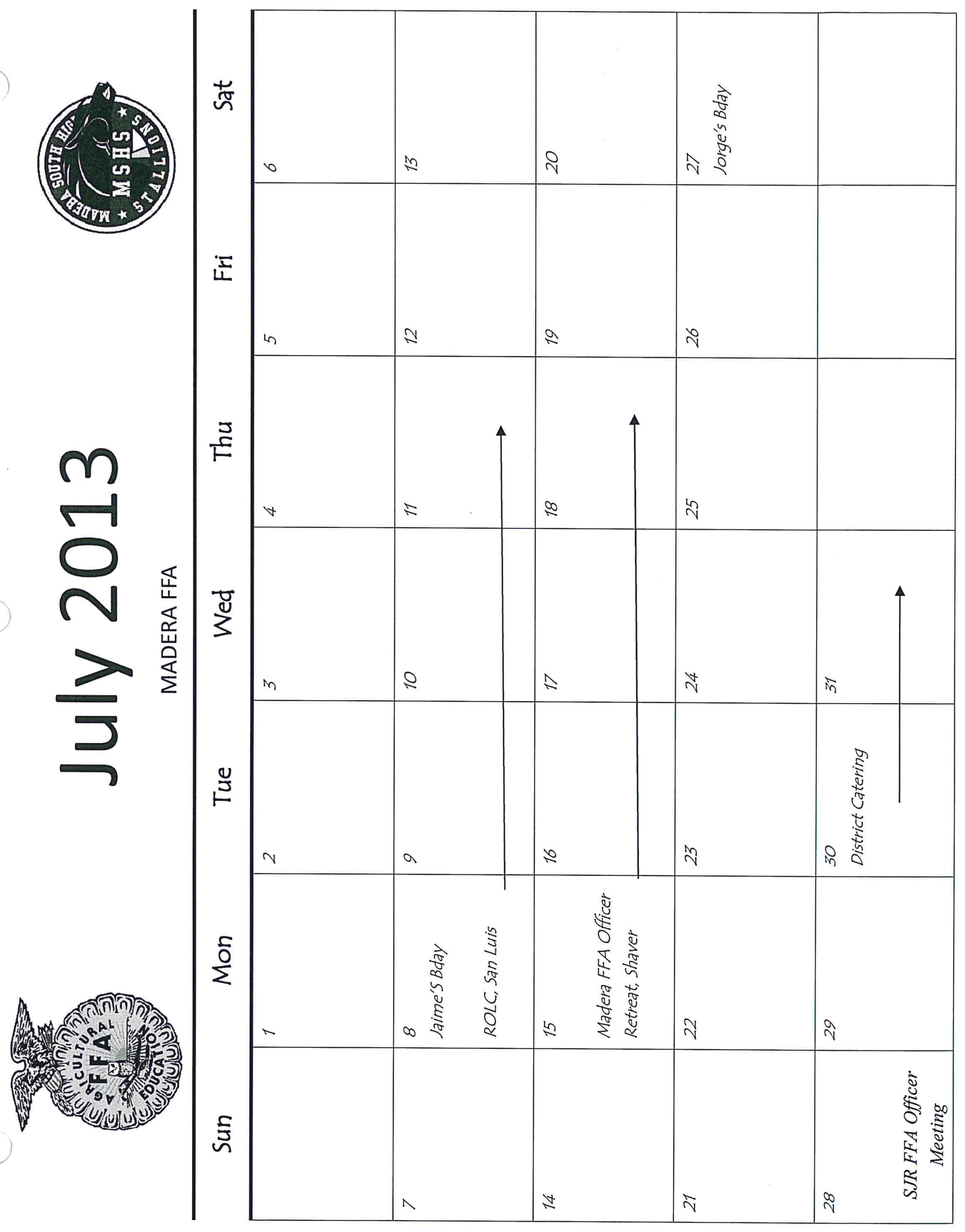




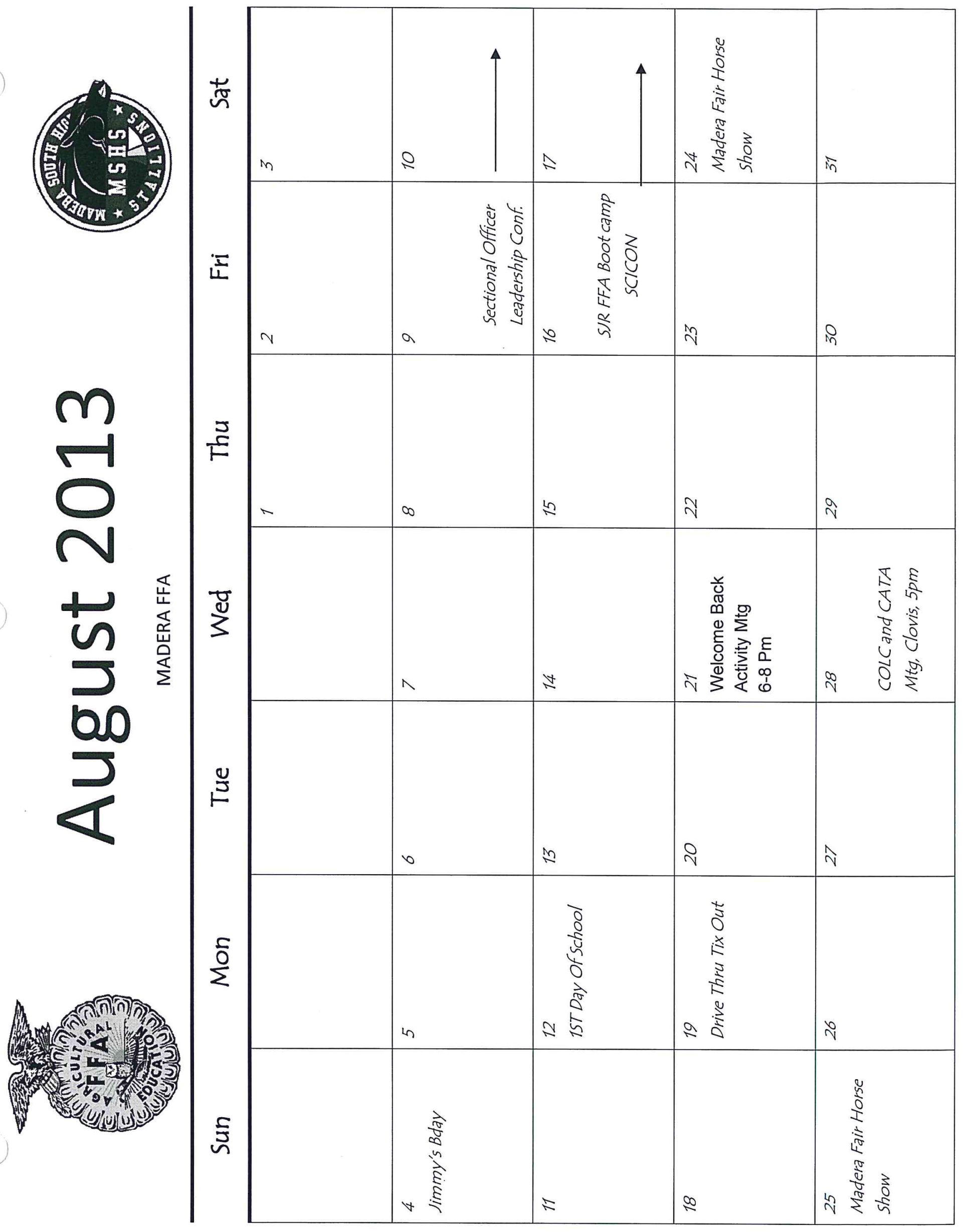




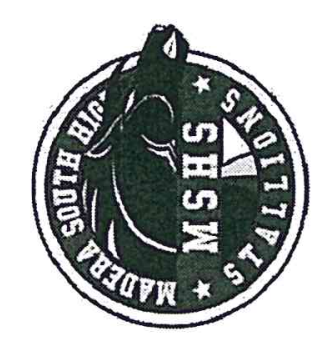

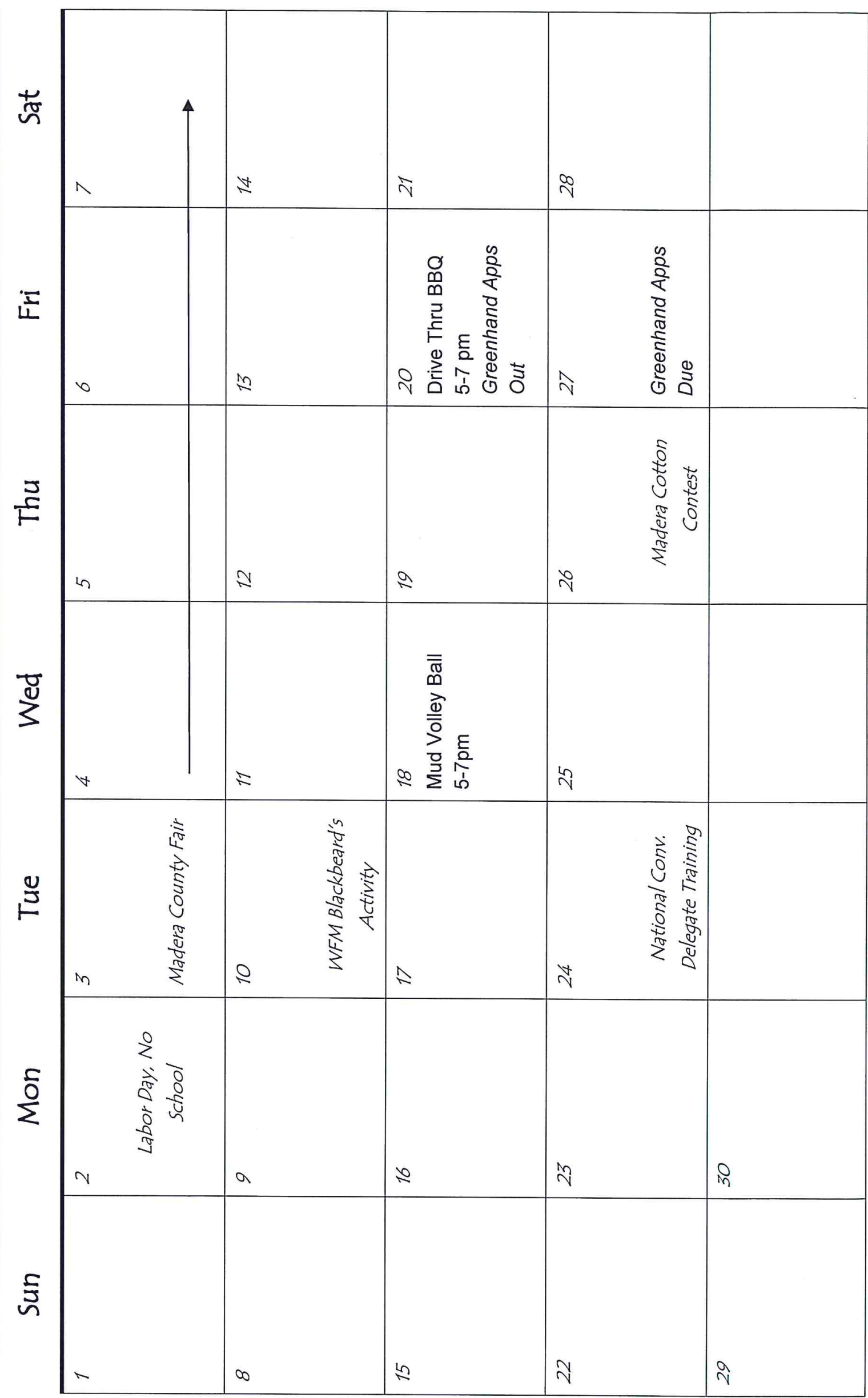




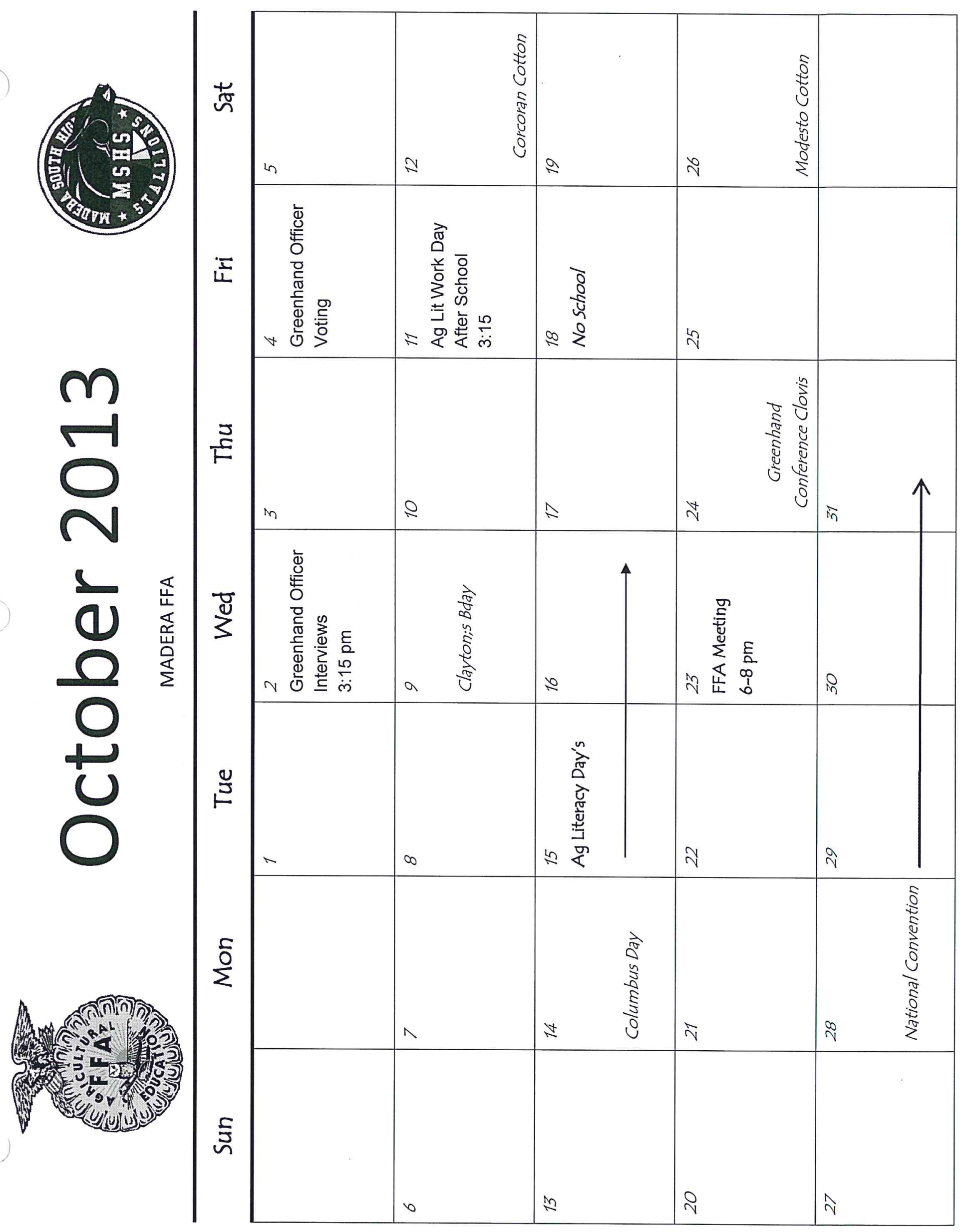




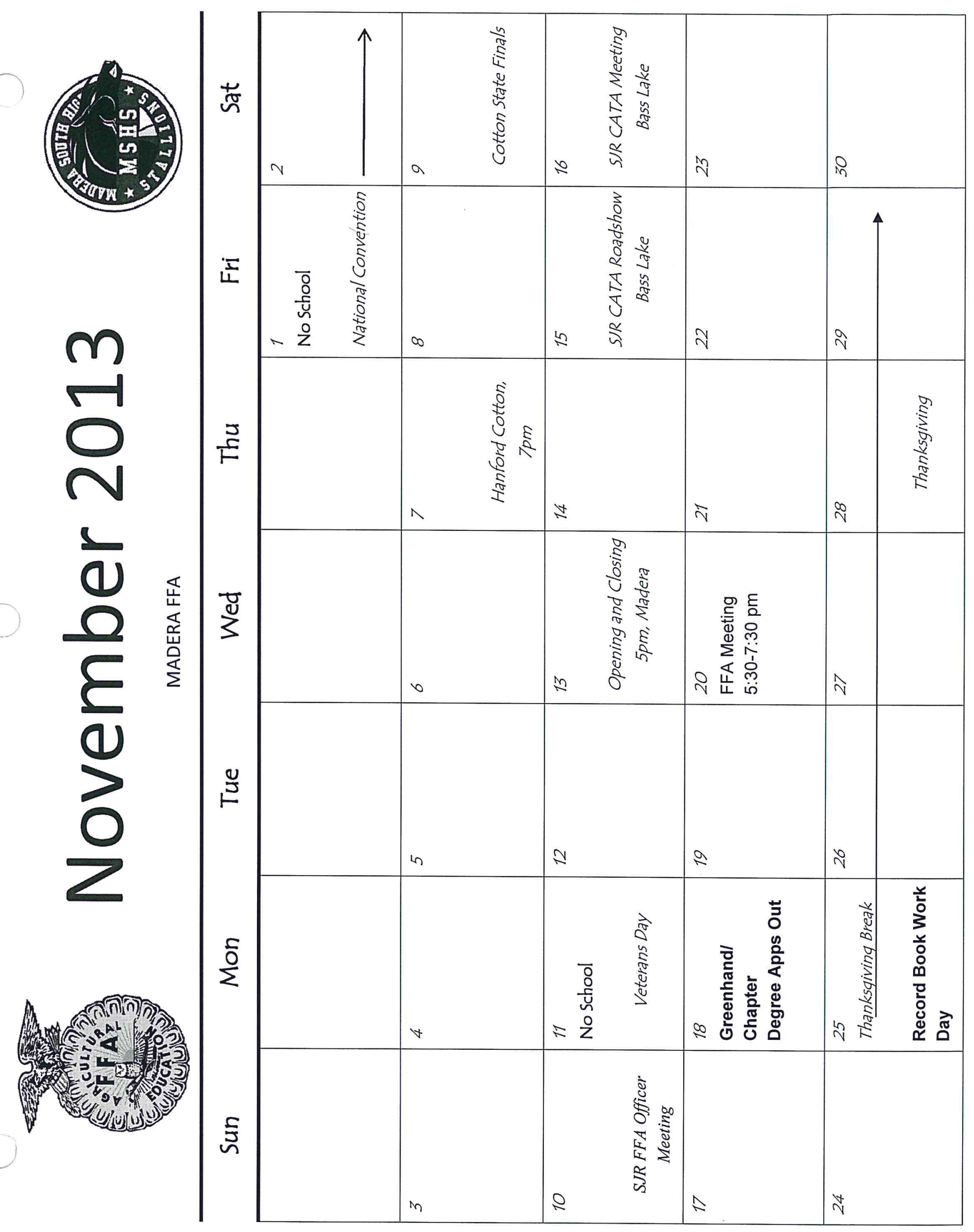




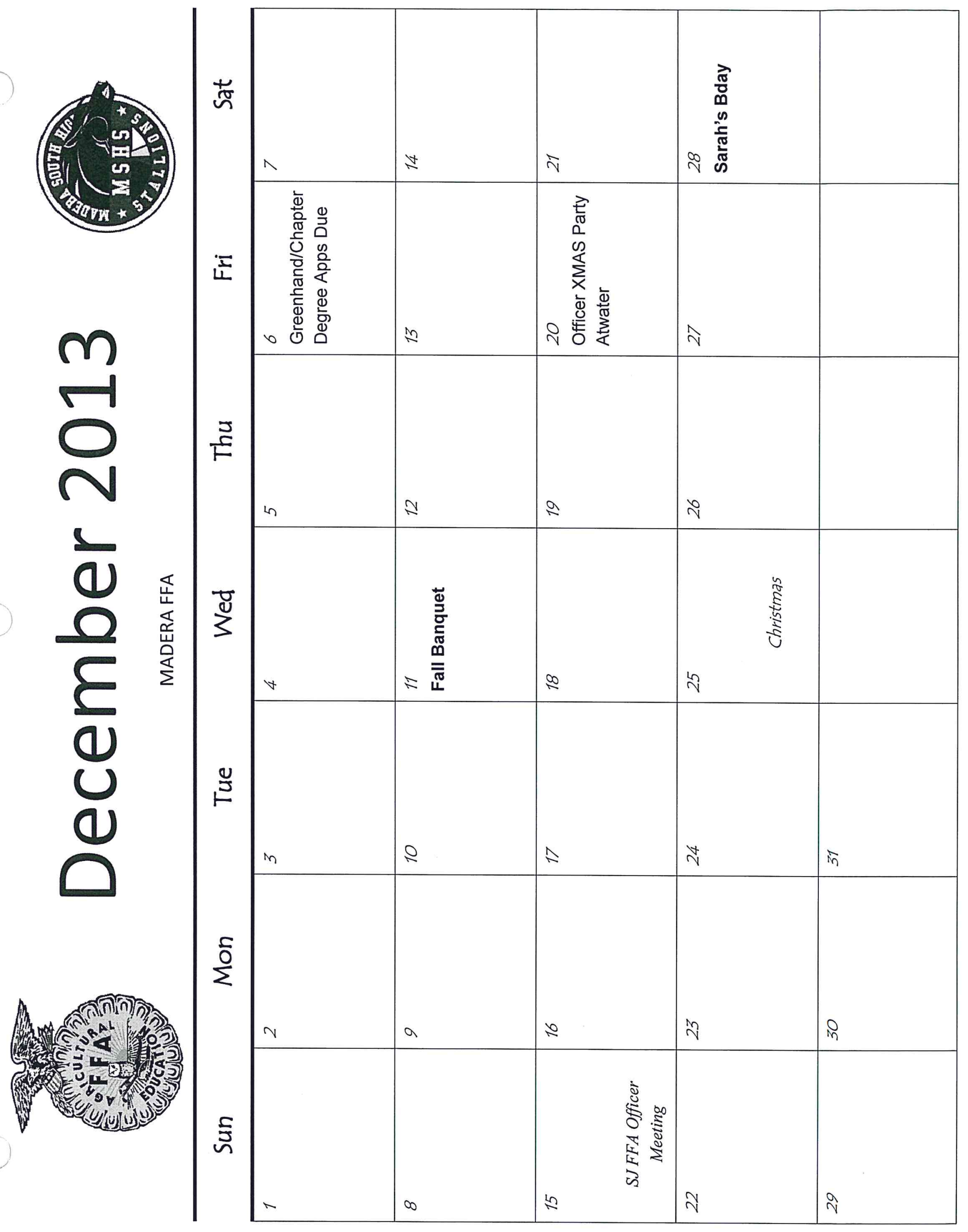




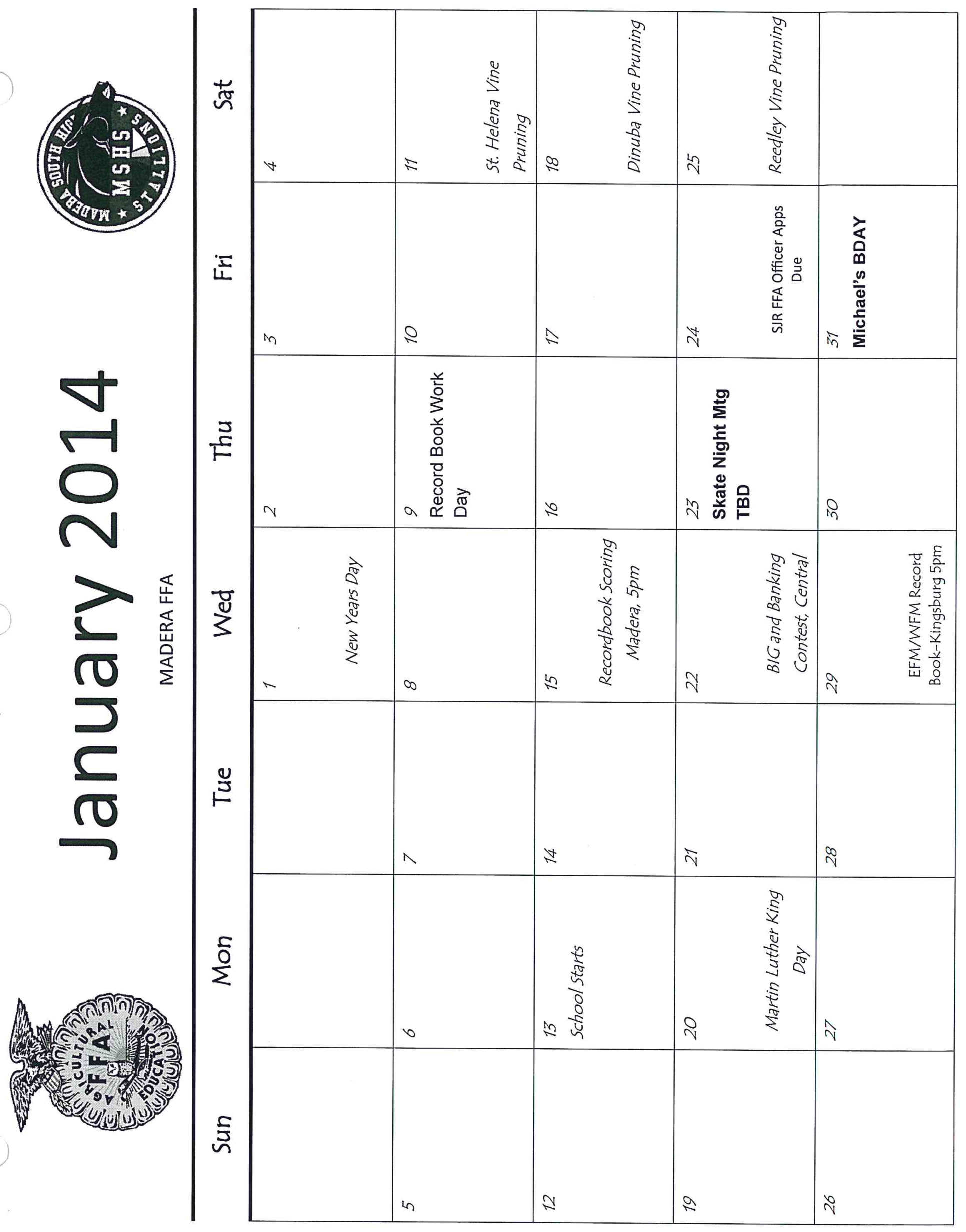




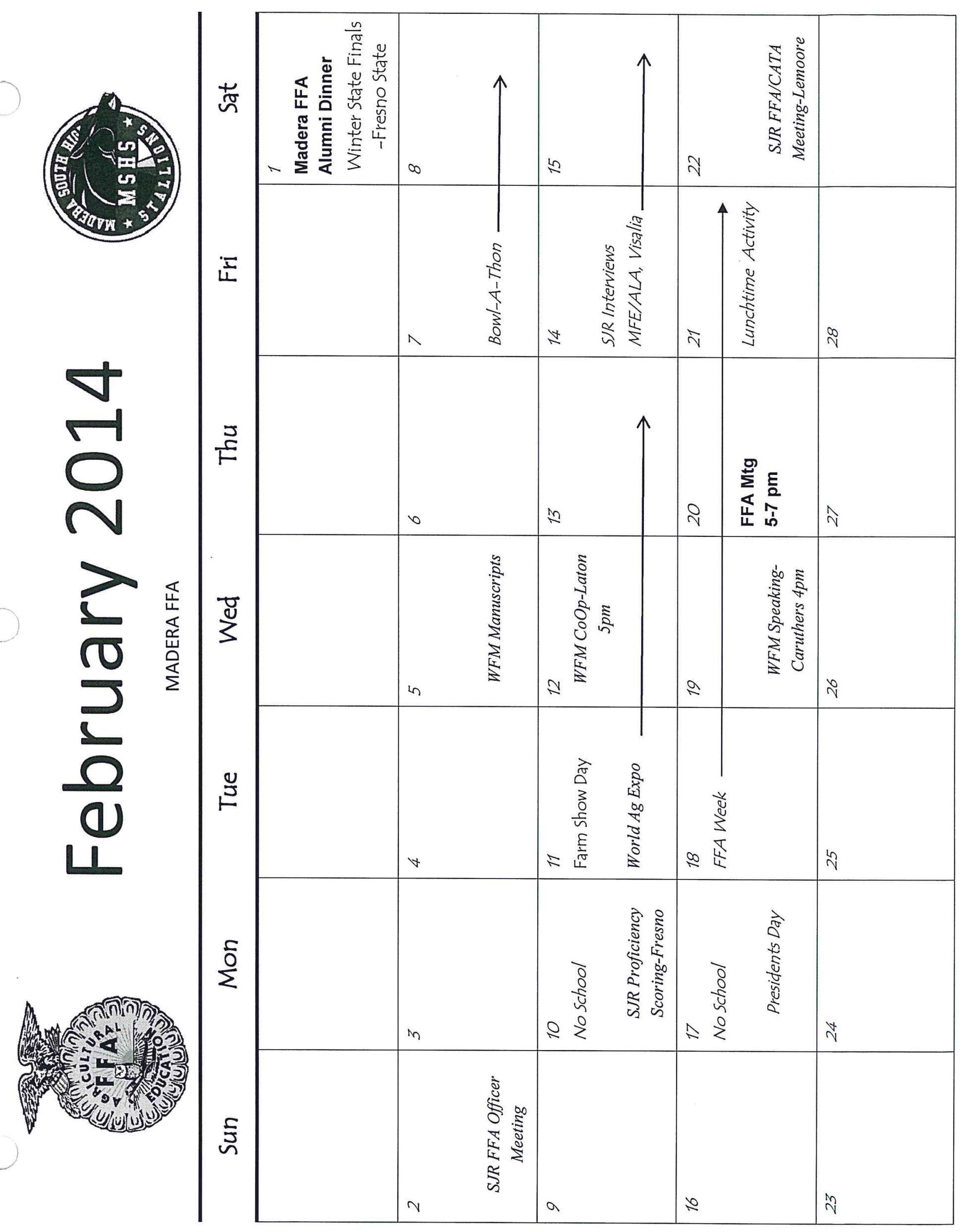




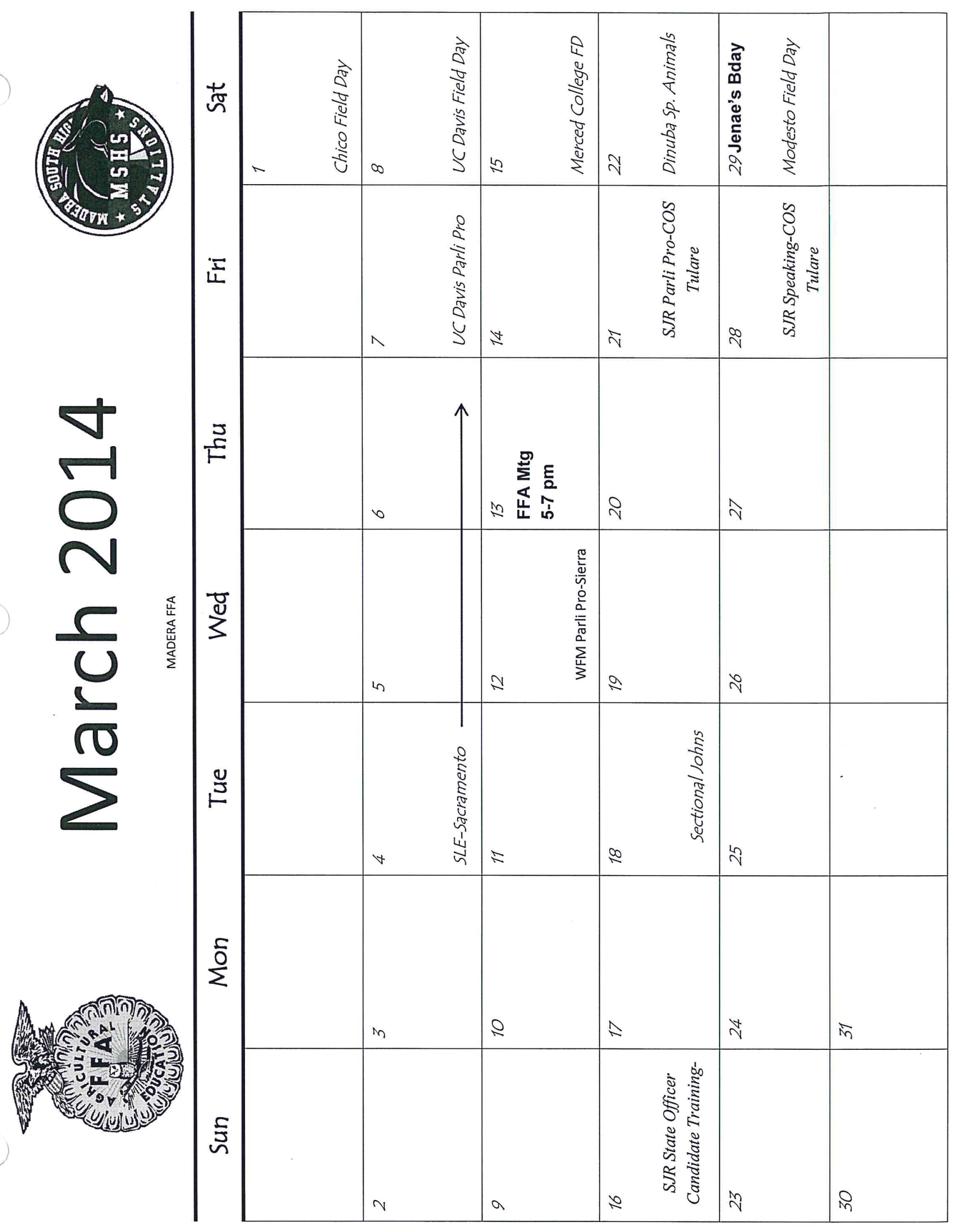




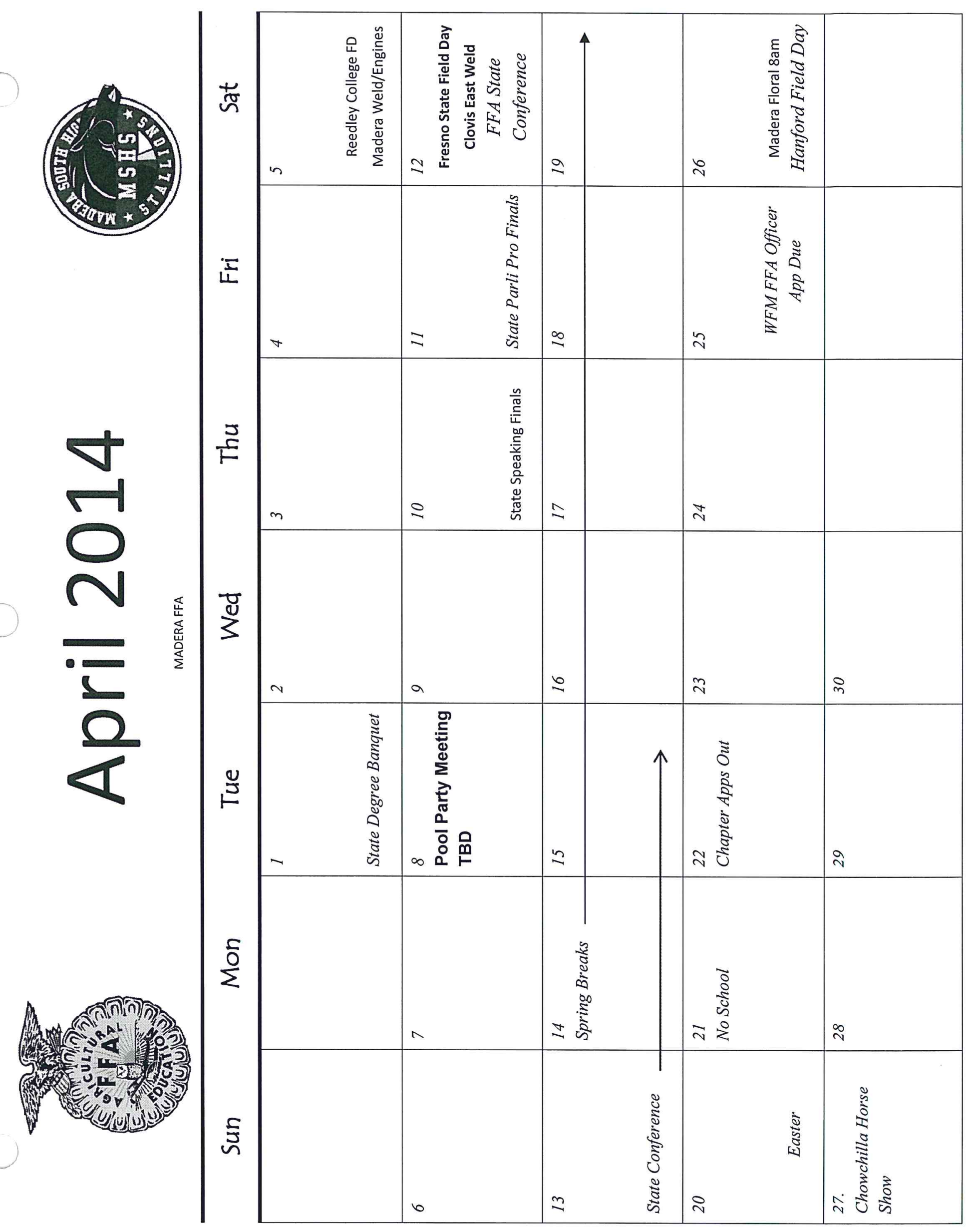




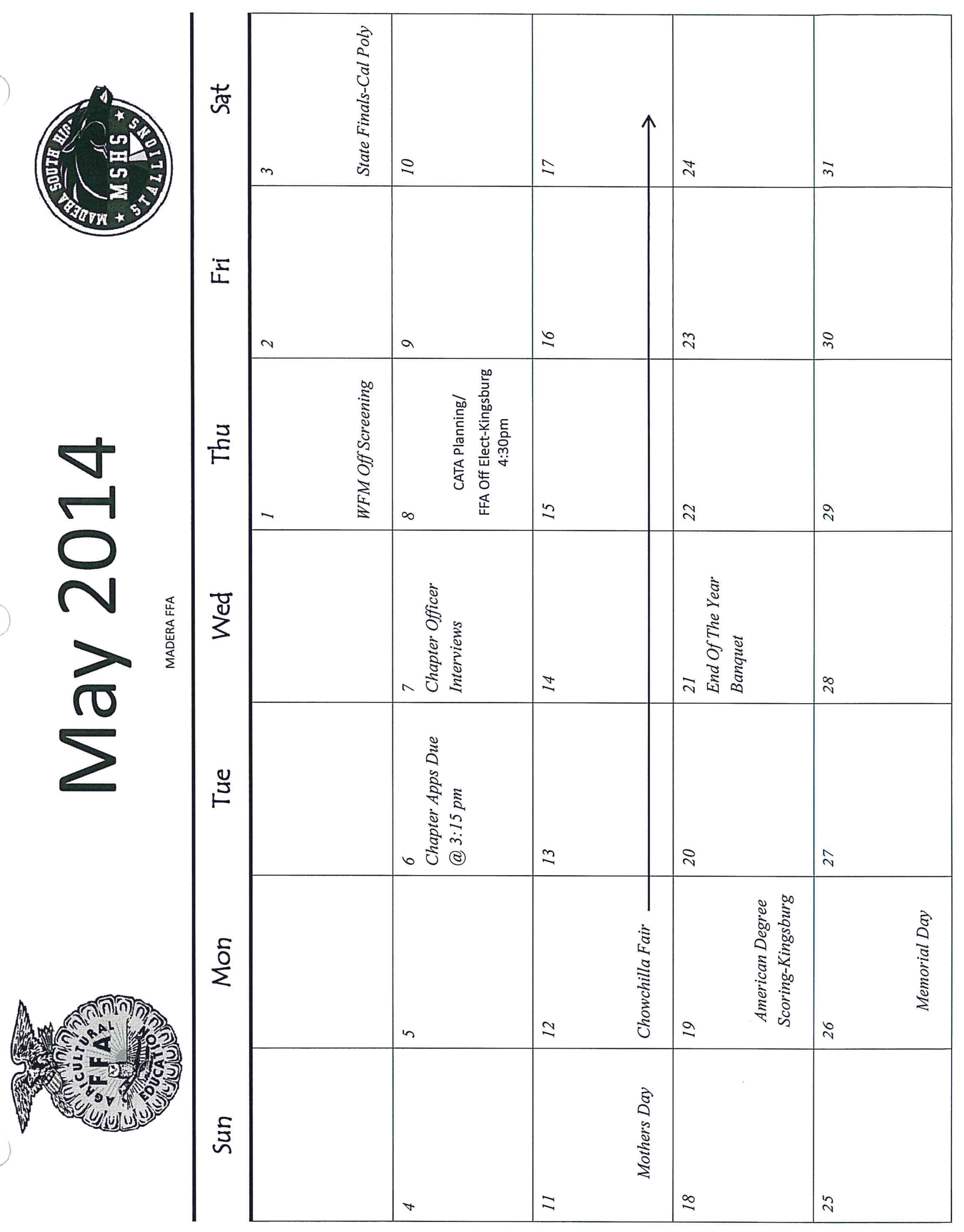




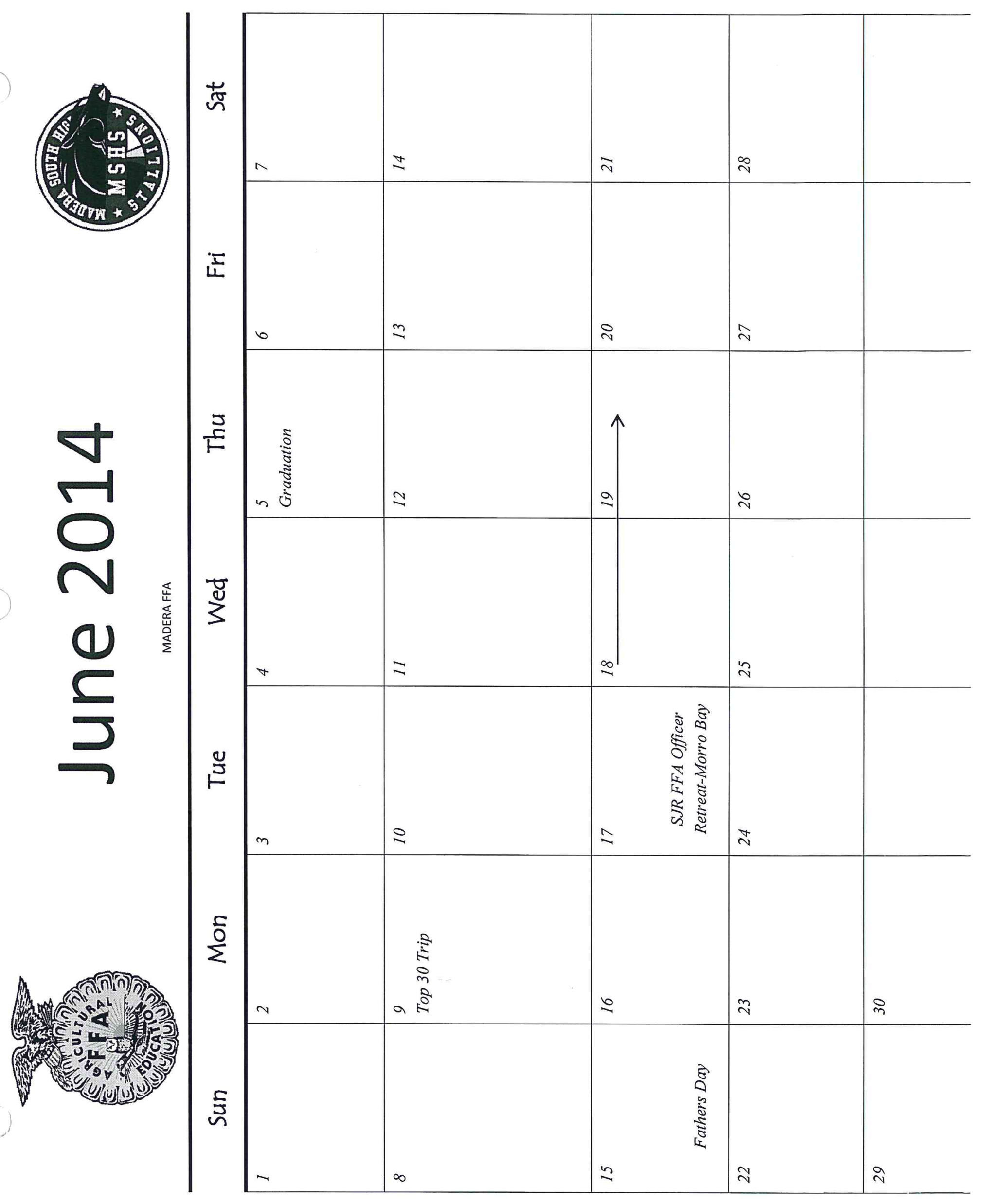


V. Copies Of Your Daily Logs For Your Current Year 
Madera South High

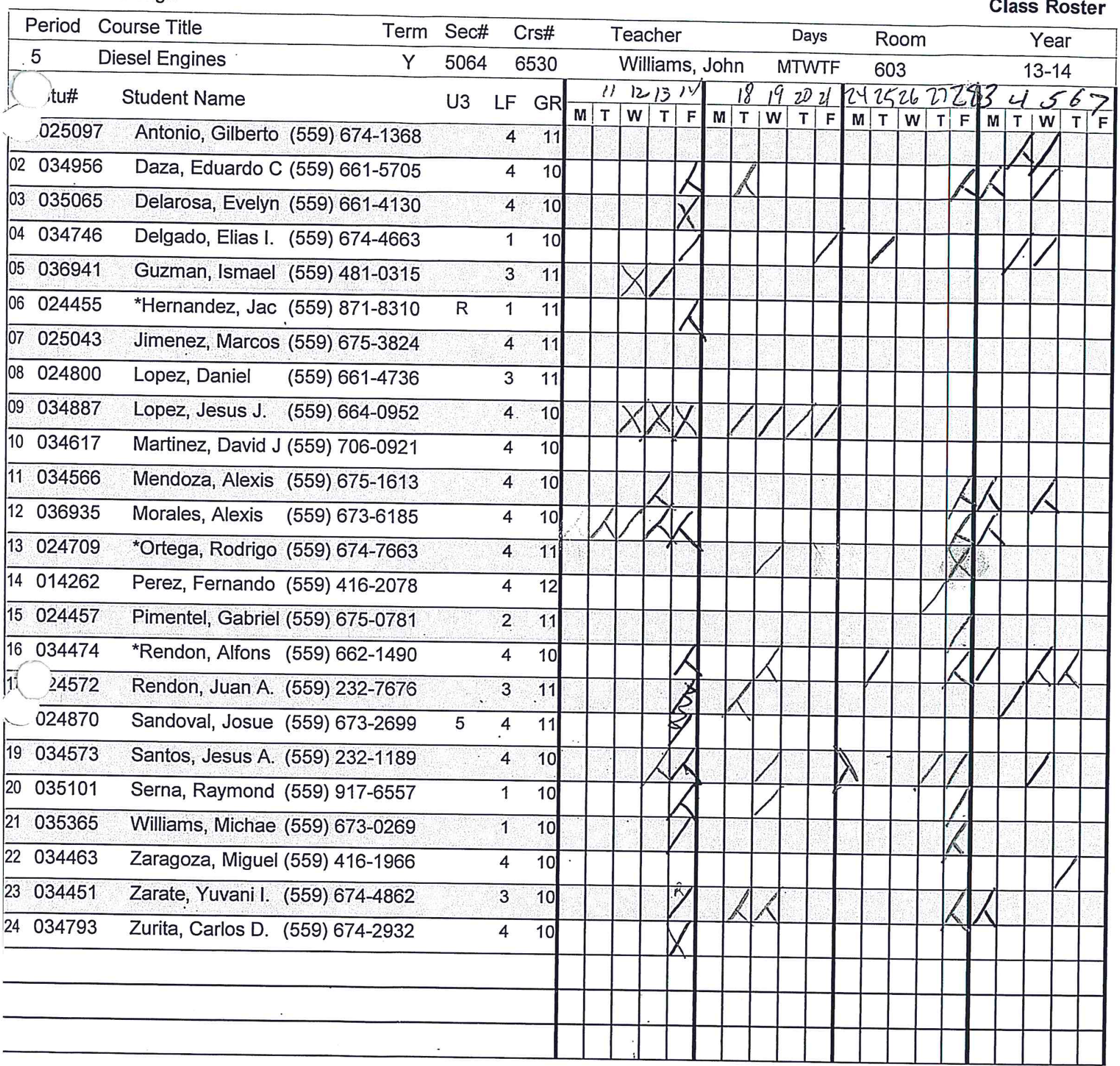


Madera South High

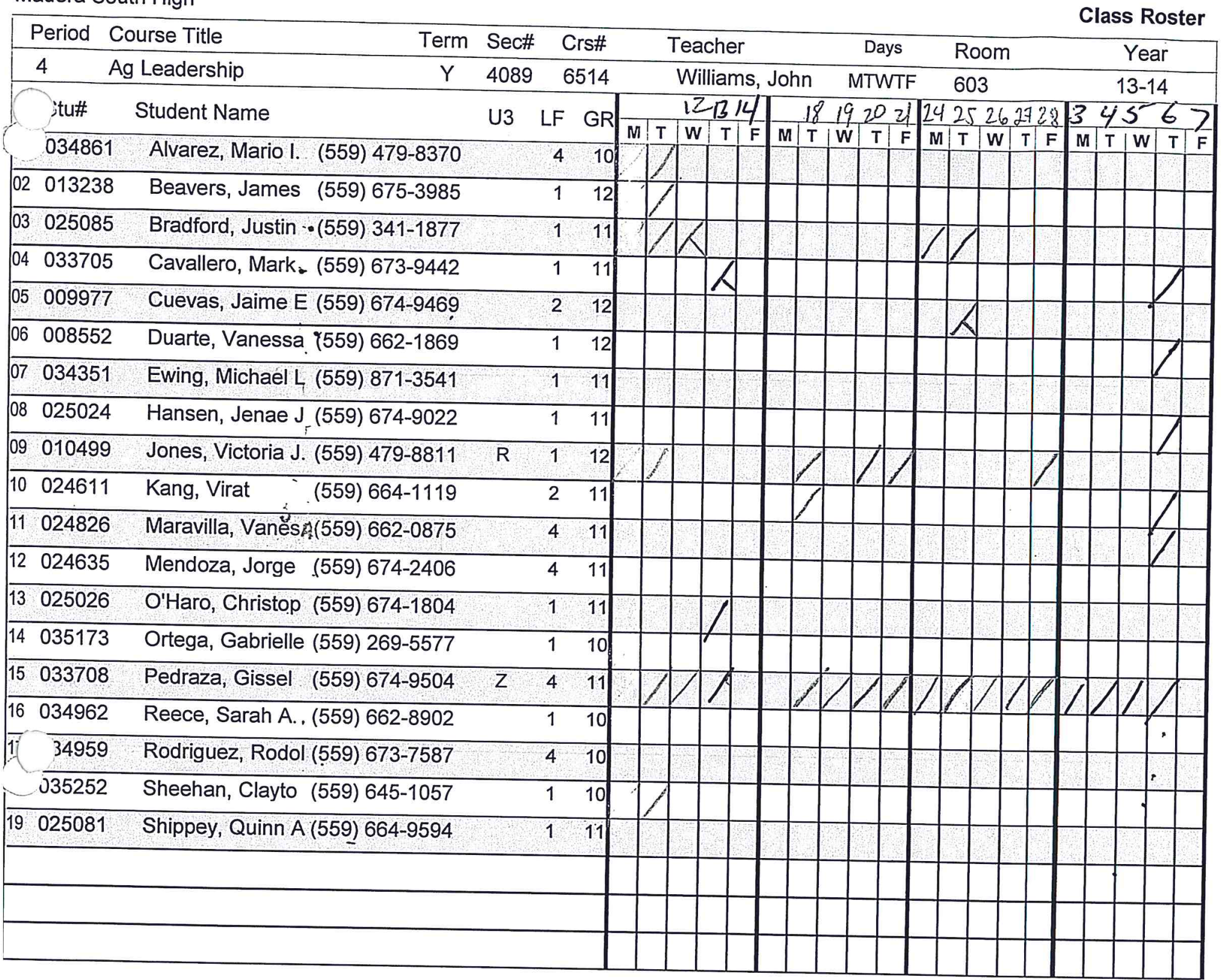


Madera South High

Class Roster

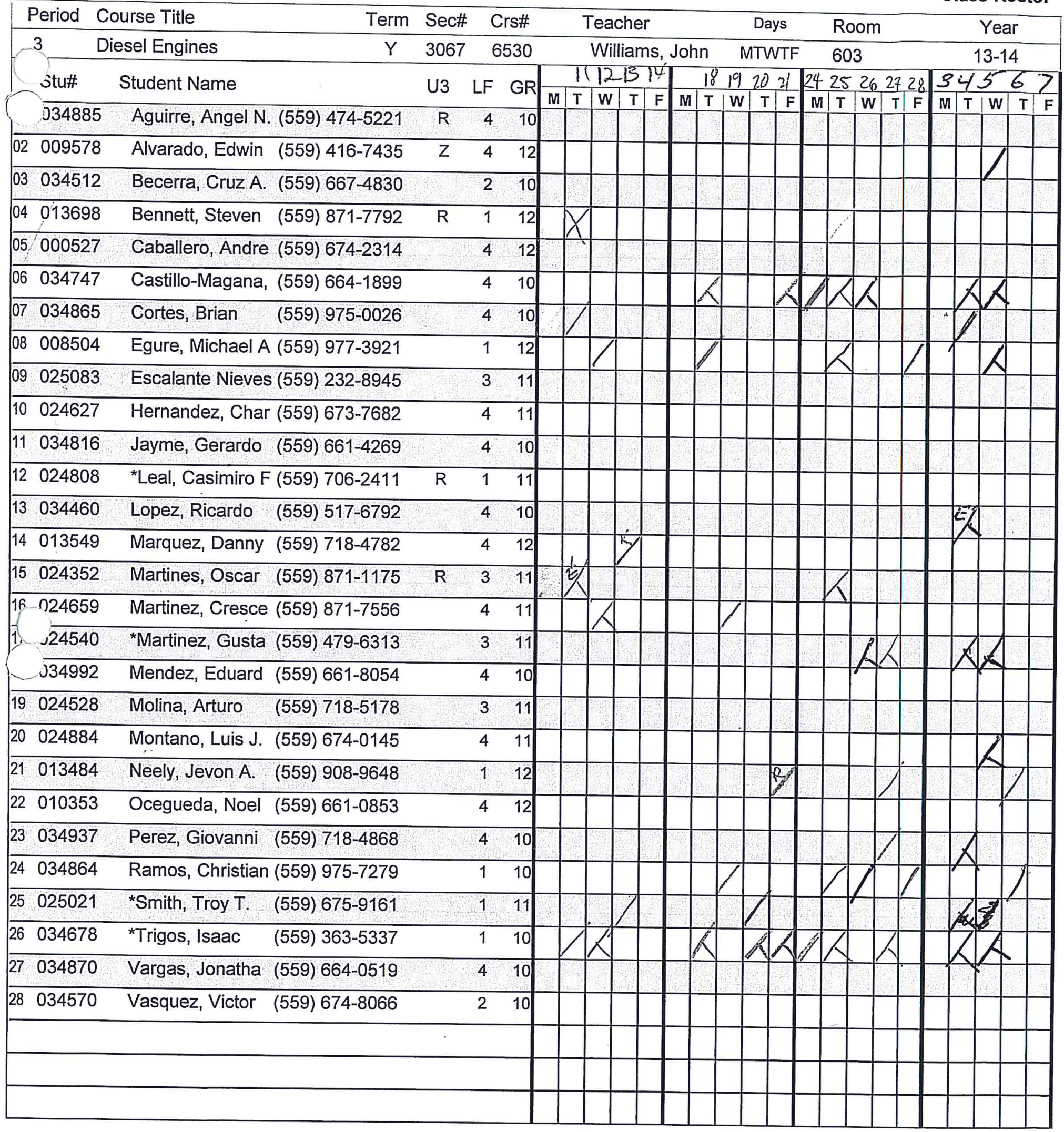


Madera South High

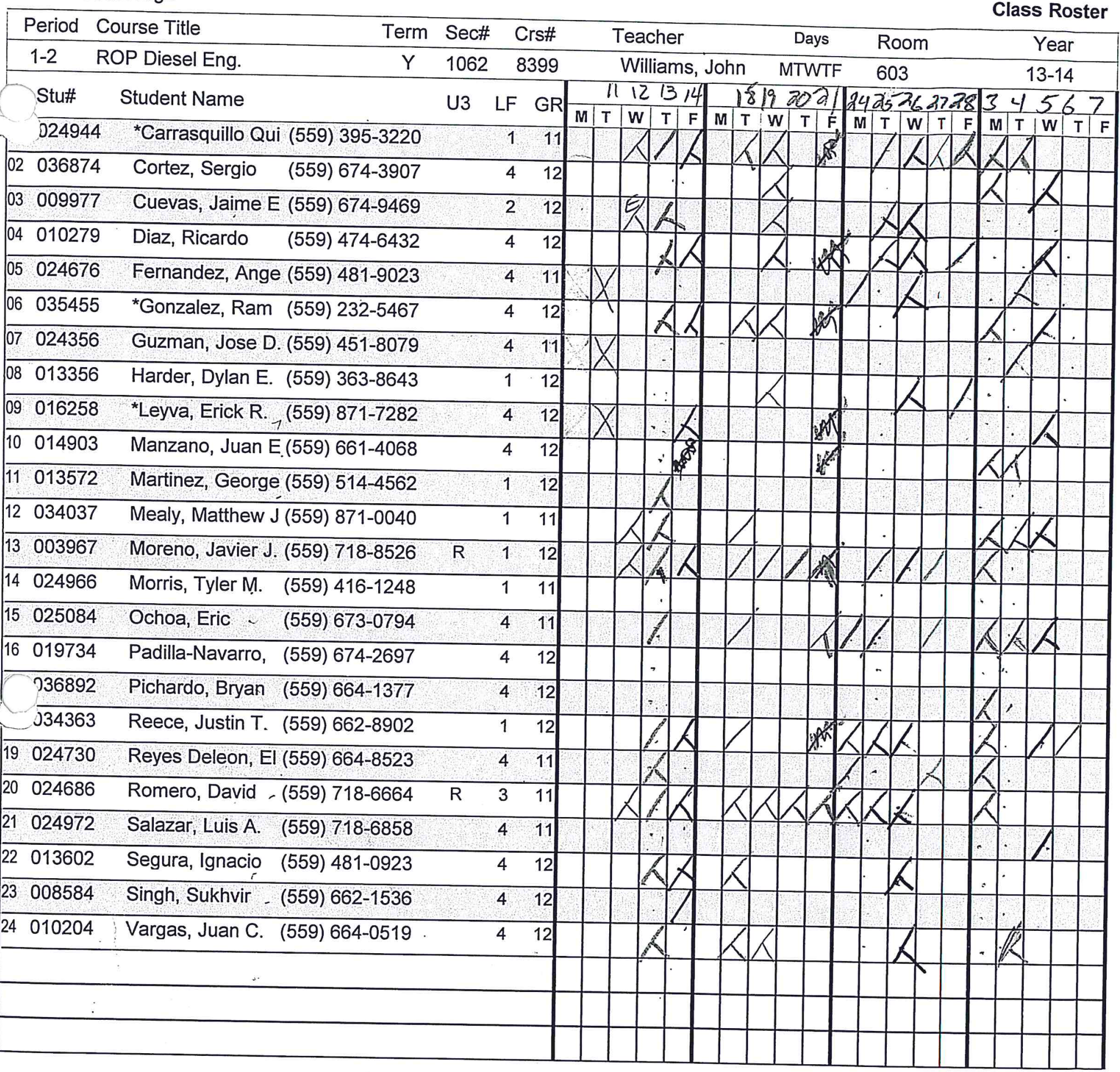




\section{W. A List Of Expected Professional Growth Activities.}




\section{Professional Growth}

- CATA Summer Conference

- SJ Region Fall and Spring Meetings

- Briggs and Stratton Advanced Technician School

- Stihl Certification

- American Vintners Conference 


\section{Copy Of Current Year's R-2 Reports}




\section{Madera South HS}

R2 Student Report

Year:2013

Gender

\begin{tabular}{|l|l|l|l|}
\hline Schnum & ProgName & Male & Female \\
\hline 170 & Ag Bus Mgt & 8 & 3 \\
\hline 170 & Ag Mech. & 303 & 9 \\
\hline 170 & Agriscience & 46 & 22 \\
\hline 170 & An. Science & 38 & 97 \\
\hline 170 & Forestry/NR & 1 & 1 \\
\hline 170 & O.H. & 7 & 48 \\
\hline 170 & PlantSoil Sci. & 23 & 16 \\
\hline
\end{tabular}

Hispanic

\begin{tabular}{|l|l|l|}
\hline ProgName & Hispanic & Non-Hispanic \\
\hline Ag Bus Mgt & 11 & 0 \\
\hline Ag Mech. & 266 & 46 \\
\hline Agriscience & 55 & 13 \\
\hline An. Science & 109 & 26 \\
\hline Forestry/NR & 2 & 0 \\
\hline O.H. & 49 & 6 \\
\hline Plant/Soil Sci. & 32 & 7 \\
\hline
\end{tabular}

Race*

\begin{tabular}{|l|l|l|l|l|l|l|l|}
\hline ProgName & White & Black & Hispanic & Americian Indian & Asian & Native Hawaiian/Pacifc Island & 2 or more \\
\hline Ag Bus Mgt & 11 & 0 & 0 & 0 & 0 & 0 & 0 \\
\hline Ag Mech. & 269 & 6 & 0 & 13 & 13 & 2 & 11 \\
\hline Agriscience & 63 & 1 & 0 & 1 & 1 & 0 & 2 \\
\hline An. Science & 124 & 1 & 0 & 3 & 3 & 1 & 5 \\
\hline Forestry/NR & 2 & 0 & 0 & 0 & 0 & 0 & 0 \\
\hline O.H. & 53 & 1 & 0 & 0 & 0 & 0 & 1 \\
\hline Plant/Soil Sci. & 38 & 1 & 0 & 0 & 0 & 0 & 0 \\
\hline
\end{tabular}

Grade Level

\begin{tabular}{|l|r|r|r|r|r|r|r|r|r|}
\hline Year In Ag & Grade9 & Grade10 & Grade11 & Grade12 & Grade13 & Grade14 & Grade15 & Grade16 & Total \\
\hline 1 & 214 & 38 & 31 & 30 & 0 & 0 & 0 & 0 & 313 \\
\hline 2 & 0 & 120 & 10 & 13 & 0 & 0 & 0 & 0 & 143 \\
\hline 3 & 0 & 0 & 85 & 10 & 0 & 0 & 0 & 0 & 95 \\
\hline 4 & 0 & 0 & 0 & 56 & 0 & 0 & 0 & 0 & 56 \\
\hline 5 & 0 & 0 & 0 & 0 & 11 & 0 & 0 & 0 & 11 \\
\hline
\end{tabular}




\begin{tabular}{|l|r|r|r|r|r|r|r|r|r|}
\hline 6 & 0 & 0 & 0 & 0 & 0 & 3 & 0 & 0 & 3 \\
\hline 7 & 0 & 0 & 0 & 0 & 0 & 0 & 1 & 0 & 1 \\
\hline Total & 214 & 158 & 126 & 109 & 11 & 3 & 1 & 0 & 622 \\
\hline \multicolumn{7}{|c|}{ Total 9-12 } & 607 \\
\hline
\end{tabular}

\section{Freshman Persistance:}

Cohort Year: 2010-2011

\begin{tabular}{|l|r|r|}
\hline Years in Ag Completed & Count & Percent \\
\hline 1 & 79 & $38 \%$ \\
\hline 2 & 59 & $29 \%$ \\
\hline 3 & 13 & $6 \%$ \\
\hline 4 & 56 & $27 \%$ \\
\hline Freshman Cohort Students & 207 & \multicolumn{1}{|c}{} \\
\cline { 1 - 2 } Average Years Completed & 2.2 & \multicolumn{1}{|l}{} \\
\cline { 1 - 2 } &
\end{tabular}

*Prior to 2010 Hispanic is listed as a race.

Printed: 3/13/2014 10:54:13 AM

Site developed and maintained by the Californa FRA Association. 


\section{FFA Roster}

\# CA0141 Madera-Madera South

Madera South HS

705 West Pecan

Madera, CA 93637

Year: 2013 Go

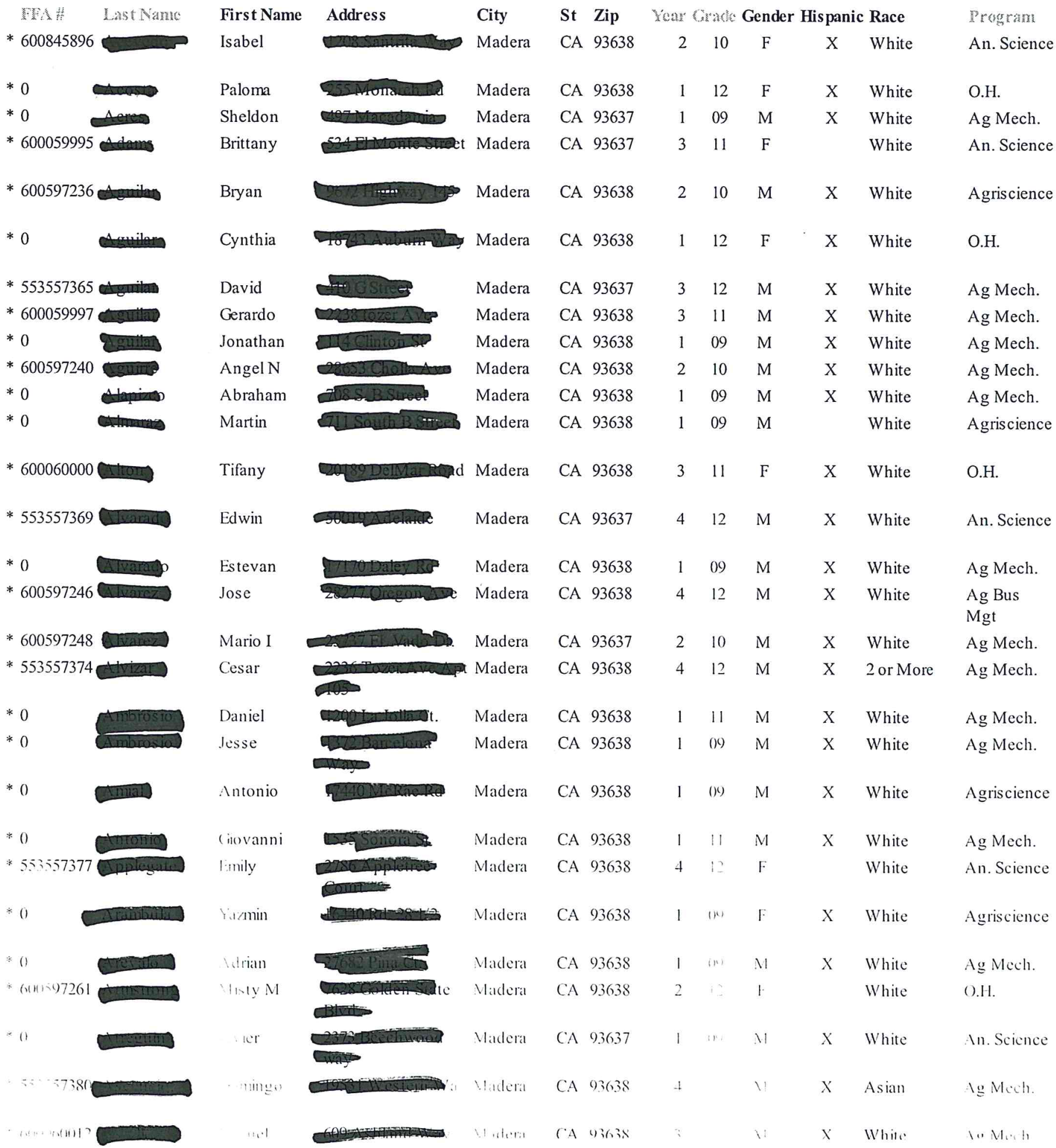




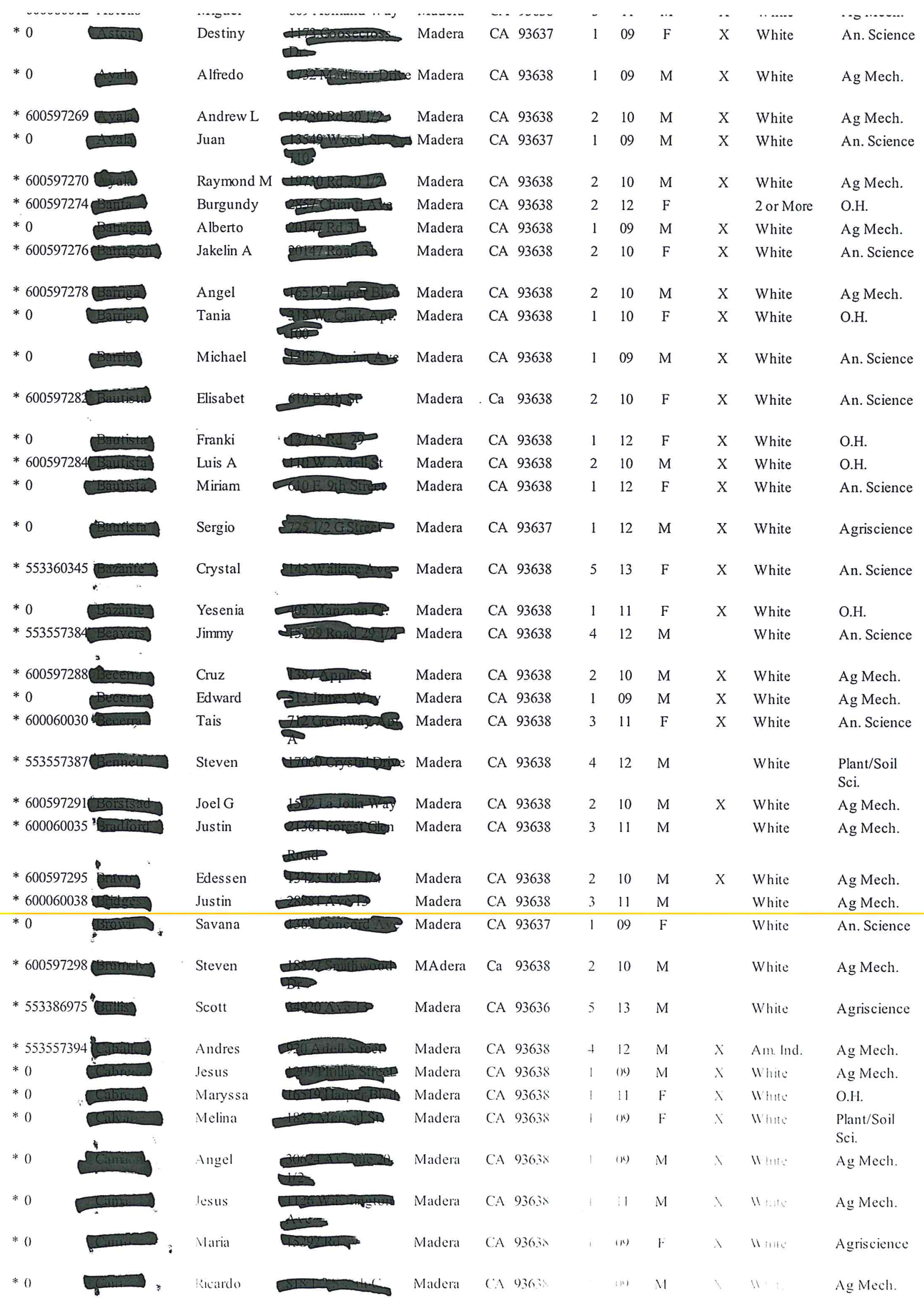




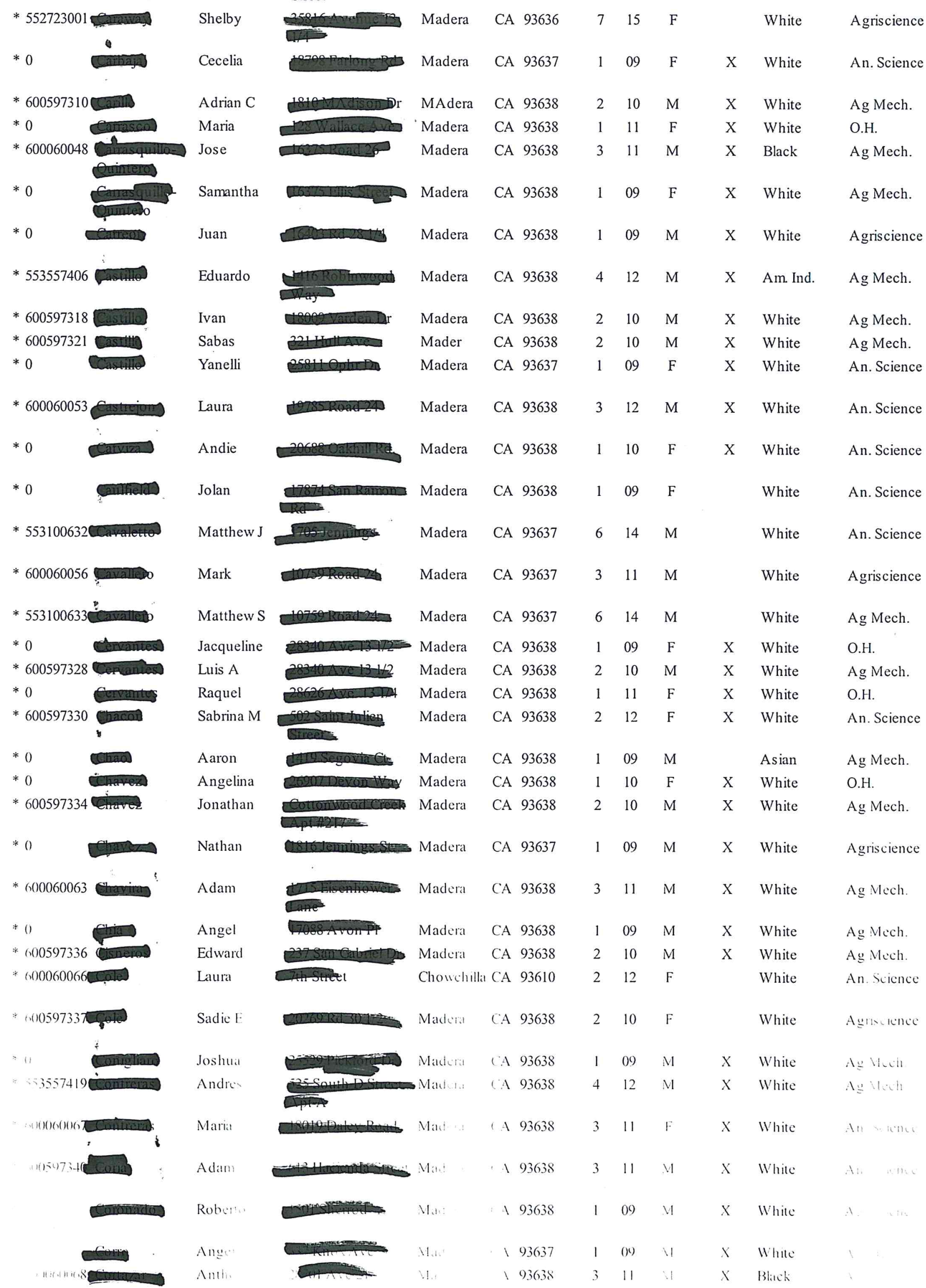




\begin{tabular}{|c|c|c|c|c|c|c|c|c|c|c|c|}
\hline * 60059734 Colfes & Brian & PSCentros & Madera & $\mathrm{CA}$ & 93638 & 2 & 10 & M & $\mathrm{X}$ & White & Ag Mech. \\
\hline$* 0$ & Jessenia & Denschoss Sta & Madera & $\mathrm{CA}$ & 93638 & 1 & 12 & $\mathrm{~F}$ & $\mathrm{X}$ & White & O.H. \\
\hline * 600597343 & Joel C & 240tyallace & Madera & $\mathrm{Ca}$ & 93638 & 2 & 10 & M & $\mathrm{X}$ & White & An. Science \\
\hline$* 0$ & Alexa & 518-Somith A Streed & Madera & $\mathrm{CA}$ & 93638 & 1 & 09 & $\mathrm{~F}$ & $\mathrm{X}$ & White & An. Science \\
\hline * 600597344CCan & Ariana & & Madera & $\mathrm{CA}$ & 93638 & 2 & 10 & $\mathrm{~F}$ & $X$ & White & Agriscience \\
\hline$* 0$ & Damaris & +306-Eresno-Streets & Madera & $\mathrm{CA}$ & 93638 & 1 & 09 & $\mathrm{~F}$ & $X$ & White & $\begin{array}{l}\text { Plant/Soil } \\
\text { Sci. }\end{array}$ \\
\hline * 55355742飞 & Daniel & & Madera & $\mathrm{CA}$ & 93638 & 4 & 12 & M & $X$ & Am. Ind. & Ag Mech. \\
\hline * $60006007 \varnothing$ & Deysi & - SISSOHth-AStrect & Madera & $\mathrm{CA}$ & 93638 & 3 & 11 & $\mathrm{~F}$ & $X$ & White & An. Science \\
\hline$* 0$ & George & mado-Drs & Madera & $\mathrm{CA}$ & 93638 & 1 & 09 & M & $X$ & White & Agriscience \\
\hline$* 0$ & Miguel & atisponDr. & Madera & $\mathrm{CA}$ & 93638 & 1 & 09 & M & $X$ & White & Agriscience \\
\hline * 553557425 & Sergio & 1174 Lamewney & Madera & $\mathrm{CA}$ & 93638 & 3 & 12 & M & $X$ & Am Ind. & Ag Mech. \\
\hline$* 0$ & Annie & 3A. TayHeFDAD & Madera & $\mathrm{CA}$ & 93638 & 1 & 09 & $\mathrm{~F}$ & & White & An. Science \\
\hline$* 0$ & Kaelan & & Madera & $\mathrm{CA}$ & 93638 & 1 & 09 & M & & White & Ag Mech. \\
\hline$* 0$ & Marlon & & Madera & $\mathrm{CA}$ & 93638 & 1 & 09 & M & & White & Ag Mech. \\
\hline$* 0$ & Maribel & & Madera & $\mathrm{CA}$ & 93638 & 1 & 11 & $\mathrm{~F}$ & $X$ & White & O.H. \\
\hline$* 0$ & Jose & 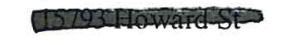 & Madera & $\mathrm{CA}$ & 93638 & 1 & 09 & M & $X$ & White & Ag Mech. \\
\hline * 600060074 & Iris & & Madera & $\mathrm{CA}$ & 93637 & 3 & 11 & F & $X$ & White & Agriscience \\
\hline * 600845987 & Araceli & & Madera & $\mathrm{CA}$ & 93637 & 2 & 10 & $\mathrm{~F}$ & $\mathrm{X}$ & White & An. Science \\
\hline * 600060076 & Drivi & Eystreet & Madera & $\mathrm{CA}$ & 93638 & 3 & 11 & F & $X$ & White & An. Science \\
\hline * 600597352 & Marcos & & Madera & $\mathrm{CA}$ & 93638 & 2 & 10 & M & $\mathrm{X}$ & White & Ag Mech. \\
\hline$* 0$ & Enayjah & $\mathrm{Se}$ & Madera & $\mathrm{CA}$ & 93638 & 1 & 09 & $\mathrm{~F}$ & $X$ & White & An. Science \\
\hline * 553557428 & Jaime & assent & Madera & $\mathrm{CA}$ & 93638 & 4 & 12 & M & $X$ & Am. Ind. & Ag Mech. \\
\hline * 553360380 & Jessica & & Madera & $\mathrm{CA}$ & 93638 & 5 & 13 & $\mathrm{~F}$ & $X$ & 2 or More & An. Science \\
\hline *600597355 & Eduardo & & Madera & $\mathrm{CA}$ & 93638 & 2 & 10 & M & $\mathrm{X}$ & White & Ag Mech. \\
\hline$* 0$ & Valentin & & Madera & $\mathrm{CA}$ & 93638 & 1 & 09 & M & $\mathrm{X}$ & White & Ag Mech. \\
\hline * 600597358 & Evelyn B & & Madera & $\mathrm{CA}$ & 93638 & 2 & 10 & $\mathrm{~F}$ & $\mathrm{X}$ & White & Ag Mech. \\
\hline$* 600597360$ & Alexis & & Madera & $\mathrm{CA}$ & 93638 & 2 & 10 & M & $\mathrm{X}$ & White & Ag Mech. \\
\hline$* 600597361$ & Elias I & & Madera & $\mathrm{CA}$ & 93638 & 2 & 10 & M & $\mathrm{X}$ & White & O.H. \\
\hline$* 0$ & Stephanie & & Madera & $\mathrm{CA}$ & 93638 & 1 & 11 & $\mathrm{~F}$ & $\mathrm{X}$ & White & O.H. \\
\hline$* 0$ & Gabino & & Madera & CA & 93638 & 1 & 09 & M & $\mathrm{X}$ & White & Ag Mech. \\
\hline$* 0$ & Joshua & 49 & Madiera & $\mathrm{CA}$ & 93637 & 1 & 09 & M & & White & A@ Mech. \\
\hline$* 0$ & Karen & (174) & Madera & $\mathrm{CA}$ & 93638 & 1 & 10 & F & $X$ & White & O.H. \\
\hline 533557435 & Ricardo & $\rightarrow$ & Madela & CA & 93638 & 4 & 12 & M & X & 2 or More & Ig Mech. \\
\hline$* 11$ & Mallhew & 08010 (f) & Minfera & $\mathrm{CA}$ & 93638 & 1 & (0) & M & $x$ & White & deriscknce \\
\hline$\Rightarrow: 3,57437$ & Myguel & $\Rightarrow$ & Madera & CA & 93638 & +4 & 12 & M & $x$ & Am. Ind. & Ig Mech \\
\hline 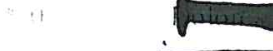 & Vatentul & & Yh⿻ 1 & $\mathrm{CA}$ & 93038 & 1 & (1) 5 & M & $x$ & White & Men \\
\hline$\cdots$ & Kinclynn & 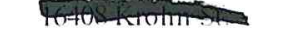 & $\because n+\cdots !$ & $\mathrm{CA}$ & 93638 & 1 & (6) & F & $x$ & White & 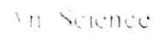 \\
\hline
\end{tabular}




\begin{tabular}{|c|c|c|c|c|c|c|c|c|c|c|c|}
\hline * 553557440 & Michael & 190mrean Streat & Madera & $\mathrm{CA}$ & 93638 & 4 & 12 & M & $\mathrm{X}$ & White & Ag Mech. \\
\hline * 553557441 & Vanessa & gorterims atre & Madera & $\mathrm{CA}$ & 93638 & 4 & 12 & $\mathrm{~F}$ & $\mathrm{x}$ & White & An. Science \\
\hline$* 0$ & Bryce & ous.BSined & Madera & $\mathrm{CA}$ & 93638 & 1 & 09 & M & & White & Ag Mech. \\
\hline * 553557444 & Michael & & Madera & $\mathrm{CA}$ & 93638 & 4 & 12 & M & $\mathrm{X}$ & Am. Ind. & Ag Mech. \\
\hline *600597371 & Ernesto & Bita & Madera & $\mathrm{CA}$ & 93638 & 2 & 11 & M & $\mathrm{x}$ & White & Ag Mech. \\
\hline * 553557445 & Luis & 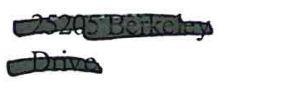 & Madera & $\mathrm{CA}$ & 93638 & 4 & 12 & M & $\mathrm{X}$ & 2 or More & An. Science \\
\hline *600060087 & Justine & & Madera & $\mathrm{CA}$ & 93638 & 3 & 11 & M & $x$ & White & An. Science \\
\hline * 600060089 & Brenda & 495 & Madera & $\mathrm{CA}$ & 93637 & 3 & 11 & M & $\mathrm{X}$ & White & $\begin{array}{l}\text { Plant/Soil } \\
\text { Sci. }\end{array}$ \\
\hline * 600597374 & Raemon A & 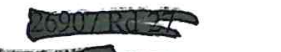 & Madera & $\mathrm{CA}$ & 93638 & 2 & 10 & M & $X$ & White & Ag Mech. \\
\hline * 600597378 & Michael L & & Madera & $\mathrm{CA}$ & 93638 & 3 & 11 & M & & White & Ag Mech. \\
\hline$* 0$ & Brandon & eorsandar & Madera & $\mathrm{CA}$ & 93637 & 1 & 09 & M & $X$ & White & Agriscience \\
\hline * 600060095 & Angel & misstrees & Madera & $\mathrm{CA}$ & 93638 & 3 & 11 & M & $\mathrm{X}$ & White & Ag Mech. \\
\hline * 600597384 & Angel E & & Madera & $\mathrm{CA}$ & 93638 & 2 & 12 & M & $\mathrm{x}$ & Am. Ind. & Ag Mech. \\
\hline * 600060097 & Cesar & Q6 220 anting Streeth & Madera & $\mathrm{CA}$ & 92638 & 3 & 11 & M & $\mathrm{X}$ & White & Ag Mech. \\
\hline *600060098 & Christopher & & Madera & $\mathrm{CA}$ & 93637 & 3 & 11 & M & $\mathrm{X}$ & White & Ag Mech. \\
\hline$* 0$ & Jenna & & Madera & $\mathrm{CA}$ & 93638 & 1 & 10 & $\mathrm{~F}$ & $\mathrm{X}$ & White & O.H. \\
\hline$* 0$ & Jose & & Madera & $\mathrm{CA}$ & 93638 & 1 & 09 & M & $X$ & White & $\begin{array}{l}\text { Plant/Soil } \\
\text { Sci. }\end{array}$ \\
\hline * 553360399 & Matthew C & 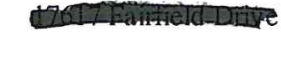 & Madera & $\mathrm{CA}$ & 93638 & 5 & 13 & M & $X$ & 2 or More & Ag Mech. \\
\hline * 600060100 & Michael & Iitherstreets & Madera & $\mathrm{CA}$ & 93638 & 2 & 11 & M & $\mathrm{X}$ & White & Ag Mech. \\
\hline$* 0$ & Roman & 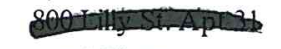 & Madera & $\mathrm{CA}$ & 93638 & 1 & 09 & M & $X$ & White & Ag Mech. \\
\hline$* 0$ & Sergio & & Madera & $\mathrm{CA}$ & 93638 & 1 & 09 & M & $\mathrm{X}$ & White & Ag Mech. \\
\hline$* 0$ & Deward & & Madera & $\mathrm{CA}$ & 93638 & 1 & 09 & M & $X$ & 2 or More & Agriscience \\
\hline$* 0$ & Juan & tra & Madera & $\mathrm{CA}$ & 93638 & 1 & 09 & M & $X$ & White & An. Science \\
\hline * 600060103 & Jose & & Madera & $\mathrm{CA}$ & 93638 & 3 & 11 & M & $\mathrm{X}$ & White & Ag Mech. \\
\hline * 600597390 & Katia J & & Madera & $\mathrm{CA}$ & 93638 & 3 & 11 & $\mathrm{~F}$ & $X$ & White & An. Science \\
\hline * 600597393 & Katheryne & & Madera & $\mathrm{CA}$ & 93638 & 2 & 10 & $\mathrm{~F}$ & & White & An. Science \\
\hline * 553557458 & Eleno & & Madera & $\mathrm{CA}$ & 93638 & 4 & 12 & M & $\mathrm{X}$ & White & Ag Mech. \\
\hline * 553557459 & Jacqueline & & Madera & $\mathrm{CA}$ & 93638 & 3 & 12 & $\mathrm{~F}$ & $\mathrm{x}$ & White & An. Science \\
\hline$* 0$ & Amaranta & & Madera & $\mathrm{CA}$ & 93638 & 1 & 10 & $F$ & $\mathrm{x}$ & White & $\mathrm{O} . \mathrm{H}$ \\
\hline$* 0$ & [Danicl & & Madera & $\mathrm{CA}$ & 93637 & 1 & (11) & M & $x$ & White & Ag Mech. \\
\hline * 600060108 & Richard & & Madera & $\mathrm{CA}$ & 93637 & : & 1 & M & $x$ & White & Ag Mech. \\
\hline$* 0$ & De:ania & & Madera & $\mathrm{CA}$ & 93638 & 1 & (n) & F & $x$ & White & An. Science \\
\hline$* 0$ & Kandion & $-2=601$ & Madera & $\mathrm{CA}$ & 93638 & 1 & (in) & M & & White & Ag Mech. \\
\hline$* 0$ & Thratidro & & Madera & $\mathrm{CA}$ & 93638 & & $\cdots$ & $M$ & $x$ & White & Agriscience \\
\hline$* 6(00597400$ & Whom & $\Rightarrow+2$ & Madera & $\mathrm{CA}$ & 93638 & & & 1 & $x$ & White & An. Science \\
\hline (n) $(0597401$ & $\operatorname{Hin}$ & WW & Vadera & $C A$ & 93638 & & & $\because$ & $x$ & White & Ag Mech. \\
\hline 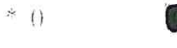 & 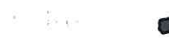 & 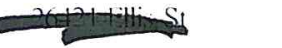 & Vadca & $C \wedge$ & 936.8 & & & U & $x$ & White & Ag Mech. \\
\hline
\end{tabular}




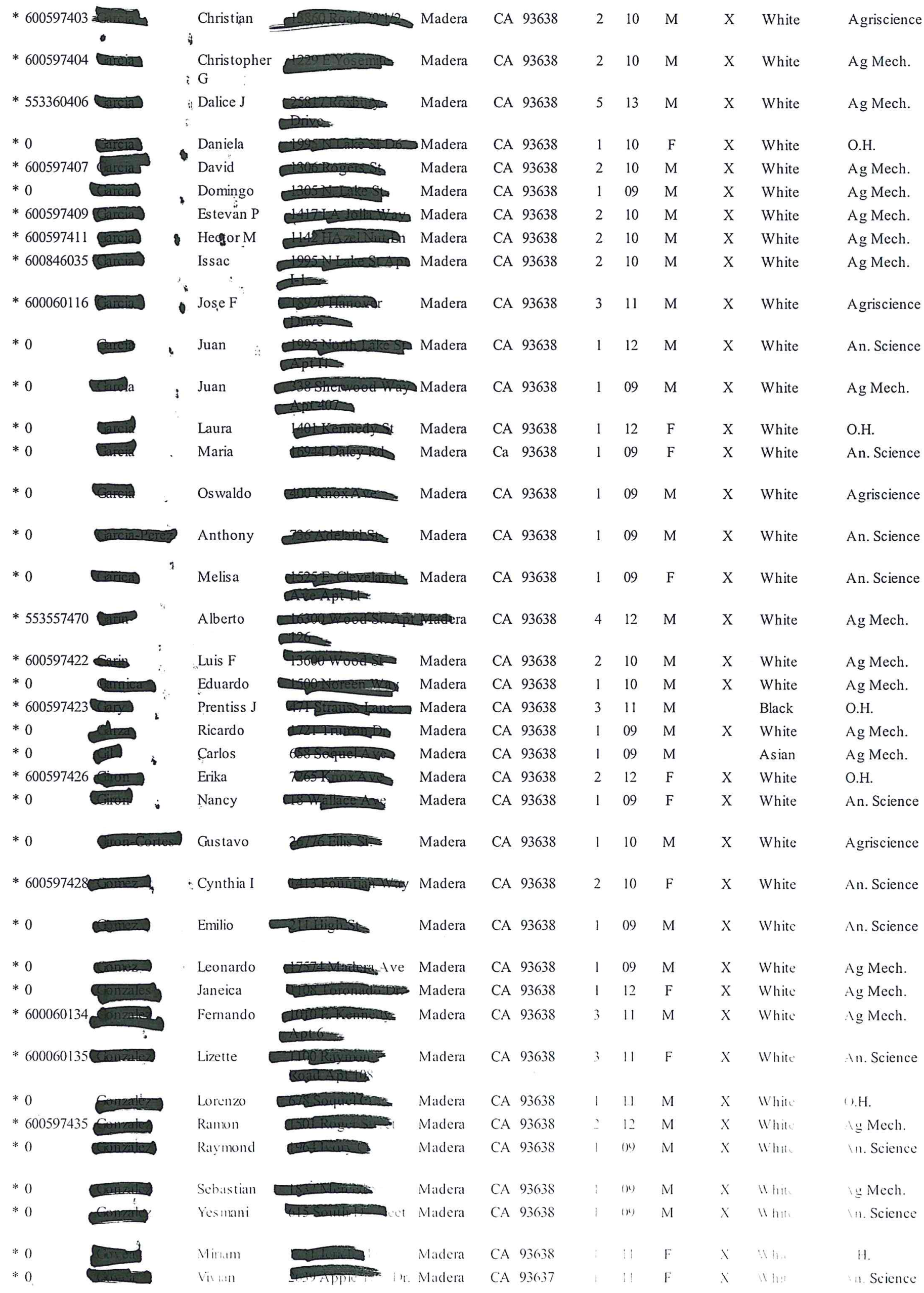




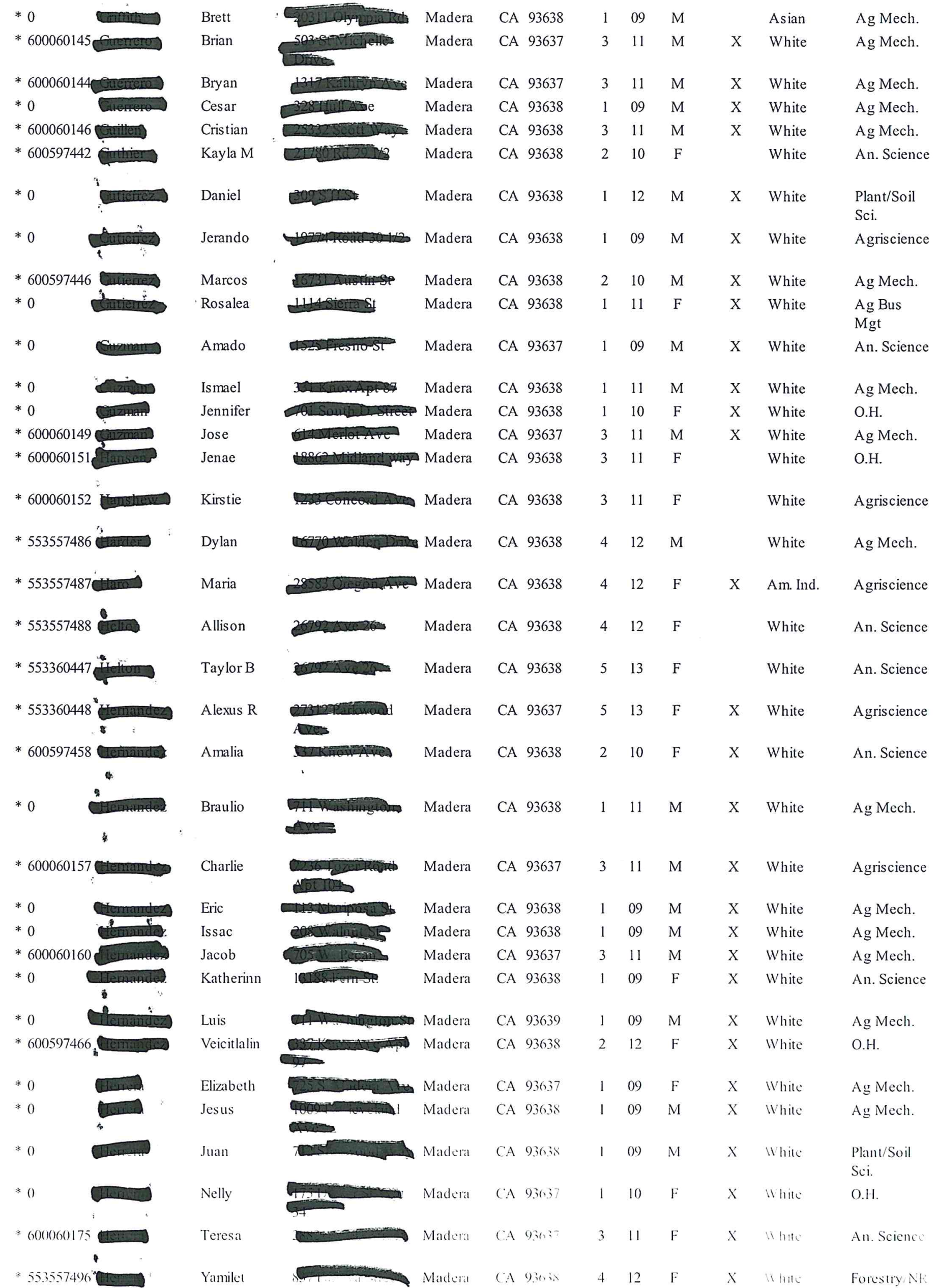




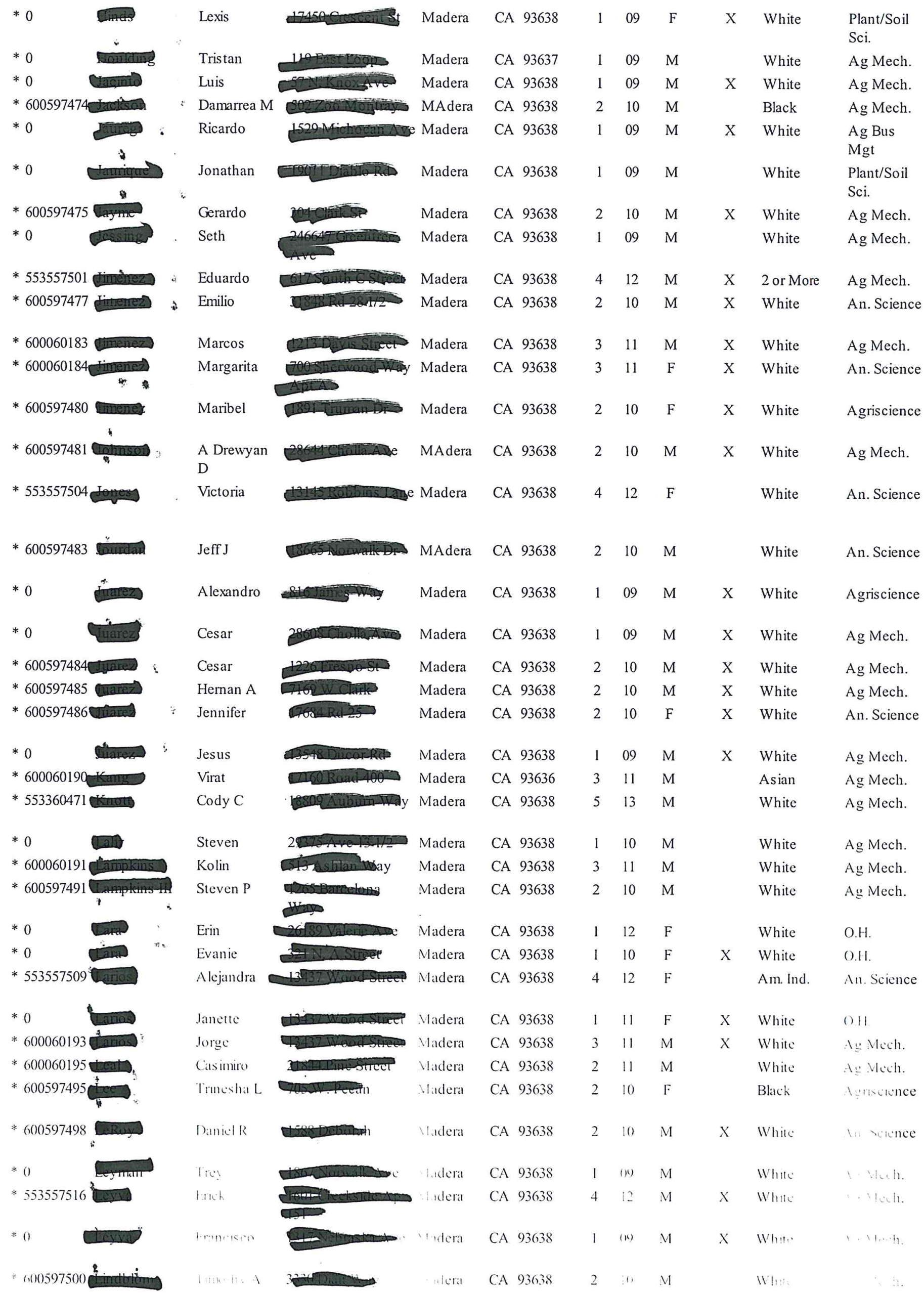




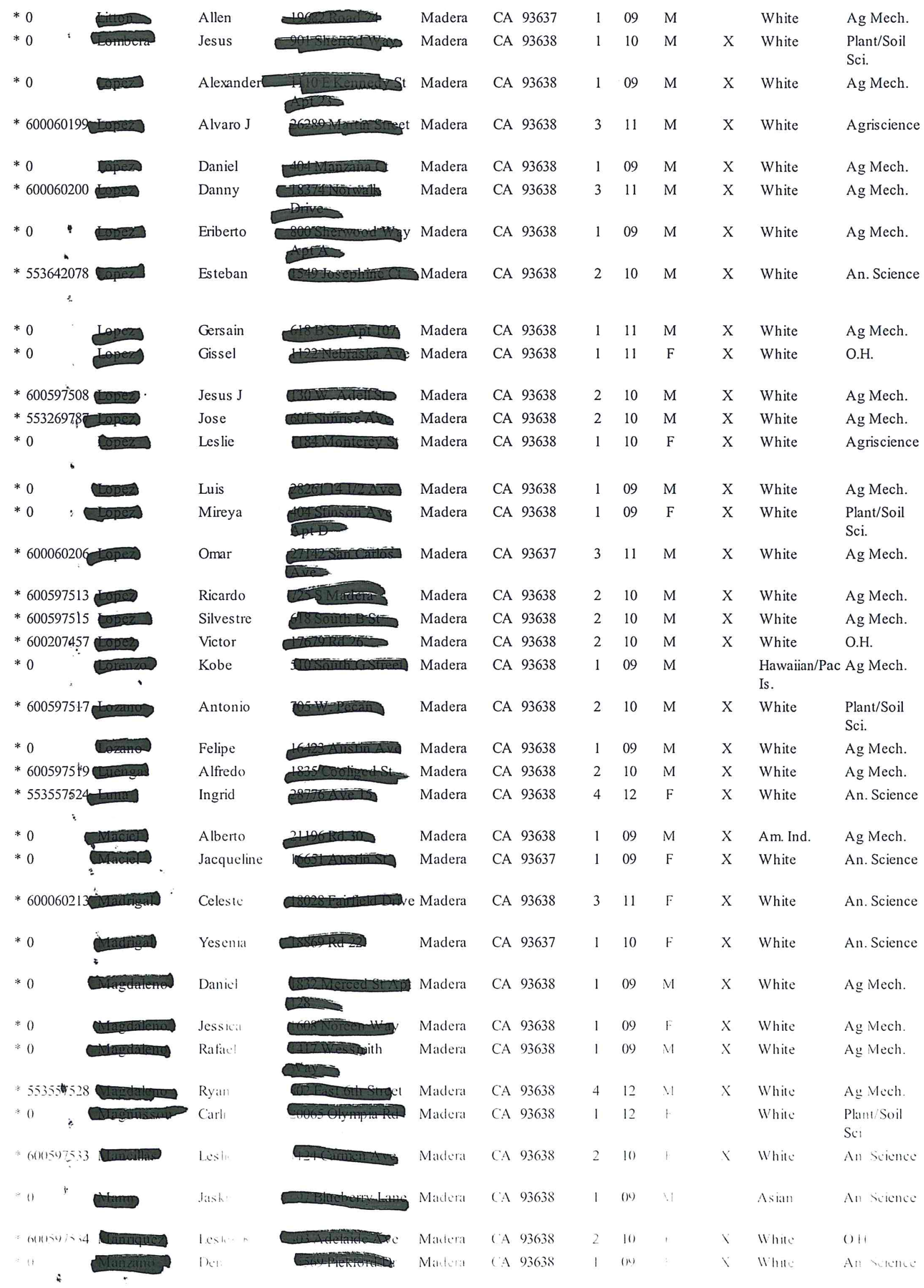




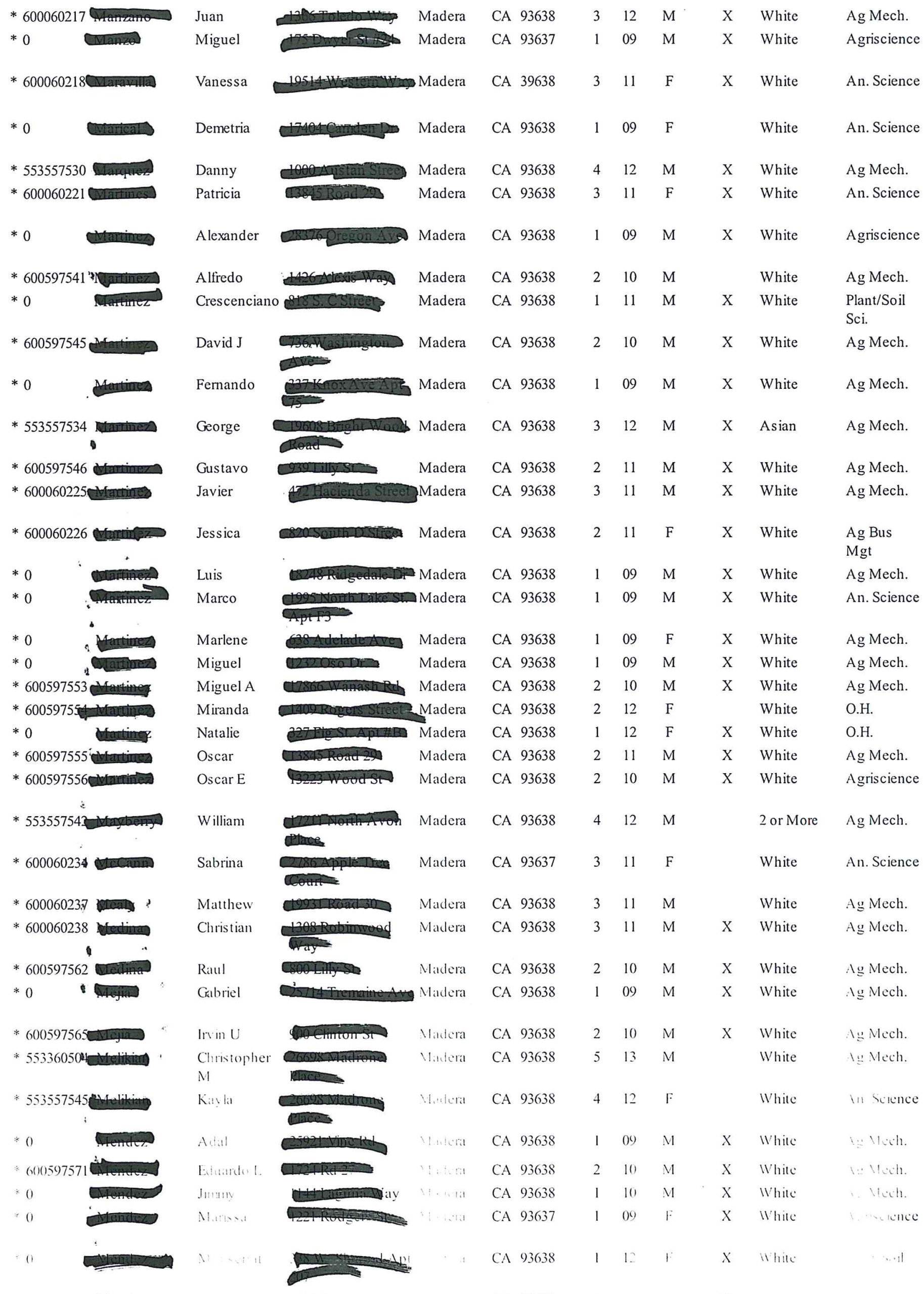




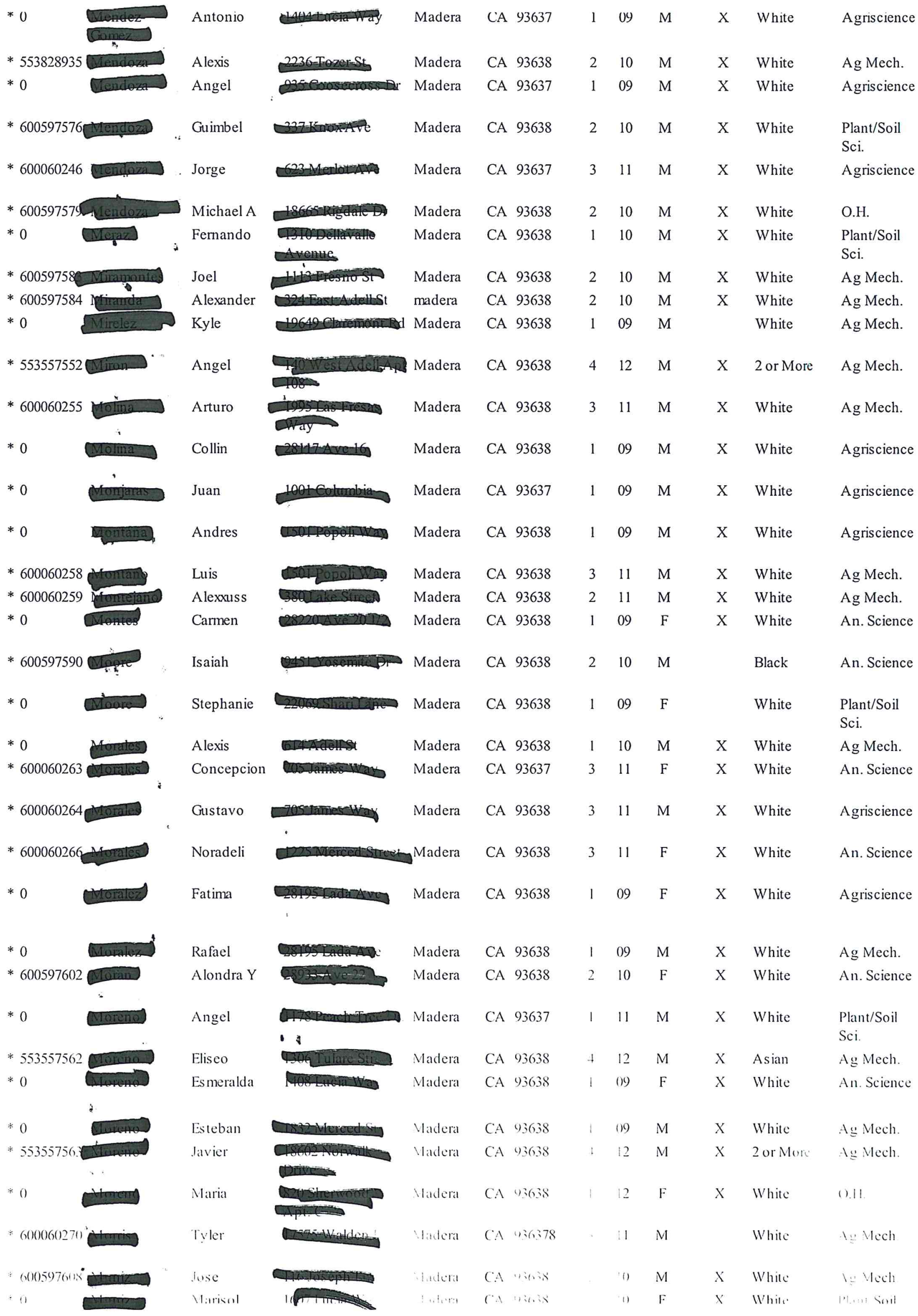




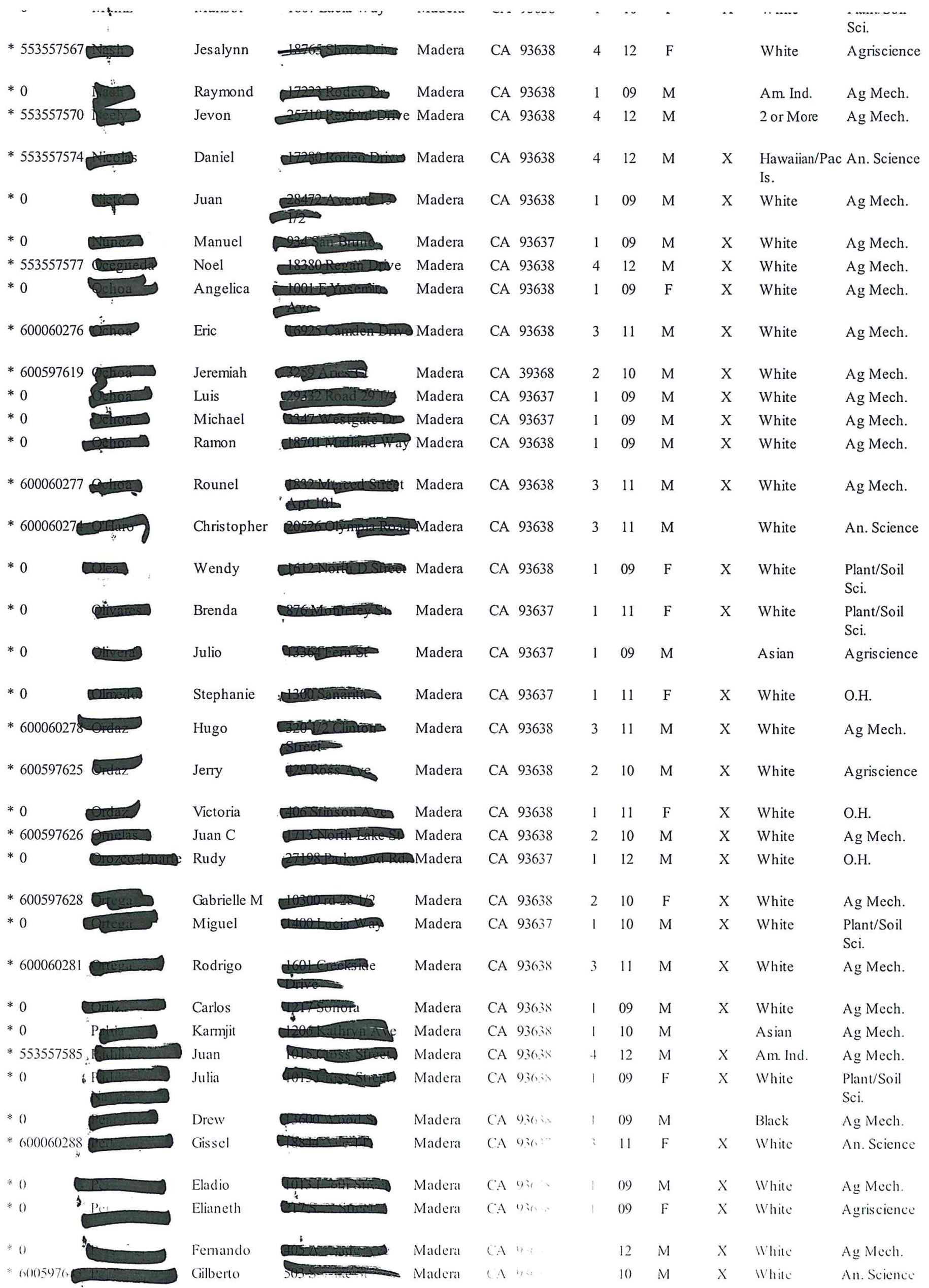




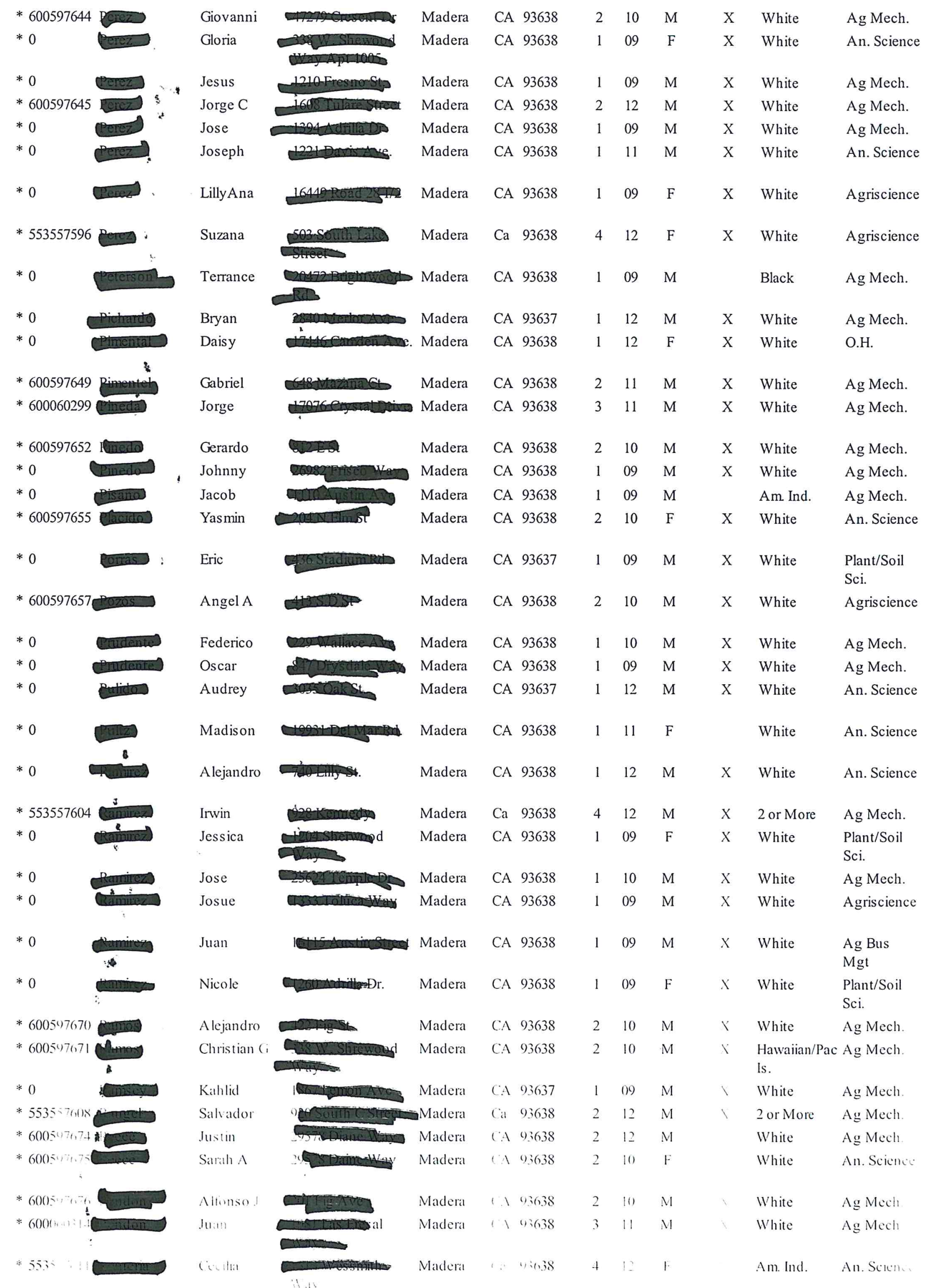




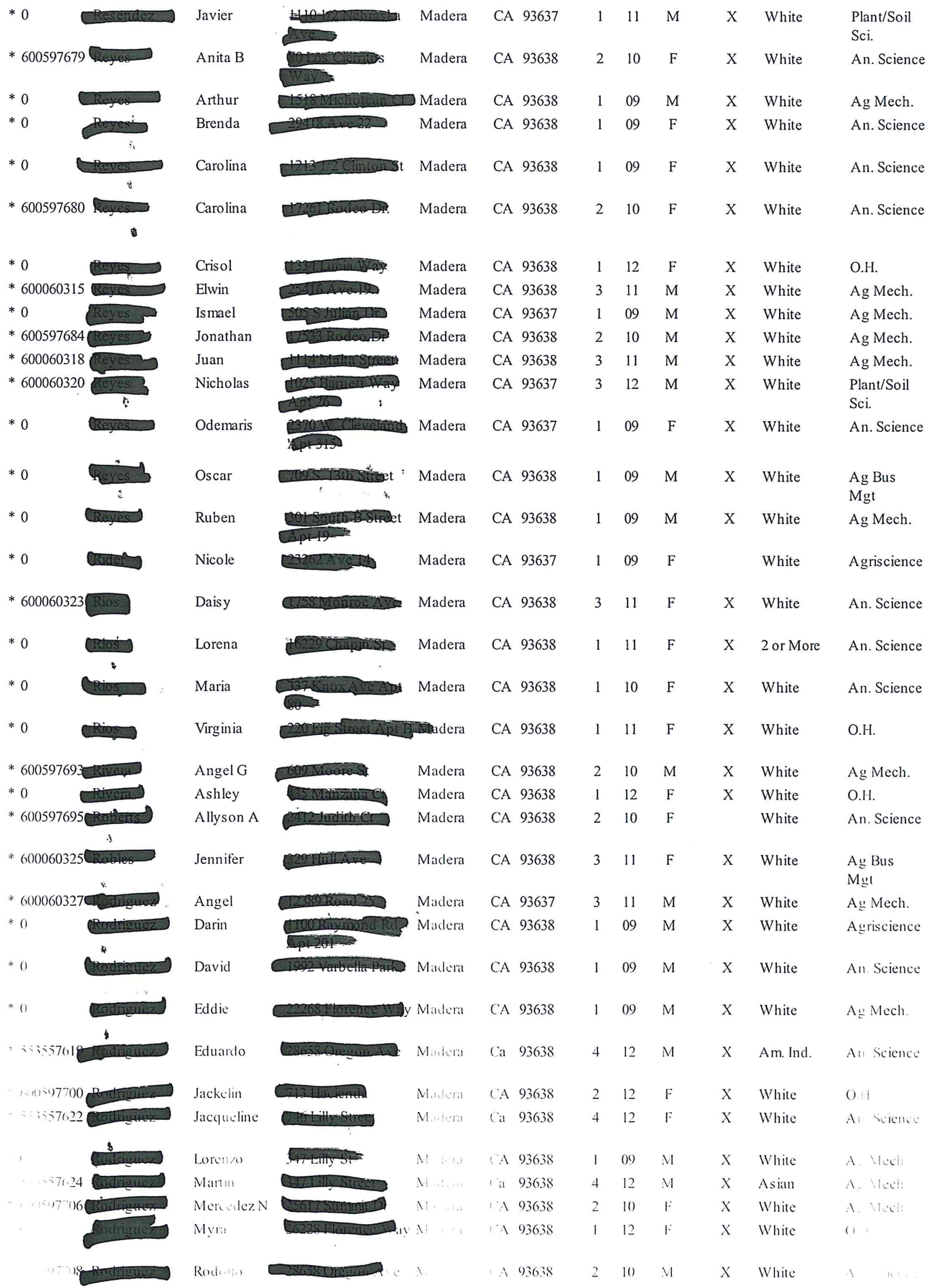




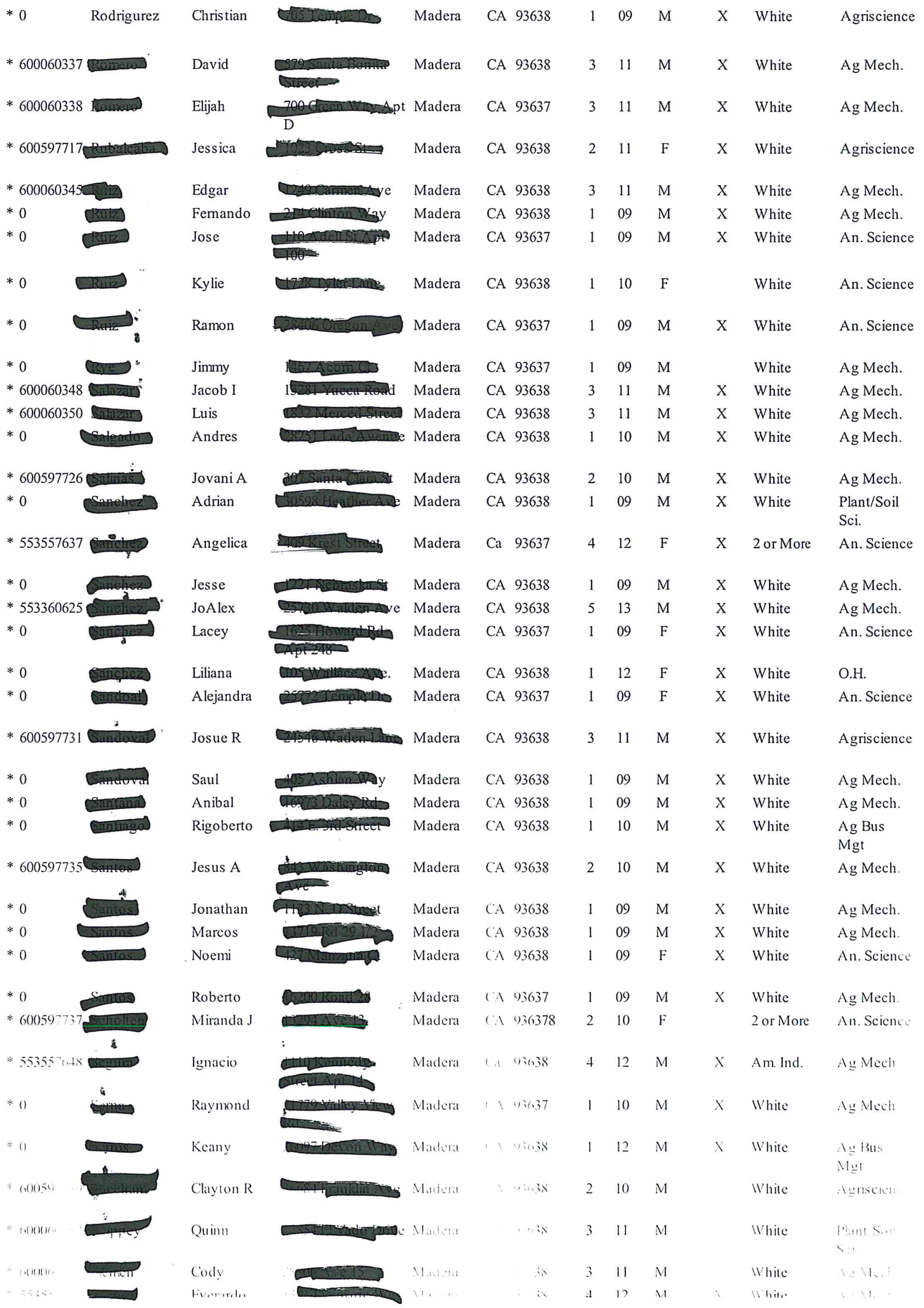




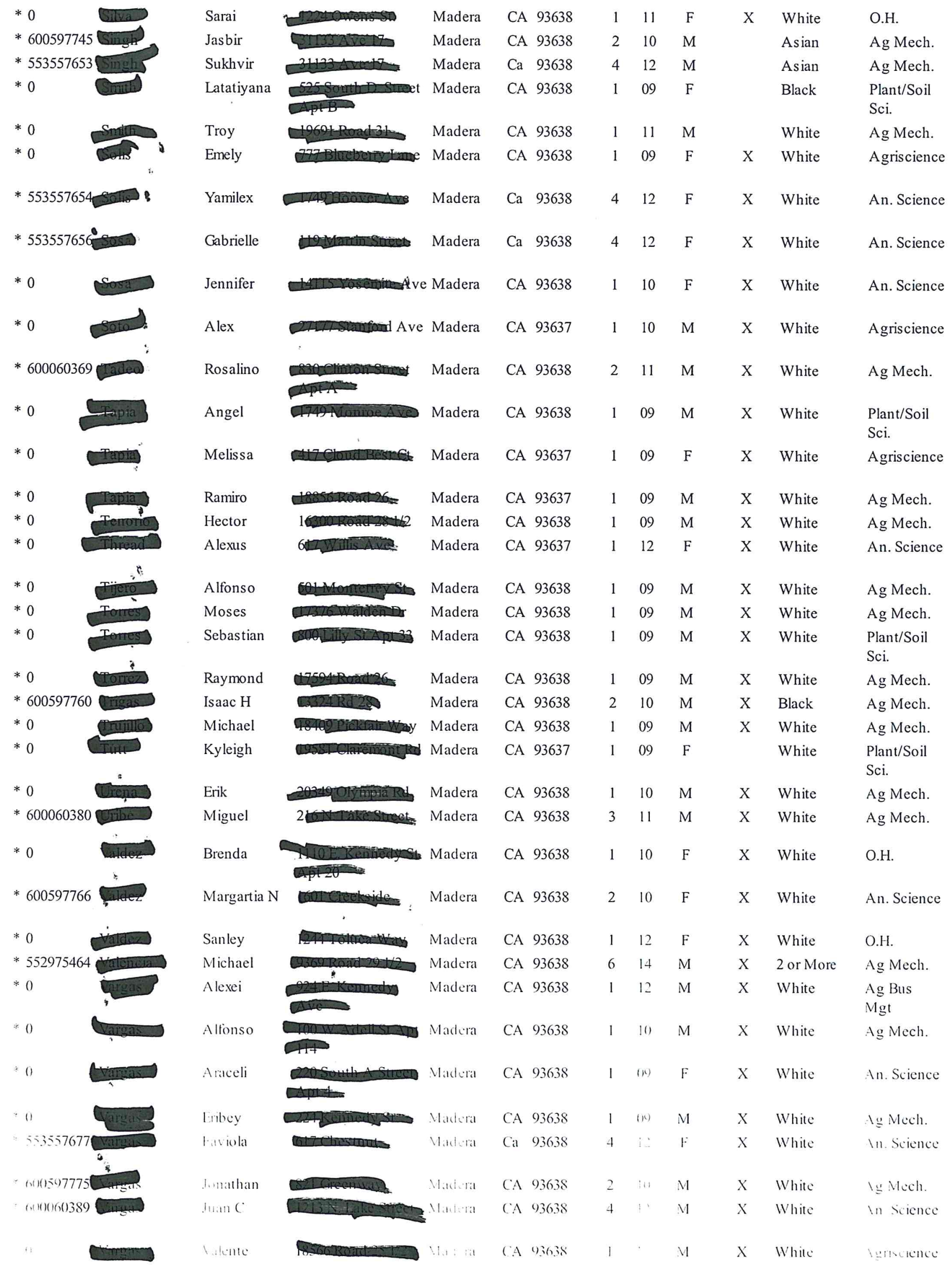




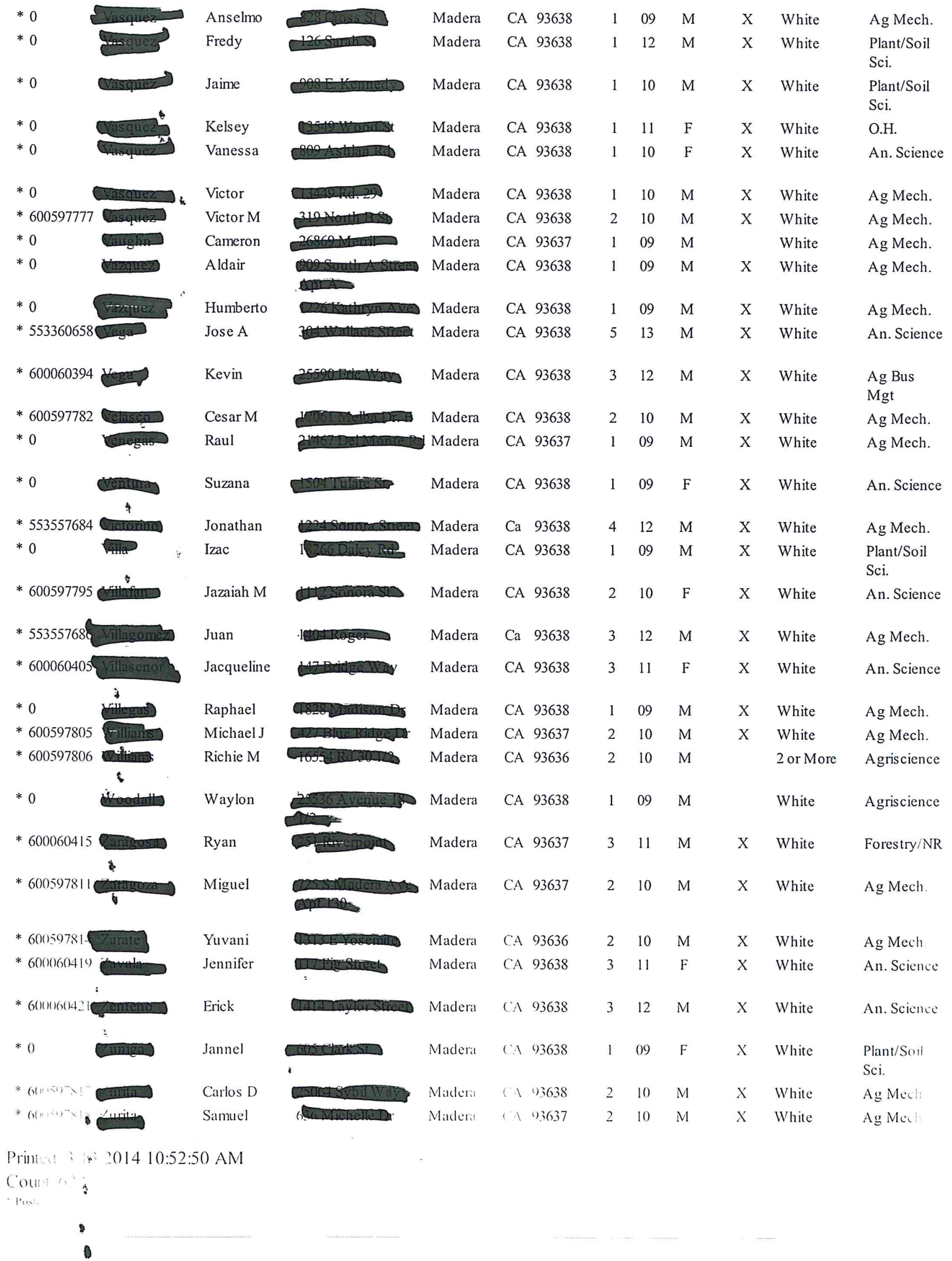

Site developed and maintained by the 


\section{Y. Copy Of extended Contract Rationale/Justification Statement Which Is On File With The Board}




\section{Madera South High School \\ Agriculture Department \\ Extended Contract Duties}

\section{Supervised Agriculture Experience Projects, Career Development Events, and FFA Responsibilities:}

1. Advise student projects in Swine, Sheep, Goats, Beef Cattle, Dairy Cattle, Horses, and Poultry.

a. Project visits at students home

b. Project visits at school farm

c. Supervise students work on the California Recordbook

d. Supervise students to ensure completion of buyers letters and thank you notes

e. Purchasing student projects from livestock producers around the state

f. Supervise students working with animals on the school farm

g. Supervise students at Madera Fair, Chowchilla Fair, State Fair, Cow Palace, and Jackpot shows.

h. Set-up equipment at the fair and removal of all equipment at the fair.

i. Operate school vehicles to transport animals and students.

j. Operate numerous farm vehicles and machinery to maintain and upkeep farm facilities.

k. Clean the pens or stalls in all facilities.

I. Treatment and care for sick or injured animals.

m. Perform proper animal restraint techniques to treat sick animals.

n. Purchase livestock feed for school farm projects and student projects kept at home.

o. Loading, hauling, and unloading grain, hay, and straw at school farm and at student's homes.

p. Be available for "on-call" emergencies of student projects at the school farm and at a student's home.

q. Trim animals hooves (sheep, goats, and horses)

r. Halter break steers and dairy heifers

s. Vaccinate, dehorn, and remove extra teats, worm, and ear tag.

t. Synchronize heifers so artificial breeding can take place.

u. Give riding lessons and take students on trail rides and to horse shows (horse projects only).

v. Supervise students in showmanship practice prior to showing at the fair.

w. Repair equipment associated with each animal unit (watering devices, feeders, gates, etc.).

x. Irrigation work, weed control, fly control, and trash cleanup for all farm areas around the animal barns. 
2. Advise student projects in agriculture mechanics, small engines, and ornamental horticulture

a. Completion of unfinished agriculture mechanics projects

b. Repair all shop equipment

i. Arc Welders

1. Disassemble \& Blow out all welders

2. Inspection of all welding cable, cleanups, electrode holders, and repair as needed.

3. Lubricate internal components to ensure ease of use

4. Basic mechanical \& working inspection of all machines

ii. MIG \& TIG Welders

1. Blow out all machines

2. Inspect MIG guns, welding cables, electrical components, regulators, MIG tips, nozzles, and replace items as needed

3. Inventory current shielding gas tanks and organize storage area

4. Inspect all welding cart tank restraints

5. Replace welding liners in MIG guns

6. Overall safety inspection \& working inspection of all machines

iii. Torches

1. Inspect all regulators, hoses, torch handles, carts, etc. for all safety hazards and function.

2. Replace parts as needed

iv. Power tools \& Equipment

1. Look over all tools for hazards, fix hazards such as cut extension cords, broken cord caps, malfunctioning shut off switches, ect.

v. Grinders

1. Inspect all guards

2. Replace broken/ worn out stones

3. Adjust all tool rest to safe standards

4. Inspect all electrical components

vi. Hand tools \& General Tools

1. Visual inspection of all tools

2. Repair items as needed

3. Organization of tool room

4. Replace broken/missing tools

c. Clean agriculture shops and clear all trash or unused equipment

d. Meets with students to plan projects for the upcoming year.

e. Meet with community members to secure projects for agriculture mechanic students

f. Assist students in the marketing of their projects 
g. Maintain safe facilities for summer student work to happen.

h. Assist students in job placement based on skill level.

i. Attend service schools to enhance classroom teaching.

j. Complete facility repairs

k. Load, haul, and unload scrap metal

I. Set up equipment for optimal and safe use by students

$m$. Clean and maintain the Horticulture unit and greenhouse

n. Irrigation work and repairs for greenhouse

o. Weed and pest control for greenhouse

3. Advise students with FFA responsibilities

a. Transport students to student leadership conferences.

b. Supervise students after school and on weekends at Judging contests around the state:

i. UC Davis

ii. CSU Chico

iii. CSU Fresno

iv. CSU, Cal Poly SLO

v. Reedley College

vi. Merced College

vii. Modesto Junior College

viii. West Hills College

c. Supervise students at judging team practices after school and on the weekends

d. Supervise students at monthly FFA meetings

e. Supervise students in the completion and preparation of Proficiency award applications

f. Supervise students in the completion and preparation of State and American Degree Awards

g. Planning and supervising students at State and National FFA Convention

h. Planning and supervising students at Officer Retreat.

i. Planning and supervising all fundraising activities in and out of school.

j. Planning and supervising of FFA banquets

k. Supervising students at all FFA activities during the week 


\section{Z. Copy Of Completed Travel Plan submitted To Administration/Board}




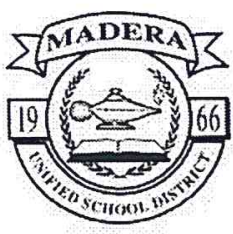

MADERA UNIFIED SCHOOL DISTRICT

1902 Howard Road a Madera, CA 93637

(559) 675-4500 o www.madera.k12.ca.us

\section{Student Out-of-Town • Overnight • Out-of-State Travel Request}

All out-of-town and overnight travel for school related events must be approved by the Madera Unified District governing board prior to travel.

\section{YOU MUST ATTACH A TRIP ITINERARY OR THIS REQUEST WILL NOT BE PROCESSED}

This form must be completed and submitted to the office of the Associate Superintendent of Educational Services prior to the cut-off date for submission of board agenda items for Cabinet review.

Incomplete request forms will be returned to the submitting party for completion.

School:

Class/team/organization:

Teacher/coach/director/advisor:

Activity/event: Location of activity/event:

Address:

Departure date:

Time:

Return date:

Time back at site:

Method of transportation:

(personal automobile; rental automobile; charter bus; school bus)

Total cost of transportation:

Transportation Special Instructions:

Lodging accommodations:

Total cost of lodging:

Total number of students traveling:

Males:

Females:

Number of chaperones:

Males:

Females:

All drivers and chaperones must have current fingerprint clearance on file with MUSD (AR 4122.2(a), AR 4222.2, BP 4122.2(a) and BP 4222.2) and must meet all requirements of MUSD regarding transportation of students (BP 3541.1).

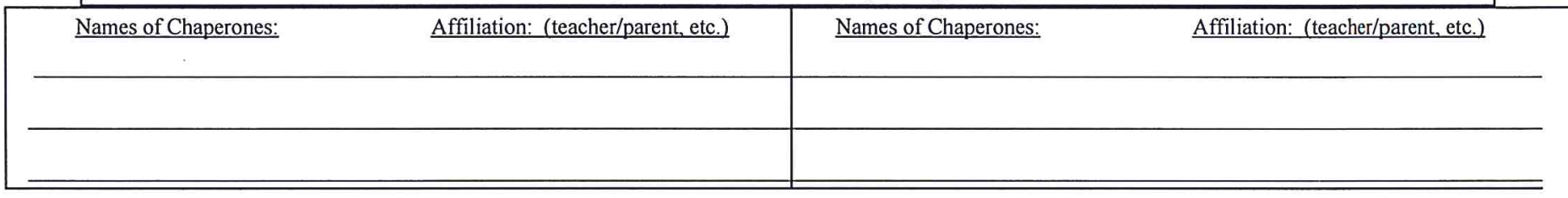

Describe the event/activity. Include how this event benefits students and how it supports the curriculum or extra-curricular activity. Justify why out-of-state travel is being requested, identify why an in-state activity could not provide the same level of benefit to students. Be complete and use an extra sheet of paper if necessary.

\section{BUDGET INFORMATION:}

Transportation to be paid by:

Lodging to be paid by:

Substitutes to be paid by:

\section{APPROVAL:}

Site Principal:

As site principal/administrator, I endorse this request and recommend this request be submitted to Cabinet

and the Board for consideration. By my signature I acknowledge that I have verified all drivers and

chaperones have current fingerprint clearance on file with MUSD (AR 4122.2(a), AR 4222.2,BP 4122.2(a)

and BP 4222.2) and have satisfied all requirements of MUSD regarding transportation of students (BP 3541.1)

Distribution: Original - Assoc. Superintendent of Education

1 Copy-Principal's Admin.. Asst.

I Copy-Originator
Date received by Associate Superintendent of Educational Services

Processed for Board Meeting date: 


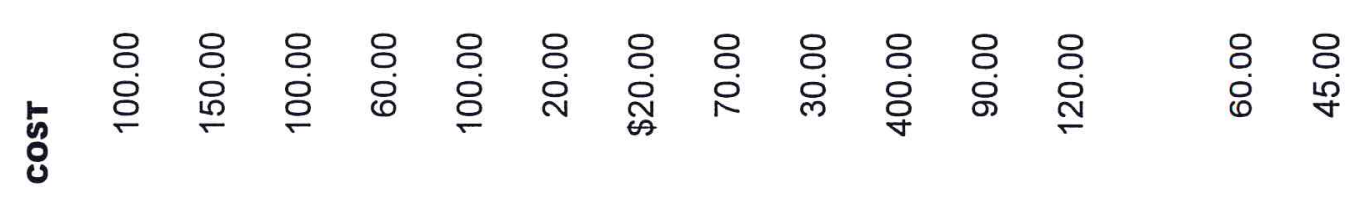

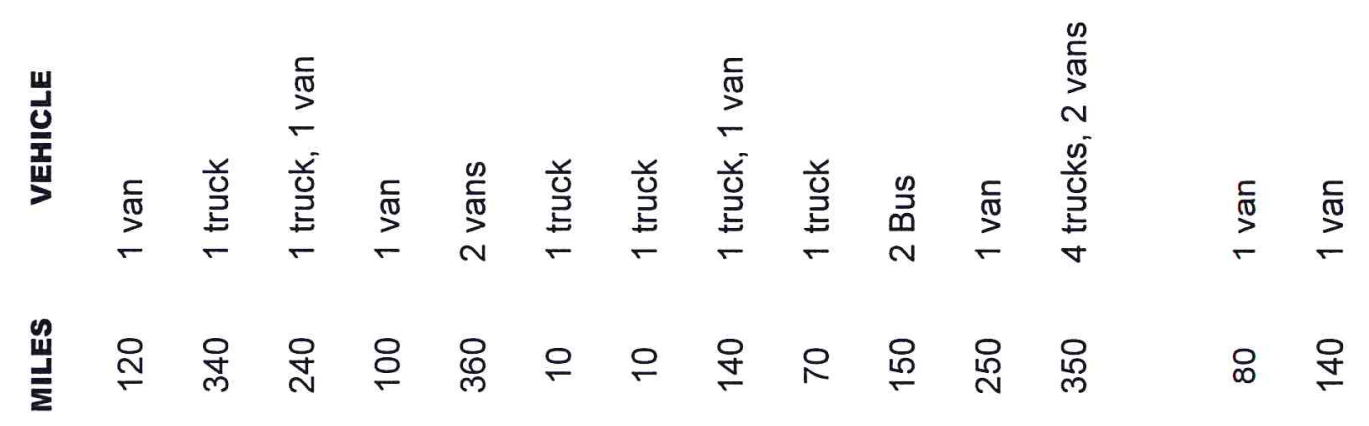

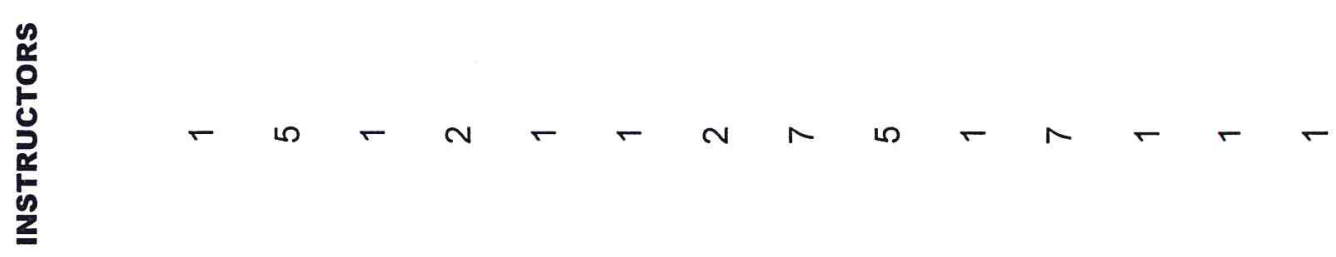

旁

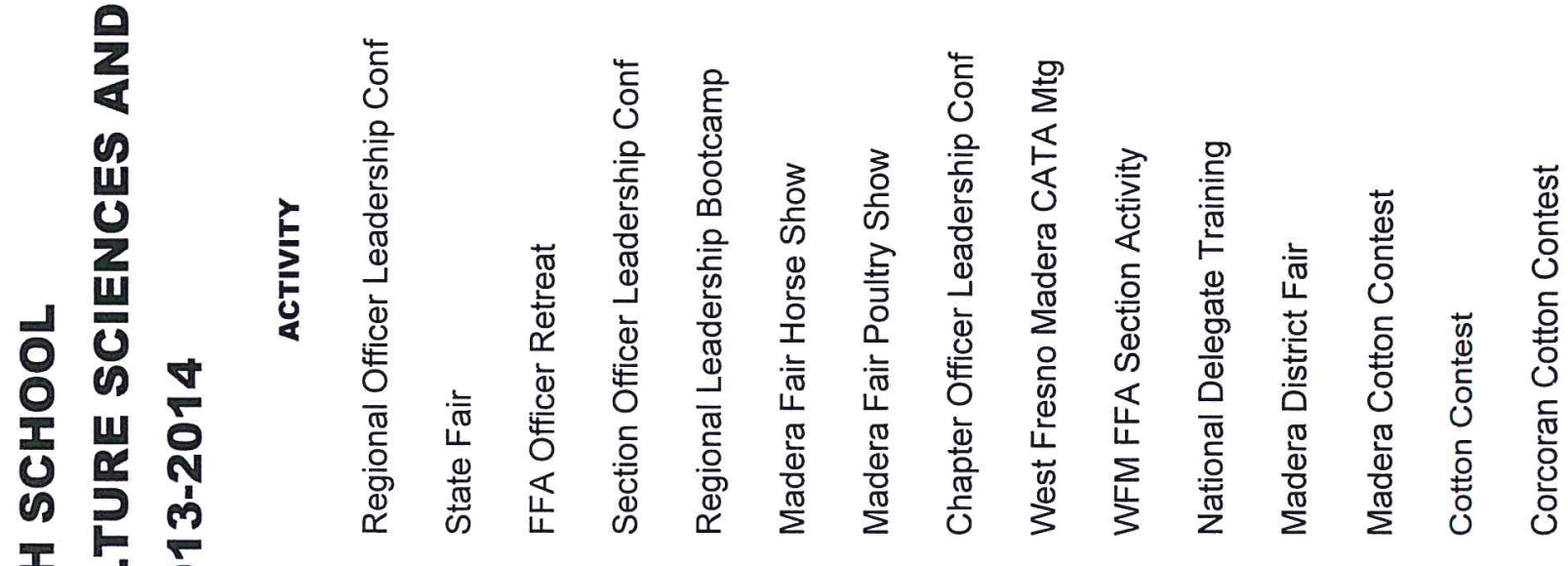

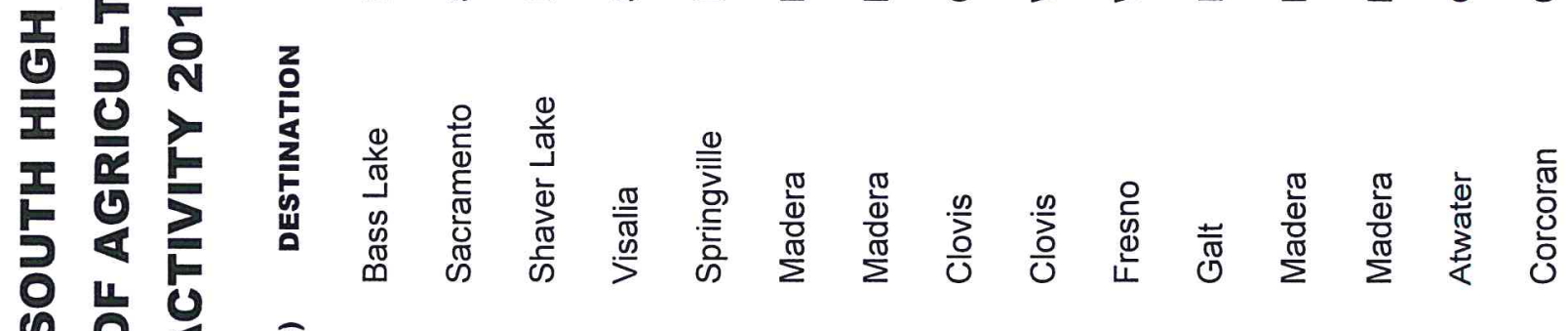

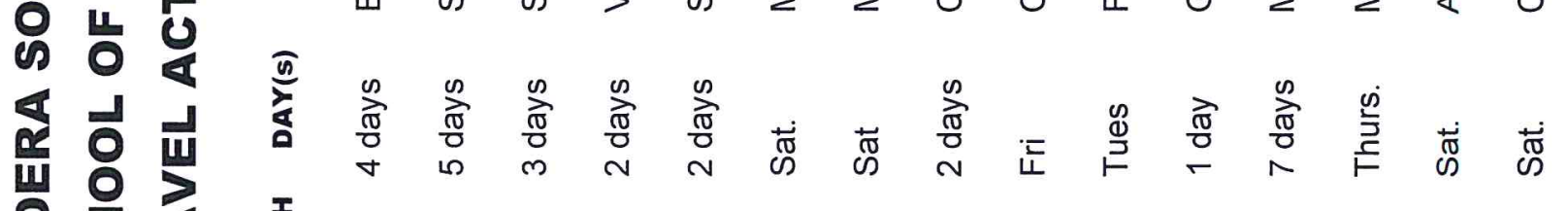

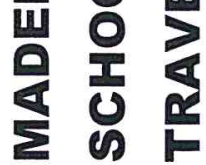

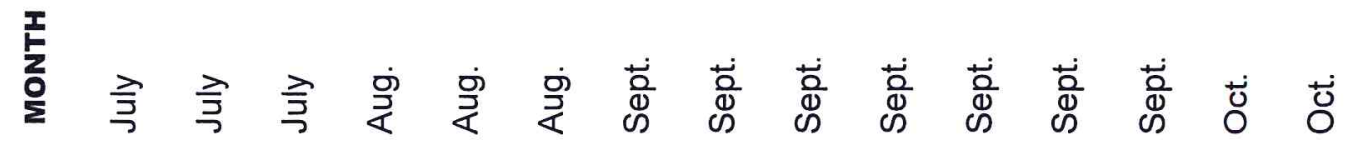




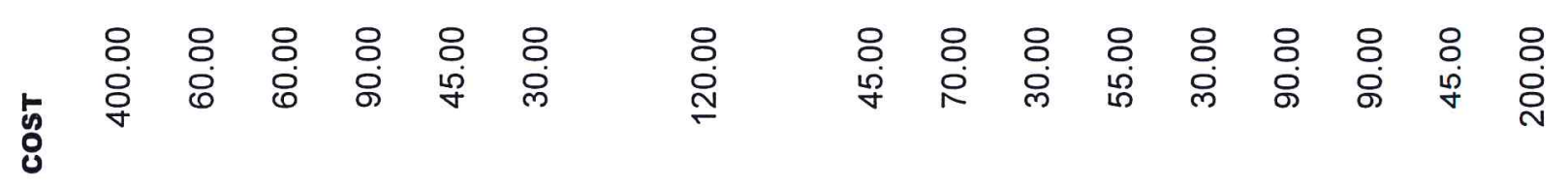

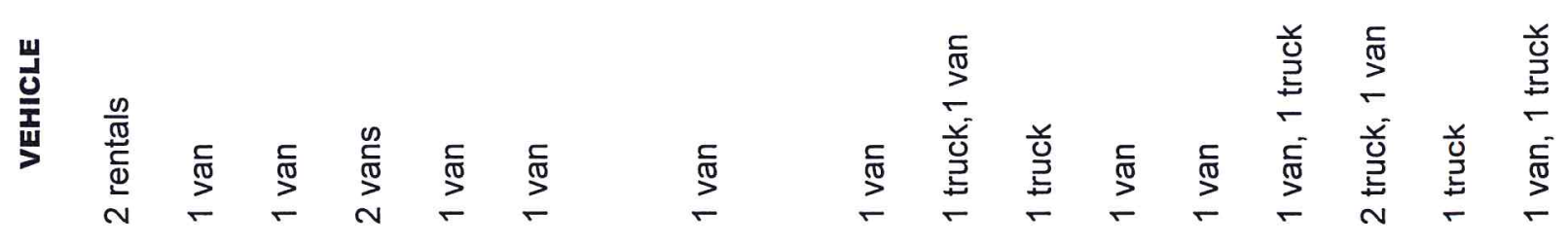

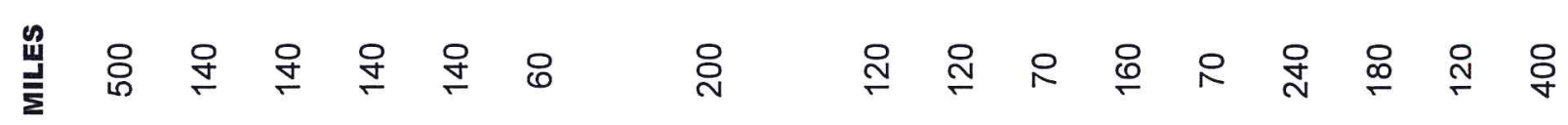

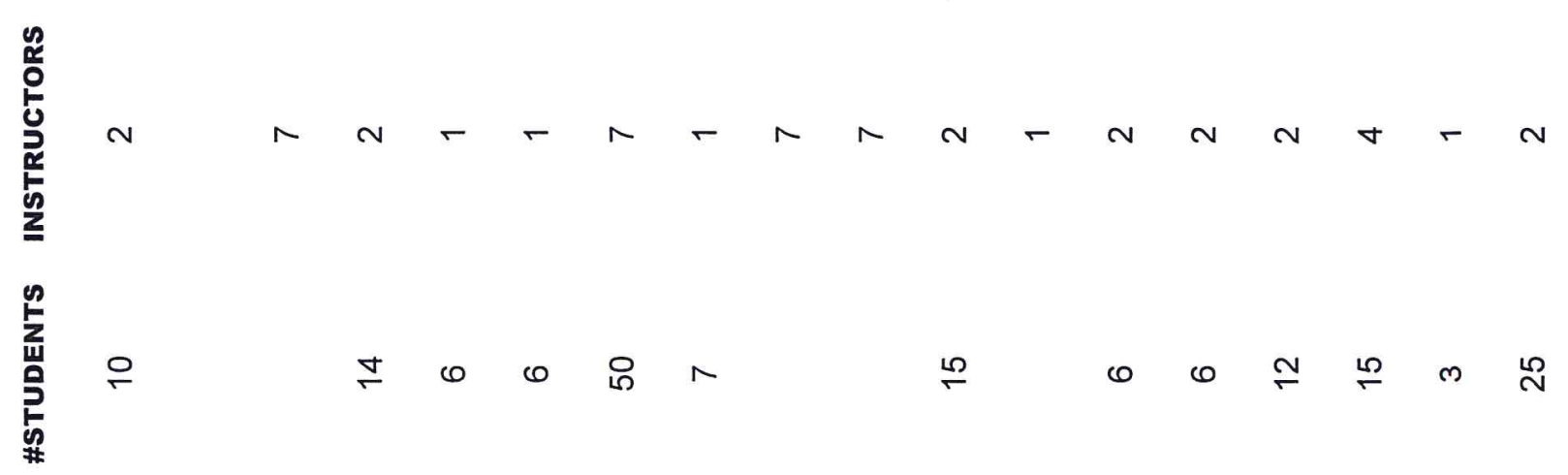

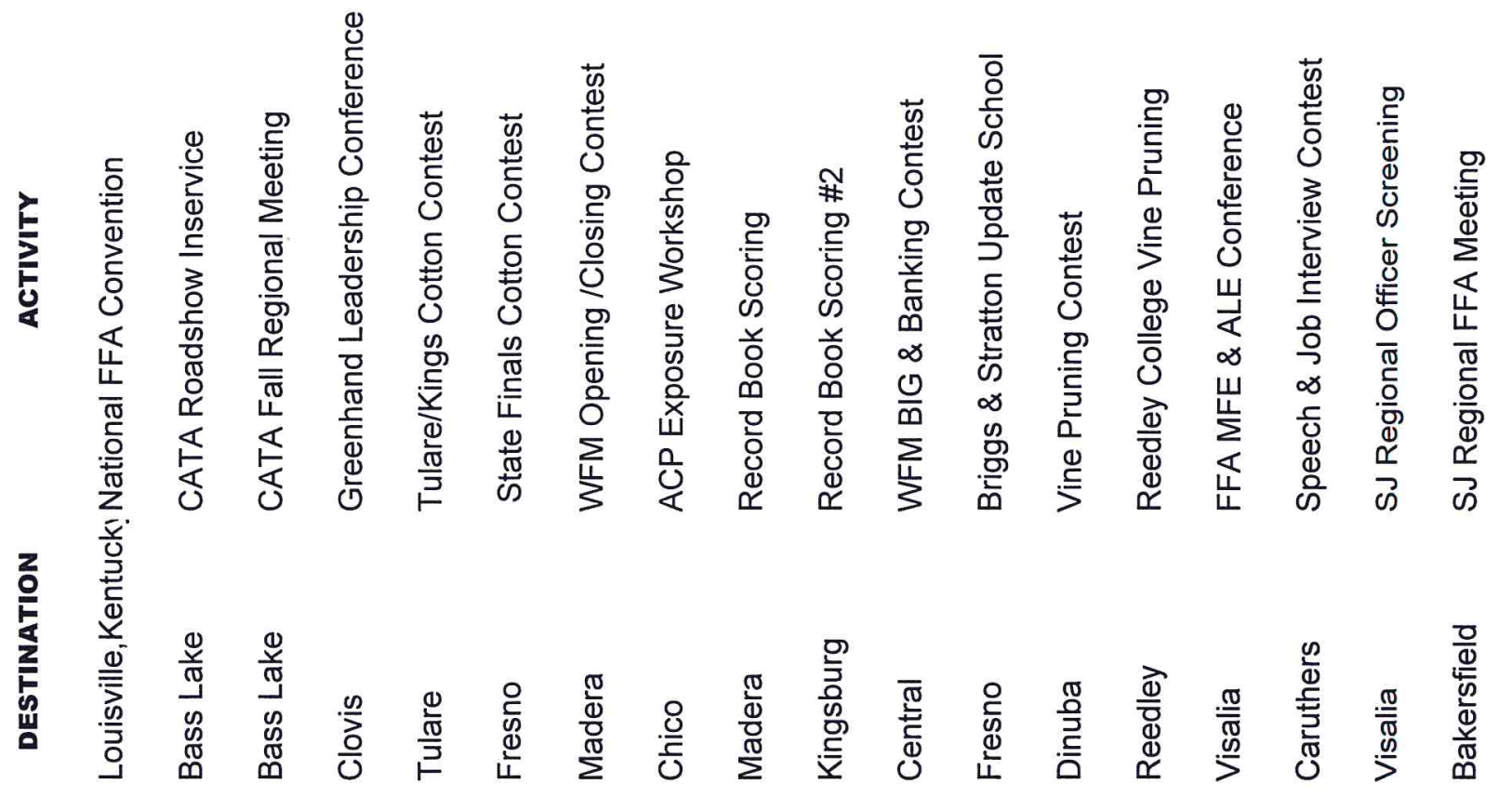

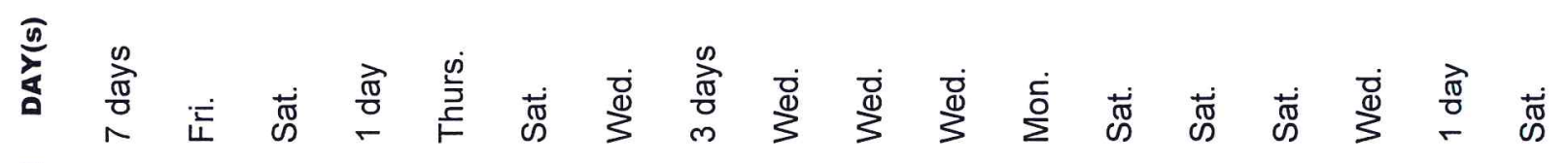

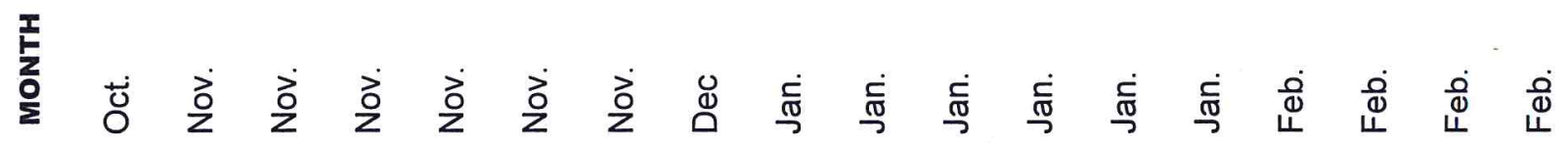




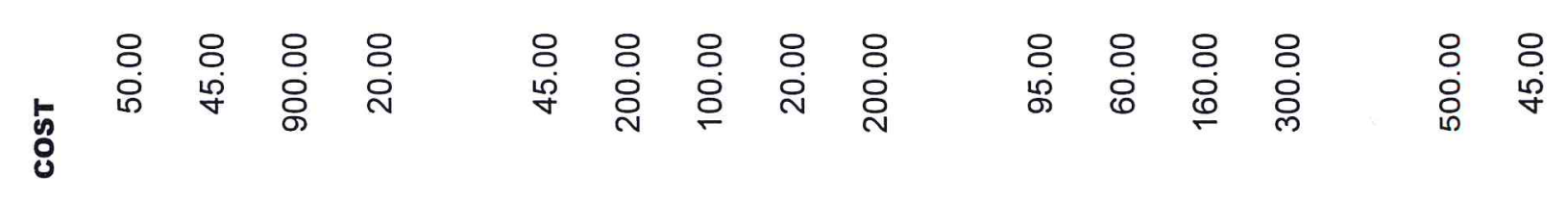

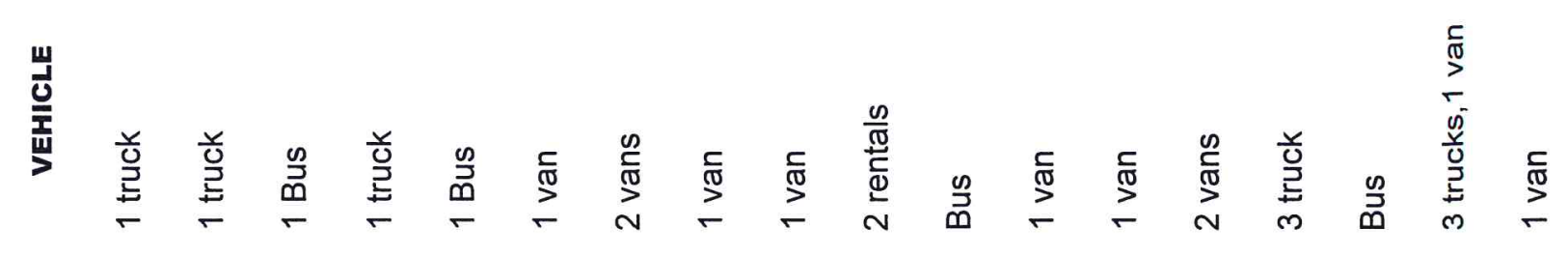

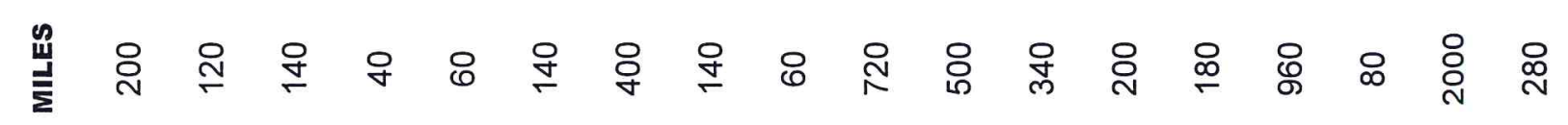

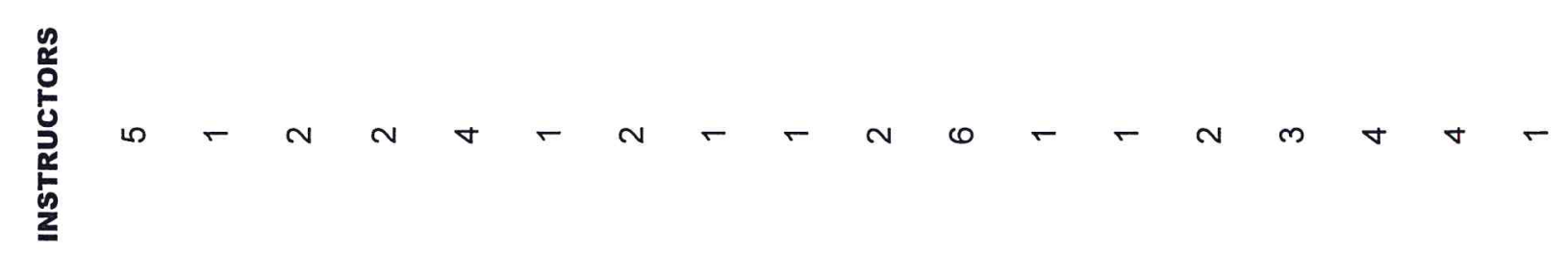

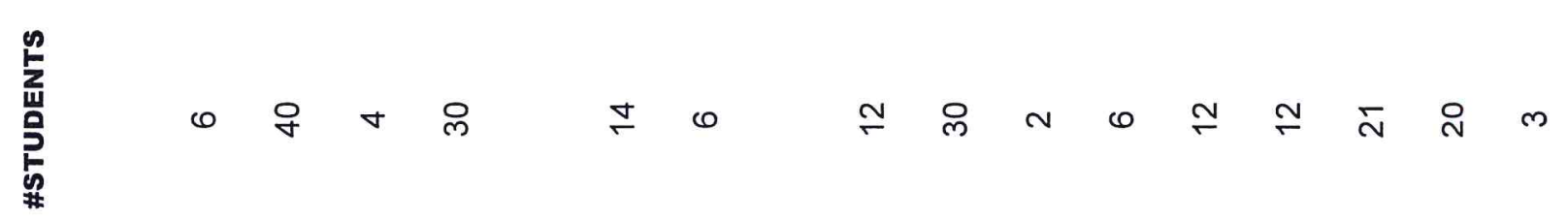

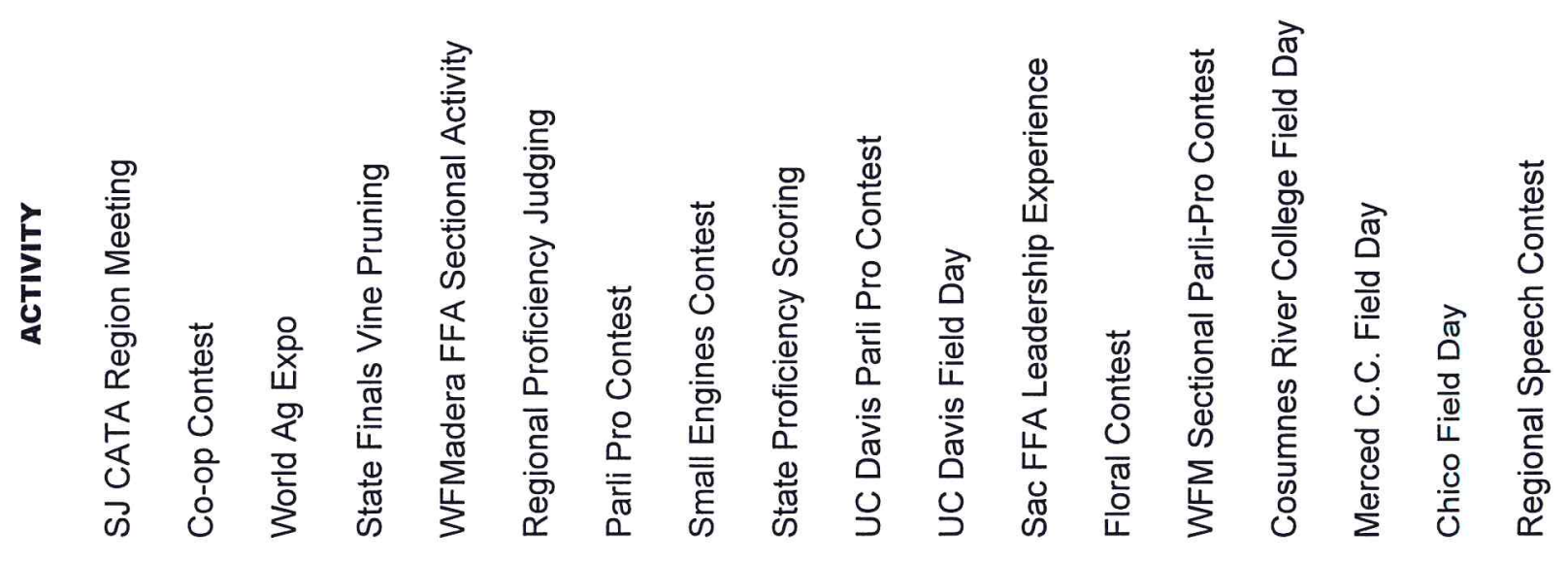

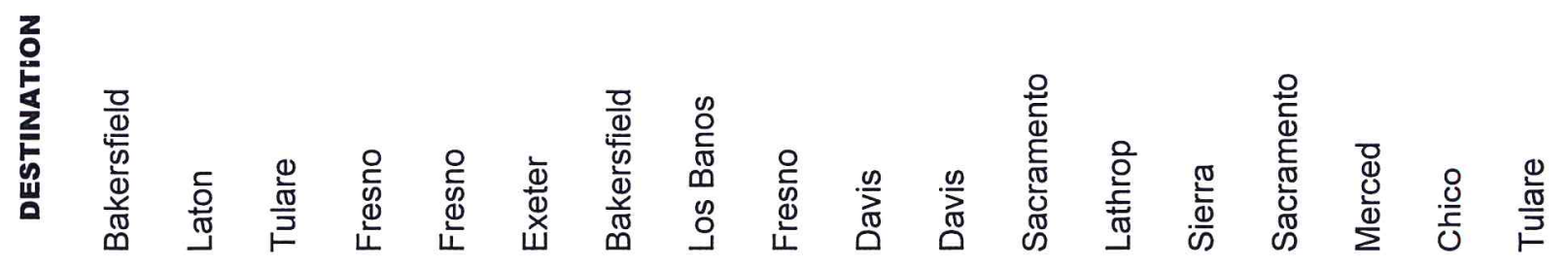

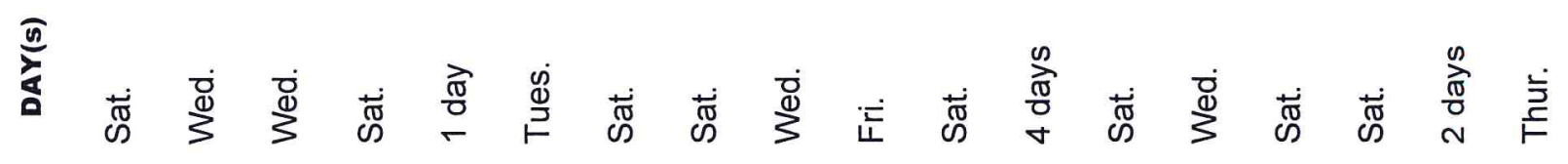

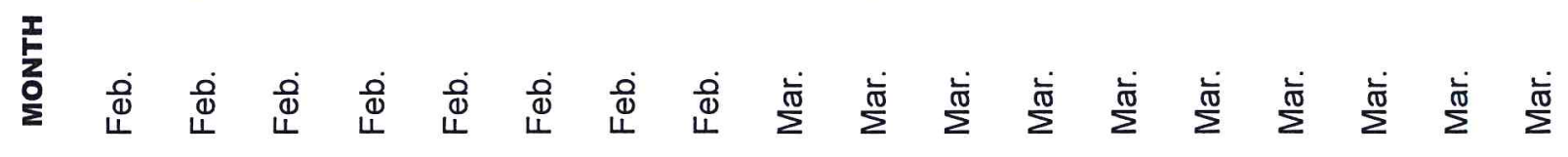




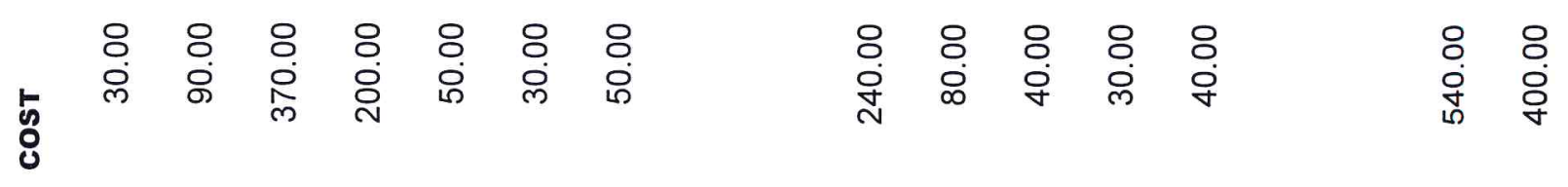

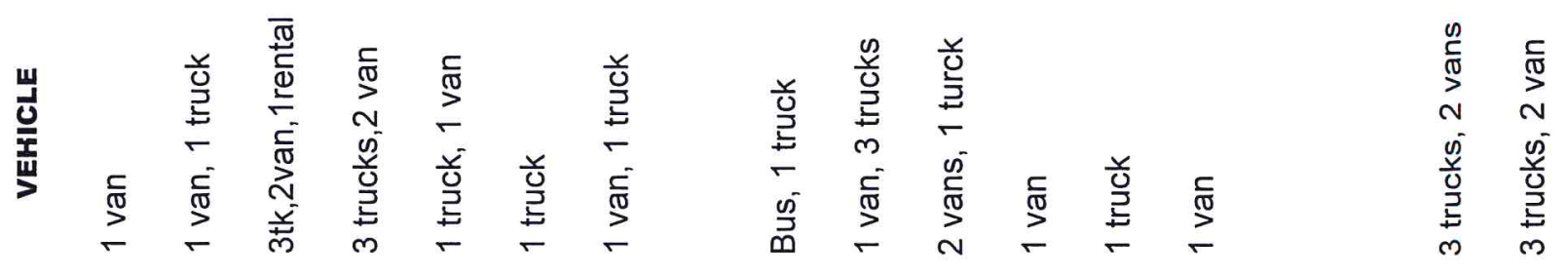

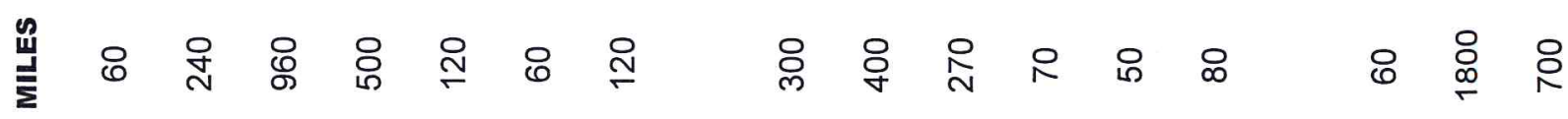

耪

竞

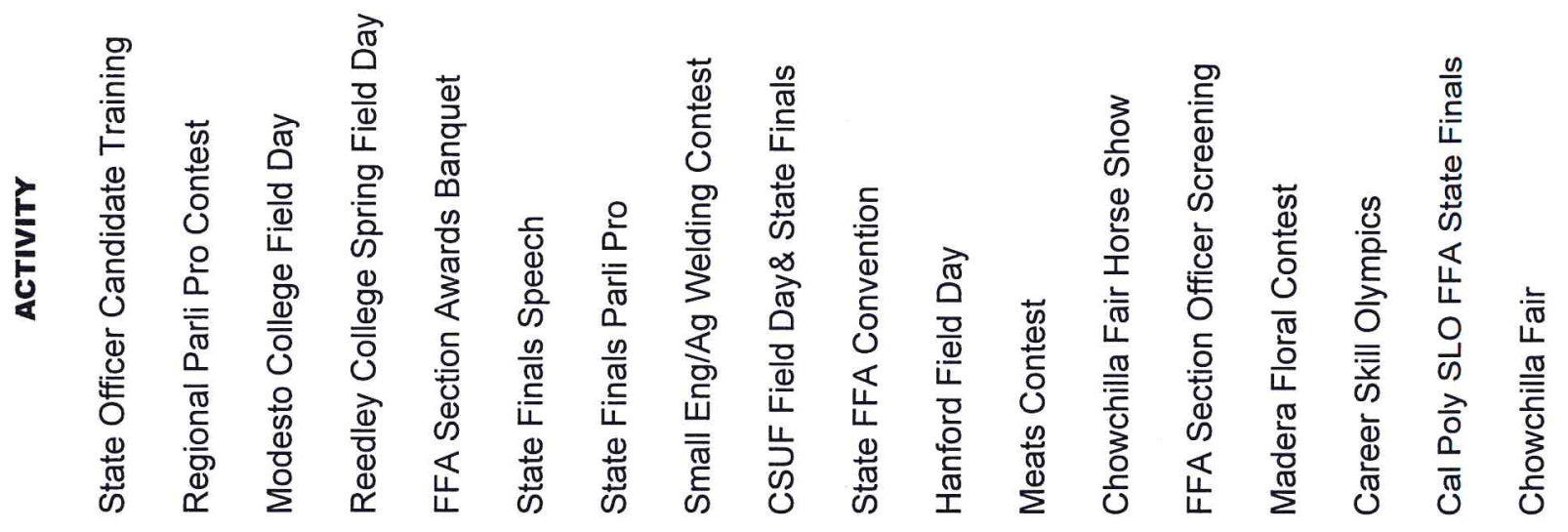

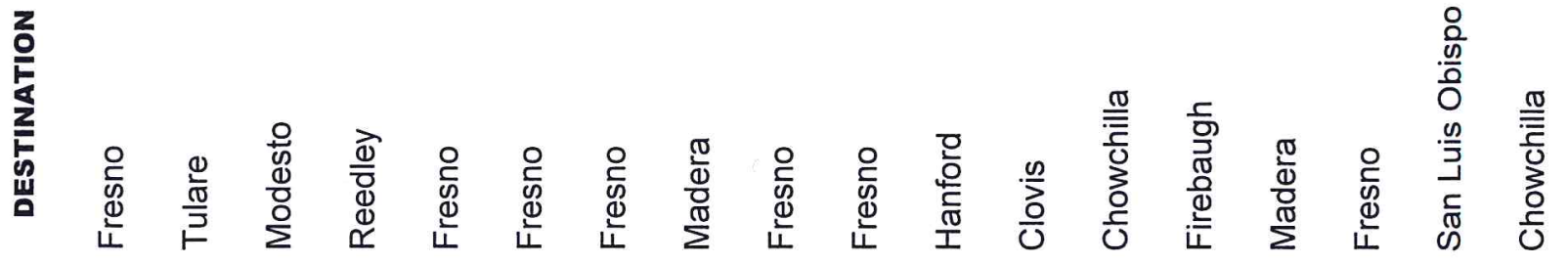

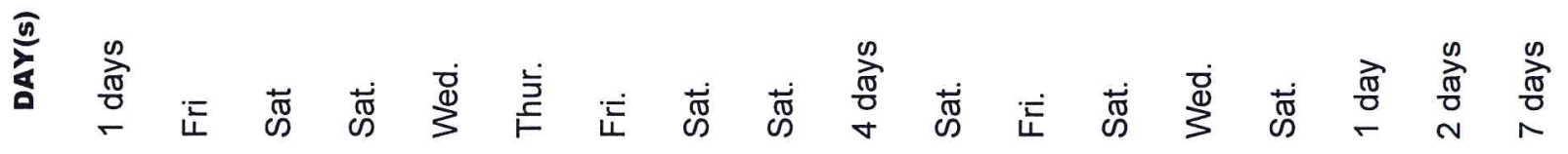

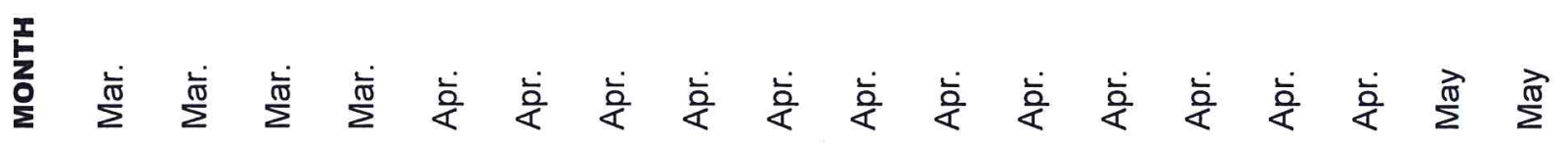




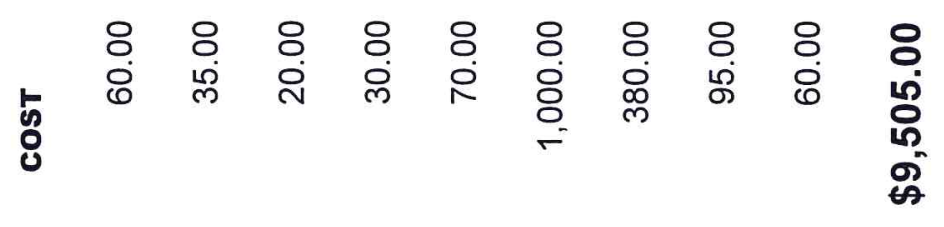

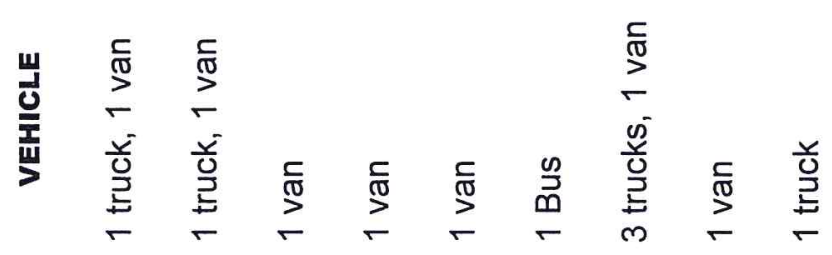

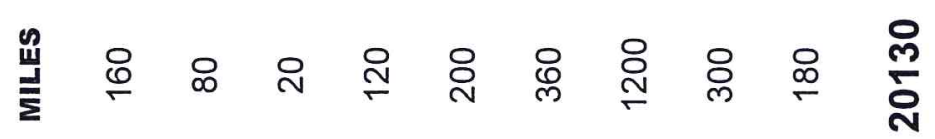

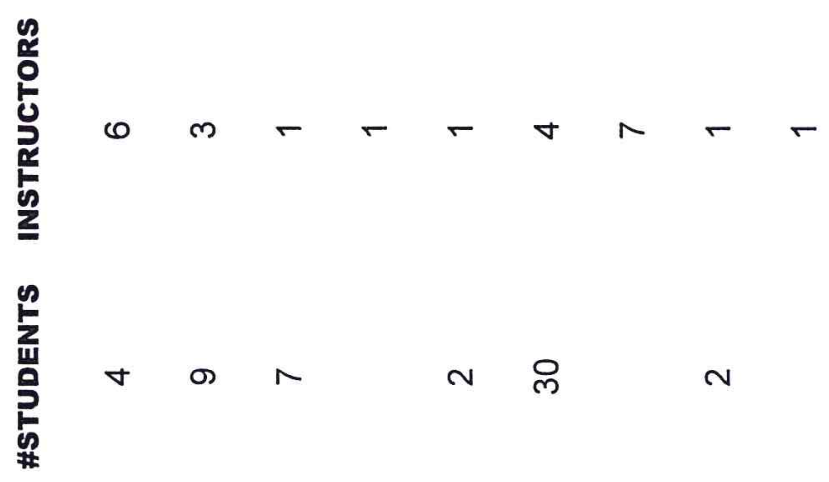

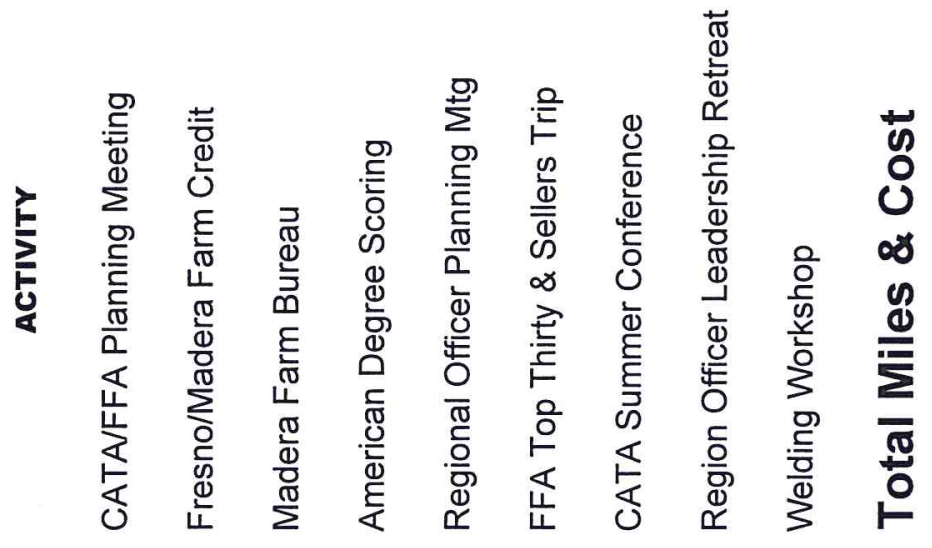

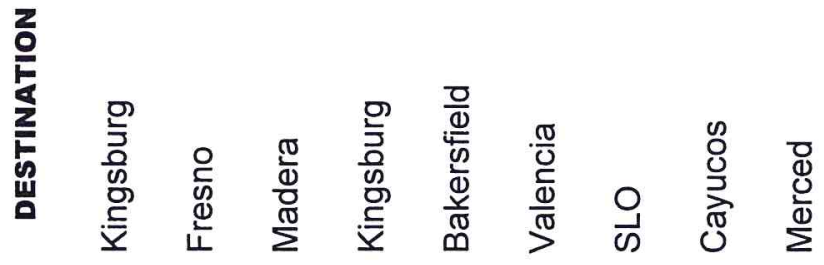

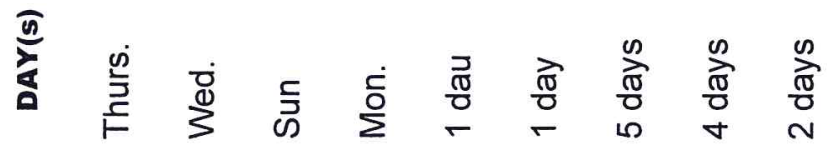

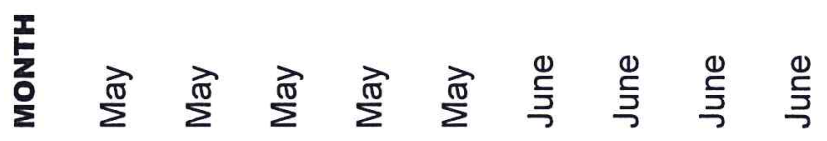


AA. CATA Membership Card 


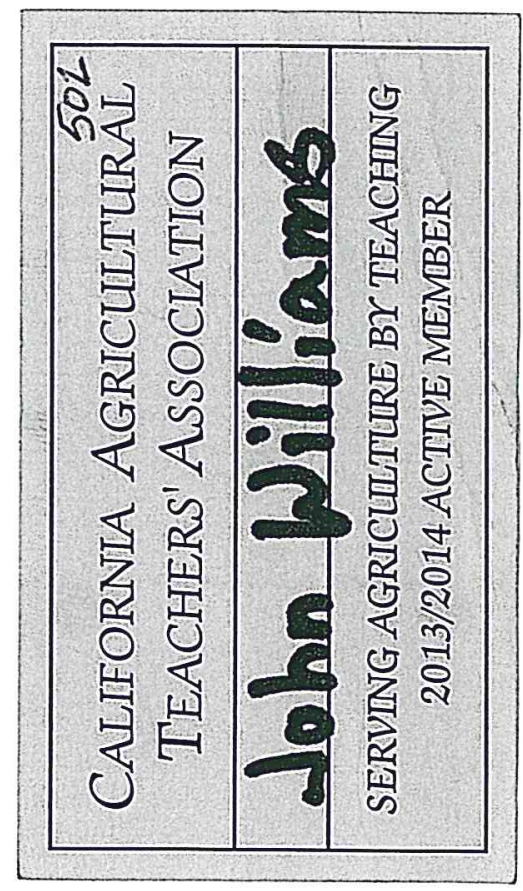




\section{BB. Meeting Reports Submitted To Administration}


Madera South High School

705 W. Pecan Ave

Madera, California 93637

(559) 675-4450

June, 2013

To: $\quad$ Dr. Anthony Monreal, Interim Superintendent

Mrs. Debie Wood, Associate Superintendent

Madera Unified School Board Members

Mrs. Shirley Woods, Career Technical Education Coordinator

Mr. Sandon Schwartz, Principal Madera South High School

Mr. Oracio Rodriguez, Vice Principal School of Agriculture MSHS

From: $\quad$ MSHS School of Agriculture Sciences and Engineering

Subject: $\quad$ Year End Report 2012-2013

This year-end information was compiled by the Agricultural Staff. Its purpose is to inform persons of the magnitude of activity generated in areas of FFA Competitions, Projects, Judging Teams and Professional Development. This information is used to evaluate, set goals, and develop a plan for the following school year. The following information on student numbers is taken from the October 15, 2012 State R-2 Report.

A. Vocational Agriculture: (7 Instructors with 35 sections as of 10/15/12)

1. Enrollment: Total: 1031 student hours (Unduplicated students) 611

2. Classes:

5 - Biology

$2-$ Ag Science I

1 - Ag Science III

1 - Animal Care/Vet Tech

1 - ROP Small Engines (2hr)

2 - Diesel Technology

5-Ag Mechanics I

2-Ag Mechanics II

1 - ROP Welding (2hr)

3 - Floral Design

1 - Advanced Floral \& Retail Floral Shop

1 - Horticulture I

1 - Horticulture II

4-Ag Earth Science

2-Ag Economics
Student Hours

188

70

26

32

56

60

162

53

30

84

20

35

34

149

50 


\section{Adult Classes:}
a. ROP Small Engines
b. ROP Diesel Engines
c. ROP Ag Welding

B. Supervised Agricultural Experience Program:

\begin{tabular}{lccc} 
Program & Students & Head & \$\$Value \\
\hline Dairy & 4 & 5 & 15,000 \\
Swine & 30 & 33 & 9,500 \\
Sheep & 12 & 16 & 6,000 \\
Beef & 2 & 2 & 8,000 \\
Horse & 5 & 7 & 8,000 \\
Poultry \& Game & 15 & 75 & 750 \\
Specialty Animal (Goat) & 5 & 5 & 1,500 \\
Ag Mechanics & 15 - Major Projects & 40,000 \\
O.H. & 1 - - Patio Garden at Fair & 1,000 \\
O.H. & 72 - Raised Plants & 2,000 \\
Floral & 17 - Floral Shop & 15,000
\end{tabular}

\section{Project Visits:}

All projects are done after school with each teacher planning to visit each project on a monthly basis with a minimum of five (5) visits per week or an average of 245 home visits per year per teacher. (These visits were normally done during an Ag Teachers' Project Supervision Period, but until monies become available, they are done after school or weekends.)

\section{Ag Mechanic Projects:}

a. 15 large projects constructed - $\$ 40,000$ cost $(\$ 75,000$ value)

b. $\quad 400$ small projects constructed $-\$ 2,000$ cost ( $\$ 4,000$ value)

c. School Projects

1. Provided metal for numerous repairs (at no cost)

2. Disc MUSD property by Desmond Elementary

3. Varies Repairs for MUSD

4. Designed \& installed mounts for fans in all animal barns

5. Welded 200 student chairs for maintenance

6. 150 small engines repairs \& rebuilds

7. Repair livestock trailer

8. Serviced 80 lawn mowers

9. Repaired disc

10. Rebuilt 12 Kubota engines

11. Repaired football water carts

12. Built \& repaired numerous field exercise equipment

13. Disc farm once/month \& spraying of farm for weeds

Other Projects:

1. Maintained \& planted 4 garden planters +4 Herb planters on campus

2. Installed new irrigation \& re-landscaped front office 
3. Agricultural Science III Class:

Castrating - goats \& pigs -20 head

Neutering - cats -4 head

Dehorning goats -5 head

Clipping \& grooming dogs -15 head

Examination of pets -30 head

Dusted chickens -75 head

Dewormed goats \& sheep -15 head

Vaccinated dogs \& cats -10 head

Vaccinated goats -8 head

Managed sheep -10 head

Managed hogs -1 sow \& 13 piglets

Processed hogs -13 head

Sheared sheep -7 head

Castrated \& vaccinated cattle -2 head

Floated horse teeth -1 head

Took apart sheep pens \& reassembled in new configuration \& cleaning, sanitizing

Cleaned out \& maintained all water troughs on farm

4. Donated plants to Elementary Schools: Nishimoto, Alpha, Sierra

Vista, Children's University

E. School Farm:

$\begin{array}{cccc}\text { 1. Horses } & 1 & \\ \text { Horses } & 6 & \text { student/boarded at farm* } & \$ 2,000 \\ & \text { *students pay for feed and care }\end{array}$

F. FFA Activities: For Supervised Agricultural Experience (SAE)

1. Fairs and Shows Attended:

Madera Fair

Chowchilla Fair

Cow Palace

Arizona National Livestock Show
State Fair

Red Wave

National Junior Swine Show

\section{Madera Fair Results:}

\section{Beef Cattle:}

Jaime Cuves

$2^{\text {nd }}$ Place Market Class

Reserve Champion FFA Market Beef

Reserve Grand Champion Market Beef

Bred and Fed Division Winner

$4^{\text {th }}$ Place FFA Intermediate Showmanship. 


\section{Ag Mechanics:}

Chris Melikian

Welding trailer $-1^{\text {st }}$ Place, Best of Show

Calf bottle trailer $-1^{\text {st }}$ Place

Shade trailer $-1^{\text {st }}$ Place

Adam Chavira

Picnic table/BBQ $-2^{\text {nd }}$ Place

Michael Cabrera

Shade trailer $-1^{\text {st }}$ Place

Jose Vega, Rickie Thacker

$6^{1 / 2} 2^{\prime} \times 18^{\prime}$ trailer $-1^{\text {st }}$ Place

Michael Valencia

3-Point Orchard Float $-1^{\text {st }}$ Place

Directors Award

Metal Design/Metal Sign $-1^{\text {st }}$ Place

Poultry:

Sabrina McCann - 3rd Place Fryer

Gissel Pedraza $-5^{\text {th }}$ Place Fryer

Jorge Mendoza - 7th Place Fryer

Spenser Smith - FFA Champion Meat Pen

Susana Perez - FFA Reserve Champion Meat Pen

Market Goats:

Eduardo Rodriguez - 2nd Place in Market Class

Rodolfo Rodriguez $-3^{\text {rd }}$ Place Market Class; Champion Novice Showman

Cecila Renteria $-1^{\text {st }}$ Place Market Class

\section{Market Sheep:}

Commercial Cross Division

Chris O' Haro $-1^{\text {st }}$ in Light Class

Tori Jones $-1^{\text {st }}$ in Light Class

Reserve Champion Commercial Cross

Allison Helton $-1^{\text {st }}$ in Light Class

Champion Commercial Cross

Hampshire Division

Justin Bradfrod $-3^{\text {rd }}$ in Class

Matt Cavallero $-3^{\text {rd }}$ in Class

Suffolk Division

Mark Cavallero - 2nd in Class

Joalex Sanchez - 4th in Class

Jorge Mendoza $-3^{\text {rd }}$ in Class

Novice Showmanship $-7^{\text {th }}$ Place

Marcelina Gonzalez $-2^{\text {nd }}$ in Class

Novice Showmanship $-9^{\text {th }}$ Place 
Crystal Bazante $-2^{\text {nd }}$ in Class

Taylor Helton $-1^{\text {st }}$ in Class

Reserve Champion Suffolk

Advanced Showmanship $-1^{\text {st }}$ Place

Round Robin $-2^{\text {nd }}$ Placed Advanced

\section{Market Sheep Chapter Group -3rd Place}

\section{Garden Display:}

2nd Place in Children's Gardens

Students: Dominique Ortega, Rebekah Lara, Quinn Shippey, Jamie Oyler, Marcos

Guterriez, Suzana Perez

\section{Market Swine:}

Michael Valencia - Reserve Champion Market Yorkshire, Champion Bred and Fed

7th Advanced Showmanship

SukVirh Singh $-2^{\text {nd }}$ Place Market, 7th Novice Showmanship

Jenae Hansen $-3^{\text {rd }}$ Place Market, 1st Novice Showmanship,

8th Intermediate Showmanship

Clayton Sheehan - 5th Place Breeding Class, Champion Market Hampshire,

Outstanding Swine Exhibitor, 1st Intermediate Showmanship,

4th Intermediate Round Robin Showmanship

Luis Cervantes - Champion Market Yorkshire, 3rd Novice Showmanship,

4th Intermediate Showmanship

Sabrina McCann - Champion Market Duroc, Reserve Champion FFA, 5th Intermediate Showmanship

Al Lopez - Reserve Champion Market Hampshire

Ryan Zaragosa $-3^{\text {rd }}$ Place Market Class, 6th Intermediate Showmanship

Chris Melikian - 2nd Place Market Class

Kayla Melikian - Champion Cross-Bred, Champion FFA, 1st Advanced Showmanship, 6th Advanced Round Robin

Vanessa Duarte $-5^{\text {th }}$ Place Market Class, 5th Advanced Showmanship

Cody Knott - Reserve Champion Cross-Bred

Scott Bullis $-1^{\text {st }}$ Place Market Class, 8th Advanced Showmanship

\section{Market Swine - Champion Chapter Group}

\section{Dairy Replacement Heifers:}

Jaime Cuevas $-6^{\text {th }}$ Place Market, $1^{\text {st }}$ Place Novice Showmanship,

$8^{\text {th }}$ place Intermediate Showmanship

Jimmy Beavers $-10^{\text {th }}$ Place Market

Scott Bullis - Reserve Supreme Champion Replacement Heifer,

$1^{\text {st }}$ Place Advanced Showmanship, 5th Place Round Robin 


\section{Chowchilla Fair Results:}

Market Sheep:

\section{Suffolk Division}

Taylor Helton $-1^{\text {st }}$ in Medium-Heavy Class, Reserve Champion Market, $5^{\text {th }}$ Place Advanced Showmanship

Feeder Results

Chris O'Haro $-2^{\text {nd }}$ in Feeder Class, 6th Place Intermediate Showmanship

$\underline{\text { Ag Mechanics: }}$

Michael Dorado

Trailer BBQ - 2nd Place

Craftsmanship Award

Outstanding Welding Project

Matthew Flores

Joey Vega

Dump Trailer $-3^{\text {rd }}$ place

Restroom Trailer $-1^{\text {st }}$ Place

Ruben Camarillo

Loading Chute $-2^{\text {nd }}$ Place

Leonel Gastellum

Farm Labor Cooling Station - Participation

Jimmy Beavers

Farm Labor Cooling Station - Participation

Dalice Garcia

Trailer BBQ - Participation

\section{Outstanding Chapter Group of 5 Projects}

\section{Dairy Heifers:}

Scott Bullis - 8th Place Yearling Heifer, 4th Advanced Showmanship

Jaime Cuevas - 6th Place Yearling Heifer, 8th Advanced Showmanship

Sukhvir Singh - 15th Place Springer Heifer, 9th Advanced Showmanship

Jimmy Beavers - 4th Place Springer Heifer, 10th Place Yearling Heifer

\section{Market Swine:}

Laura Castrejon $-10^{\text {th }}$ Place Cross-Bred Market Class

Lindsay Tasos - Champion Hampshire, Reserve FFA Champion, $1^{\text {st }}$ Advanced Showmanship

Clayton Sheehan - 5th Place Cross-Bred Market Class, 4th Place Breeding Gilt

Kayla Guthier $-3^{\text {rd }}$ Place Hampshire Market Class

Allyson Roberts $-5^{\text {th }}$ Place Hampshire Market Class, $7^{\text {th }}$ Intermediate Showmanship

Chris Melikian $-4^{\text {th }}$ Place Chester Market Class

Vanessa Duarte $-4^{\text {th }}$ Place Yorkshire Market Class

Cody Knott $-3^{\text {rd }}$ Place Hampshire Market Class

Luis Cervantes $-3^{\text {rd }}$ Place Duroc Market Class, $1^{\text {st }}$ Novice Showmanship, $3^{\text {rd }}$ Intermediate Showmanship, $10^{\text {th }}$ Advanced Showmanship 
Sabrina McCann $-2^{\text {nd }}$ Place Hampshire Market Class, $10^{\text {th }}$ Intermediate Showmanship

\section{Market Swine $-2^{\text {nd }}$ Place Chapter Group}

4. Judging Contests Attended:

Sectional BIG \& Banking Contests

Lathrop Floral Contest

Reedley Winter/Spring Field Days

Consumes River Field Day

Chico State Field Day

National FFA BIG Contest

Hanford Field Day

State Finals Speaking Contest

U. C. Davis Field Day

Clovis Ag Welding Contest

Dinuba Vine Pruning

Merced College Field Day

Cal Poly SLO State Finals

Madera Opening/Closing Contest

Sectional Co-Op Contest

Madera South Floral Contest

Sectional Parli Pro Contest

Modesto College Field Day

Regional Parli Pro Contest

Madera South Cotton Contest

Tulare/Kings Cotton Contest

Madera South Small Engines

Regional Speaking Contest

Career Skills Challenge

Madera South Ag Welding

Corcoran Cotton Contest

Sectional Speaking Contest

CSU Fresno Fall/Winter/Spring Field Days

5. Judging Team Results: The entry fees for these contests were paid entirely by Madera FFA.

Total teacher compensation from MUSD amounted to $\$ 2728$ divided among the 7 coaches.

\section{Team}

Ag Welding Team

Banking Team

Best Informed Greenhand

Cotton Team

Co-Op Team

Farm Power

Floriculture Team

Meats Team

Milk Quality \& Dairy Foods

Nursery Team

Opening \& Closing - Open

Opening \& Closing - Officer

Opening \& Closing - Frosh

Parli Pro - Novice

Scrapbook

Small Engines Team

Speaking Contests:

Creed

\section{\# Contests}

Attended

$$
5
$$

1

8

4

5

4

8

4

8

7

6 teams

5 team

2

3

1

6

2

\section{State Finals Results}

$7^{\text {th }}$

$2^{\text {nd }}$ in Section

$1^{\text {st }}$

$6^{\text {th }}$

$10^{\text {th }}$

$5^{\text {th }}$

$5^{\text {th }}$

$2^{\text {nd }}$

$14^{\text {th }}$

$5^{\text {th }}$

Gold in Section

Gold in Section

Gold in Section

$6^{\text {th }}$ in Region

$4^{\text {th }}$ in Region

$8^{\text {th }}$

$1^{\text {st }}$ Section, $2^{\text {nd }}$ Region 


$\begin{array}{cll}\text { Extemporaneous } & 1 & 1^{\text {st }}, 3^{\text {rd }}, \& 5^{\text {th }} \text { in Section } \\ \text { Impromptu } & 3 & 6^{\text {th }} \text { in State } \\ \text { Job Interview } & 1 & 5^{\text {th }} \text { in Section } \\ \text { Prepared } & 2 & 2^{\text {nd }} \text { in Section } \\ \text { Vine Pruning Team } & 3 & 1^{\text {st }} \\ \text { Vine Judging Team } & 1 & 3^{\text {rd }}\end{array}$

6. Other Chapter Awards:

5 American Degrees

3 Sectional Proficiency Awards Winners

1 Regional Proficiency Winners

1 State Proficiency Winner

1 National Delegate

1 National Proficiency Winner

Gold Star Chapter

Outstanding Gold Chapter

Gold Star Reporter - Section

20 State FFA Degrees

35 Chapter Farmer Degrees

60 Greenhand Degrees

3 Sectional Officers

1 Regional Officer

1 Silver Star Counselor

2 Gold Star Administrator

1 State Star Administrator

\section{Major FFA Activities:}

32 Executive FFA Meetings

9 Local Evening Meetings

Greenhand/Chapter Degree Potluck Dinner - 350 present

End of the Year Awards Banquet - 350 present

Ag Literacy Day - 1400 elementary MUSD students toured the MHS Farm

Top Thirty \& Seller Trip to Magic Mountain

4 Sectional Meetings 10 Sectional Officer Meetings

2 Regional Meetings

Attended State FFA Convention - 16 students, 4 advisors

Attended National FFA Convention - 19 students, 4 advisors

1 - National Delegate

Attended Greenhand Conference - 14 students

World Ag Expo Field Trip - 50 students

Old Timers Parade - pooper scoopers

Farm Bureau Scholarship Night

MSHS Senior Scholarship Night - handed out awards

3 - Canned Food Drives

Chapter Officer Leadership Conference

Sectional Officer Leadership Conference

Regional Officer Leadership Conference

Advanced Leadership Academe

Made for Excellence Conference

Sacramento Leadership Conference

State Degree Awards Dinner

1 - Tri Tip Dinner Fundraisers for 350 people

2 - Snack sales fundraiser 
West Fresno Madera Sectional FFA social event at Blackbeards in Fresno Chapter Officer Summer \& Winter Retreat

2 - Love Madera Service Projects

Toys for Tots

Kids Day Newspaper

Team Building Boot Camp

4 - Agriculture Industry Tours

West Fresno Madera Section FFA - John's Incredible Pizza

West Fresno Madera Section FFA - Bowling for Children's Hospital

CSUFresno Ag Night

Fresno Madera Farm Credit Dinner

FFA Alumni Dinner

ARC - CLC

G. Madera County Ag Booster:

35 members

Monthly meetings

Cultural Practices at Ag Center

Served on Ag Advisory Committee

Provided guidance on Ag Center

Donated Supplies for Vineyard

Donated Vehicle

2 - Donated Hoop Greenhouses

Donated for the National FFA Convention Trip

H. MSHS Ag Advisory Committee:

2 meetings

Continues to review curriculum and Career Pathways

Support reinstatement of Project Supervision Period

$\mathrm{Ag}$ Advisory Chairman in regular contact with Ag Dept. members

I. Miscellaneous Activities:

Worked with Ag Advisory Committee on future plans on Ag Center

Hosted Sectional FFA Opening/Closing Contest

Hosted Madera Floriculture/Ag Welding/Small Engines/Cotton Contests

Judges for Section/Regional

Judges for Regional/State Speech Contests

Hosted Cal Poly Student Teachers

Judged Sectional State Degree Record Books

Judged Proficiencies

Hosted Sectional CATA Record Book Scoring

FFA West Fresno Madera Section Advisor

FFA West Fresno Madera Section Financial Secretary

J. Professional Improvement Involvement (Ag Teachers):

Member of Dairy Committee 
Sectional, Regional and State CATA activities

7 - Summer Conference at Cal Poly, SLO

Farm Bureau Member - 1

Hosted Floriculture Contest at MSHS

Assisted at Small Engine Contest at Merced, Modesto, Chico, Davis

Student Teacher Mentor - 7

Master Teacher Evaluator - 3

Briggs and Stratton "Master Mechanic"

CBA - Certified Balloon Artist

Master Gardner

Private Applicators License Pesticide - 2

New Professionals

CATA Professional Development

Lincoln Electric Training

UC Kerney Research Station Horticulture Inservice

Generator \& Pressure Washer Training

CATA West Fresno Madera Sectional Vice President

CATA West Fresno Madera Sectional Secretary

CATA West Fresno Madera Sectional Treasurer

ROP Fall Conference

CATA Professional Presenter

Presenters at San Joaquin Region FFA Boot Camp

CTE Online Curriculum Writer

K. Academic/Core Professional Involvement:

Created and attended weekly School of Ag Teachers meetings

CFA Wrote \& Administered CFA's - 7

DPA's -4

PLC - Science/Social Science

BTSA Support Provider - 1

L. Community and Business Partnership Support:

Blue Scope Steel: Metal

$\$ 10,000$

Kings Valley Industries - labor, shop time

$\$ 10,000$

Producers Livestock - loaned bobcat/scales/facilities

$\$ 1,000$

Madera Tractor \& Implement Co, Inc

$\$ 150$

Madera County Ag Boosters

$\$ \$ \$ \$$

Evans Feed

$\$ 300$

Lockwood Seed \& Grain

$\$ 50$

Foster Farms

$\$ 300$

Mark Dierberger

$\$ 400$

Julius Deniz

$\$$ Equipment use

Brian Deniz

Kuckenbecker Tractor - Engines \& Equipment use

$\$$ Equipment use

$\$ 12,000$

Les Loquaci

Norm Allinder

$\$$ Equipment use

Monrovia Nursery

$\$ 150$

$\$ 1,000$ 
Prax Air

$\$ 500$

Mowers Plus

$\$ 500$

William Thorpe

$\$ 500$

David Nino

$\$ 600$

Schaffer Stakes

$\$ 3,000$

Ag Trailer

$\$ 1,000$

Duarte Nursery

$\$ 3,000$

Western Ag \& Turf

$\$ 1,500$

Bob Labrucherie

\$ Use of Livestock

Shane Giest

\$ Use of Livestock

Alumni Committee Donations

$\$ 5,000$

CSU Maritime Academy

$\$ 15,000$ 
CC. Your "Wish List" 


\section{Wish List}

- Diesel Engine Dyno

- 4 Point Hydraulic Lift

- Engine Cherry Picker

- 8 Ton Diesel Forklift

- Briggs and Stratton OHV 10 Engine School Kit

- Euro Tire Changing Machine 
EE. Advisory Committee Agendas For Current Year 


\section{Madera Agriculture Advisory Meeting Preliminary Agenda October 10, 2013 6pm, Room 706}

I. Dinner

II. Welcome and Introductions

a. Gary Geist-Advisory Chairperson

b. Kristin McKenna-Department Chairperson

III. FFA Update

a. FFA Advisors - John Williams/Brent George

IV. Agriculture Incentive Grant

a. Advisory Checklist and Verification of Criteria

V. School Farm \& Facilities Update

a. Vineyard - John Williams

VI. Curriculum Presentation

a. Welding and Fabrication Pathway-Brent George and Tim Deniz

VII. Miscellaneous, Mock Interview Panel 


\section{Madera Agriculture Advisory Meeting Preliminary Agenda March 18, 2013}

\section{Dinner}

II. Welcome and Introductions

a. Gary - Advisory Chairperson

b. Darlene - Department Chairperson

III. FFA Update

a. FFA Advisor - Kristin McKenna

IV. Perkins

a. Purposed spending plan for 2013/14 - Darlene Gilles

V. School Farm \& Facilities Update

a. Vineyard - John Williams

VI. Purposed New Class

a. Viticulture/Crops Class - Celia Casso

VII. Miscellaneous 
FF. Copy Of Advisory Committee Charter

And By-Laws 


\section{Agricultural Education}

\section{Advisory Committee Manual}

\section{Agricultural Education High School Leadership Division California Department of Education}




\section{Table of Contents}

Introduction

Forming an Advisory Committee

Functions and Duties of an Advisory Committee

Operation of Advisory Committees

Opening Session Instructions for Agricultural Education Advisory Committees

Appendixes

Appendix A -

A Typical Advisory Committee Meeting Agenda.

Appendix B -

A Typical Set of Minutes 


\section{Introduction}

The use of advisory committees is well established in the public school system. These committees were conceived in the beginning to implement the development and improvement of educational programs. This manual is written for those planning to form new advisory committees, wishing to improve those already in existence, and for newly appointed members. Advisory committees will play a vital role in agriculture programs in the future.

This manual will help prevent unnecessary errors in the development of advisory committees. These guidelines have proven successful, and may be added to and modified for local and present conditions.

Even though mandated, advisory committees are useless unless they are properly developed with practical working groups. They must be based on the needs of the people and industry for which they serve. Advisory committees are established systems for using lay persons to assist
professional educators.

With the increased need for rapid change in this technological age, there is a growing appreciation of the help provided by industry representatives serving on local advisory committees. Agriculture is a complex, highly scientific, and technological industry. Employment opportunities in agriculture are constantly changing. New technologies are continually being developed and incorporated into agricultural and educational industries.

Students must be trained for today's jobs as well as new opportunities that become available. There will be an increased need for agriculturists trained in specialized technical occupations. Advisory committees help teachers of agriculture stay abreast of these changing employment trends and opportunities. Increased interest in agriculture programs that include internships, work-study, and other types of on-the-job training will require close coordination with agricultural industry representatives.

Increased attention needs to be given to the education of at risk, disadvantaged, and other special needs individuals. Advisory committees can provide valuable assistance that is necessary for the
success of these interrelated programs.

We must remember that lay advisory groups have no administrative or legislative authority. They can not establish policy or take the place of the administration or the board of education. Their function is to provide understanding between the school and the community it serves. Advisory committees provide balanced judgment to local problems and help give continuity and support to programs.

The purpose of this manual is to provide information for Agricultural Education coordinators, school administrators, boards of trustees, teachers of agriculture, and advisory committee members. Included is information on the formation, functions, duties, and operation of advisory committees. An outline format is being used to make the information easier to find and use. 
Finally, a sample of opening session instructions, a sample agenda, and a sample set of minutes are offered for the benefit of those unfamiliar with these procedures.

\section{Forming an Advisory Committee}

Much of the success of an advisory committee is determined by the manner in which it is formed. Based on the experiences of many communities throughout the country, the following
steps are suggested:

\section{Determine and Verify the Need}

1.1 There must be a feeling of need and understanding of opportunity if an advisory committee is to succeed.

1.2 If with its help, the advisory committee can make the (department, division, district) better, it serves a usable function.

1.3 It can provide continuity of a quality program should teachers or administrative changes take place.

1.4 It is important that the school administration, agricultural education staff, parents, and other patrons of the school thoroughly understand the character and purpose of the committee.

\section{Nomination of Committee Members}

2.1 Once approval of the formation of an advisory committee by the board members is received, nominations should be made jointly by the principal or superintendent, the head of the agriculture department, and the chairperson of the school board.

2.2 Each should have an equal voice in the selections.

2.3 Avoid nomination of friends, as they may be less candid and honest in their advice. 2.4 The advisory committee should be truly representative of the district.
Members:

2.4.1 Should be successful agriculturists and/or individual/s engaged in a significant related occupation.

2.4.2 Must have recent, successful, firsthand, and practical experience in the field of agriculture

2.4.3 Should exhibit substantial interest in the agriculture program.

2.4.4 Should be representative of different important agricultural commodities, parts of district, age groups, farm organizations, \& ethnic or religious groups. 
2.4.5 Should be sought as public-spirited individuals who understand a specialized area and are willing to contribute their knowledge and advice as a member of a cooperative, constructive group.

2.4.6 From the general school staff and/or the board should only be used when special circumstances warrant their appointment.

2.4.7. Should not have frequent dealings with the department in order to minimize conflict of interest problems.

2.4.8 Should include representatives of the service areas of agriculture.

2.4.9 Should recognize the time required and express a willingness to serve on the committee.

\section{How Many Committee Members?}

3.1 No fixed number will satisfy all situations.

3.2 The group needs to be large enough to be representative of the district and to provide a quorum if several members are absent.

3.3 Should not be so large that it is unwieldy or difficult to call together.

3.4 Seven to eleven persons are suggested with nine being a workable medium.

3.5 Present only the number of names previously decided upon by the local governing board for confirmation. (When more names are presented personalities become involved yielding undesirable results.)

\section{How are Committee Members Notified of their selection?}

4.1 Notification is usually done in writing, by the principal or superintendent,

4.2 The letter should:

4.2.1 Indicate that the $\mathrm{Ag}$ teacher is supportive.

4.2.2 Indicate that the committee serves in an advisory capacity to him or her, the department, the principal, and to the school board.

4.2.3 Include a request that the member indicate whether he or she will accept.

4.2.4 Urge speed of acceptance to gain an orderly efficient start.

\section{Understanding of Responsibility}

5.1 Of greatest importance is that the committee is only advisory in character.

5.2 The advice is to the teacher, school administrator, or school board as

appropriate to accept or reject.

5.3 It has no administrative or policy forming power.

5.4 It will make suggestions on policy and procedure, but the source of its influence is in the voluntary acceptance of this advice by the proper governing authority. 
Experience has shown where all of the steps up to this point have been properly taken, a high percentage of acceptances may be expected.

\section{Functions and Duties of Advisory Committees}

1. Help to determine what type of Agricultural Education program is offered.

2. Assist the teacher(s) in finding suitable work stations (internships, work-study, cooperative learning, partnerships) for students in both production agriculture and agri-industry occupations. 3. Help the instructor establish curriculum that has a hands-on, technological
approach. 4. Help attract and encourage qualified/capable students into the Agricultural
Education program.

5. Help in recruiting and providing opportunities for special-needs students.

6. Help to evaluate the effectiveness of the Ag. Education program. Guidelines for evaluation should be developed cooperatively with the advisory committee, administration, school board, and the Agricultural Education Unit of the California Department of Education.

7. Help gain support for legislation and appropriations.

8. Help the teacher(s) develop a list of capable resource persons for use as speakers, and/or judges for both in-school and out-of-school tests and contests.

9. Help obtain sponsors for appropriating funds for awards, scholarships, or needed equipment and supplies that are useful in carrying out classroom activities and F.F.A. or other youth programs.

10. Help unify the activities of the Agricultural Education program with those of other groups and agencies interested in agriculture.

11. Assist the teacher in determining skills needed for particular jobs at entry, technical and professional levels so that he/she may be included in the instructional program. 
12. When appropriate, serve as resource person to instructor visiting work place learning sites of students and participating in classroom instruction or demonstrations and accompanying or hosting field trips.

13. Study and make recommendations on problems presented to it by the school board on which further information is needed.

14. Provide the teacher with technical assistance and keep him/her aware of new developments in the agricultural industry.

15. Provide current resources to develop and maintain an Ag library of visual aids, magazines, and books concerning agriculture and agricultural occupations.

16. Serve as speakers at civic clubs, open houses, and career days to tell the story of school-industry cooperation.

17. Identify current standards for new equipment.

18. Assist in procuring opportunities to upgrade the teacher's technical skills and
knowledge. 


\section{Operation of Advisory Committee}

It is important that correct procedures and rules be established and clearly understood by should be decided upon by the committee staffs, and the board of education. These rules should be sent to administrators and advith assistance from the school. All correspondence

\section{Number of meetings}

1.1 Must meet regularly and often enough to carry out their assignment.

1.2 Monthly or bi-monthly meetings are usually the most desirable.

1.4 Minimum number is two per year.

1.5 Practical number is between three and eight per year.

1.6 Nessity should always determine the exact numer.

1.7 Better to have fewer well planned, wem from busy individuals.

\section{Selection of Officers}

2.1 Generally a chairperson, vice chairperson, and recorder are sufficient.

2.2 Chairperson should be a lay person elected by the committee. consultant.

\section{Length of Service by Committee Members}

3.1 Three-year terms are recommended.

3.2 At formation meeting members draw for one, two, or three year terms to provide for continuity of membership.

3.3 Individual preferences in length of service need to be considered.

3.4 Limitation should be placed on reappointments.

3.5 Nominees should be submitted to board of trustees for approval. 


\section{Length and Place of Meetings}

4.1 For efficient and effective use of time, the agenda for each meeting must
be well planned.

4.2 Ample meeting notice of 10 days to 2 weeks is recommended.

4.3 Copy of agenda, minutes from previous meeting, and any reading material requiring action should be sent in advance of meeting date.

4.4 Two-hour meetings, held at a time and date chosen by the committee, are recommended.

4.5 The meeting place should provide a conference table in a quiet
environment.

4.6 Usually the agricultural department of the school provides the best meeting site, allowing members to become familiar with facilities of the

\section{Filling Committee Vacancies}

5.1 Vacancies which occur because of term completion or other reasons should be filled by nomination from the advisory committee, teacher, superintendent, department head, or principal, and approved by the board

5.2 . The committee may be asked for suggestions.

5.3 A committee should not be permitted to choose its own replacements. 5.3.1 This would be self perpetuating.

5.3.2 May become unrepresentative and unduly independent of the school administration. Rules of procedure should indicate that if a committee member misses meetings repeatedly without reason, the position be declared vacant by the chairperson, and the school board so notified.

6. Distribution of Minutes: All committee members, the career education director, the principal, school board president and the regional supervisor.

7. Making Decisions: Currently many organizations operate by consensus approval of agenda items. When consensus cannot be reached or decorum is in question, refer to
Robert's Rules of Order. 


\section{Opening Session Instructions for Agricultural Education Advisory Committees.}

\section{Instructions to Your New Advisory Committee}

1. You constitute an advisory committee for the (your school district).

2. I welcome you on behalf of the board and administration.

3. You are agents of and appointed by the (your school's board of trustees).

4. While you are not a policy making body, you are advisory to (your department), and through channels, to the principal, superintendent, and board. We need your
expertise in this area.

5. The (your district) is interested in the best possible Agricultural Education program. We need to know what is ideal for this program from the standpoint of the community. Bear in mind that what we eventually can do, while we want the ideal if possible, must be compatible with available funds and state rules and
regulations.

6. You will be a working committee and students \& school staff expects to benefit
from your work.

7. We need help to:

7.1 Review existing programs, courses of study, facilities, equipment.

7.2 Propose new programs and/or courses when needed based on solid data for this community.

7.3 Evaluate existing programs and proposed new programs.

7.4 Revise existing programs, suggest changes or deletions, and develop educational specifications for the programs. (For use in building the program and planning for equipment and facilities.)

7.5 Help develop building plans, review architects plans, etc., where new

7.6 Help point out changes needed for the future in your area of interest Keep the program up to date.

7.7 Help in placement and in evaluating performance of our Agricultural Education students at (your school or college).

8. You will be a "helping group" (as well as advisory) to the instructor, as the program is implemented and progresses. 
9. This committee serves at the pleasure of the school board and may be dissolved at any time by board action.

\section{Getting Started:}

1. Review present course offerings and majors -- catalogs, studies, data, classrooms,
labs, and other facilities.

2. Conduct studies, if needed, to get community data on which to base your
decisions.

3. Decide areas to study or review (both geographic and educational areas) and determine how to do this (formal study, informal, follow-up studies).

4. Your findings and decisions will be in the committee minutes which will be distributed to the instructors, administration, and the board.

\section{Here's What You Need To Do To Get Started:}

1. Elect a chairperson.

2. The recorder will be an instructor, or department chairperson, and he or she will also be a resource person for you to help interpret educational language and concepts, provide materials, and be the liaison person with the administration.

3. Determine rotation (1-2- or 3 years?). You will also decide length and term and who serves what term. (Subsequent appointments will be 3 years each.)

4. Decide if more than one committee is needed. Large departments may have
subcommittees.

5. Announce that any member who can not continue serving for any reason, should notify the chairperson so that a replacement appointment can be made.

Note: Be sure to start and end on time!

\section{WE NEED YOUR HELP. WE APPRECIATE YOUR WILLINGNESS TO GIVE IT AND BE OF SERVICE TO YOUR SCHOOL.}




\section{Appendix A \\ (SAMPLE) \\ Advisory Committee Meeting Agenda}

TO: List committee members here

FROM: Chairperson

DATE: Date agenda is published

RE: $\quad$ Next Advisory Committee Meeting

DATE: Date of next meeting

TIME: Time of next meeting

PLACE: Place where meeting is being held

\section{AGENDA}

1. Review and approve minutes of the previous meeting.

2. Call for additional agenda items to be added to this meeting's agenda.

3. Committee and progress reports.

4. Consideration of recommendations for a new class or activity.

5. Review of revised course of study.

6. Report and review of F.F.A. and/or other youth organization activities.

7. Set date, time, and place for next meeting.

8. Adjournment. 


\section{Appendix B}

(SAMPLE)

Set of Minutes

\section{Advisory Committee Meeting \\ January 21, 2004}

The meeting was called to order by chairperson, Joe Smith at 3:00 p.m., January 21, 2004, in room 122 at Your High School.

The minutes of the previous meeting were read, amended, by changing the word shall to should in topic \#8, and approved.

The call for additional agenda items was made.

Mr. X reported that the Field Day Committee met on January 14, 2004. It was decided that the best day for the annual field day is May 5th. It was moved, seconded, and passed that our annual field day will be held on May 5, 2004.

Mrs. Y reported on ticket sales of the coming Parent and Student Banquet. So far, 310 tickets have been sold. This is already 20 more than last year's attendance.

It was moved and seconded that a class on small gas engines be added to the Ornamental Horticulture curriculum. After a lengthy discussion, this was referred to a committee of five made up of Mrs. A, Mrs. B, Mr. C, Mr. D, and Mr. E. They are to report to the advisory committee on March 15th. Mrs. A will be the chairperson.

Mr. Z reported on the suggested revision for the Basic Plant Science class. Added topics being considered are: weeds, pathogens, and insects. Pruning practices will likely be deleted as a specific class in pruning is being considered for next Fall.

F.F.A. President, Bill G. reported on this year's calendar of events of the chapter. He was commended by the Chair for his leadership and hard work.

The next meeting is scheduled for 3:00 p.m., February 15th, in room 122 at Your High School.

The meeting was adjourned at 5:00 p.m. by chairperson Joe Smith.

Respectfully Submitted,

Mr. Q, Recorder 
GG. Current Year's Operating Budget For Vo-Ag 


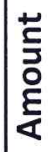

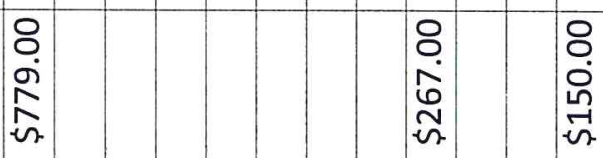

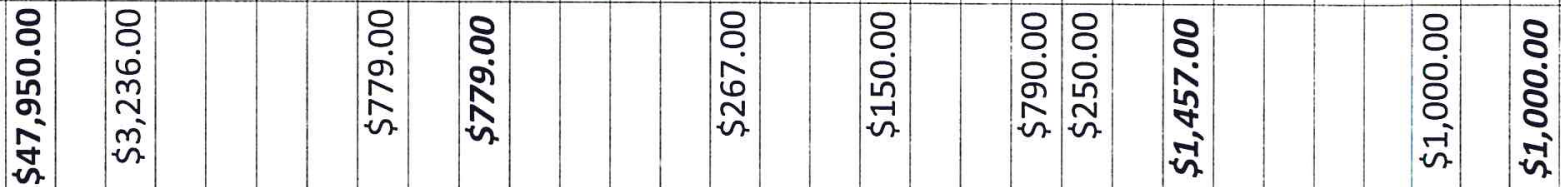

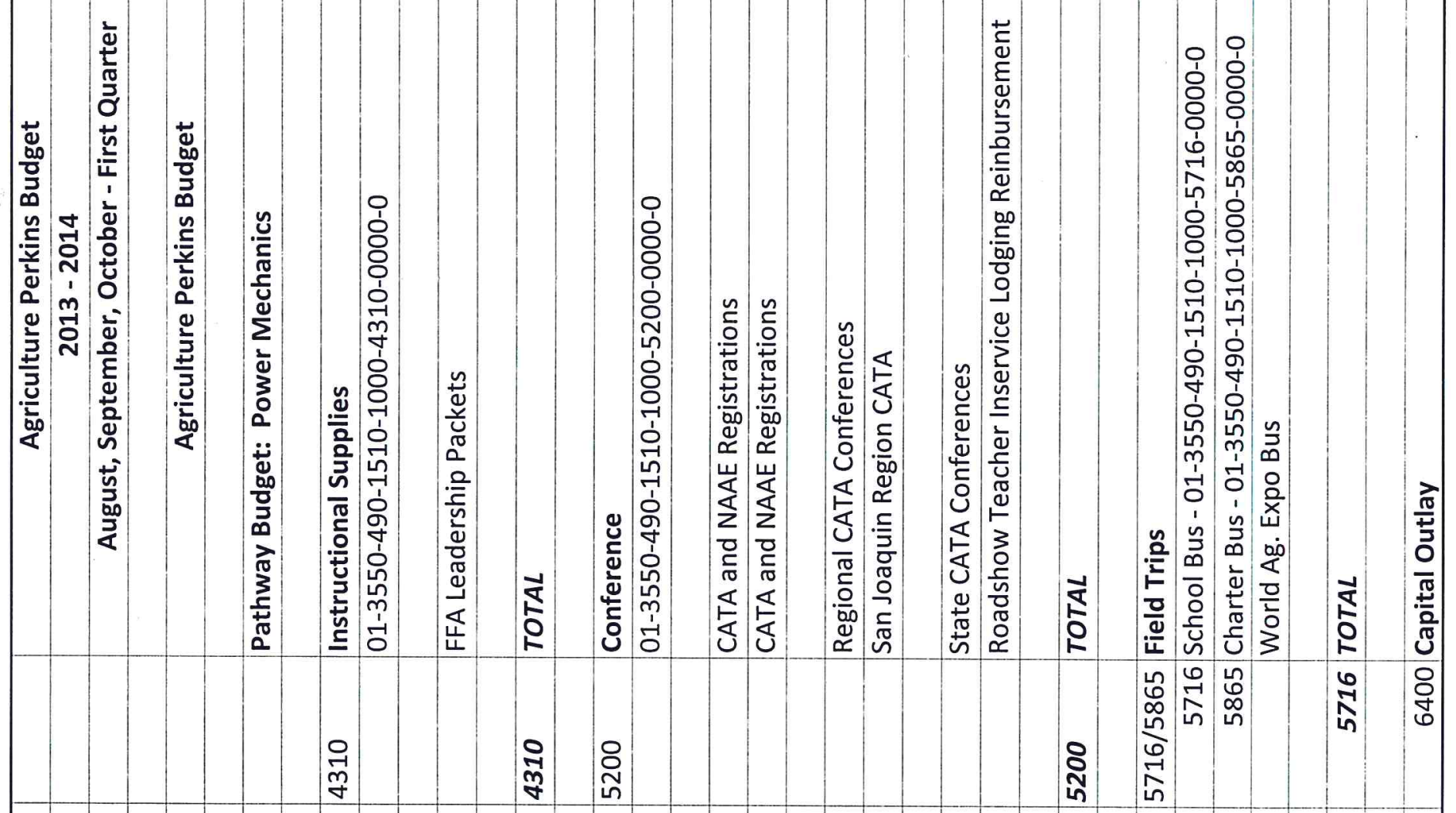

营

蒡

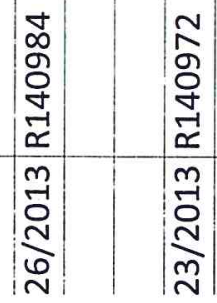




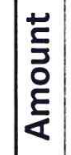

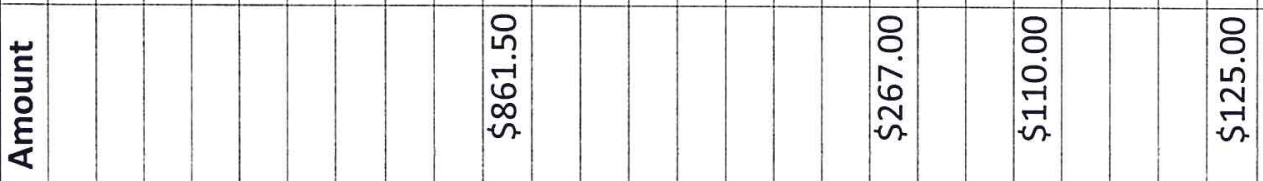

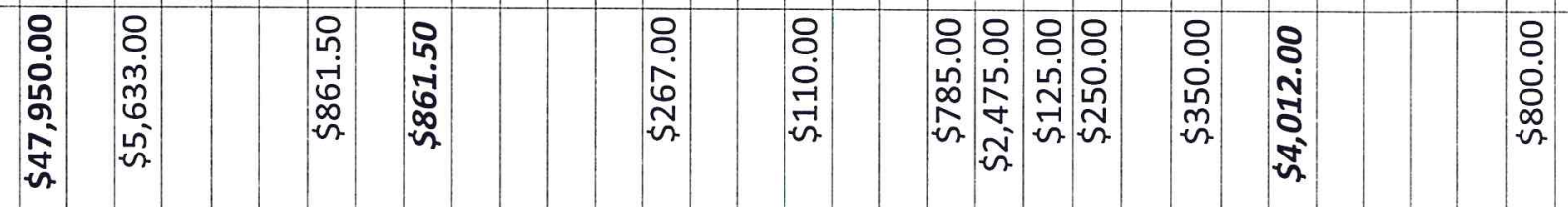

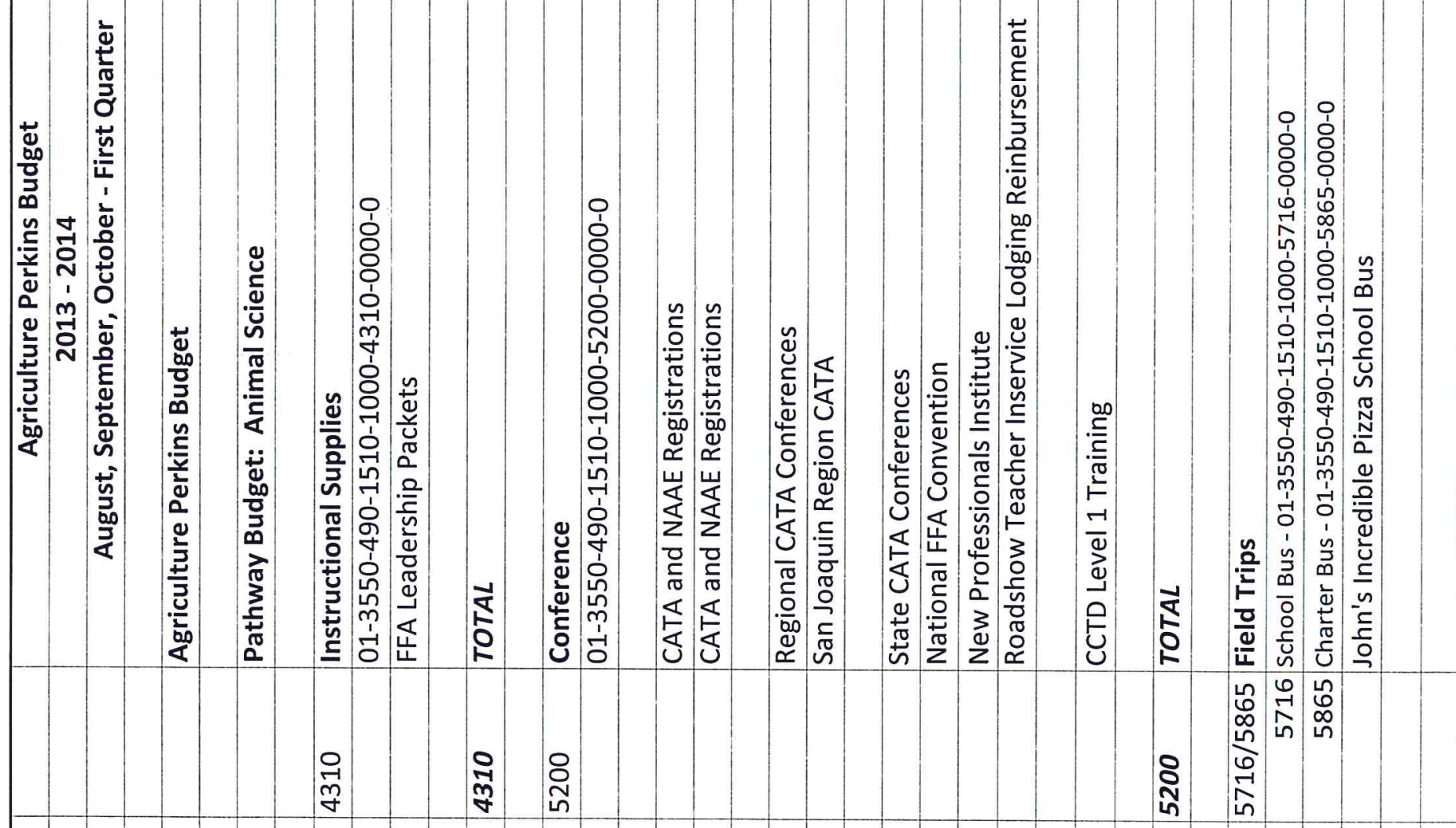

咅

葆

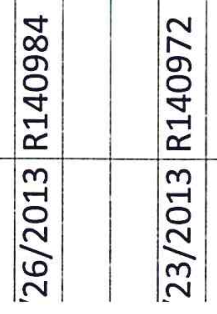




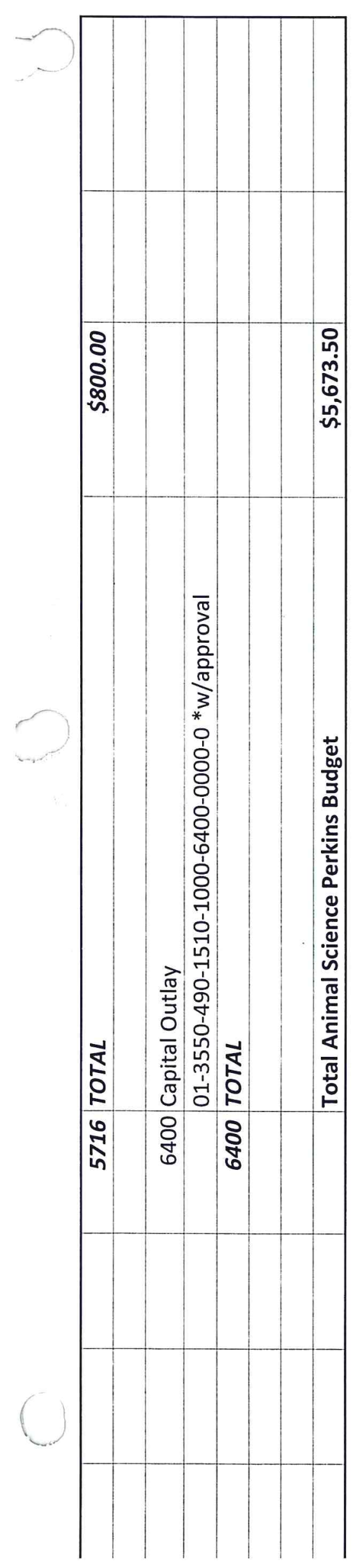




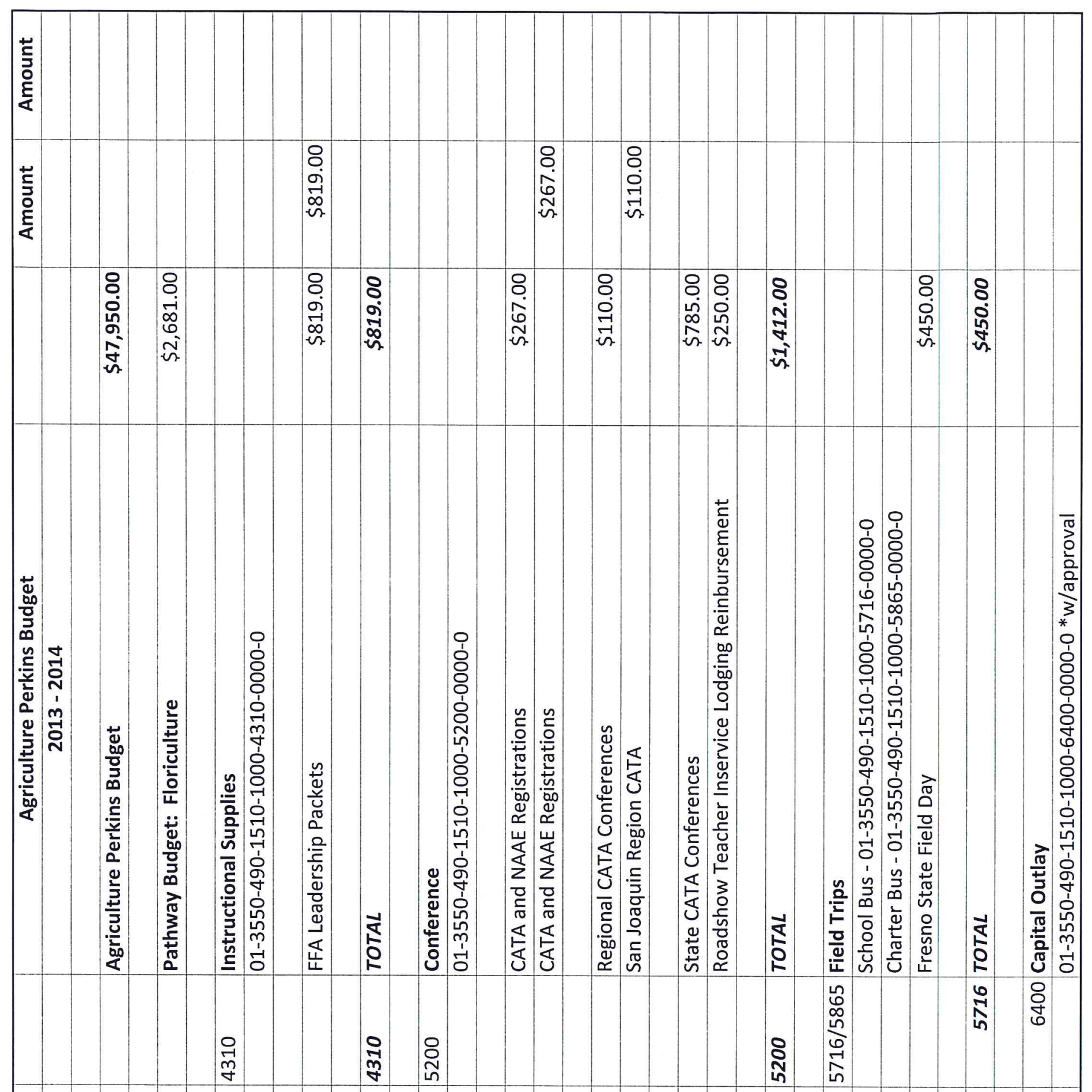

意

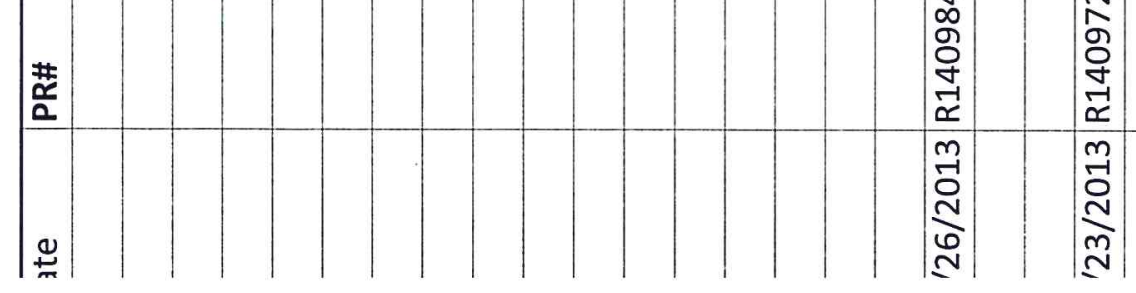




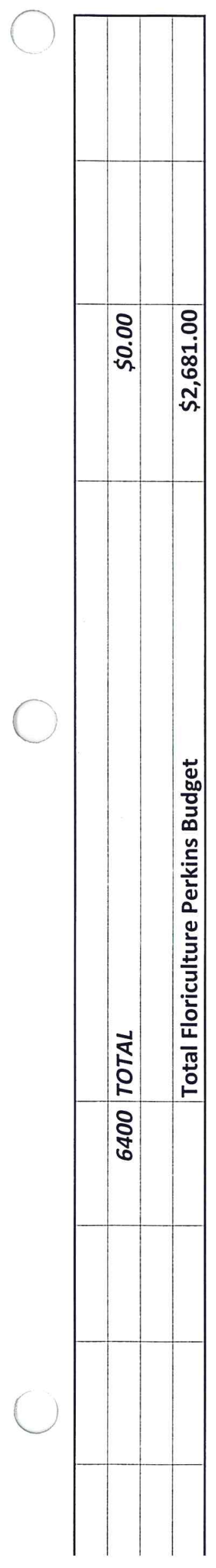


言

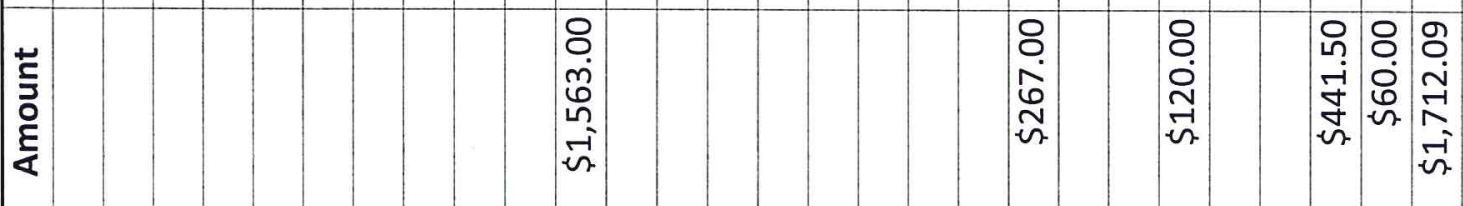

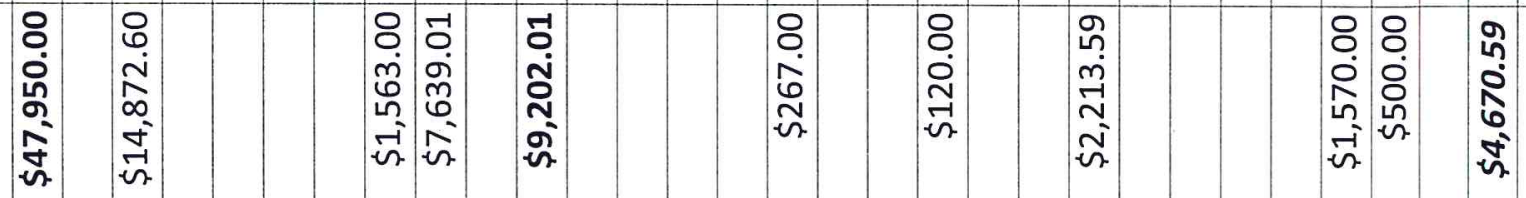

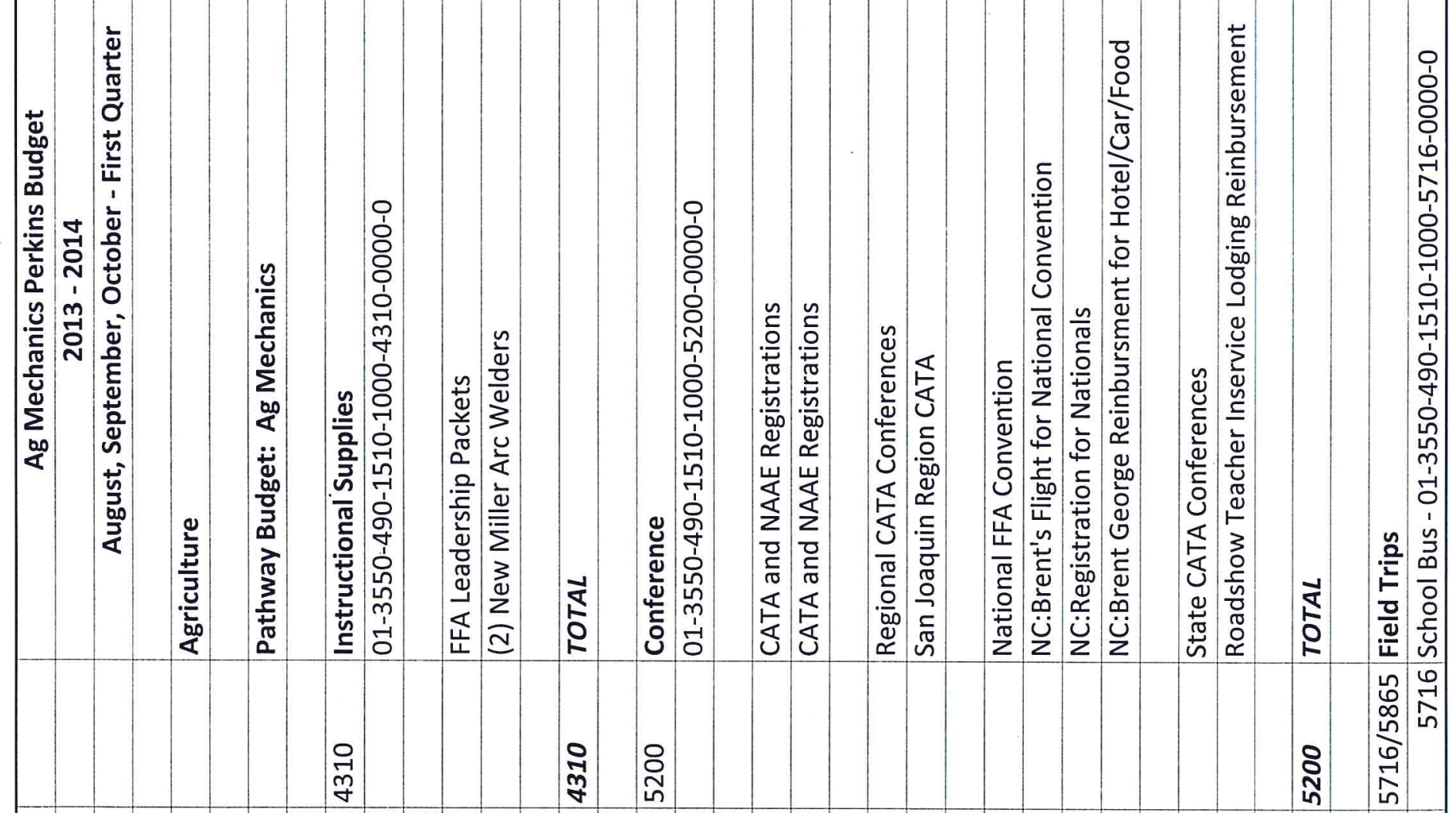

咅

若

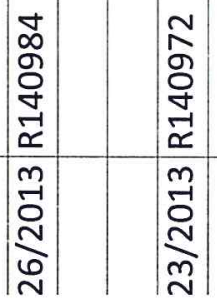




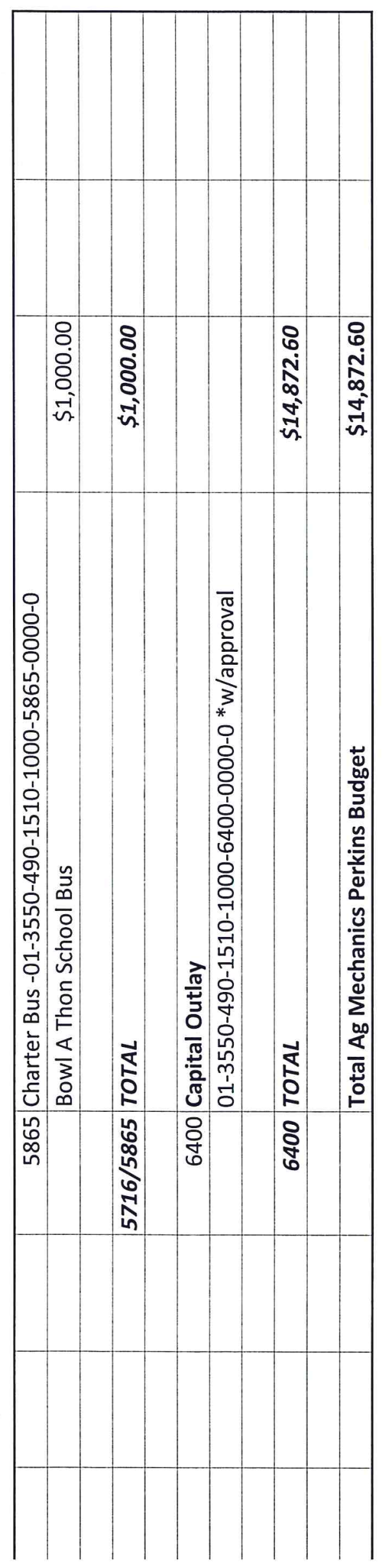




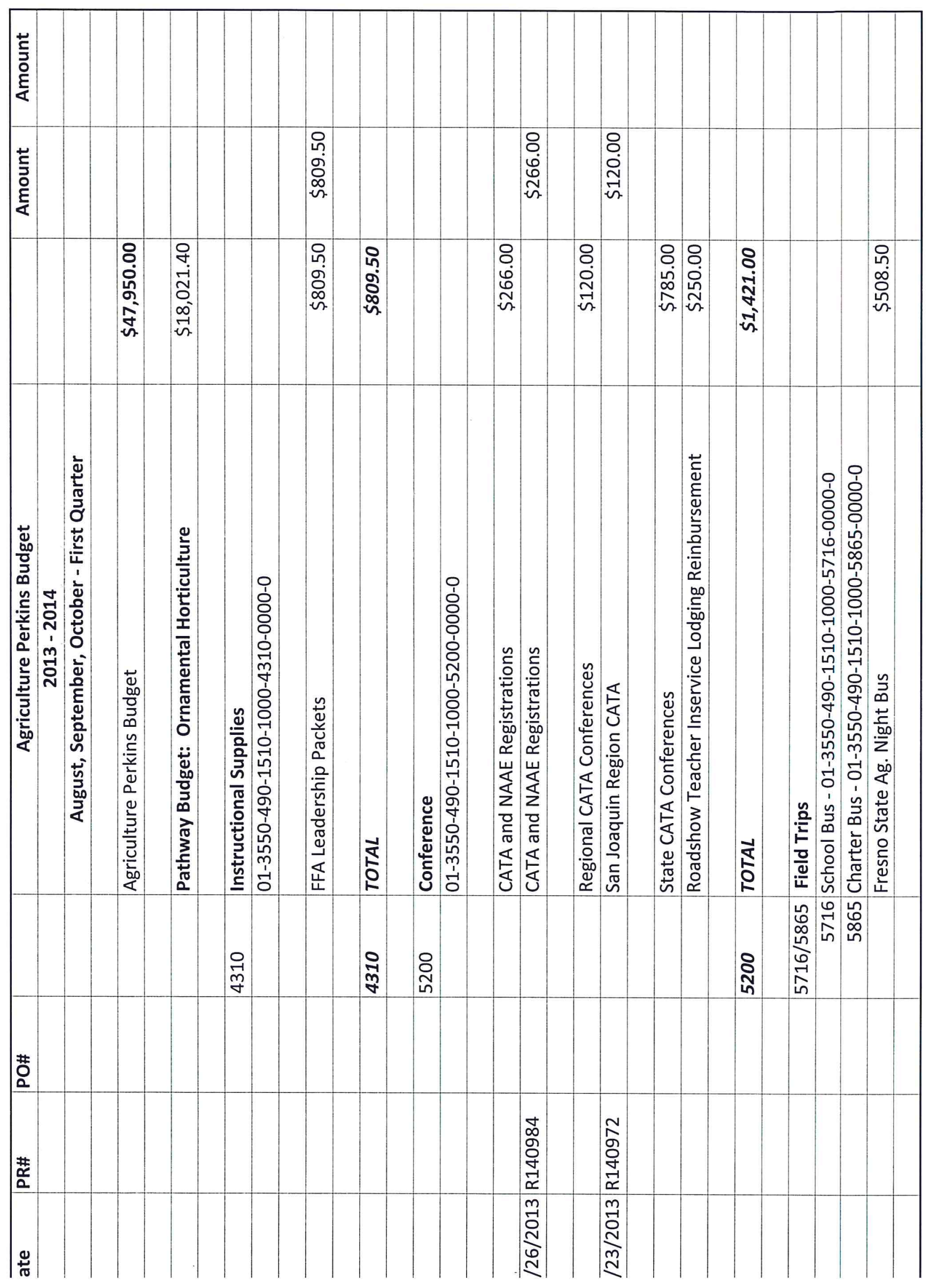


蒿
ô
है

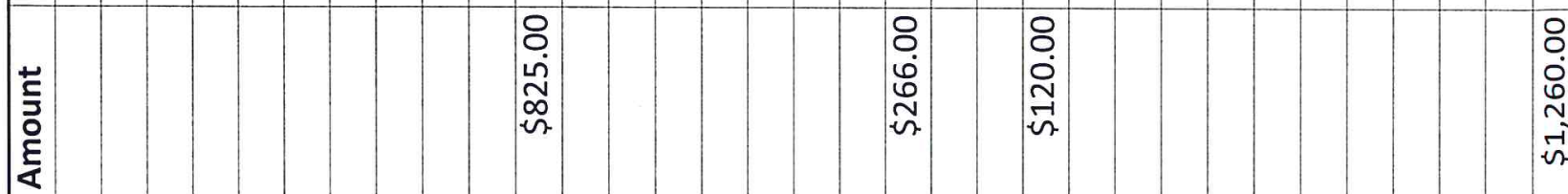

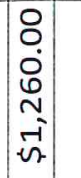

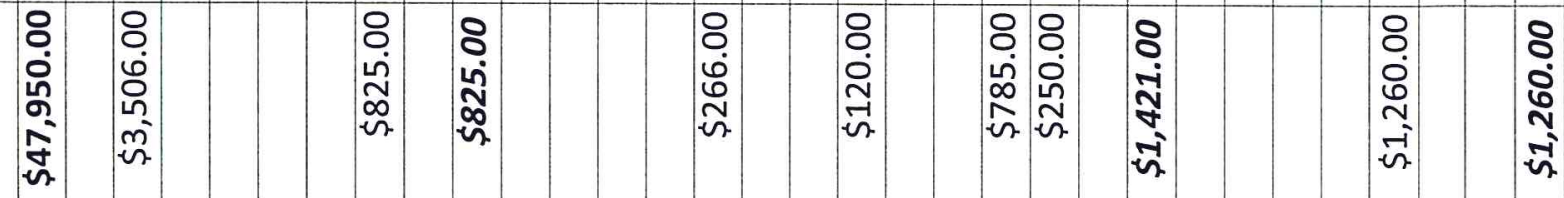

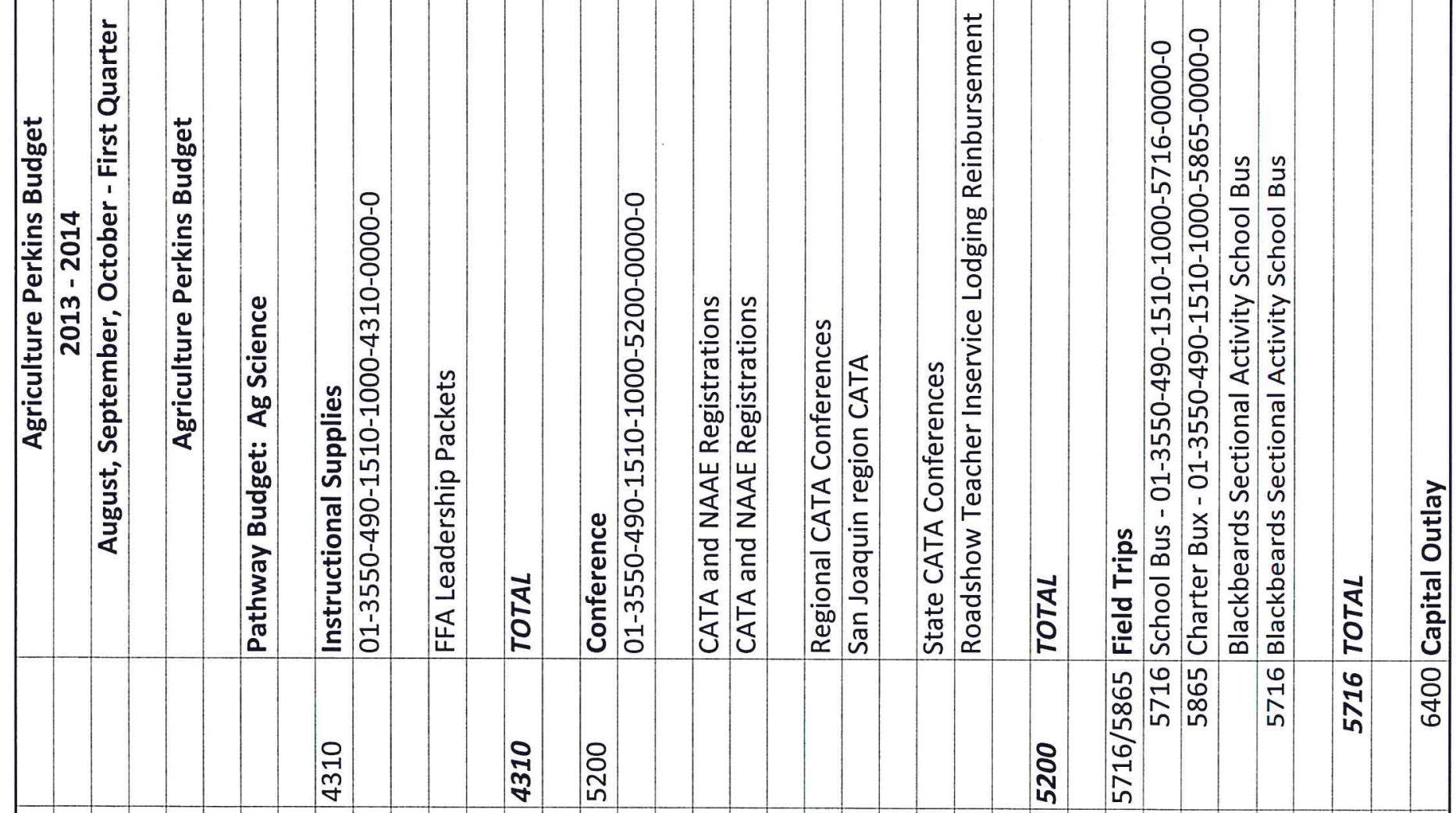

范

管

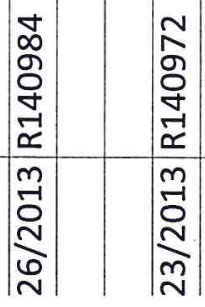

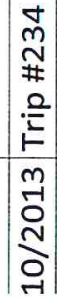




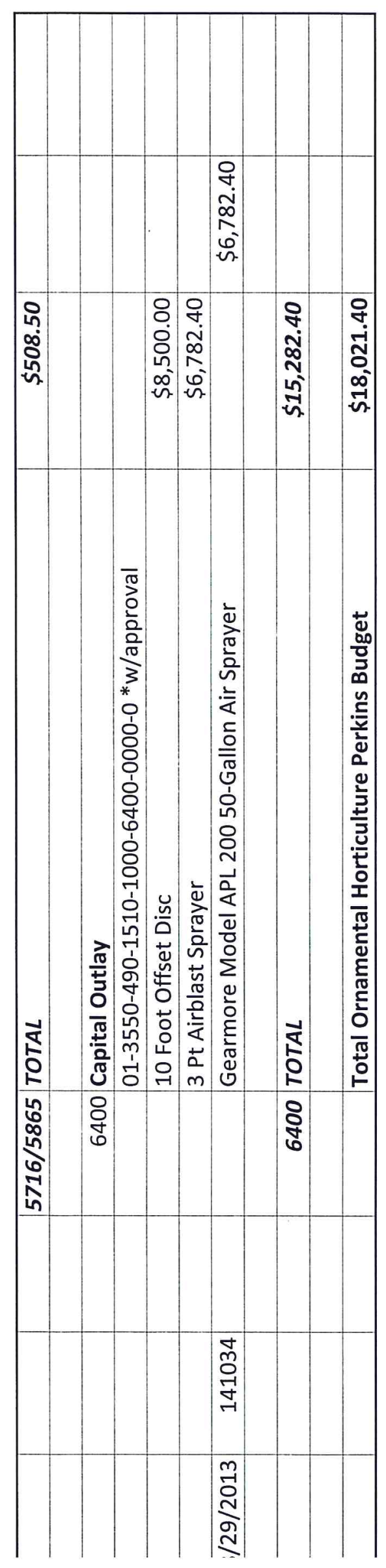


HH. Current Year's VEA District Allocation 


\section{Madera South High School Agriculture Department Budget}

Perkins 2013-2014

42,000

Books/Supplies

3,200

Services/Operating

Expenditures:

Transportation

11,800

Instruction

5,000

Professional Development

New Professionals

National FFA convention

San Joaquin region road show

Regional Meetings

CATA Summer Conference

Substitute's

8,000

8,000

FFA Leadership Packets

6,000

\section{Incentive Grant :}

26,000

Instructional Supplies

16,000

Travel

5,000

Equipment Replacement

5,000 


\section{Brief Description Of Your District/Department Budge Process}




\section{District/Department Budget Process}

Our District has always been supportive of our agriculture program; we get a school site budget of $\$ 8,000.00$ to spend on class supplies. Our Perkins allotment is $\$ 46,000.00$, and we get $\$ 25,000.00$ to spend on needs for our farm and or classroom supplies. They also match our incentive grant through subs, vehicle maintenance and fuel. The district overmatches our AIG funding and it helps us throughout the year to spend the money we get for what we need. We also have ROP budgets of $\$ 9,500.00$ for power mechanics and $\$ 15,000.00$ for the welding program. We spend well over $\$ 100,000.00$ a year on our students and classes.

To open a PO in the district we use a pink requisition forma and we have an ASB account that uses a separate PO form. (See Attached) 


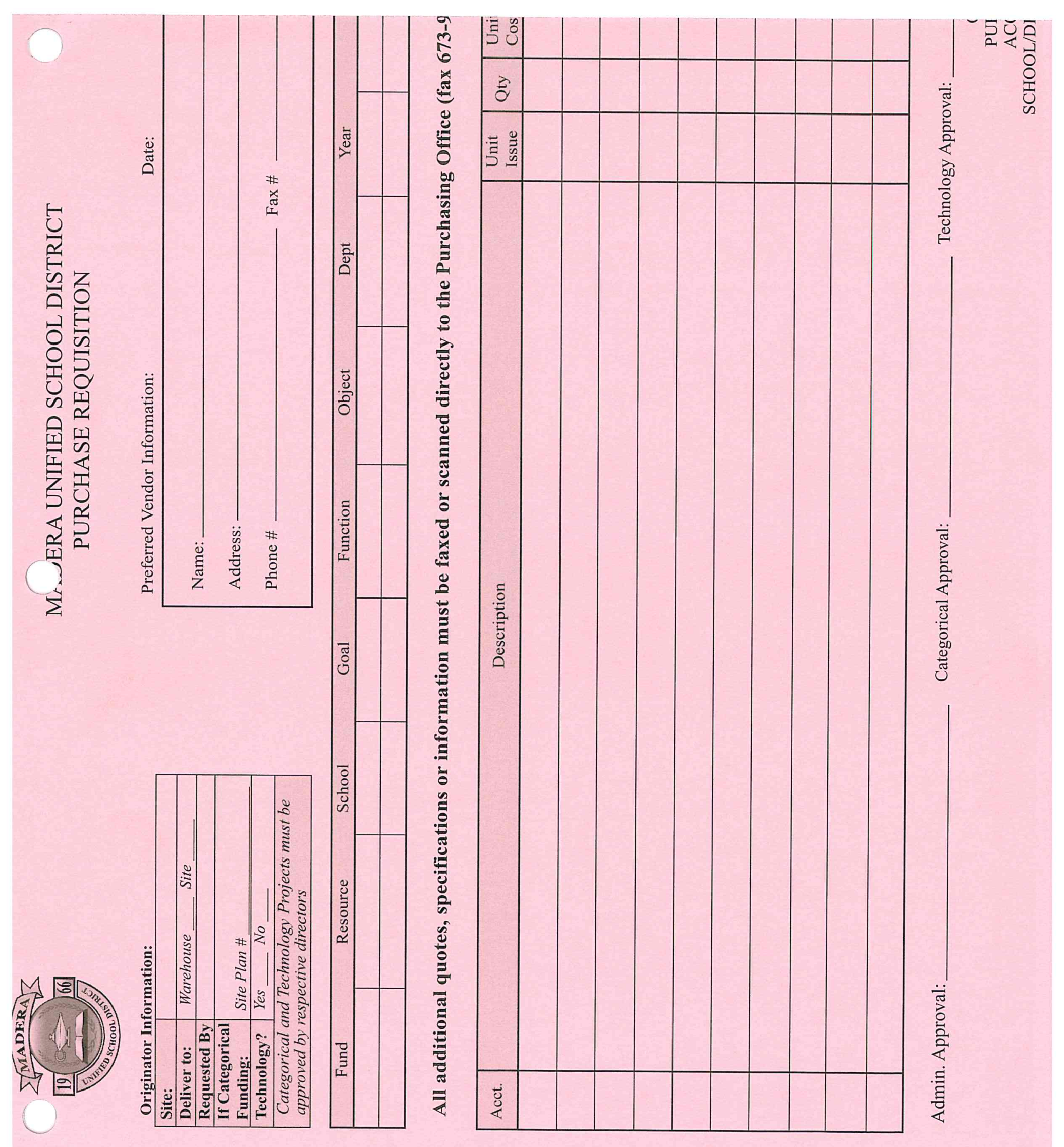




\section{MADERA UNIFIED SCHOOL DISTRICT \\ Madera South High School \\ ASSOCIATED STUDENT BODY \\ PURCHASE ORDER REQUEST/ CHECK REQUEST}

Organization (Club/Sport):

Account \#

Purchase Order $\square$ Open P.O. $\square$ One Time Use

$\square$ Check Request-invoice or original receipt must be attached

ASB is not obligated to pay for an expenditure ordered by a staff member or student who has not first received pre approval using one of the following methods:

$\square$ Purchase Order \#:

$\square$ Item Listed on Yearly Budget

Meeting Minutes Attached* *...lubs on!y.

Vendor Name Phone

Address

Reason for Purchase Order or Check

For Purchase Orders - attach a quote or list items below (Check Requests MUST have invoice attached):

\begin{tabular}{|r|r|}
\hline Quantity & Description \\
\hline & \\
\hline & Total Including Tax \& Shipping \\
Purchase Order DO NOT EXCEED Amount:
\end{tabular}

Date Needed:

Mail to Payee

Amount

Club Advisor/Athletic Coach:

Date:

Club Treasurer/Team Representative:

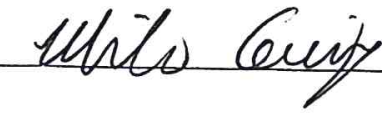

Date:

Activities Director:

Date:

Administrator:

Date:

\section{BUSINESS OFFICE USE:}

P.O. Number:

Check Number:
Vendor Code:

Amount Paid: \$
Date Issued:

Date Paid: 


\section{JJ. Copy Of Department Chairperson's Duties And Responsibilities}


KK. Copy Of Chart Of Responsibilities 

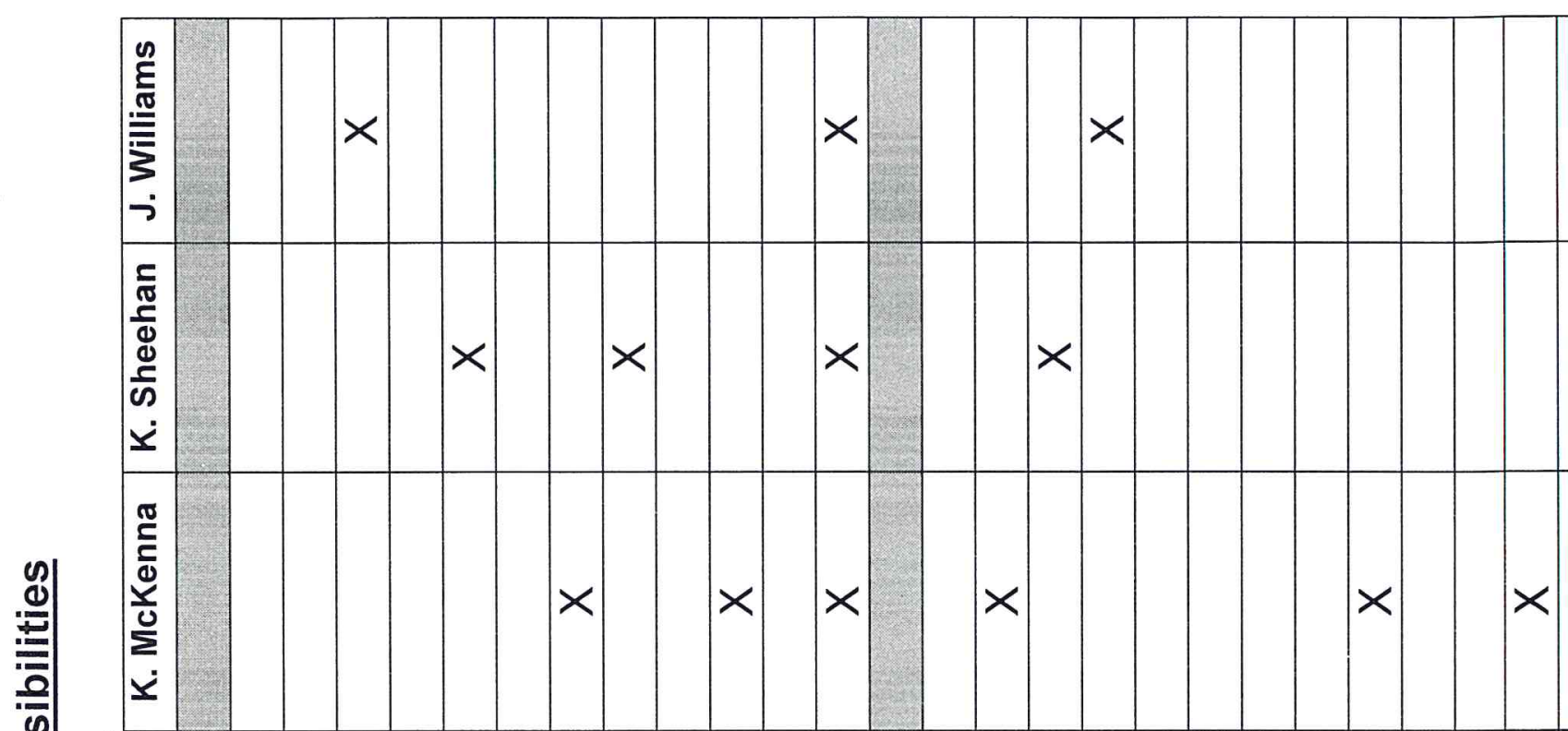

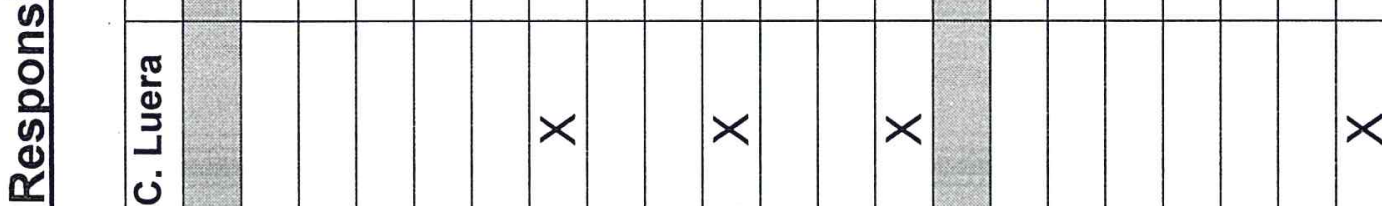

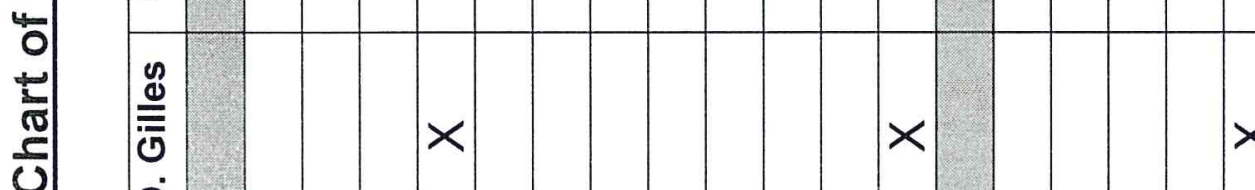

ப.

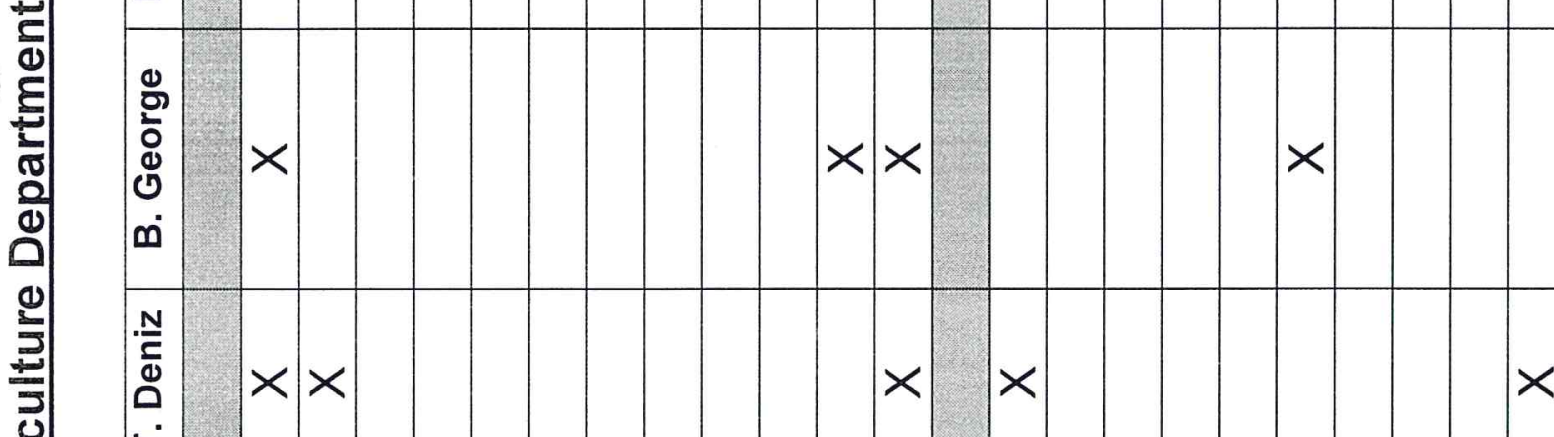

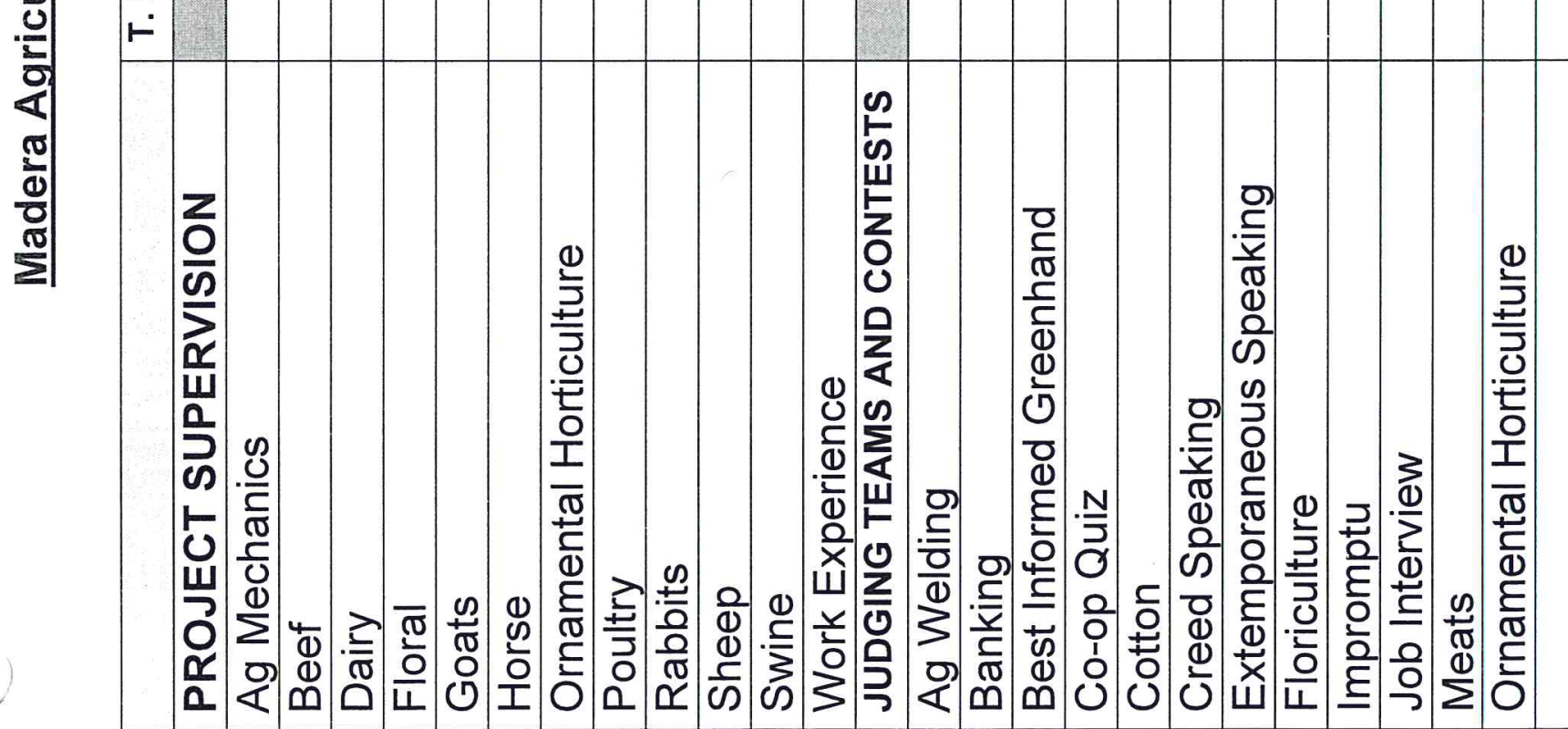



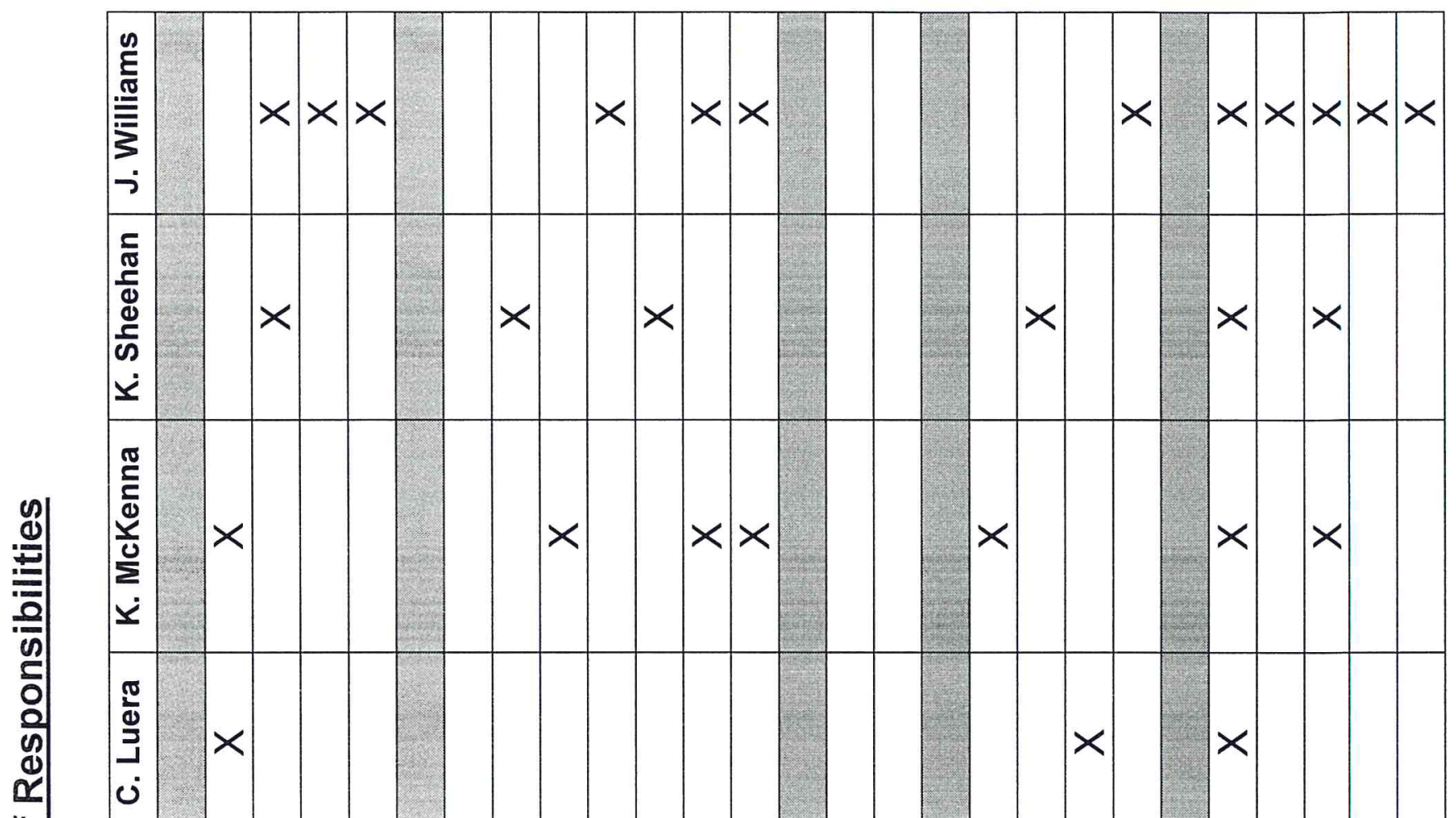

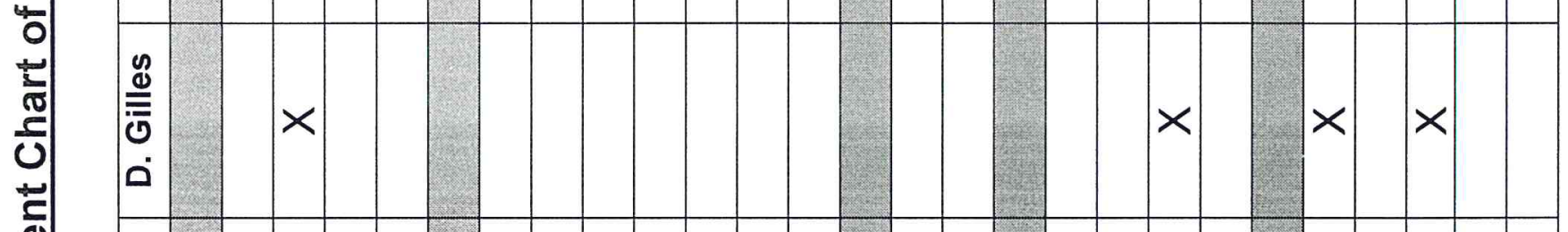

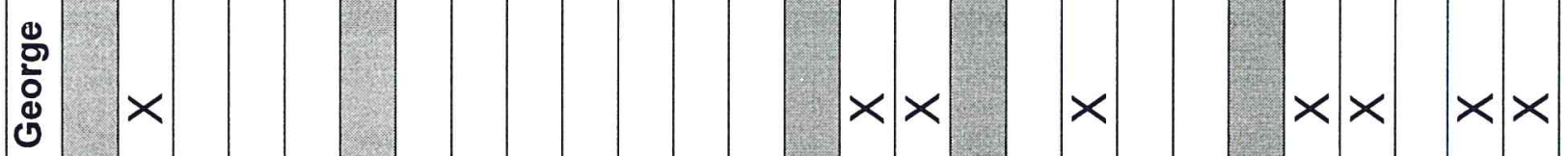

ต

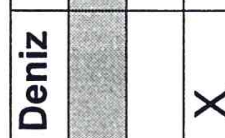

ト

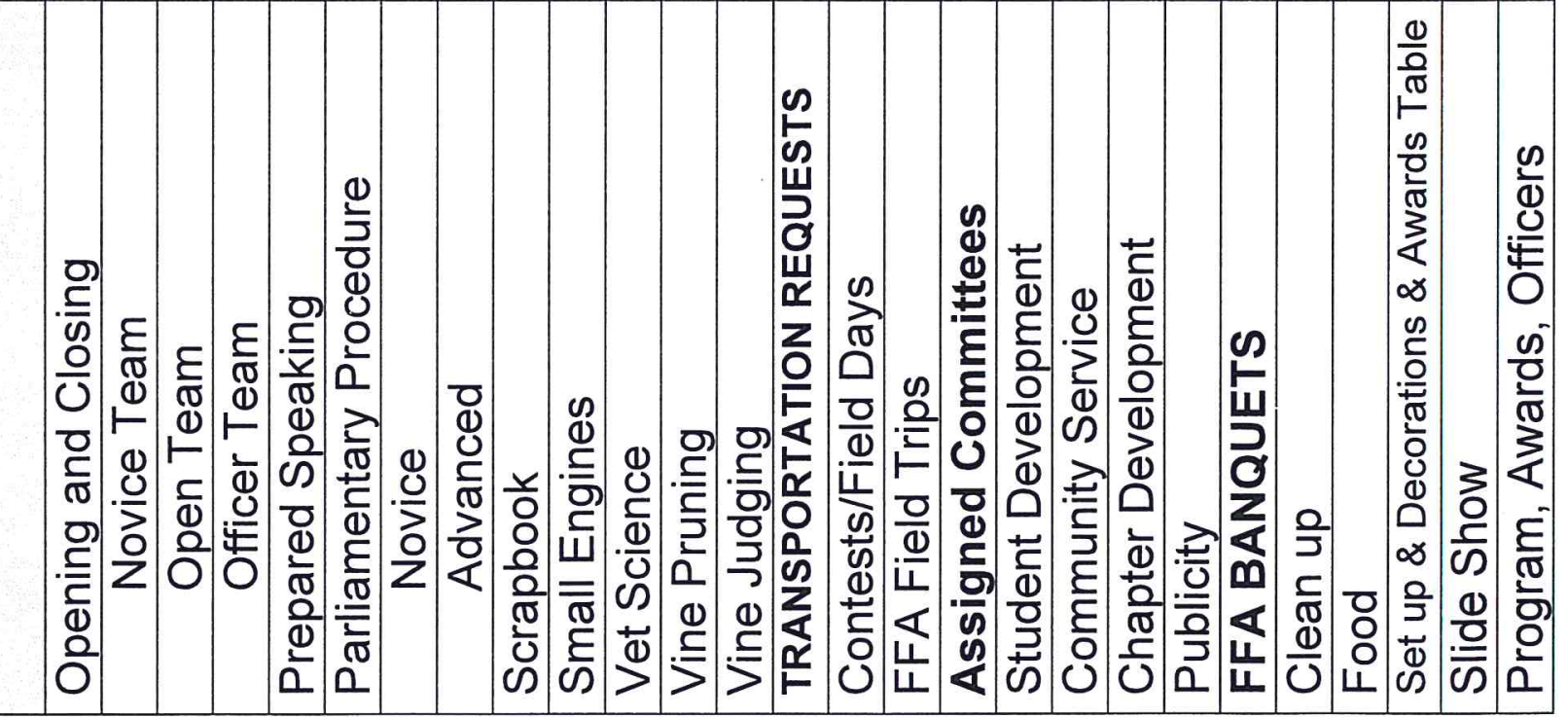




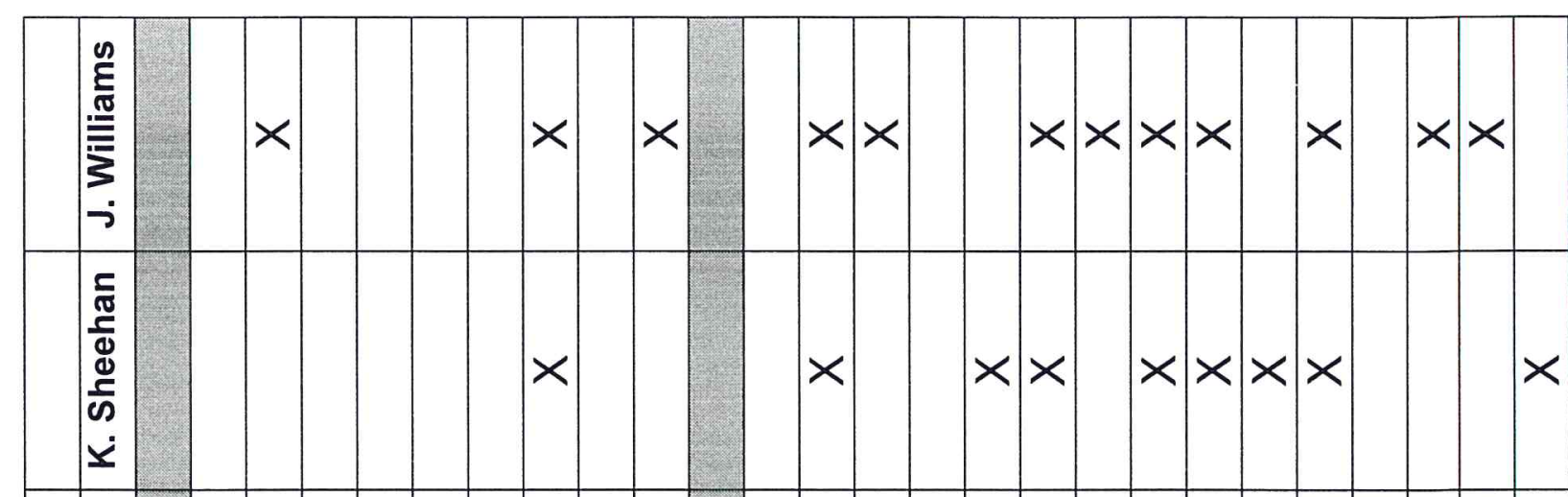

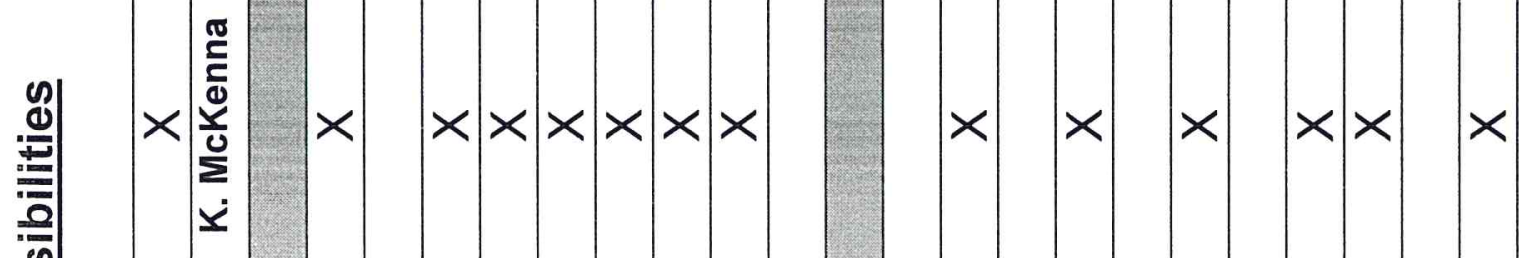

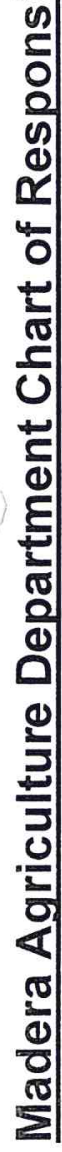

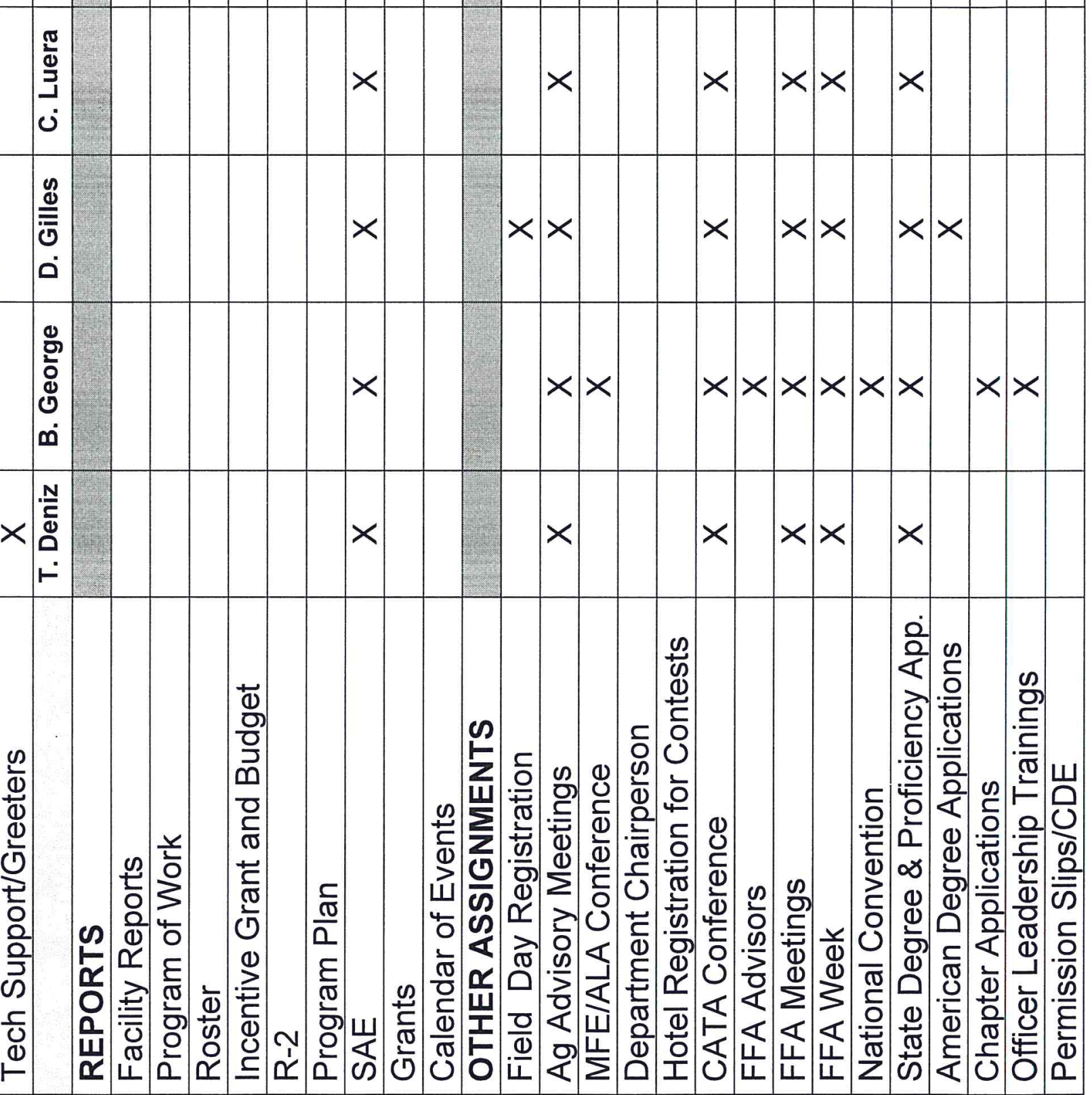



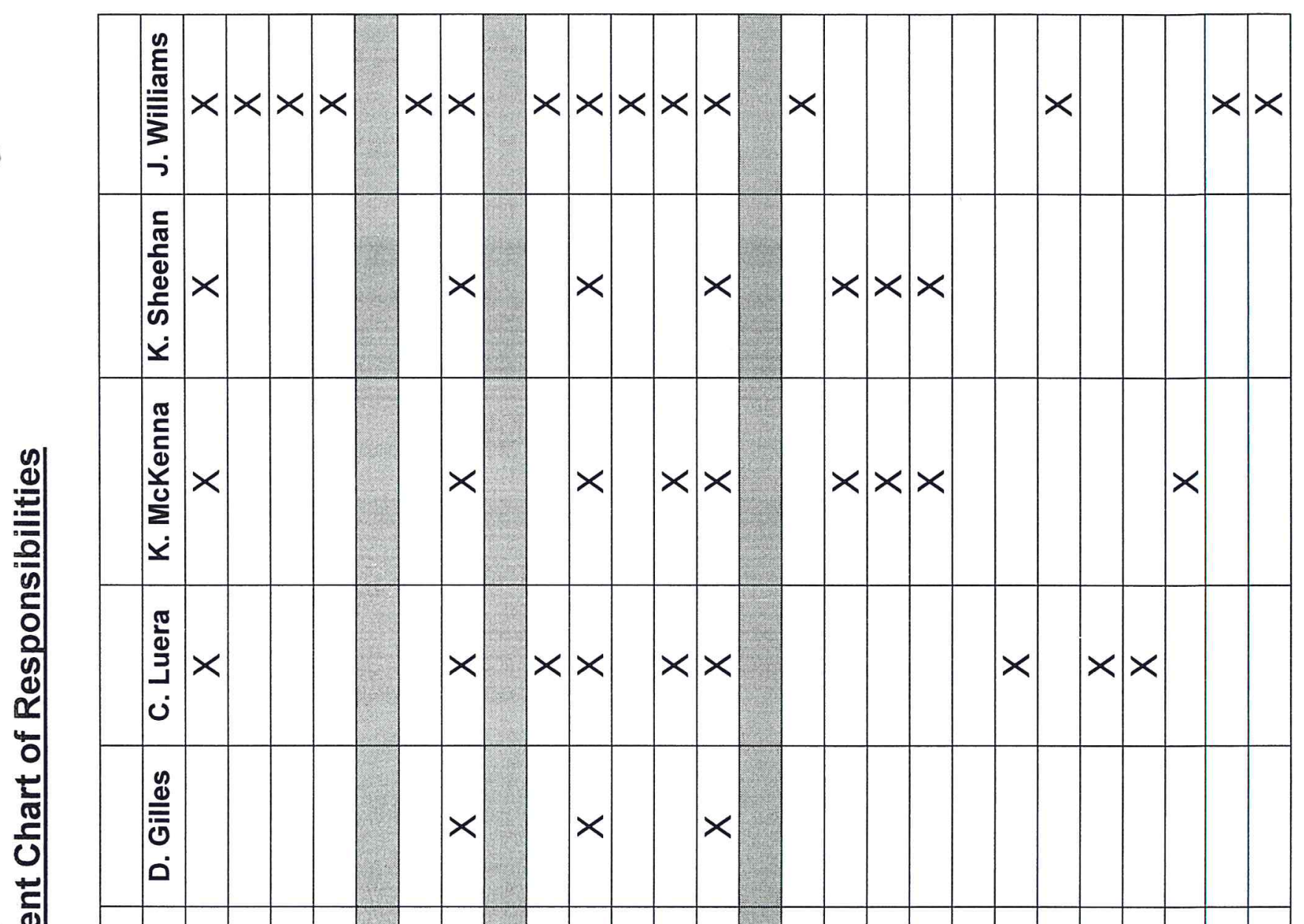
온 ๓ $x \times x \times x \quad x$ $\times \quad x$ $\times \quad \times$

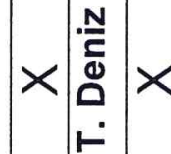
$x \quad x \quad x$ $\times$ $\times$ $\vdash$

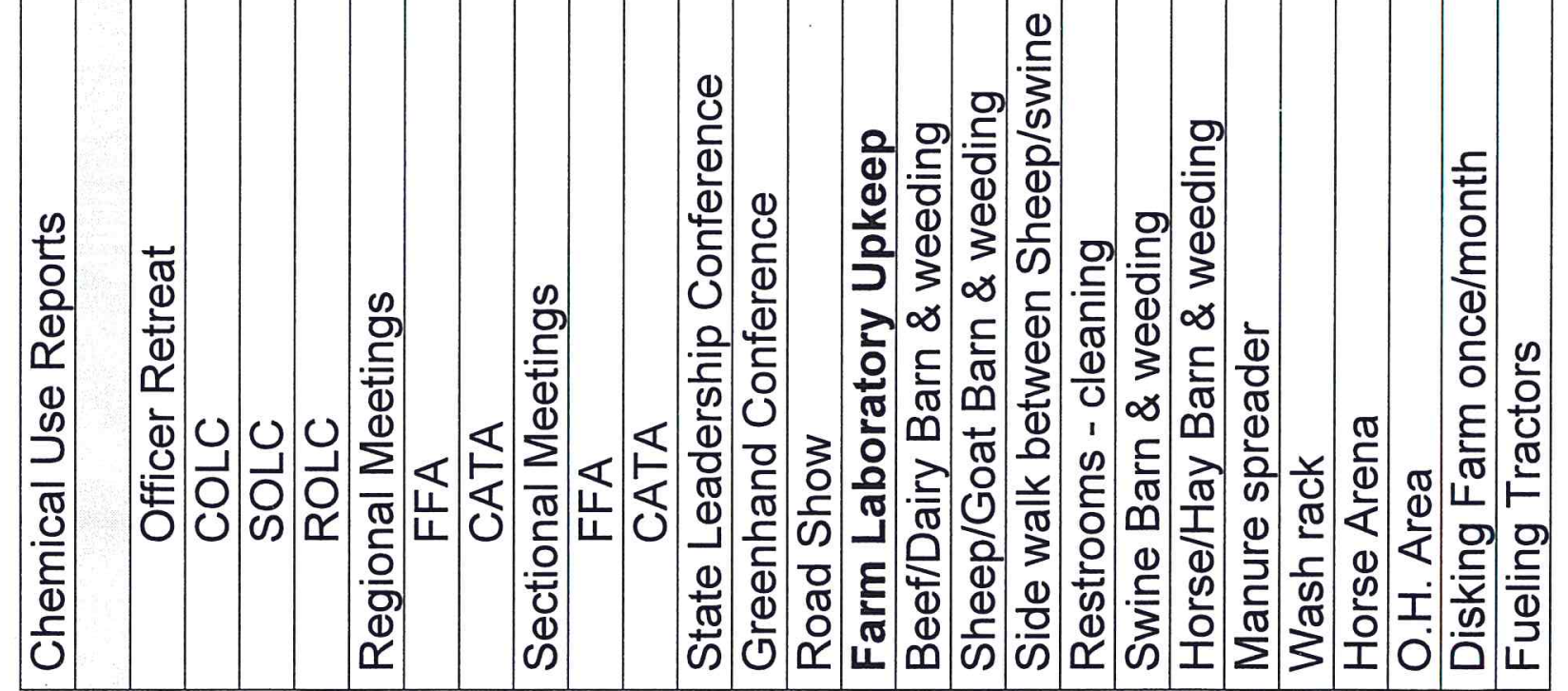



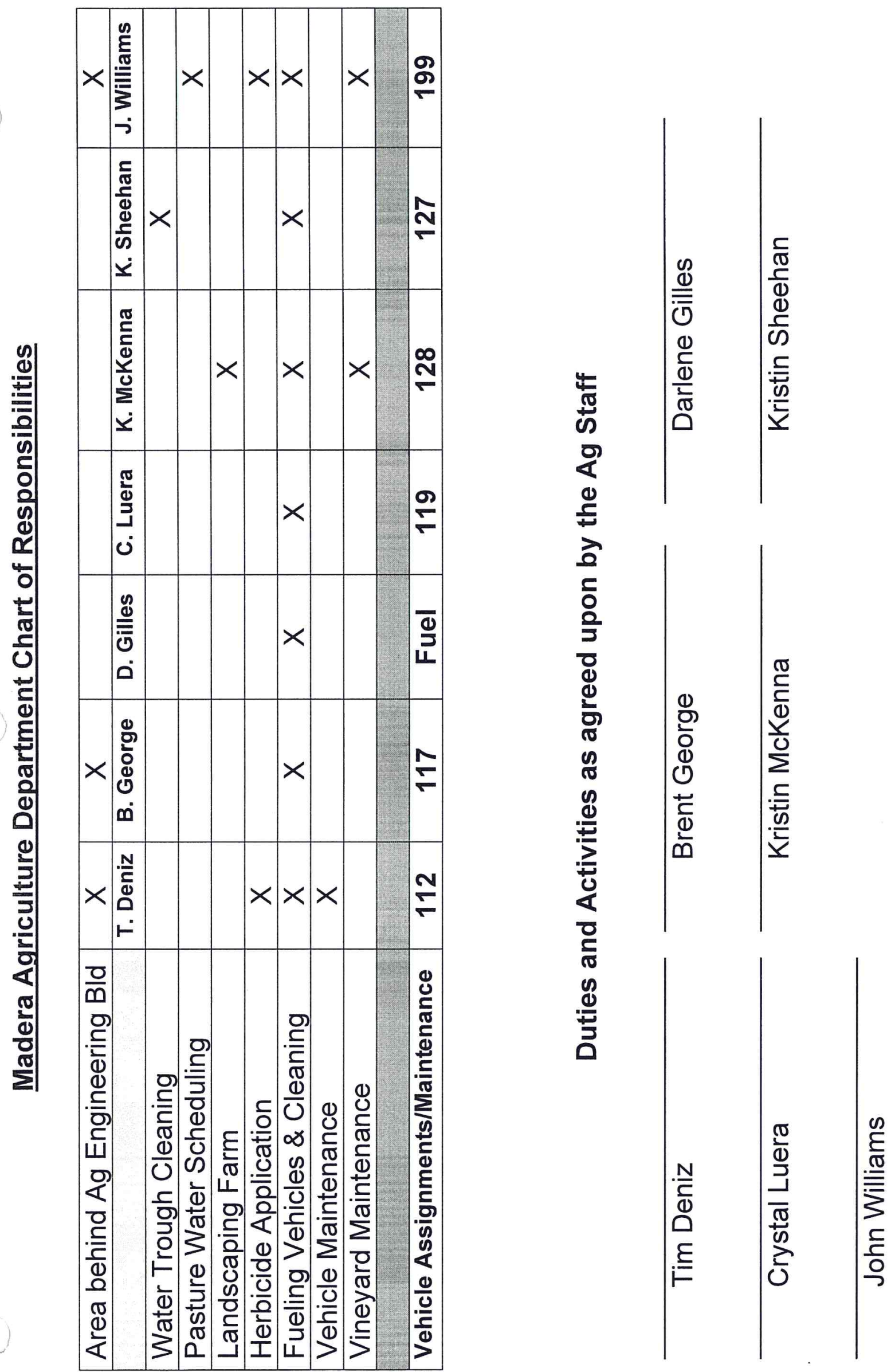


\section{LL. Copy Of Substitute Teacher Procedure And Plans}




\section{Sub Procedures}

1. Take roll using seating chart in roll book

2. Follow sub plans for each period

3. DO NOT ALLOW STUDENTS IN THE SHOP UNSUPERVISED

4. Call cleanup 10 minutes before the bell is supposed to ring

5. Have all students sitting in their seats before excusing them

In case of emergency please call Ext. 1550 to reach the closest administrator. If you have any problems with the students please leave their name on this report and send them to the office. If you have any questions call Ext. 1706 to reach Mrs. Mckenna.

Report:

Follow all school rules and procedures while in this class. 
Thanks Jim for subbing for me the next couple of days. I am at a conference trying to become a better at what I do. The next couple of days will be easy, there are two schools coming the next two days to present their programs to our kids. On Thursday it's Lincoln Tech Center and on Friday, Darryl Fishman will be here to talk about Merced College.

Thursday $11 / 18$

$1^{\text {st }}$ period- Students need to define terms on page 301 and answer questions $1-8$. This is due at the end of the period.

$2^{\text {nd }}-4^{\text {th }}$ Period, the gentleman from Lincoln School of Technology will present his information to these classes. His presentation lasts about 45 minutes and should take up the entire period.

$6^{\text {th }}$ period- Show an episode of Dirty Jobs, the students need to fill out the worksheet that is designed for Dirty Jobs.

Friday $11 / 19$

$1^{\text {st }}$ period - Have students answer questions on page 80 of the textbook. They need to write out the questions and answers.

$2^{\text {nd }}-4^{\text {th }}$ period- Fishman from Merced college will be here to present information about Merced College. He will be in Deniz's class first period, but then he will move done to your class for $2^{\text {nd }}$ through $4^{\text {th }}$ Period.

$6^{\text {th }}$ period- Have students define terms on page 99 of the Ag Mech 1 Textbook. Woodworking is our next unit in this class.

If you have any issues, give me a call, thanks again for covering for me.

Thanks,

John 


\section{Proficiency For Vocational Agriculture Students}


STATE:

Chapter \#

Place Label Here
Member ID \#

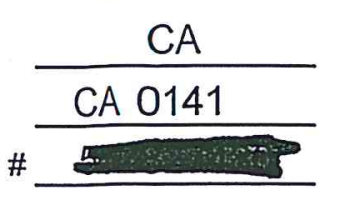

\section{DAIRY PRODUCTION}

Name of Proficiency Award Area

1. Name:

Matthew

Name on chapter FFA roster: (If Different):

2. Date of Birth:

\begin{tabular}{lcl} 
& $11-2-$ & \\
\hline (Month) & (Day) & (Year)
\end{tabular}

3. Age: 17

4. Gender: Male

6. Address: (street address required) City: Madera Female 5. E-mail:

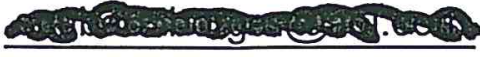

7. Home Telephone number (including area code):

8. Name of Parents/Guardians
a. Father:
Frank A.
b. Mother: Kathleen
Dairy Farmer
Homemaker

Ariso

State:

$\mathrm{CA}$ (559)

10. Complete FFA Chapter Name:

11. Name of High School:

12. School Address: (street/RR./box no.)

School City: Madera

705 W. Pecan Ave

13. School Telephone Number (including area code):

14. Chapter Advisor(s): John Williams

15. Year FFA Membership Began:

16. Years of Agricultural Education Completed:

17. Years of Agricultural Education Offered (grades 7-12) in high school last attended:

18. Year in school at time of applying for the award:

9. List Parents/Guardians Occupation Below:

Madera FFA

Madera South High School

19. If you have graduated from the high school, year graduated:

20. State/National Dues paid?

State:

$\mathrm{CA}$

School Zip:

93637

(559) $675-4450$

We have examined this application and find that the records are true, accurate, and complete. We hereby permit for publicity purposes, the use of any information included in this application with the exception of the following:

$$
\text { Candidate Signature }
$$

Parent or Guardian Signature

In addition, we certify the applicant has achieved a satisfactory record of scholastic achievement.

Chapter Advisor Signature

Superintendent or Principal Signature

(indicate which)

The information contained in this application has been substantiated by an actual visit to the site of the applicant's supervised agricultural experience program.

NOTICE: This application will not be returned by the National FFA Organization. Please make a copy for your records. 


\section{Performance Review}

\section{A. Getting Started in this activity:}

1. Briefly describe your SAE as it is related to this proficiency area. Describe how you started in this proficiency area. What interested and motivated you to begin?

My supervised agriculture experience project consists of working on my father's 5,600 cow.dairy, it's on 650 acres in western Madera County. The dairy has over 33 employees, my responsibilities consists of relief milking, bedding down pens, cleaning barns and treating cows along with numerous other tasks. I currently make $\$ 11.75$ an hour and work after school, weekends and on weekdays during the summer and holidays. I first started this project the summer before my freshman year in high school, I always loved being around the dairy and helping out my dad. So when I turned 14 my father decided to hire me as an employee. My family has always been in the dairy industry dating back to when my grandparents lived in the Azores, it has been the main source of income for our family and it is with great pride that I help my father to support our family.

2. When you were planning your supervised agricultural experience in this proficiency area, what 2 or 3 goals and objectives did you plan to achieve at this point in your development?

Goal \#1- Learn how to milk cows efficiently independently without help from the workers on the dairy. Being independent makes the operation easier for all of the employees because I won't need to have someone supervise me on a daily basis. With practice, I will be able to achieve this goal in a timely manner to increase my efficiency when milking the cows.

Goal \#2- Be able to deliver calves safely to increase the health of the new born calves. The entire dairy is based on keeping the animals healthy to provide safe milk. To achieve this goal, I will need to work with the herdsman and learn all of his techniques to increase the health of the future cows. The calves are very important to the future of the dairy.

Goal \#3- Learn how to use the equipment to increase the comfort of the herd on the dairy. The equipment at the dairy is crucial in keeping up an efficient operation. With lots of hours of practice, I will be able to be efficient on the equipment, there for the cows can be more comfortable and produce more milk.

\section{B. Progress:}

1. Describe any special advantages or disadvantages that had a major impact on your achievements in your supervised agricultural experience program.

In life, my ultimate goal is to own and operate a dairy farm of my own. I have lived on my parent's dairy farm my whole life. This is a special advantage because whenever there is a problem at the dairy $I$ am only a few minuets away from fixing the problem. Also with my father's vast knowledge of dairy cattle, he has played a major role in my employment success at Fabland Farms. My parent's have been very supportive of my decision to work for them because they know that working with dairy cattle is something that I enjoy. I started working with dairy cattle at a young age while was showing at fairs. By doing this I have been able to spot things that affect dairy cattle. Now I use my knowledge on a larger scale. Also by attending various dairy industry seminars I have also acquired knowledge of how the industry functions. My father and I also attend workshops that range from anything from heard health, cow comfort, business management, and many more. With all of this newly acquired knowledge it will benefit me in the future when I attend college and start working in the dairy industry. In my Supervised Agriculture Experience there has only been one disadvantage that I can recall. The major disadvantage I have had was learning how to properly give Dextrose to dehydrated cows. This was because the needle has to go straight into the vain; when I first started I had a difficult time trying to penetrate the vain. Once I over came this, my job in the hospital pen became much easier. 


\section{B. Progress (continued)}

2. Briefly describe your placement in this proficiency area. (Include a description of the business/ farm, working conditions, size, number of employees, type of facilities, equipment available, etc.)

I have been working at Fabland Farms since June of 2007. The dairy is located on 650 acres in Western Madera County. We milk 2,700 cows and we have an additional 2,900 head of young stock, making the dairy one of the largest in the county. The cows are milked in a double thirty parallel milking parlor. I don't have a set position on the dairy; rather I perform any task that needs to be done. During my milking shifts, there are four people in the milking pit, and one cow pusher. There are thirty three full time employees on the dairy. I spend more than seventy hours on the dairy per week during the summer. On weekends when I don't have FFA activities I relief milk both Saturday and Sunday, and the milking shift lasts twelve hours. During the summer I spend almost everyday on the dairy; I show up to work at six in the morning and I don't leave until four in the afternoon. When I first started working I got paid $\$ 9.25$ an hour, every year my pay goes up $\$ 1.25$ since I learn how to do new tasks and thus become more valuable to the operation.

\section{How has your position description and/or responsibilities changed during the time of your placement?}

Since the start of my employment at Fabland Farms over three years ago, my responsibilities have not changed. I did the bulk of my dairy training in my first year of my employment in 2007 . This prepared me to perform any tasks that I need to do in the future. My responsibilities on the dairy include relief milking, bedding freestalls, cleaning the maternity barn, pulling calves, hauling bedding, and assisting the vet when she comes to the dairy on a weekly basis. In the future I hope to obtain a more challenging role on the dairy, although I consider my current role challenging, I want to broaden my horizons. The position I really want to try, is feeding all the cows on our dairy. Preparing feed rations is something that I have not learned how to do yet. Since we have over 5,600 cows, feeding that many is going to be a challenge. By doing this, I will be able to take the next step in my goal of learning all the aspects of dairy farming.

\section{Analysis/Evaluation of Program}

1. Describe your level of achievement and progress towards your goals (such as skills, scope, etc.) in this award area as related to the goals and objectives described on page 2, question 2.

The goals that I have chosen have been a work in progress since I started this project. My first goal was to be able to milk cows efficiently without the help of the workers on the dairy. Since we work 12 hour days, I have had a lot of practice to be able to do this. Whenever it is time for me to milk, I try to keep up with the person next to me, I am more efficient than when I first started, but there is still room to improve. My second goal was to be able to deliver calves, since the dairy averages 9 calves a day; I am able to assist in any way possible when I am at the dairy. I am able to help deliver calves on my own when needed. My third goal is to be able to use equipment to increase the comfort of the herd. I have been trained on all of the equipment on the dairy, now $i$ am working on increasing the efficiency at which I am using the equipment to lay almond and rice hulls.

2. Describe the personal goals, educational goals, and career goals you would like to achieve in the next ten years.

My first goal is to graduate high school with above a 3.5 Grade Point Average. In order to accomplish this I must give every class my full effort and attention. Upon completion of high school in June 2011, I would like to attend Cal Poly San Luis Obispo or California State University, Fresno and major in dairy science with a minor in farm business management. With all of the knowledge and skills that I have I about the dairy industry I will have good foundation going into the program and will be able to learn about the more advanced topics. I would like to graduate from college in June 2016 and immediately purchase the dairy farm from my father, so I can begin my future career. I would like to maintain at least a herd of 5,000 cows and be milking at least half of those at any given time. Because I believe strongly in the community of Madera I intend on continuing my involvement with the Madera FFA Chapter by helping to coach a Dairy Cattle Judging Team. I will also make myself available to the Dairy Advisor at Madera South to help with the Dairy Replacement Projects for the county fairs. 
. Scope, Income and Expense Summary for : DAIRY PRODUCTION

Placement and Research Experimentation Type Supervised Agricultural Experience Program

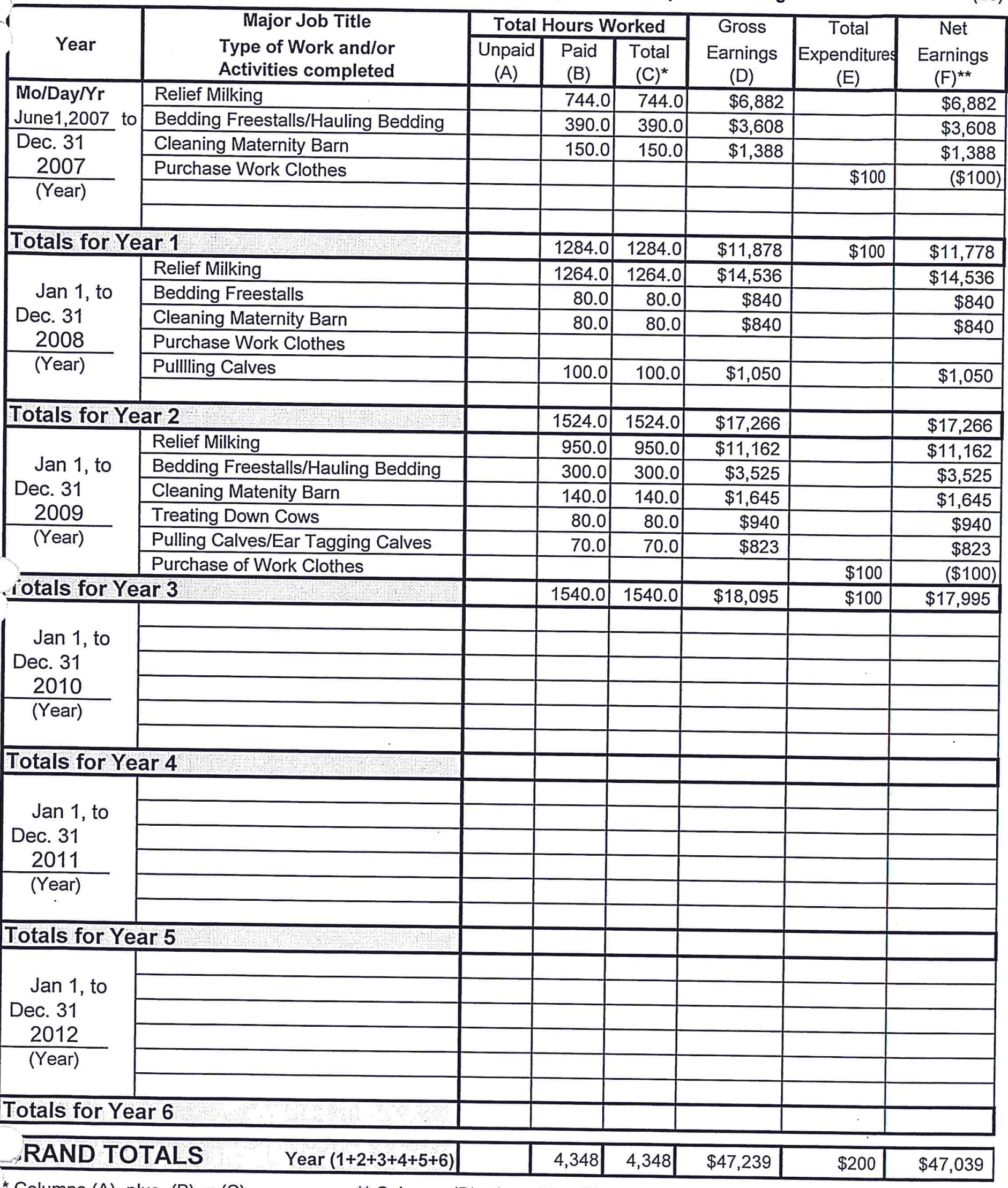

\footnotetext{
${ }^{*}$ Columns $(A)$ plus $(B)=(C)$

${ }^{* *}$ Columns $(D)$ minus $(E)=(F)$
}

DO NOT ALTER APPLICATION IN ANY WAY or APPLICATION IS SUBJECT TO DISQUALIFICATION!

Our House Enterprises 


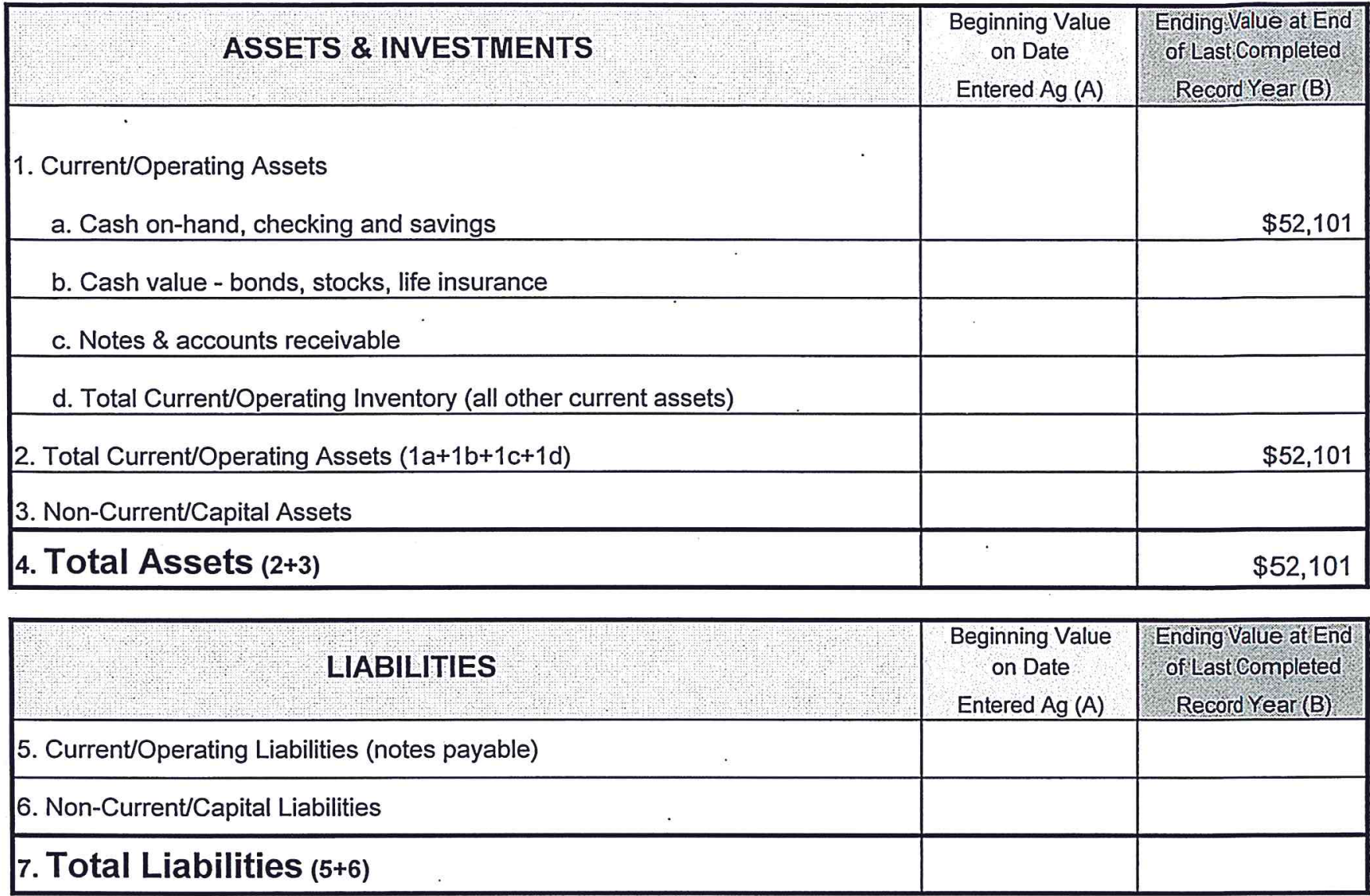

8. NET WORTH (4 minus 7)

$\$ 52,101$

\begin{tabular}{|c|c|c|}
\hline SUMMARY OF SOURCE AND USE OF FUNDS & $\begin{array}{l}\text { Beginning Value } \\
\text { on Date } \\
\text { Entered } \mathrm{Ag}(\mathrm{A})\end{array}$ & $\begin{array}{l}\text { Ending Value at End } \\
\text { of Last Completed } \\
\text { Record Year (B) }\end{array}$ \\
\hline 9. Earnings from this proficiency area & $X X X X X X X X X X X X$ & $\$ 47,039$ \\
\hline 10. Other SAE earning NOT from this area & $X X X X X X X X X X X X$ & $\$ 13,093$ \\
\hline 11. Earnings from non-SAE activities & $X X X X X X X X X X X X$ & $\$ 2,500$ \\
\hline 12. Income other than earnings & $X X X X X X X X X X X X$ & \\
\hline 13. Total Earnings $(9+10+11+12)$ & $X X X X X X X X X X X X$ & $\$ 62,632$ \\
\hline 14. Use of Funds & $X X X X X X X X X X X X$ & \\
\hline a. Total educational expenses & $X X X X X X X X X X X X$ & $\$ 8,400$ \\
\hline $\begin{array}{l}\text { b. Total other personal expenses (Includes local, state \& federal income } \\
\text { tax and FICA) }\end{array}$ & $X X X X X X X X X X X X$ & $\$ 2,131$ \\
\hline 15. Total use of funds $(14 a+14 b)$ & $X X X X X X X X X X X X$ & $\$ 10,531$ \\
\hline
\end{tabular}




\section{Skills and Activities}

DAIRY PRODUCTION

\section{A. Skills}

List your top six placement skills and give a brief description of each one and its contribution to the success of your supervised agricultural experience program.

1.Skill Number One.

\begin{tabular}{|c|l|c|c|}
\hline Year & \multicolumn{1}{|c|}{ Skill } & Where Attained & Student Hours \\
\hline 2007 & $\begin{array}{l}\text { Proper milking techniques and procedures help in } \\
\text { preventing mastitis and stress on the cows. }\end{array}$ & Fabland Farms & 100 \\
\hline
\end{tabular}

Description of Skill:

In order to make sure the cow is properly milked there are certain steps a milker must follow. When a cow enters the parlor, she is first sprayed with a pre-dip solution of $1 \%$ iodine that rids her udders of any uncleanness; I let the solution sit for two minutes, while I am applying the pre-dip to the other cows. Next, I come back to the first and make sure the udders are properly dispensing milk. Then I check the udders, I apply the machine starting with the back udders and then I attach the machine to the front udders. Once the cow is done milking, I apply a post-dip $1 \%$ iodine solution to prevent diseases such as mastitis. As one string leaves the barn, I use the same technique on every cow and string that comes through.

\section{Skill Number Two.}

\begin{tabular}{|c|l|l|c|}
\hline Year & \multicolumn{1}{|c|}{ Skill } & Where Attained & Student Hours \\
\hline 2007 & $\begin{array}{l}\text { Increasing cow comfort which in turn prevents diseases } \\
\text { and increases milk production. }\end{array}$ & Fabland Farms & 250 \\
\hline
\end{tabular}

\section{Description of Skill:}

In order for a cow to reach her full milk production potential, she needs to remain as comfortable as possible. At Fabland Farms, I bed all the freestall barns with almond shells. After the beds are filled, I come back through with a tractor that has a freestall rake attachment. By doing this the almond shells get raised up and become fluffier. All this is done while the string of cows is in the milking barn, so when they return to the pen they have fresh bedding. The freestall barns are re-filled every five days, and they are raked every day. By keeping the freestall beds clean cow comfort goes up which in turn increases milk production. Also since the cows are on a soft surface the udders stay clean and mastitis cases have decreased by $65 \%$. This has raised our milk production and decreased our medical expenses.

\section{Skill Number Three.}

\begin{tabular}{|c|c|c|c|}
\hline Year & Skill & Where Attained & Student Hours \\
\hline 2008 & $\begin{array}{l}\text { Identifying cows with medical problems, and proper } \\
\text { administration of medicine to cows in the hospital pen. }\end{array}$ & Fabland Farms & 75 \\
\hline \multicolumn{4}{|c|}{ Description of Skill: } \\
\hline \multicolumn{4}{|c|}{$\begin{array}{l}\text { When a cow in the hospital pen looks stressed, or in pain, I must first diagnose the problem. There are many different } \\
\text { ways to identify a sick or distressed cows. They can exhibit signs such as delayed movements, bloating, eye and ear } \\
\text { placement, discomfort with movement, or other indicators. Once I can see that something is wrong with the cow I } \\
\text { immediately move her to the hospital pen. Next, I either administer antibiotics, or wait a while to see if the problem can } \\
\text { fix itself. When working in the hospital pen I have many medicines at my disposal to take care of the cows illness. With } \\
\text { all the hours I have put in, this has given me a larger understanding of different ways to identify medical problems. } \\
\text { Jhen I have my own dairy in the future I can reduce my medicine costs and decrease my cull rate. }\end{array}$} \\
\hline
\end{tabular}




\section{A. Skills (continued)}

List your top six placement skills and give a brief description of each one and its contribution to the success of your supervised agricultural experience program.

4.Skill Number Four.

\begin{tabular}{|c|l|l|c|}
\hline Year & \multicolumn{1}{|c|}{ Skill } & Where Attained & Student Hours \\
\hline $2007-2009$ & $\begin{array}{l}\text { Delievering healthy live calves and proper nursing } \\
\text { procedures. }\end{array}$ & Fabland Farms & 110 \\
\hline Description of Skill: & & \\
\hline
\end{tabular}

At Fabland Farms we have an average of eight calves born each day. We bed our maternity barn with rice hulls, so when the cow is ready to calve she has a soft surface to calve on. When the calf is born it will have a warm, soft surface, to lie on until I am able to come and move it to the calf hutch area. By doing this the calf has a better chance of surviving its first few hours. After I move the calf to the hutch I immediately bottle feed the calf 24 liters of colostrum. The colostrum contains many vitamins and minerals that are critical for the calf to be healthy and to grow. I take great pride in this particular task because I am making sure that our future herd is healthy and strong. In the future we plan on raising our own calves, so I have been going ranch where we send our calves and see how they operate in order to get a better understanding.

\section{Skill Number Five.}

\begin{tabular}{|c|l|c|c|}
\hline Year & \multicolumn{1}{|c|}{ Skill } & Where Attained & Student Hours \\
\hline 2008 & $\begin{array}{l}\text { Maintain maturnity barn to prevent disease and increase } \\
\text { comfort for cows and calves. }\end{array}$ & Fabland Farms & 370 \\
\hline
\end{tabular}

At a given time, our maternity barn could have up to 30 cows calving. This can cause many problems for the calves and cows. One of my jobs is to maintain the maternity barn to prevent disease from spreading to the new calves and increase comfort to the cows. The barn needs to be cleaned weekly so that the calves being born. will have a clean environment. After the barn is cleaned, we lay rice hulls on the ground to keep the new calves warm and increase the comfort to the cow. To do this job, I use our M9000 Kubota tractor with a 6 foot box scraper. The rice hulls arrive every week as needed. This is a very important aspect to my job because the new calves need to be comfortable before we move them to the calf hutch.

\section{Skill Number Six.}

\begin{tabular}{|c|l|c|c|}
\hline Year & \multicolumn{1}{|c|}{ Skill } & Where Attained & Student Hours \\
\hline 2009 & $\begin{array}{l}\text { Using proper equipment operating procedures to haul feed } \\
\text { and fertilizer. }\end{array}$ & Fabland Farms & 50 \\
\hline
\end{tabular}

Due to our dairy's large number of cows, we have a lot of excess manure. Once I scrape up all the manure I load it up into our manure truck and haul it across the street to our ranch to be used as fertilizer for our forage at a later time. By cleaning up the excess manure, the cows are more comfortable and the cases of disease decrease. On our forage farm, we produce more feed than we can use at one time, so after the forage is harvested, it is put into a bagging machine that seals all the forage into a bag so it doesn't spoil. When we need more feed for our heifers or milk cows, I

l' ad up our dump truck and haul the feed to the silage pit where the feeder then uses it. These skills have taught me at in order to run a successful dairy, successful crop yields are needed for your cows to increase their milk production. Also by growing your own crops, your expenses decrease by not having to rely on outside sources. 


\section{B. Activities}

List your top three placement activities and give a brief description of each one and its contribution to to the success of your supervised agricultural experience program.

1.Activity Number One.

\begin{tabular}{|c|l|l|c|}
\hline Year & \multicolumn{1}{|c|}{ Activity } & Where Attained & Student Hours \\
\hline $2007-2009$ & Relief milking on weekends. & Fabland Farms & 2958 \\
\hline
\end{tabular}

\section{Description of Activity:}

At Fabland Farms we have two milking shifts from twelve in the morning to twelve at night. We have four milker's in the milking pit, and one cow pusher who bring the cows to the barn from their separate strings. On the weekend I serve as the relief milker. I arrive at the dairy on Friday night and work until twelve in the afternoon on Saturday, and then I come back at the same time on Saturday and work until twelve on Sunday afternoon. In the milking pit, we have certain procedures we must follow to keep the 2,700 milking cow operation running smoothly. Each milker has a separate station he manages. I have been trained to fix any mechanical problems that might occur during my twelve hour shift. If the problem is out of my capability our repairman is only a phone call away, however if I am able to fix the problem myself, Fabland Farms saves money.

\section{Activity Number Two.}

\begin{tabular}{|c|l|l|c|}
\hline Year & \multicolumn{1}{|c|}{ Activity } & Where Attained & Student Hours \\
\hline $2007-2009$ & Bedding Freestalls with almond shells. & Fabland Farms & 770 \\
\hline
\end{tabular}

Description of Activity:

During the duration of my work at Fabland Farms, I always strive to improve our cow's milk production. One way I do this is in the freeestall barns at our dairy, they are filled with bedding every five days. We use almond shells as bedding. This is one of my primary tasks during my summer work on the dairy. While the cows are in the milking barn I load up our freestall bedder and fill up the beds. Once I finish filling the beds, I use our tractor with a freestall rake attachment to groom the beds. This task has taught me the importance of cow comfort. It has also taught me how to properly use machinery to complete a task. On weekdays I spend the majority of my days performing this task

3. Activity Number Three.

\begin{tabular}{|l|l|l|l|}
\hline Year & \multicolumn{1}{|c|}{ Activity } & Where Attained & Student Hours \\
\hline $2007-2009$ & Cleaning the maternity barn on weekly basis. & Fabland Farms & 370 \\
\hline $\begin{array}{l}\text { Description of Activity: } \\
\text { Cleaning the maternity barn is a task that I perform on a weekly basis. I remove all the old rice hulls with a tractor } \\
\text { equipped with a rubber scraper. Once I remove all the old bedding, I stock pile the bedding outside of the barn and I } \\
\text { haul it to our ranch to be used as fertilizer at a later time. After the old material is moved, I come back with a loader and } \\
\text { refill the barn with rice hulls. This is an integral part of keeping cows comfortable and stress free during the calving } \\
\text { process. }\end{array}$ \\
\hline
\end{tabular}




\title{
VI. SUPPORTING DOCUMENTATION (continued)
}

C. Supporting Pictures

\author{
Matthew ouse
}

\section{DAIRY PRODUCTION}

\section{PHOTO \#}

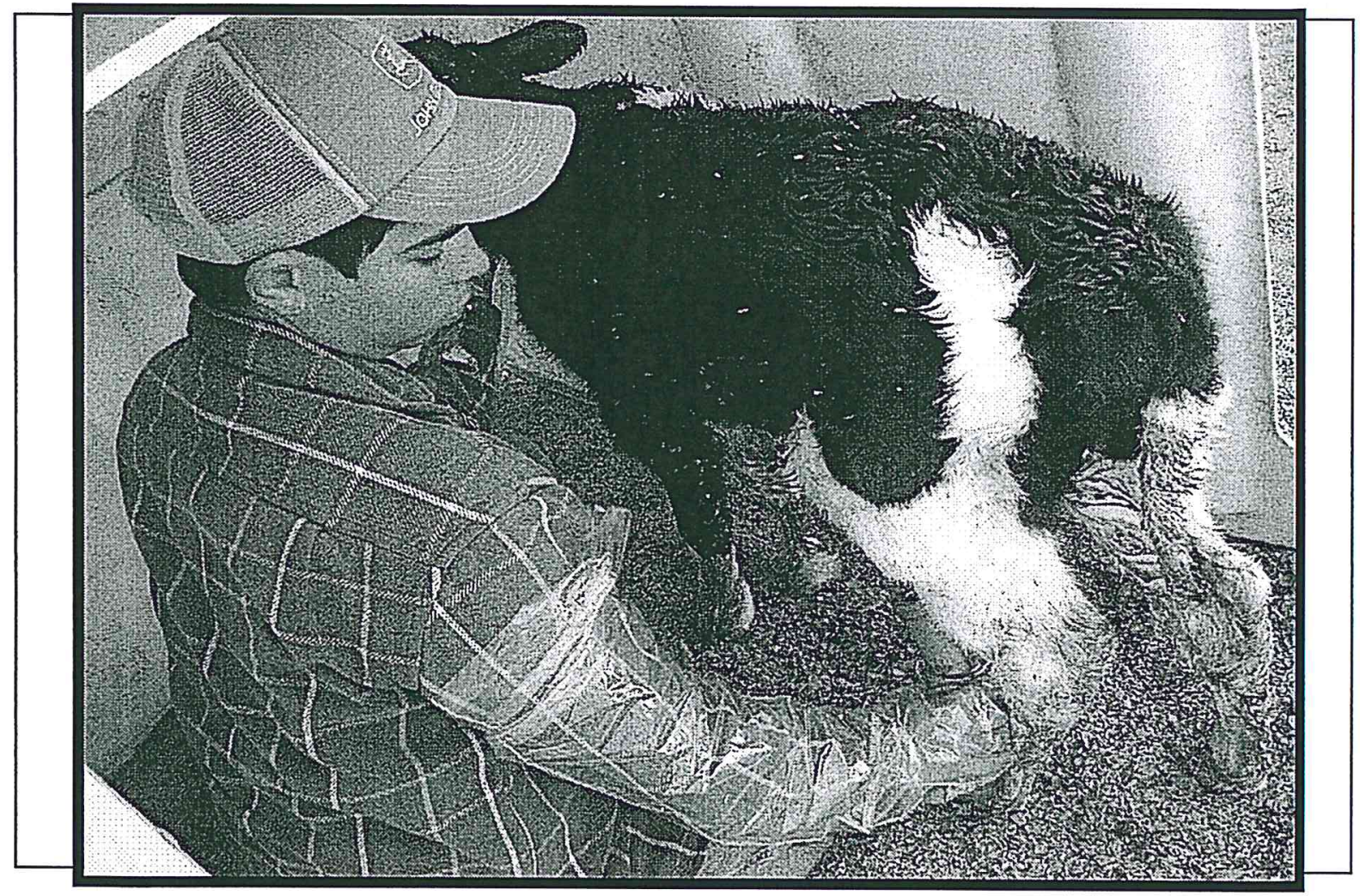

After the calf is born, it is immediately moved from the maternity barn to the calf hutch. Once the calf is there I bottle feed the calf 24 liters of colostrum. In this picture I am dipping the calfs navel in iodine to prevent infection; I typically will do this to 14 different calves in a week. The next day around 8 am the calves are sent to a calf ranch for three months. 


\section{SUPPORTING DOCUMENTATION (continued)}

C. Supporting Pictures

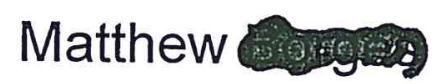

\section{DAIRY PRODUCTION}

\section{PHOTO \# 2}

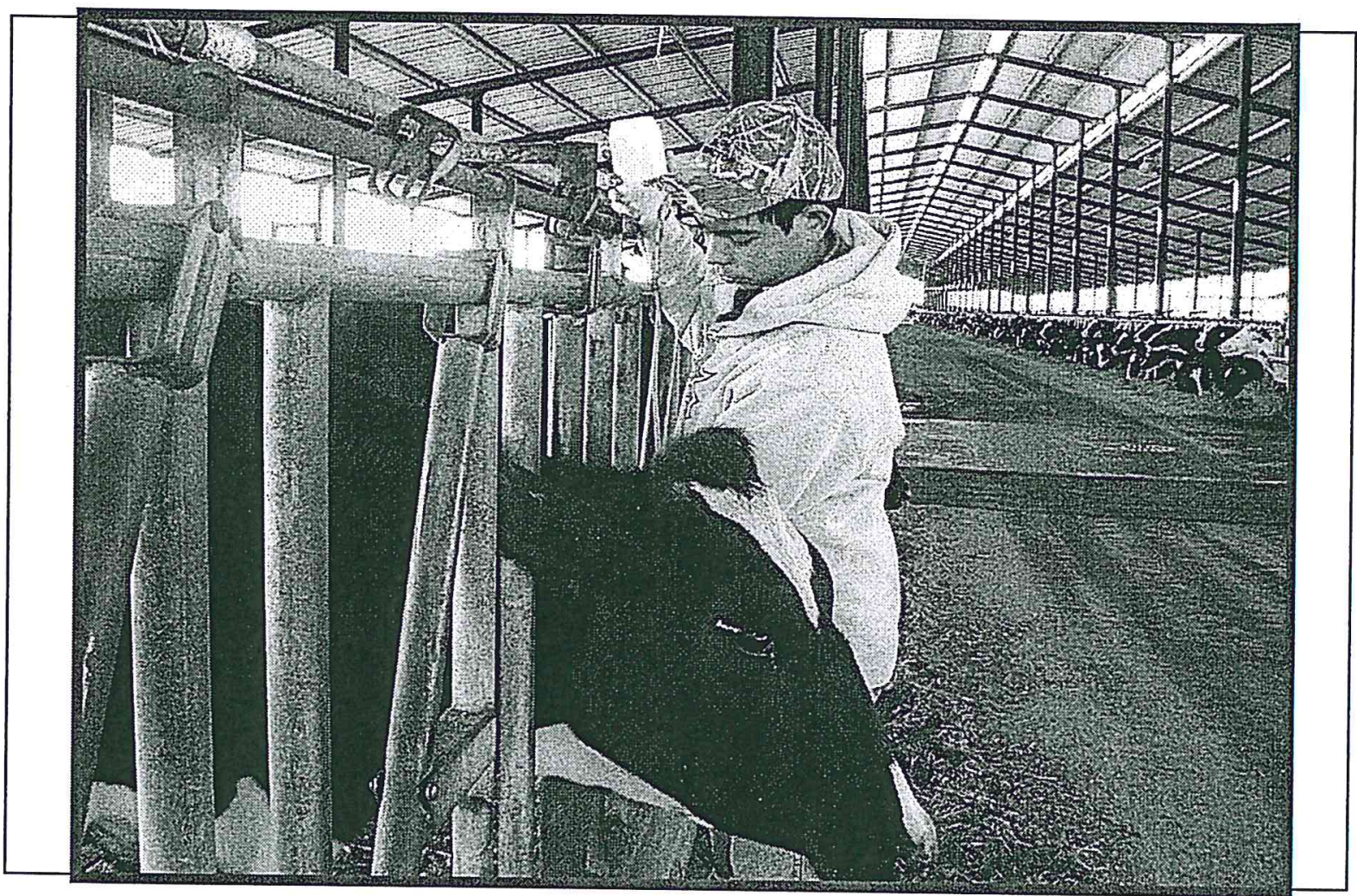

When a cow is moved in to the hospital pen, my first objective is to make a diagnosis as to what is wrong with the cow or heifer. In this particular photo I am giving Dextrose to a cow that is dehydrated in order to raise the cow's glucose level. Once the cow shows visible signs of improvement she will be moved back in to her normal milking string. 


\title{
VI. SUPPORTING DOCUMENTATION (continued)
}

\section{Supporting Pictures}

\author{
Matthew
}

\section{DAIRY PRODUCTION}

\section{PHOTO \#}

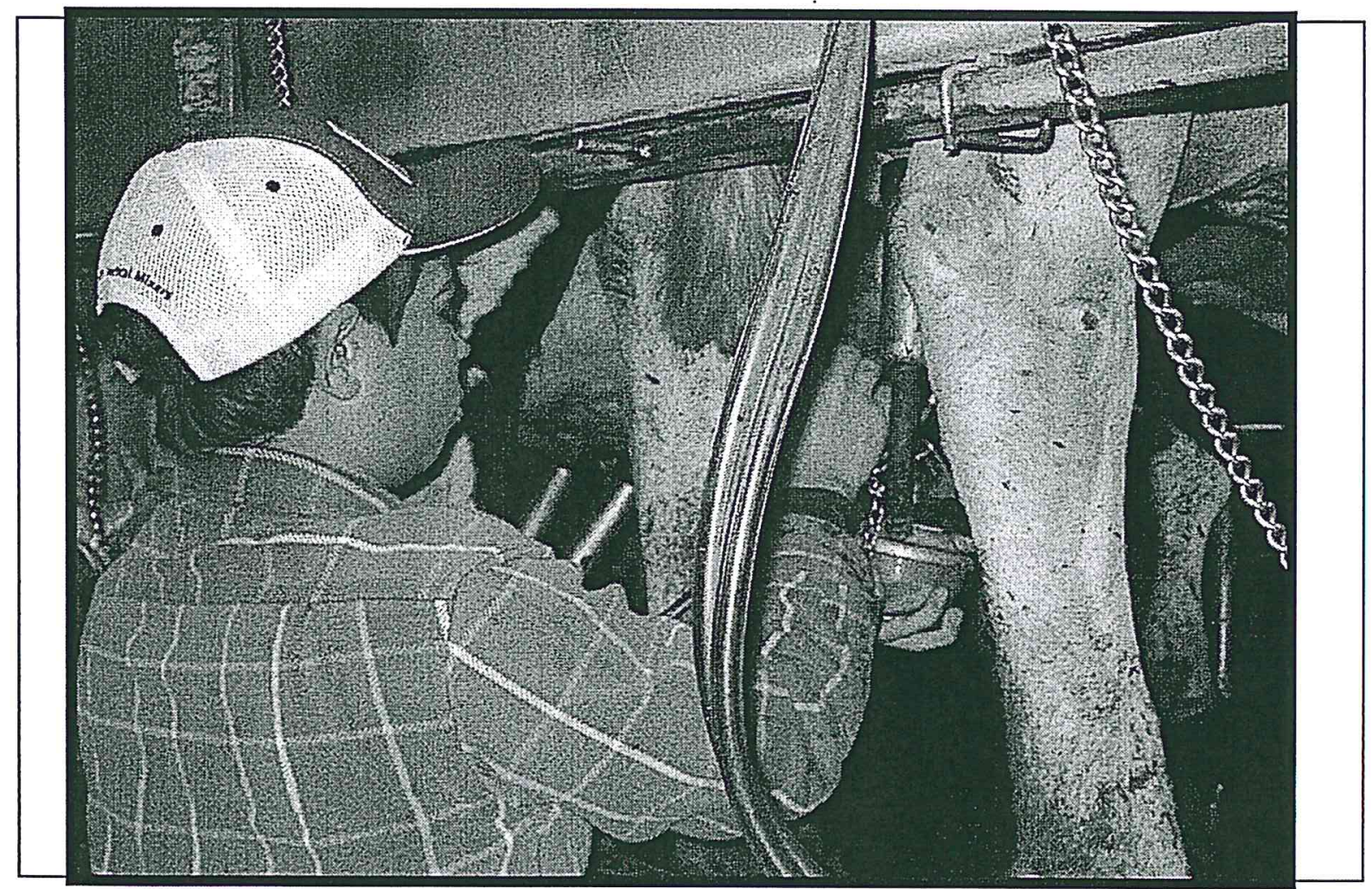

Twice, on a daily basis we have more than 2,700 milking cows come through the barn. When a cow enters the double 30 parallel milking parlor, my first step is to apply a pre dip solution to her teats and let it absorb for about three minutes. Second, I wipe the teats with rags to remove the solution and make sure milk is coming out of the teats. I apply the machine to the back teats first, then the front teats. Lastly, I apply a post dip iodine solution that prevents mastitis. 


\title{
VI. SUPPORTING DOCUMENTATION (continued)
}

\section{Supporting Pictures}

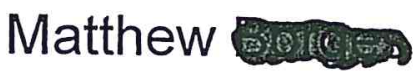

\section{DAIRY PRODUCTION}

\section{PHOTO \#}

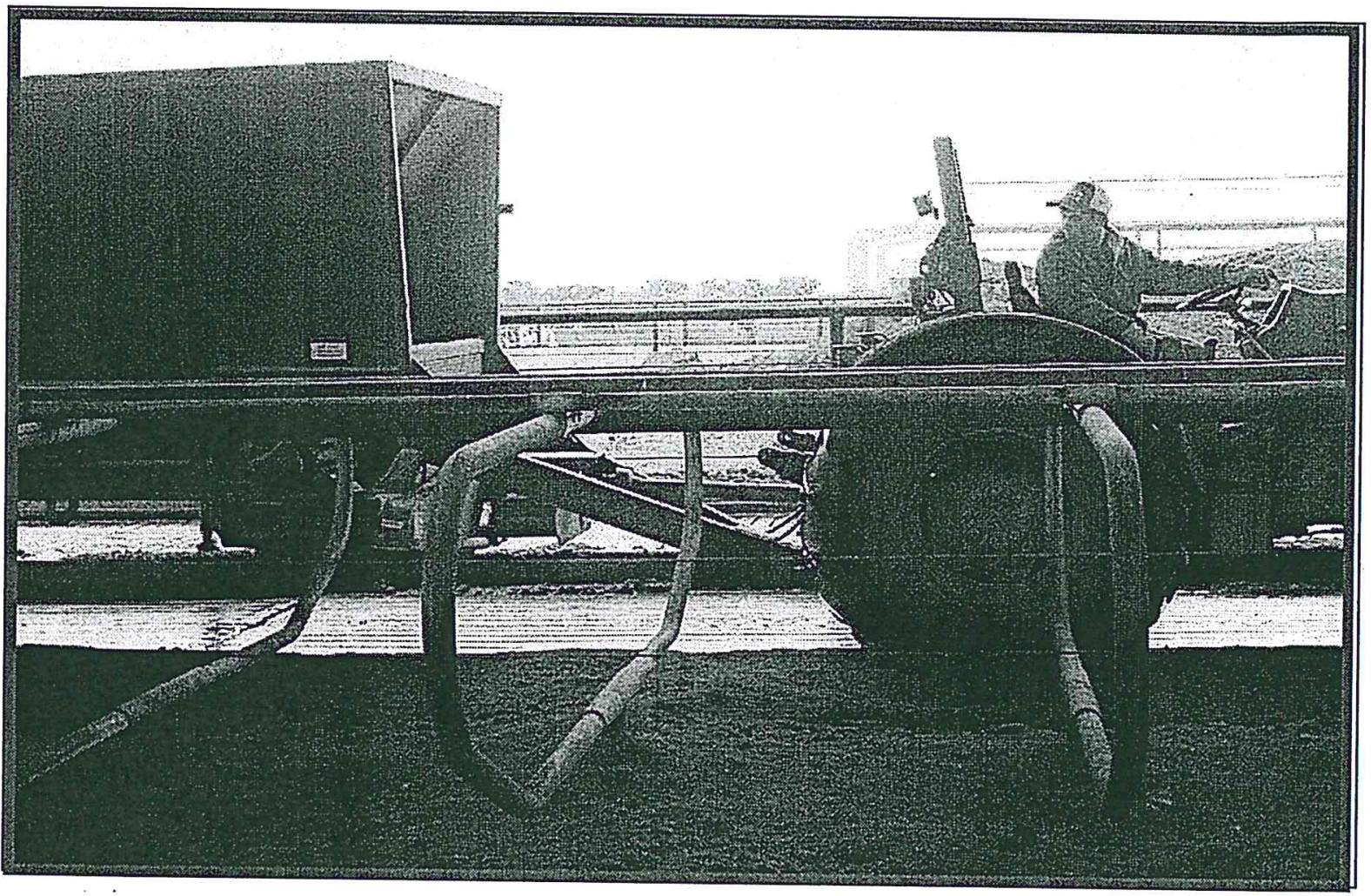

For our 2,700 milking cows, we strive to increase production from all of our cows. In order for this to happen, we need to maximize cow comfort, which in effect increases milk production and decreases diseases. In this photo I am using our free stall bedder to fill the bed with almond shells. Even though almond shells are expensive, they are the best way to increase cow comfort. By using almond shells the number of mastitis cases also decreases. 


\section{SUPPORTING DOCUMENTATION (continued)}

\section{Supporting Pictures}

\section{Matthew}

\section{DAIRY PRODUCTION}

\section{PHOTO \# \\ 5}

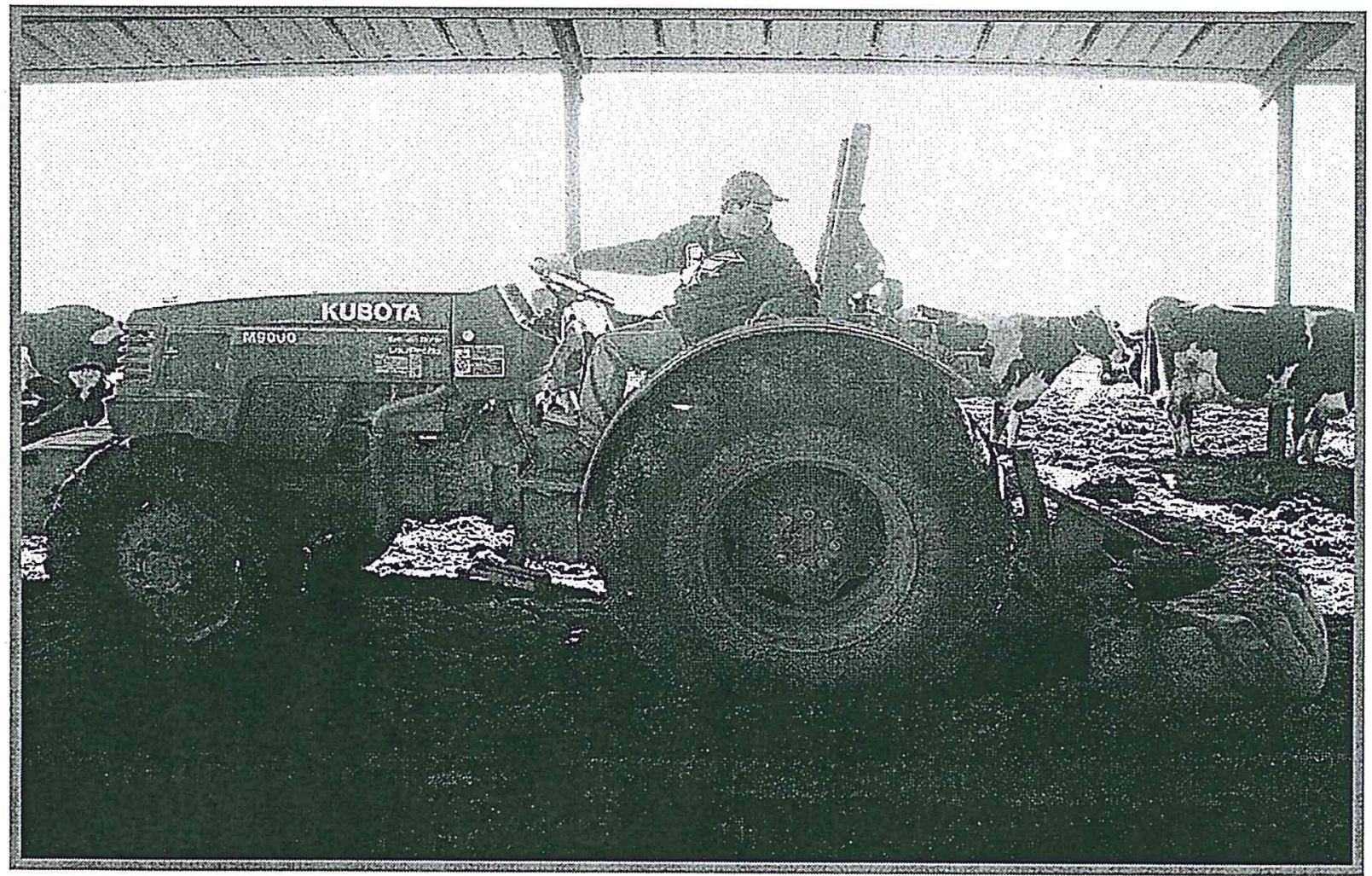

On average there are about thirty cows in our maternity barn at once. To ensure the cows comfort during the calving process we use rice hulls as bedding. On a weekly basis I clean out the barn using a tractor equipped with a rubber scraper. Once I remove all the old material, I come back with a loader and re-bed the barn with fresh rice hulls. The cows stress levels are decreased during the calving process when the cows are comfortable. 


\section{SUPPORTING DOCUMENTATION (continued)}

C. Supporting Pictures

Matthew

\section{DAIRY PRODUCTION}

\section{PHOTO \#}

6

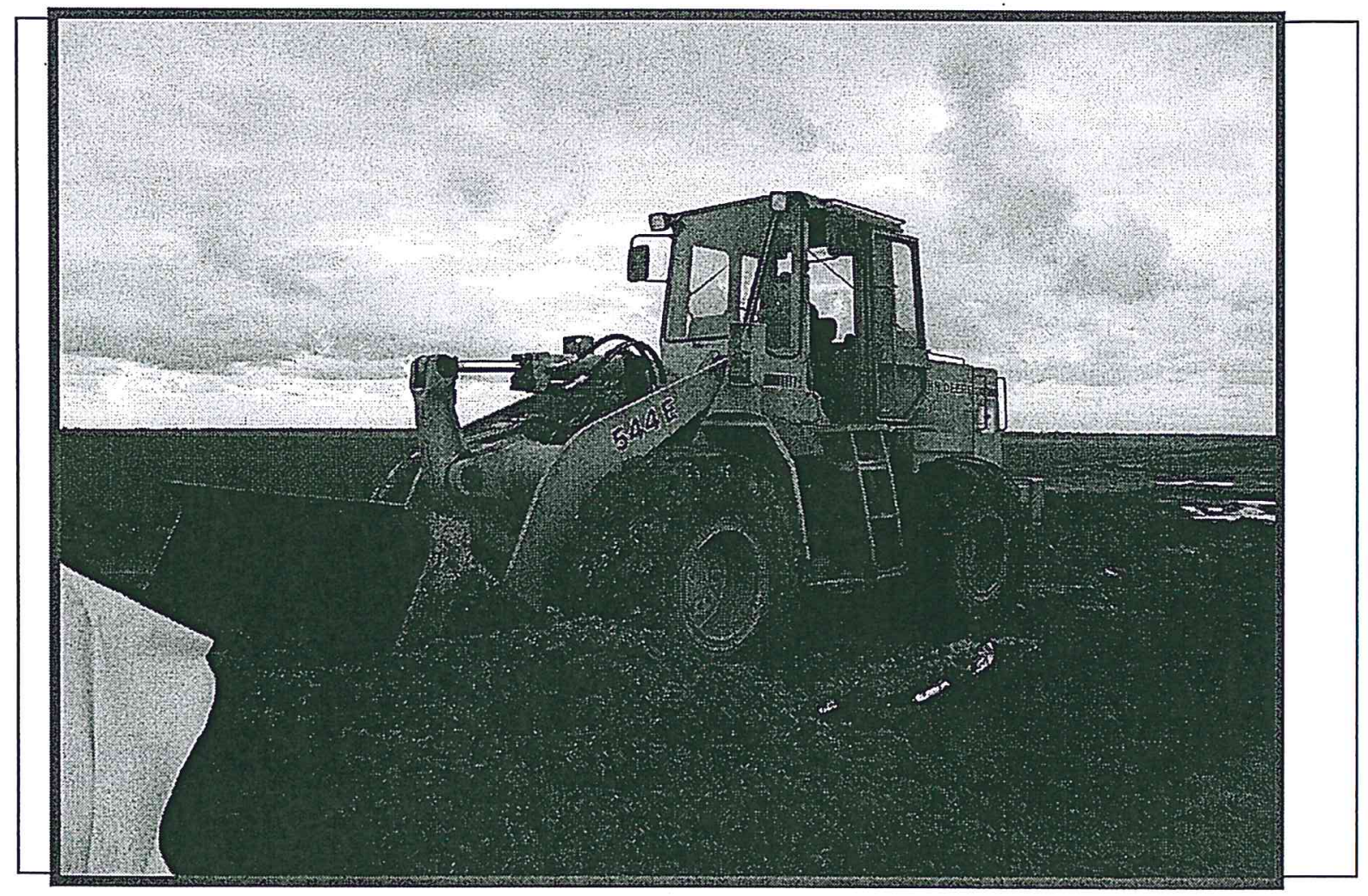

Due to our dairy's large size we store the majority of our feed in the back of the dairy. It is my job to load up our eight yard dump truck with silage that is used for our heifers. I make about four to five trips a day to haul our silage. Once the silage leaves the field it is sent to a bagging machine that packs it in a large bag and then seals it up so the silage does not spoil and we can use it in the winter months. 


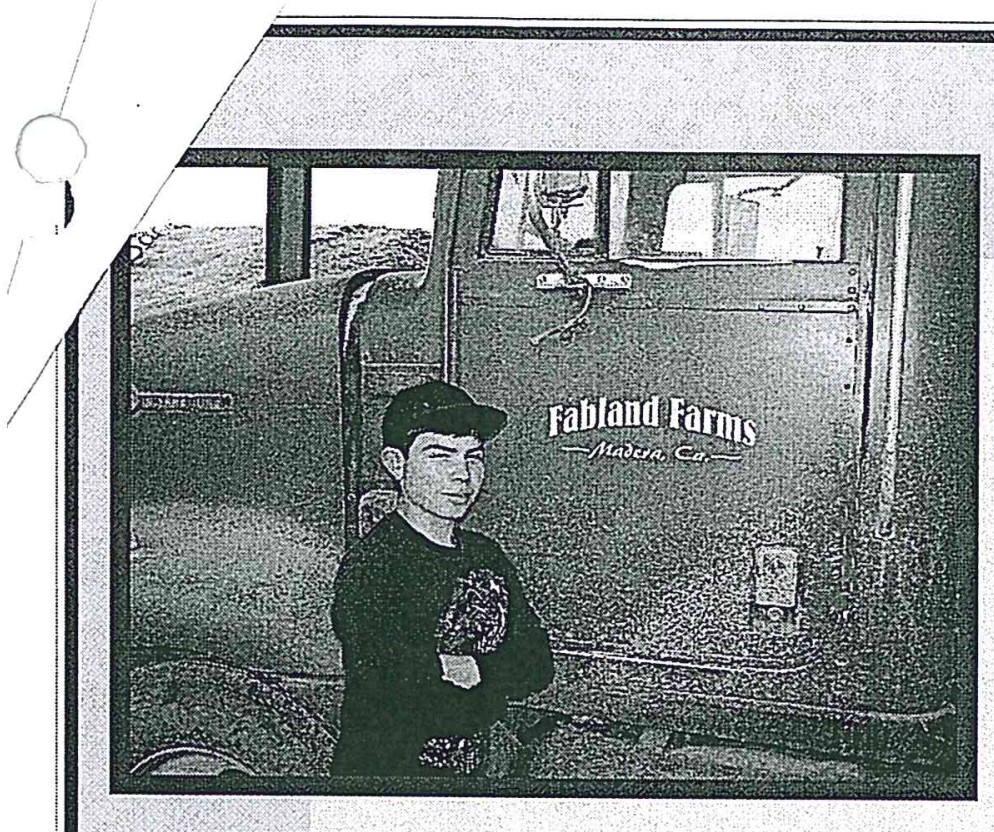

Standing next to one of 2 feed trucks that we use daily for the cows

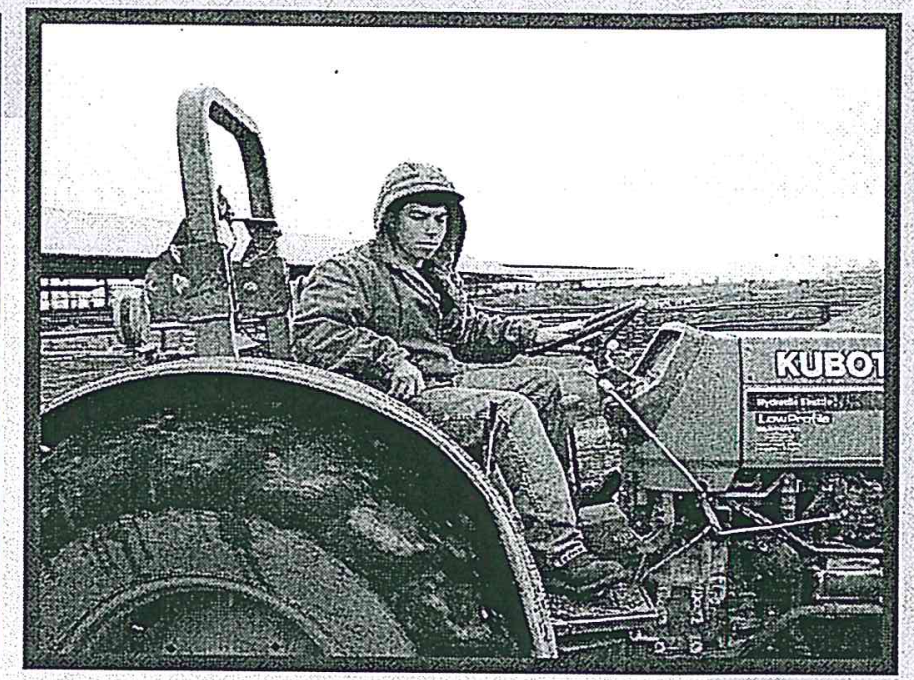

Scraping pens is necessary to keep up cleanliness and reduce disease.
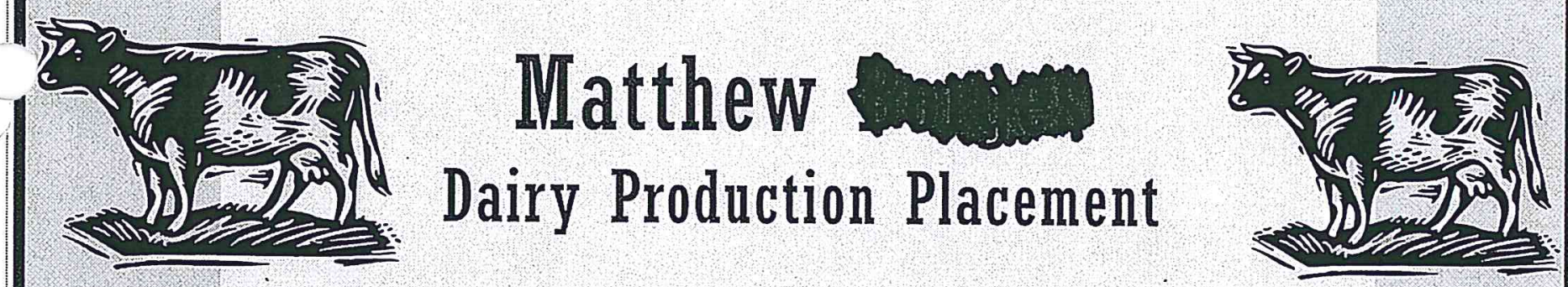

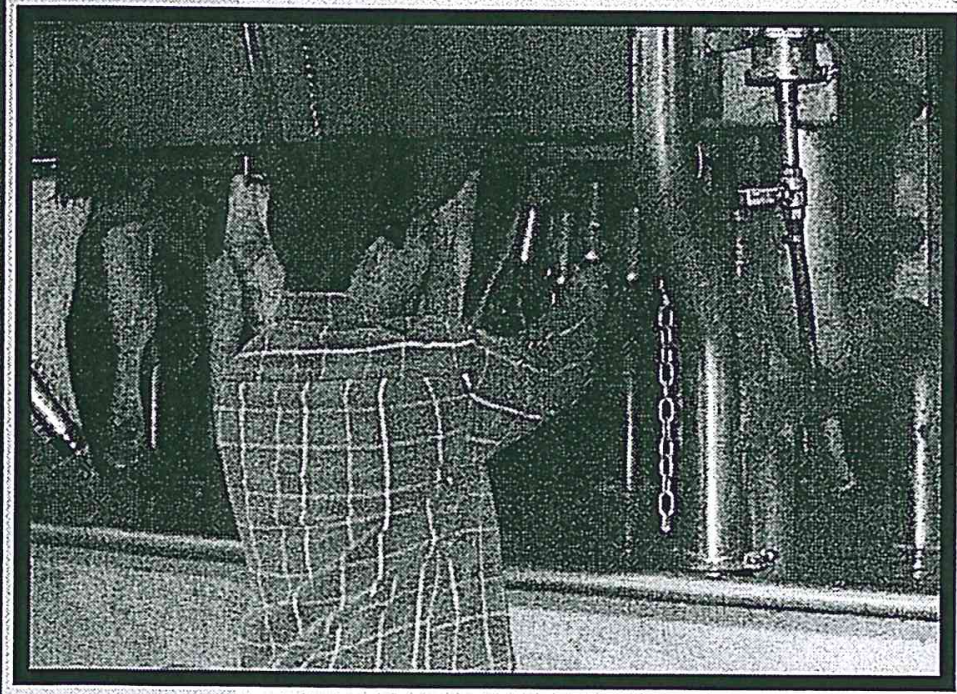

Getting ready to pull off the suckers and treat the next cow in line with lodine.

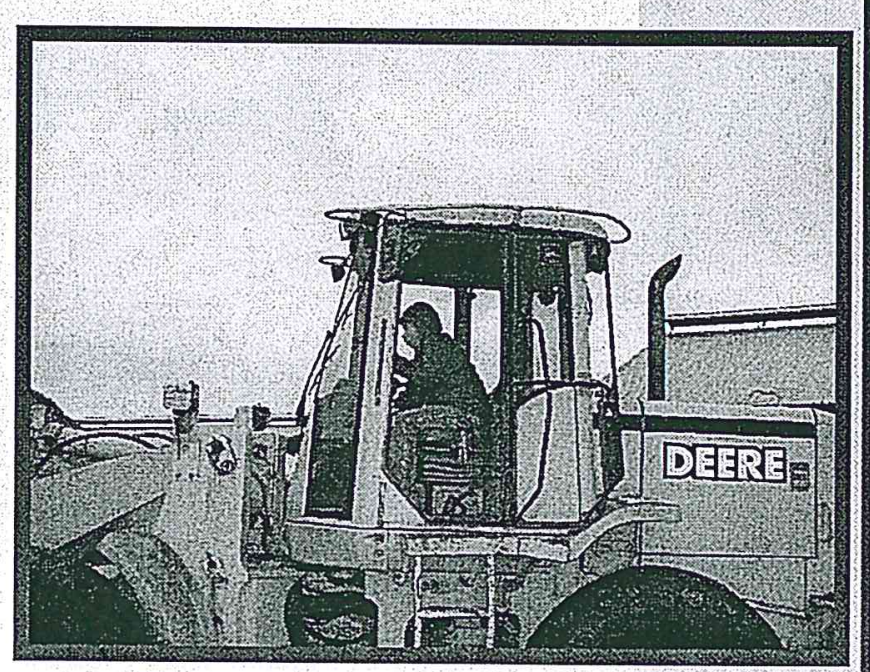

Moving the dirty almond hulls out to the compost pile after cleaning the pen. 


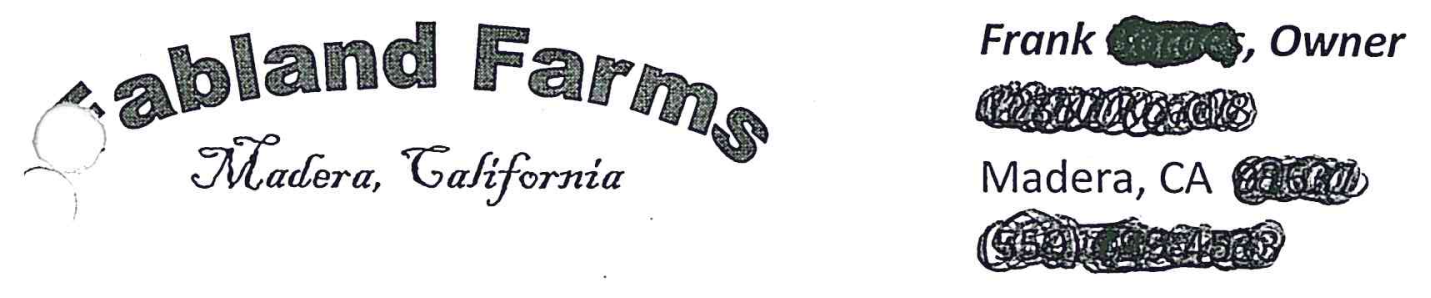

To Whom It May Concern:

It is with great pleasure to recommend Matthew for this award. Matthew has been an employee at Fabland Farms for $2 \frac{1}{2}$ years and is an excellent worker. Matthew's ability to learn quickly has made him a strong employee for my business. He has excelled from the beginning of his tenure with my farm. $\mathrm{He}$ is a hard worker, very reliable and always willing to take the necessary steps to better himself and my business. Matthew has always given $110 \%$ in every task that he has been given. His knowledge of the dairy industry makes him the ideal candidate for this award.

Matthew had always gone above and beyond when asked to do something while working on my Dairy. He has always been there when needed and will be a great dairyman when he is older. Matthew has always wanted to be involved with every aspect of my dairy and he is a great employee.

Every day that Matthew works, he learns something and he has the ability to use that knowledge to better himself as an employee. I believe that he is a great candidate for this proficiency and will be great with everything that crosses his path.

Sincerely,

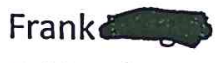

Fabland Farms 
NN. A 2+2 Agreement With A College 
OO. Reimbursement For Personal Expenses In All Integral activities Associated With The FFA, SOEP And Professional Development Examples 


\section{MADERA UNIFIED SCHOOL DISTRICT Madera South High School}

\section{ASSOCIATED STUDENT BODY \\ PURCHASE ORDER REQUEST/ CHECK REQUEST}

Organization (Club/sport): FFA-Activities

Account \#

Purchase Order $\square$ Open P.O. $\quad \square$ One Time Use

Check Request -invoice or original receipt must be attached

$A S B$ is not obligated to pay for an expenditure ordered by a staff member or student who has not first received pre approval using one of the following methods:

$\square$ Purchase Order \#:

$\square$ Item Listed on Yearly Budget

保 Meeting Minutes Attached* *Clubs only

vendor Name John Williams Phone $805-4>8-0 / 93$ Address 1813 Stoney Creek Ct Atwater Ca 95301 Reason for Purchase Order or Check Reimbursement

For Purchase Orders - attach a quote or list items below (Check Requests MUST have invoice attached):

\begin{tabular}{|c|c|c|}
\hline Quantity & Description & Amount \\
\hline & $\begin{array}{l}\text { Reimbursement for Skate Night } \\
\text { FFA Meeting Supplies }\end{array}$ & $396-00$ \\
\hline \multirow{2}{*}{\multicolumn{2}{|c|}{$\begin{array}{l}\text { Total Including Tax \& Shipping } \\
\text { Purchase Order DO NOT EXCEED Amount: }\end{array}$}} & \\
\hline & & 39500 \\
\hline
\end{tabular}

Date Needed: ASA P

Club Advisor/Athletic Coach:

Srent Greorg

Club Treasurer/Team Representative:

Activities Director:

Administrator:

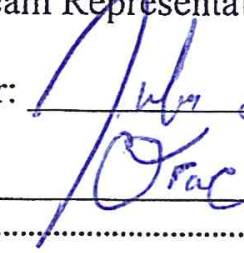

yins

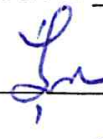

10
Mail to Payee

X'Place in Requester's Box

\section{BUSINESS OFFICE USE:}

P.O. Number:

Check Number:
Vendor Code:

Amount Paid: \$
Date: $10 / 10 /, 3$

Date: $10 / 10 / 13$

Date: $10 / 10 / 13$

Date: $10 / 10 / 13$ 